$X_{\text {sine }}$ 
$$
\bullet
$$

UNIVERSITY OF CALIFORNIA

..IIERRNIA COLLEGE OF MEDICINE - I IRRARY

\section{IIN 161971}

IDVINE. CALIFORNMA 92664 


\section{MATERIA MEDICA,}

\section{PHARMACY, PHARMACOLOGY}

AND

THERAPEUTICS. 
Digitized by the Internet Archive in 2007 with funding from

- Microsoft Corporation 


\title{
MATERIA MEDICA,
}

\section{PHARMACY, PHARMACOLOGY}

AND

\section{THERAPEUTICS.}

BY

W. HALE WHITE, M.D., F.R.C.P.,

PHYSICIAN TO AND LECTURER ON MEDICINE AT GUY'S HOSPITAL, LONDON ; AUTHOR OP A TRXT-BOOK OF GENERAL THERAPEUTICS.

\section{FIFTH AMERICAN EDITION \\ THOROUGHLY REVISED}

REYNOLD W. WILCOX, M.A., M.D., LL.D., PROPESSOR OF MEDICINE AND THERA PEUTICS AT THE NHW YORK POST-GRADUATE MEDICAL SCHOOL AND ATTENDING PHYSICIAN, TO THE HOSPITAL; VISITING PHYSICIAN TO ST. MAKK'S HOSPITAL; PRESIDENT OP THE AMERICAN THERAPEUTIC SOCIETY ; FELLOW OF THE AMERICAN ACADEMY OF MRDICINR, ETC.

\author{
PHILADELPHIA \\ P. BLAKISTON'S SON \& CO., \\ IOI 2 WALNUT STREET. \\ I 9 I I .
}

\section{Dr. J. Gaylord Goodrich}




$$
\begin{aligned}
& \text { QVम } \\
& \text { 1 } 589 \mathrm{~m} \\
& 1901
\end{aligned}
$$

Copyright, Igor, by

P. BLAKISTON'S SON \& CO. 


\section{PREFACE TO THE FIFTH EDITION.}

Endeavoring to keep this edition abreast of the times, I have revised the whole and added twelve pages of nęw matter. To Dr. E. C. Perry and other friends I am indebted for having directed my attention to some misprints.

[In presenting this edition the EDITOR would acknowledge his indebtedness, for numerous and valuable suggestions, to the many teachers, and especially to Dr. R. H. M. Dawbarn, who have used the previous editions in their classrooms, to the reviewers who have courteously, but critically, examined the book, and to Dr. Henry H. Rusby and the late. Dr. Charles Rice for important aid, particularly in regard to the definitions. The writings of most American and foreign authorities have been freely consulted. To bring this work up to date, his own journal files, which comprise all the periodicals devoted to the subjects upon which it treats, have been carefully studied. The unofficial preparations have been reviewed after consideration of the recent literature, and his practical experience with them. Notwithstanding careful condensation this edition is forty pages larger than its predecessor. For the convenience of the student a very complete index has been prepared by Dr. Ernest V. Hubbard, who has also kindly read the proof. Brackets [] are used throughout the book to indicate additions by the Editor.] 



\section{CONTENTS.}

Definitions, • • • • • • • • • • • •

Pharmacy, • • • • • • • • • • • • II

Pharmacology and Therapeutics, • • • • • $\quad 3^{8}$

Prescribing, • • • • • • • • • • 41

Actions of Drugs, . . . . . • • • . 46

Drugs acting on Processes outside the Body, . . . $44^{8}$

Drugs acting on the Blood, . . . . . • 51

Drugs acting on the Cardiac Mechanism, . . . . 55

Drugs acting on the Vessels, . $\quad . \quad$ - . 59

Drugs acting on the Skin, . • • • • • . 64

Drugs acting on the Urinary System, . . . . . . 66

Drugs acting on Bodily Heat, - • • • • • 7 I

Drugs acting on Respiration, • • • • • • • 74

Drugs acting on the Digestive Apparatus, - . $\quad$ • 79

Drugs acting on the Nervous [and Muscular] Systems, . • . 99

Drugs acting on the Organs of Generation, • • • • $~ I 12$

Drugs acting on Metabolism, • • • • • • • • $\quad$ • II5

Pharmacopqial Inorganic Materia Medica-

Group I. Water, • • • • • , I16

II. The Alkaline Metals, . • . • . • 122

III. The Alkaline Earths, . • • • • • 155

IV. Lead, Silver, Zinc, Copper, Bisnuth, Aluminum, . . 165

V. Iron and Manganese, . $\quad$ • $\quad$ • $\quad$ • $\quad$ • 188

VI. [Gold and] Mercury, . • • • • • . 209

VII. Arsenic, Antimony, Chromium, . • • • 223

VIII. Phosphorus, • • • • • • • 236

IX. Chlorine, Iodine, Bromine, • • • • 243

X. Sulphur [and its Compounds], • . • . 258

XI. Acids, . • . . • . • . 263

XII. Carbon and its Compounds, . • • . 17$)^{276}$ 
Pharmacopgeial Organic Vegetable Materia Medica-

Group I. Drugs acting on the Nervous System, . . . . 349

II. Drugs acting on the Heart, . . . . . 420

III. Drugs acting on the Respiratory Crgans, . . . 448

IV. Drugs which are Antiperiodic and Antipyretic, . . 463

V. Purgatives, . . . . . . . . 483

VI. Volatile Oils, . . . . . . . . 514

VII. Bitters, . . . . . . . . . 580

VIII. Astringents, . . . . . . . . 592

IX. Demulcents, . . . . . . . . 605

X. Parisiticides, . . . . . . . . . 622

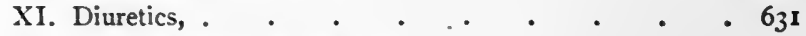

XII. Drugs acting upon the Uterus, . . . . 637

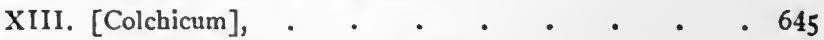

XIV. Drugs Related to Volatile Oils, . . . . . 648

XV. Drugs containing Important Acids, . . . . 654

XVI. [Flavoring Agents], . . . . . . . 66I

XVII. Coloring Agents, . . . . . . . 664

XVIII. Drugs whose Action is Mechanical, . . . . 665

XIX. Drugs [acting on Metabolism], . . . . . 669

Pharmacopoílal Organic animal Materia Medica-

[Group I. Drugs acting on the Nervous System, . . . . 679

II. Purgatives, $. \quad . \quad . \quad . \quad . \quad . \quad$. 680

III. Digestants, . $\quad . \quad$. $\quad . \quad$. $\quad 682$

IV. Drugs which are also Foods, . . . . . 684

V. Emollients, . . . . . . . . 690

VI. Coloring Agents, . . . . . . . . 692

VII. Drugs whose Action is Mechanical, . . . . 692

VIII. Organic Extracts (not official), . . . . . 70I

IX. Antitoxins and Serums (not official), . . . . 705]

Appendix I. Vegetable Natural Orders, . . . . . 713

[II. Animal Natural Orders, • • • . . 7 I8]

III. Latin Phrases used in Prescriptions, . . . 79

INDEX, • • • • • • • • • • • • • 721 


\title{
MATERIA MEDICA
}

\section{PHARMACY, PHARMACOLOGY}

\author{
AND \\ THERAPEUTICS.
}

\section{DEFINITIONS.}

Materia Medica.-[The materials used in the treatment of disease.

Therapeutics:-The application of remedial agents in the treatment of disease. It includes :

General Therapeutics.-The application of curative agents other than drugs and medicines. E.g., diet, climate, baths, venesection.

Rational Therapeutics.-Therapeutics based upon Pharmaco-dynamics. E.g., the use of digitalis for mitral disease.

Empirical Therapeutics.-Therapeutics based upon clinical experiences only. E.g., the use of colchicum for gout.

In this work we shall consider only that part of Therapeutics which is concerned with drugs.

Pharmacology.-The study of Materia Medica and Therapeutics, including the origin, history, properties and uses of drugs and medicines. It includes :

Pharmacognosy.-The study of the physical and chemical characters of drugs, and the art of identify- 
ing and selecting them in accordance with those characters.

Pharmaco-Dynamics. - The study of the action of remedial agents upon the organism of man, or the lower animals in a state of health.

Therapeutics. - Although the correct definition of this term is as given above, yet it is, for want of a better one, often used as the name of the branch of study which deals with Therapeutics. Therapo-Dynamics has been used in the same sense, but is faulty. Experimental Therapeutics has been suggested, but is not comprehensive.

Toxicology.-The study of the nature, effects and detection of poisons, substances which, introduced into the body inopportunely or in excessive amounts, are capable of destroying life. Courses of study and treatises upon Toxicology are, for convenience, commonly made to include the subject of antidotes and treatment, although this is, strictly speaking, a part of Therapeutics.

Pharmacy.-The art of preparing drugs in a form suitable for use as remedial agents and of dispensing them.

Pharmacopcia.-A code of remedial agents, usually with descriptions, definitions or directions, prepared by experts appointed by an authority of some kind, and intended to serve as a standard until superseded by a new one. By admitting certain articles to its pages, it declares them to be of importance, through the extent of their use, or to be entitled to confidence because of their value, or both, in the practice of medicine, but does not, necessarily, deny these properties to articles not admitted. It fixes their official title or titles, and often their leading synonym or synonyms. Usually it defines them, describes them with sufficient completeness to provide for identification and determination of the proper degree of purity, or strength, or both, and details and recommends such operations in preparing them as pertain to a dispensing pharmacy. It may, in addition, fix or limit doses and provide rules, formulæ, tables, and other information and directions of importance in the practice 
of pharmacy and medicine. It also fixes a date upon which its authority shall commence. Everything contained in the United States Pharmacopœia (abbreviation "U. S. P.") is said to be "official." " Not official," as used in this work, refers only to the U. S. P. Many drugs and preparations are so designated which are, however, official in the British Pharmacopœia (abbreviation "B. P.")

The United States Pharmacopœia is prepared by a committee, meeting at the beginning of each decade, consisting of delegates appointed by invitation extended by the President of the preceding Convention, to all incorporated medical and pharmaceutical societies and medical and pharmaceutical colleges, and to the United States Army, Navy, and Marine Hospital Service. By Congressional action the U.S. P. is made a legal authority in the conduct of the Department of Customs, of the Army, Navy, and Marine Hospital Service, and of the District of Columbia and other Territories within the jurisdiction of the United States laws. By legislative enactment it is also made a legal authority within the jurisdiction of many States. With these exceptions its authority is but moral. The last edition became official on January I, I 894.]

\section{PHARMACY.}

[Pharmacy covers a field of nearly as much importance, breadth and difficulty as that of medicine itself, and requires a special, extensive and thorough preparation. It should never be practiced by the physician, when the services of a competent manufacturing or dispensing pharmacist can be utilized. The physician should, however, be acquainted with the general principles and most details of the science and art of Pharmacy, that he may judge intelligently of the services rendered him by the pharmacist, and also be prepared to act with safety himself in cases of emergency. A pharmaceutical education to this extent, accompanied by dispensary practice, should be provided for in every thorough course of medical study. The more important terms pertaining to Pharmacy are defined and explained below. 


\section{DEFINITION OF TERMS AS APPLIED TO SUBSTANCES OF VEGETABLE ORIGIN.}

Alkaloids.-(Their English names terminating in ine, their Latin names terminating in ina.) Compounds of carbon, hydrogen and nitrogen, and usually containing also oxygen, either existing in the plant as proximate principles, or being derived from other alkaloids, having basic properties, and forming salts, usually crystallizable, with acids, without displacing any of the hydrogen of the latter. The chief characters are as follows:

(I) Either

(a) solid, mostly crystalline and colorless, non-volatile, or

(b) liquid and volatile.]

(2) They turn red litmus paper blue.

[(3) They are soluble in alcohol, chloroform, benzin, benzol, and often in ether. They are insoluble in water, but not so their salts, while the latter are insoluble in chloroform, ether, benzin and benzol.

(4) They are usually precipitated from saline solutions by alkalies.

(5) [One or more of the following will precipitate them: tannic, phosphomolybdic or picric acid, potassio-mercuric iodide or auric chloride.]

(6) Their solutions are usually [intensely] bitter.

[Alkaloids are, as a class, the most energetic and important medicinal constituents of plants. Examples in U. S. P. : Atropine, Morphine, Strychnine.

Glucosides.-(Their English names terminating in in, their Latin names terminating in inum.) Bodies which, heated with a diluted mineral acid and water, or by the action of a ferment, split up into glucose and some other substances (alcohols, aldehydes, phenols). Examples in U.S. P.: Salicinum, Picrotoxinum.

Amaroids or Bitter Principles (their names ending in in and inum as above) are of such varied nature that they do not admit of any chemical diagnosis. The term includes all distinctly bitter extractives of definite chemical composition other than alkaloids and glucosides.

Glucosides and Amaroids are not the only principles whose names end in in.

Fixed Oils are ethers of the higher fatty acids which] at ordinary temperatures remain liquid. The usual fatty acids 
entering into the composition of fixed oils are oleic, palmitic, and stearic.

Example: Olive oil consists of a mixture of a combination of oleic acid $\left(\mathrm{C}_{18} \mathrm{H}_{34} \mathrm{O}_{2}\right)$ with glyceryl $\left(\mathrm{C}_{3} \mathrm{H}_{5}\right)$ and palmitic acid $\left(\mathrm{C}_{16} \mathrm{H}_{32} \mathrm{O}_{2}\right)$ with glyceryl. That is to say, ordinary olive oil is a mixture of two oils having the formulæ $\mathrm{C}_{3} \mathrm{H}_{5}\left(\mathrm{C}_{18} \mathrm{H}_{33} \mathrm{O}_{2}\right)_{3}$ and $\mathrm{C}_{3} \mathrm{H}_{5}\left(\mathrm{C}_{16} \mathrm{H}_{31} \mathrm{O}_{2}\right)_{3}$ respectively. When acted upon by caustic alkalies or metallic oxides they form soaps (oleates, palmitates, or stearates of metals) and glycerin. This process is called saponification, e.g., $\mathrm{C}_{3} \mathrm{H}_{5}\left(\mathrm{C}_{18} \mathrm{H}_{33} \mathrm{O}_{2}\right)_{3}+3 \mathrm{NaOH}=3 \mathrm{NaC}_{28} \mathrm{H}_{33} \mathrm{O}_{2}$

Sodium oleate (Hard soap.) Glycerin.

Fixed oils are obtained by expression or by boiling with water and skimming off the melted oil, from the fruits or seeds of plants, or from animal tissues. When pure they are usually [colorless or pale] yellow ; they float on water and cause a greasy mark on paper. They are called fixed because they cannot be distilled without decomposition. They are soluble in ether, chloroform, [turpentine and volatile oils.

Those in U. S. P. are Oleum Amygdalæ Expressum, Lini, Morrhuæ, Olivæ, Ricini, and Tiglii.] Cretion Cris.

Fats are fixed oils which are solid at ordinary temperatures; if extracted by expression, sufficient heat to melt them must be used.

[Examples in U. S. P. : Oleum Theobromatis, Adeps.

The same definitions will apply to fixed oils and fats of animal origin.]

Waxes are chiefly composed of fatty acids combined with monohydric alcohols homologous with methyl alcohol.

Volatile or Essential Oils only resemble fixed oils in being soluble in the same media. They do not leave a greasy mark on paper. They are mostly inflammable, and mostly lighter than water. They are highly aromatic, and sufficiently soluble in water to impart their odor and taste to it. Most are prepared by distillation - that is, by passing a current of steam through the substance from which they are extracted, the steam is condensed, and the oil either floats to the top or sinks to the bottom of the water. A few, as oil of lemon, are obtained by expression. Their composition varies [greatly, and they are of four classes: 
(a) Terpenes, which consist of carbon and hydrogen; e.g., Oil of turpentine.

(b) Oxygenated, containing oxygen; e.g., Oil of eucalyptus.

(c) Sulphurated, containing sulphur; e.g., Volatile oil of mustard.

(d) Nitrogenated, containing hydrocyanic acid; e.g., Oil of bitter almond.]

They may contain aldehydes, phenol derivatives, ethers or ethereal salts, alcohols or ketones, generally associated with terpenes of varying composition.

[Elæoptens, their names ending in ene, are liquid hydrocarbons isomeric with terpene $\left(\mathrm{C}_{10} \mathrm{H}_{16}\right)$.

Stearoptens, their names usually ending in ol, are oxidized hydrocarbons, usually solid and crystalline.

Examples in U. S. P. : Camphora, Menthol.]

Resins [are of very indefinite composition]. They are among the products of oxidation of volatile oils, [being usually oxidized terpenes. They are solid, most uncrystallizable, fusible, not volatile, combustible, insoluble in water, mostly soluble in alkalies and volatile oils, and also in one or more of the following : alcohol, ether, chloroform, and fixed oils. Since] they are insoluble in water, but not in alcohol, they may be prepared by extraction with alcohol and precipitation with water. This is the reason for the precipitate which falls when water is added to a resinous tincture. Those which combine with alkalies form resin soaps. Hence the alkali in Tinctura Guaiaci Ammoniata, and Tinctura Valerianæ Ammoniata. [When occurring naturally, there are usually two or more resins mixed.

The U. S. P. resins are Resina Copaibx, Jalapæ, Podophylli, and Scammonii, Pix Burgundica, and Mastiche.]

Oleoresins are natural solutions of resins in volatile oils.

[Those in the U. S. P. are Oleoresina Aspidii, Capsici, Cubebæe, Lupulini, Piperis, and Zingiberis. ]

Balsam, [is a term used in several different ways. As to the U. S. P. articles, they are liquid or soft products containing resin, an odorous principle, and benzoic, or cinnamic acids, or both. 
Those in U. S. P. are Balsamum Peruvianum, and Tolutanum, Benzoinum, and Styrax.

Resins containing benzoic or cinnamic acids are sometimes called solid balsams.]

Gums, are exudations from plants, [having an insipid taste, insoluble in ether and alcohol, in water either dissolving to form a mucilage or swelling to form an adhesive jelly.] They consist of one or more of [the following] :

(a) Arabin or soluble gums, e.g., Acacia.

(b) Bassorin or partially soluble gums, e.g., Tragacantha.

(b) Cerasin or insoluble gum.

Solutions of gum are precipitated by alcohol.

Gum-resins are exudations from plants consisting of a mixture of [one or more] gums and [one or more] resins. When they are rubbed with water the gum dissolves and the resin remains mechanically suspended in the solution, [forming an emulsion.

The U. S. P. gum-resins are Ammoniacum, Asafœtida, Cambogia, Myrrha, and Scammonium.]

\section{PHARMACEUTICAL PROCESSES.}

Many of these, as filtration, precipitation, etc., need no explanation, but the following require a few words.

[Dialysis.-The process of separating crystalloids from colloids by bringing them in a mixed solution, into contact with one side of a membrane, such as a bladder, parchment or a parchment paper, which has water in contact with its other side, and resulting in the passage into the water of the crystalloid to form the "diffusate," the remainder constituting the "dialysate." In this way dialysed iron is prepared by diffusing the excess of the crystalloid acid solution.

Displacement.--Another name for Percolation.]

Elutriation consists in diffusing an insoluble powder in water, letting the heavier part settle, then decanting the supernatant fluid. The heavier powder in this is allowed to settle, the 
fluid decanted, and so on until a fluid containing powder of the required fineness is obtained.

Levigation consists in reducing a drug to powder by triturating it with a little water and drying the resulting paste.

Lixiviation consists in the extraction with water of the soluble matter of the ashes of anything which has been ignited, the solution being called a "lye."

Maceration.-[The extraction of the soluble portions of a substance which is not wholly soluble in the menstruum, by prolonged contact therewith.

Percolation.-The extraction in a suitable vessel (the " percolator") of the soluble constituents of a powder by the descent through it of a solvent (the "menstruum"), the resulting solution being called the "percolate."] The marc is the material after its exhaustion by maceration or percolation. [Percolation enters into the manufacture of a great majority of the official preparations of organic drugs.]

Repercolation consists in using the liquid obtained by a substance as the menstruum for percolating a second portion of the same substance, and using the liquid from this second percolation as a menstruum for percolating a third portion of the same substance, and so on as often as may be desired.

Scaling.-Scale preparations are made by drying concentrated solutions of drugs on glass plates. The solid left behind forms a thin film on the plate, and this film is broken up. Some preparations of iron are [obtained by scaling].

Standardizing.- [Specifying an upper or lower limit, or both, of the active constituent which a drug or its preparation must contain in order to be official, and prescribing an appropriate process for its determination. Satisfactory processes for standardizing are extremely difficult to establish, and have been adopted for the following only:

Cinchona, not less than 5 per cent. of total alkaloids.

Cinchona rubra, " 5 " " "

Extractum nucis vomica, 15 " " "

Extractum nucis vomicæ fluidum, 1.5 per cent. of total alkaloids.

Tinctura nucis vomicæ, 0.3 per cent. of total alkaloids. 
Opium, not less than 9 per cent. of crystallized morphine.

Opii pulvis, not less than 13 nor more than 15 per cent. of crystallized morphine.

Extractum opii, 18 per cent. of crystallized morphine.

Tinctura opii,

Tinctura opii deodorati, $\}$ I. 3 to $\mathbf{I} .5$ per cent. of crystallized morphine.]

Vinum opii,

WEIGHTS, MEASURES, AND SYMBOLS.

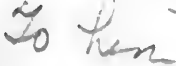

Weights [(Apothecaries' or Troy weight).

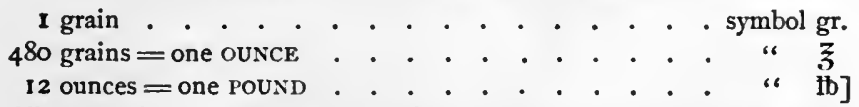

The scruple (20 grains, symbol $Э$ ) is rarely used, and the drachm (6o grains, symbol 3 ) is commonly used.

\section{Measures of Capacity.}

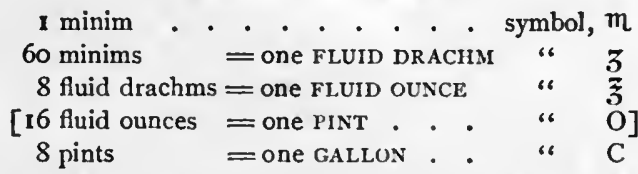

Usually $\boldsymbol{Z}$ and $\vec{\xi}$ are written $\mathrm{fl} Z$ and $\mathrm{f} \boldsymbol{\xi}$ when they stand for fluid drachms and fluid ounces.

\section{Relations of Measures to Weights.}

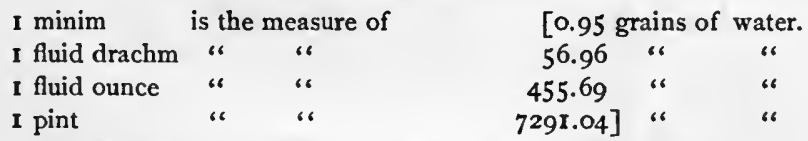

A I per cent. solution is approximately a grain in 110 minims.

A fluid grain is the volume of one grain of water at $60^{\circ} \mathrm{F} . ;\left[15.5^{\circ} \mathrm{C}\right]$; that is to say, it is a little over a minim [(1.05 $\mathrm{m})$.]

In the pharmacopoial description of the various proportions which several parts of a compound bear to one another, the word parts means parts by weight ; the term fluid parts signifies the volume of an equal number of parts of water.

Metrical System.-This, which is as follows, is official on the Continent and in the [U. S. P. and] B. P. for the making of drugs and preparations.

WEIGHTS.

1 milligramme $=0.001$ gramme.

I centigramme $=0.01$ " "

I decigramme $=0.1$ " "

1 gramme $=$ weight of I cubic centimetre of distilled water at $4^{\circ} \mathrm{C}$.; $39.2^{\circ} \mathrm{F}$. Abbreviation, gm. 
I dekagramme $=10.0$ grammes.

I hektogramme $=100.0$ "

1 kilogramme $=1000.0$ " Abbreviation, kilo.

MEASURES.

I millilitre $=$ I cubic centimetre (abbreviation, c.c.) $=$ the measure of $\mathbf{I}$

1 centilitre $=10 \mathrm{c} . \mathrm{c} .=$ the measure of $10 \mathrm{gm}$. of water. [gm. of water.

I decilitre = 100 c.c. $=$ " " 100 " "

I litre $\quad=1000$ c.c. $=$ " " 1000 " (I kilo of water).

Conversion of [United States] to Metrical System.

WEIGHTS.

\begin{tabular}{|c|c|c|}
\hline 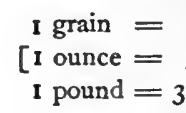 & $\begin{array}{l}0.0648 \mathrm{gm} . \\
31.103 \mathrm{gm} . \\
373.250] "\end{array}$ & \\
\hline \multicolumn{3}{|c|}{ MEASURES. } \\
\hline ninim & {$[0.0616 \mathrm{c}$} & c.c. \\
\hline uid drachm $=$ & 3.75 & \\
\hline uid ounce $=$ & 29.57 & \\
\hline$=$ & $=473.18$ & \\
\hline allon & $=3785.43]$ & \\
\hline
\end{tabular}

Conversion of Metrical to [United States Weights and Measures.] WEIGHTS.
1 milligramme $=$
0.015432 grain.
$I$ gramme $=15.43235$ grains.
I kilogramme $=15432.356$

MEASURES.

I cubic centimetre $=[16.23$ minims.

1 litre $(1000$ c.c. $)=33.81]$ fluid ounces.

In prescribing on the Continent all liquids are weighed.

The weight used for liquids and solids is grammes, and this word is not expressed. Thus-

Magnesii sulphas

$20.0=20$ grammes of magnesium sulphate.

Hydrargyri chloridum mite $0.5=$ half a gramme of mild mercurous chloride. Tinctura rhei

$1.5=$ a gramme and a half of tincture of rhubarb.

\section{Domestic Measures.}

A TEA-Spoonful is about a fluid drachm, [4 c.c]. Usually it is a little more, viz., nearly 5 c.c.

A DESSERT-SPOONFUL is about two fluid drachms, [8 c.c.].

A TABLE-SPOONFUL is about half a fluid ounce, $[15$ c.c. Usually it is almost 20 c.c.]. 
A WINE-GLASSFUl is about one and a half to two fluid ounces, [45 to 60 c.c.].

A TEA-CUPFUl is about five fluid ounces, [150 c.c.].

A BREAKFAST-CUPFUL is about eight fluid ounces, [240 c.c.].

A TUMBLERFUl is about eleven fluid ounces, [330 c.c.].

A DROP is often taken as being a minim, but drops vary so much in size that they should never be used for children, nor as a measure of powerful drugs. For example, the number of drops in a fluid drachm of the United States Syrup of Acacia is 44, of Water 6o, of Alcohol 146, of Chloroform 250.

[Spoons, glasses and cups vary so much in capacity that it is never safe to prescribe solutions of powerful drugs to be measured by them. The use of glass graduates, which can be obtained accurately marked, should be insisted upon.]

\section{PHARMACOPCIAL PREPARATIONS AND THEIR DOSES.}

Most drugs are not, in their natural state, suitable for administration. They are either too bulky, too nauseous, or contain noxious principles. Preparations suitable for administration are, therefore, prepared from them according to "official" pharmacopoial directions. The doses of the various drugs and their preparations which may safely be given to an adult [are taken from the "Tables for Doctors and Druggists," 2d ed., compiled by Eli H. Long, M.D. ;] but these doses are often not rigorously adhered to in prescribing. They vary with the purpose for which the drug is required and the age of the patient (see Doses, p. 39). The following is an account of the preparation of the pharmacopoial preparations, and the attempt has been made to arrange the doses so as to make them easy of remembrance.

[Unless otherwise specified, the preparations are for internal use.]

1 Aceta [(Vinegars). - Liquid preparations resulting from the extraction of the drug with dilute acetic acid, and filtering. The U. S. P. contains two.

$$
\begin{aligned}
& \text { Dose. } \\
& \text { Acetum Opii . . . . . . . . } 3-20 \mathrm{~m} ; .20-1.20 \text { c.c. } \\
& \text { Acetum Scillæ . . . . . . } 10-45 \mathrm{~m} ; .60-3.00 \text { c.c.] }
\end{aligned}
$$

Aquæ [(Waters).-Preparations made by impregnating water with a volatile substance.

Mostly made by triturating the substance with precipitated calcium phosphate and filtering. Those in the U. S. P. thus made are-

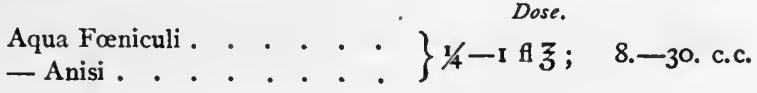




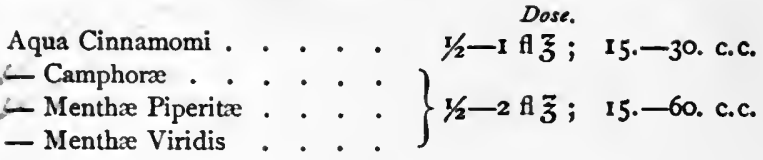

Three are made by distillation :
Aqua Aurantii Florum Fortior . . indeterminate.
- Destillata . . . . . . . indeterminate.
- Rosæ Fortior . . . . . . indeterminate.

Six are simple solutions in cold water :

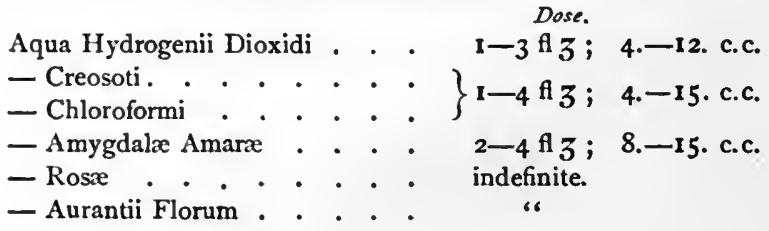

Three are made by passing gases through water:

Aqua Ammonir Fortior . . . $3-6 \mathrm{~m} ; .20-.40$ c.c.

- Ammoniæ . . . . . . 10-20 $\mathrm{m} ; .60-1.20$ c.c.

- Chlori . . . . . . I- $4 \mathrm{fl} 3 ; 4 .-15$. c.c.

1 Cerata (Cerates).-Adhesive preparations for external use, containing wax, capable of being spread at ordinary temperatures, and not melting at the temperature of the body. Six are official in U. S. P.
Ceratum
- Camphore
Ceratum Cantharidis
- Cetacei
Ceratum Resinæ
- Plumbi Subacetatis

Charta (Papers).-Non-absorbent papers coated with plaster-like preparations and used like plasters. The U. S. P. contains two :

Charta Sinapis, made from mustard.

- Potassii Nitratis, made from potassium nitrate.

Collodia (Collodions).-Preparations for external use, either simple collodion, a solution of pyroxylin in ether and alcohol, 
or impregnated with an active substance.] When applied externally a protective film is formed owing to the rapid volatilization of the solvent. [The U. S. P. contains four:

Collodium.

- Flexile.

Collodium Cantharidatum.

- Stypticum.

7 Confectiones (Confections). - Synonym._Electuaries. In England also called Boluses or Conserves. Permanent pasty preparations of powdered drugs thoroughly triturated with syrup or honey. The U. S. P. contains two:

Confectio Rosæe, used as a basis for pills.

- Sennæ ... dose, I-2 Z; 4.-8. gm.

7 Decocta (Decoctions).-Liquid preparations of organic drugs, 5 grammes of drug to 100 c.c. of decoction unless otherwise specified by the physician or Pharmacopœia, made by exhausting the drug by boiling in water, straining the resulting liquid, and adding sufficient cold water to secure the required ratio. The U. S. P. contains two :

Dose.
Decoctum Cetrariæ ... . . .
- Sarsaparillæ Compositum

Decoctions should be freshly made, as they are liable to decompose.

1 [Elixira (Elixirs). - Sweet and aromatic liquid preparations, containing 20 to 25 per cent. of alcohol. There are two in the U. S. P. :

Elixir Aromaticum . . . . dose, indeterminate.

Elixir Phosphori . . . . dose, $1 / 2-21 / 2 \mathrm{fl} 3 ; 2 .-10$. c.c.

-7 Emplastra (Plasters).-Tenacious preparations for external application, solid at ordinary temperatures, but pliable and adhesive at the temperature of the body. Those of the U. S. P. are : 
Emplastrum Plumbi

- Ammoniaci cum

- Ferri

- Hydrargyri

- Opii

- Saponis

- Resinæ

- Arnicx

- Capsici

- Belladonnæ

- Picis Burgundica

- Ichthyocollre

- Picis Cantharidatum
Lead oxide, olive oil and water. Lead oleate and glycerin are formed.

Lead plaster as the basis.

Lead plaster and wax as the basis.

Resin plaster, which is made from Lead plaster, as the basis.

Resin and soap plaster as the basis.

Wax the basis.

Court plaster.

Warming plaster.

7 Emulsa (Emulsions). - Liquid preparations consisting of oily or resinous substances held suspended in water which has been rendered mucilaginous or viscid. Mucilago Acaciæ and Tragacanthæ are frequently used to form emulsions. Mucilago Acaciæ should be recently prepared. It is incompatible with ferric chloridé, borax, and lead subacetate.

Emulsions are coagulated by acids, an undue proportion of metallic salts, and spirituous liquids. There are in U. S. P. :

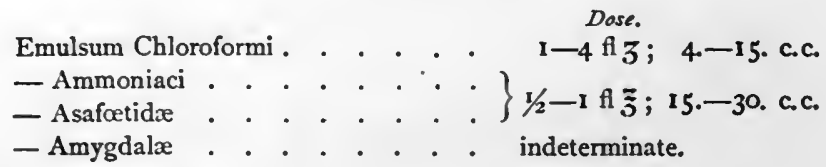

Extracta (Extracts). - Solid or soft-solid preparations not agreeing in strength, made by evaporating solutions of the active constituents of organic drugs to the required consistency.

The drug is first powdered, then macerated and percolated with the appropriate menstruum to exhaustion. The first third of the percolate is reserved, the remainder is evaporated at a temperature not exceeding $122^{\circ} \mathrm{F}$; $50^{\circ} \mathrm{C}$. ; until its weight is ten per cent. of that of the drug used, then mixed with the reserved portion, and both are evaporated at or below the above-named temperature until an extract of a pilular consistence remains. For converting extracts into powdered extracts it is sometimes necessary to add an inert dry substance. The menstrua used are : 
(a) Alcohol ; Extractum Aconiti, Cannabis Indicx, Cimicifugx, Colocynthidis Compositum, Iridis, Jalapæ, and Physostigmatis.

(b) Alcohol and water; Extractum Belladonnæ Foliarum Alcoholicum, Digitalis, Euonymi, Leptandræ, Podophylli, Rhei, and Uvæ Ursi.

(c) Alcohol, water and diluted alcohol; Extractum Cinchonæ, Hyoscyami.

(d) Diluted alcohol; Extractum Arnicæ Radicis, Cinchonæ, Colocynthidis, Juglandis, and Stramonii Seminis.

(e) Water; Extractum Aloes, Gentianæ, Glycyrrhizæ, Hæmatoxyli, Kramerix, Opii, and Quassix.

$(f)$ Water and Aqua Ammoniæ; Extractum Glycyrrhizæ Purum.

$(g)$ Acetic Acid and water ; Extractum Colchici Radicis.

(h) Acetic Acid and diluted alcohol ; Extractum Conii.

(i) Acetic Acid, alcohol, and water; Extractum Nucis Vomicæ.

Extractum Taraxaci is an inspissated juice.

Extractum Ergotæ is obtained by evaporating the fluid extract.

They are in the U. S. P.

Extractum Aconiti .

Dose.
$\frac{1}{10}-1 / 4 \mathrm{gr} . ; .006-.015 \mathrm{gm}$.

- Physostigmatis

- Belladonnæ Foliarum Alcoholicum

- Nucis Vomic

- Opii

- Cannabis Indicæ

- Stramonii Seminis

- Digitalis

- Conii

- Colocynthidis

— Colchici Radicis

- Hyoscyami

- Quassiæ

- Aloes.

- Leptandre

- Iridis

- Euonymi .

- Cimicifugæ

- Jalapæ .

- Gentianæ .

- Podophylli

- Rhei

- Arnica Radicis

- Kramerix . $\frac{1}{10}-1 / 2 \mathrm{gr} . ; .006-.03 \mathrm{gm}$. $1 / 8-1 / 2$ gr. ; .008-.03 gm. $1 / 8$ - I gr. ; .008-.06 gm.

1/6-3/4 gr. ; .01 -.045 gm.

$1 / 4-1 / 2$ gr. ; .015-.03 gm.

$1 / 4$ - I gr. ; .015-.06 gm.

$1 / 3$ - I gr. ; .02 -.06 gm.

1/2- 2 gr. ; .03- .12 gm.

$1 / 2-3$ gr. ; .03-.20 gm.

$\mathrm{r}-3 \mathrm{gr} ;$;.06-.20 gm.

I- 5 gr. ; .06- .30 gm.

$2-8$ gr. ; .12-.50 gm.

2-10 gr. ; .12-.60 gm.

$3-15$ gr. ; .20-1.00 gm.

5 -ro gr. ; $30-.60 \mathrm{gm}$.

- Colocynthidis Compositum

- Hæmatoxyli .

— Ergotæ

5-1 5 gr. ; $30-1.00 \mathrm{gm}$.

- Uvæ Ursi . 
Extractum Cinchonæ .

Dose.

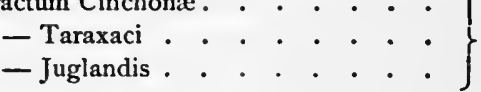

$5-30 \mathrm{gr} . ; \cdot 30-2.00 \mathrm{gm}$.

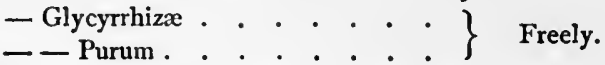

Extracta Fluida (Fluid Extracts). - Liquid preparations of organic drugs, made by completely exhausting the drug by suitable menstrua, and concentrating the resulting filtered solution, so that there shall be I c.c. of the latter for each gramme of drug extracted. They are made by percolation and partial evaporation, the menstruum employed being usually alcohol, diluted alcohol, or alcohol and water in various proportions. They are in the U. S. P. :

FLUID EXTRACTS.

Extractum Aconiti Fluidum .

- Digitalis Fluidum

- Belladonnæ Radicis Fluidum .

- Stramonii Seminis

- Nucis Vómicæ

- Ipecacuanhæ Fluidum (expect.).

“ " (emetic,).

- Sanguinariæ Fluidum (expect.). " " (emetic,).

— Veratri Viridis Fluidum . .

- Scillæ

- Colchici Seminis

- Conii

- Cannabis Indicæ

- Capsici

- Colchici Radicis

- Lobelix

- Apocyni

- Gelsemii

- Zingiberis

- Mezerei

- Sabinæ

- Hyoscyami

- Arnicæ Radicis

- Podophylli

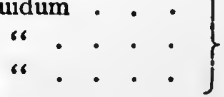

$$
\begin{aligned}
& \text { “. . . . }\} \\
& \text { “: . . . . }\}
\end{aligned}
$$$$
2-5^{m} ; .12-.30 \text { c.c. }
$$$$
2-8 \mathrm{~m} ; .12-.50 \text { c.c. }
$$$$
2-10 m ; .12-.60 \text { c.c. }
$$$$
\text { 2-20 m; .12-1.20 c.c. }
$$$$
5-10 \mathrm{~m} ; .30-.60 \text { c.c. }
$$$$
\text { 5-15 m; .30-1.00 c.c. }
$$$$
5-20 \mathrm{~m} ; .30-1.20 \text { c.c. }
$$ 
FLUID EXTRACTS.

Dose.

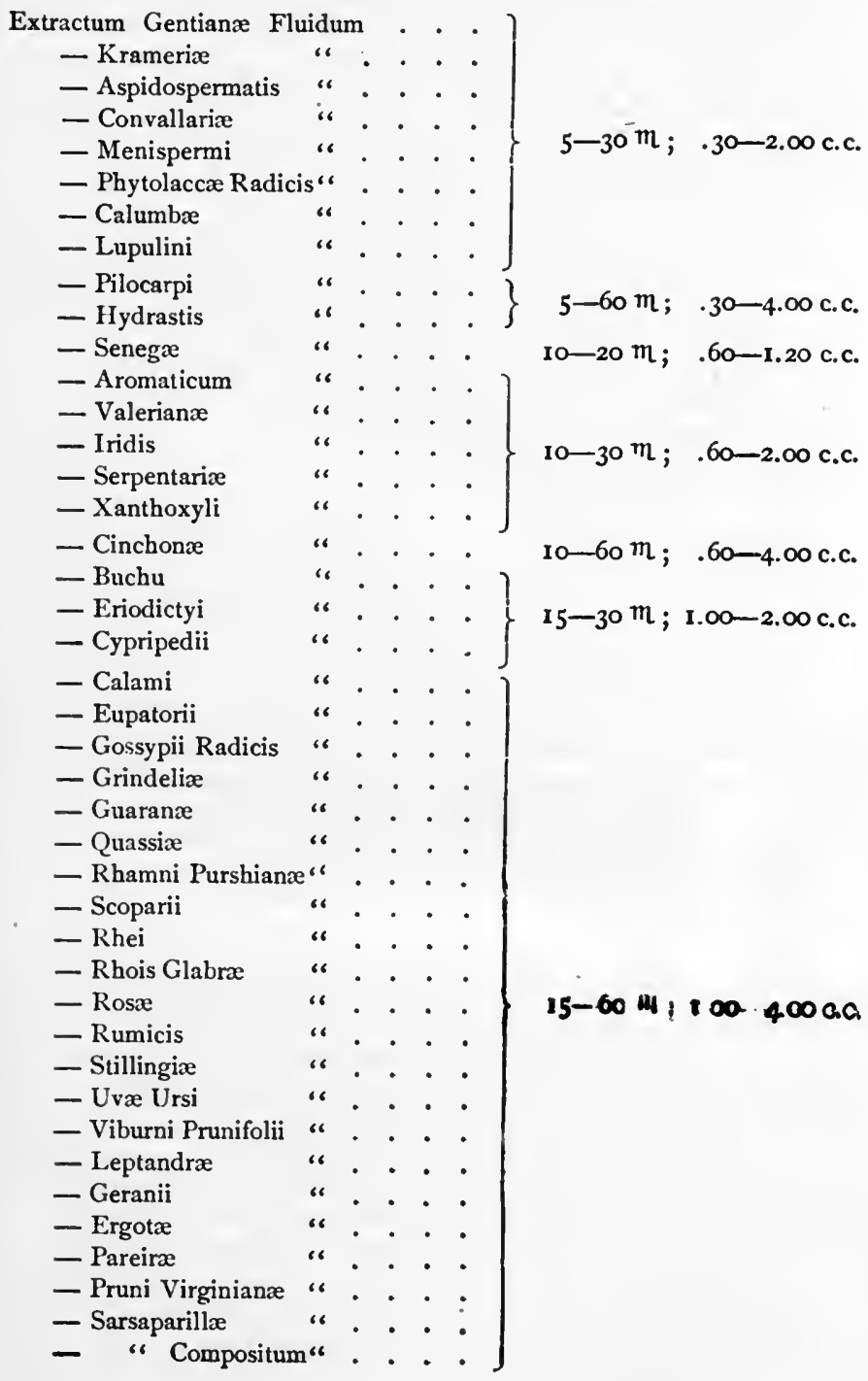




\section{FLUID EXTRACTS.}

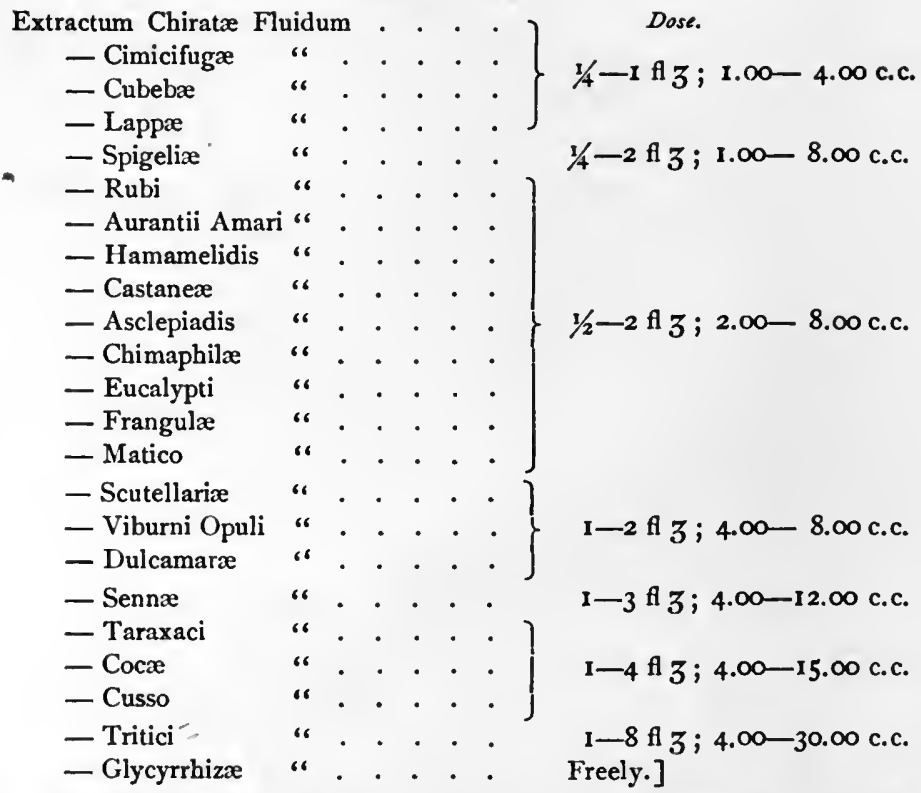

7 Glycerita (Glycerites).- Solutions of drugs in glycerin. They are all liquid preparations, [mostly] used for external application. [In the U. S. P. are six :

Glyceritum Acidi Carbolici.

- Acidi Tannici.

- Amyli.
Glyceritum Boroglycerini.

- Hydrastis.

- Vitelli.

7 Infusa (Infusions). - Liquid preparations of organic drugs, 5 grammes of drug to 100 c.c. of infusion, unless otherwise specified by the physician or Pharmacopæia, made by exhausting the drug for a specified time in a covered vessel, by water poured upon it at a boiling temperature (excepting Infusum Cinchonæ and Pruni Virginianæ which are made with cold water), straining and adding sufficient water to secure the required ratio.

The infusions of the U. S. P. are :

Infusum Digitalis . . . . . . . I-4 fl $3 ; 4$ - - I5. c.c.

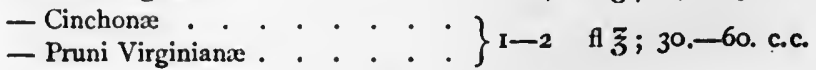


Infusum Sennæ Compositum . . . I-2 I $1 / 2$ fl $\bar{z} ; 30 .-75$. c.c. One is compound : Infusum Sennæ Compositum.

One contains acid : Infusum Cinchonæ.]

Infusions should be freshly made, as they readily decompose.

Linimenta [(Liniments).- - Solutions or mixtures for external application, liquid, at least upon application. All of which are intended to be rubbed into the skin except Linimentum Calcis. Those in the U. S. P. are :

Linimentum Ammoniæ . . Ammonia water and cotton seed oil.

- Calcis . . . . . . Solution of lime and linseed oil.

- Camphore. . . . . Camphor and cotton seed oil.

- Plumbi Subacetatis . . Solution of lead subacetate and cotton seed oil.

- Belladonnæ . . . . Fluid extract of drug and camphor.

- Cantharidis . . . Cantharides and oil of turpentine.

- Chloroformi . . . Chloroform and soap liniment.

- Saponis . . . . . Soap, camphor, oil of rosemary, and alcohol.

- Saponis Mollis . . $\left\{\begin{array}{c}\text { Soft soap, oil of lavender flowers, alcohol, and } \\ \text { water. }\end{array}\right.$ - Sinapis Compositum . $\cdot\left\{\begin{array}{c}\text { Oil of mustard, fluid extract of mezereum, cam- } \\ \text { phor, castor oil, and alcohol. }\end{array}\right.$ - Terebinthinæ . . . . Resin cerate and oil of turpentine.

Liquores (Liquors). - Solutions of chemical substances in a menstruum consisting chiefly or wholly of water. The following are the liquors of the U. S. P. :

Liquor Iodi Compositus

- Sodii Arsenatis

- Arseni et Hydrargyri Iodidi

- Potassii Arsenitis .

- Ferri Acetatis.

- Chloridi

- Nitratis .

- Subsulphatis

- Acidi Arsenosi

- Ferri Citratis

- Sodx

- Potassæ

- Sodæ Chloratæ

- Ferri et Ammonii Acetatis .

- Calcis

- Potassi Citratis

- Ammonii Acetatis

- Magnesii Citratis .

$$
\begin{aligned}
& \text { \} I-10 } m ; .06-.60 \text { c.c. } \\
& \text { 2-10 } m ; .12-.60 \text { c.c. } \\
& \text { 5-10 } \mathrm{m} ; .30-.60 \text { c.c. } \\
& 5-15 \mathrm{~m} ; .30-1.00 \text { c.c. } \\
& \text { 5-20 m; } .30-\text { I.20 c.c. } \\
& 5-30 \mathrm{~m} ; \cdot 30-2.00 \text { c.c. } \\
& \mathrm{r} / 2-\mathrm{Ifl} 3 ; 2 .-4.00 \mathrm{c.c} \text {. } \\
& \mathrm{I}-4 \mathrm{fl} 3 ; 4 .-15.00 \text { c.c. } \\
& \text { I- } 8 \mathrm{fl} 3 ; 4 .-30.00 \text { c.c. } \\
& 2-8 \text { fl } 3 ; 8 .-30.00 \text { c.c. } \\
& 2 \text { - } 8 \text { fl } 3 ; 60 \text {. } 240 \text { c.c. }
\end{aligned}
$$


The following are not used internally :

Liquor Ferri Tersulphatis.

- Hydrargyri Nitratis.

- Plumbi Subacetatis.
Liquor Plumbi Subacetatis Dilutus.

— Sodii Silicatis.

— Zinci Chloridi.

- Massæ (Masses).-Pill-masses intended for preservation in bulk until required for use. Three are official in the U. S. P. :

Massa Hydrargyri . . . . 1/2-15 gr. ; .03-1.00 gm.

- Ferri Carbonatis . . . . . $1-5$ gr. ; .06- $.30 \mathrm{gm}$.

- Copaibr . . . . . . $1 / 1 \%$ I $3 ; 1.00-4.00 \mathrm{gm}$.

7 Mellita (Honeys).-Mixtures of medicinal substances with clarified honey. The U.S. P. contains only two, one of these being simply honey purified.

Mel Despumatum. | I Mel Rosæ.

7 Misturæ (Mixtures). - Liquid preparations of insoluble, suspended, active substances, or solutions containing more than one liquid of therapeutic activity. The mixture is usually flavored, and is for internal administration. Examples in U. S. P. :

Mistura Rhei et Sodx . . . $\quad 1 / 4-2 \mathrm{fl} 5 ; 1 .-8$. c.c.

- Glycyrrhizz Composita . . I I 4 fl 3 ; 4. - I5. c.c.

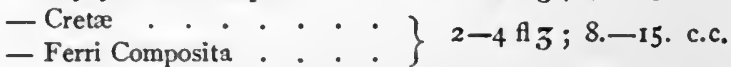

Mucilagines (Mucilages).-Adhesive liquids or jelly-like preparations, consisting of gum or starch dissolved in or fully charged with water. The U.S. P. contains four:

Mucilago Acacix.

- Sassafras Medullæ.

Mucilago Tragacanthx.

There is no fixed dose; it is usually I fl 3 ; [4. c.c.

Oleata.-Oleates are solutions in oleic acid, intended for external application. The U.S. P. contains three :

Oleatum Hydrargyri ( 20 per cent. of yellow mercuric oxide).

- Veratrinæ (2 per cent. of veratrine).

- Zinci ( 5 per cent. of zinc oxide)]. 
Olea.-There are many oils in the Pharmacopœia. They are all obtained by distillation or by expression except Oleum Phosphoratum, which is a solution of phosphorus in almond oil. [The U.S. P. olea are :

mutard Oleum Sinapis Volatile.... 1/8-1/4 Dose. Qitur almonel Amygdalix Amaræ . . . . $1 / 6-1 / 2 \mathrm{~m}$; .0r - .03 c.c.

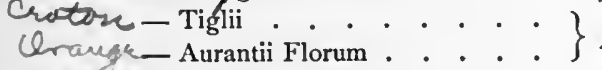

Volatill vil _ Cajuputi

- Cari.

- Anisi

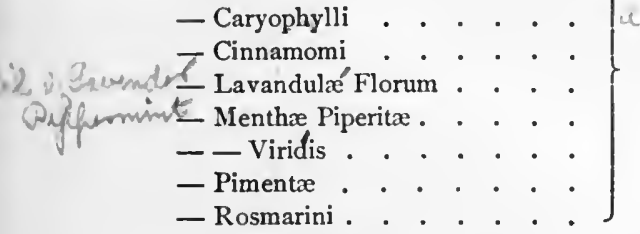

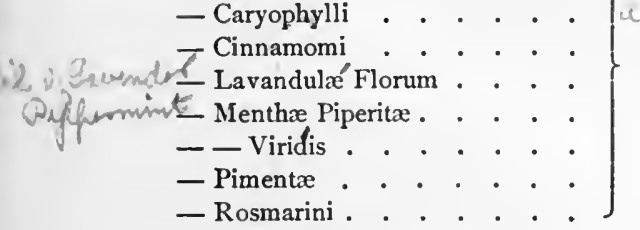

$\mathrm{I}-3 \mathrm{~m} ; .06-.20$ c.c.

$\mathrm{I}-5 \mathrm{~m} ; .06-.30$ c.c.

- Betulæ Volatile

- Sassafras

- Thymi

- Sabinæ .

- Phosphoratum

- Fœniculi

- Gaultherix

- Hedeomæ .

- Coriandri

- Chenopodii

- Santali

- Juniperi

- Copaibæ

— Erigerontis

- Cubebæ

- Eucalypti

$\mathrm{I}-5 \mathrm{~m} ; .06-.30$ c.c.

- Terebinthinæ

- - Rectificatum

- Morrliux

- Amygdalæ Expressun .

- Ricini

- Lini

- Sesami .

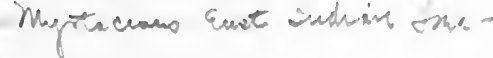

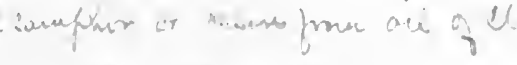

- Olivæ

$$
\begin{aligned}
& 2-5 \mathrm{~m} ; .12-.30 \text { c.c. } \\
& \text { 2-10 } m ; .12-.60 \text { c.c. } \\
& \text { 5-15 m; .30-1.00 c.c. } \\
& \text { 5-20 m; .30-1.20 c.c. } \\
& 5-30 \mathrm{~m} ; .30-2.00 \quad c . c \text {. } \\
& \mathrm{I}-4^{\mathrm{fl}} 3 ; 4.00-15.00 \text { c.c. } \\
& 1 / 4-2 \text { f } \tilde{\tilde{s}} ; 8.00-60.00 \text { c.c. } \\
& \mathrm{x} / 2-2 \mathrm{~A} \tilde{\overline{3}} ; 15.00-60.00 \text { c.c. } \\
& \text { indefinite. } \\
& \text { freely. }
\end{aligned}
$$


Oleum Gossypii Seminis

— Picis Liquidæ. . .

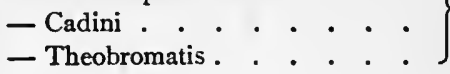

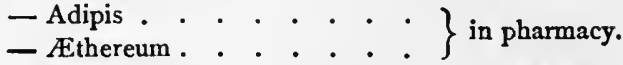

- Myrcix. . . . . . . as perfume.

- Aurantii Corticis .

- Bergamottæ

- Limonis .

- Rosæ .

Pilulæ (Pills). - Solid bodies, usually spherical or oval,] containing medicinal agents, and intended to be swallowed whole. A mass of the consistence of firm clay is made by beating medicaments together in a mortar. This mass is, with a machine, divided up and rolled into pills. In order that they may not possess a disagreeable taste, they are varnished, gilded, or sugarcoated. Unless the constituents are very heavy, each pill should not exceed 5 grains $[.3 \circ \mathrm{gm}$.] in weight, and the smaller they are the better. [Soap, mucilage of acacia or tragacanth], and confection of rose are common excipients for pills. Glycerin is often added, for it attracts moisture and prevents the pill from getting hard, but pills made of it alone are too soft. Soap is useful for creosote and for essential oils if calcium phosphate and wheaten flour be added. Liquorice powder is a good absorbent. All pills are useless unless so made that they will dissolve in the gastro-intestinal canal. If it is required that they should not be acted upon until they reach the intestine they should be coated with keratin. Pills [except when sugar-or gelatin-coated] are often kept in some powder, as lycopodium, to prevent their sticking together. [The pills in the U.S. P. are :

\section{Dose.}

Pilulæ Phosphori .

一 Opii . . . .

$$
\left\{\begin{array}{l}
\text { I } \\
\text { I gro gr. ; } .065 \mathrm{gm} . \text { in each. }
\end{array}\right\} \text { I pill. }
$$

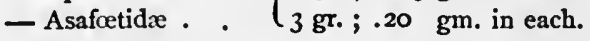

- Ferri Iodidi \} $1-2$ pills.

- Catharticæ Compositæ

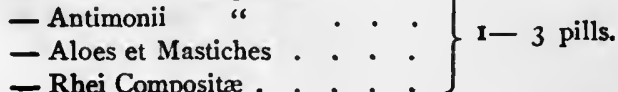


Pilulæ Aloes . . . . . .

- Aloes et Ferri . . . . . $\}$ I -4 pills.

- Rhei

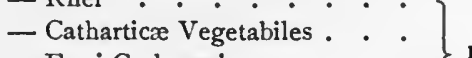

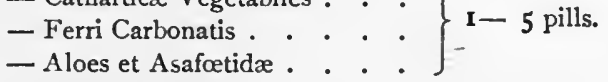

- Aloes et Myrrhæ. . . . 2-10 pills.

Pulveres (Powders).-Preparations consisting of finelypowdered drugs, or (compound powders) mixtures of the same, and frequently consisting in part of a suitable diluent or excipient.] The best diluent for powders is sugar of milk, because of its hardness and comparative insolubility. [The powders of the U.S.P. are :

Dose.

Pulvis Antimonialis . . . . . 1/2- $2 \mathrm{gr} . ; .03-.12 \mathrm{gm}$.

- Ipecacuanhæ et Opii . . . 3-I5 gr. ; .20-1.00 gm.

- Morphinæ Compositus . . . 5-15 gr. ; .30-1.00 gm.

- Cretæ "6 . . . 5-60 gr. ; $.30-4.00 \mathrm{gm}$.

- Aromaticus . . . . . I0-30 gr. ; .60-2.00 gm.

- Jalapæ Compositus . . . . 1/4-1 $3 ; 1.00-4.00 \mathrm{gm}$.

- Rhei “. . . . $1 / 2-13 ; 2.00-4.00 \mathrm{gm}$.

- Glycyrrhizæ " . . . $1 / 2-23 ; 2.00-8.00 \mathrm{gm}$.

For Pulvis Effervescens Compositus (Seidlitz Powder) see Sodium. Pulvis Ipecacuanhæ et Opii is practically a trituration.

Spiritus (Spirits).- Solutions of volatile substances in alcohol or diluted alcohol. The spirits of the U. S. P. are :

By solution :

Spiritus Glonoini

- Etheris Compositus . . .

- Phosphori

- Cinnamomi .

- Amygdalæ Amaræ

- Etheris .

- Camphoræ .

- Chloroformi

- Juniperi .

- Lavandulæ .

- Myristice

- Ammoniæ Aromaticus

- Anisi .

- Gaultheriæ .
Dose.

$$
\begin{aligned}
& 1-5 . \mathrm{m} ; .06-.30 \mathrm{c.c} . \\
& 5-60 \mathrm{~m} ; .30-4.00 \mathrm{c.c} . \\
& 8-40 \mathrm{~m} ; .50-2.50 \mathrm{c.c} . \\
& 10-30 \mathrm{~m} ; 1.00-2.00 \mathrm{c.c} . \\
& 15-45 \mathrm{~m} ; 1.00-3.00 \mathrm{c.c} . \\
& 15-60 \mathrm{~m} ; 1.00-4.00 \mathrm{c.c} . \\
& 30-60 \mathrm{~m} ; 2.00-4.00 \mathrm{c.c} . \\
& 1 / 2-2 \mathrm{fl} 3 ; 2.00-8.00 \mathrm{c.c} . \\
& 1-2 \mathrm{fl} 3 ; 4.00-8.00 \mathrm{c.c} .
\end{aligned}
$$


Dose.

Spiritus Juniperi Compositus . $\quad I-4 \mathrm{fl} 3 ; 4.00-15.00$ c.c.

- Aurantii . . . . . . . . . $\}$ as vehicle.

- Myrciæ . . . . . . as perfume.

By solution with maceration:

Spiritus Limonis . . . . as flavoring agent.

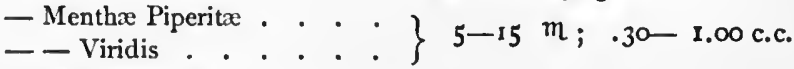

By gaseous solution :

Spiritus Ammoniæ

By chemical reaction :

Spiritus Etheris Nitrosi . . . $1 / 2-2 \mathrm{fl} 3 ; 2.00-8.00$ c.c.

By distillation :

Spiritus Frumenti . . . . . indefinite.

— Vini Gallici. . . . . indefinite.

Suppositoriæ (Suppositories).-Preparations in suitable form] for introduction into [and retention in] the rectum or vagina [and so mixed with a base (generally Oleum Theobromatis) that they shall be solid under ordinary conditions, but shall melt or liquefy after introduction. One only is official in U. S. P. :

Suppositoria Glycerini; base, stearic acid.

Syrupi (Syrups).-Liquid preparations of drugs consisting chiefly of a concentrated aqueous solution of sugar. There are 30 official in U. S. P.

Examples : Syrupus Aurantii, Syrupus Rhei.]

The dose of all is about $\mathbf{I} \mathbf{f l} \mathbf{3} ; 4$ c.c., or rather more [excepting Syrupus Ferri Bromidi, Ferri Iodidi, and Scillæ Compositus, when it is 5 to $30 \mathrm{~m}$; $.30-2.00$ c.c.

Tincturæ (Tinctures).-Solutions in alcohol or diluted alcohol of the soluble constituents of substances which are not volatile. (The U. S. P. includes also, as an exception, the alcoholic solution of iodine.)] They are prepared by-

(a) Maceration; e.g. [Tinctura Opii Camphorata.]

(b) Percolation; e.g. [Tinctura Lavandulæ Composita.]

(c) Maceration and percolation ; e.g. [Tinctura Opii.] 
(d) Simple mixing of ingredients ; e.g. [Tinctura Ipecacuanhæ et Opii.]

Tinctures containing only one active substance are simple. The rest are compound ; e.g., [Tinctura Catechu Composita.]

Some are compound, although it is not expressed in their name; [e.g., Tinctura Aloes.

The tinctures of the U. S. P. are:

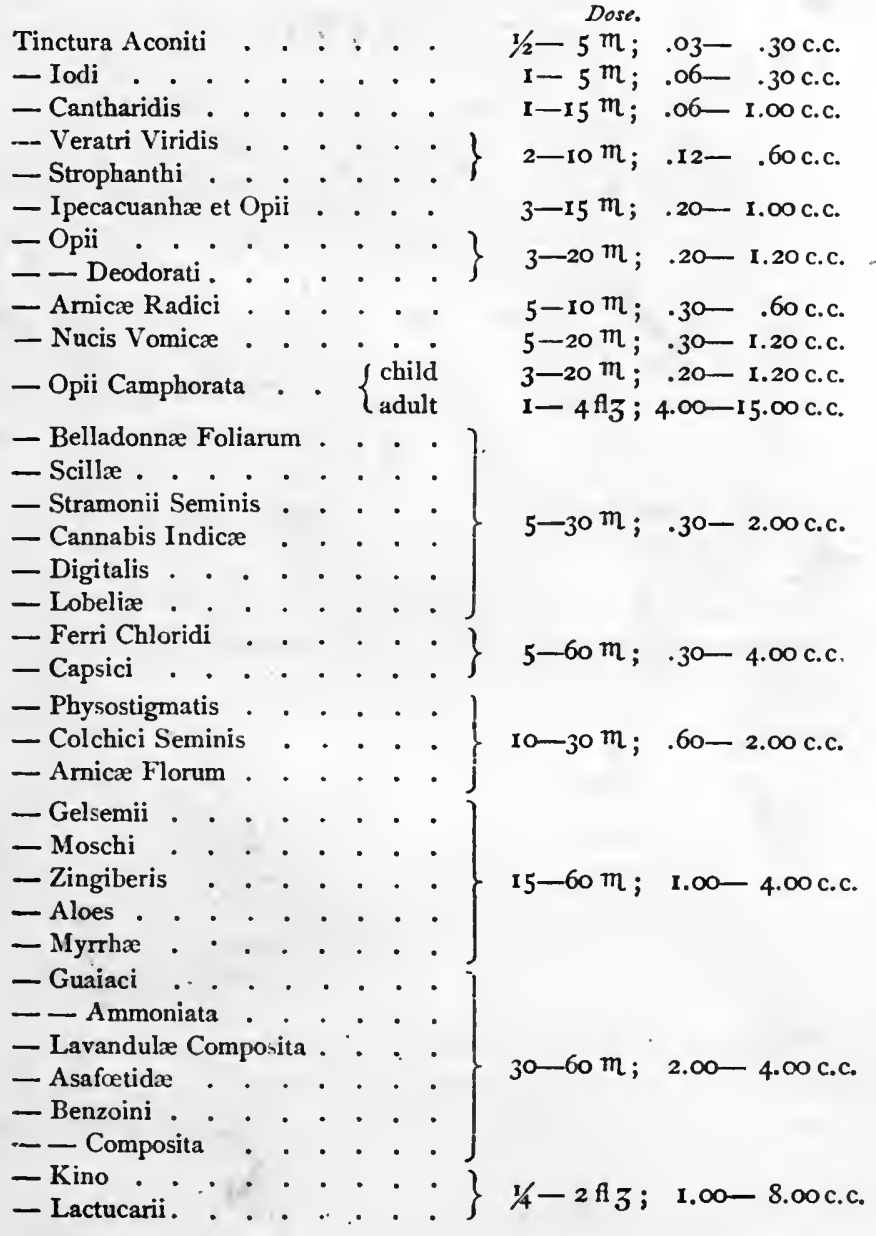




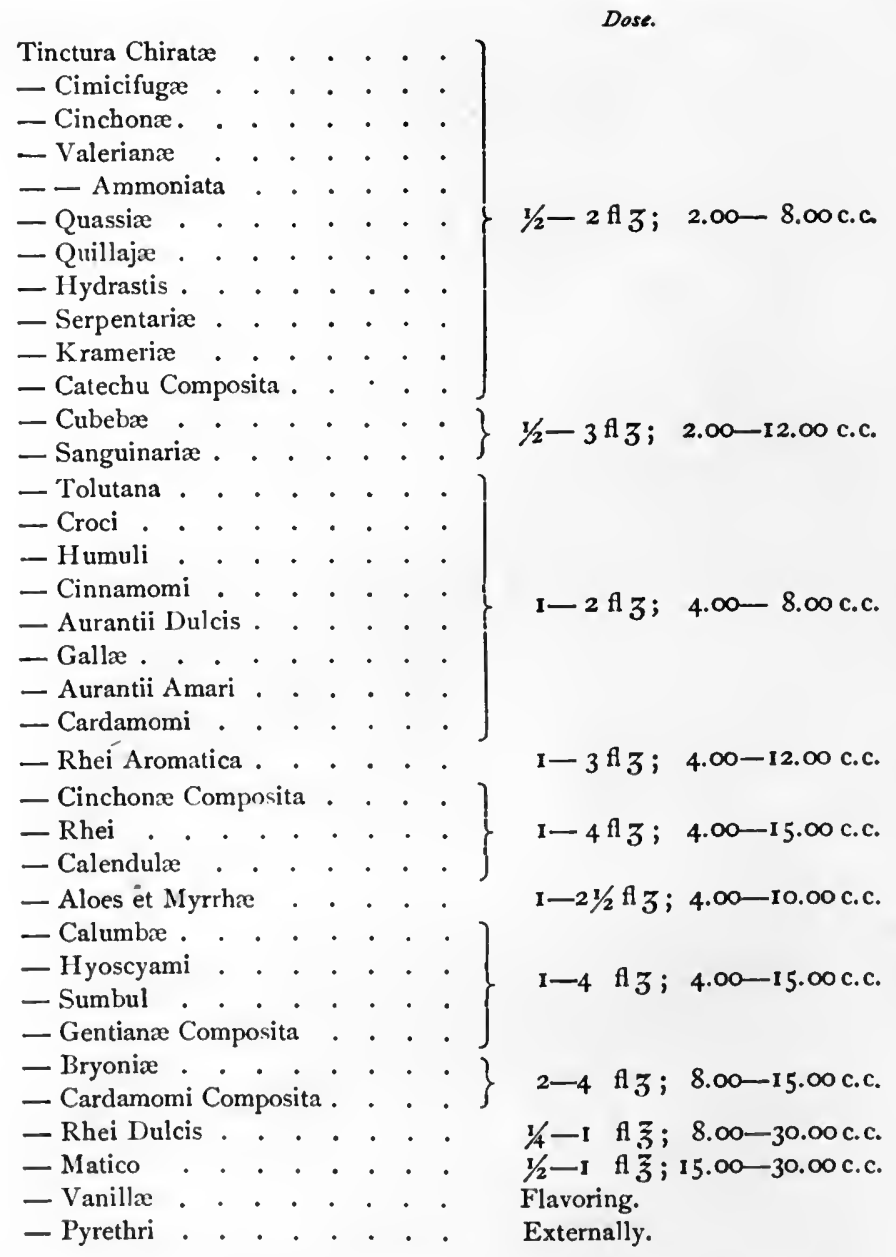

Triturationes (Triturations).--Solid preparations made by triturating a medicinal substance with a definite percentage of sugar of milk (Saccharum Lactis). The U. S. P. contains one:

Dose.

Trituratio Elaterini . . . . 1/2 to I gr.; $.03-.06 \mathrm{gm}$. 
Trochisci (Troches).- Solid preparations, usually in flattened form, consisting of one or more medicinal substances mixed with sugar or extract of liquorice and held in form by the addition of mucilage. Fifteen are official in U. S. P.

Examples : Trochisci Catechu, Trochisci Sodii Bicarbonatis. The dose of each is from I to 6 troches in the course of the day.]

Unguenta (Ointments). - [Unctuous preparations, either soft or solid at ordinary temperatures, but liquid upon being rubbed upon the skin.] They are generally spread over the skin, or may be rubbed into it. They are only intended for external use. The basis is either lard, olive oil, expressed oil of almond, lard oil, wax, suet, spermaceti, or paraffin. When it is required that the active ingredient should be absorbed, lard, which melts at about the temperature of the body, or hydrous wool fat, is the best basis; when the ointment is required for sores, paraffin is a good basis, as it only softens a little at the temperature of the body. In hot countries, if the ointment would otherwise be too soft, the basis may be replaced by indurated lard, prepared suet, white or yellow wax. Benzoinated lard is often used to prevent decomposition. [Twenty-seven are official in U. S. P.

Examples : Unguentum Hydrargyri, Unguentum Iodi.]

Vina.-Wines are weak tinctures, [the drug being extracted with white wine, containing twenty to twenty-five per cent. by weight of absolute alcohol.] 
With Vinum Antimonii and Vinum Ipecacuanhæ the dose depends upos the purpose for which the drug is used.

The following preparations [not occurring in the U.S. P.] are used :

Abstracta. - [( Abstracts) solid, dry, powdered extracts of double the strength of the crude drug. They are prepared by spontaneous evaporation of an alcoholic tincture at a low temperature, mixing with it enough Sugar of Milk to make the product weigh one-half of the original weight of the drug, and then reducing it to a fine powder.]

Bougies.-Solid cylinders impregnated with various drugs, and used for introduction into the urethra, [ uterus, ] or nose. They are made either of gelatin (to be dipped in warm water before use) or cacao butter (to be dipped in oil before use).

Cachets, made of wafer paper, consist of two watch-glass shaped halves, enclosing the drug, which adhere when moistened. The cachet is swallowed, and thus nauseous drugs are not tasted.

Capsules, [usually made of gelatin, are also used for enclosing medicines so that they shall not be tasted, and they are now made for containing both solid and liquid substances. They are either soft and elastic or hard. The "empty capsule" is of the hard form and is made in two parts, the body to be filled when required for use, and the cap to fit tightly over it when filled]. Pills, cachets and capsules should be immediately followed by enough water to wash them down.

Cataplasmáta [(Poultices). - Soft, pasty masses used as a medium for the external and local application of a moist heat, with or without the addition of active medicaments. Any bland substance which will retain its heat and moisture for a long time is applicable for this purpose, a little oil or glycerin being often added to prevent caking. The substances chiefly used are flaxseed, elm bark, bread and milk, bran, and oatmeal.

To make a poultice properly, the bowl in which it is mixed, the water, the spatula for mixing and spreading the flannel or cheese-cloth on which it is laid, must all be as hot as possible. The meal should be added gradually to the boiling water, which is continually stirred. It should not be spread so thick as to make it inconveniently heavy.]

Cigarettes. - The drug replaces the tobacco of an ordinary cigarette.

Clysters.- [Another name for Enemata.]

Collunaria. - Fluids used as nasal douches. [This term is rarely used in the United States.]

Collyria. - Fluids used as eye washes.

Cremora.-[Obsolete in the United States.] Creams are preparations having glycerin, vaseline, or some substance as a basis, and used for external application.

[Enemata (Enemas). - Liquids intended for injection into the rectum and designed to act medicinally, to evacuate the bowel mechanically, or to serve as nutrients.] When their object is to empty the bowel, they are large in bulk. to to $20 \mathrm{fl} \tilde{\tilde{J}}$ [300 to 600 c.c. ] ; when it is wished that they should be 
retained, they are small in bulk, 2 to $5 \mathrm{fl} \xi[60$ to 150 c.c.], and after injection a towel may be pressed against the anus. Mucilage, made with starch, is a good basis.

[Essentiæ (Essences).-Preparations of B. P. corresponding to Spirits, U. S. P., but of 20 per cent. strength.]

Fomenta. - Fomentations consist of flannels wrung out in hot water, to which drugs may or may not have been added.

Gargarismata (Gargles) are fluid preparations for gargling.

Granules are small pills.

Guttæ [(Drops).-In England this term is used to designate liquid] preparations to be dropped into the eye.

Haustus (Draught). - This term is used when only a single dose of a fluid preparation is required.

[Injectiones (Injections). - These are of two kinds, Rectal (see Enemata), and Hypodermatic. The latter are highly concentrated solutions intended for use by means of a bypodermatic needle. (See p. 38.)

Inbalations. - A common name for Vapors.]

Insufflationes.-Powdered medicines or medicated powders designed for blowing into the [nares], larynx or throat.

Lamellæ (B. P.).-Small, thin discs made with gelatin and glycerin, and used to drop into the eye. They each weigh $\frac{1}{50}$ th of a grain [.001 $3 \mathrm{gm}$.].

Lanolinum is an ointment having hydrous wool fat as a basis.

Linctus.- [(Never used in the United States.) A sweet mixture of a thick, syrupy consistence.] It is to be swallowed slowly, being retained some time in the mouth.

[Lotiones (Lotions). - - Liquid, usually aqueous preparations for external use, commonly applied upon lint or muslin].

Mollinum.-An ointment having for its basis mollin, a superfatted soap. It is readily absorbed, and also readily washed off with water.

Nebulæ [Sprays]. - Solutions sprayed into the throat by means of an atomizer.

[Oxymellita.-Mellita containing acetic acid.]

Paste.-A preparation to be applied as an ointment.

Pastillus (Pastils).- [A name often applied to troches, and in England limited to] those having glyco-gelatin as a basis.

Perles are small pills.

Pessus.-Pessaries are solid preparations made like suppositories for introduction into the vagina. [This term is rarely used in the United States.]

Pigmentum (A paint). - A [liquid] preparation adapted for painting on the skin, throat, etc.

Succi [(Juices). - Expressed vegetable juices preserved by the addition of a definite proportion of alcohol.]

Tabellæ.-(Tablets or Tabloids, [the latter of British usage].) Solid, disc-like or lenticular bodies made by compression. ["Tablet triturates" are composed of drugs which have been triturated before compression.] They 
are very popular, but are often useless, for they may be so hard and insoluble that they are found in the freces quite unaltered. [Tablets should always be prescribed extemporaneously and freshly made.]

Vapores.-[Liquid preparations intended for administration by inhalation in form of vapor.]

Vaselinum.-This term [in England] is applied to an ointment of which the base is vaseline.

\section{PHARMACOLOGY AND THERAPEUTICS.}

Before describing the actions and uses of drugs we must consider the manner, quantity and form in which to give them.

\section{MODES OF ADMINISTRATION OF DRUGS.}

(a) Into the blood-vessels by injection. This method is rarely used in man, excepting for transfusion of physiological saline solution (a teaspoonful of common salt to the pint of sterilized water at the temperature of the body) in cases of great loss of blood.

(b) Into the subcutaneous tissues by hypodermatic injection. The skin of the patient, where it is lax, should be raised between the thumb and forefinger of the operator's left hand ; the skin of the external surface of the forearm is often selected. In his right hand he takes a perfectly clean syringe containing the quantity of fluid to be injected, and fitted with an aseptic, hollow, silver needle, which is thrust under the raised piece of skin, but not into the muscles, for about an inch, care being taken to avoid wounding a vein. The syringe is slowly emptied, then withdrawn, and the thumb pressed lightly upon the seat of injection for a few seconds. The advantage of this method is that the drug is surely and quickly absorbed. The fluid used must not contain solid particles, nor be irritating, or abscesses will result; it must be aseptic, and therefore, if it is not freshly prepared, it may contain a little carbolic acid-or, better still, boric acid, for this is non-poisonous and nonirritating. The bulk injected should, if possible, be about five minims; [ $-3^{\circ}$ c.c.]. For injections that are not in constant use it is advisable to keep the drugs in the form of [soluble tablets or] lamellæ, and to dissolve one in a few minims of water as required.

(c) Into serous cavities by injection. This method is rarely used in man except when the object is antiseptically to wash out a serous cavity, as the pleura which has been opened, or to produce adhesive inflammation, as in the injection of irritants into the tunica vaginalis.

(d) Into mucous cavities.-Drugs are most frequently given by the mouth, to be absorbed from the mucous membrane of the stomach or intestines, but the rate of absorption is much slower than from the subcutaneous tissue, and will depend upon whether the drug is readily soluble in the gastro-intestinal secretions, and whether it is given on an empty stomach, in which case it will be quickly absorbed; or on a full one, when it will be slowly absorbed. 
[When it is intended that the drug shall act only in the intestine, pills, made purposely insoluble in the gastric fluids, are administered.] Some drugs, given by the mouth and absorbed from the stomach, probably never reach the general circulation, as they are excreted in the bile by the liver. The drug must be in a pleasant, palatable form, and generally so combined as not to irritate.

Drugs are sometimes given by the rectum-in a solid form as suppositories, in a liquid form as enemata or clysters; but they are not dissolved nor absorbed here so quickly as in the upper part of the gastro-intestinal canal.

For local effects they may be given by the urethra or vagina (injections, bougies, pessaries), or by the respiratory passages (inhalations, cigarettes, sprays or nebulæ for inbalations; insufflations for blowing into the throat and larynx ; pigmenta, gargarismata, trochisci, for a local effect on the mouth and pharynx; nasal douches for the nose). For sprays an atomizer is required. Sometimes volatile drugs, as chloroform or amyl nitrite, are inhaled for their general effect.

(e) By the skin.-Some drugs may be absorbed from the skin if mixed with some fatty substance, [especially hydrous wool fat.] In this way mercury may be absorbed by being rubbed in; but drugs are chiefly applied to the skin as ointments, plasters, etc., for their local effect.

[Some drugs may be absorbed from the skin when they are volatilized. In this way mercury is introduced into the system by fumigation.]

They are also applied to the eye and ear as drops and washes.

DOSES.

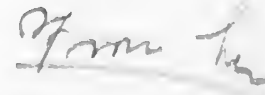

The study of doses is termed Posology. In determining the dose the following considerations have to be borne in mind :

1. Age. - The adult dose is that for a person between twenty and sixty years old. [For women the dose should be somewhat smaller than for men.]

For children under twelve, add twelve to the age, and divide the age by the number thus obtained. Thus, for a child aged eight the dose will be $\frac{8}{8+12}=\frac{2}{5}$ of an adult dose. From twelve to sixteen years from $\frac{2}{3}$ to $\frac{1}{2}$ the adult dose is required, and from seventeen to twenty years from $\frac{4}{5}$ to $\frac{2}{3}$. There are exceptions to this rule for individual drugs ; e.g., children take iron, cod liver oil, arsenic, and chloral hydrate very well, but they can take only very small doses of opium. [Cowling's rule-divide age at next birthday by twenty-four-requires rather less calculation and is generally of sufficient exactness.]

Above sixty years of age the dose should slightly diminish as age increases.

2. Weight.-In pharmacological experiments the dose should always be expressed as a proportion of the weight of the animal. In man the weight is not often considered, for it depends so much upon bone and fat, which are not active tissues. 
3. Habit.-A man who is constantly under the action of a drug becomes very insusceptible to it. Thus an opium-eater requires enormous doses of opium to produce any effect. A person who habitually takes purgatives requires very strong ones to open the bowels.

4. Idiosyncrasy.-The susceptibility to drugs varies very much. Some persons are salivated by minute doses of mercury, others bear it very well, and there is hardly a drug to which some people are not exceptionally indifferent or susceptible.

5. Time of Administration. - Drugs all act to greatest advantage when given so that their effect will be produced at its natural time. Thus soporifics act best when given in the evening, slowly acting purgatives when given overnight, quickly acting ones when given before breakfast, ergot when given during labor. [Drugs which are readily decomposed by the contents of the stomach should be given when that viscus is empty, preferably a half hour before the meal time.]

6. Mode of Administration.-We have seen that drugs are rapidly absorbed from the subcutaneous tissues. Therefore a smaller dose is required for subcritaneous injection than when the same drug is given by the stomach, for absorption is slow from the upper gastro-intestinal tract. It is slower still from the rectum [therefore, to produce effects more immediately, the dose must be larger]. Also certain drugs are excreted by the liver or destroyed in it when given by the stomach. Absorption takes place quickly from an empty, slowly from a full stomach.

7. Mental Emotion. - Sometimes if the patient's mind is particularly fixed on the action of the drug, a small dose is powerful. For example, often if the patient is convinced he will sleep, a very small dose of morphine is all that may be required.

8. Temperature.-As the action of the drug on the organism is often partly chemical, the temperature must, in cold-blooded animals and excised structures, as muscle, etc., belp to determine its action ; but the temperature of man varies within so few degrees that this is not an important factor in medicine.

9. Preparation of Drug.-A smaller dose of a soluble preparation, as a tincture, will be required than of a solid preparation, as a pill, which will have to be slowly dissolved before absorption [although in the latter case much depends upon the process of manufacture].

I0. Rate of Excretion. - It is obvious that, other things being equal, for prompt action a smaller dose will be required of a drug that is rapidly excreted than of one which is slowly excreted. [It is also true that, in order to maintain a continuous effect from drugs which are rapidly excreted, the doses must be repeated at shorter intervals.]

11. Cumulative Action. - Sometimes it is found that if a person has been taking a drug regularly, but without the production of any poisonous symptoms, these will suddenly develop. This is said to be due to the cumulative action of the drug. It may be caused by the following circumstances :

(a) The drug may be absorbed more rapidly than it is excreted. This is 
the cause of the cumulative action of mercury and lead, both of which are excreted with difficulty by the kidney.

(b) There may be a sudden arrest in the excretion of the drug. It is probable that digitalis and strychnine, when the quantity of them in the tissues reaches a certain amount, contract the renal vessels, and hence excretion is arrested.

(c) It is possible that, owing to an alteration in the intestinal contents, a drug which was previously very slowly dissolved becomes quickly dissolved, and hence rapidly absorbed.

12. Disease.-The physiological action of drugs, and consequently the dose, are profoundly modified by disease. For example, a patient with peritonitis will bear enormous doses of opium. Antipyretics, which do not affect normal temperature, powerfully depress a febrile temperature.

[The tendency of modern therapentics is towards smaller and more frequently repeated doses.]

\section{PRESCRIBING.}

The more complex prescriptions consist of -

(I) The Basis, or principal active ingredient [curare].

(2) The Adjuvans, or that which assists its action [cito].

(3) The Corrigens, or that which corrects its operation [tuto].

(4) The Constituens, vehicle, or excipient, which imparts an agreeable form [jucunde].

Thus the object of every prescription is to cure quickly, safely and pleasantly. For example [in Pilula Rhei Composita the rhubarb is the basis, the aloes and myrrh form the adjuvans, and the oil of peppermint is the corrigens to prevent the griping]. In Mistura Cretæ the cinnamon water is the vehicle. Many drugs do not require anything to assist their action or correct their operation. [The scientific physician usually prefers to administer the remedies separately, in order to more accurately observe their effect, and as well to discontinue, or change the dose of, any one which may be necessary.]

Incompatibility of ingredients should be particularly avoided in prescriptions. There are three kinds of incompatibility:

(a) Chemical Incompatibility; e.g., Glucosides should not be ordered with free acids, which decompose them; nor Alkaloids or Alkaloidal Salts with alkalies, alkaline salts, tannic acid, iodides, or bromides, for they precipitate them.

Examples of chemical incompatibility are the prescribing of (I) tannic acid or substances containing it with alkaloids or metallic salts, especially those of iron; (2) vinegars or syrups containing acetic acid prescribed with carbonates lead to the evolution of carbon dioxide; (3) strychnine sulphate is decomposed by potassium bromide, and strychnine is precipitated ; 4 ) chloral hydrate and alkalies form chloroform; (5) quinine sulphate and potassium acetate together cause a voluminous precipitate of quinine acetate; (6) lime water with mer- 
cury salts (this incompatibility is intentional in Lotio Nigra and Lotio Flava), precipitates mercuric oxides; it decomposes carbonates and bicarbonates of alkalies; it precipitates solutions of quinine and morphine salts; $(7)$ corrosive mercuric chloride is incompatible with most substances.

The following table, drawn up by Potter [Materia Medica, Pharmacy and Therapeutics, 7 th Ed., p. 540] shows the most important instances of solutions which mutually precipitate each other. The letter " $p$ " means "forms a precipitate with."

\begin{tabular}{|c|c|c|c|c|c|c|}
\hline SOLUTrONS OF & 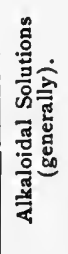 & 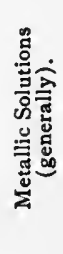 & 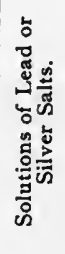 & 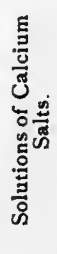 & 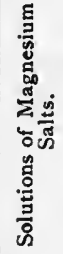 & 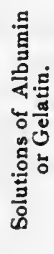 \\
\hline $\begin{array}{l}\text { Alkalies } \\
\text { Tannic acid } \\
\text { Carbonic acid and Carbonates } \\
\text { Sulphuric acid and Sulphates } \\
\text { Phosphoric acid and Phosphates } \\
\text { Boric acid and Borates } \\
\text { Hydrochloric acid and Chlorides } \\
\text { Hydrobromic acid and Bromides } \\
\text { Hydriodic acid and Iodides. } \\
\text { Sulphides } \\
\text { Arsenical Preparations : } \\
\text { Albumin. }\end{array}$ & 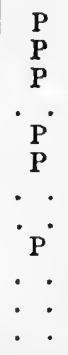 & $\begin{array}{l}\mathrm{P} \\
\mathrm{P} \\
\mathrm{P} \\
0 \\
\mathrm{P} \\
\mathrm{P} \\
\dot{P} \\
\dot{P} \\
\mathrm{P} \\
\mathrm{P} \\
\mathrm{P}\end{array}$ & $\begin{array}{l}\mathrm{P} \\
\mathrm{P} \\
\mathrm{P} \\
\mathrm{P} \\
\mathrm{P} \\
\mathrm{P} \\
\mathrm{P} \\
\mathrm{P} \\
\mathrm{P} \\
\mathrm{P} \\
\mathrm{P} \\
\mathrm{P}\end{array}$ & 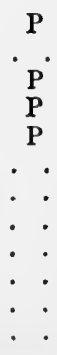 & 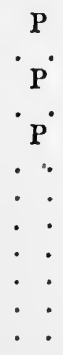 & $\begin{array}{l}\mathrm{P} \\
\vdots \\
\vdots \\
\vdots \\
\vdots \\
\vdots\end{array}$ \\
\hline
\end{tabular}

With the following drugs it is particularly difficult to avoid chemical incompatibility.

Antipyrin.

Chlorine in solution.

Liquid preparations of Iron.

Lead salts.

Zinc salts.

Silver salts.

Corrosive Mercuric Chloride (especially).

Iodine and the Iodides.

All Bromides.
Potassium Permanganate.

Potassium Acetate.

Nitrites.

Tannic Acid.

Gallic Acid.

Acidum Hydrocyanicum Dilutum.

Mineral Acids.

Liquor Potassa.

Quinine Sulphate.

Tincture of Guaiacum.

Substances rich in oxygen, as chlorates, iodates, permanganates, picrates, nitrates and bichromates should not be'mixed with readily oxidizable sub- 
stances, such as charcoal, sulphur, iodine, carbolic acid, glycerin, turpentine, and organic compounds generally, for explosive compounds are very liable to be formed.

Poisonous compounds may be formed by the admixture of substances in solution: e.g., potassium chlorate and the syrup of ferrous iodide liberate iodine; diluted hydrocyanic acid and calomel form mercuric cyanide; potassium chlorate and potassium iodide form, at the temperature of the body, a poisonous compound, probably potassium iodate. Death has occurred owing to patients having taken some of these careless prescriptions.

If, in a mixture, incompatibles are inevitable, they should both be diluted with the vehicle before they are added to each other. The careful prescriber will avoid combining any of the above incompatible substances.

(b) Physical Incompatibility.-This occurs when the mixture of the substances will not form a clear solution; e.g., insoluble powders and oils will not mix with water, the addition of which, to some spirits and all resinous tinctures, and to [fluid] extract of male fern causes a precipitate; if an acid mixture is flavored with liquorice, the acid precipitates glycyrrhizin; an alcoholic solution added to chloral hydrate causes all the chloral to rise to the top.

In such cases the aqueous solution may be thickened so that the precipitate is suspended in it to form an emulsion, but even then the mixture must be shaken before a dose is taken. Mucilage of acacia, freshly made, is the best emulsifying agent. The substances incompatible with it are mentioned on p. 22. It should be made perfectly fresh. The addition of a little almond oil improves its appearance.

I pt. of most fixed oils requires of acacia $3 / 4$ pt., water I pt.

I pt. of balsam of Peru " $\quad 2 \quad$ " $11 / 2$

I pt. of oil of turpentine " I " I

Tragacanth [because its preparations keep better] is often used to form an emulsion or a suspension, and sometimes yolk of egg or milk are employed. Liquor Potassæ much facilitates the admixture of fixed oils and water [although] it often acts chemically on the ingredients of the prescription. Tincture of senega aids the emulsification of any oil, even in small quantities, $\mathrm{m} \mathrm{x} ; .60 \mathrm{c.c}$, , being sufficient for an ounce; 30 . c.c., of a fixed oil. [Extractum quillajæ, one grain; $.06 \mathrm{gm}$., dissolved in one ounce; 30. c.c., of water, will make a tolerably permanent emulsion with one ounce; 3 o. c.c. of fixed oil, or one drachm; 4. c.c., of oleoresin.] Magnesium carbonate is employed to aid the diffusion of an oil in water through which air is to be inhaled. Resinous tinctures require an emulsifying agent; an equal part of mucilage of acacia is the best. The suspension of oil of turpentine in mucilage of acacia is a very common non-official example of an emulsion.

(c) Pharmacological Incompatibility; e.g., the combination of purgatives with astringents. Sometimes this is intentional, as in the occasional addition of atropine to a hypodermatic solution of morphine. After the description of each drug, those that are incompatible with it will be enumerated. 


\section{THE PRESCRIPTION.}

The details of a prescription should be written in the following order :

The first part is the Superscription, which is the sign R, an abbreviation for Recipe, "Take."

The second part is the Inscription, consisting of the names of the drugs in the genitive case (the vehicle in the accusative if $a d$ is used with it), and their doses in the accusative.

The third part is the Subscription, that is to say, the directions to the dispenser. This in England and most other countries is written in Latin, but in France it is in the language of the country.

The fourth part is the Signature, that is to say, the directions to the patient (from the Latin " Signetur," let it be labelled)." This is written in English.

The fifth part consists of the [physician's] name or initials at the bottom on the right, the patient's name at the bottom on the left, and under it the date; thus :

Superscription. $-\mathrm{R}$.

Inscription.-Tincture Ferri Chloridi, fl 3 iij ; [12. c.c.], (basis).

Quininx Hydrochloratis, gr. xxx; [2. gm.], (adjuvans).

Magnesii Sulphatis, 3 ij; [60. gm.], (corrigens).

Glycerini, $\mathrm{A} \tilde{\mathrm{z}} \mathrm{ij} ;[60$. c.c. $]$, (corrigens).

-Infusum [Cinchonx], ad flz $z$ viij; [240. c.c.], (excipient).

Subscription. - Fiat mistura.

Signature.-Take one table-spoonful three times daily, two hours after meals.

William Smith, Esq. (patient's name).

A. B. C. (physician's initials).

16th June, I90I (date).

[On the continent and in countries where the metric system is generally employed] the quantities, either of fluids or solids, are expressed in grammes, so that the abbreviation is omitted; [60. meaning $60 . \mathrm{gm}$. or 60 . c.c. as the substance may he solid or liquid.]

Abbreviations. - Although abbreviations are objectionable, yet this prescription could be written thus :

$$
\begin{aligned}
& \text { B. Tinct. Fer. Chlor., fl } 3 \text { iij ; [12. c.c.] } \\
& \text { Quin. Hydrochlor., gr. xxx. ; [2. gm.] } \\
& \text { Mag. Sulphat., 命 ij ; [60. gm.] } \\
& \text { Glycer., ft } z \text { ij ; [6o. c.c.] } \\
& \text { Inf. [Cinch.] ad } \mathrm{A} \tilde{\tilde{z}} \text { vij ; [240. c.c.] } \\
& \text { F. } m \text {. }
\end{aligned}
$$

S. Take one table-spoonful thrice daily, two hours after meals.
William Smith, Esq.
A. B. C. 16th June, 1901 .

$S$, ss. and $f$ s. are abbreviations for semi, 2 half, and $\overline{a a}$ for $a n a$, of each. 
The following is a prescription for a pill :

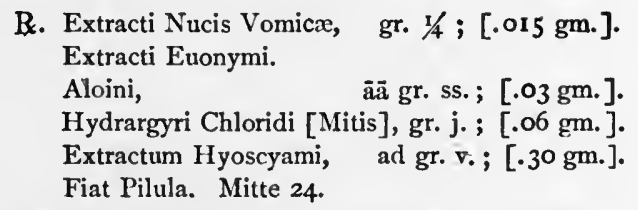

[S.] Take one immediately before dinner every evening. William Smith, Esq.

A. B. C. 16th June, rgor.

It will be observed that the quantities in the prescription are for one pill only, and the [apothecary] is directed to send 24. Often, however, the prescription is written with the quantity of each ingredient necessary to make the full number of pills. Thus :
B. Extracti Nucis Vomicæ,
Extracti Euonymi.
Aloini,
gr. vj. ; [.36 gm. $]$.
Hydrargyt
$\bar{a} a \overline{~ g r . ~ x i j . ; ~[.75 ~ g m .] . ~}$
Extractum Hyoscyami,
gr. xxiv.; [1.50 gm.].
Fiat Pilulæ 24.

[S.] Take one immediately before dinner every evening.

William Smith, Esq.

A. B. C.

I 6 th June, I90r.

Prescriptions for powders are also written in either way.

The medicine may be prescribed as a pill when it is required that the patient shall carry it about with him, when only a small dose is needed, when it is desirable that it shall act slowly, when it is required to act on the lower bowel, when it is insoluble or nauseous, or when it is difficult to prescribe in the liquid form. Kaolin is the best basis for substances, as potassium permanganate, which are decomposed by contact with organic matter.

Oils, and volatile, deliquescent or bulky substances should not be prescribed as pills, as they require much solid excipient; nor should pills be used for substances required to act immediately. Insoluble or very [disagreeable] powders are often given in cachets.

Abbreviations should be employed as little as possible. Serious mistakes have happened because the abbreviations have been ambiguous. The following are especially to be avoided :

Acid. Hydroc (may be Acidum Hydrochloricum or Acidum Hydrocyanicum).

Ext. Col. (" " Extractum Colchici or Extractum Colocynthidis).

Hyd. Chlor. ( “ "Calomel, Corrosive sublimate or Chloral hydrate). 
Hyd. (may be Hydrargyrum, Hydras, Hydriodas, Hydrochloras or Hydrocyanicus).

Sulph. (" " Sulphur, Sulphide, Sulphate, or Sulphite).

Sometimes the signature is written in Latin, and it is often abbreviated. A list of such abbreviations is given in the appendix.

In Great Britain [and in the United States] it is always understood, unless otherwise stated, that the preparations are those of the Pharmacopœia.

$A d$. - The prescriber should be careful in deciding whether or not to use this word before the vebicle. If it had been left out in the prescription given [on p. 44], the bulk of the mixture would have been nearly 10 $1 / 2$ fluid ounces; [315. c.c.], and the amount of the ingredients in each dose would have been less than was intended.

Dispensing the Prescription.-The dispenser should bear the following rules in mind : (1) Read the prescription through first. (2) Next write the directions, so that they have time to dry. (3) Solution by heat should not be used if more of the salt is ordered than will dissolve in cold water. In such case it must be suspended. (4) With fluids, measure them in such an order that the measuring glass shall be finally rinsed out with the vehicle. (5) Use glass scale pans. (6) Clean and put away everything directly after use. (7) If in the slightest doubt ask the prescriber. (8) If finally the prescription contains any insoluble matter, label "Shake the bottle." (9) If the medicine is very poisonous, label it as such and use a distinctive bottle. (10) If for outward application only, [label it as such]. (II) In dispensing substances chemically incompatible, if there is any likelihood that the new body formed is dangerous, communicate with the prescriber before dispensing (e.g., Potassium Iodide prescribed with Spiritus Ætheris Nitrosi forms free iodine; alkaloids are precipitated by alkalies). Should there be no such reason against dispensing the prescription (e.g., Liquor Potassæ and Ferrum Dialysatum), keep the incompatibles as far apart as possible by diluting each with the vehicle before mixing.

\section{PHARMACOLOGICAL AND THERAPEUTICAL ACTIONS.}

When the action of a drug is spoken of, the physiological action is usually understood.

The primary action is that due to the unaltered drug; e.g., the emetic action of zinc sulphate.

The secondary action is that due to compounds formed from the drug whilst it is in the body ; e.g., the antiseptic effect on the urine of Uva Ursi taken by the mouth is probably due to the fact that arbutin, the active principle of Uva Ursi, is in its passage through the kidney decomposed into a glucoside and hydroquinone, and the latter is a powerful antiseptic.

The direct or local action of a drug is that produced on any organ with 
which it comes in contact ; e.g., the cantharidin in cantbarides, in being excreted through the kidney, causes inflammation of it.

The indirect or remote action is a secondary effect, the result of the direct effect; e.g., curare paralyzes the respiratory muscles, consequently the blood becomes venous, and therefore convulsions take place. In this case the venosity of the blood and the convulsions are each of them indirect actions of curare.

It is clear that among drugs acting on the same parts, the total effect will depend very much upon which part is first affected. For example, atropine and curare will paralyze motor nerves, but atropine first affects the terminations of the vagus, and only late in its action the motor nerves of the voluntary and respiratory muscles; hence paralysis and asphyxia are late symptoms, and a rapid pulse is an early symptom. Curare, however, early affects the nerveendings of the voluntary and respiratory muscles, and the heart towards the end; therefore asphyxia and paralysis occur early, and a rapid pulse is a late symptom.

Relation between Chemical Constitution and Physiological Action. - There is no doubt that the physiological action of a drug often depends upon its chemical constitution. Naturally, substances which are broken up in the body in such a way as to lead to the liberation of a common element or group will have a similar action. Probably, also, the action of a drug depends upon electrolytic dissociation of its solutions.

Instances in which chemical constitution influences action are the similarity of effects of nitrites, the fact that all chlorides, bromides, and iodides of ethane and methane are anrsthetic, the similarity of action of the iodides of many metals and the similarity of action of bromides of many metals.

Substitution of one radical for another in organic compounds often strikingly modifies their action; for example, if strychnine, brucine and thebaine are converted into methyl-strychnine, methyl-brucine and methyl-thebaine, the convulsive action of each of the first three substances is replaced by a paralyzing action. The effect of substitution may be also well seen in the various derivatives of atropine and cocaine, and in the relation of aconitine to benzaconine and aconine. Another very interesting case in point is that methylglucoside is sweet, ethyl-glucoside is somewhat sweet, phenyl-glucoside is bitter, and benzyl-glucoside is intensely bitter.

Sometimes the position of the radicals in the molecule is of great physiological importance; thus resorcin (metadihydroxy-benzene) is very sweet, while pyrocatechin (orthodihydro-benzene) is bitter.

Sometimes the atomic weight appears to influence the intensity of action, for the relative toxicity of various alcohols is as follows : Methyl-alcohol, 0.8 ; ethyl-alcohol, 1.0; propyl-alcohol, 2.0; butyl-alcohol, 3.0; amyl-alcohol, 4.o. The difficulty of the whole subject is, however, so great that it is impossible at present to lay down laws suffieiently general to be of any use to the beginner. It must be remembered that dissimilarity of action is often more apparent than real, for it may be due to varying solubility, digestibility, rate 
of absorption, rate of elimination, or rate of osmosis, also to the organ which happens to be first affected, and the degree to which the drug can dissolve the constituents of tissues.

Drugs may be classified according to the parts on which they act, and before describing each individual drug, a classification on this principle will be given.

Division I.-DRugs aCting upon ORganisms which INFECT the Human Body, or upon Processes going on outside it.

Antiseptics are drugs which arrest putrefaction, either by preventing the growth of, or completely destroying the microorganism on which decomposition depends. Some authors limit the use of the word to those drugs which [restrain the development] of micro-organisms, and call those substances which destroy the [vitality of] micro-organisms, [germicides or] disinfectants. [The term disinfectant, by extension, is applied to those agents which kill non-pathogenic bacteria as well as to those which destroy disease germs.]

Statements are most discordant as to whether certain substances are antiseptics, and as to the strength of their antiseptic power. This is because antiseptics act differently on different organisms ; and the distinction has not been drawn between preventing the growth of, and destroying micro-organisms. Also because the power of antiseptics depends upon the temperature at which they act, the medium in which they are dissolved, the strength of the solution, the time given them to act, and the number of micro-organisms present in the substances to which they are added.

To properly test the value of an antiseptic the above condltions must be noted. All instruments and substances-except the fluid containing the micro-organisms to be tested-are heated so that any adventitious micro-organisms are destroyed. A cultivating medium, such as agar-agar jelly, in which the micro-organisms will grow, is selected, and two test-tubes, each containing some of it, are taken; to one of these the supposed antiseptic is added. Some fluid containing the micro-organisms is then added to both test-tubes; both are plugged with sterilized cotton to prevent the entrance of germs from the air, and it is observed whether the 
micro-organisms will grow in the tube containing no antiseptic, but not in that containing the antiseptic. As the power of an antiseptic depends on so many circumstances, no exact order of their potency can be given, but roughly the more powerful are placed first in the following list; the last are very feeble.

I. Heat. - This is the best antiseptic, but a temperature of at least $212^{\circ} \mathrm{F}$. $\left[100^{\circ} \mathrm{C}\right.$.] is required. After an infectious fever, clothing, bedding, etc., may be heated in a dry-air chamber to between $200^{\circ}$ and $300^{\circ} \mathrm{F}$. [93.5 and $149^{\circ}$ C.]; or what is far better, as dry air does not penetrate the spores nearly so well as moist, and the interior of the rolls of fabrics often bardly gets heated at all, steam under pressure may be driven through them. Another useful way is to boil the infected things in water. Surgical instruments are disinfected in this way [but one per cent. of washing soda (sodium carbonate) should be added to the water to prevent their rusting].

2. Corrosive Mercuric Chloride.-A solution of $I$ in 1000 is constantly used for washing hands, and for many other purposes connected with midwifery and surgical operations. [For most uses one part to 3 or 5000 of water or even weaker, is the limit of safety. Gauze of the strength of $\mathbf{I}$ to 2000 will blister, if the skin is damp.

3. Formaldehyde, of which the forty per cent. solution is known as Formalin, bas extraordinary power as a surface disinfectant, greater than that of any known substance. It is especially useful for the disinfection of rooms and their contents when volatilized from a specially constructed lamp.]

4. Chlorine is, as a rule, too irritating. Chlorine gas, disengaged by the action of hydrochloric acid on manganese dioxide, may be used to disinfect a room, the windows, chimneys and doors of which are sealed. It must be remembered that it attacks and bleaches many substances.

5. Chlorinated Lime [is the best antiseptic for all excreta (Sternberg)].

6. Bromine, and, 7, Iodine are rarely used, as they are too irritating.

8. Carbolic acid is used [but infrequently. If surgical instruments have been previously sterilized, the use of carbolic acid indicates a distrust, on the part of the surgeon, of his assistants. ]

9. Quinine, and, ro, Salicylic acid are too expensive for ordinary use.

II. Iodoform is commonly used to dust upon wounds, etc.

12. Boric acid is used for many surgical purposes.

13. Zinc chloride, and, 14, Potassium permanganate, are much used domestically.

15. Solution of Hydrogen dioxide is the ingredient of [various popular disinfectants].

16. Oleum Eucalypti is used in surgery.

17. Sulphurous acid, disengaged by the burning of sulphur, is used to disinfect rooms. 
18. Creosote, 19. Benzoin, 20. Zinc sulphate, 21. Ferric oxide, 22. Thymol, 23. Alcohol, 24. Balsam of Tolu, 25. Balsam of Peru, are none of them much used.

26. Lysol, Creolin [and various cresol compounds] are not pharmacopoeial, but they are powerful and much employed.

We do not know of any drugs which, when taken internally or inhaled, will certainly destroy micro-organisms, either in the gastro-intestinal tract or respiratory passages, unless they are sufficiently concentrated to be fatal to the patient. Some authorities, however, consider that naphtol, calomel, and some other substances will destroy many varieties of micro-organisms in the stornach and intestines. [The fact is often lost sight of that an infinitely small amount of a remedy which could not be administered in sufficient amounts to destroy, will often completely inhibit the growth of micro-organisms, and thus should be classed as an antiseptic.]

Deodorants, or deodorizers, are substances which destroy disagreeable smells. There are too many for enumeration. Many antiseptics are deodorizers. [Charcoal is often called a disinfectant, but it is merely a deodorizer; it is powerless if it is wet.]

Antizymotics.-This is a word sometimes applied to drugs which arrest fermentation.

Anthelmintics are drugs which kill such parasitic worms as infest the alimentary canal. Three kinds only are commonly met with in the temperate zone.

(I) Tape-worm (Tenia solium and Tenia mediocanellata). Anthelmintics: [Aspidium] (mostly used), Oleum Terebinthinæ, [Kamala,] Ḉsso, Granatum, [and Pepo.].

(2) Roynd-worm (Ascaris lumbricoides). Anthelmintics: Santonin, [and Spifgelia and Senna.]

(3) Thread-worm (Oxy'uris vermicularis). Anthelmintics: Rectal injections of salt water, infusion of quassia, solutions of iron salts, or diluted oil of turpentine. It is doubtful whether these drugs (except turpentine) relieve the patient by killing the thread-worms which inhabit the rectum, or merely, by removal of mucus, render this part unfit for them. [It is probable that rectal injections are useless. Large soap and water enemata, the patient being in the knee chest position, give the best results. (Whittaker.)]. 
Anthelmintics for the tape or round-worm should be given when the alimentary tract is empty. Hence it is a good plan to give a dose of castor oil a few hours before the anthelmintic, so as to ensure that the drug comes in contact with the worm. To expel the dead parasite a purgative should be given a few hours after the anthelmintic. [Castor oil should not be used if aspidium has been administered.] Purgatives used for this purpose are called Vermifuges. Vermicide is a term sometimes applied to drugs which kill intestinal entozoa.

Antiparasitics or parasiticides are substances which destroy parasites. The term is usually applied to those which destroy parasites infesting the skin.

(I) For the various forms of tinea the following are used: Mercurial preparations, especially the oleate, tincture of iodine, [glycerite] of carbolic acid, an ointment of pyrogallic acid, a boric acid lotion, a salicylic acid lotion, acidum sulphurosum, [formaldehyde] and thymol; and if the patches are small, severe irritants, as croton oil, cantharides, and chrysarobin ointment. Tinea versicolor never requires severe irritants.

(2) As a parasiticide for itch, sulphur ointment is generally used. Balsam of Peru and [Styrax] are also effectual.

(3) Pediculi vestimentorum will be killed by any mild parasiticide. Unguentum Staphisagriæ [unofficial; I part powdered seed, 2 parts each, olive oil and lard], is often used.

(4) Pediculi capitis and pediculi pubis are also easily killed by mild parasiticides; mercurials are commonly employed, so also is Unguentum Staphisagriæ.

Antiperiodics are drugs which arrest the return of diseases which recur periodically. Some, and probably all, act as direct poisons to the micro-organism causing the disease.

They are cinchona bark, quinine and its salts (by far the most powerful), cinchonine, arsenous acid, eucalyptus, hydrastis, salicin, salicylic acid [and berberine]. They are used for all forms of intermittent fever and neuralgia.

Division II. -DRugs acting on the Blood.

A. Drugs acting on the Plasma.-Many substances must after absorption exist in solution in the plasma, and purgatives, diuretics and diaphoretics must alter the composition of the plasma by abstracting substances from it; but while drugs are 
given with the object of acting on the plasma it is in order to render it more alkaline: we know no drugs which will make it acid, or even markedly reduce the natural alkalinity of the plasma, as the mineral acids can only exist in it in the form of neutral salts.

The alkalizers of the plasma are salts of-

(I) Potassium.

(2) Sodium.

(3) Ammonium.
(4) Lithium.

(5) Magnesium.

(6) Calcium.

This is approximately the order of their alkalizing power. Potassium is certainly the most powerful, while calcium is very feeble.

The citrates and tartrates of these metals are decomposed in the plasma into alkaline carbonates. As alkalies unite with uric acid and form soluble urates, they are given with the hope that this will occur in the plasma. The diuretic effect of the alkali aids the excretion of the urates.

Therapeutics.-The chief use of alkalies is their administration in gout, in which disease the uric acid is greatly in excess in the plasma. As the treatment has to be continued for some time, a preparation which does not [disturb] digestion, such as potassium citrate, is usually preferred, or lithium citrate, for the lithium compound of uric acid is very soluble in water; but the value of lithium is doubtful, as it does not increase the solubility of urates in the plasma. For the same purpose the [numerous] natural alkaline waters are frequently prescribed.

In lead-poisoning the lead is locked up in the tissues in a very sparingly soluble form. Potassium iodide was given because some authorities believed it increased the solubility of lead in the plasma, and consequently facilitated its excretion by the kidneys.

Alkalies have been largely used in rheumatic fever, on the assumption that there is a deleterious agent in the plasma, and that its solubility is increased by increasing the alkalinity of the plasma; but this treatment has now been abandoned in favor of that by salicylates. For the same theoretical reason alkalies have been given in rheumatoid arthritis. 
Purgatives, diaphoretics and diuretics necessarily alter the composition of the plasma, and are largely used when there is much œedema of any part, or effusion into serous cavities, in the hope that as fast as these remedies drain off fluid from the plasma it will be replaced by that which is effused pathologically. Also they are given in conditions, as uræmia, in which it is thought that there are poisons in the blood, in order that their excretion may be hastened.

The composition of the plasma can also be altered directly either by venesection or transfusion.

B. Drugs acting on the Red Corpuscles.-The most important are those which can increase the amount of hæmoglobin when that is deficient. Strictly speaking, all these have a pathological and not a physiological action, for we know of no drugs which will increase the amount of iron in perfectly healthy blood. These drugs are called hæmatinics.

They are-

(I) Iron and its salts.

(2) Arsenous acid.

(3) Potassium permanganate (doubtful).

(4) Copper salts

(5) Hydrochloric acid

(6) Potassium salts

(7) Phosphorus

(doubtful).

They not only increase the quantity of hæmoglobin in each corpuscle, but also the number of red corpuscles. Their action is much aided by good food, fresh air, and attention to the general health, and especially to the digestive organs. The mode of action of these hæmatinics is very obscure, and will be discussed under each drug. Iron is by far the most important and effectual.

Indirect hæmatinics are drugs which benefit the patient by removing some obvious cause for his deficiency in hæmoglobin, or anæmia, as it is generally termed. Such are mercury, given for syphilis, quinine for ague, etc.

Alcohol and quinine diminish the oxygenating power of the blood, for they render oxyhremoglobin a more stable compound than it usually is, but their action in this direction is slight. Citrates and tartrates of the alkaline metals are partially oxidized to carbonates at the expense of the oxygen of the red blood-corpuscles. 
The size of the red blood-corpuscles is said to be diminished by carbon [dioxide], quinine and morphine, and to be increased by oxygen, hydrocyanic acid, and quinine; and their number is said to be increased by small doses of mercury.

A large amount of sodium chloride causes the red corpuscles to pass rapidly through the walls of the capillaries.

Quinine and hydrocyanic acid diminish the ozonizing power of the blood.

There are some drugs which are not employed therapeutically for their action on the blood, which are nevertheless very important physiologically and toxicologically, for they kill by altering the composition of the hæmoglobin, thus preventing its uniting with oxygen. Such are carbonic oxide, which turns out the oxygen from oxyhæmoglobin, hydrocyanic acid, which forms cyano-hæmoglobin, and potassium chlorate. Acetanilid, antipyrin, phenacetin, and nitrites, especially amyl nitrite, convert the hæmoglobin into methæmoglobin; acetanilid, amyl nitrite, potassium chlorate, and pyrogallic acid destroy the red corpuscles.

Phosphorus, arsenic, hydrogen [sulphide], turpentine, iodine, and sulphur also reduce oxyhæmoglobin. Phosphorus is especially destructive to the blood.

When freshly drawn blood is exposed to the air its oxidization is diminished by hydrocyanic acid, alcohol, chloroform, quinine, morphine, nicotine, strychnine, and brucine.

\section{Drugs acting on the White Corpuscles.-Most if} not all drugs which are poisonous to amœbæ are poisons to white corpuscles when applied in sufficient strength, which, however, is rarely the case in the human body. All irritants which set up inflammation cause the white blood-corpuscles to wander through the capillary walls; and all the cinchona alkaloids, viz., quinine, quinidine, cinchonine, and cinchonidine, have the power of arresting this migration; of these, quinine is the most powerful. [Berberine sulphate and] acetanilid are also powerful. If the quinine is circulating in the capillaries, it prevents the white corpuscles from wandering out; if it is applied to the outside of the vessels, it prevents the corpuscles from wandering away from the vessel through the wall of which they have passed. 
Verátrine applied to white corpuscles outside the body kills them.

Camphor, myrrh and other aromatics are said to increase their production by increasing absorption from the intestine.

The following facts do not fall under any of the previous headings:-Poisonous doses of mercury increase the fluidity of the blood, impair its power of coagulation, and diminish the solids in it. Cod-liver oil increases the solids of the blood. Potassium iodide and calcium salts [especially the chloride] are said to increase the power of coagulation; other substances doing this will be described under astringents.

Division III.-Drugs acting on the Cardiac Mechanism.

The heart is capable of spontaneously originating impulses which in health begin in the sinus venosus, and spread downwards over the auricle and the ventricle to the apex. It used to be considered that these movements were due to spontaneous impulses proceeding from the cardiac ganglia surrounding chiefly the entrance of the superior and inferior venæ cavæ, the entrance of the pulmonary veins, and the auriculo-ventricular groove ; but we now know that there is no certain evidence that these ganglia originate impulses, and most of the evidence goes to show that the contraction of the muscular fibres is due to spontaneous impulses arising in them. This contractile power of the muscular fibres can be inhibited by the vagus, the fibres of which proceed from the vagal nucleus in the medulla, and can be augmented by the augmentor or accelerator nerve-fibres, which proceed downwards in the cervical spinal cord to the upper dorsal nerves, from which they pass through the first thoracic ganglion to the sympathetic, and so to the cardiac plexus, and thence to the heart. We are ignorant of any function for the cardiac ganglia; we know that medullated nerve-fibres lose their medulla in them, and that more fibres proceed from them than enter them. Possibly they have a nutritive function. We have, therefore, only to consider the action of drugs on the muscular substance of the heart, on the vagal or inhibitory fibres, on the vagal centre, on the augmentor, accelerator, or sympathetic fibres, and on the accelerator centre. The centres are remarkably easily affected by afferent impulses, proceeding from the heart itself or from almost any part of the body. Our information concerning the 
action of drugs on the heart of man is necessarily rather inexact, for many experiments are difficult to perform upon the mammalian heart, consequently the cold-blooded animals have been largely used; and as some differences are observed among them-for example, between the frog and the tortoise-it is probable that the deductions drawn from experiments upon the hearts of warmblooded animals are not wholly applicable to man. In the following account of drugs the action described is that of a moderate dose; the action of a large dose is generally the reverse of that of a moderate dose.

A. Drugs acting upon the Heart directly.-Our knowledge of these has been gained by studying the action of drugs upon excised hearts or pieces of the heart, and the action of drugs locally applied to the heart, either by gently applying a solution externally, or by means of a [transfusion] cannula. It is difficult to decide whether a drug acts upon the muscular fibre itself, or upon the fine nerves between these fibres, so that no attempt will here be made to distinguish between these actions. As the apex of the heart contains fewer nerves than the rest of the organ, it has been concluded that if a drug acts upon the apex, when it is cut off from the remainder of the heart, it acts upon the muscle only; but it would be difficult absolutely to deny the existence of fine nerve-fibres in the apex. The vagus or inhibitory nervous mechanism has been much more studied than the accelerating. The effect of stimulating the muscle may be either to increase the rate or the force of the beat, or to do both; that of stimulating the minute branches of the vagus or its terminations in the heart will be either to diminish the rate or the force of the beat, or both; and the effect of stimulating the accelerator fibres will be just the opposite; and in each of these cases the effect of paralyzing will be the reverse of stimulating. The distinction between a stimulating effect on the terminations of the vagus and a depressing effect on the terminations of the accelerator nerves might be determined by observing the effect of stimulation of each of these nerves before and after the local application of the drug, provided that it has been shown that the muscle itself is not affected by the drug; but this 
is often difficult to prove. It is easily seen that the complexity of the problem is so great that it will be most convenient to classify the drugs which act locally on the heart by the effect they produce, without attempting to say whether they act on the muscle or nerve terminations.

Drugs increasing the force of the contraction:

(I) Digitalis. Q.K.

(2) Strophantbus. Q.K.

(3) [Sparteine.]

$>$ (4) Squill. fre of rwime.

(5) Sapónin.
(6) Convallaria Majális.

(7) Caffeine.

(8) Verátrine.

(9) Erythrophloum.

(ro) Barium Salts.

In large doses these drugs in frogs always cause arrest of heart in systole; in mammals the final arrest may be in diastole with some, e.g., digitalis. They all slow the pulse.

(II) Dilute solutions of salts of the alkaline metals.

(12) Dilute solutions of copper double salts.
(13) Dilute solutions of zinc double salts.

(14) Dilute solutions of chloral.

(15) Physostigmine.

(16) Camphor. $0.1 \%$ incis

These drugs have the same action without the final arrest in systole. The rate of the pulse is not markedly altered.

Drugs the chief action of which is to decrease the force of the contraction, usually with stoppage in diastole:

(I) Diluted acids. + Stomach f pul (7) Muscarir

(2) Strong solutions of salts of the alkaline metals.

(3). " of barium salts.

(4) " of copper double salts.

(5) " of zinc double salts.

(6) Strong solutions of chloral.

(7) Muscarine.

(8) Pilocarpine.

(9) Sapónin (large doses).

(Io) A pomorphine. only for tructie.

(II) Emetine.- from y fuenc

(12) Salicylic acid (large doses). Qhemmate T

Drigs an important action of which is to inerease the rate of the cardia. beat:

- (I) Atropine.

$>$ (2) Hyoscyamine.

(3) Daturine.
(4) Duboisine.

(5) Cocaine.

(6) Saponin. 
Drugs an important action of which is to slow the rate of the cardiac beat (see also first list given above):
(1) Muscarine.
(2) Pilocarpine.

Drugs which increase both the force and the number of the beats:

$>$ (I) [Ammonium salts.]

(2) Alcohol.

(3) Ether.

(4) Chloroform.

(5) [Cactus.]
(6) Anæsthetics.

(7) Arsenical salts.

(8) Quinine.

(9) Strychnine.

Drugs which decrease both the force and the number of the beats:

(1) Antimony salts. - no.

(2) Aconite.

(3) Hydrocyanic acid.
(4) Ergot.

(5) Veratrum Viride.

(6) [Cevadilla.]

B. Drugs acting on the Vagus Centre.-If we observe that the giving of a drug to an animal alters the beat of the heart, but that this alteration can be done away with, either by cutting the vagi or stimulating the peripheral end of the nerve,-if one only of them be cut, - we may conclude that the drug acted on the vagus centre in the medulla.

Drugs which stimulate the vagus centre: that is to say, the pulse is slowed, but this slowing disappears on section of the vagi :

(I) Chloroform.

(2) Chloral hydrate.

(3) Butyl-chloral [hydrate.]

(4) Aconite.

(5) Veratrum Viride.

(6) Nicotine.

(7) Digitalis.

(8) [Sparteine.]

(9) Strophanthus.
(10) Squill. - Egiciterant.

(II) Convallaria Majalis.

(12) Hydrocyanic acid.

(13) Cocaine (large doses).

(I4) Staphisagria (Delphinine.)

(I5) Atropine. Only very

(I6) Hyoscyamine. early in their

(17) Daturine.

(18) Increased blood-pressure.

(19) Venous blood.

Drugs which depress the vagus centre: Large doses of the drugs mentioned in the last list, and drugs which diminish the blood-pressure, such as amyl nitrite [nitroglycerin, and the nitrites.]

C. Drugs acting on the Accelerating Centre.-We do not know anything of drugs which depress this. Probably some 
stimulate it, for their administration renders the pulse still more rapid after the vagi have been cut.

They are-

(I) Ammonia.

(2) Caffeine.

(3) Picrotoxin.

(4) [Cactus.]
(5) Delphinine.

(6) Any drugs which make the blood venous.

Therapeutics.-The drugs most used for their action on the heart are digitalis, strophanthus, [ammonium salts, sparteine], squill, convallaria majalis, caffeine, alcohol, ether, chloroform, [cactus], strychnine, belladonna, aconite, antimony, and hydrocyanic acid. The therapeutic indication for each of these drugs will be found given under the individual drug.

\section{Division IV.-Drugs acting on the Vessels.}

These are usually studied either by directly observing alterations in the size of the vessels in some thin structure, such as the ear of a rabbit, the mesentery, tongue, lung, web, or mylo-hyoid [muscle] of a frog, or the wing of a bat; or the rate of the flow may be observed. This can be conveniently done by cutting some part, as the toe of a frog, and noticing the rate at which the blood flows from the cut vessels with and without the administration of the drug to the animal. "It is often necessary that an artificial circulation should be maintained; for if not, it might be difficult to prove that the alteration in the quantity of blood flowing from the cut surface was not due to influences acting on the cardiac mechanism. In order to determine if the changes are due to local or central effects, it is necessary to destroy the spinal cord, or to cut the nerves going to the part. When a drug is applied locally, as to the mesentery, and the vessels alter, if the nerves going to the part are not cut, it is difficult to say whether this alteration is direct or reflex.

Drugs are applied to the interior of vessels by injecting them into the circulation.

We know that each vessel is controlled by vaso-constrictor and vaso-dilator nerves, and̆ that these proceed by different paths 
from the central nervous system, but we do not know by which set of nerves drugs act ; probably some by the vaso-constrictor, and some by the vaso-dilator. We can only classify the drugs into those which dilate or contract the vessels by local action, and those which produce these effects through their action on the central nervous system. When a drug acts locally we cannot tell whether it acts on the muscle in the wall of the vessel, or on the nerve terminations.

It of course follows that drugs acting on the heart, or on a large area, will considerably modify the blood-pressure.

\section{A. Drugs acting locally on Vessels.}

Drugs which, when locally applied to vessels, dilate them:

(I) Liquor Ammoniæ.

(2) Silver nitrate

(3) Zinc chloride

(4) Copper sulphate $\}$ (strong)

(5) Mercuric nitrate.

(6) Arsenous acid.

(7) Antimony and potassium tartrate.

(8) Iodine.

(9) Chlorine.

(IO) Mineral acids (strong).

(II) Alcohol. If prevented

(12) Ether. - from evaporat-

(13) Chloroform. ing.

(14) Carbolic acid.

(I5) Creosote.
(16) All volatile oils, as oils of turpentine, and many substances containing them, as mustard, [horse-radish] etc.

(17) Senega.

(18) Chrysarobinum.

(19) Ipecacuanha.

(20) Capsicum.

(21) Croton oil.

(22) Camphor.

(23) Cantharides.

(24) Phosphorus.

(25) Warmth, however applied, but usually as a poultice. [This is true if its evanescent effect is desired. When long applied it contracts blood-vessels.]

Irritants.-All of the above, as they dilate the vessels, are often spoken of as vascular irritants.

Rubefacients are drugs which, when locally applied to the skin, cause it to become red because of the vascular dilatation induced. All the above drugs are rubefacients.

Vesicants.-Many of these drugs are sufficiently powerful irritants to cause inflammation. If this goes no further than the exudation of plasma from the vessels, and this plasma collects 
under the epidermis to form vesicles, the drug causing the production of vesicles is said to be a vesicant ; e.g., cantharides.

Pustulants are such of the above drugs as are sufficiently powerful irritants to cause the inflammatory process to proceed to the passage of leucocytes through the-walls of the capillaries. They collect in the vesicles, which consequently become pustules; e.g., croton oil.

Escharotics or caustics are the most powerful of all the above drugs. Their local application destroys the vitality of the part to which they are immediately applied, and sets up vascular dilatation of the surrounding area ; e.g., strong nitric acid, zinc chloride, silver nitrate, and arsenous acid.

Counter-irritants. - It has been shown by experiments on animals that when the vessels of the skin are dilated by the application of an irritant, those of the subjacent viscera are often reflexly altered in size. The same is probably true of man. An irritant is called a counter-irritant when it is applied to the skin with the object of altering the size of the vessels of the subjacent viscera. It is particularly to be remembered that the action is a reflex nervous one, and is in no way due to the withdrawal of blood into the dilated vessels of the skin. them:

The following, when inhaled, dilate peripheral vessels by acting locally on

(I) Amyl nitrite.

(2) [Nitroglycerin.]

(3) Sodium nitrite, them:

Drugs which, taken by the mouth, dilate arterioles by acting locally on

(I) Caffeine.

(2) Amyl nitrite.

(3) [Nitroglycerin.]

(4) Sodium nitrite.
(4) Ethyl nitrite.

(5) Spiritus ætheris nitrosi.

(6) Erythrol tetranitrate.
(5) Ethyl nitrite.'

(6) Spiritus æetheris nitrosi.

(7) Erythrol tetranitrate.

(8) Nicotine.

Drugs which, taken by the mouth, contract arterioles by acting locally on them :

(I) Ergot.

(2) Caffeine (early in its action).

The following have been shown by experiments in the laboratory to cause contraction of small arteries through which they circulate : copper, zinc, tin, 
platinum salts all cause powerful contraction. Lithium, calcium, strontium, magnesium, cadmium, nickel, cobalt and iron salts cause slight contraction.

Drugs which, when locally applied to vessels, contract them:

These may act in two ways, either by contracting the muscular coat of the vessels, or by coagulating the albuminous fluids around them, the coagulum by its contraction constricting the vessels.

Those which act on the muscular coat of the vessels:

(I) Cold, however produced; hence rapidly volatilizing substances as ether. [This effect is but temporary. If cold is long continued it dilates blood-vessels.

(2) Cocaine.]

(3) Lead salts.
(4) Dilute solutions of silver salts.

(5) Diluted sulphuric acid.

(6) Alum.

(7) Hamamelis.

(8) [Ergot.

(9) Hydrastis.]

(I0) Acetanilid.

(II) [Antipyrin.]

Those which coagulate the albuminous fluids around the vessels:

(I) Tannic acid and all substances containing it: e.g., [nutgall,] krameria, kino, hæmatoxylon, hamamelis, cinnamon, eucalyptus gum, and catechu.

(2) Lead salts.
(3) Silver salts.

(4) Zinc salts.

(5) Copper salts.

(6) Alum.

(7) Ferric salts.

(8) Bismuth salts to a slight extent.

\section{B. Drugs which act on the Vaso-motor Centres.}

Drugs which, by their action on the vaso-motor centres, dilate the vessels:

(I) Belladonna.

(2) Stramonium.

(3) Hyoscyamus.

(4) Alcohol.

(5) Ether.

(6) Chloroform.

(7) Chloral hydrate.

(8) [Antimony and Potassium Tartrate.]
(9) Aconite.

(Io) Ipecacuanha.

(II) Lobelia.

(I2) Tobacco.

(13) Veratrine.

(I4) Hydrocyanic acid.

(r5) Opium.

Some of the substances, which in small doses contract the ressels by central action, in large doses dilate them; viz., digitalis and squill. 
Drugs which, by their action on vaso-motor centres, cause contraction of vessels :
(1) Ergot.
(2) Digitalis.
(3) Strophanthus.
(4) [Sparteine.]
(5) Squill.
(6) Physostigmine.

(7) Cocaine.

(8) Hydrastis.

(9) Hamamelis.

(I0) Strychnine.

(II) Lead salts

(12) Ammonia (slightly.) $^{2}$

Also, for a very short early period of their action, some substances whose main action is to dilate the vessels by their central action; viz., belladonna, stramonium, hyoscyamus, alcohol, ether, chloroform, hydrocyanic acid and veratrine.

Astringents are drugs which diminish the size of the vessels, and thus decrease the amount of exudation from them.

Styptics, or Hæmostatics, are drugs which stop bleeding. They comprehend all astringents, especially cold, lead and copper salts, hamamelis, ergot, [hydrastis], tannic acid, and, above all, ferric salts, for they coagulate the blood which is flowing from the vessel, and the clot prevents further bleeding. Matico leaves, because of the numerous hairs on their under surface, favor coagulation of blood when locally applied to a bleeding surface. Cobwebs act in the same way.

Therapeutics.-Drugs which locally dilate vessels are [frequently] applied to stimulate sores to heal, and to promote absorption of inflammatory products, as seen in the application of iodine over joints in certain forms of joint disease; and as counter-irritants in many forms of disease of deep-seated organs, as in the application of a blister in pleurisy. Drugs which by their central action cause dilatation of all the vessels of the body are used in certain forms of heart disease, as in the use of amyl nitrite in angina pectoris; and some suppose that the good they do is brought about by dilating the vessels, and so rendering the work of the heart easier. Amyl nitrite and nitroglycerin are much used for this purpose. Drugs causing general vascular dilatation are also employed to cause dilatation of the vessels of the skin with the object of thereby leading to an increase of perspiration and an increased radiation of heat. Alcohol, 
Spiritus Ætheris Nitrosi, and Ipecacuanha, amongst others, are used in this way.

The most important use of astringents is as styptics; they are ulso used to check excessive discharges of all sorts, as in diarrhœa, leucorrhœa, etc., and in relaxed conditions of vessels, such as are often seen in pharyngitis.

There is perhaps no better opportunity than this of mentioning emollients and demulcents.

Emollients are substances which soften and protect parts. The word is usually employed for substances applied to the skin.

Common emollients are substances soaked in warm water, as hot fomentaGons and poultices, fats of various sorts, as lard and lanolin (hydrous wool fat), non-irritating oils, as olive oil, spermaceti, [petrolatum, ] vaseline, etc.

Demulcents are substances which protect and soothe parts. They are generally applied to mucous membranes, especially when unduly dry, and thus they are often used for the mouth.

Instances of them are gelatin, isinglass, glycerin, gum, honey, flaxseed, starch, and white of egg.

\section{Division V.-Drugs acting on the Skin.}

Ail those described in the last section act on the cutaneous vessels, but in addition we have-

A. Diaphoretics, or drugs which increase the amount of perspiration. These may do so either by stimulating the sweat centres in the spinal cord, the nerves proceeding from the centres to the glands, the terminations of the nerves in the gland, or the glandular cells themselves; or dilatation of the cutaneous vessels may, by the increase in the amount of blood and the greater warmth, stimulate the glands and lead to an increase of sweat. It is difficult to tell whether drugs acting on the vessels do not also act on the other parts of the mechanism; and it is also difficult to decide whether a drug acts on the gland-cells or the termination of the nerves, so we will consider diaphoretics under two headings, those which act centrally and those which act peripherally. These are differentiated by observing whether the 
drug acts after the spinal cord is destroyed, [or] on a part of the skin after the nerves going to it are cut.

(a) Diaphoretics acting peripherally: Pilocarpine greatly increases the amount of sweat, acting in all probability on the nerve terminations in the gland-cells, but certainly not on the vessels. Nicotine also acts peripberally. Local applications of warmth, and alcohol taken internally perhaps act in the same way in addition to their vascular action.

(b) Diaphoretics acting centrally:

(I) Antimony salts.

(2) Ammonium acetate.

(3) Ammonium citrate.

(4) Ipecacuanha.

(5) Opium.

(6) Camphor.

(c) Diaphoretics whose mode of action is doubtful: Senega, cubeb, colchicum, salicin, lobelia, arnica, aconite, potassium citrate and acetate. All these, except the last two, are very fceble.

When a diaphoretic acts very powerfully it is called a Sudorific.

B. Anhidrotics, or Antihidrotics, drugs which diminish the amount of perspiration. The part on which these act is determined in the same way as in the case of diaphoretics.

(a) Anhidrotics acting peripherally: Atropine is very powerful; it acts on the terminations of the nerves in the glands; and hyoscyamus and stramonium probably act in the same way. The local application of cold has a similar action.

(b) Anhidrotics the mode of action of which is doubtful:

(x) Acids.

(2) Muscarine.

(3) Nux vomica.

(4) Quinine.
(5) Picrotoxin.

(6) Zinc salts.

(7) Salicylic acid.

[(8) Camphoric acid.]

Therapeutics.-Diaphoretics are used for three purposes: either to increase the amount of sweat because that of the urine is failing, and for this purpose pilocarpine is largely used; or in the hope that poisons may be excreted by the sweat, hence the use of pilocarpine in uræmia ; or as mild antipyretics, in order to increase the loss of heat by increased evaporation ; for this purpose alcohol, ipecacuanha, ammonium acetate, and opium were formerly much employed, but of late years much more efficient antipyretics have been discovered. 
Anhidrotics are used either for general conditions, as phthisis, or for local conditions, as sweating of the feet; but they are not of great use in medicine.

We do not know of any drugs which will alter the composition of the sweat, except in so far as that certain drugs may be excreted in the sweat when taken internally; such are iodine, potassium iodide, succinic, tartaric, and benzoic acids, the last in the form of hippuric acid.

We have no knowledge of the effects of drugs on the sebaceous secretion.

Certain drugs, when taken internally in large doses, produce a rash on the skin, possibly because in the course of their excretion through the skin they irritate it. Such are-

(I) Copaiba.

(2) Cubeb.

(3) Bromides.

(4) Iodides.

(5) Turpentine.

(6) Belladonna.

(7) Chioral [hydrate.]

(8) Opium.

(9) Quinine.

(I0) Salicylic acid.
(II) Arsenical Salts.

(12) Acetanilid.

(13) [Antipyrin.]

(14) Phenacetin.

(15) Chloralamide.

(I6) Antitoxins. C

(17) Serums. $\angle$

(18) Silver salts may discolor the skin.

(rg) [Sulphonal.]

[The following quite rarely produce an eruption :

(I) Iron.

(2) Strychnine.

(3) Creosoce.

(4) Mercury.

(5) Veratrum.
(6) Digitalis.

(7) Sulphur.

(8) Antimony.

(g) Santonin.

(10) Cod Liver Oil.]

Division VI.-Substances acting on the URINARy System.

1. Drugs increasing the quantity of urine secreted. - These are called diuretics. The kidney is a double organ with two distinct varieties of epithelium; it is particularly well supplied with vessels and vaso-motor nerves, and is also profoundly under the influence of variations in the quantity of blood flowing through it ; hence it is, with our present state of knowledge, impossible to say how many diuretics act, but the following table modified, 
from Brunton's work, shows the various ways in which they probably act, many in more ways than one :

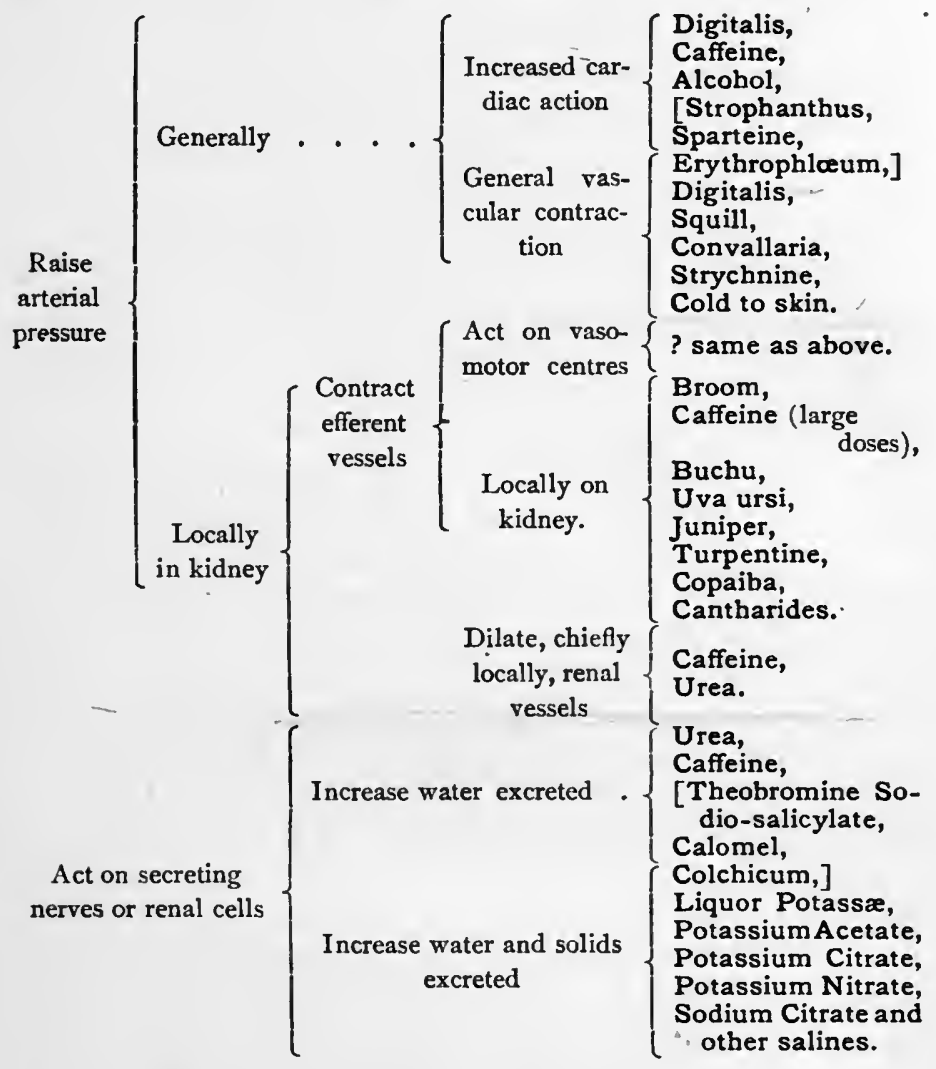

Therapeutics.-Diuretics are used in cardiac 'and pulmonary diseases when, owing to the general vascular disturbance, the quantity of urine falls below the normal standard. Also, in diseases in which there is excess of fluid in certain parts of the body ; for example, pleuritic effusion and ascites, with the object of getting rid of as much fluid as possible by the kidneys. Also, they may be used to dilute the urine, e.g., when it is prone to deposit 
its solids. Lastly, in certain forms of kidney disease, although in these maladies it is always a question how far it is desirable to stimulate diseased organs. It is of great importance to remember that diuretics may act in many different ways; that there are many causes for diminution in the quantity of urine secreted, and that it is difficult to say in any particular case what is the cause of the decrease in the quantity secreted. Therefore, it is usual to give diuretics in combination, in the hope that if one of them does not have the desired result another will.

2. Drugs diminishing the quantity of urine secreted.-These are such as produce acute inflammation of the kidney when given in large doses ; e.g., turpentine, cantharides, phosphorus. [Exalgin is reputed to diminish the quantity of urine.] They are never given for this purpose in medicine.

3. Drugs rendering the urine acid.-There is one drug that can do this, and that is benzoic acid, for in its passage through the kidney it is converted into hippuric acid. Benzoic acid is, therefore, given when from any cause the urine undergoes alkaline decomposition anywhere within the urinary passages. Salicylic acid will, to a slight extent, increase the acidity of the urine, as will very large doses of citric acid, tartaric acid [borax, and possibly saccharin. The free use of carbonated water also increases the urinary acidity (Ultzmann). Urotropin is the most reliable remedy to render an alkaline urine acid].

4. Drugs which render the urine alkaline.--[Some] salts of the metals, potassium, sodium, lithium, calcium, will do this [e.g., the carbonates, borates and hydroxides]; even the tartrates, citrates, [malates, lactates,] and acetates, for they escape by the kidney as carbonates. Nitric acid is said to increase the amount of ammonia in the urine, and thus to render it slightly alkaline. Ammonium salts given internally do not render the urine alkaline, because they are decomposed in the body, urea being probably formed; [they may even increase the acidity from the larger amount of nitric acid excreted.]

Lithontriptics are drugs which prevent the decomposition, in the urinary passages, of the solids of the urine. If this fluid be acid, uric acid often crystallizes out, forming gravel or uric 
acid calculus; less often lime oxalate crystallizes, giving rise to lime oxalate calculus. When there is any likelihood of the formation of either of these calculi, alkalies should be given. If the urine is undergoing alkaline decomposition phosphates are liable to crystallize out. In this case the object will be to render the urine acid and aseptic. This will be attained by giving benzoic acid or benzoates, salicylic acid or the salicylates, [urotropin, or the other] urinary antiseptics.

Therapeutics. - The chief use of alkalies is to diminish the acidity of the urine, so as to render the precipitation of uric acid unlikely; or to render it alkaline, so as to attempt to dissolve a uric acid calculus. We know of no drug which will dissolve a lime oxalate calculus. Alkalies are also given to gouty subjects, partly to alkalize the blood, but also partly to alkalize the urine, for such persons are very prone to deposit uric acid in the urine. Potassium urate is much more soluble than sodium urate, and lithium urate is perhaps even more soluble; citrates and acetates are not likely to upset the digestion, consequently the drugs most used are the potassium and lithium citrates. Copious draughts of water, by diluting the urine, aid in preventing the deposition of uric acid or lime oxalate calculi. Natural alkaline waters are largely used [especially those containing lithium].

5. Drugs preventing the urine from decomposing. - If the urine is retained in the bladder by stricture or from any other cause, it will undergo alkaline decomposition, and the same result may be brought about by the admixture of pus with the urine. This is generally due to inflammation of the pelvis of the kidney or the bladder. This decomposition of the urine may be prevented by giving drugs which in their excretion by the urine render it aseptic. Such are-
(I) Urotropin.
(2) Benzoic acid.
(3) Salicylic acid.
(4) Uva Ursi.
(5) Copaiba.

(6) Cubeb.

(7) Oil of Sandal Wood.

(8) [Saccharin.]

(9) Many volatile oils.

(10) Boric acid.

6. Drugs altering the composition of the urine.-Almost any drug will do this, either because it is excreted in the urine, or 
because it sets up some changes in the body, the products of which are excreted in the urine; but here we shall only refer to certain striking ones.

Turpentine, cantharides and salicylic acid in large doses will cause blood to appear in the urine, because they set up inflammation of the kidney.

Potassium cblorate, all nitrites, acetanilid, pyrogallic acid, poisoning by the mushroom, (Helvella esculenta) and transfusion of animal's blood, break up red blood-corpuscles, and the products are excreted by the urine rendering it dark. Large doses of mineral acids, arsenic, naphtol [and naphtalin] are said occasionally to produce the same result.

Pbosphorus in large doses causes leucin and tyrosin to appear in the urine, and the urea is greatly increased.

The saline diuretics increase the solids of the urine.

The chrysophanic acid in rhubarb and senna makes the urine, if it is acid, a brownish color; if it is alkaline, a purplish red. Logwood renders alkaline urine reddish or violet. Santonin colors acid urine yellow or greenish-yellow, and alkaline urine, reddish. Carbolic acid, naphtalin, creosote and other preparations of tar, as well as the arbutin in uva ursi, [chimaphila and gaultheria] make it dark greenish-brown. Picric acid makes it a bright yellow, and methyl violet a dark blue. The urine of persons poisoned with carbonic oxide remains sweet for months.

Poisoning by carbonic oxide, [curare,] amyl nitrite and turpentine, and sometimes [chloroform, camphor,] mercury, morphine, chloral, [hydrocyanic] acid, sulphuric acid, alcohol, lead compounds, and salicylic acid lead to the appearance in the urine of a body which like sugar reduces Fehling's copper solution. Some authorities state that the urine, after the administration of these drugs, does not contain glucose, but glycuronic acid ; for although it reduces blue copper solutions, it does not undergo alcoholic fermentation on the addition of yeast [or give the phenyl-hydrazin test]. The administration of phloridzin, [a glucoside from the bark of stem and root of the apple, pear, plum and cherry, which, when continuously heated with dilute mineral acids, is resolved into glucose and phloretin], leads to the production of genuine glucose in the urine.

Other drugs cause a peculiar odor in the urine; for example, the smell of violets is produced by turpentine. The aromatic odor of cubeb and copaiba can be detected in the urine after the administration of these bodies.

Lead, if taken for long periods, produces chronic interstitial inflammation of the kidney. It is stated that rarely mercury will do the same.

7. Drugs acting on the bladder and urethra.-The only ones of any practical value are sedatives to the urinary tract.

If the urine is decomposing, drugs preventing its decomposition come 
under this head. Other sedatives are opium, belladonna, hyoscyamus, pareira, buchu and uva ursi, which are direct sedatives to the vesical and urethral mucous membrane. If the urine is excessively acid, alkalies are urinary sedatives.

Urinary sedatives are used very largely in cases of cystitis and urethritis, whatever the cause may be. Local astringent and antiseptic injections are also employed.

Division ViI.-Drugs acting on the Bodily Heat.

A. Antipyretics, or Drugs which decrease the Bodily Temperature.-There are few if any drugs which can lower the temperature in health. Some, it is true, will cause the temperature to fall below normal, if given to a healthy man in large enough doses to induce severe collapse. The word antipyretic is limited to those drugs which bring down the temperature when it is raised owing to disease. We know that the greatest amount of heat is produced in the muscles, and that there is a special part of the corpus striatum presiding over this production; that heat is lost mostly by radiation from the vessels of the skin and by the evaporation of sweat, and that these vessels and the sudoriparous glands are under the control of the central nervous system. Heat is also lost through the lungs. As the production and loss are in health so accurately adjusted, many observers believe that there is a part of the cerebrum whose function is to maintain the balance between the production and the loss. Also, all parts of this complex mechanism are supplied with bloodvessels, alterations in the calibre of which must affect the activity of the parts they supply.

There is every reason for believing that the part of the central nervous system which presides over the loss of heat (thermolysis), that which presides over the production of heat (thermogenesis), and that which possibly presides over the balance between the production and the loss (thermotaxis), can, each of them be influenced by afferent impulses reaching them from various parts of the body, and thus we see each of these three functions can probably be reflexly affected (see diagram on next page).

Antipyretics which increase the loss of heat.-All sudorifics and 
all dilators of the cutaneous vessels act as antipyretics. Cold, such as a cold bath, increases the loss of heat by direct abstraction.

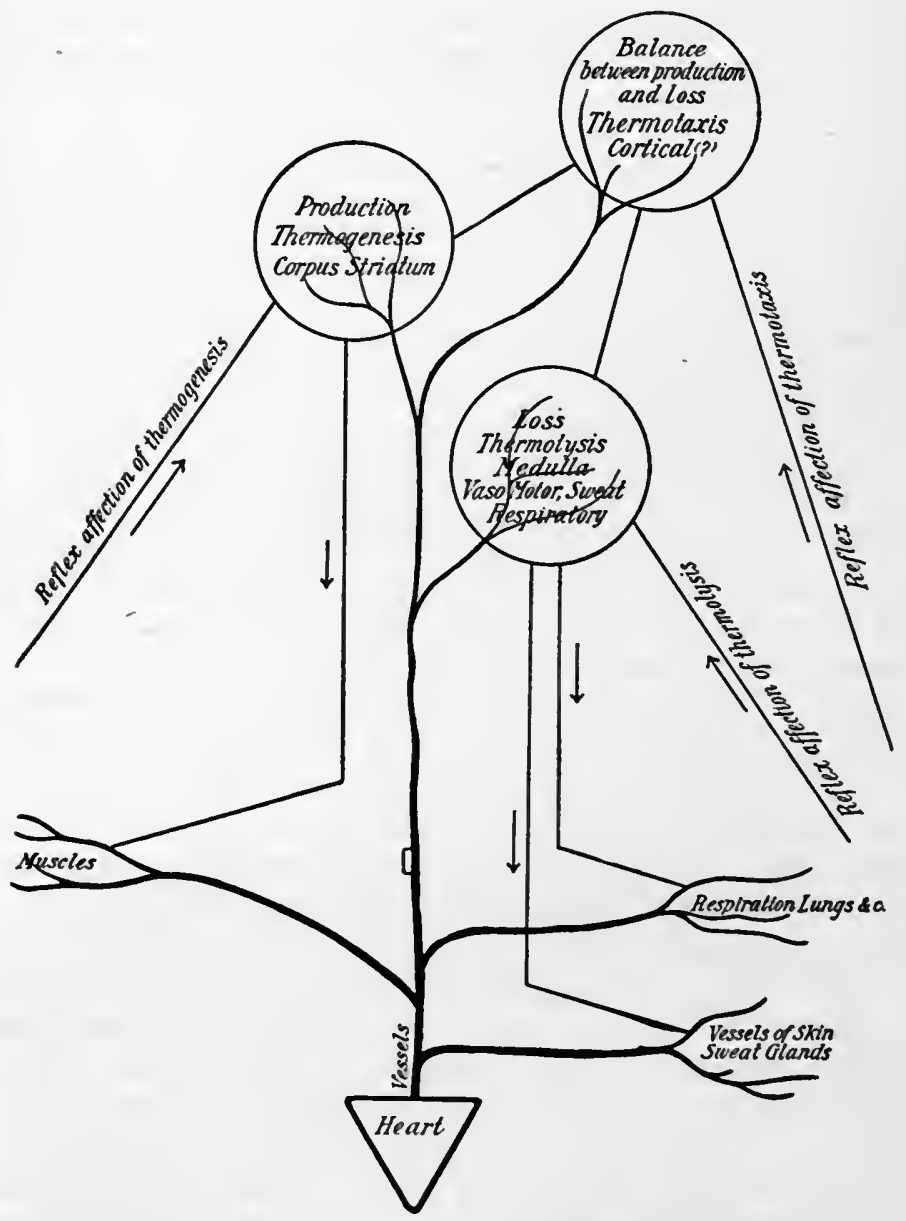

Drugs which probably diminish the production of heat.-Our knowledge about these is at present uncertain, but it is very probable that phenacetin, antipyrin, and acetanilid dimin- 
ish the production by their action on the corpus striatum; and that quinine, salicylic acid, and salicin, also diminish the production; but we do not know upon what part of the thermogenetic apparatus they act. A cold bath not only abstracts heat, but, after it has been in operation some little time, diminishes the production.

Antimony, aconite, and digitalis are probably antipyretic through their effect on the circulation, but precisely how they act is not known. Sometimes the removal of some irritation which is acting reflexly may lower the temperature. In this way purgatives are occasionally antipyretics.

Therapeutics.-The sole use of antipyretics is to lower the temperature in fever.

Drugs which increase the loss of heat were formerly popular as antipyretics, especially alcohol, spirit of nitrous ether, antimony, ipecacuanha, and opium, but now they are not mụch used. Cold is more often employed, either by cold sponging, ice, or a cold bath. Sponging with hot water will, by the vascular dilatation and subsequent sweating it induces, reduce a febrile temperature.

Of the drugs which probably alter the production, acetanilid and [antipyrin] are dangerous because of the collapse they may produce; quinine and salicylic acid are rather uncertain, except in ague and rheumatic fever respectively. [Antipyrin] and phenacetin are most in demand; they are certain antipyretics. Phenacetin is very safe, but is less powerful. They are quickly absorbed, and so act promptly ; they are far more powerful antipyretics than any drugs which act by increasing the loss of heat, and these are very uncertain in their action, often not lowering the temperature at all. Another reason for preferring drugs which diminish thermogenesis is that it is much more rational to lower the temperature by decreasing the production of heat than by increasing the loss, for then the production will, if anything, go on faster than before, in consequence of the attempt to compensate for the increased loss. Antipyretics should be rarely given, for probably fever is often beneficial.

B. Drugs which cause a rise of Temperature.-Bella- 
donna, picrotoxin, and cocaine in poisonous doses may do this. How they act is not known.

Tuberculin, various albumoses and certain animal poisons, such as that of shell-fish, will cause a rise of temperature. Their mode of action is unknown.

We know of no drugs acting on thermotaxis.

\section{Division VIII.-Drugs acting on Respiration.}

Respiration can be modified by such very various influences that it is difficult to decide upon the exact mode of action of any drug which affects it. For example, alterations in the blood and in the air will modify it; the respiratory centre itself may be influenced, either directly or reflexly, from almost any organ in the body; or, again, the movements of the respiratory muscles themselves may be interfered with; and, lastly, respiration is much under the influence of the circulatory apparatus. Furthermore, the chief object in medicine is to remove the cause of the respiratory difficulty rather than to act upon respiration itself.

We have already spoken of those drugs which produce changes in the blood and the circulation, and the consideration of those modifications of the temperature, moisture, and pressure of the air which are of value in medicine, belongs to a book on general therapeutics. We will, therefore, now consider the respiratory drugs under the following heads :

A. Drugs altering the Composition of the Air inhaled.- - This division is devoted to drugs which, inhaled, have some direct effect on the respiratory mucous membrane, on bronchial and pulmonary contents, and have certain remote effects.] It is found convenient to administer some drugs, although they are not given for their influence on respiration, by making the patient inhale them; such are anæsthetics and amyl nitrite.

Some drugs when inhaled are particularly irritating to the bronchial mucous membrane, causing dilatation of the vessels, increased secretion, and reflexly, cough from the stimulation of the sensory nerves of the bronchial mucous membrane.

Such are cold dry air, iodine, bromine, chlorine, senega, ipecacuanha, 
sulphurous anhydride, nitric acid fumes, ammonia, and tobacco. These are rarely used therapeutically as inhalations, and their inhalation is to be particularly avoided in irritable conditions of the bronchi.

The drugs which, when inhaled, are soothing to the bronchial mucous membrane, [but] are rarely employed, are-

'Hydrocyanic acid.

Conium.

Inhalations which are used to stimulate the bronchi, that is to say, to increase their vascularity, secretion, and muscular power, are-

$\left.\begin{array}{l}\text { (1) Carbolic acid } \\ \text { (2) Oil of cajuput }\end{array}\right\} m_{x x}$; [1.20 c.c. $]\left\{\begin{array}{l}\text { (4) Tinctura ben- } \\ \text { zoini composita, }\end{array}\right.$

(3) Oleum pini sylvestris, $m_{x x x}$; [2.00 c.c.]

(5) Creosote,

(6) Oil of cubeb.

fl $\xi_{\text {ss. ; }}$ I 5 c.c. $]$

The amounts given after each are the quantities that should be added to a pint $[500 \mathrm{c.c}$.$] of water at 140^{\circ} \mathrm{F}$; $\left[60^{\circ} \mathrm{C}\right.$. $]$

Inhalations which are used to disinfect foul secretions from the bronchial mucous membrane are those of-

(I) Creosote.

(2) Iodoform.

(3) Mild solutions of benzoin.

(4) Carbolic acid.
(5) Sulphurous anhydride.

(6) Oil of juniper.

(7) Oil of cubeb.

(8) [Oil of eucalyptus.]

Inhalations for relieving spasm of the bronchial tubes are those of-
(i) Conium. Frpit it.
(2) Stramonium. -
(3) Chloroform.
(4) Ether.
(5) Amyl nitrite.

B. Drugs acting on the Respiratory Centre.-If the drug, when injected into the carotid artery, very quickly produces its effect on respiration, it is concluded that it acts on the respiratory centre. Another experiment often used to determine whether the drug acts on the centre, or on the vagal terminations in the lung, is to cut the vagi and to observe whether it acts similarly before and after the section. 
Drugs which directly stimulate the respiratory centre are-

(I) Strychnine.

(2) Ammonia (very powerful).

(3) A pomorphine.
(4) Belladonna.

(5) Stramonium.

(6) Hyoscyamus.

Drugs which depress the respiratory centre are-

(I) Physostigmine (very powerful).

(2) Chloral [hydrate.]

(3) Chloroform.

(4) Ether.

(5) Alcohol.

(6) Opium.

(7) Hydrocyanic acid.

(8) Codeine.
(9) Aconite.

(I0) Veratrine.

(I I) Conium.

(12) Caffeine.

(13) Quinine.

(14) Ipecacuanha.

(15) Antimony salts (very weak).

Alcohol, ether, chloroform, caffeine, and quinine slightly excite, before they depress [the respiratory centre].

Therapeutics.-The drugs which excite the respiratory centre may be used when there is any difficulty in respiration, especially with the view of increasing the force of the respiratory act whilst other means are employed to get rid of the cause of the difficulty. They are, of course, most frequently required in diseases of the lungs, especially bronchitis. Ammonia and apomorphine are often employed, as they are also powerful expectorants ; and belladonna is useful when there is too much secretion from the bronchial tubes.

Substances which depress the respiratory centre are very little needed for this action; but the centre for the reflex act of coughing is in the close neighborhood of the respiratory centre, and opium, hydrocyanic acid, codeine, conium, and ipecacuanha are often very valuable in allaying the continual hacking cough which sn frequently accompanies disease of the heart and lungs.

The drugs which relieve cough are very numerous, for it may be reflexly set up by irritation of so many peripheral parts, viz., nose, throat, pharynx, ear, teeth, larynx, trachea, bronchi, lungs, pleura, stomach, and liver; and consequently its removal may 
depend upon the removal of peripheral irritation in any of those organs.

\section{Drugs affecting the Bronchial Secretion.}

(a) Those increasing it:

(I) [A pomorphine.]

(2) All alkalies, especially ammonium carbonate and other salts.

(3) [Cocillaña.]

(4) Ipecacuanha.

(5) Senega

(6) Squill.-

(7) Turpentine.

(8) [Terebene.]

(9) Camphor.

(IO) Benzoin.

(II) Balsam of Peru.

(I2) " "Tolu.

(13) Antimony salts.

(I4) Sulphur.

(15) Iodine.

(I6) Tobacco.

(17) Pilocarpus.

(18) Many volatile oils.

[It is probable that volatile oils and substances containing them decrease the amount of bronchial secretion as a later effect].

(b) Those decreasing it:

(I) Acids.

(2) Belladonna.

(3) Stramonium.

(4) Hyoscyamus.

Many authorities think that under some circumstances alkalies decrease the secretion.

(c) Those disinfecting it:- Urugs which, when inbaled, act in this way have already been mentioned. Copaiba, cubeb, [eucalyptus,] and many volatile oils are excreted partly by the bronchial mucous membrane, and thus will disinfect the secretion.

Therapeutics.-In bronchitis, remedies which increase the secretion are used when it is so viscid that it sticks to the tubes and cannot be coughed up; and those which decrease it are employed when it is too watery to be easily expectorated. The use of the disinfectants is obvious.

D. Drugs relaxing spasm of the Muscular Coat of the Bronchial Tubes, or Antispasmodics.-It is believed that [the symptom] asthma is due to a spasmodic contraction of the bronchial tubes, and as-

(I) Stramonium.

(2) Belladonna.

(3) Hyoscyamus.
(4) Grindelia.

(5) [Aspidosperma.]

relieve [this symptom], it is concluded that these drugs relax 
spasm of the muscular coat of the bronchial tubes. Stramonium is the most powerful. It is very likely, judging by their analogous action in other parts of the body, that the following drugs act in the same way :

Chloroform, ether, opium, chloral [hydrate], cannabis indica, amyl nitrite, and conium.

Therapeutics.-Stramonium is of great use [for relief of the symptom] asthma, and this and the other drugs may be employed for cases of bronchitis in which it is probable that the irritation caused by the inflammation of the tubes sets up spasm of them. Many of these muscular depressants in all probability depress the nerves at the same time.

E. Drugs acting on the Vessels of the Bronchi.These are the same as have been already described (p. $\left.5^{6}\right)$ as acting on the vascular system generally.

F. Expectorants.-The modes of action of drugs acting on the respiratory system are so complex that it is usual to regard most of them clinically, simply as drugs which hinder or aid the expectoration of the contents of the bronchial tubes. Those which aid it are divided into two groups, named after their action, not on the lungs, but on the circulation.

1. Stimulating expectorants. - These are stimulants to the circulation generally. They are-
(I) Acids.
(2) Ammonium salts.
(3) [Cocillaña.]
(4) Senega.
(5) Squill.
(6) Benzoin.
(7) Benzoic acid.
(8) Balsam of Tolu.

(9) Balsam of Peru.

(IO) Turpentine preparations.

(iI) Terebene.

(12) Oleum Pini Sylvestris.

(13) Nux Vomica.

(14) Sulphur.

(I5) Quillaja.

2. Depressing expectorants. - These depress the general circulation. They are-
(I) Alkalies.
(2) Antimony salts.
(3) Ipecacuanha.
(4) Lobelia.

(5) Pilocarpus.

(6) Apomorphine.

(7) Potassium Iodide. 
Therapeutics. - It is almost impossible to lay down any general directions. The prescriber must consider in any case before him whether he wishes to stimulate or to depress the circulation, to increase or to diminish or to disinfect the expectoration, to stimulate the respiratory centre, to overcome spasm of the bronchial tubes, or to allay a hacking cough; and he must combine his remedies according to the answer he makes to these questions. Warmth to the chest and warm drinks are sedative, and increase the amount of secretion. Cold and cold drinks have an opposite effect.

G. Drugs which in Man sometimes produce CheyneStokes Breathing.-These are morphine, potassium bromide, and chloral hydrate. In animals the following in addition may do it: picrotoxin, muscarine, digitalin, strychnine and ammonium carbonate.

Division IX.-Drugs acting on the Digestive Apparatus.

A. Drugs acting on the Teeth.- - Soaps and powders are used for cleaning the teeth. The basis of most tooth powders is chalk, which acts mechanically; charcoal is sometimes used in the same way. As the food is very liable to collect and decompose between the teeth, antiseptics, as quinine, borax and carbolic acid, are often mixed with tooth powders. Astringents such as [krameria] are employed if the gums are too vascular. Mineral acids and alum are injurious to the teeth if used for a long time, and iron is liable to stain them; therefore these substances are best taken through a [glass tube,] and should not be used as gargles for long [periods.]

Toothache may be relieved by local anodynes, as creosote, or [pure] carbolic acid. The tooth is plugged with [absorbent] cotton soaked in one of these. A piece of clean cotton must be placed over the carbolized cotton to prevent the carbolic acid from reaching the mouth. This method may damage the toothpulp.

B. Drugs acting on the Salivary Gland.-Much attention has been devoted to the submaxillary gland of the dog, and there is no reason for supposing that the other salivary glands 
of that animal or of other creatures, differ markedly from it. We know that the submaxillary gland is under the influence of the chorda tympani nerve, which contains vaso-dilator fibres, and also some which directly modify the secretion of the gland apart from the secondary effects, due to the alterations in the vessels, obtained when the chorda tympani is stimulated. This nerve has its centre in the medulla, and is capable of being excited reflexly by stimulation of many nerves, even the sciatic, but especially by stimulation of the gastric branches of the vagus, and by the terminations in the tongue and mouth of the glossopharyngeal and gustatory nerves. The gland is also supplied with sympathetic branches which proceed from the cervical sympathetic trunk; these are vaso-constrictor, and can also modify the quality of the secretion; but we do not know so much about them as we do about the chorda tympani. Drugs which increase the amount of saliva have been called sialogogues; those which decrease it, anti-sialogogues. It is clear that there are very many ways in which each of these might act, but here it will suffice to enumerate only those ways in which they are known to act.

I. Sialogogues acting either on the secretory cells or upon the terminations of the nerves in them.-Of these [pilocarpus] has been most studied, and by means of the experiments it has been proved to act either on the cells themselves or the terminations of the nerves in them. It acts equally well after section of all the nerves going to the gland. It acts when it is injected directly into the gland, but is prevented from reaching the general circulation. If it has been given, stimulation of the chorda or of the sympathetic produces no more effect on the amount of secretion than can be easily explained by the vascular effects.

Sialogogues falling under this heading are-

(1) Pilocarpus.

(2) Muscarine.

(3) Iodine compounds.
(4) Mercury.

(5) Nicotine.

(6) Physostigmine.

The last two probably act also by stimulating the centre in the medulla, for section of the chorda tympani decidedly lessens the secretion caused by 
them. Physostigmine soon ceases to cause an increase of the secretion, for it tightly contracts the vessels of the gland.

2. Sialogogues acting reflexly by stimulating the peripheral ends of afferent nerves. - Of these there are two important varieties :

(a) Those stimulating the gustatory and glosso-pharyngeal nerves in the mouth :

(I) All Acids and

(2) Acid Salts.

(3) Chloroform.

(4) Alcohol.

(b) Those stimulating the vagus in the stomach:

Most emetics, especially Antimony and Ipecacuanha.

3. Anti-sialogogues acting either on the secreting cells or the terminations of the nerves in them.-Of these atropine has been most studied, and it is proved to act directly on the gland by the fact that the administration of it prevents any increase of salivary secretion on stimulation of the chorda, although the vessels dilate as usual. It is highly probable that it acts upon the nerve terminations, because, even after considerable doses, quite paralyzing the secretion of chorda tympani saliva, stimulation of the sympathetic will still induce secretion.

Anti-sialogogues falling under this beading are-

(I) Belladonna.

(2) Hyoscyamus.

(3) Stramonium, and

(4) Nicotine in excess.

4. Anti-sialogogues acting reflexly by depressing the peripheral ends of afferent nerves.-Alkalies, opium, and any substances which allay irritation of the mouth. Part of the effect of opium is due to its depressing action on the medullary centre.

Therapeutics. - A deficiency in the amount of saliva secreted is seen most markedly in fever, when the mouth becomes very dry, and the patient complains of thirst. Sometimes it is a disease in itself, and the origin of this malady is then probably nervous. It is a prominent symptom of belladonna poisoning. In fever, acid drinks, especially those containing carbon dioxide gas, lemonade, etc., are of use as sialogogues. Drinks which 
relieve this febrile thirst are called Refrigerants. For [the condition known as] "dry mouth" [pilocarpus] has been used, and it will relieve the dryness due to belladonna poisoning. Excessive salivary secretion is hardly met with except as a symptom of poisoning, especially by mercury, [iodine or pilocarpus]. In some forms of indigestion the saliva has a very unpleasant taste, and may even be diminished in quantity, but then the indication is to treat the indigestion.

C. Drugs acting on the Stomach.-Strictly speaking, we ought to consider these under the following heads: $(a)$. Those drugs which, by modifying the secretion of hydrochloric acid or pepsin, influence the conversion of proteids into peptones and albumoses. (b). Those which influence the property possessed by the gastric juice of curdling milk. (c). Those which affect its antiseptic power by modifying the secretion of acid. (d). Those which modify the secretion of mucus. (e). Those which influence the nerves, the vessels, or the movements of the stomach. $(f)$. Lastly, those which are emetics. Our knowledge, however, is not sufficient to enable us to do this, and the most useful classification is into those affecting the secretion of gastric juice as a whole, the secreted contents, the vessels, the nerves, the movements, and emetics.

x. Drugs increasing the amount of gastric juice secreted.These are usually called Stomachics, and include a great many substances. Stimulants to the mouth reflexly increase the secretion of gastric juice; all bitter and aromatic substances act in this way, and they also increase the appetite. The mere presence of food in the stomach stimulates the gastric flow. Probably aromatics and bitters act also on the stomach itself.

The drugs which increase the flow of gastric juice are-

(1) Aromatics.

(2) Bitters.

(3) All alkalies (especially potassium and sodium bicarbonates, and Spiritus Ammonix Aromaticus).

(4) Alcohol.
(5) Ether.

(6) Chloroform.

(7) Magnesia.

(8) Magnesium carbonate.

(9) Pungent substances (pepper, mustard, horse-radish). 
Therapeutics.-Stomachics are very largely used for the purpose of increasing the secretion of gastric juice in cases of dyspepsia.

\section{Drugs decreasing the amount of gastric juice secreted.}

(I) Mineral acids.

(2) Acetic acid.
(3) Many of those in the last list if given in large doses, e.g., alcohol, ether and chloroform.

Therapeutics.-These drugs are never employed for this effect. It is particularly to be observed that while alkalies increase the secretion of the gastric juice, which is acid, they diminish that of the saliva, which is alkaline; but acids increase the secretion of the alkaline saliva, but diminish that of the acid gastric juice.

3. Drugs altering the composition of the gastric contents.Acids and alkalies naturally modify the reaction of the gastric contents. For this purpose diluted mineral acids are often prescribed to be taken about two hours after a meal, in cases in which the cause of indigestion is thought to be that the amount of hydrochloric acid secreted is deficient. If the acid were given at meal-time it would prevent the secretion of the natural acid; but by giving it after the meal, when all the acid that the gastric juice is capable of forming has been secreted, the drug carries on the act of digestion. In case of indigestion in which, from the nature of vomited matters or from any other reason, it is considered that there is an excess of acid in the stomach, alkalies are given at meal-times, the favorite drug being sodium bicarbonate.

Pepsin is given, usually in combination with diluted hydrochloric acid, when it is probable that the cause of the indigestion is the secretion of too small an amount of pepsin; but in this, as in every other variety of dyspepsia, it is far more important to remove the cause of indigestion than to endeavor to modify the composition of the secreted gastric juice.

Many attempts have been made to try, by the administration of antiseptics, to prevent fermentation and putrefaction from going on in the stomach, but with a limited success, for a suffcient dose of any antiseptic [may be] frequently deleterious. 
Here, even more than in the last case, the right treatment is to remove the cause of the putrefaction.

Drugs that have been used for this purpose are-
(I) Carbolic acid.
(2) Iodoform.
(3) Boric acid.
(4) Creosote.
(5) Eucalyptus.
(6) Thymol.
(7) [Resorcin.]

(8) [Salicin.

(9) Bismuth salicylate.]

(ro) Salol.

(II) Sodium hyposulphite.

(I2) Sodium sulpho-carbolate.

(13) Sulphurous Anhydride.

(14) Naphtol.

Charcoal has been employed, but when it is moist it is useless.

4. Drugs which dilate the vessels of the stomach.-The vessels of the stomach are very sensitive to irritation. They easily dilate upon mechanical irritation, and the presence of food, especially peptones, causes the vascularity of the mucous membrane to increase. Within limits greater vascularity is an advantage, for it not only favors the secretion of gastric juice, but it facilitates absorption.

The substances which increase the vascularity of the stomach are all stomachics (except alkalies), diluted mineral acids, the drugs which have been already enumerated as irritants generally, and squill, digitalis, colchicum, senega, copaiba, gamboge, guaiacum, and veratrine. This is a very long list, and many of the substances in it are never employed for their irritant effect; in fact, the only ones in common use are the stomachics; the others are far too powerful ; even small doses of them set up inflammation of the gastric wall, which is also produced by over-indulgence in stomachics, as we constantly see in the gastritis induced by alcohol. The therapeutic indications for this class of drugs are the same as those for stomachics generally.

Gastro-intestinal irritants. - In describing the individual actions of drugs the statement is frequently made that they are gastro-intestinal irritants, and this is a convenient opportunity for describing the symptoms produced in health by these drugs. If the drug has a caustic action, as many gastro-intestinal irritants have, the swallowing of it will cause considerable pain in 
the mouth and pharynx ; in a short time these parts will become severely inflamed, and consequently very much reddened, swollen and painful. The tongue will be often much enlarged. If the drug is corrosive, sloughs, generally white in color at first, with a severely inflamed area around them, will be seen; as they fall off they will leave ulcers. Owing to the pain and swelling, it will for some time be impossible to take any food, or at the best only that of a soft or fluid nature. Directly [after] the drug reaches the stomach, intense irritation is set up; consequently the patient feels severe abdominal pain, and there is violent retching and vomiting. As the poison passes on, it produces its severe irritant effects on the intestine, and diarrhœa sets in. Both the vomited matters and the motions often contain blood. The general symptoms are an anxious countenance, small feeble pulse, scanty urine, a low temperature, and all the symptoms of collapse. Later on, the gastro-intestinal irritation may be severe enough to set up general peritonitis, or a gastric ulcer may form, and then there may be added to the case all the symptoms of gastric ulcer and its sequelæ. The inflammation of the esophagus may lead to its contraction. At the postmortem examination, if the patient has died soon after the administration of the poison, the stomach will be very red and ecchymosed, with a swollen mucous membrane. Parts of the intestine will be in the same condition. This severe inflammation may, in many places, have led to the formation of sloughs. It must be remembered that many gastro-intestinal irritants have no action on the mouth.

5. Drugs which contract the gastric vcssels. - These are the same as those which have already been enumerated as being generally astringent. They are much used, more for the intestine than the stomach, and will therefore be considered in detail presently. (See p. 95.)

6. Drugs acting on the nerves of the stomach.-All drugs powerfully irritant to the stomach cause pain in it; those that are only slightly irritant give rise to a sensation of warmth. It is never desired to produce gastric pain.

Gastric sedatives.-These drugs are the same as those which 
are local sedatives to other parts of the body. Those most used for the stomach are-

(I) Bismuth subcarbonate.

(2) Bismuth subnitrate.

(3) Bismuth salicylate.

(4) Opium.

(5) Hydrocyanic acid.
(6) Carbon [dioxide.]

(7) Ice.

(8) Belladonna.

(9) Hyoscyamus.

(Io) Stramonium.

They are employed in the very many painful forms of dyspepsia. All, except perhaps stramonium, are in frequent use.

7. Drugs acting on the movements of the stomach. - It has been observed that the movements of the stomach increase as the acidity of the contents increases. If it be that the acidity is the cause of the movements, anything which causes an increase of acidity will lead to more powerful movements. Apart from this, strychnine appears directly to stimulate the [unstriped] muscle of the gastric wall. Stomachics also probably aid the movements, so that our complete list will be mineral acids, nux vomica, and the stomachics.

The proper churning up of the gastric contents is so necessary, that the value in dyspepsia, of drugs which aid the gastric movements, is very great. Hence the frequency with which nux vomica enters into anti-dyspeptic acid mixtures.

Carminatives. - This term is often applied to substances which aid the expulsion of gas from the stomach and intestines. They act by stimulating the gastric and intestinal movements. It has been found from clinical observation that the most efficient carminatives are-

(I) Stomachics generally, especially-

(2) Aromatics,

(3) Bitters,

(4) Pungent substances,
(5) Asafotida,

(6) Ammoniacum,

(7) Valerian,

(8) Camphor and

(9) Volatile oils.

8. Emetics.-It is well known that the many complicated mechanisms involved in the act of vomiting are under the control of a centre in the medulla, which is capable of being stimulated by afferent impulses reaching it from many sources, such as 
the cerebrum, as when sights or smells cause sickness, the mouth, the pharynx, the œsophagus, the lungs, the heart, the stomach, the intestines, the biliary passages, the kidney, the peritoneum, and the uterus; so that the drugs acting on any of these organs, or on the centre itself, might be emetics. But it is usual, in describing drugs which cause vomiting, to mention only those which do so either by acting on the stomach or on the centre in the medulla, and they are divided into two corresponding classes. Those acting on the stomach are sometimes called direct emetics, because they act directly on the stomach; and those influencing the medulla are called indirect; but some authors reserve the word direct for those acting on the medulla, and speak of those affecting the stomach as indirect. Considering this confusion, it is better to divide emetics into gastric and central. By means of the following experiments we determine to which group any drug belongs :

(I) The emetic is injected directly into the circulation. If very shortly after this vomiting takes place, the drug must have acted on the medulla, to which it has been carried by the circulation; but if some time elapses we conclude it acted on the stomach, and that it was first excreted into this organ before vomiting took place. This experiment may be made still more striking by injecting directly into the carotid, for then the medulla is quickly reached.

(2) If the least quantity of the drug which, when injected into the circulation, will produce vomiting is larger than is necessary when it is introduced directly into the stomach, the inference is that the drug acts primarily on the stomach, and that when it produces vomiting after injection into the circulation it only does so because some of it has been excreted into the stomach.

(3) If the drug will not produce vomiting after injection into the circulation when the stomach is replaced by a bladder, it shows that it acted directly on the stomach; but if vomiting is produced it shows that the drug acted on the medulla, and that the vomiting is caused by the contraction of the abdominal muscles. 
(4) If the drug takes a long while to act after its introduction into the stomach, it probably acts centrally; and the reason for the delay is that sufficient time must elapse for the drug to be absorbed.

In spite of these experiments it is difficult to be sure about the action of emetics, for some act in both ways, and some may, in the course of their circulation through the blood, act upon some of the many parts of the body from which the vomiting centre receives afferent impulses.

The following is a list of those emetics which are commonly used.

Emetics acting on the stomach :

(I) [Yellow mercuric subsulphate.]

(2) Alum.

(3) Ammonium carbonate.

(4) Copper sulphate.

Of these zinc and copper sulphate act slightly on the medulla.
(5) Zinc sulphate.

(6) Sodium chloride.

(7) Mustard.

(8) Warm water.

Emetics acting on the medullary centre:

(I) Apomorphine.

(2) Tartar emetic.

(3) Ipecacuanha.

(4) Senega.

(5) Squill.

Of these tartar emetic and ipecacuanha act partly on the stomach. The first three are very powerful emetics, and are much more depressant in their action than the gastric emetics.

Therapeutics.-Emetics have two uses. Firstly, to remove the contents of the stomach. Thus when that organ is over-full, and there is a feeling of nausea, an emetic by emptying the stomach may relieve. Emetics are largely used to empty the stomach in cases of poisoning, and they may benefit certain cases of sick headache. An emetic occasionally aids the expulsion of a foreign body which has become impacted in the fauces or osophagus. Secondly, emetics are used to expel the contents of the air-passages, especially in children, for they cannot expectorate well. For this purpose these drugs are given to help children to expel the morbid products in bronchitis, laryngitis, and 
diphtheria. They also aid the expulsion of foreign bodies that have become impacted in the larynx. In choosing an emetic it will be remembered that although apomorphine, ipecacuanha, and tartar emetic are the most powerful they are the most depressant, and are therefore not suitable in many cases-such, for instance, as poisoning accompanied by severe collapse. When the poison is a powerful gastro-intestinal irritant, if the condition of the mouth and œsophagus will allow of it, it is preferable to wash out the stomach rather than to use an emetic.

Emetics are not permissible for patients suffering from aneurism, hernia, prolapse of uterus or rectum, peritonitis, or a tendency to hæmorrhage, because of the straining induced by the vomiting, which should make us cautious in giving them to those who have disease of their vessels or high tension in them, for the straining may lead to hæmorrhage.

9. Anti-emetics. - The causes of vomiting are so numerous that the number of drugs which may stop vomiting is very large; therefore, as in the case of emetics, we can only consider those which act either on the stomach or on the centre in the medulla.

Anti-emetics acting on the stomach.-These are all those substances which have been already enumerated as having a sedative influence on the gastric nerves, viz. :

(I) Ice.

(2) Bismuth subcarbonate.

(3) Bismuth subnitrate.
(4) Opium.

(5) Hydrocyanic acid.

(6) Carbon dioxide.

Also some drugs which occasionally appear to have a specific local action in arresting vomiting ; such are:

(I) Cocaine.

- (2) Cerium oxalate. $0, K$ -

(3) [Menthol.]

(4) Wine of ipecac. In

$\left.\begin{array}{l}\text { (5) Tincture of iodine. } \\ \text { (6) Arsenous acid. }\end{array}\right\} \begin{aligned} & \text { minute } \\ & \text { doses. }\end{aligned}$
(7) Alcohol.

(8) Carbolic acid.

(9) Chloroform.

(I0) Creosote.

(II) Ether.

(I2) Silver nitrate.

(13) The sulphocarbolates.
In

small

doses. 
Anti-emetics acting centrally-

(1) Opium.

(2) Ammonium,

(3) Potassium, and

(4) Sodium bromides.

(5) Chloral bydrate.
(6) Amyl nitrite.

(7) Nitroglycerin.

(8) Diluted hydrocyanic acid.

(9) Alcohol.

It will be noticed that some drugs fall under both headings.

Therapeutics.-The very name of these drugs indicates their therapeutical application. At the best they are only palliative; the right way to treat vomiting is, if possible, to remove the cause. Of anti-emetics, ice, diluted hydrocyanic acid, carbon dioxide, bismuth salts, morphine, [menthol] and iodine are perhaps the most reliable, but all are very uncertain.

D. Drugs acting on the intestines.-Many secretions are poured into the intestine, the food is much altered by the time it arrives there, and it is changed in its course down the intestine; the physiology of intestinal digestion, of the movements and the nervous mechanisn of the intestine are imperfectly known; drugs nay be considerably altered by the time they come to this part of the alimentary canal, and its diseases are little understood; consequently we cannot arrange the action of drugs in a physiological classification. We know, in fact, of only [three] important divisions: purgatives, [antiseptics] and astringents.

The methods of experiments which have been used to determine the mode of action of purgatives are chiefly those of Thiry and Moreau. The first-named observer cut the intestine across in two places a short distance apart; the isolated part which was still attached to the mesentery was sewn up at one end; the other, the open end, was attached to the abdominal wall, and thus there was a test-tube-like piece of intestine into which drugs could be placed. The parts of intestine, on either side of the excised piece, were sewn together, so that the whole intestine was the same as before, but a little shorter. This method did not give very satisfactory results, and consequently Moreau devised his experiments, which seem more trustworthy. He put four ligatures around the intestine at equal distances apart, so 
that he shut off from the rest of the [intestine] and from each other three pieces of intestine, each of the same length. With a fine syringe he injected into the middle piece the drug to be experimented upon, and returned the whole into the abdominal cavity. In a few hours the animal was killed, and the state of the interior of the middle piece was contrasted with that of the pieces either side of it. Before Moreau's experiments there had been much discussion as to whether some purgatives did not act only by increasing the action of the muscular coat, and others only by stimulating the secretions, but from these experiments it appears that probably the majority act in both ways, some very slightly on the secretion and powerfully on the muscle, and others slightly on the muscle and powerfully on the secretion. We will first consider intestinal purgatives, [then intestinal antiseptics] and [finally] intestinal astringents.

Purgatives are divided into the following classes.

Laxatives. - These are substances which slightly increase the action of the bowels chiefly by stimulating their muscular coat.

They are-

(I) Whole meal bread.

(2) Honey.

(3) Treacle.

(4) Most fruits, especially-

(5) Tamarind,

(6) Fig,

(7) Prune, and

(8) Stewed apples.

(9) Manna.

(IO) Cassia [Fistula].

(II) Sulphur.

(I2) Magnesia.

(I3) Olive oil.

(I4) Castor oil (small doses).

These are all domestic remedies employed for slight cases of constipation, especially in children; some, as brown bread, fruits, honey, form articles of diet with persons who are liable to constipation. [Ergot, physostigma, nux vomica, belladonna, hyoscyamus, and stramonium are also laxatives, but are not used except under medical orders.] Nux vomica is most valuable; it is probably a direct stimulant to the muscular coat, hardly influencing secretion. It is largely used in cases of chronic constipation, especially when occurring in anæmic persons, or in those in whom, for any reason, it is likely that the intestinal peristalsis is feeble. 
Belladonna in small doses increases peristaltic movements because it paralyzes the inhibitory fibres of the splanchnics, but in moderate doses it completely arrests peristaltic movements. It is chiefly employed for this latter purpose, especially in combination with opium. Hyoscyamus acts on the intestines in the same way, and small doses of it are often given with other purgatives to prevent griping, for it gives an orderly rhythm to the irregular contractions which the stronger purgatives produce.

Ergot and physostigma are hardly ever used for their laxative effect. [Ergot, however, so often produces diarrhœea that its purgative action should be kept in mind.]

Simple purgatives. - These are rather more powerful in their action than laxatives. They stimulate peristalsis and also increase secretion. Some of the laxatives, as castor oil and magnesia, when given in large doses become simple purgatives.

The simple purgatives are-

(1) Aloes.

(2) Rhubarb.

(3) Rhamnus Frangula.
(4) [Rhamnus Purshiana.]

(5) Senna.

(6) Fel Bovis.

These are all in common use. The indications for each will be given under the individual drug.

Drastic purgatives, often called cathartics.-These excite greatly increased secretion and peristaltic movements, and if given in large doses cause severe irritation of the intestine, with much secretion of mucus, great vascular dilatation, and even hæmorrhage, severe abdominal pain and collapse, with profuse diarrhoa. The peristaltic contractions are often irregular, and hence there may be much griping pain; therefore it is usual to prescribe hyoscyamus with these drugs, which are in order of efficiency-

(I) Calomel.

(2) Podophyllum.

(3) [Leptandra.]

(4) Aloes.

(5) Jalap.

(6) Scammony.
(7) Gamboge.

(8) Oleum Terebinthinæ.

(9) Colocynth.

(xo) Elaterium.

(II) Croton oil. 
The most powerful are placed last. Some, as jalap, elaterium, scammony, are often called hydragogue, because of the large amount of secretion they excite.

Therapeutics.-Drastic purgatives are employed in obstinate constipation, and also to produce very watery evacuations with the object of removing as much fluid from the body as possible. Hence the frequent use of jalap in Bright's disease.

Saline purgatives. - The action of these is obscure, but it seems certain that they very greatly increase the secretion of intestinal fluid, and hinder its reabsorption, so that a large amount of it accumulates in the intestine. The distension due to this accumulation excites gentle peristalsis, and consequently an easy, painless evacuation of the bowels. Secretion goes on till the fluid in the intestine has become a 5 or 6 per cent. solution of the drug, so that if a very concentrated solution is given, much intestinal fluid is secreted. There is some doubt whether osmosis plays any part in the process. The action is entirely local, for no purgation follows if the salts are injected into the blood. [Magnesium sulphate, administered hypodermatically, purges. It is possible that other salines may act similarly.] The saline purgatives are-

-(r) Potassium [and sodium] tartrate.

(2) Potassium bitartrate.

(3) Potassium sulphate.

(4) Sodium sulphate.
(5) Sodium tartrate.

(6) Sodium citro-tartrate.

(7) Sodium phosphate.

$>$ (8) Magnesium sulphate and other salts.

Therapeutics.-These are very largely used as habitual purgatives, especially for persons suffering from any form of gout. They form the essential ingredient of most purgative mineral waters, as Hunyadi János, [Apenta], Pullna, Friedrichshall, Esculap, Rubinat, [Villacabras], etc. The best way of taking them is to put the required dose of the salt, or the mineral water, in a tumbler, add some [hot] water, and sip it slowly while dressing in the morning.

Cholagogue purgatives will be described when speaking of the liver. 
Enemata.-Any fluid preparation, injected into the rectum, is called an enema. When a purgative is liable to produce sickness, or it is inadvisable, because of peritonitis, intestinal obstruction, ulceration, or other diseases, to give it by the mouth, it may be given by the rectum. Castor oil, aloes, olive oil, magnesium sulphate, soap, etc., may be administered in this way. Enough of a vehicle must always be used to make a purgative enema up to three-quarters of a pint or a pint $\lceil 350$. or 500 . c.c.], for distension of the rectum greatly aids purgation. A teaspoonful [4 c.c.] of glycerin injected into the rectum, or the same amount given as a suppository, often unlocks the bowels.

[Intestinal Antiseptics.-These are believed to check fermentation and putrefaction in the intestines and are-

(I) Naphtol.

(2) Bismuth Naphtolate.

(3) Naphtalin.

(4) Bismuth Salicylate.

(5) Salol.

(6) Chlorine.
(7) Creosote.

(8) Corrosive Mercuric Chloride.

(9) Oil of Turpentine.

(10) Silver Nitrate.

Naphtol has been shown to destroy micro-organism in situ. Bismuth naphtolate has not the irritating properties of naphtol, but appears to be equally effective. When pure, naphtalin is not absorbed, it does not cause toxic symptoms, nor is there any change in the urine. Salol, a combination of salicylic and carbolic acids, decomposes only in an alkaline solution, and this is useful for action in the small intestine. Chlorine water has been used for the disinfection of the intestine in typhoid fever. Creosote is valuable if administered in the form of enteric pills, which are soluble only in the intestinal fluids. Corrosive mercuric chloride is too poisonous for use, save in exceptional cases. The late George B. Wood, of Philadelphia, achieved brilliant success with oil of turpentine in the treatment of typhoid fever. Silver nitrate has a limited use as an antiseptic, in its local application to dysenteric ulcerations within reach in the rectum and sigmoid flexure. The intelligent use of the foregoing drugs has greatly improved the success of the treatment of enteritis, diarrhoea, colitis, dysentery and typhoid fever.] 
Intestinal Astringents.-These may be described under the following heads :

Astringents acting on the vessels of the intestine.-These are the same as those acting on vessels generally. Those employed for their action on the intestine are-
(I) Lead salts.
(3) Alum.
(2) Dilute solutions of silver salts.
(4) Diluted sulphuric acid.

Astringents coagulating albuminous fluids and thus constricting the vessels:-
(I) Tannic acid, and all sub- stances containing it, as-
(2) Krameria,
(3) Kino,
(4) Hæmatoxylon,
(5) Cinnamon,
(6) Catechu, and

(7) Eucalyptus gum.

(8) Lead salts.

(9) Silver salts.

(Io) Zinc salts.

(II) Bismuth salts.

(I2) Copper salts, and especially

(13) Ferric salts.

Astringents diminishing the amount of intestinal fuid secreted :
(1) Opium.
(2) [Coto.]
(3) Lead salts.
(4) Calcium salts.

The precise action of these is obscure, but it is probable that they operate in the way indicated.

Astringents diminishing the contractions of the muscular coat of the intestines:
(1) Opium.
(2) Belladonna.
(3) Hyoscyamus.
(4) Stramonium.

(5) Lead salts.

(6) Lime.

(7) Bismuth salts.

Therapeutics.-The first proceeding in every case of diarrhœa is to remove its cause; if this can be done, it will probably subside. Often the cause is some irritating, indigestible food, and then it is advisable to give a mild purge, as castor oil, rhubarb, etc., to get rid of it. The majority of cases of ordinary diarrhoea are probably due to some slight enteritis, and then any one of the astringents that have been named will be valuable, for 
it is desirable to constrict the dilated vessels, and to diminish the secretion and the movements. Intestinal astringents are, therefore, often combined, and, when the diarrhœe is at all serious, opium is of great service. If there is a persistent cause, as tuberculous ulceration, the hope of doing good is slight. But the treatment by drugs is only a small part of the battle; if the diarrhœa is severe, absolute rest is necessary, food must be very simple and given in small quantities at a time, not much fluid should be drunk, and the patient must be kept warm.

E. Drugs acting on the Liver.-The liver has several distinct functions, viz.: $(a)$ to secrete bile; $(b)$ to form and store up glycogen; $(c)$ to form urea; $(d)$ to excrete substances absorbed from the intestine; and $(e)$ to destroy poisonous substances absorbed from the intestine.

1. Drugs infuencing the secretion of bile.-It does not follow because more bile appears in the freces that more is secreted, for it may be that the gall-bladder and ducts have been thoroughly emptied, or that the bile which has been poured into the duodenum has been swept along quickly before reabsorption, which is ordinarily brisk, has had time to take place. Drugs which increase the amount of bile actually secreted are called direct cholagogues, or hepatic stimulants; but this is a bad name, as the liver has so many distinct functions : those which simply lead to a larger amount of bile being found in the fæces without any extra secretion are called indirect cholagogues.

Direct Cholagogues. - These have been studied in fasting, curarized dogs. A cannula is inserted into the bile-duct, and is brought out of the body, the drug to be experimented upon is administered, and the amount of bile is secreted before and after the administration is noted. No food must be given during the experiment, as that alone causes a considerable increase in the biliary flow.

Direct cholagogues (the most powerful being placed first) are-
(I) Euanymus.
(2) Sodium benzoate.
(3) Sodium salicylate.
(4) Podophyllin.
(5) Iridin.
(6) [Leptandra.]
(7) [Corrosive] mercuric chlo- ride. 
(8) Sodium sulphate.

(9) Sodium phosphate.

(ro) Aloes.

(II) Ipecacuanha.

(12) Diluted nitric acid.

(13) Diluted nitrohydrochloric acid.
(14) Colocynth.

(I5) Colchicum.

(16) Potassium sulphate.

(17) Rhubarb.

(18) Jalap.

(19) Scammony.

(20) Diluted arsenous acid.

There are individual differences among direct cholagogues. Some, as sodium salicylate, make the flow very watery, others, as toluylendiamine, which is not given to man, make it so thick that it flows through the duct with the greatest difficulty. Euonymin, sodium benzoate, sodium salicylate, Harrogate old sulphur spring and Carlsbad water, all markedly increase both the total quantity and the solids. Podophyllin and iridin, on the other hand, increase the solids without affecting the quantity.

Indirect Cholagogues. - These cause no increase in the amount of bile secreted; they act by stimulating the upper part of the jejunum and the lower part of the duodenum, thus sweeping the bile on before there is time for it to be re-absorbed.

They are-(1) Mercury; (2) most Cathartic purgatives, especially Calomel.

Therapeutics.-Cholagogues are used for cases of dyspepsia in which there is reason to believe that the liver is the organ at fault, and certainly they often have a very markedly beneficial effect. It is clearly an advantage to combine direct and indirect cholagogues in order to insure that the bile shall be excreted. As bile itself is a stimulant to the peristaltic movements of the intestine, all cholagogues are purgatives, and form a distinct class of purgatives. In cases of hepatic dyspepsia attention to diet is of the greatest importance, and muscular movements, as riding, rowing, etc., aid in the expulsion of bile from the gall-bladder and ducts.

Anti-Cholagogues. - These are often called hepatic depressants. They decrease the quantity of the bile secreted. Calomel, castor oil, gamboge, magnesium sulphate, opium and lead acetate have this effect, but it is not sufficiently marked to interfere with their therapeutic use for other purposes, and they are never employed for this action. 
2. Drugs modifying the glycogenic function of the liver. $-[\mathrm{We}$ will here refer to those drugs which cause sugar to appear in the urine, and to those drugs which diminish the glycogenic function of the liver.

Drugs causing Sugar to appear in the Urine.-Until recently it was assumed that all these drugs acted on the liver, probably by increasing the amount of sugar made from the hepatic store of glycogen; but now we have reasons for thinking that sometimes the pancreas may be the organ at fault in diabetes, for its excision causes sugar to appear in the urine, and other symptoms of diabetes; also it has been suggested that perhaps some perversion of processes going on in muscles may cause diabetes, therefore it is rash to assume that all drugs causing sugar to appear in the urine (glycosuria) must act on the liver. What little can be stated as to the mode of action of these drugs will be given when each individual drug is considered.

The drugs stated to cause glycosuria have already been mentioned (See p. 7o).]

Depressants of the Glycogenic Function.-Phosphorus, arsenic, and antimony diminish and even stop the formation of glycogen by the liver; they also cause fatty degeneration of it. In certain forms of diabetes, opium, morphine, and codeine have a most marked effect in diminishing the quantity of sugar in the urine.

3. Drugs modifying the formation of urea by the liver.-It is believed that some of the nitrogenous substances, especially leucin, arriving at the liver, are there converted into urea. The quantity of urea excreted by the urine is increased by phosphorus, arsenic, antimony, ammonium chloride, and iron. Phosphorus may also lead to the appearance in the urine of leucin and tyrosin. There is some evidence that this drug causes an increase of the urea through its action on the liver, for in phosphorus poisoning that organ undergoes extreme fatty degeneration, and jaundice supervenes. Whether the other drugs act through the liver is doubtful. Antimony and arsenic, if given in large doses for some time, both produce general fatty degeneration. All these substances must be administered in almost poisonous doses in 
order to increase the urea in the urine, and they are not employed therapeutically for this purpose.

Opium, colchicum, alcohol, and quinine are said to increase the quantity of urea excreted.

Division X.-Drugs acting on the Nervous and Muscular Systems.

A. Drugs acting on the Muscles.-Pharmacologists have devoted much attention to this class of drugs; but as the facts ascertained are not used in medicine, we need not stop to consider them. Brunton gives the following classification, founded on that of Kobert:

Class I. Irritability of muscle unaffected; total amount of work it can do diminished.- The following produce this effect: Apomorphine, delphine, saponin, copper, zinc, and cadmium, and in large doses, antimony, arsenic, platinum, and iron.

Class II. Both the irritability and the capacity for work diminished.The following produce this effect: Potassium, lithium, ammonium, quinine, alcohol, chloral [hydrate], and chloroform.

Class III. Diminish the capacity for work, and make the excitability very irregular.-Lead, emetine, and cocaine.

Class IV. Alter the form of the muscle curve.-Veratrine, barium, strontium, and calcium salts, digitalis, and squill.

Class V. Increases the excitability.-Physostigmine.

Class VI. Increase the capacity for work. - Caffeine and theobromine.

Small doses of strychnine and veratrine shorten the latent period; large doses lengthen it.

Dilute alkalies diminish the extensibility ; dilute acids increase it.

B. Drugs acting on the Peripheral Endings of Motor Nerves.-Of the drugs belonging to this group the action of curare has been worked out most fully. If curare is given to an animal, it is found that the muscles will respond to a mechanical stimulus, although they will not contract when the motor nerve is stimulated. If a single muscle be removed from the circulation by ligature of its vessels before the administration of curare, afterwards it will be the only one that will respond to stimulation of its motor nerve. As this was the only muscle of the body that the drug could not reach, and it is the only one not poi- 
soned, the poison clearly acts locally on the muscles; but as the curarized muscle will respond to mechanical stimulation, curare must paralyze the motor nerves within the muscle, probably the end plates.

Drugs paralyzing the termination of the motor nerves in muscle:

(I) Curare.

(2) Conium.

(3) Belladonna (atropine).

(4) Stramonium.

$>$ (5) Hyoscyamus.

(6) Saponin.

(7) Sparteine.

(8) Amyl nitrite.

(9) Diluted hydrocyanic acid.

(Io) Cocaine.

(II) Camphor.
(I2) Lobeline.

(I3) Nicotine.

(I4) Methyl-brucine.

(I5) Methyl-cinchonine.

(I6) Methyl-codeine.

(I7) Methyl-morphine.

(I8) Methyl-quinine.

(19) Methyl-nicotine.

(20) Methyl-strychnine, and many others.

Curare and conium are by far the most important. Therapeutically we never desire to paralyze motor nerve endings.

Drugs stimulating the termination of motor nerves in muscle:

(I) Aconite.

(2) Nicotine.

(3) Pilocarpine.
(4) Pyridine.

(5) Strychnine (slightly).

Excepting that perhaps some of the beneficial action of strychnine in certain cases may be due to its slight action on motor nerves, we do not employ these drugs for this action.

\section{Drugs acting on the Peripheral Endings of} Sensory Nerves (other than those of special sense).-Our knowledge of these is derived almost entirely from observations on man, for it is very difficult to experiment upon animals, as they have such imperfect means of communicating their sensations to us.

Drugs which stimulate the termination of sensory nerves. These, when applied locally, cause pain. They are the same as the local vascular irritants which have already been enumerated (p. 6o) ; in fact, most of them give rise to pain by causing local inflammation. There is no need to repeat the list.

Therapeutics.-Local irritants are chiefly employed for 
their action on the vessels, but as they are also counter-irritants, their application to the skin, while causing some pain there, will often relieve a deep-seated pain. Although pain is always referred to the periphery, it is appreciated centrally, and therefore peripheral stimulation of nerves, which also reflexly excites the heart and respiration, is used to rouse people from unconsciousness, such as that of fainting, opium poisoning, etc. For these purposes the stimulus must be prompt, hence the application of the faradic current to the skin is a good means to employ.

Drugs which depress the terminations of sensory nerves.-Of these there are two kinds: those which only relieve pain, or local anodynes; and those which diminish sensibility, or local anæsthetics.

Local Anodynes.-These have no action unless pain be present. They are-

(1) Aconite.

(2) Carbolic acid.

(3) Menthol.

(4) Diluted hydrocyanic acid.

(5) Veratrine.

(6) Ether.

(7) Alcohol.

(8) Chloroform. $\int$ porate.
(9) Chloral [hydrate.]

(10) Belladonna.

(II) Stramonium.

(12) Hyoscyamus.

(13) Opium.

(I4) Sodium bicarbonate.

(15) Zinc oxide.

In the above list the most powerful are placed first. Many other substances are said to be local anodynes, but their claim to the title is doubtful. Cold is a powerful depressant of sensibility, and therefore it is an excellent local anodyne; so also is warmth, for heat primarily dilates the vessels, and thus relieves tension, which is a very powerful factor in causing pain.

Therapeutics.-It is clear that the scope for the employment of local anodynes is very wide. If possible, the first thing is to remove the cause of the pain, but often, as in neuralgia and many forms of pruritus, we cannot do this.

Local Anæsthetics.-These are [ethyl chloride, rhigolene], cocaine, [eucaine], carbolic acid, and extreme cold, whether produced by ice or the ether spray. This spray was formerly employed to produce local anæesthesia before doing small operations, but it has [the disadvantage of stiffening the parts so that it is only useful for single incision as for opening furuncles. 
Upon a damp day it is ineffectual. Ethyl chloride sprayed from tubes by the heat of the hand is the best method and the one most frequently employed at present. Eucaine and] cocaine, which produce a high degree of local insensibility, [are largely employed].

D. Drugs acting on the trunks of Nerves.-These are of greater pathological than pharmacological interest. If taken for a long time they produce chronic inflammation of the nerves, which is shown by the great increase of the fibrous tissue between the nerve-fibres and the fatty degeneration of the fibres themselves. During the earlier stages the irritation of the nerves causes much pain and tingling; later, as they lose their function, numbness, with loss of sensation, and paralysis set in, often accompanied by trophic lesions. For further details books on medicine must be consulted.

The drugs producing peripheral neuritis are-

(1) Lead.

(2) Mercury.
(3) Arsenic.

(4) Alcohol.

E. Drúgs acting on the Spinal Cord.-The difficulties of experiment are so great that we know nothing of the action of drugs on the sensory portions of the cord. We are also ignorant of the action of drugs on the motor fibres. The following method is adopted to discover whether a drug acts on the cells of the anterior cornua. Suppose we are studying a drug which stimulates them. After the drug has been given, a slight peripheral stimulus will produce such marked reflex action that convulsions will ensue upon the stimulation. If the cord is cut across and the convulsions follow the stimulus as before, it is clear that these cannot be of cerebral origin, for in that case they would not take place below the point of section. Again, if before injection of the drug into the circulation, the vessels of the cord are ligatured, and then the drug causes no convulsion, it is clear that it acts on the cord, and not on the muscles $o_{\text {. }}$ nerves. These results are confirmed, if, when the drug is injected into vessels by which it reaches the cord quickly, convulsions occur sooner than when it is thrown into other vessels; a!so if convulsions do not take place when the cord is destroyed; 
and lastly if, when the destruction is gradually caused by pushing a wire down the vertebral canal, the convulsions cease from above downwards as the cord is destroyed.

The drugs increasing the irritability of the anterior cornua are-
(I) Strychnine.
(2) Brucine.
(3) Ammonia.
(4) Thebaine.

(5) Chloroform.

(6) Ether.

(7) Ergot.

(8) Opium.

(The last four only slightly, and early in their action.)

Therapeutics. - It is very rarely that we can do any good in spinal diseases by attempting to stimulate the anterior cornua ; but strychnine is occasionally given for cases of paralysis due to diseases of the spinal cord.

Drugs which depress the activity of the anterior cornua:
(I) Physostigmine.
(2) Bromides.
(3) Alcohol.
(4) Chloroform.
(5) Ether.
(6) Ergot.
(7) Opium.
(8) Mercury.
(9) Zinc salts.
(10) Silver salts.
(II) Sodium salts.
(I2) Potassium salts.
(I3) Lithium salts.
(14) Antimony salts.

(15) Arsenical salts.

(16) Camphor.

(17) Amyl nitrite.

(18) Sodium nitrite.

(19) Chloral [hydrate.]

(20) Carbolic acid.

(2I) Apomorphine.

(22) Veratrine.

(23) Turpentine.

(24) Saponin.

(25) Emetine.

(26) Gelsemium.

(27) Colchicum.

Of these, apomorphine, alcohol, chloroform, ether, arsenic, camphor, morphine, carbolic acid, chloral [hydrate,] nicotine, and veratrine first excite slightly before depressing.

Therapeutics.-These drugs are of very little use in medicine for their action on the spinal cord. Physostigmine is by far the most powerful, and has been occasionally used in obscure nervous diseases accompanied by convulsions, as tetanus.

Ergot has a very peculiar action in producing sclerosis of the 
posterior columns of the cord. Lead sometimes causes atrophy of the anterior cornual cells, and long-continued abuse of alcohol probably causes slight degeneration of the cord as a whole.

F. Drugs acting on the Brain.-The action of these cannot be localized nearly so accurately as can that of drugs acting on the spinal cord and nerves. Drugs acting on the brain illustrate two very important general laws.

First, the law of dissolution, which, when stated as it applies in pharmacology, is as follows: When a drug affects functions progressively, those first affected are the highest in development; that is to say, they are the last acquired by the individual and the last to appear in the species. The next affected are those next to highest, and so on ; till finally the lowest of all from an evolutionary point of view, that is to say, the functions of respiration and circulation, are affected. This law is very well exemplified in the case of alcohol, for the first functions to be disordered are those of the intellect, especially the highest, such as judgment and reason; then follow disorders of movement, and finally death from failure of respiration and circulation.

Another law very well exemplified by drugs which act on the brain is that when a drug in moderate doses excites a function, in large doses it often paralyzes it. For example, a person under the influence of chloroform, soon after its administration, tosses his arms about in a disorderly way, but they subsequently become motionless, and cerebral stimulants may also be hypnotics.

Drugs acting on the motor centres of the brain.-To investigate these, the motor area of the cortex is exposed by trephining, and the strength of current which it is necessary to apply to the motor area to produce corresponding movements, is noted before and after the administration of the drug. Another method is to observe the strength of current necessary to evoke a movement, then to allow the wound [made by the trephine] to close, afterwards the animal is made to take the drug regularly for some weeks. The opposite motor area is then exposed, and the strength of current required to call forth movements is noted. 
It has been found that [the following] diminish the activity of the motor area.
(I) Alcohol.
(2) Anæsthetics.
(4) Potassium bromide.
(3) Chloral [hydrate.]
(5) Sodium bromide.
(6) Ammonium bromide.

Bromides are largely used in epilepsy and other convulsive disorders on account of this function.

Drugs exciting the motor cells of the cortex are-
(I) Atropine.
(3) Strychnine.
(2) Absinthium.
(4) Physostigmine.

They have no therapeutical application in virtue of this property.

General cerebral stimulants.- - It is impossible to know anything of these by experiments on animals. In man they cause general excitation of the mental faculties, followed in many cases by delirium and incoherence. The exact form of delirium differs a little in each case.

Such drugs are -
(I) Belladonna.
(2) Stramonium.
(3) Hyoscyamus.
(4) Alcohol.
(5) Chloroform.
(6) Ether.
(7) Nitrous oxide.
(8) Coffee.
(9) Tea.
(Io) Guarana.

\author{
(II) Coca. \\ (I2) Cannabis Indica. \\ (I3) Lupulus. \\ (I4) Opium. \\ (I5) Camphor. \\ (I6) Santonin. \\ (17) Quinine. \\ (18) Salicylic acid. \\ (19) Tobacco.
}

Therapeutics.-Many of these are taken habitually as cerebral stimulants; for example, alcohol, tea, coffee, tobacco, in England; opium in the East ; cannabis indica in many parts of Asia; coca in parts of South America; and if it is wished to give a cerebral stimulant as a drug, one of these is usually chosen. The rest, which are very important, are commonly employed for some other action. With very many of this class of drugs, as will be seen directly, the stimulant action soon gives way to a paralyzing influence. 
General cerebral depressants. - These are commonly divided into three classes : Hypnotics or Soporifics, Narcotics and Anæsthetics.

HypNotics OR SOPORIFICS are drugs which produce sleep, closely resembling, if not identical with, natural sleep. The brain during sleep is anæmic, and it is thought that this anæmia is the cause of sleep ; possibly some soporifics act by producing cerebral anæmia.

The hypnotics are-
(1) Opium.
(2) Morphine.
(3) Chloral hydrate.
(4) Chloralamide.
(5) Butyl-chloral hydrate.
(6) Bromides.
(7) [Trional.
(8) Pellotine.]

(9) Sulphonal.

(Io) Paraldehyde.

(II) Alcohol.

(12) Hyoscine.

(13) Cannabis Indica.

(I4) Urethane.

(I5) Lupulus.

(16) Lactucarium.

Therapeutics.-These drugs are often used for persons suffering from sleeplessness, but it is far more important to remove the cause of the sleeplessness. Sleep is often promoted by dilating the vessels of other parts of the body than the brain; for example, a warm bath or an abundant meal conduces to sleep. The use of hypnotics is greatly abused. Those who take them become habituated to them, so that at last even large doses do not cause sleep. Chloral [hydrate if used with great caution, pellotine, paraldehyde, trional], and chloralamide are perhaps the noost satisfactory.

NARCoTics are substances which not only produce sleep, but also in large doses depress the functions of respiration and circulation. Many of them fall also under the head of general anæsthetics; others are, in smaller doses, hypnotics. All must be given in considerable doses.

The following is a list of them.

(I) General Anæsthetics.

(2) Opium.

(3) Chloral hydrate.

(4) Belladonna.

(5) Stramonium.

(6) Hyoscyamus.

(7) Alcohol.

(8) Cannabis Indica.

(9) Lupulus.

All must be given in considerable doses. 
Therapeutics.-They are of great use in calming excitement of any kind; many of them, such as, for example, opium and belladonna, are beneficial in relieving distress and producing sleep in heart disease.

General Anesthetics. - These are drugs which lead to a total loss of consciousness, so that pain is no longer felt ; at the same time reflex action is abolished. They illustrate admirably the law of dissolution, and also the fact that, after excitement, paralysis often succeeds; and the stages consequent upon these laws can be readily observed in any one who is taking an anæsthetic. Firstly, in obedience to the law of dissolution the highest faculty, the imagination, becomes excited, the patient sees visions and hears noises. He next begins to chatter wildly and incoherently, for in the excitement of any function by a drug the exaltation is usually irregular, and confusion results. Next, the other motor centres of the cortex are stimulated irregularly, so that he gesticulates, throws his arms about wildly, and tosses his body. By this time the brief stimulation of the higher intellectual faculties has probably ceased, and, in obedience to the second law, vision, hearing, and touch are dulled, and he has lost control over his reason, so that he feels light-headed, as he expresses it, crying and laughing easily; now he is totally irresponsible for his actions and careless as to their results. It will be noticed that the functions are paralyzed in the order stated in the law of dissolution. Next there follows upon the stimulation of the motor areas, stimulation of the heart and respiration. The pulse and respiration both increase in number, the blood-pressure rises, the face flushes. Then comes depression of all the functions previously excited ; first the higher parts of the cerebrum give way, and the patient loses consciousness-neither bright lights, sounds, nor painful impressions arouse him; he becomes quiet, and ceases to throw his arms and legs about; the reflexes disappear, and consequently touching the conjunctiva does not produce closing of the eyelids; the feet do not move when they are tickled, the pupil is contracted, and the previous quickening of the pulse and respiration are succeeded by a slowing of their rate. It is at this period that the patient cannot feel pain, and that 
therefore operations are performed. The depression of the motor centres is followed by the depression of the muscular tone, and the muscles become quite flaccid and cease to respond to mechanical stimulation. This is the degree of narcosis that is required for the easy reduction of dislocations and for the easy manual examination of the abdominal viscera. Anæsthetics should not be pushed beyond this stage. If they are, even the involuntary muscles lose their tone and reflex excitability, so that the sphincters of the rectum and the bladder relax. The depression of the pulse and respiration continues, the movements of the chest become weaker and weaker, and slower and slower, the pulse becomes very feeble, slow, and irregular, and the heart finally stops in diastole. Death occurs partly by the heart and partly by the respiration. At any period of the administration during which recovery is possible, the functions of the body will return in just the reverse order to that in which they were lost, thus again illustrating the law of dissolution. It is often many hours before the mental faculties have recovered their equilibrium, and long after the patient can move his muscles, he cannot coordinate them. There are individual differences in the different anæsthetics and in different persons.

The general anæsthetics are-

(I) Chloroform.

(2) Ether.

(3) Nitrous oxide.

(4) [Pental.
(5) Ethyl bromide.]

(6) Many other substitution products derived from alcohols and ethers.

Therapeutics.-Anæsthetics are given to cause unconsciousness, so that pain may not be experienced during operations, to relax muscles in cases of dislocations, abdominal examinations, phantom tumors, etc., to relieve severe pain, such as that of parturition, biliary and renal colic, to quiet the body during convulsions, as in tetanus and hydrophobia.

The chief dangers of anasthetics are- $\mathrm{I}$. Death from shock. This usually takes place before the patient is fully under the influence of the anæsthetic; reflex action is not yet quite abolished, and the heart is stopped reflexly from the peripheral 
stimulus of the operation. This is one of the greatest and most common dangers of anæsthetics, especially of chloroform. It is, to a large extent, avoidable if care be taken that the patient is fully under the influence of the anæsthetic before the operation is begun; often, when it is trivial, the operator is in too great a hurry to begin, and the patient suddenly dies from failure of the heart.

2. Death from paralysis of respiration. This is usually due to a combination of circumstances. Too much of the anæsthetic may have been given, respiration may be difficult because the patient suffers from some disease of the lungs, or the operation may demand that he should lie on his side or in some other position which hampers respiration. It is not a very great danger, for it is heralded by lividity; and if then the posture is changed, the administration of the anæsthetic is stopped, and artificial respiration is performed, the patient usually quickly recovers; even if he does not, artificial respiration, with the head thrown back and the tongue pulled out, should be carried on as long as there is any evidence that the heart is beating, or if the patient draws a breath when artificial respiration is stopped for half a minute. [Patients] have recovered, although it has been necessary to keep up artificial respiration for hours.

3. Cardiac failure may occur if the vapor is too concentrated. The patient almost suddenly becomes pale, and the pulse stops. In such a case no more of the anæsthetic should be given, artificial respiration must be kept up in the manner just mentioned, [after] the patient [has been] inverted so that the head is lowest. The heart may be stimulated [by large rectal injections of hot normal saline solution or of coffee, if at hand], by the inhalation of amyl nitrite, by the plunging of electric needles into the heart, or [if the reflexes are not abolished,] by flicking the chest over the heart with hot towels and placing hot compresses over it. [The use of brandy subcutaneously is to add the effect of one poison to that of another. The application of the faradic current over the cardiac region is also objectionable.]

4. Vomited matter and, if the operation is about the mouth, blood may suffocate the patient. To avoid the first contingency 
no food should be taken for some time before the operation, and if the patient is sick, he should be turned on his side; to avoid the latter special precautions must be taken, which are described in books on operative surgery.

For the relative advantages of the different anæsthetics and the mode of giving each, the account of the different individual drugs must be consulted.

\section{G. Drugs acting on the Eye.}

I. Drugs acting on the Pupil.-The first thing to determine is whether any drug which dilates or contracts the pupil acts locally or centrally. It is dropped into one eye : if it only acts feebly and after some time on both eyes, it follows that it has acted centrally after absorption from the conjunctiva into the general circulation; but if it acts quickly, powerfully, and only on the eye into which it was dropped, its action is local. If it acts on an excised eye its action must be local. If, when all the vessels going to the eye are ligatured, the drug will act when dropped into the eye, but will not when thrown into the general circulation, this again shows that its action is local, and that when it acts after being thrown into the circulation when no vessels are ligatured, it does so because it is circulating locally through the eye. If all the arteries and veins of the eye are ligatured, and the drug will not act when locally applied, although it would before and will now, when thrown into general circulation, it shows that its action is central, and that it acts when dropped into the eye because some of it is absorbed. If it has been proved by these means to act centrally, the further investigation is difficult, for the central mechanism is complex.

If it has been proved to act locally, it may act either on the muscular fibres of the iris, on the terminations of the third nerve in them, or on the terminations of the cervical sympathetic in them. Stimulation of the third nerve causes the pupil to contract ; section of it causes the pupil to dilate. Stimulation of the sympathetic causes the pupil to dilate; section of it causes the pupil to contract. If the pupil is dilated by the local action of a drug, and stimulation of the third nerve will not cause it to 
contract, but yet the muscle is responsive to mechanical stimulation, it shows that the endings of the third nerve are paralyzed. If the pupil is contracted by the drug, and, although responsive to mechanical stimulation, will not dilate when the third nerve is cut, it shows that the ends of the third nerve are stimulated. If a drug locally dilates the pupil, but not as powerfully as stimulation of the sympathetic, it is clear that its whole effect is not due to a stimulation of the sympathetic; and if the muscle remains locally irritable, the third nerve ending must be paralyzed. A series of similar experiments may be made with regard to the sympathetic. By these means the mode of action of many drugs has been made out, but often they act both on the sympathetic and the third nerve. In the following list they will be classified under their main actions :

Mydriatics (dilate the pupil)-

A. Paralyze the termination of the third nerve.

(I) Atropine.

(2) Homatropine.

(3) Daturine.

(4) Hyoscyamine.

(5) Coniine.
(6) Gelsemine.

(7) Muscarine.

(8) Hydrocyanic Acid.

(9) Aconite.

(Io) Amyl nitrite.

B. Stimulate the terminations of the sympathetic.-Cocaine.

C. Act centrally.-Anæsthetics (late in their action).

Myotics (contract the pupil).

A. Stimulate the terminations of the third nerve.-Pilocarpine, and nicotine (probably).

B. Stimulate the muscle.-Physostigmine.

C. Act centrally.-Anæsthetics (early in their action), Opium.

Therapeutics.-Dilators of the pupils, especially atropine and homatropine, are used to dilate the pupil for ophthalmoscopic examination, and to prevent or break down adhesions of the iris. Contractors of the pupil, especially physostigmine, are used to overcome the effects of atropine, [to prevent or break down adhesions of the iris], and to prevent too much light entering the eye in painful diseases of it. 
2. Drugs acting on the Ciliary Muscle. - The following drugs impair or paralyze accommodation :

(I) Atropine.

(2) Daturine.

(3) Hyoscyamine.

(4) Homatropine.

(5) Cocaine.
(6) Physostigmine.

(7) Pilocarpine.

(8) Gelsemine.

(9) Coniine.

Intra-ocular tension is increased by atropine (large doses), hyoscyamine, and daturine. It is decreased by cocaine, hyoscine, and physostigmine.

Gelsemine paralyzes the external ocular muscles, especially the elevator palpebræ and the external rectus, by its action on the terminal nerve filaments.

Cocaine, [by] stimulating the unstriped fibres in the orbital membrane and the eyelids, causes the eye to protrude. Coniine produces ptosis [when given in large doses].

The capacity for seeing blue is increased by strychnine. Santonin causes first violet, then yellow vision.

H. Drugs acting on the Ears.-We know very little about the action of drugs on them. Quinine and salicylic acid cause noise and buzzing.

I. Drugs acting on the Sympathetic System.-Much of this subject has already been discussed when speaking of the action of drugs on vessels. The curious fact has been made out that if an animal be treated with a large dose of nicotine, or if this be applied locally to the superior cervical ganglion, stimulation of the nerve below the ganglion no longer produces its characteristic effects, although stimulation above the ganglion does.

Division XI.-Drugs acting on the Organs of Generation.

A. Aphrodisiacs.-These are substances which increase sexual desire. There are conceivably many ways in which this might take place. There is a centre in the lumbar spinal cord, irritation of which causes erection, and this is capable of being excited by afferent impulses proceeding from many parts of the body, but especially from the cerebrum, and the genital organs 
themselves, or the parts in their immediate neighborhood. The lumbar centre appears to be very dependent upon the general health, and therefore substances which improve this are indirectly aphrodisiacs.

The following drugs are known as aphrodisiacs; their mode of action is not certainly known :
(I) Strychnine.
(2) Cantharides.
(3) Alcohol.
(4) Cannabis Indica.
(5) Camphor.
(6) Phosphorus.
(7) Damiana.

B. Anaphrodisiacs.-We do not know for certain of any drugs which have a depressant effect upon the lumbar centre. Most anaphrodisiacs act by decreasing or removing some irritation which is reflexly producing an aphrodisiac effect, but some probably act centrally.

Drugs used as anaphrodisiacs are-
(I) Bromides.
(2) Potassium iodide.
(3) Opium.
(4) Belladonna.
(5) Hyoscyamus.
(6) Stramonium.
(7) Digitalis.
(8) Purgatives.

C. Ecbolics or Oxytocics are remedies which during or immediately after parturition increase uterine action.

They are-
(I) Ergot.
(2) Quinine.
(3) Hydrastis.
(4) Savine.
(5) Rue.
(6) Powerful purgatives.

Of these ergot is by far the most important. Occasionally some of these drugs will act upon the gravid uterus to produce abortion before parturition has begun. They have all of them been used criminally for this purpose.

D. Emmenagogues are substances used to increase the menstrual flow. Diminution of the menstrual flow is a symptom of so many diseases that a large number of drugs which remedy these are indirect emmenagogues, but the substances which seem to have a special action in increasing the menstrual flow are- 
(I) All Ecbolics.

(2) [Manganese dioxide.]

(6) Guaiacum.

(3) Asafœida.

(4) Apiol.

(5) Myrrh.

(7) Cantharides.

(8) Borax.

(9) [Tansy.]

Among the many indirect emmenagogues the commoner are purgatives, iron, cod-liver oil and strychnine, which act by improving the general health. Hot foot- or hip-baths, especially if mustard be added, often aid the onset of menstruation.

\section{E. Substances which depress Uterine Action.-} These are employed to restrain the contractions of the gravid uterus.

They are-

(r) Bromides.

(2) Opium.

(3) Chloral [hydrate.

(4) Viburnum.]

(5) Cannabis Indica.

(6) Chloroform.

(7) Antimony and potassium tartrate.

\section{F. Drugs acting on the Secretion of Milk.}

Galactagogues [are] drugs which increase the secretion of milk.

[Pilocarpus, Leaves of Ricinus Communis,] and Alcohol.-Of these [pilocarpus] is the most powerful, but its effects soon pass off. [The leaves of the castor-oil plant are used, applied as a poultice, and a decoction, or the fluid extract of them, given internally at the same time.] Alcohol is very feeble. The secretion is so much under the control of the general health that the best way to insure an abundant secretion is to keep the general health as good as possible.

Antigalactagogues [are] drugs which decrease the secretion of milk.

Belladonna, either given internally or applied locally, is very efficient, probably acting on the mammary gland as on the sweat glands.

The following drugs, if given, are excreted by the milk, and are therefore taken in by the child :-Oil of anise, oil of dill, garlic, oil of turpentine, oil of copaiba, and probably all volatile oils, sulphur, rhubarb, senna, jalap, scammony, castor oil, opium, iodine, indigo, antimony, arsenic, bismuth, iron, lead, mercury, zinc and potassium iodide. It is clear that these must be administered 
with care to the mother; for example, copaiba or turpentine will make the milk so [unpleasant] that the child will not take it. The above purgatives, given to the mother, may cause diarrhœa in the child. Opium should not be given in large doses to the mother. On the other hand, mercury, arsenic, and potassium iodide may be administered to the child by being given to her.

\section{Division XII.-Drugs acting on Metabolism.}

Our knowledge of the normal metabolism of the body is very imperfect, consequently we know very little more than has already been stated under other divisions, about the action of drugs on metabolism. Any further remarks which are necessary will be made when the individual drugs are considered. Two words in common use are alterative and tonic.

Alterative is a vague term of which no definition can be given. It is often used to cloak our ignorance, when we have no exact knowledge of the action of a drug. Many drugs comprehended under this term have the property of profoundly altering the body, especially if it be diseased ; for example, mercury will, if the patient be suffering from syphilis, generally cause the absorption of syphilitic exudations, but we do not know how this takes place. All that can be said about such drugs will be stated under each, for their mode of action is probably so different that no useful purpose would be served by considering them together.

Tonic.-This is a term even more vague than alterative. So ill-defined is it that it is advisable never to use it if it can be avoided. As commonly employed, it means a drug which makes the patient feel in more robust health than he did before he took it. Obviously this may happen in many ways, such as, for instance, by improving the digestion or the quality of the blood. [This definition is evidently inadequate, and none thus far proposed is entirely satisfactory. The following is suggested (Wood): A drug which so influences nutrition as to increase the reconstruction or upbuilding of the tissue or tissues concerned.] 


\section{PHARMACOPOEIAL MATERIA MEDICA.}

(All the substances about to be described are pharmacopoeial unless the contrary is stated.)

\section{PART I.-INORGANIC MATERIA MEDICA.}

\section{GROUP I.}

\section{Aqua, Hydrogenii Dioxidum, Oxygenium.} state.

I. AQUA, [Water, $\mathrm{I}_{2} \mathrm{O}=17.96 .-$ Natural water in its purest attainable

Characters. - A colorless, limpid liquid, without odor or taste at ordinary temperature, and remaining odorless while being heated to boiling.

II. AQUA DESTILLATA.-Distilled water, $\mathrm{H}_{2} \mathrm{O}=17.96$.

SourcE. - Take 1000 volumes of water, distill from a suitable apparatus provided with a block tin or glass condenser, reject the first 100 volumes, which contain volatile impurities, and preserve the next 800 in glass-stoppered bottles, rinsed with hot distilled water immediately before being filled.

Characters. - A colorless, limpid liquid, without odor or taste, and perfectly neutral to litmus paper.

TESTS. - Evaporated in a clean glass capsule on a water bath, no residue should remain. It is not affected by Hydrogen or Ammonium Sulphide (absence of metallic impurities), Ammonium Oxalate (Calcium), Silver Nitrate (Chlorides), Barium Chloride (Sulphates), or Mercuric Chloride (Ammonia), nor should its transparency be affected when mixed with twice its volume of Calcic Hydrate test solution (absence of Carbon Dioxide). In heating to boiling and acidulating with diluted Sulphuric Acid and adding a one-tenth of one per cent. solution of Potassium Permanganate, the color produced is not entirely destroyed by boiling five minutes nor by afterwards setting the vessel aside, well covered, for ten hours (absence of organic or other oxidizable matters)]. Aqua Destillata is always to be used for making up prescriptions. (116) 
Action of WATER.*

External.-An indifferent bath $\left(88^{\circ}-98^{\circ}\right.$ F.) [31. $1^{\circ}-$ $36.6^{\circ} \mathrm{C}$.] , or one in which the bather feels neither hot nor cold, produces no particular effect.

Cold baths increase the production of heat, and abstract heat from the body if they are prolonged; therefore, at first the bodily temperature may rise slightly, but when the loss exceeds the production it falls. The amount of carbon [dioxide] expired is increased. The rate of the pulse and respiration at first rise, but they soon fall. The skin becomes pale, and the condition of goose-skin is seen. After the bath (the duration and temperature suitable for different persons vary widely) there is a feeling of warmth and exhilaration, and the cutaneous vessels dilate, [reaction].

A warm bath, if sufficiently prolonged, may cause a slight rise of the bodily temperature, the skin becomes red, the pulse and respiration are more frequent, the amount of urine secreted is diminished, and after the bath there is profuse perspiration.

Internal.-Warm water gives rise to nausea and vomiting; hot water, taken in small quantities at frequent intervals, may check both. Water is quickly absorbed from the stomach, and very soon afterwards the amount of urine secreted is greatly increased, and to a less degree the amount of bile, pancreatic juice, and saliva. Large quantities of fluid should nat be drunk during meal times, as that impairs digestion. If a considerable amount of water is drunk daily, the amount of urea excreted is increased, and that of uric acid is diminished. Water not only washes out the tissues, but apparently renders tissue metamorphosis more complete.

\section{Therapeutics of Water.}

External.-Cold baths are used for the subsequent exhilarating effects, which may be increased by [brisk] rubbing with a rough towel. Persons in whom a feeling of warmth does not

* Unless otherwise stated, the word action will in this book always be taken to mean physiological action, or action in health. 
immediately follow a cold bath should not use it. The constant daily use of a cold bath probably diminishes the liability to catch cold. Cold baths are said to arrest attacks of laryngismus stridulus. They have been largely used to reduce the temperature in fever, especially typhoid fever. The first effect of putting the patient in the cold water is to cause, reflexly from the stimulation of the skin by the cold, an increased production of heat; for this reason and because of the cessation of radiation, the rectal temperature at first rises a little, but soon, owing to the direct abstraction of heat, and to the diminished production of heat which quickly sets in, it falls rapidly, and continues to do so after the patient is taken out. The temperature of a bath for a patient with typhoid fever should be between $68^{\circ}$ and $58^{\circ} \mathrm{F}$. $\left[20^{\circ}\right.$ and $\left.14.4^{\circ} \mathrm{C}.\right]$; he should be lowered into it by a sheet, and remain in ten minutes, unless before that time he shows signs of collapse; he is then lifted back to bed, where a blanket is thrown loosely over him. If this treatment is adopted, the bath ought to be given whenever the axillary temperature is $103^{\circ}$ F. $\left[39.4^{\circ} \mathrm{C}.\right]$. Sometimes the patient is placed in a bath at a temperature of $10^{\circ} \mathrm{F} .\left[5.5^{\circ} \mathrm{C}.\right]$ below his own, and the water is cooled by putting in cold water or ice, till it has fallen to about $68^{\circ} \mathrm{F} .\left[20^{\circ} \mathrm{C}\right.$.] , when he is taken out. [Brisk rubbing of the whole body should be carried out during the bath and the feet kept warm. Cold baths are no longer used in the treatment of typhoid fever with the notion that they reduce temperature. They are useful for the stimulation of the nervous system which they may possibly bring about to some degree and for the marked diuresis which they produce thus, supposedly, favoring the elimination of toxins in the urine.] Often, instead of having a bath, he is sponged with cold water as he lies in bed; this saves trouble, but both sponging and a cold pack (which consists of a sheet four folds thick, wrung out in cold water and wrapped round the naked body for five or ten minutes) are inferior to a bath. Pneumonia is often treated by the application of cold, generally by means of ice poultices applied to the chest. To make an ice poultice, put on a piece of [rubber] tissue a layer of wood wool, then one of powdered ice sprinkled 
with a little salt, turn over the edge of the [rubber tissue]which has been left wide enough-so as to cover in the poultice, and seal the edges with a little chloroform or turpentine. Put the poultice in a flannel bag, and bind it on the body when desired, with lint between it and the skin. [The term "poultice" is hardly a proper one to designate this method of applying cold. See definition of poultice on p. 36.] The immediate action of very cold baths is far the best treatment for any sudden hyperpyrexia.

Cold is applied locally either by cold water in Leiter's coils or by ice bags, in a number of conditions, with the object of arresting inflammation. Thus ice bags are put on the head in meningitis, or concussion, and on the knee-joint for acute synovitis, etc. According to most authorities, cold contracts not only the vessels of the skin to which it is applied, but by reflex action those of the organs underneath it. This explains the application of an ice bag to the chest to arrest pulmonary hæmorrhage. Cold locally applied is, therefore, hæmostatic.

Warm baths, as they liquefy the fatty secretions, are more cleansing than cold. Hot baths, like any other application of heat, soothe pain; hence they are useful for rheumatoid arthritis and colic, whether renal, biliary or intestinal. By bringing blood to the skin and lessening the amount in the internal organs, they relieve muscular spasm, such as we find in [spasmodic] stricture of the urethra, colic, laryngismus stridulus, other forms of laryngeal spasm, and infantile convulsions. In the same way they are of service in weariness from muscular or cerebral activity, and are useful in many inflammatory affections; as, for example, a cold in the head. A warm bath immediately before going to bed may sometimes cure insomnia. [The practice in asylums for the insane is to give a hot bath ( $\mathrm{ro4}^{\circ} \mathrm{F}$.; $40^{\circ}$ C.) as a remedy for sleeplessness.] The subsequent increased perspiration makes hot baths and hot packs of great value in the various forms of nephritis and in uræmia. Great care must be taken, after a hot bath which has been given to induce sweating, to see that the patient is kept warm by being wrapped quickly in a hot blanket and put into a warm bed; if 
not, the cutaneous vessels soon contract, and there is no diaphoresis. A local hot bath has the same effects, but to a less degree. A hot foot-bath is often used for a cold in the head, or for amenorrhœa. Sponging with hot water will, by the vascular dilatation and sweating it causes, reduce the temperature slightly in fever.

A cold bath is one the temperature of which is below $70^{\circ} \mathrm{F}$. [ $21^{\circ} \mathrm{C}$.] , one between $88^{\circ}$ and $98^{\circ} \mathrm{F}$. [3 $\mathrm{I} \cdot \mathrm{I}^{\circ}$ and $36.6^{\circ} \mathrm{C}$.] is properly speaking indifferent, but it is often called a warm bath. A tepid bath is intermediate between warm and cold. Anything above $98^{\circ} \mathrm{F}$. $\left[36.6^{\circ} \mathrm{C}\right.$. $]$ is a hot bath. Few people can bear a temperature much over $102^{\circ} \mathrm{F}$. $\left[3^{8} .9^{\circ} \mathrm{C}\right.$. $]$.

Internal.--The chief therapeutic use of water is to wash out the tissues, especially the kidneys, and to keep the urine diluted. Some persons who are liable to the formation of gravel or urinary calculi can, by drinking plenty of pure water, prevent their formation, for the minute collection of crystals which are the beginning of all calculi, are washed out of the ordinary system before they have time to grow to any size, and if they are composed of uric acid, the copious drinking of water diminishes the liability of their formation, for it decreases the amount of uric

2. acid excreted. The liability to the formation of gall-stones may also be kept in check by the drinking of plenty of water, [since] then the bile becomes less concentrated and flows more quickly. When large quantities of water are drunk it should be pure distilled water, and should be taken between meals. A glass of cold water taken on rising in the morning will with some persons cause the bowels to be opened. Warm water is an emetic.*

[III. AQUA HYDROGENII DIOXIDI.-Solution of Hydrogen Dioxide. Synonym.-Solution of Hydrogen Peroxide.

A slightly acid, aqueous solution of Hydrogen Dioxide $\left(\mathrm{H}_{2} \mathrm{O}_{2}=33.92\right)$, containing, when freshly prepared, about 3 per cent., by weight, of the pure Dioxide, corresponding to about ten volumes of available oxygen.

Source.-By solution of Barium Dioxide, 300 ; in cold distilled water

* It is impossible in this book to give more than a brief sketch of baths and the drinking of water and mineral waters. Further information will be found in works on "General Therapeutics." 
500 , and refrigeration to $50^{\circ} \mathrm{F}$; ; $10^{\circ} \mathrm{C}$. Phosphoric Acid, 96 ; is dissolved in distilled water 320 . The magma is added to the latter solution and thoroughly mixed, being kept acid by Phosphoric Acid. Filter and wash with distilled water. Add diluted Sulphuric Acid to the filtrate, and starch, Io; by agitation. Filter and re-filter until a clear solution is obtained. The bottle should be kept tightly corked.

Characters. - A colorless liquid, without odor, slightly acidulous to the taste, and producing a peculiar sensation and soapy froth in the mouth; liable to deterioriate by age, exposure to heat, or protracted agitation. Sp. gr. : about I.006 to I.0I 2.

Dose, I to 3 fl. dr.; 4 to 12 c.c.

\section{Action of Hydrogen Dioxide.}

Hydrogen dioxide readily yields oxygen to all oxidizable substances. When taken internally it gives oxygen to the blood, stimulates the nervous system and increases urinary secretion. It is a non-poisonous antiseptic, destroying organized fermentations and liberating oxygen. It decomposes pus and probably destroys the microbes of suppuration.

\section{Therapeutics of Hydrogen Dioxide.}

Hydrogen dioxide seems to have a favorable action in some forms of dyspepsia, and to improve digestion. In diphtheria it is useful as a cleansing agent and to absorb false membranes, but should be used in glass or hard rubber instruments. Some commercial preparations are very acid, and therefore too irritating for this purpose. This acidity can be neutralized by adding twice its quantity of lime water. It will check bleeding, but from small vessels only. It is of great value in cleansing wounds, ulcers and fistulous tracts, and for surgical dressings; the cessation of frothing indicates the destruction of pus. But the converse of this is not true, for it will froth with perfectly normal blood. It should not be injected into a suppurating cavity unless there is a free outlet for the escape of the gas which is formed. Its most popular use is for bleaching the hair.] Internally it has been recommended for many diseases, especially diabetes, epilepsy, and uræmia, but there is no proof of its efficacy. It is dangerous when given subcutaneously, for it is broken up by the blood; and if more oxygen is formed than the blood can dispose of, 
gas emboli are produced, and, these lodging in the lungs, cause death from asphyxia.

IV. [OXYGENIUM, Oxygen (not official) $O=15.96$.

SOURCE. - By exposing manganese dioxide with potassium chlorate to a strong heat. $\mathrm{KClO}_{3}=\mathrm{KCl}+\mathrm{O}_{3}$.

CharactzRs. - A colorless, odorless gas, slightly soluble in water and alcohol.]

Compressed oxygen gas is sold in [metallic] cylinders.

\section{Action and Therapeutics of Oxygen.}

Oxygen inhalations are used in pneumonia, bronchitis, heart disease, convulsions, and any other condition accompanied by great lividity. This they will often relieve, and they may help a patient to tide over a temporary risk of death from asphyxia, and even if they fail to avert death, they often render the end less distressing. The gas should be allowed to issue in a gentle stream, and the inhaler should not be held too near the patient. [In various chronic conditions as anæmia, albuminuria, glycosuria and various forms of sub-oxidation, the persistent use of oxygen has given-excellent results.]

\section{GROUP II.}

THE ALKALINE METALS.

Potassium, Sodium, Ammonium, Lithium.

I. POTASSIUM.

$$
\mathrm{K}=[39.03 \text {. }
$$

1. POTASSA. $-\mathrm{KOH}=55.99$. Synonyms. - Potassium Hydrate. Potassium Hydroxide. Caustic Potash.

SourCE.-Evaporate Liquor Potasse, fuse the residue and pour into clean cylindrical moulds which have been previously warmed.

Characters. - Dry, white, translucent pencils, or fused masses, hard and brittle, showing a crystalline fracture; odorless, or having a faint odor of lye, and of a very acrid and caustic taste. Great caution is necessary in tasting and handling it, as it rapidly destroys organic tissues. Exposed to the air, it rapidly 
absorbs Carbon Dioxide and moisture, and deliquesces. Solubilit - In about 0.5 part of water and in 2 parts of alcohol.

IMPURITIES.-Organic matter, arsenic, lead, iron, soda, alıminum, calcium, chlorides, sulphates, silicates, carbonates, and nitrates.

\section{Preparations.}

I. Liquor Potassæ. Solution of Potassa. Synonym. - \$olution of Potassium Hydrate. An aqueous solution of Potassium Hydrate $(\mathrm{KOH}=55.99)$, containing about 5 per cent. of the hydrate.

Source.-Dissolve Potassium Bicarbonate in distilled water; slake Lime, dissolve in distilled water and boil, add this to the first solution, continue to boil, strain when cold ; when it has become clear from subsidence, decant or siphon off the clear solution. $\mathrm{K}_{2} \mathrm{CO}_{3}+\mathrm{Ca}(\mathrm{OH})_{2}=2 \mathrm{KOH}+\mathrm{CaCO}_{3}$. Or it may be prepared from a solution of Potassa, $5^{6}$ parts of the full strength, directed by the U. S. P. ( 90 per cent.), in distilled water ( 944 parts).

ChARACTERS. - A clear colorless liquid, odorless, having a very acrid and

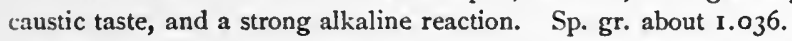

IMPUR ITIES. - See Potassa.]

INCOMPATIBLES.-Acids, acid salts, metallic salts and preparations of ammonia, belladonna, hyoscyamus and stramonium, the alkaloids of these three being decomposed by caustic potash. All alkaloids are precipitated by alkalies.

Dose, 5 to $30 \mathrm{~m}$.; [ .30 to 2.00 c.c.], freely diluted.

2. Potassa cum Calce.-[Potassa with Lime. Synonyms.-Vienna Caustic. Vienna Paste. Potassa, 500; Lime, 500. Rubbed together in a warm, iron mortar.

ChARACTERS. - A grayish-white powder, deliquescent, having a strongly alkaline reaction, and responding to the tests for Calcium and Potassium. Solubility. - In diluted hydrochloric acid without leaving more than a small residue.]

\section{Action of Potash.}

External.-It is, if concentrated, a powerful irritant and caustic, acting by abstracting water from the part to which it is applied. It dissolves fatty matters that may be present on the surface. It is antacid, and, if freely diluted, sedative.

Internal.-Mouth.-As alkalies check alkaline secretions, potash momentarily checks the secretion of saliva.

Stomach.-Because alkalies stimulate acid secretions, the flow of gastric juice is excited, if alkalies are given before a meal, but if at the end of or after a meal the gastric juice already 
secreted is neutralized. Being readily diffusible, alkalies are quickly absorbed.

Blood.-This is rendered more alkaline. Probably all alkalies circulate in the blood as carbonates, but their action as alkalizers of the blood is very transitory, for they are quickly excreted. The amount of hæmoglobin, if it is deficient, is said to be increased. The continual use of alkalies diminishes the quantity of fat.

Heart.-Large amounts of potassium salts are depressant to all muscular tissues, and therefore decrease the force of the heart, ultimately causing diastolic arrest by direct action on the cardiac muscle.

Kidney.-Potassium salts are diuretic, acting directly on the renal epithelium. They are quickly excreted in the urine, rendering it alkaline, and thus increasing its power of holding uric acid in solution.

Respiratory passages. - The bronchial secretion is increased in quantity, and it is rendered less viscid, but in some cases of bronchitis it is diminished.

Muscle. -The prolonged contraction produced by veratrine, or barium salts, is abolished by potassium salts. They are direct muscular depressants, and depress also the nervous system, especially the brain and spinal cord.

Metabolism.-Potassium salts, like all alkalies, if given in large doses, increase metabolism, leading to a greater oxidation of proteids and fats.

\section{Therapeutics of Potash.}

External.-Caustic potash is used to destroy lupus, and it was formerly employed to make issues. Care must be taken to limit its action, for it diffuses very rapidly. Liquor potassæ is used to dissolve off the fatty matters and thoroughly cleanse the skin before operations, and weaker solutions of it are employed to remove the epidermis in certain chronic skin diseases. A 40 per cent. solution is recommended to remove an ingrowing toenail, which is painted with the fluid, and in a few seconds is so softened that much can be scraped off. The procedure is 
repeated till the nail that remains is sufficiently thin to be removed with a pair of fine scissors. Dilute solutions, acting as sedatives, relieve itching.

Internal.-To obtain the effects of alkalies upon internal organs, potassium bicarbonate, citrate and acetate are preferable to potash, for that is apt to irritate the stomach; but it is occasionally used in small doses as a gastric sedative for dyspepsia.

Toxicology. - See Soda, p. I 39.

2. POTASSII CARBONAS.-[Potassium Carbonate. $\mathrm{K}_{2} \mathrm{CO}_{3}=$ 137.91.] Synonym.-Salt of Tartar.

SOURCE.-Pearlash, which is a product of the lixiviation of wood ashes, is treated with water, which dissolves little but potassium carbonate, and the solution is evaporated.

CHARACTERS. - A white, [granular powder, odorless, having a very strong alkaline taste; very deliquescent. Solubility.-In I.I parts of water, and in 0.65 part of boiling water; insoluble in alcohol.

IMPURITIES. - Sulphates, chlorides, nitrates, cyanides, earthy and metallic matters.

Potassium Carbonate is used in preparing Mistura Ferri Composita, Pilulæ Ferri Carbonatis (in which Ferrous Carbonate is formed), and Potassa Sulphurata.

Dose, 5 to $30 \mathrm{gr}$; .30 to $2.00 \mathrm{gm}$.]

\section{Action and Therapeutics of Potassium Carbonate.}

These are the same as those of potash; but the carbonate is less caustic.

3. POTASSII BICARBONAS.-[Potassium Bicarbonate. $\mathrm{KHCO}_{3}$ $=99.88$.

Source. - Pass Carbon Dioxide] through a solution of Potassium Carbonate, and let the bicarbonate crystallize out. $\mathrm{K}_{2} \mathrm{CO}_{3}+\mathrm{CO}_{2}+\mathrm{H}_{2} \mathrm{O}=$ $2 \mathrm{KHCO}_{3}$.

Characters. - [Colorless, transparent, monoclinic prisms, odorless, and having a saline and slightly alkaline taste. Permanent in the air. Solubility. -In 3.2 parts of water; almost insoluble in Alcohol.

IMPUR ITIES. - The carbonate, chlorides, and iron.

Potassium Bicarbonate is used in preparing Liquor Potassii Arsenitis, Liquor Potassii Citratis, and Potassii Citras Effervescens.

Dose, 5 to $60 \mathrm{gr}$; .30 to $4.00 \mathrm{gm}$.] 


\section{Action of Potassium Bicarbonate.}

Potassium bicarbonate is too feebly caustic to be of any use for this purpose. Otherwise its actions are those of potash.

\section{Therapeutics of Potassium Bicarbonate.}

Stomach. - Potassium bicarbonate may be given before meals to stimulate the flow of gastric juice; and as it is a gastric sedative, it is useful in painful dyspepsia accompanied by a scanty secretion of gastric juice. The increase of the antiseptic acid secretion is valuable in cases of dyspepsia associated with fermentation in the stomach. It may be taken after meals if too much acid is secreted, and the patient suffers from acid eructations, especially if pain be present also ; but it is better treatment to remove the cause of the dyspepsia. It is not a common remedy for dyspepsia, sodium bicarbonate being usually preferred. Either is beneficial when much mucus is present, for this is rendered less viscid by alkalies. It should not be used as an alkali in cases of poisoning by mineral acids, because of the evolution of carbon [dioxide,] gas. Bicarbonates are used in preference to carbonates, as the latter are far too strongly alkaline for the stomach. Potash water is often drunk as an effervescing water instead of soda water. It should be a [half of a one per cent.] solution of potassium bicarbonate in water, into which [carbon dioxide] gas under a pressure of four atmospheres has been passed.

Blood.-Potassium bicarbonate circulates in the blood as the carbonate. It was formerly much used in rheumatic fever, but is now superseded by the salicylates. Probably it did no good. In gout it is given to increase the alkalinity of the blood, which contains an excess of uric acid, but there is no evidence that it benefits gout, and the many alkaline mineral waters used for this disease are efficacious because they dilute the plasma, and so render it more capable of holding uric acid in solution. Potassium bicarbonate is believed to be hæmatinic, that is to say, it is thought to increase the amount of hæmoglobin; but as for this 
purpose it is usually given with iron, its hæmatinic power has not yet been proved.

Kidneys.--It is not much used for its diuretic effect and its alkalizing power over the urine, as the vegetable salts are preferable.

4. POTASSII ACETAS. $-\left[\right.$ Potassium Acetate. $\mathrm{KC}_{2} \mathrm{H}_{3} \mathrm{O}_{2}=97.89$.

Source._Add Acetic Acid in excess to Potassium Carbonate or Bicarbonate. Evaporate to dryness and fuse the residue. $\mathrm{K}_{2} \mathrm{CO}_{3}+2 \mathrm{HC}_{2} \mathrm{H}_{3} \mathrm{O}_{2}=$ $2 \mathrm{KC}_{2} \mathrm{H}_{3} \mathrm{O}_{2}+\mathrm{H}_{2} \mathrm{O}+\mathrm{CO}_{2}$. Or, if the bicarbonate, which is preferable, is used, $\mathrm{KHCO}_{3}+\mathrm{HC}_{2} \mathrm{H}_{3} \mathrm{O}_{2}=\mathrm{KC}_{2} \mathrm{H}_{3} \mathrm{O}_{2}+\mathrm{H}_{2} \mathrm{O}+\mathrm{CO}_{2}$.

Characters. - A white powder or crystalline masses of a satiny lustre, odorless and having a warming saline taste ; very deliquescent. Solubility.In $0.3^{6}$ part of water, and 1.9 parts of Alcohol.

IMPURITIES.-The carbonate and metallic impurities.

Dose, 5 to $60 \mathrm{gr}$. ; .30 to $4.00 \mathrm{gm}$.]

5. POTASSII CITRAS.-[Potassium Citrate. $\mathrm{K}_{3} \mathrm{C}_{6} \mathrm{H}_{5} \mathrm{O}_{7}+\mathrm{H}_{2} \mathrm{O}=$ 323.59 .

Source. - Neutralize Potassium Carbonate with a solution of Citric Acid, and evaporate to dryness. $3 \mathrm{~K}_{2} \mathrm{CO}_{3}+2 \mathrm{H}_{3} \mathrm{C}_{6} \mathrm{H}_{5} \mathrm{O}_{7}=2 \mathrm{~K}_{3} \mathrm{C}_{6} \mathrm{H}_{5} \mathrm{O}_{7}+$ ${ }_{3} \mathrm{H}_{2} \mathrm{O}+{ }_{3} \mathrm{CO}_{2}$.

CHARACTERS. - Transparent, prismatic crystals or a white, granular powder, odorless and having a cooling, saline taste. Deliquescent on exposure to the air. Solubility. - In 0.6 part of water ; sparingly in Alcohol.

IMPURITIES. - Carbonates, chlorides, and tartrates.

Dose, 5 to $30 \mathrm{gr}$; . 30 to $2.00 \mathrm{gm}$.]

\section{Preparation.}

Liquor Potassii Citratis.-Solution of Potassium Citrate. Synonym.-Mistura Potassii Citratis. Citric Acid, 6; Potassium Bicarbonate, 8 ; water to roo. The acid and the bicarbonate are dissolved separately and the solutions mixed. It contains about 9 per cent. of anhydrous Potassium Citrate.

Dose, I to 8 fl. dr.; 4. to 3o. c.c.

6. POTASSII CITRAS EFFERVESCENS.-Effervescent Potassium Citrate. Citric Acid, 63; Potassium Bicarbonate, 90 ; Sugar, 47.

SOURCE.-Powder the ingredients separately, and mix them thoroughly in a warm mortar. Dry the resulting, uniform paste rapidly, and when it is perfectly dry, reduce it to a powder of the desired degree of fineness. $\mathrm{H}_{2} \mathrm{C}_{5} \mathrm{H}_{5} \mathrm{O}_{7}$ $+{ }_{3} \mathrm{KHCO}_{3}+\mathrm{H}_{2} \mathrm{O}=\mathrm{K}_{3} \mathrm{C}_{6} \mathrm{H}_{5} \mathrm{O}_{7}+{ }_{3} \mathrm{CO}_{2}+{ }_{4} \mathrm{H}_{2} \mathrm{O}$.

Characters. - A fine, white powder, odorless, and having a sweetish. saline taste. Solubility. - Completely in water, with effervescence.

Dose, 5 to $60 \mathrm{gr}$; ; 30 to $4.00 \mathrm{gm}$.] 
Action of Potassium Citrate and Acetate.

External.-No action. Being neutral, they are not even antacid.

Internal.-These are the least irritating to the stomach of all potassium salts ; being neutral, they have no action on gastric juice. They circulate as potassium carbonate. Both are more powerfully diuretic than any other potassium salts, and act by directly stimulating the renal cells. They are diaphoretic, especially the citrate ; but neither of them causes a great increase of the perspiration. How they produce this effect is not certainly known.

\section{Therapeutics of Potassium Citrate and Acetate.}

As neither impairs digestion, they are chiefly used for remote effects.

Blood.-They have been largely given for rheumatic fever, but are now rarely employed. Many believe both salts are of great value in gout; if that is so, it is doubtful how they act, for they do not increase the power of blood plasma to dissolve sodium biurate. They are powerfully antiscorbutic; that is to say, they prevent scurvy; but they are not so efficacious as lemon-juice, lime-juice, and fresh vegetables.

Kidneys. - Although in health the diuresis produced by the potassium citrate and acetate is slight, and the urea and other solids of the urine are actually decreased, yet clinical experience points clearly to the fact that both these salts are, in chronic Bright's disease, powerful diuretics. They are frequenly used in this disease and in feverish conditions, and also to increase the amount of urine, and thus to remove pathological fluids in cases of pleuritic effusion, ascites, etc. Diuretics are best combined, and the following is a good mixture: Potassium acetate, $20 \mathrm{gr}$. [r.20 gm.] ; tincture of squill, ro $\mathrm{m}$ [.60 c.c.] ; spirit of nitrous ether, $30 \mathrm{~m}[2.00 \mathrm{c.c}$.$] ; juice of broom, I f. 3$ [4.00 c.c.]; water to $8 \mathrm{fl} 3[30.00$ c.c. ; juice of broom, B. P., is obtained by bruising fresh broom tops, expressing the juice, adding onethird part of alcohol and filtering after seven days].

They render the urine alkaline, and are much employed for 
this purpose, having the advantage over other potassium salts that they do not derange digestion. Not only do they prevent the precipitation of uric acid, and thus hinder the formation of uric acid gravel, but they will dissolve small uric acid calculi. Roberts states that to keep the urine at the alkalinity necessary for this purpose, 40 to $60 \mathrm{gr}$. [2.50 to $4.00 \mathrm{gm}$.] of the acetate or citrate should be dissolved in four ounces [120. c.c.] of water, and taken every four hours. If more than this is used, harm is done; for an insoluble biurate forms on the surface of the calculus. With many patients it suffices if such a dose in a tumbler of water be taken at bed-time; for during the night the acidity of the urine is highest, as there is no alkaline tide due to meals. Owing to the depressing action of potassium salts, they should be used with care in persons suffering from heart disease.

Skin. - Both these salts may be used in slight pyrexia, such as that of a common cold, on account of their diaphoretic properties.

Lungs. - These salts, like the carbonates and bicarbonates, are mild saline expectorants, especially suitable for cases of bronchitis with viscid, scanty expectoration, as they increase the secretion and lessen the viscidity. The iodide is, however, still more efficacious.

7. POTASSII SULPHAS. - [Potassium Sulphate. $\mathrm{K}_{2} \mathrm{SO}_{4}=173.88$.

Source. - Add Potassium Carbonate to Acid Potassium Sulphate, which is a bye-product of the manufacture of Nitric Acid. $\mathrm{K}_{2} \mathrm{CO}_{3}+2 \mathrm{KHSO}_{4}=$ $2 \mathrm{~K}_{2} \mathrm{SO}_{4}+\mathrm{CO}_{2}+\mathrm{H}_{2} \mathrm{O}$.

Characters. - Hard, colorless, transparent, six-sided, rhombic prisms terminated by pyramids, or a white powder, odorless, and having a somewhat bitter, saline taste. Solubility.-In 9.5 parts of water; insoluble in Alcohol.

Impurities. -Sodium, arsenic, lead, copper, zinc, iron, aluminum, calcium, magnesia and chlorides.

Dose, $1 / 2$ to $4 \mathrm{dr}$; 2. to $15 . \mathrm{gm}$.

8. POTASSII BITARTRAS. - Potassium Bitartrate. $\mathrm{KH}_{4} \mathrm{C}_{4} \mathrm{HO}_{6}=$ 187.67. Synonyms. - Acid Potassium Tartrate. Cream of Tartar.

Source. - Obtained from crude Tartar (argol) deposited on the sides of wine casks during the fermentation of grape juice, and purified by boiling water, filtration through charcoal and crystallization,

Characters. - Colorless or slightly opaque rhombic crystals; or a fine, white, somewhat gritty powder, odorless, and having a pleasant, acidulous taste. Solubility. - In 201 parts of water; very sparingly soluble in Alcohol. 
IMPÚRITIES.-Calcium tartrate, copper, lead, and iron.

Potassium Bitartrate is contained in Pulvis Jalapæ Compositus.]

Dose, 20 to $60 \mathrm{gr}$.; [1.20 to $4.00 \mathrm{gm}$.] (diuretic and refrigerant); $1 / 2$ to 4 dr.; [2. to I5. gm. ;] (purgative).

Action of Potassium Bitartrate and Sulphate.

External.-One of these being only slightly acid and the other neutral, they have none of the external caustic or antacid properties of other potassium salts.

Internal.-Intestines.-Both salts are typical hydragogue saline purges, producing easy, soft, watery motions without griping. They abstract fluid from the blood, and cause it to be poured into the intestine. Their mode of action has already been fully described (see p. 93).

Liver.-Potassium sulphate is a moderate cholagogue, slightly increasing the biliary flow.

Kidney. - The bitartrate is diuretic, because a small amount of it is, in the intestine, converted into a carbonate and absorbed, and this acts directly on the renal cells. Hence it renders the urine alkaline. But all the sulphate and most of the bitartrate is excreted with the fæces, and if, as seems probable, some is absorbed by the small intestine in the form in which it is taken, it is excreted again into the colon.

\section{Therapeutics of Potassium Bitartrate and Sulphate.}

Internal.-Intestines.-These excellent purgatives are frequently used, especially for habitual sluggishness of the bowels. A dose should be dissolved in a tumbler of [hot] water, and sipped during dressing. They may be employed to open the bowels in cases, such as dropsy or uræmia, in which we wish to eliminate as much fluid as possible. They should for this purpose be given in a concentrated form, for then a large amount of fluid will be secreted from the intestine to bring the solution of the salt to that degree of dilution at which it will act. Compound jalap powder [which contains potassium bitartrate] is also much used for this class of cases. The sulphate having some cholagogue action, is to be preferred when it is believed that the liver is at fault. 
Liver.-These salts are often given to those who suffer from gall-stones, although no potassium salts have any power to dissolve gall-stones, but the sulphate does good as a cholagogue.

Kidney. - The bitartrate is sometimes used as a diuretic in the same class of cases as the acetate and citrate. A very pleasant drink for feverish patients is Imperial drink. It contains potassium bitartrate, $60 \mathrm{gr}$. [4.00 gm.] ; saccharin, I gr. [.06 $\mathrm{gm}$.$] ; oil of lemon, 3 \mathrm{~m}$ [.20 c.c.]; to a pint [500. c.c. $]$ of boiling water.

9. POTASSII NITRAS.-[Potassium Nitrate. $\mathrm{KNO}_{3}=100.92$. Synonyms.-Nitre. Saltpetre.

SOURCE.-Purified native Saltpetre.

Characters.-Colorless, transparent, six-sided rhombic prisms or a crystalline powder, odorless, and having a cooling, saline and pungent taste. Solubility. - In 3.8 parts of water; very sparingly soluble in Alcohol.

IMPURITIES. - Sulphates, chlorides and lime, and the metals.

Potassium Nitrate is used to prepare Argenti Nitras Dilutus.

Dose, 5 to $20 \mathrm{gr}$; .30 to $1.20 \mathrm{gm}$.

\section{Preparation.}

Charta Potassii Nitratis.-Potassium Nitrate Paper. Potassium Nitrate, 200 ; distilled water, 800. Dissolve the Potassium Nitrate in the Distilled Water. Immerse strips of white, unsized paper in the solution, and dry them.]

\section{Action of Potassium Nitrate.}

External.-Nothing noteworthy.

Internal._Stomach and Intestines.-It is liable to cause nausea, vomiting, diarrhœa, symptomatic of the gastritis and enteritis produced by it.

Blood.-Owing to its high diffusion power it quickly passes ints the blood unchanged. External to the body, nitrates prevent the coagulation of the blood, or dissolve the clot if it be already formed; but it is not known that they have any effect on the blood in the body.

Heart.-Potassium nitrate is a powerful cardiac depressant, causing the beats to become feeble and few. Large doses lead to great weakness, fainting, and death.

Kidneys. - Small doses are diuretic from their direct action 
on the renal cells, but large ones are liable to inflame the urinary passages, causing hæmaturia. The drug is excreted unchanged in the urine.

Skin.-Potassium nitrate is a mild diaphoretic.

[Lungs. - Large doses retard respiration and tend to paralyze unstriped muscular fibre.]

\section{Therapeutics of Potassium Nitrate.}

Internal.-Blood.-On account of its supposed action in preventing the coagulation of the living blood, it has been used in rheumatic fever and many inflammatory conditions, but it is now discarded. Probably, as it is a cardiac depressant, it only does harm.

Kidney's and Skin.-It is sometimes employed as a diuretic and diaphoretic in febrile conditions, but the acetate and the citrate are much preferable.

Asthma.-[Potassium nitrate is an anti-spasmodic for the reason given above.] For the treatment of this [symptom] potassium nitrate paper, in pieces about $1 \mathrm{I} / 2$ inches $[4 . \mathrm{cm}$.] square, is lighted, one at a time, and the patient inhales the fumes. Ringer considers it better to dip the paper also into a solution of potassium chlorate, and to burn a piece large enough to fill a whole room with the fumes. This treatment often relieves, and nitre is a common ingredient of [so-called] asthma powders.

Io. POTASSII CHLORAS. - [Potassium Chlorate. $\mathrm{KClO}_{3}=\mathbf{1 2 2 . 2 8 .}$

SuURCE. - Pass Chlorine into a mixture of Potassium Carbonate and Slaked Lime ; then treat the result in boiling water and separate the Chlorate by recrystallization. $\mathrm{K}_{2} \mathrm{CO}_{3}+6 \mathrm{Ca}(\mathrm{OH})_{2}+6 \mathrm{Cl}_{2}=2 \mathrm{KClO}_{3}+5 \mathrm{CaCl}_{2}+\mathrm{CaCO}_{3}+$ $6 \mathrm{H}_{2} \mathrm{O}$.

Characters. - Colorless, lustrous monoclinic prisms or plates, or a white powder, odorless and having a cooling, saline taste. Easily explodes on trituration with many substances, especially Sugar, Sulphur, Tannic Acid, Metallic Sulphides, Phosphorus, Charcoal, and Glycerin. Solubirity._In 16.7 parts of water; insoluble in absolute Alcohol.

ImpURITIEs.-Calcium chloride and lime.

Dose, 3 to $20 \mathrm{gr}$.; .20 to $1.20 \mathrm{gm}$.]

\section{Preparation.}

Trochisci Potassii Chloratis.-[Troches of Potassium Chlorate. Potassium Chlorate, 30; Sugar, 120; Tragacanth, 6 gm. ; Spirit of 
Lemon, I c.c. ; water, a sufficient quantity to make 100 troches. Mix the Sugar with the Tragacanth and the Spirit of Lemon by trituration in a mortar; then transfer the mixture to a sheet of paper, and, by means of a bone spatula, mix with it the Potassium Chlorate, being careful, by avoiding trituration or pressure, to prevent the mixture from igniting or exploding. Lastly, with water, form a mass. Each troche contains 5 gr. ; $\cdot 30 \mathrm{gm}$.]

Dose, I to 6 troches.

\section{Action of Potassium Chlorate.}

External.-It is easily decomposed by septic tissues, and the nascent oxygen given off acts as a stimulant and antiseptic to them.

Internal.-Stomach and Intestines.-Small doses have no effect; poisonous ones produce symptoms similar to those induced by the nitrate.

Blood.-Here also small doses have no effect, but several cases of poisoning show that in large doses potassium chlorate disintegrates the red corpuscles, and converts hæmoglobin into methæmoglobin. The altered blood causes the skin to be cyanotic, it is passed by the urine, which is, therefore, darkcolored, and contains granular debris, and thus the urine is exactly like that met with in paroxysmal hæmoglobinuria. The liver and spleen are enlarged. There may be jaundice and hæmatemesis, and the marrow of the bones becomes very vascular. Nephritis is induced, the tubules are blocked by the débris of the blood, and so the urine is scanty. Death occurs from cardiac weakness or uræmia.

As potassium chlorate easily yields up its oxygen, some believe that it gives off part of its oxygen to the tissues while it is circulating in the blood, but much of it is excreted unchanged in the urine and other excretions.

\section{Therapeutics of Potassium Chlorate.}

This drug is used empirically for stomatitis, tonsillitis, and pharyngitis of all varieties, either as lozenges, gargle ( $x$ to $5 \circ$ parts of water or decoction of cinchona), or to be swallowed in solution, for it is then excreted by the saliva. Its action is there- 
fore always local, as it is beneficial [by] virtue of the nascent oxygen given off from it. It is especially valuable for ulcerative stomatitis. It has been given to women liable to miscarry.

11. POTASSII PERMANGANAS, see Manganese.

12. [POTASSII ET SODII TARTRAS, see Sodium.]

13. POTASSII IODIDUM, see Iodine.

14. POTASSII BROMIDUM, see Bromine.

15. POTASSA SULPHURATA, see Sulphur.

I6. POTASSII FERROCYANIDUM.-[Potassium Ferrocyanide. $\mathrm{K}_{4} \mathrm{Fe}(\mathrm{CN})_{6}+3 \mathrm{H}_{2} \mathrm{O}=421.76$. Synonym.-Yellow Prussiate of Potash.

Potassizem Ferrocyanide is used to prepare Acidum Hydrocyanicum Dilutum, and Potassii Cyanidum; also as a test for iron, copper and zinc.]

17. POTASSII CYANIDUM.- [Potassium Cyanide. $\mathrm{KCN}=65.01$.

SourCE. - Heat in an iron crucible dried Potassium Ferrocyanide, 8; with Potassium Carbonate, 3 ; until effervescence ceases.

Characters. - White, opaque, amorphous pieces, or a white, granular powder, odorless when perfectly dry, but in moist air exhales the odor of $\mathrm{Hy}$ drocyanic Acid. This salt is very poisonous. Solubility. - In about 2 parts of water and sparingly in alcohol.

Dose, $\frac{1}{20}$ to $1 / 7 \mathrm{gr}$; ; .003 to $.015 \mathrm{gm}$.

\section{Preparation.}

Acidum Hydrocyanicum Dilutum.-Diluted Hydrocyanic Acid.-HCN $[=26.98$. Synonym.-Prussic Acid. A liquid compound of 2 per cent., by weight, of absolute Hydrocyanic Acid, and 98 per cent. of water.]

SouRCE. - Distil a mixture of l'otassium Ferrocyanide, 20; Sulphuric Acid, 8 ; and water, 65 , into distilled water. $\mathrm{K}_{4} \mathrm{FeC}_{6} \mathrm{~N}_{6}+2 \mathrm{H}_{2} \mathrm{SO}_{4}=2 \mathrm{~K}_{2} \mathrm{SO}_{4}$ $+\mathrm{H}_{4} \mathrm{FeC}_{6} \mathrm{~N}_{6}$. On the application of heat the Hydroferrocyanic Acid reacts with the remaining Potassium Ferrocyanide and Sulphuric Acid, and Hydrocyanic Acid distils over. $\mathrm{H}_{4} \mathrm{FeC}_{6} \mathrm{~N}_{6}+\mathrm{K}_{4} \mathrm{FeC}_{6} \mathrm{~N}_{6}+\mathrm{H}_{2} \mathrm{SO}_{4}=6 \mathrm{HCN}+$ $\mathrm{K}_{2} \mathrm{SO}_{4}+\mathrm{K}_{2} \mathrm{Fe}\left(\mathrm{FeC}_{6} \mathrm{~N}_{6}\right)$. The distillate is diluted with distilled water until the official strength is obtained. [Diluted Hydrocyanic Acid may also be prepared, extemporaneously, in the following manner: Mix Hydrochloric Acid, 5 , with distilled water, 55 ; add Silver Cyanide, 6 , and shake the whole together in a glass-stoppered bottle. When the precipitate has subsided, pour off the clear liquid.] Scheele's I'russic Acid is a 4 or 5 per cent. solution.

Characters. - A colorless [liquid, of a characteristic odor and taste, resembling that of bitter almond]. Very unstable; to preserve it best, it should be kept in inverted blue-stoppered bottles. Old specimens may be inert. 
INCOMPATIBLES._Salts of silver, copper and iron, red mercuric oxide and sulphides.

IMPURITIES.-Sulphuric and hydrochloric acids.

Dose, $\mathrm{I}$ to $3 \mathrm{~m}$.; [.06 to .20 c.c.

Hydrocyanic acid belongs chemically to the Carbon compounds, but on account of its physiological and therapeutical relationship to Potassium Cyanide it is considered at this place.]

\section{Action of Potassium Cyanide and Diluted Hydrocyanic} ACID.

External.-Hydrocyanic acid can pass through the epidermis, and then it paralyzes the terminations of the sensory nerves; thus it is a local anæsthetic and sedative. It is very rapidly absorbed from raw surfaces, and may cause poisoning if applied to them. [Potassium cyanide may possibly give the same results. It also produces a dermatitis on local application to the epidermis.]

Internal.-Alimentary tract.-Hydrocyanic acid is quickly [and potassium cyanide less rapidly] absorbed by mucous membranes, and has the same anæsthetic and sedative effect on the mouth and stomach as on the skin. It must always be employed [well diluted]. A single drop of the pure acid placed inside the eye of even a moderately large animal will kill it instantly.

Blood.-If death takes place almost immediately after the administration of the drug, all the blood in the body is a bright arterial tint; but if death does not occur for some little time (within half an hour), the blood is of a dark venous color. The primary transitory reddening of the venous blood is due to the fact that the hæmoglobin in it is oxidized; we do not know the cause of this. The subsequent darkening of the arterial blood is due to the fact that it has lost its oxygen, and contains carbon [dioxide] gas; why this should be is not certain, but probably it depends upon the asphyxia consequent upon the action of hydrocyanic acid on the respiratory centre. If blood be shaken up with [hydrocyanic] acid, after some time oxyhæmoglobin is converted into cyanohæmoglobin, the oxygen being turned out. [Hydrocyanic] acid added to drawn blood alters the shape of the red blood-corpuscles. Neither of these actions is seen in 
life, for sufficient [hydrocyanic] acid to cause them would kill before they could take place.

Heart.-Large doses cause instantaneous diastolic arrest. As this is also true if the drug is applied locally, we may conclude that large doses paralyze the heart directly. But [hydrocyanic] acid acts also on the cardiac centre in the medulla. A small dose will cause a slowing of the pulse from stimulation of the vagus centre, and the stoppage from larger doses is due both to the direct action on the heart and to that on the medulla.

Vaso-motor system. - The vaso-motor centre in the medulla is first briefly stimulated, but soon profoundly paralyzed ; bloodpressure therefore falls very low.

Respiration.-The respiratory centre is paralyzed even more readily than the cardiac or vaso-motor centres, consequently the respirations quickly diminish both in force and frequency. Unless the heart has been instantaneously stopped by a large dose, asphyxia is the cause of death, and the heart goes on beating after the respirations have stopped. Occasionally, if the dose be small, all three centres may be at first very transitorily stimulated, so that for a few seconds the pulse and respiration may be increased in frequency, and blood-pressure may rise.

Nervous system.-Cerebrum.-Medicinal doses of [hydrocyanic] acid have no effect on the cerebrum. Toxic doses cause deep insensibility and coma. In man convulsions are rarely seen; in animals they are common. It is probable that the coma and convulsions are due to the direct effect on the brain, but they may in part be due to the altered circulation through it, or the asphyxia.

Peripheral nerves and muscles. - In animals dead of [hydrocyanic] acid poisoning these are unexcitable. This paralyzing effect is due to direct action on the nerves and muscles themselves, for it does not occur in the peripheral part of a limb if it is connected with the rest of the body only by its nerve. In this case, as no blood is circulating through the distal part of the limb, no [hydrocyanic] acid reaches it; but if the acid be applied locally to the severed limb, the nerve and muscles are paralyzed. This explains the local anæsthetic effect of [hydrocyanic] acid. 
Shortly before death the spinal cord is paralyzed. The pupil is dilated. We do not know of any effect of [hydrocyanic] acid on the kidneys, nor how it is excreted. It slightly reduces the temperature.

\section{Therapeutics of Potassium Cyanide and Diluted Hydro- CYANIC ACID.}

External.-Lotions of a strength of about [ $\mathrm{r}$ to 48 ] of the diluted acid in water are valuable for allaying itching due to any cause. If the skin is abraded they must not be used.

Internal.-[Hydrocyanic acid may be administered as the official diluted acid, oil of bitter almond ( 3 to $\times 4$ per cent. of acid), bitter almond water, cherry laurel water (B. P.), the fluid extract, infusion and syrup of wild cherry, and as potassium cyanide. Reference should be made to each of these.] Small doses, 2 to 3 minims [. $x 2$ to .20 c.c.] of the diluted acid, are used for their sedative effect on the nerves of the stomach, to allay vomiting, and to relieve gastric pain, whatever be their cause, and often with good effect. A useful way of giving it is in an effervescent draught. [Since the effect of the remedy is transient, it should be given at frequent intervals.] It is a common ingredient of cough mixtures, for by its depressing effect on the central nervous system it diminishes reflex excitability, and is consequently most serviceable for a dry, hacking cough by means of which nothing is expectorated. [The uses of potassium cyanide are similar to those of hydrocyanic acid.]

\section{ToXICOLOGY.}

Symptoms. - With a large dose [of hydrocyanic acid] the symptoms usually begin in a few seconds; it is rare for them to be delayed more than two minutes. The patient is perfectly insensible ; the eyes are fixed and glistening, the pupils dilated, the limbs flaccid, the skin cold and clammy. The respiration is slow, deep and convulsive; the pulse almost imperceptible. [Hydrocyanic acid kills by respiratory failure.j Post-mortem. - There may be an odor of [hydrocyanic] acid about the body, which is very livid. The fingers are clenched, the jaws firmly closed and there is froth at the mouth; the eyes are fixed and glistening and the pupils dilated. The stomach may be a little reddened; the blood is very dark.

Treatment. - Wash out the stomach immediately; [the physician almost 
never bas the opportunity.] If emetics are available, large doses must be given promptly, for every moment is important. [Vomiting may be induced by inserting the finger into the throat.] Give ether or brandy and $\frac{1}{50} \mathrm{gr}$. [.013 gm.] of atropine subcutaneously. Use inhalations of Ammonia and artificial respiration. [Cold affusions, or alternately hot and cold, may be available. Antal, from an experience of forty instances of poisoning, believes that Cobalt nitrate is the best chemical antagonist. A thirty per cent. solution of hydrogen dioxide may be employed to wash out the stomach. Intravenous injections of sodium hyposulphite (producing theoretically the relatively harmless sulphocyanide) enable animals to survive an otherwise lethal dose.]

18. POTASSII BICHROMAS, see Chromium.

19. POTASSII HYPOPHOSPHIS, see Phosphorus.

\section{SODIUM.}

$$
\mathrm{Na}=23.0 \text {. }
$$

I. [SODA. $-\mathrm{NaOH}=39.86$. Synonyms. - Caustic Soda. Sodium Hydrate. Sodium Hydroxide.

SouRCE. - Dissolve Sodium Carbonate in boiling distilled water. Slake Lime and dissolve in distilled water, adding this in small portions at a time to the solution of Sodium Carbonate, boil, strain when cold, set aside until clear and remove the clear solution. Evaporate this solution to an vily consistence and pour into moulds. $\mathrm{Na}_{2} \mathrm{CO}_{3}+\mathrm{Ca}(\mathrm{OH})_{2}=2 \mathrm{NaOH}+\mathrm{CaCO}_{3}$.

Characters. - Dry, white, translucent pencils, or fused masses, showing a crystalline fracture, odorless, and having an acrid and caustic taste.

IMPURITIES.-Lime, sulphates, chlorides and carbonates.

\section{Preparation.}

Liquor Sodæ. - Solution of Soda. Synonym. - Solution of Sodium Hydrate.

SourCE.-An aqueous solution of Sodium Carbonate is boiled with slaked lime. The supernatant liquid is then siphoned off. $\mathrm{Na}_{2} \mathrm{CO}_{3}+$ $\mathrm{Ca}(\mathrm{OH})_{2}=2 \mathrm{NaOH}+\mathrm{CaCO}_{3}$. Or it may be prepared by dissolving Soda, 56 ; in distilled water, 944 . The Soda must be of the full strength, as directed by the U. S. P. (90 per cent.).

Characters. - A clear, colorless liquid, odorless, having a very acrid and caustic taste, and a strong alkaline reaction. Sp. gr., 1.059. Strength, about 5 per cent. of the Iydrate.

IMPURITIES.-As of Soda.

Incompatibles. - The same as of Potassa. (See p. 122.)

Dose, 5 to $20 \mathrm{~m}$.; .30 to 1.20 c.c., freely diluted.

\section{ACtion of Soda.}

It is in all respects, save one, similar in its action to potash. The difference is, that sodium salts are all much less depressant 
to the cardiac, muscular, and nervous systems, and therefore far less poisonous than potassium salts.

\section{Therapeutics OF Soda.}

It is very little used. Potash is almost always preferred.]

\section{TOXICOLOGY.}

Poisoning by caustic alkalies is very rare ; usually it takes place either by Potash, Soda, Pearlash (Potassium Carbonate), or soap lees (Sodium Carbonate). (Both the last are impure. They contain caustic Soda or Potash.)

Symptoms. - A caustic taste is experienced, and is quickly followed by symptoms of gastro-intestinal irritation, viz., burning heat in the throat, vomiting, diarrhœa, and abdominal pain, together with those of depression, viz., a feeble, quick pulse, and a cold clammy skin. Soon the lips, tongue, and throat become swollen, soft and red. Post-mortem appearances.-The mucous membrane of the mouth, tongue, stomach and œesophagus, and occasionally that of the larynx, is excoriated, dark, softened and inflamed.

Treatment.-Wash out the stomach or give emetics, as Zinc Sulphate, $20 \mathrm{gr}$ [ [1.20 gm.] ; or powdered Ipecacuanha, $30 \mathrm{gr}$. [2.00 gm.] ; or Copper Sulphate, $5 \mathrm{gr}$. [.30 gm.], in half a pint [240. c.c.] of tepid water; or Vinum Ipecacuanhæ, f. 3 j [3o. c.c.] ; or mustard, a tablespoonful [16. gm.] in half a pint [240. c.c.] of tepid water; or common salt, 2 tablespoonfuls [30. gm.] in half a pint [240. c.c.] of tepid water; or $\frac{1}{10} \mathrm{gr}$. [.006 gm.] of apomorphine [hydrochlorate] hypodermatically. If none of these are handy, give plenty of warm water and tickle the back of the throat. Then give feeble acids, as diluted Lemon juice, diluted solution of Citric Acid, Vinegar, [or] diluted Acetic Acid. Then demulcents, as oil, [flaxseed] tea, or water and white of egg.

2. SODII CARBONAS. - [Sodium Carbonate, $\mathrm{Na}_{2} \mathrm{CO}_{3}+10 \mathrm{H}_{2} \mathrm{O}=$ 285.45. Synonyms. -Sal] Soda. Washing Soda.

SoURCE.-Made thus :-First stage, Sodium Chloride and Sulphuric Acid are heated together. $2 \mathrm{NaCl}+\mathrm{H}_{2} \mathrm{SO}_{4}=\mathrm{Na}_{2} \mathrm{So}_{4}+2 \mathrm{HCl}$. Second stage, the Sodium Sulphate is heated with Carbon. $\mathrm{Na}_{2} \mathrm{SO}_{4}+{ }_{4} \mathrm{C}=\mathrm{Na}_{2} \mathrm{~S}+4 \mathrm{CO}$. Third stage, the Sodium Sulphide is heated with chalk. $\mathrm{Na}_{2} \mathrm{~S}+\mathrm{CaCO}_{3}=\mathrm{Na}_{2} \mathrm{CO}_{3}$ $+\mathrm{CaS}$.

[It is also made from Cryolite, a mineral found in Greenland. Cryolite and chalk are heated to redness, producing Calcium Fluoride and Sodium Albuminate; the latter is soluble in water, and is decomposed by Carbon Dioxide, which precipitates Aluminum Hydroxide, retaining a little Sodium Carbonate, while the pure Sodium Carbonate remains in solution.

Characters. - Colorless, monoclinic crystals, odorless, and having a strong alkaline taste. In dry air the salt effloresces, and if left exposed soon 
loses about one-half of its water of crystallization and becomes a white powder. Solubility. - In 1.6 parts of water; insoluble in Alcohol and in Ether.]

IMPURITIES. - Sulphates, chlorides [and metals.

Sodium Carbonate is used to prepare Liquor Sodx Chloratx, Massa Ferri Carbonatis, and Suppositoria Glycerini.

Dose, 5 to $15 \mathrm{gr}$; .30 to $1.00 \mathrm{gm}$.

\section{Preparation.}

Sodii Carbonas Exsiccatus.-Dried Sodium Carbonate.

SOURCE. - 200 parts of Sodium Carbonate are broken into small fragments, allowed to effloresce, then gently heated until it becomes a white powder, weighing 100 parts.

Characters. - A loose, white powder, conforming to the tests and reactions given under Sodii Carbonas.]

Dose, 5 to $15 \mathrm{gr}$; ; [.30 to $1.00 \mathrm{gm}$.]

Action and Therapeutics of Sodium Carbonate and the Dried Carbonate.

The same as those of [soda,] except that the carbonate is less caustic. [A one per cent. solution of sodium carbonate is used for boiling surgical instruments in the process of sterilization in order to prevent their rusting.]

3. SODII BICAR BONAS. - [Sodium Bicarbonate. $\mathrm{NaHCO}_{3}=83$. 85. Synonyms.-Baking Soda. Sodium Sesquicarbonate. Soda.

SourCE. - Made from the Carbonate in the same way as the Potassium Bicarbonate is made. $\mathrm{Na}_{2} \mathrm{CO}_{3}+\mathrm{CO}_{2}+\mathrm{H}_{2} \mathrm{O}=2 \mathrm{NaHCO}_{3}$. Or by treating Sodium Chloride at the same time with Ammonia gas and Carbon Dioxide. $\mathrm{NaCl}+\mathrm{NH}_{3}+\mathrm{CO}_{2}+\mathrm{H}_{2} \mathrm{O}=\mathrm{NaHCO}_{3}+\mathrm{NH}_{4} \mathrm{Cl}$.

Characters. - A white opaque powder, odorless, and having a cooling mildly alkaline taste.

Solubility. - In 11.3 parts of water; insoluble in Alcohol and Ether.

IMPURITIES. - The carbonate.

INCOMPATIBLES. - It is decomposed by acids and acid salts, e.g., Bismuth Subnitrate.

Sodium Bicarbonate is used to prepare Mistura Rhei et Sodæ, Ferri Carbonas Saccharatus, and Pulvis Effervescens Compositus.

Dose, 5 to $60 \mathrm{gr}$; ; 30 to $4.00 \mathrm{gm}$.

\section{Preparation.}

Trochisci Sodii Bicarbonatis.-Troches of Sodium Bicarbonate. Sodium Bicarbonate, 20; Sugar, 60; Nutmeg, 1 gm.; Mucilage of Tragacanth, a sufficient quantity to make 100 troches. Triturate the 
Nutmeg with the Sugar, gradually added, until they are reduced to a fine powder, and mix this intimately with the Sodium Bicarbonate; then with the Mucilage of Tragacanth, form a mass. Each troche contains 3 gr. ; .20 gm., of Sodium Bicarbonate.]

Dose, I to 6 troches.

\section{Action of Sodium Bicarbonate.}

The same as that of potassium bicarbonate, except that it is much more slowly absorbed from the gastro-intestinal tract, and like all sodium salts it is only feebly depressant. Sodium salts are much less depressant to the cardiac, muscular, and nervous systems, and therefore are far less poisonous than potassium salts.

\section{Therapeutics of Sodium Bicarbonate.}

External.-A lotion of $7 \mathrm{gr}$. [.5० gm.] to $\mathrm{I}$ fl. 0z. [30. c.c.] of water is employed as a sedative to relieve itching. [Either in saturated solution or as a fine powder sodium bicarbonate locally applied is the best remedy to relieve the pain from burns. Of late it has been strongly recommended to be used for packing to prevent pain after operations upon the vagina.]

Internal.-Stomach.-Its use in disease is very similar to that of the corresponding potassium salt, but on account of the two differences just mentioned, it is much more frequently given. Hence it is a very common ingredient of medicines designed to relieve dyspepsia, being taken at or a little before meals to increase the flow of gastric juice, or some time afterwards to neutralize excessive acidity in the cases in which the patient complains of heartburn and acid eructations. Its value is also partly due to its sedative action on the gastric nerves, whereby it relieves gastric pain, and partly also to its power of liquefying tenacious mucus. A very favorite gastric sedative mixture consists of about Io gr. [.60 gm.] of sodium bicarbonate, together with the same quantity of bismuth [subcarbonate,] suspended in mucilage. A grain or two [.06 to .12 gm.] of sodium bicarbonate with a grain [.o6 gm.] of powdered rhubarb, and some sugar, forms a common stomachic powder for children. Sodium bicarbonate and gentian are also often combined together in 
stomachic mixtures. Effervescing soda water (made in the same way as potash water, see p. r22) is a mild gastric sedative. In commerce these waters contain neither potash nor soda, but even then the carbon [dioxide] gas acts as a sedative.

Sodium bicarbonate is so slowly absorbed, and is, in comparison with potassium salts, so poor a solvent of uric acid, that it is rarely used for any effects it may have after absorption. It is stated that large doses ( 150 to 500 grains [10. to $32 . \mathrm{gm}$.$] ) are$ useful for diabetic coma.

4. SODII PHOSPHAS. - [Sodium Phosphate. $\mathrm{Na}_{2} \mathrm{HPO}_{1}+12 \mathrm{H}_{2} \mathrm{O}=$ 357.32. Synonym.-Sodium Orthophosphate.

Source.-Digest Bone Ash with Sulphuric Acid; Acid Calcium Phosphate is formed. $\mathrm{Ca}_{3}\left(\mathrm{PO}_{4}\right)_{2}+2 \mathrm{H}_{2} \mathrm{SO}_{4}=\mathrm{CaH}_{4}\left(\mathrm{PO}_{4}\right)_{2}+2 \mathrm{CaSO}_{4}$. Filter and add Sodium Carbonate to the solution. $\mathrm{CaH}_{4}\left(\mathrm{PO}_{4}\right)_{2}+\mathrm{Na}_{2} \mathrm{CO}_{3}=\mathrm{Na}_{2} \mathrm{HPO}_{4}+$ $\mathrm{H}_{2} \mathrm{O}+\mathrm{CO}_{2}+\mathrm{CaHPO}_{4}$. The filtrate requires to be evaporated and the salt is obtained by crystallization.

Characters. - Large, colorless, monoclinic prisms, odorless, and baving a cooling saline taste. The crystals effloresce on exposure to the air, and gradually lose 5 molecules of their water of crystallization. Solubility.-In 5 parts of water.]

IMPUŔTIEs. - Lime phosphate [sulphates and carbonates.

Sodium Phosphate is used to prepare Ferri Phosphas Solubilis.

Dose, 5 gr. to I oz.; .30 to $30 . \mathrm{gm}$.]

5. SODII SULPHAS. - Sodium Sulphate. $\mathrm{Na}_{2} \mathrm{SO}_{4}+\left[\mathrm{1OH}_{2} \mathrm{O}=\right.$ 321.42.] Synonym.-Glauber's Salt.

SOURCE. - Neutralize with Sodium Carbonate, the residue left in the manufacture of Hydrochloric Acid from Salt. $2 \mathrm{NaHSO}_{4}+\mathrm{Na}_{2} \mathrm{CO}_{3}=2 \mathrm{Na}_{2} \mathrm{SO}_{4}+$ $\mathrm{CO}_{2}+\mathrm{H}_{2} \mathrm{O}$.

ChARACTERs. - [Large, colorless, transparent monoclinic prisms, odorless, and having a bitter, saline taste, efflorescing on exposure to air, and losing all of their water of crystallization.] Solubility. - In 3 parts of water.

ImpURITIES. - Ammonium and Iron salts.

Dose, 1 to $8 \mathrm{dr}$. [4. to $30 . \mathrm{gm}$.

6. POTASSII ET SODII TARTRAS.-Potassium and Sodium Tartrate. $\left[\mathrm{KNaC}_{4} \mathrm{H}_{4} \mathrm{O}_{6}+4 \mathrm{H}_{2} \mathrm{O}=258.5 \mathrm{I}\right.$. Synonyms. - Rochelle Salt. Tartrated Soda.

Source. - Add Acid Potassium Tartrate to a hot solution of Sodium Carbonate. $2 \mathrm{KHC}_{4} \mathrm{H}_{4} \mathrm{O}_{6}+\mathrm{Na}_{2} \mathrm{CO}_{3}=2 \mathrm{KNaC}_{4} \mathrm{H}_{4} \mathrm{O}_{6}+\mathrm{H}_{2} \mathrm{O}+\mathrm{CO}_{2}$.

Characters.-Colorless, transparent, rhombic prisms, or a white powder, odorless, and having a cooling saline taste. The crystals are slightly efflorescent. Solubility. - In 2 parts of cold water. 
ImPURity. -Acid Potassium Tartrate.

Dose, $1 / 4$ to I oz.; 8. to 30 . gm. (purgative) 30 to $60 \mathrm{gr}$; 2 2 to $4 . \mathrm{gm}$. (diuretic).

\section{Preparation.}

Pulvis Effervescens Compositus. - Compound Effervescing Powder. Synonym.-Seidlitz Powder. Take Potassium and Sodium Tartrate, 93 gm., and Sodium Bicarbonate, $3^{1} \mathrm{gm}$. ; mix, divide into twelve equal parts, and wrap each part in a separate paper of some pronounced color, as blue. Tartaric Acid, $27 \mathrm{gm}$., divided into twelve equal parts, and wrap each part in a separate paper of a color distinctly different from that used for wrapping the mixture, as white. Each powder in blue paper contains about $120 \mathrm{gr}$. $7.75 \mathrm{gm}$., of Potassium and Sodium Tartrate with $40 \mathrm{gr}$., $2.58 \mathrm{gm}$., of Sodium Bicarbonate. The white paper contains $35 \mathrm{gr}$. $2.25 \mathrm{gm}$., of Tartaric Acid.

Dose.-Dissolve the powder in the blue paper in nearly half a pint [24o. c.c.] of cold or warm water, and then add that in the white paper, and drink while effervescing.

\section{Action of Sodium Sulphate and Phosphate, and of Potassium and Sodium Tartrate.}

Internal.-Intestines.-Owing to the slowness with which, compared to the corresponding potassium salts, these sodium salts are absorbed, they pass on into the intestines and there act more efficiently than potassium salts. They are typical saline purgatives, abstracting fluid from the blood until they form a 5 per cent. solution, and then exerting a painless laxative effect, produce a soft motion about two or three hours after administration (see p. 93). The sulphate, which is the most active purgative, and the phosphate are mild cholagogues, and Carlsbad waters (see p. I44) have been shown to increase, in the human subject, the amount of bile and the solids in it.

Blood and Kidneys.-Owing to their tardy absorption the action of these salines, as alkalizers of the blood and urine and as diuretics, is more feeble than that of the corresponding potassium salts.

Therapeutics of Sodium Sulphate and Phosphate, and of Potassium and Sodium Tartrate.

Internal. - Intestines. - These salts of sodium are some of the best purgatives we possess, being especially useful for habitual 
constipation, and for constipation associated with gout, with hepatic dyspepsia, or with any of the manifestations of an excess of uric acid in the blood or the urine. The best way to take them is to dissolve the required amount in half a tumbler of [hot] water, and to drink it in successive small draughts while dressing in the morning. The bowels are then usually comfortably opened soon after breakfast. These salts, especially the phosphate and sulphate, are also cholagogues; these two are therefore to be preferred in cases of disease of the liver. The sulphate is the most powerful purgative of all. It is the chief constituent of Carlsbad, Marienbad, Tarasp, [Villacabras and Rubinat] Condal waters, and it occurs associated with much magnesium sulphate in Æsculap, Hunyadi János, Seidlitz, Pullna, Friedrichshall, Apenta, and Kissingen waters. A powder consisting of $30 \mathrm{gr}$. [2. gm.] of each of sodium sulphate and magnesium sulphate and a grain [.06 gm.] of sodium chloride and sodium bicarbonate (dose I to $4 \mathrm{dr}$.); [4. - I $5 . \mathrm{gm}$.] forms when,dissolved a good imitation of Asculap, Hunyadi János, and Franz Joseph waters. The phosphate is a milder and less unpleasant purgative than the others; it is often given to children. The effervescing preparation [Seidlitz Powder (see p. I43)] is a palatable form. If large doses are used, the evacuations are very watery, and therefore these drugs are useful to remove fluid in cases of dropsy or ascites (especially if due to disease of the liver). Sufferers from gall-stones are undoubtedly benefited by a course of water containing sodium sulphate, and therefore frequently go to Carlsbad.

7. SODII CHLORIDUM.-Sodium Chloride. $\mathrm{NaCl}[=58.37$. Synonym. - Common Salt.

SOURCE.-Occurs native.

Characters. - [Colorless, transparent, cubical crystals, or a white, crystalline powder, odorless, and having a purely saline taste. Solubility. - In 2.8 parts of water; almost insoluble in Alcohol.

IMPURITY.—Potassium Chloride.]

Dose, 5 to $60 \mathrm{gr}$.; [.30 to $4.00 \mathrm{gm}$.]

\section{Action of Sodium Chloride.}

Common salt forms an article of diet with all creatures living on vegetable food, especially if it contains large amounts of po- 
tassium, but is not used either by carnivorous animals or by tribes living solely on flesh. The importance of it is seen in the long distances herbivorous animals will wander to salt licks, and by the fact that tribes living on vegetables will go to war for the possession of it. Bunge's explanation of this desire for salt is as follows : Blood plasma contains much sodium chloride, vegetable foods contain a large amount of potassium salts; when, therefore, these salts of potassium reach the blood, potassium chloride and the sodium salt of the acid which was combined with the potassium are formed. This and the potassium chloride are excreted by the kidneys, and the blood loses its sodium chloride, which loss is therefore made up by taking sodium chloride with the food. The deprivation of salt leads to general weakness, œdema and anæmia, a series of symptoms often seen in France before the repeal of the salt tax. Quantities of a tablespoonful [15. gm.] and upwards act as an emetic, and may also purge. Rectal injections of solutions of salt [by removing mucus may make the rectum unfit for the habitation of ] the Oxyuris vermicularis.

\section{Therapeutics of Sodium Chloride.}

It is occasionally used as an emetic, also as an anthelmintic. Bathing in sea water acts as a mild general stimulant, and very concentrated hot salt baths, such as those of Droitwich and Nantwich, are useful for chronic rheumatism and sciatica. Sixty grains [4. gm.] of commoll salt in a pint [500 c.c.] of boiled water allowed to $\mathrm{cool}$ to $100^{\circ} \mathrm{F}$. $\left[37.7^{\circ} \mathrm{C}\right.$. $]$ form a normal saline solution, which is frequently injected into any convenient vein-or sometimes into loose connective tissue-in cases of collapse from hæmorrhage, often with strikingly good results. Such injections have also been used for diabetic coma, and may render the patient sensible again for a little while, but they do not avert the end.

8. SODII SULPHIS. - [Sodium Sulphite. $\mathrm{Na}_{2} \mathrm{SO}_{3}+7 \mathrm{H}_{2} \mathrm{O}=25 \mathrm{I} .58$. SOURCE. - Saturate a solution of Sodium Carbonate or Caustic Soda with Sulphur Dioxide gas. $\mathrm{Na}_{2} \mathrm{CO}_{3}+\mathrm{SO}_{2}=\mathrm{Na}_{2} \mathrm{SO}_{3}+\mathrm{CO}_{2}$.

Characters.-Colorless, transparent, monoclinic prisms; odorless, and 
having a cooling, saline, sulphurous taste ; efflorescent in air. Solubility.In 4 parts of water.

I MPURITIES. - Sulphates and chlorides.]

Dose, 5 to $60 \mathrm{gr}$; ; [.30 to $4.00 \mathrm{gm}$.

9. SODII BISULPHIS.-Sodium Bisulphite. $\mathrm{NaHSO}_{3}=103.86$.

SoURCE. - From Sodium Carbonate or Bicarbonate and Sulphur Dioxide gas. $\mathrm{NaHCO}_{3}+\mathrm{SO}_{2}=\mathrm{NaHSO}_{3}+\mathrm{CO}_{2}$. Solubility.-In 4 parts of water; and in $\mathbf{7 2}$ parts of Alcohol.

CharACTERS. - Opaque, prismatic crystals, or a granular powder, exhaling an odor of Sulphur Dioxide, and having a disagreeable, sulphurous taste.

Dose, 5 to $30 \mathrm{gr}$. ; .30 to $2.00 \mathrm{gm}$.

10. SODII HYPOSULPHIS. - Sodium Hyposulphite. $\mathrm{Na}_{2} \mathrm{~S}_{2} \mathrm{O}_{3}+$ ${ }_{5} \mathrm{H}_{2} \mathrm{O}=247.64$. Synonym.-Sodium Thiosulphate.

SoURCE. - From Sodium Bisulphate in solution with Metallic Zinc. $3 \mathrm{Na}$ $\mathrm{HSO}_{3}+\mathrm{Zn}=\mathrm{NaHSO}_{3}+\mathrm{Na}_{2} \mathrm{~S}_{2} \mathrm{O}_{3}+\mathrm{ZnO}_{2}+\mathrm{H}_{2} \mathrm{O}$. Or pass Sulphurous Anhydride into a solution of Soda (or Sodium Carbonate) with Sulphur. $\mathrm{SO}_{2}+$ $2 \mathrm{Na}(\mathrm{OH})+\mathrm{S}=\mathrm{Na}_{2} \mathrm{~S}_{2} \mathrm{O}_{3}+\mathrm{H}_{2} \mathrm{O}$.

Characters. - Colorless, transparent, monoclinic prisms, odorless and having a cooling, afterwards bitter taste. Solubility.-In 0.65 part of water; insoluble in Alcohol.

Dose, 5 to $20 \mathrm{gr}$; .30 to $1.20 \mathrm{gm}$.]

Action and Therapeutics of Sodium Sulphite, Bisulphite AND HYPOSULPHITE.

Sodium sulphite solutions ( 1 in 8 ) may be used externally as mild antiparasitics. This body is, in the stomach, decomposed by the acids there and gives off sulphurous anhydride. It may, therefore, be given to arrest fermentation. If any remains undecomposed, it is absorbed as a sulphite. No other action is known. They are very rarely given in medicine, but in sufficient doses might produce the effects of sodium sulphate.

11. SODII BROMIDUM, see Bromine.

12. SODII IODIDUM, see Iodine.

13. SODII HYPOPHOSPHIS, see Phosphorus.

14. SODII ARSENAS, see Arsenic.

15. SODII SULPHOCARBOLAS, see Acidum Carbolicum.

16. [SODII NITRAS.-Sodium Nitrate. $\mathrm{NaNO}_{3}=84.89$.

Source. - Imported from Chili and Peru.

Characters.-Colorless, transparent, rhombohedral crystals, odorless, baving a cooling, saline and slightly bitter taste. Deliquescent in moist air. 
Solubility.-In 1.3 parts of water; soluble in 100 parts of Alcohol.

Sodium Nitrate is used for preparing Sodium Arsenate.

Dose, $1 / 2$ to $1 \mathrm{Oz}$.; 15 . to $30 . \mathrm{gm}$.

\section{Action and Therapeutics of Sodium Nitrate.}

Sodium nitrate, in moderate doses, does not directly lessen the force or frequency of the pulse, nor lower the animal temperature, nor decrease the elimination of urea; in large doses, acting as a purgative, it may produce these effects. It is somewhat diuretic, but its chief medicinal virtue is that of a mild purgative.

17. SODII A CETAS.-Sodium Acetate. $\mathrm{NaC}_{2} \mathrm{H}_{3} \mathrm{O}_{2}+3 \mathrm{H}_{2} \mathrm{O}=135.74$.

SourCE. - From Sodium Carbonate and Acetic Acid. $\mathrm{Na}_{2} \mathrm{CO}_{3}+2 \mathrm{HC}_{2}$ $\mathrm{H}_{3} \mathrm{O}_{2}=2 \mathrm{NaC}_{2} \mathrm{H}_{3} \mathrm{O}_{2}+\mathrm{H}_{2} \mathrm{O}+\mathrm{CO}_{2}$. By evaporation to crystallization.

CHARACTERS. - Colorless, transparent, monoclinic prisms, or a granular, crystalline powder, odorless, and having a cooling saline taste. Efflorescent in warm, dry air. Solubility. - In 1.4 parts of water; and in 30 parts of Alcohol.

IMPURITIES.-Bilica, metals and calcium.

Sodium Acetate is used for preparing Acetic Ether and Acetic Acid.

Dose, 10 to $60 \mathrm{gr}$; .60 to $4.00 \mathrm{gm}$.

\section{Action and Uses of Sodium Acetate.}

Sodium acetate is diuretic, but it is rarely used as a medicine.]

18. SODII BENZOAS, see Acidum Benzoicum.

19. SODII NITRIS, see Nitrites.

20. SODII VALERIANAS [not official], see Valeriana.

21. SODII SALICYLAS, see Acidum Salicylicum.

22. SODII BORAS, see Acidum Boricum.

23. [SODII CHLORAS.-Sodium Chlorate. $\mathrm{NaClO}_{3}=106.25$.

SourCE. - From Acid Sodium Tartrate and Potassium Chlorate in solution; by filtration, evaporation and crystallization. $\mathrm{NaHC}_{4} \mathrm{H}_{4} \mathrm{O}_{6}+\mathrm{KClO}_{3}$ $=\mathrm{NaClO}_{3}+\mathrm{KHC}_{4} \mathrm{H}_{4} \mathrm{O}_{6}$.

ChARACTERs.-Colorless, transparent crystals (principally regular cubes with tetrahedral facets), or a crystalline powder; odorless, having a cooling, saline taste. Solubility. - In I.I parts of water; and in roo parts of Alcohol.

Dose, 5 to $15 \mathrm{gr}$; .30 to $1.00 \mathrm{gm}$.

Action and Uses of Sodium Chlorate.

Sodium chlorate has medicinal properties similar to those of the potassium chlorate, whilst its greater solubility permits the 
use of stronger solutions. It has recently been recommended in large doses for malignant diseases of the stomach.

24. SODII PYROPHOSPHAS. - Sodium Pyrophosphate. $\mathrm{Na}_{4} \mathrm{P}_{2}$ $\mathrm{O}_{7}+\mathrm{IOH}_{2} \mathrm{O}=445.24$.

Source. From heating Sodium Phosphate. $2 \mathrm{Na}_{2} \mathrm{HPO}_{4}+12 \mathrm{H}_{2} \mathrm{O}=\mathrm{Na}_{4}$ $\mathrm{P}_{2} \mathrm{O}_{7}+1_{3} \mathrm{H}_{2} \mathrm{O}$.

CHARACTERS. - Colorless, transparent, monoclinic prisms, or a crystalline powder, odorless, having a cooling, saline and feebly alkaline taste. Solubility. - In 12 parts of water; insoluble in Alcohol.

Sodium Pyrophosphate is used to prepare Ferri Pyrophosphas Solubilis.

Dose, $1 / 2$ to 4 dr.; 2. to $15 . \mathrm{gm}$.

Action and Therapeutics of Sodium Pyrophosphate.

Sodium pyrophosphate has the same therapeutical action as sodium phosphate, but its principal use is in pharmacy.]

25. SODII ETHYLAS. - [Sodium Ethylate. $-\mathrm{NaC}_{2} \mathrm{H}_{3} \mathrm{O}=67.90$. (Not official.)

Source.-By solution of Metallic Sodium in Ethylic Alcohol and crystallization. $2 \mathrm{Na}+2 \mathrm{C}_{2} \mathrm{H}_{5} \mathrm{OH}=2 \mathrm{NaC}_{2} \mathrm{H}_{5} \mathrm{O}+\mathrm{H}_{3}$.

Characters. - A deliquescent, caustic salt in white or whitish crystals.]

Preparation. [B. P. not official.]

Liquor Sodii Ethylatis, [Solution of Sodium Ethylate.]-Sodium, I ; Ethylic Alcohol, 20. Characters. - A clear, syrupy liquid, changing to brown in keeping. [This preparation has a sp. gr. of 0.567.] It should be freshly made when wanted.

\section{Action and Uses of Sodium Ethylate.}

This is used locally as a mild caustic to remove nævi and other growths. It is applied with a pointed glass rod for two or three days; then a scab forms; when this has fallen off, the treatment may be repeated. Sodium ethylate is, perhaps, our best caustic.

\section{AMMONIUM.}

$\mathrm{NH}_{3}=17.01$.

I. AQUA AMMONIÆ FORTIOR. - [Stronger Ammonia Water. 28 per cent. by weight of the gas $\left(\mathrm{NH}_{3}=17.01\right)$ dissolved in water.

SoURCE. - Generate Ammonia gas by heating Ammonium Chloride with Slaked Lime, and pass it into water. $2 \mathrm{NH}_{4} \mathrm{Cl}+\mathrm{Ca}(\mathrm{OH})_{2}=\mathrm{NH}_{3}+2 \mathrm{H}_{2} \mathrm{O}$ $+\mathrm{CaCl}_{2}$. 
Characters. - A colorless, transparent liquid, having an excessively pungent odor, and very acrid and alkaline taste and a strongly alkaline reaction. Sp. gr. 0.901.]

IMPURITIES. - Ammonium chloride, sulphide and sulphate.

Dose, 3 to $6 \mathrm{~m}$. ; [.20 to .40 c.c.], well diluted.

\section{Preparation.}

[Spiritus Ammoniæ.-Spirit of Ammonia. Contains so per cent., by weight, of the gas dissolved in Alcohol.

Dose, 10 to $60 \mathrm{~m}$.; .60 to 4.00 c.c.]

2. AQUA AMMONIÆ.-[Ammonia Water. (ro per cent. by weight of the gas $\left(\mathrm{NH}_{3}=17.0 \mathrm{r}\right)$ dissolved in water).

Source.-The same as for Aqua Ammoniæ Fortior.

Characters. - Like, but less pungent than, the stronger solution. Sp. gr. 0.960 .

Dose, 10 to $20 \mathrm{~m}$.; .60 to $\mathrm{r.20}$ c.c., well diluted.

\section{Preparations.}

I. Linimentum Ammoniæ.-Ammonia Liniment. Synonym. -Volatile Liniment. Ammonia Water, 350; Alcohol, 50; Cotton Seed Oil, 600.]

2. Spiritus Ammoniæ Aromaticus, see Ammonium Carbonate.

Action of Solutions of Ammonia.

External.-A solution of ammonia produces rubefaction with a sensation of heat, and, if strong, a sensation of pain and burning. If the vapor is confined, it causes vesication.

Internal.-Nose.-When inhaled, the vapor of ammonia is irritating to the nose and air passages, causing a pungent sensation and sneezing. The eyes and nose water. The pulse and respiration are refiexly accelerated. If very concentrated, it produces swelling and inflammation of the nose, glottis and respiratory tract.

Stomach.-Like other alkalies, given before meals, ammonia increases the flow of gastric juice; given after meals, it neutralizes it. It dilates the gastric vessels, and produces a feeling of warmth in the epigastrium. It reflexly stimulates the heart and respiration.

Blood.-Its action on the blood is not known; but it is supposed to diminish its local liability to clot in cases of thrombosis, and to dissolve [a clot which has] already formed. 
Heart. -Ammonia causes a rise of blood-pressure with an increased pulse rate, due probably to stimulation of the accelerator mechanism.

Respiration.-It increases greatly the frequency of respiration, probably from stimulation of the respiratory centre in the medulla.

Nerious system. - The brain is unaffected, and the nerves also, except for the tingling produced when a strong solution of ammonia is locally applied. Convulsions are often produced in animals poisoned by ammonia ; these are certainly central, and are probably due to stimulation of the spinal cord.

Kidneys.-Ammonia and its salts are oxidized in the body, and the nitric acid, uric acid, and urea in the urine are increased.

\section{Therapeutics of Solutions of Ammonia.}

External.-The liniment is used as a counter-irritant in numerous conditions, such as chronic joint disease, chronic rheumatism, etc., and is often rubbed on the chest in bronchitis. Ammonia is a very uncertain vesicant. Weak solutions of it are often applied to the bites of insects. [Aqua] ammoniæ is very valuable when held to the nose of any one who has fainted, for it almost instantly reflexly produces its stimulating effect on the heart and respiration.

Internal.-Ammonia in some form may be given before meals as a gastric stimulant in dyspepsia. Sal volatile (see below) is often used for this purpose, and also for its general stimulating effect on the cardiac, respiratory and spinal systems, especially in sudden collapse from any cause. [For collapse it may be used intravenously.] Ammonia has been injected subcutaneously in cases of snake-bite, [but it almost invariably produces a slough.]

3. AMMONII CARBONAS. - [Ammonium Carbonate, $\mathrm{NH}_{4} \mathrm{HCO}_{3}-$ $\mathrm{NH}_{4} \mathrm{NH}_{2} \mathrm{CO}_{2}=156.77$. Synonyms.-Bakers' Ammonia. Hartshorn. Sal Volatile.

Source. - A mixture of Ammonium Sulphate or Chloride and Calcium Carbonate is subjected to sublimation and resublimation. ${ }_{4} \mathrm{NH}_{4} \mathrm{Cl}+2 \mathrm{CaCO}_{3}$ $=2 \mathrm{CaCl}_{2}+\mathrm{NH}_{4} \mathrm{HCO}_{3}-\mathrm{NH}_{4} \mathrm{NH}_{2} \mathrm{CO}_{2}+\mathrm{NH}_{3}+2 \mathrm{H}_{2} \mathrm{O}$.

CliARACTERS. - White, hard, translucent, striated masses, baving a strongly 
ammoniacal odor without empyreuma, and a sharp saline taste. On exposure to the air it loses both Ammonia and Carbon Dioxide, becoming opaque, and is finally converted into friable, porous lumps, or a white powder. Solubility. -Slowly but completely in 5 parts of water.]

IMPURITIES. - Sulphates and chlorides.

Dose, 2 to 15 gr.; [.I2 to I.00 gm.]-(Stimulant or expectorant.)

\section{Preparation.}

Spiritus Ammoniæ Aromaticus.-[Aromatic Spirit of Ammonia. Ammonium Carbonate, 34 ; Ammonia Water, 90 ; Oil of Nutmeg, I ; Oil of Lemon, Io; Alcohol, 700 ; Oil of Lavender Flowers, I ; water to make I000. Sp. gr. about 0.905.]

Aromatic Spirit of Ammonia is used in making Tinctura Guaiaci Ammoniata and Tinctura Valerianæ Ammoniata.

Dose, $1 / 2$ to 2 fl. dr. [2. to 8 . c.c.]

\section{Action and Therapeutics of Ammonium Carbonate.}

The external and internal actions of the carbonate are the same as those of [Aqua] Ammoniæ. It is not used externally, but Spiritus Ammoniæ Aromaticus is inhaled for its reflex effects, is taken as a gastric stimulant and carminative in dyspepsia, and as a cardiac and general stimulant in syncope, etc. The carbonate is, in addition, an excellent expectorant, stimulating the respiratory movements, and by its general stimulating effect aiding the expulsion of thick mucus. It is most used for bronchitis in children and the aged. It is an emetic acting directly on the stomach.

\section{TOXICOLOGY.}

Symptoms. - Liquor Ammoniæ and the Carbonate produce symptoms like other alkalies, but are more corrosive. The air-passages are often inflamed, and the inhalation of the vapor has been known to kill from this cause.

Treatment as for other alkalies. (See p. 139.)

4. AMNONII CHLORIDUM.-[Ammonium Chloride. $\mathrm{NH}_{4} \mathrm{Cl}=$ 53.38.] Synonyms. - Sal Ammoniac, [Ammonium Muriate.

Source.-Neutralize Gas Liquor with Sulphuric Acid, converting all to Ammonium Sulphate. $2 \mathrm{NH}_{4} \mathrm{HO}+\mathrm{H}_{2} \mathrm{SO}_{4}=\left(\mathrm{NH}_{4}\right)_{2} \mathrm{SO}_{4}+2 \mathrm{H}_{2} \mathrm{O}$. After crystallization, sublime with Sodium Chloride. $\left(\mathrm{NH}_{4}\right)_{2} \mathrm{SO}_{4}+2 \mathrm{NaCl}=$ $2 \mathrm{NH}_{4} \mathrm{Cl}+\mathrm{Na}_{2} \mathrm{SO}_{4}$.

ChARACTERS. -A white, crystalline powder, without odor, having a cool- 
ing, saline taste, and permanent in the air, but volatile when heated. Solubility.-In 3 parts of water; almost insoluble in Alcohol.

IMPURITIES.-Chiefly tarry matters.

Dose, I to $30 \mathrm{gr}$; .06 to $2.00 \mathrm{gm}$.

\section{Preparation.}

Trochisci Ammonii Chloridi.--Troches of Ammonium Chloride. Ammonium Chloride, 10 ; Extract of Glycyrrhiza, 25 ; Tragacanth, 12 ; Sugar, 50 gm.; Syrup of Tolu, a sufficient quantity to make 100 troches. Each troche contains two grains; .12 gm.

Dose, I to 6 troches.]

\section{Action of Ammonium Chloride.}

Locally applied, ammonium chloride increases the secretion of mucous membranes, and to a slight extent it does the same after absorption. 'It is a feeble cholagogue, diaphoretic, diuretic, [and a general stimulant, but of less power than the carbonate.]

\section{Therapeutics of Ammonium Chloride.}

It is a very favorite remedy for local application, by means of inhalation of the vapor, to increase the secretion of mucus from the pharynx, Eustachian tubes, larynx, trachea and bronchi in cases of chronic pharyngitis, otitis media, laryngitis, and bronchitis. Many forms of apparatus for its inhalation are in the market. In most of them it is generated by the action of hydrochloric acid on ammonia. It is occasionally given by the mouth, either as a cholagogue, gastric stimulant, diaphoretic, or diuretic, but it is too feeble to be recommended, and it is very [unpleasant]; the taste may to some extent be concealed by liquorice. It is useful in chronic bronchitis with much expectoration and is then best given as a [troche or compressed tablet with chocolate]. Some authorities consider it to be, [in large doses, ] a specific for neuralgia.

5. LIQUOR AMMONII ACETATIS.-[Solution of Ammonium Acetate. Synonym.-Spirit of Mindererus. An aqueous solution of Ammonium Acetate $\left(\mathrm{NH}_{4} \mathrm{C}_{2} \mathrm{H}_{3} \mathrm{O}_{2}=76.87\right)$, containing about 7 per cent. of the salt, together with small amounts of Acetic and Carbonic Acids.

Source.-Ammonium Carbonate is gradually added to diluted Acetic Acid until it is neutralized.] 
Incompatibles.—Potash, soda, and their carbonates, acids, lime-water, lead and silver salts.

[Solution of Ammonium Acetate is used in preparing Liquor Ferri et Am. monii Acetatis.]

Dose, 2 to 8 fl. dr.; [8. to 30. c.c.]

\section{Action and Therapeutics of Ammonium Acetate.}

It is a mild diaphoretic and diuretic, and is used only for these effects. It probably acts in both cases, either on the secre tory cells or the nerves connected with them. It does not irritate the kidneys, but increases both the water and the solids excreted. It is employed in Bright's disease as a diuretic, and in febrile conditions as a diaphoretic.

6. AMMONII BENZOAS, see Acidum Benzoicum.

7. AMMONII BROMIDUM, see Bromine.

[8. AMMONII IODIDUM, see Iodine.

9. AMMONII NITRAS.-Ammonium Nitrate. $\mathrm{NH}_{4} \mathrm{NO}_{3}=97.9$.

SoUrCE. - By treating commercial Ammonium Carbonate with Nitric Acid, filtration and evaporation. $\mathrm{NH}_{4} \mathrm{HCO}_{3}-\mathrm{NH}_{4} \mathrm{NH}_{2} \mathrm{CO}_{2}+3 \mathrm{HNO}_{3}=$ $3 \mathrm{NH}_{4} \mathrm{NO}_{3}+2 \mathrm{CO}_{2}+\mathrm{H}_{2} \mathrm{O}$.

CirARACTERs. - Colorless crystals, generally in the form of long, thin rhombic prisms, or in fused masses, without odor, having a sharp, bitter taste, and somewhat deliquescent. Solubility.-In 0.5 part of water; and in 20 parts of Alcohol.

Ammonium Nitrate is used to prepare Nitrous Oxide gas, freezing mixtures and artificial cold applications.]

I0. AMMONII VALERIANAS, see Valeriana.

\section{LITHIUM.}

$$
\mathrm{Li}=7.01 \text {. }
$$

I. LITHII CARBONAS. - [Lithium Carbonate. $\mathrm{Li}_{2} \mathrm{CO}_{3}=73.87$.

SOURCE. - By action of Lithium Chloride upon Ammonium Carbonate, filtration, washing with Alcohol, and drying. $2 \mathrm{LiCl}+\mathrm{NH}_{3} \mathrm{HCO}_{3}=\mathrm{Li}_{2} \mathrm{CO}_{3}$ $+\mathrm{NH}_{4} \mathrm{Cl}+\mathrm{HCl}$.

CHARACTERS. - A light, white powder, odorless, and having an alkaline taste; permanent in the air. Solubility. -In 80 parts of water; insoluble in Alcohol.]

IMPURITIES. - Lime and aluminum.

Dose, 2 to $10 \mathrm{gr}$.; [.12 to .60 $\mathrm{gm}$.]

2. LITHII CITRAS. - [Lithium Citrate. $\mathrm{Li}_{3} \mathrm{C}_{6} \mathrm{H}_{5} \mathrm{O}_{7}=209.57$. 
Source., - By action of Lithium Carbonate upon Citric Acid, evaporation and crystallization. $3 \mathrm{Li}_{2} \mathrm{CO}_{3}+2 \mathrm{H}_{3} \mathrm{C}_{6} \mathrm{H}_{5} \mathrm{O}_{7}=2 \mathrm{Li}_{3} \mathrm{C}_{6} \mathrm{H}_{5} \mathrm{O}_{7} \quad 3 \mathrm{H}_{2} \mathrm{O}+3 \mathrm{CO}_{2}$.

ChARACTERS. - A white powder, odorless and having a cooling, faintly alkaline taste; deliquescent on exposure to air. Solubility.-In 2 parts of water; almost insoluble in Alcohol or Ether.

Dose, 5 to $20 \mathrm{gr}$; .30 to $1.20 \mathrm{gm}$.

\section{Preparation.}

Lithii Citras Effervescens.-Effervescent Lithium Citrate.

Source. - Lithium Carbonate, 70 ; Sodium Bicarbonate, 280; Citric Acid, 370 ; Sugar, a sufficient quantity, to rooo. Triturate the Citric Acid with Sugar, and dry the mixture thoroughly. Then incorporate with it, by trituration, Lithium Carbonate and Sodium Bicarbonate, and enough Sugar to make the product weigh 1000 parts.

ChARACTERs. - A white powder having a cooling, saline and sweetish taste. Solubility. - Completely in water with effervescence.

Dose, 10 to $45 \mathrm{gr}$; .60 to $3.00 \mathrm{gm}$.]

\section{Action of Lithium Carbonate and Citrate.}

These lithium salts closely resemble in their actions the corresponding potassium salts, in large doses leading to muscular and cardiac depression with gastro-intestinal irritation; but, as lithium has a strong affinity for uric acid, and lithium biurate is very soluble, they are more powerful solvents of uric acid. They are also efficacious as diuretics and render the urine very alkaline.

\section{Therapeutics of Lithium Carbonate and Citrate.}

Lithium salts are much used internally in acute and chronic gout, to promote the elimination of sodium biurate. They are also given as solvents to patients suffering from uric acid gravel and calculus. Those suffering from gravel are said often to derive great benefit. A lotion of the carbonate (I to 120 of water) applied on lint and covered with gutta-percha relieves the pain of gouty inflammation, promotes the healing of gouty ulcers, and aids the disappearance of tophi [although it does seem to prevent their formation]. Lithium salts should always be freely diluted. The citrate has the advantage of greater solubility. Although there is no doubt that the salt of lithium and uric acid is very soluble in water, much doubt has recently been cast upon 
the efficacy of lithium salts administered for gout, because the addition of a lithium salt to blood serum does not enhance its solvent power on sodium biurate.

3. [LITHII BENZOAS, see Acidum Benzoicum.

4. LITHII BROMIDUM, see Bromine.

5. LITHII SALICYLAS, see Acidum Salicylicum.]

\section{GROUP III.}

METALS OF THE AIKALINE EARTHS.

Calcium, Strontium, Barium, Magnesium, Cerium.

I. CALCIUM.

$\mathrm{Ca}=39.9 \mathrm{r}$.

1. CRETA PREPARATA.-[Prepared Chalk. $\mathrm{CaCO}_{3}=99.76$. Synonym.-Drop Chalk.

SOURCE. - From Chalk by levigation, elutriation and drying.

Characters. - A white, amorphous powder, often moulded into conical drops, odorless and tasteless; permanent in the air. Solubility.-Almost insoluble in water; insoluble in Alcohol.]

INCOMPATIBLES. -Acids and sulphates.

Dose, 10 to $60 \mathrm{gr}$. [.60 to $4.00 \mathrm{gm}$.]

\section{Preparations.}

I. Pulvis Cretæ Compositus. - [Compound Chalk Powder. Prepared Chalk, 30 ; powdered Acacia, 20 ; powdered Sugar, 50.

Dose, 5 to $60 \mathrm{gr}$; .30 to $4.00 \mathrm{gm}$.

2. Mistura Cretæ.-Chalk Mixture. Compound Chalk Powder, 200 ; Cinnamon Water, 400; Water to make 1000.

Dose, 2 to 4 fl. dr.; 8. to 15. c.c.

3. Hydrargyrum cum Creta, see Hydrargyrum.

4. Trochisci Cretæ.-Troches of Chalk. Prepared Chalk, 25 ; Acacia, $7 \mathrm{gm}$. ; Spirit of Nutmeg, 3 c.c. ; Sugar, $40 \mathrm{gm}$.; Water to make 100 troches. Each troche contains $4 \mathrm{gr} . ; .25 \mathrm{gm}$.

Dose, ad libitum.]

2. CALCII CARBONAS PRACIPITATUS.-[Precipitated Calcium Carbonate. $\mathrm{CaCO}_{3}=99.76$.

Source. - From Calcium Chloride and Sodium Carbonate, and drying the precipitate. $\mathrm{CaCl}_{2}+\mathrm{Na}_{2} \mathrm{CO}_{3}=2 \mathrm{NaCl}+\mathrm{CaCO}_{3}$. 
Characters. - A fine, white power, odorless and tasteless, permanent in the air. Solubility. - Nearly insoluble in water.

Precipitated Calcium Carbonate is used to prepare Pulvis Morphinæ Compositus and Syrupus Calcii Lactophosphatis.]

Dose, 5 to $60 \mathrm{gr}$; ; .30 to $4.00 \mathrm{gm}$.]

Action of Creta Preparata and Calcium Carbonate.

External.-It is mildly astringent and helps to dry moist surfaces.

Internal.-Stomach and Intestines.-Calcium carbonate is antacid. It is a mild but certain astringent. How it acts as an instringent is unknown. It is excreted unchanged in the fæces.

Kidneys. Because certain mineral waters containing calcium bicarbonates and sulphates amongst other salts, have been used successfully in cases of urinary gravel and calculi, it has been asserted that these salts are diuretic, and solvent for uric acid, but it is more likely that the beneficial effects of these waters are due merely to the large amount of water drunk; [at least], there is no proof that it is due to the salts. Such waters are those of Contrexéville, Vittel, [Clarendon, and Waukesha].

Therapeutics of Creta Preparata and Calcium Carbonate.

External.-Prepared chalk forms an excellent dusting powder for moist eczema.

Internal.-Alimentary canal.-Because of its mechanical action it is a good tooth powder. The following is a good formula : Potassium chlorate, 4 ; powdered soap, 8 ; carbolic acid, 2 ; oil of cinnamon, I ; precipitated calcium carbonate to 48 parts. Chalk mixture and [compound] chalk powder, particularly the former, are very valuable for checking mild diarrhœa, especially in children.

Kidneys. - There is no doubt that persons passing gravel or urinary calculi, especially if composed of uric acid, are benefited by drinking the waters of Contrexéville and Vittel. They should be taken in quantities of 3 to 6 pints [1500. to 3000 . c.c.] a day and between meals, to avoid the large amount of fluid causing indigestion. At Contrexéville the great bulk is drunk before breakfast. 
3. CALX. $-[$ Lime. $\mathrm{CaO}=55.87$. Synonym.-Burned Lime.

Source.-Made by burning white marble, oyster sbells, or the purest varieties of natural Calcium Carbonate, to expel Carbon Dioxide.

Characters. - Hard white or grayish-white masses, which in contact with air gradually attract moisture and Carbon Dioxide, and fall into a white powder (slaked lime); odorless; of a sharp, caustic taste. Solubility.-In 750 parts of water; insoluble in Alcohol.

\section{Preparations.}

1. Liquor Calcis.-Solution of Lime. Synonyms.-Lime Water. Solution of Calcium Hydrate.

SourCE.-Made from slaked lime by solution. A saturated, aqueous solution of Calcium Hydrate. The percentage of Calcium Hydrate varies with the temperature, being somewhat over 0.17 per cent. at $59^{\circ} \mathrm{F}$; $15^{\circ} \mathrm{C}$, and diminishing as the temperature rises.

Dose, I to $8 \mathrm{fl}$. dr.; 4. to 30 . c.c.

2. Linimentum Calcis.-Lime Liniment. Synonym.-Carron Oil. Solution of Lime, Linseed Oil, of each, one volume. Mix them by agitation.

3. Syrupus Calcis.-Syrup of Lime. Lime, 60 ; Sugar, 400 ; Water, to 1000 .

Dose, 15 to $60 \mathrm{~m}$.; 1 . to 4. c.c.

4. Potassa cum Calce._-See Potassium, p. 122.]

\section{Action of Lime.}

External.-Slaked lime is caustic. Lime water is astringent. Internal.-Alimentary tract.-Lime is antacid. It prevents milk from forming solid, bulky curds in the stomach. It allays vomiting, and is an antidote for poisoning by mineral acids, oxalic acid, and zinc chloride. It acts as mild intestinal astringent.

\section{Therapeutics of LiMe.}

External.-Slaked lime, employed as a caustic, is usually mixed with caustic potash, when it forms Vienna paste (see p. I23), [or with caustic soda known as London paste and is] used to destroy warts and other small growths. Lime water applied to weeping eczema is especially serviceable if mixed with glycerin. Linimentum Calcis is very valuable for burns.

Internal.-Lime water is much used to mix with milk to prevent its forming thick curds in the stomach, especially when, 
as is often the case with children, the curds cause vomiting. It is difficult to understand how it acts, for, although lime water contains so little lime, it is often efficacious. In severe cases of infantile vomiting equal parts of milk and lime water may be ordered. Lime water will check slight diarrhœa. It is a useful injection for thread-worms, for leucorrhœa, and for gleet.

4. CALCII PHOSPHAS PRECIPITATUS.-[Precipitated Calcium Phosphate. $\mathrm{Ca}_{2}\left(\mathrm{PO}_{4}\right)_{2}=309.33$. Synonym.-Precipitated Lime Phosphate.

Source.-Made from Bone Ash (impure Calcium Phosphate) digested with diluted Hydrochloric acid, made alkaline with solution of Ammonia, and by precipitation.

Characters. - A light, white amorphous powder, colorless and tasteless, and permanent in the air. Solubility.-Almost insoluble in water.

Precipitated Calcium Phosphate is contained in Pulvis Antimonialis.

Dose, 5 to $30 \mathrm{gr}$; ; 30 to $2.00 \mathrm{gm}$.

\section{Preparation.}

Syrupus Calcii Lactophosphatis.-Syrup of Calcium Lactophosphate. Precipitated Calcium Carbonate, 25; Phosphoric Acid, 36; Lactic Acid, 60 ; Orange Flower Water, 25; Sugar, 700; Water, to 1000.

Dose, I to 2 fl. dr.; 4. to 8. c.c.]

\section{Action and Therapeutics of Calcium Phosphate.}

Calcium phosphate is a most important constituent of bones, and therefore it is necessary that food should contain it. If not, the bones become soft. Calcium salts are abundant in milk, yolk of egg, vegetables, and the bones that carnivora eat. They are absorbed from the intestine, and the excess is excreted into the intestine and passed with the fæces. Calcium phosphate has been given for rickets, and for the anæmia and feebleness often seen in young children, but it is not certain that it does any good. [It is important that calcium phosphate should be made from bones when used in the treatment of rickets.] It may be given to pregnant and [nursing] women in order to provide the child with sufficient calcium salts for its bones.

It is used as a diluent for powders, as it is inert, and it prevents their agglutination. For these reasons, and because it is [almost] insoluble, it is a useful constituent of pills containing essential 
oils. The syrup of calcium lactophosphate is with many a favorite prescription for tuberculosis and other conditions of anæmia and weakness.

5. CALX SULPHURATA, see Sulphur.

6. CALCII CHLORIDUM.-[Calcium Chloride. $\mathrm{CaCl}_{2}=1$ ro. 65 .

SoURCE. - Obtained by neutralizing Hydrochloric Acid with Calcium Carbonate and evaporating. $\mathrm{CaCO}_{3}+2 \mathrm{HCl}=\mathrm{CaCl}_{2}+\mathrm{CO}_{2}+\mathrm{H}_{2} \mathrm{O}$. This is rendered anhydrous by fusion at the lowest possible temperature.

Characters. - White, slightly translucent, hard fragments, odorless, having a sharp saline taste and very deliquescent. Solubility.-In 1.5 parts of water and in 8 parts of Alcohol.]

Dose, 5 to $20 \mathrm{gr}$; ; .30 to $\mathrm{r.20} \mathrm{gm}$.]

\section{Action and Therapeutics of Calcium Chloride.}

Calcium chloride, outside of the body, increases the rate of coagulation of the blood [and produces a firmer clot. It has been employed in the treatment of chronic bronchitis and pneumonia, and has been recommended by Sée for gastric catarrh and fermentative dyspepsia. Its most important use is for the hæmorrhages of scurvy and hæmophilia. If maximum doses are administered for several days previously, it is often possible to perform operations upon bleeders. It may be of use in hæmatemesis and hæmoptysis, and, possibly, also for aneurism.]

7. CALX CHLORATA, see Chlorine.

8. CALCII HYPOPHOSPHIS, see Phosphorus.

9. [CALCII BROMIDUM, see Bromine.

ro. CALCII SULPHAS EXSICCATUS.-Dried Calcium Sulphate. Synonyms.-Dried Gypsum. Plaster of Paris.

SOURCE. - A powder containing about 95 per cent., by weight, of Calcium Sulphate $\left(\mathrm{CaSO}_{4}=\mathbf{1} 35.73\right)$, and about 5 per cent. of water, prepared from the purer varieties of native Gypsum $\left(\mathrm{CaSO}_{4}+2 \mathrm{H}_{2} \mathrm{O}=17 \mathrm{I} .65\right)$, by heating until about three-fourths of the water has been expelled.

CHARACTERS.-An amorphous white powder, without taste or odor, and when mixed with half its weight of water it forms a smooth paste, which rapidly hardens. Solubility. - In about 4 Io parts of water; insoluble in Alcohol.

Dried Calcium Phosphate is used to prepare Calx Sulphurata.

Uses of Dried Calcium Sulphate.

Dried Calcium sulphate is used for making casts of deformities and injuries, and for making immovable bandages and apparatus for injuries and diseases when immobilization is necessary. 


\section{STRONTIUM.}

$$
\mathrm{Sr}=87 \cdot 3 \text {. }
$$

I. STRONTII LACTAS.-Strontium Lactate. $\operatorname{Sr}\left(\mathrm{C}_{3} \mathrm{H}_{5} \mathrm{O}_{3}\right)_{2}+3 \mathrm{H}_{2} \mathrm{O}$ $=3 \mathrm{I} 8.76$.

SOURCE. - From the carbonate, by dissolving it in lactic acid somewhat diluted with water; if necessary, heat is applied to effect solution. After filtration the solution is evaporated with moderate heat, to dryness. $\mathrm{SrCO}_{3}+$ $2 \mathrm{HC}_{3} \mathrm{H}_{5} \mathrm{O}_{3}=\mathrm{Sr}\left(\mathrm{C}_{3} \mathrm{H}_{5} \mathrm{O}_{3}\right)_{2}+\mathrm{H}_{2} \mathrm{O}+\mathrm{CO}_{2}$.

Characters. - A white, granular powder or crystalline nodules, odorless and having a slightly bitter taste. Permanent in the air. Solubility.-In about 4 parts of water; soluble in Alcohol.

INCOMPATIBLES. - Solutions of carbonates and sulphates, and potassium chromate.

IMPURITIES.-Barium carbonate, oxalates, metallic and organic impurities.

Dose, $1 / 4$ to 2 dr.; 1 . to $8 . \mathrm{gm}$.

\section{Action of Strontium Lactate.}

The strontium salts were demonstrated by Laborde to be harmless to animals and men. He also ascribed to them a diuretic action. If given for some time and in large quantities they impair gastric digestion and subsequently the general nutrition. The lactate reduces the amount of albumin in albuminuria, and it is claimed to have a sedative effect on the heart in diseases of the valves and of the muscular tissue. It also checks fermentation and putrefaction in the small intestines.

\section{Therapeutics of Strontium Lactate.}

The strontium salts in gastric affections improve the appetite and facilitate digestion, and are useful in chronic intestinal catarrh. The lactate is diuretic and is useful in albuminuria, due to renal atony, but not in uræmia, nor in interstitial nephritis, nor in the high fever of acute parenchymatous nephritis. In the chronic form due to scrofula, rheumatism or gout it is useful. It has had a decidedly beneficial action in diabetes of hepatic origin, and in cirrhosis of the liver.

2. STRONTII BROMIDUM, see Bromine.

3. STRONTII IODIDUM, see Iodine.] 


\section{BARIUM.}

$$
\mathrm{Ba}[=\mathbf{1} 36.9 \text {. }
$$

BARII DIOXIDUM.-Barium Dioxide. $\mathrm{BaO}_{2}=168.82$. Synonym.Barium Peroxide.

SoURCE.-By conducting oxygen over Barium Oxide, heated to full redness.

Characters. -A heavy, grayish-white, or pale, yellowish-white, amorphous, coarse powder, odorless and tasteless. When exposed to the air it slowly attracts moisture and Carbon Dioxide, and is gradually decomposed. Solubility.-Almost insoluble in water.

IMPURITIES. - Sulphates and nitrates.

INCOMPATIBLES.-Hydrochloric, phosphoric and most other mineral acids. Barium Dioxide is used in preparing Aqua Hydrogenii Dioxidi.

\section{Action of Barium Salts.}

Barium chloride (not official) causes the cardiac contractions to become slower and more forcible, acting like digitalis. The blood-vessels are constricted, and the blood pressure-rises. The plain muscular fibres of the intestine may be excited, and the peristalsis is increased. In these respects it resembles ergot as well as digitalis. It acts like veratrine when applied locally to voluntary muscles, prolonging the contraction ; but this effect is done away with by the application of potassium salts.

\section{Therapeutics of Barium Salts.}

These are not often given, but the chloride (dose, $\frac{3}{10}$ to $\mathrm{x} / 2$ gr., .006 to $.03 \mathrm{gm}$.) has been used for mitral insufficiency accompanied by irregularity of the heart, for hæmorrhage, and as a stimulant in atony of the bladder or intestine. Formerly it was given in nervous diseases.] The waters of Llangammarch wells contain $6.7 \mathrm{gr}$. [.40 gm.] to the [Imperial] gallon [ 4545 c.c.] of barium chloride, and have been used in cardiac cases. a depilatory.

[The sulphide (not official) has been used as

\section{TOXICOLOGY.}

Symptoms.-Poisonous doses cause salivation, thirst, vomiting, purging, difficulty of breathing, a slow pulse, and, from its action on the spinal cord, paralysis of the limbs. The heart is arrested in systole. 
Treatment.-Poisoning should be treated by non-irritant emetics and draughts of weak solution of sodium or magnesium sulphate, followed by albuminous drinks, and diffusible stimulants. ]

\section{MAGNESIUM.}

$$
\mathrm{Mg} \cdot=\mathbf{2 4} \cdot \mathbf{3} \text {. }
$$

1. MAGNESII SULPHAS.-[Magnesium Sulphate. $\mathrm{MgSO}_{4}+\mathrm{H}_{2} \mathrm{O}$ $=245.84$. Synonym. - Epsom Salt.

Source. - It is obtained from (I) Dolomite (native Calcium and Magnesium Carbonate); or (2) Magnesite (native Magnesium Carbonate), by the action of Sulphuric Acid. (I) $\mathrm{MgCO}_{3}+\mathrm{MgSO}_{4}+2 \mathrm{H}_{2} \mathrm{SO}_{4}=\mathrm{CaSO}_{4}+\mathrm{MgSO}_{4}$ $+2 \mathrm{H}_{2} \mathrm{O}$ or (2) $\mathrm{MgCO}_{3}+\mathrm{H}_{2} \mathrm{SO}_{4}=\mathrm{MgSO}_{4}+\mathrm{H}_{2} \mathrm{O}+\mathrm{CO}_{2}$. Treat with water, filter and evaporate the filtrate to crystallization.

Characters. - Small,] colorless, rhombic prisms or acicular crystals, very like Zinc Sulphate, but moister, and of a bitter taste, whilst that of the Zinc Salt is metallic. Solubility. - In 1.5 parts of cold water.

Incompatibles. - Alkaline carbonates, phosphoric acid, phosphates, lime water, lead acetate and silver nitrate.

IMPURITIES. - Lime and Iron.

[Magnesium Sulphate is contained in Infusum Sennæ Compositum.]

Dose, $1 / 4$ to $\mathrm{x} \mathrm{oz}$; ; 8 , to $30 . \mathrm{gm}$.

2. MAGNESII CARBONAS.-[Magnesium Carbonate. $\left(\mathrm{MgCO}_{3}\right)_{4}$, $\mathrm{Mg}(\mathrm{OH})_{2}+5 \mathrm{H}_{2} \mathrm{O}=484.62$.

SourCE. - Mix strong, boiling aqueous solutions of Magnesium Sulphate and Sodium Carbonate, and evaporate. ${ }_{4} \mathrm{MgSO}_{4}+4 \mathrm{Na}_{2} \mathrm{CO}_{3}+\mathrm{H}_{2} \mathrm{O}=(\mathrm{Mg}$ $\left.\mathrm{CO}_{3}\right)_{4}, \mathrm{Mg}(\mathrm{OH})_{2}+4 \mathrm{Na}_{2} \mathrm{SO}_{4}+\mathrm{CO}_{2}$. Digest with water, filter and dry.

Characters. - Light, white friable masses, or a light white powder, without odor, and having a slightly earthy taste. Solubility.-Almost insoluble in water.

IMPURITIES. - Lime and sulphates.

Magnesium Carbonate is used to prepare Magnesia.

Dose, $1 / 4$ to $2 \mathrm{dr}$.; $\mathrm{x}$. to $8 . \mathrm{gm}$.]

\section{Preparations.}

x. Magnesii Citras Effervescens.-[Effervescent Magnesium Citrate. Magnesium Carbonate, 10; Citric Acid, 46; Sodium Bicarbonate, 34; Sugar, 8 ; Alcohol and distilled water, a sufficient quantity.

Characters. - A white, coarsely granular salt, without odor, and having a mildly acidulous, refreshing taste, deliquescent. Solubility. With copious effervescence, in 2 parts of water; almost insoluble in Alcohol.

Dose, $1 / 4$ to $\mathrm{I} 0 z . ; 8$. to $30 . \mathrm{gm}$. 
2. Liquor Magnesii Citratis.-Solution of Magnesium Citrate. Dissolve Magnesium Carbonate, 15; in a solution of Citric Acid, 30 ; add Syrup of Citric Acid, 60 ; then crystals of Potassium Bicarbonate, 25. Cork and wire immediately. It effervesces when uncorked.

Dose, 2 to 8 fl. oz.; 6o. to 240. c.c.]

3. MAGNESIA.-Light Magnesia. $\mathrm{MgO}[=40.26$. $]$ Synonym.-Calcined Magnesia.

SourCE. - [By heating the official Magnesium Carbonate; water and Carbon Dioxide are given off, and Magnesium Oxide remains behind. $4\left(\mathrm{MgCO}_{3}\right)$, $\mathrm{Mg}(\mathrm{OH})_{2}+5 \mathrm{H}_{2} \mathrm{O}=5 \mathrm{MgO}+4 \mathrm{CO}_{2}+6 \mathrm{H}_{2} \mathrm{O}$.

Characters. - A white, very light and very fine powder, without odor, and having an earthy, but not saline taste. Solubility. -Almost insoluble in water; insoluble in Alcohol.

Light Magnesia is used to prepare Heavy Magnesia and is contained in Massa Capaibæ, Pulvis Rhei Compositus and Ferri Oxidum Hydratum cum Magnesia.

Dose, 5 to $60 \mathrm{gr}$; .30 to $4.00 \mathrm{gm}$.]

4. MAGNESIA PONDEROSA.-Heavy Magnesia. [ $\mathrm{MgO}=40.26$.

SOURCE. - From Magnesia by trituration for some time in the presence of strong Alcohol, drying, and rubbing to powder.

Characters. - A white, dense and very fine powder, which should correspond to the tests for Magnesia, from which it differs in not readily uniting with water to form a gelatinous hydrate. ]

Dose, 5 to $60 \mathrm{gr}$.; [.30 to $4.00 \mathrm{gm}$.]

\section{Action of Magnesium Salts.}

External.-None.

Internal.-Stomach and Intestines.-Magnesia and magnesium carbonate are antacid, acting in many ways like the potassium and sodium alkalies. Carbon [dioxide] is given off, if the carbonate has been given, and is sedative to the stomach. They are both decomposed by the gastric juice, magnesium chloride, lactate and bicarbonate being formed. These salts, or the sulphate, if that has been taken, act in the intestine, as typical saline purgatives. The sulphate is most powerful. The mode of action of this group of purgatives has been discussed on p. 93 .

Blood and Urine.-Like other alkaline remedies, these magnesium salts increase the alkalinity of the blood, alkalize the urine, help to keep uric acid in solution, and are diuretic. But 
their action on the blood and urine is feebler than that of potassium and sodium salts, for they are with difficulty absorbed. Large doses injected into the blood of aninals are toxic, killing by their action on the heart.

\section{Therapeutics of Magnesium Salts.}

Internal.-Stomach.-Magnesia and the carbonate are mild alkaline remedies, and may be used in the same class of cases as other alkalies. They form insoluble compounds with mineral acids, oxalic acid, and mercury, arsenic and copper salts. By alkalizing the gastric contents they hinder the absorption of alkaloids. They are, therefore, antidotes to all these substances; the objection to them is their bulk. Magnesia is to be preferred, as the carbonate gives off carbon [dioxide] gas. They must be freely given. The sulphate is an antidote to lead and barium salts, forming insoluble sulphates.

Intestines. - The magnesium salts are very common purgatives. Magnesia, the carbonate, and the citrate are excellent for children. The sulphate is one of our best saline purgatives. It is very largely used, especially for the varieties of constipation that are associated with hepatic disorder, gout or excessive uric acid. Its use is then spread over some time, and it may conveniently be taken as one of the mineral waters which contain it and sodium sulphate (see p. 144). A concentrated solution, causing as it does an increased secretion of intestinal fluid, is a useful purge for dropsy or ascites. [It is useful with glycerin in concentrated enema for thorough cleansing of the bowels before surgical operations (glycerin, 1 oz.; 30 . c.c., in a saturated solution of magnesium sulphate, in hot water 3 oz. ; 90 . c.c., which is allowed to cool). It can also be used hypodermatically in dose of 3 gr. ; .20 gm., which frequently will cause a watery evacuation. In operations during which the abdomen is opened, the subsequent intestinal paralysis can be prevented from causing constipation by injecting into the small intestine through a cannula one ounce; 3 o. c.c. of a saturated solution of magnesium sulphate. The wound in the bowel should be closed by a Lembert stitch.] 
Blood and kidneys. - So little of these salts is absorbed that they are only to be given for their alkaline effects on the blood and urine in those cases of gout and uric acid gravel in which potassium or sodium salts cannot be borne.

\section{CERIUM.}

$$
\mathrm{Ce}=\mathbf{1 3 9 . 9} \text {. }
$$

CERII OXALAS.-[Cerium Oxalate. $\mathrm{Ce}_{2}\left(\mathrm{C}_{2} \mathrm{O}_{4}\right)_{3}+9 \mathrm{H}_{2} \mathrm{O}=704.78$. Synonym.-Cerous Oxalate.

SoURCE. - The powdered mineral is heated with concentrated Sulphuric Acid, ignited, then dissolved in dilute Nitric Acid and treated with Hydrogen Sulphide to remove copper; the calcium salt is held in solution by a little $\mathrm{Hy}$ drochloric Acid, and the cerite metals are precipitated as oxalates by Oxalic Acid. It is purified by calcination and solution, reduced to a cerous salt and precipitated by Oxalic Acid.

Characters. - A white granular powder, without odor or taste. Solubility. - Insoluble in water, Alcohol or Ether.]

IMPURITIES. - Aluminum and lanthanum and didymium oxalates.

Dose, I to $8 \mathrm{gr}$.; [.06 to $.50 \mathrm{gm}$.]

\section{Therapeutics of Cerium Oxalate.}

It is given empirically for vomiting, especially for that of pregnancy, and occasionally with benefit. No physiological action is known. [The dose above given is often exceeded; 30 gr. (2. gm.); have been frequently given with good results.]

\section{GROUP IV.}

\section{Plumbum, Argentum, Zincum, Cuprum, Bismuthum, Aluminum.}

The pharmacopœial Salts of these metals are powerful astringents. Many of them have some Salts which are emetic, and others which, when applied locally, are Caustic. Aluminum pharmacologically falls into this group.

\section{PLUMBUM.}

$$
\mathrm{Pb}=206.4 \text {. }
$$

I. PlUMBI OXIDUM.-[Lead Oxide. $\mathrm{PbO}=222.36$.$] Synonym.$ -Litharge.

SOURCE.-Made by roasting Lead in air. 
CHARACTERs. - [A heavy, y ellowish or reddish-yellow powder or minute scales, without odor or taste. Solubility.-Almost insoluble in water; soluble in Nitric and Acetic Acids.

IMPURITIES.-Copper, iron and carbonates.

Lead Oxide is used to make Liquor Plumbi Subacetatis.]

\section{Preparations.}

I. Emplastrum Plumbi.-Lead Plaster. This is LEAd oleate, and is sometimes called Diachylon Plaster. [Lead Oxide, 3200, is boiled in water, and Olive Oil, 6000. When the mass has acquired a whitish color and is perfectly homogeneous, it is well kneaded to remove the Glycerin and divided into rolls of suitable size.] $3 \mathrm{PbO}+$ ${ }_{3} \mathrm{H}_{2} \mathrm{O}+2\left(\mathrm{C}_{3} \mathrm{H}_{5}\left(\mathrm{C}_{18} \mathrm{H}_{33} \mathrm{O}_{2}\right)_{3}\right)=3\left(\mathrm{~Pb}_{2}\left(\mathrm{C}_{18} \mathrm{H}_{33} \mathrm{O}_{2}\right)_{2}\right)+2\left(\mathrm{C}_{3} \mathrm{H}_{5}(\mathrm{OH})_{3}\right)$.

[Lead Oxide or its Plaster is contained in Emplastrum Ammoniaci cum Hydrargyro, Ferri, Hydrargyri, Opii, Resinæ, and Saponis.

2. Unguentum Diachylon.-Diachylon Ointment. Lead Plaster, 500; Olive Oil, 490 ; Oil of Lavender Flowers, Io.]

2. PLUMBI ACETAS.-Lead Acetate. $\mathrm{Pb}\left(\mathrm{C}_{2} \mathrm{H}_{3} \mathrm{O}_{2}\right)_{2}+3 \mathrm{H}_{2} \mathrm{O}[=$ 378.0.] Synonym.--Sugar of Lead.

SOURCE. - [Metallic Lead is dissolved, in the presence of air, in Acetic Acid. $\mathrm{PbO}+2 \mathrm{C}_{2} \mathrm{H}_{4} \mathrm{O}_{2}+2 \mathrm{H}_{2} \mathrm{O}=\mathrm{Pb}\left(\mathrm{C}_{2} \mathrm{H}_{3} \mathrm{O}_{2}\right)_{2}+3 \mathrm{H}_{2} \mathrm{O}$. To obtain well-defined crystals the solution must have a distinctly acid reaction.

Characters. - Colorless, shining, transparent, monoclinic prisms or plates, or heavy, white, crystalline masses, or granular crystals, having a faintly acetous odor, and a sweetish, astringent, afterwards metallic taste. Efflorescent, and absorbing Carbon Dioxide, on exposure to the air. Solubility. - In 1.8 parts of water.]

IncompatiblFs. - Hard water, mineral acids and salts, alkalies, lime water, potassium iodide, vegetable astringents, preparations of opium, and albuminous liquids.

IMPURITY.-Lead carbonate.

Dose, $1 / 2$ to $5 \mathrm{gr}$; [ [.03 to .30 $\mathrm{gm}$.]

Preparations made from the Acetate in which Lead exists as the SUBACETATE, $\mathrm{Pb}_{2} \mathrm{O}\left(\mathrm{C}_{2} \mathrm{H}_{3} \mathrm{O}_{2}\right)_{2}=546.48$.

[1. Liquor Plumbi Subacetatis.-Solution of Lead Subacetate. Synonym.-Goulard's Extract. Lead Acetate, 170; and Lead Oxide, 100 ; are boiled together in distilled water, to make 1000 . An aqueous liquid containing approximately 25 per cent. of Lead Subacetate.

2. Liquor Plumbi Subacetatis Dilutus.-Diluted Solution of Lead Subacetate. Synonym.-Lead Water. Liquor Plumbi Subacetatis, $3^{\circ}$; distilled water to 1000 .

3. Ceratum Plumbi Subacetatis.-Cerate of Lead Subacetate. 
Synonym.-Goulard's Cerate. Solution of Lead Subacetate, 200; Camphor Cerate, 800.]

3. PLUMBI CARBONAS.-[Lead Carbonate. A mixture of Carbonate and Hydrate. $\left(\mathrm{PbCO}_{3}\right), \mathrm{Pb}(\mathrm{OH})_{2}=772.82$. Synonym. - White Lead.

Source. - Expose Lead to the vapor of Acetic Acid and to air charged with Carbon Dioxide. $6 \mathrm{~Pb}+6 \mathrm{HC}_{2} \mathrm{H}_{3} \mathrm{O}_{2}+3 \mathrm{O}_{2}+2 \mathrm{CO}_{2}=\left(\mathrm{PbCO}_{3}\right), \mathrm{Pb}(\mathrm{OH})_{2}+$ $2 \mathrm{H}_{2} \mathrm{O}+2 \mathrm{~Pb}\left(\mathrm{C}_{2} \mathrm{H}_{3} \mathrm{O}_{2}\right)_{2}$.

Characters. - A heavy, white, opaque powder, or a pulverulent mass, without odor or taste.

IMPURITY.-Lime.

Preparation.

Unguentum Plumbi Carbonatis.-Ointment of Lead Carbonate. Lead Carbonate, I0; Benzoinated Lard, 90.]

4. PLUMBI NITRAS. - [Lead Nitrate. $\mathrm{Pb}\left(\mathrm{NO}_{3}\right)_{2}=330.18$.

Source._Dissolve Lead in warm diluted] Nitric Acid.

Characters. - [Colorless, transparent, octahedral crystals, or white opaque crystals; without odor, and having a sweetish, astringent, afterwards metallic taste.

Ledoyen's Disinfecting Fluid is Lead Nitrate, 1 ; dissolved in water, 8 parts.]

5. PLUMBI IODIDUM.-[Lead Iodide. $\mathrm{PbI}_{2}=430.46$.

Sovrce.-Mix solutions of Lead Nitrate and Potassium Iodide and dry the precipitate. $\mathrm{Pb}\left(\mathrm{NO}_{3}\right)_{2}+2 \mathrm{KI}=2 \mathrm{KNO}_{3}+\mathrm{PbI}_{2}$.

Characters. - A heavy, bright-yellow powder, without odor or taste. Solubility. - In about 2000 parts of water.

\section{Preparation.}

Unguentum Plumbi Iodidi._-Ointment of Lead Iodide. Lead Iodide, 10; Benzoinated Lard, 90.]

\section{Action of Lead Salts.}

External.-The action of lead salts on the unbroken skin, if they have any, is very slight ; but when applied to the abraded skin, to sores and to ulcers, they coagulate the albumin of the discharge, thus forming a protective coat; they coagulate the albumin in the tissues themselves; and they contract the small vessels; for these three reasons they are powerfully astringent. They also soothe pain, and are therefore excellent local sedatives. It is obvious that substances so markedly astringent 
will be hæmostatics. Any salt may be irritant and caustic if enough be used, and it is sufficiently concentrated.

Internal.-Lead salts act on mucous membranes precisely as on the unbroken skin, and are therefore powerfully astringent and hæmostatic to all parts of the alimentary canal, from the mouth downwards. In the stomach they are converted into a chloride. (For other actions see Toxicology.)

\section{Therapeutics of Lead Salts.}

External.-Lead salts are applied as lotions or ointments in many conditions for which an astringent, sedative effect is desired, as in weeping eczema and many varieties of ulceration. The glycerin of the subacetate [B. P., lead acetate, 10; lead oxide, 7 ; glycerin, 40 ; water, 24 ; boiled together] diluted fourfold with glycerin or milk is useful for these conditions. The lotions may be injected in vulvitis, leucorrhœa, gleet and otorrhøa, but should not be applied for ulceration of the cornea, lest the white precipitate formed should lead to permanent opacity. The sedative effect is well seen in their use in pruritus; but of course the cause of the itching should, if possible, be removed. The Liquor Plumbi Subacetatis is rarely used, as it is strong enough to irritate; the diluted form is that usually employed when a lotion is desired. It is often applied to bruises when the skin is unbroken, but is doubtful if it is absorbed. The ointment is an excellent remedy, and a lotion of lead and opium is a favorite preparation. It may be made by mixing $5 \mathrm{gr}$. [. $30 \mathrm{gm}$.$] of extract of opium with I oz. [30. c.c.] of Liquor$ Plumbi Subacetatis Dilutus and I oz. [30. c.c.] of water. Diachylon ointment mixed with an equal quantity of zinc oleate and mercuric oleate ointments forms a transparent ointment excellent for many purposes.

Internal.-The chief uses of lead salts (the acetate is the only one given internally) are as astringents in severe diarrhœa, such as that of typhoid fever, and as hæmostatics, as in gastric ulcer, or in hæmorrhage from the intestine, especially if severe, as in typhoid fever or tuberculosis. For these purposes the Pilula Plumbi cum Opio [B. P., lead acetate, 3 gr.; .20 gm.; 
opium, I gr.; .o6 gm.] is very valuable, and suppositories containing the same amount of the ingredients may be employed for rectal hæmorrhage. Lead salts produce marked constipation. Other preparations are generally preferred, but lead subacetate may be used as a gargle when an astringent effect on the mouth or pharynx is desired.

\section{Toxicology.}

Acute Lead Poisoning.-As when applied externally, so when taken internally, the lead salts, if concentrated, are powerful irritants. Cases of acute poisoning are rare. The Acetate is most frequently taken. There is a burning, sweetish taste in the mouth, thirst, vomiting [of whitish fluid due to lead chloride], abdominal colic, and usually constipation, but if the bowels are open the fæces are black [due to lead sulphide]: the skin is cold, and there is collapse. If the patient live long enough, cramps in the legs, giddiness, torpor, coma, and convulsions are present. Post-mortem.-The stomach and intestines show signs of irritant poisoning.

Treatment._Give emetics (see p. 139), or wash out the stomach. Give Sodium or Magnesium Sulphate to form an insoluble sulphate, and to open the bowels. If collapse is present, stimulants and warmth should be used.

Chronic Lead Poisoning. - This is so common [that the sources of accidental poisoning should be borne in mind. The most important are : soft water, carbonated waters and alcoholic drinks (beer) which have passed through lead pipes or been stored in receptacles lined with lead. Occupations as painters (colica pictonum), plumbers, type-setters, gold-miners, white lead workers, potters, glaziers (Devonshire colic) because] they will not wash their hands before meals [nor use ordinary care; lead hair dyes and face powders, biting leaded white thread, eating certain canned fruits (lead solder), sheetlead (tin-foil) about tobacco, filling holes in mill-stones with lead, giving of tin (lead) soldiers to children, use of lead carbonate ointment on burns, lead bullets in flesh, white or red lead used for preparing rubber for Vulcanizing, lead plates in dentistry (Osler), the use of lead chromate to color buns yellowish, have all been followed by chronic plumbism.]

Symptoms. - The earliest are constipation and intestinal colic. Lead is certainly absorbed, for it circulates in the blood and is excreted, chiefly, by the kidneys. It is supposed to be taken up as an albuminate, but it cannot exist in the blood as such, for it would be precipitated by the alkali of that fluid. After absorption it diminishes the amount of hæmoglobin and the number of red blood-corpuscles, and produces a sallow anæmia; it checks the separation of urates from the blood and their excretion by the kidneys, hence gout is very common in those poisoned by lead. As it circulates in the gums, and the lead-impregnated plasma bathes the epithelium, through which some of the sulphur in the food and in the tartar of the teeth has diffused, a Lead Sulphide is precipitated in the gums, and forms the well-known very dark-blue 
line [known as Burton's line], at the base of the teeth. For the same reason a blue line may occasionally be seen round the anus, and, after death, deposits of pigment in the intestines. Circulating in the nervous system, lead very often produces chronic inflammation of the peripheral nerves, especially those supplying the extensors of the hand, and hence wrist-drop is a very common symptom ; but any muscle, and sometimes almost all the muscles of the body, may be paralyzed from neuritis [it is a clinical observation that such muscles are very refractory to electricity]. It is noteworthy that the supinator longus usually escapes, [the reason apparently being that the supinator is not an extensor muscle]. The sensory fibres of the nerves are not often affected, hence pain and anæesthesia are rare; but pains, especially round the joints, may occur. In exceptional cases the anterior cornua of the spinal cord waste, and lead often affects the brain, causing saturnine lunacy, and also convulsions, knowis as saturnine epilepsy. Inflammation of the optic nerve or optic neuritis, sometimes occurs, leading to blindness, which, however, may be present without any change in the nerve. The kidneys are often the seat of chronic inflammation; whether this is due to the passage of the lead through them, or to the gout caused by the lead, is an open question.

Treatment.-The treatment consists chiefly in avoidance of the source of poisoning, [the use of Sulphuric Acid lemonade, and in the administration of Potassium Iodide which] is often given, as it is supposed to increase the excretion of lead in the urine. This is probably incorrect as very little lead passes out by the urine; most leaves the body by the freces. It is said also to be excreted in the bile, sweat, and milk. For a clinical account of the symptoms and treatment a text-book of medicine must be consulted.

[The following method may be employed to determine the presence of lead in the urine. Administer potassium iodide for four days, collecting the urine. Evaporate to a pint; 500 c.c., and filter. Pass hydrogen sulphide gas through the urine thus concentrated, when a black precipitate will form if lead is present. Other substances give a black precipitate with hydrogen sulphide, but none are likely to be present in the urine. A simple test is to paint a small area of skin with a six per cent. solution of sodium sulphite. If lead is present the painted area will darken after a few days, (Cicconardi). Patients using face enamels containing lead will find the skin blackened on taking baths in water containing hydrogen sulphide (Richfield Springs).]

\section{ARGENTUM.}

$$
\text { Ag. }=[107.66 .
$$

I. ARGENTI NITRAS.-Silver Nitrate. onym. - Lunar caustic.

SourcF, - Dissolve Silver in Nitric Acid with the aid of heat. Evaporate and crystallize. $\quad\left[6 \mathrm{HNO}_{3}+3 \mathrm{Ag}_{3}=6 \mathrm{AgNO}_{3}+3 \mathrm{H}_{2}\right.$.

Characters.-Colorless, transparent, tabular, rhombic crystals, becoming gray or grayish-black on exposure to light in the presence of organic 
matter; without odor, but having a bitter, caustic and strongly metallic taste. Solubility. - In 0.6 part of water; and in 26 parts of Alcohol.] It should be kept in the dark, as light blackens it.

Incompatibles. - Alkalies and their carbonates, chlorides, acids (except nitric and acetic), potassium iodide, solutions of arsenic and astringent infusions.

IMPURITIES. - Other nitrates.

Dose, $1 / 4$ to $1 \mathrm{gr}$. ; [.015 to $.06 \mathrm{gm}$.] in a pill.

\section{Preparations.}

[1. Argenti Nitras Dilutus.-Diluted Nitrate of Silver. Synonym.-Mitigated caustic.

SouRCE. - It is a mixture made by fusing together Silver Nitrate, 30 , and Potassium Nitrate, 60 . The product is poured into moulds.

ChARACTERS. - A white, hard solid, generally in the form of pencils or cones of a finely granular fracture, becoming gray or grayish-black on exposure to light in the presence of organic matter; odorless, having a caustic, metallic taste, and neutral to litmus paper.

2. Argenti Nitras Fusus.-Moulded Nitrate of Silver. Synonyms. - Lunar caustic. Lapis infernalis.

SourCE. -By melting Silver Nitrate, 100 ; Hydrochloric Acid, 4 ; cooling in moulds.

Characters. -A white, hard solid, generally in the form of pencils of a fibrous fracture, becoming gray or grayish-black on exposure to light.]

2. ARGENTI OXIDUM.-[Silver Oxide. $\mathrm{Ag}_{2} \mathrm{O}=23 \mathrm{r} .28$.

SOURCE. - Shake a solution of Silver Nitrate with a solution of Potassa and wash the precipitate. $2 \mathrm{AgNO}_{3}+2 \mathrm{KOH}=\mathrm{Ag}_{2} \mathrm{O}+\mathrm{KNO}_{3}+\mathrm{H}_{2} \mathrm{O}$.

CHARACTERS. - A heavy, dark brownish-black powder, having a metallic taste. Solubility. - Slightly in water.]

INCOMPATIBLES. - Chlorides and organic substances, especially creosote, for it rapidly oxidizes them and forms explosive compounds.

IMPURITY.-Metallic silver.

Dose, $1 / 2$ to $2 \mathrm{gr}$; [ .03 to $.12 \mathrm{gm}$.] in a pill with kaolin.

3. [ARGENTI CYANIDUM.-Silver Cyanide. $A g C N=133.64$.

SoURCE. - From Potassium Cyanide, which reacts with Silver Nitrate, producing the precipitate of Silver Cyanide. $\mathrm{KCN}+\mathrm{AgNO}_{3}=\mathrm{AgCN}+\mathrm{KNO}_{3}$.

Characters. - A white powder, odorless and tasteless, permanent in dry air, but gradually turning brown on exposure to light. Solubility.-Insoluble in water and Alcohol.

Silver Cyanide is used to prepare extemporaneously Acidum Hydrocyanicuin Dilutum. 
4. ARGENTI IODIDUM.-Silver Iodide. AgI=234. 19.

Source.-From Silver Nitrate and Potassium Iodide, washing and drying the precipitate. $\quad \mathrm{AgNO}_{3}+\mathrm{Kl}=\mathrm{AgI}+\mathrm{KNO}_{3}$.

ChARACTERS. - A heavy, amorphous, light-yellowish powder, without odor and taste. Solubility. - Insoluble in water and Alcohol.

Dose, $1 / 4$ to $\mathrm{Igr}$; .015 to $.06 \mathrm{gm}$.]

\section{Action of Silver Salts.}

External.-The action of silver salts is very like that of lead salts, but they are more powerful. Therefore silver nitrate is much used as a caustic, but it does not act deeply; it is consequently an admirable agent when we wish a limited caustic action on any particular part. Lotions of it may be used as astringents, but they are not so useful as lead lotions, for they are more irritating and cause pain. Silver salts, like lead salts, are hæmostatic, acting in precisely the same way. Weak solutions of the nitrate stimulate to healthier action indolent ulcers and other inflamed surfaces.

Internal.-Silver salts, when locally applied to the mucous membrane of the mouth, act as upon the abraded skin. In the stomach the ritrate is decomposed; we do not know what compound is formed, but it is said to have no astringent action. Silver is absorbed from the alimentary tract, for its long-continued use leads to a bluish-slate color of the skin (argyria). This color is due to the deposition of minute granules of metallic silver. Very little is known about its further action. In acute poisoning severe vomiting and nervous symptoms, as convulsions, are met with; in the chronic form, seen more often when silver was frequently prescribed internally, is shown by paralysis like that due to lead, albuminuria, and the discoloration above mentioned. Some is passed in the frces as the sulphide; some is deposited in the internal organs, especially the kidney.

\section{Therapeutics of Silver Salts.}

External.-Silver nitrate is much used because it is, from its limited action, one of the best caustics, and may be employed to destroy warts and exuberant granulations, or to apply to bites; but it must be remembered that it is of no use when an extensive 
or deep action is required. [Silver nitrate is a dangerous caustic to employ in deep bites, for the pellicle of silver albuminate retains the poison in the wound.] Because of its combination of an irritant stimulating effect with an astringent influence, lotions of it, of generally about $5 \mathrm{gr}$. ; [.30 gm. $]$ to the fluid ounce; [30. c.c.] of water are of much benefit when applied as a paint to weak ulcers, to bedsores, to the affected parts in chronic pharyngitis or laryngitis, or as an injection in gleet or inflammation of the [cervix] uteri. [An useful injection in gonorrhœa is silver caseinate (Argonin, not official) in $\mathbf{I} .5$ per cent. solution which causes the speedy disappearance of gonococci, but since this is not astringent, other remedies must be employed to relieve the inflammation. Silver lactate (Actol, not official) is used as an antiseptic in sore throat, gonorrhœa, etc., in a 2 per cent. solution. Silver citrate (Itrol, not official) in I to 4000 solution is employed for the same purpose]. Weaker solutions (I to 240) are employed for granular lids and various forms of ophthalmia. [Ophthalmia neonatorum is best treated by early applications of a I per cent. aqueous solution of silver nitrate. This is commonly known as Credé's method, but the original formula as prescribed by him was double this strength.] Solutions of the nitrate will sometimes relieve pruritus, and may be applied to the red skin of a threatening bedsore; very strong solutions have been recommended as a local application in erysipelas. Tinea tarsi is often treated by the application of solid silver nitrate, and ulcers of the mouth and other parts may be touched with it. It is an excellent hæmostatic for leech-bites. It is also applied to smallpox vesicles to prevent pitting, to boils, and to the uterus in chronic cervical catarrh. Protargol [not official], a proteid compound containing 8 per cent. of silver easily soluble in water, is used as an injection for gonorrhœa. The usual strength is I per cent. [Argentamine (not official) a ro per cent. solution of silver nitrate in a ro per cent. solution of ethylendiamine has been used in gonorrhœe and conjunctivitis in a $I$ to 4000 solution; also as a disinfectant. This sterilizes a pure culture of gonococci in from five to seven minutes. It can be used in as strong a solution as I to I000 in the urethra, it pene- 
trates deeply into the tissues without altering them, and by the seventh day the discharge is usually quite thin and gonococci can hardly be found. It then disappears rapidly. The iodide possesses the general properties of the nitrate.

Silver, soluble in water, an allotropic form discovered by Lea about 1890 , now termed colloidal silver (not official), has recently been well received and has obtained a permanent place in therapeutics. It is employed as a I $_{5}$ per cent. ointment (Credé) by inunction. It has been used successfully for chronic furunculosis, phlebitis and other septic processes.]

Internal.-Silver salts are not much used internally, and their continuous employment is objectionable on account of the [discoloration of the skin] produced. They were formerly often given in nervous diseases; but there is no evidence that they did any good. Although it is said that the compound of silver formed in the stomach is non-astringent, silver nitrate will certainly check severe diarrhœa, especially that of children. [Colloidal silver is entirely soluble in water, and in albuminous fluids is unirritating, so that it can be administered hypodermatically and intravenously as well as by inunction, as is mentioned above. For internal use, to prevent its conversion into a chloride in the stomach, it is first dissolved in equal parts of albumin and glycerin. The dose is $\frac{1}{6} \mathrm{gr}$, , o I gm., two or three times daily. Credé claims that it has a very beneficial influence and often affords a rapid cure in recent and also in chronic sepsis, when secondary changes in the vital organs have not occurred. It seems to inhibit the action of staphylococci and streptococci or destroy them altogether. It has been used in various conditions : osteomyelitis, so-called gonorrhœal rheumatism, puerperal fever, cerebro-spinal meningitis, and diverse septic processes. Thus far no instance of argyria from its use has been reported.] Sixty grains [4. gm.] of silver nitrate dissolved in three pints [I500. c.c.] of tepid water, and injected high up the rectum, have been used with great benefit in dysentery.

\section{[TOXICOLOGY.}

The nitrate sometimes causes acute poisoning.

Symptoms. - These are intense pain in the abdomen and muscular spasm, 
followed by vomiting and purging. The face is livid and covered with perspiration. The vomited matter is black and contains coagulated mucus. Chronic poisoning or argyria shows itself by a permanent slaty discoloration of the skin, conjunctiva and labial mucous membrane and ulcerations in the digestive tract.

Treatment.-This consists of administering a solution of sodium chloride (common salt), soothing the mucous membranes by injection of milk and relieving pain with opium. The chronic form is avoided by interrupting the treatment, using eliminating remedies, and preventing staining of the skin by baths of sodium hyposulphite.]

\section{ZINCUM.}

$$
\mathrm{Zn} .=65 \cdot[\mathrm{ro}] \text {. }
$$

I. ZINCUM.-Zinc. Zn. $[=65$. I0.

SUURCE, - Roast the native Zinc Sulphide or Carbonate, and reduce the resulting Oxide with Charcoal.

Characters. - A bluish-white metal in the form of thin sheets, or irregular, granulated pieces, or moulded into thin pencils, or in a state of fine powder.]

2. ZINCI CHLORIDUM.-[Zinc Chloride. $\mathrm{ZnCl}_{2}=\mathrm{r} 35.84$.

Synonym.-Butter of Zinc.

Source.-Dissolve Zinc in Hydrochloric Acid by boiling. The solution contains Zinc Chloride, with Iron and Lead Chlorides as impurities. These are precipitated by adding first Nitric Acid, then Zinc Carbonate. Filter and finally evaporate. $\mathrm{Zn}_{2}+4 \mathrm{HCl}=2 \mathrm{ZnCl}+2 \mathrm{H}_{2}$.

ChARACTERs. - A white, granular powder, or porcelain-like masses irregular, or moulded into pencils; odorless, of such intensely caustic properties as to make tasting dangerous, unless the salt be dissolved in much water, when it has an astringent, metallic taste. Very deliquescent. Solubility.-In 0.3 part of water; very soluble in Alcohol.

IMPURITIES.-Iron and lead chlorides,] calcium and sulphates.

3. LIQUOR ZINCI CHLORIDI.-Solution of Zinc Chloride.

SourCE.-[Prepared as above, but with the addition of water.

Characters. - A clear, colorless liquid of an astringent, sweetish taste. Sp. gr. about I.535. It contains about 50 per cent. by weight of the salt.]

\section{Action of Zinc Chloride.}

External. - It is very caustic, penetrating deeply, and limited in its effect to the seat of application. It is strongly antiseptic, and a solution of it of sp. gr. 2.0, known as Burnett's fluid, is used as a domestic antiseptic.

Internal, see Toxicology. 


\section{Therapeutics of Zinc Chloride.}

External.-It is used as a powerful caustic, and is often made into sticks with plaster of Paris to destroy warts, nævi, condylomata, lupoid patches, etc. For the same purpose it may be made into a paste with equal parts of starch or flour. [Canquoin's paste is a mixture of zinc chloride in varying strength with wheat flour and water.] Either the liquor, or Burnett's fluid, may be employed to wash out bed-pans, closets, etc., but zinc chloride is not so commonly used as other antiseptics. [Platt's chlorides are said to consist of various salts of zinc, chiefly of the chloride, in saturated solution.] Zinc chloride is not given internally.

4. $Z$ INCI SULPHAS. - [Zinc Sulphate. $\mathrm{ZnSO}_{4}+7 \mathrm{H}_{2} \mathrm{O}=286.64$.

Synonym. - White Vitriol.

Source. - Made with Zinc and Sulphuric Acid as the chloride was made from Hydrochloric Acid, and with the same precautions for removing impurities.

ChARACTERs. - Colorless, transparent rhombic crystals, without odor, very like Magnesium Sulphate (see p. 162), but having an astringent, metallic taste. Solubility. - In 0.6 part of water; insoluble in Alcohol.]

IMPURITIFS. - Lead, iron, copper and arsenic.

InCOMPATIBLES. - Alkalies and other carbonates, lime water, lead acetate, silver nitrate, astringent vegetable infusions or decoctions, and milk.

Dose, $1 / 2$ to $2 \mathrm{gr}$.; $[.03$ to $.12 \mathrm{gm}$.] (tonic) : 10 to $30 \mathrm{gr}$.; [.60 to 2.00 gm.] (emetic).

5. ZINCI CARBONAS PRECIPITATUS.-Precipitated Zinc Carbonate. $\left[2\left(\mathrm{ZnCO}_{3}\right)_{3} \mathrm{Zn}\left(\mathrm{OH}_{2}\right)_{2}=546.94\right.$ ?.

SOURCE. - Boil together solutions of $\mathrm{Zinc}$ Sulphate and Sodium Carbonate. ${ }_{3} \mathrm{ZnSO}_{4}+3 \mathrm{Na}_{2} \mathrm{CO}_{3}+2 \mathrm{H}_{2} \mathrm{O}=2\left(\mathrm{ZnCO}_{3}\right)_{3} \mathrm{Zn}(\mathrm{OH})_{2}+2 \mathrm{CO}_{2}+3 \mathrm{Na}_{2} \mathrm{SO}_{4}$. Dry the precipitated Zinc Salt.

CHARACTERS. - An impalpable, white powder, of somewhat variable chemical composition, without odor or taste. Similar in constitution to Magnesium Carbonate. Solubility. - Insoluble in water and Alcohol.]

IMPURITIES.-Sulphates, chlorides and copper.

Zinc Carbonate is rarely used except to make the Oxide and Acetate.

6. ZINCI OXIDUM.-[Zinc Oxide. $\mathrm{ZnO}=81.06$.

SovrcE.-Heat the precipitated Carbonate to redness in a crucible. $2\left(\mathrm{ZnCO}_{3}\right)_{3} \mathrm{Zn}(\mathrm{OH})_{2},=3 \mathrm{ZnO}+3 \mathrm{H}_{2} \mathrm{O}+\mathrm{CO}_{2}$.

Characters. - An amorphous, white, tasteless, and odorless powder. Solubility. - Insoluble in water and Alcohol.] 
IMPURITIES. - The carbonate and its impurities.

Dose, I to $5 \mathrm{gr}$; [ [.06 to .30 $\mathrm{gm}$.]

\section{Preparations.}

I. Unguentum [ $Z$ inci Oxidi.-Ointment of Zinc Oxide. Zinc Oxide, 200 ; Benzoinated Lard, 800 ; melted together.

2. Oleatum Zinci.-Oleate of Zinc. Zinc Oxide, 50; Oleic Acid, 950.]

7. ZINCI ACETAS.-[Zinc Acetate. $\mathrm{Zn}\left(\mathrm{C}_{2} \mathrm{H}_{3} \mathrm{O}_{2}\right)_{2},+2 \mathrm{H}_{2} \mathrm{O}=218.74$. SoURCE.-Dissolve Zinc Oxide in Acetic Acid and water, and boil. $\mathrm{ZnO}+2 \mathrm{HC}_{2} \mathrm{H}_{3} \mathrm{O}_{2}=\mathrm{Zn}\left(\mathrm{C}_{2} \mathrm{H}_{3} \mathrm{O}_{2}\right)_{2}+\mathrm{H}_{2} \mathrm{O}$. Zinc Acetate crystallizes out.

ChARACTERS. - Soft, white, six-sided monoclinic plates, of a pearly lustre, having a faintly acetous odor, and an astringent, metallic taste. Solubility.In 2.7 parts of water; and in $3^{6}$ parts of Alcohol. ]

IMPURITIES. - Those of the precipitated carbonate.

INCOMPATIBLES. - The same as of the sulphate.

Dose, $1 / 2$ to $2 \mathrm{gr}$.; [.03 to .12 $\mathrm{gm}$.] (tonic).

8. [ZINCI BROMIDUM. See Bromine.

9. ZINCI IODIDUM. See Iodine.]

Io. ZINCI VALERIANAS. See Valeriana.

11. [ZINCI PHOSPHIDUM. See Phosphorus.]

12. ZINCI SULPHOCARBOLAS. See Acidum Carbolicum.

\section{Action of Zinc Salts.}

External.-These salts when applied to the broken skin or an ulcerated surface, are all astringents, acting by precipitating the albumin in the discharge and also that in the tissues. Thus they resemble lead and silver salts, but as a whole they are less powerfully astringent. The most active of them are the sulphate and acetate, whilst the [precipitated] carbonate and oxide are very weak. All these zinc salts are mild hæmostatics.

Internal.-Alimentary canal. - They all have an astringent effect on the gastric and intestinal mucous membranes. The sulphate, and to a less degree the [precipitated] carbonate, in doses of about $20 \mathrm{gr}$. [1.20 gm.] are prompt emetics. They act directly on the stomach, and have the advantage of producing very little depression.

Remote effects. - Nothing is known about the remote action of zinc salts, nor do we know how they act on the blood. It has been stated that they are depressant to the nervous system as a 
whole, and that they act as remote astringents, and will therefore arrest hæmorrhage from the uterus, kidney, etc., but this statement is probably incorrect. The prolonged administration of zinc salts causes symptoms like those of lead poisoning. Probably the symptoms of which those who work with zinc sometimes complain are due to arsenic and other metals which contaminate zinc compounds.

\section{Therapeutics of Zinc Salts.}

External.-A solution of the sulphate, generally about [ 1 to 240], usually colored red with compound tincture of lavender, and then called Lotio Rubra, is very often applied for its astringent effect to all sorts of raw surfaces and ulcers, and as an injection in gonorrhœa, leucorrhœe, vulvitis or otitis. Plain solutions of this strength may be applied to the eye for conjunctivitis. The oleate is an excellent application to sores and ulcers when a less astringent preparation is required; and the oxide and [precipitated] carbonate, either dusted on the part or used as an ointment, are in constant use for cases in which only a mild astringent effect is desirable. An ointment, often known as Unguentum Metallorum, consists of equal parts of ointments of zinc oxide, lead acetate, and diluted mercuric nitrate. This is a very good application for many varieties of eczema, sores and ulcers. Equal parts of zinc oleate, mercuric oleate and diachylon ointment (see p. 166) form an ointment which has the great advantage of being transparent, and therefore the progress of the disease can be observed, without washing off the ointment. Calamine (purified zinc carbonate) is an excellent slight astringent for skin diseases. An ointment ( 1 to 5 of benzoinated lard) or a lotion (calamine, 3 ; zinc oxide, 3 ; lime water, I6; glycerin, 4 ; water, 160 ;) are good preparations. The following often succeeds in pruritus: Zinc oxide, 25 ; gelatin, 20 ; glycerin, 60 ; water to 480 . The jelly is to be melted when used, and applied with a brush, and then covered with cotton.

Internal. - Alimentary canal. - On account of their disagreeable taste, solutions of zinc salts are not used as astringents to 
the mouth. Small doses of the oxide or sulphate may be given as astringents in diarrhœa. The sulphate is a very good emetic for cases of poisoning, for it acts promptly without causing much nausea and hardly any depression. It is occasionally given as an emetic to children suffering from laryngitis or bronchitis.

Remote effects. - Because it is believed to act as a depressant to the nervous system, zinc sulphate has been given in hysteria, epilepsy, whooping-cough and chorea in doses of I to $3 \mathrm{gr}$. [.06 to $.20 \mathrm{gm}$.] thrice a day. Its use is now generally limited to chorea, but often its effect is so slow that it is difficult to prove that the patient would not have improved quite as rapidly without any drug. It is usually said to be a tonic, but there is no trustworthy evidence for this statement. The oxide [Trousseau's pill ( $5 \mathrm{gr}$; $.30 \mathrm{gm}$. of zinc oxide with I gr. ; $.06 \mathrm{gm}$. of extract of hyoscyamus)], given internally will occasionally check the night-sweats of phthisis [but it is quite likely to interfere with the digestion].

\section{Toxicology.}

Symptoms. - Zinc Chloride is a corrosive irritant poison, causing a sensation of burning in the mouth and throat, abdominal pain, vomiting - the vomit containing blood, mucus, and shreds of mucous membrane,- -violent purging, and collapse. [Zinc Sulphate, in large doses, acts as an irritant poison producing vomiting, colicky pains, diarrhœa and prostration.] Post-mortem.-The appearances are those produced by an acute irritant.

Treatment. - Wash out the stomach, or give emetics (see p. 139), and then demulcents; [lime water, mucilaginous drinks, and albumin freely in the form of eggs or milk.]

\section{CUPRUM.}

$$
\mathrm{Cu}=63.18 \text {. }
$$

I. CUPRI SULPHAS. - [Copper Sulphate. $\mathrm{CuSO}_{4}+5 \mathrm{H}_{2} \mathrm{O}=248.8$. Synonyms. - Blue Vitriol. Bluestone. Cupric Sulphate.

SourCE.-Heat Copper and Sulphuric Acid together, and dissolve the soluble product in hot water and evaporate. $2 \mathrm{Cu}+2 \mathrm{H}_{2} \mathrm{SO}_{4}=2 \mathrm{CuSO}_{4}+2 \mathrm{H}_{2}$.

Characters. - I.arge, transparent, deep blue, triclinic crystals, odorless, of a nauseous, metallic taste. Solubility.-In 2.6 parts of water; almost insoluble in Alcohol.]

IMPURITY.-Iron.

INCOMPATIBLES. - Alkalies and their carbonates, lime water, mineral salts (except sulphates), iodides and most vegetable astringents. 
Dose, $1 / 8$ to $\mathrm{x} / 2 \mathrm{gr}$; ; [.008 to $.03 \mathrm{gm}$.], (astringent), 2 to $20 \mathrm{gr}$; [ [.12 to $1.20 \mathrm{gm}$.] (emetic).

[Copper Sulphate is used in preparing Trommer's and Fehling's test for sugar.]

\section{Action of Copper Sulphate.}

External.-In the solid form copper sulphate is, when applied to raw surfaces, a powerful caustic. In dilute solutions it is an astringent, acting like zinc sulphate, but more powerfully.

Internal.-Alimentary canal.-Here also, if very concentrated or given in large doses, copper sulphate is an acute caustic irritant, but poisoning by it is very rare. In medicinal doses it is strongly astringent. Five to ten grains [.30 to .60 gm.] of the sulphate form a powerful emetic, acting directly on the stoinach. As it is more irritating than zinc sulphate it acts more readily, but it has the disadvantage that, if it fails to act, the stomach must be promptly emptied by some other means, for if not the copper sulphate will cause inflammation of it.

Remote effects. - Copper salts are slowly absorbed, and copper is chiefly re-excreted by the liver in the bile. Nothing is known of its further effects.

\section{Therapeutics of Copper Sulphate.}

External.-The sulphate is applied as a caustic to reduce exuberant granulations, and is used for tinea tarsi, being rubbed on the edges of the lids; as it is milder than silver nitrate, it causes less pain. The Lapis Divinus, which is often used for this last purpose, consists of copper sulphate, potassium nitrate, [and alum, of each] 24 parts, [and] camphor I part. The first three are fused together. The camphor is added, and the mass is cast into cylindrical moulds. Lotions of copper sulphate, usually about [I to 240], may be applied as astringents for just the same purpose as lotions of zinc sulphate; but it must be remembered that they are more powerful. This is the usual strength for solutions which are to be dropped into the eye. Rather stronger solutions are mild hremostatics. Copper oleate [not official] made with lanolin, into an ointment of a strength of 10 to 20 per cent. is an excellent parasiticide for ringworm. 
Internal.- In small doses copper sulphate is valuable for severe diarrhœa ; usually it is given by the mouth in the form of a pill, but it may be given as a rectal injection. It is a rapid emetic, and may be employed in laryngitis and bronchitis in children, and in cases of narcotic poisoning, for which it is useful on account of its prompt action. It is [extremely doubtful if it is] particularly serviceable in phosphorus poisoning [but] if it is used, copper [may be] deposited on the phosphorus rendering it inert. It is usual to give three or four grains [. 20 to $.25 \mathrm{gm}$.] of the sulphate in water every few minutes till vomiting takes place. After emetic doses of copper sulphate, there is generally only one act of vomiting, but by that the stomach is completely emptied. Very little is known about the remote action of salts of copper, but it is stated that the sulphate will cure chlorosis, [and it has been recommended for the treatment of syphilis.]

\section{Toxicology.}

Symptoms. - In sufficient doses copper salts are violent gastro-intestinal irritants, but acute poisoning is very rare. Copper may be taken in very small quantities for a long time without producing any ill effects, for many persons habitually consume, without harm, preserved vegetables, the green color of which is due to preparation with copper. It has been thought that coppersmiths are particularly liable to phthisis, but they are not more prone to it than the followers of other dusty trades. Workers in brass may suffer from anæmia, a green line on and at the base of the teeth, wasting, weakness, dyspepsia, tremors, headache, vague pains, pharyngeal and laryngeal catarrh with occasional hæmoptysis and aphonia, and profuse secretion of sweat which may be green. These symptoms are thought to be due to the copper contained in brass. Sometimes colic is due to the contamination of copper and brass by lead.

[ Treatment.-For acute poisoning give albumin, milk or magnesia. Potassium ferrocyanide is the chemical antidote. Then promptly empty the stomach and saturate the system with potassium iodide. Chronic poisoning is best treated by the administration of fifteen drops of diluted phosphoric acid before each meal, the ingestion of large quantities of milk and thorough daily evacuation of the bowels with magnesium or sodium sulphate.]

\section{BISMUTHUM.}

$$
\mathrm{Bi}=[208.9 \text {. }
$$

1. BISMUTHI SUBCARBONAS.-Bismuth Subcarbonate. $(\mathrm{BiO})_{2}$, $\mathrm{CO}_{3}+\mathrm{H}_{2} \mathrm{O} ?=527.53$ ? 
Source. - Dissolve Purified Bismuth in Nitric Acid and water, decant and filter, mix with Ammonia Water; the precipitate is washed and dissolved in Nitric Acid, and poured into a solution of Sodium Carbonate, the resulting precipitate is collected and washed. The final reaction is $2 \mathrm{Bi}\left(\mathrm{NO}_{3}\right)_{3}+3 \mathrm{Na}_{2} \mathrm{CO}_{3}$ $+\mathrm{H}_{2} \mathrm{O}=(\mathrm{BiO})_{2}, \mathrm{CO}_{3}+\mathrm{H}_{2} \mathrm{O}+6 \mathrm{NaNO}_{3}+2 \mathrm{CO}_{2}$. The precipitated Carbonate is separated by filtration.

Characters. - A white or pale yellowish-white powder, of somewhat varying chemical composition, odorless and tasteless. Solubility.-Insoluble in water and Alcohol.]

IMPURITIES. - [Lead, arsenic, chlorides and nitrates, tellurium, the lașt giving an alliaceous odor to the breath.]

Dose, 5 to $60 \mathrm{gr}$; [ [.30 to $4.00 \mathrm{gm}$.]

2. BISMUTHI SUBNITRAS. $-\left[\right.$ Bismuth Subnitrate. $\mathrm{BiONO}_{3}+$ $\mathrm{H}_{2} \mathrm{O}$ ? $=304.7 \mathrm{I}$ ?.]

Source. - Dissolve Purified Bismuth in Nitric Acid and water, concentrate by evaporation, pour in more water, and stir thoroughly, wash and dry the precipitated Subnitrate. $\left[\mathrm{Bi}_{2}+6 \mathrm{HNO}_{3}=2 \mathrm{Bi}\left(\mathrm{NO}_{3}\right)_{3}+3 \mathrm{H}_{2}\right.$ and $\mathrm{Bi}\left(\mathrm{NO}_{3}\right)_{3}$ $+\mathrm{H}_{2} \mathrm{O}=\mathrm{BiONO}_{3}+2 \mathrm{HNO}_{3}$.

ChARACTERS. - A heavy, white powder, of somewhat varying chemical composition, odorless and almost tasteless. Solubility.-Almost insoluble in water; soluble in Alcohol.]

IMPURITIES. - As of the subcarbonate.

Dose, 5 to $60 \mathrm{gr}$.; [.30 to $4.00 \mathrm{gm}$.]

[Bismuth subnitrate is used to prepare Neylander's test for glucose in urine.]

3. BISMUTHI CITRAS. - [Bismuth Citrate. $\mathrm{BiC}_{6} \mathrm{H}_{5} \mathrm{O}_{7}=397.44$.

SoUrCE. - Boil Bismuth Subnitrate, 100 ; in Citric Acid, 70 ; dissolved in sufficient water. Wash the Precipitate and dry. $\mathrm{BiONO}_{3}+\mathrm{H}_{3} \mathrm{C}_{6} \mathrm{H}_{5} \mathrm{O}_{7}+$ $\mathrm{H}_{2} \mathrm{O}=\mathrm{BiC}_{6} \mathrm{H}_{5} \mathrm{O}_{7}+\mathrm{NO}_{4}+\mathrm{H}_{2} \mathrm{O}$.

CharaCters. - A white, amorphous micro-crystalline powder. Solubility. -Insoluble in water; soluble in Ammonia.

IMPURITIES. - As of the subcarbonate.]

Dose, 1 to $3 \mathrm{gr}$.; [.06 to $.20 \mathrm{gm}$.]

\section{Preparation.}

Bismuthi et Ammonii Citras.-[Bismuth and Ammonium Citrate.

Source. - Mix Bismuth Citrate, I00; with distilled water to make a paste, heat, add Ammonia Water to make a solution, filter, evaporate, and dry on plates of glass.

ChARACTERS.-Small, shining, pearly or translucent scales, of a slightly acidulous and metallic taste. Solubility.-Very soluble in water; sparingly soluble in Alcohol.]

Dose, 2 to $5 \mathrm{gr}$; [.12 to .30 $\mathrm{gm}$.] 
4. BISMUTHI SALICYLAS.-[Bismuth Salicylate (Not official.)$\mathrm{Bi}\left(\mathrm{C}_{7} \mathrm{H}_{5} \mathrm{O}_{3}\right)_{3} \mathrm{Bi}_{2} \mathrm{O}_{3}=1084.59$.

SOURCE. - It is prepared by diluting a Glycerin solution of crystallized Bismuthous Nitrate with water, and decomposing this with a concentrated aqueous solution of Sodium Salicylate; the precipitate is well washed with hot water and carefully dried. It contains about 76 per cent. of Bismuth Oxide and 24 per cent. of Salicylic Acid.

Characters. - It is a soft white powder. Solubility. - Insoluble in water, Glycerin and Ether, but soluble in acids.

Dose, 5 to $20 \mathrm{gr}$. ; $[.30$ to $\mathrm{x} .20 \mathrm{gm}$.]

\section{Action of Bismuth Salts.}

External.-Bismuth salts have no action on the unbroken skin. Dusted on a raw surface they form a protecting coat, are germicidal, and are very mildly astringent. [For this purpose the tribromophenolate and subgallate, the latter also known as dermatol (both unofficial), are preferable.]

Internal.-If large doses of bismuth salts are injected under the skin of animals, or if large doses of soluble salts are given to them by the mouth, they produce effects as severe as those due to antimony. The chief are gastro-intestinal irritation and fatty degeneration, and it is stated that some very susceptible persons may be poisoned by large doses by the mouth of insoluble salts; but, as a rule, any symptoms of gastro-intestinal irritation caused by the insoluble bismuth salts are due to the arsenic with which they may be contaminated. When pure, it is probable that these salts, like any bland heavy powder, act chiefly as protectives to the gastro-intestinal mucous membrane; [for gastric ulcer the subnitrate is to be chosen]. They have an astringent action, diminishing secretion, and are [especially the naphtolate (not official)] gastro-intestinal antiseptics. Bismuth is slowly absorbed and excreted in the urine [Bergeret and Mayençon], and it may be found in the liver, spleen, kidneys, and nervous system. Nothing is known of any remote effects. Bismuth leaves the rectum as the sulphide, and colors the fæces black. It may cause a purplish line on the gums.

\section{Therapeutics of Bismuth Salts.}

External.-Bismuth salts may be dusted on sores as protectives and mild astringents. The following is a good bismuth 
ointment:-Bismuth oxide [official in B. P.] I part, and oleic acid 8 parts, stirred in with 3 parts of white wax liquefied by heat, and with 9 parts of soft paraffin. The subnitrate is sometimes snuffed up the nose during a cold, and suspended in mucilage it may be used as an injection for gonorrhœea or leucorrhœa.

Internal.-The subnitrate and the subcarbonate are chiefly employed, and they seem to be more efficient than the soluble preparations. [Soluble bismuth salts when absorbed are likely to act as active irritant poisons, and for this reason should not be used.] The insoluble preparations must be suspended in mucilage [of tragacanth, for with acacia a compact mass is formed at the bottom of the bottle]; given thus they are more efficacious than as a lozenge. It is not known how the effect is produced, but either of these salts is remarkably efficient in removing gastric pain, whether due to ulcer or to gastritis, or even when no cause can be detected. Both of these drugs will often stop the vomiting due to gastritis, gastric ulcer, chloroform, pregnancy, or indeed any other cause. For their astringent action they are given in diarrhœa, doses of $60 \mathrm{gr}$. [4. gm.] being administered without any ill effect, and some believe that part of the benefit is due to the antiseptic action of bismuth salts. They appear sometimes to check the severe diarrhoea of tuberculous ulceration of the bowel. [For internal use the phenolate and naphtolate (neither are official) are preferable to the inorganic salts. They are employed in the same dose.] Their efficacy as gastric anodynes and gastric astringents is much increased by combination with a little morphine, and if given as gastric sedatives the addition of sodium bicarbonate as well as the morphine is an advantage. In such a prescription the bismuth subcarbonate is preferable to the subnitrate, for the latter may act on the sodium bicarbonate and lead to the production of sufficient carbon [dioxide] to drive the cork out of the bottle. The salicylate has been largely used in various gastric disorders. It is supposed to combine the virtues of bismuth salts with the antiseptic action of salicylic acid. [Bismuth salicylate probably passes through the stomach unchanged to be broken up in the small intestine where it acts as an unirritating antiseptic. It has 
been proved to be a valuable remedy in the treatment of diarrhœas, typhoid fever and catarrhs of the alimentary tract. Heuppe's observations in Asiatic cholera prove that the tribromophenolate is a valuable intestinal antiseptic. Bismuth subgallate (dermatol) was formerly much employed in the treatment of gastro-intestinal indigestion, but has been supplanted by the more efficient naphtolate and tribromophenolate.

Eudoxin (not official) is bismuth tetra-iodophenol-phthaleinate, and occurs as a tasteless, odorless, reddish-yellow, insoluble powder. This is decomposed in the intestines, and is claimed to be a germicide. It is certainly known that iodine is liberated. It - is employed as an intestinal antiseptic in dose of from 5 to 8 gr. ; 30 to $.50 \mathrm{gm}$.

\section{Toxicology.}

When applied in large quantity to an exterior wounded surface sufficient bismuth may be absorbed to produce poisoning. This may also occur if glycerin is used to form an emulsion for injection into closed cavities (abscesses, joint-disease).

Symptoms. - These are acute stomatitis with a peculiar blackish discoloration of the mucous membrane, generally upon the borders of the teeth and extending over the whole mouth, ulceration of the mucous membrane, intestinal catarrh, pain and diarrhoea. Even desquamative nephritis may be set up.

Trealment.-Use demulcents.]

\section{ALUMINUM.}

$$
\mathrm{Al}=27.04 \text {. }
$$

I. ALUMEN.-Alum. $\mathrm{Al}_{2} \mathrm{~K}_{2}\left(\mathrm{SO}_{4}\right)_{2}+24 \mathrm{H}_{2} \mathrm{O}[=946.46$. Synonyms. Aluminum and Potassium Sulphate. Potassium Alum.

Source.-Made from Alum Slate or Shale (a mixture of Aluminum Silicate and Iron Sulphide) by roasting and exposing to the air. By lixiviating the heaps with water, Sulphuric Acid, Aluminum and Iron Sulphates are obtained in solution. This solution is concentrated, and while hot mixed with Potassium Chloride, which, reacting upon the Ferric Sulphate, yields Potassium Sulphate and Ferric Chloride, while the Alum separates on cooling as a crystalline powder. It is then purfied by one or two recrystallizations. Also by making an Aluminum Sulphate from $\mathrm{Al}(\mathrm{OH})_{3}$, the by-product in the manufacture of Soda from cryolite.

ChARACTERS. - Large, colorless, octahedral crystals, sometimes modified by cubes, or in crystalline fragments, without odor, but having a sweetish and strongly astringent taste. On exposure to the air, the crystals are liable to absorb ammonia, and acquire a whitish coating.] Solubility.-In 9 parts of water. 
Incompatibles. - Alkalies, lime, lead, mercury, and iron salts, tartrates and tannic acid.

IMPURITIES. -Ferrous sulphate and silicates.

Dose, 5 to $30 \mathrm{gr}$. ; [.30 to $2.00 \mathrm{gm}$. ]

\section{Preparation.}

Alumen Exsiccatum. - [Dried Alum. Synonyms. - Alumen Ustum. Burnt Alum.

SourCE. - Heat 100 parts of Alum with moderate heat till aqueous vapor ceases to be disengaged, and it is reduced to 55 parts.

Characters. - A white, granular powder, without odor, possessing a sweetish, astringent taste, and attracting moisture from the air. Solubility.-Slowly but completely soluble in 20 parts of water.

2. ALUMINI HYDRAS.-Aluminum Hydrate. $\mathrm{Al}_{2}(\mathrm{OH})_{6}=\mathbf{1 5 5 . 8 4}$. Synonyms.-Aluminum Hydroxide. Hydrated Alumina.

Source.-By separate solution of Alum, IOO; and Sodium Carbonate, roo in distilled water and heat. Mix the hot solutions, wash the precipitate with hot distilled water, dry and reduce to a fine powder.

Characters. - A white, light amorphous powder, odorless and tasteless, permanent in dry air. Solubility.-Insoluble in water or Alcohol.

Dose, I to ro gr.; [.06 to .60 gm.]

3. ALUMINI SULPHAS. - Aluminum Sulphate. $\mathrm{Al}_{2}\left(\mathrm{SO}_{4}\right)_{3}+16$ $\mathrm{H}_{2} \mathrm{O}=628.9$.

Source.-Aluminum Hydroxide, $\mathrm{Al}(\mathrm{OH})_{3}$, a by-product in the manufacture of Soda from cryolite, is dissolved in diluted Sulphuric Acid ; the solution is filtered and evaporated to dryness.

Characters. - A white, crystalline powder, without odor, having a sweetish and afterwards astringent taste. Solubility.-1.2 parts of water; almost insoluble in Alcohol.]

\section{Action of Aluminum Salts.}

External.-They have no action on the unbroken skin, but coagulate the albumin of the discharges from ulcers, sores, etc., and thus form a protecting covering to the parts, and act as efficient astringents. The albumin in the tissues themselves is coagulated also. This coagulated albumin will compress and occlude the vessels, and thus alum is hæmostatic. Dried alum absorbs water, and therefore its solid form is mildly caustic. [Aluminum acetate (not official) in saturated solution is a very penetrating antiseptic.] 
Internal.-Alimentary tract.-Alum is an excellent astringent for the mouth, stomach and intestines, and will cause constipation. In large doses it is emetic, acting directly on the stomach, and in larger still, irritant and purgative. Most, if not all, is passed by the frecs; probably, in medicinal doses, it has no more remote effects on the tissues.

Nervous System.-Given to animals, in large doses, it produces paresis, loss of sensation, forced movements, drowsiness and death from respiratory paralysis.

\section{Therapeutics of Aluminum Salts.}

External.-Alum is occasionally used as a caustic to destroy weak, exuberant granulations. Kaolin [a native aluminum silicate, freed from gritty particles (not official)] is a good dusting powder. Fuller's earth [of similar chemical composition] is used [for the same purpose]. Kaolin resists most chemical reagents, and therefore it is used as a basis for making pills of such bodies as phosphorus, silver nitrate or potassium permanganate, for with them chemical reaction would occur if an ordinary basis were used. [An excellent substitute for poultices is made as follows: Kaolin, rooo parts, is sifted and sterilized by heat; glycerin, rooo parts, is added, the heat being continued and mixed by stirring for half an hour. When nearly cool add boric acid, roo parts, and oil of peppermint, $\mathbf{r}$; oil of wintergreen, $\mathbf{~}$; and oil of eucalyptus, 2 parts.] Because of its astringency alum has many uses; it may, for example, be applied to weeping eczematous surfaces, and as an injection or on [moistened] lint for vulvitis of children. Solutions of it have been used for leucorrhœa and gleet. Ten grains $[; .60 \mathrm{gm}$. $]$ to the fluid ounce; [30. c.c.] of water is a common strength for most purposes. Five grains $[; .30 \mathrm{gm}$.$] to the fluid ounce; [30. c.c.] make a$ good eye wash or a gargle. Strong solutions, or powdered alum, applied locally, stop bleeding, if it is not severe, such as occurs from piles, leech-bites, slight cuts, the gums and the nose. [Aluminum naphtol-sulphonate (alumnol, not official) in $x$ to 3 per cent. solutions is an unirritating astringent which, although precipitating albumin, dissolves it when in excess and therefore pen- 
etrates below the surface. It is used for the treatment of acute and chronic inflammations of various mucous membranes.]

Internal.-Alimentary canal. - As a mouth wash or gargle [ $\mathrm{I}-2$ to $\mathrm{r} 00]$, alum is very valuable in ulcerative stomatitis, in aphthous conditions of the mouth, and in slight pharyngitis or tonsilitis. [Alum as a mouth wash attacks the enamel of the teeth.] One part of alum with five parts of glycerin, painted on with a camel's hair brush, is excellent for these conditions. If the nose be irrigated with a solution of alum, it may remedy a chronic ozæna. It has been found that other astringents are preferable for bleeding from the stomach and for diarrhœa, but a teaspoonful [4. gm.] of alum, dissolved in syrup, and given every quarter of an hour till vomiting is produced, is an excellent emetic for children, and may be used to produce vomiting in laryngitis and bronchitis, as it is non-depressant. Alum whey, obtained from milk curdled by àlum, may be given in the diarrhœa of typhoid fever. In lead colic, alum may open the bowels, probably because, being a sulphate, it precipitates any lead salts as insoluble lead sulphates.

\section{GROUP V. \\ Ferrum, Manganum.}

It is probable that the action of these drugs is, in some respects, similar.

\section{FERRUM.}

$$
\mathrm{Fe}=55.88 \text {. }
$$

I. FERRUM.-[Metallic Iron in form of fine, bright and non-elastic wire.]

Metallic Iron is pharmacopœial in two forms, viz., this and reduced iron.

[Metallic Iron is used to prepare Ferri Iodidum Saccharatum, Ferri Chloridum, Liquor Ferri Chloridi, and Syrupus Ferri Iodidi.]

2. FERRUM REDUCTUM.-Reduced Iron. [Synonyms.-Quevenne's Iron. Iron by Hydrogen.] Metallic Iron with a variable amount of oxide.

SOURCE. - [Hydrogen gas is passed through a hot, closed tube which contains freshly prepared, thoroughly washed Ferric Oxide. $\mathrm{Fe}_{2} \mathrm{O}_{3}+3 \mathrm{H}_{2}=\mathrm{Fe}_{2}+$ $3 \mathrm{H}_{2} \mathrm{O}$. 
ChARACTERs. - A very fine grayish-black lustreless powder, strongly attracted by the magnet, without odor or taste. Solubility.-Insoluble in water or Alcohol.]

IMPURITY.-Sulphur.

[Reduced Iron is used to make Pilulæ Ferri Iodidi and Ferri Iodidum Saccharatum.

Dose, I to $5 \mathrm{gr} . ; .06$ to $.30 \mathrm{gm}$.]

The following (viz., the sulphate, the carbonate, [the iodide and the lactate)] are ferrous salts, that is to say, salts of the lower Oxide, $\mathrm{FeO}$.

3. FERRI SULPHAS. - [Ferrous Sulphate. $\mathrm{FeSO}_{4}+7 \mathrm{H}_{2} \mathrm{O}=\mathbf{2 7 7 . 4 2}$. Synonyms. - If impure, Copperas. Green Vitriol.

SOURCE.-Iron Wire is dissolved by boiling in Sulphuric Acid and water. The sulphate is crystallized out. $\mathrm{Fe}_{2}+2 \mathrm{H}_{2} \mathrm{SO}_{4}=2 \mathrm{FeSO}_{4}+2 \mathrm{H}_{2}$.

Characters. - Large pale, bluish-green monoclinic prisms, having a saline], styptic taste. Solubility.-In I.8 parts of water; insoluble in Alcohol.

IMPURITIES. - Ferric salts and copper.

[Ferrous Sulphate is used to make Ferri Carbonas Saccharatus, Liquor Ferri Subsulphatis, Liquor Ferri Tersulphatis, and Massa Ferri Carbonatis.

Dose, $1 / 2$ to $2 \mathrm{gr}$; .03 to $.12 \mathrm{gm}$.]

\section{Preparations.}

I. Ferri Sulphas Exsiccatus.-[Dried Ferric Sulphate. Approximately $2 \mathrm{FeSO}_{4}+3 \mathrm{H}_{2} \mathrm{O}=357.28$.

Source. - Allow the sulphate to effloresce at $104^{\circ} \mathrm{F} . ; 40^{\circ} \mathrm{C}$., and heat in a porcelain dish until it weighs 64 to 65 parts.

ChARACTERs. - A grayish-white powder, slowly but completely soluble in water.]

Dose, $1 / 2$ to $2 \mathrm{gr}$.; [ .03 to $.12 \mathrm{gm}$.

Dried Ferrous Sulphate is used to make Pilulæ Aloes et Ferri.]

2. [Ferri Sulphas Granulatus.-Granulated Ferrous Sulphate. $\mathrm{FeSO}_{4}+7 \mathrm{H}_{2} \mathrm{O}=277.42$.

SOURCE. - Dissolve Ferrous Sulphate, 100; in diluted Sulphuric Acid, 5 ; and Distilled Water, IO0; pour upon it Alcohol, 25; and filter, wash and dry the precipitate.

Characters.-Pale, bluish-green, crystalline powder, which conforms to the reactions of the sulphate.

IMPURITIES. - The same as of the sulphate.

Dose, $1 / 2$ to $2 \mathrm{gr}$; .03 to .12 $\mathrm{gm}$.]

3. Mistura Ferri Composita.-Compound Iron Mixture. Synonym.-Griffith's Mixture. [Ferrous Sulphate, 7 ; Potassium Carbonate, 8 ; Myrrh, 18 ; Sugar, 18 ; Spirit of Lavender, 60 ; Rose Water to 1000.] It is a dark-green mixture containing Ferrous Carbonate, 
for Ferrous Sulphate and Potassium Carbonate react on each other. Dose, 2 to 4 fl. dr.; [8. to 15. c.c.]

4. [Pilulæ Ferri Carbonatis.-Pills of Ferrous Carbonate. Synonyms.-Ferruginous pills. Chalybeate pills. Blaud's pills.

Ferrous Sulphate, 16; Potassium Carbonate, 8 ; Sugar, 4 ; Tragacanth, $\mathrm{x}$; Althæa, I gm.; Glycerin and water; to make 100 pills.

Dose, $I$ to 2 pills.]

4. FERRI CARBONAS SACCHARATUS. - [Saccharated Ferrous Carbonate. A powder containing Ferrous Carbonate $\mathrm{FeCo}_{3}$, made from Ferrous Sulphate, 50; Sodium Bicarbonate, 35 ; Sugar, and distilled water by solution and filtration.

CharActers. - A greenish-brown powder, of a sweetish, afterwards slightly ferruginous taste.] It is a very unstable compound, being easily oxidized. Solubility.-Only partially in water.

IMPURITIES.-[Sodium] Sulphate and excess of Ferrous Oxide.

Dose, 2 to $10 \mathrm{gr}$.; $[.12$ to $.60 \mathrm{gm}$.]

5. [MASSA FERRI CARBONATIS.-Mass of Ferrous Carbonate. Synonym.-Vallet's Mass. Ferrous Sulphate, 100; Sodium Carbonate, 100 ; Clarified Honey, 38 ; Sugar, 25 ; Syrup and distilled water to 100. By solution, precipitation and evaporation.

Dose, I to $5 \mathrm{gr}$; ; .06 to $.30 \mathrm{gm}$.

6. FERRI IODIDUM SACCHARATUM. - Saccharated Ferrous Iodide.

SOURCE.-From Iron Wire, 6; Reduced Iron, I ; Iodine, 17 ; distilled water, Sugar of Milk to 100 . By solution, filtration, evaporation and trituration.

Characters. - A yellowish-white powder, very hygroscopic, odorless, having a sweetish ferruginous taste. Solubility. - In 7 parts of water; only partially soluble in Alcohol.

Dose, 5 to $15 \mathrm{gr}$; .30 to $1.00 \mathrm{gm}$.]

7. SYRUPUS FERRI IODIDI.-[Syrup of Ferrous Iodide. A syrupy liquid, containing to per cent. by weight of Ferrous Iodide. Iron Wire, 25 ; Iodine, 83 ; Syrup and distilled water, a sufficient quantity to 1000.

ChARACTERs. - A transparent, pale-green liquid, odorless, having a sweet strongly ferruginous taste and a neutral reaction. Sp. gr. about 1.353 .

Dose, 5 to $30 \mathrm{~m}$.; .30 to 2.00 c.c.]

8. PILULA FERRI IODIDI__[Pills of Ferrous Iodide. Triturate Reduced Iron 4, with Iodine 5, and water; add Glycyrrhiza 1, Sugar 4, Extract of Glycyrrhiza I, and Acacia $i \mathrm{gm}$., with sufficient water, Balsam of Tolu and Ether, and evaporate to make roo pills.] To be preserved from light and air as much as possible, as they do not keep well.

Dose, $I$ to 2 pills. 
9. [FERRI LACTAS. - Ferrous Lactate. $\mathrm{Fe}\left(\mathrm{C}_{3} \mathrm{H}_{5} \mathrm{O}_{3}\right)_{2}+3 \mathrm{H}_{2} \mathrm{O}=$ 287.34.

SOURCE. - By crystallization from a solution of Lactic Acid and Iron in distilled water.

Characters. - Pale, greenish-white crusts, consisting of small needleshaped crystals, having a slight, peculiar odor, and a mild, sweetish ferruginous taste. Solubility. - In 40 parts of water, and almost insoluble in Alcohol.

Ferrous Lactate is used in Syrupus Hypophosphitum cum Ferro.

Dose, $\mathrm{I}$ to $5 \mathrm{gr}$. ; .06 to .30 $\mathrm{gm}$.]

The following (viz., the Chloride, the Tersulphate, the Nitrate, the Subsulphate, the Citrate, the Acetate, the Hydrate [the Ammonium Sulphate, the Hypophosphite and the Valerianate] are Ferric Salts: they are compounds of the higher Oxide, $\mathrm{Fe}_{2} \mathrm{O}_{3}$. Most are official in the form of liquors.

10. [FERRI CHLORIDUM. - Ferric Chloride. $\mathrm{Fe}_{2} \mathrm{Cl}_{6}+12 \mathrm{H}_{2} \mathrm{O}=$ 539.5 .

SOURCE. - Iron Wire, 15; Hydrochloric Acid, and distilled water to 60. By solution in water with heat, filtration, addition of Nitric Acid, and crystallization.

Characters.-Orange-yellow, crystalline pieces, very deliquescent, having a faint odor of Hydrochloric Acid and strongly styptic taste. Solubility. -Freely in water, Alcohol and Ether.]

II. LIQUOR FERRI CHLORIDI.-[Solution of Ferric Chloride. An aqueous solution of Ferric Chloride $\left(\mathrm{Fe}_{2} \mathrm{Cl}_{6}=323.98\right)$, containing about 37.8 per cent. of the Anhydrous Salt, or corresponding to about 13 per cent. of Metallic Iron.

SOURCE.-Dissolve Iron Wire, 15.8, in Hydrochloric Acid, 870, and water to 1000 . $\mathrm{Fe}+2 \mathrm{HCl}=\mathrm{FeCl}_{2}+\mathrm{H}_{2}$. Nitric Acid is also added, and thus the Ferrous is converted into Ferric Chloride. $6 \mathrm{FeCl}_{2}+6 \mathrm{HCl}+2 \mathrm{HNO}_{3}$ $=3 \mathrm{Fe}_{2} \mathrm{Cl}_{6}+4 \mathrm{H}_{2} \mathrm{O}+2 \mathrm{NO}$.

Characters. - A reddish-brown liquid, having a faint odor of Hydrochloric Acid, and an acid, strongly styptic taste. Sp. gr. about I.387.]

IMPUR ITIES. - Ferrous salts.

Dose, 2 to $10 \mathrm{~m} . ;$ [.12 to $.60 \mathrm{c.c}$.

\section{Preparations.}

I. Tinctura Ferri Chloridi.-[Tincture of Ferric Chloride. Solution of Ferric Chloride, 250; Alcohol to I000.

Characters. - A bright, brownish liquid, having a slightly ethereal odor, a very astringent, styptic taste, and an acid reaction. Sp. gr. about 0.960 .]

Dose, 5 to $60 \mathrm{~m}$.; [.30 to 4.00 c.c.] 
2. Liquor Ferri et Ammonii Acetatis.-[Solution of Iron and Ammonium Acetate. Synonym.-Basham's Mixture. Tincture of Ferric Chloride, 20; diluted Acetic Acid, 30 ; Solution of Ammonium Acetate, 200; Aromatic Elixir, 100; Glycerin, 120; water to 1000.

Dose, 1 to 4 fl. dr.; 4. to 15. c.c.]

12. LIQUOR FERRI TERSULPHATIS. - [Solution of Ferric Sulphate. An aqueous solution of normal Ferric Sulphate $\left(\mathrm{Fe}_{2}\left(\mathrm{SO}_{4}\right)_{3}=399.2\right)$, containing about 28.7 per cent. of the salt, and corresponding to about 8 per cent. of Metallic Iron.

Source. - A hot solution of Ferrons Sulphate, 400 ; in Sulphuric Acid, 78 ; and water, is boiled with Nitric Acid and water to $1000.6 \mathrm{FeSO}_{4}+$ ${ }_{3} \mathrm{H}_{2} \mathrm{SO}_{4}+2 \mathrm{HNO}_{3}=3 \mathrm{Fe}_{2}\left(\mathrm{SO}_{4}\right)_{3}+4 \mathrm{H}_{2} \mathrm{O}+2 \mathrm{NO}$.

Characters. - A dark reddish-brown liquid, having an acid, strongly styptic taste, and an acid reaction. Sp. gr. about 1.320.]

13. LIQUOR FERRI NITRATIS. - [Solution of Ferric Nitrate. $\mathrm{Fe}_{2}\left(\mathrm{NO}_{3}\right)_{6}=483.1$. An aqueous solution containing about 6.2 per cent. of the Anhydrous Salt, and corresponding to about 1.4 per cent. of Metallic Iron.

SourCE._Precipitate Ferric Sulphate, I80; with Ammonia water, 160; wash thoroughly and dissolve in Nitric Acid, 71 ; distilled water to 1000 .

Characters. - A clear, amber-colored or reddish liquid, having an acid, styptic taste, and an acid reaction. Sp. gr. about $\mathbf{1}$.050.]

IMPURITIES. -Ferrous salts.

Dose, 2 to $10 \mathrm{~m}$.; [.12 to .60 c.c.]

14. LIQUOR FERRI SUBSULPHATIS. - [Solution of Ferric Subsulphate. Synonym.-Monsel's solution. An aqueous solution of Basic Ferric Sulphate of variable chemical composition, corresponding to about 13.6 per cent. of Metallic Iron.

SuUrce_-From Ferrous Sulphate, 675 ; Sulphuric Acid, 65 ; Nitric Acid, 65 ; distilled water to 1000 .

Characters. - A dark, reddish-brown liquid, odorless, having an acid, strongly styptic taste, and an acid reaction. Sp. gr. about 1.550 .

Dose, 2 to $10 \mathrm{~m}$.; .12 to .60 c.c.]

15. LIQUOR FERRI CITRATIS.-[Solution of Ferric Citrate.

An aqueous solution of Ferric Citrate, corresponding to about 7.5 per cent. of Metallic Iron.

SoURCE.-From solution of Ferric Sulphate, 1050 ; in water ; precipitation with Ammonia Water, 880 ; with addition of Citric Acid, 300; filtration and evaporation of filtrate to 1000 .

Characters. - A dark-brown liquid, odorless, having a slightly ferruginous taste, and an acid reaction. Sp. gr. 1.250.

Dose, 5 to $x 5 \mathrm{~m}$.; $.30^{\circ}$ to 1.00 c.c.] 
16. LIQUOR FERRI ACETATIS.-[Solution of Ferric Acetate. $\mathrm{Fe}_{2}\left(\mathrm{C}_{2} \mathrm{H}_{3} \mathrm{O}_{2}\right)_{6}=464.92$. An aqueous solution of Ferric Acetate.

SoURCE. - Ferric Sulphate, IOOO; is precipitated with a solution of Ammonia Water, $850 . \quad \mathrm{Fe}_{2}\left(\mathrm{SO}_{4}\right)_{3}+6 \mathrm{NH}_{4} \mathrm{OH}=\mathrm{Fe}_{2}(\mathrm{OH})_{6}+3\left(\mathrm{NH}_{4}\right)_{2} \mathrm{SO}_{4}$. The resulting hydrate is dissolved in Glacial Acetic Acid, 260. $\mathrm{Fe}_{2}(\mathrm{OH})_{6}+6 \mathrm{HC}_{2} \mathrm{H}_{3} \mathrm{O}_{2}$ $\equiv \mathrm{Fe}_{2}\left(\mathrm{C}_{2} \mathrm{H}_{3} \mathrm{O}_{2}\right)_{6}+6 \mathrm{H}_{2} \mathrm{O}$.

Characters. - A dark, reddish-brown, clear liquid of an acetous odor, a sweetish, acidulous, somewhat styptic taste. Sp. gr. about I.160.

Dose, 2 to Io m.; .I2 to .6o c.c.]

17. FERRI OXIDUM HYDRATUM.-[Ferric Hydrate. $\mathrm{Fe}_{2}\left(\mathrm{OH}_{6}\right)$ =213.52. Synonyms. - Hydrated Ferric Oxide. Ferric Hydroxide.

SoURCE.-Add to a solution of Ferric Sulphate, IOO; Ammonia Water, I10. The precipitate is Ferric Hydrate.

Characters. - A reddish-brown magma, wholly soluble in Hydrochloric Acid without Effervescence.

Dose, I to 4 dr. ; 4. to I5. c.c.]

\section{Preparations.}

1. Emplastrum Ferri.-[Iron Plaster. Synonym.-Strengthening Plaster. Ferric Hydrate, 90 ; Burgundy Pitch, I40 ; Lead Plaster, 720 ; Olive Oil, 50.

2. Trochisci Ferri.-Troches of Iron. Ferric Hydrate, 30; Vanilla 1 ; Sugar, $100 \mathrm{gm}$. ; Mucilage of Tragacanth, to make roo troches.

Dose, I to 2 troches.]

18. FERRI OXIDUM HYDRATUM CUM MAGNESIA. Ferric Hydrate with Magnesia. [Synonym. - Arsenic Antidote. Solution of Ferric Hydrate, 50 ; add to the Iron, water, roo. Magnesia, ro; add water to the Magnesia to 750 . Keep in separate bottles.

UsES.-For arsenical poisoning, for which it should be mixed by adding the magnesia mixture gradually to the iron solution, and shaking until a homogeneous mass results. It should be given in large doses and frequently repeated.]

19. FERRI ET AMMONII SULPHAS. - [Ferric Ammonium Sulphate. Synonyms.-Ammonio-Ferric Sulphate. Ammonio-Ferric Aluın. $\mathrm{Fe}_{2}\left(\mathrm{NH}_{4}\right)_{2}\left(\mathrm{SO}_{4}\right)_{4}+24 \mathrm{H}_{2} \mathrm{O}=962.1$.

SourCE. - From crystallization of Ammonium Sulphate in a solution of Ferric Sulphate.

Characters. - Pale, violet, octahedral crystals, efflorescent, odorless, having an acid, styptic taste, and a slightly acid reaction. Solubility. - In 3 parts of water; insoluble in Alcohol.

Dose, 2 to $10 \mathrm{gr}$.; 12 to $.60 \mathrm{gm}$. 
20. FERRI HYPOPHOSPHIS. See Phosphorus.

21. FERRI VALERIANAS. See Valeriana.]

The following are scale preparations of Iron, so called because they are dried to form scales. They are not well-defined chemical compounds. There are [nine-the Soluble Phosphate, Iron and Potassium Tartrate,] Iron and Ammonium Citrate, [Iron and Strychnine Citrate, Iron and Ammonium Tartrate, the Citrate, Iron and Quinine Citrate, the soluble Iron and Quinine Citrate, the soluble Pyrophosphate.

22. FERRI PHOSPHAS SOLUBILIS.-Soluble Ferric Phosphate. It consists of Ferric Phosphate, with some Oxides.

Source. - Dissolve Ferric Citrate, 50; in distilled water, 100; add Sodium Phosphate, 55. Evaporate and dry on glass.

Characters. - Thin, bright green, transparent scales, having an acidulous, slightly saline taste. Solubility. - Freely and completely in water.

Dose, I to $5 \mathrm{gr}$; .06 to $.30 \mathrm{gm}$.

\section{Preparation.}

Syrupus Ferri, Quininæ et Strychninæ Phosphatum.-Syrup of the Phosphates of Iron, Quinine and Strychnine. Synonyms. Easton's Syrup. Syrupus Trium Phosphatum. Dissolve Soluble Ferric Phosphate, 20; in water, 50; and add Phosphoric Acid, 48 ; Quinine Sulphate, 30 ; and Strychnine, $\frac{2}{10}$; with Syrup, Glycerin and distilled water, to 1000 .

$$
\text { Dose, } 1 / 2 \text { to I fl. dr.; 2. to } 4 \text {. c.c. }
$$

23. FERRI ET POTASSII TARTRAS.-Iron and Potassium Tartrate. Synonyms. - Potassio-Ferric Tartrate. Tartarated Iron.

SouRCE. - Add solution of Ferric Sulphate, 100 ; in water, 1300 ; to Ammonia Water, I10; with water, 250; filter, add water, I500; heat with Potassium Bitartrate, 38 ; and filter. Dry the precipitate on glass.

Characters. - Thin, transparent, garnet-red to reddish-brown scales, having a sweetish, slightly ferruginous taste. Solubility. - Very soluble in water, insoluble in Alcohol.

IMPURITIES. - Ammonia and Ferrous Salts.

Dose, 5 to $15 \mathrm{gr}$; .30 to $\mathrm{r} .00 \mathrm{gm}$.]

24. FERRI ET AMMONII CITRAS.-[Iron and Ammonium Citrate. Synonym.-Ammonio-Ferric Citrate.

Source. - From evaporation of a solution of Ferric Citrate, IOO; with Ammonia Water, 40; to consistency of syrup. Dry the precipitate on glass.

ChARActers. - Thin, transparent, garnet-red scales, having a saline, 
mildly ferruginous taste, deliquescent. Solubility.-Very soluble in water; insoluble in Alcohol.]

IMPURITIFs.-Tartrates and alkaline salts.

Dose, I to $5 \mathrm{gr}$.; [.06 to .30 $\mathrm{gm}$.]

\section{Preparation.}

[Vinum Ferri Citratis.-Wine of Ferric Citrate. Iron and Ammonium Citrate, 40 ; Tincture of Sweet Orange Peel, I50; Syrup, 100 ; White Wine, to 1000 .

Dose, $\mathrm{I}$ to 2 fl. dr.; 4. to 8. c.c.

25. FERRI ET STRYCHNINAE CITRAS.-Iron and Strychnine Citrate.

Source. - Dissolve Iron and Ammonia Citrate, 98 ; in distilled water, I00; and Strychnine, I ; Citric Acid, I ; in distilled water, 20. Mix the solution, evaporate to the consistency of syrup and spread on plates of glass.

ChARACTERS. - Thin, transparent, garnet-red, to yellowish-brown scales, having a bitter, slightly ferruginous taste. Solubility.-Completely in water.

Dose, I to $3 \mathrm{gr}$; ; .06 to $.20 \mathrm{gm}$.

26. FERRI ET AMMONII TARTRAS.-Iron and Ammonium Tartrate. Synonym.-Ammonio-Ferric Tartrate.

Source. - Add solution of Ferric Sulphate, IOO; to Ammonia Water, 110 ; diluted with cold water, 250 ; filter, dissolve the precipitate in Tartaric Acid, 29, dissolved in distilled water ; filter and evaporate to a syrupy consistence and dry on glass.

Characters. - Thin, transparent scales, from garnet-red to reddishbrown, having a sweetish, slightly ferruginous taste. Solubility.-Very soiuble in water; insoluble in Alcohol.

Dose, 5 to $15 \mathrm{gr}$; .30 to $1.00 \mathrm{gm}$.

27. FERRI CITRAS.-Ferric Citrate.

SOURCE. - By evaporation of the Solution of Ferric Citrate to a syrupy consistency and drying on glass.

Characters. - Thin, transparent, garnet-red scales, odorless, having a slightly ferruginous taste and an acid reaction. Solubility.-Completely in water; insoluble in Alcohol.

Dose, $x$ to $5 \mathrm{gr}$.; .06 to $.30 \mathrm{gm}$.]

28. FERRI ET QUININÆ CITRAS.-[Iron and Quinine Citrate.

Source.-Dissolve Ferric Citrate, 85 ; in distilled water, 160 ; dissolve Quinine, 12 ; and Citrate Acid, 3 ; in distilled water, 20 ; mix these solutions, evaporate to a syrupy consistency and dry on glass. 
Characters. - Thin, transparent, reddish-brown scales of a bitter, mildly ferruginous taste. Solubility.-Slowly but completely in water. Contains at least II. 5 per cent. of dried Quinine.]

IMPURITIES. -Alkaline Salts and other alkaloids instead of quinine.

Dose, 2 to ro gr.; [.12 to $.60 \mathrm{gm}$.

29. FERRI ET QUININA CITRAS SOLUBILIS.-Soluble Iron and Quinine Citrate.

Source.-Dissolve Ferric Citrate, 85 ; in distilled water, 160 ; by heating; add Quinine, 12 ; Citric Acid, 3 ; previously triturated in distilled water, 20; mix these solutions and stir with Ammonia Water, 50; evaporate to a syrupy consistency and dry on glass.

Characters. - Thin, transparent scales, of a greenish, golden-yellow color, having a bitter, mildly ferruginous taste; deliquescent.

Dose, 2 to ro gr.; .12 to $.60 \mathrm{gm}$.

\section{Preparation.}

Vinum Ferri Amarum.-Bitter Wine of Iron. Soluble Iron and Quinine Citrate, 50 ; Tincture of Sweet Orange Peel, 150 ; Syrup, 300; White Wine to 1000 .

Dose, I to 4 fl. dr.; 4 . to 15 . c.c.

3o. FERRI PYROPHOSPHAS SOLUBILIS.-Soluble Ferric Pyrophosphate.

SoURCE. - By solution of Ferric Citrate, 50; Sodium Pyrophosphate, 50 ; in distilled water, IOo; evaporation and drying on glass.

CHARACTERS. - Thin, apple-green, transparent scales, odorless, having an acidulous, slightly saline taste, and a slightly acid reaction. Solubility.Freely in water; insoluble in Alcohol.

Dose, I to $5 \mathrm{gr}$; .06 to $.30 \mathrm{gm}$.]

31. FERRI ARSENAS. [B. P. Not official.] Iron Arsenate. $\left[3 \mathrm{Fe}(\mathrm{FeO}) \mathrm{AsO}_{4}+16 \mathrm{H}_{2} \mathrm{O}=1086.74.\right]$ It consists of both ferrous and ferric arsenates, with some oxide.

SoURCE. - Mix hot solutions of Sodium Arsenate and Iron Sulphate, add Sodium Bicarbonate to neutralize the free Sulphuric Acid that is formed when Iron Arsenate is precipitated.

CharACTERS. - A greenish, amorphous powder, insoluble in water.

IMPURITIES. - Sulphates.

Dose, $\frac{1}{16}$ to $1 / 2 \mathrm{gr}$; [ .004 to $.03 \mathrm{gm}$.] as a pill.

32. [FERRUM DIALYSATUM.-Dialyzed Iron. (Not official.)

SoURCE. - By heating a solution of Ferric Chloride with Ammonia, Ferric Hydrate being precipitated, this being redissolved by agitation, is placed in a 
dialyzer and suspended in water, which is renewed so long as it shows a trace of Hydrochloric Acid.

Composition. - Its composition varies from $\mathrm{Fe}_{2} \mathrm{Cl}_{6}, \mathrm{I}_{2} \mathrm{Fe}_{2} \mathrm{O}_{3}$ to $\mathrm{Fe}_{2} \mathrm{Cl}_{6}$, $95 \mathrm{Fe}_{2} \mathrm{O}_{3}$, and is a ro per cent. solution of Ferric Oxychloride in water.

Characters. - A reddish-brown liquid, free from astringent, styptic taste.

Dose, 10 to $30 \mathrm{~m}$; ; .60 to 2.00 c.c.]

INCOMPaTibles of Iron SAlts in General.-All substances containing tannic or gallic acid form an intense black with ferric salts. Preparations of iron are therefore incompatible with all vegetable astringent solutions, and the only infusions with which they can be prescribed are infusions of quassia and of calumba. It is a common mistake to forget that because of its tannin, the tincture as well as the infusion of digitalis makes an inky mixture with iron preparations. Such a mixture may be clarified with a little diluted phosphoric acid, but after a few days a slight precipitate of ferric phosphate falls. Ferric salts render mucilage of acacia gelatinous.

Alkalies and their carbonates, lime water, calcium carbonate, magnesia and [magnesium] carbonate give green precipitates with ferrous, and brown with ferric salts.

\section{Action of Iron and Its Salts.}

External:-Solutions of iron salts are antiseptic. They have no action on the unbroken skin, but when applied locally to the abraded skin, sores, ulcers, and mucous membranes, the [ferric] salts are powerful astringents, because they coagulate albuminous fluids, both those discharged from the surface and also those in the tissue itself. There is no direct effect on the walls of the vessels, but the contraction of the coagulated albumin compresses them and diminishes their calibre. Partly for this reason, but still more because these iron salts quickly cause the coagulation of blood, and the clot thus formed plugs the bleeding vessels, they are the most perfect local hæmostatics we possess, and will often arrest very severe hæmorrhage. [Ferric] chloride, the nitrate, and sulphate are all very strongly astringent ; but the scale preparations, reduced iron, the carbonate, iodide, phosphate and acetate are so very feebly astringent that they are never used as local applications; in fact, to most persons they are non-astringent. Ferric oxides have the property of converting oxygen into ozone, and are therefore disinfectant.

Internal.-Mouth. - Preparations of iron have a styptic 
taste, the teeth and tongue may be blackened when they are taken, owing to the formation of ferrous sulphide, the sulphur being derived from the food and the tartar on the teeth; hence it is advisable to take iron preparations through a glass tube [and immediately afterwards to brush the teeth. The free acid in the tincture of ferric chloride will destroy the enamel of the teeth, even if diluted with eight parts of water.] The astringent preparations have, when locally applied, the same action on the mucous membrane of the mouth as on the raw skin.

Stomach. - Whatever form of iron is given by the mouth, it is converted in the stomach into ferric chloride, with probably a iittle ferrous chloride. Long experience has shown that ferric chloride is to the physician a most valuable preparation of iron; probably this is because it will not abstract hydrochloric acid from the gastric juice, as is the case with all other preparations of iron. It is often stated that an iron albuminate is formed in the stomach; this is incorrect, and when the iron albuminate is given by the mouth it will be converted into a chloride in the stomach. Although whatever form of iron is administered ferric chloride is formed in the stomach, the choice of the preparation is a matter of great importance, for if strongly acid salts are given, the acid set free after the formation of the chloride will act as a caustic, and damage the mucous membrane; even the preparations of the chloride may do this, for they [always] contain a considerable amount of free acid. These facts explain why iron preparations, especially the acid ones, so often cause headache, nausea, loss of appetite, and other symptoms of severe indigestion. We also learn why experience has taught that the sulphate, which is so often used, should be given in the form of a pill, for this, if specially coated, is not dissolved till the intestine is reached, and the acid is harmless in the alkaline solutions of that part of the alimentary canal. Further, we see why the preparations which are either not acid at all or only very slightly acid, such as reduced iron, dialyzed iron, [not official], ferrous carbonate and the scale preparations, do not as a rule cause indigestion [but it can also be said that they are generally not so efficient as the stronger preparations. However, this free acid 
may be neutralized by the addition of sodium bicarbonate, so that the tincture of ferric chloride will be acid only so far as the basic ferric chloride has an acid reaction ; nor does this neutralization impair its therapeutic properties, for hydrochloric acid is added to it in the stomach. An effective preparation is now made, in which these disadvantages of the tincture of ferric chloride are removed, which is known as Weld's syrup of ferric chloride.] Ferric chloride is very astringent, hence the astringent effect on the stomach of iron salts. The non-astringent preparations can only be astringent in proportion to the amount of ferric chloride formed from the gastric juice; but if large quantities of astringent preparations are given, the excess which is not decomposed by the gastric juice will add its astringency to that of the ferric chloride formed in the stomach.

Intestines.-On passing into the intestines, the contents of which are alkaline from sodium carbonate, ferric chloride becomes ferric oxide, which remains in solution owing to the presence of organic substances; ferrous chloride is converted into ferrous carbonate, which is also soluble. Lower down in the intestine, by the action of the sulphur compounds, the nascent hydrogen, and other readily oxidizable products of decomposition there present, these iron compounds are converted into ferrous sulphide and tannate (the tannic acid being derived from the vegetables in the food), and as such are eliminated with the fæces, which are turned black. Large amounts of the astringent preparations have a constipating effect; this may be owing to there being an excess of them, for the oxides and carbonates are non-astringent preparations.

Absorption.-Iron is certainly taken up from the alimentary canal, for the growing child gets from its food all the iron necessary for its increase in weight, but as the total amount of iron in the adult body is only about $3^{8}$ grains [2.46 gm.], it is probably absorbed very slowly in very minute amounts, and as iron in food exists as oryanic compounds, there is no doubt about the absorption of organic iron.

Whether, however, inorganic iron salts can be absorbed has been much discussed. The prevailing opinion, founded chiefly on 
histological evidence, now is that they can be taken up by the intestinal epithelium and passed into the leucocytes of the blood in $\mathrm{mi}$ nute particles. A little of this iron is deposited in the spleen, but more goes to the liver, where it is built up into complex bodiesone of which is called hæmatin-which are the precursors of hæmoglobin; where in the body this is finally made is not known, but the red marrow utilizes it to make red blood-corpuscles.

The other opinion is that inorganic iron salts are not absorbed. The chief reason for this view is that the giving of such salts by the mouth does not lead to more iron in the urine; but we now know that this is because in such a case the excess of iron taken up is excreted into the intestine as an organic compound, and as it has been shown that under all circumstances the bile contains the merest traces of iron this excretion must take place by the intestinal mucous membrane.

Blood.-It is often stated that the administration of iron causes, in healthy subjects, an increase in the number of red blood-corpuscles, but this is very doubtful. Probably in health it has little or no effect on the blood.

Iron salts injected into animals subcutaneously or directly into the veins cause gastro-intestinal irritation and paralysis from depression of the central nervous system. Part of the iron is stored up, but much is excreted by the gastro-intestinal mucous membrane.

In certain forms of anæmia (a condition in which the amount of hæmoglobin and the number of corpuscles are diminished), especially chlorosis, the administration of iron rapidly improves the blood in both respects. It is therefore said to be hæmatinic; and as an improvement in the quality of the blood leads to an improvement in the functions of all the organs of the body, iron is also called a tonic. Tonics are drugs which indirectly improve the action of the several organs of the body; usually they act by improving the quality of blood or by aiding digestion, and thus rendering the digestion and absorption of the food more easy (see p. II 5). If, as already stated, inorganic iron is directly taken up by the intestinal epithelium and passed to the leucocytes, the benefit in anæmia is easy to understand. But 
we have seen that some believe that inorganic iron is not absorbed, and if this be so, it is at first sight difficult to understand how it can benefit anæmia. As the organic iron in food must be absorbed we may conclude that it is in some way or other protected from decomposition in the alimentary canal, if we believe that the inorganic compounds which would result if they were decomposed are incapable of absorption. Bunge's hypothesis is, that in some forms of anæmia, especially chlorosis, organic salts of iron taken in the food are in some way split up in the intestines so as to be incapable of absorption. In those anæmic conditions which can be benefited by iron the administration of the inorganic salts prevents the decomposition of the organic salts in the food by fixing the decomposing agents, which, according to Bunge, are chiefly alkaline sulphides and forming iron sulphide. This, he says, is supported by the fact that to cure chlorosis rapidly, enormous doses of iron are often found to be necessary ; for example, a patient will take $6 \mathrm{gr}$. [.40gm.] of reduced iron three times a day, or I $8 \mathrm{gr}$. [1.20 gm.] a day. Now, the whole amount of iron in the blood of an ordinary healthy woman is about $3^{8} \mathrm{gr}$. [2.46 gm.], for there is only one atom of iron in a molecule of hæmoglobin, which contains considerably over 2000 atoms. Supposing she had lost half her hæmoglobin, if the iron given were simply absorbed, one day's treatment would speedily restore her health, but it is well known that weeks are often required. But if this view were correct we should expect that bismuth, manganese, or arsenic, by fixing the decomposing agents, would cure chlorosis as efficiently as iron. It has been stated that they will, but Stockman has published results that point in a contrary direction, and he has shown that iron sulphide will cure chlorosis, although on Bunge's hypothesis, it should not; for it will not fix the decomposing agents if they are alkaline sulphides. Further, many think that it is not necessary to give large doses of iron to cure chlorosis. Iron injected subcutaneously cures chlorosis, but this does not [point] in one direction more than another, for it may be excreted into the intestine, and there fix the alkaline sulphides. On the whole, the evidence seems against Bunge's view. 
Remote effects.-As iron in anæmic subjects increases the amount of hæmoglobin, more oxygen is carried to the tissues, and thus the whole body shares in the benefit of a course of iron, which has also been thought to have a direct effect on the kidneys as a mild diuretic, and a direct effect in promoting the menstrual flow. These actions are, however, slight, and may be due to the general improvement in health. Iron salts have been given to produce abortion, but without any result. Remote astringent effects have been attributed to them, but there is no satisfactory proof that they have any; and, indeed, when we remember that very little if any iron is absorbed in an astringent form, and it cannot exist in the blood in such a form, we should hardly expect that iron salts could be remotely hæmostatic or astringent. Iron is chiefly stored in the spleen, lymphatic glands, liver and marrow ; possibly it is by stimulating the activity of this that iron cures chlorosis.

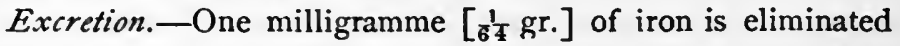
daily in the urine, and this remains constant under all circumstances. Any excess of elimination following subcutaneous injection, or excessive absorption from the intestine, takes place through the intestinal mucous membrane.

\section{Therapeutics of Iron and ItS SAlts.}

External.-Solutions of the sulphate, chloride, nitrate and Liquor Ferri Subsulphatis (Monsel's solution), are the most valuable local astringents we have. It matters very little which of these is used. In England the solution of the chloride is perhaps oftenest employed. Either is of service in many casesfor example, to stop hæmorrhage from leech-bites, from the nose, from piles, or from the uterus, as in the hæmorrhage of malignant disease. A convenient way to apply them is on lint or cotton soaked in the solution, and a cavity such as the nose or uterus may be plugged with the lint. [These preparations form very disagreeable clots, which readily decompose and give rise to septic infection.] The aqueous solution of the chloride has been used as a spray for hæmoptysis, but as it may excite coughing, it is not to be recommended. It is very useful as an astrin- 
gent for painting on the fauces, pharynx or tonsils in inflammation of these parts. It may, for this purpose, be diluted with an equal quantity of water, or a solution of I part of [ferric] chloride in 4 of glycerin may be used. It has been advised to paint erysipelatous skin with the tincture of [ferric] chloride. A solution of the sulphate ( $\mathrm{I}$ to 480 ) has been used in gleet.

Internal.-Gastro-intestinal tract.-The astringent preparations may be swallowed in cases of severe bleeding from the stomach, such as that of malignant disease, ulcer or cirrhosis. If the bleeding is profuse, a drachm [4. c.c.] of Liquor Ferri Chloridi with a drachm [4. c.c.] of glycerin, to facilitate swallowing, may be given every hour or oftener, and this will sometimes apparently save a patient's life. For less serious hæmorrhage smaller quantities will suffice. Intestinal hæmorrhage may also be treated in the same way.

The tendency of [ferric] salts to constipate is usually overcome by the addition of some purgative ; thus, magnesium sulphate is commonly given with the chloride, and aloes is often prescribed with [ferrous] sulphate in a pill. [This method, however, interferes with the time during which iron remains in the intestines, and it is better to administer the laxative separately, so that the dose can be regulated according to circumstances.] The ferric salts have been given for diarrhœa, but there are many drugs more suitable for this symptom. Chronic constipation is often very effectually treated by a pill of [ferrous] sulphate and extract of nux vomica, but probably the efficient purgative in it is the nux vomica, although some claim that large doses of [ferrous] sulphate will overcome chronic constipation. [At least] the constipating effect of the ferric salts is often much exaggerated.

A rectal injection of a fluid drachm [4. c.c.] of the tincture of ferric chloride to half a pint of water [240. c.c.] kills threadworms [the patient being in the knee-chest position].

Arsenical poisoning is best treated by the humid ferric oxide, which should be freshly prepared by mixing together $3 \mathrm{fl}$. oz. [90. c.c.] of Liquor Ferri Tersulphatis with $\mathrm{x}$ oz. [30. gm.] of sodium carbonate diluted with water. Half an ounce [15. c.c.] 
should be given every five or ten minutes. An insoluble arsenite is formed, and may be gotten rid of by a thoroughly purgative dose of magnesium sulphate or some other simple purge. A dose of common salt or of sodium bicarbonate, followed by i fl. oz. [30. c.c.] of dialyzed iron, [useless as an iron preparation], diluted with water, is also efficient in poisoning by arsenic. [A better method of using iron for this purpose is given on p. r93.] Blood. - The great use of iron salts is to restore the amount of hæmogloblin and the number of red corpuscles in anæmia, especially chlorosis. They are useless in pernicious anæmia, and generally of little value, if any, in the anæmia of leucocythæmia, exophthalmic goitre, or Hodgkin's disease. All other common forms of anæmia are secondary to some definite cause, such as hæmorrhage, lead poisoning, scurvy, etc., and are treated by the removal, if possible, of the cause of the anæmia, but recovery may be aided by the administration of iron. [Ferric] chloride and [ferrous] sulphate are two of the most efficacious preparations, and pills containing a grain [.06 gm.] of the dried sulphate, with aloes or nux vomica, if constipation is present, are very valuable. It is usual to begin with one pill containing one grain [.06 gm.] of the dried [ferrous] sulphate thrice a day, but gradually the number of pills may be increased till three or four are taken at a dose. This method of large doses of the sulphate often appears to cure more rapidly than smaller doses. If these astringent preparations cause indigestion, any of the milder preparations may be substituted. The carbonate may be given in pills in rapidly increasing doses, or the dose of reduced iron, conveniently given on bread and butter, may be pushed. Mistura Ferri Composita [Griffith's Mixture] is a disagreeable preparation to take and to look at, and the inky character of the aromatic mixture makes it undesirable. The styptic taste of some of the preparations, especially the astringent ones, may be concealed by giving them with a drachm [4. c.c.] of glycerin, which acts by its viscosity and by reducing some of the ferric to a ferrous salt. It is often added to the tincture of the chloride. The scale preparations hardly ever disagree; they are therefore used for patients with a delicate digestion, and for such it is much better to make no attempt to 
rapidly increase the dose, but to depend on small doses spread over a long period. Mineral waters containing iron (such as those of La Bourboule and Levico) or the red wines may be given in such cases. Flitwick water, however, contains a good deal [of iron]. Often iron and quinine citrate is prescribed as a pill ; powdered tragacanth and syrup form the best excipient. Treatment of anæmia by iron leads, of course, to the improvement of the numerous symptoms, such as amenorrhœa, constipation, dyspepsia, etc., which are dependent upon the anæmia. That form of neuralgia which is associated with anæmia usually yields to iron. Easton's syrup (see p. I94) is a very popular preparation ; it is used for anæmia, and to promote the health and appetite during convalescence after long illness. A pill very similar to the syrup, and containing iron phosphate, I gr. [.06 gm.]; quinine, I gr. [.06 gm.]; strychnine, $\frac{1}{32}$ gr. [.002 gm.]; concentrated phosphoric acid I I $/ 2 \mathrm{~m}$. [:10 c.c.]; liquorice powder to $5 \mathrm{gr}$. [.30 gm.] is prepared. It is called Easton's pill or Pilula Trium Phosphatum. A similar [tablet] is in the market. [Ferrous iodide] has been given, sometimes apparently with success, in cases of rheumatoid arthritis, but it must be continued thrice daily for many months. A pill is often preferable to the syrup, as that so readily changes. Two grains [. $12 \mathrm{gm}$.] may be made into a pill in the same way as that advised for the [yellow] mercurous iodide, and one or two such pills be given thrice a day. Large doses of iron ( $10,[.60$ c.c. $]$ or even 20 minims, [ 1.20 c.c.] of the tincture of the chloride every hour or two) have been given in diphtheria and other forms of bad sore throat, such as hospital sore throat, apparently with considerable benefit. Erysipelas has been treated in the same way. Fever due to other causes is said to contra-indicate the use of iron.

Kidney.-Iron salts are reported to have a feeble diuretic action, but this is doubtful. The chloride is often given empirically for all forms of Bright's disease. Whether it does good is at present undecided.

[Iron should always be administered when the stomach is full (after meals) excepting when given for follicular tonsillitis, diphtheria, erysipelas, gastric hæmorrhage or arsenical poison- 
ing.] Occasionally a patient is found who cannot take iron in any form, because of the headache and indigestion caused by it.

The different preparations of Iron.-These have already been classified into astringent and non-astringent. There are some, viz., the iodide, the phosphate, the iron and quinine citrate, and [the iron and strychnine citrate], the value of which depends in part at least upon their other ingredients. The arsenate must be given in such small doses to avoid arsenical poisoning that it is probable that the iron in it has no effect. Hence arsenous acid may just as well be given, and this is commonly done. [Ferric] phosphate, which always contains some free phosphoric acid, is an excellent hæmatinic. It is used largely for children, because the syrup of it is very pleasant in taste, and also because it was formerly believed that the phosphoric acid would aid the growth of bones, especially in cases of rickets. Parrish's food, known also as Squire's chemical food, and Dusart's syrup both have for their chief ingredient ferric phosphate; the dose of each is $1 / 2$ to $2 \mathrm{fl}$. dr.; [2. to 8. c.c.] [Ferrous] iodide has been introduced for cases in which we wish to gain the benefit of both elements, but the proportion of iron to iodine is small ( $I$ to 9). It is especially liable to damage the teeth. The iron and quinine citrate combine the virtues of both iron and quinine. It is a favorite, mild preparation for slight cases of anæmia, but must not be prescribed with alkalies, as they precipitate the quinine. [Ferratin (not official) is claimed to be the characteristic iron compound of the liver. It is an acid albuminate, prepared artificially, and is used in dose from $11 / 2 \mathrm{gr}$; , $10 \mathrm{gm}$. to $8 \mathrm{gr}$. ; $50 \mathrm{gm}$. No evidence experimental or clinical, has as yet been brought forward, which, outside of theoretical reasoning, makes the superiority of this over the older iron compounds probable, (Wood). Since it is practically tasteless it is easily administered. Practically all of the albuminates and peptonates to be found in the shops are worthless as hæmatinics.]

\section{MANGANUM.}

$\mathrm{Mn} .[=54.8$.

1. [MANGANI DIOXIDUM.-Manganese Dioxide. $\mathrm{MnO}_{2}=86.72$. Synonym.-Black Manganese Oxide.

SOURCE. - Native crude Manganese Dioxide containing at least 66 per cent. of the pure Dioxide.

Citaracters. - A heavy, grayish-black, more or less gritty powder. Solubility.-Insoluble in water or Alcohol.

Manganese Dioxide is used for making Chlorine, Corrosive Mercuric Chloride and Potassium Permanganate.

Dose, 2 to ro $\mathrm{gr}$; .12 to $.60 \mathrm{gm}$. 


\section{Uses of Manganese Dioxide.}

It has been used empirically as an emmenagogue and is probably the most certain of all when administered in maximum dose.

2. MANGANI SULPHAS.-Manganese Sulphate. $\mathrm{MnSO}_{4}+4 \mathrm{H}_{2} \mathrm{O}$ =222.46. Synonym.-Manganous Sulphate.

SUURCE. - By heating the Dioxide with sufficiently strong Sulphuric Acid, evaporation and crystallization.

Characters. - Colorless, or pale, rose-colored, transparent tetragonal prisms, having a slightly bitter and astringent taste. Solubility.-In 0.8 part of water; insoluble in Alcohol.

IMPURITIES. - Zinc, copper, iron and alkalies.

Dose, 2 to $8 \mathrm{gr}$; ; 12 to $.50 \mathrm{gm}$.

\section{Uses of Manganese Sulphate.}

It has been used as a cholagogue purgative, but on account of its irritating properties it is a very unsafe remedy.]

3. POTASSII PERMANGANAS.-Potassium Permanganate. KMn $\mathrm{O}_{4}[=157.67$.

Source. - Caustic Potash, Potassium Chlorate and Manganese Dioxide are heated together. $6 \mathrm{KOH}+\mathrm{KClO}_{3}+3 \mathrm{MnO}_{2}=3 \mathrm{~K}_{2} \mathrm{MnO}_{4}+\mathrm{KCl}+3 \mathrm{H}_{2} \mathrm{O}$. Potassium Manganate is boiled with water till the color changes to purple and the Permanganate is formed. $3 \mathrm{~K}_{2} \mathrm{MnO}_{4}+2 \mathrm{H}_{2} \mathrm{O}=2 \mathrm{KMnO}_{4}+4 \mathrm{KOH}+\mathrm{MnO}_{2}$. The liquid is neutralized with Carbon Dioxide and evaporated.

Characters. - Slender monoclinic prisms of a dark purple color, almost opaque by transmitted, and of a blue, metallic lustre by reflected light, and having a taste at first sweet, but afterwards disagreeable and astringent.] Solubility. - In 16 parts of water; a grain [.06 gm.] gives a fine purple color to a gallon of water [3775. c.c.].

INCOMPATIBLES. - It is very readily deoxidized in the presence of organic matter. It is usually given as a pill or a tabella, and should be made up with kaolin or paraffin, or an explosion will very likely take place.

IMPURITIES.-Potassium carbonate and manganese dioxide.

Dose, $1 / 2$ to $2 \mathrm{gr}$. ; [.03 to $.12 \mathrm{gm}$.]

\section{Action of Potassium Permanganate.}

External.-In a solid form it is a mild caustic, and is, when kept dry, a permanent salt. Its most important action is that when moist it rapidly gives up its oxygen in the presence of organic bodies, and its solutions therefore quickly turn dark 
brown, manganese dioxide being formed. The power possessed by its solution of giving up oxygen makes it a disinfectant, deodorant, and antiseptic, especially as much of the oxygen is in the form of ozone. But its action as a germicide is very limited, for it so readily gives up its oxygen to the organic substances in which the micro-organisms flourish that it very soon becomes inert.

Internal.-Potassium permanganate, when taken internally, must be quickly decomposed. Manganese salts are only absorbed from the intestine in extremely minute quantities. When they are injected into the blood they are excreted in the urine and into the intestine. Probably they have no important action after absorption. Formerly it was thought that they could replace iron in the body, but this is not so. The red corpuscles do not take up manganese.

\section{Therapeutics of Potassium Permanganate.}

External.-Although potassium permanganate is not of much practical use as a germicide, it is commonly employed as a deodorant [ $\mathrm{r}$ to $\mathrm{I}_{50} \mathrm{C}$ for drains, bed-pans, to wash utensils, and to wash the hands; for the last purpose it is suitable as being non-irritant. [The hands should be washed in a saturated solution of the permanganate, which stains them a dark purple, and immediately decolorized with a saturated solution of oxalic acid.] It has one advantage, namely, that it is easy by its change in color to see when it has lost its efficacy. Condy's red fluid consists of $8 \mathrm{gr}$. [.50 gm.] of potassium permanganate to the fluid ounce [3०. c.c.] of distilled water. It is expensive for purposes requiring a large quantity. It stains fabrics. The stain may be gotten out by applying sulphurous acid, but the fabric must be immediately rinsed in water, [else] sulphuric acid is formed.

Internal.-The liquor of potassium permanganate [B. P., I in 100 of distilled water], considerably diluted, can be used as a mouth wash or gargle in foul conditions of the mouth, or as an injection in cases of foul discharges, such as may occur with gonorrhœa, vaginitis, uterine disease or ozæna. Some have considered that potassium permanganate is beneficial for the same 
cases of anæmia as iron, but it probably has no effect. Others praise its power in amenorrhœa. It should be always given as a pill, for the taste of solutions of it is very [disagreeable. For amenorrhœa the black oxide is preferable. (See p. 206.) If manganese is of any use in anæmia, which has not yet been proven, it probably acts in the same way as iron. The ironmanganese preparations so much lauded, owe their efficiency, if they possess any, to the iron which they contain in varying amounts. Potassium permanganate] oxidizes morphine and is therefore an antidote to morphine poisoning. [About two grains in solution should be given for each grain (estimated) of morphine swallowed, and the stomach should be immediately and repeatedly washed out with repetitions of the antidote].

\section{GROUP VI.}

[Aurum,] Hydrargyrum.

I. $[A \cup R U M$.

$\mathrm{Au}=196.7$.

AURI ET SODII CHLORIDUM.-Gold and Sodium Chloride. A mixture composed of equal parts by weight of dry Gold Chloride $\left(\mathrm{AuCl}_{3}=\right.$ 302.81) and Sodium Chloride $(\mathrm{NaCl}=58.37)$.

CHARACTERS. - An orange-yellow powder, odorless, having a saline and metallic taste, and a slightly acid reaction. Solubility.-Very soluble in water, and at least one-half is soluble in Alcohol.

Dose, $\frac{1}{30}$ to $\frac{1}{10} \mathrm{gr}$; .002 to $.006 \mathrm{gm}$.

\section{Action of Gold and Sodium Chloride.}

In small doses gold and sodium chloride is supposed to promote appetite and digestion, to stimulate the functions of the brain and to be an aphrodisiac. Full doses cause nausea and vomiting, and finally impair nutrition. The toxic symptoms resemble those of poisoning by corrosive mercuric chloride.

\section{Therapeutics of Gold and Sodium Chloride.}

It is useful in irritative dyspepsia, gastro-duodenal catarrh, hypochondriasis, chronic ovarian irritation and ovaritis, in 
chronic albuminuria, hepatic sclerosis, and granular kidney, as it prevents hyperplasia of connective tissue. It is a valuable remedy in the tertiary manifestations of syphilis, especially of the bones, and presents fewer disadvantages than does the corrosive mercuric chloride.]

\section{HYDRARGYRUM.}

$$
\mathrm{Hg}[=\mathbf{r} 99.8 \text {. }
$$

I. HYDRARGYRUM.-Mercury. [Synonym.-Quicksilver.]

SOURCE. - Cinnabar, the native Sulphide, is roasted or distilled with Lime; [the volatile Mercury is condensed in suitable aludels.

ChARACTERS. $-A$ shining, silver-white metal, easily divisible into small globules. Sp. gr. 13.5584.]

IMPURITY.-Lead, tin, and other metals.

\section{Preparations.}

I. Hydrargyrum cum Creta.-[Mercury with Chalk. Synonym.-Gray powder.

By trituration of Mercury, 38; Prepared Chalk, 57; Clarified honey, 10; with sufficient water to 100. By keeping, the Mercury is liable to become Mercuric Oxide, which makes the powder more active. - Strength. -38 per cent. of Mercury.

Dose, $1 / 2$ to ro $\mathrm{gr}$; .03 to $.60 \mathrm{gm}$.

2. Emplastrum Hydrargyri.-Mercurial Plaster. Mercury, 300 ; Oleate of Mercury, 12 ; Lead Plaster to 1000. Strength.-30 per cent. of Mercury.]

3. Emplastrum Ammoniaci cum Hydrargyro.-[Ammoniac Plaster with Mercury. Mercury, 180; Oleate of Mercury, 8; an evaporated solution of Ammoniac. 720; in diluted Acetic Acid, 1000; and Lead Plaster a sufficient quantity to 1000 . Strength.-1 8 per cent. of Mercury.

4. Massa Hydrargyri.-Mass of Mercury. Synonyms.-Blue Mass. Blue Pill. Pilula Hydrargyri. Mercury, 33; Honey of Rose, 34; Glycyrrhiza, 5 ; Althxa, 25; Glycerin, 3. Strength.-33 per cent. of Mercury.

Dose, $1 / 2$ to $15 \mathrm{gr}$; .03 to $1.00 \mathrm{gm}$.]

5. Unguentum Hydrargyri.-[Mercurial Ointment. Synonym. - Blue Ointment. Mercury, 500; Lard, 250; Suet, 230; Oleate of Mercury, 20.-Strength. - 50 per cent. of Mercury.]

2. HYDRARGYRI OXIDUM RUBRUM.-[Red Mercuric Oxide. $\mathrm{HgO}=215.76$. Synonym.-Red Precipitate. 
Source.-Dissolve Mercury in diluted Nitric Acid. ${ }_{3} \mathrm{Hg}+8 \mathrm{HNO}_{3}=$ ${ }_{3} \mathrm{Hg}\left(\mathrm{NO}_{3}\right)_{2}+2 \mathrm{NO}+4 \mathrm{H}_{2} \mathrm{O}$. Evaporate to dryness. Triturate the Mercuric Nitrate thus formed, with Mercury, and heat. $2 \mathrm{Hg}\left(\mathrm{NO}_{3}\right)_{2}+\mathrm{Hg}_{2}=4 \mathrm{HgO}+$ $2 \mathrm{~N}_{2} \mathrm{O}_{4}$.

Characters. - Heavy orange-red, crystalline scales, or a crystalline powder; having a somewhat metallic taste. Solubility.-Almost insoluble in water.]

IMPURITIES. - Red lead, brick dust, and mercuric nitrate.

Dose, $1 / 4$ to I gr.; [.015 to $.06 \mathrm{gm}$.]

\section{Preparation.}

Unguentum Hydrargyri Oxidi Rubri.-[Ointment of Red Mercuric Oxide. Synonym. - Red Precipitate Ointment. Red Mercuric Oxide, 10 ; Castor Oil, 5 ; Ointment, 85.]

3. HYDRARGYRI OXIDUM FLAVUM. - [Yellow Mercuric Oxide. $\mathrm{HgO}=215.76$.

Source.-Precipitate a solution of Corrosive Mercuric Chloride, I000; with Soda, 4O. $\quad\left[\mathrm{HgCl}_{2}+2 \mathrm{NaOH}=\mathrm{HgO}+2 \mathrm{NaCl}+\mathrm{H}_{2} \mathrm{O}\right.$.

Characters. - A light orange-yellow, amorphous, heavy, impalpable powder, having a somewhat metallic taste.] Not given internally. It is contained in Lotio Hydrargyri Flava [B. P.-Corrosive Mercuric Chloride, I; Lime Water, 240]. It has the same composition as the Red Oxide, but is more crystalline.

\section{Preparations.}

I. Unguentum Hydrargyri Oxidi Flavi.-[Ointment of Yellow Mercuric Oxide. Yellow Mercuric Oxide, Io; Ointment, 90.

2. Oleatum Hydrargyri._-Oleate of Mercury. Yellow Mercuric Oxide, 200; Oleic Acid, 800.]

Oleate of Mercury is contained in Unguentum Hydrargyri, Em. plastrum Hydrargyri and Emplastrum Ammoniaci cum Hydrargyro.

4. HYDRARGYRI [CHLORIDUM CORROSIVUM.-Corrosive Mercuric Chloride. $\left.\quad \mathrm{HgCl}_{2}=270.54.\right]$ Synonyms.-Corrosive Sublimate. Mercuric Bichloride. Corrosive Chloride of Mercury.

Source. - Heat a mixture of Mercuric Sulphate, Sodium Chloride, and Manganese Dioxide. $\mathrm{HgSO}_{4}+2 \mathrm{NaCl}+\mathrm{MnO}_{2}=\mathrm{HgCl}_{2}+\mathrm{Na}_{2} \mathrm{SO}_{4}+\mathrm{MnO}_{2}$. The [Corrosive] Chloride sublimes and is condensed. The object of the Manganese Dioxide is to prevent the formation of Mercurous Chloride by setting free Chlorine, which will convert it into Mercuric Chloride.

Characters. - Heavy, colorless [rhombic crystals, or crystalline masses, having an acrid and persistent metallic taste.] Solubility. - In [ 16 parts of water ; in 3 parts of Alcohol.]

INCOMPATIBLES. - Alkalies and their carbonates, potassium iodide, lime 
water, tartar emetic, silver nitrate, lead acetate, albumin, soaps, vegetable preparations containing tannic acid, and in fact most substances.

Dose, $\frac{1}{60}$ to It $^{\frac{1}{6}} \mathrm{gr}$.; [.001 to $.006 \mathrm{gm}$.]

5. HYDRARGYRI [CHLORIDUM MITE.- Mild Mercurous Chloride. $\mathrm{Hg}_{2} \mathrm{Cl}_{2}=470.34$. $]$ Synonyms.-Calomel. Mild Chloride of Mercury. Subchloride of Mercury.

Source.-Rub Mercury with Mercuric Sulphate to form Mercurous Sulphate, $\mathrm{Hg}_{2} \mathrm{SO}_{4}$. Add Sodium Chloride, and then heat, the Calomel sublimes, $\mathrm{Hg}_{2} \mathrm{SO}_{4}+2 \mathrm{NaCl}=\mathrm{Hg}_{2} \mathrm{Cl}_{2}+\mathrm{Na}_{2} \mathrm{SO}_{4}$.

Characters. - [A white, impalpable powder without odor or taste.

IMPURITY.-Corrosive] Mercuric Chloride.

Dose, $1 / 2$ to $10 \mathrm{gr}$; ; .03 to $.60 \mathrm{gm}$.]

Preparations.

1. Pilulæ Cathartica Compositæ.-[Compound Cathartic Pills. Mild Mercurous Chloride, 60 ; Compound Extract of Colocynth, 80 ; Extract of Jalap, 30 ; Gamboge, 15 gm., to make 1000 pills.]

Dose, I to 3 pills.

2. Pilula Antimonii Composita.-[Compound Pills of Antimony. Synonym.-Plummer's Pills. Mild Mercurous Chloride, 4; Sulphurated Antimony, 4; Guaiac, 8 gm. ; Castor Oil, a sufficient quantity to make 100 pills.

Dose, $I$ to 3 pills.]

6. HYDRARGYRI IODIDUM RUBRUM.-[Red Mercuric Iodide. $\mathrm{HgI}_{2}=452.86$. Synonyms. - Mercury Biniodide. Red Iodide of Mercury.

SourCE. - Mix hot solutions of Corrosive Mercuric Chloride and Potassium Iodide. Filter and dry the precipitated Red Iodide. $\mathrm{HgCl}_{2}+2 \mathrm{KI}=$ $\mathrm{HgI}_{2}+2 \mathrm{KCl}$.

Characters. - A scarlet-red, amorphous powder. Solubility.-Almost insoluble in water, but freely soluble in a solution of Potassium Iodide.]

IMPURITIEs. - The same as of the Corrosive Chloride.

Dose, ชo to Ir gr.; [.001 to $.006 \mathrm{gm}$.]

\section{Preparation.}

Liquor Arseni et Hydrargyri Iodidi.-[Solution of Arsenic and Mercuric Iodide. Synonym.-Donovan's Solution. Dissolve Arsenic Iodide, IO; and Red Mercuric Iodide, 10; in distilled water, I000.

Characters. - A clear, pale-yellowish liquid, having a disagreeable, metallic taste.] Strength. $\rightarrow$ I per cent. of each Iodide.

Dose, I to $10 \mathrm{~m}$.; $[.06$ to .60 c.c. $]$

7. [HYDRARGYRI IODIDUM FLAVUM.- $-\mathrm{Hg}_{2} \mathrm{I}_{2}=652.66$. Yellow Mercurous Iodide. Synonyms.-Mercury Protiodide. Yellow or Green Mercury Iodide. 
Source. - By pouring a solution of Potassium Iodide, 24; in distilled water, 1000 ; into a solution of Mercurous Nitrate, 40 ; in Nitric Acid, Io; and distilled water, rooo. The precipitate is washed and dried. The Mercurous Nitrate is obtained from Mercury treated by a solution of Nitric Acid in distilled water, by filtration. $\mathrm{Hg}_{2}\left(\mathrm{NO}_{3}\right)_{2}+2 \mathrm{KI}=\mathrm{Hg}_{2} \mathrm{I}_{2}+2 \mathrm{KNO}_{3}$.

Characters. - A bright yellow amorphous powder, odorless and tasteless. Solubility. - Almost insoluble in water, and wholly insoluble in Alcohol and Ether.

Dose, $\frac{1}{6}$ to I gr. ; .0I to $.06 \mathrm{gm}$.]

8. LIQUOR HYDRARGYRI NITRATIS.-[Solution of Mercuric Nitrate. A liquid containing about 60 per cent. of Mercuric Nitrate. $\mathrm{Hg}\left(\mathrm{NO}_{3}\right)_{2}=323.5^{8}$, together with about II per cent. of free Nitric Acid.

Source.-Dissolve Red Mercuric Oxide, 40 ; in Nitric Acid, 45 ; with distilled water, 15 , and heat.

Characters. - A clear, nearly heavy, colorless liquid, having a faint odor of Nitric Acid. Sp. gr. about 2. 100.]

IMPURITY.-Mercurous Nitrate.

9. UNGUENTUM HYDRARGYRI NITRATIS.-[Ointment of Mercuric Nitrate. Synonym.-Citrine Ointment.

Source.-Mix a hot solution of Mercury, 70 ; in Nitric Acid, 175 ; with Lard Oil, 760.]

Characters. - A lemon-yellow ointment.

10. HYDRARGYRUM AMMONIATUM.-Ammoniated Mercury. $\mathrm{NH}_{2} \mathrm{HgCl}[=25 \mathrm{I}$.18.] Synonyms. - White Precipitate. [Mercuric AmmonioChloride.]

SOURCE.-Mix solutions of Ammonia, I00; and Corrosive Mercuric Chloride, roo. $\mathrm{HgCl}_{2}+2 \mathrm{NH}_{4} \mathrm{OH}=\mathrm{NH}_{2} \mathrm{HgCl}+\mathrm{NH}_{4} \mathrm{Cl}+2 \mathrm{H}_{2} \mathrm{O}$. Filter and wash the precipitated Ammoniated Mercury.

Characters. - [White, pulverulent pieces, or a white, amorphous powder, having an earthy, afterwards styptic and metallic taste. Solubility.-Almost insoluble in water or Alcohol.]

IMPURITIES. - The same as of the [Corrosive] Chloride.

\section{Preparation.}

Unguentum Hydrargyri Ammoniati.-Ointment of Ammoniated Mercury. Synonym. - White Precipitate Ointment. [Ammoniated Mercury, 10; Benzoinated Lard, 90.

11. [HYDRARGYRI CYANIDUM.-Mercuric Cyanide. $\mathrm{Hg}(\mathrm{CN})_{2}$ $=251.76$.

SouRcE. - By boiling pure Potassium Ferrocyanide with Mercuric Sulphate in water, and recrystallization from diluted Alcohol. $7 \mathrm{HgSO}_{4}+2 \mathrm{~K}_{4} \mathrm{Fe}$ $(\mathrm{CN})_{6}=\mathrm{Hg}+6 \mathrm{Hg}(\mathrm{CN})_{2}+4 \mathrm{~K}_{2} \mathrm{SO}_{4}+\mathrm{Fe}_{2}\left(\mathrm{SO}_{4}\right)_{3}$. 
Characters. - Colorless or white prismatic crystals, odorless, having a bitter, metallic taste. Solubility. - In 12.8 parts of water and 15 parts of Alcohol. Dose, $\frac{1}{60}$ to $\frac{1}{10} \mathrm{gr}$; ; .001 to $.006 \mathrm{gm}$.

12. HYDRARGYRI SUBSULPHAS FLAVUS.-Yellow Mercuric Subsulphate. $\mathrm{Hg}\left(\mathrm{HgO}_{2} \mathrm{SO}_{4}=727.4\right.$. Synonyms. - Turpeth Mineral. Basic Mercuric Sulphate.

Source.-By solution of Mercury, 100; Sulphuric Acid, 30 ; Nitric Acid, 25 ; water, a sufficient quantity ; decantation, drying of the residue.

ChARACTERS. - A heavy, lemon-yellow powder, odorless and almost tasteless. Solubility. - In about 2000 parts of water; insoluble in Alcohol.

Dose, 2 to $4 \mathrm{gr}$; ; 12 to $.24 \mathrm{gm}$. as an emetic.]

\section{Action of Mercury and its Salts.}

External.-[Corrosive mercuric chloride] is one of the most powerful and important antiseptics with which we are acquainted. In 1870 it was discovered that I part in 6000 would kill infusoria and spermatozoa. Now it is known to be a universal germicide. The published results of experiments with it vary very much, because the duration of the action, the solvent, and the micro-organism experimented upon, are not always the same. Evans found that anthrax spores were destroyed by [corrosive mercuric chloride] solutions of $\mathrm{I}$ in 1000 acting for a quarter of an hour, and $I$ in 3000 acting for one hour. The bacilli themselves were destroyed by solutions of $\mathrm{I}$ in 15,000 acting for one minute, and $I$ in 25,000 acting for half an hour. A solution of $\mathbf{I}$ in 70,000 prevented the growth of the spores, and one of $\mathrm{I}$ in 500,000 prevented the growth of the bacilli. A reference to carbolic acid will show how much more powerful corrosive mercuric chloride is. A solution of $I$ in $I 000$ is very commonly employed for many disinfecting purposes. If albumin be present in the fluid to be disinfected, an albuminate of mercury is formed, and the antiseptic value of the fluid is destroyed. This change may be prevented by the addition of 5 parts of either hydrochloric, [citric] or tartaric acid to $I$ of [corrosive mercuric chloride]. The [red mercuric iodide] is also a powerful antiseptic. Metallic instruments cannot be disinfected with the [corrosive chloride] for mercury deposited on them. 
Most mercurials, especially the oleate, oxide, ammoniate, nitrate and [corrosive chloride], will destroy the animal and vegetable parasites that infest the skin; they are, therefore, anti-parasitic. Also, most of them will occasionally relieve itching, even when no cause is to be found.

The mercurial preparations, especially the red [mercuric] iodide and the acid solution of the nitrate, are powerful irritants. The latter is strongly caustic. Mercurous salts are slightly irritant and stimulating; calomel is sometimes applied to sores for this property.

Metallic mercury and its salts are absorbed by the skin, especially when rubbed in either as an oleate or an ointment. These preparations are also taken up, although to a less degree, if simply applied to the skin, for minute particles of mercury or its salts pass into the hair follicles and sebaceous follicles, from which they are absorbed as an oxide or a chloride. All the symptoms of mercurial poisoning can be produced if the drug is absorbed through the skin. The vapor can be absorbed through the mucous membrane of the lungs, and mercury compounds are so volatile that when they are applied to the skin some usually enter the blood by the lungs.

Internal.-Although the different salts of mercury have different external actions, after absorption their actions are, in most respects, similar. The long-continued use of excessive doses of mercurials produces well-marked and important symptoms (see Toxicology). The actions for which mercurials are used in medicine are the following:

Stomach and intestines. - The metal mercury itself and mercurous compounds, being mildly irritant in their action, are often used as purgatives; but the mercuric compounds given in the same doses produce severe gastro-intestinal irritation. The action is chiefly on the duodenum and upper part of the jejunum; the precise mode of irritation is unknown, but it is certain that, in consequence of the administration of the mercurial, the contents of the duodenum are hurried along before there is time for the bile to be reabsorbed, and hence the motions are very dark-colored. There is probably some, but not an ex- 
cessively increased secretion from the intestinal walls, for the motions, although large and loose, are not watery. As the action of the mercurial is chiefly on the upper part of the intestine, it is greatly assisted by giving a saline purge a few hours after it, for this will act more on the lower part of the bowel. The contents are passed along so quickly, that it is doubtful whether there is time for much mercury to be absorbed if a purgative dose of it has been given. Calomel and the metallic preparations are the two forms most used as purgatives. The former is the more powerful.

Whatever compound of mercury is taken by the mouth, it, in the stomach, becomes a complex albuminate containing mercury, sodium, chlorine, and albumin. This compound, in the presence of the sodium chloride in the stomach, can exist in solution there. Precisely what happens to it in the duodenum is doubtful; but it is quite certain that if the dose is insufficient to cause purgation some mercury is absorbed, the rest passing out of the bowel as a sulphide.

Liver.-It was formerly taught that calomel increased the amount of bile formed by the liver. This is now known to be an error, but [corrosive mercuric chloride] increases it, and pos: sibly, occasionally when calomel is administered, some of it is converted into the [corrosive] chloride. Large doses of calomel are said to slightly diminish the secretion of bile. Calomel and, to a less extent, preparations of metallic mercury are, however, called indirect cholagogues, because they, in the manner already explained, aid the excretion of bile. The stools are [spinach-] green and contain calomel, mercuric sulphide, and unaltered bile.

Blood.-After absorption the mercurial compound formed in the stomach and intestines probably becomes oxidized, and circulates as an oxyalbuminate. Minute, long-continued doses of mercury slightly increase the richness of the blood in red corpuscles, and in animals may add a little to the weight of the body. I-arge doses produce anæmia. Mercury checks the emigration of white corpuscles, and this perhaps explains its antiphlogistic action. 
Remote effects.-Mercury is excreted by the saliva, bile, urine, sweat, [fæces, ] and milk. In small doses no effects can be attributed to this, but in large doses mercury irritates the salivary glands and is a powerful sialogogue. By itself it is a feeble diuretic, but it sometimes powerfully aids other diuretics. It is eliminated very slowly, and hence accumulates in the body.

\section{Therapeutics of Mercury and its Salts.}

External.-Antiseptic action.-Solutions of the [corrosive] chloride are very largely employed. A strength of $I$ in 1000 is used for washing the hands, for washing the parts to be operated upon, for soaking towels, lint, sponges, etc., used in operations, for washing infected articles, infected rooms, furniture, linen, etc. For wounds and cavities (as the uterus), the strength for a single washing should not exceed I in 2000, [and weaker. solutions are preferable]; for continual irrigation $I$ in 10,000 . Corrosive sublimate [tablets], tinted blue, made so that one dissolved in a pint of water makes a solution of $I$ to 500 , are a convenient form in which to carry the antiseptic. Corrosive sublimate solutions should always be tinted blue to render them easy to recognize. [The mixed mercury and zinc cyanide as suggested by Lister is unirritating. It is said to have but slight germicidal value, but its inhibitory power is so great that a solution of I to 1200 will permanently prevent putrefaction in animal fluids. Cyanide gauze may be made actively germicidal by impregnation with a solution of I to 4000 of corrosive mercuric chloride.]

Antiparasitic action. - White precipitate ointment, diluted [mercuric] nitrate ointment, and a wash of [corrosive] chloride are very useful for destroying lice on the head ; and these three, especially the last, are excellent for destroying the fungus in ringworm and favus. The oleate of mercury is useful for destroying that in pityriasis versicolor; if the skin is easily irritated the ointment of it should be used. Mercurials should not be applied over so large an area that there is a risk of poisoning from absorption.

Irritant action.-The acid solution of the nitrate is used to destroy warts, condylomata, etc. ; no doubt much of its caustic 
action is due to the free nitric acid it contains. Milder preparations, such as the ointment of [mercuric] nitrate, or of red [mercuric] oxide, if diluted, may be used for tinea tarsi ; and the same ointments are very beneficial to any ulcer or sore that requires a stimulant, whether or not it be syphilitic. [In ophthalmic practice the ointment of yellow mercuric oxide, known as Pagenstecher's ointment or ophthalmic salve, is largely employed.] When a milder preparation is required, calomel is often dusted on the part; and black wash [Lotio Hydrargyri Nigra, B. P.-Calomel, $\mathbf{x}$; glycerin, 8 ; mucilage of tragacanth, 20 ; lime water, to $\mathrm{I} 60$;] is very commonly used, especially for syphilitic sores and condylomata. [This reaction may be used to determine whether the corrosive mercuric chloride with which gauze has been impregnated has partially changed into the mild chloride. If a black color appears upon application of lime water, calomel is present.]

Itching.-Black wash, yellow wash [Lotio Hydrargyri Flava] (see p. $2 \mathrm{Ir}$ ), or Unguentum Hydrargyri may be employed to relieve the itching of skin diseases, such as [pruritus] senilis and urticaria, if they are not too extensive. A very favorite ointment for many skin diseases is composed of equal parts of diluted mercuric nitrate, zinc oxide and lead acetate ointments (see p. I 78 ).

Absorbent action. - All mercurial ointments and the oleate, when applied to or gently rubbed into any part which is chronically inflamed, often aid the absorption of the products of inflammation, if they are not too deep-seated. For this purpose blue ointment, or Scott's ointment [Unguentum Hydrargyri Compositum, B. P., which consists of mercurial ointment, ro; yellow wax, 6 ; olive oil, 6 ; and camphor 3 , ] or the oleate [in an] ointment are very commonly used for chronic inflammation of joints, chronically enlarged glands, and chronic peritonitis, which certainly sometimes appears to be cured by the application of a binder spread with one of these preparations or the Linimentum Hydrargyri [B. P., which consists of equal parts of mercurial ointment, solution of ammonia and camphor liniment] even when the disease is tuberculous. The ointment of the red [mer- 
curic] iodide is, in India, applied to the thyroid gland in goitre.

Internal. - Alimentary canal. - Very dilute solutions of the [corrosive] chloride (4 gr. [.24 gm.] to $10 \mathrm{fl}$. oz. [300. c.c.] water with I fl. dr. [4. c.c.] of diluted hydrochloric acid and a little glycerin may be used as a mouth wash for syphilitic ulceration. Ringer advises gray powder [Hydrargyrum cum Creta] in minute doses for the sudden vomiting immediately after food sometimes met with in children. By far the most important intestinal action of mercury is its purgative effect. Calomel and blue pill are pre-eminently the purgatives to employ when there is, from the headache, constipation, furred tongue, feeling of weight over the liver, and general lassitude, reason to suspect that the dyspepsia is hepatic. Either of these drugs at night, followed by a watery purge, in the morning, will often completely relieve the symptoms. The blue pill at night, and black draught (Infusum Sennæ Compositum) in the morning have long been a favorite combination. [Acid solutions, as lemonade, should not be taken until the purgative effects of calomel have passed.] Mercury or calomel is also one of the best purgatives for cases of cirrhosis, and for cardiac cases in which there is considerable hepatic congestion. Gray powder mixed with a little sugar is an excellent purgative for children, or even for adults, when a very mild purge is required-as, for example, after severe enteritis or peritonitis. [Calomel in small doses (10 gr. ; .006 gm.), triturated thoroughly with sugar of milk and repeated every hour until a movement is secured, is a favorite gentle purgative] or if it is desirable to open the bowels dur. ing typhoid fever. Children take mercury very well. Infants can easily bear grain [.06 gm.] doses of the gray powder. As diarrhœa, especially in children, is so often due to the presence of some irritant, a simple purgative, as gray powder, will, by removing it, often cure the diarrhœa. This preparation hardly ever causes griping, but calomel is liable to do so. Mercury compounds are, on account of their intestinal antiseptic action, much given in Germany for typhoid fever.

Remote uses. - In cases of heart disease mercury is often com- 
bined with digitalis and squill as a diuretic (as in the well-known Guy's diuretic pill : blue pill, powdered squill, powdered digitalis, of each I gr. [.०6 gm.]; extract of hyoscyamus, $11 / 2 \mathrm{gr}$. [. $10 \mathrm{gm}]$.$) , and in some cases this combination does great good.$ The [corrosive] chloride is most often used for adults, and the gray powder for children.

Syphilis.-Mercury in any form is powerfully antisyphilitic. This action is so important that it makes mercury one of the most valuable drugs we have. It has already been mentioned that it may be applied locally to syphilitic ulcerations, but to be of any use it is essential that it should also be administered so as to reach the blood. It is a direct antidote to the syphilitic virus; it can completely cure the patient; its use must be continued over a long time, but it should never be pushed to salivation. Treatment should be begun at as early a stage as possible, [as soon as the diagnosis is established.] It is especially valuable in the primary and secondary stages; authorities differ as to its value in tertiary syphilis. It is as efficacious for the congenital as for the acquired disease. It is also administered for many non-syphilitic varieties of chronic inflammation, but not so often as formerly. Patients with disease of the kidneys do not bear it well. The [yellow] iodide is very commonly prescribed for syphilis, and often succeeds when other preparations have failed. Its great disadvantage is in its instability. Mercurous tannate (dose, I to $2 \mathrm{gr}$. [.06 to . $22 \mathrm{gm}$.] in a pill) is strongly recommended by some authorities.

[Mercurol (not official) is a chemical combination of nucleinic acid and mercury, the former being obtained from yeast. It is a brownish-white powder, soluble in water but insoluble in alcohol. It is employed in a 2 per cent. solution as an injectiun in gonorrhœa. This apparently destroys the gonococci, lessens the severity of the inflammation, and tends to prevent the development of complications. It does not entirely stop the discharge in all cases.]

13. SAL ALEMBROTH.-[Not official. ] Ammonio-Mercuric Chloride. A double Mercury and Ammonium Chloride. 
Source. - Mix 27I parts of [Corrosive Mercuric Chloride] with 107 of Ammonium Chloride, both in solution, and evaporate.

CHARACTERS. - Flattened rhombic prisms, freely soluble in water or glycerin. It contains one molecule of [Corrosive Mercuric Chloride] combined with two of Ammonium Chloride. Three grains [.20 gm.] of Sal Alembroth contain two grains [. $12 \mathrm{gm}$. of Corrosive Mercuric Chloride]. It is a very powerful antiseptic, but does not combine with albumin so readily as [Corrosive Mercuric Chloride], and is therefore less irritating.

\section{[Uses of Sal Alembroth.]}

Sal alembroth gauze (containing I per cent.) and sal alembroth wool ( 2 per cent.), both tinted with aniline blue, which is bleached by the discharge, so that it is easy to see if it has soaked through, are much used to dress wounds antiseptically.

Sal alembroth injections ( $1 / 3 \mathrm{gr} . ;[02 \mathrm{gm}$.$] in ro minims,$ [.6o c.c.] ; of water) are a convenient non-irritating form in which to use mercury subcutaneously in syphilis. [The precautions mentioned below should be observed.]

14. MERCURO-ZINC CYANIDE.-(Not official.) This consists of one molecule of Mercuric Cyanide combined with four molecules of Zinc Cyanide.

Characters. - A white powder.

\section{Uses of Mercuro-Zinc Cyanide.}

Mercuro-zinc cyanide gauze and wool, each containing 3 per cent. of the salt, and each tinted pink, are much used in antiseptic surgery, as the salt is unirritating. It is also used as an ointment.

Modes of administration of mercurials. - ( 1 ) By the mouth. - Tho Liquor Hydrargyri Perchloridi [B. P. which is corrosive mercuric chloride, $\mathbf{I}$; ammonium chloride, I; water, 1000] is often given to adults, usually in doses of $x$ to $2 \mathrm{fl}$. dr. [4. to 8. c.c.] For the later symptoms of syphilis, potassium iodide is often combined with it. [Mercuric] iodide is formed and is kept in solution by the excess of potassium iodide. Mercurous iodide, known as the [yellow] iodide, is much used by some. It is insoluble in water, and is incompatible with potassium iodide, red [mercuric] iodide and metallic mercury being formed. The best preparation for children is $1 / 2$ to $1 \mathrm{gr} .[.03$ to $.06 \mathrm{gm}$.] of gray powder, given just often enough to avoid purgation.

(2) By the rectum. - Occasionally mercury is given as a suppository. [Each may contain $5 \mathrm{gr} . ; \cdot 30 \mathrm{gm}$. of mercurial ointment.] 
(3) Endermically.-Mercurials, especially calomel, are often dusted on sores and ulcers, and lotions are also locally applied. Mercury can be absorbed in this way.

(4) By inunction.-Blue ointment may be rubbed into the skin. The best position is the inner side of the thigh. Usually a piece the size of the top of the thumb, rubbed in once a day, is enough. It has been put inside the sock, for then it is rubbed into the foot during walking. A very efficient way of applying the ointment in children is to smear it on a flannel binder which is worn round the abdomen. [The oleate may be employed for inunction; this possesses the advantage of not staining the clothing.] Mercury is rapidly absorbed by these means.

(5) Hypodermatically.-One-eighth of a grain [.008 gm.] or less of the [Corrosive] Chloride dissolved in about 5 to 8 minims [.30 to .50 c.c.] of distilled water is used for a dose. The needle of a hypodermatic syringe is plunged deeply into some muscles, preferably those of the gluteal region, and to the outer side of it, so that the patient does not sit or lie on the spot. If much pain is caused, a piece of ice may be held over the part before the injection and after the needle is withdrawn. The injection should be repeated daily; before going to bed is a good time. With proper care no abscesses result. This is a very rapid and thorough way of bringing the patient under the influence of mercury. Mercuric cyanide is also a good salt for subcutaneous injection.

(6) Fumigation.-Calomel, [the black oxide, or the red mercuric sulphide known as Cinnabar (neither of the last two are official), may be] used. The patient, who is naked, sits on a cane-bottomed chair ; a blanket, which reaches to the floor, is fastened lightly round his neck. Twenty grains [1.20 $\mathrm{gm}$. of the salt] are placed in a porcelain dish, over a spirit lamp, under the chair. [The mercury] volatilizes, and is absorbed by the skin. A bath should last twenty minutes; with obvious modifications this method may be applied to patients in bed.

(7) Inhalation.-This is rarely or never used.

(8) Baths of three drachms [12. gm.] of [corrosive] chloride to thirty gallons [114 liters] of water, with one fluid drachm [4. c.c.] of hydrochloric acid added, have been used, but they are now very rarely employed.

\section{TOXICOLOGY.}

Acute poisoning is [not] rare. Salts of mercury, especially the mercuric, produce severe gastro-intestinal irritation, causing great pain, vomiting and diarrhœea. The corrosive [chloride] and white precipitate are the preparations usually taken. [In case of acute poisoning albumin, the white of an egg (one being sufficient for $4 . \mathrm{gr}$; .24 gm. of the corrosive chloride, the albuminate re-dissolving in an excess), milk and flour are useful. Vomiting should be induced by mustard and lukewarm water, apomorphine or by irritation of the fauces.] 
Chronic Poisoning.-Ptyalism by mercury or its salts produces a train of remarkable symptoms. They were very common when it was the practice to give larger doses of mercurials than are now employed, and they are occasionally seen in those who work in mercury. In the present day, when the patient shows any sign of mercurialism, the dose is reduced. The symptoms (which constitute hydrargyrism or mercurialism) may be brought about however the mercury is taken. The first indications noticed are slight foetor of the breath and soreness of the gums when the teeth are [brought forcibly together, by closure of the jaws.] Then follows a disagreeable metallic taste in the mouth, the gums become swollen and soft, and they bleed readily. Next there is a considerable increase in the amount of saliva secreted. All these symptoms gradually become more marked, and the tongue swells. The teeth are now loose, the saliva, which is thick and viscid, pours from the mouth, the parotid and salivary glands are enlarged and tender, and there is a slight rise of temperature. In olden days the symptoms occasionally ended in the falling out of the teeth, extensive ulceration of the mouth and tongue, necrosis of the jaw, great weakness, emaciation, anæmia, a watery state of the blood, a liability to hæmorrhages, exhaustion and death.

More rarely the symptoms are, for the most part, nervous. These occur, chiefly, if not entirely, among those who work in the metal and inhale the vapor. The first to be observed is tremor, beginuing in the face, then invading the arm, and afterwards the legs. Early in the case the trembling is seen only on movement ; soon it is permanent. It resembles paralysis agitans. Usually there is considerable weakness of the affected muscles ("mercurial palsy"). There may be pains, and a weak mental condition is common. Nothing has been found, post-mortem, to account for these symptoms.

\section{GROUP VII.}

\section{Arsenic, Antimony, Chromium.}

The compounds of these metals have several physiological and some chemical points in common. The oxide of each is externally a powerful caustic. Internally Arsenic, Antimony, and (as far as we know) Chromium compounds are severe gastro-intestinal irritants. Arsenic and Antimony in large doses both cause general fatty degeneration.

\section{ARSENUM.}

$$
A s=[74.9 .]
$$

I. ACIDUM ARSENOSUM.-[Arsenous Acid. $\mathrm{As}_{2} \mathrm{O}_{3}=\mathbf{1 9 7 . 6 8}$. Synonyms._Arsenic Trioxide. White Arsenic.]

SOURCE. - Arsenical ores are roasted and purified by sublimation.

Characters. - [A heavy, solid, occurring either as an opaque, white 
powder, or in irregular masses of two varieties-the one amorphous, transparent and colorless, the other crystalline, opaque or white. Solubility.-In 30 to 80 parts of cold, in 15 parts of boiling water.]

INCOMPATIBLES.-Lime water, iron salts, and magnesia.

IMPURITIES. - Lime salts.

Dose, $\frac{1}{60}$ to $\frac{1}{10} \mathrm{gr}$; [ [.00r to $.006 \mathrm{gm}$.]

\section{Preparations.}

r. Liquor [Potassii Arsenitis.-Solution of Potassium Arsenite. Synonym.-Fowler's solution. Arsenous Acid, 1o; Potassium Bicarbonate, 20; Compound Tincture of Lavender, 30. Boil in sufficient distilled water to make I000.] No decomposition occurs, but an alkaline solution of arsenous acid is formed. Strength. - I per.cent. of Arsenous Acid.

Dose, 2 to $10 \mathrm{~m}$.; [.12 to .60 c.c.]

2. Liquor [Acidi Arsenosi.-Solution of Arsenous Acid. Arsenous Acid, IO ; is boiled with diluted Hydrochloric Acid, 50 ; and distilled water to make 1000.] No decomposition occurs, but an acid solution of arsenous acid is formed. Strength. - I per cent. of Arsenous Acid.

Dose, 5 to ro m.; [.30 to .60 c.c.]

2. SODII ARSENAS.-Sodium Arsenate. $\mathrm{Na}_{2} \mathrm{HAsO}_{4}+7 \mathrm{H}_{2} \mathrm{O}[=$ 311.46.

SoURCE.-Heat to redness Arsenous Acid, Sodium Nitrate, and Sodium Carbonate; dissolve the fused mass in water and crystallize. Sodium Pyroarsenate is formed. $\mathrm{As}_{2} \mathrm{O}_{3}+2 \mathrm{NaNO}_{3}+\mathrm{Na}_{2} \mathrm{CO}_{3}=\mathrm{Na}_{4} \mathrm{As}_{2} \mathrm{O}_{7}+\mathrm{N}_{2} \mathrm{O}_{3}+\mathrm{CO}_{2}$. On adding water to the Pyroarsenate, a solution of Sodium Arsenate, which crystallizes on standing, is formed. $\mathrm{Na}_{4} \mathrm{As}_{2} \mathrm{O}_{7}+\mathrm{H}_{2} \mathrm{O}=2 \mathrm{Na}_{2} \mathrm{HAsO}_{4}$, which crystallizes with $7 \mathrm{H}_{2} \mathrm{O}$.

Characters._Colorless, transparent, monoclinic prisms, having a mild alkaline taste. Solubility.-In 4 parts of water.]

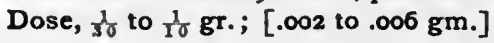

\section{Preparation.}

Liquor Sodii Arsenatis. - [Solution of Sodium Arsenate. Synonym.-Pearson's solution. (Pearson's solution is really one-fifth as strong as the official Liquor Sodii Arsenatis.)

SourCE.-Dissolve Sodium Arsenate, 1; deprived of its water of crystallization by heat, in distilled water, 100.] Strength. - I per cent. of Sodium Arsenate.

Dose, I to ro m. ; [.06 to .60 c.c. $]$

3. ARSENI IODIDUM.-[Arsenic Iodide. AsI $_{3}=454.49$. $]$

Source. - Made by the direct union of Iodine and Metallic Arsenic [or by mixing solutions of Arsenous and Hydriodic Acids and evaporating. 
Characters. - Glossy orange-red, crystalline masses, or shining orangered, crystalline scales, having an Iodine-like odor or taste. Solubility. - In 7 parts of water, and in 30 parts of Alcohol.]

Dose, $\frac{1}{30}$ to $\frac{1}{10} \mathrm{gr} . ;$ [ $[.002$ to $.006 \mathrm{gm}$.]

\section{Preparation.}

Liquor Arseni et Hydrargyri Iodidi. See Mercury, p. 212.

4. FERRI ARSENAS. See Iron, p. 196.

\section{Action of Arsenical Compounds.}

External.-Arsenous acid has no action on the skin, but applied to raw surfaces it is a powerful caustic.

Internal. - Alimentary canal.-Unless the dose be very small all preparations containing arsenic are very severe gastro-intestinal irritants (see Toxicology). Part at least of this effect is due to excretion of the arsenic into the stomach after absorption, for if given subcutaneously there may be no local effect, although there is intense gastritis soon after injection. In minute doses they are gastric stimulants, causing dilatation of the gastric vessels and an increased flow of gastric juice. Small doses also stimulate the duodenum.

Blood.-Arsenic is absorbed into the blood. Nothing is known of its physiological action there ; but it can, in some forms of anæmia, increase the hæmoglobin and the number of red corpuscles. Given to animals it considerably increases the red marrow at the expense of the yellow, and slightly stimulates the formation of compact bone.

Circulation.-In the frog the rapidity and force of the heart are lessened till it finally stops. This is a local action, for it takes place when applied to the excised heart. Large doses destroy the capillaries and lead to hæmorrhage.

Remote effects. - In many diseases arsenic evidently profoundly affects metabolism, for the patient recovers under treatment by this drug. It is doubtful whether, if given in small doses to healthy persons, it usually does more than sharpen the appetite. It has been stated by Dogiel to unite with albumin; another view, that of Binz and Schulz, is that arsenous acid becomes arsenic acid by taking oxygen from the protoplasm, but that the 
arsenic acid subsequently yields up the oxygen again, and that the activity of arsenic is due to its being a carrier of oxygen. Some of the people in Styria eat white arsenic in small quantities, and it increases their strength, weight, and appetite, and clears their complexion. It is probable that the reason why these people can take arsenic in such quantities is that an antitoxin is developed in them. Wood concludes that small doses of arsenic check tissue change and decrease nitrogenous elimination, whilst toxic doses have the opposite effect. But there is no proof of any of these statements, and we have no certain knowledge of the influence of arsenic on nutrition, nor do we know of any action to which its beneficial effects in many diseases can be referred; but as the drug certainly in some way alters the condition of the sufferer it is vaguely called an alterative. It is eliminated by the urine, the alimentary canal, the sweat, the saliva, the milk, and even the tears, but it is also stored in the body, chiefly in the liver and kidneys. It may be found many years after death in the bodies of those who have taken it'during life. It can pass from the mother to the fœetus.

\section{Therapeutics of Arsenical Compounds.}

External.-Formerly arsenous acid was used as a caustic to destroy growths, lupus, warts, etc., either pure or as a paste. [Marsden's paste consists of arsenous acid, I ; powdered acacia, 2 parts.] Arsenous acid, I ; charcoal, I; red [mercuric] sulphide, 4 parts; and water, [sufficient to make a paste,] is a formula once very popular. It must be used strong enough to make the mass of dead tissue slough out quickly, or else the patient becomes poisoned, for the arsenic is rapidly absorbed. Arsenous acid, I ; calomel, 8 ; vermilion antimony [sulphide, 8 parts ;] make a caustic powder. Liquor [potassii arsenitis] has been recommended by Ringer as an application for corns.

Internal.-Alimentary canal.-Arsenous acid is useful to destroy the tooth pulps before [filling] teeth.

In some forms of dyspepsia small doses of Liquor Potassii Arsenitis are occasionally given to stimulate the appetite. Arsenic is so liable to cause sickness, diarrhœa, and other symptoms of 
poisoning, that it is a rule always to begin a course of it with small doses, say 3 or 4 minims [.20 to .25 c.c.] of Liquor [Potassii Arsenitis,] or $\frac{1}{80}$ to $\frac{1}{40} \mathrm{gr}$. [.00I to .0015 gm.] of arsenous acid as a pill, and to gradually increase the quantity. Arsenic in any form should always be taken immediately after meals, so as to dilute it by the contents of a full stomach. Children bear it well; old people do not. Very small doses sometimes check vomiting, especially that form in which the food simply regurgitates, and in exceptional cases it may succeed in checking diarrhœa when other drugs have failed.

Remote effects.-Arsenic is of great value in chronic superficial skin diseases not owing their cause to an irritant. It is, therefore, largely used for psoriasis, pemphigus, and sometimes for chronic eczema. It is of no use in the acute stages of these maladies, nor if cutaneous inflammation is deep-seated.

Cases of anæmia which cannot be cured by iron, and which fall under the heading of primary anæmia, may be occasionally much improved by arsenic. Such are pernicious anæmia, splenic leucocythæmia, and Hodgkin's disease ; but often no drug is of any avail. In other forms of anæmia, such as chlorosis, arsenic may be tried, but not often with benefit, when iron compounds disagree. It often improves the metabolism, the appetite, and the weight in those whose general health is feeble. Arsenic is, next to quinine, the best antiperiodic we have; but it is not nearly so efficacious. It may, however, in the absence of quinine, be used for ague, and is especially valuable for the anæmia which follows ague, and for neuralgia due to the same cause. It often does distinct good in rheumatoid arthritis if given for a long while. It is frequently prescribed for chorea, [particularly in rapid increasing doses]; but it is difficult to prove that the [patients] get well more quickly than they would without any drug. Arsenic has been strongly recommended in asthma and in hay fever. For asthma it may be given by the mouth, or smoked as cigarettes, made by saturating bibulous paper in a solution of fifteen grains [I. gm.] of [potassium] arsenite to an ounce [3o. c.c.] of water. It has been given in phthisis, but without benefit. [There appears to be good evidence that arsenic 
in large doses restrains the growth of sarcomata, particularly of the fusiform-cell variety.] The springs of Levico and La Bourboule contain arsenous acid. Strong Levico contains $\frac{1}{12}$ gr. [.005 gm.] of arsenous acid and $30 \mathrm{gr}$. [2. gm.] to the pint [48o. c.c.]. Weak Levico $\frac{1}{120}$ gr. $[.0005 \mathrm{gm}$.] and $8 \mathrm{gr} .[0.5$ $\mathrm{gm}$.] respectively. La Bourboule contains $\frac{1}{12}$ gr. [.005 gm.] of arsenous acid and a trace of iron to the pint [480. c.c.]. These waters should always be drunk at meals.

[Cacodylic acid ( $\mathrm{AsO}\left(\mathrm{OH}_{3}\right) \mathrm{O}\left(\mathrm{CH}_{3}\right)_{2}$, (not official), and sodium cacodylate ( $\mathrm{AsONa}\left(\mathrm{CH}_{3}\right)_{2}$, (not official) have recently been proposed as eligible methods for the administration of arsenic. The former contains $5^{8}$ per cent. of arsenic. Their solubility, relatively small toxicity and the diminished local irritation which they produce are advantages to be borne in mind. The best form of administration is as sodium cacodylate given hypodermatically in daily amount of from $3 / 4$ to $2 \mathrm{x} / 2 \mathrm{gr}$; .05 to $.15 \mathrm{gm}$., in solution. By this method the arsenic is fully efficacious, no alliaceous odor is given to the breath or perspiration, and gastric and intestinal disturbances do not supervene. Prolonged use may set up albuminuria. By the rectum it produces less irritation and the odor of garlic is not so pronounced as after the use of Fowler's solution. This method is preferable in the treatment of tuberculosis, diabetes, Basedow's disease and leukæmia.]

\section{Toxicology.}

Acute Poisoning.-[Arsenous acid] is frequently used as a poison. [The forms most employed are Scheele's and Paris Green (cupric arsenite), and Schweinfurth's Green (a compound of cupric arsenite and arsenate). Symptoms.]-Soon after taking it the sufferer experiences faintness, nausea, sickness, epigastric pain and tenderness. These symptoms quickly increase. The romit is brown, and often streaked with blood; the pain is very severe; there is profuse diarrhoa, with much tenesmus; and there are cramps in the calves of the legs. The vomiting becomes violent and incessant; there is a burning sensation in the throat, with intense thirst. Soon severe symptoms set in ; the skin is cold, the pulse small and feeble, and the patient dies [in collapse]. The symptoms frequently bear a close resemblance to those of cholera. Post-mortem. - The stomach is intensely inflamed, even if the arsenic has not been taken by the mouth, but has been applied in large quantities to cancerous growths. This shows that arsenic is excreted from the blood into the stomach. The small intestines are also acutely inflamed. 
Treatment.-Wash out the stomach. Give emetics (see p. 139), choosing the least irritating and least depressing. The stomach must be completely emptied. Give unlimited quantities of freshly prepared humid [ferric hydrate] (see p. 193) or dialyzed iron. If neither of these is handy, give magnesia in large amounts, or large doses of castor oil and water. Give brandy or ether subcutaneously; apply hot blankets and bottles to the feet and the abdomen.

Chronic Poisoning.-Often, when arsenic is taken as a medicine, slight symptoms of poisoning are seen. They are loss of appetite, nausea, perhaps vomiting, slight abdominal pain, and mild diarrhoa. The eyelids become a little puffy, the conjunctivæ injected, the eyes and nose water, and there is slight headache. These symptoms, of course, show that the dose given is too large, and that it must be decreased.

Arsenic is so often used in the manufacture of all sorts of articles, especially wall papers and fabrics, that chronic poisoning by it is frequently seen. [The evidence in regard to chronic poisoning from occupancy of rooms decorated with arsenical wall paper is somewhat contradictory, but the facts point towards its probability. Quite as often the poisoning is due to the arsenic which is a contamination of aniline dyes as it is the arsenical pigments, so that the color should not be depended upon, but rather a chemical examination.] It is also met with in workers of arsenic, and in persons to whom it has been given with intent to murder. The symptoms produced are those already mentioned as present when large doses of arsenic are taken medicinally.

Long-continued use of arsenic may induce peripheral neuritis; the chief symptoms of arsenical neuritis are herpes zoster, paralysis of the muscles of the limbs, especially the extensors of the hands and feet, ataxic gait, severe darting pains in the limbs, and rapid muscular atrophy. Several cases are recorded in which arsenic has caused general brown pigmentation of the skin. It may also give rise to brown pigmentation of patches of psoriasis, and in quite exceptional cases cause eczema or urticaria. After death from chronic poisoning, in addition to the gastro-intestinal and nervous lesions, there is widespread fatty degeneration of most of the organs of the body. It is well seen in the liver, kidneys, stomach and muscles, including the heart.

Repeated doses given to animals abolish the glycogenic function of the liver, so that puncture of the floor of the fourth ventricle no longer causes glycosuria. In frogs poisoned with arsenic the epidermis peels off very easily. This is due to degeneration of its lower cells, the degeneration proceeding from the lowest layer outwards.

[The tests for arsenic are so simple that every physician should be able to make use of them. They are: (1) Reinsch's. - Hydrochloric acid and a clean slip of copper are boiled in the suspected liquid. Bluish spots indicate the poison. (2) Marsh's._Diluted sulphuric acid and zinc are introduced into a flask with the suspected liquid. The gas issuing from the tube is ignited and the flame allowed to impinge upon a clean porcelain plate forming a steel-white mirror if arsenic be present ; or the delivery tube may be heated when the mirror will be deposited upon it. This mirror is distinguished from 
that produced by antimony by its solubility in potassium hypochlorite if arsenic is the cause. ]

\section{ANTIMONIUM.}

$$
\text { Sb. [=1 19.6.] }
$$

I. ANTIMONII [SULPHIDUM.-Antimony Sulphide. $\mathrm{Sb}_{2} \mathrm{~S}_{3}=$ 335.14. Synonym.-Antimony Trisulphide.

Source.-Native Antimony Sulphide purified by fusion, as free from Arsenic as is possible.

Characters. - Steel-gray masses of a metallic lustre and a striated crystalline fracture, forming a black or grayish-black, lustreless powder without odor or taste. Solubility. - Insoluble in water or Alcohol.

\section{Preparations.}

I. Antimonii Sulphidum Purificatum.-Purified Antimony Sulphide. $\mathrm{Sb}_{2} \mathrm{~S}_{3}=335.14$. Synonym.-Purified Black Antimony.

Source. - Antimony Sulphide, I00; purified by Ammonia Water, 50 ; decanted and dried.

CHARACTERs.-A heavy, grayish-black, lustreless powder, without odor or taste.

IMPURITY._Silica.]

2. Antimonium Sulphuratum.-Sulphurated Antimony. [Chiefly Antimony Sulphide $\left(\mathrm{Sb}_{2} \mathrm{~S}_{3}\right)$ and with a small amount of Antimony Oxide $\left(\mathrm{Sb}_{2} \mathrm{O}_{3}\right)$. Synonym. - Kermes mineral.

Source. - Boiled Purified Antimony Sulphide, IOO; and Solution of Soda, 1 200. $\mathrm{Sb}_{2} \mathrm{~S}_{3}+4 \mathrm{NaOH}=\mathrm{NaSbO}_{2}+\mathrm{Na}_{3} \mathrm{SbS}_{3}$. Precipitate with Sulphuric Acid, wash and dry. $\mathrm{NaSbO}_{2}+\mathrm{Na}_{3} \mathrm{SbS}_{3}+2 \mathrm{H}_{2} \mathrm{SO}_{4}$ $=\mathrm{Sb}_{2} \mathrm{~S}_{3}+2 \mathrm{Na}_{2} \mathrm{SO}_{4}+2 \mathrm{H}_{2} \mathrm{O}$.

ChARACTERS. - An amorphous reddish-white powder, becoming lighter in color on exposure to light. Solubility. - Insoluble in water or Alcohol.]

Sulphurated Antimony is contained in Pilulæ Antimonii Compositx. See Mercury, p. 212.

Dose, $\frac{1}{6}$ to I gr.; [.01 to $.06 \mathrm{gm}$.]

2. ANTIMONII OXIDUM.-[Antimony Oxide. $\mathrm{Sb}_{2} \mathrm{O}_{3}=287.07$. Synonym. - Antimony Trioxide.]

Source.-Pour a solution of Antimony Chloride into water. Antimony Oxychloride is precipitated. $\mathrm{SbCl}_{3}+\mathrm{H}_{2} \mathrm{O}=\mathrm{SbOCl}+2 \mathrm{HCl}$. The precipitate is treated with Sodium Carbonate, washed, and dried. ${ }_{2} \mathrm{SbOCl}+\mathrm{Na}_{2} \mathrm{CO}_{3}$ $=\mathrm{Sb}_{2} \mathrm{O}_{3}+2 \mathrm{NaCl}+\mathrm{CO}_{2}$.

Characters. - [A heavy grayish-white powder, without odor or taste.]

IMPURITIES. - Higher oxides.

Dose, 1 to $4 \mathrm{gr}$.; [.06 to .24 gm.] 


\section{Preparation.}

Pulvis Antimonialis.--[Antimonial Powder. Synonym.-James' Powder. Antimony Oxide, 33 ; Precipitated Calcium Phosphate, 67. Dose, 3 to $15 \mathrm{gr}$; .20 to $1.00 \mathrm{gm}$.]

3. [ANTIMONII ET POTASSII TARTRAS.-Antimony and Potassium Tartrate. $2 \mathrm{~K}(\mathrm{SbO}) \mathrm{C}_{4} \mathrm{H}_{4} \mathrm{O}_{6}+\mathrm{H}_{2} \mathrm{O}=662.42$. Synonyms. - Tartar Emetic. Tartrated Antimony.

Source. - Make a paste of Antimony Trioxide $\left(\mathrm{Sb}_{2} \mathrm{O}_{3}\right)$ with Acid Potassium Tartrate and water. Let it stand twenty-four hours, boil in water, and crystallize. $\quad 2 \mathrm{~K} \mathrm{HC}_{4} \mathrm{H}_{4} \mathrm{O}_{6}+\mathrm{Sb}_{2} \mathrm{O}_{3}=2 \mathrm{~K}(\mathrm{SbO}) \mathrm{C}_{4} \mathrm{H}_{4} \mathrm{O}_{6}+\mathrm{H}_{2} \mathrm{O}$.

Characters. - Colorless, transparent crystals of the rhombic system, becoming opaque and white on exposure to air ; or a white granular powder, having a sweet, afterwards disagreeable metallic taste. Solubility.-In 17 parts of water; insoluble in Alcohol].

INCOMPATIBI.Es.-Gallic and tannic acids, most astringent infusions, alkalies, and lead salts.

IMPURITY.-Acid Potassium Tartrate.

Dose, $\frac{1}{10}$ to $1 / 2 \mathrm{gr}$. [.006 to $.03 \mathrm{gm}$.] (diaphoretic), $1 / 2$ to $\mathrm{I} \mathrm{gr}$. [.03 to $.06 \mathrm{gm}$.] (cardiac depressant), $\mathrm{r}$ to $2 \mathrm{gr}$. [.06 to $.12 \mathrm{gm}$.] (emetic).

\section{Preparations.}

1. Vinum Antimonii.-[Wine of Antimony. Antimony and Potassium Tartrate, 4 ; boiling distilled water, 65 ; Alcohol, 150 ; White Wine to 1000 .

Dose, 5 to $60 \mathrm{~m}$.; 30 to $4.00 \mathrm{c.c}$.

2. Syrupus Scillæ Compositus.-Compound Syrup of Squill. Synonym.-Hive Syrup, so called from hives, the old name of croup. Fluid extract of Squill, 8o; Fluid extract of Senega, 8o ; Antimony and Potassium Tartrate, 2; Sugar, $75^{\circ}$; Precipitated Calcium Phosphate, I0; water to 1000.

Dose, 5 to $30 \mathrm{~m}$. ; .30 to 2.00 c.c.]

\section{Action of Salts of Antimony.}

External.-Antimonial compounds are powerful external irritants. [The Liquor Antimonii Chloridi of the B. P., which is a solution of antimony chloride in hydrochloric acid is a severe caustic.] Tartar emetic produces a pustular eruption at the point of application.

Internal.-Alimentary canal.-All compounds of antimony are powerful irritants, internally as well as externally; the action of tartar emetic is best known. The first result of 
swallowing this is vomiting. The early acts of vomiting are due to the direct action of the drug on the wall of the stomach, but it is quickly absorbed, and by its action on the medulla it also produces sickness, but this action is slight. It will produce vomiting when injected into the blood, partly by its action on the medulla - for it will act if the stomach is replaced by a bladder-but also because some of it is excreted into the stomach and intestines, and thus the vomiting is continued for some time. In large doses tartar emetic is irritant to the intestine. [A round mass of metallic antimony was formerly known as the " family pill," because it could be repeatedly used as a laxative.]

Heart. - Antimony acts upon man as upon the lower animals. It is a powerful cardiac depressant, diminishing both the frequency and the force of the beat of the heart. Experiments on animals have shown that the final stoppage takes place in diastole, and that the chief action of antimony is that of a direct depressant to the cardiac muscle itself. Of course, the cardiac depression causes the arterial pressure to fall; but part of this effect is due to a coincident action upon some portion of the vaso-motor system; the probability being that antimony, by paralyzing the muscular coat of the arteries, relaxes them.

Respiration.-Respiration is depressed, the movements become weaker, and inspiration is shortened, but expiration is prolonged. Finally, the pauses become very long and the movements very irregular. The cause of this is not known; probably it is very complex.

Nervous and muscular systems. - Here also antimony acts as a powerful depressant, especially to the spinal cord, and to a less extent to the brain; hence moderate doses cause a feeling of languor, inaptitude for mental exertion, and sleepiness. Experiments on animals show that, after the administration of large doses of antimony, reflex movement is soon lost, and that this is due to a depressing effect on the sensory part of the spinal cord. This depressant influence is felt also in the muscles, and hence antimony will relieve spasm, but whether it does so by direct action on the muscles or by acting on the nervous system is doubtful. 
Temperature.-Moderate doses of antimony have little influence on the temperature, but large doses cause a considerable fall, due, no doubt, in the main to the circulatory depression, but, also, it is said, to a direct action in decreasing the amount of heat produced.

Excretion.-Antimony is excreted by the urine, bile, sweat, bronchial secretion, milk, and particularly by the fæces. We have seen that part of its emetic effect is due to its excretion into the stomach. As it passes out by the bronchial mucous membrane it increases the amount of secretion, and thus acts as an expectorant. On the skin the action is that of a profuse diaphoretic. This is chiefly a secondary result of the depression of the circulation, but is possibly in part a direct local effect. In frogs the action on the skin is very like that of arsenic, but antimony softens rather than detaches the epidermis, which thus becomes a jelly-like mass. Being excreted in the bile, it aids its flow; therefore it is a cholagogue.

In passing through the kidneys it may be slightly diuretic, but this depends upon the amount of perspiration produced by it. If its use is continued for some time it will cause, like arsenic, fatty degeneration, especially of the liver, and abolition of the hepatic glycogenic function.

\section{Therapeutics of Salts of Antimony.}

External. - [A solution of antimony chloride, known as Butter of Antimony, has been used as a caustic, but its employment has been abandoned, as the sore produced is difficult to heal.] Many years ago an ointment of tartar emetic was commonly applied as a counter-irritant, but it causes much pain, and is now seldom used.

Internal.-Alimentary canal.-Tartar emetic is not to be recommended as an emetic, for the action is slow, and the general depression of emetic doses is great. For this reason it should never be given to produce purgation. The only cases in which it is permissible are those in which an emetic is required for laryngitis, bronchitis, or some other acute inflammatory condition of the respiratory tract, for then its depressant action on the 
circulation may perhaps be beneficial, but usually ipecacuanha is preferable.

Circulation.-Antimony was formerly largely employed, especially in combination with aconite, to reduce the force and frequency of the pulse in all sorts of febrile conditions; but this is now generally thought unnecessary. If it is to be used, it is especially indicated in respiratory affections ; for then its expectorant effect may be valuable.

Respiration.-It has been very much given for the early stage of acute bronchitis; but certainly it should not be continued after a free secretion of bronchial mucus has been set up by it. After that it is, on account of its depressing influence, an undesirable expectorant.

Nervous and muscular systems.-Its use as a sedative in delirium tremens is now abandoned, and the introduction of chloroform has made it unnecessary to employ tartar emetic to relax muscular spasm in herniæ, dislocations, etc.

Remote effects. -Occasionally it is given in fevers for its diaphoretic influence and for its slight antipyretic action. Sometimes it is órdered as a cholagogue ; but because of its powerful depressant action, it is less used as a medicine than formerly.

\section{TOXICOLOGY.}

Acute Poisoning.-The symptoms are very like those of arsenical poisoning (see p. 228). Post-mortem.-The gastro-intestinal irritation is very similar, but it is not nearly so marked.

Treatment.- Unless the vomiting is very free, apomorphine [hydrochlorate] subcutaneously, or zinc sulphate by the mouth or the stomach pump, may be used. Also frequent doses of half a drachm [2. gm.] of tannic or gallic acid dissolved in water, strong tea or coffee, mucilaginous drinks, and stimulants subcutaneously. Hot water bottles and warm blankets may be necessary.

Chronic Poisoning is not sufficiently common to call for notice here. [An instance of the use of antimony for homicidal purposes has recently been the subject of judicial inquiry.]

\section{CHROMIUM.}

$$
\text { Cr. [=52.0.] }
$$

1. ACIDUM CHROMICUM.-Chromic Acid. $\mathrm{CrO}_{3}[=99.88$. Synonyms. - Chromic Anhydride. Chromic Trioxide. 
Source.-Dissolve Potassium Dichromate in Sulphuric Acid and water, decant from the Acid Potassium Sulphate, heat with more Sulphuric Acid, cool and crystallize. $\mathrm{K}_{2} \mathrm{Cr}_{2} \mathrm{O}_{7}+2 \mathrm{H}_{2} \mathrm{SO}_{4}=2 \mathrm{CrO}_{3}+2 \mathrm{KHSO}_{4}+\mathrm{H}_{2} \mathrm{O}$.

Characters. - Small needle shaped crystals, or rhombic prisms, of a dark purplish-red color and metallic lustre. Readily yields Oxygen, and will, therefore, easily explode, with either Glycerin, Ether, or Alcohol. Solubility. - Very soluble in water.]

\section{Action of Chromic Acid.}

External.-In consequence of its oxidizing power, chromic acid is a powerful deodorant and disinfectant. It coagulates albumin and oxidizes organic matter, and is therefore a powerful caustic.

Internal.-None is known.

\section{Therapeutics of Chromic Acid.}

External.-[It is used generally in the strength of $x$ per cent. to harden catgut and kangaroo tendon for surgical uses. A lotion of the same strength is used in Germany to toughen the feet of marching soldiers.] As a lotion, I to 40 , or even stronger, chromic acid has been used for its disinfectant properties to wash foul ulcers and sores, and also as a local application for ozæna, gonorrhœa, leucorrhœa, and bad ulcerations of the mouth, but a gargle should not be stronger than [I to 480]. The Liquor [of the B. P., which is one part of chromic acid in 3 of water,] is occasionally used as a caustic to destroy condylomata, etc.

2. POTASSII BICHROMAS. - [Potassium Bichromate. $\mathrm{K}_{2} \mathrm{C}_{2} \mathrm{O}_{7}=$ 293.78. Synonym.-Potassium Dichromate.

Source. - Finely-ground Chrome-iron ore mixed with Potassium Carbonate is roasted in a reverberatory furnace, which causes the separation of all iron in the form of Ferric Oxide, and the production of Potassium Bichromate. Lime or chalk is added during the roasting to prevent fusion. $2 \mathrm{FeOCr}_{2} \mathrm{O}_{3}+$ ${ }_{4} \mathrm{~K}_{2} \mathrm{CO}_{3}+4 \mathrm{O}_{2}=\mathrm{Fe}_{2} \mathrm{O}_{3}+4 \mathrm{~K}_{2} \mathrm{CrO}_{4}+4 \mathrm{CO}_{2}$. After solution in water Sulphuric Acid is added, and the two salts are separated by crystallization. $2 \mathrm{~K}_{2} \mathrm{CrO}_{4}+$ $\mathrm{H}_{2} \mathrm{SO}_{4}=\mathrm{K}_{2} \mathrm{Cr}_{2} \mathrm{O}_{7}+\mathrm{K}_{2} \mathrm{SO}_{4}+\mathrm{H}_{2} \mathrm{O}$.

Characters. - Large, orange-red, transparent, triclinic prisms or foursided tables, odorless, and having a bitter, metallic taste. Solubility. - In ro parts of water; insoluble in Alcohol.] 
INCOMPATIBLES. - Owing to the ease with which it oxidizes it readily forms explosive compounds. It is here prescribed as a pill made up with kaolin.

[IMPURITIES. - Sulphates, chlorides and calcium.

Dose, $\frac{1}{10}$ to $1 \mathrm{gr}$. ; .006 to $.06 \mathrm{gm}$.

\section{action of Potassium Bichromate.}

External.-It is an irritant caustic.] Handling the salt may produce eczema.

Internal.-Occasionally solutions of it have been taken by mistake. Symptoms of very severe gastro-intestinal inflammation with much collapse have followed.

Therapeutics of Potassium Bichromate.

External.- [It is used as a caustic for warts, venereal ulcers and mucous patches.] Its solution is caustic and antiseptic, but it is weaker than chromic acid.

Internal. - [It has been recommended for the treatment of gastric catarrh and gastric ulcer in dose of from $\frac{1}{12}$ to $\frac{1}{6} \mathrm{gr}$., .005 to .or gm., given thrice daily on an empty stomach, and is reputed to relieve nausea, vomiting and pain.]

The remaining groups of the inorganic drugs are non-metallic.

\section{GROUP VIII.}

Containing Phosphorus only.

\section{PHOSPHORUS.}

$$
\text { P. }[=30.96 .]
$$

Source. - [Treat Bone Ash or Lime Phosphate with Sulphuric Acid and water, filter and evaporate. $\mathrm{Ca}_{3}\left(\mathrm{PO}_{4}\right)+2 \mathrm{H}_{2} \mathrm{SO}_{4}=\mathrm{CaH}_{4}\left(\mathrm{PO}_{4}\right)_{2}+2 \mathrm{CaSO}_{4}$. Heat the Acid Calcium Phosphate thus formed, with Charcoal and sand. The heat first forms Calcium Metaphosphate. $\mathrm{CaH}_{4}\left(\mathrm{PO}_{4}\right)_{2}=\mathrm{Ca}\left(\mathrm{PO}_{3}\right)_{2}+2 \mathrm{H}_{2} \mathrm{O}$. This is acted on by the Charcoal and sand thus: $2 \mathrm{Ca}\left(\mathrm{PO}_{3}\right)_{2}+2 \mathrm{SiO}_{2}+\mathrm{IOC}_{2}=$ $2 \mathrm{CaSiO}_{3}+10 \mathrm{CO}+\mathrm{P}_{4}$.

Characters. - A translucent, nearly colorless solid of a waxy lustre, having, at ordinary temperatures, about the consistence of beeswax. By long keeping the surface becomes red, and occasionally black. It has a distinctive and disagreeable odor and taste (but should not be tasted except in a state of 
great dilution); when exposed to the air it emits white fumes, which are luminous in the dark, and have an odor somewhat resembling garlic. On longer exposure to air it takes fire spontaneously. Heated with Hydrogen it becomes red, amorphous, non-poisonous Phosphorus. Solubility.-Sparingly in alcohol, ether and chloroform; freely in carbon disulphide ; insoluble in water.]

Dose, $\frac{1}{100}$ to $\frac{1}{20} \mathrm{gr}$. ; [.0006 to $.003 \mathrm{gm}$.] Never given alone.

\section{Preparations.}

I. Oleum Phosphoratum.-Phosphorated Oil. [Phosphorus, I ; Expressed Oil of Almond, 90 ; (which must be heated to $25^{\circ} \mathrm{F}$.; $121^{\circ} \mathrm{C}$., and filtered to remove water and organic matter, which would otherwise oxidize the phosphorus), and Ether to roo. Strength.-I per cent.]

Dose, 1 to $5 \mathrm{~m}$.; [.06 to $.30 \mathrm{c.c}$.

2. Pilulæ Phosphori.-[Pills of Phosphorus. Dissolve Phosphorus, .06; Chloroform, 5 ; add Althrea, 6 ; and Acacia, $6 \mathrm{gm}$.; with water, and Glycerin; to make 100 pills.] Strength._Each pill contains $\frac{1}{100}$ gr. [.0006 gm.] of Phosphorus.

Dose, I to 5 pills.

[3. Spiritus Phosphori.-Spirit of Phosphorus. Synonym.Tincture of Phosphorus. Phosphorus, 1.2; absolute Alcohol to 1000. Dissolve by heating upon a water-bath.

Spirit of Phosphorus is used to prepare Elixir Phosphori.

Dose, 8 to $40 \mathrm{~m}$.; .50 to 2.50 c.c.

4. Elixir Phosphori.-Elixir of Phosphorus. Spirit of Phosphorus, 210 ; Oil of Anise, 2 ; Glycerin, 550; Aromatic Elixir to 1000.

Dose, $1 / 2$ to $2 \frac{1}{2}$ fl. dr. ; 2. to to. c.c.]

\section{Action of Phosphorus.}

The action of minute doses of phosphorus is that in animals the spongy tissue in the long and short bones is thickened by the deposition of true bone of normal composition, and the compact tissue is rendered more dense. [It diminishes tissue waste, decreasing the elimination of urea and of carbon dioxide. As it is found as a necessary element in the nervous system its action is that of a stimulant to its growth.] Phosphorus enters the blood as phosphorus, and probably acts as such, not as phosphoric acid. [The best known liquid preparation is Thompson's solution: phosphorus, I ; absolute alcohol, 300 ; glycerin, 720 ; and spirit of peppermint, 40 ; dose, $1 / 3$ to I $\mathrm{fl}$. dr.; 1.20 to 4.00 c.c.] See [also] Toxicology. 


\section{Therapeutics of Phosphorus.}

Phosphorus has been used in osteomalacia, in rickets, and in cases of ununited fracture. [Without doubt it promotes calcareous deposit in the healing of fractures. It is of value in the convalescence from exhausting diseases, in nervous exhaustion, in neuralgia when dependent upon debility, in alcoholism, in sexual exhaustion and in various suppurative diseases.]

\section{Toxicology.}

Acute Poisoning.-Phosphorus is often taken or administered criminally, either as match-heads or vermin paste.

[Symptoms.]-For the first few hours there are no effects, then the following symptoms of gastro intestinal irritation set in : Nausea, abdominal pain, and vomiting; the vomited matters smell of phosphorus and are luminous, [if heated with sulphuric acid (Wood).] There is some general depression. Diarrhoea is rare. The patient may die in collapse, but far more frequently these symptoms all pass off, and he appears quite well. But after three or four days jaundice is noticed, and this soon becomes very [marked]; there is now great prostration, the liver is enlarged, the abdomen distended, and he complains of intense thirst. [There is likely to be a garlicky odor to the breath. (Brunton, Semple).] Vomiting of altered blood and diarrhoea with bloody stools may be observed, but these two symptoms are not severe. The skin is cold, the pulse feeble and rapid. The urine is scanty, highly colored, albuminous, bile-stained, and perhaps bloody, and it may contain bile acids and crystals of leucin and tyrosin. [Sarcolactic acid found in the urine is diagnostic. (Wood).] Muscular twitchings occur, the patient becomes comatose and dies. Post-mortem.-Two results are very striking. (x.) Fatty degeneration (thus phosphorus resembles arsenic and antimony), affecting principally the liver, in which it is very marked; and if the patient lives long enough, there may be a diminution in the size of the organ. Fatty degeneration is also found in the muscles, kidneys and gastro-intestinal tract. (2.) Hæmorrhages are seen in many places, and ecchymoses are sometimes very abundant. If they occur in the gastric and intestinal mucous membranes, they may give rise to the erroneous belief that evidences of acute gastro-intestinal irritation can be found at death. The symptoms of phosphorus poisoning in many respects resemble those of acute yellow atrophy of the liver.

Treatment.-Thoroughly empty the stomach by a stomach pump, or by washing it out. [Formerly] copper sulphate [was given] as an emetic (see Copper, p. 180 ), three grains $[.20 \mathrm{gm}$.] every few minutes till vomiting is induced, then every 15 minutes; also half a drachm [2. c.c.] of oil of turpentine every half hour. A full dose of a saline purge may be administered. [Repeated and free inhalations of oxygen have been used, which suggest that 
hydrogen dioxide may be efficacious when given by the mouth.] No oils or fats should on any account be given. [Percy found that the old oil of turpentine, which contains oxygen, if administered soon after taking the poison and before it was absorbed, was an antidote (experiments upon dogs).]

Chronic Poisoning. $\rightarrow$ This, which used to be seen in those who worked among phosphorus fumes, is now of great rarity. [This is because the red or non-poisonous phosphorus is generally employed in match factories.] The chief symptoms are those of gastro-intestinal irritation and necrosis of the jaw. This Stockman has shown to be due to the fact that the phosphorus fumes, when the gum is broken, gain access to the bone and lower its vitality, so that it easily becomes the seat of tuberculous disease. Sufferers from phosphorus necrosis often die from general tuberculosis.

x. CALCII HYPOPHOSPHIS.-[Calcium Hypophosphite. $\mathrm{Ca}(\mathrm{P}$ $\left.\mathrm{H}_{2} \mathrm{O}_{2}\right)_{2}=169.67$.

SOURCE. - Heat Phosphorus with Milk of Lime. Then pass Carbon Dioxide through the liquid to remove the excess of Iime. The Hypophosphite crystallizes out of the solution. $3 \mathrm{Ca}(\mathrm{OH})_{2}+4 \mathrm{P}_{2}+6 \mathrm{H}_{2} \mathrm{O}=3 \mathrm{Ca}\left(\mathrm{PH}_{2} \mathrm{O}_{2}\right)_{2}+2 \mathrm{PH}_{3}$.

Characters. - Colorless, transparent, monoclinic prisms or small lustrous scales, or a white, crystalline powder, having a nauseous, bitter taste. Solubility. - In 6.8 parts of water; insoluble in Alcohol.]

Dose, 2 to $5 \mathrm{gr}$.; [.12 to .30 $\mathrm{gm}$.]

2. SODII HYPOPHOSPHIS.-[Sodium Hypophosphite. $\mathrm{NaPH}_{2} \mathrm{O}_{2}$ $+\mathrm{H}_{2} \mathrm{O}=$ 105.84.]

SOURCE. - Add Sodium Carbonate to a solution of Lime Hypophosphite and evaporate [the filtrate]. $\mathrm{Ca}\left(\mathrm{PH}_{2} \mathrm{O}_{2}\right)_{2}+\mathrm{Na}_{2} \mathrm{CO}_{3}=\mathrm{CaCO}_{3}+2 \mathrm{NaPH}_{2} \mathrm{O}_{2}$.

Characters. - [Small, colorless, transparent, rectangular plates of a pearly lustre, or a white granular salt, with a bitterish-sweet, saline taste. Solubility. - In I part of water; in 30 parts of Alcohol.]

Dose, 5 to $20 \mathrm{gr}$.; [.30 to $1.20 \mathrm{gm}$.]

3. [POTASSII HYPOPHOSPHIS. - Potassium Hypophosphite. $\mathrm{KPH}_{2} \mathrm{O}_{2}=103.91$.

Source.-From double decomposition of Calcium Hypophosphite and Potassium Carbonate; the Potassium Hypophosphite remains in solution. $\mathrm{Ca}\left(\mathrm{PH}_{2} \mathrm{O}_{2}\right)_{2}+\mathrm{K}_{2} \mathrm{CO}_{3}=\mathrm{CaCO}_{3}+2 \mathrm{KPH}_{2} \mathrm{O}_{2}$.

Characters. - White, opaque, hexagonal plates, or crystalline masses, or a granular powder, having a pungent, saline taste. Solubility. - In 0.6 part of water, and in 7.3 parts of Alcohol.

Dose, 5 to $20 \mathrm{gr}$; . 30 to $1.20 \mathrm{gm}$.

\section{Preparations.}

r. Syrupus Hypophosphitum.-Syrup of the Hypophosphites. Calcium Hypophosphite, 45 ; Sodium Hypophosphite, 15 ; Potassium 
Hypophosphite, 15; diluted Hypophosphorous Acid, 2; Spirit of Lemon, 5 ; sugar, 500 ; and sufficient water to make 1000 .

Dose, $x$ to 2 fl. dr.; 4. to 8. c.c.

2. Syrupus Hypophosphitum cum Ferro.-Syrup of the Hypophosphites with Iron.

Ferrous Lactate, Io; Potassium Citrate, 1o; Syrup of the Hypophosphites, to 1000 .

Dose, I to 2 fl. dr.; 4 , to 8. c.c.

4. FERRI HYPOPHOSPHIS.-Ferric Hypophosphite. $\mathrm{Fe}_{2}\left(\mathrm{PH}_{2}\right.$ $\left.\mathrm{O}_{2}\right)_{6}=501.04$.

SOURCE. - A solution of Sodium Hypophosphite is added to a solution of Ferric Chloride, the precipitate is washed and dried with moderate heat. $6 \mathrm{NaPH}_{2} \mathrm{O}_{2}+2 \mathrm{FeCl}_{6}=\mathrm{Fe}_{2}\left(\mathrm{PH}_{2} \mathrm{O}_{2}\right)_{6}+6 \mathrm{NaCl}$.

Characters.-A white, or grayish-white powder, nearly tasteless. Solubility. - Only slightly soluble in water.

IMPURITIES. - All of the Hypophosphites are liable to be contaminated with the alkaline carbonates or to contain free alkali. They explode if heated.

Dose, 2 to ro $\mathrm{gr}$; ; .12 to $.60 \mathrm{gm}$.

5. ZINCI PHOSPHIDUM.-Zinc Phosphide. $\mathrm{Zn}_{3} \mathrm{P}_{2}=257.22$.

SUURCE. - From vapor of Phosphorus in a current of dry Hydrogen over heated Zinc, after all atmospheric air is expelled.

Characters. - A gritty powder of a dark-gray color, or crystalline fragments of a dark metallic lustre, and having a faint odor and taste of phosphorus.

Dose, $\frac{1}{10}$ to $\frac{1}{3} \mathrm{gr}$; .006 to $.02 \mathrm{gm}$.

6. ACIDUM HYPOPHOSPHOROSUM DILUTUM.-Diluted Hypophosphorous Acid. A liquid composed of about ro per cent., by weight, of absolute Hypophosphorous Acid $\left(\mathrm{HPH}_{2} \mathrm{O}_{2}=65.88\right)$ and about 90 per cent. of water.

SoURCE.-Decompose Potassium Hypophosphite by Sulphuric Acid, filter and evaporate to a syrupy consistence. $2 \mathrm{KPH}_{2} \mathrm{O}_{2}+\mathrm{H}_{2} \mathrm{SO}_{4}=\mathrm{K}_{2} \mathrm{SO}_{4}+2 \mathrm{H}$ $\mathrm{PH}_{2} \mathrm{O}_{2}$.

Characters. - A colorless liquid, without odor, and having an acid taste. Sp. gr., about 1.046. Solubility.-Miscible in all proportions, with water.

Diluted Hypophosphorus Acid is used in Syrupus Hypophosphitum.]

Therapeutics of Ferric, Calcium, Sodium, and Potassium HYPOPHOSPHITES.

These drugs have been recommended for phthisis. Although in many cases there is no satisfactory evidence of their value [yet 
they are extensively used and are the basis of a large number of proprietary preparations.

Following Churchill they should be of chemical purity, neutral in reaction; the presence of free alkali or alkaline carbonates quickly giving rise to an atonic dyspepsia. The preparation, the syrup of the hypophosphites, is faulty in that each salt has a peculiar property, yet the final result being due to the hypophosphite, and its beneficial effect upon nutrition. In the early stages of phthisis (infiltration) the sodium salt should be administered and the sodium salt alone; if excavation is present the calcium salt is indicated, and that alone, provided that it does not too suddenly check expectoration, when the sodium salt should be resumed.

The potassium salt is a valuable expectorant in chronic bronchitis; but it has a very limited usefulness in phthisis. The hypophosphites, when administered intelligently, will improve nutrition and relieve some of the symptoms of phthisis. If administered in too large doses, or simultaneously with other remedies, as . arsenic, stimulants, strychnine, or cod-liver oil, they are likely to produce headaches and dyspepsia, and fail to cause improvement. The objection to the syrup, U. S. P., namely, the use of the salts in combination, applies to nearly all of the proprietary preparations, most of which contain impure drugs, contain a low percentage of hypophosphites, and are not scientific combinations. The syrup of the hypophosphites with iron is valuable as a reconstructive. Zinc phosphide is believed to have the same physiological and therapeutical effects as phosphorus.

7. CALCII GLYCEROPHOSPHATIS.-Calcium Glycerophosphate. (Not official.)

Source. - Phosphoric Acid, 30 ; mixed with Glycerin, 36 ; is kept at a little above normal body temperature for six days, being shaken several times daily. On the seventh day it is cooled, its acidity is gradually and completely neutralized with Calcium Carbonate. After two days the solution is filtered, and the salt precipitated by Alcohol. The precipitate is dissolved in cold water, the solution filtered and evaporated to dryness at a low temperature.

Characters. - A white, crystalline powder. Solubility.-In 20 parts of water, almost insoluble in boiling water, and insoluble in Alcohol.

Dose, 5 to $15 \mathrm{gr}$; .30 to $1.00 \mathrm{gm}$.

8. SODII GLYCEROPHOSPHATIS.-Sodium Glycerophosphate. (Not official.) 
Source.-By a method similar to that given above, a Sodium being substituted for a Calcium Salt.

Characters. - A white, crystalline powder which is so hygroscopic that this salt appears in commerce only as a 75 per cent. solution in water.

Dose, to to $30 \mathrm{~m}$. ; .60 to 2.00 c.c., usually hypodermatically.

Potassium Glycerophosphate (not official) is similar in physical appearance to the Sodium Salt. Iron, Lithium and Magnesium Glycerophosphates (none official) are fine, white, soluble powders.

\section{ACtion of The Glycerophosphates.}

According to Robin, the administration of these substances increases the solids of the urine, the urea, the carbon dioxide and sulphur oxidation coefficient, the chlorides, sulphates, lime, magnesia and potash, with but little effect on uric acid. They improve the nutrition of all organs, but more particularly that of the nervous system.

\section{Therapeutics of the Glycerophosphates.}

Inasmuch as the urine of neurasthenics contains relatively large amounts of incompletely oxidized phosphorus, especially in the form of glycerophosphoric acid, the effort was made to replace this loss by the introduction of phosphorus into the organism in a form approaching, so nearly as is possible, that in which it exists in the nervous system. The indications for the glycerophosphates are conditions of nerve depression. If given subcutaneously they are at least as efficacious as testicular fluid $(q . v$.$) , which owes its activity to its contained organic phos-$ phates, and possess the advantage of more accurate dosage. They are useful in various neuralgias, as sciatica, tic douloureux, Addison's disease, and in the symptom-complex, known as neurasthenia. Chlorosis, albuminuria, phosphaturia, anæmia, the latter by the iron salt, have been benefited. In diabetes the general condition improves and the amount of sugar may diminish. In various diseases of the bones, such as rachitis, osteomalacia, tuberculous affections, the lime and magnesium salts are indicated. The remedies should not be expected to rejuvenate senility, but are useful, even if slowly acting, adjuncts to the systematic treatment of impaired nervous systems]. 


\section{GROUP IX.}

\section{Chlorine, Iodine, Bromine.}

These elements, which are chemically so clusely allied, are all oi them powerful disinfectants and irritants.

\section{CHLORUM.}

[CHLORUM.-Chlorine. $\mathrm{Cl}=35 \cdot 37$.

This gas is not official under its own name, but is obtained from two sources of it, viz., Hydrochloric Acid and Chlorinated Lime.

I. AQUA CHLORI.-Chlorine water. An aqueous solution of Chlorine $(\mathrm{Cl}=35.37)$ containing at least 0.4 per cent. of the gas.

Source. - Pass washed Chlorine gas into water. The gas is obtained by heating diluted Hydrochloric Acid, 35; and Manganese Dioxide, 10. ${ }_{4} \mathrm{HCl}$ $+\mathrm{MnO}_{2}=\mathrm{Cl}_{2}+\mathrm{MnCl}_{2}+2 \mathrm{H}_{2} \mathrm{O}$.

ChARACTERS. - A clear greenish-yellow liquid, having the suffocating odor and disagreeable taste of Chlorine.

INCOMPATIBLES. - Lead and Silver salts.

Free Chlorine is contained in Acidum Nitrohydrochloricum Dilutum.

Dose, I to 4 fl. dr.; 4. to 15. c.c. diluted with water.

2. CALX CHLORATA.-Chiorinated Lime. [A compound resulting from the action of Chlorine upon Calcium Hydrate, and containing not less than 35 per cent. of available Chlorine.] Synonym.-Bleaching powder. It may be regarded either as a compound of Calcium Hypochlorite and Chloride, or as one of Lime and Chlorine.

Source.-Pass Chlorine gas over slaked Lime. ${ }_{2} \mathrm{Ca}(\mathrm{OH})_{2}+{ }_{2} \mathrm{Cl}_{2}=$ $\mathrm{CaCl}_{2} \mathrm{O}_{2}+\mathrm{CaCl}_{2}+2 \mathrm{H}_{2} \mathrm{O}$.

CHARACTERS. - A white or grayish-white granular powder, [exhaling the odor of Hypochlorous Acid, having a repulsive, saline taste, and becoming moist and gradually decomposing on exposure to air.]

3. LIQUOR SODE CHLORAT Æ.-[Solution of Chlorinated Soda. Synonym.-Labarraque's solution. An aqueous solution of several Chlorine compounds of Sodium, chiefly $\mathrm{NaCl}$ and $\mathrm{NaClO}$, containing at least 2.6 per cent., by weight, of available Chlorine.]

Source.-[A solution of Sodium Carbonate, 150 ; Chlorinated Lime, 75 ; in water to 1000 .

Characters. - A clear, pale-greenish liquid, having a faint odor of Chlorine. Sp. gr., about 1.052.]

Dose, $1 / 2$ to I fi. dr.; [2. to 4 . c.c.] 


\section{Action of Chlorine.}

External.-Chlorine is one of the most powerful disinfectants and deodorizers. It has a very great affinity for hydrogen, and hence decomposes compounds which contain hydrogen, oxygen generally being set free. Chlorine is a very active and destructive irritant to the skin and [respiratory] mucous membranes.

Internal.- [When] given internally, [some of it is converted into chlorides, but not all, for the odor of chlorine has been found in the brain after death from its inhalation (Cameron). The odor of chlorine is noticeable in the fæces after its internal administration.]

\section{Therapeutics of Chlorine.}

External.-Chlorine is largely used in the form of chlorinated lime to disinfect privies, drains, urinals, etc. It may be employed also to disinfect rooms after infectious diseases. All metals or articles such as fabrics, likely to be bleached, should be covered up or removed; the windows and chimneys should be pasted up. The gas can be evolved from common salt, $[18 ;]$ manganese dioxide, $[\mathrm{r} 5 ;]$ and sulphuric acid, [45; in iced water, 2 I parts by weight.] 'The door is then shut, and the cracks around it are pasted over with paper. [To disinfect hands moistened chlorinated lime is spread over the hands, next a large crystal of washing soda is held in the hands, and they are washed with rubbing under water until a cooling sensation is experienced. The best disinfectant for excreta is fresh chlorinated lime, I ; dissolved in water, I6. One quart ( 960 c.c.) is placed in the receptacle into which the dejecta are received and left one hour. (Sternberg.)] Chlorine water is sometimes employed as a wash for foul ulcers and discharges. The preparation known as electrozone owes its antiseptic properties to chlorine. It is sea water, the alkaline chlorides of which have been converted into hypochlorites by electrolysis. Its antiseptic strength is about the same as that of Iiquor Sodæ Chloratæ.

Internal.-Chlorine is used internally in the form of a wash 
for the mouth. A wash (strong hydrochloric acid, $5 \mathrm{~m} .\left[\cdot 3^{\circ}\right.$ c.c.]; potassium chlorate, 9 gr. [.60 gm.]; water, I fl. oz. [30. c.c.]) containing free chlorine, is very useful for syringing the fauces and nose in scarlet fever. The vapor gives rise to great irritation of the respiratory tract, and should never be inhaled. [Aqua Chlori has been successfully used, well diluted, in the diarrhœe of typhoid fever, particularly in markedly septic patients. After the administration of drachm doses every hour the temperature falls, the intellect brightens, the tongue clears and betterment goes on to recovery in many apparently hopeless cases.]

\section{IODUM.}

IODUM.-Iodine. I $[=\mathbf{1} 26.53$.

SoURCE.-Obtained from the ashes of sea-weed [and from the motherliquor of Chilian Sodium Nitrate.

CHARACTERS. - Heavy bluish-black, dry and friable rhombic plates, having a metallic lustre, a distinctive odor, and a sharp and acrid taste. Solubility.In 5000 parts of water; in 10 parts of Alcohol, freely in Ether, slightly in Glycerin, freely in a solution of Potassium Iodide or Sodium Chloride.]

INCOMPATIBLES. - Metallic salts, mineral acids, alkaloids, oil of turpentine and ammonia; with the last two explosive compounds may be formed.

IMPURITIES.-Iodine cyanide and iron.

\section{Preparations.}

[1. Liquor Iodi Compositus.-Compound Solution of Iodine. Synonym.-Lugol's Solution. Iodine, 5 ; Potassium Iodide, 10; water to roo. Sirength. -5 per cent.

Dose, I to $10 \mathrm{~m}$.; .06 to .60 c.c.]

2. Tinctura Iodi.-[Tincture of Iodine. Iodine, $70 ;$ Alcohol to rooo. Strength. -7 per cent.

Dose, 1 to $5 \mathrm{~m}$.; .06 to .30 c.c.]

3. Unguentum Iodi.-[Iodine Ointment. Iodine, 4 ; Potassium Iodide, I ; water, 2 ; Benzoinated Lard, 93. Strength. -4 per cent.]

\section{ACtion of Iodine.}

External.-The actions of iodine applied externally are the same as those of chlorine, that is to say, it is powerfully disinfectant and irritant. The latter action is the most important. Iodine applied to the skin produces a yellow stain, which can be 
removed by an alkali or sodium hyposulphite. At the same time it causes a sensation of heat and burning, dilatation of the vessels (rubefaction), some œdematous swelling, and some exuda-. tion of leucocytes, to which its energetic absorbent action is probably due. There often is an accumulation of fluid under the epidermis forming a vesicle. Preparations of jodine are rarely used strong enough to produce more powerful irritation than this. The external application of them probably reflexly contracts the vessels of the subjacent organs, and this may explain their use as counter-irritants. If they are too strong, the irritation set up by them will proceed to the formation of vesicles, and even pustules, and deep inflammation with scarring. They usually destroy the superficial cuticle, so that after the use of them the skin peels. Iodine may be absorbed from the skin, and the alkalies in the blood serum lead to the formation of sodium iodide and iodate ; thus, $6 \mathrm{NaHCO}_{3}+{ }_{3} \mathrm{I}_{2}={ }_{5} \mathrm{NaI}+\mathrm{NaIO}_{3}+$ $6 \mathrm{CO}_{2}+{ }_{3} \mathrm{H}_{2} \mathrm{O}$. These, when they meet an acid, undergo double decomposition; thus $5 \mathrm{NaI}+\mathrm{NaIO}_{3}+\mathrm{H}_{2} \mathrm{O}=6 \mathrm{NaHO}+3 \mathrm{I}_{2}$. Thus free iodine is formed in the stomach and kidneys, and so if iodine has been applied to too large an area we get gastro-intestinal irritation and vomiting. The same may happen if it is taken by the mouth, and it may cause precisely the same symptoms of iodism as potassium iodide. Iodine preparations are parasiticides to the various vegetable and animal parasites which infest the skin.

Internal.-Minute doses of the tincture occasionally stop vomiting. The vapor [B. P., which is tincture of iodine, I ; water, 8 ; to be gently heated] is very irritating to the respiratory passages.

\section{Therapeutics of Iodine.}

External.-Iodine is rarely employed for its antiseptic properties, as chlorine is cheaper. The preparations of iodine are in constant use as irritants and counter-irritants. Usually one or two coats of the liniment [B. P., I885, which contains iodine, 5 ; potassium iodide, 2 ; glycerin, I; alcohol, 40], lightly painted on the skin, suffice; if more is used the inflammation is too severe. If it causes pain, it may be washed off with a solu- 
tion of potassium iodide. The ointment, tincture and compound solution are much milder than the liniment, which is too strong for many persons. Preparations of iodine are frequently used as counter-irritants for chronic inflammation of joints, for pleurisy, chilblains, periostitis, and many other purposes. The mild preparations of iodine are applied over chronically inflamed lymphatic glands when the cause of the swelling cannot be removed. A decolorized tincture of iodine is prepared, consisting of iodine dissolved in [alcohol,] and decolorized by a strong solution of ammonia. Its strength is I in 40 , nearly, and has the advantage of not staining the skin; but it contains no iodine, for ammonium iodide and iodate are formed. Therefore it is a much milder irritant than other iodine preparations. Any effect it may have is due to excess of ammonia. For its irritant effect the official tincture may be injected into a hydrocœle or a cyst to cause adhesive inflammation, and it has been injected into joints, abscesses, and the pleural cavity after empyema; but in such cases great care must be taken that the inflammation induced is not too severe, and this treatment is now very rarely used, for the cavities, being kept aseptic, heal up without it. The tincture, or, if it can be borne, the liquor [B. P., which is iodine, ro; potassium iodide, I5; water, 200 ;] is often used as an antiparasitic for ringworm. Coster's paste, which is sometimes employed for this disease, consists of iodine dissolved in light oil of wood tar ( 1 to 4 ). Morton's fluid, which is used as an injection for spina bifida, consists of iodine, I ; potassium iodide, 3 ; glycerin, 48. [Two preparations are frequently employed in the treatment of diseases of women. These are: Churchill's tincture ; iodine, 5 ; potassium iodide, $\mathrm{x}$; water, 8 ; alcohol, 24, and Battey's fluid; iodine, 2 ; pure carbolic acid, r.]

Internal.-The vapor [B. P., see p. 246] of iodine is occasionally inhaled for diseases of the lungs; but it probably does more harm than good. One or two minims, [.06 to .r2 c.c. $]$ of the tincture in half an ounce, [I5. c.c.] of water are often given, quite empirically, every half hour, in cases of vomiting, and sometimes with distinct benefit. Preparations of seaweed have among uneducated persons a reputation for reducing obesity. 
If they have any such action it is probably because the iodine, chlorine and bromine in them set up such dyspepsia that the proper digestion and absorption of food are prevented. Extracts of Fucus vesiculosus, the bladderwrack or seawrack, have been used, and are the basis of some quack preparations.

I. POTASSII IODIDUM.-Potassium Iodide. KI $[=165.56$.

SOURCE. -Dissolve Iodine in a hot solution of Potassa in distilled water. $3 \mathrm{I}_{2}+6 \mathrm{KHO}=5 \mathrm{KI}+\mathrm{KIO}_{3}+3 \mathrm{H}_{2} \mathrm{O}$. Evaporate and heat the residue with Charcoal ; the Oxygen of the Iodate is carried off as Carbonic Oxide. $\mathrm{KIO}_{3}$ $+\mathrm{C}_{3}=\mathrm{KI}+{ }_{3} \mathrm{CO}$. Dissolve in boiling water, filter, wash and crystallize.

CirARACTERS. - [Colorless, transparent or translucent, cubical crystals, or a white, granular powder, having a peculiar, faint, iodine-like odor, and a pungent, saline, afterwards bitter taste. Solubility.-In 0.75 part of water; in 18 of Alcohol; in 2.5 of Glycerin.]

INCOMPATIBLES. - Bismuth subnitrate, sweet spirit of nitre, liquorice, and preparations containing starch.

IMPURITIES. - Iodates [and free alkalies.]

Dose, 5 to $60 \mathrm{gr}$.; [.30 to $4.00 \mathrm{gm} .$,$] or more.$

\section{Preparations.}

[1. Syrupus Acidi Hydriodici.-Syrup of Hydriodic Acid. A syrupy-liquid containing about 1 per cent., by weight, of absolute Hydriodic Acid ( $\mathrm{HI}=\mathbf{1 2 7 . 5 3})$.

Source.-Dissolve Potassium Iodide, 13 ; and Potassium Hypophosphite, I ; in water. Dissolve Tartaric Acid, 12 ; in diluted Alcohol, 25 ; mix the two solutions, cool, filter, reduce the filtrate by evaporation to 50 parts, and mix, when cold, with Syrup to 1000 .

Characters. - A transparent, colorless or not more than a pale straw-colored liquid, having a sweet and acidulous taste and an acid reaction. Sp. gr., about 1.313 .

Dose, i/ to I $\mathrm{fl}$. dr. ; r. to 4 . c.c.

2. Unguentum Potassii Iodidi.-Ointment of Potassium Iodide. Potassium Iodide, 12 ; Sodium Hyposulphite, 1; hot water, 10; Benzoinated Lard, 77.]

2. SODII IODIDUM.-Sodium Iodide. $[\mathrm{NaI}=149.53$.

Source. - Made from a solution of Soda, as Potassium Iodide is made from a solution of Potassa. $3 \mathrm{I}_{2}+6 \mathrm{NaHO}=5 \mathrm{NaI}+\mathrm{NaIO}_{3}+3 \mathrm{H}_{2} \mathrm{O}$.

Characters. - [Colorless, cubical crystals or a white crystalline powder, having a saline and slightly bitter taste. Solubility. - In 0.6 part of water; in about 3 parts of Alcohol.]

Dose, 5 to $30 \mathrm{gr}$.; [.30 to $2.00 \mathrm{gm} .$,$] or more.$ 
[3. AMMONII IODIDUM.-Ammonium Iodide. $N \quad I=1.14 .54$.

SOURCE. -Dissolve Potassium Iodide and Ammonium Sulphate in boiling water, add Alcohol, filter, wash, and evaporate the filtrate to aryness. $2 \mathrm{KI}+$ $\left(\mathrm{NH}_{4}\right)_{2} \mathrm{SO}_{4}=2 \mathrm{NH}_{4} \mathrm{I}+\mathrm{K}_{2} \mathrm{SO}_{4}$.

Characters. - Ninute, colorless, cubical crystals, or a white granular powder, very deliquescent, having a sharp, saline taste. Solubility.-In I part of water, and in 9 parts of Alcohol.

IMPURITIES. - Iodates and potassium sulphate.

Dose, 2 to $10 \mathrm{gr}$.; .12 to $.60 \mathrm{gm}$., or more.

4. STRONTII IODIDUM.-Strontium Iodide. $\mathrm{SrI}_{2}+6 \mathrm{H}_{2} \mathrm{O}=448.12$.

SOURCE. - By neutralization of freshly prepared solution of Hydriodic Acid with Strontium Carbonate; the filtrate is concentrated and the salt obtained by crystallization. $2 \mathrm{HI}+\mathrm{SrCO}_{3}=\mathrm{SrI}_{2}+\mathrm{CO}_{2}+\mathrm{H}_{2} \mathrm{O}$.

ChAracrers. - Colorless, transparent, hexagonal plates, odorless, and having a bitterish, saline taste. Deliquescent, and colored yellow by exposure to air and light. Solubility.-In 0.6 part of water; soluble in Alcohol, and slightly in Ether.

IMPURITY.-Barium salts.

Dose, 5 to $30 \mathrm{gr}$; ; 30 to $2.00 \mathrm{gm}$.

5. ZINCI IODIDUM.-Zinc Iodide. $\mathrm{ZnI}_{2}=318.16$.

SourcE. - By digesting an excess of Zinc, with Iodine diffused in water. $\mathrm{Zn}+\mathrm{I}_{2}+\mathrm{H}_{2} \mathrm{O}=\mathrm{ZnI}{ }_{2}+\mathrm{H}_{2} \mathrm{O}$.

ChARACrers. - A white, granular powder, odorless, and having a sharp, saline and metallic taste. Very deliquescent, and liable to absorb oxygen from the air, and to become brown from liberated Iodine. Solubility.-Readily in water, Alcohol, or Ether.

Dose, I to $3 \mathrm{gr}$; .06 to .20 $\mathrm{gm}$.]

\section{ACTION OF THE IODIDEs:}

External.-They have none. They do not irritate, and they are absorbed by the unbroken skin in very small quantities.

Internal.-There is much uncertainty about the action of iodides, which is not surprising when we remember the powerful chemical affinities of iodine. Binz teaches that they are decomposed in the body by small quantities of nascent oxygen (set free by living protoplasm) acting upon an iodide which is in an acidulated solution, the acid being provided by carbon [dioxide]. Thus $\mathrm{KI}+\mathrm{H}_{2} \mathrm{O}+\mathrm{CO}_{2}=\mathrm{KHCO}_{3}+\mathrm{HI}$, and then ${ }_{4} \mathrm{HI}+\mathrm{O}_{2}=2 \mathrm{H}_{2} \mathrm{O}$ $+2 \mathrm{I}_{2}$. We have just shown that iodine acts as an absorbent and that it leads to leucocytosis; and that iodides act by virtue of the iodine set free from them in the body is supported by the fact 
that the older physicians produced the same therapeutic effects by giving iodine internally as we procure with iodides, and that iodine taken internally will produce symptoms of iodism. Potassium iodide replaced iodine in therapeutics because it does not cause the same gastro-intestinal irritation. The beneficial effects of iodides are so very marked in syphilis that in this disease they must have some specific action in addition to their general power as absorbents. They also have a specific effect on the mammary gland, for they lessen the secretion of milk. In long-continued large doses they cause atrophy of the testicles and breasts. Some believe that they aid in the elimination of lead, and this may be due to the fact that lead albuminate is soluble in solutions of potassium iodide. Occasionally considerable general depression is produced by large doses of potassium iodide ; but this is probably due to the potassium, and not to the iodine, [and in this case the syrup of hydriodic acid can be substituted]. The iodides are rapidly eliminated by the urine, saliva, sweat and mucous membranes. When taken in excess they produce a number of symptoms known as Iodism.

Iodism.- [This occurs in three forms. (I) The symptoms are those of gastric irritation only.] (2) The patient complains of heavy pain over the frontal sinus, running at the nose, sore throat, increased secretion of saliva, and an eruption on the skin, consisting of patches of erythema. In rare cases there is albuminuria. The inflammation about the fauces may spread to the gums or down the trachea, setting up laryngitis, tracheitis, and bronchitis. These symptoms have been ascribed to an excessive formation of free iodine [produced] as mentioned above-and this is supported by the fact that they can be checked by large doses of sodium bicarbonate, which keep the fluids of the body alkaline, and thus prevent the formation of free iodine-and also to the decomposition of iodides by nitrites, for minute traces of these are believed to exist in saliva, nasal and bronchial mucus, and sweat, and they will liberate free iodine from potassium iodide. It is stated in support of this view that sulphanilic acid (dose, 60 to $90 \mathrm{gr}$. [4. to 6. gm.]), which forms a very stable compound with nitrous acid, will prevent iodism. 
[Nervous troubles, neuralgia, singing in the ears, convulsive movements, disturbed intellection and rarely atrophy of mammæ and testicles may be noticed. (3) Iodic cachexia, which is characterized by rapid emaciation; severe cardiac palpitation and ravenous appetite, are also prominent symptoms.] The susceptibility of people to poisoning by iodides varies very much. [Treatment.-The chewing of pellitory will hasten the elimination of iodine in the chronic forms.]

\section{Therapeutics of the IoDides.}

The most important use of iodides is for syphilis ; their value for the primary and secondary stages is comparatively slight, but they are invaluable for the tertiary stage, as they often cause the rapid absorption of nodes, gummata and other syphilitic deposits. The pharmacopœial dose may often be exceeded : patients sometimes take two, three, or even four drachms [8. to I6. gm.] a day. Large doses are especially used in syphilis of the nervous system. [Daily doses of an ounce, 30 . gm., are not infrequently necessary in these cases. No symptoms of iodism are likely to appear until the disease subsides.] Potassium iodide is often prescribed with [corrosive mercuric] chloride; [red mercuric] iodide is formed and dissolved in the excess of potassium iodide.

Chronic rheumatoid arthritis is often treated, and sometimes with benefit, by small doses of potassium iodide continued for a long while, but probably [ferrous] iodide is more useful. [Socalled] gonorrhœal rheumatism is often treated with potassium iodide [but the syrup of hydriodic acid is preferable.] It frequently aids the absorption of chronic inflammatory products, even when they are not syphilitic. Therefore certain forms of joint disease, of pleurisy and of pulmonic consolidation sometimes yield to treatment by this drug. The attempt has been made to cure aneurisms which are inaccessible to surgery by giving potassium iodide for long periods, for it is thought that it aids the coagulation of blood in them; but as at the same time the patient is always kept in bed, it is difficult to say how much of any improvement that may happen to take place is due to the iodide. Occasionally it relieves the pain of aneurism or of 
angina pectoris. It is a valuable expectorant, [particularly the ammonium salt], and sometimes cures cases of bronchitis when other remedies have failed. Lately, chronic Bright's disease has been largely treated with the drug. Lardaceous disease of the kidneys and other organs is benefited by it. [The potassium salt] is recommended for the [symptom] asthma, and in some cases does much good. It is occasionally given to decrease the secretion of milk. Potassium iodide perhaps causes an increased excretion of both lead and mercury if they exist in the body, and is therefore occasionally given in chronic poisoning by these metals. Sodium iodide is not so much used; it probably produces the same effects as the potassium salt, [but does not occasion so much depression.] Ammonium iodide may be given if the potassium salt causes depression, and it is said that rubidium iodide [not official] (dose, 5 to $20 \mathrm{gr}$. [.30 to $\mathrm{I.20} \mathrm{gm}$.$] )$ is sometimes better tolerated than the potassium salt.

[Strontium iodide has been recently introduced, and is used for the same purposes as the other iodides. It is believed that it is less likely to disturb the stomach, cause acne, and depress the heart than the remaining iodides. In many instances the syrup of hydriodic acid can be substituted with advantage for the iodides. It is not so likely to produce iodism, nor does it so readily give rise to the "iodide punishment." Its pleasant taste is grateful to most patients ; it should, however, be administered, well diluted, one-half hour before meals, or at least upon an empty stomach. Some of the commercial preparations are likely to decompose readily, especially when made from tartaric acid and potassium iodide, and are objectionable from the amount of syrup which is administered when large doses are employed.]

\section{BROMUM.}

BROMUM.-Bromine. $B r .=[79.76$.

SouRCE. - [A liquid obtained from sea water and saline springs.

Characters.-A heavy, dark, brownish-red, mobile liquid, evolving, even at ordinary temperatures, a yellowish-red vapor, highly irritating to the eyes and lungs, and having a peculiar suffocating odor, resembling that of Chlorine. Sp. gr., 2.990.] Solubility.-In 30 parts of water; [readily in Alcohol and Ether.]

IMPURITY.-Iodine. 
Action of Bromine.

Its action is like that of chlorine and iodine. It is rarely used in medicine.

I. POTASSII BROMIDUM.-Potassium Bromide. $\mathrm{KBr}[=\mathbf{I} 18.79$. Source.- - [By adding Bromine to a solution of Potassa, a solution of the Bromide and Bromate is made. $3 \mathrm{Br}_{2}+6 \mathrm{KOH}=5 \mathrm{KBr}+\mathrm{KBrO}_{3}+3 \mathrm{H}_{2} \mathrm{O}$. On evaporation to dryness, mixing the salts with Charcoal and heating to redness, the Bromate is reduced to a Bromide, while the Oxygen unites with the Carbon, forming Carbonic Oxide, which escapes. $\mathrm{KBrO}_{3}+3 \mathrm{C}=\mathrm{KBr}+3 \mathrm{CO}$. Dissolving in water, the solution yields the Bromide in crystals.

ChARACTERS.-Colorless or white cubical crystals, or granules, having a pungent saline taste. Solubility. - In about 1.6 parts water; in 200 parts of Alcohol.

IMPURITIES.—Potassium bromide and free alkali.]

INCOMPATIBLES. - Acids, acid and metallic salts.

Dose, 5 to $60 \mathrm{gr}$; ; .30 to $4.00 \mathrm{gm}$.]

2. SODII BROMIDUM.-Sodium Bromide. $\mathrm{NaBr}[=\mathrm{I02.76.]}$

Source.-Made from a solution of Soda, as Potassium Bromide is made from a solution of Potassa.

ChARACTERS.- [Colorless or white cubical crystals, or a white granular powder, having a saline, slightly bitter taste. Solubility.-In about 1.2 parts of water ; in I 3 parts of Alcohol.]

ImpURities AND Incompatibles. - Those of Potassium Bromide.

Dose, 15 to $60 \mathrm{gr}$; ; [1. to $4 . \mathrm{gm}$.]

3. AMMONII BROMIDUM.-Ammonium Bromide. $\mathrm{NH}_{4} \mathrm{Br}[=$ 97.77.]

SOURCE.-Made by neutralizing Hydrobromic Acid with Ammonia or Ammonium Carbonate, evaporating and crystallizing. $\left[\mathrm{HBr}+\mathrm{NH}_{4} \mathrm{OH}=\right.$ $\mathrm{NH}_{4} \mathrm{Br}+\mathrm{H}_{2} \mathrm{O}$.]

CHARACTERS.-Colorless [transparent, prismatic crystals, or a white crystalline product, having a pungent saline taste. Solubility.-In 1.5 parts of water; in $3 \circ$ parts of Alcohol.]

InCOMPATIBLES. - Acids, acid salts, and spirit of nitrous ether.

ImPURITIES. - Iodides and free bromine.

Dose, 5 to $30 \mathrm{gr}$.; [.30 to $2.00 \mathrm{gm}$.

4. LITHII BROMIDUM.-Lithium Bromide. $\mathrm{LiBr}=86.77$.

SourCE. - From a solution of Ferrous Bromide heated with Lithium Carbonate ; when cool the solution is evaporated, and the salt obtained by crystallization. $\mathrm{FeBr}_{2}+\mathrm{Li}_{2} \mathrm{CO}_{3}=2 \mathrm{LiBr}+\mathrm{FeCO}_{3}$.

Characters. - A white granular salt, odorless, having a sharp and 
slightly bitter taste, very deliquescent. Solubility.-In 0.6 part of water; very soluble in Alcohol.

Dose, 5 to $20 \mathrm{gr}$; .30 to $1.20 \mathrm{gm}$.

5. CALCII BROMIDUM.-Calcium Bromide. $\mathrm{CaBr}_{2}=199.43$.

SouRCE. - From pure Caltium Carbonate by solution in Hydrobromic Acid and evaporation. $\quad \mathrm{CaCO}_{3}+2 \mathrm{HBr}=\mathrm{CaBr}_{2}+\mathrm{CO}_{2}=\mathrm{H}_{2} \mathrm{O}$.

Characters. - A white granular salt, having a sharp, saline taste, very deliquescent. Solubility. - In 0.7 part of water, and in I part of Alcohol.

Dose, 15 to $30 \mathrm{gr}$; ;. to 2. $\mathrm{gm}$.

6. ZINCI BROMIDUM.-Zinc Bromide. $\mathrm{ZnBr}_{2}=224.62$.

Source.-From granulated Zinc digested in Hydrobromic Acid; the solution is concentrated, acidulated with Hydrobromic Acid, and dried upon a water-bath. $\mathrm{Zn}+2 \mathrm{HBr}=\mathrm{ZnBr}_{2}+\mathrm{H}_{2}$.

Characters. - A white granular powder, baving a sharp, saline and metallic taste, very deliquescent, and a neutral reaction. Solubility.-Readily in water and Alcohol.

Dose, I to $5 \mathrm{gr}$; .06 to $.30 \mathrm{gm}$.

7. STRONTII BROMIDUM.-Strontium Bromide.' $\mathrm{SrBr}_{2}+6 \mathrm{H}_{2} \mathrm{O}$ $=354.58$.

SOURCE. - By neutralizing diluted Hydrobromic Acid with Strontium Carbonate, filtration and evaporation. $\mathrm{SrCO}_{3}+2 \mathrm{HBr}=\mathrm{SrBr}_{2}+\mathrm{CO}_{2}+\mathrm{H}_{2} \mathrm{O}$.

Characters. - Colorless, transparent, hexagonal crystals, odorless, and having a bitter saline taste. Very deliquescent. Solubility. - In 1.05 parts of water. Readily soluble in Alcohol.

IMPURITIES. - Barium and calcium salts.

Dose, 5 to $30 \mathrm{gr}$; .30 to $2.00 \mathrm{gm}$.]

\section{- Action of the Bromides.}

External.-They have none.

Internal.-Alimentary canal.-Dolutions of any of the [first] three bromides, frequently painted on the throat, diminish its sensibility. Medicinal doses have no other effect on the alimentary canal. All bromides are quickly çonverted into sodium bromide in the stomach and intestines, and they are readily absorbed.

Nervous system.-Bromides are powerful depressants to the nervous system; [the action of the potassium salt being most marked.] Thus, if an animal be given large doses of any of them, irritation of the cortical motor areas, which before easily 
excited movements, fails to do so. Experiments also show that the reflex excitability of the cord is considerably diminished, and that the activity of the sensory mechanism is also impaired, for large doses of bromides given to frogs cause cutaneous anæsthesia. In man, at least, not only the cortical motor area, but the brain as a whole is depressed, therefore these drugs are powerful hypnotics. It is probable that in addition to the brain and spinal cord the peripheral nerves are depressed, so that bromides are well worthy to be called powerful nervous depressants. The activity of the muscles is also diminished, not only by the action of the drugs on the nervous system, but by their direct action on them. It has been definitely shown by Wright that excessive doses of potassium bromide cause in man and rabbits degeneration of the cortical cells, and that this degeneration begins at the periphery of the dendrons.

Circulation.-Here also bromides are depressant; large doses exert a direct paralyzing influence on the heart, lessening the force and the frequency of the beat and producing stoppage in diastole; [the potassium having the greatest effect, the sodium salt has less influence; the ammonium salt slightly stimulating the heart.] They are said to cause vaso-motor spasm; but the evidence of this is very unsatisfactory. Toxic doses produce a fall of temperature; this is probably in some way secondary to the depression of the circulation.

Respiration is slightly depressed by bromides.

Metabolism.-The amount of carbon [dioxide] exhaled is greatly decreased by taking large doses of bromides. The amount of urine is increased [particularly after the use of the lithium salt]; the coloring matters, the sulphur, and the nitrogen in it are increased; but the phosphorus is decreased.

Sexual organs. - If bromides are taken for a long time a failure of sexual vigor is produced, and ultimately there is a great lessening of the sexual appetite. Bromides are therefore anaphrodisiacs.

Elimination.-Bromides are rapidly eliminated by the kidneys, skin, saliva, intestinal mucous membrane, bronchial mucous membrane, and milk. 
Bromism.-If bromides are taken for too long a period, a zeries of symptoms of poisoning, to which the above name has been given, may.appear. [The hydrobromic acid (see p. 258), although containing a larger proportion of bromine, rarely gives rise to bromism.] The earliest of them is a rash, consisting of red papules, chiefly on the face and back, exactly resembling some forms of acne. This is probably the result of the excretion of bromide by the skin. The next symptoms are a general lowering of the cutaneous sensibility and also of that of the pharynx, then there is diminution of sexual power, the patient becomes low-spirited, easily fatigued, unfit for work, and his intellect is dulled, and in bad cases this passes on to dementia, melancholia, and other disorders. There may be a little conjunctivitis, and some increased secretion from the bronchi. [A coated tongue and disordered digestion are constant symptoms.]

Potassium bromide owes its action largely to the bromine in it, but probably also, to a less extent, to the potassium. In man, at least, the higher functions of the brain are depressed before the lower, and these again before the spinal. Thus the depression takes place in regular order from above downwards, in the reverse order of the physiological development of the functions, and this is commonly the case with many drugs (see Law of Dissolution, p. I04).

Those who take bromides habitually find themselves unable to sleep without them, and their intellect becomes obscured. These bad effects are intensified by the fact that gradually larger doses are required to produce sleep, and thus the unfortunate sufferer becomes more and more a slave to the drug. [Also occasionally maniacal excitement, mental confusion and even delirium are observed after continued use of moderate doses, particularly of the potassium salt.]

Therapeutics of the Bromides.

External.-None.

Internal.-Alimentary canal. - Formerly the back of the throat was painted with a solution of a bromide before a laryn- 
geal examination, but now cocaine is employed for this purpose.

Nervous system. - Because of their depressing effect bromides are largely used for many nervous diseases. They are the most valuable drugs we have for the treatment of epilepsy, acting no doubt by diminishing the excitability of the cerebral cortex. They rarely cure, but often greatly diminish the number of fits. Rubidium [and] ammonium bromide appears to be the best bromide for some cases of epilepsy (dose, $30 \mathrm{gr}$. [2. gm.]). It is impossible to say in any given case whether bromides will do good, therefore they [should] be tried in all; petit mal is more difficult to influence than grand mal. The next most common use of bromides is as hypnotics. They are most useful when there is no organic cause to explain the insomnia, and therefore they are not employed when pain keeps the patient awake, but are given with great benefit in the insomnia of overwork, worry, or that connected with the climacteric period. The sleep induced is quiet and refreshing, without dreams, and therefore these drugs are of great value in nightmare, and in the night-screaming of children, which may be regarded as allied to it. Also because of their depressant effect on the nervous system they are given in migraine, and often they are the only drugs which do any good for the intense headache of this disease. Large doses, often a drachm [4. gm.] at a time, are given in delirium tremens especially in combination with chloral [hydrate], and sometimes the patient seems quieter for this treatment. Not only the insomnia, but the other nervous symptoms that are common at the climacteric period may be relieved by bromides. For their depressing power on centres below the cortex they are used, and with good results, in laryngismus stridulus, and have been given in whooping-cough ; but the benefit is not marked. Some cases of tetanus have recovered after enormous doses of bromides. Here their value is, no doubt, due to their power of diminishing the reflex function of the spinal cord. Bromides have been given as antidotes for strychnine poisoning. Sometimes they succeed in cases of hysteria and neuralgia, and some varieties of functional disease of the heart are much improved by them. 
Sexual functions. - Because of its depressant effect potassium bromide is given for spermatorrhœa and nymphomania.

8. ACIDUM HYDROBROMICUM DILUTUM.-Diluted Hydrobromic Acid. [A liquid compound of 10 per cent., by weight, of absolute Hydrobromic Acid $(\mathrm{HBr}=80.76)$ in water.

Source.-Potassium Bromide, in solution, is mixed with Sulphuric Acid, and Potassium Sulphate allowed to crystallize, the precipitate is washed upon the filter and the filtrate is distilled nearly to dryness and then diluted to the proper strength. $2 \mathrm{KBr}+\mathrm{H}_{2} \mathrm{SO}_{4}=2 \mathrm{HBr}+\mathrm{K}_{2} \mathrm{SO}_{4}$.

Characters. - A clear, colorless liquid, having a strongly acid taste. Sp. gr., about 1.077 .

Dose, $1 / 2$ to I fl. dr.; 2. to 4 . c.c.]

\section{Action and Therapeutics of Hydrobromic Acid.}

The action of this acid appears to be the same as that of the bromides of the alkaline metals, but it is very rarely used for the same purposes. It has been employed with occasional success to relieve noises in the ears, and it is said to prevent the symptoms of poisoning by quinine; [of this drug it is an excellent solvent.]

\section{GROUP X.}

Containing Sulphur [and its compounds].

SULPHUR.

$$
\mathrm{S}[=31.98 .]
$$

Sulphur is official in [three] forms.

I. SULPHUR SUBLIMATUM.--Sublimed Sulphur. Synonym.Flowers of Sulphur.

Source.-From Crude Sulphur by sublimation.

Characters. - [A fine, yellow powder, having a slight, characteristic odor and a faintly acid taste.]

IMPURITIES.-Sulphurous and sulphuric acids, arsenic sulphide, and earthy matters.

[Sublimed Sulphur is used to make Potassa Sulphurata.]

Dose, 15 to $60 \mathrm{gr}$. ; [1. to $4 . \mathrm{gm}$.] 
2. SULPHUR PRACIPITATUM.-Precipitated Sulphur. Synonym.-Milk of Sulphur. [Lac Sulphuris.

SOURCE. - Boil Sublimed Sulphur, 100 ; with Slaked Lime, 50 ; in water. $6 \mathrm{~S}_{2}+3 \mathrm{Ca}(\mathrm{OH})_{2}=2 \mathrm{CaS}_{5}+\mathrm{CaS}_{2} \mathrm{O}_{3}+3 \mathrm{H}_{2} \mathrm{O}$. This gives a solution containing Calcium Sulphide and Calcium Hyposulphite. To it Hydrochloric Acid is added, and Sulphur is thrown down as a very fine precipitate. ${ }_{2} \mathrm{CaS}_{5}+$ ${ }_{4} \mathrm{HCl}=2 \mathrm{CaCl}_{2}+4 \mathrm{~S}_{2} \quad 2 \mathrm{H}_{2} \mathrm{~S}$. Wash and dry the precipitate.

ChARACTERS. - A fine, amorphous powder of a pale yellow color, without odor or taste.]

IMPURITY.-Calcium sulphate, which makes it gritty.

Dose, 15 to $60 \mathrm{gr}$; ; [ $\mathrm{I}$. to $4 . \mathrm{gm}$.]

[3. SULPHUR LOTUM.-Washed Sulphur.

Source.-Sublimed Sulphur, 100; with water, 100 ; and Ammonia Water, Io; is digested, filtered, drained and dried.

Characters. - A fine, yellow powder, without odor or taste. Solubility. -Insoluble in water.

IMPURITIFS._Acids, arsenous acid, and arsenous sulphide.

Washed Sulphur is contained in Pulvis Glycyrrhizæ Compositus, and is used to make Sulphuris Iodidum.

Dose, 15 to $60 \mathrm{gr}$; ; 1 . to 4 . gm.

\section{Preparation.}

Unguentum Sulphuris.-Sulphur Ointment. Washed Sulphur, 300; Benzoinated Lard, 700.]

\section{Action of Sulphur.}

External.-Sulphur itself has no action on the skin, but some of it is converted into hydrogen [sulphide], and that is a mild vascular stimulant, causing slight dilatation of the vessels, and in some persons, eczema. It kills the Sarcoptes [scabiei], and is therefore a parasiticide. When applied to raw surfaces it is converted into sulphurous and sulphuric acids, and is therefore a severe irritant.

Internal.-Alimentary canal.-It has no effect on the stomach, and most that is taken is passed out in the fæces unaltered. A certain amount is, in the intestine, converted into hydrogen [sulphide] and other sulphides. These cause a mild laxative effect, increasing the secretion of intestinal juice, and slightly stimulating the muscular coat, producing soft semi-liquid stools, sometimes accompanied by flatus of hydrogen [sulphide], 
which, if in sufficient quantity, makes sulphur an undesirable laxative.

Remote effects. - Sulphur is absorbed as sulphides and hydrogen [sulphide], which is a powerful poison, decomposing the blood, and thus producing symptoms of asphyxia. It also paralyzes the whole nervous and muscular systems, but sulphur is never given to man in sufficient doses to produce any remote effects. Patients taking sulphur get rid of some minute portion of it as hydrogen [sulphide] through the kidneys, the milk, the lungs and skin. The breath occasionally smells of it, and silver ornaments next to the skin may be discolored.

\section{Therapeutics of Sulphur.}

External.-Sulphur is commonly used to kill the Sarcoptes [scabiei], and thus to cure scabies. The skin should be well scrubbed with soft soap and hot water to lay open the burrows. Then it is thoroughly rubbed with the ointment. The patient should do this before bedtime, sleep in flannel, and wash the ointment off the next morning. This proceeding repeated three or four times will generally cure the disease. Sulphur ointment was formerly applied as a stimulant to ulcers, and was rubbed in for chronic rheumatism; but these modes of treatment are now rarely used, and their value is doubtful. [Mineral waters containing sulphur and its salts are useful for chronic rheumatism, as, for example, those of Richfield Springs.] Mild sulphur preparations are applied for acne.

Internal.-Alimentary canal.--Sulphur is a very good laxative, especially for children; as it produces a soft motion, but no pain, it is useful for cases of piles or fissure of the anus. [Washed] sulphur is contained in compound liquorice powder [see Senna], which is an excellent and popular laxative. One or two sulphur lozenges [of the B. P., each containing $5 \mathrm{gr}$. [.30 gm.] of precipitated sulphur and I gr. [.06 gm.] of acid potassium tartrate], taken at bedtime, often secure an easy evacuation of the bowels the next morning, in persons liable to slight constipation. These lozenges have been recommended for constipation associated with hepatic disease, and many mineral waters containing 
sodium and hydrogen sulphides have considerable reputation for hepatic disorders. Of these, Harrogate water has been shown to increase the amount of bile and the solids in it.

Remote effects. - Sulphur has been administered internally for all sorts of skin diseases, generally without any good result, but occasionally chronic eczema associated with much itching appears to be benefited by it, so that the sulphur lozenge is a suitable laxative for these cases. Sulphur has been also given for bronchitis, for chronic rheumatism, and rheumatic myalgia, but it is very doubtful whether in these diseases there is much relief from this treatment.

4. POTASSA SULPHURATA.-Sulphurated Potassa. Synonym.Liver of Sulphur. [A mixture consisting for the most part of Potassium Hyposulphite $\left(\mathrm{K}_{2} \mathrm{~S}_{2} \mathrm{O}_{3}\right)$ and Sulphide $\left.\left(\mathrm{K}_{2} \mathrm{~S}_{3}\right) \cdot\right]$

SOURCE. - Heat in a crucible a mixture of [sublimed] Sulphur, 100; and [dried] Potassium Carbonate, 200. $3 \mathrm{~K}_{2} \mathrm{CO}_{3}+4 \mathrm{~S}_{2}=\mathrm{K}_{2} \mathrm{~S}_{2} \mathrm{O}_{3}+2 \mathrm{~K}_{2} \mathrm{~S}_{3}+3 \mathrm{CO}_{2}$.

Characters. - [ When freshly prepared it forms irregular pieces of a liver-brown color, which, by exposure to the air, gradually absorb moisture, Oxygen, and Carbon Dioxide, and change to a greenish-yellow and finally a gray mass, containing Potassium Carbonate, Hyposulphite and Sulphate. The compound has a faint odor of Hydrogen Sulphide, and a bitter, alkaline taste. Solubility. - In 2 parts of water.]

5. CALX SULPHURATA.-Sulphurated Lime. [Synonym.-Crude Calcium Sulphide. A mixture containing at least 60 per cent. of Calcium Monosulphide $(\mathrm{CaS}=7 \mathrm{r} .89$ ), together with unchanged Calcium Sulphate $\left(\mathrm{CaSO}_{4}=135.73\right)$, and Carbon, in varying proportions.

SOURCE. - Obtained by heating a mixture of Calcium Sulphate, 70 ; Charcoal, 10; and Starch, 2.

Characters. - A pale gray powder, exhaling a faint odor of Hydrogen Sulphide, having a nauseous, alkaline taste, and gradually decomposed by exposure to air. Solubility. - Very slightly in water ; insoluble in alcohol.]

Dose, $\frac{1}{10}$ to $1 / 2 \mathrm{gr}$; ; [.006 to $.03 \mathrm{gm}$.]

6. SULPHURIS IODIDUM.-Sulphur Iodide. $\operatorname{SI}[=158.51$.

SoURCE. - By heating washed Sulphur, I20 ; with Iodine, 8o, to liquefaction; when solid after cooling, reduce the fused mass to pieces.

ChARACTERS. - Brittle masses of a crystalline fracture and a grayish or black, metallic lustre, having the odor of Iodine, and a somewhat acrid taste.] Solubility. - [Almost $]$ insoluble in water.

Dose, I to $4 \mathrm{gr}$.; [.06 to $.24 \mathrm{gm}$.] 
Action of Sulphurated Potash, Sulphurated Lime, and SUlPHUR IODIDE.

External.-These preparations are irritant, and are powerful parasiticides for the Sarcoptes [scabiei.]

Internal.-Nothing is known of their internal action.

\section{Therapeutics of Sulphurated Potash, Sulphurated Lime,} AND SUlPHUR IODIDE.

External.-An ointment of either will cure scabies and a sulphurated potash ointment ( $I$ in 80 ) is often used for this purpose in the same way as sulphur ointment. [These] drugs have been used for many chronic skin diseases; but now they are not often employed. They appear, however, occasionally to do good in cases of acne indurata. Baths containing sulphides in solution are considered by many to be very useful for chronic rheumatic arthritis and rheumatic myalgia. The famous natural sulphide baths are those of Aix-la-Chapelle, Aix-les-Bains, and there are many others, which will be found described in works on general therapeutics; but as in all of them the water is warm, and warm water is beneficial for chronic rheumatism, and the sulphides exist in infinitesimally small quantities, it is very probable that the benefit is due more to the heat of the water than to its constituents. An artificial bath (sulphurated potash, I; water, 960,) is used for chronic psoriasis.

Internal.-Sulphides have been given for chronic rheumatism, various skin diseases, and phthisis; but the evidence of good done is scanty. [Sulphurated lime has been given internally in cases of suppuration,] for boils, carbuncles, and tuberculous glands in the neck. Half a grain or a grain $[.03$ to .06 gm.] should be given every four hours. It is best made into a pill with acacia, sugar of milk and syrup.

[7. CARBONEI DISULPHIDUM.-Carbon Disulphide. $\mathrm{CS}_{2}=$ 75.93. Synonym. - Carbon Bisulphide.

SOURCE.-By combination of Carbon and Sulphur, by distillation.

ChARACTERS.-A clear, colorless, highly refractive liquid, very diffusive, having a strong characteristic, but not fetid odor, and a sharp, aromatic taste. Solubility. - In 535 parts of water; very soluble in Alcohol, Ether, Chloroform, fixed and volatile oils. Sp. gr., I.268 to 1.269. 


\section{Uses of Carbon Disulphide.}

Carbon disulphide is used as a solvent. It is the best solvent for rubber and similar bodies. It can be freed from its usual disgusting odor by repeated rectification.]

\section{GROUP XI.}

\section{Acids.}

Those acids which will be considered here may be divided into two classes.

Class I.-Those which are strongly acid, the more powerfully acid being active caustics. They are Sulphuric, Nitric, Hydrochloric, Nitrohydrochloric, Phosphoric, Acetic, Tartaric, Citric and Lactic acids. [Hypophosphorous, Hydriodic and] Hydrobromic acids might be placed here, but they have already been considered [see pp. 240, 248 and 258].

Class I1.-Those which, although feebly acid, are powerfully antiseptic. They are Sulphurous and Boric acids.

Diluted Hydrocyanic, Carbolic, Benzoic, Gallic, Tannic, Oleic and Salicylic acids are not used as acids, and will be considered under other headings.

Arsenous Acid and Chromic Acid are not true acids; they are Anhydrides, and have already been considered (see 223 and 234).

\section{Class I.}

I. ACIDUM SULPHURICUM.-[Sulphuric Acid. Synonym.Oil of Vitriol. A liquid composed of not less than :72.5 per cent., by weight, of absolute Sulphuric Acid $\left(\mathrm{H}_{2} \mathrm{SO}_{4}=97.82\right)$ and 7.5 per cent. of water.]

SourCE. - Produced by the combustion of Sulphur or Iron Pyrites, and the oxidation and hydration of the resulting Sulphur Dioxide gas by means of nitrous and aqueous vapors. $\left[2 \mathrm{HNO}_{3}+2 \mathrm{SO}_{2}+\mathrm{H}_{2} \mathrm{O}=2 \mathrm{H}_{2} \mathrm{SO}_{4}+\mathrm{N}_{2} \mathrm{O}_{3} . \quad \mathrm{N}_{2} \mathrm{O}_{3}\right.$ $+2 \mathrm{SO}_{2}+\mathrm{O}_{2}+\mathrm{H}_{2} \mathrm{O}=2 \mathrm{SO}_{2}, \mathrm{OHNO}_{2} . \quad 2 \mathrm{SO}_{2}, \mathrm{OHNO}_{2}+\mathrm{H}_{2} \mathrm{O}=2 \mathrm{H}_{2} \mathrm{SO}_{4}+\mathrm{N}_{2} \mathrm{O}_{3}$.

ChARACTERS. - A colorless liquid of oily consistence and very caustic and corrosive. Sp. gr., not below 1.835.]

IMPURITIES. - Nitric acid, lead, and arsenic.

INCOMPAT1BLES.-Alkalies, their carbonates, lead and calcium salts.

\section{Preparations.}

I. Acidum Sulphuricum Dilutum.-[Diluted Sulphuric Acid. Sulphuric Acid, 100; distilled, water, 825. Sp. gr., about 1.070. Contains 10 per cent., by weight, of absolute Sulphuric Acid.]

Dose, ro to $30 \mathrm{~m}$. ; [.60 to 2.00 c.c.] 
2. Acidum Sulphuricum Aromaticum.-[Aromatic Sulphuric Acid. Synonym.-Elixir of Vitriol. Sulphuric Acid, 100 ; Oil of Cinnamon, I ; Tincture of Ginger, 50;Alcohol, a sufficient quantity to make 1000. Sp. gr., about 0.939. Contains about 20 per cent., by weight, of official Sulphuric Acid, partly in form of Ethyl-Sulphuric Acid.]

Aromatic Sulphuric Acid is contained in Infusum Cinchonæ.

Dose, 5 to $15 \mathrm{~m}$.; [.30 to 1.00 c.c.]

2. ACIDUM NITRICUM.-Nitric Acid. [A liquid composed of 68 per cent., by weight, of absolute Nitric $A$ cid $\left(\mathrm{HNO}_{3}=62.89\right)$, and 32 per cent. of water.]

Source. - Made from Potassium Nitrate by distilling with Sulphuric Acid. $\left[\mathrm{KNO}_{3}+\mathrm{H}_{2} \mathrm{SO}_{4}=\mathrm{KHSO}_{4}+\mathrm{HNO}_{3}\right.$. $]$

Characters. - A colorless, fuming [liquid, very caustic and corrosive, and having a peculiar, somewhat suffocating odor. Sp. gr., about 1.4I4.]

IMPURITIEs. - Sulphuric acid, nitre, and lower nitrogen oxides, giving ruddy fumes.

INCOMPATIBLES.-Alcohol, alkalies, carbonates, oxides, iron sulphate, and lead acetate.

[Nitric Acid is contained in Liquor Ferri Nitratis, Liquor Zinci Chloridi, and Unguentum Hydrargyri Nitratis.]

\section{Preparations.}

I. Acidum Nitricum Dilutum.-[Diluted Nitric Acid. Nitric Acid, 100; distilled water, 580. Sp. gr., about 1.057. It contains to per cent., by weight, of absolute Nitric Acid.]

Dose, 5 to $30 \mathrm{~m}$.; [.30 to 2.00 c.c.]

2. Acidum Nitrohydrochloricum.-[Nitrohydrochloric Acid. Synonyms.-Nitromuriatic Acid. Aqua regia. Nitric Acid, I80; Hydrochloric Acid, 820.]

3. Acidum Nitrohydrochloricum Dilutum.-[Diluted Nitrohydrochloric Acid. Synonym.-Diluted Nitromuriatic Acid. Nitric Acid, 40; Hydrochloric Acid, 180; distilled water, 780. Contains Free Chlorine, Hydrochloric, Nitric and Nitrous Acids, and other compounds dissolved in water.]

Dose, 5 to $30 \mathrm{~m}$.; [.30 to 2.00 c.c.]

3. ACIDUM HYDROCHLORICUM.-[Hydrochloric Acid. Synonym.-Muriatic Acid. A liquid compound of 31.9 per cent., by weight, of Absolute Hydrochloric Acid $(\mathrm{HCl}=36.37)$ and 68 . I per cent. of water.

Source. - The fumes produced by the action of Sulphuric Acid on Sodium Chloride are dissolved in water. $2 \mathrm{NaCl}+\mathrm{H}_{2} \mathrm{SO}=\mathrm{HCl}+\mathrm{NaCl}+$ $\mathrm{NaHSO}_{4}$ and $\mathrm{NaCl}+\mathrm{NaHSO}_{4}=\mathrm{HCl}+\mathrm{Na}_{2} \mathrm{SO}_{4}$ 
Characters. - A colorless, fuming liquid, of a pungent odor, of an intensely acid taste. Sp. gr., about I. 163.]

INCOMPATIBLFS.-Lead and silver salts, alkalies and their carbonates.

[Hydrochloric Acid is contained in Liquor Ferri Chloridi and Liquor Zinci Chloridi.]

\section{Preparations.}

I. Acidum Hydrochloricum Dilutum.-[Diluted Hydrochloric Acid. Synonym.-Diluted Muriatic Acid. Hydrochloric Acid, 100; distilled water, 219. Sp. gr., about 1.050.

Diluted Hydrochloric Acid is contained in Liquor Acidi Arsenosi.] Dose, 5 to $20 \mathrm{~m}$.; [.30 to r.20 c.c.]

2. Acidum Nitrohydrochloricum.-See Nitric Acid.

3. Acidum Nitrohydrochloricum Dilutum.-See Nitric Acid.

4. ACIDUM PHOSPHORICUM.-[Phosphoric Acid. A liquid composed of not less than 85 per cent., by weight, of absolute Orthophosphoric Acid $\left(\mathrm{H}_{3} \mathrm{PO}_{4}=97.8\right)$, in water.

SourCE. - When Phosphorus is brought into contact with Nitric Acid, it is slowly oxidized and converted into Phosphoric Acid. $\mathrm{P}_{3}+5 \mathrm{HNO}_{3}+2 \mathrm{H}_{2} \mathrm{O}$ $=3 \mathrm{H}_{3} \mathrm{PO}_{4}+5 \mathrm{NO}$.

Characters. - A colorless liquid, without odor, but having a strongly acid taste. Sp. gr., 1.347.]

IMPURITIES.-Calcium preparations, and sodium carbonate.

[Phosphoric Acid is contained in Syrupus Quininæe et Strychninæ Phosphatum.]

\section{Preparation.}

Acidum Phosphoricum Dilutum.-[Diluted Phosphoric Acid. Phosphoric Acid, I00; distilled water, 750. Sp. gr., about 1.057. It contains 10 per cent., by weight, of absolute Orthophosphoric Acid.

Dose, 5 to $30 \mathrm{~m}$.; [.30 to 2.00 c.c.]

5. ACIDUM ACETICUM.-Acetic Acid. [A liquid composed of 36 per cent., by weight, of absolute Acetic Acid $\left(\mathrm{HC}_{2} \mathrm{H}_{3} \mathrm{O}_{2}=59.86\right)$ and 64 per cent. of water.

SovRCE. - By distilling Sodium Acetate with Sulphuric Acid, $\mathrm{NaC}_{2} \mathrm{H}_{3} \mathrm{O}_{2}$ $+\mathrm{H}_{2} \mathrm{SO}_{4}=\mathrm{HC}_{2} \mathrm{H}_{3} \mathrm{O}_{2}+\mathrm{NaHSO}_{4}$. The Acetic Acid distils out, and is obtained by crystallization.

IMPURITIES.-A clear, colorless liquid, having a strong, vinegar-like odor, a purely acid taste, and a strongly acid reaction. Sp. gr., about 1.048.]

IMPURITIES. - Lead and copper, sulphuric, sulphurous and hydrochloric acids.

[Acetic Acid is used to make the Aceta, and in several preparations.] 


\section{Preparation.}

Acidum Aceticum Dilutum.-Diluted Acetic Acid. [Acetic Acid, 100; distilled water, 500. Sp. gr., about 1.008. It contains 6 per cent., by weight, of absolute Acetic Acid.

Dose, I to 4 fl. dr.; [4. to 15. c.c.]

6. ACIDUM ACETICUM GLACIALE.-Glacial Acetic Acid. $\mathrm{HC}_{2} \mathrm{H}_{3} \mathrm{O}_{2}[=59.86$.

SOURCE. - Distil dry Sodium Acetate with strong Sulphuric Acid. $\mathrm{NaC}_{2} \mathrm{H}_{3} \mathrm{O}_{2}+\mathrm{H}_{2} \mathrm{SO}_{4}=\mathrm{HC}_{2} \mathrm{H}_{3} \mathrm{O}_{2}+\mathrm{NaHSO}_{4}$.

ChARACTERS. - [A clear, colorless liquid, of a strong, vinegar-like odor, and a very pungent, purely acid taste. Sp. gr., not higher than 1.058 , corresponding to at least 99 per cent, absolute Acetic Acid.]

7. ACIDUM CITRICUM.-Citric Acid, $\mathrm{H}_{3} \mathrm{C}_{6} \mathrm{H}_{5} \mathrm{O}_{7}+\mathrm{H}_{2} \mathrm{O}[=209.50$.

SOURCE. - Found in the fruits of the Lime (Citrus Bergamia) and Lemon (Citrus Limonum). Chalk is added to the boiling juice, usually lemon juice, $2 \mathrm{H}_{3} \mathrm{C}_{6} \mathrm{H}_{5} \mathrm{O}_{7}+3 \mathrm{CaCO}_{3}=\mathrm{Ca}_{3}\left(\mathrm{C}_{6} \mathrm{H}_{5} \mathrm{O}_{7}\right)_{2}+3 \mathrm{CO}_{2}+3 \mathrm{H}_{2} \mathrm{O}$. The precipitated Calcium Citrate is boiled with Sulphuric Acid. After filtration and evaporation, Citric Acid crystallizes out. $\mathrm{Ca}_{3}\left(\mathrm{C}_{6} \mathrm{H}_{6} \mathrm{O}_{7}\right)_{2}+3 \mathrm{H}_{2} \mathrm{SO}_{4}=2 \mathrm{H}_{3} \mathrm{C}_{6} \mathrm{H}_{5} \mathrm{O}_{7}+$ ${ }_{3} \mathrm{CaSO}_{4}$.

ChARACTERs. -Colorless, translucent, right-rhombic prisms, having an agreeable, purely acid taste.] Citric Acid, like Tartaric Acid, is often used to produce an effervescing mixture with [Ammonium, Sodium or Potassium] Carbonates, the two solutions being mixed immediately before taking. Carbon Dioxide which causes the effervescence is formed thus: $-3 \mathrm{KHCO}_{3}+\mathrm{H}_{3} \mathrm{C}_{6} \mathrm{H}_{5} \mathrm{O}_{7}$ $=\mathrm{K}_{3} \mathrm{C}_{6} \mathrm{H}_{5} \mathrm{O}_{7}+3 \mathrm{CO}_{2}+3 \mathrm{H}_{2} \mathrm{O}$.

INCOMPATIBLES. - Potassium tartrate, alkaline carbonates, and acetates.

IMPURITIES.-Copper, lead, sulphuric and tartaric acids, and mineral matters.

Free Citric Acid is contained in Limonis Succus.

[Citric Acid is used to make Bismuthi Citras, Ferri et Quininæ Citras, Ferri et Quininæe Citras Solubilis, Ferri et Strychninæ Citras, Liquor Ferri Citratis, Liquor Magnesii Citratis, Liquor Potassii Citratis, Lithii Citras Effervescens, and Magnesii Citras Effervescens.]

Dose, 5 to $30 \mathrm{gr}$. ; [.30 to $2.00 \mathrm{gm}$.]

\section{[Preparation.}

Syrupus Acidi Citrici.-Syrup of Citric Acid. Citric Acid, ro; water, 10; Spirit of Lemon, 10; Syrup to 1000.

Dose, $I$ to 4 fl. dr.; 4 . to 15 . c.c.]

8. ACIDUM TARTARICUM.-Tartaric Acid, $\mathrm{H}_{2} \mathrm{C}_{4} \mathrm{H}_{4} \mathrm{O}_{6}[=\mathbf{1} 49.64$. Source. - Boil Acid Potassium Tartrate with Calcium Carbonate. $2 \mathrm{~K}$ $\mathrm{HC}_{4} \mathrm{H}_{4} \mathrm{O}_{6}+\mathrm{CaCO}_{3}=\mathrm{CaC}_{4} \mathrm{H}_{4} \mathrm{O}_{6}+\mathrm{K}_{2} \mathrm{C}_{4} \mathrm{H}_{4} \mathrm{O}_{6}+\mathrm{H}_{2} \mathrm{O}+\mathrm{CO}_{2}$. Calcium Chloride 
is now added, which precipitates more Calcium Tartrate. $\mathrm{K}_{2} \mathrm{C}_{4} \mathrm{H}_{4} \mathrm{O}_{6}+\mathrm{CaCl}_{2}$ $=\mathrm{CaC}_{4} \mathrm{H}_{4} \mathrm{O}_{6}+2 \mathrm{KCl}$. The Calcium Tartrate is finally decomposed with Sulphuric Acid. $\mathrm{CaC}_{4} \mathrm{H}_{4} \mathrm{O}_{6}+\mathrm{H}_{2} \mathrm{SO}_{4}=\mathrm{H}_{2} \mathrm{C}_{4} \mathrm{H}_{4} \mathrm{O}_{6}+\mathrm{CaSO}_{4}$. Then evaporate the fluid to the Sp. gr. of I.2I. Separate the Calcium Sulphate crystals that form. Again evaporate, Tartaric Acid crystallizes out.

Characters. - Colorless translucent monoclinic prisms longer than those of Citric Acid, or crystalline crusts, or a white powder, having a purely acid taste. Solubility. -In 0.8 part of water; in 2.5 parts of Alcohol.]

INCOMPATIBLES.-Potassium salts, calcium, mercury, lead, and vegetable astringents.

IMPURITIES. - Lead, oxalic acid, lime, and potassium tartrate.

Dose, 8 to $30 \mathrm{gr}$. ; [.50 to $2.00 \mathrm{gm}$.]

9. ACIDUM LACTICUM.-Lactic Acid. $\mathrm{HC}_{3} \mathrm{H}_{5} \mathrm{O}_{3}[=89.79$.

SOURCE.-Usually obtained by subjecting milk-sugar or grape-sugar to lactic fermentation. It contains 75 per cent. by weight of absolute Lactic Acid, in aqueous solution.

CharActers. - A colorless syrupy liquid, of a purely acid taste, and absorbing moisture on exposure to damp air. Sp. gr., about 1.213. Solubility. -Freely miscible with water, Alcohol or Ether.]

IMPURITIES.-Mineral acids, sugar, lead, and iron.

[Lactic Acid is used in Syrupus Calcii Lactophosphatis.]

Action of Sulphuric, Nitric, Hydrochloric, Phosphoric, Acetic, Citric, Tartaric and Lactic Acids.

External.-All these acids are powerful irritants when applied externally. The feeblest is citric. Its concentrated solution has no action on the sound skin, but is irritant to mucous membranes and abraded surfaces. Tartaric is stronger than citric acid; it will act upon the unabraded skin, and applied to a sore it produces pain, a sensation of burning, and considerable vascular dilatation. The remaining acids are very powerful irritants, therefore even [very] dilute solutions of them may produce considerable redness and perhaps vesication, and when the solu. tion is strong they are very energetic caustics; sulphuric anc phosphoric acids, having a powerful affinity for water, are especially active. Sulphuric acid leaves the carbon untouched, therefore it blackens; nitric stains the skin a deep yellow owing to the formation of picric acid (trinitro-benzol), it does not redis. solve the albumin it precipitates, and it is consequently limited in its area of action; nitrohydrochloric is very powerful; hy. 
drochloric is the least active of the mineral acids ; glacial acetic acid is useful when a limited action is required. [Ricord's paste is composed of sulphuric acid and willow charcoal; Michel's, of sulphuric acid and asbestos.] All the stronger acids unite with and coagulate albumin; hence weak solutions, not strong enough to form a slough, which by its separation may cause bleeding, will, by coagulating the blood and so plugging the vessels, and by coagulating the albumin in the tissues and so constricting the vessels, act as astringents and hæmostatics. [Citric acid is added to tablets of corrosive mercuric chloride so that when these are dissolved in making solutions the antiseptic shall penetrate into the tissues. Tartaric acid is used for the same purpose.] Diluted solutions of acids are cooling to the flushed skin of fever, therefore they are called refrigerants.

Internal.-Mouth. - All acids have a peculiar taste, and give rise to a feeling of roughness about the teeth. As the saliva is alkaline they increase the amount secreted, consequently by keeping the mouth moist they allay thirst.

Stomach.- It is believed that, if given during a meal, acids will check the flow of gastric juice, as that is an acid secretion. Nitric acid, however, interferes with the digestion of proteids, as it combines with them. When the amount of acid secreted by the gastric mucous membrane is deficient, acids taken, after a meal, when all that the stomach can secrete has been secreted, aid digestion.

Intestine.-Acids quickly become converted into neutral salts, and are probably absorbed as such. Some, especially diluted sulphuric, preserve in the intestine their astringent action. They increase the amount of bile poured into the intestine, and are hence cholagogues; this is especially the case with nitric acid. Nitrohydrochloric acid is a still better cholagogue, as it also increases the amount of bile secreted.

Remote effects. - Acids may render the blood less alkaline, but never acid. They do this by combining with some of the alkali of the plasma. As high alkalinity of plasma and tissues favors metabolism, acids slightly diminish it. They also diminish the carbon [dioxide] in the blood. Phosphoric acid is believed to 
increase the amount of phosphates in the red blood-corpuscles. The administration of hydrochloric acid will increase the number of red corpuscles in chlorosis, but it does not alter the amount of hæmoglobin. It is probable that in their passage through the liver they check the formation of urea. The reason for this belief is that all these acids, except citric, acetic, tartaric and lactic, are excreted in the urine-especially in flesh feederschiefly as ammoniacal salts. Nitric acid is stated to be excreted to a small extent as ammonia, and hence slightly to increase the alkalinity of the urine. $\Lambda$ cetic, citric and tartaric acids are decomposed in the blood, alkaline carbonates being formed, and the alkalinity of the urine is increased. This has already been discussed (see p. r28). Lactic acid is either converted into an [alkaline] carbonate, or passed out as carbon [dioxide] in solution in the urine. Some acids depress respiration in animals, and the respiration is restored by alkalies.

Therapeutics of Sulphuric, Nitric, Hydrochloric, Phosphoric, Acetic, Citric, Tartaric and Lactic Acids.

External.-Nitric acid is more often used as a caustic than the others, for, owing to their great affinity for water, it is diffcult to limit the action of sulphuric and phosphoric acids; and the remaining acids are not so powerful as nitric acid. It is employed to destroy warts, condylomata, unhealthy phagedænic sores, cancrum oris, etc. [Nitric acid is used as Heller's test for determining the presence of albumin in the urine. At present his process is reversed, i.e., the urine is added to the acid.] Glacial acetic acid is used for small warts and corns. If this causes pain it may be diluted. Very dilute solutions are rarely employed for their irritant effects, but at some bathing establishments acid baths are used, but it is not proved that they do any good. Any well-diluted acid, especially sulphuric, may be applied to check slight bleeding, as that of leech-bites, piles, etc. Vinegar can always be obtained; even this should be diluted. In fever the skin is often bathed with vinegar as a refrigerant, and very dilute sulphuric acid is used as a local astringent in the sweating of phthisis. 
Internal.-Mouth.-As acids damage the teeth they should be taken through a glass tube. Lemon juice or citric acid itself is often used to stimulate the secretion of saliva, and hence allay the thirst of fever patients. Lemonade is a favorite drink for this purpose. Lactic acid has been strongly recommended to dissolve the membrane in diphtheria, but there is no evidence that this treatment benefits the patient. Equal parts of lactic acid and water may be applied with a mop, or a spray of a. strength of [ $\mathrm{I}$ to 8$]$ of water may be employed. Very dilute nitric acid has been used for the same purpose. Lactic acid is applied [more frequently than any other drug] with a brush in tuberculosis of the larynx, and in some cases with good results. It is usual to begin with lactic acid, 2 ; water, I ; and glycerin, $\mathbf{I}$. The strength of the solution is increased till at last lactic acid alone is used. Other accessible tuberculous ulcers-as those of the tongue and skin - may be treated in the same way.

Stomach and Intestines. - Hydrochloric, and to a less extent nitrohydrochloric acid is of the greatest value in that variety of dyspepsia in which the acidity of the gastric juice is deficient. They should, as already explained, be given some little while after a meal. A very useful stomach mixture consists of diluted nitrohydrochloric acid combined with tincture of nux vomica, and some other stomachic, as compound tincture of gentian. Lactic acid has been used for the same purpose. Acids will often alleviate that form of indigestion in which the patient complains of acid eructations and heartburn. For this purpose they should be given during a meal or before it. They then check the excessive secretion of acid and restrain fermentation. An acid mixture sometimes benefits the indigestion of pregnancy, and small doses of hydrochloric acid may be prescribed during typhoid and other fevers, because the secretion of this acid is much diminished when the temperature is raised. Vinegar is often drunk to reduce obesity, but it only does so because a long course of any acid will set up a mild gastritis, and thus hinder the digestion and absorption of food. Carbonic acid, taken as an effervescing mixture, is a common and very efficacious gastric sedative, beneficial, therefore, in painful dyspepsia 
and in vomiting. Diluted sulphuric acid may be ased as a hæmostatic in bleeding from the stomach or intestines, but its action is feeble. It is, however, successful as an astringent in . many cases of summer diarrhœa. Nitric and nitrohydrochloric acids, increasing the amount of bile poured into the intestines, are given, and sometimes with much benefit, when it is considered that dyspepsia is due to disordered function of the liver. Diluted sulphuric acid is often taken by workers in lead factories, as it forms an insoluble lead sulphate in the intestine and so prevents absorption of lead.

Remote effects. - The remote effects of salts of citric, tartaric, and acetic acids have already been described (see p. 128). They are due to the increase in the alkalinity of the blood and the urine. Phosphoric acid is often given to weak, sickly, anæmic children with the view of improving the quality of the red bloodcorpuscles, and possibly aiding the growth of bones, but it has not been proved to have any great value. The same may be said of lactic and phosphoric acids when given for diabetes; indeed, the latter is said to do harm. [There is probably no doubt as to the value of lime and lemon juice in the treatment of scurvy.] Lime juice was formerly a popular remedy for acute rheumatism, but it did little if any good. Sulphuric acid is by some said to be anhidrotic in the night-sweating of phthisis, and had some reputation as a remote hæmostatic, but it is rarely given now for these purposes. Aromatic sulphuric acid, with a little syrup and water, forms a pleasant cooling drink in fever. Röhrig found that acids diminished the tracheal secretion, and some physicians find that they diminish the secretions in bronchitis. We thus see that the remote effects of all acids, except citric, tartaric, and acetic, are unimportant.

\section{TOXICOLOGY.}

All these acids are severe gastro-intestinal irritants when given in toxic doses. Tartaric, citric, and lactic are very rarely taken as poisons.

Symptoms. - These are severe burning pain extending from the mouth to the stomach, excoriation of the mouth with the formation of sloughs, great difficulty in swallowing, vomiting of dark-brown, coffee-colored material and shreds of mucus, intense ahdominal pain aggravated by the slightest move- 
ment, generally obstinate constipation, but if the bowels are open the motions are dark, from the blood contained in them. Some of the acid generally passes down to the larynx, and causes swelling of that organ and consequently dyspncea from obstruction to respiration. The patient becones cold, collapsed and covered with a cold sweat; his pulse is very feeble, and he suffers from great thirst. Post-mortem. - The mucous membrane of the mouth and œesophagus is softened and corroded, and whitish-gray sloughs and hæmorrhages may be seen here and there. The coats of the stomach are softened. It is often contracted, and it may be perforated, the aperture being irregular. If the acid escapes into the peritoneal cavity, it may act on almost any of the abdominal organs. Should the patient have lived long enough, there may be corrosion and inflammation of parts of the small intestine. The mucous membrane of the throat and larynx is inflamed and swollen.

Treatment.-Alkalies should be given at once, e.g., soap and water, lime water, magnesia, washing soda ; and then demulcents, as milk, white of egg, oil, linseed tea. Do not use the stomach tube if sulphuric acid has been taken, otherwise wash out the stomach. Morphine may be injected subcutaneously for the pain, and brandy [given] subcutaneously for the collapse.

Class II.

1. ACIDUM SULPHUROSUM.-Sulphurous Acid. [A liquid composed of not less than 6.4 per cent. by weight of Sulphurous Acid Gas (Sulphur Dioxide, $\mathrm{SO}_{2}=63.9$ ), and not more than 93.6 per cent. of water.

SOURCE. - Sulphuric Acid, 80 ; is heated with Charcoal, 20; and the resulting Sulphur Dioxide is dissolved in water. ${ }_{4} \mathrm{H}_{2} \mathrm{SO}_{4}+\mathrm{C}_{2}={ }_{4} \mathrm{SO}_{2}+2 \mathrm{CO}_{2}$ $+4 \mathrm{H}_{2} \mathrm{O}$.

ChaRACTERS. - A colorless liquid of the characteriscic odor of burning Sulphur, and of a very acid, sulphurous taste. Sp. gr., not less than 1.035.]

IMPURITIFS. - Sulphuric acid, and mineral matters.

Dose, $1 / 2$ to 2 fl. dr.; [2. to 8. c.c.]

\section{Action of Sulphurous Acid.}

External.-Sulphurous acid is strongly deoxidizing, and as it takes up oxygen so easily from organic bodies, it readily decomposes them, becoming itself converted into sulphuric acid, and hence is irritant, but not violently so, for the amount of sulphuric acid in proportion to the water is slight. It is a disinfectant and deodorant; for in virtue of its property of absorbing oxygen, it destroys micro-organisms and arrests fermentation. When applied to the skin it is a parasiticide.

Internal.-It is believed to act as a disinfectant in the stomach and intestine, but it is very doubtful whether enough of 
it to have any appreciable action in this direction can be safely taken.

\section{Therapeutics of Sulphurous Acid.}

External.-Sulphurous acid is chiefly used as an antiseptic, disinfectant, and deodorant. Sulphur [dioxide] is employed as a disinfectant for a sick-room after a patient with an infectious disease has been in it. The chimneys and windows should be stopped up. A quarter to half a pound [1 20. to 240. gm.] or more of flowers of sulphur [or better, one or two sulphur candles, now to be found in pharmacies], are placed in an earthenware vessel and lighted; the door is shut, and the cracks around it pasted over. The room should be left untouched for six hours. Generally not enough sulphur is burned for this method to be efficacious. Sulphurous acid [I to 4] in water is locally applied to cure ringworm. Foul sores may be washed with it.

Internal.-Sulphurous acid is sometimes given internally with the object of preventing abnormal fermentation in the stomach and intestines in certain varieties of dyspepsia, but there is no clinical proof that it can do this, and it should be remembered that it is possible it may do harm by impeding the action of the normal ferments.

2. ACIDUM BORICUM.-Boric Acid. $\mathrm{H}_{3} \mathrm{BO}_{3}[=61.78$. Synonym. -Boracic Acid.

SoURCE.-Native from Northern Tuscany, or made by the action of Hydrochloric Acid on Borax by filtration and recrystallization. $\mathrm{Na}_{2} \mathrm{~B}_{4} \mathrm{O}_{7}+$ $2 \mathrm{HCl}+10 \mathrm{H}_{2} \mathrm{O}=4 \mathrm{H}_{3} \mathrm{BO}_{3}+2 \mathrm{NaCl}+5 \mathrm{H}_{2} \mathrm{O}$.

Characterś. - Transparent, colorless scales, of a somewhat pearly lustre, or, when in perfect crystals, six-sided triclinic plates, slightly unctuous to the touch, having a faintly bitterish taste. Solubility. - In 25.6 parts of water; in 10 of glycerin; in 15 of Alcohol.

Dose, 5 to $15 \mathrm{gr}$; .30 to $1.00 \mathrm{gm}$.

\section{Preparation.}

Glyceritum Boroglycerini.-Glycerite of Boroglycerin. Synonyms. - Glycerite of Glyceryl Borate. Solution of Boroglyceride. Boric Acid, 310; Glycerin to 1000 .]

3. SODII BORAS.-Sodium [Borate. $\mathrm{Na}_{2} \mathrm{~B}_{4} \mathrm{O}_{7}+\mathrm{IOH}_{2} \mathrm{O}=380.72$. Synonyms.-Borax. Sodium Pyroborate. 
SOURCE. - Native, as a saline incrustation on the shores of certain lakes and as a crystalline deposit at the bottom of the Borax lake of California, or by boiling together Boric Acid and Sodium Carbonate and crystallization. ${ }_{4} \mathrm{H}_{3} \mathrm{BO}_{3}+\mathrm{Na}_{2} \mathrm{CO}_{3}=\mathrm{Na}_{2} \mathrm{~B}_{4} \mathrm{O}_{7}+\mathrm{CO}_{2}+6 \mathrm{H}_{2} \mathrm{O}$.

ChARACTERs. - Colorless, transparent, monoclinic prisms, or a white powder, having a sweetish alkaline taste. Solubility. - In 16 parts] of water; in I of Glycerin.

Dose, 5 to $30 \mathrm{gr}$; ; [.30 to $2.00 \mathrm{gm}$.]

\section{Action of Boric Acid and Borax.}

External.-Both boric acid and borax have the power of destroying micro-organisms and are thus disinfectant and antiseptic, but their value is slight, and they are much more active in preventing than in inhibiting decomposition. The action is extremely local. Solutions of boric acid will relieve itching. Neither substance produces any irritation. Boric acid is very largely used to preserve milk, butter and animal food.

Internal.-Borax and boric acid check the action of saliva on starch, but, if anything, they increase the action of the gastric juice and the pancreatic secretion. Large amounts, however, slightly retard digestion, and still larger are gastro-intestinal irritants. Boric acid is rapidly eliminated in the urine, it is said to increase the urea and the quantity of urine. Large doses [increase] the acidity of this fluid. It is also excreted in the saliva, sweat, and fæces, and it is stated in rare cases to cause abortion. In exceptional instances where large quantities have been applied to raw surfaces or mucous membranes, reduction of temperature, depression of spirits, feeble pulse, ecchymoses and vomiting have supervened. Harmful symptoms do not follow from taking food preserved with boric acid if the amount used is small, such as anything under [one-tenth of one] per cent., but they may follow if large amounts are used. It should never be used for solid foods.

\section{Therapeutics of Boric Acid and Borax.}

As they do not irritate, both these substances are largely used to keep wounds, ulcers, and sores sweet. The action is so local that they cannot be used to dress cavities. Boric lint is em- 
ployed to dress wounds. It is made by passing lint through a hot saturated solution of boric acid. Boric cotton is made the same way. Lister's boric acid ointment consists of boric acid, I ; white wax, 1 ; paraffin, 2 ; almond oil 2 parts. A saturated solution of boric acid ( 4 per cent.), [or the glyceritum boroglycerini well diluted with water] may be used as an antiseptic wash. Such solutions are used for ozæna, vaginitis, urethritis, and ophthalmia. Colitis is often benefited by washing out the large bowel with a quart [ 960 c.c.] of a saturated solution of boric acid; sometimes tannic acid is added. Lister's ointment, or an ointment of boroglyceride, [(not official) glycerin, 92 ; boric acid, 62 ; by heating], may be used for pruritus, sunburn, etc. Powdered boric acid blown into the ear is very useful in fotid discharges from it. Thompson's fluid (borax, 1 ; glycerin, 2 ; water, 2 ), in the proportion of $\mathbf{I}$ to 8 of warm water, is commonly employed to wash out the bladder in cystitis. [One of the most important antiseptic solutions is that of Thiersch. This consists of boric acid, 12 ; salicylic acid, 2 ; water 1000$]$. The glycerin [of the B. P., which is, borax, I ; water, 2 ; glycerin, 4 ;] and the honey of borax, [of the B. P., which is, borax, 2 ; glycerin, I ; clarified honey, I 6 ;] are excellent applications for aphthous states of the mouth, especially in children. The following is a good wash for the mouth: Glycerin of borax, [see above] 6 ; tincture of myrrh, I ; water to 48 .

Borax has been given in epilepsy, and its use is gaining ground. It is often prescribed with advantage in combination with bromides, but it is decidedly inferior to them, although in exceptional cases it may succeed when they have failed. As it is an antiseptic it has been given internally in typhoid fever and phthisis, but with doubtful benefit. Taken internally, it is said to relieve irritability of the bladder. In rare cases its use has caused either psoriasis, a papular eruption especially marked near the elbows, an erythematous rash, or eczema. Nausea, loss of appetite, vomiting, and diarrhœa may be produced. It has no effect on the intelligence. The taste is best covered with syrup of orange peel.

Boric acid is not employed internally in medicine, [except- 
ing for correcting the fotor of fermentative dyspepsia and in ammoniacal cystitis, where it is also used in solution for irrigation of the bladder.]

\section{GROUP XII.}

\section{CARBON AND ITS COMPOUNDS.}

\section{Class I.-[Drugs which Act Mechanically.}

\section{Carbon, Petrolatum and Benzin.}

This class includes carbon in its three official forms, and the hydrocarbons.]

\section{CARBO.}

$$
[\mathrm{C}=11.97 \text {. }
$$

I. CARBO ANIMALIS.-Animal Charcoal, Synonym.-Bone-black.

Sourcr. - Expose bones, deprived of fat, in iron cylinders, to red heat without access of air, and then powder them.

Characters. - Dull, black, granular fragments or a dull black powder, nearly tasteless. Solubility. - Insoluble in water or Alcohol. coal.

2. CARBO ANIMALIS PURIFICATUS.-Purified Animal Char-

Source.-Digest Animal Charcoal, 100 ; with Hydrochloric Acid, 300 , and a sufficient quantity of water. Filter, wash and heat the residue to redness in a closed crucible.

Characters. - A dull black powder, odorless, tasteless, and insoluble in water or Alcohol. It should contain no salts.

Dose, 20 to $60 \mathrm{gr}$.; 1.20 to $4.00 \mathrm{gm}$.; $1 / 2 \mathrm{oz}$; $15 . \mathrm{gm}$. or more as an antidote. ]

3. CARBO LIGN1.-[Charcoal.-Synonym.]-Wood Charcoal.

SOURCE. - Wood charred without access of air.

Characters. - [A black, odorless, and tasteless powder, free from gritty matter.]

Dose, 20 to $60 \mathrm{gr}$.; [x.20 to $4.00 \mathrm{gm}$.]

\section{Action of Charcoal.}

External.-Dry charcoal absorbs gases and condenses them within its pores. It thus absorbs oxygen, and hence has an oxidizing power, parting with the absorbed oxygen to oxidize 
organic and other substances. Organic matter is believed to be decomposed by ærobic micro-organisms which act by oxidation, and anærobic which decompose directly, producing offensively smelling and toxic bodies. Wild suggests that the reason for the deodorant action of charcoal is that it converts anærobic into ærobic decomposition. It attracts and oxidizes coloring matters, and consequently decolorizes them. It has no effect on living organisms, and is not antiseptic.

Internal.-Formerly it was thought only to oxidize when dry, but to a less degree it has this power when moist, presumably because there is still some active oxygen in its interstices. It is passed in the fæces unchanged.

\section{Therapeutics of Charcoal.}

External.-Charcoal has been recommended as an antiseptic and deodorant for foul ulcers, etc., but it is a dirty preparation, and large quantities must be used. Charcoal is used in pharmacy as a decolorizing agent.

Internal.- It has been given as a powder, as lozenges, and as biscuits, with the object of preventing fermentation in the stomach, but it is not of much use. Garrod has shown that a tablespoonful or larger doses of charcoal frequently repeated, are antidotes against most active vegetable poisons, as opium, nux vomica, and aconite, for charcoal seems to have a special attraction for alkaloids. Animal charcoal is the best form to give as an antidote. Charcoal is used as a tooth powder [but it should not be recommended because it abrades the enamel of the teeth].

\section{[II. PETROLATUM.}

I. PETROLATUM LIQUidUM.-Liquid Petrolatum.

SoURCE. - A mixture of Hydrocarbons, chiefly of the Marsh-gas series, obtained by distilling off the lighter and more volatile portions from Petroleum, and purifying the residue when it has the desired consistence.

ChARACTERs. - A colorless, or more or less, yellowish, oily, transparent liquid, without odor or taste, or giving off, when beated, a faint odor of Petroleum. Sp. gr., about 0.875 to 0.945 . Solubility. - Insoluble in water; scarcely soluble in cold or hot alcohol, or in cold Absolute Alcohol; but soluble in boiling Absolute Alcohol, and readily soluble in Ether, Chloroform, Carbon Disulphide, Oil of Turpentine, Benzin, Benzol, and fixed or volatile oils. 
2. PETROLATUM MOLLE.-Soft Petrolatum. Synonym.-Soft Petroleum Ointment.

Source.-A mixture of Hydrocarbons, chiefly of the Marsh.gas series, obtained by distilling off the lighter and more volatile portions from Petroleum, and purifying the residue when it has the desired melting point.

When Petrolatum is prescribed or ordered without further specification, soft Petrolatum (Petrolatum Molle) is to be dispensed.

Characters. - A fat-like mass, of about the consistence of an ointment, varying from white to yellowish or yellow, more or less fluorescent when yellow, especially after being melted, transparent in thin layers, completely amorphous, and without odor or taste, or giving off, when heated, a faint odor of Petroleum. If a portion of Soft Petrolatum be liquefied, and brought to a temperature of $140^{\circ} \mathrm{F}$; $60^{\circ} \mathrm{C}$., it will have a specific gravity of about 0.820 to 0.840 . The melting point of Soft Petrolatum ranges between about $104^{\circ}$ and $113^{\circ} \mathrm{F}$; $40^{\circ}$ and $45^{\circ} \mathrm{C}$.

3. PETROLATUM SPISSUM.-Hard Petrolatum. Synonym.Hard Petroleum Ointment.

Source. - A mixture of Hydrocarbons, chiefly of the Marsh-gas series, obtained by distilling off the lighter and more volatile portions from Petroleum, and purifying the residue when it has the desired melting point.

Characters. - A fat-like mass, of about the consistence of a cerate, varying from white to yellowish, or yellow, more or less fluorescent when yellow, especially after being melted, transparent in thin layers, completely amorphous, and without odor or taste, or giving off, when heated, a faint odor of Petroleum. If a portion of Hard Petrolatum be liquefied, and brought to a temperature of $142^{\circ} \mathrm{F}$; $61.1^{\circ} \mathrm{C}$, it will have a specific gravity of about 0.820 to 0.850 . The melting point of Hard Petrolatum ranges between about $113^{\circ}$ and $125^{\circ} \mathrm{F}$.; $45^{\circ}$ and $51.3^{\circ} \mathrm{C}$.

IMPURITIES. - Fixed oils, fats of animal or vegetable origin, resin, and organic impurities.

\section{Action and Uses of Petrolatum.}

Petrolatum is used exclusively as a bland, neutral protective, and, because it does not become rancid nor act as an irritant, and as it is not affected by acids, alkalies or powerful reducing agents, it is employed as a substitute for fatty materials in ointments. But as it is absorbed with difficulty it is not a suitable vehicle for drugs which are intended for absorption through the skin. Liquid petrolatum has been used as a local soothing application in inflammation of the mucous membrane of the nose, throat, larynx, and even of the bronchial tubes. It is then ap- 
plied with an atomizer and may be employed as a vehicle for medicinal substances. None of the petroleums are nutritive.

\section{BENZINUM.}

BENZIN.-Synonyms.-Petroleum Benzin. Petroleum Ether. A purified distillate from American Petroleum, consisting of hydrocarbons, chiefly of the Marsh-gas series $\left(\mathrm{C}_{5} \mathrm{H}_{12}, \mathrm{C}_{6} \mathrm{H}_{14}\right.$, and homologous compounds).

ChARACTERS. - A transparent, colorless, diffusive liquid, of a strong, characteristic odor, slightly resembling that of Petroleum, but much less disagreeable, and having a neutral reaction. Sp. gr., 0.670 to 0.675 . Solubility.Insoluble in water; soluble in about 6 parts of Alcohol, and readily soluble in Ether, Chloroform, Benzol, fixed and volatile oils.

\section{UsES OF BENZIN.}

Benzin is used to obtain volatile oils by percolation, as a substitute for ether in making oleoresins, for dissolving fats, resins, caoutchouc and some of the alkaloids.]

\section{Class II.-The Anæsthetics.}

\section{Alcohol, Chloroform, Ether, and Acetic Ether.}

These substances produce local anæsthesia by evaporation. They are rubefacient if their vapor is confined. The stomach, heart and central nervous system are first stimulated and then depressed by them. [Amylic Alcohol, (B. P.) Ethyl Bromide, Bromoform and Pental, none of which are official, are considered in this class.]

\section{ALCOHOL.}

[Ethyl Alcohol. $\mathrm{C}_{2} \mathrm{H}_{5} \mathrm{OH}=45.9$.

Alcohol is official in the eight following forms :]

I. ALCOHOL.- [A liquid composed of about 9I per cent. by weight, or 94 per cent., by volume, of Ethyl Alcohol, and about 9 per cent., by weight, of water. Synonyms. - Ethylic Alcohol. Spirit of Wine.

SoURCE. - Macerate rectified spirit with Anhydrous Potassium Carbonate to remove the water, then again with freshly fused Calcium Chloride, and distil.

Characters. - A transparent, colorless, mobile and volatile liquid of a characteristic, rather agreeable odor, and a burning taste. Sp. gr., about 0.820 . Boils at $172.4^{\circ} \mathrm{F} ; 78^{\circ} \mathrm{C}$. Entirely volatilized.

IMPURITIES. - Resins or oils, detected by turbidity on dilution.

Alcohol is used to make Chloroform.

\section{Preparation.}

Alcohol Dilutum.-Diluted Alcohol. Synonym.-Proof Spirit. A liquid composed of about $4^{1}$ per cent., by weight, or about 48.6 per 
cent., by volume, of absolute Ethyl Alcohol, and about 59 per cent. of water. Alcohol, 500 ; distilled water, 500 .

Characters.-The same as those of Alcohol. Sp. gr., about 0.938 .

2. ALCOHOL ABSOLUTUM.-Absolute Alcohol. Ethyl Alcohol, containing not more than I per cent, by weight, of water.

SourCE.-By percolation of the strongest and purest Alcohol through recently burned lime, out of contact with the air, then re-distil the percolate in vacuo.

Characters. - A transparent, colorless, mobile, and volatile liquid, of a characteristic, rather agreeable odor, and a burning taste. Very hygroscopic. Sp. gr., not higher than 0.797 .

3. AlCOHOL DEODORATUM.-Deodorized Alcohol. A liquid composed of about 92.5 per cent., by weight, or 95.1 per cent., by volume, of Ethyl Alcohol, and about 7.5 per cent., by weight, of water.

SOURCE. - The foreign odors may be removed by distilling the Alcohol over about 2 per cent. of pure, fused Sodium Acetate.

Characters. - As of Absolute Alcohol. Sp. gr., about 0.816.]

4. SPIRITUS VINI GALLICI.-Brandy.

[SOURCE.-An alcoholic liquid obtained by the distillation of the fermented, unmodified juice of fresh grapes, and at least four years old.

Characters. - A pale, amber-colored liquid having a distinctive odor and taste, and a slightly acid reaction. Contains 39 to 47 per cent., by weight, of Alcohol, together with a volatile oil and several Ethers. Sp. gr., 0.925 to 0.941, which limits it should not exceed.

Dose, indefinite.

5. SPIRITUS FRUMENTI.-Whiskey.

Source. - An alcoholic liquid obtained by the distillation of the mash of fermented grain (usually a mixture of corn, wheat and rye), and at least two years old.

Characters. - An amber-colored liquid, having a distinctive taste and odor, and a slightly acid reaction. Contains 44 to 50 per cent., by weight, of Absolute Alcohol. Sp. gr., 0.917 to 0.930 , which limits it should not exceed.

Dose, indefinite.

6. VINUM ALBUM.-White Wine.

SoURCE.-An alcoholic liquid, made by fermenting the juice of fresh grapes, the fruit of Vitis Vinifera (nat. ord. Vitacea), freed from seeds, stems and skins.

Characters. - A pale, amber-colored, or straw-colored liquid, having a pleasant odor, free from yeastiness, and a fruity, agreeable, slightly spirituous taste, without excessive sweetness or acidity. Contains between Io and 14 
per cent., by weight, of Absolute Alcohol. Sp. gr., 0.990 to 1.010, which limits it should not exceed.

7. VINUM RUBRUM.-Red Wine.

SnURCE.-An alcoholic liquid, made by fermenting the juice of fresh colored grapes, the fruit of Vitis Vinifera (nat. ord. Vitacea) in presence of their skins.

Characters. - A deep red liquid, having a pleasant odor, free from yeastiness, and a fruity, moderately astringent, pleasant, and slightly acidulous taste, without excessive sweetness or acidity. Contains to to I 4 per cent., by weight, of Absolute Alcohol. Sp. gr., 0.989 to I.oro, which limits it should not exceed.]

Amount of Ethyl Alcohol by Volume in Various Important Substances.

[Alcohol Absolutum . . . . contains 99 per cent.

Alcohol Deodoratum . . . . " " 95.1] “

Alcohol . . . . . . . . " " 94 "

Spiritus Rectificatus [B. P.]. • " " 90 " "

Spiritus Tenuior (Proof Spirit) • "6 57.09 " "

Rum, Gin, Strong Liqueurs . " " 51 to 59 "

[Spiritus Frumenti . . . . " 50 to 58] "

Alcohol Dilutum . . . . . " " 48.6

Spiritus Vini Gallici . . . " " 46 to 55 "

Vinum Album Fortius (U. S. P., I880) - . . . . " " 23 to 29

[Vinum Porteuse] . . . " " 20 to 30 "

[Vinum Xericum or] Madeira . " 16 to 22 "

Vinum Album . . . . . . " 12.4 to 17.3 "

[Vinum Rubrum . . . . . " 12.4 to 17.3 ] "

Champagne . . . . . . . " 10 to 13 "

Vinum Aurantii [B:. P.] . . . " 10 to 12 "

Burgundy . . . . . . " a 9 to 12 "6

Hock . . . . . . . . . " 9 to 12 "

Claret. . . . . . . " 8 to 12 "

Cider . . . . . . . " " 5 to 9 "

Strong Ale or Stout . . . . " 5 to 9 "

Beer [or] Porter . . . . . " a 2 to 5 "

[Kumyss] . . . . . . " - 1 to 3 "

Action of Alcohol.

External.-It is a powerful antiseptic, preventing the formation of and killing putrefactive bacteria. If applied to the skin, alcohol quickly evaporates. It therefore cools the skin, which consequently becomes pale from the contraction of the 
small vessels; owing to this less sweat is secreted. Alcohol is thus refrigerant, astringent and anhidrotic. But if evaporation is prevented in any way, such as by a watch-glass or a piece of gutta-percha, or the alcohol is rubbed in, it quickly absorbs water from the skin, and thus hardens it. Having thus passed through the epidermis, it dilates the vessels, causes a feeling of warmth, and produces a rubefacient effect. It has the power of coagulating albumin, but the coagulum quickly re-dissolves. It extracts water from all tissues.

Internal.-Mouth. - When concentrated, alcohol produces a feeling of warmth, or often even a burning sensation, in the mouth. If held there for some time, the albumin of the superficial tissues is coagulated, and the mucous membrane becomes whitish, congested, and opaque ; but this appearance soon disappears, as the coagulum is re-dissolved by the fluids of the tissues. Directly [after] the alcohol is put in the mouth there is an increased flow of saliva, and the pulse may be quickened; these results are reflex, for they occur before there is time for the alcohol to be absorbed. Alcohol has a slight local anæsthetic effect.

Stomach.-Here also, if the alcohol is sufficiently concentrated, there is a sensation of warmth or even of burning. If only small quantities are given, the gastric vessels dilate, the mucous membrane becomes red, and there is an increased secretion of gastric juice. All this [has been] seen to happen in cases of gastric fistula. The result of these effects is that the appetite is sharpened, and this explains the custom, common with many people, of taking a little alcohol immediately before meals, and also the common experience that alcohol taken during meals aids digestion. It also increases the activity of the gastric movements and promotes absorption. Thus there are several ways in which moderate doses of alcohol may help the digestive process, and Binz has actually demonstrated, by removing the gastric contents at stated times after a meal, that alcohol aids digestion, and by giving potassium iodide he showed that it increased the rapidity of absorption. In some cases it produces a local anæsthesia in the stomach, and so it may relieve gastric pain. It is to a slight extent decomposed into aldehyde and 
acetic acid, and consequently some of the pepsin, peptones, and proteids are precipitated. This hinders digestion, but usually not sufficiently to outdo the aid due to the vascular dilatation, the increased secretion, and the greater movement. The effect of large doses is very harmful. The activity of the gastric juice is destroyed, the gastric walls are inflamed, large quantities of mucus are poured out, and if the over-indulgence is continued chronic gastritis ensues, the gastric glands atrophy, and consequently we get the permanent dyspepsia of drunkards.

A single dose of alcohol introduced into the stomach in a concentrated form, e.g., [clear] brandy, immediately produces important reflex effects. The heart beats more rapidly and more forcibly, the vessels of the whole body dilate, especially those of the skin; hence there is a feeling of warmth. The blood-pressure rises. These reflex effects are well seen in the immediate restoration of a fainting person by the ingestion of a single dose of brandy. Diluted alcohol, e.g., beer, does not produce them. They are quickly followed by the effects of alcohol upon the circulation due to its presence in the blood after absorption.

Intestines.-Here alcohol has a slight astringent effect, and consequently it may check diarrhœa.

Blood.-Alcohol is absorbed more largely by the blood-vessels than the lacteals. It first increases and then diminishes the amœboid movements of the white blood-corpuscles. It so acts on the red corpuscles as to prevent oxyhæmoglobin from readily yielding up its oxygen, consequently it diminishes the oxidation of the tissues. This, in habitual drinkers of large quantities of alcohol, may lead to an imperfect combustion of fat, consequently it accumulates in the tissues, and obesity, which is often increased by the amount of saccharine matters alcoholic liquids contain, results. The skin acquires a velvety feeling.

Alcohol is slightly antipyretic, lowering the temperature in fever. This is chiefly due to cutaneous vascular dilatation and rapidity of circulation, but also slightly, perhaps, to general diminished oxidation. A litre, [about a quart] of Rhine wine of average strength produces by its oxidation about as much heat as five or six 
tablespoonfuls [1 00. to I 20. c.c.] of olive oil. Neither the intake of oxygen nor the output of carbon [dioxide] is altered by alcohol, therefore as it has been oxidized in the body it saves the tissues and is a food. Repeated observations have shown the proof of this, for moderate doses of alcohol diminish the output of urea and uric acid, 6 or 7 per cent. ; and that it is a food is also proved by the fact that the weight of the body may be maintained if a large amount of alcohol is taken, even if the rest of the food is very small in amount. [Alcohol ceases to be a food when it is ingested in such large amounts that it cannot be completely oxidized. In this instance the excess is likely to be harmful.]

If only moderate doses are drunk, very little alcohol leaves the body in the urine; with large doses the case is different.

Circulation. - The effects upon the circulation reflexly produced by stimulation of the mouth and stomach have already been mentioned. After alcohol is absorbed it influences the heart markedly. It beats more powerfully and more rapidly, the pulse becomes fuller; these results are due to the peripheral árterial dilatation and to a stimulating effect on the accelerator nerves. The vaso-motor system is acted upon, all the vessels of the body dilate, especially those of the skin; therefore, if he previously felt cold, the person who has taken the alcohol feels warm. The blood-pressure rises, the increased action of the heart more than compensating for the vascular dilatation. [This is not true for dogs, as has been demonstrated in the laboratory (Long).] The direct effects of alcohol on the circulation after absorption appear more slowly and last longer ; but they are clearly similar to those due to the reflex stimulus from the stomach, and therefore they continue them. The result of the increased circulation through the various organs is that they work to greater advantage, hence the mental faculties are brightened for a time, the muscular strength seems increased, more urine is passed, and the skin perspires. The person who has taken the alcohol, in fact, usually feels generally better for it. This is by no means always so ; some persons have a headache or feel very sleepy immediately after alcohol. This is probably because the vessels of the abdomen or skin have dilated 
so excessively that almost all the blood in the body is in them, and consequently there is very little in the brain. There are many individual peculiarities in the effects of alcohol.

It has been repeatedly proved that these good results are but transitory. The heart, although at first stimulated, is more exhausted after the stimulation has passed off than it was before. This is also true of all the organs of the body stimulated by the increased circulation induced by alcohol. In many cam. paigns and arctic expeditions it has been found that although at first the men, after taking alcohol, could do more work, yet soon they felt so tired and exhausted, that on the whole they could do much more without than with the alcohol. Large doses of alcohol do not stimulate the heart at all ; they paralyze it, both reflexly from the stomach and after absorption. Enormous doses poured into the stomach kill almost immediately by reflex action. A drunkard who is "dead drunk" is, accurately speaking, one who is killed by the paralyzing effect of alcohol on the heart; but the phrase is often applied to any one who is very drunk.

Skin.-Alcohol is a mild diaphoretic, partly because of its vaso-dilator action, and perhaps also because of some direct influence on the sweat-glands. As just mentioned, the cutaneous vascular dilatation leads to a feeling of warmth if the patient's cutaneous vessels were previously contracted from cold. It may be that part of the antipyretic power of alcohol is due to increased radiation from the dilated vessels, and also to evaporation of the increased amount of sweat. If a person is in a cold atmosphere, alcohol, by increasing the radiation from the skin, leads to the loss of so much heat that he may die from cold, although at first the increased cutaneous circulation, making him feel warmer, gives him a delusive feeling of warmth.

Kidneys. - About 5 per cent. of the alcohol ingested-unless very large quantities are taken-is excreted unchanged, mostly in the urine, to a less extent in the expired air, only the merest trace in the sweat and none in the milk or fæces. Most of it is oxidized in the body. It acts as a diuretic; probably this is a secondary result of its vascular effects, but it probably also acts directly on the glomeruli. 
Nerious system.-Unless the dose be very large the whole nervous system is stimulated, perhaps to a slight extent directly, but chiefly as a secondary result of the vascular dilatation and cardiac stimulation. The highest functions are most affected. The person who has taken the alcohol talks more fluently and brilliantly, his wits are sharpened, he has a feeling of strength. If the dose has been large, the stage of exaltation of these or any other functions quickly passes into one of depression, the highest functions being affected first, and the stimulation and depression of function proceed regularly from the highest to the lowest. The action of alcohol thus illustrates both the fact that stimulation is usually succeeded by depression, and also the "law of dissolution," which (see p. 104) states that functions which have appeared latest in the animal series or the individual are the most easy to influence, those which have appeared earlier are less easy to influence; and so by regular sequence till we arrive at those functions which are first developed, which are the last to be influenced. The stimulation and subsequent depression of function, therefore, proceeds in a descending scale from the highest or least firmly fixed function to the lowest or most firmly fixed. Thus the power of judgment is abolished very early by alcohol ; this is so while the imagination, the emotions, and the power of speech still remain stimulated; but soon the power of imagination goes, the patient loses all conmand over his emotions, he cries and laughs irregularly, but this soon stops. He next begins to lose control over his speech, talking incoherently and thickly; shortly afterwards he cannot talk at all, but can only make a noise. Muscular movements, which are not so highly developed as those of speech, are next affected; delicate, lately developed movements, as writing, feeding himself, etc., are for a time performed inco-ordinately, but soon they are paralyzed. Next the muscular movements, developed before these, are implicated, and the patient cannot undress himself or walk straight, and inco-ordination of these movements passes into the inability to do them at all. Next the activity of the reflex centres of the cord is abolished, the patient passes his urine and fæces [involuntarily]. Then the respiratory centre, which was previously stimulated, becomes 
paralyzed, breathing is difficult, and the face is livid. Lastly, the heart, which was also at first stimulated, is paralyzed, and the patient dies. The depression of the reflex centres of the cord accounts for the fact that injuries which would kill a sober man do not kill a drunken one, for the heart and respiration, owing to the general central depression, are not affected reflexly by them.

\section{Therapeutics of Alcohol.}

External.-Four parts of alcohol to one of water form the Lotio Spiritus of many pharmacopœias. [Cotton] or lint dipped in it are applied to sprained joints, bruises, etc. The alcohol evaporates, cools the part, consequently the vessels contract, and inflammation may thus be checked. At the same time the local anæsthetic effect of the cold relieves the pain. In a similar way many varieties of headache may be soothed by bathing the forehead with eau de Cologne or Bay Rum. Brandy or some other form of alcohol is often used to bathe the skin in order to harden it, by abstraction of water, and thus prevents the formation of bed-sores or cracked nipples. Spirit lotions dabbed on the skin may, by means of the vascular contraction produced, stop sweating. Alcohol rubbed in, as in the use of Linimentum [Saponis], is commonly employed for its rubefacient effect, to aid the absorption of inflammatory products and relieve pain, as in chronic rheumatism, myalgia, etc.

Internal.-Mouth.-A little brandy held in the mouth will be a local anæsthetic and relieve toothache. Alcohol is used as a gargle of port wine for its power of precipitating albumin and acting as an astringent in cases of chronic sore throat, excessive salivation, or inflammation of the gums.

Stomach. - Because it increases the secretion of gastric juice, the vascularity and the movements of the stomach, alcohol aids digestion. It must only be taken in small quantities, for large amounts paralyze the secretion and cause gastritis, and ultimately lead to atrophy of the gastric glands. It should be given just before or during a meal. It is harmful in acute dyspepsia, but for the indigestion of the aged and feeble, or for those who are thoroughly exhausted by overwork, it is very valuable, as the 
stomach shares in the general exhaustion. It is also useful because it increases the appetite. Owing to its anæsthetic property it may relieve painful dyspepsia, and may check vomiting, especially if taken with carbon [dioxide], as, for example, in the form of champagne or brandy and soda-water, and because it increases the activity of the gastric movements it may relieve flatulence. A single dose of strong spirits poured into the stomach is often employed with great benefit for its reflex stimulant effects on the circulation for those who have fainted, or who are collapsed from cold or any other cause.

Intestines.-Brandy and water will often check diarrhœa. Perhaps this is owing to the astringent power of the brandy.

Fever.-Alcohol has been largely used in all sorts of febrile conditions. We have seen that it impairs oxidation by its action on the red corpuscles, that it is oxidized and is therefore a food, and that it is mildly antipyretic and diaphoretic. These results would be beneficial in fever. On the other hand, the acceleration of the pulse would be distinctly harmful, although it must be remembered that very often, for some unexplained reason, alcohol lowers the pulse in fever; the indigestion caused by the taking of large quantities, and the liability to depression of the respiratory and cardiac centres, would be very undesirable. The best rules are that while alcohol may be given, often with immense advantage in fever, either to aid digestion, to slow the pulse, as a cardiac stimulant if the patient be much collapsed, or to produce sleep, yet it may, in any of the ways alluded to, do harm. Therefore, when it is being used, the effect must be carefully watched, and if the pulse becomes quick and feeble, or, as indicating gastric irritation, the tongue becomes dry and brown, or the skin becomes hot and dry, or the breathing hurried, or the patient suffers from insomnia, the alcohol should be stopped. On the other hand, if the pulse becomes stronger and slower, the tongue and skin moist, the breathing tranquil, and the patient sleeps well, the drug is doing good, and may be continued. IVe have so many more powerful diaphoretics and antipyretics that alcohol is not often given for these purposes. Of all fevers it is most used for acute lobar pneumonia, and, speaking generally, 
it is most likely to be valuable when our object is to keep up the patient's strength for a few days only, till the termination of a specific fever of short duration; but it is often given when it is quite unnecessary.

Nervous system.-Alcohol may, as just mentioned, be used as a soporific in fever. Many persons who suffer from insomnia find that they can sleep better for a glass of whiskey and water just before going to bed, no doubt because of its depressant action upon the highest centres.

Kidneys and skin.-Alcohol is occasionally given as a diuretic. Gin is the best form, because it usually contains some juniper, which is also diuretic. Although but little alcohol is excreted by the kidneys, it seems to be particularly irritant to the urethra in cases of gonorrhœea and gleet, and some authorities consider that chronic Bright's disease may be induced by alcohol. Almost the only use made of its diaphoretic effect is as a help to cure a cold in the head, for which purpose a glass of strong spirits and water may be taken immediately before going to bed.

\section{Toxicology.}

Large doses of Alcohol will produce death, either instantly by reflex stoppage of the heart, or later by cardiac and respiratory depression after absorption.

Chronic poisoning causes so many diseases that it is really the part of a text-book upon medicine [to enumerate them.] Very often confirmed drunkards, particularly if they take much spirits, are very thin; this is probably due to the fact that strong spirits cause such marked indigestion that sufficient nourishing food is not absorbed. Other drunkards are fat, especially if they drink beer. Chronic gastritis, cirrhosis of the liver, gout, peripheral neuritis, delirium tremens, mania, and perhaps chronic Bright's disease, may all be directly due to excessive indulgence in alcohol. It renders patients particularly liable to phthisis, and makes them bad subjects for withstanding any severe illness, especially pneumonia, or to undergo severe surgical operations. Alcoholic beverages contain other bodies than alcohol, which are probably partly responsible for these evil effects.

\section{II. [ALCOHOL AMYLICUM.]}

AMYLIC ALCOHOL. (Not official.) $-\mathrm{C}_{5} \mathrm{H}_{11} \mathrm{OH}=87.81$. Synonym. -Fusel Oil.

SoURCE. - Separated during the rectification of crude spirit and re-distilled at from $250^{\circ}$ to $260^{\circ} \mathrm{F}$.; $121 . \mathrm{I}^{\circ}$ to $126.6^{\circ} \mathrm{C}$. 
ChARACTERS. -A colorless, very inflammable, oily liquid, with a peculiar odor. Sp. gr., o.818.

Amylic Alcohol is used to make Amyl Nitrite.

\section{Uses of Amylic Alcohol.}

Fusel oil is a poison, and is not used in medicine. The manufacturers of cinchona alkaloids employ it as a solvent; formerly it was required for the preparation of valerianic acid.

\section{CHLOROFORMUM.}

CHLOROFORM. $-\mathrm{CHCl}_{3}=119.08$. A liquid consisting of 99 to 99.4 per cent., by weight, of absolute Chloroform, and I to 0.6 per cent. of Alcohol. Synonyms._Chloroformum Purificatum.] Trichloromethane.

SOURCE. - Heat water and Alcohol in a still to $100^{\circ} \mathrm{F}$. $\left[37.7^{\circ} \mathrm{C}\right.$. $]$, then add Chlorinated Lime; [Chloroform distils over. It is believed that reaction takes place as follows: On bringing together Alcohol and Chlorinated Lime, the Chlorine converts the former into Chloral, which is at once decomposed by the Calcium Hydroxide, yielding Chloroform and Calcium Formate $\left(\mathrm{Ca}\left(\mathrm{CHO}_{2}\right)_{2}\right)$. The Calcium Formate is decomposed by another portion of Chlorinated Lime into Calcium Carbonate and Chloride, and water. $2 \mathrm{C}_{2} \mathrm{H}_{5} \mathrm{OH}$ $+\mathrm{roCaOCl}_{2}=2 \mathrm{CHCl}_{3}+{ }_{7} \mathrm{CaCl}_{2}+2 \mathrm{CaCO}_{3}+\mathrm{Ca}(\mathrm{OH})_{2}+4 \mathrm{H}_{2} \mathrm{O}$. In late years a Chloroform quite free from (hlorinated bye products has been obtained from the distillation of Acetone (from destructive distillation of Calcium Acetate) and Chlorinated Lime, from which Chloroform is produced, together with Calcium Acetate, Hydroxide and Chloride. $2\left(\mathrm{C}_{3} \mathrm{H}_{6} \mathrm{O}\right)+6\left(\mathrm{CaOCl}_{2}\right)=$ $\left.2\left(\mathrm{CHCl}_{3}\right)+\mathrm{Ca}\left(\mathrm{C}_{2} \mathrm{H}_{3} \mathrm{O}_{2}\right)_{2}+2 \mathrm{Ca}(\mathrm{OH})_{2}+3 \mathrm{CaCl}_{2}\right]$.

Characters. - A heavy, clear, colorless, [mobile and very diffusible liquid of a characteristic, ethereal odor, and a burning sweet taste. Sp. gr., not below 1.490. It imparts a green color to flame. Solubility. - In 200 parts of water, in which it sinks in heavy drops.]

IMPURITIFS.-Hydrocarbons, shown by darkening with sulphuric acid, non-volatile compounds, shown by not completely evaporating, and. by unpleasant odor, acids, and free chlorine.

[Purification.-Chloroform which fails to respond to tests of the Pharmacopæia should be purified by the following process: Chloroform, 400; Sulphuric Acid, 80 ; Dried Sodium Carbonate, 20.gm.; Deodorized Alcohol, 4 c.c. Add the Sulphuric Acid to the Chloroform, contained in a glass-stoppered bottle, and shake them together occasionally during twenty-four hours, avoiding exposure to bright daylight. Separate the lighier Chloroform layer; add to it the Dried Sodium Carbonate, previously rendered anhydrous by heating it in a porcelain capsule on a sand-bath until it ceases to give off aqueous vapor, and shake them together frequently and thoroughly during half an hour. Then transfer the Chloroform to a dry retort, add to it the Alcohol; 
and distil, by means of a water-bath, at a temperature not exceeding $153^{\circ} \mathrm{F}$, $67.2^{\circ} \mathrm{C}$., into a well-cooled, tarred receiver, until the distillate measures 255 c.c.]

Dose, 2 to $20 \mathrm{~m}$.; [.r2 to I.20 c.c.]

\section{Preparations.}

1. Aqua Chloroformi.-[Chloroform Water. Chroroform and distilled water, by agitation, there being al ways an excess of Chloroform present.

Dose, 1 to 4 fl. dr.; 4. to I5. c.c.]

2. Linimentum Chloroformi.-[Chloroform Liniment. Chloroform, 300; Soap Liniment, 700.

3. Emulsum Chloroformi.-Emulsion of Chlorsform. Chloroform, 40; Expressed Oil of Almond, 60; Tragacanth, 15; water to 1000 .

Dose, I to 4 fl. dr.; 4. to 15. c.c.]

4. Spiritus Chloroformi.-[Spirit of Chloroform. Synonym.Chloric Ether. Chloroform, 60; Alcohol, 940. Strength. 6 per cent.

Dose, $1 / 2$ to I fl. dr.; 2. to 4 . c.c.]

5. Tinctura Chloroformi et Morphinæ Composita, [B. P., not official. Compound Tincture of Chloroform and Morphine.]-Intended to be an imitation of the proprietary medicine called Chlorodyne. [Mix Chloroform, 75 ; Tincture of Capsicum, 25 ; Tincture of Indian Hemp, I00; Oil of Peppermint, I.5; and Glycerin, 250; with Alcohol, 450; and dissolve in this Morphine Hydrochlorate, Io; add Diluted Hydrocyanic Acid, 50 ; then mix with sufficient Alcohol to form 1000 parts.]

Strength.-10 m. [.60 c.c.] contains chloroform $3 / 4 \mathrm{~m}$. [.045 c.c.], morphine [hydrochlorate, $\frac{1}{\text { If }}$ gr., $[.0054$ gm.], diluted hydrocyanic acid $1 / 2 \mathrm{~m}$. [.03 c.c.]

Dose, 5 to $15 \mathrm{~m}$.; [.30 to I.00 c.c.]

\section{Action of Chloroform.}

External.-Chloroform in many respects acts like alcohol ; but it is more powerful. Thus if allowed to evaporate on the skin it produces cold; therefore the vessels at the point of application contract, and at the same time local anæsthesia is induced. If the vapor be confined, or if chloroform be rubbed into the skin, it acts as an irritant. The vessels dilate, the part becomes red, and there is a sense of heat. This rubefacient effect may pass on to vesication. It is a powerful antiseptic. [Chloro- 
form is an excellent antiseptic to preserve urine during transportation. Three drops, .20 c.c., to 4 oz., r 20 . c.c., of urine is sufficient. Allow the chloroform to evaporate before testing the urine.]

Internal.-Mouth. - If concentrated, it produces irritation and a burning sensation. If diluted, it has a sweetish taste, which renders Aqua Chloroformi a valuable vehicle for the administration of nauseous drugs. It reflexly gives rise to an increased secretion of saliva, and is a local anæsthetic.

Stomach.-The action of chloroform is very like that of alcohol. Large doses cause marked gastro-intestinal irritation. Small doses produce a feeling of warmth, dilatation of the gastric vessels, and increased secretion of gastric juice, and more regular and more powerful gastric movements. It is perphaps slightly astringent to the intestines.

Absorption. - It is absorbed into the blood from the stomach and intestines, and, if given as vapor, from the lungs; but it is very uncertain what changes it subsequently undergoes. Probably most of it is decomposed, but some is certainly eliminated in the breath and some in the urine, and it may be found in the blood of those who have been poisoned by it.

Temperuture. - The internal temperature falls about $\mathrm{I}^{\circ} \mathrm{F}$. $\left[0.5^{\circ} \mathrm{C}.\right]$ after the prolonged administration of chloroform.

Nervous system.-Chloroform is an excellent instance of the law of dissolution (see p. 104), and also of the well known fact that drugs which, in small doses, stimulate any part, in large doses often depress it. The phenomena resulting from the inhalation of chloroform are commonly divided into three stages.

First stage.-This is, at first, one of general stimulation, the highest functions being the most stimulated, usually unevenly, so that the patient is somewhat incoherent. The imagination is momentarily excited, and he experiences a general feeling of warmth and comfort spreading over the entire body. The mind, from the irregular excitation, is confused. Sight and hearing are stimulated, he experiences sensations of light and hears noises. The stimulation of all these higher functions is very transitory, and he quickly begins to lose consciousness; he may be aware 
that people around him are talking without knowing what they are saying, but soon he hears and sees nothing. Sometimes during the early part of this stage he may laugh or cry. The inability to see and hear is quickly followed by considerable blunting of general sensation. At the same time that these higher functions are being depressed the lower motor functions are excited ; he will kick and fight, throwing his arms and legs about, so that much strength may be required to hold him down, and he will shout and talk incoherent nonsense very louldly. Almost coincidently the stimulation of the lower centres sets in; the pulse is increased in frequency, and there is throbbing of the heart and great vessels. The first inhalation or two may produce a choking sensation and a stoppage of breathing, which is often voluntary ; but soon the respirations are increased in frequency. The bloodpressure at first rises a little, and the face may be flushed. The pupils usually dilate.

Second stage. - This is best called that of depression. Some authors call it the stage of excitement, because the excitation of the motor centres may be continued into it. It is important to remember that there is no sharp boundary-line between the various stages, they pass insensibly into one another. In this stage the depression of the highest functions continues, so that the patient becomes completely unconscious, and he appears to be in a deep sleep. He sees, hears and feels nothing, hence chloroform is called a general anæsthetic. The excitement of the motor functions passes into depression, and he ceases to shout and struggle. Some of the reflex centres are depressed, so that when the cornea is touched the eye does not shut. The pupil is contracted. The stimulation of the cardiac and respiratory apparatus gives way to depression, the pulse and respiration become less frequent and less strong. The vaso-motor centre is depressed, blood-pressure falls. As he cannot feel pain, and the reflex activity is so lowered that the heart will not be reflexly inhibited by the shock of an operation, this is the period at which to operate safely.

Third stage. - In this there is a total abolition of reflex excitability. Even the lowest reflex centres of the cord are depressed, "so that the patient may pass his urine and fæces [involuntarily]; 
all muscular tone is abolished, and consequently the muscles are quite flaccid. Some of them, as those of the arm, were probably in this condition towards the end of the second stage. The pupil is widely dilated, probably because of the commencing asphyxia. This is the period to which the administration is pushed to facilitate the reduction of dislocations, or to enable the abdominal viscera to be felt through the abdominal wall. If still more chloroform is given the depression of the cardiac, respiratory and vaso-motor centres continues, the pulse becomes feeble and irregular, and the heart finally stops in diastole. At last not only its central nervous apparatus, but its muscular tissue is depressed, so that it will not respond to mechanical stimulation. The respiratory movements become slight and irregular, with very long pauses between them, and as a result the patient is more or less asphyxiated. The blood-pressure gradually falls to zero. There has been much dispute as to whether chloroform kills by the heart or the respiration. The Commission appointed by the Nizam of Hyderabad reported that it killed by depression of the respiratory centre, that respiration always failed before the heart, and that the fall of blood-pressure was not due to any effect on the heart. But their results have been disputed, and it has been shown that the fall of blood-pressure is mainly due to a weakening effect on the heart. [In the United States it has been generally believed that death is from depression of the heart.]

The recovery from chloroform also illustrates the law of dissolution. The lowest functions, such as muscular tone, are the first to reappear; but the patient does not usually regain his mental equilibrium for hours.

With the exception of its local action on the skin and alimentary canal, and its last effect on the cardiac muscle, chloroform acts entirely on the central nervous system, and this action is not the result of any effects on the blood. The peripheral nerves are not affected, unless it be just before death. Chloroform narcotizes infusoria.

Vomiting is very liable to occur during the administration of chloroform, and its advent is often made known by pallor and 
wide dilatation of the previously contracted pupil. Immediately before death the pupil may be either dilated or contracted.

\section{Therapeutics of Chloroform.}

External.-Chloroform is employed in the form of the liniment to produce rubefacient and irritant effects in cases of chronic rheumatism, myalgia and chronic inflammations.

Internal.- It may be used as a local anæsthetic for toothache, the tooth being plugged with a piece of cotton soaked in chloroform. It disguises the taste of nauseous medicines, and therefore Aqua Chloroformi is a very common vehicle and Spiritus Chloroformi is much used as a flavoring agent. In the stomach it acts like alcohol, and is given in the same varieties of dyspepsia as are benefited by that drug. Small doses may be used as a cardiac stimulant.

Inhalation.- It is inhaled to abolish sensations of pain, whether from surgical operations, biliary, renal and intestinal colic, or parturition. In the last case [but little] need be given. It is also inhaled to relax muscular spasm, as in the reduction of dislocations or herniæ, or for the relaxation of muscles for diagnostic purposes, as, for example, when we wish to feel the abdominal viscera thoroughly, or to [ascertain] whether a swelling is a phantom tumor; or, lastly, it is inhaled to relax spasm in cases of tetanus, hydrophobia, or other varieties of convulsions. The A. C. E. mixture, which consists of alcohol, I, chloroform, 2, and pure ether, 3 vols., is very commonly employed for all these purposes. It is said to be safer than chloroform. All of its three constituents volatilize from it at an equal rate.

The following points should be attended to in the administration of chloroform :

[1. The anæsthetizer must be skilled, and give his attention exclusively to the production and maintenance of narcosis.]

2. The respiration and pulse should be carefully watched for any signs of failure.

3. The operation should never be begun till reflex action is profoundly depressed-that is to say-till the stage of muscular relaxation has commenced. Many patients have been lost from 
neglect of this precaution, for the stimulus of the knife has reflexly stopped the heart. It is a common and dangerous error to think that, because the operation is trivial, it may be begun early ; most of the deaths from chloroform have taken place when the operation has been slight.

4. Great care must be exercised if the heart is fatty or feeble from any cause, or if the patient suffer from disease of the lungs, or if he may be very old.

5. In operations about the mouth care must be taken to see that no blood gets down the trachea.

6. It is desirable to have the stomach empty, therefore no solid food should be given for some hours before the administration. The patient's head must be so directed during vomiting that no vomited matter can get into the larynx.

7. False teeth should be taken out of the mouth.

8. The chloroform must be pure.

9. It should not be too concentrated. About 5 per cent. of chloroform to 95 per cent. of air is a good mixture.

10. The head should be a little raised, and the lower jaw held up so that the tongue shall not fall back over the larynx.

I I. Special care must be taken when the operation necessitates awkward positions, especially if respiration is interfered with, as in the lateral position used in obstetrical, [gynæcological] and renal cases.

I2. Because the temperature falls the patient should be kept warm.

[13. Chloroform should never be administered without an hypodermatic syringe, in good order, being at hand. Amyl nitrite, ether and ammonia should be in readiness.]

If the breathing becomes very weak, or stops altogether, artificial respiration should at once be commenced, the tongue being pulled forward by forceps to allow free entry of air to the lungs. The face and abdomen should be flicked with wet towels, a capsule of amyl nitrite may be inhaled, and ether injected subcutaneously. [Brandy should not be administered hypodermatically. The heart may be stimulated by large rectal injections of hot normal saline solution, or of hot decoctions of coffee, if 
at hand.] It is [extremely questionable] whether galvanization over the cardiac area is of any use ; it [doubtless] does harm. [If symptoms of improvement do not appear at once, the patient should be inverted.] Artificial respiration should be maintained at least an hour or so, even if there is no sign of returning life; and if there is the slightest evidence of a cardiac beat, or a single automatic respiratory movement, artificial respiration must be persevered in even for many hours. If the face be pale, the head should be lowered, and amyl nitrite is especially likely to be useful. [In spite of all care in administration and the observance of all precautions, one death takes place in about three thousand administrations. Hare and Thornton, after a painstaking series of experiments, believe chloroform to be safe for the majority of cases, provided it be given by one skilled in its use, and who not only knows how to give it, but to detect signs of danger. The respiration should be watched, because so soon as enough chloroform is used to endanger the circulation, the respiration will show some abnormality. Death in the healthy animal is always due to respiratory failure, accompanied by circulatory depression which may be severe enough to cause death, even if artificial respiration be skillfully used. Chloroform may be chosen in hot climates; when a large number of persons are to be anæsthetized; in Bright's disease ; in aneurism ; or in great atheroma of bloodvessels ; in children or adults who already have bronchitis; and in persons who struggle violently.]

\section{IV. ÆTHER.}

ETHER.- $\left[\left(\mathrm{C}_{2} \mathrm{H}_{5}\right)_{2} \mathrm{O}=73.84\right.$. Synonyms.-Sulphuric Ether. Ethylic Ether. Ethyl Oxide. A liquid composed of about 96 per cent., by weight, of absolute Ether, and about 4 per cent. of Alcohol containing a little water.

Source. - Alcohol is distilled with Sulphuric Acid. Ethyl Sulphuric (Sulphovinic) Acid and water are first formed. $\mathrm{C}_{2} \mathrm{H}_{5} \mathrm{OH}+\mathrm{H}_{2} \mathrm{SO}_{4}=\mathrm{C}_{2} \mathrm{H}_{5} \mathrm{HSO}_{4}$ $+\mathrm{C}_{2} \mathrm{H}_{5} \mathrm{OH}=\left(\mathrm{C}_{2} \mathrm{H}_{5}\right)_{2} \mathrm{O}+\mathrm{H}_{2} \mathrm{SO}_{4}$. This process is theoretically continuous, the Sulphuric Acid last formed again acting on fresh Alcohol as it is supplied. The Ether is freed from water by re-distillation with Calcium Chloride and Lime.

Characters. - A transparent, colorless, mobile liquid, having a characteristic odor, and a burning and sweetish taste. It is very inflammable, boils at 
about $98.6 \mathrm{~F} . ; 37^{\circ} \mathrm{C}$, and burns with a white flame. Sp. gr., 0.725 to 0.728 . Solubility. - In about to volumes of water.]

IMPURITIES. - Water, alcohol and fixed impurities.

Dose, 5 to $60 \mathrm{~m}$.; [.30 to 4.00 c.c.]

\section{Preparations.}

I. Spiritus Ætheris.-[Spirit of Ether. Ether, 325; Alcohol, 675. Dose, $1 / 4$ to $x$ fi. dr. ; r. to 4 . c.c.

2. Oleum Æthereum.-Ethereal Oil. A volatile liquid composed of equal volumes of heavy Oil of Wine and Ether. Alcohol, IO0O; Sulphuric Acid, I000; distilled water, 25 ; Ether, a sufficient quantity ; by distillation.

Characters. - A transparent, nearly colorless, volatile liquid, of a peculiar, aromatic, ethereal odor, a pungent, refreshing, bitterish taste, and a neutral reaction. Sp. gr., 0.9 Io.

Ethereal Oil is used to prepare Spiritus Etheris Compositus.]

3. Spiritus Etheris Compositus. [Compound Spirit of Ether.] Synonym.-Hoffman's Anodyne. [Ether, 325; Alcohol, 650; Ethereal Oil, 25.

Dose, 5 to $60 \mathrm{~m} . ; .30$ to 4.00 c.c.]

\section{Action of Ether.}

External.-Ether evaporates very quickly, producing great cold, and consequently the part to which it has been applied becomes white from the contraction of the vessels. The cold is sufficient to cause such marked local anæsthesia that the pain of slight operations, performed upon the part anæsthetized, cannot be felt. To produce this result ether is best applied as a fine spray. If it be rubbed in, or evaporation be prevented, it, like alcohol or chloroform, is an irritant.

Internal.- In the mouth and stomach also it acts like chloroform or alcohol. Thus ether causes a burning taste in the mouth, an increase of the saliva, of the gastric secretion and gastric movements, and dilatation of the vessels of the stomach. Consequently it is carminative and aids digestion. Directly [after] it reaches the stomach it reflexly excites the heart, increasing the force and frequency of the pulse, and causing a rise of blood-pressure; it is one of the best cardiac stimulants 
we have. In the same way it excites respiration. It is quickly absorbed, and its stimulating influence on the heart and respiration is continued. It is thus a good instance of a rapidly diffusible stimulant. It is also anti-spasmodic.

Nervous system.-Ether is a powerful general anæsthetic. The phenomena and stages of ether anæsthesia are so like those of chloroform anæsthesia that the description already given (see p. 292) will suffice. The following differences, however, should be noticed:

(I) The heart is paralyzed with much greater difficulty by ether than by chloroform.

(2) The same is true of the vaso-motor centre.

(3) And also of the respiratory centre.

(4) Ether is much more irritant to the respiratory mucous membrane, and hence is more liable to increase bronchitis in those already suffering from it.

(5) [Ether is much more likely to irritate the kidneys, and those suffering from the various forms of acute or chronic renal disease, or even from renal insufficiency, should be subjected to ether anæsthesia only when it is administered with the greatest caution.]

(6) With ether the stage of stimulation is more protracted, therefore there is more struggling.

(7) For the same reason the anæsthetic stage is not reached so soon.

(8) The reduction of temperature is greater with ether.

(9) Ether must be given nearly pure, about 30 per cent. of air to 70 of ethereal vapor; hence it is more difficult to administer.

(10) The smell of ether is more disagreeable, and patients dislike it more.

(II) Ether is eliminated more slowly, and hence the [odor lingers] about the patient some time.

(i2) Ether being very inflammable cannot be used in the close neighborhood of a naked light.

[Ether, on account of its greater safety, is more generally used as an anæsthetic in the United States.] 


\section{Therapeutics of Ether.}

External.-Ether, allowed to evaporate, may be used to cause local anæsthesia in cases of neuralgia. An ether spray is occasionally employed to produce local anæsthesia for small operations, but as the ether makes the skin hard and brawny the operation must be quite superficial, and even then there is much subsequent tingling and pain.

Internal.-Stomach.-It may be used for the same classes of dyspepsia as chloroform or alcohol, and is often employed as a carminative to expel gas in flatulent dyspepsia.

Heart. - Administered subcutaneously (dose, Io to I 5 minims) [.60 to $\mathrm{r} .00 \mathrm{c.c}$.] or by the mouth, ether is an excellent cardiac stimulant of great value in fainting, cardiac failure, or palpitation; its advantage over chloroform and alcohol being that it is more rapid in its action. It is, very useful as an anti-spasmodic during an attack of asthma.

Inhalation.-Ether is inhaled for the same purpose as chloroform. There is great divergence of opinion as to which is the safer anæsthétic. All the published statistics in which the two are contrasted appear to show that ether is much safer, and this is what might have been expected from the contrast between the two already given. Chloroform is administered carelessly more often than ether, as it is easier to give; but even allowing for this, ether is probably, on the whole, safer. The nausea and vomiting which sometimes follow the administration of ether may, it is said, be checked by giving 15 grains [ $1.00 \mathrm{gm}$.] of sodium bromide. Very often anæsthesia is commenced with a few inhalations of nitrous oxide gas, and then completed with ether. This is much pleasanter for the patient than to use ether from the first.

\section{AETHER ACETICUS.}

ACETIC ETHER. $-\mathrm{C}_{2} \mathrm{H}_{3} \mathrm{C}_{2} \mathrm{H}_{3} \mathrm{O}_{2}[=87.8$. Synonym. -Ethyl Acetate. A liquid composed of about 98.5 per cent., by weight, of Ethyl Acetate and about I. 5 per cent. of Alcohol, containing a little water.

Source.-A mixture of Sodium Acetate, Sulphuric Acid and Alcohol is distilled. $\quad \mathrm{C}_{2} \mathrm{H}_{5} \mathrm{OH}+\mathrm{NaC}_{2} \mathrm{H}_{3} \mathrm{O}_{2}+\mathrm{H}_{2} \mathrm{SO}_{4}=\mathrm{C}_{2} \mathrm{H}_{5} \mathrm{C}_{2} \mathrm{H}_{3} \mathrm{O}_{2}+\mathrm{NaHSO}_{4}+\mathrm{H}_{2} \mathrm{O}$. 
The distillate is purified from acid and water by digestion with Potassium Carbonate.

Characters. - A transparent, colorless liquid, of a fragrant and refreshing slightly acetous odor, and a peculiar, acetous, and burning taste. Sp. gr., 0.893 to 0.895 . Solubility. -In 8 parts of water; freely in Alcohol or Ether.]

Dose, 20 to $60 \mathrm{~m}$.; [1.20 to $4.00 \mathrm{c.c}$. $]$

\section{Action and Therapeutics of Acetic Ether.}

It acts like ether, as a stimulant, antispasmodic, and carminative, but it has a pleasanter taste.

\section{VI. [ETHYL BROMIDUM.}

ETHYL BROMIDE (not official). $-\mathrm{C}_{2} \mathrm{H}_{5} \mathrm{Br}=108.70$. Synonyms.Ather Bromatus. Hydrobromic Ether. This must be carefully distinguished from Ethylene Bromide.

Source.-From a well-cooled mixture of Sulphuric Acid, I2; and Alcohol (sp. gr., 0.816), 7 ; to which powdered Potassium Bromide, 12, is added; this mixture is distilled. The distillate is washed by agitation, first with a 5 per cent. solution of Potassium Carbonate, then with an equal volume of water; finally it is dehydrated with Calcium Chloride and re-distilled.

Characters. - A colorless, highly refractive, very volatile liquid having a strong ethereal odor and a sweetish, warm taste. Sp. gr., I.445 to 1.450. It is easily decomposed by light and air.

\section{Action and Uses of Ethyl Bromide.}

Ethyl Bromide was introduced to the profession in 1880 as the most agreeable and rapid anæsthetic. Several fatal cases having been reported, its use was abandoned. Recently, however, Cumston has recommended its inhalation, when pure, in doses of from $3 \mathrm{fl}$. dr.; I 2. c.c. (child of two years), to $6 \mathrm{fl}$. dr.; 24. c.c. (adult), for surgical anæsthesia. The following precautions should be observed: Food, even a glass of milk, is absolutely forbidden on the day of operation. The mask should perfectly cover the mouth and nose, so that no air is allowed to enter. The entire dose should be given at once. When narcosis is complete, the mask should be removed, and under no consideration be re-applied. Do not prolong the administration over one minute. Sleep is obtained in from twenty to thirty seconds, and lasts from two to three minutes, sometimes longer. 
The contra-indications to its use are dangerous lesions of heart, lungs, or kidneys.

\section{BROMOFORMUM.}

BROMOFORM (not official). $-\mathrm{CHBr}_{3}=252.25$. Synonym.-Tribromomethane.

Source. - By the action of Sodium Hypobromite (which is obtained when Bromine is added to a solution of Sodium Hydroxide) on Acetone.

Characters. - A clear, colorless liquid, of a peculiar odor, and a sweetish taste. Sp. gr., 2.90. Solubility. - Slightly in water, but readily in Alcohol. It must be kept protected from light.

Dose, I to $5 \mathrm{~m}$.; .06 to .30 c.c.

\section{Action and Uses of Bromoform.}

Bromoform, an analogue of chloroform, is an anæsthetic. Inhaled, it produces anæsthesia in animals, but of shorter duration than that of ether or chloroform. It is a remedy of value for whooping-cough, for which its pleasant taste and convenience of administration gives it great advantage. It can be givenbromoform, I ; in a mixture of alcohol, 8 ; glycerin, 48 ; and compound tincture of cardamom, 8 . Each fluid drachm; 4 c.c., contains about 3 minims; 20 c.c. of the drug. Cases of poisoning have been reported, so that it must be used with care.

\section{PENTALUM.}

PENTAL (not official). $-\mathrm{C}_{5} \mathrm{H}_{10}=69.85$. Synonym. - Trimethylethylene.

SOURCE.-It is obtained from Amylic Alcohol by digestion with Zinc Chloride for twenty-four hours, and fractional distillation.

Characters. - A colorless liquid, very volatile, insoluble in water, but miscible in all proportions with Alcohol, Ether, and Chloroform; highly inflammable. Sp. gr., o.620.

\section{Action and Uses of Pental.}

Pental is an anæsthetic, the equal of nitrous oxide in rapidity of action and perhaps safety, but superior to it in its more prolonged action and in having no unpleasant after-effects. Even when insensibility to pain is reached, consciousness is retained sufficiently to respond to commands. The stage of exhilaration is seldom present; it does not lose its effect by repeated inhalations. It differs from chloroform in that it acts more promptly, 
and has no evil after-effects; from ethyl bromide, in that it is somewhat slower in its action, but is more lasting in its effects, and can be prolonged as may be necessary ; from nitrous oxide, in its freedom from unpleasant effects.

Two substances used to produce local anæsthesia should be considered. These are ethyl chloride (synonym, hydrochloric ether) and methyl chloride (neither official).]

Ethyl chloride is an inflammable liquid of a low boilingpoint and produces intense cold by its evaporation. It is sold in glass capsules terminating in a fine tube with a screw-capped point. When the capsule is held in the hand about eight inches from the part to be anæsthetized, the warmth of the hand volatilizes the ethyl chloride, and the vapor falling on the skin of the patient, by its evaporation produces enough local anæsthesia for the performance of small operations, such as the removal of warts, etc. All fat must be removed from the skin by soap and washing with ether. [The same objections apply to this, and the following as to ether, for the production of local anæsthesia. (See p. 300).]

Methyl chloride is used in the same way to produce local anæsthesia and to relieve the pain of neuralgia, etc. The vapor is allowed to play obliquely on the skin for four or five seconds.

CLASS III.-Drugs which Dilate Blood-Vessels.

\section{Sodium Nitrite, Amyl Nitrite, Glonoin and Erythrol Nitrate.}

All these dilate the peripheral vessels, and increase the rapidity of the heart.

\section{SODII NITRIS.}

SODIUM NITRITE.-NaNO $[=68.93$.

SouRCE. - Made by heating Sodium Nitrate with Lead, which becomes an oxide, taking Oxygen from the nitrate. $\left[\mathrm{NaNO}_{3}+\mathrm{Pb}=\mathrm{NaNO}_{2}+\mathrm{PbO}\right.$.

Characters. - White, opaque, fused masses, usually in the form of pencils, or colorless, transparent, hexagonal crystals ; odorless, and having a mild, saline taste. When exposed to the air, the salt deliquesces and is gradually oxidized to Sodium Nitrate. Solubility. - In about 1.5 parts of water; slightly soluble in Alcohol.]

Dose, 2 to $5 \mathrm{gr}$.; [.12 to $.30 \mathrm{gm}$.] 


\section{Preparation.}

Spiritus Etheris Nitrosi.-[Spirit of Nitrous Ether. Synonym. -Sweet Spirit of Nitre.

An Alcoholic Solution of Ethyl Nitrite $\left(\mathrm{C}_{2} \mathrm{H}_{5} \mathrm{NO}_{2}=74.87\right)$, yielding, when freshly prepared, not less than I times its own volume of Nitrogen Dioxide. In many commercial specimens there is very little Ethy 1 Nitrite.

SouRce.-Dissolve Sodium Nitrite, 750 , in water; adding Deodorized Alcohol, 550; introduce into the containing flask, Sulphuric Acid, 520 ; previously diluted, and distil. Wash the distillate with icecold water, remove traces of acid by Sodium Carbonate, ro, dissolved in water; agitate with Potassium Carbonate to remove all traces of water, and add sufficient Deodorized Alcohol.

Characters. - A clear, mobile, volatile, inflammable liquid of a pale yellowish or faintly greenish-yellow tint, having a fragrant, ethereal and pungent odor, free from acridity, and a sharp, burning taste. Sp. gr., o. 836 to 0.842 .]

Incompatibles. - Potassium iodide, ferric sulphate, tincture of guaiacum, gallic and tannic acids, and emulsions.

IMPURITY.-Excess of acetic acid.

Dose, $1 / 2$ to 2 fi. dr.; [2. to 8. c.c.]

Action of Sodium Nitrite and Spirit of Nitrous Ether.

External.-Spirit of nitrous ether evaporates when it is applied externally, and a slightly anæsthetic effect is produced.

Internal.-It combines the action of the ether with that of the nitrites contained in it. Because of the ether it is a diffusible stimulant, a stomachic and a carminative. Because of the nitrites it acts like amyl nitrite; but as the ethyl nitrite is so diluted, its action in this direction is feeble; thus it only moderately dilates the vessels, and except in poisonous doses probably does not affect the blood. The dilatation of the vessels leads to a diaphoretic effect on the skin, a diuretic effect on the kidney, and a lowering of arterial blood-pressure. The dilatation of the cutaneous vessels, the sweating, and perhaps the changes of the blood, produce a slight antipyretic influence. It is obvious that in these effects the nitrites will to some extent be aided by the ether. [Sodium nitrite possesses the same, but a more lasting, action as the spirit of nitrous ether.] 


\section{Therapeutics of Sodium Nitrite and Spirit of Nitrous ETHER.}

For its diaphoretic and slight antipyretic effects it is commonly given in mild febrile attacks, such as a common cold. It is also used as a diuretic in chronic Bright's disease, and cardiac and pulmonary diseases accompanied by œdema [but for these purposes the sodium nitrite is preferable.]

\section{AMYL NITRIS.}

[AMYL NITRITE. $-\mathrm{C}_{5} \mathrm{H}_{11} \mathrm{NO}_{2}=116.78 . \quad$ A liquid containing about 80 per cent. of Amyl (principally Iso-Amyl) Nitrite, together with variable quantities of undetermined compounds.]

SOURCE. - By action of Nitric Acid upon Amylic Alcohol. $\mathrm{HNO}_{3}+$ $\mathrm{C}_{5} \mathrm{H}_{11} \mathrm{OH}=\mathrm{C}_{5} \mathrm{H}_{11} \mathrm{NO}_{2}+2 \mathrm{H}_{2} \mathrm{O}$. Purify the distillate with Sodium Carbonate.

Characters. - A clear yellow, or pale-yellow liquid, of a peculiar, ethereal, fruity odor, and a pungent, aromatic taste. Sp. gr., 0.870 to 0.880 . Solubility.-Insoluble in water ;] soluble in Ether, Chloroform, or Alcohol.

IMPURITIES. - Free acid and amyl nitrate.

Dose, [ $\mathrm{I}$ to $3 \mathrm{~m}$.; .06 to .20 c.c.] cautiously inhaled from a handkerchief in which a glass capsule containing the Amyl Nitrite has been crushed; $[1 / 2$ to I $\mathrm{m} . ; .03$ to .06 c.c. internally, dissolved in alcohol.]

\section{Action of Amyl Nitrite.}

External.-Locally applied it diminishes the activity of the sensory nerves, but they quickly recover.

Internal.-Amyl nitrite is rarely given by the mouth, so the following account will refer to the effects of inhalation. The effects of a single inhalation pass off in two or three minutes.

Circulation. - From the medical point of view by far the most important effects of amyl nitrite are those produced upon the heart and vessels. Within a minute of inhalation the face flushes, the heart beats very rapidly, and violently, there is a throbbing in the head, and the vessels, e.g., the carotids, may be seen to pulsate actively. Headache, giddiness, dilatation of the pupils, and increased respiratory movements quickly supervene. All the vessels of the body rapidly dilate, hence the flushing. They may be actually seen to widen in the ear of a rabbit or in the retina. This is due to a direct action on the muscular coats of the arterioles, for it happens if the cord is destroyed. 
The blood-pressure and arterial tension, of course, fall very low. The increase in the rate of the pulse is unaccompanied by any alteration in the force of the beat ; it is apparently due to a depressing influence on the inhibitory vagus centre; [the vaso-motor paralysis will, however, produce a rapid pulse.] In toxic doses the heart may be arrested in diastole from direct action on the cardiac muscle.

Respiration.-The rapidity and depth of respiration are at first increased, probably from central stimulation; the respiratory centres are later depressed, the breathing becoming slower and shallower, and usually death finally occurs from paralytic asphyxia of central origin.

Nervous system.-Many of the symptoms referable to the nervous system are secondary effects of the dilatation of the vessels of the brain and spinal cord, Such are the throbbing, sense of fulness, giddiness and headache noticed directly after inhalation. The headache may remain some time. If much has been inhaled there is unsteadiness of gait and general restlessness. The pupil dilates, and disturbances of vision are present. The motor centres of the cord are profoundly depressed; therefore after large doses reflex actions are abolished. The function of sensory nerves, motor nerves, and muscles is depressed by the local application of the drug to them, but not after inhalation until shortly before death.

Temperature.-Amyl nitrite causes this to fall considerably, both in fever and health. The fall is due to the peripheral vascular dilatation, and if large doses are given, to the changes in the blood.

Urine.-The drug probably escapes in the urine as nitrites and nitrates; it is slightly diuretic, and may cause glycosuria, due, it is said, to dilatation of the vessels of the liver or of the medulla.

Blood.-Nitrites, given in medicinal doses, circulate as sodium nitrite. Outside the body they greatly diminish oxidation, and the same takes place in the blood. After the inhalation of a considerable amount (more than is usually given to a man) the arterial and venous blood both become a uniform chocolate color. 
This is due to the formation of methæmoglobin and another body, nitric oxide hæmoglobin. The hæmoglobin can no longer absorb oxygen, and hence its oxidizing power is abolished. It is by this action on the blood that, in man, nitrites kill, not by their vaso-dilator action; therefore the treatment for poisoning by them is inhalation of oxygen, that more may be dissolved in the plasma. In some of the lower animals they kill by acting as a direct poison to animal tissues.

\section{Therapeutics of Amyl Nitrite.}

Heart and blood-vesscls. - Brunton in 1867 observed that in a [patient suffering from] angina pectoris the peripheral vessels were strongly contracted during an attack of pain. This induced him to make the patient inhale amyl nitrite, and it was found that the vessels dilated and the pain passed off. Inhalation of amyl nitrite is now used for all sorts of cardiac pain, especially when it comes on in paryoxysms. Generally the drug affords relief in a minute or so after inhalation, but by no means always. We do not sufficiently understand the pathology of angina pectoris to know how it acts. It may be by dilating the peripheral vessels; but against that view is the fact that they are not always contracted during attacks of angina pectoris, and amyl nitrite may relieve patients in whom the vessels are not contracted. The attacks of pain common in thoracic aneurism may be relieved by it. It is used to avert the dangerous pallor sometimes seen during the administration of chloroform, and may be inhaled for other forms of syncope. The peculiar hot flushes experienced by some women during the menopause are benefited by inhalation of it ; [this is probably untrue.]

Nervous system. - If it is inhaled when the aura is felt, an epileptic fit may sometimes be prevented, and it has also been found useful during the status epilepticus. Because in migraine the vessels of the head are contracted, it has been used, and sometimes successfully, for this complaint. Its depressing action on the cord has suggested its employment in tetanus and strychnine poisoning.

Occasionally the inhalation of amyl nitrite relieves an attack 
of asthma. It has been given in whooping-cough, sea sickness. and cholera, but without much good effect.

\section{III. [GLONOINUM.}

SPIRITUS GLONOINI.-Spirit of Glonoin. Synonym.-Spirit of Nitroglycerin. An alcoholic solution of Glonoin, Glyceryl (or Propenyl) trinitrate, Nitroglycerin, or Trinitrin ; $\mathrm{C}_{3} \mathrm{H}_{5}\left(\mathrm{NO}_{3}\right)_{3}=226.58$, containing I per cent., by weight, of the substance.

SOURCE. - Nitroglycerin is prepared by gradually adding dehydrated Glycerin to Nitric and strong Sulphuric Acid, the result being Propenyl trinitrate or Trinitroglycerin. $\mathrm{C}_{3} \mathrm{H}_{5}(\mathrm{OH})_{3}+3 \mathrm{HNO}_{3}=\mathrm{C}_{3} \mathrm{H}_{5}\left(\mathrm{NO}_{3}\right)_{3}+3 \mathrm{H}_{2} \mathrm{O}$. It separates as an oily layer which is washed with water and with dilute soda solution to remove all acid.

Characters. - A clear, colorless liquid, possessing the odor and taste of Alcohol. Caution should be exercised in tasting it, since even a small quantity of it is liable to produce a violent headache. The same effect is produced when it is freely applied to the skin. Sp. gr., 0.826 to 0.832 .

Dose, I to $3 \mathrm{~m}$.; .06 to .20 c.c.]

\section{Action and Therapeutics of Glonoin.}

Its action is the same as that of amyl nitrite, except that in many animals, and probably in man, large doses do not form methæmoglobin in the blood; the effects of [spirit of] glonoin are more persistent, and as it is only suitable for internal administration, they are slower in their onset. It is largely taken by persons liable to cardiac pains with the object of warding off the attack, and often such persons require and bear large doses. [Its most important use is for the relief of symptoms associated with the high tension pulse of chronic renal degeneration. Here the dose should be rapidly increased until relief is obtained.] Occasionally it does good in asthma. It is really a glycerin nitrate, but certainly physiologically it belongs to the class of nitrites; therefore, probably, directly it gets into the body sodium nitrite is formed.

\section{ERYTHROL NITRATE.}

ERYTHROL NITRAS.-Erythrol Nitrate (not official), $\left[\left(\mathrm{CH}_{2} \mathrm{ONO}_{2}\right)\right.$ $2\left(\mathrm{Cl} 1 \mathrm{O} . \mathrm{NO}_{2}\right)_{2}=281.44$. ] Synonym.-Erythrol Tetranitrate.

[SOURCE. - By the nitration of Erythrite $\left(\mathrm{C}_{4} \mathrm{H}_{6}\left(\mathrm{OH}_{4}\right) \cdot\right.$ ] 
Characters. - Hard, colorless, acicular crystals. Solubility.-Insoluble in water; in 60 [parts] Absolute Alcohol.

Dose, $1 / 2$ to $1 \mathrm{gr}$. ; .03 to $.06 \mathrm{gm}$.]

\section{Action and Uses of Erythrol Nitrate.}

Erythrol nitrate has the same action as nitrites. It is less powerful than amyl nitrite and nitroglycerin. It is best given in chocolate tablets, and, as its action is slow, it is the best of this group for [the relief of some symptoms of] Bright's disease.

Class IV.-The Hypnotics.

\section{Chloral and Paraldehyde.}

[Besides these the following drugs are considered: Butyl-Chloral Hydrate Chloralamide, Chloretone, Chloralose, Amylene Hydrate, Sulphonal, Trional and Urethane; none of which are official].

\section{CHLORAL.}

CHLORAL. $-\mathrm{C}_{2} \mathrm{HCl}_{3} \mathrm{O}+\mathrm{H}_{2} \mathrm{O}[=\mathrm{I} 64.97$. Synonym.-Chlora Hydrate. SouRCE. - Absolute Alcohol is saturated with dry Chlorine; Aldehyde and Hydrochloric Acid are first formed. $\mathrm{C}_{2} \mathrm{H}_{5} \mathrm{OH}+\mathrm{Cl}_{2}=\mathrm{C}_{2} \mathrm{H}_{4} \mathrm{O}+2 \mathrm{HCl}$. By the continued action of the Chlorine Gas 3 atoms of Hydrogen are abstracted from the Aldehyde and replaced by 3 atoms of Chlorine, producing Chloral. $\mathrm{C}_{2} \mathrm{H}_{4} \mathrm{O}+3 \mathrm{Cl}=\mathrm{C}_{2} \mathrm{HCl}_{3} \mathrm{O}+3 \mathrm{HCl}$. It is purified by Sulphuric Acid, and afterwards by Lime.

Characters. - Separate, rhomboidal, colorless and transparent crystals, having an aromatic, penetrating and slightly acid odor, and a bitterish, caustic taste. Easily melted by gentle heat. Solubility.-Freely in water, Alcohol and Ether.] Forms a liquid when rubbed up with an equal weight of camphor.

InCOMPATIBLES. - All alkalies decompose it.

ImPURITIES. - Hydrochloric Acid and oily impurities.

Dose, 5 to $20 \mathrm{gr}$; ; [.30 to $1.20 \mathrm{gm}$.]

[The U.S. P. is in error in naming the crystalline product, as above described, chloral; it is actually chloral hydrate, for the molecule of water is necessary for crystallization. Chloral really is an oily liquid. Whenever chloral is mentioned in this book, chloral hydrate is intended.]

\section{Action of Chloral.}

External.-It is a powerful antiseptic. Locally applied it is irritant, causing vesication. [Chloral rubbed up with equal parts of menthol and cainphor is a local anodyne.]

Internal.-Alimentary canal. - Unless diluted, chloral is a 
gastric irritant; large doses, therefore, may give rise to vomiting and purging.

Blood.-It is readily absorbed, and circulates in the blood unchanged. It was formerly thought that as alkalies convert it into chloroform and formic acid, this change would take place in the blood, and consequently Liebreich suggested its use as an hypnotic. It is now known that this view is wrong, for no chloroform can be found in the blood of chloralized animals, nor in the urine unless that fluid is alkaline, in which case chloral is decomposed by the alkali in the urine.

Circulation.-Chloral depresses the heart, large doses having this action to a considerable degree. This is due to a local effect on the organ itself; probably both the muscular substance and the nerves contained in it are affected. The pulse, which may at first be slightly quickened, soon becomes slow, feeble, and irregular, and the heart finally stops in diastole. The vaso-motor centre is depressed, and consequently the vessels dilate. As a result of these actions on the heart and the vessels the blood-pressure falls.

Respiration.-After large doses the respirations become slow and full, and after toxic doses they become irregular and shallow before finally ceasing. This is due to the action of chloral on the respiratory centre.

Temperature.-Large doses cause this to fall, probably by diminishing the production of heat.

Brain.-Chloral is a powerful hypnotic, acting directly on the brain. The stage of excitation, if it exists, is very short. Soon after taking a moderate dose the patient is overcome by sleep, which lasts several hours, and is indistinguishable from natural sleep. On waking there is neither confusion nor headache, and he feels refreshed. Large doses produce coma. The pupil is always contracted.

Spinal cord.-At first the anterior cornua may be slightly stimulated, but soon they are depressed, and there is consequently paralysis and loss of reflex excitability. The motor nerves and the muscles are not affected, nor are the sensory nerves unless the dose is very large, when there may be anæsthesia. 
It will be observed that chloral is a powerful general depressant, chiefly of the cerebrum, but also of the respiratory centre, the vaso-motor centre, the anterior cornua, of the production of heat, and the heart. It is only because it depresses the cerebrum much earlier than any other part of the body that we can use it as an hypnotic.

\section{Therapeutics of Chloral.}

External.-The compound with camphor [equal weights of camphor and chloral rubbed together to form a liquid] has been employed as a local anodyne for neuralgia, and may be applied to aching teeth. [To this menthol can be advantageously added.]

Internal.-Chloral is largely used for its hypnotic effect. Its great advantages over many other hypnotics are that doses sufficient to produce a deep sleep are not large enough to cause gastro-intestinal irritation, cardiac and respiratory depression, and the other harmful effects. Chloral is certain in its action [in that] it quickly produces sleep; and there are [usually] no bad after-effects. [Chloral is far from being a safe hypnotic; it depresses the heart so markedly that the prescriber should be always upon his guard.] Children take it well. [Its unpleasant taste can be concealed by administration in bottled "lemon soda."]

It is especially useful in simple insomnia from overwork, worry, etc. Its disadvantages are that it does not relieve pain at all, and it should therefore not be used for insomnia due to this cause; and that, as it depresses the heart and respiration, it must be given carefully in diseases of the heart and lungs, and also when the stomach or intestines are diseased, as it may irritate these structures. In febrile insomnia it is very valuable in the early stages, but must be given cautiously, later, when there is any danger of cardiac weakness. It does not relieve the distress and cough of disease of the heart and lungs. It has been used as a cerebral depressant in delirium tremens, puerperal convulsions, and mania, but very large doses are required, and consequently the results must be watched with great care. [A very important use of chloral is in midwifery; here it has been designated the 
medicinal forceps. Frequently after rest has been obtained by this drug, labor proceeds vigorously and is rapidly terminated.]

From its action on the spinal cord, chloral has been used, and sometimes with success, in tetanus, whooping-cough, incontinence of urine and strychnine poisoning.

[Hypnal is a compound of chloral with antipyrin, made by mixing their solutions, and is obtained in crystalline form. It was proposed by Bardet as a hypnotic, more certain than chloral, nearly free from taste, entirely free from irritating effect upon the mucous membranes, and having distinct analgesic effects. It has been but little used. The dose is about $15 \mathrm{gr}$. ; I. $\mathbf{1} 00 \mathrm{gm}$.]

TOXICOLOGY.

Acute Poisoning.-As will be inferred from the action of chloral, the symptoms of poisoning by it [closely resemble those of opium. They] are deep coma ; a weak, feeble, irregular, slow pulse, which may become quick before death; diminished frequency of respiration and consequent lividity; and abolition of reflex movements. The surface of the skin is cold, and the temperature is subnormal. [The pulse should always be carefully watched whenever chloral has been administered. It frequently happens that symptoms of failing heart come on unexpectedly even after small doses.]

Treatment.-Give emetics (see p. 139) or wash out the stomach. Keep up the temperature by hot bottles, hot blankets, friction and massage. Prevent sleep by the injection of hot, strong coffee into the rectum, shouting at the patient, hitting him, flapping with wet towels, bathing, etc. Give a subcutaneous injection of strychnine, because of its stimulant action on the anterior cornua. Use inhalations of amyl nitrite to stimulate the heart, and artificial respiration if necessary.

Chronic Poisoning.-The taking of chloral is a vice to which many persons are addicted. A craving for it is soon established. The chief symptoms of chronic chloral poisoning are gastro-intestinal irritation, a great liability to erythematous eruptions, dyspnœa dependent upon the cardiac and respiratory depression and general weakness. There may be disturbance of the mental equilibrium, and persons have been known to become permanently weakminded. A slightly larger dose than usual may be quickly fatal.

\section{BUTYL-CHLORAL HYDRAS.}

BUTYL-CHLORAL HYDRATE. [13. P., not official]. $-\mathrm{C}_{4} \mathrm{H}_{5} \mathrm{Cl}_{3}$ $\mathrm{O}+\mathrm{H}_{2} \mathrm{O}=[\mathrm{1}$ 92.9I. $]$ Synonym. - Croton Chloral Hydrate. (This is a misnomer.)

Source.-Dry Chlorine Gas is passed through Aldehyde. Butyl-chloral $\left(\mathrm{C}_{4} \mathrm{H}_{6} \mathrm{Cl}_{3} \mathrm{O}\right)$ is formed. It is separated by fractional distillation, and water is added. 
Characters. - Pearly-white, crystalline scales, with a nauseous taste and a pungent odor like chloral. Solubility.-In 50 parts of water; freely in Al. cohol and Glycerin.

INCOMPATIBLES, - All alkalies.

Dose, 5 to $20 \mathrm{gr}$; ; $[.30$ to $1.20 \mathrm{gm}$.]

Action and Therapeutics of Butyl-Chloral Hydrate.

The action of this drug is exactly similar to that of chloral, but butyl-chloral hydrate is less certain in its effects. It is said to be less depressant to the heart, but this is doubtful. It has a specific action in relieving neuralgia of the fifth nerve.

\section{CHLORAL [FORMAMIDATUM.}

CHLORAL FORMAMIDE. (Not official.) - $\mathrm{CCl}_{3} \mathrm{CH} . \mathrm{OH} . \mathrm{CONH}_{2}$ $=$ I9I.95. Synonym. -Chloralamide.

Source. - By the combination of Chloral and Formamide. $\mathrm{CCl}_{3} \mathrm{CHO}+$ $\mathrm{CHONH}_{2}=\mathrm{CCl}_{3} \mathrm{CH} . \mathrm{OH}$. CONH${ }_{2}$.]

Characters. - Shining colorless crystals, [without odor and of a slightly bitter taste. Solubility.-Slowly, in about 20 parts of water, in 1.5 parts of Alcohol, and in weak acid solutions. It should not be heated over $140^{\circ} \mathrm{F}$.; $60^{\circ} \mathrm{C}$, , or mixed with alkalies, for, in either case, it decomposes into Chloral.

Dose, 10 to $30 \mathrm{gr}$; . 60 to $2.00 \mathrm{gm}$.]

\section{Action and Therapeutics of Chloralamide.}

Chloralamide is an excellent hypnotic, producing calm, refręshing sleep without any bad after-effects. Frequent use does not necessitate an increased dose, nor, as far as known, is any habit contracted. It does not relieve pain, but is equally serviceable for all varieties of insomnia, unless due to pain. If possible it should not be given as a powder, for it is then so very slowly absorbed that probably some of it is decomposed in the intestines or stomach; [very rarely], when powdered chloralamide has been administered in the evening, the patient has not slept during the night, but has slept all the next day, because the drug has been so slowly absorbed. The best way to give it is to dissolve it in a little alcohol. The patient may be told to dissolve $20 \mathrm{gr}$. ; [1.20 gm.] or more, in sufficient brandy, to add water not above $130^{\circ} \mathrm{F}$. ; $54.4^{\circ} \mathrm{C}$, , and drink it before going to bed. Some specimens are very insoluble, and must be suspended. It is said that ro minims [.60 c.c.] of aromatic sulphuric acid added 
to I fl. oz. ; [30 c.c.] of water will dissolve $30 \mathrm{gr} . ;$ [ $2 \mathrm{gm}$.$] of$ chloralamide, but this is not always true. It acts if given as an enema. Fifteen grains $[\mathrm{x} .00 \mathrm{gm}$. $]$ of potassium bromide and chloralamide, flavored with tincture of orange [peel] and chloroform water has been strongly recommended for insomnia and for sea-sickness. This mixture resembles a proprietary preparation called Chlorobrom.

\section{IV. [CHLORETONUM.}

CHLORETONE: (Not official.) Synonyms. - Trichlor-tertiary Butyl Alcohol. Acetone-Chloroform.

SOURCE. - From equal weights of Acetone and Chloroform when Caustic Potash is added.

ChARACTERS. - A white crystalline powder having a camphoraceous odor. Solubility. - Sparingly in water; very soluble in Chloroform, Alcohol and Ether.

Dose, 5 to $20 \mathrm{gr}$; .30 to $1.20 \mathrm{gm}$.

\section{Action of Chloretone.}

Without markedly influencing respiration or blood-pressure it produces anæsthesia and sleep. It directly affects sensory nerves, and so may be used as a local anæsthetic. If the dose by the mouth is excessive dogs may sleep for several days, finally succumbing from asphyxia. Since neither acetone nor chloroform are found in the expired air or the urine, and the chlorides are increased in the latter, it is probably broken up in the body. .

\section{Therapeutics of Chloretone.}

In one per cent. solution it may be applied as a local anæsthetic to ulcers and infected wounds. Internally, its chief use is as a hypnotic, which is both safe and generally efficient. In moderate doses it promptly relieves irritability of the stomach.]

\section{V. [ANHYDROGLUCO-CHLORAL.}

CHLORALOSE. Not official. $-\mathrm{C}_{8} \mathrm{H}_{11} \mathrm{Cl}_{3} \mathrm{O}_{6}=259.85$.

SuURCE. - By heating together Anhydrous Chloral and Glucose, the soluble Chloralose is formed which crystallizes. $\mathrm{C}_{2} \mathrm{HCl}_{3} \mathrm{O}+\mathrm{C}_{6} \mathrm{H}_{12} \mathrm{O}_{6}=\mathrm{C}_{8} \mathrm{H}_{11} \mathrm{Cl}_{3} \mathrm{O}_{6}+$ $\mathrm{H}_{2} \mathrm{O}$.

Characters. - In small crystals, having a bitter and disagreeable, but not acrid taste. Solubility. - Freely in hot, slightly in cold water.

Dose, 2 to $4 \mathrm{gr}$; . 12 to $.25 \mathrm{gm}$. 


\section{Action and Therapeutics of Chloralose.}

Chloralose on account of its bitter taste is best given in capsules. It will produce sound sleep in which sensibility is not lost, although the reflex activities are greater than usual, because as, Richet says, it excites the spinal cord while depressing the functions of the brain. The awakening is without unpleasant effects. Ten grains; .60 gm., have produced profound unconsciousness, so that caution should be exercised in prescribing.

\section{AMYLENI HYDRAS.}

AMYLENE HYDRATE. (Not official.) $-\left(\mathrm{CH}_{3}\right)_{2} \mathrm{C}_{2} \mathrm{H}_{5} \mathrm{COH}=87.8 \mathrm{r}$. Synonyms.-Dimethylethylcarbinol. Tertiary Amylic Alcohol.

SOURCE. - By the action of Sulphuric Acid on Amylene, separation of the Amylene-sulphuric Acid, dilution, filtration, neutralization with Milk of Lime or solution of Soda, and fractional distillation.

Characters. - A limpid, colorless, oily liquid, of a peculiar penetrating odor. Sp. gr., o.815. Solubility.-In 8 parts of water; readily in Alcohol, Ether or Chloroform.

Dose, $1 / 2$ to I fl. dr.; 2. to 4 . c.c.

\section{Action and Uses of Amylene Hydrate.}

Amylene hydrate is a hypnotic, about midway in power between chloral and paraldehyde, having a pleasanter taste than the latter. The sleep is generally natural, and the awakening prompt and complete. It is a safe hypnotic, having no action upon the heart or respiration, and it may have also anodyne properties. It can be administered in wine, raspberry syrup, or simply in water. It has been given hypodermatically, with onehalf its volume of alcohol. After continued use it is apt to disagree with the stomach.]

\section{PARALDEHYDUM.}

[PARALDEHYDE. $-\mathrm{C}_{6} \mathrm{H}_{12} \mathrm{O}_{3}=13 \mathrm{I} \cdot 7 \cdot$.]

SoURCE. - A product of the polymerization of [Ethylic] Aldehyde by means of various Acids or Salts. For example, [Ethylic] Aldehyde may be acted on by Hydrochloric Acid, [Carbonyl Chloride $\left(\mathrm{COCl}_{2}\right)$, Sulphur Dioxide] or Zinc Chloride ; during the action, the temperature of the mixture rises, and almost complete conversion into Paraldehyde takes place. On cooling to below $32^{\circ} \mathrm{F}$. [ $\left[0^{\circ} \mathrm{C}\right.$.], Paraldehyde crystallizes. ${ }_{3} \mathrm{C}_{2} \mathrm{H}_{4} \mathrm{O}=\mathrm{C}_{6} \mathrm{H}_{12} \mathrm{O}_{3}$.

Characters. - A colorless [transparent liquid, having a strong, charac- 
teristic, but not unpleasant or pungent odor, and a burning and cooling taste. Solubility. - In 85 parts of water; freely in Alcohol and Ether.]

Dose, $1 / 4$ to $x$ fi. dr. ; [x. to 4 . c.c.]

\section{Action of Paraldehyde.}

External.-It is antiseptic.

Internal.-Large doses increase the flow of urine and somewhat strengthen the heart, but they do not affect the gastro-intestinal tract or respiration; enormous doses of paraldehyde weaken the action of the heart, and kill by paralysis of the respiratory centre.

Nervous system.-It is a powerful hypnotic, without any unpleasant after-effects. It acts quickly, and the sleep, which lasts several hours, is quiet, refreshing, and dreamless. Paraldehyde in toxic doses paralyzes the anterior cornua of the spinal cord ; thus it abolishes reflex action and causes paralysis. It does not affect nerves or muscles.

\section{Therapeutics of Paraldehyde.}

Internal.-It is given solely as an hypnotic in the same class of cases as chloral, and as it does not act on the heart it may also be used for patients suffering from cardiac disease. It has been used largely in asylums to produce quiet in mania, and sleep in melancholia. It may produce an erythematous rash. Unless given in capsules, the great objection to its use is its [disagreeable] taste, which is best covered by prescribing it with syrup and tincture of orange peel in at least two fluid ounces [6o. c.c.] of water to insure that a usual dose will be dissolved. [It can also be administered in glycerin in a 25 per cent. solution, which renders it more palatable.] It gives an extremely offensive and persistent odor to the breath, which lasts many hours. [Instances of the paraldehyde habit have been occasionally reported. There is great emaciation, cardiac weakness, unsteady gait, mental confusion and agitation with hallucinations of sight and hearing and unpleasant delusions. Restraint for several months is necessary for cure.] 


\section{SULPHONALUM.}

SULPHONAL. [B. P., not official. $-\mathrm{C}_{7} \mathrm{H}_{16} \mathrm{~S}_{2} \mathrm{O}_{4}=227.59$. Synonym. -Diethylsulphondimethylmethane. $\left(\mathrm{CH}_{3}\right)_{2} \mathrm{C}\left(\mathrm{SO}_{2} \mathrm{C}_{2} \mathrm{H}_{5}\right)_{2}=227.59$.

SouRCE. - Mercaptan (Ethyl Hydrosulphide) is combined with Acetone to form Mercaptol, which by oxidation with Potassium Permanganate yields Sulphonal.]

Characters. - Colorless, tabular crystals, inodorous, almost tasteless. Solubility. - In 450 parts of cold; in 15 parts of boiling water; in 90 parts of Alcohol or Ether; in 3 parts of Chloroform.

Dose, 15 to $40 \mathrm{gr}$.; [1.00 to $2.40 \mathrm{gm}$.] in cachets or suspended in mucilage.

\section{Action and Therapeutics of Sulphonal.}

Sulphonal is an hypnotic. It does not depress the heart. The drug is given for the same class of cases as chloral, but as it is so insoluble, it is absorbed with difficulty and very slowly; hence it takes two or more hours to act, and its action may be prolonged into the next day. It produces its effect most rapidly if the fluid, in which it is suspended, is hot; but as they are so much more convenient it is usually given in cachets an hour and a half before bedtime. Sulphonal rarely leads to a habit or to any disagreeable after-effects. The symptoms of a sulphonal habit are general lethargy, mental, moral and muscular weakness, loss of nutrition, and dyspepsia. It has been known to produce [persistent] eruptions upon the skin and hæmatoporphyrin in the urine. [Several fatal cases of poisoning by this drug have been reported from small doses continued for long periods. Severe general functional disturbances have followed its use.] Enormous single doses produce, in addition to these symptoms, prolonged sleep, lasting many days, paralysis of sphincters, anuria, a fall of temperature, and, late in the case, depression of respiration.

\section{TRIONALUM.}

[TRIONAL. (Not official.) $-\mathrm{CH}_{3} \mathrm{C}\left(\mathrm{SO}_{2} \mathrm{C}_{2} \mathrm{H}_{5}\right)_{2} \mathrm{C}_{2} \mathrm{H}_{5}=24 \mathrm{I} .56$. Synonym.-Diethylsulphonmethylethylmethane. This contains three Ethyl groups instead of two as does Sulphonal.

SourcE. - It is prepared as in Sulphonal, except that Methylethylketone is used in place of Acetone. 
Characters. - Shining, colorless, crystalline plates, tasteless and odorless. Solubility.-In 320 parts of water; readily in Alcohol and Ether.

Dose, 5 to $30 \mathrm{gr}$. ; 30 to $2.00 \mathrm{gm}$.

\section{Action and Therapeutics of Trional.}

Trional is a prompt hypnotic, without cumulative action, and it has no injurious or unpleasant after-effects. Apparently the patients do not become habituated to its use. It has been used as a hypnotic and sedative for the insane ; for narcotic habitués, so far as is known, it is a safe remedy. It is important the daily action of the bowels be secured, an alkaline water be given daily, and weekly intermissions be insisted upon, otherwise it may give rise to disagreeable after-effects. It but rarely gives rise to hæmatoporphyrinuria.

Tetronal is of similar chemical composition, containing four instead of three ethyl groups, and is used for the same purposes, but in somewhat larger dose.

\section{URETHANUM.}

URETHANE. (Not official.)-CO. $\mathrm{NH}_{2} \cdot \mathrm{OC}_{2} \mathrm{H}_{5}=88.94$. Synonyms. -Ethyl Carbamate. Ethyl Urethane.

SourcE. - By the interaction of Urea Nitrate and Ethyl Alcohol; and crystallization on cooling.

ChARACTERS.-Colorless, columnar or tabular crystals; colorless, and having a pleasant, saline, somewhat cooling taste. Solubility.-In I part of water; 0.6 part of Alcohol ; 1 part of Ether ; 3 parts of Glycerin.

Dose, 5 to $30 \mathrm{gr}$; .03 to $2.00 \mathrm{gm}$.

\section{Action and Uses of URethane.}

Urethane is used as a hypnotic, and is believed to provide a calm, natural sleep without any disagreeable after-effects. It was formerly more frequently employed.]

Class V.-Drugs which have an Antipyretic or Analgesic Action.

\section{[The single official representative is Acetanilid.}

Antipyrin and Phenacetin (B. P.), Exalgin, Lactophenin, Phenocoll Hydrochloride and Thallin Sulphate, none of which are official, belong to this class. Alpha- and Beta-Eucaine, local anæsthetics are considered bere, for convenience]. 


\section{ACETANILIDUM.}

ACETANILID. - $\mathrm{C}_{6} \mathrm{H}_{3} \mathrm{NHC}_{2} \mathrm{H}_{3} \mathrm{O}[=\mathrm{r} 34.73$.$] \quad Synonyms. - Phenyl-$ acetamide. Antifebrin. [An Acetyl derivative of Aniline.]

Source.-Glacial Acetic Acid and [pure] Aniline are heated together [the excess of both ingredients is then distilled off, and the congealed residue is crude Acetanilid, which is purified by repeated crystallization from water.] $\mathrm{C}_{8} \mathrm{H}_{5} \mathrm{NH}_{2}+\mathrm{HC}_{2} \mathrm{H}_{3} \mathrm{O}_{2}=\mathrm{C}_{6} \mathrm{H}_{5} \mathrm{NHC}_{2} \mathrm{H}_{3} \mathrm{O}+\mathrm{H}_{2} \mathrm{O}$.

ChARACTERS. - [White, shining micaceous crystalline laminæ, or a crystalline powder, odorless, having a faintly burning taste, and permanent in the air. Solubility. - In I94 parts of water, and in 5 parts of Alcohol; also soluble in 18 parts of Ether, and easily soluble in Chloroform.

INCOMPaTIBLES.-Potassium and sodium hydrate, and chloroform.

IMPURITIES. - Aniline and its salts.

Dose, 2 to $5 \mathrm{gr}$.; .I 2 to .30 $\mathrm{gm}$.]

\section{Action of Acetanilid.}

External.--It is a local hæmostatic as it contracts bloodvessels when applied to them.

Internal.-Blood.-With ordinary doses of this drug this fluid is unaffected, but in large doses the color is changed, from the formation of methæmoglobin. The passage of this in the urine discolors it. It causes the red corpuscles to break up and arrests the movements of the white.

Heart.-This substance depresses the heart. It is not known how it does this; but what little evidence there is appears to show that it has a directly paralyzing action on the cardiac muscle.

Vessels.-Acetanilid contracts the smaller vessels from direct action on their muscular coat. The blood-pressure therefore rises.

Respiration. - This is not affected by ordinary doses. After toxic doses the force of the respiratory act progressively diminishes.

Kidneys. - This substance is a mild diuretic. The excretion of urea and uric acid is stated to be increased by it. Large doses cause the urine to be dark from the passage of altered blood. Acetanilid is said to be excreted as aniline, but this requires confirmation.

Skin.-This may produce an erythematous rash which usually 
[resembles that of measles] or is urticarial, and it is occasionally a mild diaphoretic.

Temperature.-This substance is a powerful antipyretic. It has a very slight action on the temperature of health, but it reduces it very markedly when it is raised from any cause. It was introduced into medicine for this property. We have already seen (see p. 7I) how numerous are the ways in which antipyretics may act. The fall of temperature produced by this drug is not due to any action on the blood or the circulation, and it is too marked to be entirely owing to its diaphoretic action. It decreases heat production, and it is most likely that it acts directly upon that part of the central nervous system, probably upon the corpora striata, which presides over heat production. It, to a much less extent, increases heat dissipation. The result of these two actions is that the temperature falls. The proof of these statements is too long and complicated to give here, but we may mention that it is easy to show both by a calorimeter, and by the decrease of the products of the febrile destruction of tissue, such as urea, that these drugs diminish heat production. [It also possesses anti-periodic properties.]

Nervous system.-The drug is a powerful analgesic. Acetanilid in large doses is said to produce first convulsions, then coma, and paralysis of motor nerves and muscles; but all these statements require further experiments.

\section{Therapeutics of Acetanilid.}

External.-Acetanilid has been used in form of a dusting powder [for soft and hard venereal ulcerations, in place of iodoform, ] or as an ointment ( $\mathrm{I}$ to 24 ) for chronic ulcers and eczema. [It has also been employed as an antiseptic for wounds. Too large a surface, however, should not be dusted over.]

Internal.-Pyrexia.-Originally this drug was introduced into medicine on account of the property which it has of reducing pyrexia. The opinion is, however, gaining ground that if the temperature is not dangerously high no attempt should be made to reduce it, for probably the raised temperature is an endeavor on the part of the body to defend itself against the micro-organ- 
isms which are the cause of the particular fever from which the patient is suffering; in other words, the pyrexia is a "defensive mechanism." Further, this drug is a cardiac depressant, and therefore unsuitable for patients suffering from fever, and this last consideration makes many physicians prefer to use cold water when the temperature is so high that it, of itself, is dangerous to life. In order to gain a rapid effect, when it has been decided to use an antipyretic this drug is useful. The balance of evidence is that toxic symptoms are more common after acetanilid, which, however, does not keep the temperature down quite so long as [other antipyretics.] It takes about two hours to reduce the pyrexia to its minimum. [Acetanilid] may be given per rectum. [It has no action upon the intestinal tract.]

Analgesic action. - This drug has the property of relieving pain [of neuralgia, sciatica, dysmenorrhœea, locomotor ataxia, migraine, and various headaches.]

[Under the name of Antikamnia a substance has been introduced which is probably a mixture of 20 parts of sodium bicarbonate, 70 of acetanilid and 10 of caffeine. Since acetanilid is a cardiac depressant, the addition of caffeine may be advantageous in some cases. A case of death has been reported, attributed to the ingestion of 24 grains [ $1.5 \circ \mathrm{gm}$.] of this mixture.] Antinervin contains acetanilid, sodium salicylate and potassium bromide.

\section{Toxicology.}

Symptoms. - Acetanilid occasionally produces in man collapse, cyanosis, very slow respiration, a feeble and irregular putse, vomiting, profuse sweating, and profound prostration. [Death has occurred after a dose of $5 \mathrm{gr} . ; .30 \mathrm{gm}$.] It is not known whether these symptoms are due to impurities in the drug.

Treatment.-Stimulation by alcohol and ether, subcutaneously, and by the mouth. Strychnine subcutaneously to stimulate the heart. Oxygen inhalations. Warmth to the feet and body.

\section{ANTIPYRINUM.}

ANTIPYRIN. (Not official.)-Phenazonum, [B. P.]- $\mathrm{C}_{6} \mathrm{H}_{5}\left(\mathrm{CH}_{3}\right)_{2} \mathrm{C}_{3}$ $\mathrm{HN}_{2} \mathrm{O}=[187.65$.$] Synonym.-Phenyldimethylpyrazolone.$

SOURCE. - Aceto-acetic Ether is acted upon by Phenyl-hydrazine, when Phenylmonomethylpyrazolone, Ethyl Alcohol, and water are formed. $\mathrm{CH}_{3}$ $\mathrm{COCH}_{2} \mathrm{COOC}_{2} \mathrm{H}_{5}+\mathrm{H}_{2} \mathrm{NNHC}_{6} \mathrm{H}_{5}=\mathrm{C}_{6} \mathrm{H}_{5}\left(\mathrm{CH}_{3}\right) \mathrm{C}_{3} \mathrm{H}_{2} \mathrm{~N}_{2} \mathrm{O}+\mathrm{C}_{2} \mathrm{H}_{5} \mathrm{OH}+\mathrm{H}_{2} \mathrm{O}$. 
The Monomethyl compound is treated with Methyl Iodide and Methyl Alcohol. $\mathrm{C}_{6} \mathrm{H}_{5}\left(\mathrm{CH}_{3}\right) \mathrm{C}_{3} \mathrm{H}_{2} \mathrm{~N}_{2} \mathrm{O}+\mathrm{CH}_{5} \mathrm{I}=\mathrm{C}_{6} \mathrm{H}_{5}\left(\mathrm{CH}_{3}\right)_{2} \mathrm{C}_{3} \mathrm{HN}_{2} \mathrm{O}+\mathrm{HI}$.

ChARACTERs.-Colorless, odorless, scaly crystals [of a somewhat bitter taste]. Solubility. - Freely in water, Alcohol and Chloroform.

INCOMPATIBLES.-Iron sulphate, iodide, and chloride, copper sulphate, iodine, arsenic iodide, carbolic, hydrocyanic, and nitric acids, potassium permanganate, salicylates, [corrosive mercuric chloride], spirit of nitrous ether ; all preparations of tannin give a white precipitate; chloral hydrate decomposes it unless in dilute solution.

Dose, 3 to $20 \mathrm{gr}$.; [.20 to $1.20 \mathrm{gm}$.]

\section{Action AND Therapeutics of ANTIPYRIN.}

[Antipyrin in small doses may moderately increase arterial pressure by direct stimulation of the heart (Wood); in large doses it is a cardiac depressant, the final fall of blood-pressure being certainly due, at least in part, to a direct action upon the heart.] It also contracts the blood-vessels, being therefore a local hæmostatic. It is a mild diuretic, and is quickly excreted in the urine as antipyrin. [It may produce an erythematous rash. It rapidly reduces an elevated temperature by decreasing heat production, by direct action on the centre which presides over heat production, and to a much less extent increases heat dissipation. In large doses it is said to produce convulsions, later coma, and paralysis of notor nerves and muscles.

Antipyrin is given internally as a powerful antipyretic, in fevers of various kinds. It is also used as a hæmostatic in hæmorrhoids and epistaxis. It has been used with some success in diabetes. It is largely employed as an anti-neuralgic, relieving the pains of locomotor ataxia and other nervous affections, and as anti-rheumatic. It has been highly recommended in chorea and epilepsy. Externally it is supposed to be an antiseptic, but irritation has followed its hypodermatic use. It is a local anæsthetic.

Salipyrin is prepared by the action of antipyrin upon salicylic acid in substance. It is a white, coarsely-crystalline powder with a rather sweetish taste, readily soluble in alcohol, but slightly in water. In chronic articular rheumatism and sciatica it has been successful, but it does not prevent relapses. It has 
been successfully used for spasmodic dysmenorrhœa. The dose is from 6 to $30 \mathrm{gr}$; .40 to $2.00 \mathrm{gm}$.

Iodopyrin or iodantipyrin is supposed to have a hydrogen atom in the phenyl group of antipyrin replaced by iodine. It occurs in colorless, prismatic needles, which are tasteless. It is, with difficulty, soluble in cold water or alcohol, but readily when hot. It causes a fall of temperature and perspiration, but without collapse or shivering. It is doubtful if it has any advantage over antipyrin. The dose is from 2 to $15 \mathrm{gr}$. ;. . I 2 to $\mathrm{I} .00 \mathrm{gm}$.]

\section{TOXICOLOGY.}

Symptoms.-Antipyrin occasionally produces, in man, collapse, cyanosis, very slow respiration, a feeble and irregular pulse, vomiting and profound prostration. It is not known whether these symptoms have been due to impurities in the drug, [but it is quite likely that they have arisen from improper dosage.] Many deaths have been caused by this drug; it has been stated that during one epidemic of influenza in Vienna seventeen persons were killed by it.

Treatment.-Stimulation by Alcohol and Ether subcutaneously and by the mouth; Strychnine subcutaneously to stimulate the beart; warmth to the feet and body.

\section{PHENACETINUM.}

[PHENACETIN.] (Not official.) $-\mathrm{C}_{10} \mathrm{H}_{13} \mathrm{NO}_{2}=[\mathrm{I} 78.63]$. Synonym. -Para-acetphenetidin. $\mathrm{C}_{6} \mathrm{H}_{4} \mathrm{OC}_{2} \mathrm{H}_{5} \mathrm{NHC}_{2} \mathrm{H}_{3} \mathrm{O}=[178.63]$.

Source.-Glacial Acetic Acid is made to act upon paraphenetidin, a product of Paranitrophenol. $\quad\left[\mathrm{C}_{6} \mathrm{H}_{5} \mathrm{OC}_{2} \mathrm{H}_{5} \mathrm{NH}_{2}+\mathrm{HC}_{2} \mathrm{H}_{3} \mathrm{O}_{2}=\mathrm{C}_{6} \mathrm{H}_{4} \mathrm{OC}_{2} \mathrm{H}_{5} \mathrm{NHC}_{2}\right.$ $\mathrm{H}_{3} \mathrm{O}+\mathrm{H}_{2} \mathrm{O}$.]

Characters. - Colorless, tasteless, scaly crystals. Solubility.-Very sparingly ( 1 in 1700 ) in water; in 30 parts of Alcohol ; soluble in Glycerin.

Dose, 5 to $10 \mathrm{gr}$.; [.30 to $.60 \mathrm{gm}$.] (as an antipyretic), in cachets, capsules or suspended.

\section{Action and Therapeutics of Phenacetin.}

[Phenacetin has no action externally nor on the gastro-intestinal tract, and with ordinary doses the blood is unaffected. It slightly depresses the heart, but does not in ordinary doses affect the respiration. It is a mild diuretic, but large doses cause the passage of altered blood. It is a powerful antipyretic by decreasing heat production and slightly increasing heat dissipation. It is a powerful analgesic. It is a valuable remedy for reducing fever, and because it depresses the heart but little, it is safer 
than either antipyrin or acetanilid. It is, however, very insoluble, and slower and less powerful than these remedies, but the effects last longer. As this drug possesses a marked analgesic action, it is to be preferred as a remedy for the relief of pain, as neuralgia, sciatica, locomotor ataxia, migraine and various headaches. For this purpose, the dose of $5 \mathrm{gr}$.; $.30 \mathrm{gm}$., should be administered every hour for three or four hours; this generally gives relief. This drug has been of service in the treatment of epilepsy.

\section{TOXiCOLOGY.}

Symptoms. - This drug sometimes produces severe vomiting, sweating, feeble and rapid pulse, and collapse. Treatment.-Alcoholic stimulation. Strychnine hypodermatically. External warmth.]

\section{EXALGINUM.}

EXALGIN. (Not official.) $-\left[\mathrm{C}_{6} \mathrm{H}_{5} \mathrm{~N}\left(\mathrm{CH}_{3}\right) \mathrm{CH}_{3} \mathrm{CO}=148.70.\right]$ Syno. nym.-Methyl Acetanilid.

[SOURCE.-By warming together Monomethylaniline and Acetyl Chloride.

Characters. - Colorless acicular needles, with a slightly] saline taste. Solubility.-In 60 parts of water; freely in Alcohol.

Dose, $1 / 2$ to $3 \mathrm{gr}$; ; [.03 to $.20 \mathrm{gm}$.]

\section{Action and Uses of Exalgin.}

Exalgin is a powerful analgesic and has been given with success for neuralgia. Often it relieves when many other drugs have failed. Medicinal doses hardly ever cause depression, but very large quantities may be dangerous from their breaking up the blood like acetanilid. It is best dissolved in Tinctura Aurantii [Dulcis], but may be made into a pill. [It is used for migraine, sciatica, the pains of rheumatism, and, of late, for chorea.

\section{TOXICOLOGY.}

Several severe cases of poisoning having been reported; the usual dose should not be exceeded. The symptoms are similar to those of acetanilid.

Treatment. - As for acetanilid. (See p. 32r.)

\section{LACTOPHENINUM.}

LACTOPHENIN. (Not official.) $-\mathrm{C}_{11} \mathrm{H}_{15} \mathrm{NO}_{3}=208.56$. Synonym.Lactylparaphenetidine. $\mathrm{C}_{6} \mathrm{H}_{4} \mathrm{OC}_{2} \mathrm{H}_{5} \mathrm{NHCOCH}(\mathrm{OH}) \mathrm{CH}_{3}=208.56$. 
SovrCE. - Lactophenin differs from Phenacetin only by the substitution of Lactic for Acetic Acid.

Characters. - A white, tasteless powder. Solubility. - In $33^{\circ}$ parts of water.

Dose, 10 to $15 \mathrm{gr}$; .60 to $1.00 \mathrm{gm}$.

\section{Action and Uses of Lactophenin.}

Lactophenin is an analgesic and antithermic. It is usually better borne than antipyrin. Although it may, in some instances, give rise to sweating, it does not cause collapse nor cyanosis. It produces a considerable and persistent lowering of a febrile temperature, but without abundant perspiration, and its use is not followed by chilly sensations. It has been administered in articular rheumatism, influenza, scarlet fever, septicæmia and other infectious diseases. Von Jaksch has obtained excellent results in typhoid fever, with daily doses from 7 to $15 \mathrm{gr}$.; .50 to I. $\circ 0 \mathrm{gm}$., not only in reducing the fever, but as a sedative when delirium becomes a prominent symptom.

\section{PHENOCOLLI HYDROCHLORIDUM.}

PHENOCOLL HYDROCHLORIDE. (Not official.) $-\mathrm{C}_{10} \mathrm{H}_{14} \mathrm{~N}_{2} \mathrm{O}_{2}$ $\mathrm{HCl}=230.01$.

Source.-By the intermixture of Phenetidine with Glycocoll or Amidoacetic Acid.

Characters. - It is a white micro-crystalline powder. Soluoizizity.-In 20 parts of water.

Dose, 5 to $30 \mathrm{gr}$.; .30 to $2.00 \mathrm{gm}$.

\section{Action and Uses of Phenocoll Hydrochloride.}

It is not poisonous to animals and nor does it injuriously affect the blood. It is a fairly powerful antipyretic, not followed by collapse or cyanosis; the perspiration is not more marked than after large doses of antipyrin. It has also been used as an antineuralgic; in severe acute articular rheumatism it has exercised a beneficial action upon the joints when other remedies have failed. It is rapidly excreted by the urine, to which it gives a brownish color, and it probably increases the excretion of uric acid. The reports, of which there are now a considerable number, are favorable to this remedy. 


\section{THALLIN IE SULPHAS.}

THALLINE SULPHATE. (Not official.) $-2 \mathrm{C}_{9} \mathrm{H}_{10} \mathrm{~N}\left(\mathrm{OCH}_{3}\right) \mathrm{H}_{2} \mathrm{SO}_{4}$ $+2 \mathrm{H}_{2} \mathrm{O}=459.06$.

SourcE.-By heating together Paramidoanisol, Paranitranisol, Glycerin and Sulphuric Acid.

ChARACTERS. - A white or whitish granular crystalline powder having a slightly Anise-like odor and a nauseous, bitter, saline and pungent taste. Solubility. -In 7 parts of water; in 100 parts of Alcohol.

Dose, 2 to ro $\mathrm{gr}$; ; .12 to $.60 \mathrm{gm}$.

\section{Action and Uses of Thallin Sulphate.}

This drug was introduced into medicine as an antipyretic, but it was soon abandoned because of the dangerous collapse with marked cyanosis which it produced. It is rarely given internally, because it is poisonous to the red blood corpuscles and to the nervous system. Its chief use is as an injection for gonorrhœa, in aqueous solution ( $\mathrm{I}$ or 2 to $\mathbf{I} 20$ ), or it can be used in a 2 per cent. solution in gelatin bougies. In gleet beneficial results have followed the injection of $\mathrm{I}$ to 8 aqueous solutions.

\section{ALPHA-EUCAINE HYDROCHLORAS.}

ALPHA-EUCAINE HYDROCHLORATE, (Not official.)$\mathrm{C}_{19} \mathrm{H}_{27} \mathrm{NO}_{4} \mathrm{HCl}+\mathrm{H}_{2} \mathrm{O}=386.6 \mathrm{r}$. The hydrochlorate of a synthetic alkaloid having a close chemical relationship to cocaine.

SOURCE. - By the action of one molecule of ammonia upon three molecules of acetone, triacetonamine is formed. This is then transformed into triacetonamencyanhydrine by hydrocyanic acid. This compound when saponified becomes triacetonalkamin carbonic acid, ammonia being given off, when benzylated and methylated trimethylbenzoyltetramethyl-y-oxypiperidin carbonic acid-methylester or Eucaine is formed.

Char acters. - Permanent shining scales containing one molecule of water of crystallization. Solubility. - In about 6 parts of water.

INCOMPATIBLES.-Caustic alkalies, alkaline carbonates and ammonia.

\section{Action of Eucaine Hydrochlorate.}

The general action of eucaine, both in cold and warm blooded animals, consists in a marked excitation of the entire central nervous system, followed by paralysis; in toxic doses going on to death. Small doses administered to mice and rabbits cause increased reflex excitability, and increased but weakened respira- 
tory movements. Medium doses in rabbits cause repeated tonic and clonic convulsions. The animals lie senseless on their sides, with dyspnœa, opisthotonos, and finally paresis of the posterior limbs. These phenomena are most marked when large toxic doses are administered; the convulsions return continuously, and affect all the muscles of the body. The animals finally die when the paralysis reaches the respiratory muscles. When the dose is not a fatal one, the convulsions gradually cease, the increased reflex excitability disappears, and the paresis of the hind limbs slowly improves. The effect on the central nervous system is therefore at first excitant, and later, in toxic doses, paralyzing. The paralysis is a central one, for if the sciatic nerve of a frog poisoned with eucaine is exposed, and its peripheral end irritated with the induced current, the limb reacts in a normal manner. As regards its action on the heart and the blood-vessels, the subcutaneous and intravenous injection of smail and medium doses slows the heart on the average from twenty to thirty beats per minute, but without otherwise modifying the beats, or increasing the blood pressure. This effect on the pulse is caused by the excitation of the central vagus; for section of the vagi causes an immediate increase of the pulse to the normal and above it, together with an increase of the blood pressure. . Death occurs from paralysis of the respiratory centres, for the heart continues to beat for some time thereafter (Vinci).

\section{Therapeutics of Eucaine Hydrochlorate.}

Eucaine is used in from $I$ to 5 or even ro per cent. solutions for the same purposes as is cocaine. The anæsthesia comes on somewhat more slowly, with solutions of the same strength is about equal to, and its effects last about the same time as with the latter drug. It possesses the disadvantage of causing hyperæmia of mucous membranes, and in 2 per cent. solution may irritate the conjuctiva. A I per cent. solution, however, does not cause any disturbance. It is preferable to cocaine in that its aqueous solutions are permanent and can be sterilized by heat without decomposition. It does not cause mydriasis nor disturbance of accommodation, nor does it dry the corneal epithe- 
lium, and further it is relatively safer, so far as circulation and respiration are concerned, than cocaine. Vinci claims that its solutions possess moderate antibacterial powers.

In order to avoid the burning sensations, pain and hyperæmia to which eucaine may give rise, a substance known as Benzoylvinyldiacetonalkamin hydrochlorate named Beta-eucaine (not official), a compound closely related chemically to eucaine, has been recommended. Its chemical and physiological properties, with the above exceptions, are the same. It is safe, being three and three-quarters less toxic than cocaine, does not affect the heart, and is unirritating. It does not produce, when employed in the eye, mydriasis, corneal lesions, nor disturbances of accommodation. It can be sterilized by boiling without deterioration; its solutions are permanent and do not decompose when kept. Its field is the same as that of cocaine, and it can be employed for the various operations upon the eye, nose, ears, genito-urinary tract, in minor surgery and dentistry, and for infiltration anæsthesia. For medullary anæsthesia while the aftereffects seem no greater than with cocaine the analgesia is not so uniform nor lasting. Its ease and certainty of sterilization by boiling are in its favor and some.operators are strong advocates of it. It is employed in from $1 / 2$ to 4 per cent. (saturated) aqueous solution, but of the latter not more than 30 minims; 2.00 c.c., should be employed at one time, although for a prolonged operation five times this quantity may be employed].

\section{Class VI.-The Antiseptics.}

\section{Carbolic Acid, Sodium Sulphocarbolate, Creosote, Iodoform, Naphtalin, Naphtol and Resorcin.}

[Besides these the following drugs which are not official are included in this class : Zinc Sulphocarbolate (B. P.), Guaiacol, Aristol, Iodol, Europhen, Creolin, Lysol, Asaprol, Formaldehyde, Urotropin, Saccharin, Methylene Blue and Piperazine.]

\section{I. [ACIDUM CARBOLICUM CRUDUM.}

\section{CRUDE CARBOLIC ACID.}

SOURCE. - A liquid consisting of various constituents of coal-tar, chiefly cresol and phenol, obtained by fractional distillation between the temperatures 
of $302^{\circ}$ and $392^{\circ} \mathrm{F} . ; 150^{\circ}$ and $200^{\circ} \mathrm{C}$, , and twice rectified between the temperatures of $33^{\circ}$ and $374^{\circ} \mathrm{F}$.; $170^{\circ}$ to $190^{\circ} \mathrm{C}$.

Characters. - A nearly colorless or reddish, or brownish-red liquid of a strongly empyreumatic and creosote-like odor, having a benumbing, blanching and caustic effect upon the skin and mucous membrane, and a slightly acid reaction.

Used only externally.]

\section{ACIDUM CARBOLICUM.}

CARBOLIC ACID.- $\mathrm{C}_{6} \mathrm{H}_{5} \mathrm{OH}[=93.78$.$] Synonyms.-Phenic Acid.$ Phenol. Phenyl Alcohol.

Source.-[From Crude Carbolic Acid by agitation with warm concentrated solution of Soda, heating to $33^{\circ} \mathrm{F}$.; $170^{\circ} \mathrm{C}$., solution and treatment with Hydrochloric Acid. After being agitated with Sodium Chloride, digested with Calcium Chloride, it is distilled between $336^{\circ}$ and $374^{\circ} \mathrm{F}$.; $168.8^{\circ}$ and $190^{\circ} \mathrm{C}$, , and crystallized.

ChARACTERS. - Colorless, interlaced or separate, needle-shaped crystals, or a white crystalline mass, sometimes acquiring a reddish tint, having a somewhat aromatic odor, and, when copiously diluted with water, a sweetish taste with a slightly burning after-taste. Treated with about 8 per cent. of water, it becomes fluid ; the crystals are very hygroscopic, and hence soon become semifluid on exposure to air ; it has a faintly acid reaction and coagulates albumin. Solubility. -Slowly in about 15 to 20 parts of water;] freely in Alcohol, fats and oils. [Solution in water is more easily made if an equal part of Glycerin is added.]

Dose, $1 / 2$ to I gr. ; [.03 to .06 $\mathrm{gm}$.

\section{Preparations.}

I. Unguentum Acidi Carbolici.-[Ointment of Carbolic Acid. Carbolic Acid, 5 ; Ointment, 95.

2. Glyceritum Acidi Carbolici._Glycerite of Carbolic Acid. Carbolic Acid, 20 ; Glycerin, 80.

Dose, 2 to $5 \mathrm{~m}$.; .12 to .30 c.c.]

\section{Action of Carbolic Acid.}

External.-Carbolic acid is a powerful antizymotic, rapidly destroying organized ferments, both animal and vegetable. Consequently it destroys those of septic diseases, hence it is antiseptic. It thus prevents the formation of the products of the decompositions which are set up by these organisms. For this reason it is disinfectant, and as the products of decomposition are generally foul-smelling, it is deodorant. It does not act so readily on unorganized ferments (enzymes), such as pepsin 
and ptyalin, but in large doses it likewise destroys their activity. Carbolic acid is not so powerful an antizymotic as [corrosive mercuric] chloride (see p. 214); for Evans found that anthrax spores were not killed in twenty-four hours by a solution of $I$ in I 00 , but were killed by a solution of $I$ in 20 acting for twentyfour hours, but not when it acted for only four hours. The bacilli of anthrax were killed by a solution of $I$ in 100 acting for five minutes, I in 150 acting for a quarter of an hour, I in 175 acting for half an hour, but were unaffected by a solution of $I$ in $I 5^{\circ}$ acting for one minute, $I$ in 175 acting for a quarter of an hour, $I$ in 300 acting for an hour. Strengths of $I$ in 40 and I in 20 are commonly employed in surgery. The solution in oil has no antiseptic properties. The power of carbolic acid to destroy low organisms makes it an efficient parasiticide against certain vegetable parasites infesting the skin.

When applied to the skin in weak or moderately strong solutions, it produces local anæsthesia with a feeling of numbness which lasts some hours. If concentrated, it acts as an irritant and caustic, causing a burning pain, and in a few minutes a white spot appears, which becomes red when the acid is removed. If the application is prolonged, a white eschar or slough results. There is no vesication.

Internal.-Gastro-intestinal tract.-If concenträted, carbolic acid produces the same effect on the mouth as on the skin, and is a powerful gastro-intestinal irritant (see Toxicology). In the stomach it is converted into a sulphocarbolate, and unless poisonous doses be given, it is so diluted by the gastric contents that it loses its antizymotic power.

Blood.-It is not known in what form carbolic acid circulates, probably as an alkaline carbolate.

Circulation.-Medicinal doses have no effect. Large doses paralyze the vaso-motor centre in the medulla, and the bloodpressure falls. It is not until very large doses have been given that the heart is affected, and then its activity is depressed.

Respiration.-Small doses have no infuence on respiration, but large ones accelerate it, probably from stimulation of the vagi. Ultimately respiration is paralyzed, and death results. 
Temperature. - This is unaffected by small doses of carbolic acid, but large doses cause it to fall, because they diminish the production of heat and increase its dissipation.

Nervous system.-Carbolic acid is a cerebral depressant in large doses, for coma is produced by them; they first stimulate the anterior cornua, producing convulsions, but subsequently depress them, causing paralysis.

Urine.-Much interest attaches to this, for even after moderate doses of carbolic acid, or absorption from surgical dressings, the urine may become dark. This is not due to blood, as was once thought, for Stevenson has shown that there is no increase of iron in the urine. Carbolic acid [is partially oxidized to pyrocatechin and hydroquinone, which combine in the body with sulphuric and glycuronic acids and are excreted in the urine as double (ethereal) sulphates and phenol, pyrocatechin and hydroquinone glycuronates.] Pyrocatechin [and hydroquinone are unstable bodies, and their oxidation products are doubtless] the cause of the dark urine; pyrocatechin can only exist in alkaline urine, [so that it cannot be the sole cause of the dark color.] The presence in the urine of these results of carbolic acid is recognized by distilling them over from it. The distillate gives a blue color with neutral ferric chloride, and a white crystalline precipitate of tribromophenol with bromine water, showing the presence of sulphocarbolic acid. [The inorganic sulphates are usually absent. This is determined by the use of the barium chloride test which does not precipitate the combined sulphates (sulphocarbolates). (Sonnenberg's test).] Some carbolic acid escapes in the other excretions; some is burned up in the body. When very large doses are given carbolic acid itself may appear in the urine.

\section{Therapeutics of Carbolic Acid.}

External.-Carbolic acid is largely used as a deodorant and disinfectant for drains, bed-pans, [for which the cheaper crude acid may be employed,] soiled linen, surgical instruments, the surgeon's hands, etc. Carbolic lotion ( $\mathrm{I}$ in 40 ) is used to wash wounds to keep them antiseptic, and carbolized gauze (which is 
bleached cotton gauze medicated with half its weight of a mixture of carbolic acid, $\mathrm{I}$; resin, 4 ; paraffin, 4 , ) is employed as a dressing for the same purpose. A spray of a solution of carbolic acid was formerly much used to keep the air around the wound antiseptic during an operation, but it is now discarded as unnecessary.

Glycerite of carbolic acid is a very efficient preparation to destroy the fungus of tinea tonsurans or tinea versicolor ; for the latter it should be diluted.

Because of its anæsthetic effect a strong solution ( $\mathrm{I}$ in 20) will relieve itching from any cause. Carbolized vapor has been inhaled in phthisis, but by the time it reaches the lungs it is far too dilute to have any action on the tubercle bacilli.

Internal.-Mouth. - The glycerite, if diluted, may be applied as a stimulant to the mouth in aphthous stomatitis, or when any indolent ulceration is present. A gargle (of [carbolic acid] in water, $I$ in $\mathbf{2} 20$ ) is an excellent preparation. The glycerite has been used for diphtheria, but probably it does no good, except that being a local anæsthetic it soothes pain. A piece of cotton soaked in strong carbolic acid will relieve pain if placed in a decayed tooth, but care must be taken to prevent it from coming in contact with the soft parts by putting another piece of dry cotton over it.

Stomach._Carbolic acid has been given to relieve flatulence, because it was thought that it would prevent decomposition in the stomach ; but it is powerless to do this, owing to the degree to which the gastric contents dilute it. Some state that it checks vomiting and helps to cure dyspepsia, but it is not a remedy which is universally regarded as useful for these purposes. It may, however, be tried in obstinate cases, and it will sometimes be found to be a good carminative. It has been given internally as an antiseptic in phthisis, but it does no good, and those who give it forget that probably very little carbolic acid reaches the lungs. It has been extensively tried in typhoid fever, but without any good effect.

\section{Toxicology.}

If carbolic acid is at all concentrated, immediately on swallowing it there is an intense burning sensation in the mouth, [œsophagus] and stomach, and 
white eschars form in the mouth. The patient is collapsed, his skin is cold and clammy. The breathing becomes more and more feeble and shallow, and finally stops. The urine is darkish green. Reflex movements are abolished, and ultimately he becomes insensible and comatose. [Carbolic acid taken by the mouth has proven fatal in two ninutes.] Post-mortem. - There are white, hard sloughs, with perhaps inflammatory redness round them, in the mouth, œsophagus, and stomach. The blood is dark and coagulates imperfectly. In some cases fatty degeneration of the liver and kidneys may be found.

Treatment.-Any soluble sulphate, such as an ounce [30. gm.] of magnesium sulphate or half an ounce [15. gm.] of sodium sulphate dissolved in half a pint [250. c.c.] of water, is the natural antidote, because sulphates and carbolic acid form sulphocarbolates in the blood, and these are harmless. [Saccharated lime or soap may be used as chemical antidotes.] Before the antidote is given, wash out the stomach or use some very quickly-acting emetic, as apomorphine [hydrochlorate] given hypodermatically. [It is of the utmost importance to immediately] give stimulants freely, such as ether or orandy subcutaneously. Apply hot water bottles and blankets if there are any signs of collapse. [The most important antidote to carbolic acid is pure alcohol. Success in treatment demands that the acid and alcohol should be brought in contact; therefore if the acid has been swallowed for some time alcohol måy not be efficacious.]

\section{SODII SULPHOCARBOLAS.}

SODIUM SULPHOCARBOLATE.- $\left[\mathrm{NaSO}_{3} \mathrm{C}_{6} \mathrm{H}_{4}(\mathrm{OH})+2 \mathrm{H}_{2} \mathrm{O}=\right.$ 231.56. Synonym.-Sodium Paraphenolsulphonate.

SOURCE.-Phenolsulphuric Acid is formed by adding Sulphuric Acid to crystallized Carbolic Acid; on heating this mixture it becomes Paraphenolsulphuric Acid, which yields a clear solution with water. $\mathrm{C}_{6} \mathrm{H}_{5} \mathrm{OH}+\mathrm{H}_{2} \mathrm{SO}_{4}$ $=\mathrm{C}_{6} \mathrm{H}_{5} \mathrm{HSO}_{4}+\mathrm{H}_{2} \mathrm{O}$. Barium Carbonate is then added, and Barium Sulphocarbolate is precipitated. $\quad 2 \mathrm{C}_{6} \mathrm{H}_{5} \mathrm{HSO}_{4}+\mathrm{BaCO}_{3}=\mathrm{Ba}\left(\mathrm{SO}_{3} \mathrm{C}_{6} \mathrm{H}_{4}(\mathrm{OH})\right)_{2}+\mathrm{H}_{2} \mathrm{O}$ $+\mathrm{CO}_{2}$. This is treated with water and Sodium Carbonate; a solution of Sodium Sulphocarbolate is formed, and Barium Carbonate is precipitated. $\mathrm{Ba}\left(\mathrm{SO}_{3} \mathrm{C}_{6} \mathrm{H}_{4}(\mathrm{OH})\right)_{2}+\mathrm{Na}_{2} \mathrm{CO}_{3}=2 \mathrm{NaSO}_{3} \mathrm{C}_{6} \mathrm{H}_{4}(\mathrm{OH})+\mathrm{BaCO}_{3}$. The solution is evaporated to crystallization.

Characters. - Colorless, transparent, rhombic prisms, odorless and having a cooling, saline, slightly bitter taste. Solubility.-In 4.8 parts of water; in 132 parts of Alcohol.]

Dose, 5 to $30 \mathrm{gr}$.; [.30 to $2.00 \mathrm{gm}$. ]

\section{Action and Therapeutics of Sodium Sulphocarbolate.}

This substance is antiseptic, like carbolic acid, and may be used externally for this purpose. Internally it is occasionally given in the hope of controlling gastric fermentation. 


\section{ZINCI SULPHOCARBOLAS.}

IV. ZINC SULPHOCARBOLATE.-[B. P., not official.] Zn $\left(\mathrm{C}_{6} \mathrm{H}_{5} \mathrm{SO}_{4}\right)_{2}+\mathrm{H}_{2} \mathrm{O}=[448.62$. Synonym. - Zinc Paraphenol Sulphonate. $]$

SourCE. - Sulphocarbolic Acid is formed by adding Sulphuric Acid to Carbolic Acid. This is treated with Zinc Oxide; the Zinc Sulphocarbolate crystallizes out on evaporation.

Characters.-Colorless crystals, freely soluble in water.

\section{Action and Therapeutics of Zinc Sulphocarbolate.}

Zinc sulphocarbolate is, like carbolic acid, an antiseptic, and may be used externally for that purpose. [It is used as an astringent for indolent or foul ulcers, and in subacute inflammations of mucous membrane, in solutions which are somewhat stronger than those of zinc sulphate.] It is not given internally.

\section{CREOSOTE.}

\section{CREOSOTUM.}

SourCE.- - A mixture of Phenols, chiefly Guaiacol $\left(\mathrm{C}_{7} \mathrm{H}_{8} \mathrm{O}_{2}\right)$ and Creosol $\left(\mathrm{C}_{8} \mathrm{H}_{10} \mathrm{O}_{2}\right)$ obtained during the distillation of wood tar, preferably of that derived from the beech Fagus Sylvatica Linné (nat. ord. Cupulifera.)

Characters. - An almost colorless, slightly yellowish or pinkish, highly refractive, oily liquid, having a penetrating smoky odor, and a burning caustic taste, usually becoming darker in tint on exposure to light. Sp. gr., not below 1.070. Solubility. - In I 50 parts of water; freely in Alcohol, Ether, Chloroform and glacial Acetic Acid.]

IMPURITY.-Carbolic Acid [which coagulates albumin and collodion. Creosote does not].

INCOMPATIBLE. - Explodes when mixed with Silver oxide.

Dose, $[1 / 2$ to $2 \mathrm{~m}$; .03 to .12 c.c.

\section{Preparation.}

Aqua Creosoti.-Creosote Water. Creosote, Io; distilled water, 990.

Dose, I to 4 fi. dr.; 4 . to I5. c.c.]

\section{Action and Therapeutics of Creosote.}

Creosote has the same action as carbolic acid, and before that was introduced, creosote was used externally as a stimulating antiseptic, a parasiticide and a slight local anæsthetic ; and internally it was given for vomiting and flatulence. An aching tooth may be relieved if it is plugged with cotton thoroughly moistened 
in creosote. [The most important use of creosote is as a pulmonary antiseptic, administered by the mouth, hypodermatically, or by inhalation. To Bouchard and Gimbert belongs the credit of introducing the use of creosote in cases of tuberculosis. It can be administered in the form of an emulsion with cod-liver oil and acacia; or with the hypophosphites and cod-liver oil; or with the syrup of wild cherry and acacia, two minims ; . I 2 c.c.; of the creosote being contained in a drachm; 4 c.c. of the emulsion, or in a mixture of glycerin and whiskey. The dose should be one-half to two minims; .03 to .1 2 c.c., given thrice daily, and increased to twenty to twenty-five minims; 1.20 to I. 50 c.c. in the twenty-four hours, by easy stages. Administered in the form of enteric pills (which will dissolve only in the intestinal fluids), a daily dosage of forty-five to fifty minims; 3.00 to 3.30 c.c. can be reached without inconvenience. This method is preferable to that of Sommerbrodt, which consists in the administration of one minim; .06 c.c. of creosote in two minims; 12 c.c. of cod-liver oil, in capsules. The method of hypodermatic injection in sterilized olive oil requires a special apparatus, is very tedious, somewhat painful, and altogether irksome to patient and physician. By inhalation it is employed with equal parts of alcohol and spirit of chloroform, or in alcohol, one part to eight, in a perforated zinc inhaler, of which ${ }_{5} 5$ minims; r. c.c., are placed upon a bit of cotton and used for fifteen minutes in every hour. If the best beechwood creosote is employed, no untoward results are likely to be obtained. If the dose is increased too rapidly there may occur some nausea, epigastric uneasiness, and even vomiting. Disturbance of the kidneys has been produced and the urine presents practically the same appearance as after the injection of carbolic acid (see p. 331). The stomach symptoms have been relieved by the patient placing himself upon his back, for half an hour after administration of the remedy. It is quite likely that the patient acquires a tolerance, for the daily dose of three hundred minims; 20. c.c. has been given for a considerable time, with benefit, although fifty minims; 3.30 c.c. should be considered as the maximum daily dose. Creosote is more efficient than either of 
its principal constituents, guaiacol or creosol, even if given in proportionate dose. Creosote should never be given to the aged.

Creosote carbonate (not official) which contains 92 per cent. of creosote, does not possess the caustic and irritative properties of the pure creosote, and can be administered in dose of from 15 to $60 \mathrm{~m}$. ; I. to 4 . c.c., in a wineglass of sherry after meals. As it is slowly absorbed it is probable that it is eliminated in greater part by the bronchial mucous membrane. Since it does not irritate the gastro-intestinal tract nor the kidneys it is the method of choice in the treatment of pulmonary tuberculosis.]

\section{VI. [GUAIACOLUM.}

GUAIACOL. (Not official.) $-\mathrm{C}_{6} \mathrm{H}_{4} \mathrm{OHOCH}_{3}=123.7 \mathrm{I}$.] Synonym.Methyl Pyrocatechin. [A liquid constituting from 60 to 90 per cent. of Creosote, which is mainly composed of this and Creosol.

SOURCE.-By fractional distillation of Beech-wood Tar; treated with Ammonia to remove acid compounds, and then again fractionated.

ChARACTERS. - A colorless, highly refractive liquid, of a strongly aromatic odor. Sp. gr., I.II7. Solubility.-Very slightly in water, readily in Alcohol and Ether.

Dose, 2 to $10 \mathrm{~m} . ; .12$ to .60 c.c.

\section{GUAIACOLI BENZOAS.}

GUAIACOL BENZOATE. (Not official.) $-\mathrm{C}_{6} \mathrm{H}_{4} \mathrm{OHC}_{6} \mathrm{HC}_{5} \mathrm{O}_{2}=$ 203.49. Synonyms. - Benzosol. Benzoyl Guaiacol.

SouRCE. - This is prepared by adding to an alcoholic solution of Guaiacol, Potassium Hydroxide, forming Potassium Guaiacol, which is heated in a water-bath with Benzoyl Chloride. Benzoyl Guaiacol is formed and purified by re-crystallization from Alcohol.

ChARACTERS.-A colorless, odorless and tasteless crystalline powder. Solubility. -Almost insoluble in water; readily soluble in Ether and Chloroform.

Dose, 2 to $10 \mathrm{gr}$; . 12 to $.60 \mathrm{gm}$.

\section{GUAIACOLI CARBONAS.}

GUAIACOL CARBONATE. (Not official.) $-\left(\mathrm{C}_{6} \mathrm{H}_{4} \mathrm{OCH}_{3}\right)_{2} \mathrm{CO}_{2}=$ 257.39 .

SoURCE. - By passing Phosgene gas $\left(\mathrm{COCl}_{2}\right)$ into Guaiacol, previously dissolved in a Soda solution. The Carbonate is obtained by crystallization.

ChARACTERs. - A white, neutral crystalline powder, almost odorless and tasteless. Solubility.-Insoluble in water; soluble in Ether and Chloroform.

Dose, 5 to $30 \mathrm{gr}$; . 30 to $2.00 \mathrm{gm}$. 


\section{GUAIACOLI SALICYLAS.}

GUAIACOL SALICYLATE. (Not official.) $-\mathrm{C}_{6} \mathrm{H}_{4} \mathrm{OHCO}_{2} \mathrm{C}_{6} \mathrm{H}_{4}$ $\mathrm{OCH}_{3}=243.42$.

Source.-By the action of Phosphorus Oxychloride on a mixture of Sodium Guaiacol and Salicylate.

Characters. - A white crystalline, odorless and tasteless powder. Soluoility. - Insoluble in water; soluble in Alcohol, Ether and Cbloroform.

Dose, 5 to $30 \mathrm{gr}$; .30 to $2.00 \mathrm{gm}$.]

\section{Action and Uses of Guaiacol.}

External.-Guaiacol is antiseptic. If painted on the skin over an area of from 4 to 20 square inches [10. to $50 . \mathrm{sq} . \mathrm{cm}$.$] ,$ it reduces pyrexia, but it is not used for this purpose as it causes sweating and collapse.

Internal.- [ It is less likely than creosote to irritate the intestinal canal and kidneys. Benzosol was introduced as a nearly tasteless combination for the administration of guaiacol. In the digestive tract it splits up into guaiacol and benzoic acid. As an intestinal disinfectant it has proved to be of service in the treatment of diabetes mellitus. The carbonate and salicylate have been used as substitutes for guaiacol, and are in many cases preferable to it. Guaiacol especially the carbonate] has been much given in phthisis, for it is believed to aid the destruction of the bacilli in the lungs, but although widely used there is no [absolutely] certain evidence that it [does this. The carbonate has given excellent results in the treatment of typhoid fever in limiting the decomposition in the intestines].

\section{CREOLINUM.}

[CREOLIN. (Not official.)-A solution of the higher homologues of Phenol.

SoURCE.-Creolin is derived from coal by dry distillation, and consists of a mixture of the sodium salts of some resinous acids with Creolin Oil and Pyridines.

Characters. - A dark brown, syrupy alkaline liquid. It forms a turbid, milky mixture with water, which has the characteristic, rather pleasant, odor of the preparation.

Dose, I to $5 \mathrm{~m}$.; .06 to .30 c.c. 


\section{Action and Uses of Creolin.}

Creolin is a non-irritating but powerful antiseptic, frequently used in place of carbolic acid. It is used pure, in 2 per cent. solution, in an ointment or as a soap, ro per cent. It has been given internally in gastric fermentation, dysentery and typhoid fever. Toxic symptoms have been observed, but they are rarely encountered.] Jeyes' disinfectant preparations contain creolin.

\section{LYSOLUM.}

[LYSOL. (Not official.)-A substance containing about 50 per cent. of Cresol.

SOURCE. - From tar oil by dissolving in fat and saponifying with Alcohol.

Characters. - A brown, oily-looking clear liquid, with a feeble aromatic odor. Solubility. - Soluble in all proportions in water (forming a clear, frothing, saponaceous liquid), in Alcohol, Chloroform and Glycerin.

\section{Action AND Uses of Lysol.}

Lysol is an antiseptic, about one-eighth as poisonous as car. bolic acid, and even less poisonous than creolin, used in from one-half to two per cent. aqueous solution. The literature is extensive and generally favorable.] Lysol does not affect instruments but may make them difficult to hold, as it is a solution of tar oils in a neutral soap. Izal is a coal tar derivative [chiefly used in England, possessing similar properties and used for the same purposes.]

\section{IODOFORMUM.}

IODOFORM. $-\mathrm{CHI}_{3}[=392.56$.$] Synonym. - Tri-iodomethane.$

Source. - Heat together Alcohol, Iodine, Potassium Bicarbonate, and water. $\mathrm{C}_{2} \mathrm{H}_{6} \mathrm{O}+4 \mathrm{I}+2 \mathrm{KHCO}_{3}=2 \mathrm{CHI}_{3}+2 \mathrm{KI}+3 \mathrm{H}_{2} \mathrm{O}+2 \mathrm{CO}_{2}$.

Characters. - [Small lemon-yellow, lustrous crystals of the hexagonal system, having a peculiar, very penetrating and persistent odor somewhat resembling that of Saffron and Iodine, and an unpleasant, slightly sweetish, and Iodine-like taste. Solubilitj.-Very slightly in water; soluble in 52 parts of Alcohol; freely in fixed and Volatile Oils, Ether and Chloroform.] It con. tains 96.7 per cent. of Iodine.

Dose, $\mathrm{I}$ to $3 \mathrm{gr}$.; [.06 to .20 $\mathrm{gm}$.]

\section{Preparation.}

Unguientum Iodoformi.-[Iodoform Ointment. Iodoform, IO; Benzoinated Lard, 90.] 


\section{Action of IODOForm.}

External.-Iodoform is [anæsthetic], antiseptic and disinfectant, if we may judge by the results obtained in clinical practice; but the experimental evidence that it has no power to hinder the development of Staphylococcus pyogenes, Bacillus subtilis, and other micro-organisms, is very strong, for all, except one or two experimenters state that it has no antiseptic properties. The reason for these discrepancies is this : Iodoform only acts as an antiseptic after its decomposition, which results in the liberation of free iodine. The fats always present in tissues dissolve it. When dissolved it is easily decomposed by many agents, such as light, oxygen, living cells, or ptomaines, which would have no effect on it if it were undissolved. By one or more of these it is, when dissolved after [having been] dusted upon a wound, slowly decomposed. Iodine is thus set free rapidly enough to act as an antiseptic, but not rapidly enough to act as an irritant.

Internal. - Not much is known about the internal action of iodoform. It is eliminated in all the secretions, but chiefly in the urine, as iodine, iodides, and iodates. They may be found in the urine for three days after administration of iodoform.

\section{THERAPEUTICS OF IODOFORM.}

External.-lodoform is much used as a local stimulant, antiseptic and disinfectant. The clinical testimony as to its value is overwhelming. [Before using, since iodoform itself, as above explained, is not antiseptic, it must be disinfected by washing in a I to 2000 solution of corrosive mercuric chloride and preserved while damp, in closed, sterile jars.]

Its anæsthetic influence diminishes the pain, if there is any, of the sores to which it is applied. It is an excellent application for all sorts of ulcers, sores and wounds, but especially for tuberculous and syphilitic ulcerations and chancres. Powdered iodoform is usually [dusted] upon them. Wounds and venereal sores are often painted with a solution of it in collodion ( $\mathrm{I}$ in $\mathrm{x} 2$ of flexible collodion). This is an excellent application. Mixed 
with bismuth subnitrate, it is useful as an insufflation (iodoform, I ; starch, 2 ;) for ozæna, ulcers of the mouth and throat, and tuberculous ulcers of the larynx. Often $\frac{1}{3}$ gr. [.02 gm.] of morphine acetate is added. An emulsion (iodoform, moistened with alcohol, I ; boiling water, 2 ; glycerin, 7 ;) is very useful for injection into sinuses, abscess cavities, etc. It has been used in the form of a bougie for the urethra and for the nose. The suppository [B. P., each $3 \mathrm{gr}$; .20 gm., in I $2 \mathrm{gr}$.; $.80 \mathrm{gm}$. of oil of theobroma] is useful in painful conditions of the rectum. It is occasionally employed for pruritus, and to relieve the pain of neuralgia. Many attempts have been made to get rid of its odor; balsam of Peru, musk, or creolin ( 2 per cent.) have been used, but oil of geranium ( $\mathbf{I}$ to 25 ) is best. [Some believe that the odor of iodoform is preferable to that of musk. By keeping a Tonka bean or ground roasted coffee with it, the odor is lessened.]

Internal.-Iodoform has not been found to be of any use internally. It has been tried unsuccessfully in phthisis and many other conditions.

\section{Toxicology.}

Symptoms.-Curious symptoms, often severe and sometimes ending in death, are occasionally observed after the application of iodoform to a raw surface. They are a quick pulse, gastro-intestinsl irritation, fever, rapid collapse, melancholia, ballucinations, dilated pupils, extensive erythema and perhaps eczema. These symptoms vary much in severity, and it is rare for more than two or three of them to be present at once. Fatty degeneration of the liver and muscles may occur.

[Treatment.]-Stimulants, diaphoretics and sponging the skin with warm water are recommended.

\section{XIII. [ARISTOLUM.}

ARISTOL. (Not official). $-\mathrm{C}_{20} \mathrm{H}_{24} \mathrm{O}_{2} \mathrm{I}_{2}=548.38$. Synonyms.-Dithymoldiiodide. Annidalin.

SOURCE. - It is prepared by the decomposition of a solution of Iodine in Potassium Iodide, by Thymol dissolved in a solution of Sodium Hydroxide. The precipitate is washed with water and dried. It contains .46 per cent. of Iodine.

Characters. - An amorphous brownish-red, almost tasteless powder, of a slight, peculiar, iodine-like odor. Solubility. - Insoluble in water and Glycerin ; soluble in Ether and Chloroform; with difficulty soluble in Alcohol; it is readily taken up by fixed oils and Petrolatum. 


\section{Action and Uses of Aristol.}

It is used for the same purposes as is iodoform in various skin diseases, as lupus, psoriasis and for tertiary syphilis, both in ointment, lanolin, flexible collodion and as a powder. It has the very great advantage of being nearly free from odor. In surgery when dusted upon serous membranes it tends to prevent their adhesion.

\section{IODOLUM.}

IODOL. (Not official). $-\mathrm{C}_{4} \mathrm{I}_{4} \mathrm{NH}=569.01$. Synonym. - Tetraiodopyrrol.

Source. - From the interaction of Iodine and Pyrrol in Alcoholic solution. It contains 90 per cent. of Iodine.

Characters. - A pale-yellow, bulky powder, odorless and tasteless. It becomes dark in color on exposure to light. Solubility. - Insoluble in water ; soluble in Alcohol, Ether and fixed oils.

Dose, $1 / 2$ to $3 \mathrm{gr}$; . 03 to $.20 \mathrm{gm}$.

\section{Action and Uses of Iodol.}

Iodol was introduced as an antiseptic iodine compound, and is used for the same purpose as iodoform, for which it is a frequent substitute. It has been administered internally as a substitute for potassium iodide.

\section{EUROPHEN.}

EUROPHEN. (Not official.) - Synonym.-Diisobutylorthocresol iodide. $-\mathrm{C}_{22} \mathrm{H}_{29} \mathrm{O}_{2} \mathrm{I}=450.79$. This should be distinguished from Europhin.

SOURCE.-It is prepared in the same way as is Aristol except that Isobutylorthocresol is used in place of Thymol. It contains 28 per cent. of Iodine.

CiHARACTERS.--It is an amorphous, golden-yellow powder of a slightly saffron-like odor. Solubility.-In Alcohol, Ether, and in about 4 parts of Olive Oil ; insoluble in water. or Glycerin.

\section{Action and Uses of Europhen.}

Europhen is permanent when dry, but when moistened splits up into iodine and a new soluble iodine compound. It is a powerful germicide and bactericide, and is used for wounds and for like purposes as is iodoform, and in the same quantities.]

Various iodoform-like substances (not official) are found in the market which depend for their antiseptic properties upon the iodine in them. They 
have no advantage over jodoform except that some of them have no odor. The following are the chief :

\begin{tabular}{|c|c|c|c|c|}
\hline Losophan & contains & 80.0 & $\mathrm{er} \mathrm{ce}$ & of iodine. \\
\hline Di-iodosalicylic acid & “ & 66.0 & “ & ، \\
\hline Sozoiodol & “ & 54.0 & “" & “ \\
\hline Iodosalicylic acid & “ & 50.0 & ، & “ \\
\hline
\end{tabular}

\section{XVI. [NAPHTALINUM.}

NAPHTALIN. $-\mathrm{C}_{10} \mathrm{H}_{8}=127.7$. Synonym. - Naphtalene.

SoURCE. - A Hydrocarbon obtained from Coal Tar by distillation between $35^{\circ}$ and $482^{\circ} \mathrm{F}$; $180^{\circ}$ and $250^{\circ} \mathrm{C}$. The impure Naphtalin is treated successively with Sodium Hydroxide and Sulphuric Acid, and is purified by distillation in the presence of steam, and by treating with concentrated Sulphuric Acid and distilling.

Characters. - Colorless, shining, transparent laminæ, having a strong, characteristic odor resembling that of Coal Tar, and a burning aromatic taste; slowly volatilized on exposure to air. Solubility.-Insoluble in water; soluble in 15 parts of Alcohol, very soluble in Ether, Chloroform, Carbon Disulphide, and fixed or Volatile Oils.

Dose, I to $20 \mathrm{gr}$; ; .06 to $1.20 \mathrm{gm}$.

\section{Action and Uses of Naphtalin.}

Since náphtalin is not absorbed by the system, it acts only upon the mucous membrane of the bowels. It is a true intestinal antiseptic, and is of great value in dysentery, and in catarrhal, typhoid and tuberculous diarrhcea, where it markedly lessens or entirely abolishes the fotor of the movements. It has also been used as a vermifuge (dose, 3 to $6 \mathrm{gr}$; .20 to .40 gm.). Success in the treatment of dysentery usually requires a daily dose of from one to two drachms ; 4. to 8 . gm., best administered in starch wafers with oil of bergamot.

\section{NAPHTOL.}

NAPHTOL. $-\mathrm{C}_{10} \mathrm{H}_{7} \mathrm{OH}=1$ 43.66. Synonym.-Beta-Naphtol. A phenol occurring in Coal Tar, but usually prepared artificially from Naphtalin.

SourCE. - Concentrated Sulphuric Acid is allowed to act on Naphtalin, whereby $\beta$-Naphtalin Sulphonic Acid is formed $\left(\mathrm{C}_{10} \mathrm{H}_{7} \mathrm{HSO}_{3}\right)$. This acid is dissolved in water, saturated with Milk of Lime, and the resulting Calcium Salt separated by crystallization. The crystals are re-dissolved in water and decomposed by Sodium Carbonate, yielding Sodiun Naphtalin-Sulphonate $\left(\mathrm{C}_{10} \mathrm{H}_{7} \mathrm{SO}_{3} \mathrm{Na}\right)$. The Sodium Salt is next added to fused Sodium Hydroxide and Sodium-Naphtol $\mathrm{C}_{10} \mathrm{H}_{7} \mathrm{ONa}$, and Sodium Sulphite $\mathrm{NaSO}_{3}$ is formed. The 
former is treated with Hydrochloric Acid and Naphtol is obtained, which is purified by sublimation and re-crystallization from hot water.

Characters. - Colorless, or pale buff-colored, shining, crystalline laminæ, or a white, or yellowish-white, crystalline powder, having a faint, phenol-like odor, and a sharp and pungent but not persistent taste. Solubility. - In about IO00 parts of water, and in 0.75 part of Alcohol; very soluble in Ether, Chloroform or solutions of caustic alkalies.

Dose, I to $20 \mathrm{gr}$; .06 to $1.20 \mathrm{gm}$.

\section{Action and Uses of Naphtol.}

Naphtol was introduced as an antiseptic, at fift in dermatological practice as a ro per cent. ointment, in scabies, ringworm and psoriasis ; it is, however, irritating in eczema. It is a remedy of great value in obtaining intestinal antisepsis, bacteriological investigations showing that it destroys certain micro-organisms in sith when administered to the extent of $40 \mathrm{gr} . ; 2.70 \mathrm{gm}$. per day. As it is irritating to the stomach it can be administered in keratin-coated pills.] It is useful for cases of dilated stomach.

\section{XVIII. [ASAPROLUM.}

ASAPROL. (Not official). $\left.-\left(\mathrm{C}_{10} \mathrm{H}_{6} \mathrm{OHSO}\right)_{2}\right)_{2} \mathrm{Ca}+3 \mathrm{H}_{2} \mathrm{O}=538.83$. Synonym. - Calcium Beta-naphtol alpha-monosulphonate.

Source.-By action of free Sulphuric Acid on Beta-Naphtol, neutralizing this product with Calcium Carbonate, concentration and crystallization.

Characters. - A colorless, or yellowish-white, crystalline powder, of a slightly bitter taste, becoming darker on exposure to light. Solubility. - In $\mathbf{1 . 5}$ parts of water; in 3 parts of Alcohol.

Dose, 15 to $60 \mathrm{gr}$.; 1 . to $4 . \mathrm{gm}$.

\section{Action and Uses of Asaprol.}

Asaprol is an useful, soluble and safe antiseptic. It is valuable in epidemic influenza, relieving the pain and reducing the fever, not giving rise to prostration nor interference with the heart or respiration. In atonic dyspepsia, when fermentation alternates with acid eructations, it has achieved brilliant results. Since it is not irritating to the alimentary mucous membranes, it can advantageously replace naphtol. In chronic rheumatism it will relieve the pain of an acute exacerbation. In acute rheumatism, although it does not present the disadvantages of the salicylates, it is not so useful, nor so uniformly successful.] 


\section{RESORCINUM.}

RESORCIN. $-\left[\mathrm{C}_{6} \mathrm{H}_{4}(\mathrm{OH})_{2}=109.74\right.$. Synonyms. - Resorcinol. Metadioxybenzol.

SOURCE. - This is a diatomic phenol obtained by heating Benzene with fuming Sulphuric Acid, whereby Benzene Meladisulphonic Acid is formed $\left(\mathrm{C}_{6} \mathrm{H}_{4}\left(\mathrm{HSO}_{3}\right)_{2}\right)$. The acid is dissolved in water, neutralized with Milk of Lime, the Calciun Sulphate expressed, Sodium Carbonate added, filtered and the filtrate evaporated to dryness. The residue is then heated with Caustic Soda and Sodium Resorcin $\left(\mathrm{C}_{6} \mathrm{H}_{4}(\mathrm{ONa})_{2}\right)$ is formed ; continuous boiling expels the Sulphurous Acid, and the residue is extracted with Ether and impure Resorcin obtained by distillation. This is purified by sublimation or re-crystallization from water.

Characters. - Colorless or faintly reddish, needle-shaped crystals or rhombic plates, having a faint, peculiar odor, and a disagreeable, sweetish and afterwards pungent taste, acquiring a reddish or brownish tint by exposure to light and air. Solubility.-In 0.6 part of water, and in 0.5 part of Alcohol ; also readily soluble in Ether or Glycerin; very slightly soluble in Cbloroform.

Dose, 2 to $5 \mathrm{gr}$; . 12 to $.30 \mathrm{gm}$.]

\section{ACtion AND Uses of Resorcin.}

This substance, originally introduced as an antipyretic, is now rarely employed for this purpose, as [the necessarily large doses are] too depressant to the heart. A solution of resorcin in glycerin, I to 4 , is excellent for removing epidermic scales in chronic skin diseases, and also for getting rid of the [scales] in seborrhœea sicca of the scalp; [here it doubtless inhibits the action of the bacteria which may be the cause of dandruff.] A lotion: Resorcin, I ; ether, I ; castor oil, I ; eau de cologne, 10 ; alcohol ( $9 \circ$ per cent. ), 35 ; is useful for dandruff and alopecia. Resorcin is a powerful antiseptic, and a 5 per cent. solution may be injected into the bladder in cystitis. [It is of great value in fermentative dyspepsia when administered, well diluted, one hour after ingestion of food. Dark-colored urine, often described as smoky, is sometimes seen after large doses.]

\section{FORMALDEHYDUM.}

FORMALDEHYDE. (Not official.) $-\mathrm{CH}_{2} \mathrm{O}[=29.93$. Synonyms.Formal. Formic Aldehyde. A gas soluble in water. The solution known as Formalin contains about 40 per cent. of the gas.

Source. - It is obtained by the oxidation of methylic alcohol at a moderately high temperature or by passing the vapor over red-hot metal. 
CHARACTERs. - The solution of Formaldehyde is colorless, having a pecu. liar odor and an irritative taste. Solubility. - Readily in water and in Alcohol.

\section{Action and Therapeutics of Formaldehyde.}

Formaldehyde is a powerful disinfectant. It hardens the skin and is irritant to the respiratory mucous membranes. It is used diluted with twenty-five times its bulk of water as a preservative for anatomical specimens, which do not shrink in it, and, in I to 60 aqueous solution, as a hardening agent in histological work. As a germicide it is about equal to corrosive mercuric chloride, but is not much used in surgery as it retards healing. As a powerful disinfectant it can be employed for large rooms, when volatilized in a special lamp. It does not bleach colored fabrics. The penetrating power of the gas depends largely upon the conditions of moisture, although under favorable circumstances it may be considerable. If the micro-organisms are exposed they will be destroyed within a half hour by a quart; 960 c.c., volatilized in a room fifteen feet square, and ten feet in height; if protected, within an hour and a half. Small animals accidentally confined in rooms undergoing this process rarely survive, when it is properly carried out (Harrington).

\section{UROTROPINUM.}

UROTROPIN. (Not official. ) $-\mathrm{C}_{6} \mathrm{H}_{12} \mathrm{~N}_{4}=\mathbf{r} 39.86$. Synonym.-Hexamethylentetramin.

Source. - It is obtained by the action of 4 molecules of Ammonia on 6 molecules of Formaldehyde : $4 \mathrm{H}_{3} \mathrm{~N}+6 \mathrm{CH}_{2} \mathrm{O}=\mathrm{C}_{6} \mathrm{H}_{12} \mathrm{~N}_{4}+6 \mathrm{H}_{2} \mathrm{O}$.

ChARACTERS. - It occurs as colorless, transparent, six-sided rhomboids or prisms, of a sweetish, afterwards bitter taste, which are permanent when kept dry. It is odorless at ordinary temperatures, but heating evolves a peculiar fishy odor. Solubility. - Readily in 1.25 parts of water, in Chloroform, Alcohol, Acetone, and Carbon Bisulphide ; insoluble in Ether.

INCOMPATIBLES. - All acids decompose it in the presence of warmth.

Dose, 7 to $15 \mathrm{gr}$; .50 to $1.00 \mathrm{gm}$. in full tumbler of plain or carbonated water.

\section{ACtion of URotropin.}

Large doses in man, e.g., 150 grains; 10 gm., are well borne ; intravenous injections in rabbits and dogs do not increase, but, rather, slightly lower blood-pressure. Very large doses cause 
albuminuria in rabbits and hæmaturia in dogs. Of most importance is the inhibitory action of this remedy upon microorganisms when it is split up into formaldehyde and ammonia, the former being the active agent. This takes place for the most part, after ingestion, in the urine, which is not only of the proper temperature but also contains uric acid and acid salts which are efficient. Further, as Bardet showed, it will dissolve uric acid at the temperature of the body. Lastly, it may or may not produce diuresis.

\section{Therapeutics of Urotropin.}

This remedy is of especial value in diseases of the urinary passages. In ammoniacal fermentation of the urine, which is especially frequent in the cystitis of prostatic hypertrophy, the maximum dose given for two or three successive days is efficient in clearing that excretion. Inasmuch as the growth of the micro-organisms is inhibited, the remedy should be continued in sufficient amount to maintain this result. In gonorrhœeal posterior urethritis, cystitis and pyelitis the results are equally favorable. It may be employed as a prophylactic measure before operations upon the genito-urinary tract. For the uric acid diathesis it has been used with good results by most, with failure by a very few, physicians. Since it is not always diuretic, other measures should be employed for this purpose. As a lithontriptic some success has been claimed. For phosphaturia excellent results are reported. Inasmuch as the bacillus of enteric fever is found in a very considerable percentage of urines from patients suffering from this disease, and failure to disinfect this excretion is a source of danger, urotropin should be administered not only for this purpose but as well to avoid the cystitis which sometimes supervenes in the course of the disease.]

\section{SACCHARINUM.}

SACCHARIN. (B. P., not official). $-\mathrm{C}_{6} \mathrm{H}_{4} \mathrm{COSO}_{2} \mathrm{NH}=[168.65$. Synonyms.-Glusidum, [B. P.] Benzoyl-sulphonic-imide. Glucusimide. Gluside.

Sourcen-It is derived from Toluene, $\mathrm{C}_{6} \mathrm{H}_{5} \mathrm{CH}_{3}$, a derivative of coal tar, by a complicated process. 
Characters. - A light, white, crystalline powder. In solution it has an intensely sweet taste ; 1 of Saccharin is equal to 300 of cane sugar. Solubility. -In 400 parts of cold water; in 24 parts of boiling water; in 500 parts of Chloroform; in 25 parts of Alcohol ; in 48 parts of Glycerin. It unites with alkaline hydrates and carbonates, evolving from the latter Carbon [dioxide], and yields soluble Saccharin, which has lost none of its sweetness, and is very soluble in water.

IMPURITIES.-Commercial Saccharin is not a pure or uniform product; it often contains less than 50 per cent. of actual Saccharin.

Dose, $1 / 2$ to $2 \mathrm{gr}$; ; [.03 to .12 gm.]

\section{Action and Therapeutics of Saccharin.}

[Saccharin] is an antiseptic, but it is not used as such, [excepting in the surgery of the bladder.] It is employed as a sweetening agent, when from any cause, as in diabetes [mellitus], sugar cannot be taken. It may be given in tablets, or with sodium carbonate to form soluble solutions. An elixir is prepared, containing [saccharin, 2 ; sodium bicarbonate ( 90 per cent.), I ; alcohol, 5 ; and water, 35 parts.] This is excellent for covering the taste of nauseous medicines. Usually $20 \mathrm{~m}$. $[1.20$ c.c.] are required for a four-ounce [125. c.c.] mixture.

\section{METHYLENE BLUE.}

METHYLENE BLUE. (Not official.)-Synonym.-Tetramethylthionin chloride. $\left[\mathrm{C}_{16} \mathrm{~N}_{18} \mathrm{~N}_{3} \mathrm{SCl}=318.90\right.$.

Source.-By treating dimethylparaphenylenediamine $\left(\mathrm{NH}_{2} \mathrm{C}_{6} \mathrm{H}_{4} \mathrm{~N}\left(\mathrm{CH}_{3}\right)_{2}\right)$ in an acid solution with Hydrogen Sulphide and Ferric Chloride.

Characters. - In small, blue, scaly crystals, with a copper-bronze tinge. Solubility. - Readily in water.

Dose, I to $5 \mathrm{gr}$; .06 to $.30 \mathrm{gm}$.

\section{Action and Uses of Methylene Blue.}

Methylene blue (not to be confounded with methyl blue) has been introduced into medicine as antiseptic. It has been used for rheumatism of the joints and muscles. Lately it has been given for intermittent fevers, but the reports show that it pos: sesses no advantage over quinine excepting that it is tasteless and may be substituted for it if it cannot be taken or it has been unsuccessfully used. Recent reports indicate that even when given internally it causes gonococci to rapidly disappear from the 
urine in specific urethritis. As to its effects upon inoperable neoplasms when injected into them clinical reports differ widely. It imparts a blue color to nerve substance and a like color to the urine. It may produce irritation at the neck of the bladder, which about $30 \mathrm{gr}$.; $2 \mathrm{gm}$. of powdered nutmeg is said to relieve.] It has been used as an analgesic in neuralgia, migraine, sciatica, and rheumatism.

\section{XXIV. [PIPERAZINUM.}

PIPERAZINE. (Not official). $-\mathrm{C}_{4} \mathrm{H}_{10} \mathrm{~N}_{2}=85.9$. Synonyms. -Piperazidine. Diethylenediamine. Dispermine.

(This drug is antiseptic only so far as its clearing the urine of pathological products prevents decomposition. It is placed here for convenience.)

Solrce. - By the action of Ammonia on Ethylene Chloride. The mixture of bases is fractionated, and from the fraction boiling between $266^{\circ} \mathrm{F} ; 130^{\circ}$ C., and $356^{\circ} \mathrm{F}$; $180^{\circ} \mathrm{C}$., Piperazine separates on cooling.

Characters. - It occurs in colorless, well-defined, acicular crystals, readily soluble in water. In cold, aqueous solutions, it will dissolve twelve times as much Uric Acid as will Lithium Carbonate.

Dose, 8 to $15 \mathrm{gr}$; .50 to $1.00 \mathrm{gm}$.

Action and Uses of Piperazine.

Piperazine is believed to increase slightly the amount of urea in the urine, while the uric acid co-efficient is diminished. The testimony as to its diuretic action is conflicting, but the weight of clinical evidence is in favor of its being a reliable diuretic. In ordinary doses it does not appear to have any influence upon the nervous, circulatory or respiratory systems ; nor does it irritate the gastro-intestinal or the genito-urinary tract. There is much clinical testiniony as to the value of this drug in gout, goutiness (uricacidremia) and rheumatism. It is here given in water containing carbon dioxide. It is so highly hygroscopic that it cannot be administered as pill or powder.] 


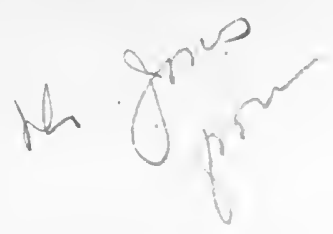

\section{Part II.- -ORGANIC MATERIA MEDICA.}

SECTION I.-PHARMACOPCEIAL SUBSTANCES DERIVED FROM THE VEGETABLE KINGDOM.

THE drugs comprehended in this section may be arranged in many ways; but there are objections to each. Inasmuch as the medical student should be well acquainted with the actions of these drugs in health and disease, those which act similarly will be grouped together. In Appendix I a list of these drugs, arranged according to their Natural Orders, will be found.

\section{GROUP I.}

\section{Vegetable Drugs acting chiefly on the Nervous System.}

These may be classified as follows :

CLASS I.-Acting on the cerebrum.

A. Cerebral depressants or soporifics :

Opium, Hops, [Lactucarium, Pellotine].

B. Cerebral excitants :

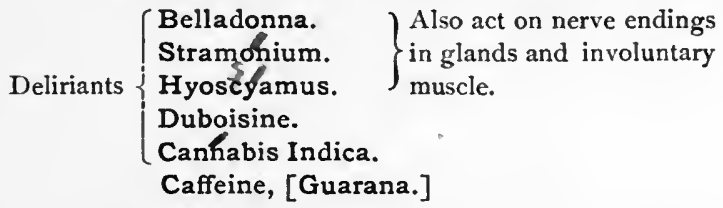

Class II.-Acting on the spinal cord.

A. Exciting the cells of the anterior cornua. [Nux Vomica,] Strychnine.

B. Depressing the cells of the anterior cornua. Calabar bean, [Muscarine], Gelsemium.

CIASS III.-Acting on the nerves.
A. Depressing the motor nerves. Coniúm, Tobacco.
в. Depressing the motor end plates. Curare. Koo- hath-re
C. Depressing the sensory nerves. [Coca], Cocaine.
D. Stimulating the secretory nerves. Pilocarópus. 


\section{Class I.-DRUGS ACTING CHIEFLY ON THE CEREBRUM.}

\section{[A. Cerebral Depressants or Soporifics.]}

\section{OPIUM.}

OPIUM.-[The concrete, milky exudation obtained by incising the unripe capsules of Papáver somniferum Linné (nat. ord. Papaveracea), and yielding, in its normal, moist condition, not less than 9 per cent. of crystal. lized morphine, when assayed. Synonym.-Poppy. Habitat.-Western Asia ; cultivated.]

Characters. - [In irregular or subglobular cakes, with the remnants of poppy leaves and fruits of a species of Rumex adhering to the surface; plastic, or of a harder consistence ; chestnut-brown or darker, and somewhat shining; internally showing some tears and fragments of regetable tissue. It has a sharp, narcotic odor, and a peculiar, bitter taste.

VARIETIES.-The above is the official opium; but the following are met with in commerce, and may be used to prepare the alkaloids:] (a) Constantinople Opium, small lenticular masses, $1 / 4$ to $1 / 2 \mathrm{lb}$. [ [ 20. to 240. gm.] in weight, and enclosed in a poppy leaf, but without the Rumex seeds. Sometimes the terms Turkey and Levant Opium include this. (b) Egyptian Opium. Flat, more or less circular cakes, two or three in. [5. to $7.5 \mathrm{~cm}$.] in diameter, reddish hue internally, covered with a leaf externally. Persian, Indian, English, French and German opiums are rarely met with in England.

Composition.-( I) Alkaloids.-At least [nineteen] in number. Most are combined with Meconic Acid, some with Sulphuric Acid, and some are free. Some Morphine Salts, and Codeine are official. These two alkaloids and Narceine and Thebaine are important. The following are the alkaloids existing in Opium :

5 Morphine [(2.5 to 22.8 per cent.) $)$.] Hydrocotarnine

Codeine $[(0.2$ to 0.7 per cent. $)$.$] Laudanine$

Thebaine $[(0.15$ to I per cent. $)$.$] Laudanosine$

Narcotine $[(\mathbf{1} .3$ to Io per cent. $)$.$] \quad Meconidine$

Narceine [( 0.1 to 0.7 per cent. $).] \quad$ Rhoadine

Papaverine [(I per cent. $)$. $]$

Codamine

Pseudomorphine [(0.2 per cent.).] Gnoscopine

Protopine

Lanthropine

Oxynarcotine $\}$ [in minute quantity. Deuteropine (not known in pure state).] Cryptopine

[Narcotine is more properly called Anarcotine. Apomorphine is an artificial alkaloid.]

(2) Neutral bodies. - Two in number :

Meconin.

Meconoiasin.

(3) Organic acids. - Two in number :

Meconic Acid, [official in B. P.] Thebolactic Acid. 
(4) Water, [about] I6 per cent.

(5) Mucilage, resin [pectin,] glucose, fats, essential oil, caoutchouc, odorous substances, and ammonium, calcium and magnesium salts.

The following analysis shows how specimens vary:

Patna Opium 3.98 per cent. of Morphine, 6.36 per cent. of Anarcotine.
Smyrna “
8.27
I.94
“6

IMPURITIES. - Water, stones, fruits, leaves, starch, [gum, and lead balls.]

Incompatibles. - Ferric chloride gives a deep red color (due to Meconic Acid). Zinc, copper and arsenic salts, silver nitrate, lead acetate and subacetate, give precipitates of meconates, sulphates and coloring matters. All tannin-containing preparations precipitate codeine tannate. Fixed alkalies, their carbonates and ammonia precipitate morphine and [a]narcotine. The small amount of glucose in opium may cause it to explode when made into a pill with silver nitrate.

Dose, $1 / 4$ to $2 \mathrm{gr}$.; [.015 to .12 gm.]

[OPII PULVIS.-Powdered Opium. Opium dried at a temperature not exceeding $185^{\circ} \mathrm{F} . ; 85^{\circ} \mathrm{C}$, and reduced to a very fine powder.

Powdered Opium, for pharmaceutical or medicinal purposes, when assayed, should yield not less than 13 nor more than 15 per cent. of Crystallized Morphine. Any Powderer Opium of a higher percentage may be brought within these limits by admixture with Powdered Opium of a lower percentage, in proper proportions.

Dose, $1 / 4$ to $2 \mathrm{gr}$.; .015 to .1 $2 \mathrm{gm}$.]

\section{Preparations.}

I. [Extractum Opii.-Extract of Opium. Powdered Opium, 100 ; Distilled Water, I000; Sugar of Milk, a sufficient quantity ; by trituration, filtration and evaporation.

Dose, $1 / 8$ to I $\mathrm{gr}$; .008 to $.06 \mathrm{gm}$.

2. Emplastrum Opii.-Opium Plaster. Extract of Opium, 6o; Burgundy Pitch, I80 ; Lead Plaster, 780 ; water, 80.

3. Trochisci Glycyrrhizæ et Opii.-Troches of Glycyrrhiza and Opium. Extract of Glycyrrhiza, 15; Powdered Opium, 0.5 ; Acacia, 12 ; Sugar, $20 \mathrm{gm}$.; Oil of Anise, 0.2 c.c. To make 100 troches. Each troche contains about $\frac{1}{12}$ gr. ; $.005 \mathrm{gm}$., of Opium.

Dose, $I$ to 4 troches.

4. Vinum Opii.-Wine of Opium. Synonym.-Sydenham's Laudanum. Powdered Opium, I00; Cassia Cinnamon, I0; Cloves 10; Alcohol, 150; White Wine to I0oo; by maceration and filtration.

Dose, 3 to $20 \mathrm{~m}$.; .20 to 1.20 c.c.

5. Pilulæ Opii.-Pills of Opium. Powdered Opium, 6.5; Soap, $2 \mathrm{gm}$. To make 100 pills. Each pill contains I gr. ; $.06 \mathrm{gm}$., of Opium.

Dose, I to 2 pills. 
6. Pulvis Ipecacuanhæ et Opii.-Powder of Ipecac and Opium. Synonym.-Dover's Powder. Powdered Opium, 10; Ipecac, Io; Sugar of Milk, 80 .

Dose, 3 to $15 \mathrm{gr}$; .20 to $1.00 \mathrm{gm}$.

7. Acetum Opii.-Vinegar of Opium. Synonym.-Black Drop. Powdered Opium, 100; Nutmeg, 30; Sugar, 200; Diluted Acetic Acid to 1000 ; by maceration and percolation.

Dose, 3 to $20 \mathrm{~m}$.; .20 to 1.20 c.c.

8. Tinctura Opii.-Tincture of Opium. Synonym.-Laudanum, Powdered Opium, 100; Alcohol, 400; Water, 400; Diluted Alcohol to 1000 ; by maceration with Precipitated Calcium Phosphate, 50 ; and percolation.

Dose, 3 to $20 \mathrm{~m}$.; .20 to I.20 c.c.

9. Tinctura Opii Camphorata. - Camphorated Tincture of Opium. Synonym.-Paregoric. Powdered Opium, 4; Benzoic Acid, 4 ; Camphor, 4; Oil of Anise, 4; Glycerin, 4; Diluted Alcohol to 1000 ; by maceration and percolation.

Dose, 1 to $4 \mathrm{fl}$. dr.; 4. to 15 . c.c.

10. Opium Deodoratum,-Deodorized Opium. Synonym.Opium Denarcotisatum. Powdered Opium (containing 13 to 15 per cent. of Morphine), 100 ; macerated with Ether, 700 ; the clear solution poured off, and macerated twice with Ether, 350. The residue is dried and mixed with Sugar of Milk by trituration to weigh 100 parts.

Dose, $1 / 4$ to $2 \mathrm{gr}$; . 015 to $.12 \mathrm{gm}$.

II. Tinctura Opii Deodorati.-Tincture of Deodorized Opium. Powdered Opium, 100 ; Ether, 200 ; Alcohol, 200 ; Water to 1000. By maceration with Precipitated Calcium Phosphate, 50; by percolation and evaporation.

Dose, 3 to $20 \mathrm{~m}$.; .20 to 1.20 c.c.

12. Tinctura Ipecacuanhæ et Opii.-Tincture of Ipecac and Opium. Tincture of Deodorized Opium, 1000 ; Fluid Extract of Ipecac, I00; Diluted Alcohol to 1000 ; by evaporation and filtration.

Dose, 3 to $15 \mathrm{~m}$; .20 to 1.00 c.c.]

It will be noticed that from [Extract of Opium there is prepared Emplastrum Opii, and from the Deodorized Tincture, Tinctura Ipecacuanhre et Opii.

The following non-official preparations are sometimes used:-Nepenthe, of the same strength as Laudanum, Jiquor Opii Sedativus (Battley's solution), which is about 50 per cent. stronger than Laudanum, Acetum Opii Crocatum (black drop), four times as strong as Laudanum, and Tinctura Opii Ammoniata, B. P. (Scotch Paregoric), about two and one-half times as strong as the Camphorated Tincture.]

The following list, in which the doses are arranged, may assist the student: 
Name.

- [Extractum Opii.

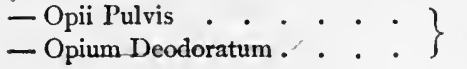

- Pulvis Ipecacuanhæ et Opii .

- Tinctura Ipecac et Opii] .

- Vinum Opii . . . . . .

- Tinctura Opii . . . . . .

- [Tinctura Opii Deodorati .

- Acetum Opii . . . . .

- Tinctura Opii Camphorata.

- Pilulæ Opii . . . . . .

- Trochisci Glycyrrhizæ et Opii]

- Emplastrum Opii . . . .
Dose.

$1 / 8$ to $\mathrm{I} \mathrm{gr.;} \mathrm{[.008} \mathrm{to} .06 \mathrm{gm}$. ]

$1 / 4$ to 2 gr. ; [.015 to. $12 \mathrm{gm}$.]

3 to 15 gr.; [. 20 to $1.00 \mathrm{gm}$.]

3 to $15 \mathrm{~m}$.; [. 20 to 1.00 c.c. $]$

3 to $20 \mathrm{~m} . ;$ [. 20 to 1.20 c.c.]

I to 4 f. dr. ; [4. to 15. c.c. ]

I to 2 pills.

I to 4 troches.

Externally.

[ACIDUM MECONICUM.-Meconic Acid (not official). $\mathrm{H}_{3} \mathrm{C}_{7} \mathrm{HO}_{7}$ $=199.5$ r.

Source.-Calcium Meconate, which is precipitated in the manufacture of Morphine Hydrochlorate, is placed in hot water and treated with Hydrochloric Acid. On cooling, Meconic Acid crystallizes out.

Characters. - Almost colorless, micaceous crystals. Solubility:-In 150 parts of water; in 45 parts of Alcohol; it is decomposed by boiling water.

\section{Preparation (not official).}

Liquor Morphinæ Bimeconatis.-Solution of Morphine Bimeconate. Morphine is precipitated by Ammonia from a solution of the hydrochlorate. It is dissolved in water, Alcohol and Meconic Acid. Strength, $I$ in 80.

Dose, 5 to $40 \mathrm{~m} . ; .30$ to 2.50 c.c.]

[MORPHINA.-Morphine. $\mathrm{C}_{17} \mathrm{H}_{19} \mathrm{NO}_{3}+\mathrm{H}_{2} \mathrm{O}=302.34$.

SourCE.-An Alkaloid obtained from Opium. Opium is macerated with distilled water, and strained, the infusion is evaporated and filtered. To the filtrate Alcohol and Water of Ammonia are added, and the Morphine crystallizes out. To purify the crystals, they are boiled with Alcohol, the solution filtered, when hot, through Animal Charcoal, and set aside to crystallize.

Characters. - Colorless or white, shining prismatic crystals, or fine needles, or a crystalline powder, having a bitter taste. Solubility. - In $435^{\circ}$. parts of water.

Dose, $1 / 8$ to $1 / 7 \mathrm{gr}$; .008 to .015 gm.]

MORPHIN E HYDROCHLORAS. - [Morphine Hydrochlorate. $\mathrm{C}_{17} \mathrm{H}_{19} \mathrm{NO}_{3} \mathrm{HCl}+3 \mathrm{H}_{2} \mathrm{O}=374.63$.

SoURCE. - ( I) Take a cold concentrated watery solution of Opium, precipitate the Meconic acid and resins with Calcium Chloride. The solution 
contains Morphine Hydrochlorate. (2) Evaporate the solution till it is solid, press to remove coloring matter, exhaust with boiling water, filter, and again evaporate and press; repeat this till the solution is nearly colorless. (3) Complete the decolorization by digesting with Charcoal. (4) Precipitate the Morphine with Ammonia and wash. (5) Dissolve in Hydrochloric Acid and crystallize out.

CHARACTERs. - White, feathery needles of a silky lustre, or minute, cubical crystals, having a bitter taste. Solubility.-In 24 parts of water; in 62 of Alcohol.]

INCOMPATIBLES.-Lead, iron, copper, mercury and zinc salts; alkaline carbonates; lime water; liquor potassii arsenitis; all substances containing tannin.

[Morphine Hydrochlorate is used in Tinctura Chloroformi et Morphinæ Composita. (See p. 291.[)

Dose, $1 / 8$ to $1 / 4$ gr.; [.008 to .015 gm.]

MORPHIN $\approx$ ACETAS.-Morphine Acetate. $\mathrm{C}_{17} \mathrm{H}_{19} \mathrm{NO}_{3} \mathrm{C}_{2} \mathrm{H}_{4} \mathrm{O}_{2}+$ ${ }_{3} \mathrm{H}_{2} \mathrm{O}=[398.12$.

SourCE. - Morphine is precipitated with Ammonia from a solution of the Hydrochlorate. It is dissolved in Acetic Acid and water, and the neutral solution is evaporated.

CHaracters. - [A white, or faintly yellowish-white, crystalline or amorphous powder, having a faint, acetous odor, and a bitter taste. Solubility.In 2.5 parts of water; in 47.6 parts of Alcohol.] Many specimens are not so soluble as this. The use of this salt is diminishing as it is unstable, losing Acetic Acid on exposure to air.

Dose, $1 / 8$ to $1 / 4$ gr.; [.008 to .015 gm.]

MORPHIN E SULPHAS.-Morphine Sulphate. $\left(\mathrm{C}_{17} \mathrm{H}_{19} \mathrm{NO}_{3}\right)_{2} \mathrm{H}_{2} \mathrm{SO}_{4}$ $+5 \mathrm{H}_{2} \mathrm{O}=\left[756.3^{8}\right.$.

SOURCE. - Morphine is dissolved in boiling distilled water; diluted Sulphuric Acid is added to neutralization, and on cooling the Sulphate appears in crystals.

ChARACTERS. - White, feathery, acicular crystals of a silky lustre, and having a bitter taste. Solubility.-In 2 parts of water; sparingly in Alcohol.

Dose, $1 / 8$ to $1 / 4 \mathrm{gr}$; .008 to $.015 \mathrm{gm}$.

\section{Preparations.}

1. Pulvis Morphinz Compositus.-Compound Powder of Morphine. Synonym.-Tully's Powder. Morphine Sulphate, I ; Camphor, 19; Glycyrrhiza, 20; precipitated Calcium Carbonate, 20; Alcohol, a sufficient quantity. By trituration.

Dose, 5 . to 15 . gr. ; 30 to $1.00 \mathrm{gm}$.

2. Trochisci Morphina et Ipecacuanha.-Troches of Morphine and Ipecac. Morphine Sulphate, 0.16; I pecac, 0.50; Sugar, 
65 gm.; Oil of Gaultheria, 0.2 c.c.; Mucilage of Tragacanth, a sufficient quantity to make 100 troches. Strength. -About $\frac{1}{40}$ gr.; .0016 gm. of Morphine in each.

Dose, I to 5 troches.

Two solutions of Morphine Sulphate should be carefully distinguished: (1) The U. S. P. Solution ( 1 to 480 of water), and (2) Magendie's ( 1 to 30 of water). Magendie's Solution as prepared in France is made from the acetate and is somewhat weaker ( 1 to $3 \mathrm{grs}$ ).]

\section{Action of Opium.}

The action and uses of opium are due almost entirely to its morphine, and therefore they may be studied together. Codeine [and other alkaloids will be considered on p. 366 . Meconic acid appears to be nearly free from physiological properties.]

External.-Opium probably has no action when applied to the unbroken skin, but it has been said to be slightly anodyne. It can be absorbed from and relieve the pain of raw surfaces.

Internal.-Alimentary canal.-Opium diminishes all the secretions of the body except the sweat. The mouth consequently becomes dry, and the patient feels thirsty, but after a small dose not markedly so. This effect is partly due to the direct action of the opium on the mouth, but to a less extent to its influence exerted after it has been absorbed. In the stomach and intestines, by the same double action, the secretion of the gastric and intestinal juices is diminished. The drug also paralyzes the peristaltic movements of the stomach and intestines. This is due to its action on the nervous or muscular structures in the wall of the intestine itself. The result of the diminution of secretion and peristalsis is that opium appeases hunger, often causes indigestion, almost always gives rise to constipation, and if vomiting or diarrhoea is present, it may prevent it. These actions are also in part due to its general sedative influence on the nervous system. If pain exists in the abdomen or elsewhere, opium is a powerful anodyne. Most of it is absorbed, but rather slowly. If injected subcutaneously it is excreted into the stomach. With some persons it causes vemiting. Whether the biliary and pancreatic secretions are diminished is not known. 
Blood.-Morphine for the most part circulates in the blood as such, and is excreted by the kidneys, but a small part of it is destroyed in the liver. The fate of the other alkaloids is not known, nor are we aware of any direct action of any of the constituents of opium on the blood itself.

Circulation. - In an ordinary healthy man small doses of opium hardly affect the heart or vessels. Large doses diminish the force and frequency of the heart, which finally stops in diastole. These effects can be produced by applying the drug to the organ; it therefore directly affects either the cardiac muscle or the nerves in it. But this local action is said to be helped by the less important influence of opium on the vagal centre; this is at first stimulated, and about the time at which the heart itself is depressed, so that both these actions make the pulse slow. Patients rarely die from the effect of opium on the heart and its nervous apparatus, this being much less important than the influence on respiration, and some of the cardiac depression may be secondary to asphyxia.

The vaso-motor centres are slightly depressed by moderate doses, hence the vessels, particularly those of the skin, dilate; with large doses the depression is considerable.

Respiration.-Opium is a direct poison to the respiratory centre. Breathing therefore becomes slow, less air is taken in at each inspiration, and death takes place from asphyxia. The secretion of bronchial mucus is decreased.

Nervous system. - Brain. - The higher faculties are at first excited even by small doses. In a few persons there is no incoordination in this excitement. The intellectual power and mental vigor are increased, and therefore the drug is taken by some people to enable them to do their mental work. Usually, however, the excitation does not affect the mind evenly ; generally the imagination is powerfully and pleasantly excited, much more so than the faculties of reason and judgment, which are a little dulled. The expression on the face is one of happiness and comfort, and this corresponds with the condition of the mind, which is in a state of peace, calm and happiness. This is soon succeeded by sleep, which is accompanied by pleasant 
dreams, generally of an impossible nature. With some persons, however, the sleep is quite dreamless. This, which is the beginning of the depression of the highest centres, is soon followed by depression of the others, the higher being influenced before the lower, so that soon the sleeper does not respond to any sound, light, or cutaneous stimulation, nor does he feel pain. It is this last fact that makes the drug so invaluable. The dose requisite to annul pain depends, of course, upon the severity of it. If a large amount is given, often there is no primary excitement, and then the first symptom that opium has been taken is drowsiness. On waking from sleep induced by opium some persons feel quite well, but usually there is a little languor, headache, and nausea. Opium-eaters take it for its stimulant effect. It is given medicinally as a hypnotic and anodyne. The pupil is contracted; this is due to the effect of the drug on the pupillary centre in the floor of the aqueduct of Sylvius. In man, just as the stimulation of the intellectual centres is brief, so is that of the cerebral motor centres-in fact, it is often difficult to detect any evidence of it. Their subsequent depression is never so marked as that of the intellectual faculties; for although there is languor and muscular weakness, and the patient always lies down, yet he can be walked about if he is supported. Vomiting is occasionally caused by transient irritation of the vomiting centre, but soon it is depressed, and therefore emetics do not act well in cases of opium poisoning.

The motor cells of the spinal cord are at first slightly stimulated, and consequently reflex excitability is exaggerated; but they are soon depressed, and it is difficult to obtain reflex movements.

The excitability of motor and sensory nerves is, perhaps, a little increased at first, but in the later stages of opium poisoning they are depressed, the sensory before the motor. The muscles remain irritable to the last.

Opium, in its action on the nervous system, illustrates the common fact that functions at first stimulated by a drug are usually subsequently paralyzed by it; and it affords an excellent example of the law of dissolution, for higher functions, such as 
the intellectual and imaginative, are first affected; motion is then disordered; next the pupillary centre, and then the medullary centres, for respiration and cardiac action are implicated. The spinal cord is influenced to a less degree, the nerves very slightly, and the muscles not at all.

In man the peculiarities of the action of morphine are its predominating influence on the higher mental functions, and the slight affection of the motor and the vaso-motor centres, the cord, the nerves, and the muscles. In frogs morphine produces violent convulsions, because its predominating action is to stimulate the spinal cord. Birds are peculiarly insusceptible to morphine. Mammals are for the most part affected in the same way as man, except that the first or excitement stage is more marked ; hence with many mammals, especially cats, morphine is a violent convulsant; dogs and rabbits require large doses to produce symptoms.

Kidneys.-Sometimes opium slightly increases, sometimes it slightly decreases, the urinary flow. Perhaps morphine is decomposed in the body, for oxydimorphine has been found in the urine of those taking morphine.

Skin.-Opium is a mild diaphoretic. It may cause itching.

Metabolism. - If the person taking opium has glycosuria, the amount of sugar he passes in the urine is diminished. General metabolism appears to be decreased also, for it is stated that the amounts of uric acid and carbon [dioxide] excreted are lessened, but some experimenters contradict this statement.

Temperature.-Large doses depress this, probably from the effect of the drug upon the thermogenetic nerve centres.

Persistent use of large doses decreases the secretion of milk and the menstrual discharge. It is excreted by the milk, and so may affect the child.

Peculiarities.-There are few drugs which have such different effects upon different people. The above description states the manner in which most human beings are affected; but in some the stage of excitation is very evident, so that they become delirious and cannot sleep. In others, vomiting and indigestion are very marked. Some of these peculiarities are due, no doubt, to 
the varying composition of opium. Children are easily poisoned by it, and therefore only small doses should be administered to them; women are more readily affected than men. Persons who take it habitually soon tolerate enormous quantities. It may produce an erythematous eruption on the skin.

Differences in action between opium and morphine.-(I) Morphine being more readily absorbed, acts more quickly. It is especially suitable for subcutaneous injection; given in this way it acts very rapidly. (2) Opium is more liable to upset the digestion and to cause constipation; but this last fact often makes it the more valuable in many abdominal diseases. (3) Opium is the better diaphoretic. (4) Morphine is more certain in its action as an anodyne and soporific; possibly this is because of the other powerful alkaloids in opium. (5) [It is less convulsant.] (6) Opium is stated to act more powerfully in reducing the amount of sugar present in the urine in glycosuria. [(7) Opium affects the bladder sphincter less. (8) Morphine causes more pruritus than opium.]

\section{Therapeutics of Opium.}

External.-Hot fomentations or poultices sprinkled with laudanum are often applied to painful parts ; but probably it is the heat and not the opium which relieves the pain. Linimentum Opii [B. P., tincture of opium and soap liniment, equal parts], rubbed into the skin diminishes the pain of chronic rheumatism and myalgia ; probably in this case the friction is more efficacious than the opium. Locally applied to sores and ulcers, it may soothe the pain due to them. The ointment of nutgall and opium [B. P., powdered opium, 2 ; gall ointment, 27 ;] will often relieve the pain of piles and anal fissures, especially if a mild laxative is given by the mouth. [The warm decoction of the white poppy is used in England as an anodyne fomentation. Poppy capsules (Papaveris Capsulæ, B. P.) are obtained from the Papaver somniferum (nat. ord. Papaveracea) which is cultivated in England. A ten per cent. decoction made from these contains but a small and uncertain amount of opium, and its beneficial effects, if any, are doubtless due to its warmth.] 
Internal.-Stomach.-Morphine is of great service for the pain of gastric ulcer, cancer, or even for simple painful dyspepsia. A solution [of morphine in water, $I$ in 480 ; dose, I fl. dr. ; 4. c.c.] is preferable to opium, as that may aggravate the indigestion. Morphine is frequently combined with preparations of bismuth, and taken immediately before or after meals. Many forms of vomiting are relieved by morphine, because it decreases pain, peristalsis, and excessive secretion.

Intestines.-Opium is invaluable for stopping many varieties of diarrhœa. If they will yield to any treatment, opium is most likely to be successful. Intestinal colic, being due to irregular, excessive peristaltic action, is generally relieved by opium,-and, indeed, so is abdominal pain of all sorts. In all inflammatory conditions of the peritoneum full doses of opium must be given, the object being so to paralyze the intestinal movements as to prevent the peritoneal surfaces rubbing against each other. [This method is quite likely to result in extensive peritoneal adhesions.] It is the great mainstay in perityphlitis, acute peritonitis, and after operations or wounds in the abdomen. Opium is far preferable to morphine for abdominal cases; if they are severe, it must be boldly pushed, the patient being kept just drowsy, with slightly contracted pupils; and it often does not matter if the bowels are not open for a week, or even more. [The more recent and better practice, however, is to keep the bowels slightly open by the use of the salines.] Morphine should be given sparingly, if an abdominal operation is impending, for it masks the symptoms.

Heart.-Much skill is required to give opium properly in heart disease. The hypodermatic injection of morphine is, on the whole, to be preferred to opium. The great indication for it. is when cardiac pain and distress keep the patient awake. Often it acts like a charm, a quiet refreshing sleep being the result of a single injection. No doubt it is a cardiac depressant, but we have to set against this the exhaustion of pain and insomnia. Still, if the patient is very ill, these two factors must be carefully balanced. [It is quite probable that in small doses administered hypodermatically morphine is a cardiac stimulant.] It likewise 
often relieves the pain of aneurism and intra-thoracic growths. Its depressant effect may be to some extent counterbalanced by combining belladonna with it.

Vessels.-Opium is an excellent hæmostatic. It is probably efficient after absorption, but its great value is in gastric and intestinal hæmorrhage, when it acts partly by stopping peristaltic movements. An excellent form in which to give it is the Pilula Plumbi cum Opio, [B. P., powdered opium, I; lead acetate, 6 ; dose, 2 to $4 \mathrm{gr}$.; . I 2 to $.25 \mathrm{gm}$.] It is also very useful in hæmoptysis, for it acts as a hæmostatic, and relieves cough.

Respiration.-It will be remembered that opium depresses the respiratory centre; therefore, by diminishing the activity of the centre for the reflex act of coughing, it will often alleviate the distressing symptoms, but it is only justifiable to give it when the irritation which reflexly sets up a cough is irremovable, as in intra-thoracic growth or aneurism, or when there is little or no lividity and yet the cough is violent, as is often the case in pleurisy. The liability to lividity and asphyxia in many diseases attended with cough must never be forgotten. Thus opium is quite inadmissible in the last stages of bronchitis and pneumonia, and, as a rule, in even the earlier stages of these diseases other means of relieving the cough should be tried first ; and if opium is given, it must be administered with great caution and judgment. But in pneumonia without lividity it is very useful, lessening cough and pain, and promoting sleep. A "linctus opiatus," a favorite remedy, is often given at night when a cough keeps the patient awake. It may consist of tincture of opium, I ; diluted sulphuric acid, I ; treacle, I 5 ; water to [30 parts]. The object of the treacle is to soothe the pharynx locally. Opium must also be given cautiously for [the relief of the symptom] asthma, as there is in this disease a great liability to the growth of a permanent opium habit. ' [Codeine is preferable to all other preparations and alkaloids of opium for relief of cough in pulmonary diseases.] An insufflation of one-half grain $[.03 \mathrm{gm}$.$] of morphine acetate with 5 \mathrm{gr}$. [.30 gm.] of starch is of great use when blown on to a larynx painful from 
organic disease. A grain [.06 gm.] of boric acid or of iodoform is often added to each insufflation.

Nervous system-Brain.-It is in its action on this organ that the marvellous value of opium is seen, its great function being to relieve pain and to produce sleep when that is prevented by pain. For these purposes it is best given hypodermatically as morphine, for that acts more quickly, more certainly, and is less liable to produce indigestion and excitement than opium. Many [prefer] to inject a solution containing $\frac{1}{56} \mathrm{gr}$. [.001 $3 \mathrm{gm}$.] of atropine sulphate to each $1 / 2 \mathrm{gr}$. [.03 gm.] of morphine salt, for by so doing the liability of morphine to upset the stomach and bowels is diminished and its efficacy as an anodyne is not sensibly lessened. It would be a long list to give all the diseases the pain of which can be relieved by morphine; cancer and fractures are typical instances. Morphine is very valuable for the insomnia of acute diseases; but it should never be prescribed for habitual sleeplessness, for fear the patient should contract the habit of opium taking-unless the disease causing the insomnia is incurable, when the use of opium is quite justifiable. It should not be given in gout, for that is often accompanied by granular kidneys; nor for hysteria, for often it does not relieve hysterical pains, and an opium habit may be formed. It is especially useful in renal and biliary colic, and for the afterpains of a confinement. In these cases it relieves the pain partly from its power as an anodyne, and also because by its paralyzing effect on unstriped muscle it relaxes the muscular contraction. This property also makes it valuable in some cases of spasmodic stricture of the urethra. It may be given as a sedative in delirium tremens and some forms of mania, but often such large doses are required that its use is not justifiable. Patients suffering great pain can take enormous doses without any symptoms of poisoning.

Spinal cord.-It has been used for pains of locomotor ataxia and occasionally in convulsive diseases, but without much success.

Kidneys. - It should always be remembered that morphine is excreted with difficulty, if the kidneys are diseased. There are several cases recorded in which persons suffering from Bright's 
disease have been killed by quite small doses of opium. But it often so markedly relieves uræmic dyspnœa, uræmic insomnia, the cardiac dyspnœa which may complicate Bright's disease, and even uræmic convulsions, that it may be justifiable to inject $1 / 6$ of a grain [.or gm.] of a morphine salt subcutaneously into a patient suffering from one of these conditions and run the slight risk there is of poisoning him. But it is clear that this treatment must be adopted very cautiously.

Skin.-Combined with ipecacuanha [as in] Dover's powder, opium is commonly given as a mild diaphoretic, in cases of slight inflammatory disorder, such as a common cold.

Metabolism. - Opium is administered to persons suffering from diabetes, and the amount of sugar in the urine certainly diminishes and the patient's general health improves [but, however, codeine is preferable.] Opium can, in the opinion of many, control all varieties of inflammation, therefore it is given for a cold in the head, for cystitis, pleurisy, etc. Occasionally persons taking [morphine] suffer from retention of urine. We have indicated the occasions on which opium and morphine are respectively preferable.

[Heroine (not official) is morphine diacetic ester, which as a hydrochlorate is freely soluble in water and alcohol. It generally produces no disagreeable symptoms, beyond headache, and is said not to give rise to habituation. It occasionally produces violent and uncontrollable vomiting. Since it diminishes the sensitiveness of the respiratory centres to an excess of carbon dioxide in the blood (Wood) it is useful in some forms of dyspnœa. It is of great value in quieting cough. The dose is $\frac{1}{20}$ to $\frac{1}{5} \mathrm{gr}$.; .003 to $.012 \mathrm{gm}$.

Dionine (not official) is morphine mono-ethyl ester hydrochlorate, which is readily soluble in water. It is somewhat hypnotic, and like heroine is useful to allay cough. It is said to check night sweats. The dose is $1 / 6$ to $\mathrm{I} / 4 \mathrm{gr}$.; . or to $.015 \mathrm{gm}$.

Peronine (not official) is morphine benzylic ester hydrochlorate, soluble in water. This is hypnotic, producing sound sleep without previous excitement, and is useful in allaying the 
cough of pulmonary tuberculosis, chronic bronchitis and pertussis. Dose, $\frac{1}{15}$ o gr.; $.0004 \mathrm{gm}$. in pill.]

\section{Toxicology.}

Acute poisoning. - There may be slight preliminary excitability; but soon drowsiness sets in. This is followed by incapacity for exertion, sleep, and finally deep coma. The pupils are minutely contracted. At first the patient can be roused; but soon no stimulation will do this. Reflex action is abolished. The skin is cold, the face and lips are livid, and toward the end bathed in sweat. The pulse is weak and slow. The respiration becomes slower and more irregular; and last it is stertorous, and the patient dies from asphyxia.

Diagnosis of poisoning by Opium.-1. From Alcoholic poisoning.-Often very difficult, especially if, as commonly happens, the man poisoned with opium has taken alcohol or had it given to him. The pupils are more contracted in opium poisoning. The patient is more easily roused in alcohol poisoning. Examine the urine for morphine and alcohol. Get a careful history. 2. From cerebral hamorrhage.-If this is in the pons Varolii, the pupils may be very contracted and the diagnosis difficult, but look carefully for local paralysis. Usually cerebral hæmorrhage takes place into the internal capsule, and then the face and the limbs on the opposite side are paralyzed. If the hremorrhage is a small one, and especially if it is in the pons, the temperature may be raised; if it is a very large one, the temperature falls for the first few hours, but may rise subsequently. If the pupils are unequal, the case is one of cerebral hæmorrbage. 3. From Carbolic Acid poisoning, in which there may be coma and contracted pupils. The acid produces white patches in the mouth, and the odor is characteristic. 4. From Chloroform and Ether poisoning, by the odor of the breath and of the vomited matters. 5. From uramia, by the signs of Bright's disease, especially albuminuria. 6 . From diabetic coma, by the smell of the breath and the glycosuria. 7. From the comatose stage of an epileptic fit, by the history, the dilatation of the pupils, and the fact that the lividity does not deepen. 8. From the same stage of a fit in general paralysis of the insane and other nervous diseases, by the same symptoms.

Post-mortem. - The appearances after death from Opium poisoning are those al ways found after fatal asphyxia.

Treatment.-Wash out the stomach. [This should be repeated at frequent intervals, because the morphine, which has been absorbed, is excreted into the stomach.] Give prompt emetics (see p. 139), as apomorphine [hydrochlorate] subcutaneously. Always arouse the patient by walking him about, flapping him with a towel, pinching him, applying the faradic current, and putting ammonia to the nose; a pint [500. c.c.] of strong coffee should be injected into the rectum, $\frac{1}{20} \mathrm{gr}$. $[.003 \mathrm{gm}$. ] of atropine sulphate given subcutaneously, or 30 minims, [2. c.c.] of tincture of belladonna by the mouth, re- 
peated every quarter of an hour. [Instances of recovery from opium poisoning followed by death from the belladonna or atropine, used as an antidote, have been observed. Great caution must be exercised if this antidote be used,if indeed it should be employed at all.] If the breathing is very difficult, artificial respiration should be employed. Oxygen or amyl nitrite inhalations may be used. The treatment must be kept up for several hours if necessary. [Potassium permanganate, well diluted, bas been successfully used in an amount equal to that of the alkaloid ingested; it almost immediately destroys the latter, through its chemical action. It is claimed that it can act upon the poison when in the blood so that a hypodermatic injection of it even for some hours after its ingestion may afford relief. Wood, however, has found that its hypodermatic injection does no good in morphine poisoning. The reports have been so generally favorable that potassium permanganate should be used immediately].

Chronic Morphine poisoning.-As many persons adninister this drug subcutaneously to themselves, chronic poisoning is very common. The symptoms are that the patient loses all sense of right and wrong, he will lie and thieve in the most degrading way, especially if his desire is to oltain the drug, and absolutely no statement that he makes can be trusted. He neglects his work, and lets his business go to ruin. He wastes and becomes anæmic, he suffers from loss of appetite, indigestion, dry month, sluggish bowels, and a foul tongue. The nails are brittle, the skin is dry, the hair turns gray early, and falls out. There is sexual impotence, no erections take place, no semen is secreted, there is amenorrhœa, and the flow of milk is stopped, but there is polyuria. The pupils are small, there is loss of muscular pawer, slight ataxia and tremor in severe cases. The arm, [leg, or portions of the body] are scarred with marks of the [needle]. Even $20 \mathrm{gr}$. [1.20 gm.] of morphine may be taken each day.

[ Treatment.-] The patient must be isolated and carefully watched [so] that he gets no morphine (he often eludes or bribes his nurse); it should be diminished gradually, so that at the end of a fortnight he is taking none. If it is stopped suddenly there may be serious collapse and wild delirium. Relapses are very common, and a complete cure after a relapse is very rare. [No reliance can be placed upon any of the advertised cures for the morphine habit; most contain morphine and the remainder are useless. The patient on entering an institution for his cure must be most thoroughly and carefully searched so that he shall not be possessed of a supply sufficient to keep him comfortable during his treatment.

\section{Antagonism.}

Atropine.-Atropine [see p. 373] is a valuable antidote to morphine, because it powerfully stimulates the respiratory centre. It also stimulates the cerebral convolutions and intestinal peristalsis, both depressed by morphine. It appears to be antagonistic to opium in other particulars, but is not really so. Thus, although it prevents perspiration and dilates the pupil, these effects are 
due to action on the peripheral nerve terminations, while morphine produces contrary results by acting on the central nervous system. Still it has been found that some of the undesirable effects that may follow the subcutaneous injection of morphine, such as indigestion, constipation and cardiac depression,

may be avoided if ${ }_{I \frac{1}{5} 0}$ to $\frac{1}{100} \mathrm{gr}$; [ .0004 to $.0006 \mathrm{gm}$.] of atropine sulphate is injected at the same time.

CODEINA.-Codeine. An alkaloid obtained from Opium. $\mathrm{C}_{18} \mathrm{H}_{21}$ $\mathrm{NO}_{3}+\mathrm{H}_{2} \mathrm{O}=[3 \mathrm{I} 6.3 \mathrm{I}$. Synonym.-Methyl Morphine.

SoURCE. - Obtained by evaporating the ammoniacal liquids, remaining after the precipitation of Morphine by Ammonia in the preparation of the Hydrochlorate, treating the residue with water, precipitation with Caustic Potash, and purifying by dissolving in Ether and letting the Codeine crystallize out on spontaneous evaporation.

Characters. - White, or nearly translucent, orthorhombic prisms, or octahedral crystals, having a faintly bitter taste, and slightly efflorescent in warm air. Solubility. - In 80 parts of water; in 3 of Alcohol.]

Dose, $1 / 4$ to $2 \mathrm{gr}$.; [.015 to .12 $\mathrm{gm}$.]

\section{Action and Therapeutics of Codeine.}

Codeine may produce tremors because it excites the cord more, and depresses the higher faculties less, than morphine, and in man its physiological action is in all respects much less than that of morphine. It often relieves the hacking cough of phthisis. It is also used for ovarian pain and to diminish the glycosuria in diabetes [which it does more effectually than opium itself and as an analgesic. It is an excellent substitute for morphine as an ingredient of cough mixture. For diabetes it is usually given as a pill, or in a syrup.] The phosphate (not official) has the advantage of being much more soluble than codeine.

[The following non-official alkaloids are sometimes used :-

Thebaine. Synonym.-Paramorphine. It is contained in Opiun, 0.15 to I per cent. It occurs in silvery scales or hard prisms, odorless and having a bitterish taste; soluble in Ether, Alcohol and Chloroform.] This produces powerful convulsions as the result of its action on the cord. Its subsequent depressant action is very slight.

[Narceine.-It is contained in Opium 0.1 to 0.7 per cent., and occurs in long quadrangular prisms, or white, silky needles, sparingly soluble in Alcohol, and in water. It closely resembles morphine in its action, but is probably more hypnotic and not so likely to be followed by disagreeable after-effects.]

Anarcotine. - This is also known as narcotine, which is an [improper] 
name, for the drug does not cause sleep. [It is contained in opium 1.3 to ro. per cent., the amount varying greatly according to the source]; it is the chief constituent of Indian opium. [It exists in a free state in Opium from which it is left behind when the drug is treated with water. It crystallizes in white, silky, flexible needles which are tasteless and odorless. It is antiperiodic in its action and is valuable not only as a preventive, but as well cures malarial fevers.]

APOMORPHINAE HYDROCHLORAS. - Apomorphine Hydrochlorate. $\mathrm{C}_{17} \mathrm{H}_{27} \mathrm{NO}_{2} \mathrm{HCl}[=302.79$.

SOURCE. - It is the hydrochlorate of an [artificial] Alkaloid obtained by heating Morphine in sealed tubes with an excess of Hydrochloric Acid. The Morphine loses one molecule of water thus: $\mathrm{C}_{17} \mathrm{H}_{19} \mathrm{NO}_{3}=\mathrm{C}_{17} \mathrm{H}_{17} \mathrm{NO}_{2}+\mathrm{H}_{2} \mathrm{O}$.

Characters. - [Minute, grayish-white, shining, acicular crystals, hav ing a faintly bitter taste, and acquiring a greenish tint on exposure to light and air. Solubility. - In 45 parts of water; in about 45 parts of Alcohol.]

Dose, $\frac{1}{25}$ to $\frac{1}{6}$ gr.; [.0027 to .or gm.] hypodermatically, $\frac{1}{20}$ to $\frac{1}{10} \mathrm{gr}$.; [.003 to $.006 \mathrm{gm}$.] by the mouth.

\section{Action of Apomorphine Hydrochlorate.}

\section{External.-None.}

Internal.-Gastro-intestinal tract. - Apomorphine is the most powerful emetic we possess. It does not act locally on the stomach, but solely on the vomiting centre in the -medulla. It is therefore an indirect emetic. This is shown by the fact that when the drug is injected subcutaneously it produces violent vomiting if the vessels are so tied that none can reach the stomach, but not if they are so tied that it cannot reach the medulla.

Circulation.-Therapeutic doses have no effect beyond the depressing action which may be attributed to the vomiting. Large doses cause a rise in the rate of the pulse, probably from stimulation of the accelerator nerves, and with fatal doses the pulse-rate falls, because the drug directly paralyzes the cardiac muscle.

Respiration. - This is at first stimulated by the act of vomiting. The effect of poisonous doses is doubtful ; probably they depress respiration. [Physiological experiments show that it produces a watery discharge from the blood-vessels of the respiratory mucous membrane, which is found to be paler after the administration of this remedy, as well as less œdematous. This effect is produced within a half hour after ingestion, and it is not in any respect the first stages of emesis.] 
Nervous system. - The first result of toxic doses is to cause delirium. Finally there is paralysis of the motor nerves, and consequently of the muscles.

\section{Therapeutics of Apomorphine Hydrochlorate.}

Internal.-Vomiting action. - The advantages of apomorphine over the other emetics are that it is certain, prompt, and powerful ; it can be given when emetics introduced directly into the stomach would not act, and it does not irritate the stomach. It is largely used in cases of poisoning. It is usually given hypodermatically, [dissolved in camphor water; 1 in 50 . Dose, 4 to 8 minims; .25 to .50 c.c. This must be prepared extemporaneously, as it will not keep.]

Expectorant action.-It is, when given by the mouth, a valuable expectorant for bronchitis. [In an adult $\frac{1}{32} \mathrm{gr}$, ,.002 gm., or I dr., 4. c.c., of the syrup (see below) will produce a watery expectoration within the time above stated, and this effect will last from two to three hours. It is particularly useful in the early stages of acute bronchitis, in chronic dry bronchitis, in chronic catarrhal pneumonia, and in old tuberculous patients who are harassed by an unproductive cough.

Soporific action.-Recently it has been claimed that when given hypodermatically at bedtime, in dose just short of producing emesis, sleep, closely approaching the normal, ensues. This is not always the case, and its hypnotic action may be due to contamination with other alkaloids.]

The British Pharmaceutical Conference recommends the following Syrup of Apomorphine: Mix Rectified Spirit, 84; with the same amount of water; dissolve in this Apomorphine $\mathrm{Hy}$ drochlorate, I ; add Diluted Hydrochloric Acid, 24 ; and finally Syrup, 1728 parts. Dose, $1 / 2$ to $I$ fl. dr. [2. to 4 . c.c.] The drug may also be given as a lozenge.

\section{HUMULUS.}

HOPS.-[The strobiles of Humulus Lupulus Linné (nat. ord. Urticacex). Habitat.-Northern temperate zone; cultivated.

Characters. - Ovate, about $3 \mathrm{~cm}$. long, consisting of a thin, hairy, undulated axis, and many obliquely ovate, membranous scales, in the upper part 
reticulately-veined, and towards the base parallel-veined, glandular, and surrounding a subglobular achene; color of the scales greenish, free from reddish or brownish spots; odor, aromatic; taste, bitter, aromatic and slightly astringent.]

Compositron.-The chief constituents are-(1) Lupulin. (2) Lupulinic Acid, 11 per cent., a bitter crystalline principle. (3) Valerol, I per cent., an aromatic volatile oil giving the odor. (4) Resin, 9 to 18 per cent. (5) Tannic acid, 3 to 4 per cent.

[INCOMPATIBLES. - Mineral acids, and metallic salts.

\section{Preparation.}

Tinctura Humuli.-Tincture of Hops. Hops, 200 ; by maceration and percolation with Diluted Alcohol to rooo.

Dose, I to 2 fl. dr.; 4. to 8. c.c.]

LUPULINUM.-Lupulin. A glandular powder, separated from the strobiles of Humulus Lupulus Linne (nat. ord. Urticacea).

[CHARACTERS. - Bright, brownish-yellow, becoming yellowish-brown, resinous, consisting of minute granules, which, as seen under the microscope, are subglobular, or rather hood-shaped, and reticulate ; aromatic and bitter.]

Dose, 5 to $30 \mathrm{gr}$.; [.30 to $2.00 \mathrm{gm}$.]

\section{Preparations.}

1. Extractum Lupulini Fluidum.-Fluid Extract of Lupulin. Lupulin, 1000 ; by maceration and percolation with Alcohol, evaporation and solution to 1000 parts.

Dose, 5 to $30 \mathrm{~m}$. ; .30 to 2.00 c.c.

2. Oleoresina Lupulini.-Oleoresin of Lupulin. Lupulin, 100; by percolation with Ether and evaporation.

Dose, $x$ to $5 \mathrm{gr}$; . 06 to $.30 \mathrm{gm}$.]

\section{Action of Hops.}

The volatile oil is stomachic and carminative like other volatile oils. [To a slight extent it reflexly excites the circulation.] The bitter principle aids the stomachic influence. Hops are decidedly soporific. Probably it is the volatile oil that produces this effect.

\section{Therapeutics of Hops.}

The pharmacopœial preparations of hops are not much used, but good beer, because of the hops contained in it, may with some persons aid digestion, and for this purpose is often given 
with meals to those whose digestion is feeble after a long illness, or from any other cause. [The alcohol in the beer increases this effect.] Many people find the soporific influence of beer very well marked. [Lupulin has been used in nervous tremors, wakefulness and in the delirium of drunkards.]

\section{LACTUCARIUM.}

[LACTUCARIUM.-The concrete milk-juice of Lactuca virosa Linné (nat. ord. Compositoc). Synonym.-Lettuce. Habitat.-Southern and Central Europe.

ChARACTERS. - In sections of plano-convex, circular cakes, or in irregular, angular pieces, externally grayish-brown or dull reddish-brown, internally wbitish or yellowish, of a waxy Iustre ; odor heavy, somewhat narcotic ; taste bitter.

Composition.- The chief constituents of Lactucarium are-(1) Lactucerin or Lactucone, $\mathrm{C}_{16} \mathrm{H}_{26} \mathrm{O}$, about $5^{\circ}$ per cent., a crystalline principle. Lactucin, $\mathrm{C}_{11} \mathrm{H}_{12} \mathrm{O}_{3}+\mathrm{H}_{2} \mathrm{O}$, resembling mannit. (3) Lactucic Acid.

Dose, 5 to $60 \mathrm{gr}$. ; .30 to $4.00 \mathrm{gm}$.

\section{Preparations.}

I. Tinctura Lactucarii.-Tincture of Lactucarium. Lactucarium, 500; Glycerin, 250; by treatment with Benzin and drying, then macerate and percolate with water, and Alcohol; evaporate, filter and add diluted Alcohol to 1000 .

Dose, $1 / 4$ to 2 fl. dr.; $x$. to 8 . c.c.

2. Syrupus Lactucarii.-Syrup of Lactucarium. Tincture of Lactucarium, I00; Sugar, 50; Precipitated Calcium Phosphate, 50; triturated together with water, filter, dissolve Sugar, 700 ; and add water to 1000 .

Dose, I to 4 fl. dr. ; 4 . to 15. c.c.

\section{Action and Therapeutics of Lactucarium.}

Lactucarium has been credited with mild hypnotic powers. Large doses of the green extract may cause mental derangement, and will dilate the pupil.]

\section{[PELLOTINUM.}

PELLOTINE (Not official). $-\mathrm{C}_{13} \mathrm{H}_{21} \mathrm{NO}_{3}=238.50$. An alkaloid obtained from the Anhalonium Williamsii (nat. ord. Cactacea). Habitat.Mexico.

Characters. - The hydrochloride, which is to be found in the shops, 
occurs as a colorless, amorphous, intensely bitter powder. Solubility.—Readily in water.

Dose, $1 / 2$ to $\mathrm{I} \mathrm{gr} . ;$; 03 to $.06 \mathrm{gm}$. (Hypodermatically.)

\section{Action of Pellotine.}

Slight narcosis follows its injection in from ten to fifteen minutes (frogs). The reflexes are somewhat diminished. After twenty to thirty minutes there comes a distinct increase of reflex irritability, followed by spasms, resembling strychnine poisoning. If large doses are administered this condition may pass into one of complete paralysis. The pulse rate is slightly diminished, drowsiness and sleep supervene after its administration to man. The drug is probably excreted by the kidneys.

\section{Therapeutics of Pellotine.}

Pellotine has been recently introduced as a hypnotic which, because it is unirritating, can be used hypodermatically. It slows the pulse slightly, induces a feeling of weariness, weight of eyelids and limbs, disinclination to mental and bodily exertion, and a quiet sleep follows. The awakening is easy and usually without untoward symptoms. In full doses, with the patient in an upright position it may give rise to vertigo. It apparently has no effect on the secretions.]

\section{[B. Cerebral Excitants.]}

\section{BELLADONNA.}

BELLADONN $\approx$ FOLIA.-Belladonna Leaves. [The leaves of Atropa Belladonna Linné (nat. ord. Solanacea). Synonym.-Deadly Nightshade. Habitat.-Europe and Asia Minor.

Characters. - Leaves from 10 to $15 \mathrm{~cm}$. long, from 5 to $10 \mathrm{~cm}$. broad, broadly ovate, equilaterally narrowed into a petiole, tapering at the apex, entire on the margin, smooth, thin, the upper surface brownish-green, the lower surface grayish-green, both surfaces whitish, punctate; odor slight, bitterish, disagreeable.] Resembling Belladonna leaves. -Stramonium leaves, more wrinkled; hyoscyamus leaves, hairy.

Composition. - The chief constituents are-(1) Atropine (see p. 373), [(2) so-called Belladonnine, a yellowish powder, is probably identical with Hyoscyamine (see p. 384).] It has been stated that atropine does not exist in Belladonna in the natural state, but that it is a conversion product of hyoscyamine, 
which is the natural alkaloid of Belladonna. [Atropine, hyoscine, (see p. 384), daturine, (see p. 382 ), duboisine and scopolamine, all derived from atropaceous plants, are nearly identical, and exist as malates in the plant. ]

Dose, $x$ to $5 \mathrm{gr}$; ; .06 to $.30 \mathrm{gm}$.]

\section{Preparations.}

1. [Extractum Belladonnæ Foliorum Alcoholicum.-Alcoholic Extract of Belladonna Leaves. By percolation with Alcohol and water, and evaporation.

Alcoholic Extract of Belladonna Leaves is used to make Emplastrum Belladonnæ and Unguentum Belladonnæ.

Dose, $1 / 8$ to $1 / 2 \mathrm{gr}$.; .008 to $.03 \mathrm{gm}$.

2. Tinctura Belladonnæ Foliorum.-Tincture of Belladonna Leaves. Belladonna Leaves, 150; Diluted Alcohol, to 1000. By maceration and percolation.

Dose, 5 to $30 \mathrm{~m}$.; .30 to 2.00 c.c.

3. Unguentum Belladonnæ.-Belladonna Ointment. Alcoholic Extract of Belladonna Leaves, Io; Diluted Alcohol, 5; Benzoinated Lard, 85.

4. Emplastrum Belladonnæ.-Belladonna Plaster. Alcoholic Extract of Belladonna Leaves, 200; Resin Plaster, 400; Soap Plaster, 400. Melt the plaster on a water-bath ; then add the extract of belladonna leaves and continue the heat, stirring constantly, until a bomogeneous mass results. ]

BELLADONN E RADIX.-Belladonna Root. The root of Atropa Belladonna [Linnê (nat. ord. Solanacea). Habitat.-Central and Southern Europe, in woods.

ChARACTERS. - In cylindrical, somewhat tapering, longitudinally wrinkled pieces, 10 to $25 \mathrm{~mm}$. or more in thickness; externally brownish-gray, internally whitish; fracture nearly smooth and mealy, not radiating or showing medullary rays in the thicker roots, only in the layer near the bark; nearly inodorous; taste sweetish, afterwards bitterish and strongly acrid.]

Composition._As of the leaves. Usually contains 0.2 to 0.6 per cent. of alkaloids.

\section{Preparations.}

1. [Extractum Belladonnæ Radicis Fluidum.-Fluid Extract of Belladonna Root. By percolation with Alcohol and Water, and evaporation.

Dose, $\mathrm{I}$ to $3 \mathrm{~m}$.; .06 to .20 c.c.

2. Linimentum Belladonnæ.-Belladonna Liniment. Fluid Extract of Belladonna Root, 950 ; Camphor, 50.] 
ATROPINA.-Atropine. $\mathrm{C}_{17} \mathrm{H}_{23} \mathrm{NO}_{3}[=288.38$. An Alkaloid obtained from Belladonna, which it contains in .06 to . 3 per cent. 'As it occurs in commerce, it is always accompanied by a small proportion of hyoscyamine extracted along with it, from which it cannot be readily separated.

Source. - Atropine is made from the root thus:-(I) Make a tincture of the root by maceration and percolation with Alcohol. (2) Add slaked lime; this splits up the Atropine Malate, Lime Malate being precipitated. (3) Filter, and add Sulphuric Acid to precipitate the excess of Lime. (4) Filter, concentrate by distillation, partially evaporate, add Potassium Carbonate; after six hours much coloring matter is precipitated. (5) Filter, add more Potassium Carbonate ; this sets free the Atropinc. (6) Shake up with Chloroform, which takes up the Atropine in solution. (7) Withdraw the Chloroform, evaporate, and Atropine is left. It is purified by digestion with warm Alcohol and Animal Charcoal.]

ChARACTERS. - [White, acicular crystals, or a more or less amorphous, white powder, without odor, having a bitter, acrid taste, and gradually assuming a yellowish tint on exposure to air. Solubility. - In 130 parts of water, 3 parts of Alcohol, 16 parts of Ether, 4 parts of Chloroform, and about 50 parts of Glycerin.] It can be decomposed into Tropine and Tropic Acid, and reconstructed by their synthesis. It is distinguished from hyoscyamine, with which it is isomeric, by its melting-point, optical properties and molecular constitution.

Incompatibles. - Caustic alkalies decompose it.

Dose, I $\frac{1}{20}$ to $\frac{1}{60} \mathrm{gr}$.; [.0005 to .001 $\mathrm{gm}$.]

ATROPIN $A$ SULPHAS.-Atropine Sulphate. $\quad\left[\left(\mathrm{C}_{17} \mathrm{H}_{23} \mathrm{NO}_{3}\right)_{2} \mathrm{H}_{2}\right.$ $\mathrm{SO}_{4}=647.5^{8}$.

SouRCE. -Dissolve Atropine in Diluted Sulphuric Acid, treat with Ether, the insoluble Sulphate is deposited.

Characters. - A white, indistinctly crystalline powder, having a very bitter nauseating taste. Solubility.-In 0.4 part of water; in 6.2 parts of Alcohol.]

Dose, $\frac{1}{20}$ to $\frac{1}{20} \mathrm{gr} . ;$ [ .0005 to $.003 \mathrm{gm}$.]

\section{Action of Belladonna.}

The action of belladonna and atropine is the same.

External.-Atropine placed by itself upon the unbroken skin cannot be absorbed, but rubbed in with substances which are absorbed, such as alcohol, glycerin, camphor, etc., or applied to a broken surface, it paralyzes the terminations of the sensory nerves, especially if pain is present. It is thus a local anæsthetic and an anodyne. These are its chief actions, but to a much less extent it locally paralyzes the terminations of the 
motor nerves, first contracts and then dilates the vessels, and renders the secretions of the skin less active.

Internal. - Gastro-intestinal tract. - It will be convenient to describe the effects of belladonna on all secretions when speaking of its action on nerves, and we need not mention here its influence on the muscular coat of the intestine, for that is secondary to its action on the nervous system.

Blood.-Atropine is quickly absorbed, but does not affect the blood. Its main action is on the nervous system, and that must be considered in detail.

Secretory nerves. - The activity of the peripheral terminations of all the secretory nerves in the body is, as far as we know, depressed. These nerves fall under the following headings :

(a) Mouth.-Even small doses of atropine make the mouth dry from lack of saliva and mucus. In health, secretion of submaxillary saliva always follows stimulation of the chorda tympani nerve, and, as is weil known, this is due to the fact that this nerve is the secretory nerve for this gland, and not to any vascular dilatation. If atropine be given to an animal, stimulation of the chorda no longer causes an increased flow of saliva, however close to the gland the nerve is excited, the reason being that atropine has paralyzed the terminations of the chorda tympani. In the same way the terminations of the secretory nerves of the other salivary glands and the mucous glands are paralyzed, and hence the mouth is dry, because normal impulses cannot reach the cells of the glands.

Stomach, liver, and intestines. - We do not know what influence atropine has on the secretions of these organs.

Sweat glands.-Atropine paralyzes the terminations of the nerves in the sudoriparous glands. Thus it causes the skin to become dry.

Kidneys. - The effect of atropine on the amount of urine secreted is necessarily uncertain, as the urinary flow depends so much on the secretion of sweat.

Bronchial mucous membrane.-The secretion of bronchial and tracheal mucus, like that of the mouth, is diminished. 
Mammary gland.-The activity of the peripheral terminations of the secretory nerves in the cells of the mammary gland is inhibited, hence the flow of milk, if any is present, is arrested, and belladonna is called an antigalactagogue.

Sensory nerves. - It has already been mentioned that belladonna rubbed into the skin depresses the function of the terminations of the sensory nerves. It does the same when given by the mouth, but its action on sensory nerves-that is to say, its anæsthetic and anodyne action-is very inferior to that on the secretory nerves, and is not powerful enough for atropine to relieve pain when given internally. It is only used as a local anodyne.

Voluntary muscles and their nerves. - Voluntary muscles are quite unaffected even by toxic doses of atropine; towards the end of a case of belladonna poisoning the motor nerves are slightly paralyzed.

Involuntary muscles and their nerves. - The splanchnics are the inhibitory nerves of the intestinal movements, and if they are stimulated, the peristaltic movements stop; impulses are constantly descending these nerves to restrain these movements. If atropine in small doses is given to animals, it is observed that the bowels are relaxed, because intestinal peristalsis is much increased, and that stimulation of the splanchnics is powerless to arrest it ; clearly the drug has paralyzed the terminations of the splanchnics in the involuntary muscles of the intestine. Larger doses stop peristalsis.

All involuntary nerve terminations, as those of the muscles of the bladder, ureters, urethra, vesiculæ seminales, uterus and vagina are paralyzed like those in the intestinal muscles.

The eye and its nerves. - Atropine acts only on the terminations of the nerves in the involuntary muscles of the eye. If it be dropped into the eye or given by the mouth the pupil dilates widely, and cannot be made to contract by stimulation of the third nerve. That this dilatation is not due to any marked action on the muscular fibres of the iris themselves is shown by the fact that the atropinized pupil will contract if the muscle itself be stimulated. Therefore it must be that the terminations of 
the third nerve in the iris are paralyzed. The ending of this nerve in the ciliary muscle is affected in the same way, and consequently accommodation is paralyzed. It is certain that this mydriasis and defective accommodation is in no part central, as is the contraction of the pupil produced by opium. So strong is the local action of belladonna that if atropine be dropped into the recently excised eye the pupil will dilate. When the third nerve is cut the pupil dilates, and if, after this, atropine be dropped into the eye it dilates still further. Some have concluded, from this and other reasons, that atropine also stimulates the terminations of the sympathetic in the iris; but this action is slight. [The pupil dilates because the elastic fibres in the iris have an opportunity to act.] The intra-ocular tension is increased by large doses. There is, as a result of the paralysis of the ciliary muscle, disturbance of vision. Atropine does not act on the pupils of birds.

The heart and its nerves. - The main action of atropine is to paralyze the terminations of the vagus in the heart, and consequently the pulse is rendered more rapid, and cannot be slowed by strongly stimulating the vagus. If the rate of the heart has been lowered by [muscarine], which can be shown to have a local stimulating influence on the termination of the vagus in the heart, the application of atropine renders the heart quick again, the two drugs being, in their effect on the heart, exactly antagonistic. This quickening of the pulse from inhibition of the vagal cardiac terminal filaments is the chief action of atropine on the heart, but the following minor actions must be noticed. The vagus centre and the trunk of the nerve are also depressed, but to a much less extent. Before the pulse is quickened it is occasionally slowed for a short time by atropine; this is probably owing to a brief excitation of the vagus centre. Some authorities believe that part of the quickening of the pulse is due to a slight stimulation of the cardiac accelerator nerves, just as we have seen that some consider that the sympathetic fibres in the iris are excited; but if the accelerator nerves are stimulated, the stimulation is quite subsidiary to the important paralysis of the vagal terminations. Although the pulse is quickened by bella- 
donna, its force is not diminished. Toxic doses abolish the function of the cardiac muscle, and the heart stops in diastole.

Vaso-motor system and its nerves. - After a considerable dose of belladonna the skin is flushed, and a scarlatiniform erythematous rash may be present in belladonna poisoning. It is thus obvious that such a dose of belladonna relaxes the peripheral vessels. The exact cause of this has not definitely been made out, but it is extremely probable that it is largely a peripheral action, quite harmonizing with the peripheral action we have seen atropine to have on the involuntary muscles of the intestines, eye and heart ; that is to say, the vaso-constrictor nerve-filaments supplying the arterioles are paralyzed, and consequently the vessels dilate. The action of atropine on the medullary vaso-motor centre is more marked than that on the cardiac medullary centre ; but it is the same, - the centre first being stimulated, and then depressed. The primary stimulation is sufficient to overcome the tendency of the peripheral vessels to dilate, so that belladonna at first contracts them; and as this stage of contraction lasts well into the period during which, owing to paralysis of the vagal terminations, the heart is accelerated, the blood-pressure rises considerably; subsequently it falls, the fall being due to the depression of the vaso-motor centre and the peripheral action of belladonna on the vessels, causing their wide dilatation. Ultimately, when the heart itself is paralyzed, the blood-pressure is very low. The spinal vaso-motor centres are acted on as powerfully and in the same way as the medullary centre.

Respiration and its nerves. - Here also belladonna paralyzes peripheral nerve-filaments, in this case those of the vagus in the bronchial tubes. Both the afferent and efferent pulmonary vagal fibres are effected. The result is that the muscular coat of the bronchial tube is relaxed, and that the secretions (the activity of the afferent fibres being depressed) do not irritate the nerves so much as before, and therefore cough is lessened. It will be remembered that the quantity of bronchial secretion is diminished. The medullary and spinal respiratory centres are influenced precisely as the vaso-motor,-that is to say, they are 
first stimulated, and so the respirations are quicker and deeper, then large doses paralyze them, and the breathing is slow and shallow. The patient becomes asphyxiated, and this contributes to the result in a fatal case.

Temperature. - This is decidedly raised by toxic doses of belladonna (it may be $4^{\circ}\left[\mathrm{F}\right.$., about $2^{\circ} \mathrm{C}$. $]$ or more). This rise is independent of the blood-pressure and of the diminution of perspiration. It is said that heat-production is greatly exaggerated. The heat-loss is also increased, probably because the flushing of the skin leads to a greater loss by radiation.

Spinal cord.-Except for the action on the vaso-motor and respiratory spinal centres, belladonna has little influence on the spinal cord in man, but has a well-marked tetanizing effect in frogs. It is said slightly to increase and afterwards diminish general reflex excitability.

Cerebrum. - A considerable dose of belladonna causes delirium, showing that the higher centres are stimulated. Generally the stimulation takes place inco-ordinately. That it is powerful is indicated by the fact that in poisoning by belladonna the delirium will last for a long while. The subsequent quietude is not more than the exhaustion of the cerebrum from the continued delirium will explain. Belladonna rarely, if ever, produces genuine coma. Other symptoms that may be observed with large doses, and which are probably due to disorder of the brain, are staggering gait, giddiness and occasionally convulsions.

Elimination. $-\Lambda$ tropine is probably eliminated entirely by the kidneys.

It will be seen that the dominant action of belladonna is to depress the activity of the terminations of nearly all varieties of nerves. In addition, it first stimulates and then depresses the three great medullary centres, and it is a deliriant. A summary of its effects on man will be given under the heading of Toxicology.

Children can take considerable doses of belladonna without any symptoms of poisoning. Pigeons and rodents are. peculiarly insusceptible to it. 


\section{Therapeutics of Belladonna.}

Externally.-Belladonna is used externally to relieve all sorts of pain,-for example, that of neuralgia, pleurodynia, and chronic osteo-arthritis. Chloroformum Belladonnæ (Brit. Pharm. Conf.), - the root being extracted with ammonia and chloroform, -diluted with a little olive oil, or the liniment is excellent for these purposes. A glycerin preparation (made by rubbing [the alcoholic] extract of belladonna [leaves], 4 ; with boiling water, I ; and then adding gradually glycerin, I 2), soothes the pain of acute inflammations. This, or the plaster, or the ointment, is very efficacious in preventing the secretion of milk in women who do not for any reason nurse their infants. Pruritus and local sweating of various parts of the body, especially the feet, may sometimes be stopped by the application of belladonna liniment. A solution of atropine sulphate, 4; boric acid, 5; in water to 480 ; will dilate the pupil for ophthalmoscopic examination. Atropine is often used in ophthalmic practice to paralyze the movements of the iris and ciliary muscle, to break down adhesions, and to prevent the formation of contractions of the iris. [It must not be used if the patient is suffering from glaucoma.] See also Homatropine [Hydrobromate, p. $3^{81}$ ].

Internal.-Alimentary canal. - [Belladonna] has occasionally been employed to check salivation, and some use it to overcome constipation and colic. The alcoholic extract [of the leaves] is then given, and is commonly combined with some purgative in a pill. [This] extract in the form of a pill is often administered with opium to patients suffering with appendicitis or peritonitis; as it is given several times a day a large amount is taken, and this, as already explained, probably paralyzes intestinal movements, and so aids the opium.

Skin.-Atropine sulphate ( $\left.\frac{1}{100} \mathrm{gr} . ; .0006 \mathrm{gm}.\right)$ injected subcutaneously, or one or two minims [.06 to .i2 c.c.] of the solution of atropine sulphate [ $\mathrm{I}$, in camphor water, I00.], by the mouth will sometimes arrest sweating, and this treatment may succeed with the night-sweats of phthisis.

Circulation.-There are many cases of heart disease in which belladonna may advantageously be combined with other drugs. 
Whenever we wish to empty the ventricle completely it is useful, for it will be remembered that it increases the rapidity of the heart without diminishing the force. But its greatest value is to remove cardiac pain and distress, which it often does most effectually. It may be conveniently applied as a plaster over the cardiac region, or it may be given internally, usually as the tincture [of the leaves].

Respiration.-As belladonna relaxes the muscular coat of the bronchial tubes, it is of great value in spasmodic affections of the respiratory passages. Thus, of all the numerous drugs that have been given for whooping-cough, it is the best. It is also very useful [to relieve the symptom] asthma, and in bronchitis with asthma-like paroxysms; in the last named [condition] its powerful stimulation of the respiratory centre and its capability of dininishing the secretion will, in properly chosen cases, render it particularly valuable. It is generally given as a tincture [of the leaves], and combined with other drugs. A useful linctus contains the tincture, 3 ; vinegar of squill, 5 ; syrup of tolu, Io; glycerin to 60 parts.

Genito-urinary diseases.-Belladonna is one of the favorite remedies for the nocturnal incontinence of children, and it occasionally overcomes this trouble in adults when it is not due to organic diseases. [It relieves eneuresis because it has an anodyne effect upon the centres in the cord and when excreted in the urine anæsthetizes the neck of the bladder.] Its power of relieving the spasm of involuntary muscle is well shown in the effectual manner in which the very painful vesical spasm which accompanies calculus, cystitis, and prostatitis may be benefited by it. It may be given internally as in the form of a suppository, or applied as a plaster to the perinæum.

It has been tried in many nervous diseases, but without any good results.

\section{ToxiCOLOGY.}

[Symptoms.]-If a person takes a moderate dose of belladonna he soon experiences dryness of the mouth and throat, and as the food, therefore, cannot be properly lubricated, there is difficulty of swallowing ; the pulse may at first be a little slower than usual. The pupil is dilated; accommodation is defective, and vision confused. The skin feels dry. If the dose has been a large one, these 
symptoms all come on quickly; the conjunctivæ and face, and perhaps other parts of the skin are flushed, and the rate of the pulse is greatly increased, it may even be doubled. The patient staggers, feels giddy, and reels when he walks; the throat soon becomes very hot, the skin still more flushed, the eyelids swell, and there may be a uniform erythematous rash. The temperature is often raised, the respirations are slow and deep. The pupils are very widely dilated. By this time the patient is quite delirious. There may be purging, but this is not common; and sometimes he complains of a frequent desire to micturate, although he is unable to pass any urine. Death takes place from cardiac failure combined with asphyxia. Post-mortem.-The organs are all in a state of venous congestion, which is due to the asphyxia. If recovery takes place the patient may have no recollection of his illness.

Treatment._Give emetics (see p. 139) or wash out the stomach. Inject pilocarpine and stimulants subcutaneously. Employ artificial respiration and hot bottles, and give strong coffee per rectum.

\section{Antagonism.}

The antagonism between atropine and morphine has already been discussed (see p. 365). It is clear that as pilocarpine stimulates the terminations of the secretory nerves in the salivary and sweat glands, and also excites the terminations of the third nerve in the iris and ciliary muscle, it is a diaphoretic, a sialagogue, and a myotic, and is in these respects antagonistic to atropine. Physostigmine also causes contraction of the pupil and spasm of the ciliary muscle by stimulation of the terminations of the third nerve, and it depresses the respiratory centre almost from the beginning. In these points it is an antagonist to atropine.

[HOMATROPINAE HYDROBROMAS. - Homatropine Hydrobromate., B. P., not official.] $\mathrm{C}_{16} \mathrm{H}_{21} \mathrm{NO}_{3} \mathrm{HBr}[=355.17]$. The hydrobromate of an alkaloid prepared from Tropine. [Homatropine is really Oxytoluyltropine.]

SouRCE. - $\left[(1)\right.$ Tropine, $\mathrm{C}_{8} \mathrm{H}_{15} \mathrm{NO}$, a derivative of Atropine, is heated with Oxytoluic Acid in the presence of Hydrochloric Acid ; (2) Ammonia is added, and pure Homatropine shaken out with Chloroform; (3) the Chloroformic solution is evaporated ; (4) the Homatropine is neutralized with Hydrobromic Acid.

Characters.--Small prismatic white crystals. Solubility.-In to parts of water ; in 133 parts of Alcohol.]

Dose, ${ }_{1}^{\frac{1}{20}}$ to $\frac{1}{20} \mathrm{gr}$; [ [.0005 to $.003 \mathrm{gm}$.] subcutaneously.

\section{Action and Therapeutics of Homatropine Hydrobromate.}

Homatropine [hydrobromate] has an action exactly similar, so far as we know, to that of atropine. It is only used to dilate 
the pupil in ophthalmic practice, the advantage over atropine being that the dilatation produced by homatropine passes off in a quarter of the time. It may be applied as a solution, [ $\mathrm{r}$ in] distilled water [1 20.] Sometimes a solution in castor oil is used, for it is less likely to be washed out by the tears, but it may be rather irritating.

\section{STRAMONIUM.}

STRAMONII SEMEN.-Stramonium Seed. [The seed of Datura Stramonium Linné (nat. ord. Solanacea). Synonyms. - Thorn apple. Stink: weed. Jamestown Weed. Habitat._Asia; naturalized in most countries.

Characters. - About $4 \mathrm{~mm}$. long, reniform, flattened, pitted and wrinkled ; testa dull, brownish-black, hard, inclosing a cylindrical, curved embryo, imbedded in a whitish, oily perisperm; of an unpleasant odor when bruised, and of an oily and bitter taste.]

Composition. - The chief constituents are-(1) Daturine (.02 to .03 per cent.) which resembles Atropine but is [probably identical with] Hyoscyamine. Usually a little Atropine is present, and the term daturine is occasionally applied to the total alkaloids of Stramonium. (2) [A fixed oil (25 per cent.), which contains Daturic Acid, $\mathrm{C}_{17} \mathrm{H}_{34} \mathrm{O}_{2}$.]

InCOMPATIBLEs. - Caustic alkalies, metallic salts, and mineral acids.

Dose, I to $3 \mathrm{gr}$; ; [.06 to .20 gm.]

\section{Preparations.}

I. Extractum Stramonii [Seminis.-Extract of Stramonium Seed. By maceration and percolation with Diluted Alcohol, and evaporation.

Extract of Stramonium Seed is used to prepare Unguentum Stramonii.

Dose, $1 / 4$ to $1 / 2 \mathrm{gr}$; .015 to $.03 \mathrm{gm}$.

2. Extractum Stramonii Seminis Fluidum.-Fluid Extract of Stramonium Seed. By maceration and percolation with Alcohol and Water, and evaporation.

Dose, 1 to $3 \mathrm{~m}$.; .06 to .20 c.c.

3. Tinctura Stramonii Seminis.-Tinctura of Stramonium Seed. Stramonium Seed, 150; Diluted Alcohol, by maceration and percolation to 1000 .

Dose, 5 to $30 \mathrm{~m} . ; \cdot 30$ to 2.00 c.c.

4. Unguentum Stramonii.-Stramonium Ointment. Extract of Stramonium Seed, Io; Diluted Alcohol, 5 ; Benzoinated Lard, 85.] 
STRAMONII FOLIA.-Stramonium Leaves. [The leaves of Datura Stramonium Linnê (nat. ord. Solanacea).

Characters. - About $15 \mathrm{~cm}$. long, petiolate, dark-green, smooth, ovate, pointed, unequal, especially at the base, coarsely and sinuately toothed ; thin, brittle, and nearly inodorous; taste unpleasant, bitter and nauseous.] Resembling Stramonium Leaves. - Belladonna leaves, but less wrinkled; hyoscyamus leaves, hairy.

Composition.-As of the seeds; the proportion of alkaloids is very inconstant.

Dose, I to $5 \mathrm{gr}$; .06 to $.30 \mathrm{gm}$.]

\section{Action of Stramonium.}

The physiological action of stramonium is precisely that of belladonna; the difference being that stramonium relaxes the muscular coat of the bronchial tubes more powerfully than belladonna, and it may cause the heart to be a little irregular, and is generally thought to be more active than belladonna.

\section{Therapeutics of Stramonium.}

There is no reason why stramonium should not be employed for the same purposes as belladonna, but it is rarely used, except in cases of [the existence of the symptom] asthma, to relieve the spasm of the bronchial tubes. For this it is very valuable. Cigarettes of the leaves may be smoked, or the drug may be given internally. The following powder, which gives off dense fumes if burnt, is said to afford great relief for asthma :-Leaves of Datura Stramonium, Datura Tatula [not official], Cannabis Indica, and Lobelia Inflata, all in powder, of each 12 ; nitre in powder, 16 ; oil of eucalyptus, $\mathbf{r}$. Mix thoroughly. Himrod's, Bliss', and other "cures" for asthma are of similar composition.

\section{HYOSCYAMUS.}

HYOSCYAMUS.-[Synonym.-Henbane. The leaves and flowering tops of Hyoscyamus Niger Linné (nat. ord. Solanacee) collected from plants of the second year's growth. Habitat.--Europe and Asia; naturalized in some parts of North America.

ChARACTERS. - Leaves ovate, or ovate-oblong, up to $25 \mathrm{~cm}$. long and ro $\mathrm{cm}$. broad; siruate-toothed, the teeth large, oblong or triangular; grayishgreen and, particularly on the lower surface, glandular-hairy; midrib promi- 
nent; flowers nearly sessile, with an urn-shaped, five-toothed calyx, and a light yellow purple-veined corolla; odor heavy, narcotic; taste bitter and somewhat acrid.]

Composition.-The chief constituents are-(I) Hyoscyamine, $\mathrm{C}_{17} \mathrm{H}_{23} \mathrm{NO}_{3}$, an alkaloid. It is very closely allied to the active alkaloids of belladonna and stramonium (see p. 382 ) and is also contained in many plants of the natural order Solanacea. It, like Atropine, with which it is isomeric, consists of Tropic Acid and Tropine. There is in commerce an amorphous impure $\mathrm{Hy}-$ oscyamine, which is a dark brown extract-like fluid having a disagreeable odor. As it is much cheaper than the crystalline alkaloid, it is often used, [but this as well as other specimens should be proscribed.] (2) Hyoscine [see p. $3^{8} 5$ ], a white crystalline alkaloid. It is [stated to be] the same as Scopolamine, an alkaloid isomeric with cocaine. Only its salts are used.

Incompatibles._Vegetable acids, silver nitrate, lead acetate, and alkalies.

Dose, 5 to $15 \mathrm{gr} . ;$ [.30 to $1.00 \mathrm{gm}$.]

\section{Preparations.}

1. Extractum Hyoscyami. - [Extract of Hyoscyamus. maceration and percolation with diluted Alcohol and evaporation.

Dose, $1 / 2$ to $2 \mathrm{gr}$; ; 03 to .12 gm.]

2. Extractum Hyoscyami Fluidum.-[Fluid Extract of Hyoscyamus. By maceration and percolation with Alcohol and water, and evaporation.

Dose, 5 to $15 \mathrm{~m} . ; .30$ to 1.00 c.c.]

3. Tinctura Hyoscyami,-[Tincture of Hyoscyamus. Hyoscyamus, I50; diluted Alcohol to 1000 . By maceration and percolation. Dose, I c.c. $(15 m)$.]

[HYOSCYAMIN $\approx$ SULPHAS.-Hyoscyamine Sulphate. $\left(\mathrm{C}_{17} \mathrm{H}_{23}\right.$ $\left.\mathrm{NO}_{3}\right)_{2} \mathrm{H}_{2} \mathrm{SO}_{4}=674.5^{8}$. The neutral sulphate of an alkaloid prepared from IHyoscyamus.

SOURCE, - Hyoscyamus is treated with Ether, to remove fat, then with Alcohol acidulated with Sulphuric Acid and distilled. The aqueous residue is neutralized with Soda, and the liquid precipitated with Tannic Acid, the precipitate mixed with Lime, then exhausted by strong Alcohol. The Alcobolic solution is treated with Sulphuric Acid, then with Soda, and finally with Ether, which dissolves the alkaloid, which is obtained from it by distillation.

Ciraracters. - White, indistinct crystals, or a white powder, without odor, and having a bitter, acrid taste; deliquescent in damp air. Solubility. In 0.5 part of water, and in 2.5 parts of Alcohol; very slightly soluble in Ether or Chloroform.

Dose, fy to sid $^{\mathrm{gr}}$; ; .001 to $.002 \mathrm{gm}$. 
HYOSCYAMINE HYDROBROMAS.-Hyoscyamine Hydrobromate. $\mathrm{C}_{17} \mathrm{H}_{23} \mathrm{NO}_{3} \mathrm{HBr}=369 . \mathrm{I} 4$. The hydrobromate of an alkaloid obtained from Hyoscyamus.

Characters. - A yellowish-white, amorphous, resin-like mass, or prismatic crystals, having, particularly when damp, a tobacco-like odor, and an acrid, nauseous, and bitter taste; deliquescent on exposure to air. Solubility. -In about 0.3 part of water, 2 parts of Alcohol, 3000 parts of Ether, or $25^{\circ}$ parts of Chloroform.

Dose, $\frac{1}{60}$ to $\frac{1}{30} \mathrm{gr}$; .001 to $.002 \mathrm{gm}$.

HYOSCINA HYDROBROMAS.-Hyoscine Hydrobromate. $\mathrm{C}_{17}$ $\mathrm{H}_{21} \mathrm{NO}_{4} \mathrm{HBr}+3 \mathrm{H}_{2} \mathrm{O}=436.98$. The Hydrobromate of an alkaloid obtained from Hyoscyamus.

Characters. - Colorless, transparent, rhombic crystals, odorless, and having an acrid, slightly bitter taste; permanent in the air. Solubility.-In I.9 parts of water, and in $\mathbf{I} 3$ parts of Alcohol; very slightly soluble in Ether or Chloroform.

Dose, $\mathrm{I}^{\frac{1}{5} \mathrm{o}}$ to $\frac{1}{100} \mathrm{gr}$.; .0004 to $.0006 \mathrm{gm}$.]

\section{Action of Hyoscyamus.}

That the action of hyoscyamus is almost identical with that of belladonna and stramonium is not surprising when we remember the close resemblance in alkaloidal composition. The following are the chief points of difference: (I) Hyoscyamus contains hyoscine in minute quantities. This is a powerful cerebral and spinal sedative, and therefore the excitation and delirium occasioned by the atropine in belladonna are not so evident when hyoscyamus is given; indeed, that may, owing to the hyoscine in it, distinctly depress the higher functions of the brain. The heart is not quite so powerfully affected by hyoscyamus as by belladonna, for hyoscine has a comparatively feeble cardiac influence. Still it is, of course, affected by the hyoscyamine, which acts like atropine. (2) Hyoscyamus increases the peristaltic contractions of the intestines more powerfully than belladonna, and at the same time it is more efficient in relieving the griping of other purgatives. (3) Hyoscyamus has a more markedly sedative action on the urinary unstriped muscle than belladonna. (4) Hyoscine diminishes intra-ocular tension, therefore hyoscyamus does not affect this so much as belladonna. 


\section{Therapeutics of Hyoscyanus.}

Hyoscyamus might be used for the same purpose as belladonna, but is chiefly employed in combination with purgatives to diminish their griping action. It is also largely given to relieve vesical spasm in calculus, cystitis, and prostatitis, usually in conjunction with other urinary sedatives, as buchu, uva ursi, or benzoic acid if the urine is alkaline. It will be noticed that the doses of the preparations of hyoscyamus are larger than those of the corresponding preparations of belladonna.

Hyoscine and hyoscyamine may, if given cautiously, be employed as cerebral depressants, and are used in acute mania, delirium tremens, febrile delirium, and insomnia, sometimes with good results. They are mostly given in asylum practice. Hyoscine is most used, and is usually given subcutaneously. Chorea, paralysis agitans, and other convulsive diseases have been treated with them, but the convulsions always recur when these drugs are discontinued. They must be given very carefully, as the activity of different specimens varies, and fatal results have followed their use. They should never be employed if the patient is weak.

DUBOISIN $\approx$ SULPHAS.-Duboisine Sulphate. (Not official.) The Sulphate of the alkaloid Duboisine obtained from the leaves of Duboisia myoporoides, [nat. ord. Solanacea]. Duboisine is probably identical with Hyoscyamine (sec p. 384).

\section{Action and Therapeutics of Duboisine.}

The action of duboisine is like that of atropine, and ophthalmic discs containing $\frac{1}{5000}$ of a grain [.00001 $3 \mathrm{gm}$.] are used to dilate the pupil.

\section{CANNABIS INDICA.}

[INDIAN CANNABIS.-Synonym.-Indian Hemp. The flowering tops of the female plant of Cannabis sativa Linné (nat. ord. Urticacea), grown in the East Indies. Habitat.-Asia; collected in India.

Characters. - Branching, compressed, brittle, about $5 \mathrm{~cm}$. or more long, with a few digitate leaves, having linear-lanceolate leaflets and numerous, sheathing, pointed bracts, each containing two small, pistillate flowers, sometimes with the nearly ripe fruit, the whole more or less agglutinated with a 
resinous exudation. It has a brownish green color, a peculiar, narcotic odor, and slightly acrid taste.]

Composition.- [The chief constituents are (I) Cannabinon, a soft resin. (2) Choline, $\mathrm{C}_{5} \mathrm{H}_{15} \mathrm{NO}_{2}$, which is contained in (3) Tetanocannabine, and with alkalies gives (4) Cannabinine. (5) Cannabin, a brown, amorphous resin, said to be very active.]

INCOMPATIBLE. - Water, which precipitates the resin.

Dose, 2 to $5 \mathrm{gr}$.; [.12 to $.30 \mathrm{gm}$.]

\section{Preparations.}

x. Extractum Cannabis Indicæ.-[Extract of Indian Cannabis. By maceration and percolation with Alcohol, and evaporation.

Dose, $\frac{1}{6}$ to $3 / 4 \mathrm{gr}$; ; .or to $.045 \mathrm{gm}$.

2. Extractum Cannabis Indicæ Fluidum.-Fluid Extract of Indian Cannabis. By maceration, percolation with Alcohol, which is distilled off, and evaporation.

Dose, 2 to $5 \mathrm{~m}$.; .12 to .30 c.c.

3. Tinctura Cannabis Indicæ.-Tincture of Indian Cannabis. Indian Cannabis, 50 ; Alcohol, by maceration and percolation, to I000.

Dose, 5 to $30 \mathrm{~m} . ; .30$ to 2.00 c.c.]

Synonymis. - Haschisch is a confection of the drug. Gunjah, or Ganga, is the dried flowering tops of the cultivated female plants which are coated with resin. Churrus or Charas is the resin scraped off the leaves. Bhang [or Siddhi] is the dried leaves [and stalks made with preserved fruits into a confection]. In some provinces it means powdered Ganga made into a drink. Ganga and Charas are often smoked like tobacco.

\section{Action of Cannabis Indica.}

External.-None is known.

Internal. - The effects of cannabis indica vary very much on different people. This is partly due to the uncertain strength of the preparations of the drug, and partly to individual peculiarities, but generally the symptoms are somewhat as follows. After some time, usually from half an hour to two or three hours, there is a pleasurable sensation of mild intoxication; the patient is particularly gay, joyous, and pleased with everything; he will laugh and smile on the slightest provocation, and is himself able to say sharp, witty things. Pleasant ideas flit through his mind with wonderful rapidity, so that time seems to him much [prolonged; space also seems to be extended]. Generally the ideas 
are quickly forgotten, but sometimes the memory of them remains after recovery. The eyes are bright, the pupils may be dilated. The limbs feel heavy, and there is a marked lowering of general sensibility, so that he scarcely feels a severe pinch; this may pass on to complete anæsthesia. There may be headache. After a time sleep, which is often accompanied by delightful dreams, comes on. [On awakening the sense of hunger is acute.] The drug is frequently taken in the East to produce the early pleasurable symptoms, and, in moderation, it causes no harm. Very few take it to excess, but in them it leads to loss of appetite and strength, trembling and much weakness. Cannabis indica is reputed to occasionally produce sexual excitement, but this is incorrect. Large doses given to a dog only made him sleepy, and uncertain on his legs, but he appeared contented and pleased. Much the same results followed when a monkey was made to inhale the smoke daily for six months.

\section{Therapeutics of Cannabis Indica.}

It has been given with success in migraine and neuralgia, but it very often fails to afford relief. Its use as an hypnotic has been discarded. The tincture is very difficult to prescribe, because of the voluminous precipitate of resin which falls on the addition of water. Mucilage must be used to suspend it, and the taste should be covered with spirit of chloroform.

\section{CAFFEINA.}

CAF FEINE. $-\mathrm{C}_{8} \mathrm{H}_{10} \mathrm{~N}_{4} \mathrm{O}_{2}+\mathrm{H}_{2} \mathrm{O}=211.68$. Synonyms. - Theine. Guaranine. [A feebly basic, proximate principle, obtained from the dried leaves of Thea sinensis Linné (nat. ord. Ternstramiacea), or from the dried seed of Coffea arabica Linné (nat. ord. Rubiacea), and found also in other plants. Habitat.-Tropical Africa ; cultivated in tropical countries.

SOURCE. - Exhaust bruised coffee by successive portions of boiling water, precipitate with Lead Acetate, decompose the excess of Lead Acetate in the filtrate by Hydrogen Sulphide, concentrate by evaporation, neutralize with Ammonia. The Caffeine crystallizes on cooling, and is purified by re-dissolving in water, treating with Animal Charcoal, and evaporation.

Characters. - Fleecy masses of long, flexible, white crystals, possessing a silky lustre, without odor, having a bitter taste, and permanent in the air. Solubility. - In 80 parts of water, 33 parts of Alcohol, 555 parts of Ether, or 
7 parts of Chloroform.] Tea contains 3 to 5 per cent. (hence the name Theine), Coffee, $\mathrm{r} .3$ per cent. (coffee leaves contain much more). Guarana (the seeds of Paullinia [Cupana]), [4 to] 5 per cent. (hence the name Guaranine). Maté (Paraguay tea, the leaves of Ilex paraguayensis), 1.2 per cent. Kola nut (which is used as a beverage in Africa), 3 per cent. ; this is the fruit of Sterculia acuminata. Caffeine is trimethyl-xanthine, theobromine is dimethylxanthine, and both can be prepared synthètically from xanthine.

[Strictly speaking, Theine is the alkaloid of tea-leaves. It differs from caffeine in that it is analgesic and constipates. Much of the caffeine in the market is really theine and is made from the sweepings of tea-warehouses.]

Incompatibles. - Potassium iodide, mercury salts, and tannic acid.

Dose, $x$ to $5 \mathrm{gr}$; ; [.06 to .30 $\mathrm{gm}$.]

\section{Preparations.}

[1. Caffeina Citrata.-Citrated Caffeine. Dissolve Citric Acid, 50 , in hot Distilled Water, 100; add the Caffeine, 50, and evaporate the resulting solution on a water-bath to dryness, constantly stirring towards the end of the operation. Reduce the product to a fine powder.

Characters. - A white powder, odorless, having a purely acid taste and an acid reaction. Solubility.-One part of Citrated Caffeine forms a clear, syrupy solution with about 3 parts of water.

Dose, 2 to $8 \mathrm{gr}$; . 12 to $.50 \mathrm{gm}$.

2. Caffeina Citrata Effervescens.-Effervescent Citrated Caffeine. Caffeine, 1o; Citric Acid, 10; Sodium Bicarbonate, 330; Tartaric Acid, 300; Sugar, $35^{\circ}$; Alcohol, to make rooo. Triturate the solid ingredients, separately well dried, to a fine uniform powder. Mix this with Alcohol to a soft paste and rub it through a sieve. Then dry it, and reduce it to a coarse, granular powder.

Dose, $x$ to $4 \mathrm{dr}$; 4 . to $15 . \mathrm{gm}$.]

\section{Action of Caffeine.}

\section{External.-None.}

Internal.-Alimentary canal.-Excessive tea-drinking may cause indigestion, but this is probably induced by the tannic acid in the tea, and not by the caffeine. The teeth of tea tasters are very liable to decay. Coffee is, with some persons, slightly laxative; it is not known to what ingredient this is due.

Circulation.-Caffeine is freely absorbed. It produces no change in the blood. Moderate doses increase the force of the cardiac contraction and the duration of the systole, the diastolic period being shortened; as a consequence of this 
the blood-pressure rises. The pulse is usually slowed. Toxic doses paralyze the heart. These effects are largely due to the direct action of caffeine on the cardiac muscle, but also to an action on the inhibitory centres.

Vessels. - Caffeine causes first a constriction and then a dilatation of the arterioles of the body, and this is due principally to its action on the muscular coat and slightly to its action on vasomotor centres.

Respiration.-In animals the rate of breathing is increased by caffeine. Medicinal doses are said to excite and toxic doses to depress it.

Nervous system.-It is well known that tea and coffee stimulate the cerebrum. This is due to the caffeine in them. The patient becomes wakeful, the mental activity and capability for work are increased, the reasoning powers being quite as much affected as the imagination. In this respect the cerebral stimulation of caffeine differs from that of opium, and also in that the excitation is not inco-ordinate, nor is it soon replaced by sleep. Very excessive tea-drinking causes trembling of all the muscles of the body, and makes the patient extremely " nervous."

In man the spinal cord and muscles are very little affected by caffeine, but in some frogs the spinal cord is decidedly stimulated, and convulsions occur; in other species the muscles are thrown into a state of rigidity, which is clearly due to an action on the muscles themselves, for it follows the application of caffeine to an isolated muscle. Sometimes the muscle curve is altered in character. It is believed that in man the powers of muscular endurance are increased by caffeine. Motor and sensory nerves are uninfluenced in all animals.

Kidneys. - As caffeine first causes a contraction of arterioles there is decrease in the urinary flow ; but soon the renal vessels dilate, the renal cells are stimulated and the flow of urine is increased. Thus caffeine is a good local diuretic.

Metabolism.-Many elaborate experiments have been made upon the action of caffeine on tissue waste ; they are all of them inconclusive, [probably] because it has no effect. It increases the excretion of xanthin in the urine; probably this xanthin is 
derived directly from it, and the increased urea said to be excreted may also proceed directly from the caffeine. Toxic doses may cause a slight rise of temperature.

\section{Therapeutics of Caffeine.}

Heart.-Caffeine has been most used in heart disease. It is given when, as in aortic or mitral obstruction, a purely stimulant effect is desired; large doses, 3 or $8 \mathrm{gr}$. [.20 to .50 gm.] a day of the [citrated, or 2 to $6 \mathrm{dr}$, , 8. to 24 . gm., of the effervescent citrated], are often easily borne, and may be combined with strychnine. [These preparations are useful, when combined with antipyrin or acetanilid derivatives, to counteract their depressing influence upon the heart.] Caffeine will not replace digitalis, for it does not slow the heart nor make it regular, and it. shortens the diastole. It is, on account of its diuretic action, especially valuable in cardiac cases in which there is dropsy. Tea and coffee are, in some persons, liable to produce irregularity of the heart.

Kidney.-Small doses of caffeine are powerfully diuretic, and are therefore used in heart disease, ascites, pleuritic effusion, etc. As the drug acts directly on the kidney, it should be given cautiously in renal disease. Many patients so soon become used to it that at the end of a week it has lost its power of producing diuresis.

Nervous system. - Occasionally it cures migraine, but it is not so useful as [antipyrin] or exalgin.

It may be rendered sufficiently soluble for subcutaneous administration by mixing it with a solution of sodium salicylate.

\section{GUARANA.}

GUARANA. - Synony'm.-Brazilian Cocoa. [A dried paste chiefly consisting of the crushed or pounded seeds of Paullinia Cupana, Kunth (Paullinia sorbilis, Martius; nat. ord., Sapindacea.) Habitat.-Northern and Western Brazil.

ChARACTERs. -Subglobular or elliptic cakes, or cylindrical sticks, hard, dark reddish-brown; fracture uneven, somewhat glossy, pale reddish-brown, showing fragments of seeds invested with blackish-brown integuments; odor slight, peculiar, resembling that of chocolate; taste, astringent and bitter. Solubility. - It is partly soluble in water, and in Alcohol. 
Compositron. - The chief constituents are (1) Guaranine, identical with Caffeine, 4 to 5 per cent. (see p 388); (2) Volatile Oil, a trace; (3) Saponin; (4) Tannic acid.

Dose, $1 / 4$ to $\mathrm{I} \mathrm{dr.;} 1$. to $4 . \mathrm{gm}$.

\section{Preparation.}

Extractum Guaranæ Fluidum.-Fluid Extract of Guarana, By maceration and percolation with Alcohol and water, and evaporation.

Dose, $1 / 4$ to $I$ fi. dr.; $x$. to 4 . c.c.]

\section{Action and Therapeutics of Guarana.}

Although there is no reason to believe that guaranine does not produce the same action on the nervous system, heart and kidneys as caffeine, yet it is rarely used except for sick headaches; but in these cases it is sometimes of the greatest service.

\section{[THEOBROMINAE SODIO-SALICYLAS.}

THEOBROMINE SODIO-SALICYLATE. (Not official). $\mathrm{C}_{7} \mathrm{H}_{7} \mathrm{NaN}_{4} \mathrm{O}_{2}+\mathrm{NaC}_{7} \mathrm{H}_{5} \mathrm{O}_{3}=361.42$. Synonym.-Diuretin.

Source.-By the interaction of Sodium Theobromine and Sodium Salicylate. It contains 49.7 per cent. of Theobromine.] It corresponds to the Caffeine Sodio-Salicylate, the salt of Caffeine most used in Germany.

[CHARACTERs. - A white powder, soluble in half its weight of warm water, the solution remaining perfect when cooled.

Dose, 15 to $3 \circ \mathrm{gr}$; r. to $2 . \mathrm{gm}$.

\section{Action and Uses of Theobromine Sodio-Salicylate.}

This is a pure diuretic acting upon the renal epithelium, without action upon the heart, and it is believed that it does not irritate the kidneys. The daily dose is from one to two drachms; 4. to 8. gm., best administered in solution with aromatic water. It has been administered with benefit in cases of severe cardiac or hepatic dropsy.] It is said that it does not produce depression, but it has occasionally given rise to severe symptoms, [which may have been due to impurities.] 
Class II.-DRUGS ACTING CHIEFLY ON THE SPINAL CORD.

\section{[A. The following excite the cells of the anterior cornua.]}

\section{NUX VOMICA.}

NUX VOMICA.-Synonyms.-Poison Nut. [Dog Button. Quaker Button. The seed of Strychnos Nux-vomica Linné (nat. ord. Loganiacea). Habitat.-India and East Indian Islands.

Characters. - About $25 \mathrm{~mm}$. in diameter, orbicular, grayish or greenishgray; soft-hairy, of a silky lustre, with a slight ridge extending from the centre of one sicle to the edge ; internally horny, somewhat translucent, very tough, with a large, circular cavity, into which the heart-shaped, nerved cotyledons project. It is inodorous and persistently bitter.]

Compositron. - The chief constituents are-(I) Strychnine (see below), 0.9 to 1.9 per cent. ; (2) Brucine, which is dimethyl oxylstrychnine, $\mathrm{C}_{23} \mathrm{H}_{26}$ $\mathrm{N}_{2} \mathrm{O}_{4}, 0.9$ to $\mathrm{I} .5$ per cent. in colorless prismatic crystals or pearly flakes. Very bitter, but less so than Strychnine. [Brucine is found in Hoang-Nan.] Solubility. - Freely in Alcohol, and in 7 parts of Chloroform. It has the same action as Strychnine, but is considerably less powerful and slower in its effects. Strong Sulphuric or Nitric Acid gives a blood-red color. (3) Igasuric Acid, with which the Strychnine and Brucine are united. (4) Loganin, $\left[\mathrm{C}_{25} \mathrm{H}_{34} \mathrm{O}_{14},\right]$ in colorless prisms, an inert glucoside.

Dose, I to $4 \mathrm{gr}$.; [.06 to $.24 \mathrm{gm}$.]

\section{Preparations.}

I. Extractum Nucis Vomicæ.-[Extract of Nux Vomica. By maceration with Acetic Acid, Alcohol and Water, percolation with Alcohol and Water, and distillation ; treat with Ether, filter, evaporate and add Sugar of Milk.

Extract of Nux Vomica is used to make Tinctura Nucis Vomicæ.

Dose, $1 / 8$ to I $\mathrm{gr}$; ; .008 to $.06 \mathrm{gm}$.

2. Extractum Nucis Vomicæ Fluidum.-Fluid Extract of Nux Vomica. By digestion with Acetic Acid, Alcohol and Water, and percolation with Alcohol and Water. Distil off the Alcohol and evaporate.

Dose, I to $4 \mathrm{~m}$.; .06 to .25 c.c.

3. Tinctura Nucis Vomicæ.-Tincture of Nux Vomica. Extract of Nux Vomica, 20 ; Alcohol and Water to 1000. By solution.

Dose, 5 to $20 \mathrm{~m}$.; .30 to $\mathrm{r} .20$ c.c.] 2 ?

STRYCHNINA.-Strychnine. $\mathrm{C}_{21} \mathrm{H}_{22} \mathrm{~N}_{2} \mathrm{O}_{2}[=333.3$ I. An alkaloid obtained from Nux Vomica, and also obtainable from other plants of the natural order Loganiacea, especially from the Strychnos Ignatia.

Source. - ( I) Comminute the Nux Vomica; (2) Extract the Strychnine with water acidulated with Hydrochloric Acid; (3) Concentrate the infusion, decompose the Strychnine with Lime; (4) Extract the Strychnine from the

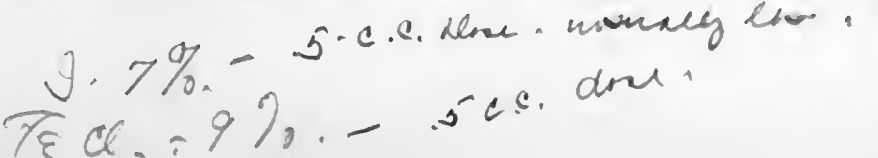


precipitate with boiling Alcohol; (5) Concentrate the solution to obtain the crystals.

ChARACTERS. - Colorless, transparent, octahedral or prismatic crystals, or a white crystalline powder, odorless, and baving an intensely bitter taste perceptible even in highly dilute ( 1 in 700,000 ) solution. Only extremely diluted solutions should be tasted. Permanent in the air. Solubility.-In 6700 parts of water; in 110 parts of Alcohol; in 2500 parts of boiling water; and in 12 parts of boiling Alcohol. Also soluble in 7 parts of Chloroform, but almost insoluble in Ether.] Gives no color with Nitric or Sulphuric Acids. Add to a crystal strong Sulphuric Acid, and then add a particle of Potassium Bichromate, a beautiful violet color, passing to brown and green, is formed. Resembling Strychnine.-Salicylic Acid ( $q . v$.)

Incompatibles.-Alkalies, Iodides and Bromides; the last are especially dangerous, for the precipitated strychnine bromide falls slowly.

IMPURITY.-Brucine, distinguished by tests.

[Strychnine is used to prepare Ferri et Strychninæ Citras and Syrupus Ferri, Quininæ et Strychninæ Phosphatum.

Dose, $\frac{1}{60}$ to $\frac{1}{20} \mathrm{gr}$; .001 to $.003 \mathrm{gm}$. in solution, or made in a pill with Sugar of Milk (thoroughly to divide it) and Glycerin of Tragacanth ; or hypodermatically. The Nitrate (not official), soluble in I in 60 of water, may be used.

STRYCHNIN $\approx$ SULPHAS.-Strychnine Sulphate. $\left(\mathrm{C}_{21} \mathrm{H}_{22} \mathrm{~N}_{2} \mathrm{O}_{2}\right)_{2}$ $\mathrm{H}_{2} \mathrm{SO}_{4}+5 \mathrm{H}_{2} \mathrm{O}=854.24$.

Source. - By the action of Diluted Sulphuric Acid on Strychnine, filtration and evaporation.

Characters. - Colorless or white, prismatic crystals, odorless and having an intensely bitter taste perceptible even in highly dilute ( $\mathrm{I}$ in 700,000 ) solution. Efflorescent in dry air. Solubility. - In 50 parts of water, and in rog parts of Alcohol; almost insoluble in Ether.

Dose, $\frac{1}{60}$ to $\frac{1}{12} \mathrm{gr} . ; .001$ to $.005 \mathrm{gm}$.]

\section{Action of Nux Vomica.}

External. - Strychnine is a very powerful antiseptic. Brucine is a local anæsthetic.

Internal.-Gastro-intestinal tract.-Being intensely bitter, nux vomica is a good stomachic, increasing the vascularity of the gastric mucous membrane, the secretion of gastric juice, and the movements of the stomach, just like calumba, gentian, or any other bitter ; consequently it aids digestion and sharpens the appetite. In the intestine it is a direct stimulant to the intestinal muscular coat, and by this means it increases peristalsis, and is therefore purgative. 
Blood.-Strychnine is absorbed into the blood, and circulates as such. If blood is mixed with strychnine and shaken with air, it contains more oxygen and less carbon [dioxide] than it would have done had the strychnine been absent; but there is no evidence that strychnine in small doses alters the oxidizing power of living blood.

Spinal cord.-Strychnine causes convulsions. They are not cerebral, for they occur if the spinal cord is separated from the brain. They do not depend upon excitation of the motor nerves or muscles, for they are absent in a limb the spinal anterior nerve-roots of which are cut. They occur if the posterior nerve-roots are cut, provided the proximal end is stimulated. Therefore they must be spinal; and this is proved by the fact that if all the vessels of the lower part of the spinal cord are [ligated] just at their entry into the cord, so that this is the only part of the body deprived of its blood supply, and strychnine is injected into the blood, convulsions occur in all the muscles except those the nerves of which spring from the part of the cord which the strychnine cannot reach. Again, if an animal be convulsed by strychnine, and a probe be slowly passed down the spinal canal, the convulsions will gradually cease from above downwards. But a peripheral stimulus, particularly if sharp and sudden, so easily excites convulsions when strychnine has been given that we are justified in assuming that every convulsion is excited by a peripheral stimulus, and often so slight as not to be evident. Further, strychnine enormously exaggerates the conduction power of the cord in such a way that general convulsions reflexly follow a very slight local stimulus. It is believed that the precise part of the spinal cord stimulated to increased excitability by strychnine is that immediately on the afferent side of the anterior cornual cells.

Muscles and neries.-Even with enormous doses the muscles and afferent nerves are unaffected. Towards the end of a case of poisoning the functional activity of the motor end-organs is depressed. This is due to direct action on them, and occurs readily in some species of frogs.

Brain.-The convolutions are quite unaffected. The 
centres in the medulla, which are really the continuation upwards of the anterior cornua of the cord, are powerfully stimulated, especially the respiratory centre. The vaso-motor centre is also considerably excited, and chiefly for this reason the blood-pressure rises from the very first. The cardiac centre is but slightly affected [although the clinical evidence is in favor of its being strongly influenced].

Circulation.-Strychnine stimulates the heart directly, either by its action on the cardiac muscles or, as most authorities think, by stimulating the motor ganglia. The blood-pressure is raised, partly no doubt by the action on the heart, but also by the contraction of the vessels all over the body, which is brought about first by the direct excitation, by the strychnine, of the medullary vaso-motor centre, and subsequently by its asphyxial stimulation, and also by the increased peripheral resistance which must occur from the frequent contraction of all the muscles. [The result is that the force of the heart is increased and the diastole lengthened.]

Respiration.-Respiration is rendered quicker and deeper because strychnine excites the spinal and medullary respiratory centres. The respiratory muscles are implicated in the general convulsions with the result that the patient ultimately becomes asphyxiated owing to exhaustion of them, and to their prolonged contraction during the convulsive spasms. The heart continues to beat after death which is entirely due to failure of respiration. The excessive muscular contractions occasionally cause a rise of temperature, but so rarely that often the loss of heat must be greatly increased.

Special senses.- Smell, hearing, touch and sight are sharpened by strychnine. The field of vision, especially for blue, is said to be enlarged.

Elimination.-Strychnine is eliminated unchanged in the urine. It is excreted very slowly, and therefore accumulates in the system. Tolerance is never established. For a clinical account of strychnine poisoning see Toxicology.

Brucine and thebaine act like strychnine, but methyl-brucine, methyl-thebaine, and methyl-strychnine do not influ- 
ence the cord, but paralyze the ends of the motor nerves like curare.

Strychnine acts on all animals in the main as on man; but some birds and guinea-pigs are less susceptible to it, for they absorb it slowly.

\section{Therapeutics of Nux Vomica.}

External.-Strychnine is so posionous that its use as an antiseptic would not be safe.

Internal.-Gastro-intestinal tract.-Tincture of nux vomica is very largely given with excellent results as a bitter stomachic and carminative, especially in cases in which the feebleness of digestion is merely part of generally feeble health. [Twenty drops of tincture of nux vomica in a wineglass of hot water will frequently at once check gastro-intestinal fermentation.] A mixture of diluted hydrochloric acid, gentian and nux vomica is of great service in these cases. As the digestion improves, the general health improves. Because of its power to stimulate peristalsis nux romica is a valuable drug for cases of constipation in which the contractile strength of the muscular coat of the intestine is weak ; usually this is a part of a general weakness of the whole body. The constipation of anæmia, which can be very successfully treated by a pill of extract of nux vomica and ferrous sulphate, is a good instance of this variety of constipation.

Circulation. - In cases of heart disease in which digitalis is inadmissible, nux vomica and strychnine are excellent cardiac stimulants, and for this purpose they may be combined with caffeine. Patients almost dead from failure of the heart in the course of chronic cardiac disease may sometimes be brought around by the subcutaneous injection of strychnine.

Respiration.-Strychnine may be combined with expectorants, because it stimulates the respiratory centre; and it is extremely serviceable when from any cause, such as severe bronchitis, the respirations are feeble and shallow. [It is also useful in pneumonia when death is imminent from dilatation of the right heart. In this condition it should be administered hypodermatically and at frequent intervals.] 
Nervous system. - It has been given for a number of nervous diseases, but with no constantly good results, for when the disease is not in the anterior cornua, strychnine is hardly indicated ; and if it is in this part of the cord, it is doubtful whether it is advisable to stimulate the part of the body which is diseased.

\section{Toxicology.}

Symptoms. - In about an hour after a poisonous dose the patient begins to feel uneasy from a sensation of impending suffocation. The tetanic convulsions then commence with great violence, nearly all the muscles of the body being affected at once. The limbs are thrown out, the hands are clenched, the head is jerked forwards and then bent backwards, and the whole body is perfectly stiff from the violence of the contractions. The pulse is very rapid ; the temperature may rise. Hearing and sight are acute. The convulsion lasts a minute or two, then the muscles relax, and the patient feels exhausted and sweats all over. The intermission is short, convulsions soon come on again, and again there is a relapse to the state of muscular relaxation. The convulsions now rapidly increase in severity, and owing to the violent contractions of the muscles of the back, the patient is in the position of opisthotonos, resting on his head and his heels. The abdominal muscles are as hard as a board, the chest is fixed, the face becomes livid, the eyeballs are staring. The contraction of the muscles of the face causes a risus sardonicus; but those of the jaw are not affected till near the end ; [this aids in distinguishing strychnine poisoning from tetanus.] Consciousness is retained till the last. The slightest noise or even a bright light will reflexly bring on the convulsions, which may jerk the patient out of bed. Ultimately he dies from exhaustion and asphyxia. The smallest dose of strychnine known to have killed is half a grain; [.03 gm.] Post-mortem.-The usual appearances of death by asphyxia are seen.

Strychnine poisoning is liable to be confounded with tetanus, but in this disease symptoms come on more slowly, the muscles of the jaw are implicated very early, and there is continuous muscular rigidity with paroxysmal exacerbations, but never complete muscular relaxation.

Treatment.-[Give emetics (see p. 139), particularly apomorphine bydrochlorate hypodermatically, or wash out the stomach if the patient is seen early enough for the passing of the tube not to cause spasm]. The stomach [may be] washed with solution of potassium permanganate as for opium [poisoning, but it is not so effective]. Inject large doses of potassium bromide and chloral per rectum. Use amyl nitrite inhalations, and if possible artificial respiration.

\section{Antagonism.}

In a sense strychnine is antidotal to chloral and morphine, but it is not a strict antidote, for they act chiefly on the cerebrum. Still chloral is valuable in strychnine poisoning, and although the antagonism with Calabar bean and 
gelsemium is more accurate, as both depress the anterior cornua, yet they are of very little use in strychnine poisoning. [Chloroform is the most practical antidote to strychnine.

\section{B. The following depress the cells of the anterior cornua.]}

\section{PHYSOSTIGMA.]}

PHYSOSTIGMA.-[Synonyms.-Calabar bean. Ordeal bean. The seed of Physostigma venenosum Balfour (nat. ord. Leguminosa). Habitat.Tropical Western Africa, near the mouth of the Niger and old Calabar.

Characters. - About 25 to $30 \mathrm{~mm}$. long, 15 to $20 \mathrm{~mm}$. broad, and 10 to $15 \mathrm{~mm}$. thick; oblong, and somewhat reniform; testa granular, chocolatebrown, with a broad, black groove extending over the entire length of the convex edge; embryo with a short, curved radicle, and two large, white, concavoconvex cotyledons; inodorous ; taste, bean-like.

Composition.-The chief constituents are: (I) Physostigmine or Eserine (see below); (2) Calabarine, a derivative of Physostigmine; (3) Eseridine; and (4) Physosterin a neutral principle closely related to cholesterine.

Dose, $1 / 2$ to $2 \mathrm{gr}$; .03 to $.12 \mathrm{gm}$. of the powdered seeds.]

\section{Preparations.}

I. Extractum Physostigmatis.-[Extract of Physostigma. By maceration, percolation with Alcohol and evaporation.

Dose, $\frac{1}{10}$ to $1 / 2 \mathrm{gr}$. .006 to $.03 \mathrm{gm}$., internally, or rather less dissolved in ro m. ; .60 c.c. of water and given subcutaneously.

2. Tinctura Physostigmatis.-Tincture of Physostigma. Physostigma, I 50. By maceration and percolation with Alcohol to 1000 .

Dose, ro to $30 \mathrm{~m}$.; .60 to 2.00 c.c.

PHYSOSTIGMINAE SALICYLAS. - Physostigmine Salicylate $\mathrm{C}_{15} \mathrm{H}_{21} \mathrm{~N}_{3} \mathrm{O}_{2} \mathrm{C}_{7} \mathrm{H}_{6} \mathrm{O}_{3}=412.17$. Synonym.-Eserine Salicylate. The Salicylate of an Alkaloid obtained from Physostigma.

SuURce. - By adding Physostigmine to a solution of Salicylic Acid in boiling Distilled Water, and allowing the salt to crystallize on cooling.

ChARACTERS. - Colorless or faintly yellowish, shining, acicular, or short, columnar crystals, odorless, and having a bitter taste. It acquires a reddish tint when long exposed to light and air. Solubility. - In I 50 parts of water, and in 12 parts of Alcohol.

Dose, $\frac{1}{100}$ to $\frac{\pi}{30} \mathrm{gr}$; .0006 to $.002 \mathrm{gm}$.

PHYSOSTIGMIN $\nRightarrow$ SULPHAS.-Physostigmine Sulphate. $\mathrm{C}_{15} \mathrm{H}_{21}$ $\left.\mathrm{N}_{3} \mathrm{O}_{2}\right)_{2} \mathrm{H}_{2} \mathrm{SO}_{4}=646.82$. Synonym.-Eserine Sulphate. The Sulphate of an Alkaloid obtained from Physostigma.

Characters.-A white or yellowish-white, micro-crystalline powder; 
odorless, and having a bitter taste. It is very deliquescent when exposed tc moist air, and gradually turns reddish by exposure to air and light. Solubility.

-Very soluble in water and in Alcohol.

Dose, $\frac{1}{100}$ to $\frac{1}{30} \mathrm{gr}$; ; .0006 to $.002 \mathrm{gm}$.]

Action of Calabar Bean.

External.-None.

Internal.-Mouth.-After physostigmine is absorbed it increases the salivary secretion; and this has been shown to be due to stimulation of the terminations of the secretory nerves in the glands. Other secretions are increased, probably in the same way. After a time the flow of saliva ceases, because the drug has so acted on the circulation as to constrict the vessels, and consequently the flow of blood through the salivary glands is diminished.

Stomach and intestines. - The muscular coat of the stomach and intestines is stimulated by the direct action of the drug circulating through it. The result is that after a large dose vomiting and purging occur. Physostigmine is quickly absorbed.

Circulation. - No influence on the blood is known. The effect on the heart is obscure, but it appears that the irritability of the peripheral terminations of the vagus is at first increased, and that consequently the heart is slowed. Very large doses are said to decrease the irritability of the vagus. In addition to its effects on the vagus, physostignine powerfully stimulates the contractile force of the heart. The beat is therefore both more forcible and slower. Ultimately the organ is paralyzed and stops in diastole.

The blood-pressure rises very much; this is largely due to the increased force of the cardiac beat, and partly to contraction of all unstriped muscle of the abdominal viscera, driving much blood out of the abdomen. It is not known for certain if the unstriped muscle of the arteries is stimulated. Analogy would leave us to suppose that it is.

Respiration.-This is first quickened, but soon retarded, and death takes place from asphyxia. Three factors at least are probably concerned in bringing about these results. The ends 
of the vagi in the lungs are stimulated, for if these nerves are cut and physostigmine is administered there is no primary quickening of respiration. Physostigmine, from its action on involuntary muscular fibre, causes contraction of that in the bronchial tubes, with consequent constriction of them. Lastly and the most important, the activity of the respiratory centres in the medulla and cord is depressed.

Nerious system.-Brain.-Even in fatal doses consciousness is unimpaired. The only part of the brain known to be affected is the respiratory centre.

Spinal cord. - It is here that physostigmine produces its most characteristic effects. Reflex activity is inhibited; by exclusion it can be shown that this is not owing to any influence on the nerves or voluntary muscles, therefore it is due to depression of the anterior cornua of the spinal cord. The most conclusive proof of this is the direct application of the drug to the cord. There is then, at first, from the irritation, which is caused by almost any substance, a slight increase of reflex excitability, but this soon gives way to complete abolition of it. Later on the posterior part of the cord is also paralyzed, so that there is a diminution of cutaneous sensibility.

Voluntary muscles and their nerves.-Muscular twitchings follow large doses in many animals. These appear to be due to the action on the motor nerve terminations; sensory nerves are unaffected.

Involuntary muscles. - We have already seen that the involuntary muscles of the intestines, stomach, and bronchial tubes are stimulated by physostigmine; so also are those of the spleen, uterus, bladder, and iris. Probably in all these instances it is the terminations of the motor nerves that are affected.

Eye.-Physostigmine applied locally to the conjunctiva or introduced into the circulation causes contraction of the pupil, spasm of accommodation from direct stimulation of the ends of the motor nerves of the iris and the ciliary muscle. There is a diminution of intra-ocular tension. Thus, as regards both secretions and the eye, physostigmine is antagonistic to atropine. 
The action of physostigmine is much more constant than that of Calabar bean, perhaps because the other active principles in the bean interfere with the action of physostigmine.

\section{Therapeutics of Calabar Bean.}

Involuntary muscles.-Because of its stimulating power on unstriped muscle Calabar bean has been recommended for chronic constipation, atony of the bladder, and chronic bronchitis with deficient power of expectoration, but it is rarely given for these purposes.

Spinal cord.-Calabar bean has been largely used for tetanus, and some cases of recovery have been reported. It must be administered boldly; the extract has often been given, but it is better to inject physostigmine sulphate under the skin. Doses of $\frac{1}{30} \mathrm{gr} .,[.002 \mathrm{gm}$.$] , frequently repeated, may be employed,$ but the patient must be carefully watched. Physostigmine has been given as an antidote for strychnine poisoning.

Eye.-[A solution of physostigmine salicylate $\mathbf{I}$ or $\mathbf{2}$, to water, 480 ; is dropped] in the eye to break up adhesions of the iris, to diminish intra-ocular tension, and to prevent prolapse of the iris after wounds, or ulcers of the cornea. It is also employed in glaucoma, in paralysis of the iris and ciliary muscles, and to prevent the entrance of light into the eye in photophobia.

\section{Antagonism.}

It will be observed that in its actions on the pupil, on secretion, on the heart, and on respiration, physostigmine is antagonistic to atropine. In its action on the spinal cord and respiratory centre it is antagonistic to strychnine.

\section{[MUSCARINA.}

MUSCARINE. (Not official). $-\mathrm{C}_{6} \mathrm{H}_{15} \mathrm{NO}_{3}=136.74$. An alkaloid obtained from Amanita Muscaria, Fly Fungus. Habilat.-Russia and Nortbern Europe.

ChARACTERS. - A liquid of the consistence of syrup, without odor or taste. Solubility. -Nearly in water and Alcohol; insoluble in Ether and Chloroform.

Dose, $1 / 8$ to $2 \mathrm{~m}$.; .008 to .12 c.c. 


\section{Action and Uses of Muscarine.}

Muscarine in its action somewhat resembles Calabar bean, and it is antagonistic to atropine. It produces free salivation, abundant perspiration, diminution of the force and frequency of the pulse, dyspnœa, paralysis and finally death. The pupil is contracted ; dilating, however, before death. The cardiac diastole is prolonged, due to action upon the inhibitory nerves. The muscles of the intestines and bladder are markedly contracted. The abdominal secretions are increased. Although it has been but little used in medicine, it is likely to be useful in intestinal torpor, duodenal catarrh, and in inflammatory effusions and exudations. As it produces contraction of pulmonary capillaries, it is indicated in pulmonary hæmorrhage and incipient pulmonary congestion.]

\section{GELSEMIUM.}

GELSEMIUM. Synonym.-Yellow Jasmine. [The rhizome and roots of Gelsemium sempervirens (Linné) Persoon (nat. ord. Loganiacea). Habitat. - Southern United States.

Characters. -Cylindrical, long, or cut in sections, mostly from 5 to $\mathbf{1 5}$ $\mathrm{mm}$. and occasionally $3 \mathrm{~cm}$. thick, the roots much thinner; externally light yellowish-brown, with purplish-brown, longitudinal lines; tough ; fracture splintery ; bark thin, with silky bast-fibres, closely adhering to the pale yellowish, porous wood, which has fine, medullary rays, and in the rhizome a thin pith ; odor, aromatic, heavy ; taste bitter.]

Composition. - The chief constituents are-(I) Gelsemine, $\left[\mathrm{C}_{54} \mathrm{H}_{69} \mathrm{~N}_{4} \mathrm{O}_{12}\right.$, a colorless, with difficulty crystallizable, bitter alkaloid, soluble in Alcohol and Ether, sparingly in water. (2) Gelseminine, a brown, amorphous, bitter alkaloid, very poisonous. (3) Gelseminic Acid. (4) A volatile oil.

Dose, 5 to $10 \mathrm{gr} . ; .30$ to $.60 \mathrm{gm}$. Of Gelsemine hydrochlorate (not official), $\frac{1}{60}$ to $\frac{1}{20} \mathrm{gr} . ; .001$ to $.003 \mathrm{gm}$.

\section{Preparations.}

I. Extractum Gelsemii Fluidum.-Fluid Extract of Gelsemium. By maceration and percolation with Alcohol and evaporation.

Dose, 5 to ro m.; .30 to .60 c.c.

2. Tinctura Gelsemii.-Tincture of Gelsemium. Gelsemium, 150 ; by maceration and percolation with Alcohol, to 1000.

Dose, $1 / 4$ to $x$ fl. dr.; 1 . to 4 . c.c.] 


\section{Action of Gelsemium.}

External.-None.

Internal.-Gelsemium produces no effect on the stomach or intestines. Its powerful general physiological effects are due to the gelseminine in it.

Brain.-In poisoning by gelsemium consciousness is maintained till the end; the drug, therefore, has no power on the higher cerebral centres.

Spinal cord. - The most marked symptom produced by gelsemium is paralysis of all the muscles of the body; and by a series of experiments, like those used for strychnine, this can be shown to be due to depression of the activity of the anterior cornua of the spinal cord. This is said to be followed by a depression of the sensory part of the cord, with consequent anæsthesia. The motor nerves are quite unaffected till just before death, when the end-plates are paralyzed. The result of the action on the cord is that the patient may be unable to walk, or, if he can, the gait is staggering; his general sensibility is much impaired. Convulsions may be produced. The cause of these cannot be made out, for they appear to be neither cerebral, spinal, nor peripheral.

Eye.-Gelsemium soon causes disturbance of vision, then follows diplopia, due to paralysis of the ocular muscles, and from the same cause the upper lid drops. The pupil is dilated. All these symptoms are probably owing to the paralysis of the motor centres in the floor of the fourth ventricle and the aqueduct of Sylvius, for these are the continuation upwards of the anterior cornua.

Circulation. - The action of moderate doses is not marked. Toxic doses are powerfully depressant; the force and rate of the pulse and the blood-pressure fall: This is owing to a direct action on the ends of the vagus. How far these effects are due also to affection of the medullary and spinal centres is not known.

Respiration.-Doon after the administration of gelsemium the respiration becomes slower and more feeble; ultimately it stops, death taking place by asphyxia. This is due to paralysis of 
the respiratory centres in the cord and medulla. Before death the temperature falls, and the skin is bathed in a cold sweat.

\section{Therapeutics of Gelsemium.}

Gelsemium was formerly given [as a circulatory depressant, but it is not now used, as its other effects are so harmful. Nor is it any longer prescribed for convulsive diseases, as tetanus, whooping-cough, chorea, etc., as it was not found to do any good. It is often successfully used for neuralgia and migraine; how it acts is quite uncertain.] A good combination for neuralgia and migraine is gelsemine hydrochlorate $\frac{1}{200} \mathrm{gr}$. [.0003 gm.] with butyl chloral hydrate, $3 \mathrm{gr}$. [.20 gm.] made into a pill with mucilage, and given every two hours until pain is relieved. Sometimes it is employed to dilate the pupil and paralyze accommodation. It will do this when applied locally, for it is quickly absorbed from the eye. [It has the advantage that its influence passes off rapidly.] Discs [of gelatin], each containing $\frac{1}{50} \sigma \mathrm{gr}$. [.0001 $3 \mathrm{gm}$.] gelsemine (not official), are made for application to the eye.

\section{Class III.-DRUGS ACTING CHIEFLY ON THE NERVES.}

\section{[A. The following depress the motor nerves.]}

\section{CONIUM.}

CONIUM.-Synonym.-[Spotted Hemlock. The full grown fruit of Conium Maculatum, Linné (nat. ord. Umbellifera), gathered while yet green. Habitat.-Europe and Asia; naturalized in North America.

Characters. - About $3 \mathrm{~mm}$. long; broadly ovate, laterally compressed; grayish-green; often divided into the two mericarps, each with five crenate ribs, without oil-tubes, and containing a seed which is grooved on the face; odor and taste slight.] Resembling conium fruit.-Caraway, anise, dill, all known by having viltr [oil-tubes].

Composition. - [The chief constituents are-(I) Coniine, $\mathrm{C}_{8} \mathrm{H}_{27} \mathrm{~N}$, the active principle; a colorless, oily, volatile alkaloid, of a disagreeable odor and acrid taste.] Solubility. - In 100 parts of water. It is easily obtained from the plant by distillation with alkalies. It is readily decomposed by light and heat, and the preparations of Conium are therefore of very varying strengths. Its salts are much more stable. (2) Methyl-coniine, $\mathrm{C}_{8} \mathrm{H}_{19} \mathrm{CN}$. A 
colorless, liquid alkaloid. (3) Conhydrine, a nearly inert crystallizable alka. loid.

InCOMPATIBLES.-Caustic alkalies, vegetable acids and astringents.

Dose, 2 to $5 \mathrm{gr}$; ; [.12 to .30 $\mathrm{gm}$.]

Preparations.

I. [Extractum Conii.-Extract of Conium. By maceration and percolation with Diluted Alcohol and Acetic Acid; and evaporation.

Dose, $1 / 3$ to $1 \mathrm{gr}$; .02 to $.06 \mathrm{gm}$.

2. Extractum Conii Fluidum.-Fluid Extract of Conium. By maceration and percolation with Diluted Alcohol and Acetic Acid, and evaporation.

Dose, 1 to $5 \mathrm{~m}$.; .12 to .30 c.c.]

\section{Action of Conium.}

External.-Coniine probably has no influence on the unbroken skin, but it has been thought to be anæsthetic when applied to painful broken surfaces. This is doubtful, for, in the first place, we have no proof that it can be absorbed from sores; and, secondly, experiments show that large doses have to be given to depress the activity of sensory nerves.

Internal.-Gastro-intestinal tract. - It has no special action here, but it may occasionally give rise to vomiting and diarrhœea.

Circulation.-Coniine is absorbed into the blood, and circulates unchanged. Probably it paralyzes the terminations of the vagus and so increases the rapidity of the cardiac beat. The heart beats long after breathing has ceased.

Respiration. - Owing to the profound paralysis of all motor nerves, and the later depression of the respiratory centre and motor part of the cord, death takes place from enfeeblement of respiration and consequent asphyxia.

Nervous system. - Nerves.-Coniine powerfully depresses all the motor nerves. This depression begins at the periphery, and gradually ascends till the whole nerve, up to the spinal cord, is incapable of responding to stimuli. This leads to paralysis of all the muscles of the body so far as voluntary and reflex motion is concerned, but they themselves are unaffected, retaining their irritability to local stimuli. The sensory nerves are not 
implicated unless the dose is very large; then their conducting power is slightly impaired. The effects on nerves are well illustrated in the death of Socrates, for he was directed to walk about until his legs felt heavy (motor paralysis), and later, when his foot was pressed he could not feel.

Spinal cord.-This remains uninfluenced till quite late; then, if poisonous doses have been given, the function of its motor cornua is feebly depressed, as is also that of the respiratory centre in the medulla. These actions are probably due to the methylconiine. As the amount of this is variable in the different specimens, the exact period at which these effects come on varies with different preparations. In some animals asphyxial convul. sions are very marked.

Brain.-Except for the respiratory centre the whole of the brain is unaffected by coniine. Consciousness is preserved until the stage of asphyxia.

Eye.-Coniine, when dropped into the eye, causes immediate contraction of the pupil reflexly from the conjunctival irritation. But soon the pupil dilates, and accommodation is paralyzed; the same usually happens when the drug is given internally. Probably these results are owing to paralysis of the terminal portions of the third nerve, for well-marked ptosis, which is due to this cause, is present.

Coniine is excreted unchanged, chiefly in the urine.

\section{Therapeutics of Conium.}

External.-Conium has been applied to yainful ulcers and sores, but it is, for the reasons already given, doubtful whether it produces any good effect. It has also been employed for myalgia and rheumatism, but it is quite useless.

Internal.-Conium is rarely given as a medicine, for $(a)$ the amount of coniine extracted by any preparation is very variable ; (b) the amount in the same part of different plants is inconstant ; (c) the amount of methyl-coniine present is also very uncertain; $(d)$ coniine is very volatile; $(e)$ it is unstable, light and air making it inert. For these reasons it is probable that 
often the pharmacopœial preparations contain no coniine at all. Ounces of the succus [B. P., which is the expressed juice of the leaves and young branches, to which 25 per cent. of alcohol has been added], and which is believed to be the most reliable preparation, have frequently been swallowed without producing any effects. The preparations of the fruit are said by some to be more reliable than those of the leaves. Conium has been given in spasmodic diseases, as whooping-cough, in chorea, tetanus, asthma, and epilepsy, but in all it does little or no good.

\section{TOXICOLOGY.}

Symptoms. - The symptoms produced by a poisonous dose are in strict accordance with the physiological action. The sufferer feels bis legs to be heavy ; on attempting to walk he staggers, and finds he can bardly move them, and finally he bas to lie down because he has no power over them. The arms become powerless, and lie motionless at his side. There is ptosis, and dimness of vision from paralysis of accommodation; the eyes are fixed, the pupil is dilated. Swallowing becomes difficult. Respiration is labored, the voice is lost, and death takes place from asphyxia. Post-morlem.-The organs are found congested with venous blood.

Trealment,-Emetics (see p. 139), and wash out the stomach. Give tannic acid and again wash it out. Stimulants subcutaneously, warmth to the feet, and artificial respiration [are necessary].

\section{TABACUM.}

[TOBACCO.-The commercial dried leaves of Nicotiana Tabacum (nat. ord. Solanacece). Habitat. - Tropical America ; cultivated.

Characters. - Up to $50 \mathrm{~cm}$. long, oval or ovate-lanceolate, acute, entire, brown, friable, glandular-hairy, of a heavy, peculiar odor, and a nauseous, bitter, and acrid taste.]

Composition. - The chief constituent is-(I) Nicotine, $\left[\mathrm{C}_{10} \mathrm{H}_{14} \mathrm{~N}_{2}(0.7\right.$ to 5, sometimes I I per cent.)]. A colorless, volatile, oily alkaloid, smelling and tasting like Tobacco leaves, darkening with age. Solubility.-Soluble in water, more so in Alcohol and Ether. Turkish Tobacco contains hardly any. (2) Nicotianine. (3) Salts and flavoring agents.

[Nicotine is decomposed by heat, consequently. Tobacco smoke contains none (Binz), but consists of small quantities of various Pyridine compounds, as Pyridine $\mathrm{C}_{5} \mathrm{H}_{5} \mathrm{~N}$, Picoline $\mathrm{C}_{6} \mathrm{H}_{7} \mathrm{~N}$, Lutidine $\mathrm{C}_{7} \mathrm{H}_{9} \mathrm{~N}$, Collidine $\mathrm{C}_{8} \mathrm{H}_{11} \mathrm{~N}$, Parvoline $\mathrm{C}_{9} \mathrm{H}_{13} \mathrm{~N}$, Coridine $\mathrm{C}_{10} \mathrm{H}_{15} \mathrm{~N}$, Rubidine $\mathrm{C}_{11} \mathrm{H}_{17} \mathrm{~N}$, and small amounts of Hydrocyanic and Acetic Acids, Creosote, Sulphur, and Carbon compounds.] 


\section{Action of Tobacco.}

Tobacco leaves, when taken internally, act entirely by virtue of their nicotine, which is one of the most powerful and rapid poisons known.

External.-Nicotine is an antiseptic.

Internal.-Gastro-intestinal tract. - Nicotine in even minute doses ( $\frac{1}{7} \mathrm{gr}$. [.009 $\left.\mathrm{gm}.\right]$ ) promptly produces greatly increased salivary flow, burning pain in the mouth, œsophagus and stomach, horrible nausea, quickly succeeded-owing to its action on the gastro-intestinal muscle-by vomiting and free purging. The marked characteristic of this gastro-intestinal irritation is the extreme collapse which accompanies it. Thus there is a rapid, very feeble pulse, intense muscular weakness, labored respiration, partial loss of consciousness, occasional convulsions, icy extremities, and profound general collapse. A dose of nicotine has been known to kill in three minutes, but in both man and animals a certain tolerance may be acquired.

Circulation.-Nicotine disintegrates the red blood-corpuscles of freshly-drawn blood, but has not this effect upon living blood, although the spectrum of hæmoglobin is altered, so that the corpuscles must be in some way affected. The action on the heart is obscure; the muscle itself is unaffected, but the rapidrunning, feeble pulse shows that some part of the cardiac apparatus is powerfully influenced. The blood-pressure falls rapidly; this is not entirely due to the action of nicotine on the heart, but is in part due to its peripheral action on the vessels.

Respiration. - This is at first accelerated and deepened; ultimately it is paralyzed from depression of the centre. Death is partly due to asphyxia.

Nervous system.-The higher faculties are depressed by large doses of nicotine, for those poisoned by it become comatose within even a minute or two of taking a large dose. The convulsions occasionally observed in man, and always in the frog, are due to spinal stimulation. All observers are agreed that ultimately the function of the motor nerves is entirely abolished. This explains the intense muscular weakness. Probably the sensory nerves, and certainly the musrles, escape. 
Eye.-A toxic dose taken internally, or the local application of nicotine to the eye, contracts the pupil of man and most animals. This will occur in excised eyes, and is therefore a local effect. With some animals nicotine is a mydriatic. We know nothing of the details of its action.

Secretion.-Nicotine first stimulates but ultimately paralyzes the secretory structures of the salivary, sweat and lachrymal glands.

Elimination. - Nicotine is eliminated partly by the lungs, but chiefly in the urine, the secretion of which it increases.

\section{Therapeutics of Tobacco.}

Tobacco is never used therapeutically. [In non-smokers it is useful to relieve the symptom asthma.] Formerly it was employed in the form of an enema of leaves to relax muscular spasm, so as to facilitate the reduction of dislocations. This enema was also sometimes given as a purgative. [Pyridine which is found in tobacco, but commercially is obtained from other sources, when administered by inhalation will frequently relieve the paroxýsms of asthma. For this purpose a fluid drachm; 4. c.c., is necessary, placed in a dish, so that it may slowly evaporate. Its persistent and abominable odor is a great obstacle to its use.]

Tobacco smoking, in those who are unaccustomed to it, produces, to a greater or less degree, the symptoms of gastro-intestinal irritation and collapse just mentioned. Even in those who are used to it the smoke may produce catarrh of the pharynx. Some persons find smoking after breakfast assists the daily action of the bowels. With many people it has an obscure effect, especially among those who lead sedentary lives, in stimulating the brain and producing a peaceable, calm state of mind. Overindulgence in it may lead to loss of appetite, irregularity of the heart, chronic laryngeal and pharyngeal catarrh, and retrobulbar neuritis of the optic nerve. The effect of this is that the sufferer complains that objects look misty, he has a central scotoma, sometimes complete, often only for red and green, and finally atrophy of his optic nerve. 


\section{[Toxicology.}

Symptoms. - The symptoms are those which we should expect from its physiological action.

Treatment.-Tannic acid followed by emetics (see p. 139). Strychnine is the true physiological antidote. Alcohol and ammonia stimulate the heart. The recumbent position must be maintained. Artificial respiration may be necessary.]

\section{[B. The following depresses the motor end-plates.]}

\section{[CURARA.]}

CURARE. (Not official.)-Synonyms. - Wourara. Ourari. Urari. Wourali. The South American arrow poison, prepared from species of Strychnos and other plants.

Characters.-A blackish-brown, dry extract, [brittle or hygroscopic, with a bitter taste. Solubility._Almost completely in diluted Alcohol.]

Composition. - It contains an extremely active poison, Curarina or Curarine, $\left[\mathrm{C}_{18} \mathrm{H}_{35} \mathrm{~N}\right.$, ] a yellowish-brown powder intensely bitter.

Dose, $\frac{1}{25}$ to $1 / 2 \mathrm{gr}$; [ [.0025 to $.03 \mathrm{gm}$.]

The British Pharmaceutical Conference [proposes for hypodermatic injection of Curara :] -Curare, $\mathbf{I}$; add distilled water to form a thin paste. Put in a funnel plugged with absorbent wool, and gradually add more water until 12 parts are obtained. Of this the dose is I to $6 \mathrm{~m}$. ; [.06 to .25 c.c.]

Lamellæ or discs, each containing $\frac{1}{26}$ gr. ; [.003 gm.] are also prepared. They are dissolved in a few drops of water before injection subcutaneously.

\section{Action and Therapeutics of Curare.}

The physiological action of curare, by which it paralyzes the end plates of the motor nerves of voluntary muscles, is well known. It has been given successfully in tetanus, and is probably the most useful of all the drugs employed for this very fatal disease.

\section{[C. The following depress the sensory nerves.]}

COCA.

COCA.-[Syrionyms.-Erythroxylon. Cuca. The leaves of Erythroxylon Coca, Lamarck (nat. ord. Linea). Habitat.-Peru and Bolivia ; cultivated.

ChiARACTERs.-Varying between ovate, lanceolate, and obovate-oblong, and from 2 to 5 or $7 \mathrm{~cm}$. in length; short-petiolate, entire, rather obtuse or emarginate at the apex, slightly reticulate on both sides, with a prominent mid- 
rib, and on each side of it a curved line running from base to apex ; odor slight and tea-like; taste somewhat aromatic and bitter. When chewed, it temporarily benumbs the lips and tongue.]

Composition. - It contains at least three alkaloids, viz., (I) Cocaine, which is methyl benzoyl ecgonine, 0.2 per cent. ; (2) Cocamine or isatrophyl cocaine ; (3) Cinnamylcocaine. Also (4) Coca-tannic acid and (5) Coca-wax. Different specimens vary very much in strength of Cocaine. Fresh specimens are stronger than those that have been kept.

Incompatibles. - Mineral acids (decompose cocaine into benzoic acid and ecgonine), sodium bromide, menthol, mercury salts and silver nitrate.

[Dose, I to $4 \mathrm{dr}$. ; 4. to $15 . \mathrm{gm}$. of the leaves infused in hot water.]

\section{Preparation.}

Extractum Cocæ [Fluidum.-Fluid Extract of Coca. By maceration and percolation with Diluted Alcohol, and evaporation.

Dose, I to 4 fi. dr.; 4 . to r5. c.c.]

COCAIN 2 HYDROCHLORAS.-Cocaine Hydrochlorate. $\mathrm{C}_{77} \mathrm{H}_{21}$ $\mathrm{NO}_{4} \mathrm{HCl}[=338.7 \mathrm{I}$. The hydrochlorate of an Alkaloid obtained from Coca.

SourCE.-Agitate with Ether an aqueous solution of an acidulated Alcoholic extract, make alkaline with Sodium Carbonate; separate and evaporate the Ethereal liquid ; purify by repetition; decolorize, neutralize with Hydrochloric Acid, and re-crystallize.

Characters. - Colorless, transparent crystals, or a white, crystalline powder, without odor; of a saline, slightly bitter taste, and producing upon the tongue a tingling sensation followed by numbness of some minutes' duration. Permanent in the air. Solubility. - In 0.48 part of water, and in 3.5 parts of Alcohol ; also soluble in 2800 parts of Ether, or in 17 parts of Chloroform.

Dose, $1 / 8$ to $2 \mathrm{gr}$; .008 to $.12 \mathrm{gm}$.]

\section{Action of Coca.}

External.-Cocaine has little action on the unbroken skin, but if injected subcutaneously or applied to mucous membranes -as, for example, those of the eye, nose, mouth, rectum, vagina -it produces complete local anæsthesia to pain, touch, heat and cold, so that small operations can be performed without the patient feeling them. A 5 to ro per cent. solution of the hydrochlorate is strong enough thus to paralyze the sensory nerves. Much larger doses must be applied to motor nerves to paralyze them.

Internal.-Gastro-intestinal tract.-Applied to the nose and tongle cocaine abolishes smell and taste respectively, and when it 
is taken internally, the gastric mucous membrane experiences its anæsthetic influence. Therefore the sensation of hunger is deadened, and persons taking cocaine can go a long time without feeling the want of food; but the drug is not a food, for the body rapidly wastes. Because of its local anæsthetic effect it sometimes stops vomiting. Very large doses may lead to diarrhœea.

Circulation.-Large doses depress the vagus, and therefore the pulse quickens, and as the vaso-motor centre is stimulated the blood-pressure rises; larger doses slow the pulse from stimulation of the vagus. [Cocaine constricts the arterioles].

Respiration. - It acts upon the respiratory centre, first stimulating it, so that the rapidity and depth of respiration are increased; but soon depression of the centre follows, the respiratory movements become feeble, and death takes place from asphyxia.

Nervous system.-Cerebrum.-Moderate doses greatly increase the bodily and mental power, and give a sense of calm and happiness, with abolition of bodily and mental fatigue. This greater physical energy renders possible the performance of long, exhausting muscular feats. For this, and for the extreme sense of peace produced, coca leaves mixed with clay or ashes are chewed by thousands of the inhabitants of Peru and the neighboring countries. It is said that forty million pounds of the leaves are annually harvested. A single large dose causes mental excitement, delirium and ataxia, with subsequent headache and depression. This ataxia is due to impairment of conduction of sensory impressions from the effect of the cocaine on peripheral sensory nerves. An excessive indulgence in the habit of coca-chewing leads to indigestion, extreme enraciation, insomnia and enfeeblement of intellect. In animals coca causes cerebral convulsions.

Eye.-When a solution of cocaine of about 4 per cent. is dropped into the eye local anæsthesia is produced, first of the conjunctiva and cornea, later of the iris. It is attained in about seven minutes, and lasts about the same time. At first there may be a transitory contraction of the pupil. This is probably due to reflex action, and soon gives way to wide dilatation. 
The maximum is attained in an hour or two. The normal state is regained in from twelve to twenty-four hours. The dilated pupil is freely responsive to light, and the dilatation is rapidly overcome by physostigmine. The ocular tension is slightly lowered, and the palpebral aperture is widened. Accommodation is partially, but never completely, paralyzed. These effects are chiefly due to irritation of the sympathetic, and as they are quickly produced by dropping the drug in the eye they are probably local. All these effects are slowly produced if large doses of cocaine are taken internally. [Strong solutions or weak solutions frequently applied desiccate the corneal epithelium.]

Muscles. - The amount of muscular work of which the body is capable is increased by cocaine, how, is not known. The excretion of urea and nitrogenous metabolism are unaltered.

Temperature. - This may rise in cocaine poisoning.

Kidneys. - Cocaine is most likely excreted by these organs. It diminishes sexual excitability.

\section{Therapeutics of Coca.}

External.-A 5 to ro per cent. solution of the hydrochlorate may be injected subcutaneously as a local anæsthetic when any small operation has to be performed. [In the infiltration method of Schleich three solutions are employed : cocaine hydrochlorate, 0.2 (strong); O.I (normal); or o.or (weak); morphine hydrochlorate, 0.025 ; sodium chloride, 0.2 ; sterilized distilled water or saturated boric acid solution to ı०o. These are injected into the substance of the skin forming wheals. This method requires less of the drug than when used subcutaneously. Yet it should be borne in mind that the anæsthetic properties of the two weaker solutions depend largely upon the mechanical anæsthesia produced by injection of water, which had been previously pointed out by Halsted.] Solutions, painted or dropped on, may be used for operations on the mouth, throat, teeth ( 4 per cent.), eye ( 1 to 4 per cent.), ear, vagina, urethra and rectum ( 4 to ro per cent.), and they may be applied to any of these parts when they are very painful. [Congestive urethral stricture may be temporarily relieved by it so that instruments may be passed, but it 
should be used with great care in urethral operations.] Cocaine will relieve vaginal pruritus, and has been used locally applied in the nose in hay fever. Painful ulcers, fissures, etc., are beneficially treated with it. Ointments, bougies, and suppositories, usually containing 2 to 5 per cent. of cocaine, which mixes better than the hydrochlorate, are very useful. A I 5 per cent. solution has been injected into the gums for tooth extraction, but is not strongly recommended. [Ophthalmic surgeons] employ it very largely to produce local anæsthesia of the eye. [If inflammation is present anæsthesia is produced with great difficulty.]

Internal.-Mouth.-A solution is useful for painting or spraying on the throat previous to laryngeal examinations. [Lozenges of the hydrochlorate, containing $\frac{1}{12}$ gr. ; .005 gm., in each, are valuable for painful sore throat. Often in addition each lozenge contains $3 \mathrm{gr}$; $.20 \mathrm{gm}$. of extract of krameria.]

Stomach.-Cocaine in some cases allays excessive vomiting, and has been said to cure sea-sickness.

It is not often used in Europe as a medicine for its restorative effects; as already mentioned, it is not a food, and the good it does is only temporary.

[Medullary Anasthesia.-It has been recently proposed to obtain surgical anæsthesia by injection of from $\frac{1}{10}$ to $\frac{1}{5} \mathrm{gr}$; ;.006 to $.012 \mathrm{gm}$., into the arachnoid space. Puncture is made between third and fourth lumbar interspace of the spine with a specially prepared needle as for diagnostic purposes. A few drops of the spinal fluid is allowed to escape and the solution is injected. Anæsthesia supervenes, gradually extending from the feet upwards, and may reach to the chest or even higher; this persists for a variable time, but generally sufficient for the performance of surgical operations. This method of anæsthesia does not interfere with labor further than abolishing its pain. Strict asepsis must be observed. Although thus far no accidents have been recorded, it is by no means clear that it will supplant ether or chloroform narcosis, nor that it can be employed when contra-indications exist to either. Beyond some nausea, vomiting and headache, after-effects are not noticed. It is yet too early to formulate an opinion as to the practical value of this method.] 


\section{ToXICOLOGY.}

Symptoms. - It is a respiratory depressant; but symptoms of poisoning have rarely been noticed unless the drug has been injected under the gums or skin. Then it may quickly cause vertigo, pallor, fainting, profound cardiac and res. piratory depression with tremors and other nervous symptoms which may per. sist for months, even if the other symptoms are overcome.

In the chronic forms, known as cocamania, the sufferer takes cocaine either for its pleasant effects or because he thinks it will help break himself from the morphine habit, or he takes it with morphine. It is usually administered subcutaneously. The pulse is rapid, and fainting is common. There is much wasting, and the patient looks pale and death-like. Usually he suffers from insomnia, and he may become acutely maniacal with delusions of persecution. Visual and other hallucinations are often present, and it is very characteristic that patients complain of little animals creeping on the skin, "cocaine bugs," they say. They are extraordinarily prolix in both conversation and writing. [Cocaine habitués are by no means infrequently met with. The moral degradation is fully equal to that of opium.eaters.

Treatment.-This should consist in stimulation, and emptying the stomach, if the drug has been ingested, by means of hypodermatic injections of apomorphine. Artificial respiration may be required. Since cocaine asphyxiates by constricting the blood-vessels at the base of the brain, strychnine hypodermatically or alcohol may remove this danger.

For the chronic forms corfinement in an asylum, with careful treatment during the period of severance, is essential.

\section{OUABAINUM.}

OUABAIN. (Not official.) $-\mathrm{C}_{30} \mathrm{H}_{46} \mathrm{O}_{12}=569.62$. A glucoside isolated from the root of a tree, said to be the Acocanthera Onabaio (nat. ord. Apocynacece.) This glucoside is also obtained from the seeds of Strophanthus glabrus. Habitat.-Africa.

Characters. - A white crystalline powder slightly bitter. Solubility.Soluble in hot, but with difficulty in cold water; insoluble in Chloroform and Ether.

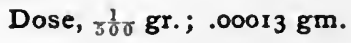

\section{Action and Uses of Ouabain.}

Ouabain paralyzes cardiac muscle by direct action, and when given hypodermatically is an emetic. According to Gley it is a local anæsthetic, having ten times the power of cocaine. It has also been recommended for all stages of whooping-cough in doses of ${ }_{1000}^{1} \mathrm{gr} . ; .00006 \mathrm{gm}$, , for children. As it is a very powerful drug, $\frac{1}{65} \mathrm{gr} . ; .001 \mathrm{gm}$., when taken into the blood, being sufficient to kill a man, it should be used with great caution. 


\section{The following stimulates the secretory nerves.] PILOCARPUS.}

PILOCARPUS.-Synonym.--Jaborandi. [The leaflets of Pilocarpus Selloanus Engler (Rio Janeiro Jaborandi), and of Pilocarpus Jaborandi Holmes (Pernambuco Jaborandi), (nat. ord. Rutacea). Habitat._Brazil, near Pernambuco.

Characters. - About to to $15 \mathrm{~cm}$. long, and 4 to $6 \mathrm{~cm}$. broad, shortstalked, oval or ovate-oblong, entire and slightly revolute at the margin, obtuse and emarginate, unequal at the base ; dull green, coriaceous, pellucid punctate, mostly smooth; when bruised, slightly aromatic; taste somewhat bitter and pungent.]

IMPUR ITIES. - Leaves of species of Piper, not oval-oblong.

Composition.-The chief constituents are-(I) A liquid, colorless, alkaloid, Pilocarpine, $\mathrm{C}_{11} \mathrm{H}_{16} \mathrm{~N}_{2} \mathrm{O}_{2}, 1 / 4$ to I per cent. (2) Jaborine, $\mathrm{C}_{22} \mathrm{H}_{32} \mathrm{~N}_{4} \mathrm{O}_{4}$, an alkaloid resembling in its physiological action Atropine, and therefore antagonistic to Pilocarpine. (3) [Pilocarpidine, $\mathrm{C}_{10} \mathrm{H}_{14} \mathrm{~N}_{2} \mathrm{O}_{2}$, a decomposition product whose action is weaker than Pilocarpine.] (4) A volatile oil [chiefly Pilocarpene, $\mathrm{C}_{10} \mathrm{H}_{16}$ ]. (5) A peculiar acid. These active principles are soluble in Alcohol, but only imperfectly so in water.

Dose, 5 to $60 \mathrm{gr}$.; $[.30$ to $4.00 \mathrm{gm}$. $]$

\section{Preparation.}

Extractum [Pilocarpi Fluidum.-Fluid Extract of Pilocarpus. By maceration and percolation with Diluted Alcohol and evaporation.

Dose, 5 to $60 \mathrm{~m}$. ; .30 to 4.00 c.c.]

PILOCARPIN $\approx$ [HYDROCHLORAS.-Pilocarpine Hydrochlorate. $\mathrm{C}_{12} \mathrm{H}_{16} \mathrm{~N}_{2} \mathrm{O}_{2} \mathrm{HCl}=243.98$. The hydrochlorate of an Alkaloid obtained from Pilocarpus.

SourCE.-Obtained by exhausting Pilocarpus with Alcohol acidulated with Hydrochloric Acid, distillation and evaporation. The filtrate is treated with a slight excess of Ammonia, and a large quantity of Chloroform. The solution is agitated with water, to which Hydrochloric Acid is added to neutralization. The Hydrocblorate is obtained on evaporation in crystals which are purified by re-crystallization.

Characters. - Small, white crystals, odorless, and having a faintly bitter taste; deliquescent on exposure to damp air. Solubility.-Very soluble in water and in Alcohol; almost insoluble in Ether or Chloroform.

Dose, $1 / 8$ to $1 / 3 \mathrm{gr}$; ; .008 to $.02 \mathrm{gm}$.]

Action of Pilocakpus.

External.-None.

Internal.-Gastro intestinal tract. - Pilocarpine is very quickly absorbed, and soon produces a great increase in the 
amount of salivary secretion. The mouth seems warm, and there may be a feeling of tenseness about the salivary glands. The saliva contains an abundance of salts and ptyalin, and can convert starch into sugar. Its increase is due to a direct stimulation of the terminal filaments of the chorda tympani and of the other nerves which end in the cells of the salivary glands, so that stimulation of these nerves can add very little to the flow produced by the drug-in fact, not more than can be accounted for by vascular alterations. This action is antagonized immediately by atropine, as that paralyzes the endings of these nerves. To a slight extent pilocarpine excites the secretion of the gastric juice, intestinal fluid, and pancreatic secretion. The unstriped muscle of the stomach and intestine is stimulated, and thus the drug may purge. The bile is unaffected. Large doses, especially of [pilocarpus], may produce vomiting.

Circulation.-Pilocarpine has no effect on the blood, but it is a cardiac depressant. The pulse-rate, it is true, may be, and in the human being always is, a little accelerated at first, but soon both it and the blood-pressure fall. This slowing of the pulse is at once set aside by atropine, but is not prevented by section of the vagus, therefore pilocarpine acts on the heart itself, probably stimulating the terminations of the vagus. The bloodvessels are at first dilated.

Respiration. - The drug has no effect on this. The amount of bronchial secretion is increased.

Skin.-[Pilocarpus], through its alkaloid pilocarpine, produces a very profuse secretion of sweat. It is the most powerful diaphoretic drug we have. A single dose may cause the flow of fifteen fluid ounces [450. c.c.] of sweat. It is said that the proportion of urea and chlorides in the sweat is greatly increased. This profuse diaphoresis is due to the action of the pilocarpine on the cells of the sweat-glands, or the terminations of the nerves in them, and is stopped by atropine. The skin may flush, but this is not the cause of the diaphoresis. Under a course of [pilocarpus] the hair grows more actively, but it becomes very coarse and dark.

Kidneys. - If the sweating is profuse, the secretion of urine is lessened. Pilocarpine is excreted unchanged in the urine. 
Temperature.-There may be a slight rise at first, but soon the temperature falls considerably. This is probably due in large part to the evaporation of the perspiration.

Eye.-Whether applied locally to the eye or given internally, pilocarpine produces great contraction of the pupil, due to stimulation of the ends of the third nerve in the eye, and this is antagonized by atropine. It also causes increased tension of the eye-ball, and an approximation of the nearest and farthest points of distinct vision.

Other actions. - It stimulates the uterus, and has in very rare cases produced abortion. It increases the secretion of milk, of tears, of nasal mucus, and, according to some authors, that of cerumen. It causes the spleen and bladder to contract.

It will be noticed that it has two main actions. (I) It stimulates the secretions-viz., those of the salivary glands, stomach, intestines, skin, kidney, bronchial mucous membrane, nose, lachrymal glands and ear. In those that have been investigated, and probably in all, it acts locally. It has not been decided in every case whether the cells of the glands or the nerve terminations in them are affected. (2) It stimulates the nerve terminations of involuntary muscles-viz., in the eye, the intestines, the stomach, the uterus, the spleen, the heart, the bladder, and it acts on the muscular coat of the vessels, although these, if affected, are usually dilated. The most important effects are the diaphoresis, the salivation, and the myosis. It is consequently antagonistic in its action to atropine. Children bear large doses of it well. Pilocarpine is much more used than [pilocarpus], as it is more prompt and more certain in its action, and is less likely to cause indigestion.

Jaborine has an action similar to that of atropine; the amount of it in [pilocarpus] varies, hence the varying effects of different specimens of the leaves, but there is never enough totally to counteract the pilocarpine.

\section{Therapeutics of Pilocarpus.}

External.-Pilocarpine has been used locally to promote the growth of hair. An ointment (Pilocarpine [hydrochlorate], 
I ; soft petrolatum, 60; hydrous wool fat, 6o, ) or a lotion (Pilocarpine [hydrochlorate], I ; quinine hydrochlorate, 4 ; glycerin, 60 ; rose water, I 80 ,) have been used.

Internal.-Pilocarpine has been employed for many conditions, but its great use is as a diaphoretic in Bright's disease. For this purpose $1 / 6$ of a gr., [.or gm.] or more of the [hydrochlorate] is injected subcutaneously in the evening. The sweating is aided by wrapping the patient, who should be naked, in several warm blankets, giving him hot drinks, and putting a hot water bottle to his feet. After the sweating has ceased, he should be dried and left in a dry blanket. As it is such a powerful cardiac depressant, it must be given with great caution when the heart is diseased. Occasionally it is employed locally in affections of the eye. Patients suffering from deafness due to disease of the auditory nerve or its terminations are sometimes relieved by pilocarpine. [It is often given internally for deafness due to otitis media sicca.] Injected subcutaneously, it has been given successfully as an antidote to belladonna poisoning.

\section{GROUP II.}

\section{Vegetable Drugs whose main Action is on the Heart.}

CIASS I.-The Digitalis group, decreasing the frequency and increasing the force of the beat of the heart :

Digitalis, Strophanthus, Convallaria, Squill, [Scoparius,] Erythrophlœum, and [Adonidin].

Class II.-The Aconite group, decreasing the frequency and force of the beat of the heart:

Aconite, [Veratrum Viride,] Veratrina.

[CLASS III.-The Cactus group, increasing the frequency and force of the beat of the heart :

Cactus Grandiflorus.] 
Class I.-THE DIGITALIS GROUP.

\section{DIGITALIS.}

DIGITALIS.-[Synonym.-Foxglove. The leaves of Digitalis Purpurea Linné (nat. ord. Scrophularinea), collected from plants of the second year's growth. Habitat.-Europe, in sandy soil and the edges of woods.

Characters. - From io to $30 \mathrm{~cm}$. long, ovate or ovate-oblong, narrowed into a petiole ; crenate; dull green, densely and finely pubescent ; wrinkled above ; paler and recticulated beneath ; midrib near the base broad ; odor slight; somewhat tea-like; taste bitter, nauseous.] Resembling Digitalis leaves. Matico leaves, which are more deeply reticulated.

Composition.-The chief constituents are-(I) Digitoxin, a glucoside, crystallizable, the most active principle, very poisonous, cumulative. Insoluble in water, sparingly in Ether, easily in Chloroform and Alcohol. Exists as minute white crystals. Dose $\frac{1}{250}$ to $\frac{1}{50} \mathrm{gr}$. [.00025 to.001 $25 \mathrm{gm}$. $]$ (2) Digitalin, a crystalline glucoside, possessing in a high degree the actions of Digitalis. It is also called Digitalinum Verum. Soluble in water, $I$ in Iooo. Dose, $\frac{1}{200}$ to $\frac{1}{100} \mathrm{gr}$. [.0003 to $.0006 \mathrm{gm}$.] subcutaneously. (3) Digitalein, an amorphous glucoside, not yet proved to be a definite chemical substance, soluble in water, and therefore suitable for hypodermatic injections; dose hypodermatically $\frac{1}{\mathrm{~T}^{\frac{1}{0}}} \mathrm{gr}$; ; .0006 gm., said to be non-cumulative. These three glucocides are said to represent the cardiac stimulating action of the drug, (4) Digitonin, $\left[\mathrm{C}_{27} \mathrm{H}_{44} \mathrm{O}_{13}\right]$ a glucoside closely allied both chemically and physiologically to, and perhaps identical with, the Saponin of Senega $(q . v$.$) . [Dose,$ $\frac{1}{300}$ to $\frac{1}{100} \mathrm{gr} . ; .0002$ to $.0006 \mathrm{gm}$.] This is a cardiac depressant, and is therefore antagonistic to the other active principles. (5) Digitin, a glucoside devoid of physiological action. All these five glucosides are non-nitrogenous. (6) Two acids, Digitalic and Antirrhinic. (7) Other usual constituents of plants, as tannic acid, volatile oil, coloring matter, starch, sugar, gum, salts. It will be noticed that Digitalis contains no Alkaloids.

The following five substances, all soluble in alcohol, are met with in commerce: (A) Homolle's Digitalin (same as Quevenne's), an amorphous yellowish-white powder or small scales, intensely bitter, inodorous, but irritating to the nostrils. [Soluble in 2000 parts of water.] Consists chiefly of Digitalin with a little Digitoxin. Possesses the action of the leaves. Granules of it are much used in France ; each usually contains $\frac{1}{65} \mathrm{gr} .$, , .0OI gm., which is equal to $\mathrm{I} 1 / 2 \mathrm{gr}$; , Io gm. of the powdered leaves. (B) Nativelle's Digitalin, $\left[\mathrm{C}_{25} \mathrm{H}_{40} \mathrm{O}_{15}\right.$, light, white, crystalline tufts of needles, very bitter. Soluble in Chloroform and in Alcohol, not in Water or Ether. It consists very largely of Digitoxin and is cumulative. Dose, $\frac{1}{60}$ to $\frac{1}{30} \mathrm{gr}$; .001 to $.002 \mathrm{gm}$. in a pill. (C) German Digitalinum Purum. Dose, $\frac{1}{60}$ to $\frac{1}{30}$ gr. [.001 to.002 gm., soluble in water. Consisting chiefly of Digitalein, with some digitalin 
and digitonin. (D) Digitoxin, already described (see p. 42I). $\quad$ (E) Digitalin. already described (see p. 42I). [None of the above constituents are official.] InCOMPATIBLES. - Ferric salts, lead acetate, and cinchona. Dose, $1 / 2$ to $3 \mathrm{gr}$.; [.03 to .20 $\mathrm{gm}$.]

\section{Preparations.}

I. [Extractum Digitalis.-Extract of Digitalis. By maceration and percolation with Alcohol and Water, distillation of the Alcohol, and evaporation.

Dose, $1 / 4$ to $\mathrm{I} \mathrm{gr}$; .015 to $.06 \mathrm{gm}$.

2. Extractum Digitalis Fluidum.-Fluid Extract of Digitalis. By maceration and percolation with Alcohol and Water, and evaporation.

Dose, $1 / 2$ to $3 \mathrm{~m} . ; .03$ to .20 c.c.

3. Infusum Digitalis.-Infusion of Digitalis. Digitalis, 15; Cinnamon Water, 150; boiling water, 260 ; Alcohol, 100; cold water to 1000 .

Dose, I to 4 fl. dr. ;] (note that it is drachms, not ounces); [4. to 15. c.c.]

4. Tinctura Digitalis.-[Tincture of Digitalis. Digitalis, $15^{\circ}$; Diluted Alcohol to 1000 . By maceration and percolation.

Dose, 5 to $30 \mathrm{~m}$.; .03 to 2.00 c.c.]

As the proportion of the many constituents varies in the preparations, some prefer always to give the powdered leaves.

\section{Action of Digitalis.}

External.-The leaves are slightly irritating, but it is doubtful whether any of their constituents can be absorbed by the skin.

Internal. - Gastro-intestinal tract. - Digitalis is a mild gastro-intestinal irritant, and even moderate doses cause vomiting and diarrhœa in some people.

Blood.-It is [not] rapidly absorbed; it is not known to affect the blood.

Heart. - The first action of digitalis is to slow the beat of the heart, the diastole is prolonged, the duration of the systole is not altered, but its force is greatly increased, so much so that after large doses the heart may, in animals, be seen to become pale, because almost every drop of blood is squeezed 
out of it. The pulse is consequently increased in force, but retarded. If before the drug was given the heart was beating irregularly, it generally becomes regular. If the drug is taken internally, the whole of both ventricles is, in mammals, affected; but in frogs one portion of the ventricle may remain spasmodically contracted during the diastole of the rest of it. Finally the ventricles are, in frogs, arrested in systole, firmly contracted, quite pale, and unable to respond to any stimuli, but in mammals the heart finally stops in diastole. If locally applied to parts of the ventricle of the frog, only those parts-to which the digitalis is applied are contracted; this is not so in mammals. The auricles are in most animals slowed by it-but the force of their beat is not much altered. In all animals large doses cause great irregularity of the auricular beat.

That these phenomena are chiefly due to the direct action of the drug on the cardiac muscle is shown by the fact that digitalis not only tonically contracts the frog's heart when applied locally, but it will even increase the force of the contraction, when applied to the isolated apex in which it is believed no nerves exist, and it acts on the embryonic heart of the chick before the nerves are developed. But the inhibitory activity of the cardiac peripheral end of the vagus is increased; for a mild stimulation of the vagi, which, before the drug was given, had no effect, will after the drug is given, stop the heart, and in warm-blooded animals digitalis does not very markedly retard the pulse, if the vagi have been cut, although it increases the force of the cardiac beat. The vagus centre in the medulla is stimulated to a less extent. Cushing has shown that the vagal action, with most of the digitalis group of drugs, begins a little before the muscular.

It has been proved that even small doses actually increase the amount of work done by the heart in a given time; thus there is a greater output at each ventricular contraction.

Vessels. - Moderate doses of digitalis produce a great rise in the blood-pressure. This is partly due to the greater cardiac force, but not entirely, for in the web of the frog's foot and the rabbit's mesentery the arterioles have been observed to 
contract vigorously when digitalis has been given. As this still occurrs in arterioles quite separated from the body, and through which an artificial circulation of blood containing digitalis is carried on, it is clear that the drug contracts the arterioles by direct action on their muscular coat. But as the contraction is greater in an intact animal than in one whose spinal cord is destroyed, or in whom the nerves going to the part experimented upon are divided, it is clear that digitalis also stimulates the medullary and spinal vaso-motor centres. With toxic doses the irritation of the centres and of the muscular coat of the arterioles passes on to depression, and the blood-pressure falls.

Kidney. - The effect of digitalis on the kidney is very uncertain. Most experimenters have found that in health it is diuretic, but some have not, and the same discrepancy in its action on the kidney exists in patients with heart disease, but generally in these cases it is a diuretic. The reasons for these discrepancies are that if the arterial vessels are, like the rest of the vessels in the body, tightly contracted by the drug, very little blood will come to the kidney, and very little urine will be secreted; but if the digitalis does not constrict the renal vessels markedly, the increased cardiac force and the general rise of blood-pressure will send more blood through the kidney and more urine will be excreted. Some observers have, probably incorrectly, stated that digitalin and digitoxin have a special effect in relaxing the vessels of the kidney. We have no certain knowledge of the effect of digitalis on the constitution of the urine.

Temperature.-Moderate doses have no influence on the temperature, but toxic doses cause it to fail even in health. The reason of this is unknown.

Respiration.-This is unaffected by digitalis unless poisonous doses have been given, when it begins to fail from the imperfect circulation through the respiratory mechanism.

Nervous system and Muscles.-Medicinal doses have no marked influence. Large doses will, because of the alterations in the cerebral circulation, cause headache, giddiness, and disturbances of sight and hearing. In many cases of poisoning all 
objects have appeared blue. [In acute poisoning there is a peculiar blue color of the sclerotic.]

The reflex activity of the cord and motor nerves is depressed independently of the action on the circulation; sensory nerves are unaffectcd. Digitalis directly paralyzes muscles if given in toxic doses.

Uterus.-This organ is said to be stimulated to contract by digitalis.

\section{Therapeutics of Digitalis.}

External.-Digitalis is [sometimes] used externally [in the form of a poultice made from the leaves, and placed over the loins in case of renal congestion].

Internal.-It is one of the most valuable drugs we have. It is chiefly given in cases of cardiac disorder.

Mitral regurgitation. - If in any case of this variety of heart disease the organ is beating feebly, irregularly, and rapidly, digitalis in moderate doses will probably strengthen, regulate, and slow the beat. It will cause the left ventricle to contract more forcibly and to act synchronously in all its parts; hence the nitral flaps will be better approximated, the regurgitation will be less, and more blood will be sent on into the arterial circulation. The prolonged diastole will also be of great advantage, for it will allow more time for the blood to flow from the dilated auricle, and from the right side of the heart and venous system generally, into the left ventricle. In mitral regurgitation, as is well known, venous engorgement and œdema of the lungs, of the right side of the heart, of the liver, the kidneys, and subcutaneous tissues is very common. Digitalis, by improving the venous flow towards the heart, will ameliorate all these symptoms. It might be supposed that by constricting all the peripheral arterioles it would impede the arterial flow, because the heart will have to contract against a greater peripheral resistance, but this disadvantage is never enough seriously to hamper the increased cardiac power; and it must be remembered that it is a great advantage to the circulation to have a proper peripheral arterial resistance, for without that, the elastic coat of the arteries cannot aid the arterial flow. If, as it usually does in these cases, digi- 
talis acts as a diuretic, this will be of great value in removing the œdema, and in causing the scanty high-colored urine to become pale and abundant. The improvement in the circulation relieves the cardiac pain and distress which so commonly accompany mitral regurgitation, the lividity passes off, the dyspnœa decreases, and usually in a day or two a wonderful improvement in the patient's condition takes place. The more any case of mitral regurgitation deviates from the above [œdematous] type, the less good, as a rule, will digitalis do. Thus cases in which there is much pain and distress, and but little regurgitation, are not so often benefited, although even of such cases many are improved. Sometimes the romiting caused by digitalis prohibits its use. Fatal syncope may occur in those taking digitalis if they are too suddenly raised from the prone to the upright posture.

Mitral constriction.-In this condition it is obvious that it will be a great advantage to lengthen the diastole, for then there will be a greater chance that the diastole will be long enough to allow the normal amount of blood to pass through the constricted orifice. In proportion as this end is attained, the œedema, lividity, and other signs of backward venous congestion will be relieved, and if the digitalis induces diuresis, this is very valuable in aiding the reduction of the œdema.

Diseases of the tricuspid valve. - In both tricuspid constriction and tricuspid regurgitation, digitalis will be beneficial in the same way as in similar affections of the mitral valve. As a rule, however, it does less good when the disease is on the right side of the heart.

Aortic regurgitation. - Often digitalis is harmful, for by prolonging the diastole more time is allowed for the blood to flow back through the imperfectly closed aortic orifice, and hence there is great danger of fatal syncope. The drug should only be given in cases of aortic regurgitation, when the heart is very rapid, or when there is evidence that not much blood regurgitates, or when there are reasons, such as the coincident presence of aortic obstruction, for wishing to strengthen and regulate the contraction. The dose must be small and the effects must be carefully watche: 
Aortic constriction.-This, unfortunately, is usually accompanied by aortic regurgitation; but sometimes when it is wished to increase the force of the beat, and so to drive more blood through the constricted aortic orifice, digitalis is useful, or when, as a result of the obstruction, mitral dilatation and consequent regurgitation, with much pulmonary and venous engorgement, have set in. Many cases of pure aortic obstruction do not require drugs, for the heart hypertrophies sufficiently to overcome the obstruction.

Bright's disease. - In cases of contracted granular kidney in which the cardiac hypertrophy has been unable to overcome the peripheral resistance, and consequently the left ventricle and with it the auriculo-ventricular orifice has dilated, and mitral regurgitation has therefore ensued, digitalis may be of service for the reasons given elsewhere (see p. 425): A diuretic pill, often used for this condition, consists of [calomel], digitalis, and squill a grain of each; [.06 gm.] made up with extract of [hyoscyamus]. Otherwise, in chronic Bright's disease, digitalis is not a suitable diuretic, for it raises the tension of the pulse, which is already high. In the earlier stages of acute Bright's disease it has been given as a diuretic, but it is questionable whether it is right to dilate the vessels of an acutely inflamed organ; further, digitalis is always, unless the heart is diseased, an uncertain diuretic, and even in the early stage of acute Bright's disease the arterial tension is somewhat raised. In chronic tubal nephritis, uncomplicated by cardiac disease, it is worse than useless, for it has no effect on the renal cells, and it raises the blood-pressure.

Diseases of the cardiac muscle. - If the heart be fatty, or otherwise degenerated, digitalis rarely does good, for it is harmful for the diseased heart to have to work against the increased arterial tension, and it is said that there is danger of rupture of some of the degenerated fatty fibres. The weakly acting heart that is met with after pericarditis, typhoid fever, scarlet fever, rheumatism, and other acute diseases, even if no valvular defects are present, is markedly strengthened by digitalis. For this purpose it nuay be combined with caffeine, or two drachms [8. c.c.] of the infusion may be given, with three minims [. 20 c.c.] of 
stronger ammonia [water], in a little water. Each contraction is more efficient, and the prolonged diastole allows more time for the muscle to rest. It is clear that in the course of twentyfour hours this additional repose, although but little in each cycle, will amount to a considerable time. Many men who have practiced rowing or other hard exercise to excess, suffer from shortness of breath, and the apex of the heart is found to be a little outside the normal position, but there is no demonstrable valvular lesion. This condition, which also occurs in soldiers after a long campaign, is much benefited by digitalis. The dilatation of the right side of the heart that so frequently accompanies chronic disease of the lungs may be, but usually is not, improved by digitalis.

Functional diseases of the heart.-The irregular, palpitating beat, often seen apart from any organic disease, may be benefited markedly by digitalis; but it must be remembered that this condition is commonly a result of indigestion, in which case the right treatment is, if possible, to cure the dyspepsia, and if digitalis is given at all, to do so cautiously, for it may excite indigestion. The functional affections of the heart met with in highly neurotic subjects may be, but are not often, benefited by digitalis.

Exophthalmic goitre may improve under a long course of digitalis; but generally this treatment fails.

Hamorrhage. - Although digitalis contracts the arterioles, it is not often given as a hæmostatic, for the increased blood-pressure may lead to greater hæmorrhage, but it may be useful in the pulmonary hæmorrhage due to disease of the mitral valve.

Alcoholism. - Moderate doses of digitalis have been said to be serviceable in chronic alcoholism on account of their stimulating effect on the circulation. Enormous doses have been given empirically in delirium tremens, but generally without any good result.

Uteris. - Because of its power to contract the uterus, digitalis may be useful in menorrhagia.

[Digitalis is said to be useless if the patient suffers from high fever.] 
It is often desirable to combine fluid preparations of digitalis with iron salts, the resulting mixture, which is usually inky from the action of the iron on the tannic acid in the digitalis, can be clarified by the addition of a little diluted [phosphoric] acid. Because of this difficulty the powdered digitalis leaves are often made into a pill with dried [ferrous] sulphate.

\section{Antagonism.}

Antagonism between Digitalis and Aconite.-Aconite is a cardiac poison, weakening instead of strengthening the beat; it dilates the peripheral vessels, it lowers the blood-pressure, and after death the heart is always found in a condition of diastole. In all these points it is antagonistic to digitalis, but the action of aconite is very rapid, that of digitalis very slow. Therefore these drugs are not practical antidotes to each other in poisoning. [Saponin and senegin are considered to be the most complete physiological antidotes.]

Digitalis is cumulative. Patients who have taken it for a long while sometimes suddenly show symptoms of poisoning without any increase in the dose. This is because the drug is not excreted by the kidneys so fast as it is absorbed, therefore it accumulates in the body. [Digitalis should be stopped so soon as symptoms of gastro-intestinal irritation supervene, or the pulse becomes abnormally slow.]

\section{STROPHANTHUS.}

STROPHANTHUS.-[The seed of Strophanthus hispidus De Candolle (nat. ord. Apocynacee), deprived of its long awn. Habitat.-Tropical Africa.

Characters. - About $15 \mathrm{~mm}$. long and 4 or $5 \mathrm{~mm}$. broad, oblong-lanceolate, flattened and obtusely edged, grayish-green, covered with appressed, silky hairs, one side with a ridge extending into the attenuated, pointed end; kernel white and oily, consisting of a straight embryo, having two thin cotyledons, and surrounded by a thin layer of perisperm; nearly inodorous; taste very bitter.]

Composition. - The chief constituents are-(I) Strophanthin, $\left[\mathrm{C}_{31} \mathrm{H}_{48} \mathrm{O}_{12}\right]$. It exists in all parts of the plant, but mostly in the seeds ( 8 to 10 per cent.). This is in all probability the same as, or closely allied to, the active principle Ouabain [see p. 416] which has been isolated from [another species of] Strophanthus. It is a transparent, white, imperfectly crystalline, bitter glucoside, (being split up by acids into glucose and Strophanthidin). [Very] soluble in water; insoluble in Chloroform and Ether. [Strophanthin, according to recent investigation, can be isolated from Strophanthus Kombe, and many other species of Strophanthus. (2) Kombic Acid, which is not identical in all varieties of Strophanthus. (3) Incine, an Alkaloid. (4) Tanghinin, $\mathrm{C}_{27} \mathrm{H}_{40} \mathrm{O}_{8}$, in rhombic prisms. 


\section{Preparation.}

Tinctura Strophanthi.-Tincture of Strophanthus. Strophanthus, 50. By digestion and percolation with Alcohol and Water to I000.

Dose, 2 to $10 \mathrm{~m}$.; .12 to .6o c.c.

Tincture of Strophanthus Kombé, from which the oil has been extracted and made from assayed material, is far more reliable. Of this the maximum dose is $5 \mathrm{~m} . ; .30$ c.c.]

\section{Action of Strophanthus.}

\section{External.-None.}

Internal.-Gastro-intestinal tract.-Like digitalis, strophanthus is liable to cause vomiting and diarrhœa, especially if the dose be large. [Generally these disturbances result from preparations from which the volatile oil contained in the seeds has not been extracted.] In small doses its bitter action may come into play, and then it will aid digestion like any other bitter stomachic.

Heart. - Strophanthus acts on the heart exactly like digitalis, for it strengthens the force without altering the duration of the systole, slows the rate of the beat, and consequently prolongs the diastole, and makes an irregular heart regular. In fatal cases of poisoning by strophanthus, the heart may be arrested either in diastole or systole. The details of its cardiac action are the same as those of digitalis.

$V$ essels.-It does not constrict the peripheral vessels, or at any rate very slightly; therefore, the slow rise of bloodpressure is almost entirely due to the action of the drug on the heart. This is the most important difference between it and digitalis, which contracts the vessels powerfully and consequently gives a greater rise of blood-pressure, [and is the reason for the greater safety of the former.]

Kidneys. - It is diuretic, [more] powerfully than digitalis. Probably the diuresis is entirely due to the increased cardiac action. No special alteration in the size of the renal vessels takes place under strophanthus.

Nervous system. - This is not affected. In toxic doses it is 
a direct poison to the voluntary muscles. Strophanthin is a powerful local anæsthetic when dropped on the conjunctivæ [of dogs].

Respiration.-No particular effect is produced. The African Kombé arrow poison is made from strophanthus.

\section{Therapeutics of Strophanthus.}

Strophanthus is used in the same varieties of cardiac disease as digitalis; that is to say, when it is desirable to slow the heart, to increase its force, to make it regular, and to prolong the diastole. It is clear, therefore, that it will be chiefly valuable in cases of mitral disease. A priori, it might be thought that as strophanthus does not contract the peripheral vessels and so increase the cardiac resistance, consequently it would be the more useful drug, [and] experience has confirmed this [for certain cases]; it will generally happen that strophanthus will not produce vomiting when digitalis does. [The advantages which strophanthus possesses over digitalis may be summed up as ( 1 ) greater rapidity, modifying pulse rate within an hour ; (2) absence of vaso-constrictor effects ; (3) greater diuretic powers ; (4) no disturbance of digestion from properly made preparations ; (5) absence of so-called cumulation ; (6) greater value in children; and (7) greater safety in the aged.

It should be the remedy of choice in all cases, (I) in which we wish to establish compensation; (2) of arterial degeneration in which a remedy which causes more energetic cardiac contraction is required ; (3) of cardiac disease when a diuretic is necessary ; (4) of weak or irritable hearts ; (5) of cardiac disease in childhood or old age.] Strophanthus is preferable to digitalis when it is wished to give one of these drugs in Bright's disease.

\section{CONVALLARIA.}

CONVALLARIA.--[The rhizome and roots of Convallaria majalis Linné (nat. ord. Liliacea). Synonym._Lily of the Valley. Habitat.United States, in the Allegheny Mountains; Europe and Northern Asia.

ChARACTERS. - Of horizontal growth and somewhat branched, about 3 mm. thick, cylindrical, wrinkled, whitish, marked with few circular scars; at the annulate joint are about eight or ten, long, thin roots; fracture somewhat 
fibrous, white ; odor peculiar, pleasant ; taste sweetish, bitter, and somewhat acrid.]

Compositios. - The chief constituents are-(I) Convallamarin, $\left[\mathrm{C}_{46} \mathrm{H}_{48}\right.$ $\mathrm{O}_{24}$ ], a glucoside the active principle; [a white, bitter-sweet powder, soluble in water and Alcohol. (2) Convallarin, $\mathrm{C}_{34} \mathrm{H}_{31} \mathrm{O}_{11}$, a glucoside, in acrid prisms, sparingly soluble in, but foaming with water, soluble in Alcohol, but not in Ether.] This is said only to purge.

\section{Preparation.}

[Extractum Convallarix Fluidum.-Fluid Extract of Convallaria. By maceration and percolation with Diluted Alcohol, and evaporation.

Dose, 5 to $30 \mathrm{~m}$.; .30 to 2.00 c.c.]

\section{Action and Therapeutics of Convallaria.}

The action of convallaria is precisely that of digitalis, and it may be given in exactly the same varieties of heart disease. It is sometimes successful when digitalis has failed. It is not so powerful as digitalis, but some find it less likely to produce sickness. [It is said to act more powerfully upon the right heart, but this is probably not true. A more extended experience seems to indicate that this drug is very unreliable.]

\section{SCILLA.}

SQUILL.-[Synonym.-Sea Onion. The bulb of Urginea maritima (Linné) Baker (nat. ord. Liliacea), deprived of its dry, membranaceous outer scales, and cut into thin slices, the central portions being rejected. Habifat. - Basin of the Mediterranean near the sea.

ChARACTERS. - In narrow segments, about $5 \mathrm{~cm}$. long, slightly translucent, yellowish-white or reddish, brittle and pulverizable when dry, tough and flexible after exposure to damp air; inodorous; taste mucilaginous, bitter and acrid.]

Composition. - The chief constituents are-(1) Scillitoxin, the most active principle; (2) [Scillipicrin, acting upon the heart; (3) Scillin, producing numbness and vomiting; (4) Mucilage.

Dose, I to $5 \mathrm{gr}$; . .06 to $.30 \mathrm{gm}$.]

\section{Preparations.}

1. Acetum Scillæ.-[Vinegar of Squill. Squill, 100; Diluted Acetic Acid, by maceration and percolation, to 1000 .

Vinegar of Squill is used to prepare Syrupus Scillæ.

Dose Io to $45 \mathrm{~m}$.; 60 to 3.00 c.c. 
2. Extractum Scillæ Fluidum.-Fluid Extract of Squill. By maceration and percolation with Alcohol and Water, and evaporation. positus.

Fluid Extract of Squill is used to prepare Syrupus Scillæ Com-

Dose, 1 to $5 \mathrm{~m}$.; .06 to .30 c.c.

3. Tinctura Scillæ.-Tincture of Squill. Squill, I50. By maceration and percolation with Alcohol and Water, to I000.

Dose, 5 to $30 \mathrm{~m}$.; .30 to 2.00 c.c.

4. Syrupus Scillæ.-Syrup of Squill. Vinegar of Squill, 450; Sugar, 800 ; water to rooo. By solution and straining.

Dose, $1 / 2$ to I fl. dr.; 2. to 4 . c.c.

5. Syrupus Scillæ Compositus.-Compound Syrup of Squill. Synonym.-Hive Syrup. See Antimony, p. 230.]

\section{Action of SQuill.}

Squill so closely resembles digitalis in its action that the account of that drug will apply to squill, with the following additions: Squill is a much more powerful gastro-intestinal irritant ; vomiting and purging result from even moderate doses, and after death, if animals are killed with it, much gastro-enteritis is found. In the second place, some constituent of squill is excreted by the bronchial mucous membrane, and in passing through it irritates it. The vascularity and the amount of secretion are thereby increased. Squill is, therefore, a powerful expectorant. Thirdly, squill in the course of its excretion through the kidneys stimulates them; it is, therefore, a more energetic diuretic than digitalis, and it may irritate the kidneys excessively.

\section{Therapeutics of Squill.}

Because of its irritating properties, squill is not given alone, but it is frequently combined with digitalis when that drug is administered for heart disease or as a diuretic. A very favorite diuretic pill is composed of squill, digitalis and calomel, I gr. [.०6 gm.] of each, made up [with extract of hyoscyamus, I I 2 gr.; .09 gm. This is sometimes known as Guy's triplex pill.]

Squill is much used as an expectorant. Here also it is always 
prescribed in combination; it is too irritating to the bronchial mucous membrane for it to be advisable to give it in acute bronchitis; nor should it be chosen in phthisis, lest it should cause dyspepsia; but it is valuable in chronic bronchitis if the secretion is scanty.

Squill should not be given in acute Bright's disease, for it is too irritating to the kidneys. [Vinegar of squill should not be prescribed with ammonium carbonate.]

\section{SCOPARIUS.}

SCOPARIUS.-Synonym.-Broom. The tops of Cytisus Scoparius [(Linnê) Link (nat. ord. Leguminosa). Habitat.-Western Asia, Southern and Western Europe; naturalized in some localities in the United States.

Characters. - In thin, flexible, branched twigs, pentangular, winged, dark green, nearly smooth, tough, usually free from leaves; odor peculiar when bruised; taste disagreeably bitter.]

Composirion.-The chief constituents are-(I) Scoparin, $\mathrm{C}_{21} \mathrm{H}_{22} \mathrm{O}_{10}$, a [tasteless, amorphous, neutral principle. (2) Sparteine, $\mathrm{C}_{15} \mathrm{H}_{26} \mathrm{~N}_{2}$, a colorless, oily, very bitter alkaloid.

(3) Tannic Acid.

Dose, $1 / 4$ to I dr. ; I. to $4 . \mathrm{gm}$.]

\section{Preparation.}

[Extractum Scoparii Fluidum.-Fluid Extract of Scoparius. By maceration and percolation with Diluted Alcohol, and evaporation.

Dose, $1 / 4$ to $\mathrm{I}$ fi. dr.; r. to 4 . c.c.

SPARTEIN $\approx$ SULPHAS. Sparteine Sulphate. $-\mathrm{C}_{15} \mathrm{H}_{26} \mathrm{~N}_{2} \mathrm{H}_{2} \mathrm{SO}_{4}+$ ${ }_{4} \mathrm{H}_{2} \mathrm{O}=403.23$.

Source. - The neutral sulphate of an alkaloid obtained from Scoparius. It is obtained by extracting the plant with water acidulated with Sulphuric Acid, concentrating, decomposing with Sodium Hydroxide, and distilling. The Sulphate is prepared from the Alkaloid by neutralization with Sulphuric Acid, and crystallization.

Characters. - Colorless, white, prismatic crystals, or a granular powder, odorless, and having a slight saline and somewhat bitter taste. Liable to attract moisture when exposed to damp air. Solubility. - Very soluble in water and Alcohol.

Dose, it to $2 \mathrm{gr}$.; .006 to $.12 \mathrm{gm}$.]

\section{Action of Broom.}

External.-Broom has no external action.

Internal. - Nervous system. - [Its alkaloid sparteine sulphate 
in poisonous doses causes, in the lower animals, tremblings, incoordination, increase of reflexes, clonic and tonic convulsions, followed by enfeeblement of all the functions, convulsions, and death from asphyxia.

Respiration.-It paralyzes the respiratory centres, causes embarrassment of the respiration and paralyzes the motor centres of the spinal cord, but has a very feeble influence upon the muscles, lessening, though not destroying, their excitability.

Circulation.-According to Laborde, under the influence of this alkaloid there is a very great increase in the size and height of the cardiac wave. If the dose has been a small one, the pulse is at first accelerated; after large doses there is a slowing followed by enfeeblement of the heart. The arterial pressure is not materially changed unless the dose is toxic, when it falls. Small doses weaken, and large ones paralyze the pneumogastric; upon the vaso-motor system it appears to have no influence, unless in very large toxic doses, when it, perhaps, acts as a paralyzant.

Kidneys. - Broom is of value as a diuretic. Scoparin probably represents the diuretic principles of the plant.]

\section{Therapeutics of Broom.}

Broom is a very useful diuretic. It is usually given in combination with other diuretics in cases of dropsy from heart disease or interstitial nephritis. If there is acute renal inflammation it should not be prescribed. [According to Sée, sparteine sulphate is of very great value in producing regularity in cases of irregular cardiac action. It accelerates the beats when a weak, atonic state is present, and has the great advantage of acting quickly, is not cumulative, on the whole is probably inferior to digitalis in power, but it is useful in uncompensated cardiac, especially mitral, disease.]

\section{ERYTHROPHLCUM.}

SASSY BARK. (Not official).-Synonyms.-[Mancona Bark.] Ordeal Bark. .Casca Bark. The bark of Erythrophloum guineense (nat. ord. Legruminose $)$. [Habitat. $]$-Africa.

Characters. - [ In flat or curved pieces of irregular size, about $6 \mathrm{~mm}$. 
thick, covered externally with an uneven warty and fissured corky layer, or deprived of the same, of a dull brown color. It is hard, brittle, of a fibrous texture, internally with pale yellowish brown spots, inodorous, of an astringent, somewhat bitter and acrid taste, and when powdered excites sneezing.]

CoMposition.-The active principle is Erythrophloine, a [colorless alkaloid, soluble in water and Alcohol; this is a local anæsthetic (Koller)].

\section{Action and Therapeutics of ERythrophloeum.}

The action of erythrophlœum [of which a ro per cent. tincture has been recommended in dose of from 5 to $10 \mathrm{~m}$. ; .30 to .60 c.c., by the British Pharmaceutical Conference] is the same as that of digitalis, and it may be used for the same class of cases. [In organic cardiac disease its effect in strengthening the pulse and in increasing the urine is by no means constant nor lasting.] It is, however, more likely to cause vomiting, and the action on the inhibitory cardiac mechanism is much more marked than that on the cardiac muscle.

\section{[ADONIDINUM.}

ADONIDIN. (Not official).-A glucoside obtained from Adonis Vernalis (nat. ord. Ranunculacea). Synonym.-False Hellebore. Habitat.Northern Europe and Asia.

CharaCters. - This glucoside: occurs as a somewhat hygroscopic, canarycolored powder, of intensely bitter taste; soluble in water and Alcohol; insoluble in Ether, Chloroform and Benzin.

Dose, $1 / 6$ to $1 / 3 \mathrm{gr}$.; .01 to $.02 \mathrm{gm}$.

\section{Action and Uses of Adonidin.}

Adonidin markedly increases the arterial pressure while decreasing the pulse rate. The primary rise is chiefly of cardiac origin, the slowing of the rate is due to stimulation of the inhibitory nerves. The late fall of blood-pressure is due, at least in great part, to vaso-motor paralysis. In its action it is more prompt than digitalis, and, according to Durand, does not have a cumulative action. If it is diuretic, it is chiefly through its effects upon the circulation. It is used for the same class of cases as digitalis. Its irritating properties prevent its subcutaneous use and even prolonged administration by the mouth.] 


\section{Class II.-THE ACONITE GROUP.}

\section{ACONITUM.}

ACONITE.-[Synonyms.-Monkshood. Wolfsbane. The tuber of Aconitum Napellus Linné (nat. ord. Ranunculacece). Habitat.-Mountainous districts of Europe, Asia, and Northwestern North America.

Characters. - From to to $20 \mathrm{~mm}$. thick at the crown; conically contracted below ; from 50 to $75 \mathrm{~mm}$. long, with scars or fragments of radicles; dark brown externally; whitish internally; with a rather thick bark, the central axis about seren-rayed; without odor; taste at first sweetish, soon becoming acrid, and producing a sensation of tingling and numbness, which lasts for some time.] Resembling Aconite. - Horseradish (q. v.).

Composition, - The active princip!e is the very poisonous alkaloid Aconitine (see [below]). Two other alkaloids are present,-Aconine $\left[\mathrm{C}_{26} \mathrm{H}_{39} \mathrm{O}_{11}\right]$ and Benzaconine. Other principles are, perhaps, Pseudo-aconitine, $\left[\mathrm{C}_{36} \mathrm{H}_{49}\right.$ $\mathrm{NO}_{11}$, or Napelline, (dose, $1 / 6$ to $1 / 2$ gr. ; .OI to .03 gm., Merck), Pseudoaconine, $\mathrm{C}_{27} \mathrm{H}_{41} \mathrm{NO}_{8}$, Picro-aconitine, $\mathrm{C}_{31} \mathrm{H}_{45} \mathrm{NO}_{10}$, combined with Aconitic Acid, $\mathrm{H}_{3} \mathrm{C}_{6} \mathrm{H}_{3} \mathrm{O}_{6}$.]

Dose, $1 / 2$ to $2 \mathrm{gr}$; ; [.03 to .1 $2 \mathrm{gm}$.]

\section{Preparations.}

[1. Extractum Aconiti,-Extract of Aconite. By maceration and percolation with Alcohol, and evaporation.

Dose, $\frac{1}{10}$ to $1 / 4 \mathrm{gr}$; .006 to $.015 \mathrm{gm}$.

2. Extractum Aconiti Fluidum.-Fluid Extract of Aconite. By maceration and percolation with Alcohol and Water, and evaporation.

Dose, $1 / 2$ to $2 \mathrm{~m}$.; .03 to .12 c.c.

3. Tinctura Aconiti.-Tincture of Aconite. Aconite, 35. By maceration and percolation with Alcohol and Water to 1000.

Dose, $1 / 2$ to $5 \mathrm{~m}$.; .03 to $.30 \mathrm{c.c}$.

It should be remembered that Fleming's Tincture of Aconite, which is found in the shops, is nearly twice as strong as the official tincture.]

ACONITINA. - Aconitine. (B. P., not official). $-\mathrm{C}_{33} \mathrm{H}_{45} \mathrm{NO}_{12}$ ? = [645.54. ?]

SOURCE. - [It is precipitated from an aqueous solution of an alcoholic extract of the powdered root by Ammonia, and then purified.]

ChARACTERS. - Colorless, hexagonal prisms of the rhombic system. Causes tingling when placed on the tongue. By partial hydrolysis it yields benzaconine, and on further hydrolysis it forms aconine and benzoic acid. Solubility. -Readily in Alcohol and Chloroform, less so in Ether; nearly insoluble in water.

[Dose, $\left.\frac{1}{260} \mathrm{gr} . ; .0003 \mathrm{gm}.\right]$ 


\section{Preparation, [B. P., not official.]}

Unguentum Aconitinæ.-[Aconitine Ointment.] Aconitine dissolved in Alcohol, 1 ; Oleic Acid, 8 ; [Benzoinated] Lard, 4 I.

\section{Action of Aconite.}

The action of aconite, which has lately been studied by Cash and Dunstan, is due chiefly to the aconitine in it, and therefore they may be considered together.

External.-Applied to the skin, to a mucous membrane, or to a raw surface, aconitine and therefore aconite, first stimulates and then paralyzes the sensory nerves; it therefore causes first tingling, then numbness and local anæsthesia, which last some time. Unless the skin is sound a dangerous quantity may be absorbed. It is intensely irritant to the nostrils, causing, when it is inhaled, sneezing and much secretion with an icy cold sensation.

Internal.-Gastro-intestinal tract.-Unless it is very dilute, numbness and tingling are produced in the mouth. There are no other gastro-intestinal symptoms unless the dose is very large, when there may be vomiting and purging.

Heart.-If small doses are given, the rate of the beat is soon very decidedly steadied and slowed, shortly after that the force and tension become less, and these effects are mainly due to a stimulation of the roots of the vagus. But after larger doses the pulse quickens, misses beats, and becomes irregular. Many of the ventricular beats have no corresponding auricular contraction, although the two auricles always contract together and the two ventricles contract together. As the irregularity and frequency of ventricular contractions increase, the bloodpressure rapidly undergoes great variations. It is not until quite the end of its action that aconite influences the heart muscle. The ventricles, always more affected than the auricles, pass into a condition of delirium. Even small doses lead to a fall of blood-pressure, but all the effects on blood-pressure are almost entirely secondary to the action on the heart or its nerves. The vaso-motor centre is only slightly affected. [Clin- 
ically it would appear that the peripheral vessels are dilated. Aconite has been named the vegetable lancet.]

Respiration.-The respiration, after a transitory quickening, is slowed, expiration and the pause after it are considerably prolonged. The movements become more slow and dyspncal, the respiratory centre is powerfully depressed, but it is not easy to decide whether death is due to this or to cardiac failure.

Nervous system.-It appears clear that aconite, whether given internally or applied locally, depresses the activity of the peripheral terminations of the nerves; the nerves of common sensation and temperature are affected before the motor. Any pain that may be present is relieved. Large doses in man cause clonic convulsions, chiefly respiratory. Later on, the paralysis of the motor nerves gives rise to muscular weakness. It is doubtful whether the cord is influenced. The brain is not. The pupil is dilated.

Temperature.-Aconite causes a febrile temperature to fall, This is in part due to its action on the circulation and respira. tion, but probably other causes are at work.

Skin.-Aconite is a mild diaphoretic; in this case also we do not understand how it acts. [The cutaneous blood-vessels are generally dilated.] Occasionally it produces an erythematous rash.

Kidneys. - It is said to be a feeble diuretic, but its effect is very slight. Aconitine is excreted in the urine.

Benzaconine is bitter, and does not cause tingling or numbness of mucous surfaces; in large doses it slows the pulse-beat very strikingly because the auricular beats are frequently not followed by ventricular contraction. Its action is probably chiefly on the heart muscle itself. It does not paralyze sensory nerves, but greatly interferes with motor nerves and causes a semi-comatose condition. The fall of temperature produced by it is very slight. It will be noticed that it is in almost all respects contrary in action to aconitine.

Aconine is bitter, but does not produce numbness. It is non-toxic as regards the heart, and opposes the cardiac incoordination and asequence caused by aconitine. It depresses 
motor nerves and respiration very strikingly, probably acting like curare.

It is worth noting that, whilst the introduction into aconitine of two additional acetyl groups (as in diacetyl-aconitine) gives rise to a derivative very similar in action to aconitine, the loss of the acetyl group, as in benzaconine, almost entirely abolishes all physiological resemblance to aconitine. On the other hand, the removal of the benzoyl radical from benzaconine (aconine remaining) produces much less alteration in action, although it does not diminish the toxicity of benzaconine.

\section{Therapeutics of Aconite.}

External.-As aconite produces local anæsthesia, it is applied externally and often with great benefit in cases of neuralgia, especially facial neuralgia. Frequently it fails, and we cannot tell beforehand whether it will succeed. A small piece of the ointment [B. P.] may be rubbed in till numbness is produced, but as this is a very expensive preparation it is usually better to paint on the liniment [B. P., a 40 per cent. solution of the powdered root in alcohol, to which 2 per cent. of camphor is added], with a camel's-hair brush. The pain of chronic rheumatism is sometimes relieved by aconite. Linimentum Aconiti Compositum, commonly called A. B. C. liniment because it contains equal parts of Aconite, Belladonna and Camphor lininsents, is an excellent preparation for external use. Aconite should never be used externally unless the skin is quite sound.

Internal.-It may be given internally for neuralgia, but it does not succeed nearly so well as when applied externally. It is not used internally as much as formerly, when it was administered in almost every febrile disease, with the object of decreasing the force and tension of the pulse. Certainly it does this very effectually, and the only reason why it is not so popular at the present time is, that it is not now thought desirable to reduce the force and frequency of the heart in these diseases. Perhaps it is used too little, for many believe that the milder febrile diseases, such as tonsilitis, laryngitis, or a common cold, are distinctly benefited by aconite, especially if they occur in children. 
In addition to retarding the pulse it increases perspiration and lowers the temperature. As large doses diminish the force of the heart, it is usually given in doses of two or three minims [. I 2 or .20 c.c.] of the tincture every hour or so till the pulse falls to nearly normal ; for the same reason it is not advisable to use it for prolonged fevers, as typhoid, nor when the heart is diseased, except in the few cases in which there is sufficient compensative cardiac hypertrophy. In such cardiac cases it is sometimes useful to slow the pulse, even when there is no fever. It will occasionally relieve the pain of aneurism. A common practice was to combine with it one or two minims; $[.06$ or .1 2 c.c.] of Vinum Antimonii, as that has much the same action on the heart. Formerly it was much used in surgery if it was feared that inflammation might set in after injuries.

\section{TOXICOLOGY.}

Symptoms. - They come on quickly; in a few minutes there is a severe burning, tingling sensation in the mouth, followed by numbness. Vomiting [is not common, but may] begin in an hour or so, and [then] is very severe. There is an intense abdominal burning sensation. The skin is cold and clammy. Numbness and tingling, with a sense of formication of the whole skin, trouble the patient very much. The pupils are dilated, the eyes fixed and staring. The muscles become very feeble, hence he staggers. His pulse is small, weak and irregular. There is difficulty of respiration. Death takes place from asphyxia, or in some cases from syncope. The patient is often conscious to the last. Post-mortem. - The usual signs of death from asphyxia are seen.

Treatment. - Wash out the stomach promptly, give emetics (see p. 139). Use artificial respiration early. Inject stimulants, as ether or brandy, subcutaneously; apply warmth. Atropine and the tincture of digitalis should be given subcutaneously. Hot blankets and bottles [are useful].

AMYL COLLOID. (Not official.)-Synonym._Anodyne Colloid. The composition of this is Amyl Hydride, 480; Aconitine, 1 ; Veratrine,6; Collodion to 960 . It is a fluid preparation.

\section{Action and Therapeutics of Amyl Colloid.}

Amyl colloid is painted on the skin over painful areas in neuralgia, sciatica, etc. It is an elegant method of obtaining the local anæsthetic action of aconitine and veratrine, which is aided by the evaporation of amyl hydride; [but it is extremely diffi- 
cult to make a clear solution.] When the collodion has formed a film, a piece of warm, moist spongiopiline helps the anæsthetic effect of the alkaloids.

\section{[VERATRUM VIRIDE.}

VERATRUM VIRIDE.-Synonyms.-American Hellebore. Green Hellebore. The rhizome and roots of Veratrum viride Solander (nat. ord. Liliacea). Habitat.-North America, in rich woods.

Characters. - Rhizome upright, obconical, simple or divided, from 3 to $8 \mathrm{~cm}$. long, and 2 to 4 or $5 \mathrm{~cm}$. thick, externally blackish-gray, internally grayish-white, showing numerous short, irregular wood-bundles. Roots emanating froin all sides of the rhizome, numerous, shrivelled, light yellowishbrown, about 10 to $20 \mathrm{~cm}$. long, and $2 \mathrm{~mm}$. thick. Inodorous, but strongly sternutatory when powdered; taste bitterish and very acrid. Resembling Veratrum.-Valerian, Serpentaria, and Arnica, but Veratrum bas thicker rootlets, and no odor.

Composition. - The chief constituents are $-(\mathrm{I})$ Jervine $\mathrm{C}_{26} \mathrm{H}_{37} \mathrm{NO}_{3}$, an alkaloid, non-sternutatory. (2) Pseudojervine, an alkaloid, resembling Jervine. (3) Veratroidine, sonetimes called Cevadine, uncrystallizable, and sternutatory, is probably mostly Rubijervine. (4) Resin.

Dose, 1 to $5 \mathrm{gr}$; .06 to $.30 \mathrm{gm}$.

\section{- Preparations.}

I. Extractum Veratri Viridis Fluidum.-Fluid Extract of Veratrum Viride. By maceration and percolation with Alcohol, and evaporation.

Dose, I to $5 \mathrm{~m}$.; .06 to .30 c.c.

2. Tinctura Veratri Viridis.-Tincture of Veratrum Viride. Veratrum Viride, 400. By maceration and percolation, with Alcohol to 1000 .

Dose, 2 to ro m.; 12 to .60 c.c.

It should be remembered that Norwood's Tincture of Veratrum Viride, which is found in the shops, is one-tenth stronger than the official.

\section{Action of Veratrum Viride.}

The action of veratrum viride is very complex, as it contains so many alkaloids, but experiments have only been made on two active principles. These are jervine, and a substance, veratroidine, which further analysis shows to consist chiefly of rubijervine, resin, and, perhaps, some other bodies. Jervine, veratroidine, and veratrum viride will be considered separately. 
Jervine.-Gastro-intestinal tract.-When administered internally this substance produces profuse salivation, but neither vomiting nor purging.

Circulation.-The pulse is markedly lessened in frequency if the animal is quiet, but often the convulsions produced by the jervine cause a rapid pulse. The force of the cardiac beat is not at first altered. The blood-pressure falls at once, and continues to fall till death. Experiments made by excluding different parts show that these effects are produced by a powerful direct depressant effect on the cardiac muscle itself, and that the vaso-motor nerve-centres are powerfully paralyzed.

Respiration.-This is 1 rofoundly depressed, and death takes place from asphyxia.

Nervous system.-Early in the case there is muscular weakness, and this becomes more and more marked, so that the animal cannot stand, and reflex action is abolished. Yet, weak as these muscles are, they are soon violently convulsed, and it is found that jervine produces these apparently contrary effects by energetically stimulating the cerebral motor centres, but at the same time paralyzing the anterior cornual cells of the spinal cord, although not sufficiently to prevent the very strong impulses from the cerebral centres reaching the muscles and causing convulsions. The muscles themselves and the motor and sensory nerves are not affected ; or, if they are, they are depressed a little, just before death. Consciousness and the pupils are uninfluenced.

Veratroidine. - Gastro-intestinal tract. - This substance always produces vomiting and sometimes purging.

Circulation.-At first it lessens the pulse-rate because it stimulates the pneumogastric, consequently the blood-pressure falls, and if artificial respiration is kept up these effects continue till, if very large doses have been given, the stimulation of the vagi passes into paralysis, and then the pulse rises in frequency. Veratroidine probably has no action on the vaso-motor centres. Its influence on the respiratory centres is so intense that if artificial respiration is not maintained, the effects of the asphyxia so 
mask those of the drug on the vagi that the blood-pressure rises, and the pulse becomes rapid.

Respiration.-The function of respiratory centres is powerfully depressed, the animal soon becomes asphyxiated and dies.

Nervous system.-The action is the same as that of jervine.

Veratrum Viride.-The symptoms produced by this drug in man are as follows. They are easily explained by the combined action of jervine and veratroidine:-The frequency and force of the pulse are profoundly depressed. There may be severe nausea and vomiting. After large doses the pulse becomes very feeble and uneasy, there is difficulty of respiration and intense muscular weakness. Convulsions are not common in man. The temperature may fall several degrees.

\section{Therapeutics of Veratrum Viride.}

It is the opinion of most authorities that veratrum viride should be prescribed with great caution, as it is such a powerful poison. Veratrum viride has been successfully employed for many years in the treatment of puerperal eclampsia, and of the drugs generally employed for this purpose it is the most reliable. It has been given as a cardiac depressant, but antimony and aconite are much safer. Some have, however, claimed that it is a better cardiac depressant than aconite, because the vomiting it induces quickly indicates that too large a dose has been administered; but if the pulse is carefully watched, too much aconite need not be given, and the vomiting itself is objectionable.]

\section{VERATRINA.}

VERATRINE.-[A mixture of alkaloids obtained from the seed of Asagrae officinalis (Schlechtendal et Chamisso, Lindley (nat. ord. Liliacea). Habitat.-Mexico to Venezuela.

Source.-(1) The seed is exhausted with Alcohol, and the Alcohol recovered by distillation. (2) The residuary liquid is diluted with water to precipitate the resins and filtered. (3) Ammonia is added to the filtrate when veratrine is precipitated. (4) It is then re-dissolved, decolorized, and re-precipitated.

ChARACTERS. -A white or grayish-white, amorphous or semi-crystalline powder, odorless, but causing intense irritation and sneezing when even a 
minute quantity reaches the nasal mucous membrane; having an acrid taste, and leaving a sensation of tingling and numbness on the tongue; permanent in the air. Solubility. - Very slightly soluble in water; soluble in 3 parts of Alcohol; also soluble in 6 parts of Ether, and in 2 parts of Chloroform.

Dose, $\frac{1}{30}$ to $\frac{1}{10} \mathrm{gr}$; .002 to $.006 \mathrm{gm}$.

\section{Preparations.}

1. Oleatum Veratrinæ.-Oleate of Veratrine. Veratrine, 2; Oleic Acid, 98.]

2. Unguentum Veratrinæ. $-[$ Veratrine Ointment. Veratrine, 4 ; Olive Oil, 6 ; Benzoinated Lard, 90.]

\section{Action of Veratrine.}

External.-Veratrine, if it is applied to the unbroken skin, and especially if it is rubbed in, produces tingling and numbness, followed by a sensation of coldness, and anæsthesia to pain, touch, and temperature. Given subcutaneously, it causes violent pain and irritation.

Internal.-Gastro-intestinal tract.-Inhalation of the minutest portion causes great irritation of the mucous membrane of the nose, violent sneezing, and a free discharge of mucus, which may be bloody. A [minute portion] upon the tongue gives rise to burning pain and profuse salivation. On arriving at the stomach and intestine it produces great epigastric pain, vomiting and diarrhœa. These results also occur if it is given subcutaneously.

Blood.-Veratrine is quickly absorbed. It is not known to affect the living blood, but it kills the white corpuscles in drawn blood.

Heart. - It acts directly on the cardiac muscle as it does upon voluntary muscle; that is to say, the contractions of the heart become fewer, but each lasts a very long while until ultimately the heart stops in systole. It also acts on the vagus as on spinal nerves, the functional activity being first exalted, and this is partly the reason of the slowing of the heart; afterwards the vagus is depressed, but this does not cause a quickening of the pulse because of the action of the veratrine on the cardiac 
muscle, but it may make the beat irregular. The blood-pressure at first rises from the increased force of the beat, but when the heart becomes very slow it falls. Possibly these effects are also, in part, owing to the action of the drug on the vaso-motor centres.

Respiration.-Small doses quicken respiration, large ones retard it, producing long pauses, and finally arresting it. These results are probably due at first to stimulation, and afterwards to paralysis of the ends of the vagus in the lung, and to paralysis of the respiratory centres. The temperature is lowered.

Nervous system.-The brain is unaffected, and probably veratrine has no influence on the spinal cord. Motor nerves are first excited and then paralyzed; and the same is true of sensory nerves and their endings, but here the primary stimulation is very marked, hence the pain produced by the local inunction of veratrine.

Muscles.-The effect of veratrine is pecular and characteristic. In animals to which it has been given, or in excised muscles to which it is applied, it is found that the period during which a single contraction lasts is enormously prolonged. If a tracing of the contraction be taken it will be seen that the latent period and the time of the ascent of the curve are unaltered, that the height is greatly increased and the descent is extraordinarily extended. This is a genuine lengthened contraction, which is neither rigor nor tetanus, but it almost exactly resembles the contraction of the muscles met with in Thomsen's disease. This effect of veratrine disappears if the muscle is cooled.

\section{Therapeutics of Veratrine.}

External.-Veratrine [as an oleate or ointment] has been much used as an inunction for neuralgia, and sometimes it succeeds admirably, generally in the same class of cases as are benefited by the local application of aconite. [See also amyl colloid, p. 44 I.]

Internal.-It is rarely given internally, as it has such a powerful and pecular action on the heart. 


\section{[CLASS III.-THE CACTUS GROUP.}

\section{CACTUS.}

CEREUS GRANDIFLORUS.-Synonym.-Night-blooming Cereus. The stems of Cactus Grandiflorus Linné (nat. ord. Cactacea). Habitat.Tropical America; cultivated.

CHARACTERS.-Its branches, or sterns, are scandent, diffuse, radicant, slightly 5 to 7 angular; areolæ, 5 to 12 spinulose ; spinules short, 4 to $6 \mathrm{~mm}$. long, nearly equalling the whorl ; flowers large, nocturnal, white, pleasantly and strongly fragrant; the calyx is about 15 to $20 \mathrm{~cm}$. in diameter; the inside being of a splendid yellow, the outside is of a dark brown; the petals of a pure white; and there is a vast number of recurved stamens in the centre.

Constituent.-It is believed to contain an alkaloid, Cactine, but this has not been satisfactorily demonstrated.

\section{Preparation.}

Extractum Cacti Fluidum.-Fluid Extract of Cactus. By maceration and percolation of the fresh flowering branches with Alcohol and Water and evaporation.

Dose, 10 to $30 \mathrm{~m} . ; .60$ to 2.00 c.c.

\section{Action and Uses of Cactus.}

The action of cactus is upon the intra-cardiac ganglia and accelerator nerves, through the cardiac plexus of the sympathetic, and there is not any interference with the inhibitory nerves, nor, indeed, does its administration produce any very marked vasomotor changes. It shortens the ventricular diastole, thus quickening the pulse, and increases the blood-pressure. It is useful in cardiac weakness, that is, relative incompetency ; in convalescence from typhoid fever; in simple eccentric cardiac dilatation; in functional cardiac diseases, from tea, coffee, tobacco and alcohol, dyspepsia, neurasthenia of the climacteric, sexual exhaustion; in the "slow heart," from over-stimulation of the pneumogastric or degeneration of the muscular wall of the ventricles. It is of very great use in aortic regurgitation, but is absolutely contra-indicated in mitral stenosis, thus being of value in those cases where the use of digitalis is inadmissible. It has a sphere of action entirely of its own, not, however, replacing other remedies used for cardiac disease, but it is useful in many cases where these drugs are not only dangerous, but absolutely 
contra-indicated. Failures to obtain results depend upon the fact that many adulterated specimens are found in the shops, or upon the uses of inert, dried material. If made from the green plant, as it should always be, the fluid extract is of peculiar green color.]

\section{GROUP III.}

Vegetable Drugs employed for their action on the Respiratory Organs and not falling among Volatile Oils. (See Group VI.)

\section{Senega, [Sanguinaria, Pulsatilla,] Ipecacuanha, [Allium,] Lobelia, [Asclepias, Castanea, Inula, Aspidosperma, Eridictyon, Cocillaña,] and Prunus Virginiana.}

Senega, Ipecacuanha and Lobelia are gastro-intestinal irritants. Senega and Ipecacuanba are both excreted by the bronchial mucous membrane, which they irritate. Group II. is connected with this group by Senega, which acts on the heart like Squill, and like it is excreted by the bronchial mucous membrane.

\section{SENEGA.}

[SENEGA.-The root of Polygala Senega Linné (nat. ord. Polygalea). Synonym.-Senega Snakeroot. Habilal._United States, westward to Minnesota.

Characters.-About $10 \mathrm{~cm}$. long, with a heavy knotty crown, and spreading tortuous branches, keeled when dry, fleshy and round after having been soaked in water; externally yellowish-gray or brownish-yellow; bark thick, whitish within, enclosing an irregular, porous, yellowish wood; odor slight, but unpleasant ; taste sweetish, afterwards acrid.] Resembling Senega root.-Arnica, Valerian, Serpentaria and Green Hellebore, but none of these have a keel.

Composition. - The active principle is Senegin $\left(\mathrm{C}_{32} \mathrm{H}_{34} \mathrm{O}_{18}\right)$. Also called Saponin, which is found in Quillaja $(q . v$. ). It is a colorless, amorphous glucosicle, insoluble in Alcobol, but forming a soapy emulsion when mixed with boiling water, and is decomposed by Hydrochloric Acid into glucose and Sapogenin. It exists as a white powder, which forms a soapy emulsion when mixed with boiling water. It acts like Digitonin (see p. 42I), and is found in many plants.

IMPURITIES. - Other roots are mixed with it.

[Dose, 10 to $20 \mathrm{gr}$; ; 60 to $1.20 \mathrm{gm}$.] 


\section{Preparations.}

[1. Extractum Senegæ Fluidum.-Fluid Extract of Senega. By maceration and percolation with Alcohol, Ammonia Water and Water, and evaporation.

Fluid Extract of Senega is used to prepare Syrupus Senegxe and Syrupus Scillæ Compositus.

Dose, 10 to $20 \mathrm{~m}$.; .60 to r.20 c.c.

2. Syrupus Senegæ.-Syrup of Senega. Fluid Extract of Senega, 200; Ammonia Water, 5; Sugar, 700 ; Water to 1000 . Filter before adding the Sugar.

Dose, I to 2 fl. dr.; 4. to 8. c.c.

3. Syrupus Scillæ Compositus.-See Antimony, p. 23I.]

\section{Action of Senega.}

External.-Senega is an irritant to the skin.

Internal.-Alimentary canal. - Senega in large doses is an irritant here also, producing salivation, vomiting and diarrhœa. Even small doses often cause indigestion. It is absorbed with difficulty.

Circulation.-Senegin circulates as such in the blood. It arrests the heart in diastole. It is excreted by the skin, the bronchial mucous membrane, and the kidneys.

Respiration. - When the powdered root is inhaled it acts as a violent irritant to the nose, causing much sneezing and cough, together with hyperæmia and increased secretion from the respiratory mucous membrane. If senega is taken internally; the bronchial mucous membrane is irritated because of excretion through it of senegin, which causes vascular dilatation, greater secretion, and reflexly, cough. Senega is, therefore, a stimulating expectorant.

Kidney.-It is a diuretic, because excretion of senegin through the kidneys causes irritation of them.

\section{Therapeutics of Senega.}

Senega is only used as a stimulating expectorant. It is evident that it will be useful in bronchitis, when the secretion is scanty, and when the power to cough is feeble. As it is an irritant to the bronchial mucous membrane, it must not be given in 
acute bronchitis, nor, on account of its gastro-intestinal action, when there is indigestion. It has been employed as a diuretic, but it is not powerful, and is uncertain.

\section{[SANGUINARIA.}

SANGUINARIA.-Synonym.-Bloodroot. The rhizome of Sanguinaria canadensis Linné (nat. ord. Papaveracea), collected in autumn. Habitat. - North America, in rich woods.

Characters. - Of horizontal growth, about $5 \mathrm{~cm}$. long, and $1 \mathrm{~cm}$. thick, cylindrical, somewhat branched, faintly annulate, wrinkled, reddish-brown; fracture short, somewhat waxy, whitish with numerous small, red resin-cells, or of a nearly uniform, brownish-red color; bark, thin; odor, slight ; taste persistently bitter and acrid.

Compositios. - Its chief constituents are-(I) Sanguinarine, $\mathrm{C}_{20} \mathrm{H}_{15} \mathrm{NO}_{4}$ a white substance, soluble in Alcohol. (2) Chelerythrine, $\mathrm{C}_{20} \mathrm{H}_{15} \mathrm{NO}_{1}$. (3) Protopine. $-\mathrm{C}_{20} \mathrm{H}_{17} \mathrm{NO}_{5}$, also present in Opium. (4) Homochelidonine, $\mathrm{C}_{23}$ $\mathrm{H}_{21} \mathrm{NO}_{4}$. (5) Resins. (6) Citric and Malic Acids.

Dose, 2 to $30 \mathrm{gr}$; .12 to $2.00 \mathrm{gm}$.

\section{Preparations.}

I. Extractum Sanguinariæ Fluidum.-Fluid Extract of Sanguinaria. By maceration and percolation with Alcohol, Water and Acetic Acid, and evaporation.

Dose, I to $5 \mathrm{~m}$.; .06 to .30 c.c. (expectorant); $1 / 4$ to I f. dr.; I. to 4 . c.c. (emetic).

2. Tinctura Sanguinariæe--Tincture of Sanguinaria. Sanguinaria, $15^{\circ}$; by maceration with Alcohol, Acetic Acid and Water, and - percolation to rooo.

Dose, 5 to $3 \circ \mathrm{m}$.; .30 to 2.00 c.c. (expectorant); I to $3 \mathrm{fl}$. dr. ; 4. to I2. c.c. (emetic).

\section{Action and Uses of Sanguinaria.}

Sanguinaria is an acrid emetic with stimulant, and in large doses, narcotic powers; it is also expectorant and said to be an emmenagogue. It is chiefly used as a stimulating expectorant in chronic bronchitis or in advanced stages of the acute disease.

\section{PULSATILLA.}

PUlsatilla.-The herb of Anemone Pulsatilla and of Anemone pratensis Linné (nat. ord. Ranunculacea), collected soon after flowering. Habitat._Europe. 
Characters. - Leaves radical, petiolate, silky-villous, twice or thrice deeply three-parted or pinnately cleft, with linear, acute lobes, appearing after the large, purple flowers; inodorous, very acrid.

Composition.-The chief constituent is Anemonin, $\mathrm{C}_{10} \mathrm{H}_{8} \mathrm{O}_{4}$, which is colorless, inodorous and, when distilled with water, acrid.

Dose, I to $5 \mathrm{gr}$; .06 to $.30 \mathrm{gm}$.

\section{Action and Uses of Pulsatilla.}

It is said to be very nearly the equivalent of senega, and also to paralyze the heart and respiratory centres. It has been used for the treatment of the symptom of asthma, convulsive coughs and in bronchitis. Pulsatilla is a favorite remedy for dysmenorrhœa in its various forms, obstructive excepted. The fluid extract (not official) in dose of $\mathrm{I}$ to 5 minims; .06 to .30 c.c., has been highly recommended for orchitis and epididymitis.]

\section{IPECACUANHA.}

IPECACUANHA. - Ipecac. [The root of Cephaẽlis Ipecacuanha (Brotero) A. Richard (nat. ord. Rubiacea). Habitat.-Brazil to Bolivia and New Granada, in damp forests ; cultivated in India.

Characters. - About $10 \mathrm{~cm}$. long, and 4 or $5 \mathrm{~mm}$. thick ; mostly simple, contorted, dull grayish-brown or blackish, finely wrinkled ; closely and irregularly annulated, and often transversely fissured; bark thick, brittle, brownish, easily separated from the thin, whitish, tough, ligneous portion; odor slight, peculiar, nauseous ; taste bitterish, acrid, nauseating.]

Composition.-The chief constituents are-(I) Emetine, $\mathrm{C}_{30} \mathrm{H}_{44} \mathrm{~N}_{2} \mathrm{O}_{4}$, from I to 2 per cent., an uncrystallizable alkaloid. It is colorless (turns yellow on keeping), odorless, bitter, and soluble in Alcohol, Ether and Chloroform, slightly soluble in water, not in caustic alkali. (2) Cephaéline, $\mathrm{C}_{28} \mathrm{H}_{38}$ $\mathrm{N}_{2} \mathrm{O}_{4}$, an amorphous, bitter alkaloid, colorless (turns yellow on keeping), soluble in caustic alkali, less soluble in Ether than Emetine, but freely in Alcohol and Chloroform. (3) A third alkaloid in minute quantities. (4) A mixture called Cephaëlic or Ipecacuanhic Acid. (5) Tannic acid, Volatile Oil, Starch, Gum, etc.

Ipecacuanha contains about 2 per cent. of alkaloids. The proportion of each varies in different specimens of the root, but as a rule there is twice as much Emetine as Cephaëline. Emetine hydrochlorate and hydrobromate are in the market; a solution of either in sherry [ 1 to 3840 ] is of about the same strength as Vinum I pecacuanhre.

IMPURities.-Hemidesmus, which is cracked, not annulated. AlmondPowder, occasionally found mixed with powdered I pecacuanha root, gives odor of [Hydrocyanic] Acid when moistened. 
Dose, of powdered root, $1 / 2$ to $2 \mathrm{gr}$; ; $[.03$ to $.12 \mathrm{gm}$. (expectorant), 15 to $30 \mathrm{gr}$.; I. to 2. gm. (emetic).]

\section{Preparations.}

I. [Extractum Ipecacuanhæ Fluidum.-Fluid Extract of Ipecac. By maceration and percolation with Alcohol and Water, distillation of the Alcohol, addition of water to residue, evaporation and addition of Alcohol.

Fluid Extract of Ipecacuanha is used to make Syrupus Ipecacuanhæ, Tinctura I pecacuanhæ et Opii, and Vinum Ipecacuanhæ.

Dose, I to $5 \mathrm{~m}$.; .06 to $.30 \mathrm{gm}$.

2. Pulvis Ipecacuanhæe et Opii.-See Opium, p. 352.

3. Trochisci Ipecacuanhæ.-Troches of Ipecac. Ipecac, 2; Tragacanth, 2 ; Sugar, $65 \mathrm{gm}$.; Syrup of Orange, a sufficient quantity for top troches. Each troche contains $1 / 3 \mathrm{gr}$.; $.02 \mathrm{gm}$. Ipecacuanha.

Dose, I to 6 troches.

4. Trochisci Morphinæ et Ipecacuanhæ.-See Morphine, p. 354 .

5. Syrupus Ipecacuanhæ.-Syrup of Ipecac. Fluid Extract of Ipecac, 75; Acetic Acid, 10; Glycerin, 100; Sugar, 700; Water to 1000.

Dose, $5 \mathrm{~m}$. to $2 \mathrm{fl}$. dr. ; .30 to 8.00 c.c.

6. Tinctura Ipecacuanhæe et Opii.-See Opium, p. 352.

7. Vinum Ipecacuanhx. -Wine of Ipecac. Fluid Extract of Ipecac, I00; Alcohol, 100; White Wine, 800.

Dose, $I$ to $60 \mathrm{~m}$.; .06 to $4.00 \mathrm{c.c}$.]

\section{Action of Ipecacuanha.}

External.- Ipecacuanha powder is a powerful irritant to the skin, producing redness, vesication, and pustulation. It has some antiseptic powers, for it can destroy anthrax bacilli, but it has no effect on the spores. This property is not due to its emetine, but to some other constituent.

Internal.-Alimentary canal.-Here also the irritating action of ipecacuanha is seen. It increases the flow of saliva, dilates the gastric vessels, and stimulates the secretion of gastric juice. Therefore small doses are distinctly stomachic, and aid digestion. Large doses are, however, powerfully emetic. This is partly due to their jrritant effect upon the stomach, but 
still more to the fact that emetine acts directly upon the vomiting centre in the medulla, as can be proved by observing that, when the alkaloid is thrown directly into the circulation, vomiting follows before there is time for it to have been excreted into the stomach. Ipecacuanha is, therefore, both a direct and indirect emetic. It produces a certain amount of depression, but not more than the mere act of vomiting will explain. It does not usually cause nausea. The irritant effect is continued in the intestine, and hyperæmia, excessive secretion, and purging result. In dysentery there is a peculiar tolerance of ipecacuanha. Ipecacuanha increases the amount of bile secreted; and is therefore a direct cholagogue.

Circulation.-Large doses of emetine depress the heart powerfully, the blood tension falls, and the heart finally stops in diastole.

Respiration.-This is unaffected. Ipecacuanha powder when inhaled, or ipecacuanha taken internally, when it is excreted by the bronchial mucous membrane, causes hyperæmia of it, together with an increased secretion of bronchial mucus, and therefore, reflexly, coughing is stimulated. It is, consequently, an expectorant; and because it depresses the circulation a little, it is called a depressant expectorant; but this is a complete misnomer, considering that the bronchial mucous membrane is stimulated. Animals to which large doses of ipecacuanha or of emetine have been given show, after death, considerable hyperæmia of the bronchial mucous membrane, of the lungs, and of the stomach and intestines, and the,same - condition of the respiratory passage is seen if ipecacuanha powder has been inhaled.

Skin. - Ipecacuanha is a mild diaphoretic.

\section{TherapeUtics of IPECACUANHa.}

External.-Ipecacuanha is never, at the present day, employed for its external irritant effect. It has been used with success, as an antiseptic, in cases of anthrax. It is directed that the wound should be dressed with the powder, and that $5 \mathrm{gr}$. [. $30 \mathrm{gm}$.$] should be taken by the mouth, every two hours.$ 
Internal.-Stomach.-Occasionally in small doses, such as 4 or 5 minims $[.25$ to .30 c.c.] of the wine or $1 / 4$ gr. [.or $5 \mathrm{gm}$. ] of the powder, it is employed as a stomachic, and these quantities may even stop vomiting when other drugs have failed. . A usual prescription to arrest the vomiting of pregnancy is a minim [.o6 c.c.] of ipecacuanha wine in water every half hour. The powder of ipecac [and opium] has been praised in cases of gastric ulcer; no doubt any good effect it may have is due to its stimulating power. Ipecacuanha is a very common emetic. It should not be given when it is desired, as in cases of poisoning, to empty the stomach quickly, for some time elapses before it is absorbed and influences the medulla; nor should it be given to the very feeble, for it has no action that will counteract the depression of the vomiting. But it is an excellent emetic when it is wished, by the act of vomiting, to empty the air-passages, as in bronchitis, the early stages of diphtheria, tracheitis, and laryngitis, for not only the vomiting, but the effect of ipecacuanha on the respiratory tract and the slight subsequent depression will be beneficial. It is chiefly employed for this purpose in children, as they cańnot cough well, and often it seems to act like a charm. It used to be given in the early stage of fevers, to empty the stomach of undigested food. A good emetic powder for an adult consists of $20 \mathrm{gr}$. [1.20 gm.] of powdered ipecacuanha with $1 / 2 \mathrm{gr}$. [.03 gm.] of tartar [emetic.]

Ipecacuanha is said to be a specific for dysentery. How it acts is not known. Very large doses must be given-60 to 90 gr. [4. to 6. gm.] of the powder in a single dose, or $20 \mathrm{gr}$. [1.20 gm.] every four hours. Ipecacuanha from which the emetine has been removed (de-emetized ipecacuanha) has been much employed (dose, ro to $30 \mathrm{gr}$. [.60 to $2.00 \mathrm{gm}$.]); on the other hand, it has been stated that the efficient agent in the treatment of dysentery is the emetine.

Half a grain to a grain $[.03$ to $.06 \mathrm{gm}$.] or more is often combined in a pill with other cholagogues to relieve cases of hepatic dyspepsia, and sometimes with excellent results.

Respiration. - Ipecacuanha is a very common expectorant. [The troches] may be [dissolved in the mouth or the syrup], or 
wine may be given internally. It is suitable in cases of bronchitis or phthisis in which the secretion is scanty, and therefore there is much purposeless cough; and also when the disease is of long standing, for then the stimulation of the chronically inflamed mucous membrane will aid the cure of it. Its power of exciting the act of coughing adds to its usefulness. [Cephaëline seems to possess the expectorant properties of ipecacuanha.]

The inhalation of ipecacuanha powder [or wine] by means of an atomizer has been recommended in cases of asthma, and for the asthma-like paroxysms which often accompany chronic bronchitis. Sometimes it does good, but it may make the trouble worse.

Skin.-Dover's powder [see p. 352] is very commonly used as a diaphoretic in mild feverish attacks.

\section{[ALLIUM.}

GARLIC.-The bulb of Allium sativum Linné (nat. ord. Liliacea). Habitat.-Asia and Southern Europe, cultivated.

Characters. - Bulb subglobular, compound, consisting of about eight compressed, wedge-shaped bulblets, which are arranged in a circle around the base of the stem, and covered by several dry membranous scales. Odor pungent and disagreeable ; taste warm and acrid.

Composition. - The chief constituents are-(I) A volatile oil, $1 / 4$ per cent., which consists of Allyl $\left(\mathrm{C}_{3} \mathrm{H}_{5}\right)$ oxide and sulphide. (2) Mucilage. (3) Albumin.

Dose, 15 to $30 \mathrm{gr}$. ; I. to $2 . \mathrm{gm}$.

\section{Preparation.}

Syrupus Allii.-Syrup of Garlic. Garlic, 200; Sugar, 800 ; diluted Acetic Acid to I000.

Dose, I to 4 fl. dr.; 4 . to 15 . c.c.

\section{Action and Uses of Garlic.}

The effects of garlic are those of a general stimulant, quickening the circulation, exciting the nervous system, and pronoting. expectoration. It is beneficial in impaired digestion, in chronic catarrhal and other pectoral affections in which symptoms of inflammation have subsided and a relaxed state of the vessels remains.] 


\section{LOBELIA.}

LOBELIA.- - The leaves and tops of Lobelia inflata Linné (nat. ord. Lobeliacea), collected after a portion of the capsules have become inflated. Synonym.-Indian Tobacco. Habitat.-North America, in the fields and open woods.

Characters. - Leaves alternate, petiolate, the upper ones sessile, ovate or oblong, about $5 \mathrm{~cm}$. long, irregularly toothed, pubescent, pale green ; branches hairy, terminating in long racemes of small, pale blue flowers, having an adherent five-toothed calyx, which is inflated in fruit, a bilabiate corolla, and five united stamens; odor slight, irritating ; taste mild, afterwards burning and acrid.]

CoMPosition. - The chief constituents are-(I) Lobeline, [an alkaloid, as a yellowish, oily liquid of pungent taste, having an odor resembling that of tobacco. (2) Lobelacrin (probably Lobeline Lobelate). (3) Lobelic Acid.]

Incompatibles.-Caustic Alkalies, as they decompose Lobeline.

Dose, I to ro $\mathrm{gr}$; ; $[.06$ to $.60 \mathrm{gm}$.]

\section{Preparations.}

1. [Extractum Lobeliæ Fluidum.-Fluid Extract of Lobelia. By maceration and percolation with Diluted Alcohol, and evaporation.

Dose, I to $10 \mathrm{~m}$. ; .06 to .60 c.c.

2. Tinctura Lobeliæ.-Tincture of Lobelia. Lobelia, 200. By percolátion with diluted Alcohol to 1000 .

Dose, 5 to $30 \mathrm{~m}$.; 30 to 2.00 c.c.]

\section{Action of Lobelia.}

External.-Lobelia has no effect on the skin, but it is stated that poisonous symptoms may occur from absorption of it through the epidermis.

Internal.- Gastro-intestinal tract.-Moderate or large doses are powerfully irritant, and thus they cause.violent vomiting and purging. A peculiarity of the action of lobelia is that these results are accompanied by very intense prostration, as shown by the feeble pulse, cold sweats, pale skin, and great muscular relaxation.

Circulation.- In the frog the heart is at first stimulated, but soon depressed, and it finally stops in diastole. The bloodpressure falls. This is due partly to the action on the heart, and partly to paralysis of the vaso-motor centres.

Respiration.-Small doses slow respiration, large doses 
strongly depress the respiratory centre, and death takes place from respiratory failure. The muscular coat of the bronchi is said to be relaxed.

Nervaus system.-Toxic doses are required to affect the higher cerebral centres, and then coma and convulsions are produced, but it is not clear how far these results are due to asphyxia. The respiratory and vaso-motor centres, and probably the cardiac, are, as already mentioned, depressed. Experiments seem to show that the motor centres of the cord are also depressed. Muscles and nerves are unaffected.

Lobeline is probably excreted by the kidneys and skin, and is said to have diuretic and diaphoretic properties.

\section{THERAPEUTICS OF LOBELIA.}

Lobelia has been recommended as a purgative, and as an emetic, but it should not be used for these purposes, because of its great liability to produce collapse. It is employed [for the relief of the symptom] asthma to relax the muscular coat of the bronchial tubes. A [drachm; 4. c.c.] of the tincture should be given till nausea is experienced, but it should never be pushed beyond that point. It may also be prescribed for bronchitis accompanied by spasmodic dyspnœa. [As an external application tincture of lobelia with an equal quantity of glycerin is a most useful remedy for the relief of pain of acute epididymitis.

\section{ASCLEPIAS.}

ASCLEPIAS.-Synonym.-Pleurisy root. The root of Asclepias tuberosa Linne (nat. ord. Asclepiadea). Habitat.-United States, near the Atlantic Coast.

Characters. - Root large and fusiform, dried in longitudinal or transverse sections, from 2 to $15 \mathrm{~cm}$. long, and about $2 \mathrm{~cm}$. or more in thickness ; the head knotty, and slightly but distinctly annulate, the remainder longitudinally wrinkled, externally orange-brown, internally whitish; tough and having an uneven fracture; bark thin and in two distinct layers, the inner one whitish ; wood yellowish, with large, white, medullary rays. It is inodorous, and has a bitterish, somewhat acrid taste.

Constituents. - (I) A crystalline Glucoside, soluble in Alcohol, Ether, and somewhat in water. (2) Asclepion, a bitter crystalline principle. Two Resins.

Dose, $1 / 4$ to $2 \mathrm{dr}$; 2. to 8 . $\mathrm{gm}$. 


\section{Preparation.}

Extractum Asclepiadis Fluidum.-Fluid Extract of Asclepias. By maceration and percolation with Diluted Alcohol, and evaporation.

Dose, $1 / 2$ to 2 fl. dr.; 2. to 8 . c.c.

\section{Action and Uses of Pleurisy Root.}

Pleurisy root possesses diaphoretic, carminative, and expectorant properties, without being stimulant. It is used in the disease which gives its name, and in various pectoral affections.

\section{CASTANEA.}

CASTANEA.-Synonym. - Chestnut. The leaves of Castanea dentata (Marshall) Sudworth (nat. ord. Cupulifera), collected in September or October, while still green. Habitat.-North America, from Ontario south to Florida, and west to Arkansas and Michigan.

Characters. - From 15 to $25 \mathrm{~cm}$. long, about $5 \mathrm{~cm}$. wide, petiolate, oblong-lanceolate, acuminate, mucronate, feather-veined, sinuate-serrate, smooth ; odor slight; taste somewhat astringent.

Constituents. - (I) Tannic acid, about 9 per cent. (2) Albumin. (3) Resin. (4) Gum.

Dose, $1 / 2$ to $2 \mathrm{dr}$; ; 2 to 8 . gm.

\section{Preparation.}

Extractum Castaneæ Fluidum.-Fluid Extract of Castanea. By maceration with Boiling Water, percolation, evaporation, and addition of Glycerin and Alcohol.

Dose, $1 / 2$ to 2 fl. dr.; 2. to 8 . c.c.

\section{Action and Uses of Chestnut Leaves.}

Chestnut Leaves are mildly sedative, they are used only in whooping-cough, because they are supposed to possess anti-spasmodic and expectorant properties.

\section{INULA.}

INULA.-Synonym.-Elecampane. The root of Inula Helenium Linné (nat. ord. Compositce). Habitat.-Central and Southern Europe, and eastward to central Asia ; spontaneously in the United States; cultivated.

Characters. - In traverse, concave slices or longitudinal sections, with overlapping bark, externally wrinkled and brown; flexible in damp weather; when dry, breaking with a short fracture; internally grayish, fleshy, slightly 
radiate, and dotted with numerous shining, yellowish.brown resin cells; free from starch ; odor peculiar, aromatic ; taste bitter and pungent.

Conposition.-The chief constituents are-(I) Inulin, $3 \mathrm{C}_{12} \mathrm{H}_{20} \mathrm{O}_{10}+$ $\mathrm{H}_{2} \mathrm{O}$, sometimes called Alantin, which is found in 19 to 44 per cent., according to the season; (2) Alantic Acid, $\mathrm{C}_{15} \mathrm{H}_{20} \mathrm{O}_{2} ;$ (3) Hilenin, $\mathrm{C}_{6} \mathrm{H}_{8} \mathrm{O}$. (4) A volatile oil, consisting of alantic anhydride, and alantol or alant camphor.

Dose, $1 / 4$ to $\mathrm{Idr}$. ; r. to $4 . \mathrm{gm}$.

\section{Action aNd Uses of InUla.}

It is chiefly used in diseases of the lungs, especially when complicated with general debility. Recently, some laboratory experiments have suggested that helenin may be of value in the treatment of tuberculosis, since it is believed to be a bactericide.

\section{ASPIDOSPERMA.}

ASPIDOSPERMA.-Synonyms.-Quebracho. Iron Wood. The bark of Aspidosperma Quebracho-blanco Schlechtendal (nat. ord. Apocynacea). Habitat. - Argentine Republic.

Characters. - In nearly fiat pieces, about 1 to $3 \mathrm{~cm}$. thick; the outer surface yellowish-gray or brownish, deeply fissured; inner surface yellowishbrown or reddish-brown, distinctly striate; fracture displaying two sharply defined strata, of about equal thickness, and both marked with numerous whitish dots and strix arranged in tangential lines; the fracture of the outer, lighter-colored layer rather coarsely granular, and that of the darker colored, inner layer short-splintery; inodorous ; taste very bitter and slightly aromatic.

Composition,-(I) Aspidospermine, $\mathrm{C}_{22} \mathrm{H}_{28} \mathrm{~N}_{2} \mathrm{O}_{2}$, (2) Quebrachamine, (3) Aspidospermatine, (4) Aspidosamine, $\mathrm{C}_{22} \mathrm{H}_{28} \mathrm{~N}_{2} \mathrm{O}_{2}$, (5) Hydroquebrachine, $\mathrm{C}_{21} \mathrm{H}_{29} \mathrm{~N}_{2} \mathrm{O}_{2}$, all being Alkaloids, (6) Quebrachit, a peculiar sugar, (7) Tannic acid.

Dose, 5 to $3 \circ \mathrm{gr}$; .30 to $2.00 \mathrm{gm}$.

\section{Preparation.}

Extractum Aspidospermatis Fluidum.-Fluid Extract of Aspidosperma. By maceration with Glycerin and Alcohol, and percolation with Glycerin, Alcohol and Water, and evaporation.

Dose, 5 to $30 \mathrm{~m}$.; .30 to 2.00 c.c.

\section{Action of Aspidosperma.}

None of the alkaloids fully represent the drug. In the lower animals large doses of the bark produce motor paralysis, with dyspnœa and finally death from asphyxia. The breathing early 
becomes slower, but deeper; the blood-pressure is not affected until late. Penzoldt believes that the relief of dyspnœa, which is obtained clinically, is caused by its increasing the power of the blood to take up oxygen.

\section{Therapeutics of Aspidosperma.}

It is a bitter which may aid the appetite, and is a valuable remedy when the respiration is embarrassed by emphysema, chronic bronchitis, or chronic pneumonia ; even uræmic asthma is benefited by it. It is not of benefit in dyspnoea of cardiac origin. The commercial aspidospermine, which is an impure mixture of all the alkaloids, and therefore represents their combined action, may be given in doses of from $1 / 4$ to $1 / 2 \mathrm{gr}$; .015 to $.03 \mathrm{gm}$.

\section{ERIODICTYON.}

ERIODICTYON.-Synonyms.-Yerba Santa. Mountain Balm. Consumptive's Weed. The leaves of Eriodictyon glutinosum, Bentham (nat. ord. Hydrophyllacea). Habitat.-California.

ChARACTERs.-Oblong lanceolate, 5 to $10 \mathrm{~cm}$. long, acute at the apex, and below narrowed into a short petiole, the margin sinuately toothed to nearly entire; upper surface green, smooth, and covered with a brownish resin; lower surface reticulate and minutely white-tomentose; odor somewhat aromatic; taste balsamic and sweetish.

Composition. - The chief constituents are-(I) Volatile Oil, (2) Resin, acrid, greenish-yellow, containing Ericolin, $\mathrm{C}_{34} \mathrm{H}_{56} \mathrm{O}_{21}$, (3) Tannic acid, 8 per cent.

Dose, 15 to $30 \mathrm{gr} . ; 1$. to 2. gm.

\section{Preparation.}

Extractum Eriodictyi Fluidum.-Fluid Extract of Eriodictyon. By maceration and percolation with Alcohol and Water, and evaporation.

Dose, I5 to $30 \mathrm{~m} . ;$ I. to 2. c.c.

\section{Action and Uses of ERIodictyon.}

Eriodictyon has long been used in California as a bitter tonic, and as a stimulating expectorant. It has been found useful in chronic bronchitis. It is an excellent vehicle for quinine, concealing its bitter taste. 


\section{COCILlaña.}

COCILlaña. (Not official).-The bark of Sycocarpus Rusbyi (nat. ord. Meliacę). Habitat.-Bolivia.

Characters. - The bark is thick, and ash-colored, becoming rough only with considerable age; inner surface is grayish-yellow ; the odor is slight, but peculiar ; taste unpleasant (not bitter), slightly nauseous.

Composition. - Its chief constituents are-(I) Rusbyine (Eccles), an alkaloid. (2) Two Resins. (3) Tannic Acid. (4) Calcium Oxalate.

\section{Preparations.}

I. Extractum Cocillañæ Fluidum.-Fluid Extract of Cocillaña. By mäceration and percolation with Alcohol and Water, and evaporation.

Dose, 5 to $25 \mathrm{~m}$.; $\cdot 30$ to $\mathrm{r} .50$ c.c.

2. Syrupus Cocillañz.-Syrup of Cocillaña. By maceration and percolation with Alcohol and Water, with addition of Sugar.

Dose, $r$ to 2 fl. dr.; 4. to 8. c.c.

3. Tinctura Cocillañæ.-Tincture of Cocillaña. By maceration and percolation with Alcohol and Water, and addition of Alcohol.

Dose, I to 2 fl. dr. ; 4. to 8. c.c.

\section{Action and Uses of Cocillaña.}

Cocillaña acts upon muciparous glands, increasing their activity; on the bronchial mucous membrane, causing expectoration; on the intestinal mucous membrane, producing a laxative effect; it also slightly increases the appetite; it slightly strengthens the heart beat, and the pulse, but does not stimulate the respiratory centre. The syrup does not act as a laxative, while the resins are distinctly purgative. It is of very great value as an expectorant, preferable to ipecacuanha, in that it does not so readily cause nausea and a metallic taste in the mouth, and assists the regular movement of the bowels. If, however, nausea should be produced, it is very persistent. Its action is fully established three to six hours after administration, and persists at least for six hours. It can, in many cases, be substituted for apomorphine, ammonium carbonate and for many other drugs, classed, with more or less reason, as expectorants]. 


\section{PRUNUS VIRGINIANA.}

[WILD CHERRY.- The bark of Prunus serotina Ehrhart (nat. ord. Rosacex), collected in autumn. Habitat.-North America, westward to Minnesota and Louisiana; in woods.

Characters. - In curved pieces or irregular fragments, $2 \mathrm{~mm}$. or more thick, outer surface greenish-brown, or yellowish-brown, smooth and somewhat glossy, marked with transverse scars; if the bark is collected from old wood and deprived of the corky layer, the outer surface is nut-brown and uneven; inner surface somewhat striate or fissured. Upon maceration in water it develops a distinct bitter-almond odor; its taste is astringent, aromatic and bitter.

Compositron.-(1) Amygdalin, which yields with water, Glucose, $\mathrm{Hy}$ drocyanic Acid and the Essential Oil of Bitter Almond. (2) Emulsin, probably identical with the emulsin of Bitter Almond. The action of this ferment is destroyed at a boiling temperature.

Dose, $1 / 2$ to I dr. ; 2. to $4 . \mathrm{gm}$.

(3) Tannic Acid.

\section{Preparations.}

1. Extractum Pruni Virginianæ Fluidum.-Fluid Extract of Wild Cherry. By maceration and percolation with Glycerin, Alcohol and Water, and evaporation.

Dose, $1 / 2$ to I fl. dr.; 2. to 4 . c.c.

2. Infusum Pruni Virginianæ.-Infusion of Wild Cherry. Wild Cherry, 40. By maceration and percolation to 1000 .

Dose, I to 2 fl. oz. ; 3o. to 6o. c.c.

3. Syrupus Pruni Virginianæ.-Syrup of Wild Cherry. Wild Cherry, 150; Sugar, 700 ; Glycerin, I50; Water to 1000. By maceration and percolation.

Dose, I to 4 fl. dr.; 4. to 15 . c.c.]

\section{Action and Therapeutics of Wild Cherry.}

When this drug is treated with water diluted hydrocyanic acid is formed, and that is probably the reason why it is efficacious in relieving cough, especially a hacking cough, by which nothing is expectorated, for [hydrocyanic] acid diminishes reflex excitability. [Wild cherry] is a very favorite remedy, and the syrup is a very useful flavoring agent for cough mixtures. [It also, as a vehicle for tincture of digitalis, renders it less likely to produce gastric disturbance.] 


\title{
GROUP IV.
}

\author{
Vegetable Drugs having Antiperiodic, Antipyretic, and Antiseptic \\ Properties.
}

\section{Cinchona, Quinine, Salicin, Salicylic Acid, [Gaultheria,] Salol, [Salophen.]}

\section{CINCHONA.}

[CINCHONA.-The bark of Cinchona Calisaya Weddell, Cinchona officinalis Linné and of hybrids of these and of other species of Cinchona (nat. ord. Rubiacea), yielding, when assayed, not less than 5 per cent. of total alkaloids, and at least 2.5 per cent. of Quinine $\left(\mathrm{C}_{20} \mathrm{H}_{24} \mathrm{~N}_{2} \mathrm{O}_{2}+\mathrm{IH}_{2} \mathrm{O}=34 \mathrm{I} \cdot 3\right)$. Habitat.-South America, on the Eastern slope of the central chain of the Andes, thence spreading northward into Colombia ; cultivated in Java, India, Jamaica, and other countries; to a limited extent also in South America.

Characters. - In quills or in curved pieces, varying in length, and usually 2 or 3 , or sometimes $5 \mathrm{~mm}$. thick; the outer surface covered with a gray or brownish-gray cork, usually slightly wrinkled, marked with transverse, and also with intersecting, longitudinal fissures ( $C$. Calisaya), and sometimes with scattered warts and slight longitudinal ridges; inner surface light cinnamonbrown, very finely striate; fracture short and granular in the outer layer, and finely fibrous in the inner layer; powder light trown or yellowish brown; odor slight, somewh

Composition. - The chief constituents of Cinchona Bark are five alkaloids, two acids, a glucoside, Tannic acid, a coloring matter and a volatile oil.

(I) Quinine.-An Alkaloid, exists as the Hydrate. Gives a green color with Chlorine water and Ammonia ; turns the plane of polarization to the left, solutions of its salts are fluorescent. Forms salts with acids. (See p. 464.)

(2) Quinidine.-An Alkaloid, $\mathrm{C}_{20} \mathrm{H}_{24} \mathrm{~N}_{2} \mathrm{O}_{2}$, isomeric with Quinine, differing from it only in crystallizing in prisms, turning the plane of polarization to the right, and not being soluble in ammonia except in excess.

(3) Cinchonine.-An Alkaloid. $\mathrm{C}_{20} \mathrm{H}_{24} \mathrm{~N}_{2} \mathrm{O}$. Colorless prisms, inodorous, bitter. No green color with Chlorine water and Ammonia. Turns the plane of polarization to the right. Not fluorescent. (See p. 456.)

(4) Cinchonidine.-An Alkaloid, $\mathrm{C}_{19} \mathrm{H}_{22} \mathrm{~N}_{2} \mathrm{O}$, isomeric with Cinchonine, differing from it in turning the plane of polarization to the left, being sparingly soluble in Ether, and being slightly fluorescent.

(5) Quinamine. - An Alkaloid $\left[\mathrm{C}_{19} \mathrm{H}_{24} \mathrm{~N}_{2} \mathrm{O}_{2}\right]$. Not important.

(6) Kinic or Quinic Acid.- $\mathrm{C}_{7} \mathrm{H}_{12} \mathrm{O}_{6}$. Large, colorless prisms. It and its salts are soluble in water, and thus Quinine may be given subcutaneously as Quinine Quinate. This acid is found in the Coffe Bean and other plants. It is allied to Benzoic Acid, and appears in the urine as Hippuric Acid. 
(7) Kinovic or Quinovic Acid, $\left[\mathrm{C}_{31} \mathrm{H}_{48} \mathrm{O}_{6}\right]-A$ white amorphous substance related to Kinovin.

(8) Kinovin or Quinovin.-A Glucoside, $\left[\mathrm{C}_{30} \mathrm{H}_{48} \mathrm{O}_{8}\right.$, ] which easily decomposes into Glucose and Kinovic Acid.

(9) Cinchotannic Acid.- [ 2 to 4$]$ per cent. It is the astringent principle of Cinchona Bark. It differs from Tannic Acid in becoming green with ferric salts. It is easily oxidized to Cinchona Red.

(10) Cinchona Red.-The coloring matter of the bark. It is almost insoluble in water.

(II) A Volatile Oil.-This exists in minute quantities. Cinchona Bark owes its smell to it.

Remijia Bark yields, in addition, Homoquinine, which is a compound of Quinine and another alkaloid, Cupreine, $\left[\mathrm{C}_{19} \mathrm{H}_{22} \mathrm{~N}_{2} \mathrm{O}_{2}\right.$. $]$

IMPURITIES. - Inferior barks, known by their not yielding the full strength of Quinine and Cinchonine.

[The true yellow Cinchona Bark must not be confounded with other Cinchona Barks of a similar color, but having the bast fibres in bundles or raised rows, and breaking with a splintery or coarsely fibrous fracture.]

IncoMpatibles.-Ammonia, lime water, metallic salts and gelatin.

Dose, 10 to $60 \mathrm{gr}$; ; .60 to $4.00 \mathrm{gm}$.]

\section{Preparations.}

[1. Infusum Cinchonæ.-Infusion of Cinchona. Cinchona, 60; Aromatic Sulphuric Acid, I0; by percolation with Water to 1000 .

Dose, 1 to 2 fl. oz.; 30. to 6o. c.c.

2. Extractum Cinchonæ.-Extract of Cinchona. By maceration and percolation with Alcohol and Water, distillation of the Alcohol and evaparation.

Dose, 4 to $30 \mathrm{gr}$; .30 to $2.00 \mathrm{gm}$.

3. Extractum Cinchonæ Fluidum.-Fluid Extract of Cinchona. By maceration and percolation with Alcohol, Glycerin and Water, and evaporation, addition of Alcohol.

Dose, ro to $60 \mathrm{~m}$.; .60 to 4.00 c.c.

4. Tinctura Cinchonæ.-Tincture of Cinchona. Cinchona, 200; Glycerin, 75. By maceration and percolation with Alcohol and Water to ICOO.

Dose, $y$ to 2 f. dr. ; 2. to 8 . c.c.]

\section{CINCHONA RUBRA.}

[RED CINCHONA.-The bark of Cinchona succirubra Pavon (nat. ord. Rubiacea), containing not less than 5 per cent. of its peculiar alkaloids. Habitat.-Ecuador, west of Chimborazo.

Cinaracters. - In quills or incurved pieces, varying in length, and from 
2 to 4 or $5 \mathrm{~mm}$. thick; the outer surface covered with a grayish-brown cork, more or less rough from warts and longitudinal warty ridges, and from few, mostly short transverse fissures; inner surface more or less deep reddish-brown and distinctly striate ; fracture short fibrous in the inner layer; powder reddish-brown; odor slight ; taste bitter and astringent.

Constituents. - See Cinchona.

Dose, 1o to $60 \mathrm{gr}$. ; .60 to $4.00 \mathrm{gm}$.

\section{Preparation.}

Tinctura Cinchonæ Composita.-Compound Tincture of Cin. chona. Red Cinchona, roo; Bitter Orange Peel, 8o; Serpentaria, 20; Glycerin, 75. By maceration and percolation with Alcohol, Glycerin and Water to 1000 .

Dose, I to $4 \mathrm{fl}$. dr.; 4 . to I5. c.c.

\section{QUININA.}

I. QUININE. $-\mathrm{C}_{20} \mathrm{H}_{24} \mathrm{~N}_{2} \mathrm{O}_{2}+3 \mathrm{H}_{2} \mathrm{O}=377.22$. An Alkaloid obtained from the bark of various species of Cinchona (nat. ord. Rubiacece).

Source. - By adding to a solution of the Sulphate a sufficient quantity of Ammonia Water to precipitate the Alkaloid.

CharaCters. - A white, flaky, amorphous or crystalline powder, odorless, and having a very bitter taste; permanent in the air. Solubility.-In 1670 parts of water, and in 6 parts of Alcohol; in 23 parts of Ether, 5 parts of Chloroform and 200 parts of Glycerin; also soluble in Carbon Disulphide, Benzin, Benzol, Ammonia Water, and diluted acids.

IMPURITIES. - Other alkaloids, cinchonine, cinchonidine, quinidine and cupreine. Lime, chalk, magnesia, starch and other white powders. Salicin, detected by its giving a blood-red color with sulphuric acid.

Quinine is used to prepare Ferri et Quininæ Citras and Ferri et Quininæ Citras Solubilis.

Dose, $x$ to $20 \mathrm{gr}$; .06 to x.20. gm.]

2. QUININ $A$ SULPHAS.-Quinine Sulphate. $\left(\mathrm{C}_{20} \mathrm{H}_{24} \mathrm{~N}_{2} \mathrm{O}_{2}\right)_{2} \mathrm{H}_{2}$ $\mathrm{SO}_{4}\left[+7 \mathrm{H}_{2} \mathrm{O}=870.22\right.$.

SOURCE. - By boiling Cinchona in water acidulated with Hydrochloric Acid and straining. Add Lime to the decoction, and wash the precipitate. Digest in boiling Alcohol, and distil off the Alcohol. Dissolve the residue in Distilled Water and Sulphuric Acid, boil with Animal Charcoal, filter and set aside to crystallize.

Characters. - White, silky, light and fine, needle-shaped crystals, fragile and somewhat flexible, making a very light and easily compressible mass, lustreless from superficial efflorescence after being for some time exposed to the air, odorless, and having a persistent, very bitter taste. The salt is liable to lose water on exposure to warm air, to absorb moisture in damp air, and to become colored by exposure to light. Solubility. - In 740 parts of water, 
and in 65 parts of Alcohol; also in 40 parts of Glycerin, in about 680 pars of Chloroform, and freely in dilute acids.]

INCOMPATIBLES.-Alkalies and their carbonates, and astringent infusions.

[Quinine Sulphate is used to prepare Syrupus Ferri, Quininæe et Strychninæ Phosphatum.

Dose, $\mathrm{I}$ to $5 \mathrm{gr}$.; .06 to $.30 \mathrm{gm}$., or 5 to $20 \mathrm{gr}$; .30 to $1.20 \mathrm{gm}$.] (antipyretic and antiperiodic).

3. [QUININA BISULPHAS.-Quinine Bisulphate. $-\mathrm{C}_{20} \mathrm{H}_{24} \mathrm{~N}_{2} \mathrm{O}_{2}$ $\mathrm{H}_{2} \mathrm{SO}_{4}+7 \mathrm{H}_{2} \mathrm{O}=546.88$.

SOURCE.-By suspending Quinine Sulphate in water, adding Sulphuric Acid, filtering and crystallizing.

Characters. -Colorless, transparent or whitish, orthorhombic crystals, or small needles, odorless, and having a very bitter taste. Efflorescing on exposure to air. Solubility. - In 1o parts of water, and in 312 parts of Alcohol.

Dose, I to $20 \mathrm{gr}$; .06 to $1.20 \mathrm{gm}$.

4. QUININ AY HYROBROMAS.-Quinine Hydrobromate. $\mathrm{C}_{20}$ $\mathrm{H}_{24} \mathrm{~N}_{2} \mathrm{O}_{2} \mathrm{HBr}+\mathrm{H}_{2} \mathrm{O}=422$.06.

SourCE. - By suspending Quinine Sulphate in Water, adding Barium Bromide in solution, filtering, evaporating, and crystallizing.

Characters. - White, light, silky needles, odorless, and having a very bitter taste. The salt is liable to lose water on exposure to warm or dry air.

Solubility.-In 54 parts of. water, and in 0.6 part of Alcohol; also soluble in 6 parts of Ether, and in 12 parts of Chloroform.

Dose, I to $20 \mathrm{gr}$; . .06 to $1.20 \mathrm{gm}$.]

5. QUININ $A$ HYDROCHLORAS.-Quinine IIydrochlorate. $\mathrm{C}_{20}$ $\mathrm{H}_{24} \mathrm{~N}_{2} \mathrm{O}_{2} \mathrm{HCl}\left[+2 \mathrm{H}_{2} \mathrm{O}=395.63\right.$.

Source. - By treating the Alkaloid with diluted Hydrochloric Acid, and crystallization.

Cinaracters. - White, silky, light and fine, needle-shaped crystals, odorless, and having a very bitter taste. The salt is liable to lose water when exposed to warm air. Solubility.-In 34 parts of water, and in 3 parts of Alcohol; also soluble in 9 parts of Chloroform.]

Dose, I to $20 \mathrm{gr}$; ; [.06 to $1.20 \mathrm{gm}$.]

6. [QUININ E VALERIANAS. See Valeriana.

7. QUINIDIN $\approx$ SULPHAS.—Quinidine Sulphate. $\left(\mathrm{C}_{20} \mathrm{H}_{24} \mathrm{~N}_{2} \mathrm{O}_{2}\right)_{2}$ $\mathrm{H}_{2} \mathrm{SO}_{4}+2 \mathrm{H}_{2} \mathrm{O}=780.42$. The neutral sulphate of an alkaloid obtained from the bark of several species of Cinchona (nat. ord. Rubiacea).

SOURCE. - From the mother liquors after the crystallization of Quinine, by the same process as for Quinine.

Characters. - White, silky needles, odorless, and having a very bitter taste; permanent in the air. Solubility. -In 100 parts of water, and in 8 
parts of Alcohol; also in 14 parts of Chloroform, and in acidulated water; almost insoluble in Ether.

Dose, 1 to $20 \mathrm{gr}$; .06 to $1.20 \mathrm{gm}$.

8. CINCHONINA.-Cinchonine. $\mathrm{C}_{19} \mathrm{H}_{22} \mathrm{~N}_{2} \mathrm{O}=293.4 \mathrm{I}$.

SouRCE.-By precipitation of the aqueous solution of the Sulphate by Ammonia Water.

CHARACTERs. - White lustrous prisms' or needles, without odor, at first almost tasteless, but soon developing a bitter after-taste; permanent in the air. Solubility. - In 3760 parts of water, and in I 16 parts of Alcohol ; also soluble in 526 parts of Ether, and in 163 parts of Chloroform.

Dose, I to $30 \mathrm{gr}$; .06 to $2.00 \mathrm{gm}$.

9. CINCHONINA SULPHAS.-Cinchonine Sulphate. $\left(\mathrm{C}_{19} \mathrm{H}_{22} \mathrm{~N}_{2}\right.$ O) ${ }_{2} \mathrm{H}_{2} \mathrm{SO}_{4}+2 \mathrm{H}_{2} \mathrm{O}=720.56$.

SouRCE.-Obtained from the mother liquors after the crystallization of the Quinine, Quinidine, and Cinchonidine Sulphates by further concentration, precipitating the alkaloids by Caustic Soda, washing with Alcohol till free from other alkaloids, dissolving in Sulphuric Acid, purifying with animal charcoal, and crystallizing.

Characters. - Hard, white, lustrous, prismatic crystals, without odor, and having a very bitter taste ; permanent in the air. Solubility. - In 66 parts of water, and in ro parts of Alcohol; also soluble in 78 parts of Chloroform, but almost insoluble in Ether.

Dose, I to $30 \mathrm{gr}$; .06 to $2.00 \mathrm{gm}$.

ro. CINCHONIDINE SULPHAS.-Cinchonidine Sulphate. ( $\mathrm{C}_{19}$ $\left.\mathrm{H}_{22} \mathrm{~N}_{2} \mathrm{O}\right)_{2} \mathrm{H}_{2} \mathrm{SO}_{4}+3 \mathrm{H}_{2} \mathrm{O}=738.52$. The neutral Sulphate of an Alkaloid obtained from the bark of various species of Cinchona.

SoURCE.-Obtained from the mother liquors after the crystallization of Quinine Sulphate by further concentration, purifying by crystallization from Alcohol, and finally from hot water.

ChARACTERS. - White, silky, acicular crystals, without odor, and having a very bitter taste; slightly efflorescent on exposure to air. Solubility._In 70 parts of water, and in 66 parts of Alcohol; also soluble in 1316 parts of Chloroform, and almost insoluble in Ether. The presence of sulphates of other Cinchona Alkaloids increase its solubility in Ether and Chloroform.

Dose, I to $30 \mathrm{gr}$. ; .06 to $2.00 \mathrm{gm}$.]

\section{Action of Cinchona and its Alkaloids.}

The action of cinchona bark is due almost entirely to the quinine in it ; the other alkaloids act in much the same way as this alkaloid, the sulphate, [bisulphate, hydrobromate] and hydrochlorate of which produce the same effect as quinine itself. The following description will be that of the action of quinine sul- 
phate, which is often called quinine. Any differences between it and the bark or the other alkaloids will be mentioned in the course of this description.

External.-Quinine is a very powerful antiseptic. A solution of I to 500 destroys many forms of micro-organisms, and a solution of $\mathrm{I}$ to $25^{\circ}$ prevents fermentation and putrefaction. Quinine is very fatal to all low forms of animal and vegetable life. A solution of I to rooo kills many infusoria. No effect is produced upon the sound skin by quinine, but it is irritant to a raw surface.

Internal.-Alimentary canal.-Quinine acts like any other bitter, such as calumba. The bitter taste is very marked; in the mouth the gustatory nerves, and in the stomach the gastric nerves, are stimulated. This leads reflexly to an increase of the salivary and gastric secretions, and to greater vascularity and peristalsis of the stomach, the appetite is sharpened and digestion is aided. Quinine is, therefore, a stomachic. These effects, of course, bring about a better absorption of food; and hence, if digestion was previously feeble, the patient feels stronger after a course of quinine. In the stomach any quinine salt is converted into a chloride, some of which is probably absorbed here ; for in the intestines it would be precipitated by the alkaline secretions. It is often excreted unchanged in the frecs.

Blood.-Quinine, as the chloride, is readily absorbed into the blood; and although this is alkaline, it is not precipitated, being probably held in solution by the gases of the blood. It is not known that it undergoes any alteration there, but it produces some remarkable changes.

(a) White corpuscles. - If the movements of the white corpuscles are being watched in a drop of blood on the warm stage of the microscope, and some quinine is added, they at once cease. Again, if the mesentery of a living frog be put under the microscope, and slightly irritated so as to set up inflammation, emigration of the white corpuscles through the capillary walls, or diapedesis, as it is called, will be observed; if now some quinine be injected int? the circulation this ceases, but those white corpuscles that b sve already passed out wander further from their 
capillary. If the quinine be applied locally to the mesentery, directly the white corpuscles have passed through their capillaries their movement is stopped, and the motionless corpuscles collect in large numbers around the capillaries. It is clear, therefore, that quinine has the power of arresting the movements of white blood-corpuscles. In sufficient quantity it appears actually to destroy them, for in a cat killed by quinine they are much fewer in number than in a healthy cat.

(b) Red corpuscles. - Quinine is said to cause a diminution in the size of these, but this is most likely not strictly correct. In fever, if the temperature is high, the red corpuscles are probably a little larger than natural. If the temperature be reduced by any means the corpuscles regain their normal size. Quinine will reduce the temperature, but it probably has no special action on the corpuscles.

(c) Acidity of the blood.-Blood outside the body gradually becomes acid. Quinine prevents this.

(d) Ozonizing power.-If ozonized oil of turpentine be mixed with a tincture of guaiacum, nothing occurs; but if a drop of blood be added, that transfers the ozone to the guaiacum, oxidizes it, and turns it blue. This ozonizing power of blood is prevented by the addition of quinine.

(e) The stability of oxyhamoglobin is strengthened by quinine, so that the blood does not yield up its oxygen as easily as normally, consequently it cannot absorb oxygen readily. This inability of hæmoglobin to take up oxygen in the presence of quinine is parallel with its action on other varieties of protoplasm. For example, fungi absorb oxygen slowly if quinine be present, and thus fermentation may be prevented. Phosphorescent infusoria (the phosphorescence is due to rapid oxidation) lose this property in the presence of quinine. The ozonizing power of fresh vegetable juices is retarded by it. Quinine is, therefore, very constant and very powerful in interfering with oxidation.

Circulation.-Small doses of quinine probably increase the activity of the heart reflexly because they stimulate the stomach; but large doses (larger than are given to man medicinally), either applied to the excised heart or circulating through it, directly 
paralyze the organ; the pulse becomes slower and more feeble, and the heart is finally arrested in diastole. Whether it acts on the muscle or the ganglia is not known. Iarge doses lower the blood-pressure considerably ; this is owing partly to the effect on the heart, but it is probable that this fall of arterial pressure is due in part also to the action of quinine on the blood-vessels. If the spleen is enlarged as a result of malarial fever, the administration of quinine, curing the fever, leads to a decrease in the size of the spleen, but it has no direct effect on this organ, as is often asserted.

Respiration.-Although, as we have seen, quinine must, because of its retardation of oxidation, have a powerful influence on internal respiration, diminishing the activity of metabolism, it has but a moderate effect on respiratory movements. Small doses slightly increase, large doses depress them.

Temperature.-Quinine has very little power over the healthy temperature, but that of fever is markedly reduced; it is, therefore, an energetic antipyretic. Considering its direct capability of diminishing metabolism in the tissues, it seems fair to assume that the drug diminishes heat production, and that it does so by acting directly on the thermogenetic tissues; but, whether it decreases heat production by also influencing the cerebral thermogenetic centres is not known.

Cerebrum.-Small doses are believed to stimulate cerebral activity. The results of experiments upon the action of quinine on the brain are so discordant as to be at present valueless. The effects of a large dose in man will be described under Cinchonism.

Spinal cord and nerves.-In frogs, quinine causes a lessening of reflex excitability, which is removed by section below the medulla; but in large doses it produces a permanent diminution of reflex excitability. In these animals quinine also first excites and then paralyzes the sensory nerves or their peripheral endings. The muscles are uninfluenced. These effects are not seen in man.

Uterus.-It has often been stated that quinine will lead to abortion, that it will, when labor has commenced, aid the expul- 
sion of the fœtus, and that it will increase the menstrual flow if that is scanty. It appears that the first statement is certainly incorrect, and that the second and third are only correct for some women.

Kidneys. - After a full dose of quinine it is found in the urine in half an hour, and is slowly excreted for several days, but by far the greater part is eliminated within the first fortyeight hours. The excretion of uric acid is greatly diminished, and that of urea and other nitrogenous bodies in the urine is also considerably lessened. This confirms the statement already made that quinine retards considerably the metabolism of the body, but it should be stated that very little alteration is observed in the excretion of carbon [dioxide] by the lungs. It is said that minute quantities of quinine are gotten rid of by all the secretions, as it may be detected in milk, saliva, bile, tears, etc., and it may be found in dropsical fluids if the patient has been taking it.

Cinchonism.- In many persons a dose of ten grains; [.60 $\mathrm{gm}$.] or more of quinine produces a train of physiological symptoms, chiefly from its influence on the nervous system. The patient soon complains of ringing in the ears, fullness in the head, and slight deafness. With larger doses these symptoms increase, disturbances of vision and giddiness are added, he may stagger when he walks, and the headache may be very intense.

Quinine is hardly ever given as a poison, but if it should be, all these symptoms of cinchonism will be very severe; the patient may be delirious and comatose, quite deaf and blind, and if he die it will be from collapse due to cardiac and respiratory failure. Great congestion of the middle ear and labyrinth is found in animals poisoned by quinine. The mild degrees of cinchonism pass off directly [after] the drug is discontinued. Rarely quinine causes an erythematous rash, and it has been known to give rise to epistaxis. Those who work among cinchona barks may have a rash on their skin from the mechanical irritation of the powder. Both hydrobromic acid and ergotin are said to diminish the liability to cinchonism. 
Relative Action of the Alkaloids.-The other alkaloids are quite similar in their action to quinine, but they are not so powerful. Their relative antipyretic effect is quinine roo, quinidine 90 , cinchonidine 70 , cinchonine 40.

\section{Therapeutics of Cinchona and its Alkaloids.}

External.-Quinine is too expensive for use as an antiseptic.

Internal.-Gastro-intestinal tract.-It is very largely used on account of its stomachic properties, chiefly for that variety of indigestion which is the outcome of general ill-health, want of fresh air, anæmia, etc., and not often when the stomach is the organ primarily at fault. The preparations of cinchona bark are very useful for this variety of dyspepsia; they contain quite enough of the alkaloids. The compound tincture has the advantage of containing other stomachics. Iron is very commonly given at the same time to correct the general condition. Quinine is frequently prescribed with the tincture of [ferric] chloride; there is always enough free acid in this to dissolve any preparation of quinine. Alkalies, especially sal volatile, are often prescribed with solutions of quinine sulphate, but they precipitate the quinine, and therefore mucilage must be used to suspend it. The dose of quinine sulphate or hydrochlorate as a stomachic bitter is $1 / 2$ to $2 \mathrm{gr}$. [.03 to. $12 \mathrm{gm}$.] The hydrochlorate is often preferred, as it is the more soluble. [Cinchonidine salicylate (not official) is generally preferable to the sulphate as a tonic and an antiperiodic in dose of from 5 to $10 \mathrm{gr}$.; .30 to. $.60 \mathrm{gm}$.]

Antipyretic effect.—Quinine was commonly used as an antipyretic, but for the rare occasions on which antipyretic drugs are required, it has now been replaced by more certain drugs, as phenacetin, acetanilid, and [antipyrin]. It is, however, a very fairly certain antipyretic. It is best given for this purpose in a single dose of 20 to $40 \mathrm{gr}$. [1.20 to $2.40 \mathrm{gm}$.] for an adult. Such large doses may be prescribed either in cachets, or as a solution of the hydrochlorate, or as the sulphate suspended in milk [and at the same time sodium or potassium bromide should be administered to avoid the disagreeable tinnitus which is set up. The diluted hybrobromic acid is an excellent solvent, and, 
at the same time, will relieve the ringing in the ears]. About one or two hours elapse before the temperature begins to fall. Quinine is more efficacious in reducing a temperature just beginning to fall than a rising one. Hence, if possible, it should be administered two or three hours before the time at which previous experience of the particular case shows the temperature will probably attain its maximum; then the fall will be more marked and last longer than if the drug had not been given.

Specific action.-Quinine, and to a less extent the other cinchona alkaloids, have the remarkable property of arresting the paroxysms of malaria fever, [because it prevents the entrance of spores into the red blood-corpuscles where only their cycle of development occurs.] If 15 to $30 \mathrm{gr}$. [1. to 2. gm.] be taken about one or two hours before the attack is due, it will not take place, or it will be very mild. [If a more prompt effect is desired, quinine carbamide (not official), which is very soluble, can be administered hypodermatically; a smaller dose, 5 to $8 \mathrm{gr}$.; .30 to $.50 \mathrm{gm}$., in an hour or two, is almost invariably successful in preventing the next immediate chill.] The same effect [may] be produced if smaller doses, about $5 \mathrm{gr}$. $[.30 \mathrm{gm}$. $]$ have been taken four or five times a day during the period between the attacks. Not only is it thus prophylactic, but the continued use of it is curative. It is also preventive, even if the persons to whom it has been given have never had ague. For this purpose it is administered to soldiers and sailors who have to enter malarious regions, and it is then found that few of them get ague. If the disease is very severe it is best to give single large doses. [Clark's powder consists of quinine, ro; powdered capsicum, 4 ; powdered opium, I part. This is given in I $5 \mathrm{gr}$.; $\mathbf{I} .00 \mathrm{gm}$., doses, and is said to be more efficacious in the treatment of ague than larger doses of quinine when given alone.]

If a person has once had ague, illnesses that he subsequently suffers from are liable to assume a malarial type. This is especially the case with neuralgia, which is then peculiarly paroxysmal. It is often on the forehead, when it is called brow-ague. In such cases the effect of quinine is frequently very well-marked, and a cure speedily takes place. Sometimes neuralgia which is 
not malarial is temporarily benefited. Quinine cures ague by acting, while circulating in the blood, as a direct poison to the hæmatozoa (protozoa), the [plasmodium malariæ,] which infests the blood and is the cause of ague. It has been given for a host of diseases, especially septicæmia, but there is not any evidence that it does good to any except those mentioned. The preparations of the bark contain so little quinine that they cannot be used as antipyretics or antiperiodics.

Lately it has been stated that quinine causes black-water fever ; this is a pernicious error, as it will cure this form of malaria.

Quinine should, if possible, be avoided in (I) persons suffering from acute or subacute disease of the middle ear ; (2) those suffering from gastro-intestinal irritation, which it may increase ; (3) those people, occasionally met with, in whom quite small doses produce very severe symptoms of cinchonism; (4) [Meningitis and (5) Inflammation of genito-urinary tract.]

Warburg's tincture is a medicine which has a very high reputation in India for malaria. It has been called Tinctura Antiperiodica. The published formula states that it is a proof-spirit tincture, containing Quinine Sulphate, 80 ; Socatrine Aloés, I00 ; Opium, I ; Rhubarb, 32 ; Camphor, S ; with Angelica, Elecampane, Saffron, Fennel, Gentian, Zedoary, Cubeb, Myrrh, and Agaric, as aromatics, with menstruum to 4000 . [This contains about $91 / 2$ gr. ; .60 gm., to the ounce; 3o. c. c., of menstruum.] Dose, I to 4 fl. dr. [4. to 15. c.c.] It is often prescribed to be made without the Aloes.

\section{SALICINUM.}

SALICIN. $-\mathrm{C}_{13} \mathrm{H}_{18} \mathrm{O}_{7}[=285.33$. A neutral principle (glucoside) obtained from several species of Salix and Pofulus (nat. ord. Salicacea). Synonym.-Willow. Habitat.-Europe, naturalized in North America; cultivated.

Source-(I) Make a strong decoction of willow bark. (2) Remove the tannic acid by warming and agitating the decoction with Lead Oxide. (3) Evaporate the solution. Salicin crystallizes out, and is purified by repeated solution, and crystallization.

Ciiaracters. - Colorless, or white, silky, shining crystalline needles, or a crystalline powder, odorless, and having a very bitter taste. Permanent in the air. Solubility. - In $2 S$ parts of water, and in $3^{\circ}$ parts of Alcohol; almost insoluble in Ether or Chloroform.]

Dose, 5 to $30 \mathrm{gr}$; ; .30 to $2.00 \mathrm{gm}$.] 


\section{ACIDUM SALICYLICUM.}

SALICYLIC ACID. $-\mathrm{HC}_{7} \mathrm{H}_{5} \mathrm{O}_{3}[=137.67$. An organic acid, existing naturally, in combination, in various plants, see Oleum Gaultheriæ and Oleum Betulæ Volatile; but most largely prepared synthetically from Carbolic Acid.]

SourcE.-[Made by combining Sodium Carbolate with Carbon Dioxide Gas. Thus, dry Carbon Dioxide is passed through Sodium Carbolate heated to $4^{2} 8^{\circ} \mathrm{F}$. ; $220^{\circ}$ C. ]. $2 \mathrm{NaC}_{6} \mathrm{H}_{5} \mathrm{O}+\mathrm{CO}_{2}=\mathrm{Na}_{2} \mathrm{C}_{7} \mathrm{H}_{4} \mathrm{O}_{3}$ (Sodium Salicylate), $+\mathrm{C}_{6} \mathrm{H}_{6} \mathrm{O}$ (Phenol). This is treated with Hydrochloric Acid. $\mathrm{Na}_{42} \mathrm{C}_{7} \mathrm{H}_{4} \mathrm{O}_{3}$ $+2 \mathrm{HCl}=2 \mathrm{NaCl}+\mathrm{HC}_{7} \mathrm{H}_{5} \mathrm{O}_{3}$ (Salicylic Acid).

Characters. - [Light, fine, white, prismatic needles, or a light, white, crystalline powder; odorless, having a sweetish, afterwards acrid taste, and permanent in the air. Solubility.-In about $45^{\circ}$ parts of Water, and in 2.4 parts of Alcohol ; also soluble in 2 parts of Ether, 2 parts of Absolute Alcohol, and 80 parts of Chloroform.] Resembling Artificial Salicylic Acid.-Strychnine, but the crystals of Strychnine are larger, colorless, non-irritating, less soluble, and the solution is very bitter.

INCOMPATIBLE. - Spirit of Nitrous Ether.

IMPURITIFS. - Orthocreosotic, metacreosotic, and paracreosotic acids, only in artificial salicylic acid. In the best specimens they are absent.

Dose, 5 to $60 \mathrm{gr}$. ; $[.30$ to $4.00 \mathrm{gm}$. $]$

SODII SALICYLAS.-Sodium Salicylate. $\mathrm{NaC}_{7} \mathrm{H}_{5} \mathrm{O}_{3}[=159.67]$.

Source.-Obtained by acting on Sodium Carbonate with Salicylic Acid. $\left[2 \mathrm{HC}_{7} \mathrm{H}_{5} \mathrm{O}_{3}+\mathrm{Na}_{2} \mathrm{CO}_{3}=2 \mathrm{NaC}_{7} \mathrm{H}_{5} \mathrm{O}_{3}+\mathrm{H}_{2} \mathrm{O}+\mathrm{CO}_{2}\right.$. The solution may be strained through muslin and heated to expel the Carbon Dioxide.]

Characters. - [A white, amorphous powder, odorless, and having a sweetish, saline taste. Permanent in cool air. Solubility.-In 0.9 part of Water, and in 6 parts of Alcohol ; also soluble in Glycerin.]

InCOMPATIBLE. - Hydrobromic acid, for sodium bromide is formed and salicylic acid is precipitated.

IMPURITIFS. - Orthocreosotic, metacreosotic, and paracreosotic acids.

Dose, 5 to $60 \mathrm{gr}$; .30 to $4.00 \mathrm{gm}$.

[LITHII SALICYLAS.-Lithium Salicylate. $\quad\left(\mathrm{LiC}_{7} \mathrm{H}_{5} \mathrm{O}_{3}=143.68.\right)$

Source.-Obtained by heating Salicylic Acid, Lithium Carbonate, and Water, until the effervescence ceases, filtering and evaporating. $\mathrm{Li}_{2} \mathrm{CO}_{3}$ $+2 \mathrm{HC}_{7} \mathrm{H}_{5} \mathrm{O}_{3}=2 \mathrm{LiC}_{7} \mathrm{H}_{5} \mathrm{O}_{3}+\mathrm{H}_{2} \mathrm{O}+\mathrm{CO}_{2}$.

ChARACTERS. - A white, or grayish-white powder, odorless, and having a sweetish taste; deliquescent on exposųre to air. Solubility.-Very soluble in Water and in Alcohol.

Dose, 1 to $15 \mathrm{gr}$.; .06 to $1.00 \mathrm{gm}$.]

For Bismuth Salicylas see p. 183 , and for Salol see p. $48 \mathrm{r}$. 


\section{Action of Salicin, Salicylic Acid and of Sodium [and Lithium] Salicylates.}

External.-Salicin and salicylic acid are antiseptics rather more powerful than carbolic acid. They are stimulant and mildly irritant to the skin. Locally applied, they check sweating. The salts of salicylic acid are not [strongly] antiseptic. Salicylic acid softens and removes epithelium.

Internal.-Alimentary tract. - When inhaled or applied to the throat, salicylic acid is irritating, causing sneezing and cough. In the stomach also it is irritant, giving rise to pain, nausea, and vomiting unless well diluted. The sodium [and lithium] salts and salicin are much less irritating. The glucoside, salicin, is in the bowel converted into glucose and saligenin $\left(\mathrm{C}_{7} \mathrm{H}_{8} \mathrm{O}_{2}\right)$, and this is further decomposed into salicylic acid, salicyluric acid $\left(\mathrm{HC}_{9} \mathrm{H}_{8} \mathrm{NO}_{4}\right)$, and salicylous acid $\left(\mathrm{HC}_{7} \mathrm{H}_{5} \mathrm{O}_{2}\right)$. Liver. - The bile is rendered much less viscid ; [in fact salicylic acid is probably the strongest cholagogue known]. Sodium salicylate, like sodium benzoate, increases both the amount and the solids of the bile.

Blood.-Salicylic acid, whether taken directly or formed in the bowel from the decomposition of salicin, is rapidly absorbed in spite of its insolubility, and therefore it is probably taken up as sodium salicylate; [at least], this is the form in which it circulates in the blood, and consequently the following description will apply whether salicin, salicylic acid, or sodium salicylate has been taken. It has been thought also to exist in the blood as an albuminate, but of this there is no evidence, nor for the theory that when the sodium salicylate meets with carbon [dioxide], salicylic acid is set free. Some of the salicylic acid of the sodium salt unites with glycocoll, forming salicyluric acid, which appears in the urine. Thus: $\mathrm{HC}_{7} \mathrm{H}_{5} \mathrm{O}_{3}+\mathrm{C}_{2} \mathrm{H}_{5} \mathrm{NO}_{2}$ (glycocoll) $=\mathrm{HC}_{9}$ $\mathrm{H}_{8} \mathrm{NO}_{4}$ (salicyluric acid) $+\mathrm{H}_{2} \mathrm{O}$. It will be noticed that this change is precisely analogous to the conversion of benzoic into hippuric acid by its union with glycocoll. Binz has suggested that the specific beneficial effect of the salicylates in acute rheumatism-is due to setting free of salicylic acid in the inflamed 
part by the carbon [dioxide] in it. The beneficial effect of this acid is also seen in a disease of bees known as foul brood, and due to certain schizophytes, for feeding the creatures on syrup containing salicylic acid cures them.

Heart.- Salicin and salicylic acid are often stated to depress the force of the heart and cause a fall of blood-pressure. Careful comparison shows that salicin is not nearly so depressant as the acid-in fact, it is probable that it has not this action at all, unless given in toxic doses. Further, natural salicylic acid is not so depressant as the artificial variety. For example, Charteris found that $30 \mathrm{gr}$. [2. gm.] of salicin, or ro gr. [.60 gm.] of natural salicylic acid, or $32 \mathrm{gr}$. [2.12 gm.] of natural sodium salicylate had no injurious effect on a rabbit, but that much smaller doses than these of the artificial acid or its salt killed the animal. The artificial variety was found to contain orthocreosotic and paracreosotic acids, and these are powerful cardiac depressants. Thus it seems probable that the depressing effects commonly ascribed to salicylic acid are really due to the impurities occasionally present in the artificial form.

Respiration.-Moderate doses have very little effect on respiration. Toxic doses strongly depress it.

Temperature.-In medicinal doses salicin and salicylic acid have no influence on the temperature of man, in toxic doses they slightly lower it ; but they readily depress a febrile temperature, and are therefore called antipyretics. They cause a slight increase of perspiration, but this is not sufficient to explain the fall.

Salicylic acid and salicin are antiperiodic.

Nervous system. - We know little of the effect of salicylic acid on the individual parts of the nervous system. The clinical symptoms known as salicylism will be described presently.

Kidney.-Salicylic acid escapes chiefly through the kidneys. It, to a much less extent, also leaves the body by the sweat, the saliva, the bronchial secretions, and the freces. It appears in the urine very soon after its ingestion (in from ro to 30 minutes), but the elimination goes on slowly. It is excreted as salicyluric acid and sodium salicylate, which is split up by the phosphoric 
acid in the urine, yielding salicylic acid. The dark greenish color of the urine sometimes seen is due to small quantities of either indican or pyrocatechin. Occasionally salicylic acid causes hæmaturia, due to congestion of the kidneys. Large doses increase the nitrogenous elimination, the uric acid being especially increased. The sulphur also is increased. It renders the urine aseptic, and the salicyluric acid in that fluid will reduce Fehling's Solution. The urine of patients taking it gives a purple color with [ferric] chloride.

Salicylism.-In some persons to whom salicylic acid or its salt is given a train of symptoms is produced to which the above name has been applied. They are very like those produced by quinine. It is probable that the cause of at least some of them is the impurities existing in artificial salicylic acid, but it is stated that the natural acid may rarely give rise to them. Orthocreosotic acid is certainly toxic, metacreosotic acid has no action, and it is doubtful whether paracreosotic acid is toxic. The commonest is deafness, which is often accompanied by ringing in the ears; [these symptoms may be relieved by the administration of a small amount of alcoholic stimulant fifteen minutes before each dose.] Headache is also very frequent. The administration of the drug is usually stopped when these symptoms show themselves ; but if it is continued the patient becomes violently delirious, there is nausea and vomiting, the face is flushed, and the other symptoms increase in severity. The pulse falls in both frequency and force, it becomes irregular, epistaxis is common, and hæmorrhages from other parts of the body have been recorded, such as hæmaturia and retinal hæmorrhages. Albuminuria without hæmaturia has been observed. One of the rarest symptoms is erythema or urticaria. Very large doses may cause the breathing to become weaker, and death may take place either from cessation of the heart or the respiratory movements.

\section{Therapeutics of Salicin, Salicylic Acid and of Sodium [and Lithium] Salicylates.}

External.-The ointment [official in B. P., I part of the acid to 9 of hard, and 18 of soft paraffin] may be used when an 
antiseptic stimulating ointment is required. A collodion composed of salicylic acid, I ; flexible collodion, 8 ; or a glycerin containing ro per cent. of salicylic acid; or a plaster, also ro per cent., are good preparations. Strong applications of salicylic acid are very useful for removing excess of epidermis, warts, or corns [because it softens epithelium]. Salicylic acid, I I ; extract of cannabis indica, 2 ; flexible collodion, 87 parts, form an excellent remedy (commonly known as green solution) for corns. Powdered salicylic acid mixed with starch or chalk may be employed to check profuse perspiration of the feet and axillæ. The German Pharmacopœia has for this purpose a Pulvis Salicylicus cum Talco (salicylic acid, 3 ; wheaten starch, ro; talc, in powder, 87). The sweats of phthisis may be treated in the same way. A little salicylic acid is often added to Thompson's fluid (see p. 275). [Salicylic acid is the principal ingredient in Thiersch's solution. For this formula see p. 275.]

Internal.-Salicylic acid is a specific for [many cases of] rheumatic fever; it lowers the temperature, lessens the swelling, leads to a rapid cessation of pain, and [may] diminish the liability to pericarditis and other complications. It must be given well diluted to prevent dyspepsia. The sodium salt is often preferred as being the most soluble, but in order to diminish the risk of salicylism it should be prepared either from pure artificial or from natural salicylic acid. If the attack is severe, 20 gr. [1.20 gm.] every two or three hours should be given for the first twelve or twenty-four hours; then, if the patient is doing well, the frequency of the dose may be gradually diminished, but it should be continued thrice daily for ten days after the temperature is normal and the pain has ceased. Salicin is not so powerful as sodium salicylate, but it is said to be less depressant than the synthetic acid.

These preparations are of no use for gout or severe osteoarthritis, but occasionally the pains of chronic rheumatism are relieved.

Salicylic acid or salicin may produce a fall of temperature in any fever, but, as we have more certain antipyretics, they are not used except for rheumatic fever. Some writers have found 
salicylic acid useful in migraine, sciatica, diabetes, and diphtheria, but it is probably of little value for these disorders. [The action of the salicylates in eliminating uric acid explains their usefulness in migraine and sciatica which is incontestible in some cases. So far as they limit intestinal fermentation they are beneficial in the treatment of diabetes. For the glycosuria of patients afflicted with gout or goutiness they are useful.] Salicylic acid has been given to render the urine acid in cases of alkaline urine and cystitis, but there are better remedies for this purpose. It has also been given in cases of gallstone with the object of rendering the urine less viscid.

[Aspirin (not official) is acetyl salicylic acid, which occurs as a white, insoluble, crystalline powder, or in needles, of an agreeable taste. In an alkaline fluid it breaks up and sets free salicylic acid. It has been employed for acute polyarticular rheumatism in the same doses as sodium salicylate, over which it is believed to possess the advantage of not deranging digestion.

\section{OLEUM BETULAE VOLATILE.}

VOLATILE OIL OF BETULA.-Synonym.-Oil of Sweet Birch. A volatile oil obtained by distillation from the bark of Betula lenta Linné (Sweet Birch) ; (nat. ord. Betulacea). Habitat.-Northern United States. It is identical with Methyl Salicylate $\left(\mathrm{CH}_{3} \mathrm{C}_{7} \mathrm{H}_{5} \mathrm{O}_{3}=151.64\right)$, and nearly identical with Oil of Gaultheria.

Charactres. - It has the same properties and conforms to the same reactions and tests as Methyl Salicylate (see Methyl Salicylas; also Oleum Gaultheriæ).

Dose, I to $5 \mathrm{~m}$.; .06 to .30 c.c.

\section{OLEUM GAULTHERIE.}

OIL OF GAULTHERIA.-Synonym.-Oil of Wintergreen. A volatile oil distilled from the leaves of Gaultheria procumbens Linne (Wintergreen); (nat. ord. Ericacea), consisting almost entirely of Methyl Salicylate $\left(\mathrm{CH}_{3} \mathrm{C}_{7} \mathrm{H}_{5} \mathrm{O}_{3}=151.64\right)$ and nearly identical with Volatile Oil of Betula. Habitat.-North America, west to Minnesota, and south to Georgia.

CiIARACTERS. - A colorless or yellow, or occasionally reddish liquid, having a characteristic, strongly aromatic odor, and a sweetish, warm and aromatic taste. Sp. gr. : 1.175 to 1.185 . Boiling point : $218^{\circ}$ to $22 \mathrm{r}^{\circ} \mathrm{C} .\left(424.4^{\circ}\right.$ F. to $429.8^{\circ} \mathrm{F}$.). It deviates polarized light slightly to the left. In other 
respects it has the same properties and conforms to the same reactions and tests as Methyl Salicylate (see Methyl Salicylas; also Oleum Betulæ Volatile).

Dose, I to $5 \mathrm{~m}$. : .06 to .30 c.c.

\section{Preparation.}

Spiritus Gaultheriæ.-Spirit of Gaultheria. Oil of Gaultheria, 50 ; Alcohol, 950.

Dose, I to 2 fl. dr.; 4 . to 8 .'c.c.

METHYL SALICYLAS. - ceje hut man

METHYL SALICYLATE. $-\mathrm{CH}_{3} \mathrm{C}_{7} \mathrm{H}_{5} \mathrm{O}_{3}=151.64$. Synonym.Artificial (or Synthetic) Oil of Wintergreen. Methyl Salicylate, produced synthetically by distilling Salicylic Acid, or a Salicylate, with Methyl Alcohol and strong Sulphuric Acid.

CHARACTERS. - A colorless or slightly yellowish liquid, baving the characteristic, strongly aromatic odor and the sweetish, warm and aromatic taste of the Oil of Gaultheria, with the essential constituent of which it is identical. It is wholly identical with Volatile Oil of Betula (see Oleum Betulæ Volatile). Sp. gr. : I.183-1.185. Boiling point : $219^{\circ}-221^{\circ} \mathrm{C}$. $\left(426.2^{\circ}-429.8^{\circ} \mathrm{F}\right.$.). It is optically inactive. Solubility. - In all proportions in Alcohol, Glacial Acetic Acid, or Carbon Disulphide.

Dose, I to $5 \mathrm{~m}$; .06 to .30 c.c.

\section{Action and Uses of Volatile Oil of Betula, Oil of Wintergreen and Methyl Salicylate.}

The action of these substances is the same as of salicylic acid. When taken in moderate quantities, they are, like that acid, broken up and eliminated as salicyluric acid. It is asserted that methyl salicylate can be produced of more uniform quality and is more certain and definite in its action than either of the natural oils. The uses of these drugs is the same as of salicylic acid, and they possess the additional advantage that they are not liable to contamination with impurities (ortho- and paracreosotic acids), the former of which is a powerful cardiac depressant; both are found in the artificial salicylic acid.]

\section{SALOL.}

SALOL. $-\left[\mathrm{C}_{6} \mathrm{H}_{5} \mathrm{C}_{7} \mathrm{H}_{5} \mathrm{O}_{3}=213.49\right.$. Synony'n. - Phenyl Salicylate. The Salicylic Ether of Phenol.

Source. - By heating Salicylic Acid with Phenol in the presence of Phosphorous Pentachloride; this action dehydrates and withdraws the elements of water, and unites the Phenyl group with the Salicylic Acid radical. 
ChARACTERs. - A white, crystalline powder, odorless, or having a faintly aromatic odor, and almost tasteless. Permanent in the air. Solubility. Almost insoluble in water; soluble in ro parts of Alcohol ; also soluble in 0.3 part of Ether, and readily in Chloroform, and in fixed or volatile oils.

- Dose, 5 to $30 \mathrm{gr}$; .30 to $2.00 \mathrm{gm}$.]

\section{Action and Therapeutics of Salol.}

External.-It is an antiseptic, and when mixed with talc (I to 5) may be used as a dusting powder.

Internal.-In the body [presumably in the small intestine) salol splits up into [phenol, about 36, and salicylic acid, 64 per cent.] and the former may render the urine dark. In rheumatic fever it is efficacious on account of the salicylic acid it contains, [although somewhat slower in action] but it has no advantage over salicin or salicylic acid, and the carboluria may be troublesome.

[It is an antiseptic, and since this decomposition takes place in the alkaline fluid, it has been used as an intestinal antiseptic in acute diarrhœa, dysentery, cholera and other diseases; also in affections of the urinary tract.] For the last the following is a good way of prescribing it:-Salol, I; Almond Oil, 2 ; Powdered Acacia, I; Syrup, 2; water, 24. The emulsion should be made in a warm mortar with water at $150^{\circ} \mathrm{F}$. $\left[65.5^{\circ} \mathrm{C}\right.$. It is a remedy of very great value in the treatment of typhoid fever, for by the active disinfection of the contents of the intestine and of the ulcerations, it favors their healing and prevents reinfection, thus lowering temperature, diminishing the liability to relapse and to permanent damage to tissues. It is the logical treatment, because it destroys the cause of the symptoms, at their point of origin. On account of the large proportion of phenol which it contains it is more dangerous than the corresponding amount of salicylic acid, and it is especially to be used. with great caution if the kidneys are diseased. Sometimes, in fever, on account of the lessened alkalinity of the intestinal contents it is not decomposed into its constituents, and for that reason becomes very much less effective. In this case an alkali should be administered at the same time. 


\section{SALOPHEN.}

SALOPHEN. (Not official.) $-\mathrm{C}_{6} \mathrm{H}_{4}(\mathrm{OH}) \mathrm{CO}_{2} \mathrm{C}_{6} \mathrm{H}_{4} \mathrm{NHCOCH}_{3}=270.40$. Synonym.-Acetylparamidophenol Salicylate.

SOURCE. - From Paranitrophenol Salicylate by a complicated process, and purifying by crystallization from Alcohol.

Characters. - Minute white scales, free from odor or taste. It contains 50.9 per cent. of Salicylic Acid. Solubility.-Almost insoluble in water; freely soluble in Alkalies, Alcohol and Ether.

Dose, 5 to $15 \mathrm{gr}$; . 30 to $1.00 \mathrm{gm}$.

\section{Action and Uses of Salophen.}

In a warm alkaline solution salophen is broken up into salicylic acid and acetylparamidophenol, the latter being harmless. It is decomposed in the intestines, even when given hypodermatically. It is used as a substitute for salicylic acid in acute rheumatism, and as an intestinal antiseptic. It is probably quite as efficient, and much safer than salol. The fact that it is tasteless renders it easy of administration.]

\section{GROUP V.}

\section{Vegetable Purgatives.}

Class I. - Laxatives.

Prune, Fig, Tamarind, Cassia [Fistula, Manna, Phytolacca, Eupatorium,] Castor Oil (small doses).

Class II.--Simple purgatives.

Castor Oil, Rhubarb, [Juglans,] Senna, Frangula, [Rhamnus Purshiana,] Aloes.

Class III._Drastic purgatives.

Scammony, Jalap, [Bryonia,] Croton Oil, Colocynth, Elaterium, Gamboge.

Class IV.-Cholagogues.

Podophyllum, [Leptandra, Chelidonium, Iris,] Euonymus.

$$
\text { Class I.-LAXATIVES. }
$$

\section{PRUNUM.}

PRUNE.-[The fruit of Prunus Domestica Linné (nat. ord. Rosacea). Habitat.-Western Asia; cultivated in many varieties. 
Characters. -Oblong or subglobular, about $3 \mathrm{~cm}$. long, shrivelled blackish-blue, glaucous ; the sarcocarp brownish-yellow ; sweet and acidulous ; putamen hard, smooth, or irregularly ridged; the seed almond-like in shape, but smaller, and of a bitter-almond taste.]

Composition. - The chief constituents are-(I) Sugar, [ 12 to 15 per cent.], (2) Malic Acid, and (3) A purgative principle.

Prunes are contained in Confectio Sennæ.

[Dose. - They can be administered freely.]

\section{Action and Therapeutics of Prunes.}

Prunes are demulcent and slightly laxative. They may be eaten as articles of diet in cases of slight constipation. [They are used as a corrective for senna in the confection.]

\section{FICUS.}

FIG.-[The fleshy receptacle of Ficus Carica Linné (nat. ord. Urticaced), bearing fruit upon its inner surface. Habitat.-Western Asia; cultivated in subtropical countries.

Characters. - Compressed, of irregular shape, fleshy, brownish or yellowish, covered with an efflorescence of sugar ; of a sweet, frui y odor, and a very sweet, mucilaginous taste. When softened in water, figs are pearshaped, with a scar or short stalk at the base, and a small scaly orifice at the apex; hollow internally; the inner surface covered with numerous yellowish, hard achenes.]

Composition.-The chief constituents are-(I) Sugar, 62 per cent.; (2) Gum ; (3) [Fat and Salts.]

Figs are contained in Confectio Sennæ.

[Dose.-They can be administered freely.]

\section{Action and Therapeutics of Figs.}

Figs are a pleasant food and mildly purgative, forming a convenient remedy for slight constipation.

\section{TAMARINDUS.}

TAMARIND.-[The preserved pulp of the fruit of Tamarindus indica Linné (nat. ord. Leguminosa). Habitat.-India and tropical Africa; naturalized in the West Indies.

CharActers. - A reddish-brown, sweet, subacid, pulpy mass, containing strong, somewhat branching fibres, and polished, brown, flattish-quadrangular seeds, each enclosed in a tough membrane; taste sweet and refreshingly acidulous.] 
IMPURITY.-Copper.

Composition.-(I) Tartaric, [Citric, Malic and Acetic Acids; (2) Sugar;

(3) Pectin ; (4) Tannic acid and (5) Potassium compounds.]

Tamarind is contained in Confectio Sennx.

[Dose.-It can be administered freely.]

Action and Therapeutics of Tamarind.

Tamarind is pleasant and acid to the taste, and a mild laxative. It may be made into tamarind whey ( $I$ part of tamarind to 30 of milk) and given as an acid, cooling, slightly purgative drink in fevers. It is a good purgative for children, and may be spread on bread and butter.

\section{CASSIA [FISTULA].}

CASSIA [FISTULA.-Synonym.-Purging Cassia. The fruit of Cassia Fistula Linné (nat. ord. Leguminosa). Habitat.-East India ; naturalized in tropical Africa and America.

Characters. - Cylindrical, 40 to $60 \mathrm{~cm}$. long, nearly $25 \mathrm{~mm}$. in diameter, blackish-brown, somewhat veined, the sutures smooth, forming two longitudinal bands; indehiscent, internally divided transversely into numerous cells, each containing a reddish-brown, glossy, flattish-ovate seed imbedded in a blackish-brown, sweet pulp; odor resembling that of prunes.]

Composition.-The chief constituents are-(I) A purgative principle, closely allied to Cathartic Acid. (See Senna, p. 492.) [(2) Sennapicrin, a Glucoside, $\mathrm{C}_{34} \mathrm{H}_{58} \mathrm{O}_{17 .}$ ] (3) Sugar, 60 per cent. (4) [Pectin. (5) Calcium Oxalate.]

Cassia Fistula is contained in Confectio Sennæ.

[Dose, I to $8 \mathrm{dr}$; 4 . to 3o. gm.]

\section{Action and Therapeutics of Cassia Fistula.}

[The pulp is administered as] a laxative, given only in confection of senna.

\section{[MANNA.}

MANNA.-The concrete, saccharine exudation of Fraxinus Ornus Linnê (nat. ord. Oleacee). Habitat.-Basin of the Mediterranean.

Characters. - In flattish, somewhat three-edged pieces, occasionally 20 $\mathrm{cm}$. long, and $5 \mathrm{~cm}$. broad, usually smaller; friable; externally yellowishwhite, internally white, porous and crystalline; or in fragments of different sizes, brownish-white and somewhat glutinous on the surface, internally white and crystalline: odor honey-like ; taste sweet, slightly bitter and faintly acrid. 
Composition. - The chief constituents are--(1) Mannit, $\mathrm{C}_{6} \mathrm{H}_{8}(\mathrm{OH})_{6}, 90$ per cent. (2) Glucose. (3) Fraxin, $\mathrm{C}_{32} \mathrm{H}_{36} \mathrm{O}_{20}$. (4) Mucilage. (5) Resin.

Manna is contained in Infusum Sennæ Compositum.

Dose, $1 / 2$ to 2 oz.; 15 . to 60 . gm.

\section{Action and Therapeutics of Manna.}

Manna is given as a mild laxative to children. It dissolves easily in milk, and is pleasant to the taste.

\section{PHYTOLACCA.}

PHYTOLACCA FRUCTUS.-Phytolacca Fruit. Synonym.-Poke Berry. The fruit of Phytolacca decandra Linné (nat. ord. Phytolaccacea.) Habitat.-North America; naturalized in Europe.

Characters. - A depressed globular, dark purple compound berry, about $8 \mathrm{~mm}$. in diameter, composed of ten carpels, each containing one lenticular, black seed; juice purplish red; inodorous; taste sweet, slightly acrid.

Constituents. - (I) Phytolaccin. (2) Phytolaccic Acid. (3) Sugar. (4) Gum.

Dose, 5 to $30 \mathrm{gr}$; .30 to $2.00 \mathrm{gm}$.

PHYTOLACC E RADIX.-Phytolacca Root. Synonym.-Poke Root. The root of Phytolacca decandra Linné (nat. ord. Phytolaccacea).

Ciraracters. - Large, conical, branched and fleshy; mostly in transverse or longitudinal slices, wrinkled, grayish, hard ; fracture fibrous, the woodbundles in several distinct, concentric circles; inodorous; ' taste sweetish and acrid.

Constituents. - (1) Resin. (2) Probably a Glucoside. (3) A volatile acid.

Dose, I to $5 \mathrm{gr}$; ; .06 to $.30 \mathrm{gm}$.

\section{Preparation.}

Extractum Phytolaccæ Radicis Fluidum.-Fluid Extràct of Phytolacca Root. By maceration and percolation with Alcohol and water, and evaporation.

Dose, 5 to $30 \mathrm{~m}$.; .30 to 2.00 c.c.

\section{Action and Uses of Phytolacca.}

Phytolacca is used as a laxative, and in large doses it possesses also some narcotic properties. Recently a preparation made from the berries has been used to reduce adipose tissue. 


\section{EUPATORIUM.}

EUPATORIUM.-Synonyms. - Thoroughwort. Boneset. The leaves and flowering tops of Eupatorium perfoliatum Linné (nat. ord. Compositce). Habitat. - North America, west to Dakota ; in low grounds.

Characters. - Leaves opposite, united at the base, lanceolate, from to to I $5 \mathrm{~cm}$. long, tapering, crenately serrate, rugosely veined, rough above, downy and resinous dotted beneath; flower heads corymbed, numerous, with an oblong involucre of lance-linear scales, and with from ten to fifteen white florets, having a bristly pappus in a single row; odor weak and aromatic; taste astringent and bitter.

Composition.-Its principal constituents are - (I) Eupatorin, a bitter glucoside. (2) Volatile Oil. (3) Resin.

Dose, $1 / 4$ to I dr.; $\mathrm{r}$. to 4 . $\mathrm{gm}$.

\section{Preparation.}

Extractum Eupatorii Fluidum.-Fluid Extract of Eupatorium. By maceration and percolation with diluted Alcohol, and evaporation.

Dose, $1 / 4$ to I fl. dr.; r. to 4 . c.c.

\section{Action AND Uses of Thoroughwort.}

Thoroughwort is tonic, diaphoretic (in infusion), and mildly laxative. It is a domestic remedy for the commencement of a catarrh, influenza, or muscular rheumatism.]

\section{Class II.-SIMPLE PURGatives.}

\section{OLEUM RICINI.}

CASTOR OIL.- - A fixed oil expressed from the seed of Ricinus communis Linné (nat. ord. Euphorbiacece). Habitat.-India; cultivated.

ChARACTERS. - A pale yellowish or almost colorless, transparent, viscid liquid, having a faint, mild odor, and a bland, afterwards slightly acrid, and generally offensive taste. Sp. gr., 0.950 to 0.970 . Solubility.-In an equal volume of Alcohol, and, in all proportions, in absolute Alcohol, or in glacial Acetic Acid; also soluble in 3 times its volume of a mixture of 19 volumes of Alcohol and I volume of water (absence of more than about 5 per cent. of most other fixed oils).

Compositron. - The chief constituents are-(I) Ricinolein, $\mathrm{C}_{3} \mathrm{H}_{5}\left(\mathrm{C}_{18} \mathrm{H}_{34}\right.$ $\left.\mathrm{O}_{3}\right)_{3}$, which is the Ricinoleic Acid $\left(\mathrm{C}_{18} \mathrm{H}_{34} \mathrm{O}_{3}\right)$, Glyceride.] This constitutes the chief bulk. (2) Other fixed oils, as palmitin, stearin, etc. (3) Possibly an alkaloid, Ricinine, not purgative. (4) According to some authorities an active principle which has not yet been isolated.

[Castor oil is contained in Collodium Flexile, Linimentum Sinapis Compositum, Pilulæ Antimonii Compositæ and Unguentum Hydrargyri Oxidi Rubri.

Dose, $1 / 4$ to 2 fl. oz. ; 8. to 60 . c.c.] 
Castor oil seeds are not official, but it is important to recognize them. They are $[17 \mathrm{~mm}$.] long and $[8 \mathrm{~mm}$.] wide, ovoid, flattened. The seed is prolonged into a sharp beak. Epidermis shiny gray, marked by brownish bands and spots. Kernel white. They contain 50 per cent. of the oil, and an acrid substance which makes them poisonous. Three Castor oil seeds have been known to kill an adult man. [Quite likely the seeds are not poisonous when matured.]

\section{Action of Castor Oil.}

External.-Castor oil is, like olive oil, protective and sedative, and may be used to drop into the eye when the conjunctiva is inflamed, and as a solvent for homatropine, but this solution is occasionally a little irritating.

Internal.-Gastro-intestinal tract.-The so-called [unpleasant taste] of castor oil is mostly due to the smell, and is not noticed much if the nose is held when the oil is drunk. Medicinal doses produce no effect on the stomach. Reaching the intestine the oil is an excellent simple laxative or mild purgative, acting in about five hours, and causing no griping nor subsequent constipation. The motion is soft, but not liquid. Castor oil will purge even when rubbed into the skin. How it acts is unknown [but it is said to stimulate unstriped muscular fibre whenever found.] It has been thought that the [ricinolein (ricinoleic acid glyceride)] in the oil is decomposed in the duodenum, and the ricinoleic acid purges, but this is probably incorrect. The most likely view is that the oil contains some purgative principle which has not yet been isolated. Probably the seeds contain much more of this than the oil, for they are ten times more purgative, a fact which it is impossible to explain if it is the ricinoleic acid which purges. Castor oil will purge when given per rectum.

Mammary glands. - [The leaves] applied locally to the breasts are said to be galactagogue.

\section{Therapeutics of Castor Oil.}

Castor oil is perhaps the best simple purgative we have, and is very useful in cases in which there is slight temporary constipation. Being mild in its action it is very suitable for getting rid of undigested food that is causing diarrhœa, and a dose of 
castor oil with a minute quantity of laudanum in it is a favorite remedy for certain forms of diarrhœa. It is also especially convenient in pregnancy, after delivery, and when in any abdominal disease, as typhoid fever, peritonitis, or when, after abdominal operations, the irritation caused by the fæces makes it absolutely necessary to get the bowels open. Also, it is very useful for children, or for very old or infirm persons, or for those suffering from piles or fissures. It is a good purgative to give before and after the use of anthelmintics [aspidium being excepted. It should not be given during the later months of pregnancy owing to its action on unstriped muscle fibres.]

Its [nauseous taste] is the only objection to it. As already mentioned, this can largely be overcome by holding the nose, and there are many forms of castor oil in the market so prepared as to be almost colorless and odorless. It may be taken in capsules, but they are bulky. Lemon juice or coffee conceals the taste to some extent, or the oil may be added to a teaspoonful [4. c.c.] of peppermint water, and then a little brandy added till the oil neither sinks nor [floats]. If the inside-and rim of the glass are moistened with the vehicle, the oil, which should, if possible, be between two layers of the vehicle, is hardly tasted. [It is best administered in the beverage known as sarsaparilla.]

As an enema (castor oil, I ; warm olive oil, 5 ; mixed thorroughly) it is useful when a mild injection is required.

Breasts. - The leaves of the castor-oil plant applied to the breasts will sometimes induce the secretion of milk. A fluid extract of them [not official] may also be taken three or four times a day.

\section{RHEUM.}

RHUBARB. - [The root of Rheum officinale Baillon (nat. ord. Polygonacea). Habitat.-Western and Central China.

ChARACTERS. - In cylindrical, conical or flattish segments, deprived of the dark brown, corky layer, smoothish or somewhat wrinkled, externally covered with a bright yellowish-brown powder, marked with white, elongated meshes, containing a white, rather spongy tissue, and a number of short reddish-brown or brownish-yellow striæ ; compact, hard ; fracture uneven; internally white, with numerous red, irregularly curved and interrupted medullary rays, which are radially parallel only near the cambium line; odor somewhat jeculiar. aromatic; taste bitter, somewhat astringent. 
Compusition.-The chief constituents are-(I) Chrysophan, $\mathrm{C}_{27} \mathrm{H}_{30} \mathrm{O}_{14}$, which yields Chrysophanic Acid, $\mathrm{C}_{15} \mathrm{H}_{10} \mathrm{O}_{4}$, about 3 per cent. Synonyms.Rhein. Chrysarobin, see Chrysarobinum.] It is not known whether, [in the living plant], Rhubarb contains any Chrysophanic Acid, for when kept the Chrysophan quickly oxidizes to Chrysophanic Acid. The purgative properties are due to Ghrysophan which also gives the yellow color. (2) [Erythroretin. (3) Emodin. (4) Phaoretin. (5) Aporetin.] (6) Rheotannic Acid, $\mathrm{C}_{26} \mathrm{H}_{26} \mathrm{O}_{14}$, to which the astringency of Rhubarb is due. (7) Lime Oxalate, 35 per cent., to which the grittiness is due.

IMPURITIES.-English Rhubarb; different taste, smell, and excess of starch. Turmeric, which is turned brown by Boric Acid.

Dose, I to $5 \mathrm{gr}$.; [.06 to .30 gm. (stomachic); 10 to $30 \mathrm{gr}$.; .60 to 2.00 gm.] (purgative).

\section{Preparations.}

I. Extractum Rhei.-[Extract of Rhubarb. By percolation with Alcohol and Water, and evaporation.

Dose, 3 to $5 \mathrm{gr}$; . 20 to $1.00 \mathrm{gm}$.

2. Extractum Rhei Fluidum.-Fluid Extract of Rhubarb. By maceration and percolation with Alcohol and Water, and evaporation. Fluid Extract of Rhubarb is used to make Mistura Rhei et Sodæ and Syrupus Rhei.

Dóse, $1 / 4$ to I f. dr.; I. to 4 . c.c.

3. Pilulæ Rhei.-Pills of Rhubarb. Rhubarb, 20 ; Soap, 6 gm., to make 100 pills. Each pill contains $3 \mathrm{gr}$; . $20 \mathrm{gm}$. of Rhubarb.

Dose, 3 to 5 pills.

4. Pilulæ Rhei Compositæ.-Compound Pills of Rhubarb. Rhubarb, 13 ; Purified Aloes, Io; Myrrh, $6 \mathrm{gm}$. ; Oil of Peppermint, 0.5 c.c., to make 100 pills. Each pill contains $2 \mathrm{gr}$.; . $13 \mathrm{gm}$. of Rhubarb.

Dose, I to 3 pills.

5. Pulvis Rhei Compositus.-Compound Powder of Rhubarb. Synonym.-Gregory's powder. Rhubarb, 25 ; Magnesia, 65; Ginger, 10.

Dose, $1 / 2$ to I dr. ; 2. to $4 . \mathrm{gm}$.

6. Tinctura Rhei.-Tincture of Rhubarb. Rhubarb, 100; Cardamom, 20; Glycerin, 100; Alcohol and Water to 1000; by macera. tion and percolation.

Dose, $1 / 2$ to I fi.dr.; 2. to 4 . c.c. (stomachic); I to 4 fl. dr.; 4 . to 15. c.c. (purgative).

7. Tinctura Rhei Aromatica.-Aromatic Tincture of Rhubarb. Rhubarb, 200; Cassia Cinnamon, 40; Cloves, 40; Nutmeg, 20; Glycerin, I00; Diluted Alcohol, to I000. By maceration and percolation. 
Aromatic Tincture of Rhubarb is used to make Syrupus Rhei Aromaticus.

Dose, I to 3 fl. dr.; 4. to I2. c.c.

8. Tinctura Rhei Dulcis.-Sweet Tincture of Rhubarb. Rhubarb, 100; Glycyrrhiza, 40 ; Anise, 40 ; Cardamom, 10 ; Glycerin, 100 ; Diluted Alcohol to I000. By maceration and percolation.

Dose, $1 / 2$ to I fl. oz.; 15 . to 30 . c.c.

9. Mistura Rhei et Sodæ.-Mixture of Rhubarb and Soda. Sodium Bicarbonate, 35; Fluid Extract of Rhubarb, 15; Fluid Extract of Ipecac, 3 ; Spirit of Peppermint, 25 ; Glycerin, $35^{\circ}$; Water to 1000. By solution.

DoSe, 1/4 to 2 fl. oz.; 8. to 6o. c.c.

ro. Syrupus Rhei.-Syrup of Rhubarb. Fluid Extract of Rhubarb, 100 ; Potassium Carbonate, 10 ; Spirit of Cinnamon, 4 ; Glycerin, $5^{\circ}$; Water and Syrup to 1000 . By solution.

Dose, I to 4 fl. dr. ; 4 . to 15 . c.c.

II. Syrupus Rhei Aromaticus.-Aromatic Syrup of Rhubarb. Aromatic Tincture of Rhubarb, 150; Syrup, 850. By mixture.

Dose, I fl. dr.; 4. c.c. for a child.]

\section{Action of Rhubarb.}

External.-Probably rhubarb would have, to a mild degree, the same action as [chrysarobin], but it is never applied externally.

Internal.-Alimentary canal. - In the mouth, rhubarb increases the flow of saliva; and in the stomach, in small doses, it, like any other bitter substance, stimulates the flow of gastric juice, and the vascularity and peristaltic movements of the stomach. It is, therefore, a stomachic, and will aid digestion. In large doses it causes purgation, producing in from four to eight hours a liquid motion, colored yellow by the [chrysarobin]. The resinous constituents of rhubarb are said to increase the flow of bile, but certainly its cholagogue action is not sufficiently. powerful to explain completely its purgative properties. It is commonly stated to exaggerate very actively intestinal peristalsis, but there is no adequate proof of this. It is liable to gripe. The purgation is followed by constipation; this is ascribed to the rheotannic acid : if so, it is probably absorbed and subsequently 
re-excreted into the intestines, otherwise it would be all swept away in the purging.

Kidneys. - The coloring matter is excreted in the urine, and stains it yellow. The urinary flow is slightly increased.

\section{Therapeutics of Rhubarb.}

Rhubarb is commonly given to children as a stomachic purgative in indigestion, especially when caused by errors of diet, for it clears away any undigested food, and its stomachic and after-astringent effects are valuable. In the same way, it is useful in diarrhcea due to irritation caused by undigested food; here the after-astringency is especially serviceable. A powder of powdered rhubarb and sodium bicarbonate (which [conceals] the taste) equal parts, with some powdered gentian, or a similar [liquid] medicine, forms an excellant stomachic for young children. Rhubarb should never be given alone, because of the griping it causes.

\section{[JUGLANS.}

JUGLANS. - Synonym.-Butternut. The bark of the root of Juglans cinerea Linne (nat. ord. Juglandacere), collected in autumn. Habitat.-North America.

Characters. - In flat or curved pieces, about $5 \mathrm{~mm}$. thick; the outer surface dark gray and nearly smooth, or deprived of the soft cork and deep brown; the inner surface smooth and striate; transverse fracture short, delicately checkered, whitish and brown ; odor, feeble ; taste bitter and somewhat acrid.

Composirion.-(1) Nucin, $\mathrm{C}_{10} \mathrm{H}_{6} \mathrm{O}_{8} . \quad$ (2) Juglandic Acid. (3) Fixed Oil, 4 per cent.

Dose, I to $2 \mathrm{dr}$; ; 4 . to $8 . \mathrm{gm}$.

\section{Preparation.}

Extractum Juglandis.-Extract of Juglans. By maceration and percolation with Diluted Alcohol, and evaporation.

Dose, 5 to $30 \mathrm{gr}$; ; 30 to $2.00 \mathrm{gm}$.

\section{Action and Uses of Butternut.}

Butternut is a mild cathartic, and resembling rhubarb in the property of evacuating, without debilitating, the alimentary canal. It was much employed during the war of the Revolution.] 


\section{SENNA.}

SENNA.-[The leaflets of Cassia acutifolia Delile (Alexandria Senna), and of Cassia angustifolia Vahn (India Senna); (nat. ord. Leguminosa).

Characters. - Alexandria Senna consists of leaflets about $25 \mathrm{~mm}$. long and $10 \mathrm{~mm}$. broad, lanceolate, or lance-oval, subcoriaceous, brittle, rather pointed, equally oblique at the base, entire, grayish-green, somewhat pubescent, of a peculiar odor, and a nauseous, bitter taste. Habitat._Eastern and Central Africa.]

Resembling Senna.-Argel leaves (the leaves of Solenostemma Argel [Hayne, (nat. ord. Asclepiadea), which are frequently present; these leaves are thicker, one-veined, wrinkled, glaucous] and are equal at the base, as also are the leaves of Uva Ursi and Buchu.

[India Senna.-Synonym.-Tinnivelly Senna. Consists of leaflets from 3 to $5 \mathrm{~cm}$. long and Io to $15 \mathrm{~mm}$. broad; lanceolate, acute, unequally oblique at the base, entire, thin, yellowish green or dull green, nearly smooth ; odor peculiar, somewhat tea-like; taste mucilaginous, bitter and nauseous. Habitat. -Eastern Africa to India ; cultivated.

IMPURITIES. - Stalks, discolored leaves, and other admixtures.]

Composition of Bотн Kinds. - The chief constituents are-(I) Cathartic Acid, $\mathrm{C}_{180} \mathrm{H}_{192} \mathrm{~N}_{82} \mathrm{SO}_{2}$, an amorphous sulphurated Glucoside. It exists as salts of earthy bases, such as Calcium and Magnesium, which are soluble in water. Cathartic Acid is capable of decomposition into Glucose and Cathartogenic Acid. It is the chief purgative principle in Senna and other purgatives. (2) [Sennacrol and Sennapicrin, $\mathrm{C}_{34} \mathrm{H}_{58} \mathrm{O}_{17}$, ] glucosides, which do not, in most preparations, contribute to their action, as they are insoluble in water. (3) Chrysophanic Acid in small amounts as a coloring matter (see Rhubarb and Chrysarobinum). (4) Cathartomannit, $\left[\mathrm{C}_{21} \mathrm{H}_{44} \mathrm{O}_{19}\right.$, a peculiar unfermentable] sugar.

Dose, I to $3 \mathrm{dr}$.; [4. to 12. gm.]

\section{Preparations.}

1. Confectio Sennæ.-[Confection of Senna. Senna, 100; Oil of Coriander, 5; Fig, I20; Tamarind, I00 ; Cassia Fistula, 160; Prune, 70 ; Sugar, 555 ; Water to make 1000. By sifting, digestion and evaporation.

Dose, I to $2 \mathrm{dr}$. ; 4 . to $8 . \mathrm{gm}$.

2. Extractum Sennæe Fluidum.-Fluid Extract of Senna: By maceration, percolation with Diluted Alcohol, and evaporation.

Fluid Extract of Senna is used to prepare Syrupus Sarsaparillæ Compositus.

Dose, I to 3 fl. dr.; 4 . to I2. c.c.

3. Infusum Sennz Compositum.-Compound Infusion ut Senna. Synonym.-Black Draught. Senna, 60; Manna, 120; Mag- 
nesium Sulphate, I20; Fennel, 20; Boiling Water, 500; Cold Water to 1000 .

Dose, I to 2 fl. oz.; 30. to 6o. c.c.

4. Pulvis Glycyrrhizæ Compositus.-Compound Powder of Glycyrrhiza. Synonym. - Compound Liquorice Powder. Senna, 180; Glycyrrhiza, 236; Oil of Fennel, 4; Washed Sulphur, 80; Sugar, 500.

Dose, $1 / 2$ to $2 \mathrm{dr}$; 2. to $8 . \mathrm{gm}$.

5. Syrupus Sennæ.-Syrup of Senna. Alexandria Senna, 25; Oil of Coriander, 5; Sugar, 700; Alcohol, 150; Water to 1000 . By digestion, straining, evaporation and filtration.

Dose, 1/4 to 1 fl. oz.; 8. to 3o. c.c.]

\section{Action of Senna.}

External.-None.

Internal.--Senna, because of the cathartic acid in it, stimulates the muscular coat of the intestine, especially the colon, and produces some hyperæmia. Consequently the fluid contents of the small intestine are hurried through the colon, and pale yellow watery stools, containing some undigested food, are the result. Senna acts very feebly or not at all on the biliary secretion. Large doses open the bowels several times and produce griping, but not much hyperæmia. Probably there are other substances in senna, besides cathartic acid, having a purgative property, but it is by far the most important. Purgation by senna does not subsequently cause constipation. Some constituents of it are absorbed, and may cause the urine to be red. It will purge, if injected into the veins, and will impart its purgative properties to the milk of nursing women.

\section{Therapeutics of SenNa.}

Senna is a safe, useful purgative for cases of simple constipation. It is, because of its tendency to gripe and its nauseous taste, rarely.given alone. The compound liquorice powder is to be preferred to the compound infusion of senna ("black draught" ), as this is a [disagreeable] mixture. Senna is largely used to complete the effect of duodenal purgatives, as we see in the old prescription of a blue pill at night and a black draught 
in the morning. Acting on the colon, it is valuable in slight cases of frecal collection. Compound liquorice powder is much used in habitual constipation and the constipation of pregnancy. Confection of senna, coated with chocolate, forms the wellknown purgative, Tamar Indien, and in this form can be taken by children. It is said that an infusion contains more of the active principles than the other preparations; it soon decomposes, but nitre [ $\mathrm{I}$ to 480 ] will prevent this.

\section{FRANGULA.}

[FRANGULA.-Synonym.-Buckthorn. The bark of Rhamnus Frangula Linné (nat. ord. Rhamneœ), collected at least one year before being used. Habitat.-Europe and Northern Asia.

Characters. -Quilled, about I mm. thick; outer surface grayish-brown, or blackish-brown, with numerous small, whitish, transversely elongated lenticels; inner surface smooth, pale, brownish-yellow ; fracture in the outer layer short, of a purplish tint; in the inner layer fibrous and pale yellow; when masticated, coloring the saliva yellow; nearly inodorous; taste sweetish and bitter.

Composition. - Fresh bark contains a glucoside, Frangulin, $\mathrm{C}_{20} \mathrm{H}_{20} \mathrm{O}_{10}$. This in the old bark has become converted into Emodin, $\mathrm{C}_{15} \mathrm{H}_{10} \mathrm{O}_{5}$ (also found in Rhubarb), to which the value of the bark is due. Two products are obtained from Frangulin by hydrolysis, Emodin and Rhamnose, $\mathrm{C}_{6} \mathrm{H}_{12} \mathrm{O}_{5}$.

Dose, $1 / 2$ to I dr.; 2. to $4 . \mathrm{gm}$.

\section{Preparation.}

Extractum Frangulæ Fluidum.-Fluid Extract of Frangula. By maceration and percolation with Alcohol and Water, and evaporation.

Dose, $1 / 2$ to 2 fl. dr.; 2. to 8 . c.c.

\section{Action and Therapeutics of Frangula.}

The fresh bark is a violent gastro-intestinal irritant, but that which has been kept a year is a mild laxative, acting like senna; it is suitable for children, and for use in chronic constipation.

\section{RHAMNUS PURSHIANA.]}

CASCARA SAGRADA.-Synonyms.-Sacred Bark. [Chittem Bark. The bark of Rhamnus Purshiana De Candolle (nat. ord. Khamnea). Habitat.-Northern Idaho, and westward to the Pacific Coast.

Characters. - In quills or curved pieces, about 3 to $10 \mathrm{~cm}$. long, and about $2 \mathrm{~mm}$. thick; outer surface brownish gray and whitish; the young bark having numerous, rather broad, pale-colored warts; inner surface yellowish to 
light brownish, becoming dark brown by age ; smooth or finely striate ; fracture short, yellowish, in the inner layer of thick bark somewhat fibrous; inodorous; taste bitter.]

Composition. - The chief constituents are-(1) Cascarin, [a glucoside said to be identical with Frangulin (see above). (2) Three resins. (3) Acids. (4) A volatile oil. The fresh bark causes much griping, but this unpleasant effect is lost if the bark is kept and properly cured.]

Dose, $1 / 4$ to $1 \mathrm{dr}$.; [1. to $4 . \mathrm{gm}$.]

\section{Preparation.}

Extractum [Rhamni Purshianæ Fluidum.-Fluid Extract of Rhamnus Purshiana. By maceration and percolation with Diluted Alcohol, and evaporation.

Dose, $1 / 4$ to I fl. dr.; x. to 4 . c.c.]

\section{Action and Therapeutics of Cascara Sagrada.}

Cascara Sagrada is a simple laxative and aperient, not causing much griping, and resembling in its action [buckthorn], but it is more certain and more active. The bitter principle gives it stomachic properties. It is very serviceable for constipation, especially if chronic. A single dose may either be taken in the evening, or ro to $\mathrm{x} 5 \mathrm{minims} ; .60$ to $\mathrm{r} .00 \mathrm{c.c}$., of the fluid extract may be given three times a day before meals. One advantage of its use is that gradually increasing doses are not required. The [fluid] extract is very bitter ; this taste may be concealed by aromatics, liquorice or sal volatile, and it may be given in chloroform water. The aromatic syrup [B. P.] conceals the taste very well. [This consists of the fluid extract, 8 ; tincture of orange, 2 ; alcohol, I; cinnamon water, 3 ; syrup, 6.] A preparation known as Tinctura Laxativa, dose 20 to $60 \mathrm{~m}$. [1.20 to 4.00 c.c.] (Extractum [Rhamni Purshianæ Fluidum], Spiritus Ammoniæ Aromaticus, Spiritus Chloroformi, Tinctura Belladonnæ [Foliorum], Tinctura Nucis Vomicæ, equal parts of each), is miscible with water, and is a pleasant simple purge which is especially useful for chronic constipation and may cure it.

\section{ALOE.}

ALOE BARBADENSIS.-Barbadoes Aloes. [Synonym.-Curacoa Aloes. The inspissated juice of the leaves of Aloe vera (Linné) Webb (nat. ord. Liliacea). Habitat. - Island of Barbadoes. 
CHARACTERS. - In hard masses, orange-brown, opaque, translucent on the edges; fracture waxy or resinous, somewhat conchoidal; odor saffron-like ; taste strongly bitter.

Dose, $1 / 2$ to ro $\mathrm{gr} . ; .03$ to $.60 \mathrm{gm}$.]

ALOE SOCOTRINA.-Socotrine Aloes. [The inspissated juice of the leaves of Aloe Perryi Baker (nat. ord. Liliacea). Habitat.-Eastern Africa.

CHARACTERs. - In hard masses, occasionally soft in the interior, opaque, yellowish-brown, orange-brown or dark ruby-red, not greenish, translucent on the edges ; fracture resinous, somewhat conchoidal. When breathed upon, it emits a fragrant saffron-like odor. Taste peculiar, strongly bitter. Solubility. -Almost entirely soluble in Alcohol and in 4 parts of boiling water. The aqueous solution becomes turbid on cooling and yields a deposit. Moistened with Alcohol and examined in a thin stratum under the microscope, Socotrine Aloes exhibits numerous crystals. Socotrine Aloes is less deep in color, less opaque, its powder is brighter and more reddish, and its odor is not nearly so disagreeable as that of Barbadoes Aloes. Some specimens of Socotrine Aloes are more or less opaque and liver-colored, and then are called Hepatic Aloes.] The variety known as Zanzibar Aloes is included under Socotrine Aloes.

Composition. - (I) Aloin, see p. 498. (2) A Resin. (3) A trace of Gallic Acid. (4) A trace of a volatile oil giving the odor. Resembling Aloes.-Resins of Jalap, Guaiacum [and Catechu.]

Dose, $1 / 2$ to $10 \mathrm{gr}$.; $[.03$ to $.60 \mathrm{gm}$.

\section{Preparations.}

x. [Aloe Purificata.-Purified Aloes. Socotrine Aloes, 1000; Alcohol, 200. Heat, strain, and evaporate.

Characters.-In irregular, brittle pieces, of a dull brown or reddish-brown color, and having the peculiar, aromatic odor of Socotrine Aloes. It is almost entirely soluble in Alcohol.

Purified Aloes are contained in Extractum Colocynthidis Compositum, Pilulæ Rhei Compositæ and Tinctura Benzoini Composita.

Dose, $1 / 2$ to $10 \mathrm{gr}$; .03 to $.60 \mathrm{gm}$.

2. Extractum Aloes.-Extract of Aloes. Socotrine Aloes; by disintegration by boiling in distilled water; filtration and evaporation.

Dose, $1 / 2$ to $3 \mathrm{gr}$; .03 to .20 $\mathrm{gm}$.

3. Pilulæ Aloes.-Pills of Aloes. Purified Aloes, 13; Soap, I3 $_{3}$ gm. ; to make 100 pills. Each pill contains 2 gr. ; .13 gm., of Aloes. Dose, $I$ to 4 pills.

4. Pilulæ Aloes et Asafœtidæ.-Pills of Aloes and Asafetida. Purified Aloes, 9; Asafetida, 9; Soap, $9 \mathrm{gm}$.; to make 100 pills. Each pill contains I $1 / 3$ gr. ; .09 gm., of Aloes.

Dose, I to 5 pills. 
5. Pilulx Aloes et Ferri.-Pills of Aloes and Iron. Purified Aloes, 7 ; Dried Ferrous Sulphate, 7 ; Aromatic Powder, 7 gm. ; Confection of Rose, a sufficient quantity to make 100 pills. Each pill contains about I gr. ; $.07 \mathrm{gm}$., of Aloes.

Dose, $I$ to 4 pills.

6. Pilulæ Aloes et Mastiches.-Pills of Aloes and Mastic. Synonym._Lady Webster's pill. Purified Aloes, 13 ; Mastic, 4 ; Red Rose, $3 \mathrm{gm}$. ; to make 100 pills. Each pill contains 2 gr. ; . 13 gm., of Aloes.

Dose, I to 3 pills.

7. Pilulæ Aloes et Myrrhæ.-Pills of Aloes and Myrrh. Purified Aloes, 13 ; Myrrh, 6 ; Aromatic Powder, 4 gm. ; Syrup, a sufficient quantity to rnake roo pills. Each pill contains $2 \mathrm{gr}$. ; .1 $3 \mathrm{gm}$., of Aloes.

Dose, 2 to ro pills.

8. Tinctura Aloes.-Tincture of Aloes. Purified Aloes, 100; Liquorice Root, 200. Macerate with Diluted Alcohol and percolate to I000.

Dose, $1 / 4$ to I fl. dr.; I. to 4 . c.c.

9. Tinctura Aloes et Myrrhæ.-Tincture of Aloes and Myrrh. Synonym.-Elixir Proprietatis Paracelsi. Purified Aloes, 100; Myrrh, 100; I iquorice Root, 100. Macerate with Alcohol and Water, and percolate to 1000 .

Dose, I to $2 \frac{1}{2}$ fi. dr. ; 4 . to ro. c.c.]

ALOINUM.-Aloin. [A neutral principle obtained from several varieties of Aloes, chiefly Barbadoes Aloes (yielding Barbaloin) and Socotrine Aloes (yielding Socaloin),--differing more or less in chemical composition and physical properties according to the source from which it is derived. Besides these varieties we have Nataloin (Natal) and Zanaloin (Zanzibar).

Barbaloin, $\mathrm{C}_{17} \mathrm{H}_{20} \mathrm{O}_{7}$, is soluble in about 60 parts of water, 20 parts of Alcohol, or 470 parts of Ether.

Socaloin, $\mathrm{C}_{15} \mathrm{H}_{16} \mathrm{O}_{7}$, is soluble in about 60 parts of water, 30 parts of absolute Alcohol, $3^{80}$ parts of Ether, or 9 parts of Acetic Ether.

Characters. - Minute, acicular crystals, or a microcrystalline powder, varying in color from yellow to yellowish-brown, odorless or possessing a slight odor of Aloes, a characteristic bitter taste, and permanent in the air.

Dose, $1 / 2$ to $2 \mathrm{gr}$; .03 to $.12 \mathrm{gm}$.]

\section{Action of Aloes.}

External.-Aloes has no external action on the unbroken skin, but it can be absorbed from a raw surface, for aloes sprin- 
kled on an ulcer, to which it is a slight stimulant, will lead to purging.

Internal.-Gastro-intestinal tract.-In the stomach the bitter principle of aloes causes it to act as a stomachic, like other bitters. In the intestine it increases the rate of the flow of bile, and probably the amount secreted. It produces little influence in the small intestine, but the muscular coat of the colon is powerfully stimulated, and the intestinal secretion from that part slightly accelerated. Aloes, therefore, purges, and naturally takes some time, usually fifteen to twenty hours, to act ; the motion is well formed, not very soft, as there is so little increased secretion of fluid, and it is dark colored from the bile in it. Sometimes the drug gripes somewhat, because the muscular contraction it produces is irregular. As it acts chiefly on the lower bowel the habitual use of it may lead to piles.

Female genital organs. - Aloes will aggravate the menstrual flow; it is therefore an emmenagogue. It is excreted by the milk, for aloes given to the mother may purge the child. It is stated also to be excreted in the urine, [and it is quite likely that the habitual use of aloes will result in irritation of the kidneys.]

Barbadoes aloes is more purgative than Socatrine aloes, and contains a greater proportion of substances soluble in water. As a rule aloin acts like aloes, but it does not gripe so much. Some specimens, however, have very little action. This may be owing to adulteration, or differences in composition of different specimens.

\section{Therapeutics of Aloes.}

Aloes is an excellent purgative for cases of habitual constipation, many of which are due to an imperfect contraction of the muscular coat of the large intestine. It is very commonly given as a dinner pill ( $\mathrm{rgr}$; $.06 \mathrm{gm}$., of extract of aloes and $1 / 4 \mathrm{gr}$.; $.015 \mathrm{gm}$., of extract of nux vomica) to sufferers from chronic constipation, and in these cases its bitter principles acting as stomachics aid digestion. If the fæces are hard, $1 / 2$ gr. [.03 gm.] of powdered ipecacuanha should be added. To avoid 
griping it is well to combine a little extract of hyoscyamus or a little extract of belladonna with it. One great advantage of aloes is that the dose need not be gradually increased. It is also very commonly given as a pill with nux vomica and a grain or two; [.06 or . I $2 \mathrm{gm}$.] of dried [ferrous] sulphate to persons suffering from chlorosis. and other forms of anæmia. It overcomes the chronic constipation so common in these cases, and some regard this as very important for the cure of the disease. The amenorrhoea so frequently associated with chlorosis is often benefited by aloes, and amenorrhœa due to other causes may also be relieved. Aloes is of great service in many cases of chronic constipation of children. A warm aqueous solution of aloin purges when injected subcutaneously. Aloes must not be given in pregnancy or menorrhagia. [If a patient suffers from hæmorrhoids which are not inflamed, aloes can be safely administered, and even with marked benefit if they are due to a relaxed rectal mucous membrane, (Barker).] An enema [Socotrine aloes, 8; potassium carbonate, 3 ; mucilage of starch, 960$.$] is anthelmintic.$

\section{ClasS III.-DRASTIC PURGATIVES.}

\section{SCAMMONIUM.}

SCAMMONY.- [A resinous exudation from the living root of Convolvulus Scammonia, Linné (nat. ord. Covolvulacece). Habitat.-Western Asia.

ChARActr.RS. - In irregular, angular pieces or circular cakes, greenishgray or blackish, internally porous, and breaking with an angular fracture, of a resinous lustre; odor peculiar, somewhat cheese-like; taste slightly acrid; powder gray or greenish-gray.]

IMPURITIES.-Chalk and Starch.

Composition. - The chief constituents are-(1) The resin (see below), 75 to 80 per cent. (2) Gum, Io to 20 per cent. (3) Starch.

Dose, 5 to $15 \mathrm{gr}$.; [.30 to $\mathrm{x} .00 \mathrm{gm}$.]

\section{Preparation.}

Resina Scammonii.-Resin of Scammony.

SourcE.-[Digest Scammony wilh successive portions of boiling Alcohol, distil off the Alcohol, precipitate the Resin with Water, wash it several times, and dry with gentle heat. 
Characters. - Yellowish-brown or brownish-yellow masses or fragments, breaking with glossy, resinous fracture, translucent at the edges; or a yellowish-white or grayish-white powder, having a faint peculiar odor, and a slight, peculiar taste. Solzebility. - In Alcohol in all proportions; also wholly soluble in Ether and in Oil of Turpentine.

Composition. - The chief constituent is Scammonin, $\mathrm{C}_{88} \mathrm{H}_{156} \mathrm{O}_{42}$, probably the same as Jalapin, see p. 502.]

IMPURITIES.-Guaiacum resin, which blues potato. Resin of jalap, insoluble in Ether.

Resin of Scammony is contained in Extractum Colocynthidis Compositum.

Dose, [ $\mathrm{r}$ to $8 \mathrm{gr} . ; .06$ to $.50 \mathrm{gm}$.

\section{Action of Scammony.}

Gastro-intestinal tract.-Scammony has no effect till it reaches the duodenum. With the bile it forms a strongly purgative compound, powerfully stimulating the intestinal. glands and causing a profuse secretion of intestinal fluids. There is some exaggeration of vascularity, some irregular stimulation of the muscular coat, but these are comparatively slight, and there is little if any addition to the biliary flow. As a result of these actions, in about four hours there is a profuse watery evacuation of the bowels. The drug is, therefore, a powerful hydragogue cathartic, and, in large doses, a strong gastrointestinal irritant. Its action is attended with some griping. It produces no effect if injected into the blood, and therefore acts only locally on the intestine. It is anthelmintic to both roundworms and tape-worms.

\section{Therapeutics of Scammony.}

Scammony being a prompt purgative, obstinate constipation in either children or adults may be treated with it. It may also be given as an anthelmintic.

JALAPA.

JALAP.-[The tuberous root of Ipomaa Jalapa, Nuttall (nat. ord. Convolvulacea). Habitat.-Eastern Mexico.

Characters. - Napiform, pyriform or oblong, varying in size, the large roots incised, more or less wrinkled, dark brown, with lighter-colored spots, 
and short, transverse ridges; hard, compact ; internally pale grayish-brown, with numerous concentric circles composed of small resin-cells; fracture resinous, not fibrous; odor slight, but peculiar, smoky and sweetish; taste sweetish and acrid. On exhausting Ioo parts of Jalap by Alcohol, concentrating the tincture, and pouring it into water, a precipitate of resin should be obtained, which, after washing with water and drying, should weigh not less than 12 parts, and of which not over to per cent. should be soluble in Ether].

Composirion.-The chief constituents are: (1) The official resin (see below ), 7 to 22 per cent. mostly Jalapurgin, $\mathrm{C}_{62} \mathrm{H}_{100} \mathrm{O}_{32}$. (2) A soft resin.

Dose, 5 to $20 \mathrm{gr}$.; [.30 to $1.20 \mathrm{gm}$.]

\section{Preparations.}

I. Extractum Jalapæ.-[Extract of Jalap. By maceration and percolation, with Alcohol, and evaporation.

Extract of Jalap is contained in Pilulæ Catharticæ Compositæ and Pilulæ Catharticæ Vegetabiles.

Dose, 2 to $8 \mathrm{gr}$.; .12 to $.50 \mathrm{gm}$.

2. Pulvis Jalapæ Compositus.-Compound powder of Jalap. Synonym.-Pulvis Purgans. Jalap, 35 ; Potassium Bitartrate, 65.

Dose, $1 / 4$ to I dr.; I. to $40 . \mathrm{gm}$.

3. Resina Jalapæ.-Resin of Jalap.

SOURCE. - By maceration with Alcohol, percolation, distillation of the Alcohol, and precipitation with water, and drying.

Characters. - Yellowish-brown, or brown masses or fragments, breaking with a resinous, glossy fracture, translucent at the edges, or a yellowish-gray or yellowish-brown powder, having a slight, peculiar odor, and a somewhat acrid taste].

Compositrion. - The chief constituents are-(1) Jalapurgin, or Convolvulin $\left[\mathrm{C}_{62} \mathrm{H}_{100} \mathrm{O}_{32}\right]$, a g]ucoside, a hard substance insoluble in Ether, more irritant than Jalapin, and probably the most active ingredient of Jalap. (2) Jalapin [probably identical with Scammonin]. This is a soft resinous substance, soluble in Ether. It is found in Jalap wood and Jalap stalk. (3) Starch and Gum. Resembling Jalop Resin.Aloes, which is bitter.

Dose, I to $5 \mathrm{gr}$. ; [.06 to $.30 \mathrm{gm}$.]

\section{Action of Jalap.}

The mode of action of jalap is precisely the same as that of scammony, with only two exceptions. It causes a greater secretion of intestinal juice, and is therefore more hydragogue ; it stimulates the vessels and muscular coat less, and therefore is less irritant and griping. 


\section{Therapeutics of Jalap.}

Jalap is very largely used as a hydragogue purgative when we want to draw off large quantities of fluid ; therefore it is especially suitable for patients with Bright's disease, for those suffering from uræmia, and for those with dropsy from any cause. Large doses should not be given if the intestinal mucous membrane is liable to inflame easily. It is occasionally employed for severe constipation. [An old prescription consists of equal parts of powdered jalap and calomel, well triturated. The dose is from 5 to ro gr. ; .30 to $.60 \mathrm{gm}$. This is known as Rush's thunderbolt. Curiously enough, it does not gripe..

\section{BRYONIA.}

BRYONIA.-Synonym.-Bryony. The root of Bryonia alba, and of Bryonia dioica Linné (nat. ord. Cucurbitacea). Habitat.-Central and Southern Europe.

Characters. - In transverse sections about $5 \mathrm{~cm}$. in diameter, the bark gray-brown, rough, thin, the central portion whitish or grayish, with numerous, small, projecting wood-bundles arranged in circles and radiating lines; fracture short ; inodorous ; taste disagreeably bitter.

Composition. - The chief constituents are-(I) Bryonin, $\mathrm{C}_{34} \mathrm{H}_{48} \mathrm{O}_{9}$, a bitter glucoside; (2) Resin; (3) Starch ; (4) Gum.

Dose, 10 to $60 \mathrm{gr}$; ; 60 to $4.00 \mathrm{gm}$.

\section{Preparation.}

Tinctura Bryoniæ.-Tincture of Bryonia. Bryonia, I00; by maceration and percolation with Alcohol to 1000 .

Dose, 2 to 4 fl. dr.; 8. to 15. c.c.

\section{Action and Uses of Bryonia.}

Bryonia is an active hydragogue cathartic, which was formerly much employed, but has been superseded by jalap.]

\section{OLEUM [TIGLII.]}

CROTON OIL.-[A fixed oil expressed from the seed of Croton Tiglium Linné (nat. ord. Euphorbiacea). Habitat.-India and Philippine Islands; cultivated.

ChARACTERS.-A pale yellow or brownish-yellow, somewhat viscid, and slightly fluorescent liquid, having a slight fatty odor, and a mild, oily, afterwards acrid and burning taste; when applied to the skin, it produces rubefac- 
tion or a pustular eruption. Sp. gr., 0.940 to 0.960 . Solubility. -When fresh, in about 60 parts of Alcohol, the solubility increasing by age ; feebly soluble in Ether, Chloroform, Carbon Disulphide, and in fixed or volatile oils. The oil should be at least two years o.d ; when fresh it is of no value.]

Compositrox. - The chief constituents are-(I) Several volatile acids (I per cent. in all); these give the odor. [Tiglinic Acid, $\mathrm{C}_{5} \mathrm{H}_{8} \mathrm{O}_{2}$, is the characteristic one ; the others are Acetic, Isobutyric, Isovalerianic, Formic, Lauric, Myristic, Palmitic, Stearic, existing as glycerides. (2) Several fatty acids, both free and combined to form fats. (3) Crotonol, $\mathrm{C}_{18} \mathrm{H}_{28} \mathrm{O}_{4}$ ] a substance which is non-purgative, but is capable of causing cutaneous irritation.

Dose, $1 / 4$ to $2 \mathrm{~m}$.; [.015 to .12 c.c.] on a lump of sugar, or mixed with Castor Oil and placed at the back of the mouth, so that it may be quickly swallowed.

Croton seeds are not official, but it is important to recognize them. They are [ $13 \mathrm{~mm}$.] long, [ $8 \mathrm{~mm}$.] broad, ovoid and bluntly oblong, covered with a brown shell, which on scraping becomes black. The kernel is white and oily. They yield 50 to 60 per cent. of Croton Oil. They are known from Castor-oil seeds, which are like them, by the fact that the Castor-oil seeds are bright, polished and mottled.

\section{Action of Croton Oil.}

External.-Croton oil is one of the most powerful irritants in the pharmacopœia. A drop placed on the skin causes redness, burning pain, and quickly a crop of vesicles form (vesication); these rapidly become pustules (pustulation), and the surrounding subcutaneous tissue is red and odematous. [The pustules may be umbilicated, but differ from variolous pustules in that they vary greatly in their size.]

Internal._Gastro-intestinal tract. - Very soon after a drop has been taken there is considerable griping and abdominal pain. In an hour or two the bowels are opened, and this may subsequently occur several times, the motions becoming. more and more fluid. The croton oil greatly aggravates the vascularity of the stomach and intestines, the mucous membrane of which becomes red, œdematous and angry-looking; there is a great increase of the intestinal secretion, but none of the bile. The drug produces, in fact, severe enteritis, and to a less extent gastritis. The motions may contain blood. These effects are all due to the local [action] of the croton oil. It is probable that the peristaltic movements are increased also; whether this 
is a result of the irritation, or of some action of the drug exerted after alssorption, is not known. Croton oil applied to the skin may cause free purgation.

\section{Therapeutics of Croton Oil.}

External.-Croton oil was formerly employed externally as an irritant and a counter-irritant for inflamed joints, pleurisy, bronchitis, phthisis, etc.; but it is not often so used now, as the scars left after the suppuration are very unsightly, the application is too painful and the inflammation induced too severe. [Corson's paint is a 5 to 15 per cent. solution of croton oil in ether, to which a small quantity of tincture of iodine is added to color it]. A little croton oil spread over an area not exceeding that of a [dime] may be applied to set up suppuration in the scalp, and so destroy an inveterate patch of ringworm if it is wished to cure it quickly. The croton oil will certainly do this, but the resulting suppuration is so severe that the remedy should be used with care, and only when all others have failed. The liniment [of the B. P., I 5 per cent. of croton oil in equal parts of oil of cajuput and alcohol], well diluted, is occasionally employed to stimulate the skin in alopecia.

Internal.-Croton oil should only be given in very obstinate constipation, not due to organic obstruction, and only one dose should be administered. Not more than one or two drops should be prescribed. Constipation due to lead poisoning and fæcal impaction are sometimes suitable cases. Placed on the back of the tongue, it is, on account of its small bulk, a useful purgative for lunatics who refuse to take anything, and for unconscious patients, because in such cases it is quickly swallowed reflexly; hence also it is commonly given to those who are unconscious from apoplexy. It must never be administered to children, to pregnant women, to feeble subjects, to those with hæmorrhoids, nor to those suffering from peritonitis, gastritis, or enteritis.

\section{COLOCYNTHIS.}

COLOCYNTH.-[Synonyms.-Bitter Apple. Bitter Gourd. Bitter Cucumber. The fruit of Citrullus Colocynthis, Schrader (nat. ord. Cucur- 
bitacea), deprived of its rind. Habitat.--Southern and Western Asia, Northern and Southern Africa, Greece and Spain.

Characters. - From 5 to $10 \mathrm{~cm}$. in diameter; globular; white or yellowish-white; light, spongy; readily breaking into three wedge-shaped pieces, each containing near the rounded surface, many flat, ovate, brown seeds; inodorous ; taste intensely bitter. The pulp only should be used, the seeds being separated and rejected.]

IMPURITIES. - Seeds and cortex.

Composition.-The chief constituents are-( 1 ) Colocynthin, $\left[\mathrm{C}_{56} \mathrm{H}_{84} \mathrm{O}_{23}\right.$ about 2 per cent. ,] an amorphous or crystalline, bitter, active glucoside, readily soluble in water and Alcohol. (2) Resinous matter having the names of Citrullin, Colocynthein and Colocynthitin, insoluble in water.

Dose, 2 to $8 \mathrm{gr}$. ; [.12 to $.50 \mathrm{gm}$.]

\section{Preparations.}

[I. Extracturn Colocynthidis. - Extract of Colocynth. By maceration with Diluted Alcohol, expression and straining; percolation and evaporation.

Dose, $\mathrm{g} / 2$ to $2 \mathrm{gr}$; .03 to $.12 \mathrm{gm}$.

2. Extracturn Colocynthidis Compositum.-Compound Extract of Colocynth. Extract of Colocynth, 160 ; Purified Aloes, 500; Cardamom, 60; Resin of Scammony, 140; Soap, 140; Alcohol, 100. By melting, straining and reducing to powder.

Dose, 5 to $15 \mathrm{gr}$; .30 to $1.00 \mathrm{gm}$.

3. Pilulæ Catharticæ Compositæ.-See Mercury, p. 212.

4. Pilulæ Catharticæ Vegetabiles.-Vegetable Cathartic Pills. Compound Extract of Colocynth, 60; Extract of Hyoscyamus, 30 ; Extract of Jalap, 30 ; Extract of Leptandra, I5 ; Resin of Podophyllum, 15 gm.; Oil of Peppermint, 8 c.c.; Water, to make 1000 pills.

Dose, I to 5 pills.]

\section{Action of Colocynth.}

In small doses colocynth acts as a simple bitter, increasing the gastric and intestinal secretions and improving the appetite. In larger doses it augments considerably the flow of bile and succus entericus, stimulates the muscular coat, causes a little griping, and leads to the evacuation of a watery motion. In still larger doses the hypersecretion is excessive and the griping is severe because the muscular coat is powerfully irritated, and 
several abundant watery motions result. The drug may therefore be called drastic, hydragogue, and cathartic. The depression produced may be considerable.

\section{Therapeutics of Colocynth.}

Colocynth should never be given alone, because of the griping it causes. In the colocynth and hyoscyamus pill, [I part of colocynth, 2 parts of hyoscyamus,] which is often prescribed, the hyoscyamus prevents this painful result. Colocynth is an excellent purgative for producing a single abundant evacuation of the bowels in chronic constipation, such as that so often met with in persons suffering from hepatic disorder, and in those confined to bed. Because of the watery character of the motions it may be given in ascites or Bright's disease, but jalap or scammony is usually preferred. It is too irritant for habitual use. It should never be administered if there is any suspicion of intestinal or gastric inflammation, nor in pregnancy. It is often combined with milder purgatives. A diuretic action has been claimed for it, but this is unimportant.

\section{ELATERINUM.}

[ELATERIN. $-\mathrm{C}_{20} \mathrm{H}_{28} \mathrm{O}_{5}=347.20 . \quad \Lambda$ neutral principle obtained from Elaterium, a substance deposited by the juice of the fruit of Ecballium Elaterium (Linné) A. Richard (nat. ord. Cucurbitacea). Synonym.-Squirting Cucumber. Habitat.-Western Asia, Northern Africa and Southern Europe ; cultivated.

SuURCE. - Exhaust Elaterium with Chloroform. Add Ether, wash the resulting precipitate with Ether; purify by re-crystallization with Chloroform.

Characters. - Minute, white, hexagonal scales, or prismatic crystals, without odor, and having a slightly acrid, bitter taste; permanent in the air. Solubility. - In 4250 parts of water, and in 337 parts of Alcohol; also soluble in 543 parts of Ether, or in 2.4 parts of Chloroform.

Dose, $\frac{1}{26}$ to $\frac{1}{12}$ gr.; .003 to $.005 \mathrm{gm}$.

\section{Preparation.}

Trituratio Elaterini.-Trituration of Elaterin. Elaterin, Io ; Sugar of Milk, 90.

Dose, $1 / 2$ to I gr.; .03 to $.06 \mathrm{gm}$.] 


\section{Action of Elaterin.}

Elaterin is violently purgative, producing profuse watery evacuations, attended with griping and much prostration. It acts like colocynth, and except that it is much more energetic, the description of that drug will apply to it. It increases the salivary secretion. When injected subcutaneously it purges. It is the most powerful hydragogue purgative in the Pharmacopœia.

\section{Therapeutics of Elaterin.}

Elaterin should not be given in ordinary constipation, as it is too violent in its effects, but on account of the large amount of fluid it brings away it is in suitable cases very useful in ascites and in Bright's disease. The same cautions as were enumerated for colocynth are still more necessary here. It should not be given, or only with great care, in heart disease, on account of the depression produced.

\section{GAMBOGIA.}

[GAMBOGE.-A gum resin obtained from Garcinia Hanburïi Hooker filius (nat. ord. Guttifere). Habitat.-Anam, Camboja and Siam.

Characters. - In cylindrical pieces, sometimes hollow in the centre, 2 to $5 \mathrm{~cm}$. in diameter, longitudinally striate on the surface ; fracture flattishconchoidal, of a waxy lustre, orange-red ; in powder bright yellow ; inodorous ; taste very acrid; the powder sternutatory. Solubility. - It is partly soluble in Alcohol and in Ether.]

Composition. - The chief constituents are-(I) A brilliant yellow Resin, Gambogic Acid, [65 to 80 per cent. (2) Gum, 16 to 26 per cent.] This is soluble, so that an emulsion of Gambogic Acid is formed with water.

IMPURITIES. - Starch, woody fibre.

[Gamboge is contained in Pilulæ Catharticæ Compositx.]

Dose, I to $5 \mathrm{gr}$.; [.06 to .30 gm.]

\section{Action of Gamboge.}

Gamboge is a drastic, hydragogue purgative, causing much griping, and, in large doses, great irritation of the alimentary canal. Most of it passes in the fæces, but some is absorbed, causing the urine to be yellow. It is slightly diuretic. 


\section{Therapfutics of Gamboge.}

It is not often prescribed, as it is uncertain, and gripes considerably. It should never be given alone. It has been used as an anthelmintic.

\section{Class IV.-CHÓl.AGOGUES.}

\section{PODOPHYLLUM.}

PODOPHYLLUM. - [Synonyms.-May Apple. Mandrake. The rhizome and Roots of Podnphyllum peltatum Linné (nat ord. Berberidea). Habitat.-North America, in rich woods and thickets.

Characters. - Of horizontal growth, consisting of joints about $5 \mathrm{~cm}$. long, flattish cylindrical, about $5 \mathrm{~mm}$. thick, but somewhat enlarged at the end, which has a circular scar on the upper side, a tuft of about ten, nearly simple, fragile roots on the lower side, and is sometimes branched laterally; smooth or somewhat wrinkled, orange-brown, internally white and mealy, with a circle of small wood-bundles; pith large; nearly inodorous; taste sweetish, somewhat bitter and acrid.]

Composition. - The chief constituents are-(I) The official Resin (see below), 4 to 5 per cent. (2) Podophyllic Acid, a coloring principle.

Dose, 5 to $20 \mathrm{gr}$; .30 to $1.20 \mathrm{gm}$.

\section{Preparations.}

I. Extractum Podophylli.-Extract of Podophyllum. By maceration and percolation with Alcohol and Water, distillation of the Alcohol, and evaporation.

Dose, 2 to Io gr.; .12 to .6o gm.

2. Extractum Podophylli Fluidum.-Fluid Extract of Podophyllum. By maceration and percolation with Alcohol and Water, distillation of the Alcohol and solution.

Dose, 5 to $20 \mathrm{~m}$.; .30 to $x .20$ c.c.]

3. Resina Podophylli.-[Resin of Podophyllum. Synonym.Podophyllin.

SOURCE. - By maceration and percolation in Alcohol, distillation of the Alcohol, precipitation of Resin in Hydrochloric Acid and Water; wash and dry.

ChARACTERS. - An amorphous powder, varying in color from grayish-white to pale greenish-yellow or yellowish-green, turning darker when exposed to heat ; having a slight, peculiar odor, and a peculiar, faintly bitter taste. Permanent in the air. Solubility. - In Alcohol in all proportions: Ether dissolves 15 to 20 per cent. of it. ] 
Composition.-The Resin consists mainly of Podophyllotoxin, $\left[\mathrm{C}_{23} \mathrm{H}_{24} \mathrm{O}_{9}+2 \mathrm{H}_{2} \mathrm{O}\right.$, which is said to be a mixture of Picropodophyllin, 75 to 80 per cent., the purgative principle, and Picropodophyllic Acid, both existing free in the rhizome ; with these are associated minor resins, and Podophylloquercitin, a coloring principle.]

Incompatibles. - Water precipitates it from Alcohol; acids precipitate it from Ammonia.

[Resin of Podophyllum is contained in Pilulæ Catharticæ Vegetabiles.]

Dose, $1 / 8$ to $1 / 2 \mathrm{gr}$; [ [.008 to $.03 \mathrm{gm}$.]

\section{Action of Podophyllin.}

External.-It has no external action unless applied to raw surfaces, from which it may be absorbed, and then it will purge.

Internal. - Gastro-intestinal tract.-Podophyllin has a bitter taste. It is in large doses a powerful gastro-intestinal irritant, and has caused death. In medicinal doses it gives rise to much griping pain, perhaps some nausea, and in about ten hours there is an evacuation of the bowels ; the motion, which is liquid, is deeply stained with bile. The pain shows that the muscular coat is stimulated, the liquidity that probably more intestinal fluid is secreted, and the color that more bile is poured into the intestine. In small doses podophyllin probably increases the secretion of bile, and certainly the solids in it are greater; in purgative doses it is said not to increase the quantity, although more bile is poured from the bladder into the intestine. It is thus a direct and indirect cholagogue. It probably acts after absorption, for all its effects can be produced if it is injected subcutaneously.

\section{Therapétics of Podophyllum.}

Podophyllum is only used for its cholagogue purgative action. [An old name for this drug is Vegetable Mercury.] It is especially suitable for constipation due to hepatic disorder, whether functional, as in the hepatic dyspepsia which commonly goes by the name of biliousness, or organic, as in hepatic cirrhosis and cancer. It must be remembered that as it causes much griping, it should be combined with hyoscyamus or some other 
drug to overcome this; that it takes a long while to act, and will therefore be swept away before it has produced any effect if given with quickly acting purgatives; and that it is better to begin with small doses, as people are very unequally affected by it. It may be advantageously combined with calomel in a pill. It is so disagreeable to the taste that it is better to dissolve the resin in aromatic spirit of ammonia ( 1 to 480 ).

\section{[LEPTANDRA.}

LEPTANDRA.-Synony'm.-Culver's Root. The rhizome and roots of Veronica virginica Linné (nat. ord. Scrophularinea). Habitat.-United States, south to Georgia, and west to Minnesota, in low grounds.

Characters. - Of horizontal growth, from 10 to $15 \mathrm{~cm}$. long, and about $5 \mathrm{~mm}$. thick, somewhat flattened, bent and branched, deep blackish-brown, with cup-shaped scars on the upper side, hard, of a woody fracture, with a thin, blackish bark, a hard, yellowish wood, and a large, purplish-brown, about six-rayed pith; roots thin, wrinkled, very fragile; inodorous; taste bitter and feebly acrid.

Composition.-The chief constituents are-(I) Leptandrin, a bitter, crystalline glucoside. (2) A saccharine principle having the properties of Mannit. (3) Possibly a Volatile Alkaloid.

Dose, $1 / 4$ to $1 \mathrm{dr}$. ; $x$. to $4 . \mathrm{gm}$.

\section{Preparations.}

1. Extractum Leptandræ.-Extract of Leptandra. By maceration and percolation with Alcohol and water, and evaporation.

Extract of Leptandra is contained in Pilulæ Catharticæ Vegetabiles.

Dose, $\mathrm{I}$ to $3 \mathrm{gr}$; .06 to $.20 \mathrm{gm}$.

2. Extractum Leptandræ Fluidum.-Fluid Extract of Leptandra. By maceration and percolation with Alcohol and Water, and evaporation.

Dose, $1 / 4$ to I fl. dr.; 1 . to 4 . c.c.

\section{Action and Uses of LeptandRa.}

Recent leptandra root acts as a violent cathartic, and sometimes as an emetic. It is an excellent cholagogue, appears to have a special influence upon the muciparous follicles of the intestine, and it acts very advantageously in cases of duodenal indigestion and chronic constipation. 


\section{CHELIDONIUM.}

CHELIDONIUM.-Synonym.-Celandine. The entire plant, Chelidonium majus Linné (nat. ord. Papaveracee). Habitat.-Europe; naturalized in North America.

Characters. - Root several-headed, branching, reddish-brown; stem about $50 \mathrm{~cm}$. long, light-green, hairy ; leaves about $15 \mathrm{~cm}$. long, thin, petiolate, the upper ones smaller and sessile, light-green, on the lower side glaucous, lyrate-pinnatifid, the pinnæ ovate-oblong, obtuse, coarsely crenate or incised and the terminal one often three-lobed; flowers in small, long peduncled umbels with two sepals and four yellow petals ; capsule linear, two-valved and many seeded. The fresh plant contains a saffron-colored milk-juice, and has an unpleasant odor and acrid taste.

Composition. - (1) Chelerythrine, $\mathrm{C}_{21} \mathrm{H}_{17} \mathrm{NO}_{4}$. (2) Sanguinarine, $\mathrm{C}_{29} \mathrm{H}_{15} \mathrm{NO}_{4}$ (see p. 450.) (3) Chelidonine, $\mathrm{C}_{20} \mathrm{H}_{19} \mathrm{NO}_{5}$. (4) Protopine, $\mathrm{C}_{20} \mathrm{H}_{17} \mathrm{NO}_{5}$, also contained in Opium and Sanguinaria. (5) Chelidoxanthin, Chelidonic and Chelidoninic Acids.

Dose, 15 to $60 \mathrm{gr}$; 1. to 4 . gm.

\section{Action and Uses of Celandine.}

Celandine has been found useful in jaundice, apparently possessing a stimulating effect upon the hepatic secretions. It is a somewhat irregularly acting purgative, giving rise to watery motives, but at the same time to griping pains. It was the chief ingredient in the old Decoctum ad Ictericos of the Edinburgh Pharmacopœia.

\section{IRIS.}

IRIS.-Synonym.-Blue Flag. The rhizome and roots of Iris versicolor Linné (nat. ord. Iridea). Habitat.-North America, in swampy localities.

Characters. - Rhizome of horizontal growth, consisting of joints, 5 to $10 \mathrm{~cm}$. long, cylindrical in the lower half, flattish near the upper extremity, and terminated by a circular scar, annulated from the leaf-sheaths, grayishbrown; roots long, simple, crowded near the broad end; odor slight; taste acrid and nauseous.

Composition.-The chief constituents are-(1) An acrid resin. (2) Possibly an alkaloid. (3) Fat. (4) A camphoraceous body.

Dose, ro to $30 \mathrm{gr}$; .60 to $2.00 \mathrm{gm}$.

\section{Preparations.}

1. Extractum Iridis.-Extract of Iris. By maceration and percolation with Alcohol, and evaporation.

Dose, $x$ to $3 \mathrm{gr}$; .06 to .20 $\mathrm{gm}$. 
2. Extractum Iridis Fluidum.-Fluid Extract of Iris. By maceration and percolation with Alcohol, and evaporation.

Dose, 10 to $30 \mathrm{~m}$.; .60 to 2.00 c.c.]

\section{IRIDINUM.}

IRIDIN.-(Not official.)-Synonym.-Irisin. The powdered extractive obtained from the root of Iris versicolor,

Characters.-A dark-brown, bitter, nauseous powder.

Dose, I to $5 \mathrm{gr}$; [ [.06 to $.30 \mathrm{gm}$.]

\section{Action and Therapeutics of Iris.}

[The extract and fluid extract of iris are infrequently used.] Iridin is a cholagogue, and as it rarely gripes, it may be given when it is required to use a cholagogue purgative daily for some time. It may be combined with euonymin, calomel, podophyllin and other cholagogue purgatives.

\section{EUONYMUS.}

EUONYMUS.-Synonyms. - Wahoo. Spindle Tree. [The bark of the root of Euonymus atropurpureus Jacquin (nat. ord. Celastrinea). Habitat.United States, southward to Florida, and westward to Wisconsin, in shady woods.

Characters. - In quilled or curved pieces, from 2 to $8 \mathrm{~mm}$. thick, outer surface, ash-gray, with blackish patches, detached in thin and small scales; inner surface whitish or slightly tawny, smooth; fracture smooth, whitish, the inner layers of a laminated appearance ; nearly inodorous; taste sweetish, somewhat bitter and acrid].

Composition. - The chief constituents are-(I) Euonymin [an amorphous resin, very bitter. (2) Atropurpurin, a crystalline glucoside. (3) Citric, Tartaric and Malic Acids.

Dose, I to $2 \mathrm{dr}$.; 4. to 8. $\mathrm{gm}$.]

\section{Preparation.}

Extractum Euonymi.-Extract of Euonymus. [Synonym.Euonymin. By maceration and percolation with Alcohol and Water, distillation of the Alcohol, and evaporation.

Dose, 1 to $5 \mathrm{gr}$; ; .06 to $.30 \mathrm{gm}$.]

\section{Action and Therapeutics of Euonymus.}

In small doses euonymin stimulates the appetite and flow of gastric juice; in larger, it is irritant to the intestine and is 
cathartic. An ordinary dose increases the amount of bile excreted into the intestine, but does not gripe or cause much intestinal secretion. It has slight diuretic and expectorant effects; but its only use is as a purgative for those cases of constipation in which the liver is disordered, and for which it is particularly efficacious. It is usually combined with other cholagogues as iridin and calomel.

\section{GROUP VI.}

\section{Volatile Oils.}

These, when applied externally, stimulate the skin, and thus cause redness, sometimes even vesication, tingling, and subsequent numbness. Taken internally, they stimulate the gastro-intestinal tract, increasing its vascularity, the flow of saliva, of gastric juice, and of succus entericus ; and they excite its unstriped muscular fibres. Thus in moderate doses they are stomachics and carminatives; in large doses they are gastro-intestinal irritants. Their irritation of the stomach reflexly stimulates the heart and the central nervous system. They are absorbed and excreted by the skin, which they may thus irritate, and by the bronchial mucous membrane, while they consequently stimulate, increasing the amount of secretion from it, its vascularity, the expulsive power of its unstriped muscles, and reflexly this irritation leads to coughing; consequently they are expectorants, [although they may later limit the amount of secretion formed]. They are also largely excreted by the kidneys, which are stimulated even to inflammation, and hence these drugs are often diuretic; and by the genito-urinary mucous membrane, which is also stimulated, often so energetically that it may lecome inflamed. [Some are antiseptic.] Some volatile oils act strongly in all these ways; others act much more powerfully in some than in others. [Volatile and fixed oils are usually mutually soluble in all proportions. Volatile oils are generally slightly soluble in water.]

They will be classified according to the tissue on which they chiefly act, or for the action for which they are mostly used.

Class I.-Volatile oils (or substances containing them), acting chiefly upon, or used chiefly for their stimulation of the skin.

[Turpentine,] Oil of Turpentine, [Oil of Erigeron, Pix Liquida,] Oil of Cade, Burgundy Pitch, Resin, Frankincense, Canada [Turpentine,] Mustard, Oil of Cajuput, Eucalyptus, Oil of Rosemary, Arnica, Mezereum, [Elemi.]

CLASS II. - Volatile oils (or substances containing them) acting chiefly upon, or used chiefly for their stimulation of the gastro-intestinal tract. 
Pyrethrum, Cloves, Pimenta, Pepper, Nutmeg, [Mace,] Cinnamon, Horseradish, Capsicum, Ginger, Cardamom, Sumbul, Oil of Lavender, [Oil of Lavender Flowers, Oil of Bergamot,] Peppermint, Spearmint, Anise, [Illicium,] Coriander, Fennel, Caraway, Dill, Sambucus, [Oil of Pennyroyal, Absinthium], Chamomile, [Matricaria, Rose.]

CLASS III.-Volatile oils (or substances containing them) acting chiefly upon the stomach, so as to reflexly stimulate the heart and central nervous systems, or chiefly used for this purpose.

Valerian, [Cypripedium, ] Asafœtida, Galbanum, Ammoniacum, Myrrh.

Class IV.--Volatile oils (or substances containing them) acting chiefly upon, or used chiefly for their stimulation of the bronchial mucous membrane.

Terebene, [Terpin Hydrate], Balsam of Peru, Balsam of Tolu, Storax, Fir Wood Oil, Grindelia.

Class V.--Volatile oils (or substances containing them) acting chiefly upon, or used chiefly for their stimulation of the kidneys and genito-urinary tract.

Oil of Juniper, Buchu, Copaiba, [Oil of Thyme], Cubeb, Oil of Santal, [Matico, Damiana.]

[Class VI._-Volatile oils (or substances containing them) acting chiefly upon, or used chiefly for their stimulation of the female genital organs.

Savine, Tansy, Oil of Rue.]

\section{Class I.-THOSE USED CHIEFLY FOR THEIR ACTION ON THE SKIN.}

\section{TEREBINTHINA.}

[TURPENTINE.-A concrete oleoresin obtained from Pinus palustris Miller, and from other species of Pinus (nat. ord. Conifera). Habitat.United States; in the Atlantic and Gulf States from Virginia to Texas.

Characters. - In yellowish, opaque, tough masses, brittle in the cold, crumbly-crystalline in the interior, of a terebinthinate odor and taste.]

\section{OLEUM TEREBINTHIN E.}

OIL OF TURPENTINE.-[A volatile oil distilled from Turpentine.

Characters. - A thin, colorless liquid, having a characteristic odor and taste, both of which become stronger and less pleasant by age and exposure to the air. Sp. gr., 0.855 to 0.870 . Dissolves Resins (the solution forms varnish), Wax, Sulphur, Phosphorus and Iodine. Solubility. - In 3 times its volume of Alcohol, the solution being neutral or slightly acid to litmus paper: also soluble in an equal volume of Glacial Acetic Ácid.] It is easily oxidized. 
Old Oil of Turpentine is an oxidizing agent ; it readily absorbs Oxygen, and becomes converled into an Oleoresin. French Oil of Turpentine is lavorotatory, some of it comes from Pinus maritima; English Oil of Turpentine, which mostly comes from America, and Russian Oil of Turpentine are dextrorotatory.

Composition.-Oil of Turpentine is a mixture of ( $\mathbf{I})$ several isomeric bydrocarbons (terpenes), all having the formula $\mathrm{C}_{10} \mathrm{H}_{16}$. The chief of them found in the oil are pinene, phellandrene, limonene, and dipentene. They vary in their boiling points and the direction in which they rotate the plane of polarization. The principal terpene in American oil of turpentine is dextro-pinene; the principal terpene in French oil of turpentine is lropopinene. (2) Sesquiterpenes, $\mathrm{C}_{15} \mathrm{H}_{24}$. (3) Bornyl acetate. Most turpentine contains [from 20 to 30 per cent. ] of the Oil of Turpentine. Many official volatile oils, viz., Oils of Lavender, [Cubeb, Juniper,] Peppermint, Chamomile, Caraway, Cloves, contain various terpenes, all isomeric, and all having the formula, $\mathrm{C}_{10} \mathrm{H}_{16}$. An oxidation product of terpene is Camphor, $\mathrm{C}_{10} \mathrm{H}_{16} \mathrm{O}$, which is pharmacopoeial (see Camphor). Sanitas (see p. 518 ) is another product of the oxidation of a terpene.

Dose, 5 to $30 \mathrm{~m}$.; [ .30 to 2.00 c.c., or $1 / 2$ to 4 fl. dr. ; 2. to 15. c.c.] (anthelmintic).

Two parts of mucilage, with thorough trituration, emulsify one part of Oil of Turpentine with sixteen parts of water.

\section{Preparations.}

[1. Oleum Terebinthinæ Rectificatum.-Rectified Oil of Turpentine.

Source.-Oil of Turpentine, I ; Lime water, 6 volumes. By shaking and distillation.

ChARACrers. - A thin, colorless liquid, having the general properties mentioned under Oil of Turpentine. Sp. gr., 0.855 to 0.865 .

Dose, 5 to $30 \mathrm{~m}$.; .30 to 2.00 c.c., or $1 / 2$ to $4 \mathrm{fl}$. dr.; 2. to 15 . c.c. (anthelmintic).

2. Linimentum Terebintbinæ.-Turpentine Liniment. Resin Cerate, 650; Oil of Turpentine, 350. By melting and mixing.]

\section{Action of Turpentine.}

External.-Oil of turpentine has, to a marked degree, the action of other volatile oils. Thus applied to the skin, especially if rubbed in, it causes the ressels to dilate, there is a sense of warmth, the part becomes red, and subsequently common sensation is blunted. The oil is therefore rubefacient, irritant, and counter-irritant.' If enough is applied, it is a vesicant. 
Like the other volatile oils it is antiseptic and disinfectant. It is absorbed by the unbroken skin.

Internal.-Alimentary canal.-Oil of turpentine has the same stimulant effect when locally applied to the mouth and pharynx as it has on the skin, and in the stomach it powerfully dilates the vessels, increases peristalsis and the gastric secretion, and reflexly stimulates the heart, but on account of its nauseous taste it is not used for these properties, which it has in common with other volatile oils. Its effects on the intestine are the same as those on the stomach, the most marked being its energetic stimulation of the muscular coat, hence it is a strong carminative, expelling gas from the bowels. If a large amount is given, the excitation of the muscular coat leads to purging, the motions often containing much blood, hæmorrhage resulting from the great vascular dilatation. Oil of turpentine is anthelmintic, killing the tapeworm when administered in doses of [1/2] to 4 fl. dr. [2. to I5. c.c.]; but this treatment may cause severe symptoms. When given as an enema it kills the threadworm.

Circulation.-Oil of turpentine is readily absorbed. We do not know in what form it circulates. Statements concerning its action on the heart and vessels are very discordant, probably because different experimenters have used different varieties of oil of turpentine; but most specimens appear first to stimulate the heart, in some degree at least, directly, for oil of turpentine locally applied will excite the excised heart, increasing the force and frequency of the cardiac beat. It contracts the vessels, and therefore it is a hæmostatic. The blood-pressure rises. After a large dose of any variety this stimulation is followed by depression, the heart beats feebly, the vessels dilate, and the blood-pressure falls.

Respiration.-When inhaled, oil of turpentine acts on the bronchial mucous membrane as it does on the skin, irritating it, dilating the vessels, increasing and disinfecting the secretion, stimulating the muscles of the bronchi, and reflexly exciting cough. If given internally, [since] some of it is excreted by the bronchial mucous membrane, similar effects are produced. At 
the same time the activity of the respiratory movements is in. creased, so that the drug is a powerful expectorant.

Nervous system.-Oil of turpentine in large doses is a severe depressant to the nervous system, producing languor, dulness, sleepiness, and unsteady gait. Toxic doses cause coma and paralyze the sensory nerves; consequently reflex action is abolished.

Kidneys.-It acts more powerfully on these than almost any other volatile oil. Even moderate doses may lead to pain in the loins, scanty, high-colored urine, albuminuria, and hæmaturia. The urinary passages are also irritated ; consequently, owing to muscular spasm, there is difficulty in passing water, micturition is painful, and a sensation of heat in the perinæum is present (these symptoms constitute strangury). If a large dose has been given, the urine may be completely suppressed. Turpentine causes the urine to smell of violets.

Skin.-Oil of turpentine is excreted by the skin, and may cause an erythematous rash.

Some is probably excreted by the milk, bile and intestinal mucous membrane.

It is said to be a mild antipyretic. [Old] oil of turpentine [containing oxygen (see p. 239)] is an antidote to phosphorus, and it is stated that [that] and [the] French oil are preferable, but this is doubtful.

\section{Therapeutics of Turpentine.}

External.-Oil of turpentine is very largely employed as an irritant or counter-irritant in various forms of chronic inflammation, such as osteo-arthritis, bronchitis, or pleurisy. The liniment forms a useful application. It may also be rubbed in over painful areas, as in neuralgia, myalgia, rheumatic pains, lumbago, etc. Sometimes it is used as a parasiticide for ringworm. Sanitas [not official] is an aqueous solution of common turpentine, which has been allowed to oxidize in the air. Its active antiseptic principle is hydrogen [dioxide, ] and it contains a little thymol. It is a very pleasant disinfectant, but is not so strong as carbolic acid. [Oil of turpentine is an excellent antiseptic for old suppurat- 
ing wounds. Care must be taken that it does not blister the skin.]

Internal.-Stomach and intestines.-[For internal use the rectified oil only should be prescribed.] It is not often prescribed for its carminative and stomachic effects, though given either by the mouth or as an enema ( $\mathrm{I}$ to $\mathrm{I}_{5} \mathrm{fl}$. Oz. of mucilage of starch) it is often very efficacious in removing the intestinal distension due to gas. If it is used as an anthelmintic, $[1 / 2$ to 4 fl. dr., 2. to I 5. c.c.], emulsified in mucilage and followed by a dose of castor oil, should be given. Sometimes it promptly relieves intestinal hæmorrhage, such as that due to typhoid fever. [It is also used in this disease as an antiseptic.] Whenever it is prescribed as a hæmostatic, considerable doses, 30 to 60 minims [2. to 4. c.c.] should be administered every hour for a few hours.

Circulation.-It is not employed to influence this, except as a hæmostatic. It has the reputation of being fairly efficacious in arresting hæmorrhage. It may be given in hæmoptysis and other conditions attended with bleeding.

Respiration.-It is not much used as an inhalation, for the [Vapor Olei Pini Sylvestris $(q . v .)$,$] is much pleasanter, but it$ might be employed to disinfect foul bronchial secretions, and to stimulate the mucous membrane in chronic bronchitis.

It should be remembered that oil of turpentine inust be given internally with great care because of its liability to cause inflammation of the kidneys; indeed, this fact and its [unpleasant] taste account for its not being so often administered as would otherwise be the case. It should never be given to the subjects of Bright's disease.

\section{[OLEUM ERIGERONTIS.}

OIL OF ERIGERON.-Synonym.-Oil of Fleabane. A volatile oil distilled from the fresh, flowering herb of Erigeron canadense Linné (nat. ord. Compasitce) Habitat.-North America, in fields and waste places; naturalized in other countries.

Characters. - A pale yellow, limpid liquid, becoming darker and thicker by age and exposure to the air, having a peculiar aromatic, persistent odor, and an aromatic, slightly pungent taste. Sp. gr., about 0.850 , increasing with age. Solubility. - In an equal volume of Alcohol.

Dose, 5 to $15 \mathrm{~m}$.; .30 to $x .00$ c.c. 


\section{Action and Uses of the Oil of Erigeron.}

Oil of erigeron is less irritant and also less efficient than oil of turpentine. Externally it is often applied to prevent insects from injuring the skin. It has been used in diarrhœa, dysentery and hæmorrhages, in much the same way as oil of turpentine.]

\section{PIX LIQUIDA.}

TAR.-[An empyreumatic oleoresin obtained by the destructive distillation of the wood of Pinus palustris, Miller, and of other species of Pinus (nat. ord. Conifera). Habitat.-United States.

Characters. - Thick, viscid, semi-fluid, blackish-brown, heavier than water, transparent in thin layers, becoming granular and opaque with age; odor empyreumatic, terebinthinate ; taste sharp, empyreumatic. Solubility.Slightly, in water; soluble in Alcohol, fixed and volatile oils, and solution of Potassium or Sodium Hydrate.] On distillation it gives off an empyreumatic oil (oil of tar), which is official (see below), and pyroligneous acid. What remains behind is pitch. This is black, solid, melting in boiling water.

Composition. - Tar is a very complex substance. The chief constituents are-(I) Oil of Turpentine (see p. 5I 5). - (2) Creosote (see p. 334). (3) Phinols (see p. 324). (4) Pyrocatechin, or Catechol, $\mathrm{C}_{6} \mathrm{H}_{6} \mathrm{O}_{2}$ (which see). (5) Acetic Acid. (6) Acetone. (7) Xylol. (8) Toluol. (9) Methylic Alcohol. (IO) Resins.

Dose, $1 / 4$ to $x \mathrm{dr}$.; [x. to $4 . \mathrm{gm} .$, in the form of pill.

\section{Preparations.}

I. [Syrupus Picis Liquidæ.-Syrup of Tar. Tar, 75; Water, I50; Boiling Distilled Water, 420; Sugar, 800; Glycerin, 100; Dis. tilled Water to 1000 . By solution, decantation and filtration.

Dose, I to $4 \mathrm{fl}$. dr. ; 4 . to I5. c.c.]

2. Unguentum Picis Liquidæ.-[Tar Ointment. Tar, 500; Yellow Wax, I25; Lard, 375.

OLEUM PICIS LIQUIDA.-Oil of Tar. A volatile oil distilled from Tar.

Characters. - An almost colorless liquid when freshly distilled, but soon acquiring a dark, reddish-brown color, and having a strong, tarry odor and taste. Sp. gr., about 0.97o. Solubility.—Readily in Alcohol.]

\section{ACTION OF TAR.}

External.-Tar has precisely the same action as oil of turpentine, but it is not so powerful, therefore the vascular dilata- 
tion rarely proceeds to the stage of vesication; but pustules may result if the tar is rubbed in.

Internal.-It is very liable to upset digestion; in large doses it causes epigastric pain, vomiting, severe headache, dark urine, and other symptoms of carbolic acid poisoning (see p. 332). Some of its constituents are excreted by mucous membranes, especially the bronchial, on which it acts as a disinfectant, stimulating expectorant.

\section{Therapeutics of TAR.}

External.-Tar ointment, which is [sometimes] rather hard, and nay be softened by half the wax with almond oil, is often applied as a stimulant to chronic skin diseases, such as psoriasis and chronic eczema. Because of its mildly anæsthetic action, it is sometimes useful in pruritus.

[Wood tar is the only official form of tar, but coal tar is often used in medicine. The prepared form of it is made by simply heating and stirring coal tar at $120^{\circ} \mathrm{F} .48^{\circ} \mathrm{C}$. for an hour.] Liquor Picis Carbonis [not official] is a favorite preparation for many skin diseases. It may be made thus: Dissolve resin soap, I (see below) in alcohol, 8 ; add prepared coal tar, 4 ; digest at $125^{\circ} \mathrm{F}$. [ $5 \mathrm{I}^{\circ} \mathrm{C}$. $]$ for two days, allow it to cool, then decant and filter. An ointment of 3 parts of lard with $\mathrm{I}$ of this solution may be made. Liquor Carbonis Detergens [not official] is an alcoholic solution of ordinary coal tar. [It is used externally in skin diseases, diluted in 20 parts of water.]

Internal.-Coal tar is rarely prescribed for internal use. Wood tar is only given as an expectorant, and it is very valuable for chronic bronchitis. It may be prescribed as a pill or as the syrup, or as Vinum Picis [not official] (a saturated solution of wood tar in sherry, dose I to $4 \mathrm{fl}$. dr. [4. to I 5. c.c.]), or as the French preparation, Eau de Goudron. Tar water is made by stirring wood tar with water [ $\mathrm{I}$ to 4$]$ for fifteen minutes and decanting. The dose is a pint [480. c.c.] daily. It may be used externally as a wash. The syrup with syrup of wild cherry (see p. 462) and apomorphine hydrochlorate $\frac{1}{20} \mathrm{gr} . ; .003 \mathrm{gm}$. , forms an excellent cough mixture. 


\section{OLEUM CADINUM.}

OIL OF CADE.-[Synonyms. - Huile de Cade. Juniper Tar Oil. A product of the dry distillation of the wood of Juniperus Oxycedrus Linné (nat. ord. Conifera). Habitat.-Mediterranean districts of North Africa, Spain, Portugal and France ; in waste places and on stony hill-sides.

Characters. - A brownish or dark brown, clear, thick liquid, having a tarry odor, and an empyreumatic, burning, somewhat bitter taste. Sp. gr., aloout 0.930 . Solubility.-Almost insoluble in water; only partially soluble in Alcohol, but is completely soluble in Ether, Chloroform, or Carbon Disulphide. ]

Composition.-Probably much the same as that of Tar.

\section{Action and Therapeutics of Oil of Cade.}

Oil of cade has the same action on the skin as tar, but it is preferable, as the odor is pleasanter. The diseases treated by the application of it are psoriasis, chronic eczema, and pruritus. A usual formula is oil of cade, $\mathrm{I}$; soft soap, 4 ; alcohol, 4 ; but an ointment made by melting with it an equal part of yellow wax, is a more agreeable preparation.

\section{PIX BURGUNDICA.}

BURGUNDY PITCH.-[The prepared, resinous exudation of Abies excelsa Poiret (nat. ord. Conifera). Habitat.-Europe, in the Southern parts, in mountainous districts.

Characters. - Hard, yet gradually taking the form of the vessels in which it is kept ; brittle, with a shining, conchoidal fracture, opaque or translucent, reddish-brown or yellowish-brown, odor agreeably terebinthinate; taste aromatic, sweetish, not bitter. Solubility._Almost entirely in Glacial Acetic Acid, and partly soluble in cold Alcohol.]

Composition.-[The chief constituents are-(I) Resin; (2) A volatile oil, a mixture of several isomeric terpenes in variable proportion.]

IMPURITIES. - Palm Oil and Resin, which are detected by not being soluble in Glacial Acetic Acid.

[Burgundy Pitch is contained in Emplastrum Ferri and Emplastrum Opii.]

\section{- Preparations.}

x. Emplastrum Picis [Burgundicæ.-Burgundy Pitch Plaster. Burgundy Pitch, 800 ; Yellow Wax, 150; Olive Oil, 50.

2. Emplastrum Picis Cantharidatum.-Cantharidal Pitch Plaster. Synonym.-Warming Plaster. Cerate of Cantharides, 80; Burgundy Pitch to 1000 . Heat the cerate and strain; melt the pitch with the strained liquid.] 


\section{Action and Uses of Burgundy Pitch.}

Burgundy pitch is used as a basis for plasters. ' It is mildly stimulant to the skin.

\section{RESINA.}

RESIN.-[Synonyms.-Colophony. Rosin. The residue left after distilling off the Volatile Oil from Turpentine.

Characters. - A transparent, amber-colored substance, hard, brittle, pulverizable; fracture glossy and shallow-conchoidal ; odor and taste faintly terebinthinate. Sp. gr., 1.070 to 1.080. Solubility.-In Alcohol, Ether, and fixed or volatile oils; also in solution of Potassium or Sodium Hydrate.

Composition. - Resin may be considered as containing Abietic Acid Anhydride, $\mathrm{C}_{44} \mathrm{H}_{64} \mathrm{O}_{5}, 80$ to 90 per cent.

Resin is contained in Ceratum Cantharidis.]

\section{Preparations.}

[1. Ceratum Resinæ.-Resin Cerate. Synonym.-Basilicon Ointment. Resin, 350; Yellow Wax, 150; Lard, 500.

Resin Cerate is contained in Linimentum Terebinthinx.

2. Emplastrum Resinæ.-Resin Plaster. Synonym.-Adhesive Plaster. Resin, I40; Lead Plaster, 800; Yellow Wax, 60.

Resin Plaster is contained in Emplastrum Arnicx, Emplastrum Belladonnæ, and Emplastrum Capsici.]

\section{Action and Therapeutics of Resin.}

Resin is antiseptic and slightly stimulant, and is, therefore, an excellent application for indolent ulcers, sores and wounds. Resin soap is formed by boiling together in an evaporating dish for two hours : resin, 6 ; caustic soda, $\mathrm{I}$; and water, 2.5 ; separating the soap by a strainer, and drying on a water-bath. It may be used as an emulsifying agent, but the taste is very disagreeable.

\section{THUS AMERICANUM.}

FRANKINCENSE. [B. P., not official.]-The concrete oleo-resin scraped off the trunks of Pinus tada, Frankincense Pine, and Pinus australis, the Swamp Pine (nat. ord. Conifera). Habitat.-Southern United States.

Characters. - When fresh it is a soft, yellow, opaque, tough, solid, becoming darker, dry and brittle by keeping. Odor and taste as of other Turpentines. 


\section{USES OF Frankincense.}

Frankincense is used for the same purposes as resin (see p. $523)$.

\section{TEREBINTHINA CANADENSIS.}

CANADA TURPENTINE.-Synonyms._Canada Balsam. [Balsam of Fir. A liquid oleo-resin obtained from Abies balsamea (Linné) Miller (nat. ord. Conifera). Habitat.-Canada end Northern United States, west to Minnesota, and south along the mountains to Virginia.

Characters. - A yellowish or faintly greenish, transparent, viscid liquid, of an agreeable, terebinthinate odor, and a bitterish, slightly acrid taste. When exposed to the air, it gradually dries, forming a transparent mass. Solubility. -Completely in Ether, Chloroform or Benzol.

Compositron.-(r) A volatile oil, 20 to 30 per cent. (2) Resin. (3) A bitter principle soluble in water.]

Canada Turpentine is contained in Collodium Flexile.

\section{Action and Therapeutics of Canada Turpentine.}

Canada turpentine is rarely used except for its physical property of drying to form an adhesive varnish. It has the same action as oil of turpentine.

\section{SINAPIS.}

1. SINAPIS ALBA. - White Mustard. [The seed of Brassica alba (Linné) Hooker filius et Thompson (nat, ord. Crucifera). Habitat.-Asia and Southern Europe; cultivated.

Characters. - About $2 \mathrm{~mm}$. in diameter, almost globular, with a circular hilum; testa yellowish, finely pitted, hard; embryo oily, with a curved radicle, and two cotyledons, one folded over the other; free from starch; inodorous; taste pungent and acrid.]

Composition. - The chief constituents are-(I) A bland fixed oil, [20 to 25 per cent.] (2) Sinalbin, $\left[\mathrm{C}_{30} \mathrm{H}_{44} \mathrm{~N}_{2} \mathrm{~S}_{2} \mathrm{O}_{16}\right.$, $]$ and Myrosin; the latter is an Enzyme, and in contact with water converts Sinalbin, which is a Glucoside, into a fixed pungent body, very acrid, called Acrinyl Sulphocyanide, $\left[\mathrm{C}_{7} \mathrm{H}_{7} \mathrm{ONCS}\right]$, Glucose, and Sinapine Sulphate, $\left[\mathrm{C}_{16} \mathrm{H}_{23} \mathrm{NO}_{5} \mathrm{H}_{2} \mathrm{SO}_{4}\right]$.

2. SINAPIS NIGRA.-Black Mustard. [The seed of Brassica nigra (Linné) Koch (nat. ord. Crucifera). Habilat.-Asia and Southern Europe; cultivated.

ChARACTERS. - About $1 \mathrm{~mm}$. in diameter, almost globular, with a circular hilum; testa blackish-brown or grayish-brown, finely pitted, hard, embryo oily, with a curved radicle, and two cotyledons, one folded over the other; free from starch; inodorous when dry, but when triturated with water, of a pungent, penetrating, irritating odor: taste pungent and acrid.] 
Compositron.-The chief constituents are-(I) The same fixed oil as the white seeds, about 35 per cent. (2) Sinigrin (which is Potassium Myronate, $\mathrm{C}_{10} \mathrm{H}_{18} \mathrm{KO}_{10} \mathrm{NS}_{2}$, a potassium salt of myronic acid, which is a crystalline glucoside) and Myrosin, an enzyme which on contact with water converts Sinigrin into the official volatile Oil of Mustard, (see below) $\left(\mathrm{C}_{3} \mathrm{H}_{5} \mathrm{CNS}\right.$, which is Allyl Sulphocyanide), Glucose, and Potassium Sulphate. The volatile oil is very pungent and highly volatile, and its development on the addition of water explains the pungency of ordinary mustard.

Resembling black mustard seeds. - Colchicum seeds, which are larger, lighter and not quite globular.

\section{Preparation.}

[Charta Sinapis.-Mustard Paper. Black Mustard, 100. Percolate the Mustard with a sufficient quantity of Benzin. Remove the powder and dry; add this to the solution; India rubber, 10; Benzin, 100 ; and Carbon Disulphide, 100 ; and with a brush apply to one side of a piece of rather stiff, well-sized paper, and dry.]

3. OLEUM SINAPIS VOLATILE.-Volatile Oil of Mustard. [Allyl Sulphocyanide, $\mathrm{C}_{3} \mathrm{H}_{5} \mathrm{CNS}$. A volatile oil obtained from Black Mustard by maceration with Water and subsequent distillation.

Characters. - A colorless or pale yellow, limpid, and strongly refractive liquid, having a very pungent and acrid odor and taste. Sp. gr., 1.or8 to 1.029. Solubility. - Freely in Alcohol, Ether or Carbon Disulphide.]

\section{Preparation.}

Linimentum Sinapis [Compositum.-Compound Liniment of Mustard. Volatile Oil of Mustard, 30 ; Fluid Extract of Mezereum, 200 ; Camphor, 60; Castor Oil, 150 ; Alcohol, to 1000; by solution.]

\section{Action of Mustard.}

External.-Mustard is a typical powerful local irritant. Thus it first produces dilatation of the vessels, which causes redness of the skin (rubefacient effect) and a sensation of warmth. Because of the irritant action of mustard on the sensory nerves, a severe burning pain is soon felt. The irritation of the nerves is followed by their paralysis, consequently there is a local loss of sensibility, and a diminution both of the pain produced by the mustard and of any that may have been present before its application. The irritation of the vessels leads to the transuda. tion of plasma through them ; this, collecting under the epidermis, raises it, and thus vesicles, blebs, or blisters, are formed (vesicant effect.) Mustard is also a counter-irritant (see p. 6r); 
that is to say, the stimulation of the cutaneous nerves reflexly leads to an alteration in the size of the vessels of the viscera under the seat of application.

This excitation of the sensory nerves is sufficiently powerful to reflexly stimulate the heart and respiration, and sometimes to restore consciousness after fainting.

Internal.-Gastro-intestinal tract. - Mustard also acts here as an irritant. Taken in the usual snıall quantities as a condiment, it causes a sense of warmth in the stomach, it moderately stimulates the secretion of gastric juice and the peristaltic movements, and therefore sharpens the appetite. A dose of one to four teaspoonfuls [4. to I 5. gm.] stirred up in a tumbler of [lukewarm] water is sufficiently irritating to be a direct stomachic emetic, causing prompt vomiting without the depression which usually attends emetics, because the mustard reflexly stimulates the heart and respiration.

\section{Therapeutics of Mustard.}

External.-A poultice made with flaxseed and having a little mustard ( $\mathrm{I}$ to $\mathrm{I} 6$ of flaxseed) sprinkled on it is a very "common and efficacious application as an irritant and counter-irritant in rheumatism, pleurisy, pneumonia, bronchitis, pericarditis, and many inflamnatory diseases. In the manner already explained, it will, when applied to the skin, soothe pain in gastralgia, colic, painful diseases of the chest, neuralgia, lumbago, etc. The paper or any of the mustard leaves that are sold, moistened in water, form an excellent application. Often the local application of mustard over the stomach relieves vomiting. A large mustard poultice applied to the legs was formerly used as a reflex stimulant in cases of syncope, asphyxia, and coma.

Common colds and febrile conditions, especially in children, are often treated by placing the feet and legs or the whole body in mustard and water as hot as can be borne [ 1 to 128 ], the object being by the cutaneous dilatation to withdraw blood from the inflanied part. A mustard sitz-bath may be taken at the time of the expected period, to induce menstruation.

Internal.-Mustard is used as a condiment, and also as an 
emetic. It is especially valuable for poisoning by narcotics, because of its reflex stimulant effects.

\section{OLEUM CAJUPUTI.}

[OIL OF CAJUPUT.-A volatile oil distilled from the leaves of Melaleuca Leucadendron Linné (nat. ord. Myrtacea). Habitat._East Indian Islands.

Characters. - A light, thin, bluish-green, or after rectification, colorless liquid, having a peculiar, agreeable, distinctly camphoraceous odor, and an aromatic, bitterish taste. Sp. gr., 0.922 to 0.929 . Solubility. - Readily in Alcohol.

Composition.-The chief constituents are-(x) Cajuputol, $\mathrm{C}_{10} \mathrm{H}_{18} \mathrm{O} ;(67$ per cent.) said to be identical with Cineol, and is isomeric with Eucalyptol. (2) Terpineol, $\mathrm{C}_{10} \mathrm{H}_{18} \mathrm{O}$, and (3) Several terpenes, $-\mathrm{C}_{10} \mathrm{H}_{16}$ (cajuputene,) and $\mathrm{C}_{15} \mathrm{H}_{24}$.

IMPURITIES.-Copper, and other oils.

Dose, $\mathrm{I}$ to $5 \mathrm{~m}$.; [.06 to .30 c.c.]

\section{Action of Oll of Cijuput.}

The action of oil of cajuput is exactly the same as that of the oil of cloves (q. v.)

\section{Therapeutics of Oil of Cajuput.}

External.-Oil of cajuput is used as a stimulant, irritant, and counter-irritant-usually diluted with sweet oil-for all sorts of purposes when any of these effects are needed. Thus it is rubbed in for chilblains, myalgia, rheumatic pains, chronic inflammatory conditions of the joints or periosteum. It has also been employed as a parasiticide for Tinea tonsurans. The only objection to its use is its strong smell.

Internal.- It is occasionally given in dyspepsia, usually combined with other remedies, for the sake of its carminative, stomachic, and anti-spasmodic effects; it may be taken on sugar.

\section{EUCALYPTUS.}

[EUCALYPTUS.-The leaves of Eucalyptus globulus Labillardière (nat. ord. Myrtacea), collected from the older parts of the tree. Habitat.Australia ; cultivated in subtropical countries.

Characters. - Petiolate, lanceolately scythe-shaped, from 15 to $30 \mathrm{~cm}$. long, rounded below, tapering above, entire, leathery, grayish-green, glandu- 
lar, feather-veined between the midrib, and marginal veins; odor strongly camphoraceous; taste purgently aromatic and somewhat cooling, bitter and astringent.

Composition.-(I) A volatile oil (see below); (2) Cerylic Alcohol ; (3) A crystallizable Fatty Acid; (4) A crystallizable Resin.

Dose, $1 / 2$ to 2 dr.; 2. to $8 . \mathrm{gm}$.

\section{Preparation.}

Extractum Eucalypti Fluidum.-Fluid Extract of Eucalyptus. By maceration and percolation with Alcohol and Water, and evaporation.

Dose, $1 / 2$ to 2 fl. dr.; 2. to 8 . c.c.]

OLEUM EUCALYPTI.-[O1] of Eucalyptus. A volatile oil distilled from the fresh leaves of Eucalyptus globulus Labillardière, Eucalyptus oleosa F. v. Mueller, and some other species of Eucalyptus (nat. ord. Myrtacea).

Characters. - A colorless or faintly yellowish liquid, having a characteristic, aromatic, somewhat comphoraceous odor, and a pungent, spicy, and cooling taste. Sp. gr., 0.915 to 0.925 . Solubility. - In all proportions, in Alcohol, Carbon Disulphide, or Glacial Acetic Acid.] The oils from different species of Eucalyptus vary very much.

Composition. - The chief constituents are-(I) Eucalyptol $\mathrm{C}_{10} \mathrm{H}_{18} \mathrm{O}$ or Cineol (about 7o per cent.); [(2) Cymene, $\mathrm{C}_{10} \mathrm{H}_{14}$; (3) Eucalyptene, $\mathrm{C}_{10} \mathrm{H}_{16}$; (4) Tannic Acid.]

InCOMPATIBLES. - Alkalies, mineral acids, and metallic salts.

Dose, 5 to $30 \mathrm{~m}$.; [.30 to 2.00 c.c.

EUCALYPTOL.-Eucalyptol. $\mathrm{C}_{10} \mathrm{H}_{18} \mathrm{O}=153.66$. A neutral body obtained from the volatile oil of Eucalyptus globulus Labillardière, and of some oiher species of Eucalyptus (nat. ord. Myrtacea).

SourcE. - In the distillation of Eucalyptus leaves, crude Eucalyptol comes over between $33^{\circ}$ and $352.4^{\circ} \mathrm{F}$; $170^{\circ}$ and $178^{\circ} \mathrm{C}$., and is purified by re-distillation from Caustic Potash or Calcium Chloride.

Characters. - A colorless liquid, having a characteristic, aromatic, and distinctly camphoraceous odor, and a pungent, spicy, and cooling taste. Sp. gr., o.930. Solubility. - In all proportions, in Alcohol, Carbon Disulphide, and Glacial Acetic Acid.

Dose, 5 to $30 \mathrm{~m}$.; .30 to $2.00 \mathrm{c.c}$.]

\section{Action of Eucalyptus.}

External.-Oil of eucalyptus is much less irritant when applied externally than other volatile oils, but if its vapor is confined it will produce vesication and pustulation. It is power- 
fully antiseptic and disinfectant. Old oil is more antiseptic than new, probably from the greater amount of ozone it contains.

Internal._Gastro-intestinal tract. - In medicinal doses oil of eucalyptus is stomachic, having the same action as oil of cloves. In large doses it produces severe gastro-intestinal irritation, as shown by vomiting, diarrhœa, and abdominal pain.

Circulation.-It, like quinine, arrests the movements of the white blood-corpuscles; and it likewise resembles this drug in its antipyretic and its antiperiodic actions, and also, it is said, in causing contraction of spleen; but quinine is in all respects the more energetic. In medicinal doses the heart is stimulated by oil of eucalyptus, and the blood-pressure rises; probably these effects are reflex from the stomach. After large quantities the action of the heart is enfeebled, and temperature falls.

Respiration.-Small doses slightly accelerate, poisonous doses slow respiration.

Nervous system.—Large doses are powerfully depressant to the brain, to the medulla, and to the spinal cord, abolishing reflex action. Death occurs from paralysis of respiration.

Mucous membranes, kidneys, and skin.-Like other volatile oils, eucalyptus is excreted by all these channels. It imparts its odor to and disinfects the breath and the urine. It stimulates the organs by which it is excreted, consequently it is a diaphoretic, a stimulating expectorant, a diuretic, and a stimulant to the genitourinary tract. Large doses cause renal congestion.

\section{Therapeutics of Eucalyptus.}

External.-It is used as an antiseptic for wounds, sores, and ulcers. It is three times as powerful as carbolic acid, and is therefore preferred by some surgeons. A eucalyptus gauze has been prepared as a dressing for wounds, which may be washed with a weak solution of the oil in alcohol. An ointment of oil of eucalyptus, 8 ; iodoform, I ; hard paraffin and vaseline, of each 40 , is applied to chancres. An emulsion of the oil is used as an urethral injection. It would probably be an efficient parasiticide.

Internal.-A vapor or the spray of oil of eucalyptus has 
been recommended for diphtheria and fetid bronchitis, and it is sometimes given by the mouth to correct the fœetor of the expectoration. Occasionally it is used for its stomachic and carminative effects, especially if the fæces are very foul smelling, and some employ it in cystitis and pyelitis. It has been prescribed in septicæmia. As an antiperiodic for ague and an antipyretic it is far inferior to quinine. [In most cases eucalyptol can be substituted for the oil with advantage.]

\section{OLEUM ROSMARINI.}

OIL OF ROSEMARY.- [A volatile oil distilled from the leaves of Rosmarinus officinalis Linnê (nat. ord. Labiata). Habitat.-Basin of the Mediterranean; cultivated.

Characters. - A colorless or pale yellow, limpid liquid, having the characteristic pungent odor of Rosemary, and a warm, somewhat camphoraceous taste. Sp. gr., 0.895 to 0.915 . Solubility. - In an equal volume of Alcohol ; also soluble in an equal volume of Glacial Acetic Acid.]

Composition.-The chief constituents are-(I) The terpene, Pinene, $\left[\mathrm{C}_{10} \mathrm{H}_{16}, 80\right.$ per cent. $]$ (2) Cineol, $\left[\mathrm{C}_{10} \mathrm{H}_{18} \mathrm{O}\right]$. (3) Borneol, $\left[\mathrm{C}_{10} \mathrm{H}_{16} \mathrm{O}\right]$, an alcohol isomeric with Geraniol $(q . v$.$) . (4) Linalool (see p. 545). (5)$ Menthol (see p. 547).

Oil of Rosemary is contained in Linimentum Saponis and Tinctura Lavandulæ Compositæ.

Dose, I to $5 \mathrm{~m}$.; [.06 to .30 c.c.]

Action and Therapeutics of Oil of Rosemary.

Oil of rosemary has an action similar to that of other aromatic volatile oils. It is very commonly used to give a pleasant scent to lotions and other preparations which are used externally.

\section{ARNICA.}

[ARNICA FLORES.-Arnica Flowers. The flower heads of Arnica montana Linné (nat. ord. Composita). Synonym.-Leopardsbane. Habitat. -Europe and Northern Asia; in mountainous districts.

Characters.-Heads about $3 \mathrm{~cm}$. broad, depressed-roundish, consisting of scaly involucre in two rows, and a small, nearly flat, hairy receptacle, bearing about sixteen yellow, strap-shaped, ten-nerved ray-florets, and numerous yellow, five-tootbed, tubular disk-florets, having slender, spindle-shaped achenes, crowned by a hairy pappus. Odor feeble, aromatic; taste bitter and acrid.

Composition.-(1) Arnicin, an amorphous, yellow, acrid, bitter principle; 
easily soluble in Alcohol and Ether.

(2) Volatile Oil.

(3) Caprylic and Capronic Acids. (4) Resin. (5) Tannic acid.

Dose, ro to $20 \mathrm{gr}$.; .60 to $1.20 \mathrm{gm}$.

\section{Preparation.}

Tinctura Arnicæ Florum.-Tincture of Arnica Flowers. Arnica Flowers, 200; by percolation with Diluted Alcohol to 1000 .

Dose, 10 to $30 \mathrm{~m} . ;$.60 to 2.00 c.c.]

ARNIC E [RADIX.-Arnica Root. The rhizome and roots of Arnica montana Linné (nat. ord. Composite).

Characters. - Rhizome about $5 \mathrm{~cm}$. long, and 3 or $4 \mathrm{~mm}$. thick; externally brown, rough from leaf-scars; internally whitish, with a rather thick bark, containing a circle of resin-cells, surrounding the short, yellowish woodwedges, and large spongy pith. The roots numerous, thin, fragile, grayishbrown, with a thick bark containing a circle of resin-cells. Odor somewhat aromatic ; taste pungently aromatic and bitter.] Resembling Arnica.--Valerian and Serpentaria, each having a characteristic odor; Veratrum Viride, having thicker rootlets.

Composition.-The same as of the flowers.

Dose, 5 to $20 \mathrm{gr}$.; [.30 to $1.20 \mathrm{gm}$.]

\section{Preparations.}

[1. Extractum Arnicæ Radicis.-Extract of Arnica Root. By maceration and percolation with Diluted Alcohol, and evaporation.

Extract of Arnica Root is used to make Emplastrum Arnicæ.

Dose, 5 to ro $\mathrm{gr}$; .30 to $.60 \mathrm{gm}$.

2. Extractum Arnicæ Radicis Fluidum.-Fluid Extract of Arnica Root. By maceration and percolation with Alcohol and Water, and evaporation.

Dose, 5 to $20 \mathrm{~m}$.; .30 to $\mathrm{r.20}$ c.c.

3. Tinctura Arnicæ Radicis.-Tincture of Arnica Root. Arnica Root, 100; by maceration and percolation with Alcohol and Water to 1000.

Dose, 5 to $10 \mathrm{~m} . ; .30$ to $.60 \mathrm{c.c}$.

4. Emplastrum Arnicæ.-Amica Plaster. Extract of Arnica Root, 330; resin plaster, 670.]

\section{Action and Therapeutics of Arnica.}

External.-The action of arnica is the same as that of vola. tile oils generally. Externally the tincture is used as an application to bruises, but it is very doubtful how far its good effects 
are owing to the [alcohol] and how far to any increase of cuta. neous vascularity due to the volatile oil of the arnica.

Internal.-It is rarely given internally, but in small doses it is a stomachic, a carminative, and a reflex stimulant, and in larger doses causes vomiting and purging. It is excreted by the kidneys and mucous membranes, and it has been credited with obscure effects on the central nervous system.

\section{MEZEREUM.}

[MEZEREUM.-Synonym.-Mezereon. The bark of Daphne Mezereum Linné, and other species of Daphne (nat. ord. Thymelaacea). Habitat. -Europe in mountainous regions, eastward to Siberia ; spontaneous in Canada and New England.

CHARACTERS. - In long thin bands, usually folded or rolled into disks; outer surface yellowish or brownish yellow, with transverse scars, and minute, blackish dots, underneath of a light greenish color; inner surface whitish, silky ; bast in transverse layers, very tough ; inodorous ; taste very acrid.

Composition.-The chief constituents are-(I) Mezerein, a soft, acrid resin. (2) An acrid, rubefacient, volatile oil. (3) Daphnin, $\mathrm{C}_{15} \mathrm{H}_{16} \mathrm{O}_{9}+$ $2 \mathrm{H}_{2} \mathrm{O}$, a bitter glucoside in fine needles or rectangular plates. (4) Coccogin, $\mathrm{C}_{20} \mathrm{H}_{22} \mathrm{O}_{8}$, a bitter principle.

Mezereum is contained in Decoctum Sarsaparillæ Compositum and Extractum Sarsaparillæ Compositum Fluidum.

\section{Preparation.}

Extractum Mezerei Fluidum.-Fluid Extract of Mezereum. By maceration and percolation with Alcohol and evaporation.

Fluid Extract of Mezereum is used in Linimentum Sinapis Com. positum.

Dose, 5 to $5 \mathrm{~m}$.; .30 to r.oo c.c.]

\section{Action and Therapeutics of Mezereum.}

[External.-Mezereum has the same action as volatile oils generally. It is a powerful rubefacient and vesicant externally, and is used chiefly in the compound mustard liniment, where it excites the same effects and is employed for the same purposes as the oil of mustard. Almost its only use at present is to keep open an issue, a procedure which is very rarely employed.

Internal.- It is a gastric stimulant, producing, in large doses, vomiting and diarrhœa.] 


\section{ELEMI.}

[MANILLA ELEMI (not official.)-A concrete resinous exudation, probably from Canarium commune (nat. ord. Bursaracea). Habitat.Manilla.

Characters. - A soft unctuous mass, becoming harder and yellowish by age. Strong fennel-like odor. Resembling Elemi. -Asafotida, Galbanum, and Ammoniacum, but Elemi is known by its odor.

Composition. - The chief constituents are-(I) Resinous borlies, 80 per cent. (2) A volatile oil.

\section{Action and Therapeutics of Elemi.}

Elemi acts like volatile oils generally, but is only used as a stimulating disinfectant ointment which was formerly official in B. P. as elemi, I; ointment, 4 .]

\section{Class II.-THOSE USED CHIEFLY FOR THEIR ACTION ON THE GASTRO-INTESTINAL TRACT.}

\section{PYRETHRUM.}

PYRETHRUM.-Synonym.-Pellitory. [The root of Anacyclus $P_{y}$ rethrum (Linné) De Candolle (nat. ord. Composita). Habitat._Highlands of Northern Africa.

Characters. - From 5 to $10 \mathrm{~cm}$. long, and $\mathrm{I}$ to $2 \mathrm{~cm}$. thick, somewhat fusiform, nearly simple, annulate above, wrinkled below, externally dark grayish-brown ; internally brownish-white ; fracture short ; bark rather thick, containing two circles of resin-cells, and surrounding the slender wood bundles and medullary rays, the latter having about four circles of shining resin-cells; inodorous, pungent and very acrid.] Resembling Pyrethrum.-Taraxacum, which is darker and has not a burning taste.

Composition. - The chief constituents are-(1) Two volatile oils. [(2) An acrid, brown Resin. (3) Inulin, 50 per cent., which in many plants replaces starch.

Dose, $1 / 2$ to I dr.; 2. to $4 . \mathrm{gm}$.]

\section{Preparation.}

Tinctura Pyrethri.-[Tincture of Pyrethrum. Pyrethrum, 200; by maceration and percolation with Alcohol and Water to 1000.]

Action and Therapeutics of Pyrethrum.

Pyrethrum is a powerful sialogogue, and causes a burning sensation in the mouth, followed by numbness and tingling. Small quantities give a pleasant taste to tooth powders. 


\section{CARYOPHYLLUS.}

CLOVES.-[The unexpanded flowers of Eugenia aromatica (Linné) O. Kuntze (nat. ord. Myrtacea). Habitat.-Molucca Islands; cultivated in tropical countries.

Characters. - About $15 \mathrm{~mm}$. long, dark brown, consisting of a subcylindrical, solid and granular calyx-tube, terminated by four teeth, and surmounted by a globular head, formed of four petals, which cover numerous curved stamens, and one style. Cloves emit oil, when scratched, and have a strong, aromatic odor, and a pungent, spicy taste.]

Composition.-The chief constituents are-(I) Oleum Caryophylli [(see below), 18 per cent.] (2) Eusenin $\left[\mathrm{C}_{10} \mathrm{H}_{12} \mathrm{O}_{2}\right]$, a crystalline body. Caryophyllin, $\left[\mathrm{C}_{10} \mathrm{H}_{16} \mathrm{O}\right.$, ] n neutral body isomeric with Camphor.

[Cloves are contained in Vinum Opii, Tinctura Rhei Aromatica, and Tinctura Lavandulæ Composita.

Dose, 5 to $30 \mathrm{gr}$; . 30 to $2.00 \mathrm{gm}$.]

OLEUM CARYOPHYLLI.-[Oil of Cloves. A volatile oil distilled from Cloves.

Characters. - A pale yellow, thin liquid, becoming darker and thicker by age and exposure to the air, having a strongly aromatic odor of Cloves, and a pungent and spicy taste. Sp. gr., 1.060 to 1.067. Solubility.-Soluble in an equal volume of Alcohol; also soluble in an equal volume of glacial Acetic Acid.]

Composition.-The chief constituents are-(1) Eugenol (Synonym.Eugenic Acid), $\mathrm{C}_{10} \mathrm{H}_{12} \mathrm{O}_{2}, 85$ per cent., which chemically resembles Phenol, and forms permanent Salts with Alkalies. This is also found in Oil of Pimenta. (2) A terpene (Caryophyllene), $\mathrm{C}_{15} \mathrm{H}_{24}$.

InCOMPATIBI.ES. - Lime water, iron salts, mineral acids, and gelatin.

Dose, I to $5 \mathrm{~m}$.; [.06 to .30 c.c.]

\section{Action of Cloves and Oil of Cloves.}

Oil of cloves is a typical example of a volatile oil the most important actions of which are exerted in the stomach.

External.-When rubbed into the skin it is stimulant, rubefacient, irritant, and counter-irritant, and gives rise to considerable vascular dilatation. At first it causes a sensation of tingling and pain, which afterwards is replaced by local anæsthesia. It is a parasiticide and antiseptic.

Internal.-Mouth. - In the mouth, oil of cloves produces the same effects as on the skin: there is a burning sensation accompanied by vascular dilatation and an increased flow of 
saliva, and followed by local anæsthesia. Cloves stimulate the nerves of taste, and being volatile and aromatic, those of smell also; by both these means taste is sharpened.

Stomach.-The stimulant effect of cloves is experienced here. The vessels are dilated, peristalsis is accelerated, the secretion of gastric juice is excited, and as cloves are pleasant and aromatic, they do not ordinarily produce nausea; consequently the appetite is increased. The combined effect of these actions is to aid digestive processes-therefore oil of cloves is stomachic; and to facilitate the expulsion of gas - thus it is carminative. The stimulation of the gastric nerves to a slight extent reflexly affects the heart in the same way as alcohol; therefore the rate and force of the pulse are moderately increased.

Intestines.-Here likewise oil of cloves dilates the vessels, and stimulates the secretion and the muscular coat of the intestine; consequently colicky pains due to irregular contraction of it are relieved, and flatus is expelled.

Circulation.-Oil of cloves is readily absorbed from the intestine, circulates in the blood, and is said to increase the number of white corpuscles. It may, to a slight extent, stimulate the heart directly, but the greater part of the stimulation of the heart excited by it is reflex from the stomach. It is credited with the power of arresting painful spasmodic contractions in various parts of the body. It can, as we have seen, do this in the intestine, and possibly it may have to a slight extent the same action in the bronchial tubes, heart, etc. This causes it to be called antispasmodic.

Mucous membranes.-Like other volatile oils it is excreted by the kidneys, skin, bronchi, and genito-urinary tract, and in passing through these structures will act as a stimulating disinfectant to their secretion; but oil of cloves is never used for these purposes.

Therapeutics of Cloves and Oil of Cloves.

External.-Oil of cloves is too [expensive] for frequent external application, but on account of its local anæsthetic effect it 
has been used for neuralgia. It is employed to give a pleasant scent to liniments.

Internal.-The oil is sometimes dropped into decayed teeth to relieve pain. Cloves are frequently employed in cookery for their taste, and because they stimulate the appetite and aid digestion. The oil or infusion [B. P., I to 40] may be used medicinally as a stomachic, as a carminative, as an anti-spasmodic, or to relieve colicky pains in indigestion. It will be noticed that oil of cloves is [sometimes combined with preparations of scammony, of castor oil, and of] colocynth. This is to prevent the griping these purgatives might otherwise cause.

\section{PIMENTA.}

PIMENTA.- [Synonym.-Allspice. The nearly ripe fruit of Pimenta officinalis Lindley (nat. ord. Myrtacee). Habitat.-Tropical America; cultivated.

Characters. - About $5 \mathrm{~mm}$. in diameter, nearly globular, crowned with the short, four-parted calyx or its remnants, and a short style; brownish or brownish gray, granular and glandular, two-celled; each cell containing one brown plano-convex, roundish-reniform seed; odor and taste pungently aromatic, clove-like.] Resembling Pimenta. - Pepper, which has no calyx; Cubeb, which is stalked.

Composition.-The chief ingredient is [Oleum Pimentre (see below), which is chemically almost identical with the volatile oil found in cloves.

Dose, 5 to $30 \mathrm{gr}$; ; 30 to $2.00 \mathrm{gm}$.]

OLEUM PIMENT Æ.-[Oil of Pimenta. Synonym.-Oil of Allspice. A volatile oil distilled from Pimenta.

Characters. - A colorless or pale yellow liquid, having a strong, aromatic, Clove like odor, and a pungent, spicy taste. It becomes darker and thicker by age and exposure to the air. Sp. gr., 1.045 to 1.055. Solubility. -With an equal volume of Alcohol it forms a clear solution.

Composition.-(1) Eugenol, 7o per cent. (2) A sesquiterpene.

Oil of Pimenta is used in Spiritus Myrciz.

Dose, I to $5 \mathrm{~m}$.; .06 to .30 c.c.]

\section{Action and Therapeutics of Allspice.}

The action and uses of pimenta and its oil are precisely the same as those of cloves and oil of cloves. 
PIPER.

PEPPER. - [Synonym. - Black Pepper. The unripe fruit of Piper Nigrum, Linné (nat. ord. Piperacee). Habitat.-India; cultivated in the tropics.

Characters._Globular, about $4 \mathrm{~mm}$. in diameter, reticulately wrinkled, brownish-black, or grayish-black, internally lighter, hollow, with an undeveloped embryo; odor aromatic; taste pungently spicy.] Resembling Black Pepper.-Pimenta, which has a calyx ; Cubeb, which is stalked.

Conpositiox. - The chief constituents are - (I) An Oleoresin, readily yielding a volatile oil, [ $\mathrm{I}$ to 2 per cent.] with the odor of pepper, and a resin. (2) Piperin, (see below), 6 to 8 per cent.

Dose, 5 to $20 \mathrm{gr}$; .30 to $1.20 \mathrm{gm}$.

\section{Preparation.}

Oleoresina Piperis.-Oleoresin of Pepper. By percolation with Ether, distillation and evaporation of the residue.

Dose, $1 / 4$ to I gr.; .015 to $.06 \mathrm{gm}$.

PIPERINUM.-Piperin. $\mathrm{C}_{17} \mathrm{H}_{19} \mathrm{NO}_{3}=284.38$. A neutral principle obtained from Pepper, and obtainable also from other plants of the natural order Piperacece.

Characters. - Colorless or pale yellowish, shining, prismatic crystals, odorless and almost tasteless when first put in the mouth, but on prolonged contact producing a sharp and bitter sensation. Permanent in the air. Solubility. - Almost insoluble in water; soluble in 30 parts of Alcohol; also slightly soluble in Ether. Isomeric with Morphine, it decomposes into Piperic Acid, $\mathrm{C}_{12} \mathrm{H}_{10} \mathrm{O}_{4}$, and a liquid Alkaloid Piperidine, $\mathrm{C}_{5} \mathrm{H}_{11} \mathrm{~N}$.

Dose, I to $10 \mathrm{gr} . ; .06$ to $.60 \mathrm{gm}$.]

\section{Action of Pepper.}

Pepper, because of its volatile oil, acts like other substances containing volatile oils; thus externally it is at first rubefacient and counter-irritant, and subsequently it acts as an anodyne. Internally it increases the secretions of the mouth, and in the stomach it is stomachic and carminative. During its excretion it stimulates the mucous membrane of the genito-urinary tract. Piperin is believed to be a feeble antipyretic and antiperiodic.

\section{Therapeutics of Pepper.}

Occasionally pepper is used externally as an irritant for the same class of cases as mustard. Internally it may be applied in the form of a gargle, as a stimulant for the relaxed conditions of 
the throat. It is taken in the form of a condiment for its stomachic properties. The confection [B. P., Pepper, 2 ; caraway, 3 ; honey, I5. Synonym.-Ward's paste], or pepper lozenges are given empirically to relieve hæmorrhoids, ulcers of the rectum, and fissures of the anus.

\section{MYRISTICA.}

NUTMEG.-[The seed of Myristica Fragrans Houttuyn (nat. ord. Myristicace(e), deprived of its testa. Habitat.-Molucca Islands; cultivated in tropical countries.

Characters. - Oval or roundish-ovate, about $25 \mathrm{~mm}$. long, light brown, reticulately furrowed, with a circular scar on the broad end; internally pale brownish, with dark orange-brown veins, and of a fatty lustre; odor strongly aromatic ; taste aromatic, warm, and somewhat bitter.]

Composition. - The chief constituents are-(I) The fixed oil, 25 to 30 per cent. (see below). (2) The volatile oil (see below), 2 to 8 per cent.

Nutmeg is contained in [Pulvis Aromaticus, and Tinctura Lavandulæ Composita.]

Dose, 5 to $15 \mathrm{gr}$.; [.30 to $\mathrm{r} .00 \mathrm{gm}$.]

[OLEUM MYRISTICE EXPRESSUM. (Not official).-Expressed Oil of Nutmeg. A concrete oil obtained by expression and heat from Nutmeg.

Characters. -Orange-brown or orange-yellow, mottled, of a firm consistence; odor like Nutmeg.

Composition.-The chief constituents are-(I) Glyceryl Oleate, $\mathrm{C}_{3} \mathrm{H}_{5}$ $\left(\mathrm{C}_{18} \mathrm{H}_{33} \mathrm{O}_{2}\right)_{3}$. (2) Glyceryl Butyrate, $\mathrm{C}_{3} \mathrm{H}_{5}\left(\mathrm{C}_{11} \mathrm{H}_{7} \mathrm{O}_{2}\right)_{3}$. (3) Glyceryl, Myristate, $\mathrm{C}_{3} \mathrm{H}_{5}\left(\mathrm{C}_{18} \mathrm{H}_{27} \mathrm{O}_{2}\right)_{3}$. (4) A little volatile oil. (5) A little resin.]

OLEUM MYRISTICAE.-[Oil of Nutmeg. A volatile oil distilled from Nutmeg.

Characters. - A thin, colorless or pale yellowish liquid, having the characteristic odor of Nutmeg and a warm, spicy taste. It becomes darker and thicker by age and exposure to the air. Sp. gr., 0.870 to 0.900. Solubility. - In an equal volume of Alcohol.].

Composition. - The chief constituents are-(1) Myristicene $\left[\mathrm{C}_{10} \mathrm{H}_{16},\right]$, a terpene. (2) Myristicol, $\left[\mathrm{C}_{10} \mathrm{H}_{16} \mathrm{O}\right.$, a stearopten, isomeric with Carvol.

Oil of Nutmeg is contained in Spiritus Ammoniæ Aromaticus.]

Dose, I to $3 \mathrm{~m}$.; [.06 to .20 c.c.]

\section{Preparation.}

Spiritus Myristicæ.-[Spirit of Nutmeg. Synonym.-Essence of Nutmeg. Oil of Nutmeg, 50 ; Alcohol, 950.

Dose, $1 / 2$ to I fi. dr.; 2. to 4 . c.c.] 


\section{MACIS.}

[MACE.-The arillode of the seed of Myristica fragrans Houttuyn (nat. ord. Myristicacea). Habitat.-Molucca Islands; cultivated in the tropical countries.

ChARACTERS. - In narrow bands, $25 \mathrm{~mm}$. or more long, somewhat branched and lobed above, united into broader bands below; brownishorange; fatty when scratched or pressed; odor fragrant, taste warm and aromatic.

Composition.-The chief constituents are-(I) A Volatile Oil, 8 per cent., a greater portion of which is Macene, $\mathrm{C}_{10} \mathrm{H}_{16}$. (2) A red fixed Oil. (3) Resin.

Dose, 5 to $20 \mathrm{gr}$; .30 to $\mathrm{x} .20 \mathrm{gm}$.]

\section{Action and Therapeutics of Nutmeg and Mace.}

The action of oil of nutmeg is the same as that of other aromatic oils. [The expressed oil of nutmeg is used in plasters as a sweet-smelling stimulant.] Nutmeg [and mace] are much employed in cookery for the sake of their volatile oil, which is an agreeable stomachic. A liniment, containing one part of the expressed oil to three of olive oil, is an elegant antiparasitic for mild cases of ringworm.

\section{CINNAMOMUM.}

[CINNAMOMUM SAIGONICUM.-Saigon Cinnamon. The bark of an undetermined species of Cinnamomum (nat. ord. Laurinea). Habitat. -China.

Characters. - In quills about $15 \mathrm{~cm}$. long, and 10 to $15 \mathrm{~mm}$. in diameter, the bark 2 or $3 \mathrm{~mm}$. thick; outer surface gray or light grayish-brown with whitish patches, more or less rough from numerous warts and some transverse ridges and fine longitudinal wrinkles; the inner surface cinnamon-brown or dark brown, granular and slightly striate; fracture short, granular, in the outer layer cinnamon-colored, having near the cork numerous whitish strixe forming an almost uninterrupted line ; odor fragrant ; taste sweet, warmly aro. matic, somewhat astringent.

CINNAMOMUM ZEYLANICUM.-Ceylon Cinnamon. The inner bark of the shoots of Cinnamomum zeylanicum Breyne (nat. ord. Laurinea). Habitat.-Ceylon ; cultivated.

Characters. - Long, closely-rolled quills, composed of eight or more layers of bark of the thickness of paper; pale yellowish-brown; outer surface smooth, marked with wavy lines of bast-bundles; inner surface striate ; fracture short splintery; odor fragrant ; taste sweet and warmly aromatic. 
Composition. - The chief constituents are-(1) A Volatile Oil, $1 / 2$ to $11 / 2$ per cent. (2) Tannic acid. (3) Sugar. (4) Mannit.]

IMPURITY.-Cassia Bark.

\section{Preparations.}

[1. Pulvis Aromaticus.-Aromatic Powder. Ceylon Cinnamon, 35 ; Cardamom, 15 ; Ginger, 35 ; Nutmeg, 15.

Aromatic Powder is used to make Extractum Aromaticum Fluidum.

Dose, 10 to $30 \mathrm{gr}$; ; 60 to $2.00 \mathrm{gm}$.

2. Tinctura Cinnamomi.-Tincture of Cinnamon. Ceylon Cinnamon, 100; Glycerin, 50; Alcohol and Water to 1000. By percolation.

Dose, I to 2 f. dr.; 4. to 8. c.c.

3. Extractum Aromaticum Fluidum.-Aromatic Fluid Extract. Aromatic Powder, by maceration and percolation with Alcohol, and evaporation.

Dose, to to $30 \mathrm{~m}$.; .60 to $2.00 \mathrm{c.c}$.

CINNAMOMUM CASSIA.-Cassia Cinnamon. Synonym.-Cassia Bark. The bark of the shoots of one or more undetermined species of Cinnamomum grown in China (Chinese Cinnamon) (nat. ord. Laurinea). Habitat. -China.

Characters. - In quills of varying length and about $\mathbf{I} \mathrm{mm}$. or more in thickness; nearly deprived of the corky layer; yellowish-brown; outer surface somewhat rough; fracture nearly smooth; odor fragrant; taste sweet, and warmly aromatic.

Composition.-(I) Volatile Oil (see below)

(2) Tannic acid. Sugar. (4) Mannit.

Cassia Cinnamon is contained in Tinctura Cardamomi Composita, Tinctura Catechu Composita, Tinctura Lavandulæ Composita and Vinum Opii.]

OLEUM CINNAMOMI.--[Oi] of Cinnamon. Synonym.-Oil of Cassia. A volatile oil distilled from Cassia Cinnamon.

Cinaracters. - A yellowish or brownish liquid, becoming darker and thicker by age and exposure to the air, having the characteristic odor of Cinnamon, and a sweetish, spicy, and burning taste. Sp. gr., I.055 to r.065. Solubility. - In an equal volume of Alcohol.]

Composition.-The chief constituents are-(I) Cinnamic Aldchyde, $\mathrm{C}_{9} \mathrm{H}_{8} \mathrm{O}$, which makes up the greatest part. (2) Eugenol [found also in the oils of cloves and nutmeg. (3) In old oil, Cinnamic Acid, $\mathrm{C}_{9} \mathrm{H}_{8} \mathrm{O}_{2}$.

Oil of Cinnamon is contained in Acidum Sulphuricum Aromaticum.

Dose, I to $5 \mathrm{~m}$. ; .06 to .30 c.c.]

\section{Preparations.}

[1. Aqua Cinnamomi.-Cinnamon Water. Oil of Cinnamon, 2; by trituration with precipitated Calcium Phosphate and addition of Distilled Water to 1000. 
Cinnamon Water is contained in Infusum Digitalis.

Dose, $1 / 2$ to I fl. oz.; 15 . to 30 . c.c.

2. Spiritus Cinnamomi.-Spirit of Cinnamon. Oil of Cinnamon, 100 ; Alcohol, 900.

Dose, ro to $30 \mathrm{~m}$.; .60 to 2.00 c.c.]

\section{Action and Therapeutics of Cinnamon.}

Oil of cinnamon has the same action as other aromatic volatile oils, and is therefore stomachic and carminative. Cinnamon bark in addition has, in virtue of its tannic acid, some astringent action, and is consequently a common flavoring vehicle for astringent stomachic powders and mixtures, except such as contain iron. Finely powdered cinnamon ( 60 to $90 \mathrm{gr}$. [4, to 6 . gm.]), is given night and morning in acute dysentery.

\section{ARMORACIA.}

HORSE-RADISH. [B. P., not official.]-The fresh root of the Cochlearia armoracia (nat. ord. Crucifera). Habitat.-Cullivated in [the United States and Britain. It is most active in the autumn and early spring, before the leaves have appeared.]

Characters. - A long, cylindrical, fleshy root, enlarged at the upper end, where it is marked by the scars of fallen leaves, [ 12 to $25 \mathrm{~mm}$.] in diameter, and usually $[30 \mathrm{~cm}$.] or more long. Pale yellowish or brownish-white externally; whitish and fleshy within. Taste very pungent. Inodorous unless bruised or scraped. Resembling Horse-Radish Root.-Aconite root, which is shorter, conical, not cylindrical, darker, and causes tingling and numbness when chewed.

Composition. - The chief constituent is a substance which, by the action of an enzyme, yields a volatile oil, Butyl Sulphocyanide, $\mathrm{C}_{4} \mathrm{H}_{9} \mathrm{CNS}$.

\section{Action AND UsES OF HoRse-RAdish.}

Horse-radish is a condiment, having the same action as mustard.. It has been used as a counter-irritant. The [compound] spirit [B. P., scraped horse-radish root, ro; bitter orange peel, ı ; nutmeg, I ; alcohol, I 92 ; water, I 96 ; dose, I to 2 fl. dr. ; 4. to 8. c.c.] is a pleasant flavoring and carminative agent.

\section{CAPSICUM.}

CAPSICUM.-[Synonyms. - Cayenne Pepper. Guinea Pepper. African Pepper. The fruit of Capsicum fastigiatum Blume (nat. ord. Solanacea). Habitat. - Tropical America ; cultivated in tropical countries. 
Characters. - Oblong-conical, from 10 to $20 \mathrm{~mm}$. long, supported by a flattish, cup-shaped, five-toothed calyx, with a red, shining membranous and translucent pericarp, enclosing two cells, and containing flat, reniform, yellowish seeds attached to a thick, central placenta. It has a peculiar odor, and an intensely hot taste.] Dried and powdered it constitutes red pepper.

Composition.-The chief constituents are-(1) Capsaicin, $\left[\mathrm{C}_{9} \mathrm{H}_{14} \mathrm{NO}_{2}\right]$, a crystallizable acrid substance. (2) Capsicin, a volatile Alkaloid smelling like Coniine. (3) A fixed oil. (4) A Resin. (5) Fatty matter.

IMPURITIFs. - Various red substances, e.g., red-lead.

Dose, I to $8 \mathrm{gr}$; ; [.06 to $.50 \mathrm{gm}$.]

\section{Preparations.}

[1. Extractum Capsici Fluidum.-Fluid Extract of Capsicum. By maceration and percolation with Alcohol, and evaporation.

Dose, I to $8 \mathrm{~m}$.; .06 to .50 c.c.

2. Oleoresina Capsici.-Oleoresin of Capsicum. By percolation with Ether and distillation, and evaporation of the residue.

Dose, $1 / 4$ to I m.; .015 to .06 c.c.

3. Tinctura Capsici.-Tincture of Capsicum. Capsicum, 50. By percolation with Alcohol and Water to 1000 .

Dose, 5 to $60 \mathrm{~m}$. ; .30 to 4.00 c.c.

4. Emplastrum Capsici.-Capsicum Plaster. Resin Plaster and Oleoresin of Capsicum. Cover muslin with the melted plaster, coat it when cool, by brushing on the Oleoresin.]

\section{Action of Capsicum.}

The action of capsicum is like that of volatile oils generally. Thus externally it is a powerful rubefacient, irritant, and counterirritant. Internally in small doses it stimulates the gastric secretions, causes dilatation of the gastric vessels, and excites the muscular coat. It is therefore stomachic and carminative.

\section{Therapeutics of Capsicum.}

External.-Capsicum ointment [B. P.-Capsicum, 6 ; spermaceti, 3 ; olive oil, 22 ; which resembles Smedley's paste] is used as a counter-irritant for pleurisy, sciatica, neuralgia and rheumatic pains.

Internal.-Capsicum is used as a condiment. Medicinally it is given as a stomachic and carminative in dyspepsia, particularly that of drunkards when it is required either to excite the appetite and digestion, or to cause the evacuation of gas. 


\section{ZINGIBER.}

GINGER.-[The rhizome of Zingiber officinale Roscoe (nat. ord. Scitaminea). Habitat. - India; cultivated in the tropics.

Characters. - About 5 to $10 \mathrm{~cm}$. long, Io to $15 \mathrm{~mm}$. broad, and 4 to 8 $\mathrm{mm}$. thick, flattish, on one side lobed or clavately branched; deprived of the corky layer; pale, buff-colored, striate, breaking with a mealy, rather fibrous fracture, showing numerous small, scattered resin-cells and fibro-vascular bundles, the latter enclosed by a nucleus sheath ; agreeably aromatic, and of a pungent and warm taste.] Resembling Ginger.-Turmeric, which is yellow.

Composition. - The chief constituents are-(1) An aromatic volatile oil, [3/4 to 2 per cent.] giving the flavor. (2) Resin. [(3) Gingerol, to which the pungent taste is due (Thresh).

Ginger is contained in Pulvis Rhei Compositus and Pulvis Aromaticus.

Dose, 5 to $15 \mathrm{gr}$; .30 to $\mathrm{x} .00 \mathrm{gm}$.]

\section{Preparations.}

I. Extractum Zingiberis Fluidum.-Fluid Extract of Ginger. By maceration and percolation with Alcohol, and evaporation.

Fluid Extract of Ginger is used in Syrupus Zingiberis.

Dose, 5 to $15 \mathrm{~m}$.; .30 to 1.00 c.c.

2. Oleoresina $Z$ ingiberis.-Oleoresin of Ginger. By percolation with Ether, distillation, and evaporation of the residue.

Dose, $1 / 2$ to $2 \mathrm{~m}$.; .03 to .12 c.c.

3. Tinctura Zingiberis.-Tincture of Ginger. Ginger, 200. By percolation with Alcohol to I000.

Tincture of Ginger is used in Trochisci Zingiberis.

Dose, $1 / 4$ to I fl. dr.; r. to 4 . c.c.

4. Syrupus Zingiberis.-Syrup of Ginger. Fluid Extract of Ginger, 30 ; Sugar, 850 ; Water to 1000 . By trituration with Precipitated Calcium Phosphate, solution and filtration.

Dose, $1 / 2$ to 2 fl. dr.; 2. to 8 . c.c.

5. Trochisci Zingiberis.-Troches of Ginger. Tincture of Ginger, 20; Tragacanth, 4 ; Sugar, 130 gm. ; Syrup of Ginger in sufficient quantity to make 100 troches.

Dose, freely.]

\section{Action and Therapeutics of Ginger.}

Its action is the same as that of other substances containing aromatic volatile oils. It is chiefly used as a stomachic, carminative, and flavoring agent. The oleoresin is a useful addition to purgative pills to prevent griping. 


\section{CARDAMOMUM.}

CARDAMOM. - [The fruit of Elettaria repens (Sonnrat) Baillon (nat. ord. Scitaminea). Habitat. -Malabar; cultivated in India.

Characters. - Ovoid or oblong, from 10 to $15 \mathrm{~mm}$. long, obtusely triangular, rounded at the base, beaked, longitudinally striate; of a pale buff color, three-celled, with a thin, leathery, nearly tasteless pericarp, and a central placenta. The seeds are about $4 \mathrm{~mm}$. long, reddish-brown, angular, rugose, depressed at the hilum, surrounded by a thin, membranous arillus, and have an agreeable odor and a pungent, aromatic taste.] -

Composition. - The chief constituents are-(I) A volatile oil, 4 to 5 per cent., which contains a terpene, $\mathrm{C}_{10} \mathrm{H}_{16}$, called Terpinene. (2) A fixed oil, Io to I I per cent. The pericarp is medicinally inactive.

Cardamom is contained in Extractum Colocynthidis Compositum, [Pulvis Aromaticus, Tinctura Gentianæ Composita, Tinctura Rhei, and Tinctura Rhei Dulcis].

Dose, 10 to $15 \mathrm{gr}$; [ $[.60$ to $1.00 \mathrm{gm}$.

\section{Preparations.}

I. Tinctura Cardamomi.-Tincture of Cardamom. Cardamom, 100. By maceration and percolation with Diluted Alcohol to 1000.

Dose, I to $2 \mathrm{fl}$. dr.; 4. to 8. c.c.

2. Tinctura Cardamomi Composita.-Compound Tincture of Cardamom. Cardamom, 20; Caraway, I0; Cassia Cinnamon, 20; Cochineal, 5 ; Glycerin, 50. By percolation with Diluted Alcohol to I000.

Dose, 2 to 4 fl. dr.; 8. to I5. c.c.]

\section{Action and Therapeutics of Cardamom.}

Cardamom, because of its volatile oil, acts like cloves or pepper; therefore it is carminative and stomachic. As it has a pleasant taste, and the [compound] tincture is of a red color, it is much used as a coloring and flavoring agent. A good flavoring carminative is the Tinctura Carminativa of the British Pharmaceutical Conference. It contains cardamom, 6 ; tincture of ginger, 6 ; oil of cinnamom, oil of caraway, oil of cloves, of each, I; rectified spirit to $9^{6}$. Dosie, 2 to 10 minims [. I 2 to $.60 \mathrm{c.c}$.

\section{SUMBUL.}

SUMBUL.-Synonym.-Musk Root. [The root of Ferula Sumbul (Kauffmann) Hooker filius (nat. ord. Umbellifera). Habitat.-Central and Northeastern Asia. 
Characters. - In transverse segments, varying in diameter from about 2 to $7 \mathrm{~cm}$., and in length from 15 to $30 \mathrm{~mm}$.; light, spongy, annulate or longitudinally wrinkled; bark thin, brown, more or less bristly fibrous; the interior whitish, with numerous brownish-yellow resin dots and irregular, easily separated fibres; odor strong, musk-like ; taste bitter and balsamic.]

Composition. - The chief constituents are-(I) A volatile oil. (2) Two Resins. (3) Valerianic Acid. (4) Sumbulic and Angelic Acids.

\section{Preparation.}

Tinctura Sumbul.-[Tincture of Sumbul. Sumbul, I00; by maceration and percolation with Alcohol and Water to 1000 .

Dose, I to 4 fi. dr.; 4 . to I5. c.c.]

\section{Action and Therapeutics of Sumbul.}

The action of sumbul is the same as that of volatile oils in general. It is only used internally, and is given as a carminative in flatulence. It is also employed in much the same class of cases as valerian-that is to say, in neurotic conditions, hysteria, etc. In Russia it is given chiefly as a stimulant in typhoid fever, dysentery, diarrhœa, etc., for the same purposes as musk is employed in many other countries.

\section{OLEUM LAVANDULA [FLORUM.]}

OIL OF LAVENDER [FLOWERS. -A volatile oil distilled from the fresh flowers of Lavandula officinalis Chaix (nat. ord. Labiata). Habitat. - Southern Europe; cultivated.

Characters. -A colorless or yellowish liquid, having the fragrant odor of Lavender Flowers, and a pungent and bitter taste. Sp. gr., 0.885 to 0.897 . Solubility. - In all proportions in Alcohol.

Composition.-The chief constituents are-(I) Linalool Acetate (also found in Oil of Bergamot). (2) Linalool, $\mathrm{C}_{10} \mathrm{H}_{18} \mathrm{O}$, which is an alcohol and an oxidation product of the terpene, Myrcene, $\mathrm{C}_{10} \mathrm{H}_{16}$. It is isomeric with Borneol (see p. 530), Geraniol ( $q . v$.) and Menthol (see p. 547). (3) Cineoli also found in Oil of Eucalyptus (see p. 528) and other volatile oils.

IMPURITY. - Oil of Spike.

Oil of Lavender Flowers is contained in Linimentum Saponis Mollis, Spiritus Ammonix Aromaticus, and Únguentum Diachylon.

Dose, I to $5 \mathrm{~m}$.; .06 to .30 c.c.

\section{Preparations.}

1. Spiritus Lavandula.-Spirit of Lavender. Oil of Lavender Flowers, 50 ; Deodorized Alcohol, 950.

Dose, $1 / 2$ to I fl. dr.; 2. to 4 . c.c. 
2. Tinctura Lavandula Composita.-Compound Tincture of Lavender. Synonym. - Compound Spirit of Lavender. Oil of Lavender Flowers, 8; Oil of Rosemary, 2 ; Cassia Cinnamon, 20 ; Cloves, 5 ; Nutmeg, 10 ; Red Saunders, 10 ; Alcohol, 700 ; Water, 250 ; diluted Alcohol to I000. By mixing and percolation.

Compound Tincture of Lavender is contained in Liquor Potassii Arsenitis.

Dose, $1 / 2$ to I fl. dr.; 2. to 4 . c.c.]

Action and Therapeutics of Oil of Lavender Flowers.

Oil of lavender flowers has the same action as other aromatic volatile oils. It is used externally as a pleasant stimulating component of liniments, and most red lotions (see p. 178) are colored with the compound tincture. This given internally makes a very agreeable gastric stimulant, and carminative agent.

[OLEUM BERGAMOTTEE.

OIL OF BERGAMOT.-Synonym.-Oleum Bergamii. A volatile oil obtained by expression from the rind of the fresh fruit of Citrus Bergamia, Risso et Poiteau (nat. ord. Rutacea). Habitat.-Sicily; naturalized in subtropical countries.

Characters. - A greenish or greenish-yellow, thin liquid, having a peculiar: very fragrant odor, and an aromatic, bitter taste. Sp. gr., o.880 to 0.885. Solubility. - In Alcohol and in Glacial Acetic Acid.

Compositios. - By fractional distillation is obtained-(1) Limonine. (2) Dipentene, $\mathrm{C}_{10} \mathrm{H}_{16}$. (3) Linalool, $\mathrm{C}_{10} \mathrm{H}_{18} \mathrm{O}, 25$ per cent. (4) Linalool acetale, $\mathrm{C}_{12} \mathrm{H}_{20} \mathrm{O}_{2}$, about 20 per cent., and to which the odor is probably due.

\section{Action and Uses of Oil of Berganot.}

Although possessed of the stimulant properties of volatile oils in general, it is used chiefly, if not exclusively, as a perfume

\section{MENTHA PIPERITA.}

PEPPERMINT.-The leaves and tops of Mentha piperita Smith (nat. ord. Labiata. Habitat.-Wild in Asia, Europe, and North America; cultivated.

Characters. - Leaves about $5 \mathrm{~cm}$. long, petiolate, ovate-lanceolate, acute, sharply serrate, glandular, nearly smooth, the few hairs containing crystals of Menthol in one or more thin cells; branches quadrangular, often purplish; flowers in terminal, conical spikes, with a tubular, five-toothed, often purplish, calyx, a purplish, four-lobed corolla, and four short stamens; odor aromatic; taste pungent and cooling. 
Composition.--Its chief constituents are-(I) A volatile oil, (see below); (2) A liquid, and (3) a crystalline Menthol.

\section{Preparation.}

I. Spiritus Menthæ Piperitæ.--Spirit of Peppermint. Synonym. -Essence of Peppermint. Oil of Peppermint, roo; Peppermint, ro. By maceration and percolation with Alcohol to Iooo.

Spirit of Peppermint is contained in Mistura Rhei et Sodæ.

Dose, 5 to $15 \mathrm{~m}$.; .30 to 1.00 c.c.]

OLEUM MENTH E PIPERIT $Æ$.-Oil of Peppermint. [A volatile oil distilled from Peppermint.

Characters. - A colorless, or yellowish, or greenish-yellow liquid, becoming darker and thicker by age and exposure to the air, having the characteristic, strong odor of Peppermint, and a strongly aromatic, pungent taste, followed by a sensation of cold when air is drawn into the mouth. Sp. gr., 0.900 to 0.920 .

Composition.-The chief constituents are-(I) Menthene, $\mathrm{C}_{10} \mathrm{H}_{18}$, the liquid Terpene obtained by distillation. (2) Menthol, the solid Stearopten (q. v.), 50 to 65 per cent.

Oil of Peppermint is contained in Pilulæ Rhei Compositæ.

Dose, $\mathrm{I}$ to $5 \mathrm{~m}$.; .06 to $.30 \mathrm{c.c}$.

\section{Preparations.}

I. Aqua Menthæ Piperitæ.-[Peppermint water. Oil of Peppermint, 2. By trituration with precipitated Calcium Phosphate, and filtration with distilled water to 1000 .

Dose, $1 / 2$ to 2 fl. oz.; 15 . to 6o. c.c.

2. Spiritus Menthæ Piperitæ.-See above.

3. Trochisci Menthæ Piperitæ.-Troches of Peppermint. Oil of Peppermint, I ; Sugar, $80 \mathrm{gm}$. ; Mucilage of Tragacanth to make 100 troches.

Dose, Freely.]

\section{Action and Therapeutics of Peppermint.}

External.-The action of oil of peppermint is the same as that of volatile oils generally, but the cool, numb feeling often produced by volatile oils after the sensation has passed off is especially well marked with oil of peppermint; and this effect, which is due to the menthol in it, has caused it to be applied externally in neuralgia. Like many other volatile oils it is a 
powerful antiseptic. [It is in common use as the "peppermint test" for defective plumbing.]

Internal.-It is often used as a powerful stomachic and carminative, and also as a flavoring agent.

\section{[MENTHA VIRIDIS.}

SPEARMINT.-The leaves and tops of Mentha viridis Linne (nat. ord. Labiate). Habitat.-IVild in Europe and North America ; cultivated.

Characters.-Leaves about $5 \mathrm{~cm}$. long, subsessile, lance-ovate, acute serrate, glandular, nearly smooth; branches quadrangular, mostly light green ; flowers in terminal, interrupted, narrow, acute spikes, with a tubular, sharply five-toothed calyx, a light-purplish, four-lobed corolla, and four rather long stamens; odor aromatic, taste pungent.

Composition.-(1) A volatile oil (see below). (2) Resin. (3) Gum.

\section{Preparation.}

Spiritus Menthæ Viridis.-Spirit of Spearmint. Synonym.Essence of Spearmint. Oil of Spearmint, Ioo; Spearmint, 10. By maceration with Alcohol and filtration to 1000.

Dose, 5 to $15 \mathrm{~m}$.; .30 to 1.00 c.c.]

OLEUM MENTHAE VIRIDIS.-[Oil of Spearmint. A volatile oil distilled from Spearmint.

Characters. - A colorless, yellowish, or greenish-yellow liquid, becoming darker and thicker by age and exposure to the air, having the characteristic, strong odor of Spearmint, and a hot, aromatic taste. Sp. gr., 0.930 to 0.940. Solubility._Freely in Alcohol.]

Composition.-The chief constituents are-(I) Menthene, the same terpene as in Peppermint. (2) [Carvol, $]{ }^{\circ} \mathrm{C}_{10} \mathrm{H}_{14} \mathrm{O}$, a Stearopten isomeric with Thymol $(q \cdot v$.$) .$

Dose, I to $5 \mathrm{~m}$.; [.06 to $.30 \mathrm{c.c}$.

\section{Preparations.}

I. Aqua Menthæ Viridis.-[Spearmint water. 'Oil of Spearmint, 2. By trituration with precipitated Calcium Phosphate, addition of Distilled Water and filtration to 1000.

Dose, $1 / 2$ to 2 fl. oz.; 15 . to 6o. c.c.]

2. Spiritus Menthæ Viridis.-(See alıove).

\section{Action and Therapeutics of Spearmint.}

These are the same as those of [peppermint and] oil of peppermint, [but oil of spearmint is not so agreeable.] 


\section{ANISUM.}

ANISE.-[The fruit of Pimpinella Anisum Linne (nat. ord. Umbellifera). Habitat. - Western Asia, Egypt, Southeastern Europe ; cultivated.

Characters. - About 4 or $5 \mathrm{~mm}$. long, ovate, compressed at the sides, grayish, finely bairy, and consisting of two mericarps, each with a flat face, and five light, brownish, filiform ridges, and about 15 thin oil tubes, which can be seen in a transverse section by the microscope. It has an agreeable aromatic odor, and a sweet, spicy taste. -Resembling Anise.-Conium, which has single mericarps, smooth, grooved upon the face, and having crenate ridges with wrinkles between them, and no oil-tubes.

Composition.-The chief constituent is the official volatile oil (see below). Anise is contained in Tinctura Rhei Dulcis.

Dose; 10 to $30 \mathrm{gr}$; ; 60 to $2.00 \mathrm{gm}$.

ILLICIUM.-Synonym.-Star Anise. The fruit of Illicium verum Hooker filius (nat. ord. Magnoliacee). Habitat.-Northern Anam.

Characters. - The fruit is pedunculate and consists of eight stellately arranged carpels, which are boat-shaped, about $10 \mathrm{~mm}$. long, rather woody, wrinkled, straight-beaked, brown, dehiscent on the upper suture, internally reddish-brown, glossy, and containing a single, flattish, oval, glossy, brownishyellow seed ; odor anise-like; taste of the carpels sweet and aromatic, and of the seeds oily. Resembling Star Anise.-Illicium anisatum Linné (Illicium religiosum Siebold), the carpels of which are more woody, shrivelled, and have a thin, mostly curved beak, a faint, clove-like odor, and an unpleasant taste.

Composition.-The chief constituent is the volatile oil.

Oleun Anisi may be distilled from this as well as from Pimpinella Anisum.

Dose, 5 to $30 \mathrm{gr}$. ; .30 to $2.00 \mathrm{gm}$.]

OLEUM ANISI.-[Oil of Anise. A volatile oil distilled from Anise.

Characters. - A colorless or pale yellow, thin, and strongly refractive liquid, having the characteristic odor of Anise, and a sweetish, mildly aromatic taste. That from the Pimpinella Anisum solidifies at $59^{\circ} \mathrm{F} .\left[15^{\circ} \mathrm{C}.\right]$; that from Illicium verum (Star-anise) at about $50^{\circ} \mathrm{F}$. [10 $\left.10^{\circ} \mathrm{C}.\right]$. Sp. gr., about 0.980 to 0.990 . Solubility. - In an equal volume of Alcohol.]

Composition. - The chief constituents are-(I) A Terpene, $\left[\mathrm{C}_{10} \mathrm{H}_{16}\right.$, in small quantity. (2) A Stearopten, anethol, $\mathrm{C}_{10} \mathrm{H}_{12} \mathrm{O}, 80$ per cent.

Oil of Anise is contained in Tinctura Opii Camphorata, Spiritus Aurantii Compositus, Syrupus Sarsaparillæ Compositus, and Trochisci Glycyrrhize et Opii.

Dose, I to $5 \mathrm{~m} . ; .06$ to $.30 \mathrm{c.c}$.]

\section{Preparations.}

1. [Aqua Anisi.-Anise water. Oil of Anise, 2. By trituration with precipitated Calcium Phosphate, addition of Water and filtration to 1000 .

Dose, $1 / 4$ to I fl. oz.; 8. to 3 o. c.c. 
2. Spiritus Anisi.-Spirit of Anise. Oil of Anise, 100; Alcohol, 900 .

Dose, I to 2 fl. dr.; 4. to 8. c.c.]

\section{Action and Therapeutics of Anise.}

The action of oil of anise is the same as that of aromatic oils generally. It is specially used to get rid of flatulence in children, and, on account of its slightly exfectorant action, as a basis of cough mixtures.

\section{CORIANDRUM.}

CORIANDER.-[The fruit of Coriandrum sativum Linnè (nat. ord. Umbellifera). Habitat.—Central Asia and Southern Europe; cultivated.

Characters. - Globular; about $4 \mathrm{~mm}$. in diameter; crowned with the calyx-teeth and stylopod; brownish-yellow, with slight, longitudinal ridges; the two mericarps cohering, enclosing a lenticular cavity, and each furnished on the face with two oil-tubes; odor and taste agreeably aromatic.]

Composition.-The chief constituent is the official volatile oil (see below).

Dose, 10 to $30 \mathrm{gr}$; [ [.60 to $2.00 \mathrm{gm}$.]

OLEUM CORIANDRI.-[Oil of Coriander. A volatile oil distilled from Coriander.

Characters. - A colorless or slightly yellowish liquid, having the characteristic, aromatic odor of Coriander, and a warm, spicy taste. Sp. gr., 0.870 to 0.885 .

Composition.-(1) Pinene, the chief terpene of Oil of Turpentine, 5 per cent. (2) Coriantrol, $\mathrm{C}_{10} \mathrm{H}_{18} \mathrm{O}$, which is isomeric with Borneo Camphor $\left(q . v_{0}\right)$.]

Oil of Coriander is contained in Syrupus Sennæ, [Confectio Sennæ, and Spiritus Aurantii Compositus.

Dose, 2 to $5 \mathrm{~m}$.; .12 to .30 c.c.]

\section{Action and Therapeutics of Coriander.}

Oil of coriander has the same action as other volatile oils. It is chiefly used as a stomachic and carminative, and to disguise the taste of rhubarb and senna.

\section{FCENICULUM.}

FENNEL.- [The fruit of Faniculum capillaceum Gilibert (nat. ord. Umbellifere). Habitat.-Levant and Southern Europe; cultivated.

CharActers. - Oblong, nearly cylindrical, slightly curved, from 4 to 8 $\mathrm{mm}$. long, brownish or greenish-brown; readily separable into the two prominent mericarps, each with five light-brown, obtuse ribs, four oil-tubes on the 
back, and two or four oil-tubes upon the flat face; odor and taste aromatic, anise-like.] Resembling Fennel.-Conium fruit (Fennel is larger and has prominent vittæ, [oil-tubes]), Caraway and Anise fruits.

Composimion. - The chief constituent is the official volatile oil, probably [chemically] identical with Oil of Anise (see p. 549).

[Fennel is contained in Infusum Sennæ Compositum.

Dose, 15 to $30 \mathrm{gr}$; ; 1 . to $2 . \mathrm{gm}$.

OLEUM FENICULI.-Oil of Fennel. A volatile oil distilled from Fennel.

Characters. - A colorless or pale yellowish liquid, having the characteristic, aromatic odor of Fennel, and a sweetish, mild and spicy taste. Sp. gr., not less than 0.960. Solubility. - In an equal volume of Alcohol.

Oil of Fennel is contained in Spiritus Juniperi Compositus and Pulvis Glycyrrhizx Compositus.

Dose, I to $5 \mathrm{~m}$.; .06 to .30 c.c.

\section{Preparations.}

Aqua Fœniculi.-Fennel water. Oil of Fennel, 2. By trituration with precipitated Calcium Phosphate, addition of Distilled Water; and filtration to 1000 .

Dose, $1 / 4$ to I fl. oz.; 8. to 3o. c.c.]

\section{Action and Therapeutics of Fennel.}

These are same as of oil of anise or of coriander.

\section{CARUM.}

CARAWAY.-[The fruit of Carum Carvi Linne (nat. ord. Umbelliferce). Habitat.-Central and Western Asia; cultivated.

Characters.-Oblong, laterally compressed, about 4 or $5 \mathrm{~mm}$. long, usually separated into the two mericarps, which are curved, narrower at both ends, brown, with five yellowish, filiform ribs, and with six oil-tubes. Caraway has an agreeable odor, and a sweetish, spicy taste.] Resembling Caraway.-Conium and Fennel. Caraway is known by its small ridges and spicy taste.

Compositron.-The chief constituent is the official volatile oil (see below), [5 to 7 per cent.

Carazvay is contained in Tinctura Cardamomi Composita.]

Dose, 15 to $30 \mathrm{gr}$.; [1. to 2. gm.]

OLEUM CARI.-[Oil of Caraway. A volatile oil distilled from Caraway.

Characters. - A colorless, or pale yellow, thin liquid, having the characteristic, aromatic odor of Caraway, and a mild, spicy taste. Sp. gr., o.9ro to 0.920 . Solubility. - In an equal volume of Alcohol.] 
Composition. - The chief constituents are-(I) Cymene, $\mathrm{C}_{10} \mathrm{H}_{14}$; also found in Oil of Eucalyptus (see p. 528). (2) [Carvol, ] $\mathrm{C}_{10} \mathrm{H}_{14} \mathrm{O}$, isomeric with Thymol (q. v.), also found in Oil of Spearmint. (3) Limonene, a ter. pene, $\mathrm{C}_{10} \mathrm{H}_{16}$; also found in Oil of Lemon $\left(q . v_{0}\right)$.

[Oil of Caraway is contained in Spiritus Juniperi Compositus.]

Dose, I to $5 \mathrm{~m}$. ; [.06 to .30 c.c.]

\section{Action and Therapeutics of Caraway.}

The action and uses of oil of caraway are the same as those of other aromatic volatile oils. It is employed as a carminative, stomachic and flavoring agent.

\section{ANETHUM.}

DILL.-[B. P., not official.]-The dried fruit of Peucedanum gravcolens (nat. ord. Umbelliferce). Habitat.-Middle and Southern Europe; [cultivated.]

Characters.-Broadly oval, [12 mm.] long, brown, fiat, with a pale, broad membranous border. Mericarps distinct, odor and taste agreeable and aromatic. Resembling Dill.-Conium, Anise, Fennel, Caraway; but Dill is winged.

Compositron.-The chief constituent is the volatile oil (see below.)

OLEUM ANETHI.-[Oil of Dill. B. P., (not official)]. The oil distilled from Dill fruit.

Charácters. - Pale yellow, odor pungent, taste hot and sweetish. Sp. gr., 0.905 to 0.920 .

CoMposiriox.-The chief constituents are almost identical with those of Oil of Caraway (see p. 55I).

Dose, I to $4 \mathrm{~m}$. ; [.06 to .25 c.c. $]$

\section{Action and Therapeutics of Dill.}

The same as those of anise and caraway. Dill water [B. P.; dill fruit, I; water, Io; dose, I to 2 fl. oz., 30 . to 6o. c.c.] is a common carminative for children, and it covers very well the taste of sodium salts.

\section{SAMBUCUS.}

SAMBUCUS.-Synonym.-Elder. [The flowers of Sambucus canadensis Linne (nat. ord. Caprifoliacea). Habitat.-North America, west to the Rocky Mountains, in damp places.

Characters. - The flowers, when fresh, about $5 \mathrm{~mm}$. broad, and after drying shrivelled; calyx superior, minutely five-toothed; corolla originally 
cream-colored, after drying pale brownish-yellow, wheel-shaped and fivelobed, with five stamens on the short tube; odor peculiar; taste sweetish, somewhat aromatic and bitterish.]

Compositron.-The chief constituents are-(I) A resin. (2) Valerianic Acid. (3) A volatile oil.

Dose, $1 / 2$ to I dr. ; [2. to $4 . \mathrm{gm}$.]

\section{Action and Therapeutics of Sambucus.}

Elder flowers are used to flavor medicines, [and are gently stimulant and diaphoretic.

\section{HEDEOMA.}

HEDEOMA.-Synonym.-Pennyroyal. The leaves and tops of Hedeoma pulegioides (Linné) Persoon (nat. ord. Labiata). Habitat.-North America, south to Georgia, and west to Dakota ; in sandy fields.

Characters.-Leaves opposite, short-petioled, about $12 \mathrm{~mm}$. long, oblong-ovate, obscurely serrate, glandular beneath; branches roundish-quadrangular, hairy; flowers in small axillary cymules, with a tubular ovoid, bilabiate and five-toothed calyx, and a pale blue, spotted, bilabiate corolla, containing two sterile and two fertile, exserted stamens; odor strong, mintlike; taste warm and pungent.

Composition.-The chief constituent is the official volatile oil.

OLEUM HEDEOM $\mathbb{E}$.-Oil of Hedeoma. Synonym.-Oil of Pennyroyal. A volatile oil distilled from Hedeoma.

Characters. - A pale yellowish, limpid liquid, having a characteristic, pungent, mint-like odor and taste. Sp. gr., 0.930 to 0.940. Solubility.Freely in Alcohol.

Dose, $\mathrm{I}$ to $5 \mathrm{~m}$.; .06 to $.30 \mathrm{c.c}$.

\section{Action and Uses of Pennyroyal.}

Pennyroyal is a gentle aromatic stimulant, and may be given in flatulent colic and sick stomach. The oil is in common use locally applied as a remedy for mosquito bites.

\section{ABSINTHIUM.}

ABSINTHIUM.-Synonym.-Wormwood. The leaves and tops of Artemisia Absinthium Linne (nat. ord. Composita). Habitat.-Northern Asia, Europe and Northern Africa ; naturalized in North America; cultivated.

Characters. - Leaves about $5 \mathrm{~cm}$. long, hoary, silky-pubescent, petiolate, roundish-triangular in outline; pinnately two- or three-cleft, with the segments lanceolate, the terminal one spatulate; bracts three-cleft or entire; 
heads numerous, about $3 \mathrm{~mm}$. long, subglobose, with numerous small, pale yellow florets, all tubular and without pappus; odor aromatic; taste persistently bitter.

Composition.-The principal constituents are-(I) A volatile oil, about I per cent., mainly Absinthol, $\mathrm{C}_{10} \mathrm{H}_{16} \mathrm{O}$. (2) A bitter glucoside, Absinthin, $\mathrm{C}_{15} \mathrm{H}_{20} \mathrm{O}$. (3) Absinthic Acid. The volatile oil mixed with alcohol and oil of anise is known as absinthe, a beverage much esteemed in France.

Dose, 8 to $40 \mathrm{gr}$; .50 to $2.40 \mathrm{gm}$.

\section{Action AND Uses of Wormwood.}

Wormwood is used in an infusion as an aromatic tonic in atony of stomach or intestines.]

\section{ANTHEMIS.}

ANTHEMIS.-Synonym.-Chamomile. [The flower-heads of Anthemis nobilis Linné (nat. ord. Composita), collected from cultivated plants. Habitat.-Southern and Western Europe; cultivated; naturalized in a few localities in the United States.

Characters. - Heads subglobular, about $2 \mathrm{~cm}$. broad, consisting of an imbricated involucre, and numerous white, strap-shaped, three-toothed florets, and few, or no, yellow tubular disk florets, inserted upon a chaffy, conical, solid receptacle. It has a strong, agreeable odor, and an aromatic, bitter taste.

Composition.-The chief constituent is the volatile oil.

Dose, $1 / 2$ to $2 \mathrm{dr}$; 2 2. to 8 . $\mathrm{gm}$.]

OLEUM ANTHEMIDIS.-[B. P., not official].-Oil of Chamomile.-The volatile oil distilled from Chamomile flowers.

Characters. - Pale blue or greenish-blue, becoming yellowish-brown. Odor and taste like Chamomile.

Composition. - The chief constituents are-( $\mathrm{I})$ A terpene, $\mathrm{C}_{10} \mathrm{H}_{16}$. [Anthemol, $\mathrm{C}_{10} \mathrm{H}_{16} \mathrm{O}$.] (3) [Anthemene, $\mathrm{C}_{18} \mathrm{H}_{36}$, in tasteless needles.] A bitter principle.

Dose, I to $4 \mathrm{~m}$.; [.06 to .25 c.c.]

\section{Action and Therapeutics of Chamomile.}

External.-A poultice made with chamomile flowers is a poptular domestic remedy. Its virtues are due to its warmth.

Internal.-Like other volatile oils, oil of chamomile is a stomachic and carminative. The infusion [ $I$ to 20$]$, in large doses $[5$ to 10 fl. oz.; 1 50 . to 300 . c.c. $]$ is a simple emetic. 
[MATRICARIA.

MATRICARIA.-Synonym.-German Chamomile. The flower-heads of Matricaria Chamomilla, Linné (nat. ord. Composite). Habitat.-Europe and Western Asia.

Characters. - About $\mathrm{I} 5$ to $20 \mathrm{~mm}$. broad, composed of a flattish, imbri. cate involucre, a conical, hollow, naked receptacle, which is about $5 \mathrm{~mm}$. high, about fifteen white, ligulate, reflexed ray florets, and numerous yellow, tubular, perfect disk-florets without pappus; strongly aromatic and bitter. Resembling Matricaria.-Anthemis Arvensis and Anthemis Cotula, but these have conical, solid, and chaffy receptacles.

Composition.-(1) Volatile Oil $1 / 4$ per cent. (2) Anthemic Acid. Anthemidin, probably a glucoside. (4) Tannic acid.

Dose, $1 / 4$ to $\mathrm{I} \mathrm{dr.;} \mathrm{I.} \mathrm{to} 4$. $\mathrm{gm}$.

\section{Action and Uses of Matricaria.}

The actions and uses are identical with those of chamomile.]

ROSA GALLICA.-Red Rose. [The petals of Rosa gallica Linné (nat. ord. Rosacea), collected before expanding. Habitat.-Asia Minor and Southern Europe; cultivated.

CHARACTERS. - Usually in small cones, consisting of numerous imbricated, roundish, retuse, deep purple-colored, yellow-clawed petals, having a roseate odor and a bitterish, slightly acidulous and distinctly astringent taste.

Composition.-The chief constituents are-(I) A volatile oil, in minute quantities. (2) Tannic Acid. (3) Mucilage. (4) Sugar.

Red Rose is contained in Pilulæ Aloes et Mastiches.]

\section{Preparations.}

1. Confectio Rosæ.-[Confection of Rose. Red Rose, 80; Sugar, 640 ; Clarified Honey, 12 ; Stronger Rose Water, 16o.

Dose, $1 / 2$ to I dr.; 2 . to 4 . $\mathrm{gm}$.

2. Extractum Rosæ Fluidum.-Fluid Extract of Rose. By maceration with Glycerin and Diluted Alcohol, and evaporation.

Fluid Extract of Rose is used to make Mel Rosie and Syrupus Rosæ.

Dose, $1 / 4$ to I fl. dr.; I. to 4 . c.c.

3. Mel Rosæ.-Honey of Rose.-Fluid Extract of Rose, I20; Clarified Honey, to Iooo.

Dose, freely.

4. Syrupus Rosa.-Syrup of Rose. Fluid Extract of Rose, 125 ; Syrup, 875.

Dose, I to 2 fl. dr. ; 4. to 8. c.c.

ROSA CENTIFOLIA.-Pale Rose. The petals of Rosa centifolia Linné (nat. ord. Rosacea). Habitat.-Western Asia; cultivated. 
Characrers. - Roundish-obovate and retuse, or obcordate, pink, fragrant, sweetish, slightly bitter and faintly astringent.

Composition. - (I) Volatile oil, in minute quantities. (2) Mucilage. Tannic Acid. (4) Malates and Tartrates. .

OLEUM ROS E.-Oil of Rose. Synonym.-Attar of Rose. A volatile oil distilled from the fresh flowers of Rosa damascena Miller (nat. ord. Rosace(e). Habitat._Cultivated in Bulgaria.

Characters. - A pale yellowish, transparent liquid, having the strong fragrant odor of Rose, and a mild, slightly sweetish taste. Sp. gr., 0.865 to o.88o. Solubility. - It is but slightly soluble in Alcohol.]

Composirtion.-Its principal constituent is Khodinol, or Geraniol, $\mathrm{C}_{10} \mathrm{H}_{18} \mathrm{O}$, a volatile oil, 12 to 14 per cent., very fragrant, It is an alcohol and is related to Linalool which occurs in Oil of Lavender flowers.

[IMPURITIEs. -Oil of Ginger-grass or Turkish Oil of Geranium, Oil of Rose Geranium, Spermaceti and Paraffin.]

\section{Preparations.}

[I. Aqua Rosæ Fortior.-Stronger Rose Water. Synonym.Triple Rose Water. Water saturated with the volatile oil of Rose petals, obtained as a by-product in the distillation of Oil of Rose.

Stronger Rose Water is used to make Confectio Rosx.

Dose, indefinite.

2. Aqua Rosæ.-Rose Water. Stronger Rose Water, and Distilled Water, of each, one volume.

Dose, indefinite.

3. Unguentum Aqux Rosæ.-Ointment of Rose Water. Synonym.-Cold Cream. Spermaceti, 125; White Wax, 120; Expressed Oil of Almond, 600 ; Stronger Rose Water, 190; Sodium Borate, 5.]

\section{Action and Therapeutics of Rose.}

The preparations of rose are pleasant vehicles, the confection for pills and the water for lotions. The infusion ([B. P., not official] dried petals, 2 ; diluted sulphuric acid, I; water, 80) is mildly astringent. [The ointment of rose water is a favorite soothing application for the skin.]

\section{CIASS III.-THOSE USED CHIEFLY FOR THEIR ACTION ON THE HEART AND CENTRAL NERVOUS SYSTEM.}

\section{VALERIANA.}

VALERIAN. - The rhizome and roots of Valeriana officinalis [Linne (nat. ord. Valerianea). Habitat.-Europe and Northern Asia; naturalized in England; cultivated. 
Characters. - Rhizome from 2 to $4 \mathrm{~cm}$. long, and I to $2 \mathrm{~cm}$. thick, upright, subglobular or obconical, truncate at both ends, brown or yellowish. brown, internally whitish or pale brownish, with a narrow circle of white wood under the thin bark. Roots numerous, slender, brittle, brown with a thick bark, and slender, ligneous cord. Odor peculiar, becoming stronger and unpleasant on keeping ; taste camphoraceous and somewhat bitter.] Resembling Valerian.-Serpentaria, Arnica, Green Hellebore; but Valerian is known by its odor.

Composition. - The chief constituents are-(I) A volatile oil, $1 / 2$ to 2 per cent., consisting of Borneol, $\mathrm{C}_{10} \mathrm{H}_{18} \mathrm{O}$, and Pinene, a terpene (see p. 516). (2) Valerianic Acid, $\mathrm{C}_{5} \mathrm{H}_{10} \mathrm{O}_{2}$. It is colorless, oily, with the odor of Valerian, and strongly acid, with a burning taste. Solubility. - In 30 parts of water; readily in Alcohol and Ether. The amount of it in Valerian increases by keeping, while that of the oil decreases. It can be derived from Amylic Alcohol, $\mathrm{C}_{5} \mathrm{H}_{12} \mathrm{O}$ (Valeryl Aldehyde). [(3) Formic, Acetic and Malic Acids.

(4) Tannic acid. (5) Resin.

Dose, yo to $30 \mathrm{gr}$.; .60 to $2.00 \mathrm{gm}$.]

\section{Preparations.}

1. [Extractum Valerianæ Fluidum.-Fluid Extract of Valerian. By maceration and percolation with Alcohol and Water, and evaporation.

Dose, 10 to $30 \mathrm{~m} . ; .60$ to 2.00 c.c.

2. Tinctura Valerianæ.-Tincture of Valerian. Valerian, 200; by maceration and percolation with Alcohol and Water to 1000 .

Dose, $1 / 2$ to 2 fl. dr.; 2. to 8 . c.c.

3. Tinctura Valerianæ Ammoniata.-Ammoniated Tincture of Valerian. Valerian, 200; by maceration and percolation with Aromatic Spirit of Ammonia to 1000 .

Dose, $1 / 2$ to 2 fl. dr.; 2. to 8 . c.c.

AMMONII VALERIANAS.-Ammonium Valerianate. $\mathrm{NH}_{4} \mathrm{C}_{5} \mathrm{H}_{9} \mathrm{O}_{2}$ $=118.78$.

Source.-By saturating Valerianic Acid with Gaseous Ammonia, obtained from a mixture of Ammonium Chloride and Lime, and crystallization.

ChARACTERS. - Colorless, or white, quadrangular plates, emitting the odor of Valerianic Acid, of a sharp and sweetish taste, and deliquescent in moist air. Solubility.-Very soluble in Water and in Alcohol; also soluble in Ether.

Dose, 2 to $8 \mathrm{gr}$; ;.12 to $.50 \mathrm{gm}$.

FERRI VALERIANAS.-Ferric Valerianate.

SOURCE.-By precipitating a diluted solution of Ferric Sulphate, with a solution of Sodium Valerianate and washing the precipitate. 
Characters. - A dark, brick-red, amorphous powder of somewhat varying chemical composition, having the odor of Valerianic Acid, and a mildly styptic taste; permanent in dry air. Solubility.-Insoluble in cold water, but readily soluble in Alcohol.

Dose, I to $3 \mathrm{gr}$; .06 to $.20 \mathrm{gm}$.

SODII VALERIANAS.-(Not official.)-Sodium Valerianate. $\mathrm{NaC}_{5}$ $\mathrm{H}_{9} \mathrm{O}_{2}=123.77$.

Sou RCE. - Make Valerianic Acid by distilling a mixture of Amylic Alcohol, Sulphuric Acid, and Potassium Bichromate. Saturate the distillate which contains Valerianic Acid, with Caustic Soda, and evaporate.

Characters. - White masses of a powerful Valerian-like odor. Solubility. -Easily in both Alcohol and Ether.

Dose, I to $5 \mathrm{gr}$; .06 to $.30 \mathrm{gm}$.

QUININAE VALERIANAS.--Quinine Valerianate. $\mathrm{C}_{20} \mathrm{H}_{24} \mathrm{~N}_{2} \mathrm{O}_{2}$ $\mathrm{C}_{5} \mathrm{H}_{10} \mathrm{O}_{2}+\mathrm{H}_{2} \mathrm{O}=443.07$.

Source.-By decomposing Quinine Sulphate by Ammonia, combining directly with Valerianic Acid, and crystallizing from a cold solution.

Characters. - White, or nearly white, pearly, lustrous, triclinic crystals, having a slight od'r of Valerianic Acid, and a bitter taste. Permanent in the air. Solubility.-In 100 parts of Water, and in 5 parts of Alcohol.

Dose, I to $30 \mathrm{gr}$. ; .06 to $2.00 \mathrm{gm}$.]

ZINCI VALERIANAS.-Zinc Valerianate. $\mathrm{Zn}\left(\mathrm{C}_{5} \mathrm{H}_{9} \mathrm{O}_{2}\right)_{2}\left[+2 \mathrm{H}_{2} \mathrm{O}\right.$ $=302.56$.

SOURCE.-From hot solutions of Zinc Sulphate and Sodium Valerianate; evaporate and Zinc Valerianate crystallizes out.

ChAracters. - White, pearly scales, having the odor of Valerianic Acid, and a sweetish, astringent and metallic taste. On exposure to the air it slowly loses Valerianic Acid. Solubility.-In about 100 parts of water, and in 40 parts of Alcohol.]

INCOMPATIBLES.-All acids, soluble carbonates, most metallic salts and vegetable astringents.

Dose, $1 / 2$ to $3 \mathrm{gr}$; [.03 to $.20 \mathrm{gm}$. $]$

\section{Action of Valerian and the Valerianates.}

Neither valerianic acid, [ammonium, ferric, sodium, quinine] nor zinc valerianates are known to have any action [although their extensive use warrants the belief that they are valuable remedies.]

Valerian itself acts in virtue of its volatile oil, which has the same properties as other volatile oils. Valerian is therefore an irritant when applied externally; internally it stimulates the 
mouth, stomach, and intestines; consequently it increases the appetite and the vascularity, the secretion, and the peristaltic action of the stomach, and intestines; and in its excreticn, which takes place chiefly through the bronchial mucous membrane, kidneys and genito-urinary mucous membrane, it excites the flow of fluids excreted through these parts. Acting reflexly from the stomach, it stimulates the circulation rather more powerfully than most volatile oils.

Therapeutics of Valerian and the Valerianates.

Preparations of valerian, or still better the oil [not official, 2 to $5 \mathrm{~m}$., . I 2 to .30 c.c.] suspended in mucilage with cinnamon water, are often given as carminatives in cases of flatulence, and as reflex stimulants in fainting, palpitation, etc. Valerian and [the] valerianates sometimes relieve neuralgia, and they are often prescribed for hysteria and other neurotic conditions, but frequently without benefit.

\section{[CYPRIPEDIUM.}

CYPRIPEDIUM.-Synonym.-Ladies' Slipper. The rhizome and roots of Cypripedium pubescens Swartz, and of Cypripedium parviflorum Salisbury (nat. ord. Orchideæ). Habitat.-North America; in swampy places.

ChaRACTERS.-Of horizontal growth, bent, Io $\mathrm{cm}$., or less, long ; from 3 to $5 \mathrm{inm}$. thick; on the upper side beset with numerous circular, cup-shaped scars; closely covered below with simple, wiry roots, varying from 10 to 15 $\mathrm{cm}$. in length ; brittle, dark brown, or orange-brown; fracture short, white; odor peculiar, heavy ; taste sweetish, bitter and somewhat pungent.

Composition.-It contains-(1) A volatile oil. (2) A volatile acid. Two resins. (4) Tannic acid.

Dose, 15 to $30 \mathrm{gr}$; ; 1 to 2. gm.

\section{Preparation.}

Extractum Cypripedii Fluidum.-Fluid Extract of Cypripedium. By maceration and percolation with Diluted Alcohol, and evaporation.

Dose, 15 to $30 \mathrm{~m}$; 1 . to 2. c.c.

\section{Action and Uses of Cypripedium.}

Cypripedium is a gentle, nervous stimulant, resembling valerian in its action. It has been used for nervous diseases, epilepsy, hypochondriasis and neuralgia.] 


\section{ASAFCTIDA.}

ASAFETIDA.-A gum-resin obtained [from the root of Ferula fatida (Bunge) Regel (nat. ord. Umbellifere). Habitat.-Persia, Turkestan and Afghanistan.

Characters. - In irregular masses composed of whitish tears, which are imbedded in a yellowish-gray or brownish-gray, sticky mass. The tears, when hard, break with a conchoidal fracture, showing a milk-white color, which changes, gradually, on exposure, to pink, and finally to brown. It has a persistent, alliaceous odor, and a bitter alliaceous acrid taste. Solubility.-Partly in Ether and Alcohol.] Resembling Asafetida.-Galbanum, Ammoniacum, and Benzoin, distinguished by their peculiar odors, which differ markedly from that of Asafetida.

CoMposition. - The chief constituents are-(I) A volatile oil, 5 per cent., the most important ingredient of which is [Allyl sulphide (see p. 455).] This gives Asafetida its very unpleasant odor. (2) Gum, 25 per cent. (3) Bassorin resin, 65 per cent., [which contains Ferulaic Acid, $\mathrm{C}_{10} \mathrm{H}_{10} \mathrm{O}_{4}$.]

IMPURITIES. - Earthy matter [or Calcium Sulphate and Carbonate, and sand.]

\section{Preparations.}

r. [Emulsum Asafœtidæ.-Emulsion of Asafetida. Synonyms. -Mistura Asafcetidx. Milk of Asafetida. Asafetida, 40 ; by rubbing in a warmed mortar with Water, and straining to 1000 .

Dose, $1 / 2$ to $x$ fl. oz. ; 15 . to 30 . c.c.

2. Pilulæ Aloes et Asafœtidæ.-See Aloes, p. 497.

3. Pilulæ Asafœetidæ. - Pills of Asafetida. Asafetida, 20; Soap, $60 \mathrm{gm}$. ; to make 100 pills. Each pill contains $3 \mathrm{gr}$; .20 gm. of Asafetida.

Dose, $x$ to 4 pills.

4. Tinctura Asafcetidæ.-Tincture of Asafetida. Asafetida, 200. By maceration with Alcohol, and filtration to 1000 .

Dose, $1 / 2$ to 1 fl. dr. ; 2. to 4 . c.c.]

\section{Action of Asafetida.}

Both internally and externally, asafetida, in virtue of its volatile oil, acts like volatile oils generally. Its action as a stimulant to the intestinal muscle is especially well marked, hence it is combined with aloes in Pilılæ Aloes et Asafœtidæ; and the enema, [ $\mathrm{r}$ to 64 of water,] will relieve flatus. Owing to its containing [allyl sulphide] it is extremely [unpleasant], and therefore it is not, like many volatile oils, available as a condiment. Its taste is credited with some mental effect in cases of hysteria. 


\section{Therapeutics of Asafetida.}

Asafetida is not used externally. Internally it is prescribed to aid the action of other purgatives, and also to stimulate the muscular coat to expel flatus. It may be given by the mouth or as an enema. Partly on account of its reflex stimulating effect, but also on account of its very [unpleasant] taste, it is used to control hysteria, emotional, and other mental disturbances, but it often fails. For this purpose it may be combined with valerian. Cases of malingering may sometimes be cured by making the patient take, three times a day, an effervescing draught containing a few [drops] of each of the tinctures of valerian and asafetida, with some mucilage to suspend the precipitated resin. The effervescence makes the [unpleasant] taste of these medicines "repeat" in the mouth for some time after taking them. Asafetida oil would in the course of its excretion disinfect the urine and the expectoration, but its smell forbids its use for these purposes.

\section{GALBANUM.}

GALBANUM. [B. P., not official.] - A gum-resin obtained from Ferula galbanifua, Ferula rubricaulis (nat. ord. Umbellifera), and probably other species. [Habitat.]-Persia and the Levant.

Characters. - Tears or masses of agglutinated tears. Tears roundish, about the size of a pea, yellowish-brown or yellowish-green. Translucent, rough, and dirty. Hard and brittle in the cold, softening with heat and becoming sticky. Masses contain pieces of root, stem, etc. They are hard, compact yellowish-brown. Odor peculiar, aromatic. Taste bitter, unpleasant. Resembling Gabbanum.-Ammoniacum, Asafotida, and Benzoin ; known by their different odors.

Composition. - The chief constituents are-(I) Volatile oil, $\mathrm{C}_{10} \mathrm{H}_{16}, 6$ to 9 per cent., consisting chiefly of a terpene, $\mathrm{C}_{10} \mathrm{H}_{16^{\circ}}$ (2) A sulphurous resin, 60 to 67 per cent. (3) Gum, 19 to 22 per cent. (4) Umbelliferone, $\left[\mathrm{C}_{9} \mathrm{H}_{6} \mathrm{O}_{3}\right.$ in acicular crystals].

\section{Action and Therapeutics of Galbanum.}

Galbanum acts like other substances containing volatile oils; it is always combined with ammoniacum or asafetida. It has been used externally as a plaster [Galbanum, I ; ammoniacum, I; yellow wax, I ; lead plaster, 8 ;] for its irritant effect, to aid the absorption of old inflammatory products, and internally it is given with asafetida as a carminative. 


\section{AMMONIACUM.}

AMMONIAC. - A gum-resin [obtained from Dorema Ammoniacum Don (nat. ord. Umbellifera). Habitat.-Eastern Persia and Turkestan.

Characters. - In roundish tears, from 2 to $6 \mathrm{~mm}$. or more in diameter ; externally pale yellowish-brown, internally milk-white, brittle when cold, and breaking with a flat, conchoidal, and waxy fracture; or the tears are superficially united into irregular masses without any intervening, dark-colored substance. It has a peculiar odor, and a bitter, acrid and nauseous taste.] Resembling Ammoniacum. - Asafœtida, Galbanum, Benzoin, known by their odor.

Compositron.-The chief ingredients are-(I) Volatile oil, to per cent. (2) Resin, 70 per cent. (3) Gum, 20 per cent.

Dose, 5 to $30 \mathrm{gr}$; ; [.30 to $2.00 \mathrm{gm}$.]

\section{Preparations.}

I. Emplastrum Ammoniaci cum Hydrargyro.- $[$ See Mercury, p. 210.

2. Emulsum Ammoniaci.-Emulsion of Ammoniac. Ammoniac, 40 ; water added gradually to 1000 . It forms a milk-like emulsion.

Dose, $1 / 2$ to I fl. oz.; 15. to 30. c.c.]

\section{Action and Therapeutics of Ammoniacum.}

The action of ammoniacum is precisely the same as that of volatile oils generally. It is employed externally to aid, by its mildly irritating effects, the absorption of chronic inflammatory products, and internally in chronic bronchitis with offensive expectoration for the sake of the remote disinfectant expectorant effect that it has, in the course of its excretion through the bronchial mucous membrane.

\section{MYRRHA.}

MYRRH.-A gum-resin [obtained from Commiphora Myrrha (Nees) Engler (nat. ord. Burseracea). Habitat.-Eastern Africa and Southwestern Arabia.

ChARACTERS. - In roundish or irregular tears or masses, brownish-yellow or reddish-brown ; fracture waxy, somewhat splintery, translucent on the edges, sometimes marked with whitish veins; odor balsamic ; taste aromatic, bitter and acrid. ]

Composition. - The chief constituents are-(I) Myrrhin $\left[\mathrm{r}_{48} \mathrm{H}_{38} \mathrm{G}_{10}\right]$, a resin, 23 per cent. (2) Myrrhol, $\mathrm{C}_{10} \mathrm{H}_{14} \mathrm{O}$, a volatile oil. 2 [to 4] per cent. (3) Gum, 60 per cent. (4) A bitter principle. 
IMPURITIES. -Many varieties of gum and gum-resins.

[Myrrh is contained in Mistura Ferri Composita and Pilulæ Rhei Com. positæ.

Dose, 5 to $30 \mathrm{gr} . ; .30$ to $2.00 \mathrm{gm}$.]

\section{Preparations.}

I. Tinctura Myrrhæ.-[Tincture of Myrrh. Myrrh, 200; by maceration with Alcohol and filtration to 1000 .

Dose, $I / 4$ to I fl. dr.; I. to 4 . c.c.

2. Tinctura Aloes et Myrrhæ.-See Aloes, p. 498.

3. Pilulæ Aloes et Myrrhæ.-See Aloes, p. 498.]

ACtion OF MYrRh.

External.-Both externally and internally, myrrh has the same action as other substances containing a volatile oil. It is a mild disinfectant, and a stimulant to sores and ulcers.

Internal.-It has the same effect in the mouth. It is a stomachic carminative, exciting the appetite, the flow of gastric juice, and the vascularity and peristalsis of the stomach and intestines. The number of leucocytes in the blood is said to be increased by the administration of myrrh. It is excreted by mucous membranes, especially the genito-urinary and the bronchial, and it stimulates and disinfects their secretions in its passage through them. Thus it becomes an expectorant, a uterine stimulant, and an emmenagogue.

\section{Therapeutics OF Myrrh.}

External.-Occasionally myrrh has been employed as a stimulant to sores and ulcers.

Internal.-It is, in the form of the tincture diffused through water [ $\mathrm{I}$ to $\mathrm{I} 6]$, used as a mouth-wash and gargle for sore spongy gums, relaxed throat, and other similar conditions, for which it is often combined with borax, as in the following formula:Myrrh, I; eau de Cologne, I6; borax, I; water, 3 ; syrup, 3. It is frequently given with purgatives for the sake of its carminative and stomachic properties. It is also commonly combined with iron when this drug is given for anæmia, but the reason for this is not clear. It is prescribed for amenorrhoea, and has been 
given for cystitis, and as a disinfectant expectorant for chronic bronchitis.

ClasS IV.-THOSE USED CHIEFLY FOR THEIR ACTION ON THE BRONCHIAL MUCOUS MEMBRANE.

TEREBENUM.

[TEREBENE. $-\mathrm{C}_{10} \mathrm{H}_{16}=135.7$. A liquid consisting chiefly of Pinene, ard containing not more than very small proportions of Terpinene and Dipentene.

Source.-From acting upon Oil of Turpentine with Sulphuric Acid, and distilling.

Characters. - A colorless or slightly yellowish, thin liquid, having a rather agreeable thyme-like odor, and an aromatic, somewhat terebinthinate taste. Sp. gr., about 0.862 . Solubility. - Only slightly soluble in Water, but soluble in an equal volume of Alcohol, glacial Acetic Acid, or Carbon Disulphide.

Dose, 5 to ${ }_{5} \mathrm{~m} . ; .30$ to 1.00 c.c.]

Action and Therapeutics of Terebene.

Terebene is an excellent stimulating disinfectant expectorant for chronic bronchitis. It may be used as an inhalation thus :Pure terebene, 2 ; magnesium carbonate, 1 ; distilled water, 24.

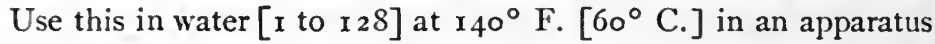
so arranged that air can be drawn through it and inhaled. Or it may be given with other expectorants in a mixture; many patients find five drops several times a day on sugar quite sufficient to cure a slight winter cough [although it is said to form an insoluble compound with sugar.]

\section{[TERPINI HYDRAS.}

TERPIN HYDRATE. $-\mathrm{C}_{10} \mathrm{H}_{18}(\mathrm{OH})_{2}+\mathrm{H}_{2} \mathrm{O}=\mathrm{I} 89.58$. The Hydrate of the Diatomic Alcohol Terpin.

Source.-Rectified Oil of Turpentine, Alcohol and Nitric Acid are allowed to stand together for three or four days in shallow porcelain disises. The crystals which have formed are collected, drained thoroughly, dried by absorbent paper, and re-crystallized in a cold solution of Alcohol.

Characters.-Colorless, lustrous, rhombic prisms, nearly odorless, and having a slightly aromatic and somewhat bitter taste. Permanent in the air. Solubility. - Soluble in about 250 parts of water, and in 10 parts of Alcohol ; also soluble in about 100 parts of Ether, 200 parts of Chloroform.

Dose, 2 to $30 \mathrm{gr}$; . 12 to $2.00 \mathrm{gm}$. 


\section{Action and Uses of Terpin Hydrate.}

Terpin hydrate is an antiseptic, and it is believed that it will arrest the development of tubercle bacilli. It increases the secretion of the mucous membrane, and the functional activity of the kidneys. It has been given as an antiseptic in acute and chronic bronchitis, when the secretion is unusually free, in whoopingcough, and rarely in the treatment of chronic nephritis, chronic cystitis and gonorrhœa.

\section{BALSAMUM PERUVIANUM.}

BALSAM OF PERU.-A balsam [obtained from Toluifera Pereirce (Royle) Baillon (nat. ord. Leguminosa). It is obtained from the bark after it has been beaten, scorched and removed. Habitat.-Central America. Balsam of Peru is named from its place of export.

CHARACTERS. - A liquid having a syrupy consistence, free from stringiness or stickiness, of a brownish-black color in bulk, reddish-brown and transparent in thin layers, of an agreeable vanilla-like, somewhat smoky odor, and a bitter taste, leaving a persistent after-taste. On exposure to air it does not become hard. Sp. gr., I.I 35 to I.150. Solubility.-Miscible, in. all proportions, with absolute Alcohol, Chloroform, or Glacial Acetic Acid; only partially soluble in Ether or Benzin. It is completely soluble in 5 parts of Alcohol.]

Composition.-The chief constituents are-(I) A volatile oil. This is present in large quantities; it consists of [Cinnamein (Benzylic Cinnamate), $\mathrm{C}_{9} \mathrm{H}_{7}\left(\mathrm{C}_{7} \mathrm{H}_{7}\right) \mathrm{O}_{2}$, about 60 per cent. (2) Cinnamic Acid, $\mathrm{C}_{9} \mathrm{H}_{8} \mathrm{O}_{2}$. (3) Resin, about 32 per cent., which on dry distillation yields Benzoic Acid, $\mathrm{HC}_{7} \mathrm{H}_{5} \mathrm{O}_{2}$. Small quantities of (4) Benzylic Benzoate, $\mathrm{C}_{7} \mathrm{H}_{5}\left(\mathrm{C}_{7} \mathrm{H}_{7}\right) \mathrm{O}_{2}$, (5) Benzyl Alcohol, $\mathrm{C}_{7} \mathrm{H}_{8} \mathrm{O}$, (6) Stilbene, $\mathrm{C}_{14} \mathrm{H}_{12}$, (7) Styrol, $\mathrm{C}_{8} \mathrm{H}_{8}$, and (8) Styracin or Cinnamyl Cinnamate, $\mathrm{C}_{9} \mathrm{H}_{7}\left(\mathrm{C}_{9} \mathrm{H}_{9}\right) \mathrm{O}_{2}$.

IMPURITIES. - Fixed oils, resins, oleoresins and alcohol.]

Dose, ro to $30 \mathrm{~m}$. ; [.60 to $2.00 \mathrm{c.c}$.]

\section{Action and Therapeutics of Balsam of Peru.}

External.-Like most substances containing a volatile oil, balsam of Peru is a disinfectant, and also a stimulant when rubbed into the skin or applied to raw surfaces. Formerly it was much used for these purposes, chiefly as an application to indolent sores and chronic eczema. [As a stimulating dressing for sluggish granulations a 5 to so per cent. solution in castor oil is frequently employed.] A mixture of balsam of Peru, I; lard, 7 ; is very useful for sore nipples and cracked lips. It is 
not often employed at present except externally as antiparasitic for pediculi, scabies and ringworm. [An ointment consisting of balsam of Peru, 20; olive oil, 50; petrolatum, I00; may be used.] For scabies it should be applied in the way already described for sulphur ointment [see p. 259]; it is a more agreeable preparation.

Internal.-Like most volatile oils balsam of Peru is carminative and stomachic, and after absorption is excreted by, and stinulates and disinfects the mucous membranes. For this reason it is used as an expectorant in chronic bronchitis. It is also excreted by the skin and the kidneys.

\section{BALSAMUM TOLUTANUM.}

BALSAM OF TOLU.-A balsam [obtained from Toluifera Balsamum Linné (nat. ord. Leguminosa). Habitat.-Venezuela and New Granada.

ChARACTERS. - A yellowish-brown, semi-fluid or nearly solid mass, becoming more brittle when exposed to cold, transparent in thin layers, having an agreeable odor recalling that of Vanilla, but distinct from it, and a mild, aromatic taste. Solubility.-Readily and completely soluble in Alcohol. Also completely soluble in Chloroform, and in solutions of the fixed alkalies; almost completely soluble in Ether, but nearly insoluble in water, Benzin, or Carbon Disulphide.]

Composition.-The chief constituents are-(I) Toluene, $\mathrm{C}_{10} \mathrm{H}_{16}$, [ $\mathrm{I}$ per cent., a thin, colorless, aromatic oil. (2) Benzylic Benzoate, $\mathrm{C}_{7} \mathrm{H}_{5}\left(\mathrm{C}_{7} \mathrm{H}_{7}\right) \mathrm{O}_{2}$, a colorless, aromatic oil. (3) Benzylic Cinnamate, $\mathrm{C}_{9} \mathrm{H}_{7}\left(\mathrm{C}_{7} \mathrm{H}_{7}\right) \mathrm{O}_{2}$. (4) Benzoic Acid, $\mathrm{HC}_{7} \mathrm{H}_{5} \mathrm{O}_{2}$. (5) Cinnamic Acid, $\mathrm{C}_{9} \mathrm{H}_{8} \mathrm{O}_{2}$. (6) Resins.

IMPURITIES. - Turpentine, storax, sweet gum, and resins.

Balsam of Tolu is contained in Tinctura Benzoini Composita.

Dose, Io to $30 \mathrm{~m}$. ; .60 to $2.00 \mathrm{c.c}$.

\section{Preparations.}

1. Syrupus Tolutanus.- [Syrup of Tolu. Balsam of Tolu, Io; Alcohol, 50; Precipitated Calcium Phosphate, 50; Sugar, 850; Water to 1000 . By solution and filtration.

Dose, $1 / 2$ to I fl. oz.; 15. to 3o. c.c.

2. Tinctura Tolutana.-Tincture of Tolu. Balsam of Tolu, Ioo. By maceration with Alcohol and filtration to 1000 .

Dose, I to $2 \mathrm{fl}$. dr.; 4. to 8. c.c.] 


\section{Action and Therapeutics of Balsam of Tolu.}

Although it has an action in all respects similar to that of balsam of Peru, it is only used as an expectorant in cough mixtures.

\section{STYRAX.}

STORAX.- [A balsam prepared from the inner bark of Liquidambar orientalis Miller (nat. ord. Hamamelacee). Habitat.-Asia Minor.

Characters. -A semi-liquid, gray, sticky, opaque mass, depositing on standing a heavier, dark-brown stratum ; transparent in thin layers, and having an agreeable odor and a balsamic taste. Solubility.- Insoluble in water, but completely soluble in an equal weight of warm Alcohol.

Composition. - The chief constituents are-(I) Styrol, $\mathrm{C}_{8} \mathrm{H}_{8}$, or Cinnamene, a volatile oil. (2) Cinnamic Acid, $\mathrm{C}_{9} \mathrm{H}_{8} \mathrm{O}_{2}$, colorless, odorless, crystalline; this can be oxidized to Benzoic Acid, and is also found in Cinnamon, and Balsams of Tolu and Peru. (3) Styracin, or Cinnamyl Cinnamate, $\mathrm{C}_{9} \mathrm{H}_{7}\left(\mathrm{C}_{9} \mathrm{H}_{9}\right) \mathrm{O}_{2}$. (4) Phenylpropyl Cinnamate, $\mathrm{C}_{9} \mathrm{H}_{7}\left(\mathrm{C}_{9} \mathrm{H}_{17}\right) \mathrm{O}_{2}$. (5) Ethyl Cinnamate, $\mathrm{C}_{9} \mathrm{H}_{7}$ $\left(\mathrm{C}_{2} \mathrm{H}_{5}\right) \mathrm{O}_{2}$. (6) Storesin, $\mathrm{C}_{36} \mathrm{H}_{58} \mathrm{O}_{3}$, in considerable quantity. (7) Vanillin, having a fragrant odor.]

Storax is contained in Tinctura Benzoini Composita.

Dose, 5 to $20 \mathrm{gr}$.; [.30 to $\mathrm{r.20} \mathrm{gm}$.]

\section{Action and Therapeutics of Storax.}

Storax has the same action as balsams of Tolu and Peru and benzoin, and may be employed for the same purposes. It is not often given internally except in the compound tincture of benzoin. Mixed with an equal part of olive oil it may be used to kill the [Sarcoptes scabiei] and pediculi.

\section{OLEUM PINI.}

OIL OF PINE. [B. P., not official.]-The oil is distilled from the fresh leaves of Pinus pumilio (nat. ord. Conifere). Synonyms._Pinol. Pumiline. [Habitat.-Russia.]

Characters. - Almost colorless. Odor aromatic. Taste pungent. Sp. gr., 0.865 to 0.870 . [Solubility. - In 7,parts of Alcobol. ]

Composition.-(1) Various terpenes." (2) Boruyl Acetate.

\section{[Preparation.}

Vapor Olei Pini (not official)._-Vapor of Oil of Pine. Oil of Pine, 2 ; rub with Magnesium Carbonate, I ; add Water, 24. Put I fl. dr. ; 4. c.c. of this in balf a pint, 240 . c.c. of cold and half a pint, 240 . c.c. of boiling water, in a vessel so arranged that air, drawn through the liquid, can be inbaled.] 


\section{Action and Therapeutics of Fir-wood Oil.}

The action of oil of pine is the same as that of oil of turpentine (see p. $5^{\mathrm{I}} 5$ ). But it is pleasanter to inhale, and forms a useful, stimulating, disinfectant, expectorant inhalation in chronic bronchitis or laryngitis.

\section{GRINDELIA.}

GRINDELIA.-[The leaves and flowering tops of Grindelia robusta Nuttall, and of Grindelia squarrosa Dunal (nat. ord. Composita). Habilat.(I) G. robusta, North America, west of the Rocky Mountains, in salt marshes. (2) G. squarrose, Western Plains to the Sierra Nevada and south to Texas.

Characters. - Leaves about $5 \mathrm{~cm}$. or less long, varying from broadly spatulate or oblong to lanceolate, sessile or clasping, obtuse, more or less sharply serrate, often spinosely toothed, or even lacinate-pinnatifid, pale green, smooth, finely dotted, thickish, brittle; heads many-flowered, subglobular or somewhat conical; the involucre hemispherical, about $10 \mathrm{~mm}$. broad, composed of numerous imbricated, squarrosely-tipped or spreading scales; rayflorets yellow, ligulate, pistillate ; disk-florets yellow, tubular, perfect ; pappus consisting of two or three awns of the length of the disk-florets ; odor balsamic, taste pungently aromatic and bitter.]

Composition. - The chief constituents are-(1) A volatile oil. (2) A resin, resembling Saponin in its action. (3) Probably an alkaloid, [Grindeline.]

Dose, $1 / 4$ to I dr.; I. to $4 . \mathrm{gm}$.

\section{Preparation.}

Extractum Grindelize [Fluidum.-Fluid Extract of Grindelia. By maceration and percolation with Alcohol, and evaporation.

Dose, $1 / 4$ to $I$ fl. dr.; $x$. to 4 . c.c.]

\section{Action and Therapeutics of Grindelia.}

In small doses, grindelia is a mild stomachic and cardiac sedative, but its main action depends upon the fact that in its excretion by the bronchial mucous membrane it acts as an expectorant, and also relaxes the muscular coat of the bronchial tubes, and this explains its efficacy [in the symptom] asthma. Two or three doses of twenty minims; [r.20 c.c.], of the fluid extract in milk, which prevents precipitation of the resin, given every twenty minutes, will often allay the paroxysms of asthma. Between the attacks, this dose should be taken three times daily. The same quantity may with advantage be added to mixtures 
prescribed for chronic bronchitis, for not only is grindelia an expectorant, but it relieves the asthmatic paroxysms which so frequently accompany bronchitis. It is very bitter; its taste is best concealed by Spiritus Chloroformi. Linen soaked in a lotion of the fluid extract in water, I to 48 , is applied to the skin for the dermatitis caused by Rhus toxicodendron, the poison ivy. The same lotion is used in burns and as an injection in gleet and leucorrhœa.

ClasS V.-THOSE USED CHIEFLY FOR THEIR ACTION ON THE KIDNEYS AND GENITO-URINARY TRACT.

\section{OLEUM JUNIPERI.}

OIL OF JUNIPER.-A volatile oil distilled [from the fruit (berry) of Juniperus communis Linné (nat. ord. Coniferce). Habitat.-North America throughout Canada, the Northern United States, and in the Rocky Mountains south to New Mexico.

CharACTERS. - A colorless or faintly greenish-yellow liquid, becoming darker and thicker by age and exposure to air, having the characteristic odor of Juniper, and a warm, aromatic, somewhat terebinthinate and bitterish taste. Sp. gr., 0.850 to 0. 890 . Solubility. - In about 4 times its volume of Alcohol.]

Composition.-Oil of Juniper is composed chiefly of terpenes, which are mostly Pinene and Cadinene.

Dose, 2 to $10 \mathrm{~m}$.; [.1 2 to .60 c.c.]

Preparations.

I. Spiritus Juniperi.-[Spirit of Juniper. Oil of Juniper, 50; Alcohol, 950.

Dose, 30 to $60 \mathrm{~m}$.; 2. to 4 . c.c.

2. Spiritus Juniperi Compositus. - Compound Spirit of Juniper. Oil of Juniper, 8; Oil of Caraway, I; Oil of Fennel, $\mathbf{1}$; Alcohol, 1400 ; Water to 2000.

Dose, I to 4 f. dr.; 4 . to I5. c.c.]

\section{Action of Oil of JUNiper.}

Oil of juniper has much the same action as oil of turpentine; but it is not so liable to upset the digestion; and although it is a powerful renal stimulant and diuretic, it does not easily cause hrematuria and albuminuria. [Because of its antiseptic properties it is employed for the preservation of cat-gut.] 


\section{Therapeutics of Oil of Juniper.}

Internal._-Occasionally it is given as a pleasant carminative and stomachic, but its main use is as a diuretic in heart disease, hepatic ascites, and chronic Bright's disease. It must not be given in the acute form, and should always be combined with other diuretics. It certainly markedly increases the quantity of the urine, which it causes to smell like violets. As [it is] a constituent of Holland and [other] gins these are good forms of alcohol for persons suffering from the above diseases.

\section{BUCHU.}

BUCHU. - Synonym. - Bucco. The leaves of Barosma betulina [(Thunberg) Bartling et Wendland, and Barosma crenulata (Linné) Hooker (nat. ord. Rutacece). Habitat.-Southern Africa.

Characters. - About $15 \mathrm{~mm}$. long, roundish-obovate, with a rather wedge-shaped base, or varying between oval and obovate, obtuse, crenate or serrate, with a gland at the base of each tooth, dull yellowish-green, thickish, pellucid-punctate; odor and taste strongly aromatic, somewhat mint-like, pungent and bitterish.] Resembling Buchu.-Senna and Uva Ursi, which have entire leaves.

IMPURITV.-Leaves of Emplanum serrulatum, which have no glands.

Composition. - The chief constituents are-(I) A yellowish-brown volatile oil, from the glands. $\left[(2)\right.$ A stearopten (Diosphenol, $\mathrm{C}_{10} \mathrm{H}_{16} \mathrm{O}_{2}$ )] in solution in a liquid hydrocarbon. The stearopten is deposited on exposure to air. (3) A [glucoside Barosmin, soluble in alcohol (scarcely so, if cold) and in ether, volatile oils, dilute acids and alkalies. (4) Rutin, a bitter principle. Mucilage.

Dose, 15 to $30 \mathrm{gr}$; 1. to $2 . \mathrm{gm}$.]

\section{Preparation.}

[Extractum Buchu Fluidum.-Fluid Extract of Buchu. By maceration and percolation with Alcohol, and evaporation.

Dose, 15 to $30 \mathrm{~m}$.; 1 . to 2. c.c.]

\section{Action and Therapeutics of Buchu.}

A medicinal dose of buchu causes a slight feeling of warmth in the stomach, and a large one gives rise to vomiting. The volatile oil diffuses into the blood and is excreted by the bronchial mucous membrane, which it stimulates, and buchu is therefore occasionally given as an expectorant. Most of the oil is 
excreted by the kidneys, which are also stimulated, and thus buchu is a mild diuretic. In the process of excretion it gives a peculiar odor to the urine, and acts as an astringent and disinfectant to the urinary tract, especially the bladder. It has consequently been administered for cystitis, irritable bladder, pyelitis, gonorrhœa, etc. Large doses continued for a long time are said to damage the kidney. The infusion [B. P., I to 20 , dose $x$ to $2 \mathrm{fl}$. oz., 30. to 60 . c.c.] contains very little of the oil. The fluid extract does not mix well with water on account of the oil in it. The action of the buchu is much the same as that of pareira, $(q . v$.) but it is pleasanter to take, and is a good vehicle for diuretics.

\section{COPAIBA.}

COPAIBA.-Synonyms. - Copaiva. [Balsam of Copaiba. (This is not a true balsam because it does not contain cinnamic or benzoic acid). The oleoresin of Copaiba Langsdorffii (Desfontaines) $O$. Kuntze, and of other species of Copaiba (nat. ord. Leguminosa). Habitat.-Brazil, Venezuela and New Granada.

CIIARACTERS. - A transparent or translucent, more or less viscid liquid; of a pale yellow to brownish-yellow color, having a peculiar, aromatic odor, and a bitter acrid taste. Sp. gr., 0.940 to 0.990 . Solubility.-Insoluble in water ; readily soluble in absolute Alcohol, Ether, Chloroform, Carbon Disulphide, Benzin, and fixed and volatile oils.]

Composition.-The chief constituents are-(I) The volatile oil, 48 to 85 per cent. (see below). (2) The Resin, 15 to 52 per cent. (see below), which exists dissolved in the oil. It consists of two Resins: (a) Copaivic Acid, $\left[\mathrm{C}_{20} \mathrm{H}_{30} \mathrm{O}_{22}\right.$, $]$ the chief constituent, a crystalline Resin, with a faint odor, a bitter taste, insoluble in water, easily soluble in absolute Alcohol and Ammonia ; (b) a non-crystallizable, viscid Resin, $1 \frac{1}{2}$ per cent.

IMPURITIES. - Turpentine, detected by the smell on heating. Fixed oils; these leave a greasy ring round the resinous stain when heated on paper. Gurjun Balsam, which coagulates at $270^{\circ} \mathrm{F}$; $\left[1 \mathrm{I}^{\circ}{ }^{\circ} \mathrm{C}\right.$; $]$ Copaiba does not.

Dose, $1 / 4$ to I fl. dr.; [I. to 4 . c.c.]

\section{Preparation.}

[Massa Copaibre.-Mass of Copaiba. Synonym.-Solidified Copaiba. Copaiba, 94; Magnesia, 6. By trituration with water, and heating.

Dose, $1 / 4$ to I dr.; I. to $4 . \mathrm{gm}$.]

OLEUM COPAIBAE.-[Oil of Copaiba. ] A volatile oil distilled from Copaiba. 
Ciraracters. - [A colorless or pale yellowish liquid, having the characteristic odor of Copaiba, and an aromatic, bitterish and pungent taste. It is isomeric with Turpentine, $\mathrm{C}_{10} \mathrm{H}_{16^{\circ}}$ Sp. gr., 0.890 to 0.910 . Solubility.-In about ro times its volume of Alcohol.]

Composition. - It consists chiefly of the hydrocarbon, Caryophyllene (see p. 534).

Dose, 5 to $15 \mathrm{~m}$.; [. 30 to 1.00 c.c.] suspended in Mucilage of Acacia ( $\mathrm{r} / 2$ fl. oz. [45. c.c.] for every fl. oz. [3o. c.c.] of Oil of Copaiba) or yolk of egg. Cinnamon or Peppermint Water, with Tincture of Orange or Ginger, covers the taste. It may be dissolved in water with the aid of Liquor Potass with which it forms a soap, or it may be given in capsules.

[RESINA COPAIBAE.-Resin of Copaiba. The residue left after distilling off the volatile oil from Copaiba.

Characters. $-\Lambda$ yellowish or brownish-yellow, brittle resin, having a slight odor and taste of Copaiba. Solubility.-In Alcohol, Ether, Chloroform, Carbon Disulphide, Benzol, or Amylic Alcohol.

Dose, 5 to $15 \mathrm{gr}$; . 30 to $1.00 \mathrm{gm}$.]

\section{Action of Copaiba.}

External.-Copaiba is a stimulant to the skin.

Internal.-Gastro-intestinal tract.-It acts like other volatile oils. Small doses produce a feeling of warnth in the epigastrium; but with large doses its irritant effect leads to vomiting and diarrhœa. Its taste is [unpleasant], and the eructations it may cause are very disagreeable.

Mucous membrane.-Here also it acts like other volatile oils. It is quickly absorbed, and then is excreted by all the mucous membranes, which it stimulates in its passage through them, increasing their vascularity and the amount of their secretion, which, if foul, is disinfected. Because of these actions it is a disinfectant expectorant, and a stimulating disinfectant to the whole of the genito-urinary tract. It imparts a powerful odor to the breath and mucous secretions. It is also excreted by the skin, and its irritant effect here is seen in the erythematous rash it often produces. Some, too, passes out by the milk.

Kidneys. - Copaiba has a more marked action on the kidneys than most substances containing volatile oils, and this is in great part due to the resin, which is particularly stimulating to the 
renal organs, and copaiba is therefore a useful diuretic. Large doses of it greatly irritate the kidney, as is shown by pain in the loins and blood and albumin in the urine. The oil and the resin are excreted in the urine, and the resin can be [precipitated] from it by nitric acid; but this precipitate is known not to be albumin by the fact that it is evenly distributed through the fluid and is dissolved by heat. [It also leads to confusion when Trommer's test is employed to detect glucose.] If the renal congestion is severe, the urine may be very scanty.

\section{Therapeutics of Copalba.}

Internal.-Genito-urinary tract. - Copaiba, or more usually its oil, is largely used to stimulate and disinfect this part of the body in cases of pyelitis, vaginitis, and gonorrhœa. It is often prescribed for this last disease, and is best given when the acute symptoms have subsided, otherwise it may increase them.

Kilneys. - The resin is an admirable diuretic for hepatic and cardiac dropsy, but because of its liability to irritate the kidneys should not be given in Bright's disease. After a time patients seem to become accustomed to it, for the diuresis is not so marked as at first. It is [disagreeable] and difficult to make palatable. Fifteen grains [ $\mathrm{r} . \mathrm{gm}$.] of the resin with 20 minims [I.20 c.c.] of alcohol, I 5 grains [I. gm.] of tragacanth to suspend it, and a fluid drachm [4. c.c.] of syrup of ginger in an ounce [30. c.c.] of water may be given for a dose.

Bronchial mucous membrane.-Copaiba is occasionally used as a disinfectant expectorant when the secretion is very foul-as, for example, when the bronchial tubes are dilated.

Skin.-Copaiba has been given in chronic skin diseases, as psoriasis, for the cutaneous stimulation caused by it, but it is now quite discarded.

The reasons why it is rarely used except in gonorrhœea, for which it would not be employed if it had not such a strongly marked beneficial action, are that the smell of the breath of those taking it is very disagreeable, it is very [unpleasant] to the taste, and often causes indigestion. 


\section{[OLEUM THYMI.}

OIL OF THYME.-A volatile oil distilled from the leaves and flowering tops of Thymus vulgaris Linne (nat. ord. Labiata). Habitat.-Southern France.

Characters. - A yellowish or yellowish-red liquid, having a strong odor of Thyme, and an aromatic, pungent, afterwards cooling taste. It becomes darker and thicker by age and exposure to the air. Sp. gr., 0.900 to 0.930 .

Solubility. - In half its volume of Alcohol, forming a clear solution, which is neutral or only very slightly acrid to litmus paper. Also soluble in all proportions, in Carbon Disulphide, and in Glacial Acetic Acid.

Composition.-Its chief constituents are the hydrocarbons-(1) Cymene, $\mathrm{C}_{10} \mathrm{H}_{18}$. (2) Thymene, $\mathrm{C}_{10} \mathrm{H}_{16}$.

Dose, $\mathrm{I}$ to $5 \mathrm{~m}$.; .06 to $.30 \mathrm{c.c}$.

\section{Action and Uses of the Oil of Thyme.}

Its action is similar to that of copaiba. The chief use of oil of thyme is as a source of thymol. It has been employed in the treatment of bronchitis, gonorrhœa, gleet, leucorrhœa, and vesical catarrh.]

\section{CUBEBA.}

CUBEB.-The unripe fruit of Piper Cubeba [Linné filius (nat. ord. Piperacea). Habitat.-Java; cultivated.

ChARACTERS.-Globular, about 4 or $5 \mathrm{~mm}$. in diameter, contracted at the base into a rounded stipe about 6 or $8 \mathrm{~mm}$. long, reticulately wrinkled, blackish-gray, internally whitish and hollow; odor strong, spicy; taste aromatic and pungent.] Resembling $C_{u b e b .-P e p p e r}$ and Pimenta; neither has a stalk.

CoMposition.-The chief constituents are-(1) The volatile oil [5 to] 15 per cent. (see below). (2) The Oleoresin, 6 per cent. (see below), which contains Cubebin, a white, crystalline, odorless substance, and Cubebic Acid. (5) A little Piperine.

Dose, 30 to $60 \mathrm{gr}$.; [2. to $4 . \mathrm{gm}$.]

\section{Preparations.}

1. [Extractum Cubebæ Fluidum.-Fluid Extract of Cubeb. By maceration and percolation with Alcohol, and evaporation.

Dose, $1 / 2$ to $x$ fl. dr.; 2. to 4 . c.c.

2. Oleoresina Cubebr.-Oleoresin of Cubeb.

SourCE. - By percolation with Ether; distil off, and evaporate the Ether.

Oleoresin of Cubeb is used to make Trochisci Cubebæ.

Dose, 5 to $30 \mathrm{~m}$.; .30 to $2.00 \mathrm{c.c}$. 
3. Tinctura Cubebæ.-Tincture of Cubeb. Cubeb, 200. By maceration and percolation with Alcohol to 1000 .

Dose, $1 / 2$ to 3 fl. dr.; 2. to I2. c.c.

4. Trochisci Cubebæ.-Troches of Cubeb. Oleoresin of Cubeb, 4 ; Oil of Sassafras, I ; Extract of Glycyrrhiza, 25 ; Acacia, 12 gm. ; Syrup of Tolu, sufficient quantity to make Ioo troches. Each troche contains $2 / 3 \mathrm{~m}$. ; .04 c.c., of the Oleoresin.]

Dose, I to 6 troches.

OLEUM CUBEBA. - Oil of Cubeb. A volatile oil distilled from Cubeb.

Characters. - A colorless, [pale greenish, or yellowish liquid, having the characteristic odor of Cubeb, and a warm, camphoraceous, aromatic taste. Sp. gr., about 0.920. Solubility. - Soluble in an equal volume of Alcohol.]

Composition. - The chief constituents are - (I) Cubeb Camphor, [ $\mathrm{C}_{15}$ $\mathrm{H}_{26} \mathrm{O}$, a Stearopten. (2) Two oils, $\mathrm{C}_{15} \mathrm{H}_{24^{*}}$ (3) A small amount of a Terpene. ]

Dose, 5 to $20 \mathrm{~m}$.; [.30 to 1.20 c.c.] suspended in Mucilage.

\section{Action of Cubeb.}

External.-Like other substances containing a volatile oil, cubeb is rubefacient when rubbed into the skin.

Internal.-Small doses are stomachic and carminative, and improve digestion, but moderate doses are very liable to cause dyspepsia. Cubeb enters the blood, and, like many volatile oils, slightly stimulates the heart, and also excites the organs through which it is excreted. Occasionally, therefore, it causes an erythematous eruption on the skin; it increases and disinfects the bronchial secretion, and is consequently an expectorant; but its main action is on the genito-urinary passages, the mucous membrane of which is powerfully stimulated. and the secretions of which are disinfected. The kidneys are also irritated, hence cubeb is a diuretic. It appears in the urine in a form (probably as a salt of cubebic acid) which may be precipitated by nitric acid.

\section{Therapeutics of Cubeb.}

It is sometimes employed as [troches], or as a powder, or as the smoke of cubeb cigarettes, to stimulate the mucous membrane in cases of slight bronchitis, chronic sore throat, or follicular pharyngitis. Chronic nasal catarrh and hay-fever have been treated by 
insufflations of the powder. [The symptom] asthma is sometimes relieved by the cigarettes. Many popular bronchial troches contain cubeb; in them it exercises its expectorant action. Cubeb is rarely used as a stomachic or cardiac stimulant, because it is so liable to upset digestion; but as it is less likely to do so than copaiba, is a little pleasanter to take, and is almost as powerful a stimulant to the genito-urinary mucous membrane; it is largely used in gleet, gonorrhœa, and chronic cystitis.

\section{OLEUM SANTALI.}

OIL OF SANTAL.-Synonym.-[Oil of Sandal Wood. A volatile oil distilled from the wood of Santalum album Iinné (nat. ord. Santalacece). Habitat.-Southern India.

Characters. - A pale yellowish or yellow, somewhat thickish liquid, having a peculiar, strongly aromatic odor, and a pungent, spicy taste. Sp. gr., 0.970 to 0.978 . It deviates polarized light to the left : distinction from Australian (Sp. gr., 0.953) and West Indian (Sp. gr., 0.965) Sandal Wood Oil, which deviate polarized light to the right. Solubility._Readily in Alcohol.

Composition. - Santalol, $\mathrm{C}_{15} \mathrm{H}_{26} \mathrm{O}$, an alcohol, 90 per cent.

Dose, 2 to $10 \mathrm{~m}$.; [.12 to .60 c.c.]

\section{Action and Therapeutics of Oil of Santal.}

The action of the oil of santal is the same as that of volatile oils in general, but, like that of the oils of copaiba and cubeb, it is especially manifested in the genito-urinary mucous membranes, which are stimulated and disinfected. The drug is used in gonorrhœa and gleet. [It is best administered in capsules, or in an emulsion.] It is pleasanter than copaiba, but more expensive. It appears in the urine half an hour after administration. Some of it is excreted by the bronchial mucous membrane; it is, therefore, a stimulating disinfectant expectorant. Two or three drops on sugar will frequently relieve the hacking cough so often met with when but little sputum is expectorated.

\section{[MATICO.}

MATICO.-The leaves of Piper angustifolium Ruiz et Pavon (nat. ord. Piperacee). Habilat.-Tropical America.

Characters. -From 10 to $15 \mathrm{~cm}$. long, short petiolate, oblong-lanceolate, apex pointed, base unequally heart-shaped, margin very finely crenulate, tessei- 
lated above, reticulate beneath, the meshes small, and the veins densely brownish-hairy; aromatic, spicy and-bitterish. Resembling Matico leaves.-Digitalis leaves, which are less deeply reticulated.

Composition. - The chief constituents are-(I) Volatile oil, [2 $1 / 2$ per cent. (2) A pungent resin. (3) A bitter principle. (4) Artanthic Acid. (5) Tannic acid.

Dose, $1 / 2$ to 2 dr. ; 2. to $8 . \mathrm{gm}$.

\section{Preparations.}

I. Extractum Matico Fluidúm.-Fluid Extract of Matico. By maceration and percolation with Alcohol and Water, and evaporation.

Dose, $1 / 2$ to 2 fl. dr.; 2. to 8 . c.c.

2. Tinctura Matico.-Tincture of Matico. Matico, 100, by maceration and percolation with Diluted Alcohol to 1000 .

Dose, $1 / 2$ to I fl. oz.; 15 . to 30 . c.c.

\section{Action and Therapeutics of Matico.}

The volatile oil of matico probably has much the same action as that of cubeb, influencing chiefly the genito-urinary passages. It has been given for the same cases, but is now rarely used. The leaves are sometimes placed upon a bleeding surface. Their numerous hairs promote the clotting of the blood, and thus they are hæmostatic.

\section{DAMIANA.}

DAMIANA. (Not official.)-The leaves of several plants, principally Bigelovia veneta Gray and Turnera microphylla De Candolle, var., aphrodisiaca (nat. ord. Turneracea). Habilat. - Western North America.

Composition. - The chief constituents of the last as given by Rantzer are -(I) A volatile oil, I per cent., amber-colored, baving an aromatic odor, and a warm camphoraceous taste. (2) Tannic acid. (3) Two resins.

Dose, $1 / 2$ to $10 z$; 15 . to $30 . \mathrm{gm}$.

\section{Action and Uses of Damiana.}

Damiana has enjoyed considerable reputation as a remedy for sexual atony. Some observers believe it to be only tonic. It is best administered as a fluid extract, in the dose of $1 / 2 \mathrm{fl}$. dr., 2. cc.

\section{ClasS VI.-THOSE USED CHIEFLY FOR THEIR ACTION ON THE FEMALE GENITAL ORGANS.}

\section{SABINA.}

SAVINE.-The tops of Juniperus Sabina Linné (nat. ord. Conifere). Habitat.-Siberia, Europe, Candda and Northern United States. 
ChARACTERS. -Short, thin, subquadrangular branchlets; leaves rather dark green, in four rows, opposite, scale-like, ovate-lanceolate, more or less acute, appressed, imbricated on the back with a shallow groove containing an oblong or roundish gland ; odor peculiar, terebinthinate ; taste nauseous, resinous and bitter.

Composition.-The chief constituent is the volatile oil (see below), about 2 per cent.

Dose, 5 to $15 \mathrm{gr} . ; .30$ to $\mathrm{r} .00 \mathrm{gm}$.

\section{Preparation.}

Extractum Sabinæ Fluidum.-Fluid Extract of Savine. By maceration and percolation with Alcohol, and evaporation.

Dose, 5 to $15 \mathrm{~m}$.; .30 to 1.00 c.c.

OLEUM SABINÆ.-Oil of Savine. A volatile oil distilled from Savine.

Characters. - A colorless or yellowish liquid, having a peculiar terebinthinate odor, and a pungent, bitterish and camphoraceous taste. It becomes darker and thicker by age and exposure to the air. Sp. gr., 0.9 Io to 0.940. Solubility.-Soluble in equal volume of Alcohol.

Composition.-It contains several terpenes.

Dose, I to $5 \mathrm{~m}$.; .06 to .30 c.c.

\section{Action of Savine.}

Oil of savine has the same action as oil of turpentine, but it is more marked. Thus externally it causes great redness, pain, vesication, and even pustulation. Internally it may produce severe gastro-intestinal irritation, with vomiting, abdominal pain and purging. In its excretion through the kidney and the mucous membranes of the genito-urinary tract it severely irritates them ; thus hrematuria, scanty urine, and pain on micturition may follow its use. The point in which the action of oil of savine differs from that of the oil of turpentine is that it powerfully irritates the ovaries and uterus, causing hyperæmia of them and accelerating menstruation. It also induces contractions of the pregnant uterus, and therefore it is an ecbolic.

\section{Therapeutics of Savine.}

The cerate made from the fluid extract, $\mathbf{I}$; in resin cerate, 4 , has been used as a powerful irritant and counter-irritant, and internally savine may be given as an emmenagogue; but, on the whole, its use is to be discouraged, as it is so liable to cause 
serious gastro-enteritis. It has often been administered as an ecbolic with criminal intent, but it is rarely used in medicine.

\section{TANACETUM.}

TANSY.-The leaves and tops of Tanacetum vulgare Linné (nat. ord. Composita). Habitat.-Asia and Europe; naturalized in North America; cultivated.

Characters. - Leaves about $15 \mathrm{~cm}$. long; bipinnatifid, the segments oblong, obtuse, serrate, or incised, smooth, dark green and glandular; flowerheads corymbose, with an imbricated involucre, a convex, naked receptacle, and numerous yellow, tubular florets; odor strongly aromatic; taste pungent and bitter.

Composition.-(I Tanacetin, $\mathrm{C}_{11} \mathrm{H}_{16} \mathrm{O}_{4}$, a bitter principle. (2) Volatile oil, $1 / 4$ per cent. (3) Tannic acid.

Dose, $1 / 4$ to $1 \mathrm{dr}$. ; $\mathrm{I}$. to $4 . \mathrm{gm}$.

\section{ACtion and Uses of Tansy.}

Tansy possesses the properties of an aromatic bitter, and is an irritant narcotic. It has been used as an abortifacient, but is dangerous in large doses, several fatal cases having been recorded.

\section{OLEUM RUTA.}

OIL OF RUE. (Not official.)-The volatile oil distilled from the fresh berb of Ruta graveolens (nat. ord. Rutacee). Habitat.-Britain.

Characters. - A light yellow oil, becoming brown on keeping. Taste bitter. Odor aromatic, disagreeable. Soluble in an equal weight of Alcohol. Sp. gr., about 0.880 .

Composition.-It consists mainly of Methyl-nonyl Ketone, $\mathrm{CH}_{3}, \mathrm{CO}$. $\mathrm{C}_{9} \mathrm{H}_{19}$.

Dose, I to $4 \mathrm{~m}$.; .06 to .25 c.c.

Action and Therapeutics of Oil of Rue.

External.-Oil of rue is irritant and vesicant.

Internal.-In large doses it is a powerful gastro-intestinal irritant. It is eliminated in, and may be recognized by its odor in the urine, breath and perspiration. It is irritant to the kidneys, ovaries and uterus, and excites the menstrual flow; consequently it is given in amenorrhœea. From its stimulating action on the uterus rue has been used as an abortifacient, and fatal cases of poisoning from gastro-intestinal irritation have been recorded. It is very rarely given as a medicine. In all points its action resembles that of savine.] 


\section{GROUP VII. \\ Vegetable Bitters.}

All these substances contain a bitter principle which stimulates the functions of the stomach.

Calumba, [Canella, Calamus,] Gentian, Quassia, Cascarilla, Chirata, Cusparia, Serpentaria, Cimicifuga, Dandelion, Orange Peel.

[Caluunba, Canella, Calamus, Gentian, Quassia, Chirata, Cusparia and Dandelion, do not contain Tannic Acid.]

\section{CALUMBA.}

CALUMBA.-[Synonym.-Columbo. The root of Jaieorrhiza palmata (Lamarck) Miers (nat. ord. Menispermaced). Habitat.-Eastern Africa; cultivated in some East Indian Islands.

Cilaracters. - In nearly circular disks, 3 to $6 \mathrm{~cm}$. in diameter, externally greenish-brown and wrinkled, internally yellowish or grayish-yellow, depressed in the centre, with a few interrupted circles of projecting wood-bundles, distinctly radiate in the outer portion; fracture short, mealy; odor, slight ; taste mucilaginous, slightly aromatic, very bitter.]

Composition.-The chief constituents are-(I) Calumbin, $\mathrm{C}_{21} \mathrm{H}_{22} \mathrm{O}_{7}$, a neutral, bitter principle crystallizing in white needles. (2) Berberine $\left[\mathrm{C}_{20}\right.$ $\left.1 \mathrm{I}_{17} \mathrm{NO}_{4}\right]$, an alkaloid $(q . v$.$) , giving the yellow color. (3) Calumbic acid,$ $\mathrm{C}_{21} \mathrm{H}_{22} \mathrm{O}_{6}$ ]. (4) Starch, 33 per cent. No Tannic Acid is present, so Calumba can be prescribed with iron salts.

Dose, 5 to $30 \mathrm{gr}$.; [.30 to $2.00 \mathrm{gm}$.]

\section{Preparations.}

1. [Extractum Calumbæ Fluidum.-Fluid Extract of Calumba. By maceration and percolation in Alcohol and Water and evaporation.

Dose, 5 to $30 \mathrm{~m}$.; .30 to 2.00 c.c.

2. Tinctura Calumbæ.-Tincture of Calumba. Calumba, roo, by maceration and percolation in Alcohol and Water to 1000 .

Dose, 1 to 4 fl. dr.; 4. to 15 . c.c.]

\section{Action of Calumba.}

External.-Calumba is a mild antiseptic and disinfectant.

Internal.-Mouth.-Calumba is a typical bitter; the appetite is sharpened because the gustatory nerves are stimulated; 
this reflexly leads to dilatation of the gastric vessels and to an increase in the gastric and salivary secretions.

Stomach.-The effects on the gastric mucous membrane which were brought about reflexly by the stimulation of the gustatory nerves are further exaggerated by the arrival of the saliva in the stomach, and by the direct action of the calumba on it, for although the immediate effect of a bitter in the stomach is to diminish the flow of gastric juice, it is [rapidly] absorbed, and after absorption it has the power to quickly increase the flow of gastric juice. The result of these actions is to cause a feeling of hunger, an extra secretion of gastric juice and greater vascular dilatation, and all this helps the digestion of the food. Peristalsis in the stomach and intestine is made slightly more active, and thus calumba is carminative. Large doses have a paralytic effect on the secretion, and are very powerful. The long continued use of bitters leads to gastric catarrh and consequent indigestion.

Most of these substances, like volatile oils, cause an increased migration of leucocytes from the intestinal glands into the blood.

Injected [into] the rectum bitters are anthelmintic, destroying the threadworm.

\section{Therapeutics of Calumba.}

Calumba is only employed to stimulate the gastric functions and improve the appetite in cases of chronic indigestion due to a general weakness of action on the part of the stomach. It is thus a type of the large class of stomachics. It is especially valuable in that form of dyspepsia in which the stomach participates in a general feebleness of all the organs of the body, such as we see in anæmia, starvation, convalescence from acute diseases, tuberculosis and general exhaustion. Bitters should never be used when there is acute or subacute gastritis, a gastric ulcer or pain. They will obviously make all these conditions worse. They must not be too concentrated, nor given for too long a time, lest they should over-irritate the stomach. They should always, as far as possible, be combined with modes of 
treatment designed to relieve the cause of the dyspepsia. Often they are called tonics; all that is meant by this is that, as they render the digestion of food more easy, the general health will improve. Most bitters, when given as rectal injections [administered when the patient is in the knee-chest position], are anthelmintics for the Oxyuris vermicularis. Half a pint [250. c.c.] of the infusion [B. P., Calumba, I; cold water (to avoid extracting the starch), 20 ;] may be thrown [into] the rectum of an adult.

\section{[CANELLA.}

CANELLE CORTEX.-(Not official.)-Canella Bark. The bark of Canella alba (nat. ord. Canellacea) deprived of its corky layer and dried. Habitat. - South Florida and the Bahamas.

Characters. - Quills, 8 to $20 \mathrm{~cm}$. long, or flattish pieces. Externally, orange-brown or buff, with sometimes remains of corky layer as silver-gray patches; whitish internally. Agreeable odor, like Cloves and Cinnamon; bitter taste.

Compositron.-The chief constituents are: (I) A volatile oil (1 per cent.), consisting of several oils, one of which is identical with Eugenic Acid, obtained from Oil of Cloves. (2) A bitter principle, Canellin. No Tannic Acid is present.

Dose, 15 to $60 \mathrm{gr}$. ; I. to $4 . \mathrm{gm}$.

\section{Action and Uses of Canella.}

Canella is an aromatic bitter stomachic, not often prescribed.

\section{CALAMUS.}

CALAMUS.-Synonym.-Sweet Flag. The rhizome of Acorus Calamus Linne (nat. ord. Aroidea). Habitat.-Europe and North America, on the banks of streams and ponds.

Characters. - In sections of various lengths, unpeeled, about $2 \mathrm{~cm}$. broad, subcylindrical, longitudinally wrinkled; on the upper surface marked with leaf-scars forming triangles, and on the lower surface with the circular scars of the rootlets in wavy lines; externally reddish-brown, somewhat annulate from remnants of leaf-sheaths; internally whitish, of a spongy texture, breaking with a short, corky fracture, showing numerous oil-cells and scattered wood-bundles, the latter crowded within the subcircular endoderm. It has an aromatic odor, and a strongly bitter taste. As found in the shops it is generally peeled.

Composition. - The chief constituents are-(I) Acorin $\mathrm{C}_{36} \mathrm{H}_{60} \mathrm{O}_{6}$, a bitter glucoside, as a syrupy, yellow liquid.

Calamine, an alkaloid. (4) Choline.

(2) Volatile oil, I to 2 per cent. 


\section{Preparation.}

Extractum Calami Fluidum.-Fluid Extract of Calamus. By maceration and percolation with Alcohol, and evaporation.

Dose, 15 to $60 \mathrm{~m}$.; I. to 4 . c.c.

\section{Action and Uses of Calamus.}

Sweet flag is a simple bitter and feeble aromatic, and is used with advantage in pain or uneasiness in the stomach or bowels arising from flatulence, or as an adjuvant to purgative medicines.]

\section{GENTIANA.}

GENTIAN.-[The root of Gentiana lutea Linné (nat. ord. Gentianea). Habitat.-Mountains of Central and Southern Europe.

Characters. - In nearly cylindrical pieces or longitudinal slices, about $25 \mathrm{~mm}$. thick, the upper portion closely annulate, the lower portion longitudinally wrinkled; externally deep yellowish-brown; internally lighter ; some. what flexible and tough when damp; rather brittle when dry ; fracture uneven; the bark rather thick, separated from the somewhat spongy meditullium by a black cambium line ; odor peculiar, faint, more prominent when moistened; taste sweetish and persistently bitter.]

Composition.-The chief constituents are-(I) Gentiopicrin, an active, very bitter glucoside, soluble in water and Alcohol. Can be split up into glucose and Gentiogenin. (2) Gentisic Acid, $\left[\mathrm{C}_{14} \mathrm{H}_{10} \mathrm{O}_{5}\right.$, in yellow, tasteless needles,] united with Gentiopicrin. (3) A trace of a volatile oil. (4) Gentianose, a sugar. Gentian contains no Tannic Acid, but cannot be prescribed with iron, because that darkens the coloring matter.

INCOMPATIBLES.-Iron salts, silver nitrate, and lead salts.

Dose, 5 to $30 \mathrm{gr}$.; [.30 to $2.00 \mathrm{gm}$.]

\section{Preparations.}

r. Extractum Gentianæ.-[Extract of Gentian. By maceration and percolation with Water, and evaporation.

Dose, 2 to ro $\mathrm{gr}$. ; .12 to $.60 \mathrm{gm}$.

2. Extractum Gentianæ Fluidum.-Fluid Extract of Gentian. By maceration and percolation with Diluted Alcohol, and evaporation.

Dose, 5 to $30 \mathrm{~m}$; ; 30 to 2.00 c.c.

3. Tinctura Gentianæ Composita.-Compound Tincture of Gentian. Gentian, I00; Bitter Orange Peel, 40; Cardamom, ro. By maceration and percolation with Alcohol and Water to $x 000$.

Dose, $x$ to 4 fi. Ar. ; 4 . to 15 . c.c.] 


\section{Action and Therapeutics of Gentian.}

Gentian has the same action as other bitters, such as calumba, and is employed for the same class of cases. It is more used than any other bitter, because its taste is pleasant and it is not astringent.

\section{QUASSIA.}

QUASSIA.- [The wood of Picrena excelsa (Swartz) Lindley (nat. ord. Simarubece). Habitat.-Jamaica.

CHARACTERS. - In billets of various sizes, dense, tough, of medium hardness, porous, with a minute pith and marrow, medullary rays; inodorous, and intensely bitter. In the shops it is usually met with in the form of chips or raspings of a yellowish-white color.] Resembling Quassia.-Sassafras, but this is aromatic and not bitter.

Composition. - The chief constituents are-(I) [Quassiin, $\mathrm{C}_{10} \mathrm{H}_{12} \mathrm{O}_{3}$, a bitter principle occurring in crystalline rectangular plates.] (2) $\mathrm{A}$ volatile oil. No Tannic Acid being present, Quassia can be prescribed with iron salts.

\section{Preparations.}

I. [Extractum Quassix.-Extract of Quassia. By percolation with Water, and evaporation.

- Dose, $1 / 2$ to $3 \mathrm{gr}$. ; 03 to $.20 \mathrm{gm}$.

2. Extractum Quassiz Fluidum.-Fluid Extract of Quassia. By maceration and percolation with Alcohol and Water, and evaporation.

Dose, $1 / 4$ to $x$ fl. dr. ; x. to 4 . c.c.

3. Tinctura Quassiæ.-Tincture of Quassia. Quassia, 100; by maceration and percolation with Alcohol and Water to 1000 .

Dose, $1 / 2$ to 2 f. dr.; 2. to 8 . c.c.]

\section{Action and Therapeutics of Quassia.}

Quassia is an aromatic bitter stomachic, acting in the same way as calumba. As it contains no tannic acid it is often prescribed with iron. The only objection to it is that some persons find it too bitter. Injected per rectum, it is an excellent anthelmintic for Oxyuris vermicularis; half a pint [250 c.c.] of the infusion [ $\mathrm{I}$ to 100 of cold water to avoid extraction of too much of the bitter principle,] may be given for this purpose, [the patient being in the knee-chest position.] 


\section{CASCARILLA.}

CASCARILLA.- [The bark of Croton Eluteriz Bennett (nat. ord. Euphorbiacece). Habitat.-Bahama Islands.

CHARACTERS. - In quills or curved pieces about $2 \mathrm{~mm}$. thick, having a grayish, somewhat fissured, easily detached, corky layer, more or less coated with a white lichen, the uncoated surface being dull brown, and the inner surface smooth. It breaks with a short fracture, having a resinous and radially striate appearance. When burned, it emits a strong, aromatic, somewhat musk-like odor; its taste is warm and very bitter.] Resembling Cascarilla.Pale Cinchona, which is less white, smooth and small.

Composition. - The chief constituents are-(I) Cascarillin, a bitter, neutral crystalline substance. (2) Volatile oil, [1.5 per cent.] (3) Resin. Tannic acid.

Incompatibles. - Mineral acids, lime water and metallic salts.

Dose, to to $30 \mathrm{gr}$.; [.60 to $2.00 \mathrm{gm}$.]

\section{Action and Therapeutics of Cascarilla.}

Cascarilla, because of its bitter principle cascarillin, like other vegetable bitters, improves the digestion, and this stomachic and carminative action is aided by the volatile oil in it. It is pleasant to take, and is suitable for the same cases as calumba. The infusion [ $\mathrm{I}$ to 20 ] will not keep good for more than a day unless the tincture [ $\mathrm{I}$ to $\mathrm{I} 5]$ is added to it. Mineral acids precipitate the resin from the tincture; therefore the infusion should be prescribed with them.

\section{CHIRATA.}

CHIRATA.-Synonym.-Chiretta. [The entire plant, Swertia Chirata Hamilton (nat. ord. Gentianea). Habitat.-Mountains of Northern India.

Characters. - Root nearly simple, about $7 \mathrm{~cm}$. long; stem branched, nearly I meter long, slightly quadrangular above; containing a narrow woodcircle and a large yellowish pith. Leaves opposite, sessile, ovate, entire fivenerved. Flowers numerous, small, with a four-lobed calyx and corolla. The whole plant smooth, pale brown, inodorous, and intensely bitter.] Resembling Chirata.--Lobelia, which is not bitter.

Composition. - The chief constituents are-(I) Chiratin, $\left[\mathrm{C}_{26} \mathrm{H}_{48} \mathrm{O}_{15}\right.$, ] an active, bitter principle, as a yellow, hygroscopic powder. (2) Ophelic Acid, $\mathrm{C}_{13} \mathrm{H}_{20} \mathrm{O}_{11}$,] with which it is combined. No Tannic Acid is present.

Dose, $1 / 2$ to I dr. ; [2. to $4 . \mathrm{gm}$.]

\section{Preparations.}

[1. Extractum Chiratæ Fluidum.-Fluid Extract of Chirata. By maceration and percolation with Alcohol and Water, and evaporation.

Dose, $1 / 2$ to $1 \mathrm{fl}$. dr.; 2. to 4 . c.c. 
2. Tinctura Chiratz.-Tincture of Chirata. Chirata, 100; by maceration and percolation with Alcohol and Water to roo.

Dose, $1 / 2$ to 2 fl. dr.; 2. to 8 . c.c.]

\section{Action and Therapeutics of Chirata.}

Chirata has the same actions and uses as gentian, calumba and other bitters. As it contains no tannic acid, it can be given with iron. It is more [frequently] used in India.

\section{CUSPARIA.}

CUSPARIAE CORTEX. [B. P., not official.]-Cusparia Bark. Synony'n.-Angustura Bark. The bark of Cusparia febrifuga (nat. ord. Rutacea). [Habitat. - ] Tropical South America.

Characters. - Flat or curved pieces or quills, [16 cm.] or less long, [4 $\mathrm{mm}$.] thick, obliquely cut on the inner edge. Externally a yellowish-gray, mottled, corky layer, which can be scraped off, and shows a dark brown resinous layer; inner surface light brown, flaky. Fracture short, resinous, and showing, under a lens, white points or lines. Taste bitter, aromatic. Odor musty, disagreeable. [Resembling Cusparia.-Canella Alba, but this is darker, and has pared edges.]

IMPURITY.-Bark of Strychnos nux-vomica (false Angustura bark); its inner surface gives bright blood-red color with Nitric Acid, showing Brucine; Cusparia does not.

Composition.-The chief constituents are-(I) Cusparine, or Angusturine, a crystalline bitter alkaloid. (2) An alkaloid, Galipeine. (3) An alkaloid, Galipidine. (4) An alkaloid, Cusparidine. (5) An aromatic oil. It is stated that no Tannic Acid is present, but Iron Salts are incompatible with Cusparia.

INCOMPATIBLES. - Mineral acids and metallic salts.

Dose, 10 to $40 \mathrm{gr}$.; [.60 to $2.40 \mathrm{gm}$.]

\section{Preparation.}

Infusum Cuspariæ. [B. P., not official.-Infusion of Cusparia], Cusparia, 5 ; Water at $120^{\circ} \mathrm{F}$; $\left[48.8^{\circ} \mathrm{C}\right.$. ] (to avoid extraction of nauseous principles), 100.

Dose, I to 2 fl. 0z.; [30. to 6o. c.c.]

\section{Action and Therapeutics of Cusparia.}

Cusparia bark is an aromatic bitter, having a similar action to calumba. It is used to make Angustura Bitters. In South America it is given [in large doses] as an antiperiodic. 


\section{SERPENTARIA.}

SERPENTARIA.-Synonym.-[Virginia Snakeroot. The rhizome and roots of Aristolochio Serpentaria Linné, and of Aristolochia reticulata Nuttall (nat. ord. Aristolochiacere). Habitat.-United States, in hilly woods.

Characters. - The rhizome is about $25 \mathrm{~mm}$. long, thin, bent; on the upper side with approximate short stem bases; on the lower side with numerous, thin, branching roots about $10 \mathrm{~cm}$. long; dull, yellowish-brown, internally whitish; the wood-rays of the rhizome longest on the lower side; odor aromatic, camphoraceous; taste warm, bitterish, and camphoraceous. The roots of Aristolochia reticulata are coarser, longer, and less interlaced than those of Aristolochia Serpentaria.] Resembling Serpentaria.-[Veratrune Viride, (see p. 442),] Arnica, (see p. 530), and Valerian, (see p. 556.)

Composition.-The chief constituents are-(1) A bitter principle, Aristolochine [in light-yellow needles. (2) A volatile oil, $1 / 2$ per cent. containing a Terpene, and mainly $\mathrm{C}_{15} \mathrm{H}_{25} \mathrm{O}_{2}$, Borneol Ether. (3) Resin. (4) Tannic Acid in small quantity.

Serpentaria is used to prepare Tinctura Cinchonæ Composita.]

Dose, 10 to $30 \mathrm{gr}$. ; [.60 to $2.00 \mathrm{gm}$.]

\section{Preparations.}

[1. Extractum Serpentariæ Fluidum.-Fluid Extract of Serpentaria. By'maceration and percolation with Alcohol and Water, and evaporation.

Dose, 10 to $30 \mathrm{~m}$; ; 60 to 2.00 c.c.

2. Tinctura Serpentariæ.-Tincture of Serpentaria. Serpentaria, I00; by maceration and percolation with Alcohol and Water to 1000.

Dose, $1 / 2$ to 2 fl. dr. ; 2. to 8. c.c.]

\section{Action and Therapeutics of Serpentaria.}

In the small doses in which serpentaria is given in medicine it is a bitter stomachic, acting just like calumba and cascarilla, and is used for the same class of cases. It is rarely prescribed alone. In large doses it produces vomiting and purging. Many virtues have been attributed to it which it [quite likely] does not possess.

\section{CIMICIFUGA.}

CIMICIFUGA.- [Synonyms. - Black Snakeroot. Black Cohosh. The rhizome and roots of Cimicifuga racemosa (Linné) Nuttall (nat. ord. Ranun(ulucec). Habitat.-North America ; in rich woodlands, westward to Eastern Kansas. 
Chinacters. - The rhizome is of horizontal growth, hard, $5 \mathrm{~cm}$. or more long, about $25 \mathrm{~mm}$. thick, with numerous stout, upright or curved branches, terminated by a cup-shaped scar, and with numerous wiry, brittle, obtusely quadrangular roots, about $2 \mathrm{~mm}$. thick; the whole brownish-black, of a slight but heavy odor, and of a bitter, acrid taste. Rhizome and branches have a smooth fracture, with a rather large pith, surrounded by numerous sublinear, whitish wood-rays, and a thin, firm bark. The roots break with a short fracture, have a thick bark, and contain a ligneous cord expanding into about four rays.

Composition.-The chief constituents are-(I) An acrid, crystalline principle, soluble in Chloroform and Alcohol. (2) Tannic and Gallic Acids. Two Resins.]

Cimicifugin or Macrotin is an impure resin deposited from the tincture on aarling water.

\section{Preparations.}

I. Extractum Cimicifugæ.-[Extract of Cimicifuga. By maceration and percolation with Alcohol, and evaporation.

Dose, I to $5 \mathrm{gr}$. ; .06 to $.30 \mathrm{gm}$.

2. Extractum Cimicifugæ Fluidum.-Fluid Extract of Cimicifuga. By maceration and percolation with Alcohol, and evaporation.

Dose, $1 / 2$ to I f. dr.; 2. to 4 . c.c.

3. Tinctura Cimicifugæ.-Tincture of Cimicifuga. Cimicifuga, 200 ; by maceration and percolation with Alcohol to 1000 .

Dose, $1 / 2$ to 2 fl. dr.; 2. to 8 . c.c.]

\section{Action of Cimicifuga.}

Cimicifuga has two chief actions. It influences the gastric secretion like any other bitter, and, to a slight extent, it depresses the rate, but increases the force of the pulse, like digitalis. The arterial tension rises. It is said to cause contractions of the uterus and to increase the menstrual flow.

\section{Therapeutics of Cimicifuga.}

It has been used for chorea, dyspepsia, bronchitis, amenorrhœea, dysmenorrhœa, rheumatism, neuralgia, and many other diseases. The evidence that it does much good [beyond thar accomplished by a simple bitter], is slight.

\section{TARAXACUM.}

TARAXACUM.-[Synonym.-Dandelion. The root of Taraxarum officinale Weber (nat. ord. Composibe), gathered in autumn. Habitat.-Grassy places and roadsides in Europe; naturalized in the United States. 
Characters. - Slightly conical, about $30 \mathrm{~cm}$. long, and 1 or $2 \mathrm{~cm}$. thick above, crowned with several short, thickish heads, somewhat branched, bark brown, longitudinally wrinkled, when dry breaking with a short fracture, showing a yellowish, porous central axis, surrounded by a thick, white bark, containing numerous milk vessels arranged in concentric circles; inodorous; bitter.] Resembling Taraxacum.-Pellitory, which is pungent when chewed.

Composition. - The chief constituents are- $[(\mathbf{I})$ Taraxacin, a crystalline bitier principle, soluble in water and Alcohol. (2) Taraxacerin, $\mathrm{C}_{8} \mathrm{H}_{16} \mathrm{O}$ ]. (3) Asparagin (found in asparagus, marsh-mallow, liquorice, euonymus) of no therapeutic value. (4) Inulin. (5) Resin (which gives the juice its milky appearance).

[IMPURITY. - The root of the Chicorium Intybus, which is paler, and has the milk vessels in radiating lines.]

Dose, $I$ to $3 \mathrm{dr}$.; [4. to $12 . \mathrm{gm}$.]

\section{Preparations.}

[1. Extractum Taraxaci.-Extract of Taraxacum. By expression and straining and evaporation.

Dose, 5 to $30 \mathrm{gr}$; .30 to $2.00 \mathrm{gm}$.

2. Extractum Taraxaci Fluidum.-Fluid Extract of Taraxacum. By maceration and percolation with diluted Alcohol and evaporation.

Dose, I to 4 fl. dr. ; 4 . to $15 . \mathrm{gm}$.]

\section{Action and Therapeutics of Dandelion.}

Dandelion is a simple bitter, and acts as a stomachic, just like calumba. It is also slightly laxative. It was formerly much more used than at the present day. It has been said to stimulate the flow of bile, but this is incorrect. [The vulgar name by which dandelion is known both in England and France suggests that it may be diuretic.]

\section{AURANTII CORTEX.}

[AURANTII AMARI CORTEX.-Bitter Orange Peel. The rind of the fruit of Citrus vulgaris Risso (nat. ord. K'utacece). Habitat.-Northern India; cultivated in subtropical countries.

Characters. - In narrow, thin bands, or in quarters; epidermis of a dark, brownish-green color, glandular, and with very little of the spongy, white inner layer adhering to it ; it has a fragrant odor, and an aromatic, bitter taste.

Composirion.-The chief constituents are-(I) A volatile oil, consisting mainly of Hesperidene, $\mathrm{C}_{10} \mathrm{H}_{16}$, with a small portion of Geraniol, $\mathrm{C}_{10} \mathrm{H}_{16} \mathrm{O}$. (2) Three glucosides, Hesperidin, Isohesperidin and Aurantiamarin, the bitter principle. Both Bitter and Sweet Orange Peel contain a substance which reacts with iron salts and Tannic Acid. 
Bitter Orange Peel is used in preparing Tinctura Cinchonæe Composita and Tinctura Gentianæ Composita.

\section{Preparations.}

I. Extractum Aurantii Amari Fluidum. - Fluid Extract of Bitter Orange Peel. By maceration and percolation with Alcohol and water, and evaporation.

Dose, $1 / 2$ to $\mathrm{I}$ fl. dr.; 2. to 4 . c.c.

2. Tinctura Aurantii Amari.-Tincture of Bitter Orange Peel. Bitter Orange Peel, 200. By maceration and percolation with Alcohol and water to 1000 .

Dose, I to 2 fl. dr.; 4. to 8. c.c.

AURANTII DULCIS CORTEX.-Sweet Orange Peel. The rind of the fresh fruit of Citrus Aurantium Linne (nat. ord. Rutacea). Habitat. -As of the Citrus vulyaris.

Characters. - Closely resembling Bitter Orange Peel, but having an orange-yellow color. It has a sweetish, fragrant odor, and an aromatic, slightly bitter taste.

Composition. - As of the Bitter Orange Peel.

\section{Preparations.}

I. Syrupus Aurantii.-Syrup of Orange. Sweet Orange Peel, 50;-Precipitated Calcium Phosphate, 50; Sugar, 700. By boiling with Alcohol, mixture of the tincture (thus formed) in Sugar with Water, addition of Water and filtration to 1000 .

Dose, as vehicle.

2. Tinctura Aurantii Dulcis.-Tincture of Sweet Orange Peel. Sweet Orange Peel, 200. By maceration and percolation with Alcohol to 1000 .

Dose, I to 2 fi. dr.; 4. to 8. c.c.

OLEUM AURANTII CORTICIS.-Oil of Orange Peel, a volatile oil obtained by expression from the fresh peel of either the Bitter Orange, Citrus vulyurus Risso, or the Sweet Orange, Citrus Aurantium Linné (nat. ord. Rutacea).

Characters. - A pale, yellowish liquid, having the characteristic, aromatic odor of Orange, and an aromatic and, when obtained from the Bitter Orange, somewhat bitter taste. Sp. gr., about 0.850 . Solubility. - In four times its volume of Alcohol.

IMPURITIES. - Oil of Turpentine or other oils containing Pinene.

Oil of Orange Peel is contuined in Spiritus Myrcix. 


\section{Preparations.}

I. Spiritus Aurantii._Spirit of Orange. Oil of Orange Peel, 50; Deodorized Alcohol, 950.

Dose, as vehicle.

2. Spiritus Aurantii Compositus.-Compound Spirit of Orange. Oil of Orange Peel, 200; Oil of Lemon, 50; Oil of Coriander, 20; Oil of Anise, 5 ; Deodorized Alcohol to 1000.

Dose, as vehicle.

3. Elixir Aromaticum.-Áromatic Elixir. Compound Spirit of Orange, 12; Syrup, 375 ; Precipitated Calcium Phosphate, 15; Deodorized Alcohol, Distilled Water, each, a sufficient quantity to make 1000. By solution of the Compound Spirit of Orange in Deodorized Alcohol, to 250; addition of Syrup and Precipitated Calcium Phosphate, and filtering, with Distilled Water to rooo.

Dose, as vehicle.

OLEUM AURANTII FLORUM.-Oil of Orange Flowers. Synonym.-Oil of Neroli. A volatile oil distilled from fresh flowers of the Bitter Orange, Citrus vulgaris Risso (nat. ord. Rutacea).

Characters. - A yellowish or brownish, thin liquid, having a very fragrant odor of Orange Flowers, and an aromatic, somewhat bitter taste. Sp. gr., 0.875 to 0.890 . Solubility. - In an equal volume of Alcohol.

\section{Preparations.}

I. Aqua Aurantii Florum Fortior.-Stronger Orange Flower Water. Synonym. - Triple Orange Flower Water. Water saturated with the volatile oil of fresh Orange Flowers.

Dose, indeterminate.

2. Aqua Aurantii Florum.-Orange Flower Water. Stronger Orange Flower Water, 500; Distilled Water to 1000.

Dose, indeterminate.

3. Syrupus Aurantii Florum.-Syrup of Orange Flowers. Sugar, 850; Orange Flower Water to 1000.

Dose, indeterminate.]

\section{Action and Therapeutics of Orange.}

The various preparations of the orange are used largely as flavoring agents. They are slightly bitter and stomachic. [The aromatic elixir is an excellent flavoring agent and vehicle for liquid medicines.] 


\section{GROUP VIII.}

\section{Vegetable Drugs containing Tannic Acid.}

These are all astringent.

[Oak Bark,] Nutgall, [Tannic Acid, Gallic Acid, Pyrogallol,] Catechu, Krameria, Kino, Hæmatoxylon, Hamamelis, [Rhus Glabra, Geranium, Rubus, Rumex,] Eucalyptus Gum, [Coto.]

[QUERCUS ALBA.-White Oak. The bark of the Quercus alla Linné (nat. ord. Cupulifera). Habitat.-North America, westward to Minnesota, Kansas, and Mississippi ; in woods.

Characters. - In nearly flat pieces, deprived of the corky layer, about 5 $\mathrm{mm}$. thick ; pale brown; inner surface with short, sharp, longitudınal ridges; tough ; of a coarse, fibrous fracture, a faint, tan-like odor, and a strongly astringent taste. As met with in the shops it is usually an irregularly coarse, fibrous powder, which does not tinge the saliva yellow.

CoMpositiox. - The chief constituents are-(I) Quercitannic acid, $\left[\mathrm{C}_{28} \mathrm{H}_{24}\right.$ $\mathrm{O}_{12}, 6$ to 1 I per cent., a variety of Tannic Acid. (2) Quercin, a bitter principle. (3) Quercite, a sugar. (4) Resin.

INCoMPATibles. - See Tannic Acid, p. 593.]

GALLA.-[Nutgall. An excrescence on Qucrcus lusitanica Lamarck (nat. ord. Cupulifer(E), caused by the punctures and deposited ova of Cynips Galle tinctorice Olivier (class Insecta; order Hymenoptera. Habitat Levant.

Cilaracters. - Subglobular, 1 or $2 \mathrm{~cm}$. in diameter, more or less tuberculated above, otherwise smooth, heavy, hard ; often with a circular hole near the middle, communicating with the central cavity; blackish olive-green or blackish-gray; fracture granular, grayish; in the centre a cavity containing either the partly developed insect, or purulent remains left by it; nearly inodorous, taste strongly astringent.]

Composition. - The chief constituents are-(1) Tannic Acid, [50 to 60] per cent. (2) Gallic Acid, 2 to [3] per cent. (3) Sugar. (4) Resin.

INCOMPATIBLES. - See Tannic and Gallic Acids, [p. 593 and p. 596.]

\section{Preparations.}

1. Tinctura Gallæ.-[Tincture of Nutgall. Nutgall, 200; by maceration with Glycerin, I00, and Alcohol to I000.

Dose, $I$ to 2 fl. dr.; 4 . to 8 . c.c.

2. Unguentum Gallæ.-Nutgall Ointment. Nutgall, 20; Benzoinated Lard, 8o.] 
ACIDUM TANNICUM.-Tannic Acid, $\left[\mathrm{HC}_{14} \mathrm{H}_{9} \mathrm{O}_{9}=321.22\right.$. Synonyms. -Tannin. Gallotannic Acid. Digallic Acid. An organic acid extracted from Nutgall.]

Source. - (I) Expose powdered Nutgall to a damp atmosphere for twentyfour hours. (2) Add Ether to form a paste, and let it stand, closely covered, for six hours. (3) Express this in a close canvas cloth, between tinned plates, reduce the resulting cake to powder and mix with sufficient Ether and express as before. (4) Mix the expressed liquids and allow the mixture to evaporate spontaneously. Tannic Acid remains. $\left[2 \mathrm{HC}_{7} \mathrm{H}_{5} \mathrm{O}_{5}-\mathrm{H}_{2} \mathrm{O}=\mathrm{HC}_{14} \mathrm{H}_{9} \mathrm{O}_{9}\right]$

GHARACTERS. - [A light yellowish, amorphous powder, usually cohering in form of glistening scales or spongy masses, odorless, or having a faint characteristic odor, and a strongly astringent taste; gradually turning darker when exposed to air and light. Solubility. - In about 1 part of water, and in 0.6 part of Alcohol; also in about I part of Glycerin, with the intervention of a moderate heat; freely soluble in diluted Alcohol, sparingly in absolute Alcohol ; almost insoiuble in alssolute Ether, Chlorofurm, Benzol or Benzın.]

InCOMPATIBLES. - Mineral acids, alkalies, antimony, lead, silver, and ferric salts, alkaloids, gelatin, and einulsions.

Dose, $\mathrm{r}$ to $20 \mathrm{gr}$; ; [.06 to $\mathrm{r} .20 \mathrm{gm}$.]

\section{Preparations.}

[r. Collodium Stypticum.-Styptic Collodion. Tannic Acid, 20; Alcohol, 5 ; Ether, 25; Collodion, to 100. By solution.

2. Trochisci Acıdi Tannici.-Troches of Tannic Acid. Tannic Acid, 6; Sugar, 65; Tragacanth, 2 gm.; Stronger Orange Flower Waler, a sufficient quantity to make 100 troches. Each troche contains about I gr.; $.06 \mathrm{gm}$. of Tannic Acid.

Dose, $\mathrm{I}$ to 3 troches.

3. Unguentum Acıdi Tannici.-Ointment of Tannic Acid. Tannic Acid, 20; Benzoinated Lard, 80.

4. Glyceritum Acidi Tannici._Glycerite of Tannic Acid. Tannic Acid, 20; Glycerin, So.]

\section{Action of Tannic Acid.}

External.-Tannic acid is one of the most important drugs, because it coagulates albumin and gelatin with great readiness; that is to say, it tans the tissues, for it is by coagulating the interstitial fluid in skins that tannic acid converts them into leather. The coagulated albumin or gelatin powerfully resists putrefaction. If an albuminous discharge is taking place from a sore or mucous surface and tannic acid is applied, the excreted 
fluid is coagulated, and the coagulum forms a solid protecting layer which prevents further discharge. As the tannic acid soaks into the tissues it coagulates the albuminous fluids there also, and this still further hinders the discharge of fluid, therefore it is an energetic astringent. If bleeding is taking place, tannic acid of course coagulates the blood as it flows and the clots plug the vessels; at the same time the coagulum formed within the tissues, by its contraction, constricts the blood-vessels, and thus tannic acid becomes a powerful hæmostatic. It has no noteworthy direct effect on the blood-vessels themselves. Tannic acid is mildly depressant to sensory nerves. Like other acids it is irritant, but it is very feebly so, and consequently its action in this direction is more than counterbalanced by its strongly astringent effects.

Internal.-Gastro-intestinal tract.-Because tannic acid coagulates the mucous secretions and the fluids in mucous membranes, it makes the mouth dry and feel stiff when locally applied; in the stomach large doses prevent the secretion of gastric juice, decrease the flow of mucus, and may cause vomiting. For these reasons, and also because it precipitates pepsin, it interferes with digestion. It will check gastric hæmorrhage. In the intestine is is either converted into gallic acid, or forms alkaline tannates, and until these alterations it acts as an intestinal astringent, controlling intestinal bleeding and causing constipation; but this acid and these salts have no astringent properties, therefore when, [as is often the case,] drugs containing large amounts of tannic acid act as powerful intestinal astringents, we must suppose that the amount of tannic acid taken is large enough for the conversion of it, into salts or gallic acid, to take place slowly. It is absorbed chiefly as gallates, and to a much less extent as tannates.

Remote effects. - Gallates and undecomposed alkaline tannates circulate in the blood, but they have no power to coagulate albumin, nor have they any astringent influence when locally applied, therefore it is difficult to believe that tannic acid has any remote astringent or hæmostatic effects ; some claim that it has, but they have not proved their case. It is excreted in the urine 
of animals as gallates with traces of tannates, but in man no derivative of it can be detected in the urine or other excretions, so that any which has been absorbed is entirely decomposed in the body. Any excess passes out in the fæces as tannates and gallates. Many vegetable substances, as logwood, depend for their astringent properties on the tannic acid they contain.

\section{Therapeutics of Tannic Acid.}

The therapeutical applications of tannic acid are very numerous, It is used as an astringent for uIcers, sores, various moist eruptions, tonsillitis, pharyngitis, nasal catarrh, otorrhœa, gastric catarrh, diarrhœa (large doses of $30 \mathrm{gr}$. ; [ $2 \mathrm{gm}$.] may be given, and catechu and logwood are favorite remedies), leucorrhœa, gonorrhœa, rectal ulcers, fissures, and prolapse. It is employed as a hæinostatic in bleeding from small wounds, ulcers, the gums, the pharynx, the nose, the stomach, the intestine, hæmorrhoids, and the bladder. Whenever practicable a good method of application is to dust it on the part, especially for a hrmorrhage ; if this is gastric or intestinal, $3 \circ \mathrm{gr}$. ; [2. gm.] or more should be frequently given by the mouth. For external use or application to the throat the glycerite is useful. A gargle of the glycerite in water, [ $\mathrm{I}$ to 8$]$ may be made. The [troches] are convenient for pharyngitis. A spray ( 1 to 2 in 96 of water) or an insufflation of tannic acid and starch may be used for the mouth and larynx. The ointment of [nutgall] and opiun, [I to 14 of ointment of nutgall,] is a favorite application for piles. The suppositories [3 gr.; . $20 \mathrm{gm}$. each] are useful for rectal discharges. Solutions [I to 48 ] in water may be injected into the urethra for urethritis and gonorrhœa, and into the bladder for cystitis. [It should never be used hypodermatically]. The decoction of oak bark, employed as a [high] rectal injection, destroys the threadworm. [A preparation of nutgall dissolved in glycerin was formerly used as an injection into hernial sacs (Heaton's method). The temporary results were excellent, but sooner or later failures occurred in a very large percentage of cases.

Tannigen (not official), the acetic acid ester of tannic acid, is prepared by the action of glacial acetic acid on tannic acid. 
It is a tasteless, odorless powder, insoluble in water, and is believed to pass unchanged through the stomach and to be slowly decomposed in the intestines, thus exerting an astringent effect in them. The dose is 5 to $30 \mathrm{gr}$., 30 to $2.00 \mathrm{gm}$. in wafers.

Tannalbin (not official) is a tannin albuminate which has been subjected to a dry heat of $230^{\circ}-248^{\circ} \mathrm{F}$. ( $110^{\circ}-120^{\circ} \mathrm{C}$.) for several hours. It is a faintly yellow, tasteless powder containing about $5 \circ$ per cent. of tannic acid. Laboratory experiments have shown that it is not easily decomposed by an artificial gastric juice, but it is rapidly separated into its constıtuents in an alkaline medium or by an artificial solution of the pancreatic ferments. This preparation then passes through the stomach unchanged, and may not be broken up until it has passed well down into the intestine. The dose is $5 \mathrm{gr} ., .30 \mathrm{gm}$., given in wafers at frequent intervals.

ACIDUM GALLICUM.-Gallic Acid. $\quad \mathrm{HC}_{7} \mathrm{H}_{5} \mathrm{O}_{5}+\mathrm{H}_{2} \mathrm{O}[=187.55$. An organic acid, usually prepared from Tannic Acid.

SoUrCE. - By exposing a unixture of Nutgall and distilled water in the form of a thin paste, to the air for a month, expressing and rejecting the liquor, boiling the residue with distilled water and filtering, when hot, through purified Animal Charcoal. $\quad \mathrm{HC}_{14} \mathrm{H}_{9} \mathrm{O}_{9}+\mathrm{H}_{2} \mathrm{O}=2 \mathrm{HC}_{7} \mathrm{H}_{5} \mathrm{O}_{5}$.

Characters. - White, or pale fawn-colored, silky, interlaced needles, or triclinic prisms; odorless; having an astringent or slightly acidulous taste; permanent in the air. Solubility. - In 100 parts of water, and in 5 parts of Alcohol; also soluble in 40 parts of Ether, and in 12 parts of Glycerin. Very slightly soluble in Chloroform, Benzol, or Benzin.]

IxсомPATI81.FS. - Ferric and metallic salts generally, and Spiritus Etheris Nitrosi.

Dose, 5 to $20 \mathrm{gr}$; [ [.30 to $1.20 \mathrm{gm}$.]

\section{Action and Uses of Gallic Acid.}

Gallic acid has no power to coagulate albumin, and therefore possesses none of the local properties of tannic acid. 'If it is wished to produce the supposed remote astringent effects of tannic acid, gallic acid may be administered, for tannic acid is in the intestine converted into it.

\section{PYROGALLOL.}

[PYROGALLOL. $-\mathrm{C}_{6} \mathrm{H}_{3}(\mathrm{OH})_{3}=125.7$. Synonym.-Pyrogallic Acid. SOURCE. - A triatomic phenol obtained chiefiy by the dry distillation of Gallic Acid. $\quad \mathrm{IC}_{7} \mathrm{H}_{5} \mathrm{O}_{5}=\mathrm{C}_{6} \mathrm{H}_{3}(\mathrm{OH})_{3}+\mathrm{CO}_{2}$. 
Characters. - Light white, shining laminx, or fine needles, odorless, and having a bitter taste; acquiring a gray or darker tint on exposure to air and light. Solubility._In 1.7 parts of water, and in I part of Alcohol; also soluble in 1.2 parts of Ether.]

\section{Action and Therapeutics of Pyrogallol.}

It is used externally as an ointment (Jarisch's ointment is pyrogallic acid, $\mathrm{r}$; lard, 8) for the treatment of chronic psoriasis. It is also an excellent parasíticide for ringworm. [Before pyrogallol is used, vaseline should be applied thoroughly, and wiped off, to remove scales and other morbid products.] It must not be applied over too large a surface, as it may be absorbed, and then it breaks up the blood corpuscles, causing methæmoglobinuria and jaundice. Jarisch's ointment is very strong; a more usual strength is I or 2 [or even less] to 48 of lard. [When it is applied in solution or ointment, it stains the skin, but not permanently; linen and clothing are, however, permanently darkened. To avoid the staining it has been proposed to dissolve the remedy in flexible collodion, I or 2 to 24.]

\section{CATECHU.}

CATECHU.-[Synonym.-Cutch. An extract prepared from the wood of Acacia Catechu (Linné filius) Willdenow (nat. ord. Leguminosce). Habitat. -India.

ChARACTERS.-In irregular masses, containing fragments of leaves, dark brown, brittle, somewhat porous and glossy when freshly broken. It is nearly inodorous, and has a strongly astringent and sweetish taste.]

Composirio.s. - The chief constituents are-(1) Catechutannic Acid, about 45 per cent., the active principle, isomeric with Catechin, and converted into it by boiling or by the saliva, a red color being formed. (2) Catechin or Catechuic Acid $\left[\mathrm{C}_{21} \mathrm{I}_{20} \mathrm{O}_{9}+5 \mathrm{H}_{2} \mathrm{O}\right]$, probably inactive. Both constituents give a green precipitate with Ferric Salts. (3) Pyrocatechin or catechol gives a green color with ferric chloride.

INCOMPATiBLes.-Alkalies, metallic salts, and gelatin.

IMPURITY. - Starch.

Dose, 10 to $30 \mathrm{gr}$; ; .60 to $2.00 \mathrm{gm}$.]

\section{Preparations.}

I. [Tinctura Catechu Composita.-Compound Tincture of Catechu. Catechu, 100 ; Cassia Cinnamon, $5^{\circ}$; by maceration and percolation with diluted Alcohol, to 1000 .

Dose, $1 / 2$ to 2 fl. dr. 2 . to 8 . c.c. 
2. Trochisci Catechu.-Troches of Catechu. Catechu, 6; Sugar, 65 ; Tragacanth, 2 gm.; Stronger Orange Flower Water, a sufficient quantity to make 100 troches. Each troche contains about I gr.; $.06 \mathrm{gm}$.

Dose, I to 6 troches.]

\section{Action and Therapeutics of Catechu.}

Catechu is a powerful astringent, acting in virtue of its tannic acid, and having a precisely similar action to it. It is. used as a [troche] for sore throat, and the compound [tincture] is very efficacious for diarrhœa.

\section{KRAMERIA.}

[KRAMERIA.-Synonym.-Rhatany. The root of Krameria triandra Ruiz et Pavon, and of Krameria Ixina Linné (nat. ord. Polygalea). Habitat. -Peru and Bolivia.

Characters. - From I to $3 \mathrm{~cm}$. thick, knotty and several-headed above, branched below, the branches long; bark smooth or, in the thinner pieces, scaly, deep rust-brown, I to $2 \mathrm{~mm}$. thick, very astringent, inodorous; wood pale brownish red, tough, with fine medullary rays, nearly tasteless. The root of Krameria Ixina (Savanilla Rhatany) is less knotty and more slender, and has a dark purplish-brown bark, about $3 \mathrm{~mm}$. thick.]

Compositron.-The chief constituents are-(I) [Kramerotannic Acid, $\mathrm{C}_{34} \mathrm{H}_{24} \mathrm{O}_{21}$, 20 per cent. (.2) Rhatanin. (3) Rhatanic red, $\mathrm{C}_{26} \mathrm{H}_{22} \mathrm{O}_{11}$ ], the coloring matter.

INCOMPATIBLES.-Alkalies, lime water, iron and lead salts, and gelatin.

Dose, 5 to $30 \mathrm{gr}$.; [.30 to $2.00 \mathrm{gm}$.]

\section{Preparations.}

I. Extractum Krameriæ.-[Extract of Krameria. By percolation with water, straining and evaporation.

Dose, 5 to $10 \mathrm{gr}$.; .30 to $.60 \mathrm{gm}$.

2. Extractum Kramerix Fluidum.-Fluid Extract of Kramerir. By maceration and percolation with Glycerin and Diluted Alcohol, and evaporation.

Fluid Extract of Krameria is used to make Syrupus Kramenx.

Dose, 5 to $30 \mathrm{~m}$. ; .30 to 2.00 c.c.

3. Tinctura Krameriæ.-Tincture of Krameria. Krameria, 200; by maceration and percolation with Diluted Alcohol to 1000.

Dose, $1 / 2$ to $2 \mathrm{fl}$. dr.; 2. to 8 . c.c.

4. Trochisci Kramerix.-Troches of Krameria. Extract of Krameria, 6; Sugar, 65; Tragacanth, 2 gm.; Stronger Orange Flower 
Water, a sufficient quantity to make Ioo troches. Each troche contains about I gr.; $.06 \mathrm{gm}$.

Dose, I to 5 troches.

5. Syrupus Krameriæ.-Syrup of Krameria. Fluid Extract of Krameria, $45^{\circ}$; Syrup, $55^{\circ}$.

Dose, $1 / 2$ to 4 fi. dr.; 2. to 15 . c.c.]

\section{ACTION OF Krameria.}

The action of krameria is due entirely to the tannic acid it contains. It is therefore a powerful astringent.

\section{Therapeutics of Krameria.}

The extract is the important ingredient of many tooth powders which are useful when the gums are liable to bleed. An infusion [B. P., I to 20] is an excellent gargle for a relaxed throat, and the troches are also efficacious. [The B. P. has a
[The troche each containing I gr.; .06 gm. of the extract with $\frac{1}{20} \mathrm{gr}$.; $.003 \mathrm{gm}$. of cocaine hydrochlorate, with a fruit basis.] Bleeding from the nose or the rectum may be stopped by applying powdered krameria locally; the infusion may be used as an injection in leucorrhœa or gonorrhœa. Any of its preparations, especially [the fluid extract] are powerful astringents for all varieties of diarrhœea, and may be taken to stop bleeding from the stomach and intestines. They are also given as remote hæmostatics for hæmoptysis and hæmaturia, but they are not reliable for these purposes.

\section{KINO.}

KINO.-[The inspissated juice of Pterocarpus Marsupium Roxburgh (nat. ord. Leguminosa). Habitat.-East Indies.

Characters. - Small, angular, dark brownish-red, shining pieces, brittle, in thin layers ruby red and transparent, inodorous, very astringent and sweetish, tingeing the saliva deep red. Solubility. - Soluble in Alcohol, nearly insoluble in Ether, and only slightly soluble in cold water.]

Composition.-The chief constituents are-(I) Kinotannic Acid, $\left[\mathrm{C}_{18}\right.$ $\mathrm{H}_{18} \mathrm{O}_{8}$, 75 per cent. (2) Kinoin, a crystalline neutral principle. (3) Pyrocatechin, $\mathrm{C}_{6} \mathrm{H}_{4}\left(\mathrm{OH}_{2}\right)$, a substance also found pathologically in the urine, and giving it a dark color. It reduces blue copper solutions. (4) Kino red, formed from kinotannic acid by oxidation. (5) Gum.

IncóMPATIBles. - Mineral acids, alkalies, all metallic salts, carbonates, and gelatin.

Dose, 5 to $30 \mathrm{gr}$; [ $[30$ to $2.00 \mathrm{gm}$.] 


\section{Preparation.}

Tinctura Kino.- [Tincture of Kino. Kino, I00; Glycerin, 150. By maceration and filtration with water, 200 ; and Alcohol to 1000.

Dose, $1 / 4$ to 2 fl. dr. ; I. to 8 . c.c.]

\section{Action and Therapeutics of Kino.}

Kinotannic acid acts like tannic acid, and therefore kino is a powerful astringent. It is used in astringent gargles, and also in diarrhœa mixtures.

\section{HAEMTOXYLON.}

HEMATOXYLON.-Synonym.-Logwood. [The heart-wood of Hématoxylon campechianum Linné (nat. ord. Leguminosa). Habitat.Central America; naturalized in the West Indies.

Characters. - Heavy, hard, externally purplish-black, internally brownish-red, and marked with irregular, concentric circles, splitting irregularly; odor faint, agreeable; taste sweetish, astringent. When chewed, it colors the saliva dark pink. Logwood is generally met with in the form of small chips or coarse powder of a dark brownish-red color, often with a greenish lustre.] Resembling Logwood.-Red Saunders, which is more dense and less astringent.

Composition.-The chief constituents are-(1) Tannic Acid. (2) Hamatoxylin, $\mathrm{C}_{16} \mathrm{H}_{14} \mathrm{O}_{6}, \mathrm{I} 2$ per cent. Occurring in [sweet], colorless crystals, which bécome dark-red on exposure to light. Solutions of it are used to stain histological specimens. [(3) Hamatein, $\mathrm{C}_{16} \mathrm{H}_{12} \mathrm{O}_{6}$, a product of oxidation of the former, having a green, metallic lustre.]

INCUMPATIBLES. - Mineral acids, lime water, and tartar emetic; metallic salts give a blue color.

\section{Preparation.}

[Extractum Hæmatoxylon.-Extract of Hæmatoxylon. By maceration in Water, boiling, straining and evaporation.

Dose, 5 to $15 \mathrm{gr}$; . 30 to $1.00 \mathrm{gm}$.]

\section{Action and Therapeutics of Logwood.}

In virtue of its tannic acid, logwood is a powerful astringent, and for this purpose is used to control diarrhœa of all sorts. It may be combined with other astringents, as chalk and [with] opium [to check peristalsis.] It does not easily produce constipation. It colors the urine and fæces dark red. One disadvantage of it is that it stains linen, if [it comes in contact with] it. 


\section{HAMAMELIS.}

HAMAMELIS.-[Synonym. - Witchhazel. The leaves of Hamamelis virginiana Linné (nat. ord. Hamamelacea), collected in autumn. Habitat.North America, in thickets.

Characters. - Short petiolate, about $10 \mathrm{~cm}$. long, obovate or oval, slightly heart-shaped and oblique at the base, sinuate-toothed, thickish, nearly smooth; inodorous; taste astringent and bitter.]

Composition. - The chief constituents are - (I) Tannic Acid, 8 per cent. (2) A bitter principle not yet isolated. - (3) Resin.

\section{Preparation.}

[Extractum Hamamelidis Fluidum.-Fluid Extract of Hamamelis. By maceration and percolation with Glycerin, Alcohol and Water, and evaporation.

Dose, $1 / 2$ to $2 \mathrm{fl}$. dr.; 2. to 8 . c.c.]

\section{Action and Therapeutics of Witchhazel.}

Hamamelis is, because of its tannic acid, astringent and hæmostatic. The [fluid] extract is used for capillary hæmorrhage from wounds, for bleeding from the nose, the sockets of the teeth, the gums, or from piles, and it may be injected into the bladder in vesical hæmorrhage. For all these purposes it is diluted with water; the fluid extract in ro or 20 parts of water is commonly employed. Locally applied, hamamelis, either as the ointment [B. P., I to Io, made from the fluid extract] or the diluted fluid [extract], is used as an astringent in bruises, sprains, pharyngitis, and nasal catarrh. The ointment is often used for piles. Given by the mouth, hamamelis may check diarrhœea, dysentery, etc.; and it is reputed to be a remote hæmostatic and astringent, but this is probably incorrect. Hazeline is a distilled extract from the leaves. [A preparation of witchhazel in popular use is known as Pond's extract.

\section{RHUS GLABRA.}

RHUS GLABRA.-Synonym.-Sumach. The fruit of Rhus glabra Linné (nat. ord. Anacardiea). Habitat. - North America, west to Colorado and Idaho; in barren soil.

Characters. - Subglobular, about $3 \mathrm{~mm}$. in diameter, drupaceous, crimson, densely hairy, containing a roundish-oblong, smooth putamen; inodor. ous; taste acidulous. 
Composition.-The chief constituents are-(1) Tannic Acid, of which it contains from 6 to 27 per cent. (2) Gallic Acid. (3) Acid calcium and potassium malates. (4) A red coloring matter.

\section{Preparation.}

Extractum Rhois Glabræ Fluidum.-Fluid Extract of Rhus Glabra. By maceration and percolation with Glycerin and Diluted Alcohol, and evaporation.

Dose, $1 / 4$ to 1 fi. dr. ; I. to 4 . c.c.

\section{Action and Uses of Rhus Glabra.}

Sumach fruit is astringent and refrigerant. The fluid extract, when diluted, affords a very useful and effective gargle for inflammation and ulceration of the throat.

\section{GERANIUM.}

GERANIUM.-Synonym.-Cranesbill. The rhizome of Geranium maculatum Linné (nat. ord. Geraniacece). Habitat.-North America, in woods and thickets.

ChARACTERS. - Of horizontal growth, cylindrical, 5 to $7 \mathrm{~cm}$. long; about I $\mathrm{cm}$. thick; rather sharply tuberculated, longitudinally wrinkled, dark brown; fracture short, pale reddish-brown; bark thin; wood-wedges yellowish, small, forming a circle near the cambium line; medullary rays broad; central pith large ; roots thin, fragile ; inodorous ; taste strongly astringent.

Composition.-(1) Tannic Acid, I2 to 17 per cent. (2) Gallic Acid. (3) Pectin.

\section{Preparation.}

Extractum Geranii Fluidum.-Fluid Extract of Geranium. By maceration and percolation with Glycerin and Diluted Alcohol, and evaporation.

Dose, $1 / 4$ to I fl. dr.; I. to 4 . c.c.

\section{Action and Uses of Geranium.}

Geranium is one of the best indigenous astringents, and is of great use in diarrhœa and dysentery, and in the various hæmorrhages.

\section{RUBUS.}

RUBUS.-Synonym.-Blackberry. The bark of the root of Rubus villosus Aiton, Rubus canadensis Linné, and Rubus trivialis Michaux (nat. ord. Rosacea). Habitat.-North America, in fieids and thickets. 
Characters. - In thin, tough, flexible bands, outer surface blackish or blackish-gray, inner surface pale brownish, sometimes with strips of whitish, tasteless wood adhering; inodorous; taste strongly astringent, somewhat bitter.

Composition.-(1) Tannic Acid, 10 to 13 per cent. (2) Gallic Acid, 0.4 per cent. (3) Villosin, a bitter crystalline glucoside, soluble in Alcohol.

\section{Preparations.}

1. Extractum Rubi Fluidum.-Fluid Extract of Rubus. By maceration and percolation with-Glycerin, Alcohol and Water, and evaporation.

Fluid Extract of Rubus is used to make Syrupus Rubi.

Dose, $1 / 2$ to 2 fl. dr.; 2. to 8. c.c.

2. Syrupus Rubi.-Syrup of Rubus. Fluid Extract of Rubus, 250; Syrup, 750.

Dose, I to 2 fl. dr.; 4 . to 8 . c.c.

\section{ACtion ANd UsEs OF BlackBerRy.}

The preparations made from blackberry root are tonic and slightly astringent. They are used for diarrhœa; blackberry brandy is a common domestic remedy.

\section{RUMEX.}

RUMEX.- Synonym.-Yellow Dock. The root of Rumex crispus Linné, and of some other species of Rumex (nat. ord. Polygonacea). Habitat.-Europe ; naturalized in North America, in grassy places and along roadsides.

Characters. - From 20 to $30 \mathrm{~cm}$. long, about to to $15 \mathrm{~mm}$. thick, somewhat fusiform, fleshy, nearly simple, annulate above, deeply wrinkled below ; externally rusty brown, internally whitish, with fine, straight, interrupted, reddish, medullary rays, and a rather thick bark; fracture short; odor slight, peculiar; taste bitter and astringent.

Composition.-(I) Tannic Acid. (2) Rumicin, identical with Chrysophanic Acid. (3) Calcium Oxalate, and other salts.

Dose, $1 / 4$ to I dr. ; I. to $4 . \mathrm{gm}$.

\section{Preparation.}

Extractum Rumicis Fluidum.-Fluid Extract of Rumex. By maceration and percolation with Diluted Alcohol, and evaporation.

Dose, $1 / 4$ to 1 fl. dr.; r. to 4 . c.c.

Action AND Uses of Rumex.

Rumex is astringent, slightly tonic and alterative. It has been used in svrhilis. scorbutic disorders, and cutaneous eruptions.] 


\section{EUCALYPTI GUMMI.}

EUCALYPTUS GUM - [B. P., not official. ] Synonym.-Red Gum. A ruby-colored exudation from the balk of Eucalyptus rostrata (nat. ord. Myrtacea) and from other species. [Habitat.-] Australia.

Characters.-An inspissated secretion forming semi-translucent and garnet-colored grains or small masses. Tough and difficult to powder. Adheres to the teeth when chewed. Taste very astringent. Soluble in water. Resembling Eucalyptus Gum.-Kino, which is darker and feebly soluble in water.

Composition. - The chief constituents are-(1) Kinotannic Acid. Catechin. (3) Pyrocatechin.

Dose, 2 to ro $\mathrm{gr}$; ; $[.12$ to $.60 \mathrm{gm}$.] in powder, or in an aqueous solution or made into a pill with mucilage of Acacia.

\section{Action and Therapeutics of Red Gum.}

Red gum is, by virtue of its tannic acid, powerfully astringent and is used in diarrhœa and dysentery. Lozenges, $x \mathrm{gr}$. [.06 gm.] in each, with fruit paste, or a decoction of $x$ in 40 , as a gargle, are employed for relaxed throats. This decoction may also be given in 2 to 4 [fl.] dr. [8. to I 5. c. c.] doses for diarrhœa. A fluid extract (red gum, 7 ; water, 2 I ; alcohol, $\mathbf{I}$;) dose, I/2 to I fl. dr. [2. to 4. c.c.] is a useful preparation. Injected into the nose it stops epistaxis. Mixed with $x$ to 10 of water it may be injected into the rectum or vagina, or may be used as a mouth wash. Suppositories, each containing $5 \mathrm{gr}$; [ $.30 \mathrm{gm}$.$] of red$ gum, are prepared, and may be employed for piles.

\section{coto.}

COTO BARK. [Not official.]-The bark [of Drimys Winteri, Foster, var. granatensis, Eichler. Habitat.-Venezuela.

Characters. - In irregular pieces, outer surface irregular as well as the inner surface; color cinnamon-brown; upon fresh cross-section the bark is seen to be filled with yellowish spots, except in the outer portions ; odor aromatic, especially if bruised; the powder is very pungent. Resembling Coto Bark.Paracoto Bark, which has a less powerful odor and taste; and is marked with deep, whitish furrows upon its surface.

Composition. - The chief constituents are-(I) Cotoin (see p. 605). (2) A volatile oil. (3) A resin in large amount. (4) A volatile alkaloid of a peppery taste, probably propylamine. (5) Piperonylic Acid, $\mathrm{C}_{8} \mathrm{H}_{6} \mathrm{O}_{4}$.] Coto is placed here provisionally among the drugs containing Tannic Acid, until its composition [can be ascertained.]

Dose, I to $10 \mathrm{gr} . ;[.06$ to $.60 \mathrm{gm}$. 
[COTOIN. $-\mathrm{C}_{22} \mathrm{H}_{18} \mathrm{O}_{6}=377$. xo.

ChARACTERS. - A glucoside occurring as a pale yellow, amorphous powder, or in minute, curved, white, fusible prisms. Solubility. - Slightly in water; soluble in Alcohol, Ether, and Chloroform. It has a biting taste, and its powder is irritating to the nostrils.

Dose, I to $2 \mathrm{gr}$. ; .06 to .I $2 \mathrm{gm}$.

\section{Action AND Uses of Coto.}

Coto is not astringent, but bécause it produces absorption, coto bark and cotoin have established a reputation as remedies for diarrhœa, whether infantile, in phthisis or in typhoid fever. It also checks salivation and night-sweats. A ro per cent. tincture of coto has been recommended by the British Pharmaceutical Conference. Dose, Io m.; .60 c.c. every 2 hours, with mucilage or syrup to suspend the large amount of resin which it contains. It should not be combined with Mistura Cretæ.]

\section{GROUP IX. \\ Vegetable Demulcent Substances.}

Many of these are nutritive.

Olive Oil, Soap, [Chondrus,] Glycerin, Oleic Acid, [Althæa,] Almond, Tragacanth, Acacia, [Ulmus,] Liquorice, Linseed, [Pearl Barley,] Sugar, Malt, Soja Bean, [Cetraria, Raisins.]

\section{OLEUM OLIVE.}

OLIVE OIL.- [Synonym.-Sweet Oil. A fixed oil expressed from the ripe fruit of Olea europaa Linné (nat. ord. Oleacea). Habitat._Asia and Southem Europe; cultivated.

Characters. - A pale yellow, or light greenish-yellow, oily liquid, having a slight, peculiar odor, and a nutty, oleaginous taste, with a faintly acrid aftertaste. Sp. gr., 0.915 to 0.918 . Solubility. -Very sparingly soluble in Alcohol, but readily soluble in Ether, Chloroform, or Carbon Disulphide.]

Composirion.-The [three] constituents are-(I) Olein, 72 per cent., a fluid oil, a compound of Oleic Acid and Glyceryl, thus : $\mathrm{C}_{3} \mathrm{H}_{5}\left(\mathrm{C}_{38} \mathrm{H}_{33} \mathrm{O}_{2}\right)_{3}$. (2) Palmitin, 28 per cent., a solid oil, a compound of Palmitic Acid, and Glyceryl, $\mathrm{C}_{3} \mathrm{H}_{5}\left(\mathrm{C}_{16} \mathrm{H}_{31} \mathrm{O}_{2}\right)_{3}$. The formula for Oleic Acid is $\mathrm{HC}_{18} \mathrm{H}_{33} \mathrm{O}_{2}$; and for Palmitic, $\mathrm{HC}_{16} \mathrm{H}_{31} \mathrm{O}_{2}$. [(3) Arachin, $\mathrm{C}_{20} \mathrm{H}_{40} \mathrm{O}_{2}$.

IMPURITIES.-Cotton seed and other oils, especially Sesame.

Dose, freely.] 
Olive Oil is contained in [Emplastrum Plumbi, Emplastrum Ferri, Em. plastrum Picis Burgundicæ, Ceratum Cetacei, Unguentum Diachylon, and Unguentum Veratrinæ.]

\section{Action and Therapeutics of Olive Oil.}

External.-Olive oil is used to facilitate the rubbing of parts ; for this purpose it is employed in massage. It is a common soothing protective to burns [being used in place of linseed oil in Linimentum Calcis (see p. r 57)], and may be mixed with poultices to prevent their [adhering] to the skin. If rubbed in vigorously, it can be absorbed through the epidermis, and might be thus used as a food when nourishment cannot be given by the mouth.

Internal.-For its soothing protective qualities it may be swallowed after corrosive poisons have been taken. It is an excellent mild laxative, and can be given with food for this purpose. Some persons like it; with others it excites nausea and vomiting. An olive oil enema (olive oil, ${ }_{5}$; with or without warn mucilage of starch, I8); [or a soap enema (soap, I ; warm water, $\left.3^{2}\right)$ ], is often used to open the bowels when a mild non-irritating injection is required. A gall stone placed in pure olive oil at the temperature of the body is slowly dissolved, because cholesterin, which is the chief constituent of gall stones, is soluble in olive oil. It is also soluble in oleic acid and in animal soaps. Many patients suffering from gall stones derive much benefit from taking olive oil. This is chiefly because the oil or some of its constituents are excreted by the bile, and to a much less extent because the intestinal peristalsis set up by the olive oil extends to the bile ducts. From 2 to $8 \mathrm{fl}$. oz. [6o. to 240 . c.c.] should be taken daily. It may be mashed with fish or potato. Some [patients] take it better if a [small quantity] of menthol and a drachm [4. c.c.] of brandy are added to each half pint [240. c.c.] of oil. Eunatrol, or pure sodium oleate, which is given a special name to distinguish it from the ordinary impure forms, has been successfully used in cases of gall stones. Thirty to forty grains [2.00 to $2.40 \mathrm{gm}$.] may be given daily. It is best prescribed as $5 \mathrm{gr}$. $[.3 \circ \mathrm{gm}$.$] pills.$ 
Olive oil is a food, but it is not often used in this country as such. The history of fats and oils in the body is discussed in works on physiology.

\section{SAPO.}

SOAP.-Sodium Oleate, $\mathrm{NaC}_{18} \mathrm{H}_{33} \mathrm{O}_{2}$. [Synonyms._White Castile Soap, Hard Soap.

SOURCE. - Soap is prepared from Soda and Olive Oil. $\mathrm{C}_{3} \mathrm{H}_{5}\left(\mathrm{C}_{18} \mathrm{H}_{33} \mathrm{O}_{2}\right)_{3}+$ ${ }_{3} \mathrm{NaOH}=2 \mathrm{NaC}_{18} \mathrm{H}_{33} \mathrm{O}_{2}$ (Hard Soajp) $+\mathrm{C}_{3} \mathrm{H}_{5}(\mathrm{OH})_{3}$ (Glycerin).

CHARACTERS. - A white or whitish solid, hard, yet easily cut when fresh, having a faint, peculiar odor free from rancidity, a disagreeable, alkaline taste, and an alkaline reaction. Solubility.-Soluble in water and in Alcohol, more readily with the aid of heat.

Soap is contained in Pilulæ Aloes, Pilulæ Aloes et Asafotidæ, Pilulæ Asafœtidæ, Pilulæ Opii, and Pilulæ Rhei.]

\section{Preparations.}

[I. Emplastrum Saponis.-Soap Plaster. Soap, 100; Lead Plaster, 900; by solution in Water and evaporation.

2. Linimentum Saponis.-Soap Liniment. Synonym.-Opodeldoc. Soap, 70; Camphor, 45 ; Oil of Rosemary, 10; Alcohol, $75^{\circ}$; Water to 1000 .

Soap Liniment is contained in Linimentum Chloroformi.]

\section{SAPO MOLLIS.}

SOFT SOAP._Potassium Oleate. [Synonyms._Sapo Viridis. Green Soap. A soap prepared from Potassa and fixed oils, generally from Olive Oil.

Source.-By heating Linseed Oil, 400 ; adding to this Potassa, 90; dissolved in Water, 450; and Alcohol, $40^{\circ}$; until the mixture is soluble in boiling Water without the separation of oily drops.

Ciraracters.-A soft, unctuous mass, of a yellowish-brown or brownishyellow color. Solubility.-In about 5 parts of hot Water to a nearly clear liquid; also in 2 parts of bot Alcohol, without leaving more than 3 per cent. of insoluble residue.

Preparation.

Linimentum Saponis Mollis.-Liniment of Soft Soap. Synonym. - Tinctura Saponis Viridis. Soft Soap, 650; Oil of Lavender Flowers, 20; Alcohol, 300; Water to 1000 . By filtration.]

USES OF SOAP.

Hard soap may be used for medicated soaps. The prescriber should state the percentage of the drug, e.g.; ichthyol, tar, 
sulphur, he wishes the soap to contain. The dispenser planes the soap into thin shavings, dries them at $100^{\circ} \mathrm{F} .\left[37.8^{\circ} \mathrm{C}\right.$.], powders them in a mortar, then thoroughly mixes and beats up the soap powder, the drugs, and one part of alcohol ( 60 per cent.) to 8 parts of soap powder. The whole is put into a soap press and stamped. [Soaps are used for cleansing. The plaster is protective against bed-sores. The liniment is used as a cutaneous stimulant. That of soft soap is employed for psoriasis, lichen and eczema.] Hard soap forms a basis for many pills. Either soap is frequently made into a lather-with about a pint [500. c.c. $]$ of water at $100^{\circ} \mathrm{F}$. $\left[37.8^{\circ} \mathrm{C}\right.$. $]$ and used as a purgative enema. Soft soap is much preferred; about I fl. oz. [3o. c.c.] is commonly used. All enemata, but perhaps especially those made with hard soap, may produce an erythematous rash, probably due to the solution and consequent absorption of some fæcal toxin.

\section{[CHONDRUS.}

CHONDRUS.-Synonyms.-Irish Moss. Carragheen. The entire plant of Chondrus crispus Stackhouse, and Gigartina mamillosa J. Agardh (class $A l_{g}($ ). Habitat.-Atlantic Ocean.

Characters. - Yellowish or white, horny, translucent; many-times forked; when softened in water, cartilaginous; shape of the segments varying from wedge-shape to linear; at the apex emarginate or two-lobed. It has a slight sea-weed odor, and mucilaginous, somewhat saline taste.

Composition. - (I) Mucilaginous Compounds, 90 per cent. (2) Albuminoids. (3) Chlorides, sulphates and phosphates, with traces of bromides and iodides.

Dose, 2 to $4 \mathrm{dr}$. ; 8. to $15 . \mathrm{gm}$.

\section{Action and Uses of Irish Moss.}

Irish Moss is not only a demulcent, but as well, when made into a jelly, is a useful article of diet. It is also used in making preparations of bone marrow.]

\section{GLYCERINUM.}

GLYCERIN. $-\left[\mathrm{C}_{3} \mathrm{H}_{5}(\mathrm{OH})_{3}=91.79\right.$. A triatomic alcohol. Synonym.Glycerol.

SOURCE. - A liquid obtained by the decomposition of vegetable or animal fats or fixed oils (see pp. 13 and 605), and containing not less than 95 per cent. of absolute Glycerin. 
Characters. - A clear, colorless liquid, of a thick, syrupy consistence, oily to the touch, odorless, very sweet and slightly warm to the taste. When exposed to the air, it slowly abstracts moisture. Sp. gr., not less than I.250. Solubility.-In all proportions, in Water or Alcohol; also soluble in a mixture of 3 parts of Alcohol and I part of Ether, but insoluble in Ether, Chloroform, Carbon Disulphide, Benzin, Benzol, and fixed or volatile oils.

Glycerin is contained in Elixir Phosphori, Liquor Ferri et Ammonii Acetatis, Mucilago Tragacanthæ, Massa Hydrargyri, Pilulæ Phosphori, in the Glycerita and in many Extracta, Extracta Fluida, Syrupi and Tincturx.]

Dose, 5 to $60 \mathrm{~m}$.; [.30 to $4.00 \mathrm{c.c}$. $]$

\section{Preparations.}

I. [Glyceritum Amyli.-Glycerite of Starch. Starch, Io; Water, Io; Glycerin, 80.

Dose, freely.

2. Glyceritum Vitelli._Glycerite of Yolk of Egg. Synonym.Glyconin. Fresh Yolk of Egg, 45; Glycerin, 55.

Dose, freely.

3. Glyceritum Acidi Carbolici.-See Carbolic Acid, p. 329.

4. Glyceritum Acidi Tannici.-See Tannic Acid, p. 593.

5. Glyceritum Boroglycerini.-See Boric Acid, p. 273.

6. Glyceritum Hydrastis.-See Hydrastis, p. 640.

7. Suppositoria Glycerini. -Suppositories of Glycerin. Glycerin, 60 ; Sodium Carbonate, 3 ; Stearic Acid, $5 \mathrm{gm}$. By solution with heat, pouring into ten moulds, and wrapping in tin-foil, when cold. Each suppository contains $90 \mathrm{gr}$; $6 . \mathrm{gm}$. of Glycerin.

Dose, as required.]

\section{Action of Glycerin.}

External.-As glycerin is an excellent solvent for numerous [substances], such as iodine, bromine, alkalies, tannic acia, many neutral salts, alkaloids, salicin, etc., it is a good vehicle for applying these substances to the skin and to sores. It does not evaporate nor turn rancid, and is powerfully hygroscopic.

Internal.-In man the only visible effect produced by its administration is purging. This occurs with quite small doses if it is given by the rectum, but large doses are necessary if given 
by the mouth. It is absorbed from the alimentary canal, and is to a slight extent a food, for some of it is oxidized in the body. Sometimes its administration leads to the appearance in the urine of a body which reduces cupric oxide and gives the fermentation test for sugar. There has been much dispute as to whether glycerin can control nitrogenous metabolism, but it appears that it cannot in any way save the waste of nitrogenous tissues. It probably has some influence on the amount of glycogen in the liver. It has also been thought to prevent artificial glycosuria, but this is doubtful.

Very large doses in animals cause the urine to be dark from the presence of the coloring matter of the blood, although there are no corpuscles in it; they also lead to loss of muscular strength, lethargy, dryness of mucous membranes, collapse and death.

\section{Therapeutics of Glycerin.}

External.-Glycerin is much employed as a basis for applications to the skin and the eye. It is commonly used for chapped hands and slight excoriations. It is readily absorbed when rubbed into the skin, therefore it is a convenient vehicle for the absorption of substances by the skin. Belladonna mixed with glycerin is often rubbed in when we desire its local anodyne action (see p. 379.) [Glycerin as well as boroglycerin (see p. 273 ) is used extensively in various local applications in the treatment of diseases of women].

Internal.-As glycerin is sweet, it is an excellent flavoring agent. It is demulcent, and is used as a vehicle for applying substances, such as tannic acid, to the throat. It is rarely given by the nouth for any medicinal virtue. It has been administered for dyspepsia, for diabetes, and as a nutritive agent, but in each case without any good result. One to two fl. dr. [4. to 8. c.c.] injected [into] the rectum, or a glycerin suppository, form an excellent means of opening the bowels in simple constipation, especially when the fæces are in the sigmoid flexure and rectum. The result is prompt, often occurring within less than half an hour. No pain nor constitutional disturbance is produced. 


\section{ACIDUM OLEICUM.}

OLEIC ACID. $-\mathrm{HC}_{18} \mathrm{H}_{33} \mathrm{O}_{2}[=28 \mathrm{I} .38$.

SoURCE.-An organic acid, prepared in a sufficiently pure condition by cooling commercial Oleic Acid to about $4 x^{\circ} \mathrm{F} . ; 5^{\circ} \mathrm{C}$, then separating and preserving the liquid portion.] In case [that it is obtained from] Olive Oil the reaction is $\mathrm{C}_{3} \mathrm{H}_{5}\left(\mathrm{C}_{18} \mathrm{H}_{33} \mathrm{O}_{2}\right)_{3}+3 \mathrm{H}_{2} \mathrm{O}=3 \mathrm{HC}_{18} \mathrm{H}_{33} \mathrm{O}_{2}+\mathrm{C}_{3} \mathrm{H}_{5}\left(\mathrm{OH}_{3}\right)$ (Glycerin).

Characters. - - A yellowish or brownish-yellow, oily liquid, having a peculiar, lard-like odor and taste; becoming darker and absorbing Oxygen on exposure to air. It becomes semi-solid at $40^{\circ} \mathrm{F}$. ; $4.4^{\circ} \mathrm{C}$. Sp. gr., about 0.900. Solubility.-Insoluble in water; soluble in Alcohol, Chloroform, Benzol, Benzin, Oil of Turpentine, and fixed and volatile oils.]

IMPURITIES. - It is rarely pure, usually containing Stearic and Palmitic Acids.

[Oleic Acid is used to prepare Oleatum Hydrargyri, Oleatum Veratrinæ, and Oleatum Zinci. There is some doubt whether the pharmacopoial oleates are chemical combinations or simple solutions.]

\section{Action and Therapeutics of Oleic Acid.}

Oleic acid is used as a solvent for remedies which it is desired to apply by means of cutaneous inunction, for it more readily penetrates the skin than fats and oils. [Oleates are made from the alkaloids, not from their salts. If metals are employed, the oxides only are chosen.] Copper oleate is also used for ringworm and indolent sores.

\section{ALTH EA.}

[ALTHÆA.-Synonym.-Marshmallow. The root of Althac officinalis Linné (nat. ord. Malvacea). Habitat._Europe, Western and Northern Asia ; naturalized in the Eastern United States and in Australia, in salt marshes; culivated in Europe.

Characters. - In cylindrical or somewhat conical pieces, from 10 to $\mathrm{X}_{5}$ $\mathrm{cm}$. long, to to $15 \mathrm{~mm}$. in diameter, deeply wrinkled; deprived of the brown, corky layer and small roots; externally white, marked with a number of circular spots, and of a somewhat hairy appearance from the loosened bastfibres; internally whitish and fleshy. It breaks with a short granular and mealy fracture, has a faint, aromatic odor, and a sweetish, mucilaginous taste.

Resembling Althaa.-Young anil peeled Belladonna roots, but these have no hair-like bast-fibres upon the surface.

Composition.-(I) Asparagin, I per cent. (2) Bassorin, a mucilage, 35 per cent. (3) Sugar, 8 per cent. (4) Pectin, Io per cent. 


\section{Preparation.}

Syrupus Althæx.-Syrup of Althæa. Althæa, 50; Alcohol, 30 ; Glycerin, 100; Sugar, 700; Water to 1000 .

Dose, freely.

\section{Action and Uses of Marshmallow.}

Marshmallow is used as a demulcent, for irritation and inflammation of mucous membranes.]

\section{AMYGDALA.}

AMYGDALA AMARA. - Bitter Almond. The seed of Prunus Amygdalus, var. amara [De Candolle (nat. ord. Rosacea). Habitat.-Western Asia; naturalized in the Mediterranean basin; cultivated.

Characters. - About $25 \mathrm{~mm}$. long, oblong-lanceolate, flattish, covered with a cinnamon-brown, scurfy testa, marked by about sixteen lines emanating from a broad scar at the blunt end. The embryo has the shape of the seed, is white, oily, consists of two plano-convex cotyledons, and a short radicle at the pointed end, and has a bitter taste. When triturated with water, Bitter Almond yields a milk-white cmulsion, which emits an odor of Hydrocyanic Acid.]

Composition.-The chief constituents are-(I) Oleum Anygdala [F.x. pressum (see p. 613), 45 per cent., the same fixed oil as in the sweet variety.] (2) Emulsin. (3) Amygdalin, $\left[\mathrm{C}_{20} \mathrm{H}_{27} \mathrm{NO}_{11}\right.$, a crystalline glucoside, having a sweetish bitter taste,] which yields Oleum Amygrala Amara. [It is very important-to distinguish it from the Oleum Amysdalce Expressum, which is harmless. The oil of bitter almond is u*ually very poisonous from admixture of Hydrocyanic Acid ; for if moisture has had access to the glucoside Amygdalin, on which in the presence of water, the emulsin in the almond acts as a ferment, the volatile oil of bitter almond (benzaldehyde), glucose and Hydrocyanic Acid are formed.] $\mathrm{C}_{20} \mathrm{H}_{27} \mathrm{NO}_{11}+2 \mathrm{H}_{2} \mathrm{O}=\left[\mathrm{C}_{2} \mathrm{H}_{6} \mathrm{O}\right]$ (the volatile oil) + $\mathrm{HCN}+2 \mathrm{C}_{6} \mathrm{H}_{32} \mathrm{O}_{6}$. The oil when separated from the [Hydrocyanic] Acid is not poisonous, and is used to flavor sweets. An artificial oil of bitter almond called Nitrobenzol is often substiluted, and bas caused death.

Bitter Almond is contained in Syrupus Amygdalæ.

[OLEUM AMYGDAL $Æ$ AMAR E.-Oil of Bitter Almond.

Source. - A volatile oil obtained from Bitter Almond by maceration with water, and subsequent distillation.

Ciiaracters. - A clear, colorless or yellowish, thin, and strongly refractive liquid, having a peculiar, aromatic odor, and a bitter and burning taste. Sp. gr., 1.060 to 1.07o. Boiling point, about $35^{\circ} \mathrm{F}$; $180^{\circ} \mathrm{C}$. Optically inactive. Solubility. - In 300 parts of water, and in Alcohol or Ether in all proportions; also soluble in Nitric Acid at ordinary temperatures without the evolution of nitrous vapors.

Dose, $\frac{1}{6}$ to $1 / 2 \mathrm{~m}$.; .or to .03 c.c. 


\section{Preparations.}

1. Aqua Amygdalæ Amaræ.-Bitter Almond Water. Oil of Bitter Almond, I ; Distilled Water, 999. By solution and filtration.

Dose, 2 to 4 fl. dr. ; 8. to r5. c.c.

2. Spiritus Amygdalæ Amaræ. - Spirit of Bitter Almond. Synonym.-Essence of Bitter Almond. Oil of Bitter Almond, 10; Alcohol, 800 ; Distilled Water, to 1000.

Dose, 15 to $45 \mathrm{~m}$.; I. to 3. c.c.]

AMYGDALA DULCIS.-Sweet Almond. Synonym.-Jordan Almond. [The seed of Prunus Amygdalus, var. dulcis De Candolle (nat. ord. Rosacea.) Habilat.-Western Asia ; naturalized in the Mediterranean basin; cultivated.

Cilaracters. - Closely resembling the bitter almond (see Amygdala Amara), but having a bland, sweetish taste, free from rancidity. When triturated with water, it yields a milk-white emulsion, free from the odor of Hydrocyanic Acid.]

Composition.-The chief constituents are-(I) Oleum Amygdala Ex. pressum (see below), 56 per cent., a fixed oil. (2) Emulsin, and other albuminous bodies.

IMPURITY.-The bitter almond, giving an odor of [Hydrocyanic] Acid when rubbèd with water.

\section{Preparations.}

[1. Emulsum Amygdalæ.-Emulsion of Almond. Synonyms. -Mistura Amygdalæ. Milk of Almond. Sweet Almond, 60; Acacia, Io ; Sugar, 30; Distilled Water, I000.

Dose, indeterminate.

2. Syrupus Amygdalæ.-Syrup of Almond. Sweet Almond, 140; Bitter Almond, 40; Sugar, 200; Orange Flower Water, 100; Water, 130 ; Syrup to 1000.

Dose, indeterminate.]

\footnotetext{
mond.

OLEUM AMYGDALA [EXPRESSUM.-Expressed Oil of Al-

Source.-A fixed oil expressed from Bitter or Sweet Almond.

Characters. - A clear, pale straw-colored or colorless, oily liquid, almost inodorous, and having a mild, nutty taste. Sp. gr., 0.915 to 0.920. Solubility.-Only slightly soluble in Alcohol; soluble in Ether and in Chloroform in all proportions.

Expressed Oil of Almond is contained in Emulsum Chloroformi and Unguentum Aquæ Rosæ.

Dose, I to 4 fi. dr. ; 4 . to I5. c.c.]
} 


\section{Action and Therapeutics of Almond.}

The sweet almond is demulcent. Its most important medicinal use is that it is made into flour to replace starchy food in cases of diabetes. Biscuits are made of the flour. These are very palatable, are a good nutritive food, and contain very little starch. The only objection to them is their price. With a little care they can be made at home. The flour of other nuts, as Brazil nuts, has been used, but it is not nearly so palatable. The almond [emulsion] is a very pleasant vehicle for the suspension of insoluble substances, and its powder [B. P., sweet almond, 8 ; sugar, 4 ; acacia, I] is a palatable basis for powders. The [expressed] oil of almond might be used for the same purposes as olive oil. It is pleasanter, but very expensive.

\section{TRAGACANTHA.}

TRAGACANTH.-A gummy exudation from [Astragalus gummifer Labillardière, and from other species of Astragalus (nat. ord. Leguminosa). Habitat.-Western Asia.

ChARACTERS. - In narrow or broad bands, more or less curved or contorted, marked by parallel lines, or ridges, white or faintly yellowish, translucent, horn-like, tough, and rendered more easily pulverizable by a heat of $122^{\circ} \mathrm{F}$; $50^{\circ} \mathrm{C}$. Very sparingly soluble in cold water, but swells into a gelatinous mass, which is tinged violet (not so deep as the color given by starch by tincture of iodine.] Resembling Tragacanth.-Squill, which is thicker and opaque.

IMPURITIES.-Other gums.

Composition. - The chief constituents are-(1) Bassorin $\mathrm{CH}_{10} \mathrm{O}_{5}$, a gum 33 per cent., only slightly soluble in water, unfermentable. (2) Arabin, 53 per cent., which resembles, but is not identical with the Arabin of Acacia. Precipitated by lead acetate. (3) A little starch.

[Tragacanth is contained in several Trochisci.]

\section{Preparation.}

Mucilago Tragacanthæ.-[ Mucilage of Tragacanth. Tragacanth, 6; Glycerin, 18; Water to 100. By heating, maceration and straining. Dose, indeterminate.]

\section{Action and Therapeutics of Tragacanth.}

Tragacanth is a demulcent, and as such may be soothing when applied to a sore throat. Its chief use is to suspend insoluble 
bodies, as resins, oils, and insoluble powders. The mucilage is better for this purpose than the compound powder [(B. P., not official), tragacanth, $\mathbf{r}$; acacia, I; starch, $\mathbf{r}$; sugar, 3 ;], which, because of its starch, is liable to ferment.

\section{ACACIA.}

[ACACIA.-Synonym.-Gum Arabic, A gummy exudation from Acacia Senegal Willdenow (nat. ord. Leguminosa). Habilat.-Eastern Africa, principally Kordofan; Western Africa, near the river Senegal.

CHARACTERS. - In roundish tears of various sizes, or broken into angular fragments, with a glass-like, sometimes iridescent fracture, opaque from numerous fissures, but transparent and nearly colorless in thin pieces; nearly inodorous; taste insipid, mucilaginous; insoluble in Alcohol, but soluble in water, forming a thick, mucilaginous liquid. Solubility. -Slowly but com. pletely soluble in 2 parts of water; insoluble in Alcohol.]

Composition.-The chief constituent is Arabin, $\mathrm{C}_{12} \mathrm{H}_{22} \mathrm{O}_{11}$; combined with Calcium, Magnesium and Potassium. This is not affected by lead acetate.

IMPURITIES. - Starch, and gum resins.

INCOMPATIBLES. -Alcohol, sulphuric acid, borax, ferric salts and lead subacetate.

[Acacia is contained in Emulsum Amygdalæ, Pulvis Cretæ Compositus, and in some Trochisci.]

\section{Preparations.}

I. Mucilago Acaciæ.-[Mucilage of Acacia. Acacia, 340; Water, to 1000.

Mucilage of Acacia is contained in Mistura Glycyrrhizze Composita and Syrupus Acaciæ.

Dose, freely.

2. Syrupus Acaciæ.-Syrup of Acacia. Mucilage of Acacia, 25 ; Syrup, 75 .

Dose, freely.]

Action and Therapeutics of Acacia.

Acacia is demulcent., It is used to suspend insoluble substances, as oils, resins, and insoluble powders. A fluid ounce [30. c.c.] of most oils or resinous tinctures requires 3 fl. dr. [ 12. c.c.] of mucilage of acacia for suspension, but copaiba requires ro fl. dr. [40. c.c.]. A disadvantage of it is that it is liable to undergo acetous fermentation, which greatly diminishes its emulsifying powers. This may be overcome, to some extent, by 
making it with tolu or clove water. It may give rise to indigestion and diarrhœa.

\section{ULMUS.}

[ELM.-Synonym.-Slippery Elm Bark. The inner bark of Ulmus fulva Michaux (nat. ord. Urticacea). Habitat.-North America, west to Louisiana and Nebraska, in woods.

ChARACTERs. - In flat pieces, varying in length and width, about $3 \mathrm{~mm}$. thick, tough, pale brownish white, the inner surface finely ridged; fracture fibrous and mealy; the transverse section delicately checkered; odor slight, peculiar; taste mucilaginous, insipid.

Compositron. - It contains-(1) Mucilage. (2) Some Tannic Acid.

Dose, 2 dr. ; 8. gm., or more.

\section{Preparation.}

Mucilago Ulmi.-Mucilage of Elm. Elm, 6; Boiling Water, 100. By digestion and straining.

Dose, freely.

\section{Action and Uses of Slippery Elm Bark.}

Slippery Elm bark is an excellent demulcent. It is especially recommended in dysentery, diarrhœe and diseases of the urinary passages. It is often employed to make poultices, especially for use upon children, because it is lighter than flaxseed.]

\section{GLYCYRRHIZA.}

GLYCYRRHIZA.-[Synonym.-Liquorice Root. The root of Glycyrrhiza glabra Linné, and of the variety glandulifera (Waldstein et Kittaibel) Reger et Herder (nat. ord. Legruminosie). Habitat.-Southern Europe and Western Asia ; cultivated.

Characters. - In long, cylindrical pieces, from 5 to $25 \mathrm{~mm}$. thick, longitudinally wrinkled, externally grayish-brown, warty; internally tawnyyellow; pliable, tough; fracture cnarsely fibrous; bark rather thick; wood porous, but dense, in narrow wedges; medullary rays linear; taste sweet, somewhat acrid. The underground stem, which is often present, has the same appearance, but contains a thin pith. The drug derived from the variety glandulifera (so-called Russian Liquorice), consists usually of roots or rootbranches, 1 to $4 \mathrm{~cm}$. thick, 15 to $30 \mathrm{~cm}$. long, frequently deprived of the corky layer, the wood rather soft, and usually more or less cleft.] Resembling Liquorice.- Pyrethrum and Taraxacum, which are not sweet.

Composition. - The chief constituents are-(I) Gilycyrrhizin $\left[\mathrm{C}_{24} \mathrm{H}_{36}\right.$ $\mathrm{O}_{9}$, about 6 per cent.], a yellow amorphous glucoside, probably in combination with Ammonia. With acids this yields a very bitter substance, Glycyrrhe- 
tin, and glucose. (2) Asparagin, [about 3 per cent. (3) Glycyramin. (4) An acrid Resin.] (5) Starch.

[Glycyrrhiza is used in Decoctum Sarsaparillæe Compositum, Extractum Sarsaparillæ Fluidum Compositum, Massa Hydrargyri, Pilulæ Ferri Iodidi, Pulvis Morphinæ Compositus, Tinctura Aloes, and Tinctura Aloes et Myrrhæ.]

Liquorice or its preparations are contained in many preparations, generally to cover their nauseous taste. They [conceal] very well that of Aloes [Rhamnus Purshiana], Ammonium Chloride, Hyoscyamus, Senega, Senna, Turpentine, and very bitter substances, as Quinine Sulphate.

\section{Preparations.}

1. Extractum Glycyrrhizæ.-[Extract of Glycyrrhiza. Synonym.-Extract of Liquorice.

Characters. - In flattened, cylindrical rolls, from 15 to $18 \mathrm{~cm}$. long, and from $\mathrm{I} 5$ to $30 \mathrm{~mm}$. thick; of a glossy, black color. It breaks with a sharp, conchoidal, shining fracture, and has a very sweet peculiar taste. Not less than 60 per cent. of it should be soluble in cold water.

Extract of Glycyrrhiza is contained in Trochisci Ammonii Chloridi and Trochisci Glycyrrhizæ et Opii.

Dose, freely.

2. Extractum Glycyrrhizz Purum.-Pure Extract of Glycyrrhiza. By maceration and percolation with Water of Ammonia and Distilled Water, and evaporation.

Pure Extract of Glycyrrhiza is used to make Mistura Glycyrrhizæ Composita.

Dose, freely.

3. Extractum Glycyrrhizz Fluidum.-Fluid Extract of Glycyrrhiza. By maceration and percolation with Water of Ammonia, Alcohol and water, and evaporation.

Dose, freely.

4. Glycyrrhizinum Ammoniatum.-Ammoniated Glycyrrhizin.

Source.-Glycyrrhiza, by maceration and percolation with Water of Ammonia and Water; precipitation with Sulphuric Acid, solution with Water of Ammonia, and drying.

Characters. - Dark-brown or brownish-red scales, without odor, and having a very sweet taste. Solubility.-Readily in Water and in Alcohol.

Dose, 5 to $15 \mathrm{gr} . ; .30$ to $1.00 \mathrm{gm}$.]

5. Pulvis Glycyrrhizæ Compositus.-See Senna, p. 493.

6. [Mistura Glycyrrhizæ Composita.-Compound Mixture of Glycyrrhiza. Synonym.-Brown Mixture. Pure Extract of Glycyr- 
- rhiza, 30 ; Sugar, 50 ; Mucilage of Acacia, 100 ; Camphorated Tincture of Opinm, 120; Wine of Antimony, 60; Spirit of Nitrous Ether, 30; Water, to 1000 .

Dose, I to 4 fi. dr.; 4. to 15. c.c.]

\section{Action and Therapeutics of Liquorice.}

Liquorice is an excellent demulcent for sore throats. It is used to [conceal] the taste of [disagreeable] medicines, and as a basis for pills. The compound liquorice powder is laxative by virtue of its senna [although liquorice itself has distinct laxative properties. Brown Mixture is much used in dispensary practice as an expectorant.]

\section{LINUM.}

[LINSEED.-Synonym.-Flaxseed. The seed of Linum usitatissimum Linné (nat. ord. Linece). Habitat._Levant and Southern Europe ; cultivated and spontaneous in most temperate countries.

Characters. - About 4 or $5 \mathrm{~mm}$. long, oblong-ovate, flattened, obliquely pointed at one end, brown, glossy, covered with a transparent, mucilaginous epithelium, which swells considerably in water; the embryo whitish or pale greenish, with two large, oily, plano-convex cotyledons, and a thin perisperm; inodorous ; taste mucilaginous, oily and bitter.

Composition.-(I) A viscid, fixed vil, 30 to 35 per cent. in the embryo. (2) Mucilage, 15 per cent. in the epithelium. (3) Proteids, 25 per cent. (4) Amygdalin, a minute quantity.]

OLEUM LINI.-[Linseed Oil. Synonym.-Oil of Flaxseed. A fixed oil expressed from Linseed without the use of heat.

Characters.-A yellowish or yellow, oily liquid, having a slight, peculiar odor, and a bland taste. When exposed to the air it gradually thickens, and acquires a strong odor and taste. Sp. gr., 0.930 to 0.940. Solubility.In about 10 parts of Alcohol, and in all proportions in Ether, Chloroform, Benzin, Carbon Disulphide, or Oil of Turpentine.

Composition.-Its most characteristic principles are-(I) Linolein. Myristin. (3) Palmitin. (4) Albumin, a large percentage, to which . its drying properties are due.)

Dose, $y / 2$ to 2 fi. oz. ; 15 . to 6o. c.c.]

\section{Action and Therapeutics of Flaxseed.}

A flaxseed poultice [4 to ro of boiling water with constant stirring and the basin being kept hot] is a very common means of applying warmth and moisture to a part. It is used to relieve 
pain, and as a mild irritant to accelerate inflammation and the bursting of an abscess, or as a counter-irritant in all sorts of deepseated inflammations. The poultices should not be too thick, and should be smeared with oil to prevent their sticking to the skin. The vascular dilatation caused by the flaxseed poultice may be increased by adding $I$ part of mustard to 16 of flaxseed. [Oil of flaxseed] is applied to burns. Mixed with an equal quantity of solution of lime it forms Carron Oil (see p. 157.) [Flaxseed tea flaxseed, 3 ; liquorice, $\mathrm{r}$; boiling water, 100 ; infuse for two hours] is a common domestic demulcent; the large quantity of mucilage it contains forms a coating for the pharynx and mouth, and thus relieves cough due to sore throat. It is said to be slightly diuretic.

\section{SACCHARUM.}

[SUGAR. $-\mathrm{C}_{12} \mathrm{H}_{22} \mathrm{O}_{11}=34$ 1.2. Synonyms. -Cane Sugar. Sucrose. The refined Sugar obtained from Saccharum officinarum Linné, and from various species or varieties of Sorghum (nat. ord. Graminea); also from one or more varieties of Beta vulgaris Linné (nat. ord. Chenopodiacea). Habitat.Southern Asia ; cultivated in tropical and subtropical countries.

Characters. - White, dry, hard, distinctly crystalline granules, odorless, and having a purely sweet taste. Permanent in the air. Solubility.-In $0: 5$ part of water, and in $\mathbf{1} 75$ parts of Alcohol ; but insoluble in Ether, Chloroform, or Carbon Disulphide.

Dose, indefinite.

\section{Preparation.}

Syrupus.-Syrup. Sugar, $85^{\circ}$; Distilled Water, by solution with heat, and straining to 1000 .

Syrup thus prepared has the Sp. gr., 1.317. It is used for compound syrups.

Dose, indefinite.]

\section{Action and Therapeutics of Sugar.}

Sugar is used as a sweetening agent. [Syrup is used as a vehicle.] Syrupus Glucosi [(B. P., not official.) Syrup, 2; liquid glucose of commerce, $\mathrm{I}$;] is used in pharmacy, especially in the making of pills, as it forms a neutral basis. 


\section{[HORDEUM DECORTICATUM.}

PEARL BARLEY. (Not official.)-The dried seed of Hordeum dis. tichum (nat. ord. Graminacece) divested of its integuments. Habitat.Britain.

Characters. - White, rounded, with a light longitudinal furrow.

\section{Action and Therapeutics of Pearl Barley.}

Barley water ( $I$ to $I 5$ of boiling water) forms a pleasant demulcent drink, especially if the throat be dry and sore. It is also given for the diarrhœas of infants.]

\section{MALTUM.}

MALT. (Not official.) Synonym.-Byne. The seed of common barley, Hordeum distichum (nat. ord. Graminacea), caused to enter the incipient state of germination by artificial means, and dried. It contains the ferment Diastase, which can convert starch into Dextrin and Maltose. Thus Io $\left(\mathrm{C}_{6} \mathrm{H}_{10} \mathrm{O}_{5}\right)+4 \mathrm{H}_{2} \mathrm{O}=4 \mathrm{C}_{12} \mathrm{H}_{22} \mathrm{O}_{11}$, Maltose $+\left(\mathrm{C}_{12} \mathrm{H}_{20} \mathrm{O}_{10}\right)$, Dextrin.

EXTRACTUM MALTI.-[(Not " official).-Extract of Malt. By maceration, dilution with Water, straining and evaporation in vacuo of the strained liquid.]

Characters. - It is a sweet, thick, brownish liquid, like honey, forming an emulsion with oils. [Most specimens are too viscid for prolonged use.]

Compositios. - This varies very much. The chief constituent is Maltose $\left(\mathrm{C}_{12} \mathrm{H}_{22} \mathrm{O}_{11}\right)$; there is also some Dextrin $\left(\mathrm{C}_{12} \mathrm{H}_{20} \mathrm{O}_{10}\right)$, some Diastase (unless destroyed by boiling), Albumin, [Inorganic] salts [contained in] barley, and sometimes Alcohol.

[Dose, I to 4 fl. dr.; 4. to I5. c.c.]

\section{Action and Therapeutics of Malt.}

Maltose is a very valuable food, especially for persons who are suffering from wasting diseases, and have a feeble digestion. It is easily retained by the stomach, even when, as often is the case in phthisis, other food, especially cod-liver oil, is rejected. In such a case a malt extract is an excellent substitute for cod liver oil. Maltose as a food leads to the formation of fat. The diastase contained in the malt extract, acting upon the starch in farinaceous food, converts it into dextrin and maltose, and thus, if the secretion of saliva and pancreatic juice is feeble, the malt extract to some extent supplies their place. Like the ferments in pancreatic juice and saliva, diastase can only act in ar. 
alkaline medium, and therefore should not be given until, at least, two hours after a meal. [Inasmuch as diastase is a most important constituent, all malts should be rejected which do not contain at least 4 per cent. of diastase. The liquid malts containing alcohol, which destroys this ferment, are worthless for assisting starch digestion and are usually only beers of an inferior quality. Malt extract, to which a suitable amount of fluid extract of rhamus purshiana has been added, is an excellent laxative.] Emulsions of cod-liver oil in it are frequently useful. Bynol is an example of these. They should contain I part of oil to 4 of malt. A mixture of extract of malt and iron is also valuable (ferric pyrophosphate, 2 ; water, 3 ; dissolve and add extract of malt, 95 . Dose, I to $4 \mathrm{fl}$. dr. [4. to I $_{5}$ c.c.]).

\section{SOJA.}

SOJA BEAN.-(Not official.)-The bean of Soja hispida. [Habitat. -Japan ; cultivated in Southern Asia.

Compositiox.-Soluble Casein, 30 ; Albumin, 0.5 ; Insoluble Casein, 7 ; Fat, 18; Cholesterin, 2; Water, 10; Dextrin, 10; Starch, 5; Cellulose, 5; Ash, 5 ; and a powerful amylolytic ferment. ] These are powdered and made into a flour, from which bread and biscuits are prepared. The flour contains but very little starch or sugar, sometimes not more than 2 or 4 per cent.

\section{action and Therapeutics of Soja Bean.}

Bread and biscuits made from the flour are used in the treatment of diabetes as a substitute for gluten bread; and many patients prefer the taste. They are quite as efficacious in reducing the amount of sugar passed in the urine.

\section{[CETRARIA.}

CETRARIA.-Synonym.-Iceland Moss. Celraria islandica (Linné) Acharius (class Lichenes). Habitat.-Northern Hemisphere.

Characters. -From 5 to $10 \mathrm{~cm}$. long, foliaceous, irregularly branched into fringed and channelled lobes, brownish above, whitish beneath, and marked with small, depressed spots; brittle and inodorous; when softened in water, cartilaginous, and having a slight odor; its taste is mucilaginous and bitter.

Composition.-The chief constituents are-( 1 ) Lichenin, $\mathrm{C}_{12} \mathrm{H}_{20} \mathrm{O}_{10}, 70$ per cent. (2) Cetraric Acid, $\mathrm{C}_{18} \mathrm{H}_{18} \mathrm{O}_{8}$, a bitter principle, 2 per cent. 
Lichenstearic Acid, $\mathrm{C}_{14} \mathrm{H}_{34} \mathrm{O}_{3}$, I per cent. (4) Fumaric Acid. (5) Oxalic Acid.

IMPURITIES. - Pine leaves, mosses and other lichens.

\section{Preparation.}

Decoctum Cetrariæ.-Decoction of Cetraria. Cetraria, 50. By boiling in water and straining to 1000 .

Dose, I to 4 fl. Oz.; 30. to I20. c.c.

\section{Action and Therapeutics of Iceland Moss.}

The decoction is demulcent, and may be given in sore throat. The moss is a food, and the jelly formed by boiling may, when suitably flavored, be taken by diabetics.]

\section{UVAE.}

[RAISINS. (Not official.)-The ripe fruit of Vitis vinifera (nat. ord. Ampelida), the grape-vine, dried in the sun, or partly by artificial heat. Habitat.-Spain.

Compositron. - The chief constituents are-(1) Grape Sugar. (2) Acid Potassium Tartrate. (3) Other acids and salts.

Action and Therapeutics of Raisins.

Raisins are used as sweetening agents, and are demulcent.]

\section{GROUP X.}

Vegetable Drugs which are used to kill parasites.

Class I. Anthelmintics for the various species of Tapeworm.

[Aspidium,] Pomegranate, Kousso, [Kamala, Pepo.]

Class II. Anthelmintics for the Roundworm (Ascaris lumbricoides).

Santonica, [Spigelia, Chenopodium.]

Class III. Parasiticides used for Pediculi.

Stavesacre, Picrotoxin.

Class IV. Anthelmintics for the Threadworm (Oxyuris vermicularis).

[Calumba, Quassia, Oak Bark.]

These have been described [in Groups VII and VIII, to which reference should be made. ] 


\section{ClASS I.-ANTHELMINTICS USED FOR TAPEWORMS.}

\section{ASPIDIUM.}

[ASPIDIUM. - Synonym.-Male Fern. The rhizome of Dryopleris Filix-mas Schott, and of Dryopteris marginalis Asa Gray (nat. ord. Filices). Habitat.-North America, Northern Asia, Europe.

Characters. - From 5 to $15 \mathrm{~cm}$. long, to to $25 \mathrm{~mm}$. in thickness, and, together with the closely imbricated, dark brown, roundish, and slightly curved stipe-remnants, 50 to $75 \mathrm{~mm}$. in diameter ; densely covered with brown, glossy, transparent, and soft, chaffy scales; internally pale green, rather spongy ; vascular bundles about ten (Dryopteris Filix-mas) or six (Dryopteris marginalis) in number, arranged in an interrupted circle; odor slight, but disagreeable; taste sweetish, acrid, somewhat bitter, astringent, and nauseous.]

Composition.-The chief constituents are-(I) Filicic acid, $\left[\mathrm{C}_{35} \mathrm{H}_{42} \mathrm{O}_{13}\right.$, a white, amorphous or] crystalline body, said to be the active principle. (2) A fixed oil, 6 to 7 per cent. (3) [Filicin, $\mathrm{C}_{35} \mathrm{H}_{40} \mathrm{O}_{12}$, a crystalline substance, soluble in Chloroform, Benzol and fixed and volatile oils. (4) Filix-red.] (5) Resins.

Dose, $1 / 2$ to $1 \frac{1}{2} \mathrm{dr}$.; 2. to $6 . \mathrm{gm}$.]

\section{Preparation.}

[Oleoresina Aspidii.-Oleoresin of Aspidium. By percolation with Ether, distillation of the Ether, and evaporation.

Dose, $1 / 4$ to I fl. dr.; I. to 4 . c.c.]

\section{Action and Therapeutics of Male Fern.}

[Aspidium] is the most certain anthelmintic we have for the common tapeworm (Tania solium), and the Bothriocephalus latus. It may be flavored with ginger or peppermint. The intestine should first be emptied with a mild purge to ensure the worm not being protected by food. Then the [aspidium] should be administered, and about twelve hours afterwards another dose of the purgative should be given to clear away the dead worm. Very little food should be taken during the treatment, and the head of the worm should be carefully searched for in the motion. [Recently several cases of poisoning have been reported, presumably not due to an excessive dose, but to the fact that castor oil was administered at the same time with it, notably increasing the absorption of filicic acid.]

This remedy also kills the Anchylostoma duodenale outside the body, and has been successfully given to persons suffering from this parasite. 


\section{GRANATUM.}

[POMEGRANATE. - The bark of the stem and root of Punica Granatum Linnè (nat. ord. Lythrariece). Habitat.-India and Southwestern Asia ; cultivated and naturalized in subtropical countries.

Characters. - In the quills or fragments, from 5 to $10 \mathrm{~cm}$. long, and from 1 to $3 \mathrm{~mm}$. thick; outer surface yellowish-gray, somewhat warty, or longitudinally and reticulately-ridged; the stem-bark often partly covered with blackish lichens; the thicker pieces of the root-bark more or less scaly externally; inner surface smooth, finely striate, grayish-yellow; fracture short, granular, greenish-yellow ; indistinctly radiate; inodorous; taste astringent, very slightly bitter.]

Composition. - The chief constituents are- $\left[(1)\right.$ Pellctierine, $\mathrm{C}_{8} \mathrm{H}_{13} \mathrm{NO}$, $1 / 2$ per cent., a colorless, oily, aromatic alkaloid, soluble in water, Alcohol, Ether and Chloroform. (2) Three allied alkaloids, Methyl-, Pseudo, and Lsopelletierine. (3) Punicotannic Acid, $\mathrm{C}_{20} \mathrm{H}_{16} \mathrm{O}_{13}, 20$ per cent.]

Incompatibles.-Alkalies, lime water, metallic salts, and gelatin.

Dose, $1 / 2$ to $1 \frac{1}{2}$ dr. ; [2. to $6 . \mathrm{gm}$.]

\section{Action and Therapeutics of Pomegranate.}

Pomegranate is a powerful astringent, and the decoction [B. P., I to 5 ; dose, $1 / 2$ to 2 fl. oz. ; 15 . to 6o. c.c.] may be used as a gargle for a sore throat. In large doses it is emetic and purgative. It is an anthelmintic for the tapeworm. It is so [disagreeable] that it is rarely used, but if employed the decoction may be given every hour for four hours before breakfast. A purge should be administered the night before and a few hours after breakfast. Pelletierine [dose of tannate, (not official), 3 to $6 \mathrm{gr}$; .20 to $.40 \mathrm{gm}$. $]$ is the active anthelmintic principle, and is said to be very efficient for killing the common tapeworm. Large doses of pelletierine given to animals act like curare.

\section{cusso.}

KOUSSO.-Synonyms.-[Brayera. Kooso. The female inflorescence of Hagenia abyssinica (Bruce) Gmelin (nat. ord. K'osacea). Habitat. Abysinia.

Characters. - In bundles, rolls, or compressed clusters, consisting of panicles about $25 \mathrm{~cm}$. long, with a sheathing bract at the base of each branch; the two roundish bracts at the base of each flower, and the four or five obovate, outer sepals are of a reddish color, membranous and veiny ; calyx top-shaped, hairy, enclosing two carpels or nutlets; odor slight, fragrant and tea like; taste bitter, acrid, and nauseous.] 
Compositron.-The chief constituents are-(I) [Kosin or] Koussin, a neutral, active principle, [in yellow crystals, tasteless, soluble in Chloroform, Benzol, Ether and Alcohol; insoluble in water. (2) Oil. (3) Gum. (4) Tannic Acid. (5) Two Resins.

Dose, 2 to 4 dr.; 8. to 15. gm.]

\section{Preparation.}

[Extractum Cusso Fluidum.-Fluid Extract of Kousso. Synonym.-Extractum Brayere Fluidum. By maceration and percolation with Alcohol, and evaporation.

Dose, I to 4 fl. dr. ; 4 . to I5. c.c.]

\section{Action and Therapeutics of Kousso.}

Kousso is used as an anthelmintic for all species of tapeworm. [Koussin (not official) has been given with good results. Dose, 20 to $40 \mathrm{gr}$. ; 1.20 to $2.40 \mathrm{gm}$.]

\section{KAMALA.}

[KAMALA.-Synonym. - Rottlera. The glands and hairs from the capsules of Mallotus philippinensis (Lamarck) Mueller Arg. (nat. ord. Euphorbiacea). Habilat. - India, China and Philippine Islands.

CHARACTERS. - A granular, mobile, brick-red or brownish-red powder, inodorous and nearly tasteless, imparting a deep red color to alkaline liquids, Alcohol, Ether, or Choloroform, and a pale, yellow tinge to boiling water. Under the microscope it is seen to consist of stellately arranged, colorless hairs, mixed with depressed-globular glands, containing numerous red, clubshaped vesicles. Solubility. - Insoluble in hot and cold water.

Composition. - The chief constituents are-(I) Rottlerin, $\left[\mathrm{C}_{22} \mathrm{H}_{20} \mathrm{O}_{6}\right.$, a neutral principle in yellowish needles, soluble in hot Alcohol, Ether, Benzol, and Carbon Disulphide. (2) Resins, nearly 8o per cent.

Dose, 1 to $2 \mathrm{dr}$. ; 4. to 8 . $\mathrm{gm}$.

\section{Action and Therapeutics of Kamala.}

Kamala is an anthelmintic. It will kill the Tenia solium, and probably the Oxyuris vermicularis and the Ascaris lumbricoides. It is also purgative.]

\section{PEPO.}

[PUMPKIN SEED.-The seed of Cucurbita Pepo Linné (nat. ord. Cucurbitacea). Habitat.--Tropical Asia and America; cultivated.

Cilaracters. - About $2 \mathrm{~cm}$. long, broadly ovate, flat, white, or whitish, nearly smooth, with a shallow groove parallel to the edge; containing a 
short, conical radicle and two flat cotyledons; inodorous; taste bland and oily.

Composition.-(I) Fixed oil, 44 per cent. (2) An Acrid Resin, considered to be the tæniafuge principle. (3) Two Proteids (Myosin and Vitellin). (4) Fatty Acids.

Dose, I to 3 oz.; 30. to $90 . \mathrm{gm}$.

\section{Action and Uses of Pumpkin Seed.}

Pepo administered as an emulsion is one of the most efficient and at the same time harmless tæniafuges.]

\section{Class II.-ANTHELMINTICS USED FOR THE ROUNDWORM.}

\section{SANTONICA.}

SANTONICA. - [Synonym. - Levant Wormseed. The unexpanded flower-heads of Artemisia pauciflora Weber (nat. ord. Composita). Habitat. -Türkestan.

Characters.-From 2 to $4 \mathrm{~mm}$. long, oblong-ovoid, obtuse, smooth, somewhat glossy, grayish-green, after exposure to light brownish-green, consisting of an involucre of about 12 to 18 closely imbricated, glandular scales with a broad midrib, enclosing four or five rudimentary florets; odor strong, peculiar, somewhat camphoraceous ; taste aromatic and bitter.

Composition. - The chief constituents are-(I) Santonin (see below). (2) A volatile oil, 2 per cent., consisting mainly of Cineol, $\mathrm{C}_{10} \mathrm{H}_{18} \mathrm{O}$.

Dose, 15 to $60 \mathrm{gr}$. ; 1 . to $4 . \mathrm{gm}$.]

SANTONINUM. - Santonin. $\mathrm{C}_{15} \mathrm{H}_{18} \mathrm{O}_{3}[=245.43$. A neutral principle obtained from Santonica.

SoURCE.-By exhausting Santonica, mixed with Lime, with Diluted Alcohol, distilling off the Alcohol, and adding Acetic Acid to the residue. The precipitated Santonin is purified by dissolving it in Alcohol, treating with A nimal Charcoal, and crystallizing.

Characters. -Colorless, shining, flattened, prismatic crystals, odorless and nearly tasteless when first put in the mouth, but afterwards developing a bitter taste; not altered by exposure to air, but turning yellow on exposure to light. Solubility. - Nearly insoluble in water; soluble in 40 parts of Alcohol ; also soluble in 140 parts of Ether, in 4 parts of Chloroform, and in solutions of Caustic Alkalies.]

Dose, $1 / 4$ to $\times \mathrm{gr} . ;$ [.015 to $.06 \mathrm{gm}$. (child), $x$ to $5 \mathrm{gr}$; .06 to $.30 \mathrm{gm}$.] (adult).

\section{Preparation.}

Trochisci Santonini.-[Troches of Santonin. Santonin, 3; Sugar, 110 ; Tragacanth, $3 \mathrm{gm}$.; Stronger Orange Flower Water, to make roo troches. Each troche contains about $1 / 2 \mathrm{gr}$, ; .03 gm.

Dose, 2 (child) to ro troches (adult).] 


\section{Action of Santonin.}

Santonin is anthelmintic, killing the roundworm, Ascaris lumbricoides, and according to some authorities the Oxyuris vermicularis, but this is doubtful, for it does not kill these worms outside the body, and therefore if efficient must be changed in the intestine. It has no action on tapeworms. Some of the santonin is absorbed as sodium-santoninate. Medicinal doses will usually cause the urine, if it is acid, to be a greenish-yellow or saffron color, and if it is alkaline to be purplish-red. This is due to the excretion in that fluid of some substance resulting from the changes undergone by santonin in the body. It is slightly diuretic. Often even small doses lead to xanthopsy -that is to say, everything the patient sees has a yellow tint; this is not the result of the staining of the tissues of the eye yellow, but is a direct effect on some other part of the visual path.

\section{Therapeutics of Santonin.}

Santonin is used solely to kill intestinal worms. It should be given on an empty stomach, and should be followed in two hours by a purgative, such as calomel, which acts on the small intestine, for this is the part inhabited by the worms killed by santonin. It is certainly very efficacious for the Ascaris lumbricoides. The lozenge [B. P., I gr., .06 gm. each] is not to be recommended, for it may not dissolve, and then will probably fail to kill the worm. A good way to give santonin is to suspend it and castor oil in mucilage flavored with peppermint. As already mentioned, probably it has no effect on the Oxyuris vermicularis, when given by the mouth, but a suppository made with oil of theobroma, and containing $4 \mathrm{gr}$; $25 \mathrm{gm}$. of santonin, is said to kill this parasite.

\section{[TOXICOLOGY.}

Symploms.]-Several cases of fatal poisoning by santonin are on record. Cerebral symptoms are very prominent. Thus convulsions, accompanied by unconsciousness, trismus, and dilated pupils, are generally present. The surface becomes cold, there is sweating, there may be trembling, the pulse and respiration become weaker and weaker, and death takes place from cardiac and respiratory failure. 
[Treatment.-Ammonia, or strychnine sulphate hypodermatically. If the drug is given with castor oil the danger of poisoning is lessened.

\section{SPIGELIA.}

SPIGELIA.-Synonyms. - Pinkroot. Carolina Pink. The rhizome and roots of Spigelia marilandica Linne (nat. ord. Loganiacea). Habitat.Southern United States; westward to Texas and IVisconsin, in rich woods.

Citaracters. - Of horizontal growth, about $5 \mathrm{~cm}$. or more long, 2 or 3 $\mathrm{mm}$. thick, dark purplish brown, bent, somewhat branched, on the upper side with cup-shaped scars; on the lower side with numerous, thin, brittle, lighter- . colored roots, about ro $\mathrm{cm}$.long; the rhizome internally with a whitish wood and a pith which is usually dark-colored or decayed ; odor somewhat aromatic; taste sweetish, bitter and pungent. Resembling Spigelia root.-Phlox Carolina, but the rootlets are brownish-yellow, rather coarse, straight, and contain a straw-colored wood underneath a readily removable bark.

Composition.-(1) Spigeline, a volatile alkaloid. (2) A little volatile oil. (3) Bitter principle. (4) Resin.

Dose, $1 / 4$ to $2 \mathrm{dr}$.; 1. to 8 . gm.

\section{Preparation.}

Extractum Spigeliæ Fluidum.-Fluid Extract of Spigelia. By maceration and percolation with Diluted Alcohol, and evaporation.

Dose, $1 / 4$ to 2 fl. dr.; r. to 8 . c.c.

\section{Action and Uses of Spigelia.}

Spigelia is one of the most powerful anthelmintics. It may give rise to symptoms of narcotic poisoning, which, however, may be obviated by its combination with a cathartic like senna.

\section{CHENOPODIUM.}

CHENOPODIUM.-Synonym.-American Wormseed. The fruit of Chenopodium ambrosioides Linne, and variety anthelminticum Gray (nat. ord. Chenopodiacea). Ifabitat. - West I udia and Central America ; naturalized in the United States.

Characters. - Nearly $2 \mathrm{~mm}$. in diameter, depressed-globular, glandular, dull greenish or brownish, the integuments friable, and containing a lenticular, obtusely-edged, glossy, black seed. It has a peculiar; somewhat terebinthinate odor, and a bitterish, pungent taste.

Composition. - It is composed chiefly of a volatile oil, consisting of (I) a hydrocarbon, $\mathrm{C}_{10} \mathrm{H}_{16}$, and (2) a liquid, oxygenated oil $\mathrm{C}_{10} \mathrm{II}_{16} \mathrm{O}$.

Dose, 15 to $30 \mathrm{gr}$. ; 1 . to $2 . \mathrm{gm}$. 
OLEUM CHENOPODII.-Oil of Chenopodium. Synonym.-Oil of American Wormseed. A volatile oil distilled from Chenopodium.

ChARACTERs. - A thin, colorless or yellowish liquid, having a peculiar, penetrating, somewhat camphoraceous odor, and a pungent and bitter taste. Sp. gr., about 0.970 .

Dose, 2 to $10 \mathrm{~m}$.; .12 to .60 c.c.

\section{Action and Uses of Chenopodium.}

Wormseed is one of the most efficient anthelmintics, particularly against $A$ scarides, but it should be followed by a cathartic.]

\section{Class III.-PARASITICIDES USED FOR PEDICULI.}

\section{STAPHISAGRIA.}

STAPHISAGRIA.--[Synonym.-Stavesacre. The seed of Delphinium Staphisagria Linné (nat. ord. Ranunculacere). Habitat.-Basin of the Mediterranean; cultivated.

Characters. - About $5 \mathrm{~mm}$. long, 3 or $4 \mathrm{~mm}$. broad, flattish-tetrahedral, one side convex, brown or browuish-gray, with reticulate ridges, containing a whitish, oily albumen and a straight embryo; nearly inodorous; taste bitter and acrid. ]

Composition.-The chief constituents are-(r) Delphinine, $\left[\mathrm{C}_{22} \mathrm{H}_{35} \mathrm{NO}_{6}\right.$, a white, crystalline, poisonous alkaloid, soluble in Alcohol, Ether and Chloroform, resembling Aconite in its action. (2) Delphinoidine, $\mathrm{C}_{42} \mathrm{H}_{68} \mathrm{~N}_{2} \mathrm{O}_{7}$, an amorphous alkaloid ; solubility as of Delphinine. (3) Delphisine, $\mathrm{C}_{2 \mathrm{~J}} \mathrm{H}_{46} \mathrm{~N}_{2} \mathrm{O}_{4}$, a crystalline alkaloid of the same solubility. (4) Staphisagrine, $\mathrm{C}_{22} \mathrm{H}_{32} \mathrm{NO}_{5}$, an alkaloid but slightly soluble in water. (5) Fixed oil, 25 per cent.]

\section{Action and Therapeutics of Staphisagria.}

[Staphisagria] is only used as a parasiticide to kill pediculi. The affected part is rubbed with the ointment, [B. P., 4 ; yellow wax, 2 ; benzoinated lard, 17 ] which, in the case of pediculi vestimentorum, is allowed to soak, day and night, into the garments next to the skin, for the parasite inhabits them. It is often employed, but it will be remembered that many other parasiticides for pediculi have been mentioned (see p. $5 \mathrm{r}$ ).

\section{PICROTOXINUM.}

PICROTOXIN. $-\mathrm{C}_{30} \mathrm{H}_{34} \mathrm{O}_{13}[=600.58$. A neutral principle obtained from the seed of Anamirta paniculuta Colebrooke (nat. ord. Menispermacea). Synonyms.-Cocculus Indicus. Fishberry. Habitat.-East India. 
Source. - Obtained by exhaustion with hot Alcohol, evaporation and purification by re-crystallization, after decolorizing with Animal Charcoal.

Characters. - Colorless, flexible, shining, prismatic crystals, or a microcrystalline powder, odorless, and having a very bitter taste; permanent in the air. Solubility.-Soluble in 240 parts of water, and in 9 parts of Alcohol ; also soluble in solutions of the alkalies, and in acids. Very slightly soluble in Ether or Chloroform.]

Dose, $\frac{1}{20}$ to $\frac{1}{60} \mathrm{gr}$. ; [.0005 to .001 gm.]

\section{Action of Picrotoxin.}

External.-Picrotoxin is very destructive to lower forms of life, and is therefore antiparasitic.

Internal.-It is a powerful poison, causing severe gastro-intestinal irritation, collapse, lightheadedness, convulsions, hyperpyrexia, slowing of the pulse and stimulation of the respiratory centre. Death results from asphyxia, partly due to convulsions and partly to ultimate paralysis of the respiratory centre.

\section{Therapeutics of Picrotoxin.}

External.-An ointment of the seeds in lard [ $I$ to 6$]$ has been applied to the scalp to kill pediculi. It must be employed with caution, for this strong poison can be absorbed if the skin be broken. It is an expensive ointment.

Internal._Picrotoxin is used empirically, to check the night sweating of phthisis. A single dose should be given in the evening. Its action is uncertain, but sometimes it succeeds. Many other diseases have been treated with it, but there is no evidence that it has benefited them. [Tablets], each containing ${ }_{10}^{\frac{1}{0}} 0$ of a grain [.0006 gm.], are prepared for subcutaneous injection. One should be dissolved in a few drops of water immediately before use. 


\title{
GROUP XI.
}

\section{Vegetable drugs apparently having only a diuretic action.}

\author{
Uva Ursi, [Apocynum, Zea,] Triticum, [Marrubium, Pareira, \\ Dulcamara, Chimaphila, Fabiana.]
}

\section{UVA URSI.}

UVA URSI.-Synonym.-Bearberry. [The leaves of Arctostaphylos Uva Ursi (Linné) Sprengel (nat. ord. Ericacea). Habitat.-Northern Hemisphere, in dry and sandy or rocky places; in the United States, south to Pennsylvania, New Mexico, and California.

ChARACTERS. - Very short-stalked, obovate or oblong-spatulate, coriaceous, from 15 to $20 \mathrm{~mm}$. long, and 5 to $8 \mathrm{~mm}$. broad, obtuse, slightly revolute on the margin, upper surface with depressed veins; lower surface distinctly reticulate; odor faint, bay-like; taste strongly astringent, and somewhat bitter. Resembling Uva Ursi._Senna (see p. 493) and Buchu (see p. 570.)]

Composition.-The chief constituents are-(I) Arbutin, $\left[\mathrm{C}_{12} \mathrm{H}_{18} \mathrm{O}_{7}\right.$, ] a bitter, crystalline glucoside yielding glucose, hydroquinone and methyl-hydroquinone. (2) Ericolin $\left[\mathrm{C}_{10} \mathrm{H}_{16} \mathrm{O}\right.$, a bitter, crystalline glucoside. (3) Ursone, a tasteless neutral body. (4) Tannic Acid, 6 to 7 per cent. (5) Gallic Acid.

INCOMPATIBLES. - Iron, lead and silver salts, alkaloids, and gelatin.]

Dose, $1 / 4$ to I dr.; [r. to $4 . \mathrm{gm}$.]

\section{Preparations.}

[1. Extractum Uvæ Ursi.-Extract of Uva Ursi. By maceration and percolation with Alcohol and Water, and evaporation.

Dose, 5 to $15 \mathrm{gr}$; ; 30 to $1.00 \mathrm{gm}$.

2. Extractum Uvæ Ursi Fluidum.-Fluid Extract of Uva Ursi. By maceration and percolation with Glycerin, Alcohol and Water, and evaporation.

Dose, $1 / 4$ to I fl. dr.; I. to 4 . c.c.]

\section{Action of Uva URSI.}

Uva ursi is a well-marked diuretic, and is astringent and disinfectant to the urinary mucous membrane. Its disinfectant action is probably due to the decomposition of the arbutin into glucose and hydroquinone, for after uva ursi is given this substance is found in the urine, and it is a very energetic antiseptic. This decomposition must take place in the kidneys, 
for hydroquinone is a powerful poison. Against this being the reason of the disinfectant action of the uva ursi, it is urged that giving arbutin does not disinfect the urine; but others deny this, and the probability is that the first-mentioned view is correct. Arbutin itself is a diuretic. The urine may be a pale greenish to dark greenish-brown color. Hydroquinone is also found in the urine in carbolic acid poisoning (see p. 332). [The fluid extract is an excellent remedy for ardor urinæ of acute gonorrhœa.] The astringent action of uva ursi on the urinary tract is usually ascribed to the gallic and tannic acids, but as these are not remote astringents this is most likely wrong.

\section{Therapeutics of Uva URSI.}

Uva ursi is given to disinfect the urine in the same class of cases as buchu-that is to say, in pyelitis, cystitis and gonorrhœa.

\section{[APOCYNUM.}

APOCYNUM.-Synonym.-Canadian Hemp. The root of Apocynum cannabinum Linné (nat. ord. Apocynacea). Habitat.-United States, on the border of thickets and in grassy places.

Characters. - Long, cylindrical, somewhat branched, 5 to $10 \mathrm{~mm}$. thick, gray or brownish-gray, longitudinally wrinkled and transversely fissured; brittle ; fracture short, white ; the bark rather thick ; the wood porous, spongy, with delicate, medullary rays; inodorous; taste bitter, disagreeable.

Composition.-The chief constituents are-(I) Apocynein, a glucoside, soluble in water, acting like digitalin. (2) Apocynin, an amorphous, resinous glucoside.

Dose, 2 to $5 \mathrm{gr}$; . 12 to $.30 \mathrm{gm}$.

\section{Preparation.}

Extractum Apocyni Fluidum.-Fluid Extract of Apocynum. By maceration and percolation with Glycerin, Alcohol and Water, and evaporation.

Dose, 2 to $20 \mathrm{~m}$.; .12 to 1.20 c.c.

\section{Action and Uses of Apocynum.}

Canadian hemp has been found to be beneficial in dropsy, because of its action which is not only diuretic, but as well resembling that of strophanthus and similar drugs, when used as an infusion ( $x$ to $x 6$ ), of which the dose is one to two fluid 
ounces; 30. to 60. c.c., twice or three times daily. This is a more valuable drug than its limited use would indicate. It frequently produces copious diuresis after other, and better known drugs, have failed. It is also, in larger doses, a hydragogue cathartic.

\section{ZEA.}

ZEA.-Synonym.-Corn-silk. The styles and stigmas of Zea Mays Linné (nat. ord. Graminea). Habitat.-Tropical America; cultivated in the warm, temperate zone.

Characters. - Thread-like; about $15 \mathrm{~cm}$. long, and $0.5 \mathrm{~mm}$. broad, yellowish or greenish, soft silky, finely hairy, and delicately veined longitudinally; inodorous; taste sweetish.

Composition.-Its chief constituents are-(I) Maizenic Acid, about 2 per cent. (2) Fixed oil. (3) Resin. (4) Salts.

Dose, I to $2 \mathrm{dr}$; 4 4 to 8 . $\mathrm{gm}$.

\section{ACtion AND USES OF CORN-Silk.}

Corn-silk is a mild but fairly certain diuretic when given in full doses. It is useful in acute and chronic cystitis and in the bladder irritation of uric acid and for phosphatic gravel. It is possibly as well a cardiac stimulant in the dropsy of heart disease. It is best administered in the form of an infusion, in boiling water ( $\mathrm{I}$ to 8), taken almost ad libitum.]

\section{TRITICUM.}

TRITICUM.-[Synonym.-Couch-Grass. The rhizome of Agropyrum repens (Linné) Beauvois (nat. ord. Graminea), gathered in the spring and deprived of the roots. Habitat.-Europe and North America.

ChARACTERS. - Very long and creeping, about $2 \mathrm{~mm}$. thick; as met with in the shops, cut into sections about $1 \mathrm{~cm}$. long; smooth, but wrinkled; hollow in the centre, straw-yellow; inodorous, taste sweetish.

Composition.--(1) Triticin, about 8 per cent., a gummy substance resem. bling Inulin. (2) Inosit. (3) Malates.

Dose, I to $8 \mathrm{dr}$.; 4 . to $30 . \mathrm{gm}$.

\section{Preparation.}

Extractum Tritici Fluidum.-Fluid Extract of Triticum. By percolation with Boiling Water, evaporation, addition of Alcohol and filtration.

Dose, I to $8 \mathrm{fl}$. dr.; 4. to 3o. c.c.] 


\section{Action and Therapeutics of Triticum.}

Triticum is a diuretic, and is used as a sedative in inflammation of the genito-urinary membranes.

\section{[MARRUBIUM.}

MARRUBIUM. - Synonym. - Horehound. The leaves and tops of Marrubium vulgare Linné (nat. ord. Labiata). Habitat.-Europe, Central Asia ; naturalized in North America; cultivated.

Characters. - Leaves about $25 \mathrm{~mm}$. long, opposite, petiolate, roundishovate, obtuse, coarsely crenate, strongly rugose, downy above, white-hairy beneath; branches quadrangular, white, tomentose ; flowers in dense, axillary woolly whorls, with a stiffly ten-toothed calyx, a whitish, bilabiate corolla, and four included stamens; aromatic and bitter.

Composition.-(1) Marrubiin, possibly a glucoside, in crystalline scales or prisms, soluble in Ether, Chloroform and Alcohol. (2) Volatile Oil, in small amount. (3) Resin.

Dose, I to $2 \mathrm{dr}$.; 4. to 8 . $\mathrm{gm}$.

\section{Uses AND ACtion of HoREhound.}

Horehound, used as a decoction or an infusion, is in moderate doses a diuretic, in large doses laxative, and may be so given as to increase the action of the skin and kidneys; but its action is not marked. It is probably also a bitter stomachic. Confection of horehound slowly dissolved in the mouth relieves the relaxed throat of public speakers.]

\section{PAREIRA.}

PAREIRA.- [Synonym.-Pareira Brava. The root of Chondodendron tomentosum Ruiz et. Pavon (nat. ord. Menispermacea). Habitat.-Brazil.

Characters. - Subcylindrical, somewhat tortuous pieces, about ro to $\mathbf{1 5}$ $\mathrm{cm}$. long, varying in thickness from 2 to $10 \mathrm{~cm}$.; externally dark brownishgray, with transverse ridges and fissures and longitudinal furrows; internally pale brown, and, when freshly cut, having a waxy lustre; bark thin; wood porous, in two or more somewhat irregularly concentric circles, with rather large medullary rays, and no distinct central pith; modorous; taste bitter.

Composition. - The chief constituent is Pelosine, an alkaloid, about 0.5 per cent., identical with Berberine (see p. 640) and Buxine].

InCOMPATIBLES.-Ferric and lead salts, and tincture of iodine.

Dose, $1 / 2$ to $\mathrm{I} \mathrm{dr}$.; [2. to $4 . \mathrm{gm}$.] 


\section{Preparation.}

[Extractum Pareiræ Fluidum.-Fluid Extract of Pareira. By maceration and percolation with Glycerin, Alcohol, and Water, and evaporation.

Dose, $1 / 2$ to I fl. dr.; 2. to 4 . c.c.]

\section{Action and Therapeutics of Pareira.}

Pareira [besides its diuretic, in which it closely resembles buchu, is not known to have any physiological action.] It is used empirically in chronic inflammation of the genito-urinary tract, such as pyelitis, cystitis, gonorrhœa, and gleet. [It was formerly renowned as a lithontriptic.]

\section{[DULCAMARA.}

DULCAMARA.-Synonyms. - Bittersweet. Woody Nightshade. The young branches of Solanum Dulcamara Linné (nat. ord. Solanacea). Habitat.-Europe and Asia; naturalized ia North America.

Characters. - About $5 \mathrm{~mm}$, or less, thick, cylindrical, somewhat angular, longitudinally striate, more or less warty, usually hollow in the centre, cut into short sections. The thin bark is externally pale greenish, or light greenish brown, marked with alternate leaf-scars, and internally green; the greenish or yellowish wood forms one or two concentric rings. Odor slight; taste bitter, afterwards' sweet.

Compositrion.-(1) Solanine, $\mathrm{C}_{42} \mathrm{H}_{87} \mathrm{NO}_{15}$, the active alkaloid. (2) Dulcamarin, $\mathrm{C}_{22} \mathrm{H}_{34} \mathrm{O}_{10}, 0.4$ per cent. a glucoside, soluble in water and Alcohol, and yielding frothy solutions.

Dose, I to 2 dr.; 4. to 8 . $\mathrm{gm}$.

(3) Resin. (4) Gum.

\section{Preparation.}

Extractum Dulcamaræ Fluidum.-Fluid Extract of Dulcamara. By maceration and percolation with diluted Alcohol and evaporation:

Dose, I to 2 fl. dr.; 4 . to 8 . c.c.

\section{Action and Uses of Dulcamara.}

Dulcamara increases the secretions, particularly those of the kidneys and skin, with some diminution of sensibility. In large doses it is an acro-narcotic poison. It has been employed chiefly for cutaneous eruptions, particularly of a scaly character, but is seldom prescribed. 


\section{CHIMAPHILA.}

CHIMAPHILA.-Synonyms. - Pipsissewa. Prince's Pine. The leaves of Chimaphila umbellata (Linnè) Nuttall (nat. ord. Ericacea). Habitat.Northern Continent.

Characters. - About $5 \mathrm{~cm}$. long, oblanceolate, sharply serrate above, wedge-shaped and nearly entire towards the base; coriaceous, smooth, and dark-green on the upper surface. It is nearly inodorous, and has an astringent and bitterish taste.

Composition.-(1) Arbutin (see p. 631). (2) Ericolin. (3) Chimaphilin, in yellow, tasteless, volatile crystals. (4) Ursone. (5) Tannic Acid, 4 per cent.

Dose, $1 / 2$ to 2 dr.; 2. to 8 . c.c.

\section{Preparation.}

Extractum Chimaphilæ Fluidum.-Fluid Extract of Chimaphila. By maceration and percolation with Diluted Alcohol, and evaporation.

Dose, $1 / 2$ to 2 fl. dr.; 2. to 8 . c.c.

\section{Action and Uses of Chimaphila.}

This plant is diuretic and diaphoretic. It is used for rheumatism and nephritic affections.

\section{FABIANA.}

PICHI.-(Not official.) - The branches of the woody shrub, Fabiana imbricata (nát. ord. Solanacea). Habitat.-Peru and Chili.

Characters. -A woody shrub growing on rocky hill-tops, with plumelike sprays, the small densely-crowded leaves much resembling those of a conifer. The branches and leafy branchlets are resinous, with an aromatic odor and taste.

Composition. - It contains (I) Fabianine, a bitter alkaloid. (2) A Resin. (3) A crystalline substance. (4) An essential oil.

\section{Preparation.}

Extractum Fabianæ Fluidum.-Fluid Extract of Fabiana. By maceration and percolation with Diluted Alcohol, and evaporation,

Dose, 5 to $40 \mathrm{~m}$.; .30 to 2.50 c.c.

\section{Action and Therapeutics of Pichi.}

Pichi is a diuretic of great value in inflammation of the bladder and catarrh of the urinary tract. It should not be used in organic disease. It is best prescribed in combination with an alkali, as sodium carbonate.] 


\title{
GROUP XII.
}

\section{Vegetable drugs acting locally on unstriped muscle, especially that of the uterus.}

\author{
Ergot, Hydrastis, [Cotton Root Bark, Caulophyllum, Viburnum, \\ Apiol.]
}

\section{ERGOTA.}

ERGOT.-[Synonym.-Ergot of Rye. The sclerotium of Claviceps purpurea (Fries) Tulasne (class Fungi), replacing the grain of rye, Secale cereale Linné (nat. ord. Graminea). Habitat._Spain and Russia.

Characters. - Somewhat fusiform, obtusely triangular, usually curved, about 2 or $3 \mathrm{~cm}$. long, and $3 \mathrm{~mm}$. thick; three-furrowed, obtuse at both ends, purplish-black, internally whitish with some purplish striæ, breaking with a short fracture ; odor peculiar, heavy ; taste oily and disagreeable.]

Composition. - The chief constituents are- $\left[(\mathrm{I})\right.$ Ergotine, $\mathrm{C}_{50} \mathrm{H}_{52} \mathrm{~N}_{2} \mathrm{O}_{3}$, an amorphous alkaloid, feebly bitter; soluble in Alcohol and water. (2) Ecboline, an amorphous alkaloid, slightly bitter; soluble in water and Alcohol. (3) Ergotinine, $\mathrm{C}_{33} \mathrm{H}_{40} \mathrm{~N}_{4} \mathrm{O}_{6}$, a crystalline alkaloid, slightly bitter.] (4) Sphacelic Acid [known also as Sphacelotoxin], a non-nitrogenous, unstable body, insoluble in water, soluble in alkalies. It is believed to be the active agent in contracting the blood-vessels. (5) Cornutine, an active alkaloid, [not only contracts the blood-vessels but] is believed to be the agent which contracts the uterus. This is the chief active constituent of alcoholic extracts of Ergot. (6) [Sclerolinic, Ergotinic or Ergotic Acid, I to 4 per cent., soluble in water and alkalies, having ecbolic properties]. This is really a mixture of Sphacelic Acid and Cornutine. (7) A fixed oil, $3^{\circ}$ per cent. (8) Trimethylamine, to which the odor is due. (9) Tannic Acid. Many other bodies have been found in Ergot, but those given are believed to be the more important; the composition of Ergot is not yet certainly made out.

Dose, 30 to $60 \mathrm{gr}$.; [2. to $4 . \mathrm{gm}$.]

\section{Preparations.}

I. [Extractum Ergotæ Fluidum.-Fluid Extract of Ergot. By maceration and percolation with Acetic Acid and Diluted Alcohol, and evaporation.

Dose, $1 / 2$ to I f. dr.; 2. to 3. c.c.

2. Extractum Ergotæ.-Extract of Ergot. Synonym.-Ergotin. Fluid Extract of Ergot. By evaporation to a pilular consistence.

Dose, 5 to $15 \mathrm{gr}$; .30 to $1.00 \mathrm{gm}$. 
3. Vinum Ergotæ.-Wine of Ergot. Ergot, I 50. By percolation with White Wine, I50; and Alcohol, to 1000.

Dose, I to 4 fl. dr. ; .4 to .r. 5 c.c.

It is said that Ammonia is the best solvent for the active principles of Ergot. It may be extemporaneously prescribed as follows : Ergot, I; Aro matic Spirit of Ammonia, 2. Dose, 10 to $60 \mathrm{~m}$. ; .60 to 4.00 c.c.]

\section{Action of ERgot.}

The chief action of cornutine is on voluntary and involuntary muscle, which is stimulated to contract, hence prolonged muscle curves, increased peristalsis of the [intestines], tonic contraction of the uterus, and consequent rise of blood-pressure. It also causes convulsions. Sphacelic acid also acts directly on the muscular tissue of arterioles and the uterus, causing it to contract, and in addition it produces an actual thickening of the walls of the arterioles. These substances are rarely given separately, and the following account refers to the action of ergot itself.

\section{External.-None.}

Internal.-Gastro-intestinal tract.-The unstriped muscle of the intestine is stimulated by ergot, and this leads to greatly increased peristaltic movements, sometimes strong enough to cause relaxation of the bowels. The vessels of the intestine are constricted, in part because of the contraction of their own muscular fibres, and in part because of the contraction of those of the intestinal muscular coat. The result is that the intestine is blanched.

Blood.-The active principles of ergot are readily absorbed, but they are not known to produce any effect on the blood.

Heart. - The activity of the heart muscle is depressed by ergot; therefore the rate of the pulse falls, and consequently at first the blood-pressure may fall.

Vessels. - But the fall of blood-pressure is soon followed by a great rise, and this is due to the general contraction of the arteries all over the body; they can, in some parts, be seen to become smaller. The rise of pressure is most marked in the pulmonary artery, and here there is no primary fall. The veins 
are contracted to a less extent. This vascular contraction is less if the spinal cord is destroyed, from which it is fair to infer that it is partly due to the action of ergot on the vaso-motor centres in the cord, but the drug acts largely directly on the muscular coat of the vessels. [Because it contracts the arterioles it is hæmostatic.] If the ergot be taken for a long time the contraction of the arterioles, together with the associated thickening of their walls produced by sphacelic acid, lead to gangrene of various parts of the body, and this was a prominent symptom of the ergotism (chronic poisoning by ergot) which used to be seen in the very poor who could get no better food than rye infected with Claviceps purpurea. Enormous single doses of ergot appear to paralyze the vaso-motor centres, and then the blood-pressure falls from vascular dilatation and cardiac depression.

Nerious system. - Medicinal doses, or even an enormous single dose, very rarely affect the nervous system, but if ergot be taken for a long time a peculiar train of symptoms sets in ; they constituted the second variety of chronic ergotism in the [times] when diseased bread was eaten. The sufferer first complained of itching and tingling, and a sensation of insects running over the skin; this was followed by numbness and local anæsthesia. These symptoms first appeared in the hands and feet, but spread over the whole body. They were followed by tonic contractions of various muscles, especially those of the extremities. The muscular power was lessened, and the gait was staggering. Dimness of vision and loss of hearing, and epileptiform convulsions were sometimes present. This variety of ergotism was usually accompanied by vomiting and diarrhœa. Death occurred from [exhaustion].

Uterus.-Ergot powerfully excites the pregnant uterus of women and lower animals to contract and expel its contents. It is therefore called an ecbolic. It is not decided whether this effect is due to the action of the drug on the organ itself or on the spinal centres. Ergot has very little power to cause contraction of the unimpregnated uterus.

The flow of urine, of saliva, of sweat, and of milk is dimin- 
ished by ergot, probably because of the general vascular constriction.

\section{Therapeutics of ERgot.}

The chief use of ergot is to cause efficient contraction of the uterus after labor, and so to diminish the risk of post-partum hæmorrhage. If there is any likelihood of profuse bleeding it should be given subcutaneously, so that it may act rapidly.

Ergot should [not] be administered before the child is expelled, for the contraction produced by it not only gradually becomes more severe, but more prolonged, so that ultimately the uterus remains tightly contracted for several minutes; that is, of course, dangerous to the life of the child, and if the resistance be very great, may lead to rupture of the uterus. [The modern practice is to forbid its use until after the expulsion of the placenta.]

This drug has often been given as a hæmostatic in hæmoptysis and other hæmorrhages from different parts of the body. Some authors claim great success. Frequently it fails, and it may, by the general rise of blood-pressure, do more harm than good. It is difficult to [estimate] its value, for so many hæmorrhages will stop, even if no drugs are given. It has been used to check the night-sweats of phthisis, and as an antigalactagogue.

It is often desirable to combine the [fluid] extract of ergot with [ferric] chloride. Because of the tannic acid in the ergot an inky mixture results; but this may be clarified by the addition of a little citric acid, and the taste may be covered with chloroform water.

\section{HYDRASTIS.}

HYDRASTIS. - [Synonyms.-Golden Seal. Yellow Puccoon. - The rhizome and roots of Hydrastis canadensis Linne (nat. ord. Ranunculacea.) Habitat.-North America, west to Missouri and Arkansas, in woodlands.

Characters. - Rhizome about $4 \mathrm{~cm}$. long and $6 \mathrm{~mm}$. thick; oblique with short branches, somewhat annulate and longitudinally wrinkled; externally brownish-gray ; fraciure short, waxy, bright reddish-yellow, with a thickish bark, about ten narrow wood-wedges, broad medullary rays, and large pith. Roots thin, brittle, with a thick, yellow bark and subquadrangular, woxdy centre. Odor slight ; taste bitter.]

Compusition. - It contains- $(\mathrm{r})$ Berberine, $\left[\mathrm{C}_{20} \mathrm{H}_{18} \mathrm{NO}_{4}\right.$, an alkaloid ex- 
isting as yellowish prismatic crystals, and is found in many plants (Berberis, Calumba, Coptis, Menispermum, Xanthorrhiza, Xanthoxylum, etc.), chiefly in the orders Berberacea, Menispermaica, and Ranunculacea. It is identical with Buxine, the alkaloid of Buxus sempervirens, and Pelosine, that of Chondodendron tormentosum (Pareira). (2) Hydrastine, $\mathrm{C}_{21} \mathrm{H}_{21} \mathrm{NO}_{6}$, a colorless alkaloid, soluble in Alcohol and Ether.] (3) Canadine, $\mathrm{C}_{21} \mathrm{H}_{21} \mathrm{NO}_{4}$, in white needles.

Dose, 5 to $60 \mathrm{gr}$; ; $[.30$ to $4.00 \mathrm{gm}$.

\section{Preparations.}

I. [Extractum Hydrastis Fluidum.-Fluid Extract of Hydrastis. By maceration and percolation with Alcohol, Glycerin and water, and evaporation.

Dose, 5 to $60 \mathrm{~m} . ; .30$ to 4.00 c.c.

2. Tinctura Hydrastis.-Tincture of Hydrastis. Hydrastis, 200 ; by maceration and percolation with diluted Alcohol to 1000 .

Dose, $1 / 2$ to 2 fl. dr.; 2. to 8. c.c.

3. Glyceritum Hydrastis.-Glycerite of Hydrastis. Hydrastis, by percolation and maceration with Alcohol, distil off the Alcohol, add Water, filter, and to the filtrate add an equal volume of Glycerin.

Dose, 5 to $60 \mathrm{~m}$.; .30 to 4.00 c.c.

HYDRASTININE HYDROCHLORAS. - Hydrastinine Hydrochlorate. $\mathrm{C}_{11} \mathrm{H}_{11} \mathrm{NO}_{2} \mathrm{HCl}=224.97$. The Hydrochlorate of an artificial alkaloid derived from Hydrastine, the latter being obtained from Hydrastis.

SOURCE. - By acting upon Hydrastine by oxidizing agents, as when Man ganese Dioxide and Sulphuric Acid are used together, or when Platinic Chloride is employed.

Characters. - Light yellow, amorphous granules, or a pale yellow, crystalline powder, odorless, and having a bitter, saline taste; deliquescent on exposure to damp air. Solubility. - In 0.3 part of water, and in 3 parts of Alcohol; with difficulty soluble in Ether or Chloroform.

Dose, $\frac{1}{12}$ to $\frac{1}{6} \mathrm{gr}$.; .005 to .01 $\mathrm{gm}$.]

\section{Action of Hydrastis.}

Hydrastis, in moderate doses, acts as a gastric bitter, promoting the appetite, stimulating the gastro-intestinal secretions, and peristalsis. It increases the flow of bile. It contracts the peripheral arterioles chiefly, if not entirely, from its action on the vaso-motor centre in the medulla. In moderate doses it diminishes the rate and depresses the force of the cardiac contraction, but the contraction of the arterioles causes a rise of blood-pres- 
sure. It is said to increase uterine contractions and to produce abortion, but this is doubtful. In poisonous doses it still more depresses the heart, causing a great fall of blood-pressure; it produces convulsions similar to those of strychnine, and kills by paralysis of respiration. Its action is mainly due to the alkaloid hydrastine. It has been stated that this, before it acts, is oxidized into hydrastinine, but against this is the fact that it is excreted unchanged in the urine.

\section{Therapeutics of Hydrastis.}

External.-Hydrastis is employed empirically as a local stimulating application in chronic inflammations, such as unhealthy ulcers. It is used also as a lotion in hyperidrosis, acne, and seborrhœa. Any of the preparations may be employed, if diluted with water.

Internal.-The chief use of hydrastis is that it is empirically administered for chronic inflammations of mucous membranes. It is said to be especially valuable for uterine affections, particularly menorrhagia and dysmenorrhœa. It is given to stop uterine hæmorrhage and to arrest the growth of uterine tumors. For all thesé diseases hydrastinine hydrochlorate has been much used. Hydrastis is also employed in the chronic gastritis of drunkenness, and to a rather less degree in other forms of chronic gastrointestinal catarrh. As an injection or lotion it is employed (any preparation diluted with an equal part of water) for chronic nasal catarrh, otorrhœa, leucorrhœa, gonorrhœa, and as a mouth wash in aphthous stomatitis, chronic pharyngitis, etc. Some authors claim that it is useful for the same diseases of the heart as are benefited by digitalis. As an antiperiodic, hydrastis is inferior [but next in value to] quinine.

\section{[GOSSYPII RADICIS CORTEX.}

COTTON ROOT BARK.-The bark of the root of Gossypium herbaceum Linné, and of other species of Gossypium (nat. ord. Maljacex). Habitat,-Subtropical Asia and Africa; cultivated in the United States.

Characters. - In thin, flexible bands or quilled pieces; outer surface brownish-yellow, with slight longitudinal ridges or meshes, small, black, circular dots, or short, transverse lines, and dull, brownish-orange patches, from 
the abrasion of the thin cork ; inner surface whitish, of a silky lustre, finely striate ; bast-fibres long, tough, and separable into papery layers; inodorous; taste very slightly acrid and faintly astringent.

Composition. - (1) A yellow Resin. Acid. (4) Yellow coloring matter.

Dose, $1 / 4$ to I dr.; I. to 4. gm.

\section{Preparation.}

Extractum Gossypii Radicis Fluidum. - Fluid Extract of Cotton Root Bark. By maceration and percolation with Glycerin and Alcohol, and evaporation.

Dose, $1 / 4$ to $I$ fl. dr.; r. to 4 . c.c.

Action and Uses of Cotton Root Bark.

Cotton Root Bark has the same action as ergot, and is an emmenagogue and an abortifacient. It is used as an uterine hæmostatic in the treatment of menorrhagia and metrorrhagia from various causes, and particularly from uterine fibroids.

\section{CAULOPHYLLUM.}

CAULOPHYLLUM.-Synonyms.-Blue Cohosh. Squaw Root. The rhizome and roots of Caulophyllum thalictroides (Linné) Michaux (nat. ord. Berberidacea). Habitat.-North America, southward to Kentucky, in rich woodlands.

Characters. - Rhizome of horizontal growth, about $10 \mathrm{~cm}$. long, and about 6 to $10 \mathrm{~mm}$. thick, bent; on the upper side with broad, concave stem; scars and short, knotty branches; externally grayish-brown, internally whitish, tough and woody. Roots numerous, matted, about $10 \mathrm{~cm}$. long, and $1 \mathrm{~mm}$. thick, rather tough ; nearly jnodorous; taste sweetish, slightly bitter and somewhat acrid.

Composition.-Its chief constituents are-(I) Caulophylline, an alkaloid, which is colorless, odorless and almost tasteless, and crystallizes with difficulty. (2) Saponin, (see p. 448). (3) Resins, 2 per cent. (4) Tannic Acid.

Dose, 5 to $30 \mathrm{gr}$; ; 30 to $2.00 \mathrm{gm}$.

Action and Uses of Caulophyllum.

Caulophyllum is used to increase the force of uterine contractions ; it has been employed as a remedy for deficient labor-pains: and is believed to be useful in dysmenorrhœea.

\section{VIBURNUM.}

VIBURNUM PRUNIFOLIUM. - Synonym. - Black Haw. The bark of Viburnum prunifolium Linné (nat. ord. Caprifoliaced). Habitat.United States, westward to Kansas and Mississippi ; in thickets. 
Characters. - In thin pieces or quills, glossy purplish-brown, with scattered warts and minute black dots; when collected from old wood, grayishbrown; the thin, corky layer easily removed from the green layer; inner surface whitish, smooth; fracture short; inodorous, somewhat astringent and bitter.

Composition.-Its chief constituents are-(I) A brown, bitter Resin. (2) Viburnin, a greenish yellow, bitter principle.

Tannic Acid. (5) Oxalates, Citrates and Malates.

Dose, $1 / 4$ to I dr. ; I. to $4 . \mathrm{gm}$.

\section{Preparation.}

Extractum Viburni Prunifolii Fluidum.-Fluid Extract of Viburnum Prunifolium. By maceration and percolation with Alcohol and Water, and evaporation.

Dose, $1 / 4$ to $x$ fl. dr.; r. to 4 . c.c.

VIBURNUM OPULUS.-Synonyms.-Cramp Bark. High Bush Cranberry. The bark of Viburnum Opulus Linne (nat. ord. Caprifoliacea). Habitat.-North America, from New Brunswick westward, and southward to Pennsylvania, in low grounds.

Characters. - In flattish or curved bands, or occasionally in quills, sometimes $30 \mathrm{~cm}$. long, and from I to $1.5 \mathrm{~mm}$. thick, outer surface ash-gray, marked with scattered somewhat traversely elongated warts of a brownish color, due to abrasion, and more or less marked with blackish dots, and chiefly in a longitudinal direction with black, irregular lines or thin ridges; underneath the easily-removed corky layer of a pale brownish or somewhat reddishbrown color; the inner surface dingy white or brownish; fracture tough, the tissue separating in layers; inodorous; taste somewbat astringent and bitter.

Composition. - The same as of Viburnum Prunifolium.

Dose, $x$ to 2 dr.; 4. to 8. gm.

\section{Preparation.}

Extractum Viburni Opuli Fluidum.-Fluid Extract of Vibur. num Opulus. By maceration and percolation with Alcohol and Water, and evaporation.

Dose, I to 2 fl. dr.; 4. to 8. c.c.

\section{ACtion and Uses of Viburnum.}

Viburnum is believed to be an antispasmodic, diuretic and tonic. It is especially used in the nervous diseases of pregnancy and to prevent miscarriage. It has considerable reputation as a remedy for spasmodic dysmenorrhœa, in the treatment of after- 
pains, and in menorrhagia. This remedy has been in extensive use for more than twenty years and is undoubtedly of value.

\section{APIOLUM.}

A PIOL.-(Not official. $)-\mathrm{C}_{12} \mathrm{H}_{14} \mathrm{O}_{4}=22 \mathrm{I} .48$. A substance obtained from the fruit of Petroselinum salivum (nat. ord. Umbellifera). Synonym.Parsley. Habitat.- Southern Europe; cultivated.

SourCE. - The fruit is exhausted with Petroleum Benzin, the solvent evaporated, the residue treated with strong Alcohol, on the evaporation of which Apiol is left.

CHARACTERS. - A colorless liquid resembling a fixed oil, but not saponifiable, becoming turbid without congealing at $12^{\circ} \mathrm{C}$.; $10.4 \mathrm{~F}$., having an acid reaction, the odor of parsley, and a pungent taste. Sp. gr., 1.07o. Solubility. - Easily in Alcohol, Ether, Chloroform, and Glacial Acetic Acid.

Dose, 10 to $15 \mathrm{~m}$; .60 to 1.00 c.c., (in capsules.)

\section{Action and Uses of ApIol.}

Apiol in large doses acts as a cerebral and circulatory stimulant. It is useful in amenorrhœe, scanty menstruation and dysmenorrhœa when administered immediately before the expected period and these conditions are due to a want of ovarian activity; that is, where direct emmenagogues are required.]

\section{GROUP XIII.}

\section{Colchicum.}

[The sole value of this drug is that it is generally a specific for gout.]

\section{COLCHICUM.}

[COLCHICI RADIX.-Colchicum Root. The corm of Colchicum au. tumnale Linné (nat. ord. Liliacea). Synonym.-Meadow Saffron. Habitat. -Southern and Central Europe.

Characters. - About $25 \mathrm{~mm}$. long, ovoid, flattish and with a groove on one side ; externally brownish and wrinkled; internally white and solid; often in transverse slices, reniform in shape, and breaking with a short, mealy fracture; inodorous ; taste sweetish, bitter and somewhat acrid.]

Composition, - The chief constituents are-(I) Colchicine, $\left[\mathrm{C}_{23} \mathrm{H}_{25} \mathrm{NO}_{6}\right.$, about 0.5 per cent., the active principle; a bitter alkaloid, usually amorphous, but may be obtained in prismatic crystals, soluble in water and Alcohol, and Chloroform, ] but changed by most acids into Colchiceine, $\left[\mathrm{C}_{22} \mathrm{H}_{23} \mathrm{NO}_{6}\right.$. $]$ 
Veratrine (see p. 444), in traces combined with Gallic Acid. (3) Starch. (4) Sugar. (5) Gum.

InCOMPATIBLES.-All astringent preparations, tincture of iodine, and tincture of guaiacum.

Dose, 2 to $8 \mathrm{gr}$.; .12 to .50 gm.,] in powder.

\section{Preparations.}

[r. Extractum Colchici Radicis.-Extract of Colchicum Root. By maceration and percolation with Acetic Acid and Water, and evaporation.

Dose, $1 / 2$ to $2 \mathrm{gr}$; .03 to $.12 \mathrm{gm}$.

2. Extractum Colchici Radicis Fluidum.-Fluid Extract of Colchicum Root. By maceration and percolation with Alcohol and Water, and evaporation.

Dose, 2 to $8 \mathrm{~m}$; ; 12 to .50 c.c.

5. Vinum Colchici Radicis.-Wine of Colchicum Root. Colchicum Root, 400; by percolation with Alcohol, 150; and with White Wine to 1000 .

Dose, 5 to $15 \mathrm{~m} . ; .30$ to 1.00 c.c.]

COLCHICI SEMEN.-Colchicum. Seed. The seed of Colchicum autumnale [Linné (nat. ord. Liliacea).

Characters. - Subglobular, about $2 \mathrm{~mm}$. thick, very slightly pointed at the hilum; reddish brown, finely pitted, internally whitish; very hard and tough; inodorous; taste bitter and somewhat acrid.] Resembling Colchicum seed.-Black mustard seed (see p. 524.)

Composition. - The chief constituents are-(I). The same as of the corm [root], but the proportion of the active alkaloid Colchicine is [smaller (0.3 per cent.). (2) A fixed oil, 6 to 8 per cent.]

Dose, $x$ to $5 \mathrm{gr}$; ; [.06 to .30 $\mathrm{gm}$.]

\section{Preparations.}

[1. Extractum Colchici Seminis Fluidum.-Fluid Extract of Colchicum Seed. By inaceration and percolation with Alcohol and Water, and evaporation.

Dose, I to $5 \mathrm{~m}$; ; .06 to .30 c.c.

2. Tinctura Colchici Seminis.-Tincture of Colchicum Seed. Colchicum Seed, 150 ; by maceration and percolation with Alcohol and Water to 1000 .

Dose, ro to $30 \mathrm{~m}$.; .60 to 2.00 c.c.

3. Vinum Colchici Seminis.-Wine of Colchicum Seed. Colchicum Seed, 150; by maceration with Alcohol, 150; and with White Wine to 1000 .

Dose, 10 to $30 \mathrm{~m}$.; .60 to 2.00 c.c.] 


\section{Action of Colchicum.}

External.-When applied to the skin colchicum acts as an irritant, causing hyperæmia and smarting, and the dust inhaled gives rise to sneezing.

Internal.-Gastro-intestinal tract. - In moderate medicinal doses colchicum produces no effect on most persons beyond slightly increasing the secretion of bile, but with others it causes loss of appetite, and a little purging, nausea and colic. In larger doses it gives rise, in all persons, to great abdominal pain, vomiting and profuse diarrhœa with the passage of blood. It is in fact a powerful gastro-intestinal irritant. There is also great prostration, the pulse becomes small, rapid, and thready, the skin cold and bedewed with sweat, and the respiration slow; death is due to collapse. It is probable that these results are not, to any great extent, owing to the effect of colchicine on the heart or respiration, but that they are merely the consequence of the severe gastro-enteritis, which, it is well known, will cause fatal collapse. These effects are produced if colchicine is injected subcutaneously, a circumstance which shows that this alkaloid is an active principle of the drug, and that it is excreted into the intestine. It is a curious fact that after a certain point, increasing the quantity does not lead to an increase of the symptoms. In animals the action on the heart is not marked, but diarrhœa and vomiting are severe.

Nervous system. - Medicinal doses have no effect. Even a fatal dose does not impair consciousness. Cold-blooded animals bear much larger proportionate doses than warm, but in all, after large quantities, sensation is paralyzed, and ultimately the spinal motor centres are powerfully depressed, death taking place from respiratory paralysis. Colchicine is said to act on muscles like veratrine.

Kidney. - The most discordant statements have been made about the action of colchicum on the urine, but it has not been definitely shown that either the quantity or composition, even in the amount of uric acid, is altered. After death by poisoning, the alkaloid is found in the blood and in most of the organs of the body. 


\section{Therapeutics of Colchicum.}

Colchicum is hardly ever used except for gout. Given during the attack, it most markedly relieves the pain ; in smaller doses given between the attacks it diminishes their severity. It is often very useful for dyspepsia, eczema, headache, neuritis, conjunctivitis, bronchitis, and other conditions which, when occurring in those suffering from gout, are probably related to it. How it acts is not known. Occasionally it is combined with other cholagogues, especially if $i:$ is desired to give these remedies to a person who is the subject of gout. If any symptoms of gastric or intestinal irritation appear, its use must be discontinued for a time. As it is a cardiac depressant, those who take it should keep the bowels well open, lest it accumulate in the body. Hence it is commonly combined with magnesium sulphate. The seed is said to be [less] active than the corm. [A well-known formuld is : extract of colchicum root, calomel, powdered aloes, powdered ipecacuanha, of each I gr., $.06 \mathrm{gm}$., with $1 / 4$ to $1 / 2 \mathrm{gr}$. ; . OI 5 to .03 gm. of extract of nux vomica.]

\section{GROUP XIV.}

\section{The Stearoptens.}

The three bodies in this group are white solids, all very closely related to volatile oils, all are antiseptic (two very powerfully so) and two at least, and probably all three, are local anæesthetics.

\section{Camphor, Thymol, Menthol.}

\section{CAMPHORA.}

CAMPHOR.-[Synonyms. - Gum Camphor, Laurel Camphor, $\mathrm{C}_{10} \mathrm{H}_{16} \mathrm{O}$ $=151.66$. A stearopten (having the nature of a ketone) obtained from Cinnamomum Camphora) (Linné) Nees et Ebermaier (nat. ord. Laurinea), and purified by sublimation. Habitat.-China and Japan.

SOURCE. - The branches and chipped wood are exposed to the vapors of boiling water, the volatilized Camphor is condensed, drained and pressed from the adherent volatile oil (Oil of Camphor), and subsequently refined by sublimation in vessels of glass or iron. Camphor is now obtained by tapping the trees and collecting the exudation. 
Citaracters. - White, translucent masses, of a tough consistence and a crystalline structure, readily pulverizable in the presence of a little Alcohol, Ether, or Chloroform, having a penetrating, characteristic odor, and a pungently aromatic taste. Sp. gr., 0.995. Burns with a smoky flame. Volatilizes slowly at ordinary temperatures. Sublimes entirely when heated. Solubility. - Very sparingly soluble in water, but readily soluble in Alcohol, Ether, Chloroform, Carbon Disulphide, Benzin, and in fixed and volatile oils. When Camphor is triturated in about molecular proportions with Menthol, Thymol, Phenol, or Chloral hydrate, liquefaction ensues.]

Composition. - Camphor is an oxidation product of Pinene (see Oil of Turpentine, [p. 515]), and may also be derived from Cymene found in Oil of Caraway [see p. 55I] and Oil of Eucalyptus [see p. 527]. The [official] camphor is called Laurel Camphor, and is dextro-rotary. Borneol, known as Borneo, [Sumatra or Barus] Camphor, see p. 557,-is often in commerce substituted for the official camphor, which it closely resembles, is derived from Dryobalanops [Camphora], and known from the official variety by sinking in water-is $\mathrm{C}_{10} \mathrm{H}_{18} \mathrm{O}$; that is to say, an alcohol. The common form of Borneol is dextro-rotary, but levo-rotary and inactive varieties are known.

[Camphor is contained in Linimentum Belladonnæ, Linimentum Sinapis Compositum, Linimentum Saponis, Tinctura Opii Camphorata and Pulvis Morphine Compositus.]

Dose, 3 to $20 \mathrm{gr}$.; [.20 to $1.20 \mathrm{gm}$.]

\section{Preparations.}

1. [Aqua Camphoræ-Camphor Water. Camphor, 8 ; by trituration with Alcohol, 5 ; and precipitated Calcium Phosphate, 5 ; ad. dition of Water, and filtration to 1000 .

Dose, $1 / 2$ to 2 fl. oz.; 15 . to 60 . c.c.

2. Linimentum Camphoræ.-Camphor I.iniment. Synonym.Camphorated Oil. Camphor, 200 ; Cotton Seed Oil, 800.

3. Spiritus Camphoræ.-Spirit of Camphor. Campbor, 10; Alcohol to 1000 . By solution and filtration.

4. Ceratum Camphoræ.-Camphor Cerate. Camphor Liniment, 100 ; White Wax, 300 ; Lard, 600.

CAMPHORA MONOBROMATA.-Monobromated Camphor, $\mathrm{C}_{10}$ $\mathrm{H}_{15} \mathrm{BrO}=230.42$.

SourCE. - By heating Bromine and Camphor, at $172^{\circ} \mathrm{F}$; $77.7^{\circ} \mathrm{C}$., solution in Benzin, and re-crystallization from hot Alcohol. $\mathrm{C}_{10} \mathrm{H}_{16} \mathrm{O}+2 \mathrm{Br}=\mathrm{C}_{10}$ $\mathrm{H}_{15} \mathrm{BrO}+\mathrm{HBr}$.

Characters. - Colorless, prismatic needles or scales, having a mild, camphoraceous odor and taste, permanent in the air, unaffected by light, and 
neutral to litmus paper. Solubility._Almost insoluble in water; freely solu. ble in Alcohol, Ether, Chloroform, hot Benzin, and fixed and volatile oils; slightly soluble in Glycerin. It is also soluble without decomposition in cold, concentrated Sulphuric Acid, from which it separates again unaltered, when the solution is poured into water.

Dose, 2 to $10 \mathrm{gr}$; .12 to $.60 \mathrm{gm}$.

ACIDUM CAMPHORICUM. (Not official.)-Camphoric Acid. $\mathrm{C}_{10} \mathrm{H}_{16} \mathrm{O}_{4}=\mathbf{r} 99.44$.

SOURCE. - From Camphor by oxidation with Nitric Acid.

Characters. - White, acicular, odorless crystals having a faintly acid taste. Solubility.-Nearly insoluble in cold, readily soluble in hot water, Alcohol, Ether and oils.

Dose, 10 to $30 \mathrm{gr}$; ; .60 to $2.00 \mathrm{gm}$.]

\section{Action of Camphor.}

External.-Camphor, although not a volatile oil, acts very much like one. Thus it is a direct cutaneous stimulant, dilating the vessels of the skin, and at first causing a sensation of warmth, but subsequently a slight degree of local anæsthesia. It is a feeble antiseptic.

Internal.-Gastro-intestinal tract.-In the stomach it is mildly stimulant, dilating the vessels, increasing the flow of gastric juice and the peristalsis. Hence it is stomachic and carminative. It has a slight reflex stimulating effect on the heart. In medicinal doses it has little action on the intestines.

Absorption.-It is quickly absorbed, both from the intestines and the skin, and two bodies formed in the body from it are known. One, camphoral (one atom of $\mathrm{H}$ in camphor being replaced by $\mathrm{OH}$ ), combines with glycuronic acid and is excreted in the urine as campho-glycuronic acid. Another, an amido. derivative, is also found in the urine.

Circulation.-It increases the number of leucocytes in the blood. To a slight extent the heart is excited directly by it in addition to the reflex stimulation just mentioned. And so the pulse becomes fuller and stronger; the rate is not much affected. The face may be flushed.

Respiration.-Probably some camphor or some derivative from it is excreted by the bronchial mucous membrane, the vas- 
cularity and secretion of which it consequently stimulates. It has the reputation of being a feeble expectorant.

Skin.-It is a mild diaphoretic. This effect is believed to be due to the action of the drug on the central nervous system. Probably some of the camphor is excreted by the skin, for the sweat may smell of it.

Nervous system.-Different people are differently susceptible to the effects of camphor. Five to ten grains [.30 to .60 gm.] will in some persons produce a feeling of exhilaration, or in others a sense of comfort and quietness. Larger doses cause excitement, giddiness, a slow pulse, and ultimately headache, burning pain in the stomach, faintness, confusion of ideas, delirium, violent convulsions, insensibility, a small, feeble pulse, and finally death from collapse. It is a mild antipyretic.

Sexual organs. - Camphor is reputed to be an aphrodisiac, but this is probably incorrect.

\section{Therapeutics of Camphor.}

External.-Its stimulating effects make camphor a favorite ingredient of many liniments. It is constantly rubbed into the skin in some form or another as a mild irritant or counter-irritant in, for example, chronic rheumatism, chronic inflammatory indurations, and the slighter chest complaints of children; and also in myalgia, neuralgia, lumbago, and sciatica, in which cases, because of its property of causing local anæsthesia, it relieves pain. In addition to the pharmacopoial preparations, a Chloroformum Camphoræ (camphor, 2, dissolved in chloroform, I) may be used. The liquid preparations with chloral [hydrate,] carbolic acid, and thymol are excellent local anodynes for neuralgia, and may be dropped into a tooth to relieve toothache.

Internal.-Camphor is used as a carminative, especially in neurotic subjects. It is a common remedy for a cold in the head, and is probably beneficial on account of its stimulation of the circulation and its slight antipyretic and diaphoretic effects. Many expectorant mixtures contain camphor. It has been given as an antispasmodic in hysteria and allied conditions, and some state that it is of use in cholera.

[Monobromated Camphor re- 
sembles, but is not identical with, the bromides in its therapeutical action, being used as a nervous sedative. Camphoric acid is successfully administered for colliquative sweating, $e . g$., that of pulmonary tuberculosis. The daily amount of from ${ }^{2} 5$ to 75 gr.; I. to 5. gm., should be given in the evening in divided doses at short intervals, either dry upon the tongue or in starch wafers.]

\section{THYMOL.}

THYMOL. $-\mathrm{C}_{10} \mathrm{H}_{14} \mathrm{O}[=149.66 . \quad \Lambda$ phenol (classified in the B. $\mathrm{P}$. as a stearopten) occurring in the volatile oils of (1) Thymus vulgaris Linné, (2) Monarda punctata Linné (nat. ord. Labiatie), and (3) Carum Ajowan (Roxburgh) Bentham et Hooker (nat. ord. Umbellifera). Habitat.-(1) Southern Europe; cultivated. (2) United States, west to Texas and Colorado; in sandy fields. (3) India, Persia, Egypt.

SouRcE. - Thymol is separated from the terpenes in the volatile oils by fractional distillation, agitated with solution of Soda to remove more of the terpenes and cooled. The compound of Soda and Thymol is decomposed by Hydrochloric Acid, and Thymol is re-crystallized from an alcoholic solution.

CHARACTERS. - Large, colorless, translucent crystals of the hexagonal system, having an aromatic, thyme-like odor, and a pungent, aromatic taste, with a very slight caustic effect upon the lips. Sp. gr., as a solid, is $\mathbf{1} .069$, but when liquefied by fusion it is lighter than water. When triturated with about equal quantilies of Camphor, Menthol, or Chloral hydrate, it liquefies. Solubility.- Soluble in about 1200 parts of water, and in less than its own weight of Alcohol, Ether, or Chloroform ; also readily soluble in Carbon Disulphide, Glacial Acetic Acid, and in fixed or volatile oils.

IMPURITIES. - Paraffin, and spermaceti.

Dose, I to $3 \mathrm{gr}$; . 20 to $1.00 \mathrm{gm}$.]

\section{Action and Therapeutics of Thymol.}

Thymol is a more powerful antiseptic than carbolic acid, but its insolubility is a drawback. It has been used in antiseptic surgery. A saturated solution, thymol gauze, and thymol ointment are employed. It is non-irritating. It has considerable antiparasitic powers, and solutions in alcohol or ether ( $I$ in $I_{5}$ ) have been used in ringworm. A solution in glycerin ( $I$ in 200) has been recommended for sore throat. A little alcohol is very useful for facilitating the aqueous solution of thymol. [The odor is likely to attract house flies.] Thymol in $30 \mathrm{gr}$; [ [2. $\mathrm{gm}$.] doses has been given as an anthelmintic for the Anchylos- 
toma duodenale. After its administration the urine may become green.

\section{MENTHOL.}

MENTHOL. $-\mathrm{C}_{10} \mathrm{H}_{19} \mathrm{OH}[=155.66$. A stearopten (having the character of a secondary Alcohol), obtained from the official oil of peppermint (from Mentha piperita Smith), or from Japanese or Chinese oil of peppermint (from Mentha arvensis Linné, var. piperascens Holmes, and Mentha canadensis Linné, var. glabrata Holmes; nat. ord. Labiata).

Source.-By fractional distillation of the volatile oil and freezing the higher boiling product, and crystallization.

Characters. - Colorless, acicular or prismatic crystals, having a strong and pure odor of peppermint, and a warm, aromatic taste, followed by a sensation of cold, when air is drawn into the mouth. Solubility.-Slightly soluble in water, but imparts to the latter its odor and taste. It is freely soluble in Alcohol, Ether, Chloroform, Carbon Disulphide, or glacial Acetic Acid. When it is triturated with about an equal weight of Camphor, Thymol, or Chloral hydrate, the mixture becomes liquid.]

IMPUR ITIES. - Glass and magnesium sulphate.

Dose, $1 / 2$ to $2 \mathrm{gr}$. ; [.03 to $.12 \mathrm{gm}$.]

\section{Action and Therapeutics of Menthol.}

Menthol is chiefly employed externally, for it produces local anæsthesia, a feeling of coldness and numbness, and thereby alleviates the pain of neuralgia, especially if it involves a superficial nerve. It is very efficacious in some cases. The solid menthol, [in the form of a cone,] may be drawn [over] the skin, or an alcoholic solution may be painted on, or a plaster; [menthol, 3 ; yellow wax, 1 ; resin, 15 ; spread upon rubber cloth] may be applied. In very hot weather this may be too fluid, and then more wax should be added. A solution made by heat with oleic acid [ $\mathrm{I}$ to 2.4] is an excellent preparation, and a good liniment is formed of menthol, 3 ; chloroform, 4 ; olive oil, 9 . The local application often relieves itching. Menthol has been applied locally to carious teeth, and has been inhaled with advantage [for the relief of the symptom] asthma. For teeth it is best rubbed up with an equal part of [pure carbolic acid], camphor or chloral hydrate. The oily liquid formed in either case may be put in the tooth. For [the symptom] asthma it is readily volatilized by the addition of hot water. Solutions of it 
have been painted upon the throat in diphtheria. A pigment of $I$ to 4 in olive oil is employed for painting the larynx in tuberculous ulceration. [It has been used internally in small doses (10 $\mathrm{gr} . ; .006 \mathrm{gm}$.) to relieve nausea and vomiting.] It is a powerful antiseptic, and is excreted in the urine, rendering it aseptic and giving it a pleasant smell. Menthol should be preserved in closed tin boxes.

\section{GROUP XV.}

\section{Vegetable drugs acting by virtue of important acids they contain.}

Lemon juice (Citric Acid), Laurocerasus (Hydrocyanic Acid), Benzoin (Benzoic Acid), Chrysarobinum (Chrysophanic Acid), [Rhus Toxicodendron (Toxicodendric Acid). Wild Cherry] (see p. 462) and Bitter Almond (see p. 612), both of which yield Hydrocyanic-Acid, have already been considered.

\section{LEMON.}

LIMONIS CORTEX.-Lemon Peel. [The rind of the recent fruit of Citrus Límonum Risso (nat. ord. Rutacea). Habitat.-Northern India; cultivated in subtropical countries.

CilARACTERS. - In narrow, thin bands or in elliptical segments, with very little of a spongy, white, inner layer adhering to them; outer surface deep lemon-yellow, and ruggedly glandular; odor fragrant; taste aromatic and bitterish.]

Composition.-The chief constituents are-(1) The official oil, $\mathrm{C}_{10} \mathrm{H}_{16}$ (see below). (2) Hesperidin, $\left[\mathrm{C}_{22} \mathrm{H}_{26} \mathrm{O}_{12}\right.$, a bitter principle.

Lemon Peel is used in Spiritus Limonis.]

OLEUM LIMONIS.-Oil of Lemon. [A volatile oil obtained by expression from fresh Lemon Peel.

Ciraracters. - A pale yellow, limpid liquid, having the fragrant odor of lemon, and an aromatic, somewhat bitterish taste. Sp. gr., 0.858 to 0.859 . Solubility. - In three times its volume of Alcohol; also soluble, in all proportions, in absolute Alcolol, Carbon Disulphide, or glacial Acetic Acid.

Composition. - The chief constituents are-(I) A terpene called Citrene, or Limonene, $\mathrm{C}_{10} \mathrm{H}_{16}$, 90 per cent., strongly dextro-rotary. This is also found in Orange Peel and Oil of Caraway. (2) Geranial or Citral, $\left[\mathrm{C}_{10} \mathrm{H}_{26} \mathrm{O}\right.$, ] an aldehyde derived from Geraniol found in Oil of Rose (see p. 556). (3) Citronellal, an aldehyde of the alcohol, Citronellol. 
[Oil of Lemon is contained in Spiritus Ammonix A romaticus, and Spiritus Aurantii Compositus.

\section{Preparation.}

Spiritus Limonis.-Spirit of Lemon. Synonym.-Essence of Lemon. Oil of Lemon, 50 ; Lemon Peel, 50 ; by maceration and filtration with Deodorized Alcohol to rooo.

Spirit of Lemon is contained in Syrupus Acjdi Citrici.

Dose, $1 / 2$ to 2 fl. dr.; 2. to 8 . c.c.]

\section{Action and Therapeutics of Lemon.}

The same as those of orange. The oil applied externally is rubefacient.

LIMONIS SUCCUS.-Lemon Juice. The freshly expressed juice of the ripe fruit of Citrus Limonum [Risso (nat. ord. Rutacee).

CHARACTERS. - A slightly turbid, yellowish liquid, usually having an odor of lemon due to the accidental presence of some of the volatile oil of the rind. Taste acid, and often slightly bitter. Sp. gr., not less than 1.030.]

Composition.-Lemon juice contains-(I) Citric Acid $\left[\left(\mathrm{H}_{3} \mathrm{C}_{6} \mathrm{H}_{5} \mathrm{O}_{7}+\right.\right.$ $\mathrm{H}_{2} \mathrm{O}$ ), about 7 per cent., ] both free, and combined to form Potassium and other salts. (2) Malic Acid. (3) Phosphoric Acid.

Dose, $1 / 2$ to 2 fl. oz.; [15. to 6o. c.c.]

\section{Action and Therapeutics of Lemon Juice.}

Lemon juice is used to relieve thirst, and to make effervescing mixtures and drinks. Its action in the body is the same as that of citric acid (see p. 266). Three or four ounces [90. or I 20. c.c.] of lemon juice daily are of great benefit in scurvy. Why this is we do not certainly know. Lemon juice is probably more efficacious than citric acid.

\section{LAUROCERASUS.}

CHERRY LAUREL. [B. P., not official.]-The fresh leaves of Prunus laurocerasus (nat. ord. Rosacee). [Habitat.-Europe.]

Characters. - Thick coriaceous, on short, strong petioles. Oblong or ovate, [ 12 to $16 \mathrm{~cm}$.] long, tapering towards each end, recurved at the apex, distinctly but sharply serrated, dark-green, smooth and shining above, pale beneath. Prominent midrib with, on either side of it at the base, one or two granular depressions. Inodorous, except on bruising, when they emit a ratafialike odor. 
Composition.-The chief constituents are-(1) Laurocerusin, a glucoside; it is a compound of Amygdalin and Amygdalic Acid. (2) Emulsin. By the same changes as in the case with Bitter Almond (see p. 6r2), in the presence of moisture, an oil, [Hydrocyanic] Acid and Glucose are formed.

\section{Preparation.}

Aqua Laurocerasi. [B. P., not official.—Cherry Laurel Water.] Made by distillation and standardized so that its strength is 0 . I per cent. of absolute Hydrocyanic Acid.

InCOMPATIBLES. - Metallic salts.

Dose, $1 / 2$ to 2 fi. dr.; [2. to 8 . c.c.]

\section{Action and Therapeutics of Cherry Laurel.}

Its action is the same as that of diluted hydrocyanic acid (see p. I34). Aqua laurocerasi is not often employed; for owing to the volatilization of the [hydrocyanic] acid, its strength is not constant. It is used as a flavoring agent.

\section{BENZOINUM.}

BENZOIN.-Synonym.- (ium Benjamin. A balsamic resin obtained from Styrax Benzoin [Dryander (nat. ord. Styracere). Habitat.-Sumatra, Java, probably also Siam.

ChARACTERS. - In lumps consisting of agglutinated, yellowish-brown tears, which are internally milk-white, or in the form of a reddish-brown mass, more or less mottled from whitish tears imbedded in it. It is almost wholly soluble in 5 parts of moderately warm Alcohol, and in solutions of the fixed alkalies. When heated, it gives off fumes of Benzoic Acid. It has an agreeable, balsamic odor, and a slight, aromatic taste. Solubility. - In 5 parts of Alcohol]; easily in Ether [or Solution of] Potassa.

Composition. - The chief constituents are-(1) Benzoic Acid (see p. 657), 12 to 20 per cent. (2) Cinnamic Acid, $\mathrm{C}_{9} \mathrm{H}_{8} \mathrm{O}_{2}$, a trace. (3) Resin. (4) Volatile Oil.

\section{Preparations.}

1. Adeps [Benzoinatus.-Benzoinated Lard. Benzoin, 20; Lard, 1000; by melting and straining.

2. Tinctura Benzoini.-Tincture of Benzoin. Benzoin, 200; by maceration and filtration with Alcohol to 1000 .

Dose, $3 / 2$ to I fi. dr.; 2. to 4 . c.c.

3. Tinctura Benzoini Composita. - Compound Tincture of Benzoin. Synonym.-Friar's lBalsam. Benzoin, 120; Storax, 80; 
Balsam of Tolu, 40; Purified Aloes, 20; by digestion with Alcohol, and filtration to 1000 .

Dose, $1 / 2$ to I fl. dr.; 2. to 4 . c.c.]

ACIDUM BENZOICUM.-Benzoic Acid. $\mathrm{HC}_{7} \mathrm{~N}_{5} \mathrm{O}_{2}[=121.7 \mathrm{I}]$.

SOURCE. - From Benzoin by sublimation. [Toluene (toluol) is generally the source of this substance.

Characters. - White, or yellowish-white, lustrous scales or friable needles, odorless, or having a slight, characteristic odor resembling that of Benzoin, and of a warm, acid taste; somewhat volatile at a moderately warm temperature, and rendered darker by exposure to light. Solubility. - When pure, in about 500 parts of water, and in 2 parts of Alcohol. Also soluble in 3 parts of Ether, 7 parts of Chloroform, and readily soluble in Carbon Disulphide, Benzol, fixed and volatile oils, but sparingly soluble in Benzin.

Benzoic Acid is contained in Tinctura Opii Camphorata.

Dose, 5 to $15 \mathrm{gr}$; .30 to $\mathrm{r} .00 \mathrm{gm}$.]

AMMONII BENZOAS. - Ammonium Benzoate. $\mathrm{NH}_{4} \mathrm{C}_{7} \mathrm{H}_{5} \mathrm{O}_{2}[=$ 138.72.

SOURCE. -Dissolve Benzoic Acid in Water of Ammonia and distilled water, and evaporate, set aside to crystallize. $\mathrm{HC}_{7} \mathrm{H}_{5} \mathrm{O}_{2}+\mathrm{NH}_{4} \mathrm{OH}=\mathrm{NH}_{4}$ $\mathrm{C}_{7} \mathrm{H}_{5} \mathrm{O}_{2}+\mathrm{H}_{2} \mathrm{O}$.

ChARACTERs. - Thin, white, four-sided, laminar crystals, odorless or having a slight odor of Benzoic Acid, a saline, bitter, afterwards slightly acrid taste, and gradually losing Ammonia on exposure to the air. Solubility._-In 5 parts of water, and in 28 parts of Alcohol.]

I NCOMPa'tibles. - Ferric salts, Liquor Potassæ, and acids.

Dose, 5 to $20 \mathrm{gr}$; ; [.30 to $1.20 \mathrm{gm}$.]

SODII BENZOAS.-[Sodium Benzoate. $\mathrm{NaC}_{7} \mathrm{H}_{5} \mathrm{O}_{2}=143.71$.

SourCE.-Benzoic Acid is added to a hot concentrated solution of pure Sodium Carbonate, the solution is evaporated, cooled and allowed to crystallize. $2 \mathrm{HC}_{7} \mathrm{H}_{5} \mathrm{O}_{2}+\mathrm{Na}_{2} \mathrm{CO}_{3}=2 \mathrm{NaC}_{7} \mathrm{H}_{5} \mathrm{O}_{2}+\mathrm{CO}_{2}+\mathrm{H}_{2} \mathrm{O}$.

ChARACTERS. - A white, amorphous powder, odorless, or having a faint odor of Benzoin, and a sweetish, astringent taste; permanent in the air. Solubility. -In 1.8 parts of water, and in 45 parts of Alcohol.]

Dose, 5 to $60 \mathrm{gr}$. ; [.30 to $4 . \mathrm{gm}$. $]$

[LITHII BENZOAS.-Lithiuin Benzoate. $\mathrm{LiC}_{7} \mathrm{H}_{5} \mathrm{O}_{2}=\mathbf{1 2 7 . 7 2}$.

SoURCE. - By decomposing Lithium Carbonate with Benzoic Acid. $\mathrm{Li}_{2} \mathrm{CO}_{3}+2 \mathrm{HC}_{7} \mathrm{H}_{5} \mathrm{O}_{2}=2 \mathrm{LiC}_{7} \mathrm{H}_{5} \mathrm{O}_{2}+\mathrm{CO}_{2}+\mathrm{H}_{2} \mathrm{O}$.

Characters. - A light, white powder, or small, shining, crystalline scales ; odorless, or of faint benzoin-like odor, and of a cooling, sweetish taste; permanent in the air. Solubility. - In 4 parts of water, and in 12 parts of Alcohol.

Dose, 5 to $20 \mathrm{gr}$; ; 30 to $1.20 \mathrm{gm}$.] 


\section{Action of Benzoic Acid.}

So far as is known, the action of benzoic acid, its salts, and benzoin, is the same. We shall therefore here describe only the action of benzoic acid.

External.-Benzoic acid is a powerful antiseptic. The growth of many forms of bacteria is completely inhibited by a solution of $I$ in 1000 . In a concentrated form it is a stimulant and irritant when applied to the skin.

Internal.- The chief fact about the internal action of benzoic acid that has been worked out is that when it is given by the mouth, hippuric acid appears in the urine. This happens by combination with a molecule of glycocoll, $\mathrm{HC}_{7} \mathrm{H}_{5} \mathrm{O}_{2}+\mathrm{C}_{2} \mathrm{H}_{3}$ $\mathrm{NO}_{2}=\mathrm{C}_{9} \mathrm{H}_{9} \mathrm{NO}_{3}$ (hippuric acid) $+\mathrm{H}_{2} \mathrm{O}$. . The source of the glycocoll is not known. The conversion takes place in the kidneys, for after giving large doses of benzoic acid, it alone can be found in the blood, and if the renal arteries are tied, no hippuric acid is formed, but if only the ureters are tied it is formed. Also benzoic acid has been successfully converted into hippuric acid by passing blood, containing benzoic acid, but no glycocoll, slowly through the kidneys removed directly after death. Further researches show that the conversion is probably effected by the renal cells. Hippuric acid has been found in the urine of new-born children when benzoic acid has been given to the mother shortly before delivery. If hippuric acid is given by the mouth benzoic acid is found in the blood, but hippuric [acid] reappears in the urine. The hippuric acid in the urine renders alkaline urine acid, and it stimulates and disinfects the urinary mucous membrane. Occasionally succinic as well as hippuric acid appears in the urine.

Benzoic acid or some derivative of it is probably excrèted in the bronchial secretion, for the bronchial mucous membrane is stimulated by the administration of benzoic acid; the mucus being increased in quantity and disinfected. The acid is therefore expectorant. The same effects are brought about if the vapor of benzoic acid is inhaled. It is said also to be excreted by the skin and salivary glands, and thereby to increase their activity. It is slightly diuretic. Medicinal doses do not pro- 
duce any effect on the stomach, intestines, circulation, or nervous system.

Benzoic acid and its salts are antipyretic, and it is stated that they are even more powerful than salicylic acid. How they produce a fall of temperature is not known. Metabolism is believed to be generally increased. Sodium and ammonium benzoate increase both the quantity and the solids of the bile.

\section{Therapeutics of Benzoic Acid.}

External.-Lint soaked in the compound tincture is a favorite dressing for wounds and sores of all sorts. Its chief advantage is the antiseptic power it possesses. Its stimulating effect is also valuable. Benzoinated lard is a common basis for ointments when it is wished that the active ingredient should be absorbed, for the lard melts on the body, especially if covered with a bandage ; the benzoin prevents the decomposition of the lard. If the benzoin irritates, which it is likely to do if near the eye, 3 parts of oil of cloves or 2 of oil of gaultheria to [480 parts] of lard makes a non-irritant basis which keeps indefinitely.

Internal.-Lungs. - Benzoin, benzoic acid and its compounds are very commonly employed as stimulating, disinfecting expectorants in cases of bronchitis or phthisis in which the expectoration is foul and scanty. The vapor from a mixture of a pint, $[500$ c.c. $]$ of water at $140^{\circ} \mathrm{F} .,\left[60^{\circ} \mathrm{C}\right.$. $]$ and a fluid drachm, [4. c.c.] of compound tincture of benzoin is often inhaled for bronchitis and laryngitis.

Urinary organs.-Benzoic acid is a most valuable drug for acidifying the alkaline decomposing urine which is formed in pyelitis and cystitis, and for stimulating and disinfecting the urinary tract in the same conditions. Ammonium benzoate is so much more soluble than benzoic acid that it is to be preferred to it. Spirit of chloroform covers the taste. It may with advantage be combined with the urinary sedatives, as tincture of hyoscyamus. The conversion to hippuric acid has been said not to take place when the kidney is diseased.

Benzoic acid has been used in Germany instead of salicylic acid for rheumatic fever. 


\section{CHRYSAROBINUM.}

CHRYSAROBIN. - [A neutral principle, in its commercial, more or less impure form, extracted from Goa or Araroba Powder, a substance found deposited in the wood of Andira Araroba Aguiar (nat. ord. Leguminosa). Habitat.-Brazil.

Characters. - A pale, orange-yellow, microcrystalline powder, odorless and tasteless; turning brownish-yellow on exposure to air. Solubility.-Very slightly soluble in cold water or Alcohol; soluble in 33 parts of boiling Benzol, in Chloroform, and in solutions of the alkalies.

Composition.- The chief constituent of Goa powder is Chrysarobin, $\mathrm{C}_{27} \mathrm{H}_{30} \mathrm{O}_{14}$. $]$ Synonyms. - Rhein. Chrysophan (see p. 490). In the fresh plant it probably exists as a Glucoside, but this is slowly oxidized into Chrysophanic Acid, $\mathrm{C}_{15} \mathrm{H}_{10} \mathrm{O}_{4}$, and glucose.

\section{Preparation.}

Unguentum Chrysarobini.-[Chrysarobin Ointment. Chrysarobin, 5 ; Benzoinated Lard, 95.]

\section{Action of Chrysarobin.}

External.-It is a powerful irritant to the skin, which it stains yellowish brown. Linen is stained the same color. (The stain may be removed by a weak solution of caustic soda or chlorinated lime.) It is antiparasitic.

Internal.-It is cathartic and very irritating to the stomach and bowels, causing vomiting and purging. It is excreted by the kidneys, and stains the urine yellow.

\section{Therapeutics of Chrysarobin.}

It is used as an antiparasitic in ringworm, and to excite healthy inflammation in chronic cutaneous diseases, especially psoriasis and acne rosacea. A pigment (chrysarobin, I; solution of gutta percha, 9 [solution of gutta percha is made by decantation of gutta percha, $\mathrm{x}$; lead carbonate, $\mathrm{x}$; chloroform, 9 ;]) is more cleanly than the ointment and does not stain the clothes. Chrysarobin has also been given internally for skin diseases, but as it is so irritating this practice is not advisable.

\section{[RHUS TOXICODENDRON.}

RHUS TOXICODENDRON.-Synonyms.-Poison Ivy. Poison Oak. The fresh leaves of Rhus radicans Linné (nat. ord. Anacardiea). Habitat. - North America, west to Wyoming and Texas; in thickets. 
Characters. - Long-petiolate, trifoliolate; the lateral leaflets sessile or nearly so, about $10 \mathrm{~cm}$. long, obliqueiy ovate, pointed; the terminal leaflets stalked, ovate or oval, pointed, with a wedge-shaped or rounded base; the leaflets entire and glabrous, or variously notched, coarsely toothed, or lobed, more or less downy; when dry, papery and brittle ; inodorous ; taste somewhat astringent and acrid. Resembling Rhus Toxicodendron.-The leaves of Ptelea trifoliata, which are similar in appearance, but have all the leaflets sessile.

Composition. - The chief constituents are-(I) Toxicodendric Acid, a volatile Acid. (2) Tannic Acid. (3) Fixed Oil.

Dose, 1 to $5 \mathrm{gr}$; .06 to $.30 \mathrm{gm}$.

\title{
Action and Uses of Rhus Toxicodendron.
}

The tincture of the fresh leaves (dose, $\frac{1}{10}$ to $2 \mathrm{~m} . ; .006$ to . 2 c.c.) has been used in paralysis, nocturnal incontinence of urine and cutaneous diseases; but the remedy is dangerous and probably, an useless one, for these purposes. Largely diluted it has been used as a lotion for bruises and burns.]

\section{GROUP XVI.}

\section{[Vegetable drugs only used as Flavoring Agents.}

\author{
Balm, Raspberry, Vanilla, Sage, Oil of Bay, Oil of Sesamum.]
}

\section{[MELISSA.}

MELISSA.-Synonym.-Balm. The leaves and tops of Melissa officinalis Linné (nat. ord. Labiatue). Habitat.-Asia Minor, Southern Europe; naturalized in the United States; cultivated.

Characters. - Leaves long, petiolate, ovate, obtuse rounded or subcordate at the base, crenate, somewhat hairy, glandular ; branches quadrangular; flowers in about four-flowered cymules, with a tubular, bell-shaped, fivetoothed calyx, a whitish or purplish bilabiate corolla, and four stamens; fragrant, aromatic ; somewhat astringent and bitterish.

Composition.-(I) Volatile Oil, $1 / 4$ per cent. (.3) Tannic Acid. Bitter principle.

Dose, I to 2 dr.; 4. to 8 . gm.

\section{USES OF BALM.}

Balm is used as a flavoring agent. 


\section{RUBUS IDEUS.}

RASPBERRY.-The fruit of Rubus idaus Linné (nat. ord. Rosacece). Habitat._Europe and Asia; cultivated.

Characters. - Deprived of the conical receptacle, and therefore hollow at the base; hemispherical, red, finely hairy, composed of from twenty to thirty coalesced, small drupes, each one crowned with the withered style; juice red; of an agreeable odor, and a pleasant, acidulous taste.

Composition.-(I) Volatile oil, a trace. (2) Citric and Malic Acids. (3) Sugar, about 5 per cent. (4) Pectin.

\section{Preparation.}

Syrupus Rubi Idæi.-Syrup of Raspberry. Fresh ripe raspberries (filtered juice), $4^{\circ}$; Sugar, 60.

Dose, indefinite.

\section{USES OF RASPBERRY.}

Raspberry syrup is used chiefly as a flavoring agent.

\section{VANILLA.}

VANILLA.-The fruit of Vanilla planifolia Andrews (nat. ord. Or(hidea). Habitat.-Eastern Mexico, in hot, damp woods; cultivated in the tropics.

CHARÁCTERS.-From I 5 to $25 \mathrm{~cm}$. long, and about $8 \mathrm{~mm}$. thick, linear, narrowed and bent or hooked at the base, rather oblique at the apex, wrinkled, somewhat warty, dark brown, glossy-leathery, one-celled, and containing a blackish-brown pulp, with numerous, minute seeds, and more or less acicular crystals; odor and taste peculiar, fragrant.

Composition.-Its chief constituents are-Vanillin, $\mathrm{C}_{8} \mathrm{H}_{8} \mathrm{O}_{3}$, a crystakline principle, 1.7 to 2.75 per cent., which develops upon the ripening of the pod. (2) Fixed Oil, I I per cent. (3) Resin. (4) Sugar. (5) Mucilage. Vanillin is the Aldehyde of Methylprotocatechuic Acid; and can be prepared artificially from Coniferin, Carbolic Acid, Guaiacol and Eugenol, $\mathrm{C}_{10} \mathrm{H}_{12} \mathrm{O}_{2}$, the last being found in Oil of Cloves (see p. 534).

\section{Preparation.}

Tinctura Vanillæ.-Tincture of Vanilla. Vanilla, 100; Sugar, 200 ; by maceration with Alcohol and Water to 1000.

Dose, as flavoring agent.

\section{USES OF VANilla.}

Vanilla is used solely as a flavoring agent. 


\section{SALVIA.}

SALVIA.-Synonym.-Sage. The leaves of Salvia officinalis Linné (nat. ord. Labiatre). Habitat.-Southern Europe; cultivated.

Characters.-About $5 \mathrm{~cm}$. long, petiolate, ovate-oblong, obtuse or subacute at the apex, rounded or somewhat beart-shaped at the base, finely crenulate, thickish, wrinkled, grayish.green, soft-hairy and glandular beneath; odor aromatic ; taste aromatic, bitterish, and somewhat astringent.

Composition.- It contains-(I) A volatile oil, containing Salviol, $\mathrm{C}_{10}$ $\mathrm{H}_{18} \mathrm{O}$, Cineol and Pinene. (2) Resin. (3) Tannic acid.

Dose, $1 / 4$ to I dr. ; I. to $4 . \mathrm{gm}$.

\section{Action and Uses of Sage.}

It is used chiefly as a condiment, and it is said to be beneficial in checking the perspiration of hectic fever.

\section{OLEUM MYRCIE.}

OIL OF MYRCIA.-Synonym.-Oil of Bay. A volatile oil distilled from the leaves of Myrcia acris De Candolle (nat. ord. Myrtacea). Habitat. -West Indies.

ChARACTERS. - A yellow or brownish-yellow liquid, having an aromatic, somewhat clove-like odor, and a pungent, spicy taste. Sp. gr., 0.975 to 0.990. Solubility. - With an equal amount of Alcohol, glacial Acetic Acid, or Carbon Disulphide, it yields slightly turbid solutions.

\section{Preparation.}

Spiritus Myrciæ.--Spirit of Myrcia. Synonym.-Bay Rum. Oil of Myrcia, 16; Oil of Orange Peel, I ; Oil of Pimenta, I ; Alcohol, I220; Water to 2000.

\section{UsES OF MYRCIA.}

Oil of myrcia is used solely as a perfume. Bay rum is used as a refrigerant lotion.

\section{OLEUM SESAMI.}

OIL OF SESAMUM.-Synonyms.--Sesame Oil. Teel Oil. Benne Oil. A fixed oil expressed from the seed of Sesamum indicum Linne (nat. ord. Pedaliaceat). Habitat.-India; cultivated.

Characters. - A yellowish or yellow, oily liquid, inodorous or nearly so, and having a bland, nut-like taste. Sp. gr., 0.919 to 0.923 .

\section{Uses of Oil of Sesamum.}

Benne oil is used in preparing hair oil.] 


\title{
GROUP XVII.
}

\section{Vegetable drugs used only as coloring agents.}

\author{
Saffron, Red Saunders.
}

CROCUS.

SAFFRON.--[The stigmas of Crocus sativus Linné (nat. ord. Iridea). Habitat.-Western Asia ; cultivated in Spain and France.

Characters. - Separate stigmas, or three, attached to the top of the style, about $3 \mathrm{~cm}$. long, flattish-tubular, almost thread-like, broader and notched above ; orange-brown; odor strong, peculiar, aromatic; taste bitterish and aromatic.]

Composition.-The chief constituents are-(1) Polychroite, $\left[\mathrm{C}_{44} \mathrm{H}_{70} \mathrm{O}_{28}\right.$, an amorphous, brown-yellow Glucoside, soluble in Alcohol and water, splitting into Sugar (Crocose), and red Crocetin, (formerly called Crocin), $\mathrm{C}_{34} \mathrm{H}_{46} \mathrm{O}_{9}$, soluble in Ether and Alcohol. (2) Picrocrocin, $\mathrm{C}_{38} \mathrm{H}_{66} \mathrm{O}_{17}$, in colorless, bitter needles, readily soluble in Alcohol and water. (3) A volatile oil, $\mathrm{C}_{10} \mathrm{H}_{16}, \mathrm{I}$ per cent. (4) Fixed oil.]

IMPURITIES.-Marigold, saffron petals, chalk, and oil.

Dose, 5 to $30 \mathrm{gr}$. ; [.30 to $2.00 \mathrm{gm}$.]

\section{Preparation.}

Tinctura Croci.-[Tincture of Saffron. Saffron, I00; by maceration and percolation with Diluted Alcohol to 1000 .

Dose, 1 to 2 fl. dr. ; 4. to 8. c.c.]

\section{USES OF SAFFRON.}

Saffron is only used to color [pharmaceutical] preparations, but it is expensive.

\section{SANTALUM RUBRUM.}

[RED SAUNDERS. - The wood of Pterocarpus santalinus Iinne filius (nat. ord. Leguminosa). Habitat.-Madras; cultivated.

ChARACTERS. - A hard, heavy, dark reddish-brown, coarsely splintery wood, deprived of the light-colored sap-wood; usually met with in chips, or as a coarse, irregular, brownish-red powder, nearly inodorous and nearly tasteless.] Resembling Red Saunders. - I ogwood, which is less dense.

Composition. - The chief constituents are- $\left[(\mathrm{I})\right.$ Santalin, $\mathrm{C}_{15} \mathrm{H}_{14} \mathrm{O}_{5}$, in red needles. (2) Santal, $\mathrm{C}_{8} \mathrm{H}_{6} \mathrm{O}_{3}$, in colorless scales. (3) Pterocarpin, $\mathrm{C}_{20} \mathrm{H}_{16} \mathrm{O}_{6}$. (4) Homopterocarpin, $\mathrm{C}_{24} \mathrm{H}_{24} \mathrm{O}_{6}$, in colorless crystals. ]

Red Saunders is contained in Tinctura Lavandulæ Composita.

\section{Uses of Red Saunders.}

Red Saunders is used to color preparations, [but is of no value medicinally.] 


\section{GROUP XVIII.}

\section{Vegetable substances whose action is mechanical.}

Cotton, [Pyroxylin, Cotton Seed Oil], Oil of Theobroma, [Mastic], India-rubber, Starch, Lycopodium, Quillaja.

\section{GOSSYPIUM [PURIFICATUM.}

PURIFIED COTTON.-Synonym.-Absorbent Cotton. The hairs of the seed of Gossypium herbaceum Linné, and of other species of Gossypium (nat. ord. Malvacece), freed from adhering impurities, and deprived of fatty matter. Habitat.-Tropical Asia and Africa; cultivated in tropical and subtropical countries.

Characters. - White, soft, fine filaments, appearing under the microscope as hollow, flattened and twisted bands, spirally striate, and slightly thickened at the edges; inodorous and tasteless; insoluble in ordinary solvents, but soluble in Copper Ammonium Sulphate solution.]

PYROXYLINUM.-Pyroxylin. Synonyms.-Gun Cotton. [Soluble Gun Cotton. Calloxylin. Purified Cotton, IOO; is immersed in a mixture of Sulphuric, 2200 ; and Nitric Acids, 1400 ; washed with a large quantity of Water, drained and dried.

\section{Preparations.}

r. Collodium.-Collodion. Pyroxylin, 30; dissolved in Ether, $75^{\circ}$; and Alcohol, 250.

2. Collodium Flexile.-Flexible Collodion. Collodion, 9zo; Canada Turpentine, 50 ; Castor Oil, 3 .

3. Collodium Cantharidatum.-Cantharidal Collodion. Synonym.-Blistering Collodion. Cantharides, 60 ; by percolation with Chloroform, evaporation and solution of residue in Flexible Collodium, 85.

4. Collodium Stypticum.-See Tannic Acid, p. 593.]

\section{Action and Therapeutics.}

The uses of cotton are well known. Cotton, lint and gauze are frequently medicated, e.g., Sal Alembroth, 2 per cent.; Boric Acid, 5 or so per cent.; Salicylic Acid, 5 per cent.; Carbolic Acid, 5 per cent. ; Iodoform, 5, 10 and 50 per cent.

Pyroxylin is only used to make collodion. Collodion, when painted on the skin, rapidly dries from evaporation of the ether, 
and covers the skin with a thin protective film. Flexible collodion has the same properties, but it does not crack, as collodion often does. These preparations are protective to small wounds, and are used after slight operations. If the end of the urethra or prepuce is closed at night with collodion, nocturnal incontinence may sometimes be cured.

\section{[OLEUM GOSSYPII SEMINIS.}

COTTON SEED OIL.-A fixed oil expressed from the seed of Gossypium herbaceum Linné, and other species of Gossypium (nat. ord. Malvacea), and subsequently purified. Habitat.-Asia and Africa ; cultivated.

Characters. - A pale yellow, oily liquid, without odor, and baving a bland, nut-like taste and neutral reaction. Sp. gr., 0.920 to 0.930. Solubility.-Slighly soluble in Alcohol, but readily soluble in Ether, Chloroform, or Carbon Disulphide.

Composition.-(1) Olein. (2) Palmitin. (3) Coloring matter.

Cotton Seed Oil is used in Linimentum Ammonix and Linimentum Camphoræ.

\section{Action and Uses of Cotton Seed Oil.}

This is used simply as a bland, nutritious oil, and in liniments.]

\section{OLEUM THEOBROMATIS.}

OIL OF THEOBROMA.-Synonym.-Cacao Butter. [A fixed oil expressed from the seed of Theobroma Cacao Linné (nat. ord. Sterculiacea). Habitat.-South America.

Characters. - A yellowish-white solid, having a faint, agreeable odor, and a bland, chocolate-like taste. Sp. gr., 0.970 to 0.980 . Solubility.Readily in Ether or Chloroform; also soluble in 100 parts of Alcohol.

Composition.-The chief constituents are-(1) Stearin. (2) Olein. (3) Theobromine, an alkaloid, $\mathrm{C}_{7} \mathrm{H}_{8} \mathrm{~N}_{4} \mathrm{O}_{2}$. (4) Formic, Acetic and Butyric Acid Glycerides. ]

\section{Uses OF OiL of Tileobroma.}

Oil of theobroma is used to make suppositories, [and as a source of stearic acid. It is also used by inunction to improve the nutrition of the body.]

\section{[MASTICHE.}

MASTIC.-A concrete resinous exudation from Pistacia Lentiscus Linné (nat. ord. Anacardiea). Habitat.-Mediterranean basin.

Criaracters.-Globular or elongated tears, of about the size of a pea, sometimes covered with a whitish dust, pale yellow, transparent, having a 
glass-like lustre and an opalescent refraction; brittle; becoming plastic when chewed; of a weak, somewhat balsamic, resinous odor, and a mild terebinthinate taste. Solubility. - Completely in Ether, and, for the most part, soluble in Alcohol. Resembling Mastic.-Acacia, which is larger, rougher, and more opaque.

Composition. - The chief constituents are-(1) A resin, $\mathrm{C}_{20} \mathrm{H}_{32} \mathrm{O}_{2}$, Mastichic Acid, 90 per cent.; soluble in Alcohol. (2) Masticin, a resin, insoluble in Alcohol. (3) Volatile oil, $\mathrm{C}_{10} \mathrm{H}_{16}, \mathrm{I}$ to 2 per cent.

IMPURITY.-Sandarac.

\section{Preparation.}

Pilulæ Aloes et Mastiches.-See Aloes, p. 498.

\section{Action and Uses of Mastic.}

Mastic is a mild stimulant, mostly used as a masticatory, for filling decayed teeth, and for cements and varnishes.

\section{ELASTICA.}

INDIA-RUBBER.-Synonym.-Caoutchouc. The prepared milkjuice of various species of Hevea (nat. ord. Euphorbiacea), known in commerce as Para Rubber. Habitat.-In tropical countries.

ChARACTERS. - In cakes, balls, or hollow, bottle-shaped pieces, externally brown to brownish-black, internally brownish or of lighter tint ; very elastic. Solubility. - Insoluble in water, diluted acids, or diluted solutions of alkalies; soluble in Chloroform, Carbon Disulphide, Oil of Turpentine, Benzin, and Benzol. When heated to about $257^{\circ} \mathrm{F}$; $125^{\circ} \mathrm{C}$., it melts, remaining soft and adbesive after cooling. Odor faint, peculiar; nearly tasteless.

Composition.-(I) A solid Hydrocarbon, $\mathrm{C}_{20} \mathrm{H}_{32}$, (2) Fat. (3) Volatile oil. (4) Coloring matters. On combining it with ro per cent. of Sulphur, Vulcanized Rubber is obtained; with 50 per cent., and hardening by pressure Vulcanite or Ebonite is produced.

\section{USES OF INDIA-RUBbER.}

India-rubber is used for making plasters, bougies, pessaries, and syringes.]

\section{AMYLUM.}

STARCH.-[The fecula of the seed of Zea Mays Linné (nat. ord. Graminea). Habitat.-Tropical Asia and Africa; cultivated in tropical and subtropical countries.

CHARACTERS. - In irregular, angular masses, which are easily reduced to a fine powder ; white, inodorous, and tasteless; insoluble in Ether, Alcohol, or cold water. Under the microscope appearing as granules, nearly uniform 
in size, more or less angular in outline, with indistinct strix and with a distinct hilum near the centre.

Composition. - Its ultimate composition is $\mathrm{C}_{6} \mathrm{H}_{10} \mathrm{O}_{5}$, but it consists of a mixture of various modifications of Starch-cellulose and Starch-granulose.]

\section{Preparation.}

[Glyceritum Amyli._See Glycerin, p. 6og.]

\section{Action and Therapeutics of Starch.}

Starch is chiefly employed for its mechanical properties, on account of which it is used as a basis for dusting powders and insufflations. The glycerite is a basis for suppositories. The mucilage ( $I$ to 40 of water, gradually added and then boiled and stirred for a few minutes) is a basis for ointments; and may be used to suspend insoluble powders or oils; it is very [convenient] as a basis for enemata, but does not keep well and is therefore not suitable as a vehicle for a mixture.

\section{LYCOPODIUM.}

LYCOPODIUM.-[Synonyms. - Vegetable Sulphur. Club Moss. The spores of Lycopodium clavatum Linné, and of other species of Lycopodium (nat. ord. Lycopodiacea). Habitat.-Europe, Asia, and North America, in dry woods.

Characters. - A fine powder, pale yellowish, very mobile, inodorous, tasteless, floating upon water and not wetted by it, but sinking on being boiled with it, and burning quickly when thrown into a flame. Under the microscope the spores are seen to be sphæro-tetrahedral, the surfaces marked with recticulated ridges, and the edges beset with short projections.

Composition.-(1) Fixed oil, 47 to 49 per cent. (2) Cane Sugar, 2 per cent. (3) A volatile base, Methylamine, in minute quantities.

IMPURITIES._Pollen, starch and sand.]

\section{Action and Therapeutics of Lycopodium.}

Lycopodium has a great power of absorbing oils and oleoresins. It is excellent as a basis of pills, especially as it protects hygroscopic substances, for it is powerfully repellant to water. It is useful as a dusting powder, and also as a basis for insufflations.

\section{QUILLAJA.}

QUILLAJA.-Synonyms. - [Panama Bark. Soap Bark. The inner bark of Quillaja Saponaria Molina (nat. ord. Rosacea). Habitat.-Chili and Peru. 
Characters. -Flat, large pieces, about $5 \mathrm{~mm}$. thick; outer surface brownish-white, often with small patches of brown cork attached, otherwise smooth; inner surface whitish, smooth; fracture splintery, checkered with pale, brownish bast fibres, imbedded in white tissue; inodorous; taste persistently acrid ; the dust very sternutatory.]

Composition.-(I) Saponin, about 9 per cent., [(see p. 448) a mixture of the Glucosides, Quillaic Acid, $\mathrm{C}_{19} \mathrm{H}_{30} \mathrm{O}_{10}$, and Sapotoxin, $\mathrm{C}_{17} \mathrm{H}_{28} \mathrm{O}_{10}$.]

Dose, $1 / 4$ to $1 / 2$ dr. ; [r. to $2 . \mathrm{gm}$.]

\section{Preparation.}

Tinctura Quillajæ.-[Tincture of Quillaja. Quillaja, 200; by boiling with Water, straining and washing, addition of Alcohol, 350; filtration and addition of Water to 1000 .

Dose, $1 / 2$ to 2 fl. dr.; 2. to 8. c.c.]

\section{Action and Uses of Quillaja.}

The tincture of quillaja, on account of its soapy nature, is largely employed to make a lather for shampooing, and may be used to aid the diffusion of oils and other insoluble bodies, but the fact that it contains the active body saponin is an objection to its employment for emulsifying medicines for internal administration. On the other hand, because of the saponin, it might probably be used more largely as an expectorant. Those who have employed it speak very favorably of it, especially in cases in which the object is to promote free expectoration of mucus which is accumulating in the chest.

\section{GROUP XIX.}

\section{Vegetable substances [acting on Metabolism].}

Guaiacum [Xanthoxylum], Sarsaparilla, [Menispermum, Stillingia, Lappa,] Sassafras, Hemidesmus, [Calendula, Scutellaria, Tonga], Bael Fruit, Oleum Gynocardix.

\section{GUAIACUM.}

GUAIACI LIGNUM.-Guaiacum Wood. Synonym.-Lignum vitx. The heart-wool of Guaiacum officinale [Linne, and of Guaiacum sanctum Linné (nat. ord. Zygophyllea). Habitat.-West Indies, North and South America. 
ChARACTERS. - Heavier than water, hard, brown or greenish-brown, resin ous, marked with irregular, concentrated circles, surrounded by a yellowish alburnum, splitting irregularly ; when beated, emitting a balsamic odor; taste slightly acrid. Guaiacum Wood is generally used in the form of raspings or turnings, which should be greenish-brown, containing few particles of a whitish color, and should acquire a dark bluish-green color on the addition of Nitric Acid.]

Composition.-The principal constituent is the Resin (see below), 20 to 25 per cent.

[Guaiacum Wood is used to make Decoctum Sarsaparillæ Compositum.]

Dose, $1 / 4$ to I dr. ; [ $\mathrm{I}$. to $4 . \mathrm{gm}$.]

GUAIACI RESINA.-Guaiac. [Synonym.-Gum Guaiac. The resin of the wood of Guaiacum officinale Linné (nat. ord. Zygophyllea).

SOURCE. - By melting the resin of the heartwood by fire.

ChARACTERS. - In irregular masses, or subglobular pieces, externally greenish-brown, internally of a glassy lustre, and, in recent Guaiac, usually reddishbrown, transparent in thin splinters, fusible, feebly aromatic, the odor becoming stronger in heating; taste somewhat acrid; powder grayish, turning green on exposure to air.] Guaiacum Resin on dry distillation yields Cresol and Guaiacol, also found in Creosote (see p. 334). Resembling Guaiacum Resin. - Myrrb, Scammony, Benzoin, Aloes, and Resin, but these have no greenish tinge.

Compositron. - The chief constituents are three resins-(I) Guaizconic Acid, $\mathrm{C}_{19} \mathrm{H}_{20} \mathrm{O}_{5}$ (7o per cent.). (2) Guaiacic Aciul, resembling Benzoic Acid. (3) Guaiaretic Acid, $\left[\mathrm{C}_{20} \mathrm{H}_{26} \mathrm{O}_{4}\right.$ (about 10 per cent.).] These are insoluble in water, soluble in alkalies, but precipitated on neutralization.

InCOMPATIBLES.-Mineral acids and spirit of nitrous ether.

[Guaiac Resin is contained in Pilulx Antimonii Compositx.]

Dose, 5 to $30 \mathrm{gr}$; ; [.30 to $2.00 \mathrm{gm}$.]

\section{Preparations.}

[I. Tinctura Guaiaci.—Tincture of Guaiac. Guaiac, 200. By maceration with Alcohol, and filtration to 1000 .

Dose, $1 / 2$ to I fl. dr.; 2. to 4. c.c.]

2. Tinctura Guaiaci Ammoniata. - [Ammoniated Tincture of Guaiac. Guaiac, 200; by maceration with Aromatic Spirit of Ammo nia, and filtration to 1000 .

Dose, $y / 2$ to I fl. dr.; 2. to 4 . c.c.]

\section{Action of Guaiac.}

External.- [The tincture of guaiac is used for the detection of blood stains.] 


\section{VEgETABLE SUBSTANCES ACTING ON METABOLISM. $67 \mathrm{I}$}

Internal.-Guaiacum resin gives rise to an acrid feeling in the throat and a sensation of heat in the epigastrium. It increases the secretions and movements of the intestine and stomach. Large doses are gastro-intestinal irritants, causing vomiting and purging. It reflexly stimulates the heart.

\section{Therapeutics of Guaiac.}

Internal.-Guaiacum resin is so [disagreeable] and its value so doubtful that it is rarely ordered. It is used empirically, sometimes successfully, for chronic sore throat, especially if the subject has had syphilis. Lozenges ( $13 \mathrm{gr}$. $[.20 \mathrm{gm}$. ] of the resin with a fruit basis) are preferred. The mixture $[B$. P., Guaiacum resin, 6 ; sugar, 6 ; tragacanth, 1 ; cinnamon water, 240 ; dose, $1 / 2$ to I fl. oz., I 5 . to 3 o. c.c.] is said to be a more efficacious preparation than that of the tincture. Thirty grains [2. gm.] of the powder itself may be placed on the back of the throat and swallowed. Guaiacum is a mild purgative, and it has been given as a pill in chronic constipation; this property accounts for its presence in compound [cathartic] pills. Lately it has been strongly recommended by Garrod as a means of warding off attacks of gout. For this purpose $\mathrm{I} 2 \mathrm{gr}$., [.75 gm.] of the powdered resin may be taken in a cachet for an indefinite period, even several years. It is well to follow it by a draught of effervescent lithium citrate. It was formerly employed in chronic rheumatism.

\section{[XANTHOXYLON.}

XANTHOXYLON.-Synonym.-Prickly Ash Bark. The bark of Xanthoxylum americanum Miller, and of Xanthoxylum Clava-Herculis Linné (nat. ord. Rutacea). Habilat.-North America.

Characters.-Xanthoxylum americanum (Northern Prickly Ash) is in curved or quilled fragments, about I mm. thick; outer surface brownish gray, with whitish patches, and minute, black dots, faintly furrowed, with some brown, glossy, straight, two-edged spines, linear at the base, and about $5 \mathrm{~mm}$. long; inner surface whitish, smooth ; fracture short, non-fibrous, green in the outer and yellowish in the inner layer; inodorous; taste bitterish, very pungent.

Xanthoxylum Clava-Herculis (Southern Prickly Ash) resembles the preceding, but is about $2 \mathrm{~mm}$. thick, and is marked by many conical, corky pro. jections, sometimes $2 \mathrm{~cm}$. high, and by stout, brown spines, rising from a 
corky base. Resembling Xanthoxylum.-Aralia spinosa, which is nearly smooth externally, and beset with slender prickles in transverse rows.

Composirion.-It contains-(I) An acrid, green oil. (2) A resin, crys. talline, white and tasteless. (3) An acrid, soft resin. (4) A bitter substance, probably an alkaloid. (5) Tannic acid, in small quantity.

Dose, 10 to $30 \mathrm{gr}$; .60 to $2.00 \mathrm{gm}$.

\section{Preparation.}

Extractum Xanthoxyli Fluidum.-Fluid Extract of Xanthoxylum. By maceration and percolation with Alcohol, and evaporation.

Dose, 10 to $30 \mathrm{~m}$.; .60 to 2.00 c.c.

\section{Action and Uses of Prickly Ash Bark.}

Xanthoxylum has about the same action as guaiac. It produces, when swallowed, a sensation of heat. It enjoys some reputation as a remedy for chronic rheumatism, and has been used in syphilis and chronic hepatic disorders. For patients suffering from chronic syphilis who do not tolerate either mercury or the iodides, McDade's formula may be employed. This is equal parts of the fluid extracts of sarsaparilla, stillingia, lappa, phytolacca root and tincture of xanthoxylon. The dose is from I to 4 fl.-dr., 4 . to I 5. c.c., thrice daily. The bark, used as a masticatory, is a popular remedy for tooth-ache.]

\section{SARSAPARILLA.}

SARSAPARILLA.-[The root of Smilax officinalis Kunth, Smilax medica Chamisso et Schlechtendal, Smilax papyracea Dubamel, and of other undetermined species of Smilax (nat. ord. Liliacea). Habitat.-Tropical America, from Mexico to Brazil.

Characters. - About 4 or $5 \mathrm{~mm}$. thick, very long, cylindrical, longitudinally wrinkled, externally grayish-brown or orange-brown ; internally showing a whitish and mealy, or somewhat horny, cortical layer, surrounding a circular wood-zone, the latter enclosing a broad pith; nearly inodorous; taste mucilaginous, bitter, and acrid.] Resembling Sarsaparilla.-Senega, which is twisted and keeled; Hemidesmus, which is cracked transversely.

Composition. - The chief constituents are - (1) Parillin [also named Smilacin, Parillinic Acid and Pariglin, about 0.2 per cent., an acrid glucoside, soluble in hot water and Alcohol, insoluble in Ether], closely resembling Saponin. (2) Resin. (3) Trace of a Volatile Oil. [(4) Calcium Oxalate and other salts. ]

INCOMPATIBLES. $-\lambda$ lkalies.

Dose, $1 / 2$ to I dr. ; [2. to $4 . \mathrm{gm}$. 


\section{Preparations.}

I. [Decoctum Sarsaparillæ Compositum.-Compound Decoction of Sarsaparilla. Sarsaparilla, I00; Sassafras, 20; Guaiacum Wood, 20; Glycyrrhiza, 20; Mezereum, 10. By boiling, maceration in Water and straining to 1000 .

Dose, $x$ to 4 fl. oz.; 30 . to r 20. c.c.

2. Extractum Sarsaparillæ Fluidum.-Fluid Extract of Sarsaparilla. By maceration and percolation with Alcohol and Water, and evaporation.

Dose, $1 / 2$ to $\times$ fi. dr. ; 2. to 4 . c.c.

3. Extractum Sarsaparillæ Fluidum Compositum.-Compound Fluid Extract of Sarsaparilla. Sarsaparilla, 750; Glycyrrhiza, I20 ; Sassafras, 100; Mezereum, 30. By maceration and percolation in Glycerin, Alcohol and Water, and evaporation, to 1000.

Dose, $1 / 2$ to $x$ fl. dr.; 2. to 4 . c.c.

4. Syrupus Sarsaparillæ Compositus.-Compound Syrup of Sarsaparilla. Fluid Extract of Sarsaparilla, 200; Fluid Extract of Glycyrrhiza, 15 ; Fluid Extract of Senna, 15 ; Oil of Sassafras, $\frac{1}{10}$; Oil of $\Lambda$ nise, $\frac{1}{10}$; Oil of Gaultheria, $\frac{1}{10}$; Sugar, 650 . By mixing, filtering, dissolving the Sugar, and straining with Water to 1000 .

Dose, $1 / 2$ to $x$ fl. oz.; 15 . to 30. c.c.]

\section{Action and Therapeutics of Sarsaparilla.}

Sarsaparilla is not known to have any physiological action. It is never given alone, therefore we are ignorant of its therapeutical effects. Probably it has none.

\section{[MENISPERMUM.}

MENISPERMUM.-Canadian Moonseed. Synonym.-Yellow Parilla. The rhizome and roots of Menispermum canadense Linné (nat. ord. Menispermacea). Habitat.-North America, in moist thickets.

Characters. - Rhizome several feet long, about $5 \mathrm{~mm}$. thick, brown or yellowish.brown, somewhat knotty, finely wrinkled, longitudinally and beset with numerous thin, rather brittle roots; fracture tough, woody; internally yellowish, the bark rather thick, the wood rays broad, porous, and longest on the lower side ; pith distinct ; nearly inodorous ; taste bitter.

Composition.-The chief constituents are-(I) Menispine, a white alkaloid, insoluble in Benzol and alkalies. (2) Berberine (see p. 640). Tannic acid. (4) Resin.

Dose, 5 to $30 \mathrm{gr}$; .30 to $2.00 \mathrm{gm}$. 


\section{Preparation.}

Extractum Menispermi Fluidum.-Fluid Extract of Menispermum. By maceration and percolation with Alcohol and water, and evaporation.

Dose, 5 to $30 \mathrm{~m} . ; \cdot 30$ to 2.00 c.c.

\section{Action and Uses of Canadian Moonseed.}

The action and uses of menispermum are similar to those of sarsaparilla.

\section{STILLINGIA.}

STILLINGIA.-Synonyms.-Queen's Root. Queen's Delight. The root of Stillingia sylvatica Linné (nat. ord. Euphorbiacea). Habitat.-Southern United States, in sandy soil.

Characters. - About $30 \mathrm{~cm}$. long, and nearly $5 \mathrm{~cm}$. thick, subcylindrical, slightly branched, compact, wrinkled, tough, grayish-brown, breaking with a fibrous fracture, showing a thick bark and porous wood, the inner bark and medullary rays having numerous yellowish-brown resin-cells; odor peculiar, unpleasant; taste bitter, acrid, and pungent.

Composition. - (1) Sylvacrol, an acrid resin, soluble in Alcohol and Chloroform. (2) Probably a glucoside. (3) Resin. (4) Volatile Oil. (5) Tannic Acid.

Dose, $1 / 4$ to I dr. ; I. to $4 . \mathrm{gm}$.

\section{Preparation.}

Extractum Stillingiæ Fluidum.-Fluid Extract of Stillingia. By maceration and percolation with Diluted Alcohol, and evaporation.

Dose, $1 / 4$ to I fl. dr.; r. to 4 . c.c.

\section{Action and Uses of Stillingia.}

Stillingia is in large doses emetic and cathartic, but in smaller ones, alterative. It is a valuable remedy in syphilis and in the cutaneous and hepatic diseases which are benefited by so-called alterative medicines.

\section{LAPPA.}

LAPPA.-Synonym.-Burdock. The root of Arctium Lappa Linne and some other species of Arctium (nat. ord. Composita). Habilat.-Europe and Northern Asia; naturalized in North America, in waste places.

Characters. - About $30 \mathrm{~cm}$. or more long, and in its thickest portion, from 1 to $2 \mathrm{~cm}$. thick; nearly simple, fusiform, fleshy, longitudinally wrinkled, crowned with a tuft of whitish, soft, hairy leaf-stalks; grayish-brown, internally paler; fracture somewhat horny; bark rather thick, the inner part and the wood radially striate, the parenchyma free from starch, - often with cavi 
ties lined with white remains of tissue; odor feeble and unpleasant; taste mucilaginous, sweetish and somewhat bitter.

Composition.-The chief constituents are-(r) Possibly a glucoside. Inulin. (3) Resin. (4) Tannic Acid, in small quantity.

Dose, $1 / 2$ to $\mathrm{I} \mathrm{dr}$; 2. to $4 . \mathrm{gm}$.

\section{Preparation.}

Extractum Lappæ Fluidum.-Fluid Extract of Lappa. By maceration and percolation with Diluted Alcohol, and evaporation.

Dose, $1 / 2$ to I fl. dr.; 2. to 4 . c.c.

\section{Action and Uses of Burdock.}

Burdock is considered to be a diuretic and a diaphoretic alterative. It has been recommended in the treatment of various chronic skin diseases, especially in psoriasis and acne.]

\section{SASSAFRAS.}

SASSAFRAS.-[The bark of the root of Sassafras variifolium (Salisbury) O. Kuntze (nat. ord. Laurinea). Habitat. - North America from Eastern Texas and Kansas eastward to Florida and Ontario ; in woods.

CilARACTERS. - In irregular fragments, deprived of the gray, corky layer, bright rust-brown, soft, fragile, with a short, corky fracture; the inner surface smooth ; strongly fragrant; taste sweetish, aromatic, and somewhat astringent.

Composition. - The chief constituents are-(I) A volatile oil (see below), about 5 per cent. (2) Sassafrid, a peculiar decomposition product of Tannic Acid. (3) Resin. (4) Tannic Acid.]

Sassafras is contained in [Decoctum Sarsaparillæ Compositum, and Extractum Sarsaparillæ Fluidum Compositum.

Dose, $1 / 4$ to $\mathrm{I} \mathrm{dr}$; ; [I. to $4 . \mathrm{gm}$.]

[SASSAFRAS MEDULLA.--Sassafras Pih. The pith of Sassafras variifolium (Salisbury) O. Kuntze (nat. ord. Laurinea).

Characters. - In slender, cylindrical pieces, often curved or coiled, light, spongy, white, inodorous and insipid. Macerated in water it forms a mucilaginous liquid, which is not precipitated on the addition of Alcohol.

\section{Preparation.}

Mucilago Sassafras Medullæ.-Mucilage of Sassafras Pith. Sassafras l'ith, 2; Water, 100. By maceration and straining.

Dose, freely.

OLEUM SASSAFRAS.-Oil of Sassafras. A volatile oil distilled from Sassafras.

CharaCters. - A yellowish or reddish-yellow liquid, baving the characteristic odor of Sạssafras without the odor of Camphor, and a warm, aromatic 
taste. It becomes darker and thicker by age and exposure to the air. Sp. gr., 1.070 to 1.090. Solubility. - Soluble, in all proportions in Alcohol, in Glacial Acetic Acid, and in Carbon Disulphide.

Oil of Sassafras is contained in Syrupus Sarsaparillæe Compositus.

Dose, 1 to $5 \mathrm{~m}$.; .06 to .30 c.c.]

\section{Action and Therapeutics of Sassafras.}

The external and internal action of sassafras is, so far as is known, the same as that of volatile oils generally. [The mucilage is somewhat stimulant in its action, and is an excellent vehicle.]

\section{HEMIDESMUS.}

HEMIDESMUS. [B. P., not official.]-The dried root of Hemidesmus Indicus (nat. ord. Asclepiadacea). Synonym.-Indian Sarsaparilla. [Habitat.-India.]

Characters. - Cylindrical, twisted, longitudinally furrowed ; [15. cm.] long; their yellowish-brown corky layer easily separable from the rest of the bark, which is annularly cracked. Odor fragrant, taste sweetish, slightly acid. Resembling Hemidesmus. - Sarsaparilla, Ipecacuanha, and Senega, but they have no cracks.

Composition. - The chief constituents are-(r) [Coumarin] (2) Hemidesmine. (3) Tannic Acid.

\section{Preparation.}

Syrupus Hemidesmi. [B. P., not official.-Syrup of Hemidesmus]. Hemidesmus, 4 ; Sugar, 28; Boiling Water, 16.

Dose, $1 / 2$ to $I$ fil. dr.; [2. to 4 . c.c.]

\section{Action and Therapeutics of Indian Sarsaparilla.}

Hemidesmus is used chiefly in India, and for the same purposes as sarsaparilla. It is doubtful whether it has any particular action. The syrup may be given as a flavoring agent.

\section{[CALENDULA.}

CALENDULA.-Synonym.-Marigold. The florets of Calendula officinalis Linné (nat. ord. Composita). Habitat.-Levant and Southern Europe; cultivated.

CHARACTERS. - Florets about $12 \mathrm{~mm}$. long, linear and strap-shaped, delicately veined in a longitudinal direction, yellow or orange-colored, threetoothed above, the short hairy tube enclosing the remnants of a filiform style terminating in two elongated branches; odor slight and somewhat heavy; taste somewhat bitter and faintly saline. 
Composition.-The chief constituents are-(I) A peculiar principle, Calendulin, which is regarded as analogous to Bassorin. (2) An amorphous bitter principle. (3) Gum.

Dose, 15 to $60 \mathrm{gr}$; 1 . to $4 . \mathrm{gm}$.

\section{Preparation.}

Tinctura Calendulæ.-Tincture of Calendula. Calendula, 200. By maceration and percolation with Alcohol to 1000 .

Dose, I to 4 fl. dr.; 4 . to 15 . c.c.

\section{Action and Uses of Marigold.}

Marigold was formerly supposed to be antispasmodic, sudorific and emmenagogue, but now it is believed to have no therapeutic value.

\section{SCUTELLARIA.}

SCUTELLARIA.-Synonym.--Skullcap. The herb of Scutellaria lateriflora Linné (nat. ord. Labiata). Habitat.-North America; west to Alabama, New Mexico and Oregon, in damp thickets.

ChARACTERS. - About $50 \mathrm{~cm}$. long, smooth; stem quadrangular, branched ; leaves opposite, petiolate, about $5 \mathrm{~cm}$. long, ovate-lanceolate or ovate-oblong, serrate; flowers in axillary, one-sided racemes, with a pale blue corolla, and bilabiate calyx, closed in fruit, the upper lip helmet-shaped; odor slight; taste bitterish.

Composition.-(I) A bitterish principle. (2) Volatile oil, a trace.

Dose, $\mathrm{I}$ to $2 \mathrm{dr}$.; 4 . to $8 . \mathrm{gm}$.

\section{Preparation.}

Extractum Scutellariæ Fluidum.-Fluid Extract of Scutellaria. By maceration and percolation with Diluted Alcohol and evaporation.

Dose, I to 2 fl. dr.; 4. to 8 . c.c.

\section{USES OF SCUTELlaRIA.}

Scutellaria has little medicinal effect. It is used as a nervous sedative; formerly it was given in decoction for epilepsy.

\section{TONGA.}

TONGA.-The bark of Raphidorphora vitiensis (nat. ord. Aracea) and Premna taitensis (nat. ord. Verbenacea). Habitat.-Fiji Islands.

Composition.-The chief constituents are-(I) Tongine, a volatile alkaloid. (2) A volatile oil.

Dose, $1 / 4$ to $\mathrm{r}$ dr. ; 1 . to $4 . \mathrm{gm}$. 


\section{Action and Therapeutics of Tonga.}

Tonga as a fluid extract ; dose, $\mathrm{I} / \mathrm{t}$ to $\mathrm{I}$ fl. dr.; r. to 4 . c.c., undoubtedly relieves some cases of intractable neuralgia. Combined with salicylates it is of great value for the treatment of socalled muscular rheumatism. In large dosẹs it is purgative.]

\section{BELAE FRUCTUS.}

BAEL FRUIT.-[B. P., not official.]-The dried half-ripe fruit of Aigle marmelos (nat. ord. Rutacea). [Habitat.]-Malabar and Coromandel.

Characters. - Roundish fruit the size of a large orange, usually imported in fragments of the hard, woody rind, with adherent dried pulp and seeds.

Composition. - [Tannic Acid in small quantity.

Preparation, not official.

Extractum Belæ Liquidum.-Fluid Extract of Bael Fruit. Bael Fruit, 16; water, 17.

Dose, I to 2 fl. dr. ; 4 . to 8. c.c.]

Action and Therapeutics of Bael Fruit.

The imported bael fruit is probably useless. In India the extract of the fresh fruit is used for the treatment of diarrhoea and dysenterý. It contains but little tannic acid, and its mode of action is not known.

\section{OLEUM GYNOCARDIÆ.}

CHAULMOOGRA OIL.-[Not official.]-The expressed oil from the seed of Gynocardia odorata (nat. ord. Bixinea). [Habitat.-East India.]

Characters. - [This is a whitish substance which is solid at $107^{\circ} \mathrm{F}$, $42^{\circ} \mathrm{C}$, and is of an acid reaction. Sp. gr., at above temperature, o.930.] Solubility. - In ether, chloroform, and alcohol.

Compositron.-Chiefly Gynocardic Acid, $\left[\mathrm{C}_{14} \mathrm{H}_{24} \mathrm{O}_{2}\right.$, $]$ a yellowish oily body with a burning taste.

Dose, 5 to $20 \mathrm{~m}$.; [.30 to $\mathrm{r} .20$ c.c.] in capsules.

\section{Action and Uses of Chaulmoogra Oil.}

Chaulmoogra oil has been much used in leprosy, for the bacilli present in the blood have diminished in number during its administration, but it does not cure the disease. An ointment (3 to 8 of lanolin) has been used as a stimulant in chronic eczema 
ANIMAL DRUGS ACTING ON THE NERVOUS SYSTEM. 679

and psoriasis. [It has been largely employed as a local application for bruises, sprains and stiffness by athletes.]

\section{SECTION II.-PHARMACOPCIAL SUBSTANCES DERIVED FROM THE ANIMAL KINGDOM.}

[The drugs in this section which act similarly will be grouped together. In Appendix II. a list of these drugs, arranged according to their Natural Orders, will be found.]

\section{GROUP I.}

\section{[Animal Drugs acting chiefly on the Nervous System.]}

\section{MOSCHUS.}

MUSK.-The dried secretion from the preputial follicles of Moschus moschiferus [Linné (class Mammalia; order Ruminantia). Habitat.Central Asia.

CHARACTERS. - In irregular, crumbly somewhat unctuous grains, dark reddish-brown, having a peculiar, penetrating and persistent odor, and a bitterish taste. It is contained in oval or roundish sacs about 4 to $5 \mathrm{~cm}$. in diameter, on one side invested with a smoothish membrane, on the other side covered with stiff, appressed, grayish hairs, concentrically arranged around two orifices near the centre. Solubility._About 1o per cent. of Musk is soluble in Alcohol, the tincture being light brownish-yellow, and on the addition of water becoming slightly turbid. About 50 per cent. of Musk is soluble in water, the solution being deep brown, faintly acid, and strongly odorous.

Composition.--(1) Ammonia. (2) An acid. (3) Cholesterin. (4) Fats and Oils. (5) Wax. (6) Gelatinous and albuminous principles. The odoriferous principle has not been isolated,] but it is probably a product of decomposition, being constantly formed; complete drying destroys the odor, but it returas after water is added.

IMPURITIES. -Dried blood, [resin, lead and other substances.]

Dose, 2 to xo $\mathrm{gr}$; $[.12$ to $.60 \mathrm{gm}$. $]$

\section{Preparation.}

[Tinctura Moschi. - Tincture of Musk. Musk, 50; Alcohol, $45^{\circ}$; Water, $45^{\circ}$; by maceration and filtration with Diluted Alcohol, to 1000 .

Dose, $1 / 4$ to I fi. dr.; x. to 4 . c.c.]

Action of Musk.

Musk is a very powerful diffusible stimulant, especially to the heart and nervous system. It also stimulates the respiratory 
centre. How it acts is not known. Occasionally it produces headache and nausea.

\section{Therapeutics of Musk.}

It has been used, and apparently with great success, in the collapse and prostration of long continued severe diseases, such as typhoid fever and pneumonia. Various functional nervous diseases, as hysteria, are occasionally treated with it. Its high price limits its use. It is usually given as a pill.

\section{GROUP II.}

\section{[Animal Purgatives.}

\section{Oxgall and Honey.]}

\section{FEL BOVIS.}

[OXGALL.-Synonym.-Fel Tauri. The fresh bile of Bos Taurus Linné (class Mammalia; order Ruminantia). Habitat.-Domesticated.

CHARACTERS. - A brownish-green or dark-green, somewhat viscid liquid, having a peculiar, unpleasant odor, and a disagreeable, bitter taste. Sp. gr., I.018 to 1.028 .]

Dose, 5 to $15 \mathrm{gr} . ;$ [.30 to $1.00 \mathrm{gm}$.]

\section{Preparation.}

[Fel Bovis Purificatum.-Purified Oxgall.

Sourck. - Evaporate Oxgall, 300, to 100; add Alcohol, 100. Decant, filter, and after distillation of the Alcohol, evaporate.

Characters. - A yellowish-green, soft solid, having a peculiar odor, and a partly sweet and partly bitter taste. Solubility.-Very soluble in water and in Alcohol.

Dose, 5 to $15 \mathrm{gr}$; . 30 to $1.00 \mathrm{gm}$.]

\section{Action of Oxgall.}

[Oxgall when added to albuminous solutions delays their decomposition. It aids in the absorption of fats. If given by the mouth it is mostly absorbed from the intestine and acts as a cholagogue.] 


\section{Therapeutics of Oxgall.}

Oxgall has been used as a cholagogue purgative in cases of constipation, in which the pale color of the fæces indicates a deficient secretion of bile. [It has also been used as an antiseptic in typhoid fever and in intestinal fermentation.] An enema of twenty grains [1.20 gm.] or more dissolved in an ounce or two [30. to 6o. c.c.] of water is very useful in case of impacted fæces, in which the rectum is so full that there is not sufficient room for a larger enema. [One to two ounces; 30 . to 6o. gm. of oxgall in a pint; 500 c.c., of water would be much more likely to be successful. According to Fraser bile has some antitoxic power with reference to the poisons produced by pathogenic micro-organisms.]

\section{MEL.}

[HONEY.-A saccharine secretion deposited in the honey-comb by $A$ pis mellifica Linné (class Insecta; order Hymenoptera). Habitat. - Domesticated.

Characters. - A syrupy liquid of a light yellowish to pale yellowishbrown color, translucent when fresh, but gradually becoming opaque and crystalline, having a characteristic, aromatic odor, and a sweet, faintly acrid taste.

Composition.-The chief constituents are-(1) Dextrose or Grape Sugar. (2) Glucose or Fruit Sugar. (3) Wax. (4) Volatile oil. (5) Formic Acid, a minute quantity.]

\section{Preparation.}

Mel Despumatum.-Clarified Honey. Melt Honey in a waterbath, and strain while hot, adding 5 per cent. of Glycerin.

Clarified Honey is contained in Confectio Rosæ and Mel Rosæ.

[Dose, freely.]

\section{Action and Therapeutics of Honey.}

Honey is a demulcent, relieving dryness of the mouth and facilitating swallowing. Oxymel, [clarified honey 8, acetic acid $\mathbf{r}$, water $\mathbf{r}]$, is a useful preparation. It is a common ingredient of cough mixtures. Honey is a mild laxative, and may be given to children for this purpose. 


\section{GROUP III. \\ [Animal Digestants.}

\section{Pepsin and Pancreatin.]}

\section{PEPSINUM.}

PEPSIN.- [A proteolytic ferment or enzyme obtained from the glandular layer of fresh stomachs from healthy pigs, (Sus scrofa Linné ; class Mammalia; order Pachydermata), and capable of digesting not less than 3000 times its own weight of freshly coagulated and disintegrated egg albumin. Habital. -Domesticated.

Source. - The mucous membrane of a pig's stomach, dissected off and finely chopped, is macerated in water acidulated with Hydrochloric Acid for several days, with frequent stirring. The strained liquor is decanted and Sodium Chloride mixed with it. After several hours the floating mixture is skimmed from the surface and placed in cotton cloth to drain, and finally submitted to strong pressure to get rid of the saline solution.

Characters.-A fine white, or yellowish-white, amorphous powder, or thin, pale yellow or yellowish, transparent or translucent grains or scales, free from any offensive odor, and having a mildly acidulous or slightly saline taste, usually followed by a suggestion of bitterness. It slowly attracts moisture when exposed to the air. Solubility. - Soluble, or for the most part soluble, in about 100 parts of water, with more or less opalescence; more soluble in water acidulated with Hydrochloric Acid; insoluble in Alcohol, Ether or Chloroform.

Dose, 5 to $60 \mathrm{gr}$; ; 30 to $4.00 \mathrm{gm}$.

\section{Preparation.}

Pepsinum Saccharatum.-Saccharated Pepsin. Pepsin, 10; Sugar of Milk, 90. By trituration. Saccharated Pepsin should digest 300 times its own weight of freshly coagulated and disintegrated egg albumin.

Dose, 5 to $60 \mathrm{gr}$; .30 to $4.00 \mathrm{gm}$.]

\section{Action and Therapeutics of Pepsin.}

Pepsin may be given to help gastric digestion in those in whom from old age or long illness the secretion of gastric juice is deficient. 'Thus, for example, it is useful in convalescence from acute illness or in cases of cancer of the stomach. It is of no use as an aid to the digestion of fatty or carbo-hydrate food. It should be given in a powder or a pill directly after meais and should be followed in about half an hour by a dose of hyaro 
chloric acid. The pepsin should be tested before use, as many preparations in the market are inert powders.

Pepsin may be used to predigest albuminous food, either for administration by the mouth or the rectum. Often this is better than giving pepsin internally, for morbid processes may be going on in the stomach which prevent digestion. The rectum has very feeble powers of digestion, and therefore nutrient enemata or suppositories should always be predigested. It is found that for predigestion pancreatin (see below) is usually a more reliable preparation than pepsin. Both should be employed with judgment, for there is a likelihood that if artificial digestion be used too long, the digestive functions of the stomach may [become incapable of action] from want of use.

The following directions for peptonizing meat may be followed. Take one pound [450. gm.] of lean meat, reduce to a fine pulp, add six times its weight of water containing 0.2 per cent. of hydrochloric acid and $120 \mathrm{gr}$. [8. gm.] of pepsin. Digest at $120^{\circ} \mathrm{F} .\left[48 .^{\circ} \mathrm{C}\right.$.] in a porcelain digester for five or six hours with frequent stirring. Then neutralize with sodium carbonate, boil and filter. Evaporate the filtrate on a water bath till it is of the consistency of a soft extract.

Peptonized meat suppositories are often very valuable. To make one suppository $30 \mathrm{gr}$. [2. gm.] of the above extract is mixed with $40 \mathrm{gr}$. [2.40 gm.] of oil of theobroma, and moulded in a conical mould.

\section{[PANCREATINUM.}

PANCREATIN.-Synonym.-Zymine. A mixture of the enzymes naturally existing in the pancreas of warm-blooded animals, usually obtained from the fresh pancreas of healthy pigs (Sus scrofa Linné; class Mammalia; order Pachydermata). Habitat.-Domesticated.

SoURCE. - Macerate the cut-up pancreas in water acidulated with Hydrochloric Acid for forty-eight hours, add a saturated solution of Sodium Chloride, allow to stand until the Pancreatin rises to the surface; skim this, drain in a muslin filter, wash with a less concentrated solution of salt until nearly dry ; then rub up with Sugar of Milk, dry thoroughly without heat, and dilute with Sugar of Milk, until ro gr.; .60 gm., will just emulsify 2 fl. dr.; 8. c.c. of Cod Liver Oil.

Characters. -A yellowish, yellowish-white, or grayish, amorphous 
powder, odorless, or having a faint, peculiar, not unpleasant odor, and a somewhat meat-like taste. Solubility.-Slowly and almost completely soluble in water, insoluble in Alcohol.

Dose, 5 to $15 \mathrm{gr}$; .30 to $1.00 \mathrm{gm}$.]

Action and Uses of Pancreatin.

Pancreatin has the power of converting starch into sugar, albumin and fibrin into peptones, and first curdling and then peptonizing milk. It will not act in an acid medium nor above $\mathrm{I} 40^{\circ} \mathrm{F}$. $\left[60^{\circ} \mathrm{C}.\right]$. The directions for peptonizing milk are given on p. 688. [It is used as an artificial agent to assist the digestion of invalids and of old persons, or those prostrated by fever or exhaustion. Also by means of this, food may be partially or wholly digested previous to administration. It should be used in combination with an alkali, as sodium bicarbonate, in the proportion of I to 4 . Nutritive enemata should be thoroughly pancreatized.]

\section{GROUP IV.}

[Animal Drugs which are also Foods.

Cod Liver Oil, Milk, Extract of Meat.]

\section{OLEUM MORRHUA.}

COD LIVER OIL.-Synonym.-[Oleum Jecoris Aselli. A fixed oil obtained from fresh livers of Gadus Morrhua Linné, and of other species of Gadus (class Pisces: order Teleostia; family Gadida.) Habitat.-North Atlantic Ocean. An oil obtained from the Candle fish (Thaleichthys Pacificus) is found in the markets under the name of Eulachon Oil and is sometimes sold as Cod Liver Oil.

SoURCE. - The fresh livers are slowly heated, and the oil is decanted from the water, and sometimes deprived of the solid fat by partial freezing.

Characters. - A pale yellow, thin, oily liquid, having a peculiar, slightly fishy, but not rancid odor, and a bland, slightly fishy taste. Sp. gr., 0.920 to 0.925 . Solubility. - Scarcely soluble in Alcohol, but readily soluble in Ether, Chloroform, or Carbon Disulphide ; also in 2.5 parts of Acetic Ether.

Composition.-The chief constituents are-(I) Olein, 70 per cent., which is a fluid fixed oil, and is Glycerin Oleate the most abundant constituent of Cod Liver Oil. (2) Palmitin, with some Stearin, 25 per cent. (3) Free 
fatty acids, as Oleic, Palmitic, Stearic. (4) Gaduin, $\mathrm{C}_{35} \mathrm{H}_{46} \mathrm{O}_{9}$, a peculiar principle, very insoluble in ordinary menstrua. (5) Morrhuol, a crystalline substance of uncertain composition, containing Phosphorus, Iodine and Bromine. (6) Traces of Iodine and Bromine. (7) Biliary principles. The socalled alkaloids of Cod Liver Oil are decomposition products, ptomaines or cadaveric alkaloids, and are fuund in larger quantities in the brown oils. Their existence in fresh oil obtained from healthy livers has not been demonstrated.]

Dose, I to 4 fl. dr.; [4. to 15. c.c.]

\section{Action of Cod Liver Oil.}

External.-Cod liver oil is a bland unirritating oil. If it is desired to administer it in cases in which it is rejected by the stomach, it may be rubbed into the skin. The oil is certainly absorbed when applied in this way.

Internal._Gastro-intestinal tract.—Cod liver oil, even more than other oils, is liable to cause indigestion, nausea, and sickness. Large doses may set up diarrhœa. It is more readily absorbed than other oils. Loops of intestine have been isolated in the lower animals, and into each loop different oils have been injected. The intestines are returned to the abdominal cavity, and after some time the animal is killed and the loops are opened. It is always found that the cod liver oil has been more rapidly absorbed than any other oil. The facility with which cod liver oil is absorbed is also shown by the fact that it often cannot be recognized in the fres, although equal quantities of other oils taken by the mouth are passed nnaltered. Some authorities believe that the superior absorbability of cod liver oil depends on the biliary principles contained in it, but this is doubtful ; others think that it is because the presence of free acids facilitates saponification and emulsion. Certainly it contains more free fatty acids than other oils, and it also emulsifies much more easily.

Tissues. - Not only is cod liver oil more readily absorbed than other oils, but it is a better food. All oils lead to an increased formation of fat, but cod liver oil is the most powerful in this respect. It reduces the color of a solution of potassium permanganate more readily than other oils-that is to say, it is more readily oxidized. Thus, as it is more easily absorbed and more 
easily oxidized, we have a partial explanation of its peculiar value in increasing the weight of the body; but the general belief is that these two facts do not wholly explain the action of cod liver oil, and that it has some peculiar specific action not yet understood, especially upon those suffering from phthisis for whom it is a very valuable drug. [If it is true, as has been stated, that iodine may occur in the proportion of I to 2000 of the oil, the influence of this remedy is not to be ignored.]

\section{Therapeutics of Cod Liver Oil.}

External.-The smell of cod liver oil is so disagreeable that it should not be rubbed in externally unless this treatment be absolutely necessary.

Internal.-Cod liver oil is of the greatest service in all varieties of tuberculous disease, the contra-indications being high temperature, severe hæmoptysis and dyspepsia, vomiting, or diarrhœa, whether primary or induced by the oil. Patients often improve in every way under its influence. With the same exceptions it may be administered with great advantage in rickets, and in any chronic disease associated with loss of flesh, such as suppuration, convalescence from acute disease, tertiary syphilis and starvation. It is often of benefit in the chronic bronchitis and the chronic eczema of childhood. It is frequently given with success in neuralgia, general feebleness, despondency and other nervous conditions. Formerly it was often prescribed for chronic rheumatism. Many persons cannot, or imagine they cannot, take it on account of its [unpleasant] taste. There are in the market several preparations of cod liver oil in which, by careful preparation, the disagreeable taste is almost abolished. Ten mininis [.6o c.c.] of pure ether, with a [minim] or two [.06 to .12 c.c.] of oil of peppermint or cloves, will, when mixed with a dose of cod liver oil, often render it more palatable. Sometimes it is taken in [soft] capsules, or made into a jelly with isinglass, or a little salt is put into the mouth after the oil is taken, or the mouth is rinsed out with brandy beforehand. Sometimes it is taken in coffee, but perhaps the best way is to form an emulsion of it. A very nutritious one is made by rub. 
bing together equal parts of [extract of malt] and cod liver oil, and in this the oil can hardly be tasted [but it is iikely to repeat.]

The British Pharmaceutical Conference advises tne following emulsion:-Cod liver oil, 8 fl. oz. [240. c.c.]; the yolk of two eggs ; tragacanth in powder, 16 gr. [1. gm.]; elixir of [saccharin (saccharin,] $24 \mathrm{gr}$; [1.50 gm.]; sodium bicarbonate, I $2 \mathrm{gr}$. [.75 gm.]; alcohol, I fl. dr. [4. c.c.]; distilled water, 7 fl. dr. [28. c.c.]), I fl. dr. [4. c.c.]; tincture of benzoin, I fl. dr.; [4. c.c.]; spirit of chloroform, 4 fl. dr.;-[15. c.c.]; oil of bitter almond, $8 \mathrm{~m}$.; $[.50$ c.c. $]$; distilled water to 16 A. oz.; $[500$. c.c.]. Dose, 2 to $8 \mathrm{fl}$. dr.; [8. to 3o. c.c.]. It is frequently desirable to give cod liver oil with iron. In that case the following preparation, in which the oil is emulsified with an alkali, will be found useful :-Cod liver oil, 4 fl. dr.; [r5. c.c.]; iron and ammonium citrate, $5 \mathrm{gr}$; [.3० gm. $]$; potassium carbonate, 3 gr.; [.20 gm. ; saccharin,] 1/4 gr. [.01 5 gm.]; oil of caraway, $1 / 4$ m.; [.015 c.c. $]$; water to I fl. oz. ; [30. c.c.].

\section{LAC.}

[MILK.-Not official.-The fresh milk of the cow, Bos Taurus, (Ord. Ruminantia.) Habitat.-Domesticated.]

ARTIFICIAL HUMAN MILK.-(Not official.)

Preparation. - Take half a pint [240. c.c.] of skimmed milk; heat it to about $96^{\circ} \mathrm{F} . ;\left[35.5^{\circ} \mathrm{C}\right.$.] and put into the warmed milk a piece of rennet an inch [2.5 cm.] square, or a teaspoonful [4. c.c.] of essence of rennet. Put the milk in a fender, or over a lamp, until it is quite warm. As soon as it is set, remove the rennet, break up the curd into small pieces with a knife, and let it stand for ten or fifteen minutes; the curd will then sink. Then pour the whey into a saucepan and boil quickly. Measure one-third of a pint [160. c.c.] of this whey, and dissolve in it, while it is hot, I Io gr. [7. gm.] of Sugar of Milk. When this third of a pint [16o. c.c.] of whey is cold, add to it twothirds of a pint [320. c.c.] of new milk and two teaspoonfuls; [8. c.c.] of cream and stir. The food should be made fresh every twelve hours, and warmed as required. Care should be taken to select an essence of rennet which does not make the milk taste.

Action and Therapeutics of Artificial Human Milk.

Artificial human milk is invaluable as a food for infants whose mothers cannot suckle thein. Many cases of infantıle diarrhœa, 
indigestion, and sickness can be cured by substituting this milk for the usual milk and water, or infants' food. Some large dairy firms supply it, but it is cheaper to make it at home, and the above directions are easily carried out. When bought it is often sterilized and sold in air-tight bottles. It should be remembered that a long-continued diet of sterilized milk may, in children, cause rickets.

\section{PEPTONIZED MILK.-(Not official.)}

Preparation.-Mix a pint [48o. c. c.] of milk with 5 fl. oz. [150. c.c.] of water. Heat to $140^{\circ} \mathrm{F} .\left[60^{\circ} \mathrm{C}\right.$. $]$, and add Liquor Pancreatis, [B. P.] I fl. dr. [4. c.c.]; and Sodium Bicarbonate, $20 \mathrm{gr}$; ; $[1.20 \mathrm{gm}$.]. Leave the mixture at the ordinary temperature of the room for three hours, or if kept at about $135^{\circ} \mathrm{F}$. [57.2 ${ }^{\circ} \mathrm{C}$.] for about half an hour, then heat for a moment to boiling point. If too much pancreatic solution is used the milk is bitter. [This preparation should be kept on ice until required.]

\section{Action and Therapeutics of Peptonized Milk.}

Peptonized milk is used in many conditions in which it is thought that the gastric digestion is too feeble to digest ordinary milk, or in which it is desired, as sometimes, in typhoid fever, for instance, to avoid the curdling of milk in the stomach. Milk should always be peptonized before being introduced into an enema. An usual nutrient enema consists of the yolk of an egg and milk up to four fluid ounces [r20. c.c.]. This mixture may be peptonized in just the same way and with the same quantity of peptonizing agents as the pint [480. c.c.] of milk. Thirty grains; [2. gm.] of common salt should be added to the enema before use.

KUMYSS.-(Not official). - [This is frequently written, Koumiss.]

Preparation.-Dissolve 4 dr. [ $15 \mathrm{gm}$.] of grape sugar in $4 \mathrm{fl}$. oz. [ 120. c.c.] of water and $20 \mathrm{gr}$. [ $1 \mathrm{I} .20 \mathrm{gm}$.] of yeast in $4 \mathrm{fl}$. oz. [120. c.c.] of cow's milk. Pour both into a bottle holding a quart [960. c.c.], which is then filled up with milk, corked, wired, and put in a cool place and frequently shaken for four days.

\section{USES OF KuMYSS.}

Kumyss contains a little alcohol and is extremely useful as a stimulant food in convalescence, in phthisis, and other conditions of exhaustion. [It is also used for the same purposes as milk; it 
is usually more agreeable to the patient and is often borne by the stomach when all other food is vomited. The above is a very good substitute for the kumyss drunk by the Tartars, who prepare it by fermenting mare's milk.]

\section{[EXTRACTUM CARNIS.}

EXTRACT OF MEAT.-(Not official).-This preparation has for its object the obtaining the nutritive matters of the flesh of animals in a permanent concentrated state.

SOURCE. - Equal parts of meat and cold water. Straining, evaporation and straining.

Characters. - Reddish-brown in color, of a slightly acrid taste, and frequently of a disagreeable odor.

Composition. - 100 parts of meat yield 25 of extract. It is rich in nitrogenous principles, but contains neither fat nor gelatin. The method of Liebig has been followed, with variations, by a large number of manufacturers. The product, however, by no means represents the nutritive qualities of the meat itself, because the albumin and fibrin are largely wanting, besides an endeavor is put forth to get rid of the gelatin and fat. Of late the attempt has been made to retain these important nutritive principles. The beef meal which was used by Debove in his method of forced feeding (gavage) was practically desiccated beef; but it did not have its highest nutritive value because it was, to a considerable extent, composed of insoluble matters. The more recent preparations are based upon the classical studies of Wurtz which showed that in the juice of the Carica papaya (nat. ord. Passiflora) was a principle, called papain, capable of converting albuminoids into soluble albumoses and peptones. The fact that a similar vegetable digestive principle exists in the juice of the pine-apple and allied plants of the nat. ord. Bromeliacece, as was first shown by Marcaño, has been utilized in the manufacture of the Mosquera-Julia beef meal, where the process of digestion is carried on before desiccation, or it may be made as a jelly. The advantages of a vegetable over an animal pepsin, the greater acceptability to weak stomachs, the avoidance of the unpleasant taste and the disagreeable odor, and absence of bitterness are very important. By this method the preparation contains only ten per cent. of water, has four times the amount of albuminoid matter present in average lean beef, one-half of which is already in an assimilable form, and about three times as much fat, in addition. Besides, it is very convenient for administration.

\section{Action and Uses of Extract of Meat.}

Extract of meat is useful as a nutrient and a stimulant in relieving prostration and fatigue. The solution seasoned with capsicum is valuable in alcoholic excess and delirium tremens. In 
the infantile bowel disturbances, when milk must be forbidden, it is often indicated. In phthisis it will frequently sustain the patient; in the aged it will support life without taxing the digestive powers. The amount to be used should be regulated by the age and condition of the patient.]

\section{GROUP V.}

\section{[Animal Emollients.}

\section{Lard, Spermaceti, Egg.]}

\section{ADEPS.}

LARD.-[The prepared internal fat of the abdomen of Sus scrofa Linne (class Mammalia; order Pachydermata), purified by washing with water, melting, and straining. Habitat.-Domesticated.

Characters. - A soft, white, unctuous solid, having a faint odor free from rancidity, and a bland taste. Solubility. - Insoluble in water; very slightly soluble in Alcohol; readily soluble in Ether, Chloroform, Carbon Disulphide or Benzin. Sp. gr., about 0.932 at $59^{\circ} \mathrm{F} . ; 15^{\circ} \mathrm{C}$.]

Composition.-(1) Olein, 60 per cent. (2) Stearin. (3) Palmitin. Adeps Induratus (Indurated Lard), which is ordinary lard deprived of a portion of its oil by pressure, may be used in [hot climates] when the high temperature renders ordinary lard too soft for use in ointments.

\section{Preparations.}

1. [Adeps Benzoinatus.-See Benzoin, p. 656.

2. Ceratum. - Cerate. White Wax, 300 ; lard, 700.

3. Ceratum Resinæ.-See Resin, p. 523.

4. Unguentum.-Ointment. Lard, 800 ; Yellow Wax, 200.

OLEUM ADIPIS.-Lard Oil. A fixed oil expressed from Lard at a low temperature.

Characters. - A colorless, or pale yellow oily liquid, having a peculiar odor, and a bland taste. Sp. gr., 0.910 to 0.920 .

Composition.-(1) Olein. (2) Palmitin. (3) Stearin.]

\section{ACtion and Therapeutics of LARD.}

Lard is an emollient, and is used as a basis for ointments when it is wished that the active ingredient should be absorbed, for 
lard melts at the temperature of the body, especially if bandaged on. The [benzoinated] lard has the advantage of not quickly hecoming rancid.

\section{CETACEUM.}

SPERMACETI.-[A peculiar, concrete, fatty substance, obtained from the sperm whale, Physeter macrocephalus Linné (class Mammalia; order Cetacea. Habitat.-Pacific and Indian Oceans.

CHARACTERS. - White, somewhat trańslucent, slightly unctuous masses of a scaly-crystalline fracture and a pearly lustre; odorless, and having a bland, mild taste. It becomes yellowish and rancid by exposure to air. Sp. gr., about 0.945. Solubility.-Insoluble in water, and nearly so in cold Alcohol ; also in Ether, Chloroform, Carbon Disulphide, fixed and volatile oils; only slightly soluble in cold Benzin.]

Composition.- It is mainly Cetylic Alcohol, $\mathrm{C}_{16} \mathrm{H}_{33} \mathrm{OH}$, which in combination with Palmitic Acid, $\mathrm{HC}_{16} \mathrm{H}_{31} \mathrm{O}_{2}$, forms a fat, Cetin, $\mathrm{C}_{16} \mathrm{H}_{33} \mathrm{C}_{16} \mathrm{H}_{31} \mathrm{O}_{2}$.

\section{Preparations.}

[1. Ceratum Cetacei.-Spermaceti Cerate. Spermaceti, I0 White Wax, 350 ; Olive Oil, 550.

2. Unguentum Aquæ Rosæ.-See Rose, p. 556.]

\section{[USES OF Spermaceti.]}

Spermaceti is used as an emollient and as a basis for ointments [and cerates].

\section{[VITELLUS.}

YOLK OF EGG.-The yolk of the egg of Gallus Bankiva, var. domestica Temminck (class Aves; order Gallina). Habitat.-Java and Cochin China ; domesticated.

Composition.-(1) Vitellin, I6 per cent. (2) Fat, 30 per cent. (3) Inorganic Salts, Cholesterin, Lecithin, and coloring matter.

\section{Preparation.}

Glyceritum Vitelli.-See Glycerin, p. 608.

Action and Therapeutics of Yolk of Egg.

Yolk of egg is used to make emulsions and is nutritive and emollient.

\section{OVI ALBUMIN.}

EGG ALBUMIN.-Not official.-The liquid white of egg of Gallus Bankiva, var. domestica. Habitat.-Java and Cochin China; domesticated. 


\section{Action and Therapeutics of Egg Albumin.}

Egg albumin is an antidote to poisoning by corrosives and irritants, especially corrosive mercuric chloride, copper sulphate, lead salts and silver nitrate. Like the yolk it is nutritive and emollient.]

\section{GROUP VI.}

\section{[Animal Coloring Agents.}

\section{coccus.]}

COCHINEAL.- [Synonyms. - Cochineal Bug. Red Scale Insect. The dried female of Coccus cacti Linné (class Insecta; order Hemiptera). Habitat. -Mexico and Central America; upon Opuntia cochinillifera, Miller and other species.

CharACTERS. - About $5 \mathrm{~mm}$. long, of a purplish-gray or purplish-black color; somewhat oblong and angular in outline; flat or concave beneath; convex above ; transversely wrinkled ; easily pulverizable, yielding a dark red powder ; odor faint ; taste slightly bitterish.]

Composition. - The chief constituents are-(I) Carminic Acid, $\left[\mathrm{C}_{17} \mathrm{H}_{18}\right.$ $\mathrm{O}_{10}$, Io per cent., a glucoside. ' (2) Coccerin, a wax. (3) Fat, consisting of myristin, and fatty acids.] Sulphuric acid and several other reagents precipitate from itś decoction the well-known coloring matter carmine.

Cochineal is contained in Tinctura Cardamomi Composita.

\section{Uses of Cochineal.}

Cochineal is only used as a coloring agent. [The B. P. directs a tincture to be made by maceration of cochineal, I; alcohol ( 45 per cent.), IO.]

\section{GROUP VII.}

\section{[Animal Drugs whose action is Mechanical.}

Suet, Curd Soap, Stearic Acid, Wool Fat, Isinglass, Sugar of Milk, Wax, Gelatin, Cantharides, Ichthyol.]

SEVUM.

SUET.- [Synonym.- Mutton Suet. The internal fat of the abdomen of Ovis Aries Linné (class Mammalia; order Ruminantia), purified by melting and straining. Habilat.-Domesticated. 
Characters. - A white, solid fat, nearly inodorous, and having a bland taste when fresh, but becoming rancid on prolonged exposure to the air. Solubility.-Insoluble in water or cold Alcohol ; soluble in about 60 parts of Ether, and slowly in 2 parts of Benzin.

Compositrion.-Its chief constituents are-(1) Stearin. (2) Palmitin. (3) Olein. (4) Hircin.

Suet is contained in Unguentum Hydrargyri.

\section{USES OF SUET.}

Suet is used chiefly in cerates.]

\section{SAPO ANIMALIS.}

CURD SOAP [B. P., not official.] -Soap made with sodium [hydrate] and a purified animal fat consisting principally of Stearin. It is chiefly Sodium Stearate, but contains some Sodium Palmitate and about 30 per cent. of water.

\section{[USES OF CURd SoAP.]}

Curd soap is used as a basis [for plasters, liniments, pills and suppositories. Emplastrum Saponis consists of curd soap, ro ; lead plaster, 36; resin, 12. Emplastrum Saponis Fuscum (brown soap plaster) is curd soap, 20 ; yellow wax, 25 ; olive oil, 40 ; lead oxide, 30 ; vinegar, 320 .]

\section{[ACIDUM STEARICUM.}

STEARIC ACID. $-\mathrm{HC}_{18} \mathrm{H}_{35} \mathrm{O}_{2}=283.38$.

SOURCE. - An organic acid, in its commercial, more or less impure form, usually obtained from the more solid fats, chiefly tallow from the ox, Bos Taurus Linné; (class Mammalia; order Ruminantia). Habitat.-Domes. ticated. By boiling with soda-lye, the Stearin is decomposed, Sodium Stearate being formed with the liberation of Glycerin. $\mathrm{C}_{3} \mathrm{H}_{5}\left(\mathrm{C}_{18} \mathrm{H}_{35} \mathrm{O}_{2}\right)_{3}+3 \mathrm{NaOH}$ $=\mathrm{C}_{3} \mathrm{H}_{5}(\mathrm{OH})_{3}+{ }_{3} \mathrm{NaC}_{18} \mathrm{H}_{35} \mathrm{O}_{2}$. The soap is decomposed by heating with water and Sulphuric Acid, setting free the fatty acids which are removed and purified with hot Alcohol. On cooling, Stearic Acid will separate.

Characters. - A hard, white, somewhat glossy solid, odorless and tasteless, and permanent in the air. Solubility.-Insoluble in water; soluble in about 45 parts of Alcohol at $59^{\circ} \mathrm{F} . ; 15^{\circ} \mathrm{C}$., readily soluble in boiling Alcohol, and in Ether.

\section{Uses of Stearic Acid.}

Stearic acid is used in the manufacture of glycerin suppositories. In combination with zinc and copper, as stearates of those metals, unofficial preparations have been introduced and used 
with success in the treatment of various diseases of the skin and mucous membranes.]

\section{ADEPS LAN}

WOOL FAT.-[B. P., not official.]-The purified cholesterin fat obtained as a bye-product in the dressing of sheep's wool.

Source. - Sheep's wool, washed with cold water, then subjected to heat and pressure, yields impure wool fat. This is purified by melting, washing with alkali, and then washed with an acid while it is heated.

ChARACTERS. - Semi-transparent, pale yellow, tenacious body. Ignited, it burns with a sooty flame. Melts between $100^{\circ}$ and $112^{\circ} \mathrm{F}$.; $\left[37.7^{\circ}\right.$ and $44.4^{\circ} \mathrm{C}$.] ; odor like sheep's wool. Solubility. -Freely in Chloroform and in Ether, partially in Alcohol. Insoluble in water, but on vigorous stirring takes up $11 \frac{1}{4}$ times its weight.

Composition. - Before the separation of the fatty acids it consists of ( 1 ) Cholesterin and Isocholesterin, 70 per cent. (2) Fatty acids, 30 per cent.

\section{ADEPS LANE HYDROSUS.}

HYDROUS WOOL-FAT.-Synonyms.-Lanolin. [Esypum. The purified fat of the wool of sheep (Ovis Aries, Linne ; class Mammalia; order Ruminantia), mixed with not more than 30 per cent. of water. Habitat.Domesticated.

SoUrCE. - Sheep's wool is treated with a weak soda solution, and the solution acidulated. The remaining wool is treated with Benzin, the liquid distilled, and the residue deprived of color by oxidizing agents, or sunlight.

CHARACTERS. - A yellowish-white or nearly white, ointment-like mass, having a faint, peculiar odor. Solubility.-Insoluble in water, but miscible with twice its weight of the latter, without losing its ointment-like character.

Composition. - Its chief constituents are-(I) Cholesterin, $\mathrm{C}_{26} \mathrm{H}_{43}(\mathrm{OH})$. (2) Ethers of Stearic, Palmitic, Oleic, Valerianic and other acids.]

\section{Action and Therapeutics of Hydrous Wool-Fat.}

Hydrous wool-fat when gently rubbed in the skin is more quickly absorbed than most fats, hence it is a useful basis if we wish to administer substances-as, for example, mercury-by inunction. [It is undoubtedly soothing to the skin, and often makes an excellent basis for ointments expected to act especially upon the skin.]

\section{[ICHTHYOCOLLA.}

ISINGLASS. - The swimming-bladder of Acipenser Huso Linné, and of other species of Acipenser (class Pisces; order Sturiones). Habitat.Caspian and Black Seas and tributary rivers.

Characters. - In separate sheets, sometimes rolled, of a horny or pearly 
eppearance; whitish or yellowish, semi-transparent, iridescent, inodorous, and insipid. Solubility. - Almost entirely soluble in boiling water and in boiling diluted Alcohol. A solution of Isinglass in 24 parts of boiling water forms, on cooling, a transparent jelly.

Composition.-(1) Gelatin (Gluten), 98 per cent. (2) Insoluble membrane, about 2 per cent.

Dose, freely.

\section{Preparation.}

Emplastrum Ichthyocollæ.- - Isinglass plaster. Synonym.Court plaster. Isinglass, 10; Alcohol, 40; Glycerin, I; Hot Water sufficient for solution. To be applied with a brush upon taffeta, previously coated on the back with Tincture of Benzoin.

\section{USES OF ISINGLASS.}

Isinglass is an emollient and nutritive substance, and is chiefly used externally as a protective. A better court plaster has goldbeaters' skin as a base.]

\section{SACCHARUM LACTIS.}

SUGAR OF MILK.-Sy'nonym.-Lactose. $\mathrm{C}_{12} \mathrm{H}_{22} \mathrm{O}_{11}+\mathrm{H}_{2} \mathrm{O}[=359.16$. A peculiar, crystalline sugar, obtained from the whey of cow's milk by evaporation, and purified by re-crystallization (Bos Taurus Linné ; class Mammalia ; order Ruminantia). Habitat.-Domesticated.

ChARACTERs. - White, hard, crystalline masses, yielding a white powder feeling gritty on the tongue, odorless and having a faintly sweet taste. Permanent in the air. Solubility. - In about 6 parts of water; insoluble in Alcohol, Ether or Chloroform.]

Sugar of Milk is used in [Pulvis Ipecacuanhæ et Opii, and Trituratio Elaterini.

Dose, indefinite.]

\section{[Uses of Sugar of Milk.]}

Sugar of milk is used as a vehicle for triturations of substances, because being very hard it thoroughly divides them, and also it is but slightly deliquescent. For these reasons it is used as a diluent to get extracts to the required strength. It is employed to sweeten infants' foods. [It is a non-nitrogenous, bland article of diet and has been used in consumption and other wasting diseases. According to Sèe, it is a diuretic which may be employed to advantage in cardiac dropsy.] 


\section{CERA.}

[CERA FLAVA.-Yellow Wax. A peculiar, concrete substance, prepared by Apis mellifica Linné (class Insecta; order Hymenoptera).

Characters.-A yellowish to brownish-yellow solid, having an agree. able, honey-like odor, and faint balsamic taste. Sp. gr., 0.955 to 0.967 . Solubility.-Insoluble in water, sparingly soluble in cold Alcohol. It is completely soluble in Ether, Chloroform, and in fixed and volatile oils.

Composition.-The principal constituents are-(I) Hydrocarbons (probably $\mathrm{C}_{27} \mathrm{H}_{56}$ and $\mathrm{C}_{34} \mathrm{H}_{64}$ ) about $\mathrm{I}_{2}$ per cent. (2) Cerin or Cerotic Acid, $\mathrm{C}_{27} \mathrm{H}_{54} \mathrm{O}_{2}$. (3) Myricin or Myrical Palmitate, $\mathrm{C}_{30} \mathrm{H}_{61}, \mathrm{C}_{16} \mathrm{H}_{31} \mathrm{O}_{2}$, the principal constituent. (4) An Alcohol, $\mathrm{C}_{25} \mathrm{H}_{52} \mathrm{O}$, in small quantities. (5) Cerylic Alcohol, $\mathrm{C}_{27} \mathrm{H}_{56} \mathrm{O}$.

Yellow Wax is used in Ceratum Cantharidis, Ceratum Resinæ, Unguentum, and Unguentum Picis Liquidæ.

CERA ALBA.-White Wax. Made by bleaching yellow wax by exposure to moisture, air and light.

CHARACTERs. - A yellowish-white solid, somewhat translucent in thin layers, having a slightly rancid odor, and an insipid taste. Sp. gr., 0.965 to 0.975 .

Composition.-As of yellow wax.

White Wax is used in Ceratum, Ceratum Camphore, Ceratum Cetacei and Unguentum Aquæ Rosæ.]

\section{USES OF WAX.}

Yellow and white wax are only used as bases for many plasters, [cerates] and ointments.

\section{GELATINUM.}

GELATIN.-[B. P., not official]. - The air-dried product of the action of boiling water on gelatinous tissues, as skin, tendons, ligaments, and bone.

CHARACTERs. - In translucent sheets or shreds. The solution in lot water is colorless and inodorous; it solidifies to a jelly on cooling. It is insoluble in Alcohol and Ether. Its aqueous solution is precipitated by Tannic Acid.]

\section{Uses of Gelatin.}

Gelatin is useful as a basis for suppositories, pessaries, bougies, capsules, lozenges, and as a coating for pills. Glycogelatin (gelatin, 2 ; glycerin, 5 ; orange flower water, 5 ; colored with carmine) is an excellent basis for throat pastilles. Each should weigh 30 gr. [2. gm.]. Almost any drug can be incorporated in such pastilles. Medicated gelatin is often melted and painted on the skin in cutaneous diseases. 


\section{CANTHARIS.}

CANTHARIDES.-[Synonyms.-Spanish Flies. Blister Beetles. The Cantharis vesicatoria De Geer (class Insecta; order Coleoptera.) Habitat.Southern and Central Europe, mainly on Oleaceæ and Caprifoliacæ.

Characters. - About $25 \mathrm{~mm}$. long and $6 \mathrm{~mm}$. broad; flattish cylindrical, with filiform antennæ, black in the upper part, and with long wing-cases and ample, membranous, transparent, brownish wings; elsewhere of a shining, coppery-green color. The powder is grayish-brown, and contains green shining particles. Odor strong and diságreeable; taste slight, afterwards acrid.]

Composition. - The chief constituents are-(I) Cantharidin, $\mathrm{C}_{10} \mathrm{H}_{12} \mathrm{O}_{4}$, 0.4 to I per cent., the active principle, a crystallizable body forming colorless plates, soluble in Alcohol, Ether, Acetic Ether, Glacial Acetic Acid, Chloroform, and oils. It is found especially in the generative apparatus, the eggs, and the blood. (2) A volatile oil giving the odor and said to have the same action as Cantharidin. (3) A green oil, the coloring principle, closely allied to chlorophyll. (4) [Various extractives and salts.]

\section{Preparations.}

[1. Ceratum Cantharidis.-Cantharides Cerate. Cantharides, 320; Yellow Wax, r80; Resin, 180; Lard, 220 ; Oil of Turpentine, 150 .

Cantharides Cerate is contained in Emplastrum Picis Cantharidatum. See Burgundy Pitch, p. 522.

2. Collodium Cantharidatum.-See Pyroxylin, p. 665.

3. Tinctura Cantharidis.-Tincture of Cantharides. Cantharides, 50 ; by percolation with Alcohol to 1000.

Dose, I to $15 \mathrm{~m}$. ; .06 to I.00 c.c.]

\section{ACtion OF CANTHARIDES.}

External.-Cantharides is a powerful irritant; but it is slower in its action than most [irritants]. If any of its preparations are applied to the skin, no effect is noticed for two or three hours; then a tingling, burning pain is perceived. Soon the part becomes red from vascular dilatation, the drug now producing its rubefacient effect. The next stage is the formation of several vesicles. These soon run together to form one large bleb full of clear serum. Not only is cantharides an irritant and vesicant, but it is a powerful counter-irritant, probably dilating by reflex action the vessels of the deep-seated organs under the point of application. 
Cantharidin can be absorbed by the skin in sufficient quantity to produce internal effects.

Internal.-Cantharides is hardly used internally in medicine, as it is such a powerful irritant.

Gastro-intestinal tract.-It produces severe gastro-intestinal irritation, the patient suffering from abdominal pain, diarrhœa and vomiting. There may be a burning pain in the throat ; the motions and vomited matters may contain blood. These symptoms naturally cause much general depression.

Genito-urinary tract.- The active principle is absorbed into the blood, and a few hours after the gastro-intestinal symptoms have set in the patient complains of great pain in the loins and strangury - that is to say, there is an urgent desire to [urinate]; the effort is very painful from vesical tenesmus, and the quantity of urine passed is very small; it may contain albumin and blood.

\section{Therapeutics of Cantharides.}

External.-Cantharides is the basis of many preparations the object of which is to stimulate the growth of hair, such as the following: Acetum cantharidis [B. P., cantharides, 1 ; glacial acetic acid, 5 ; water], $\mathrm{r}$; glycerin, $\mathrm{x}$; spiritus rosmarini, I; water, Io. Cantharides is very largely employed to raise a blister, and it is of all drugs the most commonly used counterirritant. It is applied to the chest in pleurisy, over the pericardium in pericarditis, over the inflamed nerves in neuritis, over the mastoid process in disease of the ear, over joints with chronic effusion into them, over the stomach when there is gastric pain, vomiting, etc. A blister applied over the nerve will often relieve pain in neuralgia. If a further counter-irritant effect is desired, the blister, which is usually pricked, may be irritated by the application of any irritating ointment. [Unguentum Sabinæ ; savine, 8 ; yellow wax, 3 ; benzoinated lard, 16 ;] was formerly much used for this purpose.] This, however, is very painful, and nowadays after the pricking some bland ointment is usually applied. The cantharides preparation should not be left on after the d-velopment of the bleb, lest the cantharidin should be absorbra Cantharides should not be applied to a part on which 
the patient lies, for a bed-sore may form ; nor must it be used in renal disease ; and it should be carefully employed in children or debilitated persons. It ought not to be applied to paralyzed limbs. [The cuticle raised by a blister may be used for skingrafting.]

Internal.-The drug is rarely given internally, but it has been used with success in small doses in cases of chronic gleet. Sometimes, [but not often,] it relieves chordee. [Small doses are sometimes useful in the late stage of acute desquamative nephritis. It has been recommended for diabetes insipidus. Cantharidin, in the form of potassium cantharidinate ( $1 \frac{1}{00} \mathrm{gr}$.; $.0006 \mathrm{gm}$. hypodermatically) has been used as a remedy for pulmonary tuberculosis, and for lupus. For all that cures of both of these diseases have been claimed, the value of this drug is by no means established.]

\section{TOXICULOGY.}

[Symptoms. - ] In severe cases of poisoning there may be greatly increased sexual desire, numerous seminal emissions, violent priapism, with swelling and heat of the genital organs. In women cantharides may cause abortion or induce menstruation. Post-Mortem.-Intense gastro-intestinal inflammation is present, consequently swelling, ecchymoses, and hpyeræmia of the mucous membrane of the alimentary canal are observed. The kidneys are found to be very congested, and in the early stage of acute nephritis. There is also much inflammation of the genito-urinary mucous membrane.

[ Treatment.-There is no chemical nor physiological antidote to Cantharides. The stomach should be emptied by emetics (see p. 139), or washed out by the stomach-pump. Mucilaginous and demulcent liquids should be freely given. Opium is indicated to relieve the pain and gastro-enteritis.]

\section{ICHTHYOLUM.}

ICHTHYOL.-[Not official. $]$-Synonym.-Ammonium Ichthyol-Sulphonate.

SoURCE, - A bituminous quartz containing the fossil remains of fish is distilled with [concentrated Sulphuric Acid, and the distillate is treated with a concentrated solution of Sodium Chloride, whereby the Sulphuric and Sulphurous Acids are removed.] The distillate is then saturated with Ammonia.

Characters. - A viscous, reddish-brown, almost black substance, having a tarry odor and containing 15 per cent. of Sulphur. Solubility.-Soluble in Water, Glycerin, Oils and Fats.

Dose, yo to $20 \mathrm{gr}$. ; [.60 to $\mathrm{x} .20 \mathrm{gm}$. 
Lithium, Sodium, and Zinc Icbthyol Sulphonates are prepared. [The name Ichthyol is understood to refer to the Ammonium Salt.]

\section{ACtion aNd Therapeutics OF IChTHYol.}

[Ichthyol is an active reducing agent.] It is chiefly used externally for chronic eczema and psoriasis. An ointment with lanolin and ichthyol 20 to 50 per cent. [is recommended by Von Nussbaum for the treatment of erysipelas. It has been used in the form of a suppository for chronic prostatitis.] Ichthyol paste (starch, 40, moisten with water, 20, rub in ichthyol, 40, and then a strong solution of albumin, $I$ or more) is recommended for acne rosacea. Ichthyol has been given as a pill for chronic rheumatism in dose from to to $30 \mathrm{gr}$; ; [.60 to $2.00 \mathrm{gm}$. Under the name of Thiol, a mixture of sulphuretted hydrocarbons has been used as a substitute for ichthyol, because it is less offensive. It has been applied as an ointment in vaseline, (I to 8$)$.]

\section{HIRUDO.}

THE LEECH.-[B. P., not official.]-Two species are employed: (I) Sanguisuga medicinalis, the speckled leech (belly greenish-yellow, spotted with black); (2) Sanguisuga officinalis, the green leech (belly olivegreen, not spotted), (nat. ord., Annelida).

Characters of both species.-Body soft, smooth, [5] or more [cm.] long, tapering to each end plano-convex, wrinkled transversely, back olivegreen, with six rusty-red longitudinal stripes. Each leech has a muscular disc at each end. In the centre of the anterior one is a tri-radiate mouth, provided with three saws and two rows of teeth.

[The leech, although not a drug, is considered with this group for convenience.]

\section{Action and Therapeutics of the LeEch.}

Leeches are used to remove blood. They are usually applied over deep-seated organs when they are congested, and great re: lief is often afforded. For example, three or four leeches near the liver, when that organ is enlarged in heart disease, or one or two behind the ear, when the tym panic cavity is inflamed frequently do good. The leech being applied to the skin, the animal fixes itself by its sucker-like disc, makes a tri-radiate cut with its mouth, and draws into its body, which consequently be- 
comes swollen, about a drachm and a half [6. c.c.] of blood. If this is not sufficient, a hot fomentation put on, after the animal is removed, may increase the quantity to half a fluid ounce; [15. c.c.]. The skin should be well washed with a little milk before the leech is applied. Occasionally the hæmorrhage requires pressure, or some local styptic, as ferric chloride [or better styptic collodion], to stop it. If leeches have to be applied to the mouth, rectum, or uterus, leech glasses, which only allow the head to protrude, should be used.

\section{GROUP VIII. \\ [The Organic Extracts.}

Thyroid, Suprarenal, and Brain Extracts, and Testicular Juice.

None of these are official.]

THYROIDEUM SICCUM.-Dry Thyroid [B. P., not official]. A powder prepared from the fresh and healthy thyroid gland of the sheep.

SourCE. - Remove the fat and connective tissue directly the sheep is killed. Reject cystic, hypertrophied or otherwise abnormal glands. Mince. Dry at $90^{\circ}$ to $100^{\circ} \mathrm{F}$. $\left[32.2^{\circ}\right.$ to $37.7^{\circ} \mathrm{C}$. $]$. Powder the dried product. Remove all fat by washing with petroleum spirit and again dry.

Characters. - Light dull-brown powder with faint meat-like odor and taste and free from odor of putrescence. Liable to become damp and then it deteriorates.

Composition.-The chief constituent is a proteid, which exists in the colloid matter and is called Thyroidin; it contains 9.3 per cent. of iodine and 0.5 per cent. of phosphorus.

Dose, 3 to ro gr.; [.20 to .6o $\mathrm{gm}$.]

LIQUOR THYROIDEI.-[Solution of Thyroid. B. P., not official.] A liquid prepared from the fresh and healthy thyroid gland of the sheep.

SOURCE. - The fresh healthy glands are bruised with, for each gland, 34 minims [2.20 c.c.] of glycerin and 34 minims [2.20 c.c.] of a 0.5 per cent. solution of [carbolic acid]. Let stand for 24 hours, strain and add enough of the carbolic acid [solution] to make 100 minims [6.70 c.c.].

Characters. - A pinkish, turbid liquid, free from odor of putrescence. To be freshly prepared and kept in stoppered bottles. Strength.-100 minims [6.70 c.c.] represent one gland.

Composition.-As of the powder.

Dose, 5 to 15 m.; [.30 to r.00 c.c.] 


\section{Action of Thyroid Extract.}

Circulation.-Thyroid administered to man increases considerably the rate of the pulse, causes palpitation, enfeebles the cardiac beat, and makes the skin flushed and moist. Experiments on animals have failed to reveal the precise cause of this. The blood-pressure falls when a decoction of the gland is injected ; the fall is vasomotor, for the heart is not affected. Ordinary doses produce no effect on the blood except an increase of lymphocytes.

Excretion.-The active constituents of thyroid gland are probably excreted entirely through the kidneys. Large doses may cause diarrhœa.

Metabolism.-The administration of thyroid leads to a greatly increased oxidation of all the tissues, consequently an excess of urea, uric acid and xanthin bases are excreted in the urine and more [carbon dioxide] by the lungs. It follows that large doses of thyroid reduce the body weight.

Kidneys. - The quantity of urine is increased by thyroid gland, which may cause sugar to appear in the urine.

Nervous system. - Occasionally a fine tremor, restlessness, and insomnia are caused by large doses.

\section{Therapeutics of Thyroid Extract.}

It is known that human beings or monkeys whose thyroid is excised become myxœdematous, and that all sufferers from myxœdema have atrophied thyroid glands. If a preparation of sheep's thyroid is given to patients suffering from myxœdema, all the symptoms disappear, usually in about six weeks. The effect is as striking as anything in medicine. The [solution] is better than the powder, for that may decompose. It is best to begin with $5 \mathrm{~m} .,[.30 \mathrm{c.c}$.$] thrice daily, in water, gradually to increase$ the dose till $10 \mathrm{~m} .,[.60 \mathrm{c.c}$.$] are given, and when all symp-$ coms have disappeared it will be necessary for about ro m., [.60 c.c.] to be taken twice a week for the rest of the patient's life to prevent recurrence. When the treatment was first introduced the glands were eaten, or transplanted under the skin, or the ex- 
tract was administered subcutaneously ; but equally good results are obtained by giving the [solution] or the powder by the mouth: compressed tablets of the powder are very convenient and much used. A diminution of certain goitres follows the giving of thyroid, but it is useless in exophthalmic goitre. Cretinism is also marvellously benefited, both mentally and bodily, by thyroid preparations, especially if given early in the patient's life. A few cases of imbecility in children, a few of climacteric insanity, and a few of tetany have been much improved by thyroid. Chronic psoriasis, which has resisted all other treatment, often disappears if the patient be put to bed and take daily enough of thyroid preparations to keep him on the brink of poisoning by them, but unfortunately the disease often returns when the treatment is discontinued.

Thyroid preparations have been much used for obesity, but the practice is not to be recommended. They must be carefully given to those suffering from cardiac disorder. A preparation called Iodothyrine, which contains the active principles of the gland, has been used lately. [A milk-sugar triturate of this is given in daily dose of 15 to 3 o gr.; I. to 2 . gm.]

Poisoning.-An overdose of a thyroid preparation causes an exaggeration of the effects already described. The most evident are rapid pulse, slight pyrexia, headache, nausea, diarrhœa, restlessness, pains in the limbs, pruritus, and rarely delirium. These symptoms are termed "Thyroidism." If large doses be given to monkeys for a long period of time, a condition termed "Chronic Thyroidism" is produced. The symptoms of it are emaciation, muscular weakness, paresis, some alopecia, erection of some of the hairs on the head, proptosis, dilatation of pupils, widening of palpebral fissure, and death from asthenia. In some respects these monkeys resemble patients suffering from exophthalmic goitre. [Very large doses taken for a long time make patients thin and also produce degeneration of the cardiac muscle so that permanent disability may result. Surgeons are especially liable to make this error.]

SUPRARENAL EXTRACT [Not official] is prepared from the supra- 
renal capsules of the sheep, [ox and pig]. The active principle exists only in the medulla of the gland. It may be given in tablets.

[Dose, 5 to $20 \mathrm{gr}$. ; .30 to $\mathrm{r} .20 \mathrm{gm}$.]

\section{Action and Therapeutics of Suprarenal Extract.}

This has been shown experimentally [to increase the tone of all muscular tissue, mainly if not entirely by direct action (Oliver and Schäfer), to constrict the small arteries through its action on the vasomotor centre (Szymonowicz and Cybulski), and to raise blood-pressure more than any other known substance.] It inhibits the heart through the vagus and produces a muscle curve like veratrine. [It may be used as a local vaso-constrictor in minor surgery. On account of this property it may be applied to inflamed tissues so that these may be rendered anæsthetic by cocaine. For topical application a filtered, freshly-made aqueous solution should be employed; it may be sterilized by heat without destroying its active principle. All antiseptics, used as preservatives, are disappointing. The active principle has been isolated by Abel for which he proposed the name epinephrin. Its salts produce an exceedingly powerful effect on blood-pressure.] Treatment should begin with $1 \mathrm{gr}$; [.०6 gm.], of the extract as [powder dry on the tongue and swallowed without water] thrice daily, but the dose should be rapidly pushed [to $5 \mathrm{gr} . ; .30 \mathrm{gm}$.$] . The gastric contents have no effect upon the$ extract. [It should never be given hypodermatically on account of the collapse which it causes when administered in this way. It is of great value in the treatment of "Hay-fever" given internally and also applied locally. It is useful in the treatment of acute and chronic bronchitis, bronchial asthma, congestion and œdema of the lungs, hæmoptysis, and œdema of the glottis. It may be cautiously used in diseases of the heart, which it stimulates from direct action on the heart muscle.] Some writers claim that it benefits Addison's disease, but others have failed to notice any improvement.

[BRAIN EXTRACT.-(Not official.) The gray matter of a sheep's brain is sterilized, macerated in aseptic glycerin and filtered by the method of 
D'Arsonval, being sterilized under carbon dioxide gas of a pressure of thirty atmospheres.

Dose, 15 m., r.00 c c., hypodermatically.

USES OF Brain Extract.

Paul has made use of this for the treatment of various nervous disorders and claims excellent, although not always constant, results. Febrile reaction, prostration, and in some cases cardiac weakness, has followed its administration.

TESTICULAR JUICE (Not official) is prepared in the same way as brain extract.

Dose, 10 to $20 \mathrm{~m}$.; .60 to r.20 c.c., hypodermatically.

\section{Uses of Testicular Juice.}

Brown-Séquard believed that certain organs supply to the body a natural ferment which is essential to health. When this ferment is absent the vital forces degenerate. Under the use of this fluid the functions of organic life are performed with new vigor. Favorable reports of its use in many hundreds of patients suffering from organic nervous diseases are on record.]

Extracts of many other organs, especially bone marrow and thymus, have been employed in medicine, but there is so little evidence that they are of any use that they need not be referred to here.

\section{GROUP IX. \\ [The Antitoxins and Serums.}

Diphtheria and Tetanus Antitoxins, Antistreptococcic, Antipneumococcic, and Antivenomous Serums, Plague, Cholera and Typhoid

Serums and Inoculations, Nutrient Serum, and Hydrophobia Antidote.

None of these are official.]

DIPHTHERIA ANTITOXIN.-[Not official.]-When the bacillus of diphtheria grows in the body it produces toxins, albumoses, and an organic acid, and provokes the formation of a substance (called an antitoxin) which is found in the blood. This antitoxin is an antidote to the toxin of the diphtheria bacillus, and it is largely owing to the production of it that the patient 
is enabled to survive, and his chances of surviving are enhanced if antitoxin is administered to him to aid that which is formed in his body.

SOURCE. - Diphtheria bacilli are grown in a flask containing some nutrient broth (e.g., meat brothi), to which 0.5 per cent. of sodium chloride and 2 per cent. of commercial peptone have been added. At the end of some weeks the bacilli are filtered off, and the fluid left contains a large amount of diphtheria toxin, and it should be of such strength that .or c.c. $[11 / 2 \mathrm{~m}$.] of it will kill a good-sized guinea pig. From .02 to 1.00 c.c. [3 to $15 \mathrm{~m}$.] of it is aseptically injected into the jugular vein of a horse; this produces șlight symptoms. As soon as they are past a larger dose is injected, and so the dose is gradually in. creased until Ioo c.c. [25 f. dr.] or more are given at each injection. This leads to the formation of a large amount of antitoxin in the blood serum. At the end of some months the horse is bled to 8 liters [quarts] in a sterilized vessel, the blood coagulates, and the antitoxic serum is put into sterilized bottles and hermetically sealed, a little carbolic acid or other antiseptic being added to prevent decomposition.

The details may be modified, as horses vary in their reaction to the toxin, and toxins vary in strength, but the essentials of the method always remain the same.

Mode of Administration. - The antitoxic serum has been shown to be useless when given by the mouth, perhaps because it is destroyed in the liver. Therefore it is always injected subcutaneously; usually between the shoulders or on the side of the abdomen. Before injection the skin must be thoroughly washed with an-antiseptic, and all ordinary antiseptic precautions should be taken. After injection the puncture should be healed with antiseptic gauze, jodoform and collodion. The antitoxin should be taken from a fresh bottle. A special syringe, so constructed that all the parts of it can be boiled before use is employed.

Dose.-It is better to give a small dose of a concentrated rather than a large dose of a dilute antitoxin. The dose is the same for children and adults. The strength of it, which is stated on the bottle, varies between 200 and 2500 units per cubic centimeter, but it is usually about 500 . The antitoxin must be obtained from a reliable source. The quantity given should be such that from 4000 to 12,000 units or even more are injected in the first twenty-four hours after the patient comes under treatment. This amount may be divided into two or three doses, but should be repeated on the second and third days, if necessary. A unit is the smallest quantity of antitoxic serum, which when mixe with a certain quantity of a standard diphtheritic toxin and with it injected into the subcutaneous tissue of a healthy guinea-pig weighing from 250 to 300 grammes [ 8 to $10 \mathrm{oz}$.], protects the animal from death within four days.

It is impossible in a work like this to give a more precise definition, for all diphtheritic toxin consists of a toxin proper, which is poisonous, and toxoid bodies which, although not poisonous, will neutralize the antitoxic properties of antitoxic serum. Therefore, to standardize antitoxic serum it must be tested against diphtheritic toxin in which the proportion of toxins proper and toxoids 
and the neutralizing activity of the latter as regards antitoxin are known and constant. Such a standard toxin is kept in the Government testing department in Berlin, and the strength of all diphtheritic antitoxins should be expressed in terms of it.

\section{Action and Therapeutics of Diphtheria Antitoxin.}

Antitoxin serum diminishes all the symptoms of diphtheria, and in particular it greatly lessens the liability to sudden heart failure. If the diphtheria toxin be-administered to animals fatty degeneration of the heart is found after death, but if they have also had antitoxin this is absent. Both clinical and experimental evidence show that after antitoxin is given, although the bacilli continue to exist in the throat, the formation of membrane ceases and that which is present rapidly disappears; therefore laryngeal diphtheria rarely follows faucial if antitoxin is used early, the patient becomes less anæmic, his pulse improves, and his temperature may fall a little, although this is less influenced by antitoxin than are the other symptoms of diphtheria. The maximum effect of the antitoxin is not seen until twenty-four hours after injection. All reliable collections of cases show that the mortality, especially in children, is much less when the antitoxin is used. It should be given at the earliest possible moment, even if it is only likely that the patient is suffering from diphtheria, for the number of fatal cases is less when antitoxin is used early in the illness. The benefit is more marked in laryngeal than in other varieties of diphtheria, the mortality of tracheotomy cases falling by one half. The frequency of the occurrence of paralysis is not diminished, but the percentage of recoveries in cases with paralysis is slightly increased.

Symptoms of poisoning are sometimes seen after this antitoxin has been given, but they are unimportant. They are not due to the antitoxin in, but to some other constituent of the serum, for they may follow when the simple serum of animals is injected into the human subject. The most common is a rash, met with in about 35 per cent. of the patients injected; it may appear as late as the end of the third week after injection, but it is usually seen at the end of the first week. In a few cases a second rash is observed after the first has faded. Usually it is a 
mere erythema, but it may be papular or uticarial. Commonly it disappears in three days. Pains in the joints and slight swelling of them are occasionally present [and finally somewhat rarely there has been observed an irregular temperature range and consecutively emaciation and death; evidently pointing toward an acquired septicæmia. Further in a few cases an early fatal result has been reported. Browne and Benda both have shown that in a fatal issue, nephritis, in the majority of cases, is the cause of death, and clinically hæmorrhagic nephritis is by no means rare. Inasmuch as this remedy militates solely against the infection of the Klebs-Lœffler bacillus and clinically most cases of diphtheria are cases of mixed infection, the usual local antiseptic and general supporting measures must not be omitted. The danger of antitoxin lies in the horse-serum, for, many years before antitoxin was made, the results of injection of an alien serum had been pointed out. Concentrated serums then should be preferred in that they give the largest amount of antitoxin with the smallest amount of serum.

So far as prophylaxis is concerned, the question is still sub judice, many failures are reported, and indeed instances of reinfection have occurred even after suitable doses of antitoxin have been used during a previous attack.]

TETANUS ANTITOXIN.-[Not official.]-This is prepared on the same principles as diphtheria antitoxin, and is administered in the same way or directly into the brain. No marked success has attended its use, perhaps because tetanus is not usually [recognized] till long after infection, and perhaps because the tetanus toxin is very firmly united with the proteids of the central nervous system. [Recent reports indicate that this tntitoxin, particularly when injected directly into the brain, will soon take its place as a valuable and trustworthy remedy for this disease. ]

ANTISTREPTOCOCCIC SERUM.-(Not official.) Streptococci do not cause the diseases due to them by developing a toxin which circulates in the blood, but by being themselves carried all over the body, which attempts to kill them by developing a toxin fatal to them. To prepare antistreptococcic serum the virulence of the streptococci is increased by their passage through several rabbits; they are then grown on a medium which preserves their virulence. A horse is next treated with successive doses of cultivations of these living streptococci, each more potent than the former. 
At the end of the year the strength of the antitoxic serum of the horse is powerful enough for use. This is always given subcutaneously and the dose varies with different specimens of serum.

\section{Action and Therapeutics of Antistreptococcic Serum.}

Our experience of the value of antistreptococcic serum is limited, but it suggests itself as useful for those diseases which are principally due to infection by streptococci. Such are malignant endocarditis, erysipelas, surgical septicæmia, disease of the middle ear, thrombosis of the lateral sinus, and puerperal septicæmia. Successful cases of its use in these disorders have been recorded, and it might be used with advantage in any of the many diseases in which streptococci can be found. One great disadvantage of it is that it is always impossible to foretell whether it will be of use; this is because different cultivations of apparently the same streptococcus vary so widely in their properties that serum which is antibactericidal to one cultivation is not to another.

ANTIPNEUMOCOCCIC SERUM.-(Not official.) This is prepared in the same way as antistreptococcic serum, and like it is antibactericidal, not antitoxic. It has been used for diseases due to the pneumococcus, but the evidence of its efficacy is slight.

ANTIVENOMOUS SERUM.-(Not official.) Probably the poison of all venomous snakes is the same. Horses are rendered immune to snake poison by repeated injections of snake-venom, and Calmette and Fraser have shown that the hypodermatic injection of serum from such horses will cure those bitten by a venomous snake and also render persons immune to snake poison.

PLAGUE SERUM AND INOCULATION.-(Not official.) A serum obtained from horses inoculated with plague has been used in the treatment of human beings affected with plague. Its efficacy is doubtful. If it does good it is in virtue of its antibactericidal properties, for it is not antitoxic.

An emulsion of dead plague bacilli has been largely used to vaccinate those exposed to plague. Haff kine has shown that those thus vaccinated rarely contract plague when exposed to it.

CHOLERA SERUM AND INOCULATION.-(Not official.) This serum is of no therapeutic value, but there is some evidence that vaccination with cholera micro-organisms affords some protection. 
TYPHOID SERUM AND INOCULATION.-(Not official.) There is no evidence that the serum treatment of typhoid fever is of benefit. Inoculation by injecting dead bacilli in the abdominal wall with all antiseptic precautions has been practiced. Local inflammatory reaction with some pyrexia follows in a few hours, but usually soon passes off. Therefore it is best not to give the injection in the evening. It has not yet been shown whether this inoculation confers immunity, but the blood serum of an inoculated person [agglutinates] typhoid bacilli.

NUTRIENT SERUM.-(Not official.) When diphtheritic antitoxic serum was first introduced it was very dilute, and therefore large quantities (e.g., 320 c.c. [10 fl. oz.] in two days for a child five years old) were injected. Such quantities produced no ill-effects, and this fact suggested to Salter that serum might be injected subcutaneously as a food in human beings. He has shown by experiments on animals that while the injection of small quantities, by increasing the katabolism of the body, leads to an increase of urinary nitrogen and a loss of weight, when large quantities are injected the serum is utilized as a food to such an extent as more than to counterbalance the loss due to the induced increased katabolism, for life is much prolonged if animals are allowed no food nor drink except such as is provided by the subcutaneous injection of serum.

When the serum of one animal is injected into another of a different species, unimportant toxic effects, such as transitory pyrexia, a rash, and joint pains are frequently observed. Salter states that if serum be heated to $65^{\circ} \mathrm{C}$. $\left[149^{\circ} \mathrm{F}\right.$.] the bodies producing these effects and those exciting increased nitrogenous katabolism are destroyed, although the value of the serum as a subcutaneous food is not impaired.

Such a heated horse- or sheep-serum may be injected into man when, after severe abdominal operations, it is impossible or undesirable to introduce any food into the stomach or intestines, in certain severe cases of gastro-intestinal disease, as gastric ulcer, typhoid fever, infantile diarrhœa, or during the vomiting that occurs with severe post-diphtheritic paralysis. For an infant $3)$ to 40 c.c., [8 to $10 \mathrm{fl}$. dr.], for a child 60 to 80 c.c., [ 16 to $20 \mathrm{fl}$. dr.], and for an adult 100 to 120 c.c., [ 3 to $4 \mathrm{fl}$. oz.], may be used and repeated according to the severity of the case. If it is intended to administer repeated daily doses, the most satisfactory plan is to inject the liquid into the loose subcutaneous tissue of, say one loin on the first occasion, the opposite loin on the second, then into either axilla, then between the shoulder blades, and so on, thus ringing the changes and giving each shot a few days' rest before practicing a second inoculation at the same place.

HYDROPHOBIA ANTIDOTE.-[Although this is not an antitoxin nor a serum it is conveniently considered here.] A rabbit is inoculated from the spinal cord of an animal dead of hydrophobia, other rabbits are inoculated from this, and so through a series until the spinal cord (which is the chief seat 
of the virus in hydrophobia) contains a virus the incubation period of which is seven days. The spinal cord loses its virulence when exposed to the air, so that a series of spinal cords (each of which originally contained a virus the incubation period of which was seven days) can be prepared of greater or less virulence according to the time during which they have been exposed to the air.

Uses of Hydrophobia Antidote.

It is found that if a patient who has been bitten by a rabid dog is inoculated first with a rabbit's spinal cord of a low degree of virulence, and the next day with one of higher degree, and so on increasing the virulence of the injection, hydrophobia does not usually develop in him if the treatment is begun soon after the bite. [The reports of the use of this suggest that some of the deaths after treatment may be due to it rather than to the bite. Inasmuch as the incubation period of hydrophobia is so extremely variable, the pathological findings so inconstant and the symptoms so diverse, there is some reason for doubting the existence of the disease in man. At least the majority of alleged cases have been shown to have been those of various diseases. For this reason the antidote possesses but little interest save to those who are interested in spreading hydrophobia-phobia.] 



\section{APPENDIX I.}

\section{THE PHARMACOPGEIAL VEGETABLE DRUGS ARRANGED ACCORDING TO THEIR NATURAL ORDERS.}

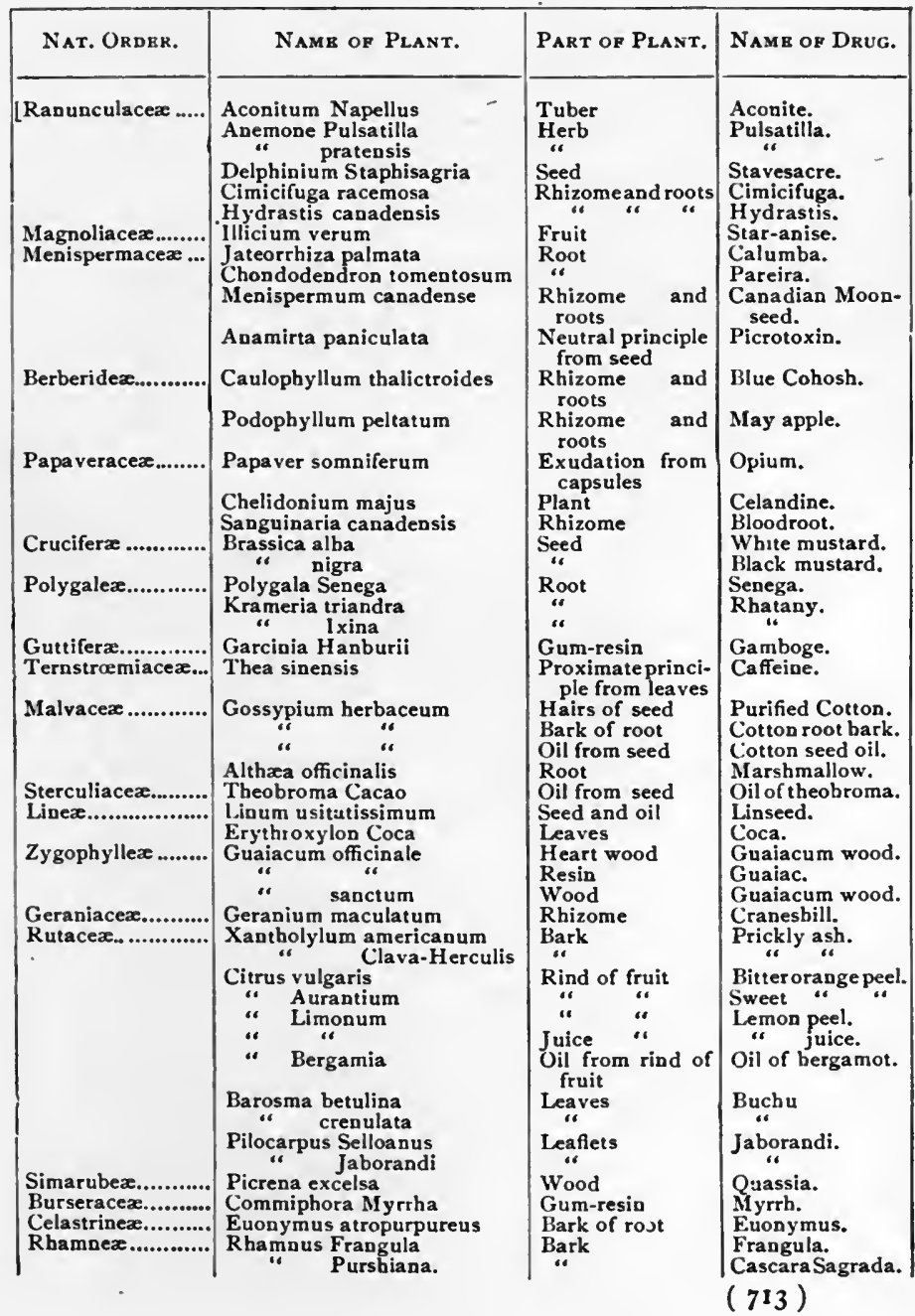




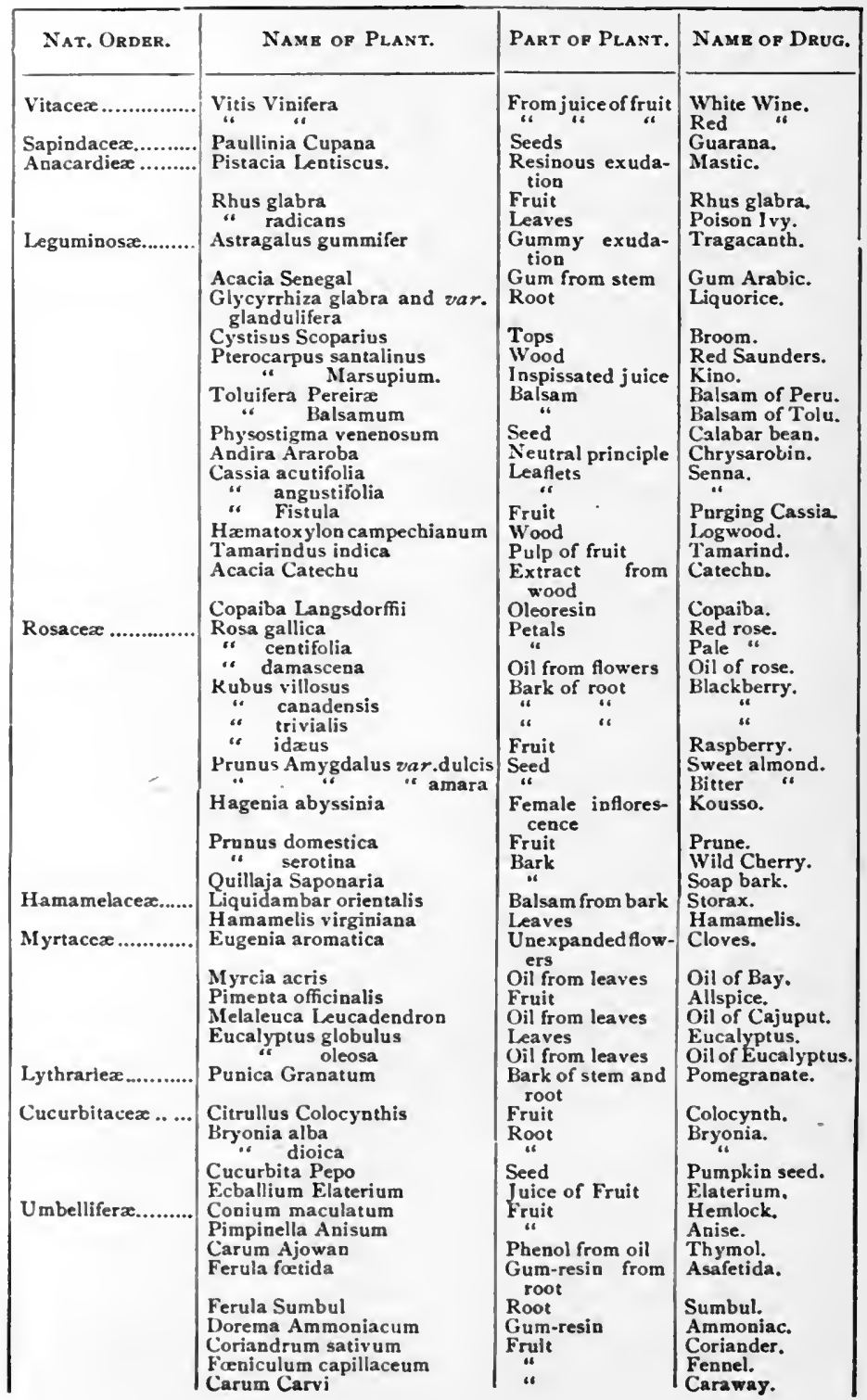




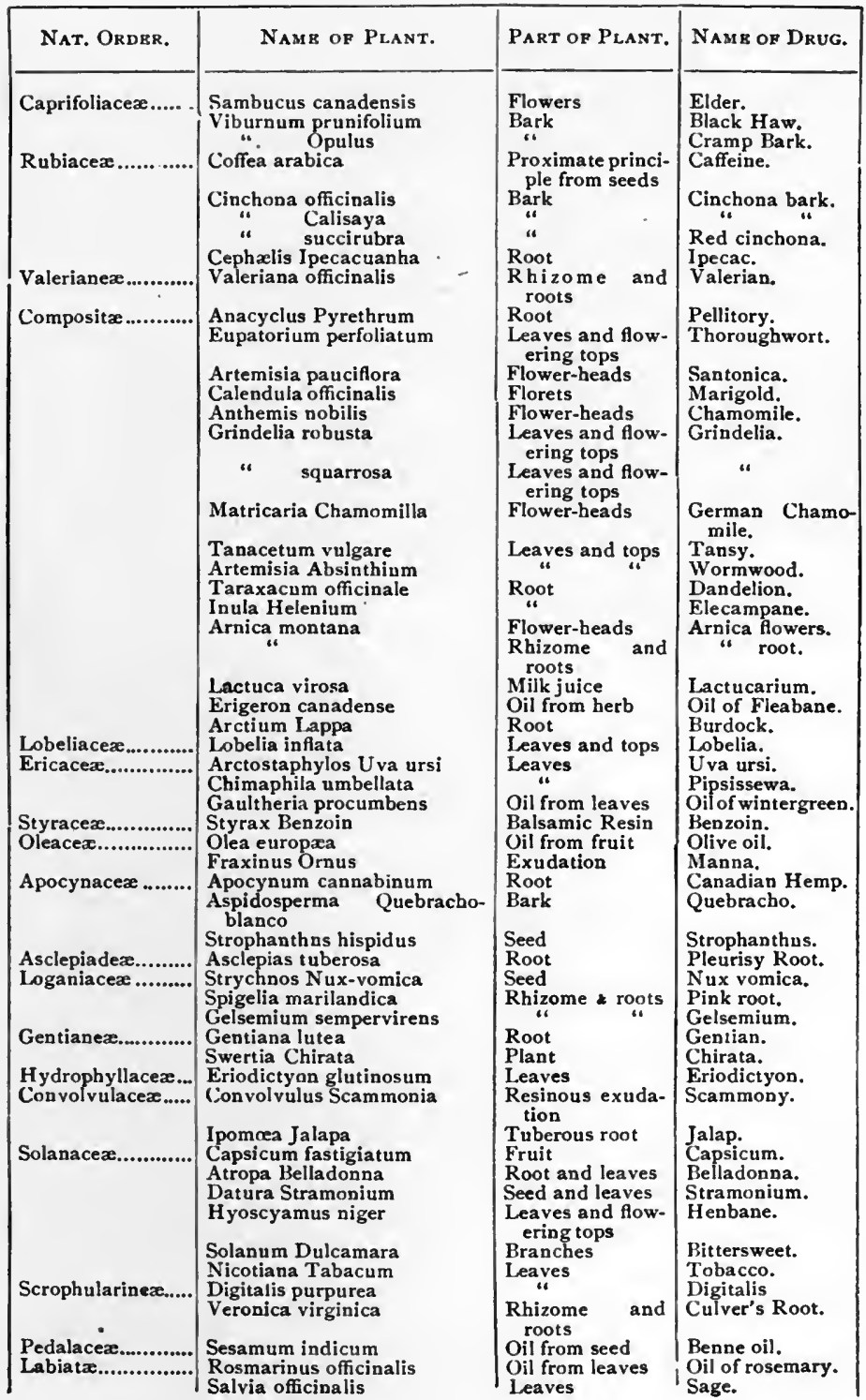




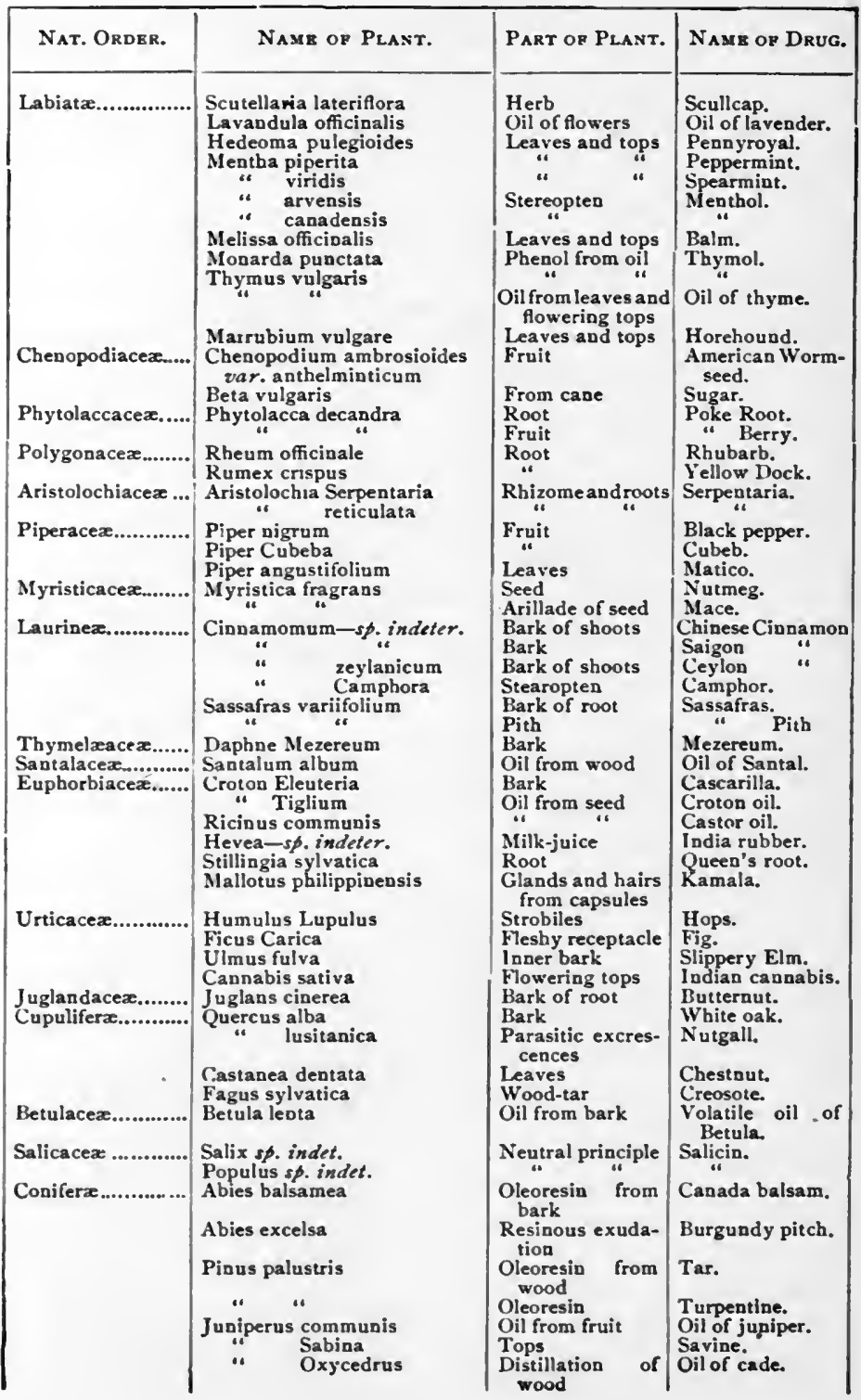




\begin{tabular}{|c|c|c|c|}
\hline Nat. ORder. & Name of Plant. & Part of Plant. & Name of Drug. \\
\hline 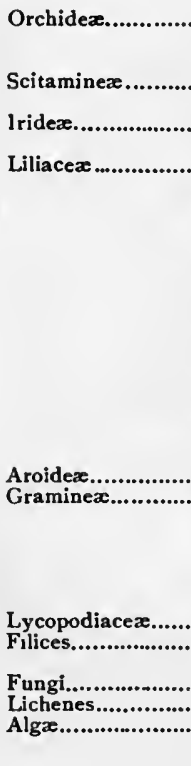 & $\begin{array}{l}\text { Cypripedium pubescens } \\
\text { Vanilla planifolia } \\
\text { Elettaria repens } \\
\text { Zingiber officinale } \\
\text { Crocus sativus } \\
\text { Iris versicolor } \\
\text { Allium sativum } \\
\text { Colchicum autumnale } \\
\text { “" } \\
\text { Convallaria majalis } \\
\text { Smilax officinalis } \\
\text { “ medica } \\
\text { Asagreapyracea } \\
\text { Veratrum viride } \\
\text { Urginea maritima } \\
\text { Aloe Perryi } \\
\text { "vera } \\
\text { Acorus Calamus } \\
\text { Agropyrum repens } \\
\text { Zea Mays } \\
\text { “ } \\
\text { Hordeum distichum } \\
\text { Saccharum officinarum } \\
\text { Lycopodium clavatum } \\
\text { Dryopteris Filix-mas } \\
\text { Claviceps marginalis } \\
\text { Cetraria islandica } \\
\text { Chondrus crispus } \\
\text { Gigartina mamillosa }\end{array}$ & $\begin{array}{l}\text { Rhizome * roots } \\
\text { Fruit } \\
\text { Rhizome } \\
\text { Stigmas } \\
\text { Rhizome roots } \\
\text { Bulb } \\
\text { Corm } \\
\text { Seed } \\
\text { Rhizome and } \\
\text { roots } \\
\text { Root } \\
\text { “" } \\
\text { Alkaloids from } \\
\text { seed } \\
\text { Rhizome and } \\
\text { roots } \\
\text { Bulb } \\
\text { Juice of leaves } \\
\text { Rhizome } \\
\text { "“ } \\
\text { Styles and stig- } \\
\text { mas } \\
\text { Fecula of seed } \\
\text { Seed } \\
\text { From cane } \\
\text { Spores } \\
\text { Rthizome } \\
\text { Sclerotium } \\
\text { Thallus } \\
\text { Plant } \\
\text { " }\end{array}$ & $\begin{array}{l}\text { Ladies" slipper. } \\
\text { Vanilla. } \\
\text { Cardamom. } \\
\text { Ginger. } \\
\text { Saffron. } \\
\text { Blueflag. } \\
\text { Garlic. } \\
\text { Colchicum root. } \\
\text { “" seed. } \\
\text { Convallaria. } \\
\text { Sarsaparilla. } \\
\text { "“ } \\
\text { Veratrine. } \\
\text { Veratrum Viride. } \\
\text { Squill. } \\
\text { Socatrine Aloes. } \\
\text { Barbadoes "“ } \\
\text { Sweet Flag. } \\
\text { Couch-grass. } \\
\text { Corn-silk. } \\
\text { Starch. } \\
\text { Pearl barley. } \\
\text { Sugar. } \\
\text { Lycopodium. } \\
\text { Male fern. } \\
\text { “" Ergot. } \\
\text { Iceland Moss. } \\
\text { Irish Moss. } \\
\text { " ] }\end{array}$ \\
\hline
\end{tabular}




\section{APPENDIX II.}

\section{[THE PHARMACOPCEIAL ANIMAL DRUGS ARRANGED ACCORDING TO THEIR NATURAL ORDERS.}

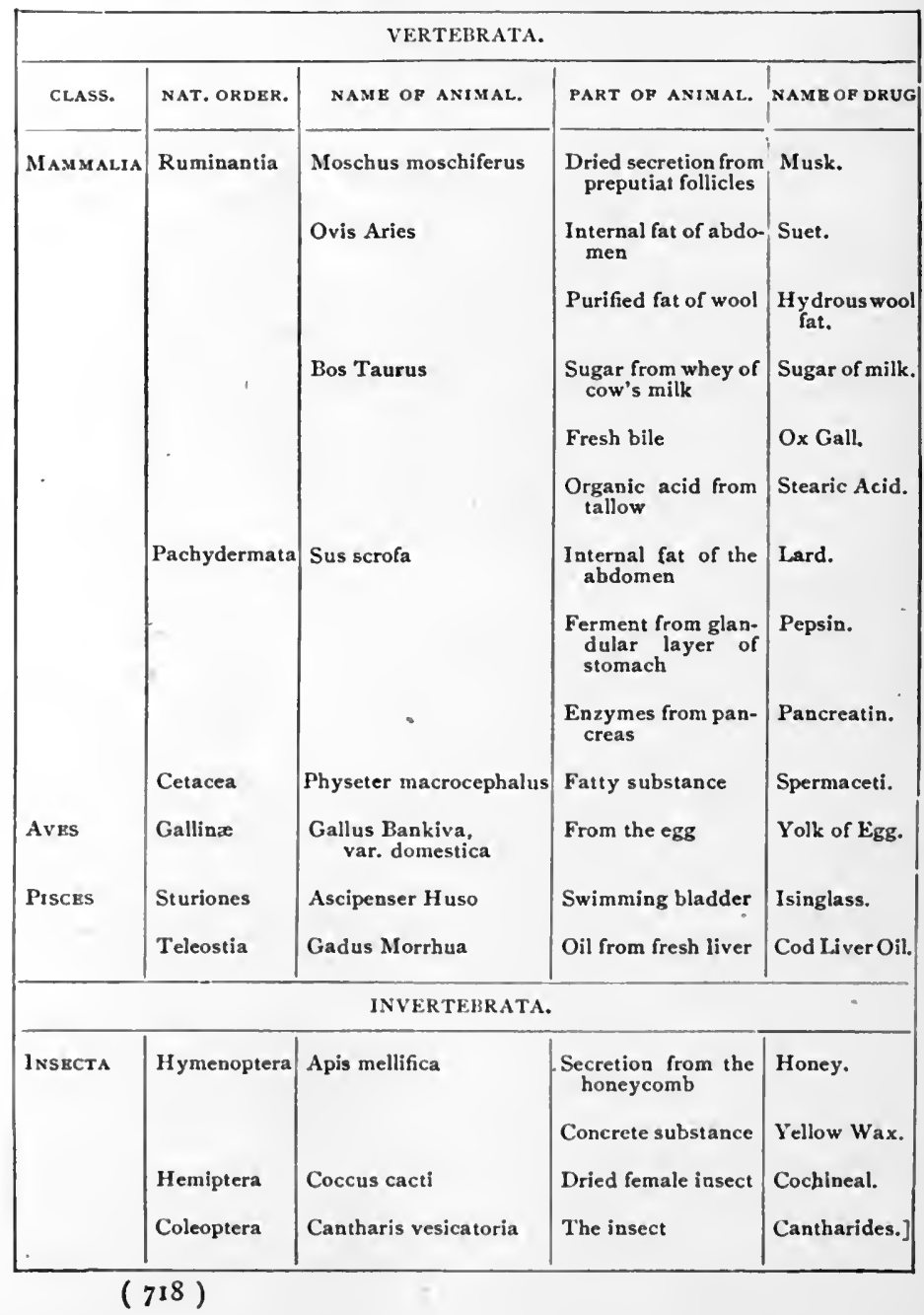




\section{APPENDIX III.}

\section{A LIST OF LATIN PHRASES COMMONLY USED IN THE WRITING OF PRESCRIPTIONS.}

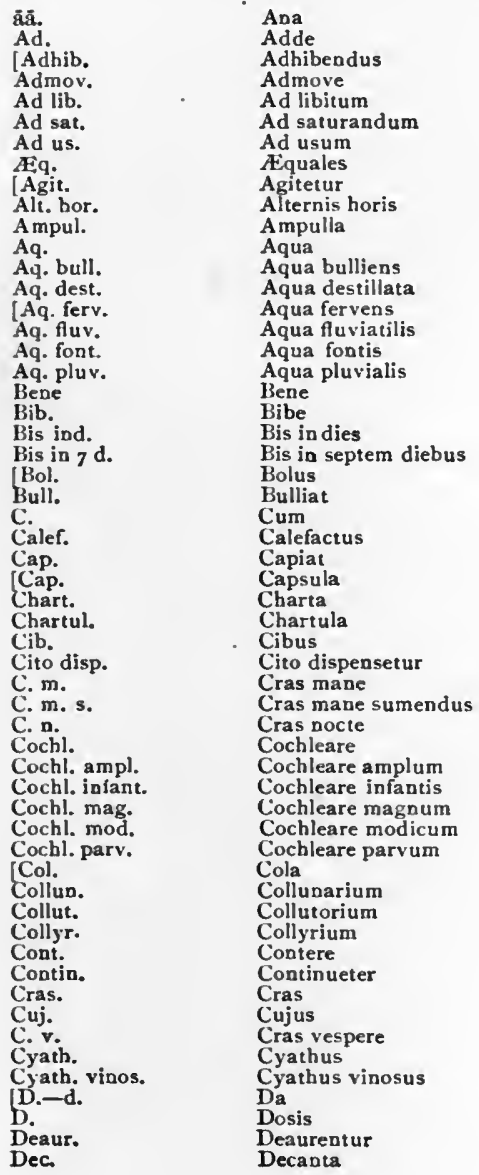

of each.

add.

to be administered.

apply.

to the desired amount.]

to saturation.

according to custom.

equal.

let it be shaken.

every other hour.

a large bottle.]

water.

boiling water.

distilled water.

hot water.

river water.

spring water.

rain water.

well.]

drink.

twice a day.

twice a week.

a large pill.

let it boil.]

with.

warmed.

let him take.

a capsule.

a paper.

a small paper.

food.

let it be dispensed quickly.]

to-morrow morning

to be taken to-morrow morning

to-morrow night.

a spoonful.

a table-spoonful.

a teaspoonful.

a table-spoonful.

a dessert-spoonful.

a teaspoonful.

strain.

a nasal wash.

a mouth wash.

an eye wash.

rub together.]

let it be continued.

to-morrow.

of which.

to-morrow evening.

a glassful.

a wine glassful.

give.

a dose.

let (the pills) be gilded.

decant.] 
D. d. in d.

Det.

Dieb. alt.

Dim.

Div.

D. in p. $æ$.

Exhib.

F. or ft.

F. h.

F. $m$.

F. pil.

Form.

Fs.a.

Garg.

Gtt.

Habt.

Hor. decub.

Hor. intermed.

H. s.

Iil.

Imp.

Ind.

IInstar

involv. gelat.

Lat, dol.

Len.

Len. ter.

Mane

Mane primo

Mic. pan.

Mit.

Mode dict.

Mod. præscript

[Non repetat.

O. $\mathrm{m}$.

Omn, bih.

Omn. hor.

O. $\mathrm{n}$.

$P$. or pt.

Part. aeq.

Penicil. cam.

Pil.

P. r. n.

[Q. hor.

Q.1.

Q. $s$.

Q. v.

k.

[Red. in pulv.

Rep.

[Sat.

Semel

Semi b.

Sesq. $h$.

Sic.

Sin.

Sing.

Si op. sit.

Sum.

T.d.

[Ter.

Ter. bene

Trit.

Utere

Vehic.

Ver.

Vesp.
De die in diem

Detur

Diebus alternis

Dimidius

Divide

Divide in parts æequales

Exhibiatur

Fiat

Fiat haustus

Fiat mistura

Fiat pilula

Formula

Fac secundum artem

Gargarysma

Gutta or guttæ

Habeat

Horâ decubitûs

Horis intermediis

Horâ somni

Illico

Impone

Indies

Instar

Involve gelatino

Lateri dolenti

Lenitur

Lenitur terendo

Mane

Mane primo

Mica panis

Mitte

Mode dictu

Modo prascripto

Non repetatur

Omni mane

Omni bihorå

Omni horâ

Omni nocte

Perstetur

Partes aquales

Penicillum camelinum

Pilula

Pro re natâ

Quâquå horâ

Ouantum libet

Quantum sufficit

Quantum volueris

Recipe

Redactus in pulverem

Repetatur

Satis

Semel

Semi hora

Sesqui hora

Sicetur

Sine

Singulorum

Si opus sit

Sumat or sumendum

Ter in die

Tere

Tere bene

Trituretur

Utere

Vehicnlum

Verus

Vesper from day to day.

let it be given.

on alternate days.

one-half.

divide.

divide into equal parts

let it be ziven.

let it be made.

make a draught.

make a mixture.

make a pill.

a prescription.

make according to art.

a gargle.]

drop or drops.

let him have.

at bed time.

at intermediate hours.

at bedtime.

immediately.

apply.]

daily.

the size of.

coat with gelatin.]

to the painful side.

easily.

by rubbing gently.

in the morning.

early in the morning.

bread crumb.]

send.

in the manner directed.

in the manner prescribed.

let it not be repeated.]

every morning.

every two hours.

every hour.

every night.

continue.

equal parts.

a camel's hair pencil.

a pill.]

when required.

every hour.]

as much as is requisite.

a sufficient quantity.

at will.

take.

reduced to powder.]

let it be repeated.

sufficient.

once.

half an hour.

an hour and a half.

let it be dried.

without.]

of each.

if necessary.]

let him take or let it be taken.

three times a day.

Rub.

Rub well.

let it be triturated.

make use of.

menstruum.

genuine.

the evening.] 


\section{N D E X.}

[In all Latin titles of more than one syllable, the accented syllable is distinguished by the sign' placed after the corresponding vowel.

\section{ABREVIATIONS, 45, 719}

Absinthe, 553

Absinthic acid, 554

Absinthin, 554

Absin'thium, 553

Absinthol, 554

Abstra'cta, 36

Abstracts, 36

Absolute alcohol, 280

Absorbent cotton, 665

Aca'cia, 615

Accelerating centre, drugs acting on, 58

Ace'ta, 19

Acetanilid, 319

Acetanili'dum, 3r9

Acetylparamidopheno]

salicylate, 483

Acetic acid, 265

$$
\text { glacial, } 266
$$

ether, 300

Acetone-chloroform, 290

Ace'tum o'pii, 352 sci'llae, 432

Acetyl salicylic acid, 480

Acid, acetic, 265 diluted, 266 glacial, 266

arsenous, 223 solution, 224

benzoic, 656

boracic, 273

boric, 273

cacodylic, 228

camphoric, 650

carbolic, 329

crude, 328

ointment, 329

citric, 266
Acid, citric, syrup of, 266

chromic, 234

chrysophanic, 490,493

digallic, 593

di-iodosalicylic, $34 x$

gallic, 596

gallotannic, 593

hydriodic, syrup of, 248

hydrobromic, diluted, $25^{8}$

hydrochloric, 264

hydrocyanic, diluted, 134

hypophosphorous, di-

$$
\text { luted, } 24^{\circ}
$$

iodosalicylic, 342

lactic, 267

meconic, 350, 353

muriatic, 264

nitric, 264

diluted, 264

nitrohydrochloric, 264

nitrom uriatic, 264

oleic, $6 x$

phenic, 329

phosphoric, 265 diluted, 265

prussic, 134

pyrogallic, 596

salicylic, 475

stearic, 693

sulphanilic, 250

sulphuric, 263 diluted, 263 aromatic, 264

sulphurous, 272

tannic, 593 ointment, 593

troches, 593

tartaric, 266
Acids, 263.

A'cidum ace'ticum, 265

dilu'tum, 266

gla'ciale, 266

arseno'sum, 223

benzo'icum, 657

bo'ricum, 273

campho'ricum, $65^{\circ}$

carbo'licum, 329

cru'dum, $3^{28}$

chro'micum, 234

ci'tricum, 266

ga'llicum, 596

bydrobro'micum dilu'-

tum, 258

hydrochlo'ricum, 264 dilu'tum, 265

hydrocya'nicum dilu'tum, 134

bypophosphor'osum dilu'tum, 240

la'cticum, 267

meco'nicum, 353

ni'tricum, 264 dilu'tum, 264

nitrohydrochlo'ricum, 264 dilu'tum, 264

ole'icum, 611

phospho'ricum, 265 dilu'tum, 265

salicy'licum, 475

stea'ricum, 693

sulphu'ricum, 263

dilu'tum, 263

aroma'ticum, 264

sulphuro'sum, 272

ta'nnicum, 593

tarta'ricum, 266

Aconine, 437

(72I) 
Aconite, 437

Aconiti'na, 437

Aconitine, 437

Aconi'tum, 437

Acorin, $5^{82}$

Acrinyl sulphocyanide, 524

Actions, pharmacological, 46 therapeutic, 46 cumulative, $4^{\circ}$

Actol, 173

$A^{\prime}$ deps, 690

benzoina'tus, 690

indura'tus, 690

la'n $x, 694$ hydro'sus, 694

Adhesive plaster, 523

A'djuvans, 4 I

Administration of drugs, $3^{8}$

Adonidin, 436

Adonidi'num, 436

Esculap, 93, 144

E'ther, 297

ace'ticus, 300

broma'tus, 301

E'thyl bro'midum, 30r

African pepper, $54 \mathrm{I}$

Air, 74

Aix-les-Bains, 262

la-Chapelle, 262

Alantin, 459

Albumin Egg, 69I

A'Icohol, 279

absolute, 280

absolu'tum, 280

amylic, 289

amy'licum, 289

deodora'tum, 280

diluted, 279

dilu'tum, 279

deodorized, 280

ethyl, 279

ethylic, 279

phenyl, 329

tertiary amyl, $3^{1} 5$

Aldehyde, formic, 344

Ale, 281

Alkaline earths, 155

metals, 122

Alkaloids, 12

A'llium, 455

Allyl oxide, 455

sulphide, 455
Allyl sulphocyanide, 525

Allspice, 536

oil of, $53^{6}$

Almond, bitter, 612

Jordan, $6 x_{3}$

sweet, 6 ז 3

A'loe, 496

barbaden'sis, 496

curacoa, 496

purifica'ta, 497

socatri'na, 497

Aloes, Zanzibar, 497

Aloin, 498

Aloi'num, 498

Alpha-eucaine, 326

Alterative, 115

Althr'a, 6iI

Alum, 185

ammonio-ferric, 193

burnt, 186

dried, 186

potassium, 185

Alu'men, 185

exsicca'tum, 186

u'stum, 186

Alu'mina, hydrated, 186

Alu'mini hy'dras, 186 su'lphas, 186

Aluminum, 185

acetate, 186

hydroxide, 186

naphtol sulphonate, 187

and potassium sulphate, 185

sulphate, 186

Alumnol, 187

Amaroids, 12

American bellebore, 442 wormseed, 628

Ammonia, aromatic spirit of,

$$
149
$$

bakers', 150

liniment, 149

spirit of, 149

water, 149

Ammoniac, $5^{62}$ emulsion, 562

plaster, with mercury, 2 ro

Ammoni'acum, 562

Ammoniated glycyrrhizin, 617

mercury, 213
Ammo'nii be'nzoas, 657

bro'midum, 253

ca'rbonas, 150

chlo'ridum, 15

io'dum, 249

pi'tras, 153

valeria'nas, 557

Ammoniated mercury ointment, 223

Ammonio-ferric alum, 193 citrate, $x 94$

sulphate, 193

tartrate, 195

mercuric chloride, 220

Ammonium, acetate, solution of, 153

benzoate, 657

bromide, 253

carbonate, 150

chloride, 151

troches, 152

Ichthyol sulphonate, 699

iodide, 249

nitrate, 153

valerianate, 557

Atnmo'nium, 148

Amy'gdala ama'ra, 612 du'lcis, 613

Amygdalin, 462, 612, 656

Amyl colloid, 44r

ni'tris, 305

nitrite, 305

Amylene hydrate, 315

Amyle'ni hy'dras, 315

Amylic alcohol, 289

A'mylum, 667

Anzsthetics, general, 106

local, 201

dangers of, 108

Analgesics, 318

Anaphrodisiacs, $x 13$

Anarcotine, 350,366

Anethene, $55^{2}$

Anethol, 549

Ane'thum, 552

Anemonin, 45x

Angelic acid, 545

Angustura bark, $5^{86}$

Anhidrotics, 65

Anhydrogluco-chloral, 324

Animal charcoal, 276

Anise, 549

Ani'sum, 549 
Annidalin, $34^{\circ}$

Anodyne, Hoffman's, 298 colloid, 44:

Anodynes, local, sor

Anthelmintics, 50

Anthemene, 554

Anthemidin, 555

A'nthemis, 526

Anthemol, 526

Anticholagogues, 97

Antidote, arsenic, 193 hydrophobia, 710

Anti-emetics, 89

Antifebrin, 319

Antigalactagogues, 114

Antihidrotics, 65

Antikamnia, 321

Antimonial powder, 23x

Antimo'nium, 230

Antimo'nii et pota'ssii tar'tras, $23 x$

o'xidum, $23^{\circ}$

su'lphidum, $23^{\circ}$ purifica'tum, $23^{\circ}$

Antimo'nium sulphura'tum, 230

Antimony and potassium tartrate, 23I

black purified, $23^{\circ}$

oxide, $23^{\circ}$

pills, compound, 212

sulphide, 230 purified, $23^{\circ}$

sulphurated, 230

tartrated, $23 \mathrm{I}$

trioxide, $23^{\circ}$

trisulphide, 230

wine of, 231

Antinervin, $32 x$

Antiparasitics, 51

Antiperiodics, $5 \mathbf{x}$

Antipneumococcic serum, 709

Antipyretics, 7x, 318

Antipyrin, 321

Antipyri'num, 321

Antiseptics, 48, 328 gastric, 83 intestinal, 94

Antisialogogues, 80

Antispasmodics, 77

Antistreptococcic serum, 708

Antitoxin, diphtheria, 705
Antitoxin, tetanus, 708

Antivenomous serum, 709

Antizymotics, 50

Apenta, 93

Aphrodisiacs, 113

Apiol, 645

Apio'lum, 645 -

Apocynein, 632

Apocynin, 632

Apo'cynum, 632

Apomorphi'næ bydrochlo'. ras, $3^{6} 7$

Apomorphine hydrochlorate, $3^{6} 7$

Aporetin, 490

Appendix, 7r3

$A^{\prime}$ qua, 116 amm'oniæ, 149 fo'rtior, 148

amy'gdale am'arx, 6r2 an'isi, 549

aura'ntii flo'rum, 591 fo'rtior, $59 x$

campho'ræ, 649

chlo'ri, 243

chlorofo'rmi, $29 x$

cinnamo'mi, $54^{\circ}$

creoso'ti, 334

destilla'ta, I 6

foni'culi, 55 I

hydroge'nii dio'xidi, 120 me'nthæ piperi'tæ, 547

me'ntha vi'ridis, 548

re'gia, 264

ro'sæ, $55^{6}$ fo'rtior, 556

A'qux, 19

Arabin, 15, 614, 615

Arachin, 605

Araroba powder, 660

Arbutin, 63x, 636

Arge'nti cya'nidum, 17x

io'didum, $x 7^{2}$

ni'tras, 170 dilu'tus, I7

fu'sus, I $7 x$

o'xidum, $17 x$

Argentamine, 173

Arge'ntum, $x 70$

Argol, 129

Argonin, 173

Aristol, $34^{\circ}$

Aristo'lum, 340
Aristolochine, $5^{87}$

Armora'cia, 54r

A'rnica, $53^{\circ}$.

A'rnicæ ra'dix, 531

flo'res, $53^{\circ}$

Arnica root, 531

flowers, $53^{\circ}$

plaster, 539

Arnicin, 530

Aromatic elixir, 59r

fluid extract, $54^{\circ}$

powder, $54^{\circ}$

Arsenic, 223

antidote, 193

and mercuric iodide, solution of, 212

iodide, 224

trioxide, 223

white, 223

A'rseni io'didum, 224

Arsenous acid, 223 solution of, 224

A'rsenum, 223

Artanthic acid, 577

Artificial human milk, 687 oil of wintergreen, $4^{8 x}$

Asafetida, 560

Asafoe'tida, 560

Asaprol, 343

Asapro'lum, 343

Asçle'pias, 457

Asclepion, 457

Ash, prickly, 67x

Asparagin, 6ri, 6r7

Aspi'dium, 623

Aspidosamine, 459

Aspidospe'rma, 459

Aspidospermatine, 459

Aspidospermine, 459

Aspirin, 480

Astringents, 63 intestinal, 95

Atropi'na, 373

Atropi'næ sul'phas, 373

Atropine, 372, 373 sulphate, 373

Atropurpurin, $5^{13}$

Attar of rose, 556

Aurantiamarin, $5^{8} 9$

Aura'ntii ama'ri co'rtex, $5^{89} 9$ du'lcis co'rtex, $59^{\circ}$

Au'riet so'dii chlo'ridum,209

Au'rum, 209 
$\mathrm{B}^{\mathrm{AEL} \text { fruit, } 678}$

Baker's ammonia, 150

Baking soda, 140

Balm, 66r

Balsam, of copaiba, 571

of fir, 524

Friar's, 656

of Peru, 565

of Tolu, 566

Ba'lsamum Peruvia'num, $5^{65}$

Toluta'num, 566

Balsams, 14

Barbadoes aloes, 496

Barbaloin, 498

Ba'rii dio'xidum, 16x

Barium, $16 \mathrm{x}$

dioxide, $16 x$

peroxide, $16 \mathrm{x}$

sulphide, $16 x$

Barley, pearl, 620

Barosmin, $57^{\circ}$

Barus camphor, 649

Basham's mixture, 192

Basilicon, ointment, 523

Basis, 4I

Bassorin, 15, 61x, 6x4

Baths, 117

cold, 120

hot, Irg

indifferent, 117

tepid, 120

warm, II

Battey's fluid, 247

Battley's solution, 352

Bay oil, 663

rum, 663

Bearberry, 631

Beer, $28 \mathrm{x}$

Be'lx fruc'tus, 678

Bellado'nna, $37 x$

Bellado'nnx fo'lia, $37 x$ ra'dix, $37^{2}$

Belladonine, $37 x$

Benne oil, 663

Ben raconine, 437

Benzin, 279

Benzi'num, 279

Benzoic acid, $565,566,656$

Benzosol, 336

Benzoyl-guaiacol, 336

Benzoyl-sulphonic-imide, 346
Benzoylvinyldiacetonalkamin hydrochlorate, 328

Benzoin, 656

Benzoinated lard, 656

Ben roi'num, 656

Benzylic benzoate, 566

cinnamâte, 566

Berberine, 580, 640, 673

Bergamot, oil of, 546

Beta-eucaine, 328

Beta-naphtol, 342

Be'tula, volatile oil of, 480

Bhang, 38,

Bile, drugs acting on, 96

Bismuth, 181

Bismuth and ammonium ci.

trate, 182

Bismuth citrate, 182

naphtolate, 184

oxide, 184

phenolate, 184

naphtolate, $18_{4}$

salicylate, 183

subcarbonate, $18 \mathrm{x}$

subgallate, 183

subnitrate, 182

tetra-iodo-phenol-

-phthaleinate, 185

tribromophenolate, 183

Bismu'thi ci'tras, $18 z$

et ammon'ii, ci'tras, 182

salicy'las, 183

subca'rbonas, 181

subni'tras, 182

Bismu'thum, 18

Bitter almond, 612

apple, 505

cucumber, 505

gourd, 505

orange peel, 589

principles, 12

wine of iron, ' 196

Bitters, 580

Bittersweet, 635

Black antimony purified, 230

berry, 602

cobosh, 587

draught, 493

drop, 352

haw, 643

mustard, 524

pepper, 537

snake root, $5^{8} 7$
Black wash, $2 \times 8$

Bladder,action of drugs on, 70

Blaud's pills, Igo

Bleaching powder, 243

Bliss' cure, $3^{8} 3$

Blister beetles, 697

Blistering collodion, 665

Blood, drugs acting on, 5r root, 450

Blue cohosh, 643

flag, $5 \times 2$

mass, 210

methylene, 347

ointment, 2 ro

pill, 2 ro

stone, 179

vitriol, 179

Bodily heat, drugs acting on, $7^{1}$

Boluses, 2x

Boneset, 487

Bone-black, 276

Boracic acid, 273

Borax, 273

Boric acid, 273

Borneo camphor, 550, 649

Borneol, 530, 557

Boro-glyceride, solution of, 273

Boro-glycerin, glycerite of, 273

Bougies, 36

Bourboule water, 205

Brain, drugs acting on, 104 extract, 704

Brandy, 280

Braye'ra, 624

Brazilian coca, 398

Bromides, 252

Bromine, 252

Bromoform, 302

Bromofo'rmum, 302

Bro'mum, 252

Bronchial secretion, drugs affecting the, 77 vessels, drugs acting on, 78 spasm, 77

Broom, 434

Brown mixture, $6 \times 7$

Brucine, 393

Bryo'nia, 503

Bryonin, $5>3$ 
Bryony, 503

Bucco, 570

Buchu, 570

Buckthorn, 495

Burdock, 674

Burgundy, 281 pitch, 522 plaster, $5^{22}$

Burned lime, 157

Burnt alum, 186

Burnett's fluid, 176

Burton's line, I 70

Butternut, $49^{2}$

Butter of cacao, 666

of antimony, 233

of zinc, $x 75$

Butyl-chloral hy'dras, $3^{12}$ hydrati, $3^{12}$

Byne, 620

Bynol, 62x

ACAO butter, 666

Cactine, 447

Ca'ctus, 447

Cachets, 36

Cade, oil of, 522

Caffei'na, $3^{88}$

citra'ta, $3^{8}$ 9

efferve'scens, $3^{8} 9$

Caffeine, $3^{88}$ citrated, 389 effervescent, $3^{89}$

Cajuput, oil of, 527

Cajuputene, 527

Cajuputol, 527

Calabar bean, 399

Calabarine, 399

Calamine, $5^{82}$

Ca'lamus, $5^{82}$

Ca'lcii bro'midum, 254 car'bonas præcipita'tus, 155

chlo'ridum, 159

bypopho'sphis, 239

pho'sphas precipita'tus, $x 5^{8}$ su'lphas exsicca'tus, 159

Calcined magnesia, 163

Ca'lcium, $x 55$

beta-naphtol alpha-monosulpbonate, 343
Ca'lcium, bromide, 254

carbonate, precipitated, $\times 55$

chloride, 159

glycerophosphate, $24 \mathrm{I}$

hydrate solution of, 157

hypophosphite, 239

lacto-phospbate, syrup of, $15^{8}$

phosphate, precipitated, $15^{8}$

sulphate, dried, $x 59$

sulphide, crude, $26 \mathrm{x}$

Cale'ndula, 676

Calendulin, 676

Calloxylin, 665

Calomel, 212

Calu'mba, 580

Calumbic acid, $5^{80}$

Calumbin, $5^{80}$

Calx, 157

chlora'ta, 243

sulphura'ta, 26x

Cambo'gia, 508

Camphor, 648

Barus, 649

Borneo, 649

laurel, 648

monobromated, 649

Sumatra, 649

$\mathrm{Ca}^{\prime}$ mphora, 648 monobro'mata, 649

Camphorated oil, 649

Camphoric acid, $65^{\circ}$

Cannabin, $3^{8} 7$

Cannabinine, $3^{8} 7$

Cannabinon, $3^{8} 7$

Canada balsam, 524 turpentine, 524

Canadian bemp, $6{ }^{2}$ moonseed, 673

Canadine, $64 x$

Candle fish, 684

Cane sugar, $6 \times 9$

Cane'lla, $5^{82}$ bark, $5^{82}$

Canellin, $5^{82}$

Ca'nnabis, Indian, $3^{86}$ i'ndica, $3^{86}$

Cantharidal collodion, 665 pitch plaster, 522

Cantha'rides, 697

Cantharidin, 697
Ca'ntharis, 697

Caoutchouc, $66_{7}$

Capronic acid, $53 x$

Caprylic acid, $53^{1}$

Capsaicin, $54^{2}$

Capsicin, $54^{2}$

Ca'psicum, $54 \mathrm{r}$

Capsules, 36

Canquoin's paste, 176

Caraway, 551

oil of, 551

Ca'rbo, 276 anima'lis, 276 purifica'tus, 276

li'gni, 276

Carbolic acid, 328

Carbon, 276 bisulphide, 262 disulphide, 262

Carbo'nei disu'lphidum, 262

Cardamom, 544

Cardamo'mum, 544

Cardiac mechanism, drugs acting on, 55

Carlsbad water, 144

Carminic acid, 692

Carminative tincture, 544

Carminatives, 86

Carolina pink, 628

Carragheen, 608

Carron oil, 157

Ca'rum, 551

Carvol, 548, $55^{2}$

Caryophyllene, 534

Caryophyllin, 534

Caryo'phyllus, 534

Casca bark, 435

Casca'ra sagra'da, 495

Cascari'lla, $5^{8} 5$

Cascarillin, $5^{8} 5$

Cascarin, 496

Ca'ssia bark, '540

cinnamon, $54^{\circ}$

$\mathrm{f}^{\prime}$ stula, 485

oil of, $54^{\circ}$

purging, 485

Casta'nea, $45^{8}$

Castile soap, white, 607

Castor oil, 487

seeds, $4^{87}$

Catapla'smata, 36

Catechin, 597, 604

Ca'techu, 597 
Ca'techu, tannic acid, 597

Catechuic acid, 597

Cathartic acid, 485,493

pills, compound, 212 vegetable, 506

Cathartics, 92

Cathartogenic.acid, 493

Cathartomannit, 493

Cantharidal pitch plaster, 522

Caullophylline, 643

Caullophy'llum, 643

Caustic, lunar, 170 mitigated, $17 x$ potash, 122 soda, $13^{8}$

Vienna, 123

Caustics, 6r

Cayenne pepper, 54I

Celandine, $5^{12}$

Cephaëlic acid, $45 x$

Cephaëline, 45x

Ce'ra a'lha, 696 fla'va, 696

Cerasin, I5

Cera'ta, 20

Cerates, 20

Cerebral depressants, 106, $35^{\circ}$

Cerebral excitants, $37 \mathrm{I}$ stimulants, ro5

Ce'reus grandiflo'rus, 447

Ce'rii o'xalas, ${ }^{6} 6$

Cerin, 696

Ce'rium, 165 oxalate, 165

Cerous oxalate, 165

Cerylic alcohol, 696

Ceta'ceum, 69x

Cetin, 6gr

Cetra'ria, 621

Cetraric acid, $62 \mathrm{I}$

Cetylic alcohol, 69x

Cevadine, 442

Ceylon cinnamon, 539

Chaik, drop, 155 mixture, 155 powder, compound, 155 prepared, 155 troches of, 155

Chalybeate pills, 190

Chamomile, 554

German, 555

oil of, 554
Champagne, 28x

Charcoal, animal, 276 purified, 276 wood, 276

Charas, $3^{8} 7$

Cha'rta, 20 pota'ssii nitra'tis, 131 si'napis, 521

Chaulmoogra oil, $67^{8}$

Chelidonine, 512

Chelido'nium, 512

Chelerythrine, 450, 512

Chemical constitution, 47 incompatibility, $4 \mathrm{I}$

Chenopo'dium, 628

Cherry laurel, 655 wild, 462

Chestnut, $45^{8}$

Cheyne-Stokes breathing, drugs which produce, 79

Chima'phila, 636

Chimaphilin, 636

Chinese cinnamon, 540

Chira'ta, $5^{85}$

Chiratin, $5^{85}$

Chire'tta, 554

Chittem bark, 495

Chloral, 309

Chloral formamide, 313 hydrate, 309

Chlorolamide, 313

Chlora'lum formamida'tum, $3 \times 3$

Chloralose, $3^{14}$

Chloretone, 314

Chloreto'num, 314

Chloric ether, 291

Chlorinated lime, 243 soda, solution of, 243

Chlorine, 243 water, 243

Chlorobrom, 314

Chlorodyne, 291

Chloroform, 290

Chlorofo'rmum, 290 bellado'nnx, 379 purifica'tum, $29^{\circ}$

Chlo'rum, 243

Cholagogues, 96

Cholera serum and inoculation, $7 \circ 9$

Choline, $3^{87}$

Cholesterin, 694
Cho'ndrus, 608

Chromic acid, 234 anhydride, 234 trioxide, 234

Chro'mium, 234

Chrysophan, 490, 660

Chrysophanic acid, 490, 493 , 603,660

Chrysarobin, 490, 660

Chrysarobi'num, 490, 660

Churrus, $3^{8} 7$

Churchill's tincture, 247

Cicconardi's test, 170

Cigarettes, 36

Ciliary muscle, drugs acting on, 112

Cimici'fuga, $5^{8} 7$

Cimicifugin, $5^{8} 7$

Cincho'na, 463

red, $46_{4}$

ru'hra, 464

Cinchotannic acid, 464

Cinchonidi'næ su'Iphas, ${ }_{46} 6$

Cinchonidine, 463 sulphate, 467

Cinchoni'na, 467

Cinchoni'n $x$ su'lphas, 467

Cinchonine, 463,467 sulphate, 467

Cinchonism, 471

Cineol, 527, 528, 530, 626, 663

Cider, 28I

Cinnabar, 210, 222

Cinnamein, 565

Cinnamene, ${ }^{6} 7$

Cinnamo'mum ca'ssia, $54^{\circ}$ saigo'nicum, 539 zeyla'nicum, 539

Cinnamon, 539

cassia, $54^{\circ}$

Ceylon, 539

Chinese, $54^{\circ}$

oil of, $54^{\circ}$

saigon, 539

Cinnamic aldehyde, $54^{\circ}$ acid, 540, 565, 566, 656

Cinnamyl cinnamate, 566 cocaine, 412

Citrated caffeine, 389

Citral, 654 
Citrene, 654

Citrine ointment, 213

Citric acid, 266, 655

Citrullin, 506

Clarendon, 156

Claret, 28r

Clarified honey, 68x

Clark's powder, 473

Club moss, 668

Cloves, 534 oil of, 534

Clysters, 36

Co'ca, 4 II

Cocamania, 416

Cocai'na bydrochlo'ras, 412

Cocaine, 412

bydrochlorate, 412

Cocamine, 412

Coccogin, 532

Cocculus Indicus, 629

Co'ccus, $69 z$

Cochineal, 692 bug, $69^{2}$

Cocilla'ña, $46 x$

Codamine, 350

Codei'na, 366

Codeine, 366 phosphate, 366

Cod liver oil, 684

Coffee, 389

Cohosh, blue, 643 black, $5^{8} 7$

Colchiceine, 645

Colchicine, 645,646

Co'lchicum, 645

root, 645 seed, 646

Cold cream, 556 pack, 118

baths, 117

Collidine, 408

Collo'dia, 20

Collodion, 665 blistering, 665 cantharidal, 665 styptic, 593

Collodions, 20

Collo'dium, 665 cantharida'tum, 665 flex'ile, 665 sty'pticum, 593

Colloidal silver, 174
Colluna'ria, $3^{6}$

Colly'ria, 36

Colocynth, 505

Colocynthein, 506

Colocynthin, 506

Colocy'nthis, 505

Colocynthitin, 506

Colophony, 523

Coloring agents, 664

Columbo, 580

Common salt, 144 -

Compound antimony pills, $2 \times 2$

cathartic pills, 212

Condal, 144

Condy's fluid, 208

Confe'ctio ro'sa, 556 se'nnz, 493

Confection of rose, 555 senna, 493

Confe'ctiones, $2 x$

Confections, 21

Conhydrine, 406

Coniine, 406

Coni'um, 405

Conserves, $2 x$

Consti'tuens, $4 \mathrm{I}$

Consumptive's weed, 460

Contrexéville, 156

Convallamarin, $43^{2}$

Convalla'ria, 43 I

Convallarin, 432

Convolvulin, 502

Copa'iba, 57x

Copaiva, $57 \mathrm{I}$

Copaivic acid, $\mathbf{5 7 x}$

Copper, 179

oleate, 180

sulphate, $x 79$

Copperas, 189

Coriander, $55^{\circ}$

Coriandrol, $55^{\circ}$

Coria'ndrum, 550

Coridine, 408

Corn-silk, $63^{6}$

Cornutine, 637

Co'rrigens, $4 \mathrm{I}$

Corrosive mercuric chloride, $2 x 1$

sublimate, $22 x$

Corson's paint, 505

Coster's paste, 247

Coto, $60_{4}$
Cotoin, 605

Cotton, 665

absorbent, 665

purified, 665

root bark, 643

soluble gun, 665

seed oil, 666

Couch grass, 633

Coumarin, 676

Counter-irritant, $6 \mathrm{x}$

Court plaster, 695

Cramp bark, 644

Cranberry, high bush, 644

Cranesbill, 602

Cream of tartar, 129

Creams, 36

Credè's method, $x 73$ ointment, 174

Cremora, $3^{6}$

Creolin, 337

Greoli'num, 337

Creosol, 334

Creosote, 334

Creoso'tum, 334

Cre'ta prapara'ta, 155

Crocetin, 644

Crocin, 644

Crocose, 644

Cro'cus, 644

Croton chloral hydrate, $3^{12}$ oil, 504 seeds, 504

Crotonol, 504

Crude calcium sulphide, 261 carbolic acid, 328

Cubeb, 574 camphor, 575

Cnbe'ba, 574

Cubebic acid, 574

Cubebin, 574

Cu'ca, 4 II

Cucumber, squirting, 507 bitter, 506

Culver's root, 5 xx

Cumulative action, 40

Cupreine, ${ }_{464}$

Cu'pri su'lphas, 179

Cupric sulphate, 179

Cu'prum, 179

Curacoa aloes, 496

Cura'ra, 4rx

Curare, 4 II

Curari'na, 4IX 
Curarine, 4 II

Curd soap, 693

Cuspa'ria, $5^{86}$ co'rtex, 586

Cusparidine, 586 .

Cusparin, 586

Cusso, 624

Cutch, 597

Cymene, 528, 574

Cypripe'dium, 559

Cryptopine, 350

\section{DAIA'NA, 577}

Dandelion, 588

Daphnin, 532

Daturic acid, 382

Daturin, $3^{82}$

Deadly nightshade, 37I

Deco'cta, 21

Deco'ctum ad icte'ricos 512

Decoctions, $2 \mathrm{I}$

Definitions, 9

Deliriants, 105

Delphinine, 629

Delphinoidine, 629

Delpbisine, 629

Demulcents, 64

Deodorants, 50

Deodorized alcohol, 280 opium, 352

Depressants, cerebral, 106

Dermatol, 183

Deuteropine. 350

Dextrin, 620

Dextropinene, 550

Dextrose, 68r .

Diachylon ointment, 166 plaster, $\mathbf{I} 66$

Dia'lysis, 15

Dialyzed iron, 196

Diaphoretics, 64

Diastase, 620

Dichloromethane, 682

Diethylenediamine, $34^{8}$

Diethylsulphondimethylmethane, 317

Diethylsulphonmethylethylmethane, 3I 7

Digallic acid, 593

Digestants, 682

Digestive apparatus, drugs acting on, 79
Digitalein, 42 I

Digitalin, $42 \mathrm{I}$

Digitali'num pu'rum, rar ve'rum, $42 x$

Digita'lis, 42 I

Digitonin, $42 x$

Digitoxin, 421

Di-iodosalicylic acid, 342

Di-isobutylorthocresol

iodide, $34 \mathrm{r}$

Dill, $55^{2}$

Diluted alcohol, 279

Dimethylethylcarbinol, 315

Dinner pill, 499

Dionine, 363

Diosphenol, $57^{\circ}$

Dipentene, 546

Diphtheria antitoxin, 705

Direct action, 46

Disinfectants, 48

Dispensing the prescription, ${ }_{4} 6$

Dispermine, 348

Displacement, 15

Dissolution, law of, rot

Distilled water, Ir6

Dithymoldiiodide, $34^{\circ}$

Diuretics, 66

Diuretin, 392

Dock, yellow, 603

Dog button, 393

Domestic measures, 18

Donovan's solution, 212

Doses, 19, 39

Dover's powder, 352

Drastic purgatives, 92

Draught, 37

Dried alum, 186 gypsun, 159 sodium carbonate, 140

Droitwich water, 145

Drop chalk, 155

Drops, 37

Drugs, administration of, $3^{8}$

acting on the heart, 420 nervous system, 349, 679

Dubo'isinæ su'lphas, 386

Duboisine sulphate, $3^{86}$

Dulcama'ra, 635

Dulcamarin, 635

Dusart's syrup, 206
FARS, drugs acting on, 112 pills, 205

Easton's syrup, 205

Eau de goudron, $52 x$

Ebonite, 667

Ecbolics, 108

Ecboline, 637

Ecgonine, 412

Effervescent citrated caffeine, 389

lithium citrate, 153 magnesium citrate, 162 potassium citrate, 127 powder, compound, 143

Egg albumin, 6gr yolk of, $69 x$

Elaoptens, 14

Ela'stica, 667

Elaterin, 507

Elateri'num, 507

Elder, 552

Elecampane, $45^{8}$

Electrozone, 244

Electuaries, 22

Elemi, 533

Eli'xir aroma'ticum, 59x pho'sphori, 237 proprieta'tis Parace']si, $49^{8}$

Eli'xira, 2 I

Elixiss, 21

Elm, 6r6 slippery, 616

Elutriation, 15

Emetics, 86, 129

Emetine, $45 \mathrm{I}$ hydrobromate, $45 \mathrm{I}$ hydrochlorate, $45 \mathrm{I}$

Emmenagogues, Ir3

Emodin, 490, 495

Emollients, 64

Empirical therapeutics, 9

Empla'stra, 2r

Emu'lsa, 22

Emulsin, 612, 6r3

Emulsions, 22

Enemas, 36

Ene'mata, 36, 94

Epinephrin, $7{ }^{\circ} 4$

Epsom salt, 162

Ergot, $6_{37}$ of rye, 637

Ergo'ta, 637 
Ergotic acid, 637

Ergotin, 637

Ergotine, 637

Ergotinic acid, 637

Ergotinine, 637

Ergotism, 639

Ericolin, 460, 631 , 635

Eri'geron, oil of, 519

Eriodi'ctyon, 460

Erythrophloeine, 436

Erythro'phloeum, 436

Erythroretin, 490

Erythrol ni'tras, 308 nitrate, 308 tetranitrate, 308

Erythro' $x y$ lon, $4 x x$

Escharotics, 61

Eseridine, 399

Eserine, 399 salicylate, 399 sulphate, 399

Essence of nutmeg, $53^{8}$ of peppermint, 547 of spearmint, $54^{8}$

Essences, 37

Esse'ntix, 37

Essential oils, 13

Ether, 297

acetic, 300

chloric, 291

ethylic, 297

hydrobromic, 301

nitrous, spirit of, 304

spirit of, compound, 298 sulphuric, 297

Ethereal oil, 298

Ethyl acetate, 300

alcohol, 279

bromide, 301

carbamate, 318

chloride, $3^{\circ} 3$

cinnamate, $5^{6} 7$

oxide, 297

urethane, $3^{3} 8$

Ethylic alcohol, 279 ether, 297

Eucai'na bydrochlo'ras, 326 alpha, 326 beta, 328

Eucaine hydrochlorate, 326

Eucalyptene, 528

Eucalyptol, 528

Eucal'yptus, 527
Encal'yptus gum, 604

Eudoxin, 185

Eugenic acid, 534

Eugenin, 534

Eugenol, 534

Eulachon oil, 684

Eunatrol, 606

Euonymin, 513

Euo'nymus, 513

Eupatorin, 487

Eupato'rium, 487 -

Europhen, 34I

Exalgin, 324

Exalgi'num, 324

Expectorants, 78 depressing, 78 stimulating, $7^{8}$

Experimental therapeutics, 10

Expressed oil of nutmeg, $53^{8}$ of almond, $6 x_{3}$

Extract of meat, 689

Extra'cta, 22 flu'ida, 24 organ'ica, 701

Extracts, 22

Extra'ctum ca'rnis, 689 pancreatis, 688

Eye, drugs acting on, 110

$\mathrm{F}^{\mathrm{ABIA}} \mathrm{NA},{ }_{36}$

Fabianine, 636

False hellebore, 436

Family pill, 232

Fats, definition of, 13

Fel bo'vis, 680 purifica'tum, 680 tau'ri, 680

Fennel, 550

Fern, male, 623

Ferratin, 206

Fe'rri a'rsenas, 196 ca'rbonas sacchara'tus, roo

chlo'ridum, $19 x$

ci'tras, $x 92$

et ammo'nii ci'tras, $x 94$

su'lphas, 193

ta'rtras, 194

pota'ssii ta'rtras, 194

quini'na ci'tras, 195

solu'bilis, 195

strychni'na citras, 195
Fe'rri bypopho'sphis, 240

jo'didum sacchara'tum, 190

la'ctas, $29 x$

o'xidum hydra'tum, 193 cum magne'sia, 193

pho'sphas solu'bilis, 194 pyropho'sphas solu'bilis, 196

su'lphas, 189

exsicca'tus, 189

granula'tus, 189

valeria'nas, 557

Ferric acetate solution, 193

ammonium sulphate, 193

chloride, 191

solution of, $19 x$

tincture of, $19 x$

Weld's syrup of, 199

citrate, solution of, 192

bydrate, $x 93$

with magnesia, 193

hydroxide, 193

hypophosphite, 240

nitrate, solution of, 192

phosphate, soluble, 194

pyrophosphate, soluble, 196

subsulphate, solution of,

192

valerianate, 557

Ferrous carbonate, mass of,

190

iodide, pills of, sgo

saccharated, 190

syrup of, $x 90$

lactate, $19 x$

sulphate, 189

dried, 189

granulated, 189

Ferruginous pills, 190

Fe'rrum, 188

dialysa'tum, 106

redu'ctum, 188

Ferulic acid, 560

Fi'cus, 484

Fig, $4^{84}$

Filicic acid, 623

Filicin, 623

Fi'lix-mas, 623

Fir wood oil, 567

balsam of, 524 
Fish berry, 629

Fixed oils, 12

Flag, sweet, $5^{82}$

Flaxseed, $6 \times 8$ oil of, 618

Fleabane, oil of, 519

Fleming's tincture, 437

Flexible collodion, 665

Flies, Spanish, 697

Flitwick water, 205

Flowers of sulphur, $25^{8}$

Floid extracts, 24

Fly fungus, 402

Foni'culum, $55^{\circ}$

Fome'sta, 37

Fomentations, 37

Foods, 684

Formal, 344

Formaldehyde, 344

Formaldehy'dum, 344

Formalin, 344

Formic aldehyde, 344

Foxglove, 421

Fowler's solution, 224

Fra'ngula, 495

Frangulin, 495

Frankincense, 523

Franz Joseph, 144

Friedrichshall, 144

Friar's balsam, 656

Fuller's earth, 187

Fusel oil, 289

ADUIN, 685

Galactagogues, 114

Galba'num, 56s

Galipeine, $5^{86}$

Galipidine, 586

Gall, ox, 680

Ga'lla, 592

Gallic acid, 592, 596, 602, 603

Gallotannic acid, 593

Gamboge, 508

Gambogia, 508

Gambogic acid, 508

Ganga, $3^{8} 7$

Gargari'smata, 37

Gargles, 37

Garlic, 455

Gastric antiseptics, 84

juice, action of drugs on, 82

sedatives, 85
Gastro-intestinal irritants, 84

Gault the'ria, oil of, 480

Gelatin, 696

Gelati'num, 696

Gelsemine, 403

Gelseminic acid, 403

Gelseminine, 403

Gelse'mium, 403

General therapeutics, 9

Generation, action of drugs on, 112

Gentian, $5^{8} 3$

Gentia'na, $5^{8} \mathbf{3}$

Gentisic acid, $5^{8}{ }_{3}$

Gentiogenin, $5^{8} 3$

Gentiopicrin, $5^{8} 3$

Geraniol, $5^{8} 9$

Gera'nium, 602

German chamomile, 555

Gin, 28I

Ginger, 543

Gingerol, 543

Glacial acetic acid, 266

Glauber's salt, 142

Glonoin, 308 spirit of, 308

Glonoi'num, 308

Glucose, 68I

Glucosides, 12

Glucusimide, 346

Gluside, 346

Glusi'dum, 346

Gluten, 695

Glycerin, 608 suppositories, 609

Glyceri'num, 608

Glyceri'ta, 26

Glycerites, 26

Glyceri'tum a'cidi carbo'lici, 329

a'cidi ta'noici, 593

a'myli, 609

boroglyceri'ni, 273

hydra'stis, 641

vite'lli, 609

Glycerol, 608

Glyceryl borate, glycerite of, 273

butyrate, $53^{8}$

myristate, $53^{8}$

oleate, $53^{8}$

trinitrate, 308

Glycogelatin, 696
Glycogenic function, 98

Glyconin, 609

Glycyramin, 617

Glycyrrhetin, 616

Glycyrrhi'za, $6 \times 6$

Glycyrrbizin, 616 ammoniated, $6 \mathrm{I} 7$

Glycosuria, 98

Gnoscopine, 350

Goa powder, 660

Gold, 209 and sodium chloride, 209

Golden seal, $64^{\circ}$

Gossy'pii ra'dicis co'rtex, 642

Gossy'pium purifica'tum, $66_{5}$

Goulard's cerate, 167 extract, 166

Grana'tum, 624

Granulated ferrous sulphate, 189

Granules, 37

Gray powder, 2 so

Gregory's powder, 490

Green mercurous iodide, 212

hellebore, 442

Paris, 228

Scheele's, 228

Schweinfurth's, 228

soap, 607

solution, 479

vitriol, 189

Griffilh's mixture, 189

Grinde'lia, 568

Grindeline, 568

Guaiac, 670 .

resin, 670

wood, 669

Guai'aci li'gnum, 67o resi'na, 670

Guaiacic acid, 670

Guaiacol, 336, 670

benzoate, 336

carbonate, 336

salicylate, 337

Guaiaco'li be'nzoas, 336

ca'rbonas, 336

sali'cylas, 337

Gua iaconic acid, 670

Guaiaretic acid, 670

Guara'na, 39r

Guaranine, 389, 392

Guinea pepper, 542

Gum arabic, 625 
Gum Benjamin, $65^{6}$ camphor, 648 guaiac, 670 resins, 15

Gums, is

Gun cotton, soluble, 665

Gunjah, $3^{87}$

Gu'tta, 37

Guy's pill, 220, 433

Gynocardic acid, 678

Gypsum, dried, 159

$\mathrm{H}^{\text {EMATIN, } 600}$

$H_{\text {Hrmatinles, } 53}$ indirect, 53

Hrmatoxylin, 600

Hrmato'xylon, 600

Hamostatics, ${ }_{3}$

Hamame'lis, 6or

Habit, 40

Hard soap, 607

Hard petrola'tum, 278

Harrogate water, 261

Haschisch, 387

Hartshorn, 150

Hau'stus, 37

Hazeline, 6or

Heart, drugs acting on, 56

Heavy magnesia, 163

Hedeo'ma, 553

Helenin, 459

Hellebore, American, 442 green, 442 false, 436

Hemidesmic acid, 676

Hemidesmine, 676

Hemide'smus, 676

Hemlock, 405

Hemp, Indian, 386 Canadian, 632

Henbane, 383

Hepatic stimulants, 96 aloes, 497

Heroine, 363

Hesperidene, 589

Hesperidin, 589

Hexamethylentetramine, 345

High bush cranberry, 644

Himrod's cure, $38_{3}$

Hircin, 693

Hiru'do, 700

Hive syrup, 232

Hoang-Nan, 393
Hock, 281

Hoffmann's anodyne, 298

Homatropi'nx hydrobro'- Hydrocyanic acid, diluted, mas, $3^{81}$

Homatropine hydrobromate, Hydrogen dioxide, solution $3^{38 x}$

Homochelidonine, $45^{\circ}$

Homoquinine, 464

Homolle's digitalin, $42 x$

Homopterocarpin, $66_{4}$

Honey, 68I

clarified, $68 \mathrm{x}$

of rose, 555

Honey, 28

Hops, 368

Ho'rdeum decortica'tum, 620

Horehound, 634

Horseradish, 54I

Hot pack, Irg bath, 119

Huile de Cade, 522

Human milk, artificial, 687

H u'mulus, 368

Hunyadi János, 93

Hydragogues, 93

Hydra'rgyri chlo'ridum corrosi'vum, 213

chlo'ridum mi'te, 212

cya'nidum, $2 \times 3$

empla'strum, 2 ro

io'didum fia'vum, 212

io'didum ru'brum, 212

o'xidum fla'vum, 211

o'xidum ru'brum, 210

Hydra'rgyri subsu'lphas

flavus, 214

Hydra'rgyrum, 210

ammonia'turn, 213

cum cre'ta, 210

Hydrastine, 64I

Hydrastini'næ hydrochlo' ras, $64 \mathrm{I}$

Hydrastinine bydrochlorate, $64 \mathrm{I}$

Hydra'stis, $64 x$

Hydrated alu'mina, 186 ferric oxide, 193

Hydriodic acid, syrup of, 248

Hydrobromic acid, diluted, 258

ether, zor

Hydrochloric acid, 265
134

Hydrochloric ether, 303

Hydrocotarnine, 350

(ndion peroxide solution, 120

Hydrous wool-fat, 694

Hydroquebrachine, 459

Hydroxide, ferric, 193

potassium, 123

sodium, $13^{8}$

Hydrophobia antidote, 7 ro

Hyosci'næ hydrobro'mas, $3^{8} 5$

Hyoscine hydrobromate, 385

Hyoscyami'nze hydrobro'mas, 385 su'lphas, $3^{8} 4$

Hyoscyamine hydrobromate, $3^{8} 5$

sulphate, $3^{8} 4$

Hyoscy'amus, 384

Hypnal, 312

Hypnotics, 106, 309

Hypodermatic injections, $3^{8}$

Hypophosphites, 239 syrup of, 239 with iron, 240

Hypopbosphorus acid, diluted, 240

\section{[CE BAGS, II poultices, 118}

lceland moss, $62 x$

Ich thyoco'lla, 694

Ichthyol, 699

1chthy'olum, 699

I diosyncrasy, 40

Igasuric acid, 393

Illi'cium, 549

Imperial drink, ${ }^{2} \mathrm{x}$

Incompatibility, chemical, $4 I$

pharmacological, 43 physical, 43

Index, 721

India rubber, 667

senna, 493

Indian cannabis, 386

hemp, 386

tobacco, $45^{6}$ 
lndian sarsaparilla, 676

Indifferent bath, 117

Indirect action, 47

Indurated lard, 690

Ineine, 429

Ivfu'sa, 26

Infusions, 26

Inhalations, 37,74

Inje'ctiones, 37

Injections. 37

Insuffla'tiones, 37

Insufflations, 37

Intestinal antiseptics, 94 astringents, 95

Intestines, drugs acting on, 90

Intra-ocular tension, 113

I'nula, $45^{8}$

Inulin, 459

Jodides, 247

lodine, 245

compound solution of, 245

ointment, 245

tincture of, 245

$10^{\prime} \mathrm{dum}, 245$

lodoform, $33^{8}$

lodofo'rmum, $33^{8}$

Iodol, $34 \mathrm{I}$

Iodo'lum, 34:

lodantipyrin, 323

lodopyrin, 323

Iodosalicylic acid, $34^{2}$

lodothyrin, 703

Ipecac, 45 I

Ipecacua'nha, 451

Ipecacuanhic acid, 45I

Iridin, $5^{1} 3$

Iridi'num, 513

Iris, $5^{12}$

Irish moss, 608

Irisin, 513

Iron, 188

and ammonium acetate, solution of, $\mathrm{Jg} 2$

and ammonium citrate,

194
tartrate, 195
and potassium tartrate,
$194 \quad$ soluble, 195
and quinine citrate, 195
and strychnine citrate,
195

Iron arsenate, 196

by hydrogen, 188

dialyzed, 196

glycerophosphate, 242

metallic, 188

mixture, compound, 189

plaster, 193

Quevenne's, 188

quinine, strychnine phos-

phates, syrup of, 194

reduced, 188

tartrated, 194

troches of, 193

wood, 459

Irritants, 61 gastro-intestinal, 84

Isinglass, 694

I sohesperidin, $5^{8} 9$

Isopelletierine, 624

Isotrophylcocaine, 412

Itrol, 73

Ivy, poison, 660

I zal, $33^{8}$

\section{JABORANDI, 417 \\ Pernambuco, 417}

Rio Janeiro, 417

Jaborine, 417

Jalap, 501

Jala'pa, 501

Jalapin, 501, 502

Jalapurgin, 502

James' powder, 23I

Jamestown weed, $3^{82}$

Jarisch's ointment, 597

Jasmine, yellow, 403

Jervine, $44^{2}$

Jeyes' disinfectant, $33^{8}$

Jordan almond, $61_{3}$

Juglandic acid, 492

Ju'glans, 492

Juices, 37

Juniper tar oil, 522

KAMA'LA, 625

K Kaolin, 187

Kermes mineral, $23^{\circ}$

Kinic acid, 464

Ki'no, 599

Kinoin, 599

Kino-tannic acid, 599, 604
Kinovic acid, 464

Kinovin, 464

Kissiogen, 144

Ko'la nut, $3^{89}$

Kombé poison, 431

Kombic acid, 429

Kooso, 624

Kosin, 625

Koumiss, 688

Koussin, 625

Kousso, 624

Krame'ria, 598

Kramero-tantic acid, 598

Kumyss, 281, 688

I ABARRAQUE'S solu-

$$
\text { tion, } 243
$$

La Bourboule, 205

Lac, 687 su'lphuris, 259

Lactic acid, 267

Lactophenin, 324

Lactopheni'num, 324

Lactose, 695

Lactuca'rium, 370

Lactucerina, $37^{\circ}$

Lactucin, $37^{\circ}$

Lactucon, $37^{\circ}$

Lactylparaphenetidine, 324

Lady Webster's pill, $49^{8}$

Ladies' slipper, 559

Lame'lla, 37

Lanolin, 694

Lanthropine, 350

La'pis divi'nus, 180 inferna'lis, 17

La'ppa, 674

Lard, 690 benzoinated, 656 indurated, 690 oil, 690

Latin phrases, 7rg

Laudanine, $35^{\circ}$

Laudanosine, 350

Laudanum, 352

Laurel camphor, 648

Laurocerasin, 656

Lauroce'rasus, 655

Lavender, oil of, 545 flowers, oil of, 545

Law of dissolution, 104

Laxatives, $g \mathrm{I}$ 
Lead, 165

acetate, 166

carbonate, 166 ointment, 167

iodide, 167 ointment, 167

nitrate, 167

oxide, 165

plaster, 166

poisoning, 169

subacetate, cerate of, $\mathrm{I} 66$ solution of, 167

sugar of, 166

white, 167

water, 166

Ledoyen's disinfecting fluid, 167

Leech, 700

Leiter's coils, II9

Lemon, 654

juice, 654

oil of, 654

peel, 654

Leopardsbane, $53^{\circ}$

Lepta'ndra, 5 II

Leptandrin, $5^{11}$

Letruce, $37^{\circ}$

Levant wormseed, 626

Levigation, 16

Levico water, 205

Lichenin, 621

Lichenstearic acid, 622

Light magnesia, 163

Li'gnum vi'tx, 669

Lily of the valley, 431

Lime, 157

chlorinated, 243

liniment, 157

phosphate, precipitated, $15^{8}$

solution of, 157

syrup of, $x 57$

water, 157

Limonene, 654

Limonine, 546

Limo'nis co'rtex, 654 su'ccus, 655

Linalool, $54^{6}$ acetate, 546

Li'nctus, 37 opia'tus, $36 \pm$

Liniment, volatile, 149

Linime'nta, 27
Liniments, 27

Linime'ntum acon'iti compositum, $44^{\circ}$

chlorofo'rmi, $29 x$

Linolein, 618

Linseed, 618

oil of, 618

Li'num, 618

Liquid petrola'tum, 277

Liqueurs, 28I

Li'quor ca'lcis, 157

carbo'nis dete'rgens, $52 \mathrm{I}$

pancrea'tis, 688

pi'cis carbo'nis, 521

pota'ssæe, 123

so'd $\mathrm{d}, 13^{8}$

thyroi'dei, 7or

Liquo'res, 27

Liquorice root, 616

powder, compound, 494

Russian, 616

Lister's ointment, 275

Litharge, 165

Li'thii be'nzoas, 657

bro'midum, 253

ca'rbonas, 153

ci'tras, 153

efferve'scens, 154

sali'cylas, 475

Lithium, 153

benzoate, 657

bromide, 253

carbonate, 153

citrate, 153

effervescent, 154

glycerophosphate, 242

salicylate, 475

Lithontriptics, 68

Liver, drugs acting on, 96 of sulphur, 261

Lixiviation, 16

Llangammarcb wells, 161

Lobelacrin, 456

Lob'elia, 456

Lobeline, 456

Local action, 46 anodynes, ror

anæsthetics, ror

Loganin, 393

Logwood, 600

London paste, 157

Losophan, 342

Lo'tiofla'va, 218
Lo'tio n'igra, 218

ru'bra, 178

spi'ritus, 287

Lotio'nes, 37

Lotions, 37

Lugol's solution, 245

Lunar caustic, 170

Lupulin, $3^{69}$

Lupulinic acid, $3^{69}$

Lupuli'num, 369

Lutidine, 408

I.ycopo'dium, 668

Lye, 16

Lysol, $33^{8}$

Ly'solum, $33^{8}$

$\mathrm{M}^{\mathrm{ACE}, 539}$

$M$ Macene, 539

Maceration, 16

Macis, 539

Madeira, 28r

Macrotin, 588

Magendie's solution, 355

Magne'sia, 163

calcined, 163

heavy, 163

light, 163

pondero'sa, 163

Magne'sii ca'rbonas, 162

ci'tras efferve'scens, 162

su'lphas, 162

Magne'sium, 162

carbonate, 162

citrate effervescent, 162 solution of, 163

glycerophosphate, 242

sulphate, 162

Maizenic acid, 633

Male fern, 623

Malic acid, 557

Malt, 620

Maltose, 620

Ma'ltum, 620

Mancona bark, 435

Mandrake, $5 \circ 9$

Manganese, 206

black oxide, 206

dioxide, 206

sulphate, 207

Manga'ni dio' xidum, 206 su'lphas, 207

Manganous sulphate, 207

Manga'num, 206 
Manilla elemi, 533

Ma'nna, 485

Marc, 16

Marienbad, 144

Marigold, 676

Marrubiin, 634

Marru'bium, 634

Marsden's paste, 226

Marsh's test, 229

Marshmallow, $6 \times 2$

Mass, blue, 210

of copaiba, 57

of ferrous carbonate, 190 Vallet's, 190

Ma'ssa copa'iba, $57^{x}$

fe'rri carbona'tis, ryo

hydr'argyri, 210

Ma'ssæ, 28

Masses, 28

Mastic, 666

Mastiche, 666

Mastichic acid, 667

Masticin, 667

Maté, $3^{89}$

Mat'eria me'dica, 9

Ma'tico, $57^{6}$

Matrica'ria, 555

May apple, 509

McDade's formula, 672

Measures, 18 domestic, 18

Meadow saffron, 645

Meat extract, 689

Meconic acid, 350, 353

Meconidine, 350

Meconin, 350

Meconoiasin, 350

Mel, 68I despuma'tum, 68r ro's $x, 555$

Me'llita, 28

Meli'ssa, 66 I

Menispe'rmum, 673

Menispine, 673

Mental emotion, 40

Me'ntha piperi'ta, 546 vi'ridis, 548

Menthene, $547,54^{8}$

Menthol, 547, 652

Mercurial ointment, $2 x 0$ plaster, 210

Mercuric ammonium chlo. ride, 213
Mercuric chloride, cor-

rosive, $2 \mathrm{rr}$

cyanide, 213

iodide, red, 212

nitrate, ointment of, $2 x_{3}$ solution of, 213

oxide, red, 210 ointment of, $21 x$

yellow, 2rx ointment of, $21 x$

subsulphate, yellow, 224

sulphate, basic, 214

Mercurous chloride, mild, 212

iodide, yellow, 212

tannate, 220

Mercuro-zinc cyanide, 221

Mercurol, 220

Mercury, 210

administration of, $22 x$

( ammoniated, 213 ointment, 213

bichloride, 211

biniodide, 212

chloride, corrosive, $2 \mathrm{II}$ mild, 212

iodide, green, 212 red, 212 yellow, 212

mass of, 210

oleate of, 212

protiodide of, 212

subchloride, 212

tannate, 220

with chalk, 2 ro

Metabolism, drugs acting on, 115

Metadioxybenzol, 344

Methyl acetanilid, 324

amine, 663

benzoylecgonime, $4^{12}$

coniine, 406

morphine, 366

pelletierine, 624

pyrocatechin, 336

salicy'las, $48 \mathrm{x}$

salicylate, $48 \mathrm{x}$

Methylene blue, 347

Metrical system, I?

Mezerein, 532

Mezereon, 532

Mezere'um, 532

Michel's paste, 268
Milk, 687

artificial human, 687

drugs acting on, 114

drugs excreted by, $\mathrm{II}_{4}$

of almond, $6 \mathrm{r}_{3}$

of asafetida, 560

of sulphur, 259

peptonized, 688

sugar of, 695

Mindererus, spirit of, 152

Mistu'ra amy'gdale, 6r 3

Mistu'ræe, 28

Mixtures, 28

Mitigated caustic, $17 x$

Molli'num, 37

Monkshood, 437

Monobromated camphor, 649

Monsel's solution, 192

Moonseed, Canadian, 673

Morphi'na, 353

Morphi'nze ace'tas, 354 hydrochlo'ras, 353 su'lphas, 354

Morphine, 353

acetate, 354

benzylic ether hydrochlorate, $36_{3}$

diacetic ether, $3^{6} 3$

hydrochlorate, 353

monoethyl ether hydro. chlorate, $36_{3}$

sulphate, 354

Morrhuol, 685

Morton's fluid, 247

Mo'schus, 679

Mosquera-Julia beef meal,

689

Moss, Iceland, 62

Irish, 608

Motor nerves, drugs acting on, 99

Moulded silver nitrate, $x 7 \mathrm{x}$

Mountain balm, 460

Mucilage, 28

Mucila'gines, 28

Muriatic acid, 264 diluted, 265

Muscari'na, 402

Muscarine, 402

Muscles, drugs acting on,

99

Musk, 679 
Musk root, 544

Mustard, 524

black, 524

paper, 525

white, $5^{24}$

Mutton suet, 692

Mydriatics, III

Myosin, 626

Myotics, III

My'rcia, oil of, $66_{3}$

Myricin, 696

Myri'stica, 538

Myristicine, 538

Myristicol, $53^{8}$

Myristin, 618

Myrosin, 524, 525

Myrrh, 562

My'rrha, 562

Myrrhin, 562

Myrrhol, ${ }^{6}$ 2

NANTWICH water, 145

Napelline, 437

Naphtalin, 342

Naphtali'num, $34^{2}$

Naphtol, 342

Narceine, 350,366

Narcotics, 106

Narcotine, 350,366

Nativelle's digitalin, 421

Natural orders, 692

Ne'bula, 37

Nepenthe, $35^{2}$

Neroli, oil of, $59 \mathrm{I}$

Nerves, drugs acting on, 99 405

Neuritis, drugs causing, 102

Nicotianine, 408

Nicotine, 408

Night blooming cereus, 447

Nightshade, deadly, 37r woody, 635

Nitric acid, 264 diluted, 264

Nitrites, $3 \circ 3$

Nitre, $13^{1}$ sweet spirit of, 304

Nitroglycerin, 308 spirit of, 308

Nitrohydrochloric acid, 264 diluted, 264
Nitromuriatic acid, 264 diluted, 264

Nitrous ether, spirit of, 304

Norwood's tincture, $\mathbf{4 4 2}$

Nucln, $49^{2}$

Nutgall, 592

Nutmeg, $53^{8}$

Nutrient serum, y10

Nux vo'mica, 393

$\mathrm{A}_{\text {poison, } 660}^{\mathrm{AK} \text { bark, } 592}$ white, 592

E'sypum, 694

Oil of allspice, 536

almond, expressed, $6 \mathbf{r}_{3}$

American wormseed, 629

anise, 549

bay, $66_{3}$

benne, 663

bergamot, 546

betula, volatile, 480

bitter almond, 6r 2

cade, $5^{22}$

cajuput, 527

caraway, 551

carron, 157

cassia, 540

castor, 487

chamomile, 555

chaulmoogra, $67^{8}$

chenopodium, 629

cinnamon, $54^{\circ}$

cloves, 534

cod liver, 684

copaiba, $57 \mathrm{I}$

coriander, $55^{\circ}$

cotton seed, 666

croton, 503

of cubeb, 575

dill, $55^{2}$

erigeron, 519

ethereal, 298

eucalyptus, 528

eulachon, 684

fennel, 551

firwood, $5^{6} 7$

flaxseed, 618

fleabane, $5^{19}$

fusel, 290

gaultheria, 480

bedeoma, 553
Oil of juniper, 569

lard, 690

lavender, 545

flowers, 545

lemon, 654

linseed, 618

mustard, volatile, 525

myrcia, 663

neroli, 591

nutmeg, 538

expressed, $53^{8}$

olive, 605,606

orange flowers, $59 \mathrm{I}$

peel, 590

pennyroyal, 553

peppermint, 547

Chinese, 653

Japanese, 653

phosphorated, 237

pimenta, 535

pine, 567

rose, 556

rosemary, $53^{\circ}$

rue, 579

sandal wood, 576

santal, 576

sassafras, 675

savine, 578

sesame, 663

sweet, 605

spearmint, $54^{8}$

sweet birch, 480

tar, 520

teel, $66_{3}$

theobroma, 666

thyme, $\mathbf{5 7 4}$

turpentine, 515

rectified, ${ }_{51} 6$

vitriol, 263

wintergreen, 480

artificial, $48 z$

synthetic, 480

Oils, 29

essential, $I_{3}$

fixed, 12

nitrogenated, 34

oxygenated, 14

sulphurated, 14

volatile, 33

Oint ments, 35

$O^{\prime}$ lea, 29

Olea'ta, 28

Oleate of mercury, $21 x$ 
Oleate of veratrine, 445 zinc, 177

Oleates, 28

Olea'tum hydra'rgyri, 211 veratri'næ, 445 zi'nci, 177

Oleic acid, $6 \mathrm{rI}$

Olein, 666, 684, 693

Oleoresins, if

O'leum a'dipis, 690 xthe'reum, 298 amy'gdala ama'ræ, 612 expre'ssum, 613 ane'thi, $55^{2}$ ani'si, 549 anthe'midis, 354 aura'ntii co'rticis, 590 flo'rum; 59 I berga'mii, 546 bergamo'ttæe, 546 be'tula vola'tile, 480 cadi'num, 522 cajupu'ti, 527 ca'ri, 55 I caryo'phylli, 534 chenopo'dii, 629 cinnamo'mi, 540 copai'br, 57 I coria'ndri, $55^{\circ}$ cube'báe, 575 erigero'ntis, 5 I9 eucaly'pti, 528 fœeni'culi, 551 gaulthe'rix, 480 gossy'pii se'minis, 666 gynoca'rdia, $67^{8}$ hede'omx, 553 jecoris ase'lli, $68_{4}$ juni'peri, 569 empyreuma'ticum, 522

lave'ndulax, 545

lavendulæ florum, 545 limo'nis, 654 li'ni, 6i 8 me'ntha piperi'tx, 547 vi'ridis, $54^{8}$

mo'rrhux, $68_{4}$ my' rcia, $66_{3}$ myri'sticx, 538 expre'ssum, $53^{8}$ oli'vac, 605
O'leum phosphora'tam, 237

pi'cis li'quidx, 520

pime'ntx, $53^{6}$

pi'ni, 567

ri'cini, 487

ro'sæe, 556

rosmar'ini, $53^{\circ}$

ru'ta, 579

sabi'nx, $57^{8}$

sa'ntali, 576

sa'ssafras, 675

se'sami, 663

si'napis vola'tile, 525

terebi'nthina, 515 rectifica'tum, 5 1ó

theobro'matis, 666

thy'mi, 574

ti'glii, 503

Olive oil, 605

Opbelic acid, 585

O'pii pu'lvis, 35r

Opium, 350

Constantinople, 350

Egyptian, 35०

Levant, 350

Patna, 35I

Smyrna, 35 I

Turkey, 350

Opodeldoc, 607

Orange peel, $5^{8} 9$

bitter, 589

oil of, 590

sweet, 590

Ordeal bark, 435

bean, 399

Organic extracts, yor

Organs of generation, drugs acting on, 112

Ouabain, 416

Ouabai'num, 416

Ourari, 4 I I

$\mathrm{O}^{\prime}$ vi a'lbumin, 6gr

Oxgall, 680 purified, 680

Oxygen, 122

Oxyge'nium, rz2

Oxymel, 68x

Oxyme'llita, 37

Oxynarcotine, 350

Oxytocics, $\mathrm{xI}_{3}$

Oxytoluyltropine, $3^{8 \mathrm{r}}$
PACK, cold, 118 hot, IIg

Paint, 37

Pale rose, 555

Palmitic acid, 60 r

Palmitin, 6:8, 666, 684, 693

Panama bark, 668

Pancreatin, $68_{3}$

Pancreati'num, $68_{3}$

Papa'ver, $35^{\circ}$

Papaverine, 350

Paper, mustard, 525 potassium nitrate, $x_{3}$

Papers, 20

Para-acetphenetidin, 323

Paraguay tea, 389

Paraldehyde, 315

Paraldehy'dum, 315

Paramorphine, 366

Para rubber, 667

Parasiticides, 5r

Paregoric, $35^{2}$

Scotch, 352

Pare'ira, 634 brava, 634

Pariglin, 672

Pari'lla, yellow, 673

Parillin, 672

Parillinic acid, 672

Paris green, 228

Parish's food, 206

Parsley, 645

Parvoline, 408

Paste, 37

Coster's, 247

London, 157

Marsden's, 226

Ricord's, 268

Vicana, 123

Pastillus, 37

Pastils, 37

Pearl ash, 139

barley, 620

Pearson's solution, 224

Pellitory, 533

Pelletierine, 624

Pelosine, 634

Pellotine, 370

Pelloti'num, 370

Pennyroyal, 553

Pental, 302

Pe'ntalum, 302

Pe'po, 625 


\section{Pepper, 537}

African, 54x

black, 537

cayenne, $54 \mathrm{I}$

guinea, $54 \mathrm{I}$

Peppermint, 546

Pepsin, 682 saccharated, 682

Pepsi'num, 682 sacchara'tum, 1682

Peptonized milk, 688

Percolation, 16

Pe'rles, 37

Pernambuco Jaborandl, $4^{1} 7$

Peronine, 363

Pessaries, 37

Pe'ssus, 37

Petrola'tum, 277

hard, $27^{8}$

liquid, 27

li'quidum, 277

mo'lle, $27^{8}$

soft, 278

spi'ssum, 278

Petroleum benzin, 279

ether, 279

ointment, soft, 278 hard, $27^{8}$

Phzoretin, 490

Pharmaceutical processes, I5

Pharmacognosy, definition, 9

Pharmacological incompati-

bility, 43

Pharmacology, definitlon, 9, $3^{8}$

Pharmacopœia, definition, ro

preparations of the, 19

Pharmaco-dynamics, 10

Pharmacy, definition, 10

Phellandrene, 5 r 6

Phenacetin, 323

Phenaceti'num, 323

Phenazo'num, 321

Phenic acid, 329

Phenocoll hydrochloride, 325

Phenoco'lli bydrochlo'ridum, 325

Phenol. 329

Phenylacetamide, 319 dimethylpyrazolone, $32 x$ alcohol, 329 propylcinnamate, 567
Phenylacetamide, salicylate, 48x

Physical incompatibility, 43

Phosphorated oil, 237

Phosphoric acid, 265 diluted, 265

Pho'sphorus, 236

elixir of, 237

pills of, 237

spirit of, 237

Physosti'gma, 399!

Physostigmi'næ salicy'las, 399

su'lphas, 399

Physostigmine, 399

salicylate, 399

sulphate, 399

Phytola'cca, 486

fruit, 486

root, 486

Phytolaccic acid, 486

Phytolaccin, 486

Pichi, 636

Picoline, 408

Picro-aconitine, 437

crocin, $66_{4}$

podophyllin, 5 ro

Picrotoxin, 629

Picrotoxi'num, 629

Pigme'ntum, 37

Pills, 30

$$
\begin{aligned}
& \text { of aloes, } 497 \\
& \text { and asafetida, } 497 \\
& \text { and mastic, } 498 \\
& \text { and myrrh, } 498 \\
& \text { antimony, compound, } \\
& 212 \\
& \text { asafetida, } 560 \\
& \text { Blaud's, 190 } \\
& \text { blue, } 210 \\
& \text { cathartic, compound, } 212 \\
& \text { vegetable, } 506
\end{aligned}
$$

chalybeate, 190.

ferrous carbonate, 190 iodide, 190

ferruginous, 190

opium, 35I

phosphorus, 237

Plummer's, 212

rhubarb, 490 compound, 490

saccharated iron carbon.

ate, $x$ o
Pilocarpidine, 417

Pilocarpi'næ hydrochlo'ras, 417

hydrochlorate, 417

Pilocarpine, 4I7

Piloca'rpus, 4I 7

Pi'lula hydra'rgyri, 210

tri'um phospha'tum, 205

Pi'lulæ, 30

Pime'nta, 536

Pink, Carolina, 628

Pink root, 628

Pinene, $66_{3}$

Pinol, 567

Pi'per, 537

Piperidine, 537

Piperazidine, 348

Piperazine, 348

Pipera'zinum, 348

Piperin, 537

Piperi'num, 537

Piperonylic acid, 604

Pipsi'ssewa, 636

Pitch, Burgundy, $5^{22}$

plaster, 522

cantharidal, 522

Pix Burgu'ndica, 522

li'quida, $5^{20}$

Plague serum and inoculation, 709

Plasma, drugs acting on, $5^{r}$

Plaster, adhesive, 523

diachylon, 166

lead, 166

of Paris, 159

Plasters, 2I

Platt's chlorides, $x 76$

Pleurisy root, 457

Plu'mbi ace'tas, 166

ca'rhonas, 167

io'didum, $x 6_{7}$

ni'tras, 167

o'xidum, 165

Plu'mbum, 165

Plummer's pills, 212

Pneumogastric, drugs acting on, 57

Podophyllin, 509

Podophyllic acid, 509, 510

Podophy'llum, 509

Podophyllotoxin, 510

Poison ivy, 660

nut, 393 
Poison oak, 660

Poke berry, 486 root, 486

Polychroite, 664

Pomegranate, 624

Pond's extract, 601

Poppy capsules, 359

Port, 28I

Porter, 28I

Posology, 39

Potash, caustic, I22 yellow prussiate of, 134

Pota'ssa, Izz cum cal'ce, $x 23$ solution of, 123 sulphura'ta, 26r with lime, 123

Pota'ssii ace'tas, 127 bica'rbonas, 126 bichro'mas, $x 38$ bita'rtras, 129 bro'midum, 253 ca'rbonas, 125 chlo'ras, 132 ci'tras, 127 efferve'scens, 127

cya'nidum, $\times 34$ et so'dii ta'rtras, $14^{2}$ ferrocya'nidum, 134 hypopho'sphis, 239 io'didum, 248 ni'tras, 132 perma'nganas, 207 su'lphas, 129

Potassio-ferric tartrate, 194

Pota'ssium, 122

acetate, 127

alum, 185

and sodium tartrate, 142 arsenite, solution of, 224 bicarbonate, 126 bichromate, $13^{8}$ bitartrate, 129 bromide, 253 cantharidinate, 699 carbonate, 125

cblorate, 132 troches of, 122

citrate, 127 effervescebt, 127 solution of, 127

cyanide, 134

dichromate, 235
Pota'ssium ferrocyanide, 134

glycerophosphate, 242

hydrate, 122 solution of, 123

hydroxide, 122

bypophosphite, 239

iodide, 248 ointment of, 248

oleate, 607

nitrate, $13 \mathrm{I}$ paper, $13 x$

permanganate, 207

sulphate, 129

tartrate, acid, 129

Powder, antimonial, 231

aromatic, $54^{\circ}$

compound chalk, $x 55$ effervescent, 143 glycyrrbiza, 494 jalap, 502 . morphine, 351

Dover's, 352 ipecac and opium, $35^{2}$ James', 231 opium, $35 \mathrm{r}$

Seidlitz, 143

Tully's, 354

Powders, 31

Poultices, $3^{6}$

Precipitate, red, $2 x 0$ white, $2 \times 3$

Precipitated calcium carbonate, I 55 phosphate, 158 sulphur, 259

Preface, 5

Preparations, standardized, 16

pharmacopocial, 19

Prepared chalk, $x 55$

Prescribiog, 4I

Prescription, 44

Prickly ash, 67 r

Primary action, 46

Prince's pine, 636

Proof spirit, 279

Propenyl trinitrate, 308

Protopine, 350, 450, 512

Protargol, 173

Prune, 483

Pru'vum, 483

Pru'nus Virginia'na, 462
Prussiate of potash, yellow, 134

Prussic acid, 134

Pseudo-aconitine, 437

aconine, 437

jervise, 442

morphine, 350

pelletierine, 624

Pterocarpin, 664

Pullna, 93, 144

Pulsati'lla, 456

Pu'lvis antimo'nialis, $23 x$

aroma'ticus, 540

cre'tx compo'situs, I 55

efferve'scens compo'situs, 143

glycyrrbi'zx compo'situs, 494

ipecacua'nbe et o'pii, $35^{2}$

jala'pe compo'situs, 502 morphi'nx compo'situs, 354

pu'rgans, 500

rhe'i compo'sit us, 490

salicy'licus cum ta'lco, 479

Pu'lveres, $3 x$

Pumiline, 567

Pumpkin seed, 625

Punicotannic acid, 624

Pupil, drugs acting on, I ro

Purgatives, $91,4^{83}$

drastic, 92

saline, 93

simple, 92

Purging cassia, 485

Purified cotton, 665

oxgall, 680

Pustulants, 6r

Pyre'thrum, 533

Pyridine, 408

Pyrocatechin, 599, 604

Pyrogallic acid, 596

Pyrogallol, 596

Pyroxylin, 665

Pyroxyli'num, 665

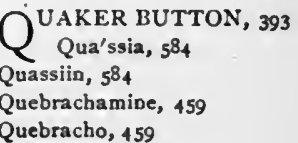


Qneen's delight, 674 root, 674

Quercin, 592

Quercitannic acid, 592

Quercite, 592

Que'rcus a'lba, 592

Quevenne's digitalin, 421 iron, 188

Quicksilver, 230

Quillaic acid, 669

Quilla'ja, 668

Quinamine, 463

Quinidi'næ su'lphas, 466

Quinidine, ${ }_{463}$ sulphate, 466

Quinic acid, 463

Quini'na, 465

Quini'nx bisu'lphas, 466 hydrobro'mas, 466 hydrochlo'ras, 466 su'lphas, 465 valeria'nas, $55^{8}$

Quinine, 463,465 bisulphate, 466 carbamide, 473 hydrobromate, 466 hydrochlorate, 466 sulphate, 465 valerianate, $55^{8}$ Quinovic acid, 464 Quinovin, 464

RAISINS, 622

$R$ Rash, drugs producing, 66

Raspberry, 662

Rate of excretion, $4^{\circ}$

Rational therapeutics, 9

Rectified oil of turpentine, 5 56

Red corpuscles, drugs acting on, 53

cinchona, $4^{64}$

gum, 604

lotion, 178

precipitate, 210

rose, 555

saunders, 664

scale insect, 692

wine, 285

Reduced iron, 188

Refrigerants, 82

Reiusch's test, 229
Remote action, 47

Remijia hark, 464

Repercolation, $x 6$

Resin, 523

of copaiba, $57^{2}$

of jalap, 502

of podophyllin, 509

of scammony, 500

soap, 523

Resi'na, 523

Resins, 14

Resorcin, 344

Resorcinol, 344

Resorci'num, 344

Respiration, drugs acting on, 74

Respiratory centre, drugs acting on, 75

depressants, 76

disinfectants, 75

stimulants, 76

Rha'mnus, purshia'na, 495

Rhamnose, 495

Rhatanin, 598

Rhatany, 598

Rheotannic acid, 490

Rhe'um, $4^{89}$

Rhein, 490

Rhine wine, 283

Rnodinal, 556

Rhœeadine, $35^{\circ}$

Rhubarb, 489

Ricinine, 487

Ricinolein, 487

Rhus gla'bra, $60 x$ toxicode'ndron, 660

Richfield Springs, 260

Ricord's paste, 268

Rio Janeiro jaborandi, 4I 7

Rise of temperature, drugs causing, 73

Rochelle salt, 142

Ro'sa, 556

ga'llica, 555

centifo'lia, 555

Rose, 555

attar of, $55^{6}$

pale, 555

red, 555

water, 556

Rqsemary, $53^{\circ}$ oil of, $53^{\circ}$

Rosin, 523
Rottle'ra, 625

Rottlerin, 625

Rubber, India, 667

Para, 667

Rubefacients, 60

Rubidine, 408

Rubidium and ammonium bromide, 257 iodide, 252

Rubijervine, $44^{2}$

Rubinat, 93

Condal, 144

Ru'bus, 602

idx'us, 662

Rusbyine, 46I

Rush's thunderbolt, $\mathrm{SO}_{3}$

Rum, 28x

Ru'mex, 603

Rumicin, 603

Russian liquorice, 6.6

Rutin, $57^{\circ}$

SABI'NA, 577

Saccharated ferrous carbonate, xgo

ferrous iodide, 190

pepsin, 682

Saccharin, 346

Sacchari'num, 346

$\mathrm{Sa}^{\prime}$ ccharum, $6 \mathrm{rg}$

la'ctis, 695

Sacred bark, 495

Saffron, $66_{4}$

meadow, 645

Sage, $66_{3}$

Saigon cinnamon, 539

Sal alembroth, 220

ammoniac, $15 \mathrm{I}$

soda, 139

volatile, 150

Salicin, 474

Salici'num, 474

Salicylic acid, 475

ether of phenol, $48 \mathrm{x}$

Salicylism, $47^{8}$

Saline purgatives, 93

Salipyrin, 322

Salivary glands, drugs act

ing on, 79

Salol, $48 \mathrm{I}$

Salophen, 483

Salt, common, 144 
Salt, Epsom, 162

Glauber's, 142

Rochelle, 142

Salts of tartar, 125

Saltpetre, $1_{31}$

Sa'lvia, $66_{3}$

Salviol, 663

Sambu'cus, $55^{2}$

Sandalwocd, oil of, 576

Sanguina'ria, 450

Sanguinaripe, 450, 512

Sanitas, 518

Santal, $66_{4}$ oil of, 576

Santalal, 576

Santalin, 664

Santalol, 576

Sa'ntalum ru'brum, 664

Santo'nica, 626

Santonin, 626

Santoni'num, 626

$\mathrm{Sa}^{\prime} \mathrm{po}, 607$

anima'lis, 693

mo'llis, 607

vi'ridis, 607

Sapogenin, $44^{8}$

Saponin, 421, 448, 643, 669

Sapotoxin, 669

Sarsapari'lla, $67^{2}$ Indian, 676

Sa'ssafras, 675 medu'la, 675

oil of, 675 pith, 675

Sassy bark, 435

Saunders red, 664

Savanilla rhatany, 598

Savine, 577

Scaling, 16

Scammonin, 501, 502

Scammo'nium, 500

Scammony, 500

Icheele's prussic acid, 134 green, 228

Bchleich's infileration, 414

Schweinfurth's green, 228

Sci'lla, 432

Scillin, 432

Scillipicrin, 432

Gollitoxin, 432

Srerotinic acid, 603

- oparin, 434
Scopa'rius, 434

Scopolamine, $3^{84}$

Scotch paregoric, 352

Scott's ointment, 228

Scullcap, 677

Scutella'ria, 677

Sea onion, 432

Secondary action, 46

Secretion of milk, drugs acting on, 214

Sedatives, gastric, 85

Seidlitz powder, 143 water, 343

Se'nega, $44^{8}$ root, $44^{8}$

Senegin, $44^{8}$

Se'nna, 493

Alexandria, 493

Tinnivelly, 493

India, 493

Sennacrol, 493

Sennapicrin, 485,493

Sensory perves, drugs acting on, 100

Serpenta'ria, 587

Serum, antipneumococcic, 709 antistreptococcic, 708 antivenomous, 709 nutrient, 710 and inoculation, cholera, $7 \circ 9$

plague, 709 typhoid, 7 to

Sesame, oil of, 663

Se'vum, 692

Sherry, 28I

Sialogogues, 80

Siddhi, $3^{8} 7$

Silver, 170

caseinate, 173

citrate, 173

colloidal, 174

cyanide, 171

iodide, $37^{2}$

lactate, 173

nitrate, $x>0$ diluted, $17 x$ moulded, $87 x$

oxide, 175

soluble, 174

Sinalbin, 524

Stnapin, sulphate, 524
Si'napis, 524

a'1ba, 524

ni'gra, 524

Sinigrin, 525

Skin, drugs acting on, 64

Slippery elm bark, 6.6

Smedley's paste, $54^{2}$

Smilacin, $6 \gamma^{2}$

Snakeroot, black, 587

senega, $44^{8}$

Virginia, 587

Soap, 607

bark, 668

constitution of, 23

curd, 693

green, 607

hard, 607

lees, 139

soft, 607

white castile, 607

Socaloin, 498

Socotrine aloes, 497

Soda, 138

baking, 140

caustic, 138

solution of, 138

washing, 139

tartrated, 142

So'dii ace'tas, 147

a'rsenas, 224

be'nzoas, 657

bica'rbonas, 140

bisu'lphis, $x+6$

bo'ras, 273

bro'midum, 253

ca'rbonas, 139

exsicca'tus, 140

chlo'ras, 147

chlo'ridum, 244

e'thylas, $14^{8}$

hypopho'sphis, 239

hyposu'lphis, 146

io'didum, 248

ni'tras, 147

ni'tris, 303

pho'sphas, 142

pyropho'sphas, 148

salicy'las, 475

su'lphas, 142

su'phis, 145

sulphoca'rbolas, 333

valeria'nas, 558

Sodium, $13^{8}$ 
Sodium acetate, 347

arsenate, 224 solution of, 224

benxoate, 657

blcarbonate, 140 troches, 140

bisulphite, 146

borate, 273

bromide, 253

cacodylate, 228

carbonate, 139 dried, $14^{\circ}$

chlorate, 147

chloride, 144

ethylate, 148

glycerophospbate, $24 \mathrm{I}$

bydrate, $13^{8}$ solution, $13^{8}$

hydroxide, 138

hydrophosphite, 239

hyposulphite, $x_{4} 6$

iodide, 248

nitrate, 147

vitrite, 303

oleate, 607

orthophosphate, 142

paraphenolsulphonate,

333

phosphate, 142

pyroborate, 273

pyrophosphate, 148

salicylate, 475

sesquicarbonate, 140

sulphate, 142

sulphite, 145

sulphocarbolate, 333

thiosulphate, 146

valerianate, $55^{8}$

Soft petrola'tum, 278 petroleum ointment, 278 soap, 607 liniment, 607

Solanine, 635

Solidified copaiba, $57^{x}$

Soluble silver, 174

Soporifics, 350

Soja bean, $62 x$

Sorghum, 6xg

Soro-iodol, 342

Spanish flies, 697

Spearmint, $54^{8}$

Spartei'nx su'lphas, 434

Sparteine, 434
Sparteine sulphate, 434

Spermace'ti, $69 \mathrm{x}$

Spige'lia, 628

Spigeline, 628

Spinal cord, drugs acting on, 102, 393

Spindle tree, 513

Sphacelic acid, 637

Sphacelotoxin, 637

Spirit of nitrous ether, 304 mindere'rus, $\times 52$ of wine, 279

Spirits, 3x, 28x

Spi'ritus, 3 I $x^{\prime}$ theris nitro'si, 304

frume'nti, 280

rectifica'tus, $28 \mathrm{r}$

te'nuior, 28x vi'ni ga'llici, 280

Sponging, cold, 118 hot, 120

Spotted hemlock, 405

Sprays, 37 .

Squaw root, 643

Squill, 432 compound syrup of, $23 x$

Squire's chemical food, 206

Squirting cucumber, 507

Standardizing, 16

Staphisa'gria, 629

Staphisagrine, 629

Star anise, 549

Starch, 667

Stavesacre, 629

Stearic acid, 693

Stearin, 666, 693

Stearoptens, 14, 648

Stilli'ngia, 674

Stimulants, cerebral, 105 hepatic, 96

Stinkweed, $3^{82}$

Stomach, drugs acting on, 82

Stomachics, 82

Sto'rax, 567

Storesin, 567

Stout, 28I

Stramo'nium, $3^{82}$

Stramo'nii fo'lia, $3^{8} 3$ se'men, $3^{82}$

Strengthening plaster, 193

Streptococcus antitoxin, 708

Stro'ntii bro'midum, 254 io'didum, 249
Stro'ntii la'ctas, 160

Stro'ntium, 160

bromide, 254

iodide, 249

lactate, 160

Strophanthidin, 429

Strophanthin, 429

Stropha'nthus, 429 kombè, 429

Strychni'na, 393

Strychni'næe su'lphas, 394

Strychnine, 393 sulphate, 394

Styptic collodion, 593

Styptics, 63

Styracin, 567

Styrax, 567

Styrol, $5^{6} 7$

Sublimate, corrosive, 211

Sublimed sulphur, $25^{8}$

Su'cci, 37

Sucrose, 619

Sudorifics, 65

Suet, $6 g_{2}$ mutton, 692

Sugar, $6 \times 9$

cane, 6rg

drugs causing it in urine, 70

of milk, 695

of lead, 166

Sulphanilic acid, 250

Sulphonal, $3^{17}$

Sulphona'lum, 317

Su'lphur, $25^{8}$

flowers of, 258

iodide, $26 \mathrm{x}$

liver of, $26 x$

lo'tum, 259

milk of, 259

ointment, 259

precipitated, 259

precipita'tum, 259

sublima'tnm, $25^{8}$

sublimed, 258

vegetable, 668

washed, 259

Sulphuric acid, 263 aromatic, 264

diluted, 263

ether, 297

Sulphurated lime, 26r

potassa, 26x 
Sulphurated antimony, $23^{\circ}$

Su'lphuris io'didum, 261

Sulphurous acid, 272

Sumach, 60 r

Sumatra camphor, 649

Sumbul, 544

Sumbulic acid, 545

Supposito'ria, 32

Suppositories, 32

Suprarenal extract, 704

Sweet almond, 6r3

flag, $5^{82}$

oil, 605

orange peel, $59^{\circ}$

spirit of nitre, 304

Sydenham's laudanum, 35I

Sylvacrol, 674

Symbols, 17

Sympathetic system, drugs acting on, 112

Synthetic oil of wintergreen, 480

Syrup, 619

Sy'rupi, 32

Syrups, 32

Sy'rupus, 619

gluco'si, 6rg

sci'llx compo'situs, $23 x$

hypophosphi'tum cum

fe'rro, 240

tri'um phospha'tum, 194

TABA'CUM, 408

Tabe'llx, 37

Tablet triturates, 37

Tablets, 37

Tabloids, 37

Tamar indien, 495

Tamarind, 484

Tamar'indus, 484

Tanacetin, 579

Tanace'tum, 579

Tannalbin, 596

Tannic acid, 592, 593, 600, $602,603,604$

Tannigen, 595

Tannin, 593

Tansy, 579

Tar, 520

Tarasp, 144

Taraxacin, 589

Taraxacerin, 589

Ta'raxacum, 588
Tartar emetic, $23 \mathrm{I}$ cream of, 129 salts of, 125

Tartaric acid, 266

Tartrated antimony, 231 iron, 195

Tea, ${ }^{889}$

Teel oil, 663

Teeth, drugs acting $<\mathrm{a}, 79$

Tepid bath, 120

Terebene, ${ }^{6}{ }_{4}$

Terebe'num, 564

Terebi'nthina, 515 canade'nsis, 524

Terpenes, 14

Terpin hydrate, 564

Terpi'ni hy'dras, 564

Terpinene, 544

Tertiary amylic alcohol: 315

Testicular juice, 705

Tetanocannabine, $3^{8} 7$

Tetanus antitoxin, 708

Tetraiodopyrrol, 34I

Tetronal, $3^{18}$

Tetramethylthlonin chloride, 347

Thalli'nx su'lphas, 326

Thalline sulphate, 326

Thebaine, 350,366

Thebolactic acid, 35x

Theine, 388

Theobro'ma, oil of, 666

Theobromi'næ sodio-salicy'las, 392

Theobromine sodio-salicylate, 392

Therapeutics, $3^{8}$ definition of, 9 general, 9 rational, 9 empirical, 9 experimental, so

Therapo-dynamics, 10

Thermogenesis, 7 I

Thermolysis, $7 x$

Thermotaxis, $7 x$

Thiol, 700

Thiersch's solution, 275

Thompson's fluid, 275 solution, 237

Thorn-apple, 382

Thoroughwort, 487
Thus America'num, 524

Thyme, oil of, 574

Thymene, 574

Thymol, 652

Thyroid, dry, $70 x$ solution of, $70 x$

Thyro'ideum si'ccum, 70r

Thyroidin, 70x

Tiglinic acid, 504

Tinctu'ra antiperio'dica, 474 carminati'va, 545

chloro'formi et morphi'næ compo'sita, 29x ergo'tz ammonia'ta, $63^{8}$. laxati'va, 493

o'pii ammonia'ta, 352

sa'ponis vi'ridis, 607

Tinctu'ræ, 32

Tinctures, 32

Tobacco, 408 Indian, 456

Tolu, balsam of, 566

Toluene, ${ }_{566}^{66}$

Tonga, 677

Tongine, 677

Tonic, definition of, 115

Toxicodendric acid, 66x

Toxicology, definition of, ro

Tragacanth, $6 x_{4}$

Tragaca'ntha, 6r4

Tribromomethane, 302

Trichloromethane, 290

Trichlor-tertiary butyl-alcohol, 314

Tri-iodomethane, $33^{8}$

Trimethylethylene, $3^{\circ} 2$

Trinitrin, 308

Trional, 318

Triona'lum, 388

Triple orange flower water, $59 \mathrm{x}$ rose water, 556

Triticin, 633

Tri'ticum, 633

Trituratio'nes, 34

Triturations, 34

Troches, 35

Trochi'sci, 35

Tropine, $3^{8} \mathrm{r}$

Trousseau's pill, 179

Trunks of nerves, drugs acting on, 102

Tully's powder, 354 
Turpentine, $5 \times 5$

Canada, 524

liniment, $5^{16}$

rectified oil of, 516

Turpeth mineral, 254

Typhold serum and inoculation, 7 ro

U'LMUS, $6 \times 6$

Ungue'nta, 35

Ungue'ntum metallo'rum, $17^{8}$

United States Pharmacopoeia, II

Urari, 4 II

Urea, drugs acting on, 98

Urethane, ${ }_{318} 8$

Uretha'num, $3^{1} 8$

Urethra, drugs acting on, 70

Urioary system, drugs acting on, 66

sedatives, 70

antiseptics, 69

Urine, composition altered, 69

increased, 66

diminished, 68

rendered acid, 68

alkaline, 68

aseptic, 69

Urotropin, 345

Urotropi'num, 345

Uterus, drugs acting on, II3

Uterine action, substances which depress, 114

U'va u'rsi, 63 i

$U^{\prime}$ va, 622

$V^{A G U S}$ centre, drugs acting on, $5^{8}$

Valerian, $55^{6}$

Valeria'na, 556

Valerianic acid, 545, 553, 557, 644

Valerol, 369

Vallet's mass, rgo

Vani'lla, 662

Vanillin, $5^{6} 7,662$

Vapo'res, $3^{8}$

Vascular irritants, 6r

Vaseli'num, 38

Vasomotor centre, drugs acting on, 62
Vegetable cathartic pills, Wash, yellow, 218$$
506
$$

drugs, 349

mercury, 5 ro

Datural orders, 692

sulphur, 668

Veratri'na, 444

Veratrine, 444

Verotroidine, $44^{2}$

Vera'trum, vi'ride, $44^{2}$

Vermicides, $5 \mathbf{x}$

Vermifuges, $5^{I}$

Vesicants, 60

Vessels, drugs acting on, Whiskey, 280 59

Viburnin, 644

Vibu'raum o'pulus, 644 prunifo'lium, 643

Vienna caustic, 123 paste, 123

Villacabras, 93,144

Villosin, 603

Vi'na, 35

Vinegars, 19

Vi'num, 280

antimo'nii, 23i

a'lbum, 280 fo'rtius, 281

aura'ntii, 28x

ru'brum, 281

Virginia snake root, $5^{8} 7$

Vitellin, 626

Vite'llus, $69 x$

Vitriol, blue, 179

elixir of, 264

green, 189

oil of, 263

white, 176

Vittel, 156

Volatile liniment, 149

oils, 13,514

oil of betula, 480

oil of mustard, 525

Vulcanite, 667

$W_{\text {Warburg's }}^{\text {AHOO, 5x3 }}$

tincture,

474

Ward's paste, $53^{8}$

Warm baths, rig

Warming plaster, 522

Wash, black, 218

Willow, 474
Washed sulphur, 259

Washing soda, 139

Water, 116 distilled, 116

Waters, 19

Waukesha, 156

Wax, 696 yellow, 696 white, 696

Weights, 17

Weld's syrup of ferric chloride, 199

White corpuscles, drugs act. ing on, 54

arsenic, 223

castile soap, 607

lead, 167

mustard, 524

oak, 592

precipitate, 213 ointment, $2 \times 3$

vitriol, 176

wax, 696

wine, 280

Wild cherry, 462

Wine of iron, bitter, 196 red, 281

Rhine, 283

white, 280

Wines, 35

Wintergreen, oil of, 480 artificial oil of, 480 synthetic oil of, 480

Witchhazel, 6or

Wolfsbane, 437

Wood charcoal, 276

Woody nightshade, 635

Wool-fat, 694 hydrous, 694

Wormseed, American, 628 oil of, 629

Levant, 626

Wormwood, 553

Woorara, 4II

Wourara, 4II

Wourali, 4 II

Wourari, 4II

XANTHOXYLON, 6 7

$X$ Xantho'xylum, 671 


\section{$Y^{\text {ELLOW DOCK, } 60_{3}}$ \\ jasmine, 403}

mercuric oxide, $21 x$ subsulphate, 214

mercurous iodide, 212 parilla, 673

prusslate of potash, ${ }_{34}$ puccoon, 640

wash, 218

wax, 6,6

Ye'rba Sa'nta, 460

Yolk of egg, 691

7 ANZIBAR aloes, 498
Zinc, 175

acetate, 177

bromide, 254

butter of, 175

carbonate, precipitated, 176

chloride, 175 solution, 175

iodide, 249

oleate, 177

oxide, $x 76$

ointment, 177

paraphenol sulphonate, 334

phosphide, 240

sulphate,
Zinc sulphocarbolate, 334 valerianate, $55^{8}$

Zi'nci ace'tas, 177

bro'midum, 254

ca'rbonas precipita'tus, ז76

chlo'ridum, 175

io'didum, 249

o'xidum, 176

pho'sphidum, 240

su'lphas, 176

sulphoca'rbolas, 334

valeria'nas, $55^{8}$

Zi'ncum, 175

Zi'ngiber, $5+3$

Zymine, 683] 


\section{Notices of the Press of Previous American Editions.}

From the Boston Medical and Surgical Journal.-This book is a model of its kind. It gives a concise and thorough review of the subject of materia medica, just such an one as should be of most use to the physician in practical work. The therapeutics is thoroughly modern, and quite as practical as the conditions of a work upon therapeutics pure and simple allow.

From the New York Medical Record.-This well-known and really excellent work has been made more useful by the thorough revision of the latest English edition which the American editor has given us.

From the New York Medical Journal.-The well-deserved success this book has had is not likely to suffer diminution from any fault of the fourth American edition. Such revision as pharmacological progress has demanded has been conscientiously done, and the work is an admirable and useful presentation of a none too easy subject. For these reasons the popularity of the bouk with teachers is not to be wondered at.

From the Medical News.-As a note-book to refresh the memory of the student, and as a quick reference-book for the experienced physician, who merely wishes a concise statement of some certain point, the book is admirable. Evidently no pains have been spared to make it accurate, and its arrangement is scientific. It is the best of its class in the market, and it has been made so in no slight degree by the additions and emendations of the American editor.

From the Vermont Medical Monthly.-As a condensed, yet complete work, containing in small compass the essential details of the whole subject, his book stands without an equal.

From the Southern Practitioner.-A most excellent, comprehensive, complete, yet condensed work, containing in small compass the common and accepted facts essential to the student and medical practitioner. The brevity of expression throughout the work is remarkable, yet it is so practical and clear that the dull and monotonous subject of Materia Medica is really made attractive. The work of the American editor has been well and satisfactorily executed, and the more recent remedial agents of even this go-ahead country have not been overlooked.

From the Texas Courier-Record of Medicine.-By the labor and skill of the editor, the work has been thoroughly adapted for the use of American physicians. The editor's additions and corrections are copious and noteworthy, and change the character of the work more or less completely, so far as American readers are concerned. These additions are distinguished from the text by the use of brackets. The book has thus been transformed into a work of the highest practical value, almost every drug in use, even down to the most recent, having received due attention. The best results of all the more recent developments in Therapeutics have been incorporated, and Dr. Wilcox's labors have been performed with excellent judgment and skill.

From the Cleveland Medical Gazette.-The care with which Dr. Wilcox has performed his work is conspicuous on every page, and it is evident that no recent drug possessing any merit has escaped his eye. We believe, on the whole, this is the best book on Materia Medica and Therapeutics to place in the hands of students, and the practitioner will find it a most satisfactory work for daily use.

From the Medical Age.-It is among the very best of reference textbooks, equally available for the student or the experienced physician, and its conciseness is certainly very far from being the least of its merits. Another notable feature is that the volume abounds in italicized notes calling attention to errors or misunderstandings which experience has shown to be likely to 
arise in a work of this kind; these notes constitute very important danger signals.

From the Denver Medical Times.-This is an excellent manual for students particularly, being arranged systematically and showing, as well as the present state of the science allows, the reasons for the administration of each certain drug. The subject is presented in a terse, rational and perspicuous manner.

From the Pharmaceutical Era.-We have taken considerable pains to compare this book with the Pharmacopoia, for we firmly believe that any work to be used or studied as a text-book by the pharmacist or pharmacy student should conform in its statements to those given by the official standard in definition, description, solubilities, etc. And in this particular the editor is to be congratulated, for he has painstakingly and satisfactorily done his work. For study in the class-room or reference in the store, the book will certainly be appreciated. We know of none better.

From the Virginia Medical Semi-Monthly.-There is a conciseness and clearness about the descriptions of drugs and their therapeutic effects and uses that makes the book easy reading, and thus permits the teachings to be easily remembered. It is in every respect a first-rate text-book.

From the Medical and Surgical Reporter.-It is a well-arranged and accurate text-book, especially in Therapeutics.

From the American Practitioner and News.-It is a live book, condensed it is true, but one in which every line tells. As a reference-book to refresh the memory on all important points it has no superior. It is representative of the highest authority and the most scrupulous care.

From the Buffalo Medical and Surgical Journal.- The fourth edition of this valuable work shows that its worth and excellence has been appreciated by the profession, and in this new edition the confidence put in the former editions may be safely continued. The manner in which the action of various drugs upon the various systems is discussed in the book is an admirable one, thus enabling the busy practitioner to seek the desired information in a few moments.

From the Chicago Medical Recorder.-In point of excellent arrangement, accuracy, conciseness, practical usefulness and completeness, this handbook is without a peer. The author and editor both deserve congratulations.

From the Alumni Journal, New York.-Preparations into which articles or their preparations enter, though not mentioned in the list of preparations named in the Pharmacopoia, are carefully introduced. Secondly, the entire work abounds in italicized notes, calling attention to errors or misunderstandings which experience has shown to be likely to arise in the work of either the pharmacist or the physician, and constitute a very important collection of danger signals. It will thus be seen that the second edition is made even more characteristically a multum in parvo than its predecessor, and that properly used it must prove an invaluable aid to students of pharmacy.

From the Brooklyn Medical Journal. - The editor's practical experience makes his reviewing of the unofficial preparations of especial value. Taken as a whole, the volume seems to us to be most admirably adapted to be both a text-book for students and a reference-book for physicians, a combination which is not found in all the works treating of these subjects.

From the Physician and Surgeon.-Brevity and lucidity of expression characterize the descriptive writing, and careful and scientific arrangement enable the reader to avail himself expeditiously of the resources placed at his command.

\footnotetext{
***The price of this book is $\$ 3.00$ net in cloth binding, or $\$ 3.50$ net in full leather. It may be obtained through any bookseller, or upon receipt of price will be sent, postpaid, to any address, by the publishers.
} 
<smiles>CC(C)COCC(C)(C)C</smiles> 





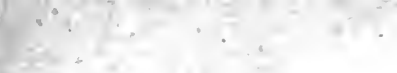


Ho xinus $-\therefore P_{1} y_{6}-t_{0} 30^{\circ}-$

$$
\text { P. } 349-368
$$

Date Due

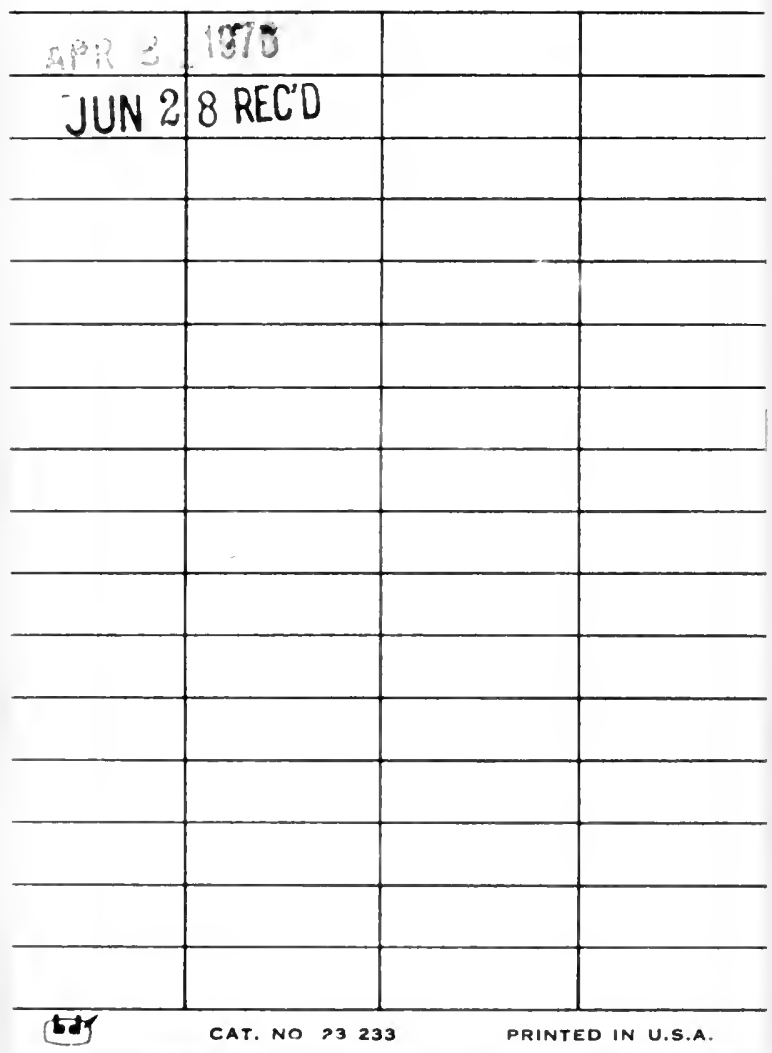


White.

\section{QV4 \\ W589m \\ 1901}

Materia medica, pharmacy, pharmacology and therapeutics

CALIFORNIA COLLEGE OF MEDICINE LIBRARY UNIVERSITY OF CALIFORNIA, IRVINE IRVINE, CALIFORNIA 92664 
